\title{
Early microlithic technologies and behavioural variability in southern Africa and South Asia
}

\author{
Laura Lewis \\ Keble College, University of Oxford
}

Thesis submitted for the degree of Doctor of

Philosophy in Archaeology

Hilary Term 2015 



\title{
Abstract
}

\section{Early microlithic technologies and behavioural variability in southern Africa and South Asia}

\author{
Laura Lewis \\ Keble College, University of Oxford \\ Thesis submitted for the degree of Doctor of Philosophy in Archaeology \\ Hilary Term 2015
}

Microlith production is a distinctive and significant stone tool technology.

However, inter-regional comparative analyses of microlithic industries are rare, and have tended to homogenise these industries by focussing analytical attention on retouched tool typologies alone. This thesis provides the first demonstration and exploration of variability in two of the earliest microlithic industries in the world - the Howiesons Poort of southern Africa and the Late Palaeolithic of South Asia. Analysis of this variation has implications for the long-standing debates concerning modern human behaviour and dispersals.

In order to assess variability in underlying technological processes and manufacturing trajectories, detailed attribute analyses were conducted on lithic assemblages. Metric and qualitative variables were recorded on cores, debitage and tools from three southern African Howiesons Poort sites (Rose Cottage Cave and Umhlatuzana, South Africa, and Ntloana Tsoana, Lesotho) and four South Asian Late Palaeolithic sites (Batadomba-lena and Kitulgala Beli-lena, Sri Lanka, and Patne and Jwalapuram 9, India).

Analysis of the results reveals variability within sites, over time, and between sites and regions, demonstrating that microlith production is not a homogenous technology. Underlying technological processes are shown to differ more between regions than do retouched tool forms. It is argued that this pattern is more parsimoniously explained by independent innovation of microlithic technology situated within local lithic traditions, rather than by cultural diffusion.

Additionally, the exploration of variability in microlithic assemblages highlights the benefits of using a methodological approach to the modern human behaviour debate which focusses on technological variability rather than the presence of particular tool types. It is this behavioural and technological variability that is key to understanding our species. 


\section{Acknowledgements}

I would like to thank my supervisor, Mike Petraglia, for his support, advice, guidance and patience, and for inspiring me to up sticks from Cambridge and move to The Other Place in search of new challenges and ideas. I would also like to extend my appreciation to my Transfer and Confirmation reviewers, Peter Mitchell, Rick Schulting and Nick Barton, for invaluable comments and discussions.

This research was funded by the Arts and Humanities Research Council UK. Additional funding for research trips and conferences was provided by the Wenner-Gren Foundation, the Boise Fund, the Faith Ivens-Franklin Travel Fund, the Meyerstein Fund, the Keble Graduate Study Support Fund and the Keble Association.

I have been very fortunate in having the opportunity to work in some fascinating parts of the world. For granting access to the Sri Lankan materials I am grateful to the Department of Archaeology, Sri Lanka, and especially Senarath Dissanayake, Siran Deraniyagala, Nimal Perera and Oshan Manjula. I would also like to thank Nimal and his family for their hospitality during my stays. For their assistance in accessing the South African materials I thank Lyn Wadley, Marlize Lombard, Karim Sadr, Amanda Esterhuysen, Nicci Sherwood, Carolyn Thorp and Gavin Whitelaw, as well as staff at the Kwa-Natal Museum in Pietermaritzburg and the University of the Witwatersrand in Johannesburg. For the chance to work on the new material from Lesotho I am very thankful to Peter Mitchell, Charlie Arthur, Rachel King, Lebo Mohapi and the rest of the Metolong Dam team. Finally, I am indebted to Chris Clarkson and Hannah James for the use of their data from Jwalapuram 9 and Patne, India.

This thesis owes its existence to two additional sources of inspiration. Firstly, my uncle Ian Morris, who is largely to blame for my addiction to archaeology. I am also indebted to Paul Mellars, who I have to thank for my fascination with all things Palaeolithic. My research has also benefited from conversations with countless other inspirational academics I have met in the field, the conference centre and the pub over the years, particularly those I had the pleasure of meeting through PalQuat.

My most heartfelt thanks go to my parents for their support, encouragement and unfailing good humour; without them none of this would have been possible. I also owe a debt of gratitude to Sol, for keeping me sane throughout. Finally, thanks go to the rest of my friends and family, particularly my fellow cantab expat and thesis buddy Luíseach, the ever-cheerful Margaret, the all-knowing Paddy, and everyone from Team New Barnett House.

All photos are by the author unless otherwise stated. 


\section{Table of contents}

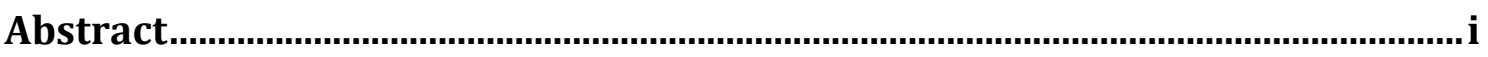

Acknowledgements ......................................................................................... ii

Table of contents ................................................................................................. iii

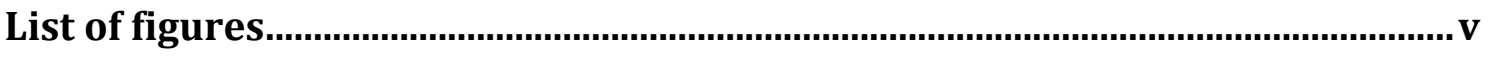

List of tables ........................................................................................................

Chapter 1: Introduction ...............................................................................1

Chapter 2: $\quad$ Microliths and their theoretical context ........................................

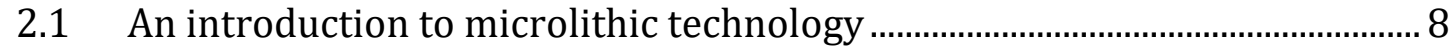

2.2 Microliths and modern human behaviour........................................................34

2.3 Microliths and modern human dispersals .......................................................49

Chapter 3: Study areas and site backgrounds..............................................65

3.1 The Howiesons Poort of Southern Africa...............................................................65

3.1.1 Rose Cottage Cave, South Africa ……………………………………......72

3.1.2 Umhlatuzana, South Africa............................................................................76

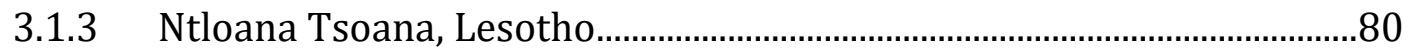

3.2 The Late Palaeolithic of South Asia …………………………………………..85

3.2.1 Batadomba-lena, Sri Lanka ......................................................................91

3.2.2 Kitulgala Beli-lena, Sri Lanka .....................................................................97

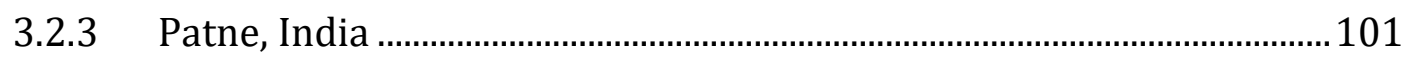

3.2.4 Jwalapuram 9, India .............................................................................

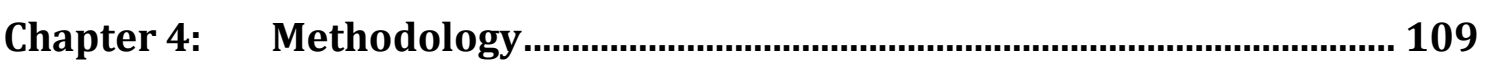

4.1 Data collection and sampling strategies.........................................................109

4.2 Attribute analysis .....................................................................................111

4.3 Statistical analysis................................................................................123

Chapter 5: Howiesons Poort microlithic technology ..................................... 128

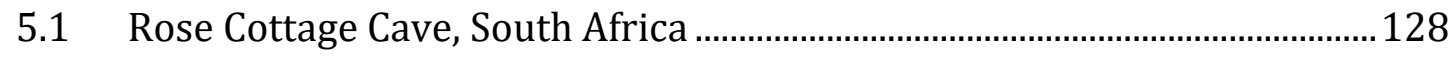

5.2 Umhlatuzana, South Africa ..............................................................................

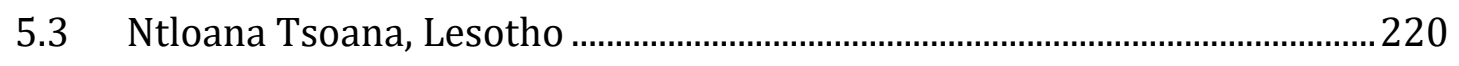

Chapter 6: South Asian Late Palaeolithic microlithic technology .............. 247

6.1 Batadomba-lena, Sri Lanka.......................................................................24

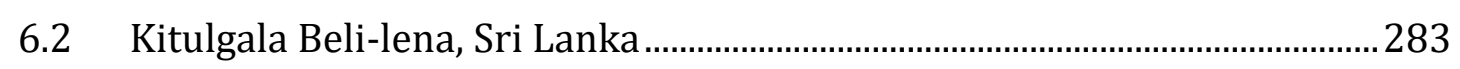

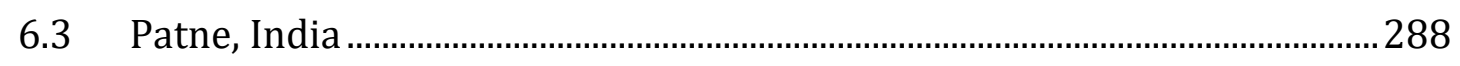

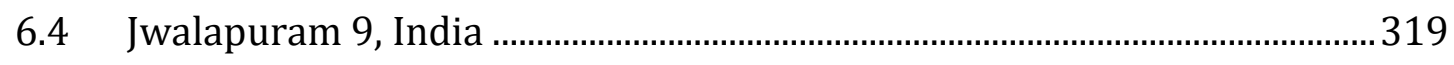


Chapter 7: The southern African and South Asian early microlithic industries compared.

7.1 Comparison between sites and summary of variability in the southern African Howiesons Poort .

7.2 Comparison between sites and summary of variability in the South Asian

Late Palaeolithic 378

7.3 Comparison between early microlithic technology in southern Africa and South Asia. 397

Chapter 8: Conclusions and wider implications....................................... 421

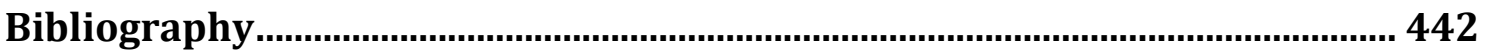

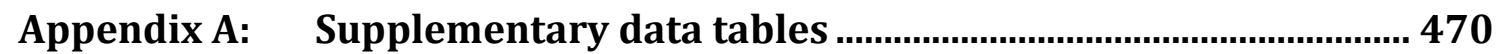

A.1 Rose Cottage Cave, South Africa ………….....................................................470

A.2 Umhlatuzana, South Africa ............................................................................... 480

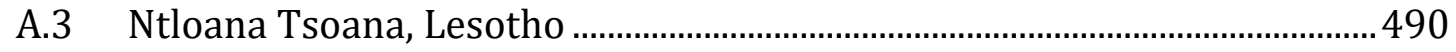

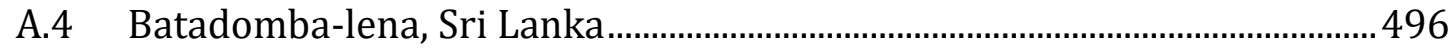

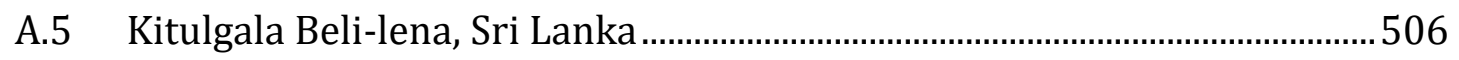

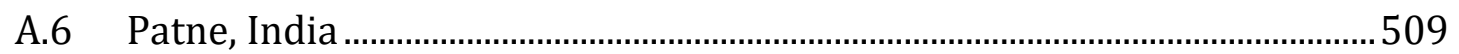

A.7 Jwalapuram 9, India ....................................................................................... 518

Appendix B: Results of statistical tests of temporal changes......................526

B.1 Rose Cottage Cave, South Africa ……………....................................................526

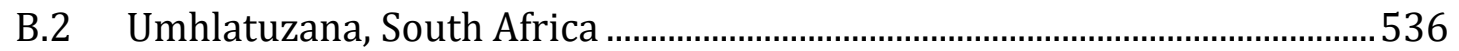

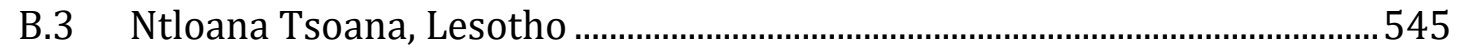

B.4 Batadomba-lena, Sri Lanka........................................................................550

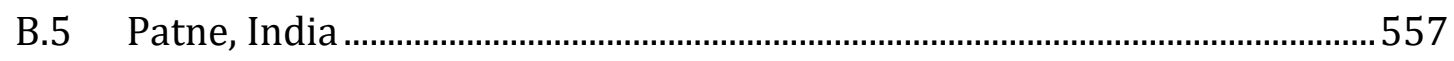

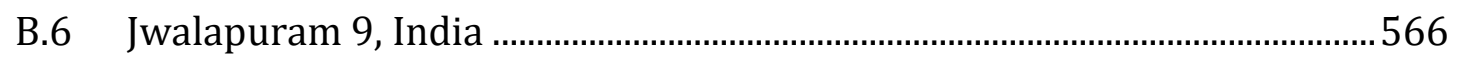

Appendix C: $\quad$ Notes on comparisons with the Indian datasets...................... 574

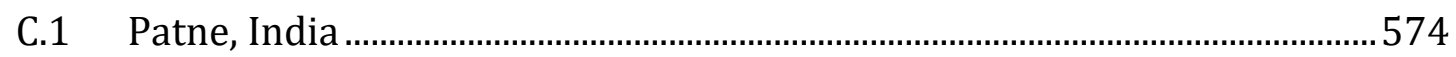

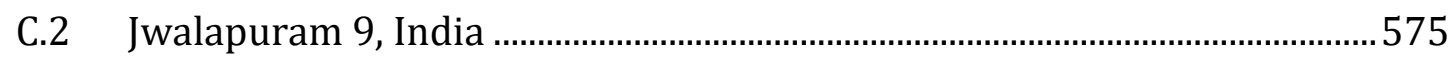




\section{List of figures}

\section{Chapter 2}

Figure 2.1.1: M. Seidler's comparison of minimum microlith lengths at sites in the Vindhya hills, India, and Scunthorpe, England

Figure 2.1.2: Examples of different applications of the microburin technique in Levantine Epipalaeolithic industries

Figure 2.1.3: A microlithic composite tool from the Mesolithic assemblage at Rönneholms Mosse, Sweden

Figure 2.2.1: A linear model (a) and a 'branching tree' model (b) of cognitive evolution

Figure 2.3.1: A basic schematic for distinguishing between homology and analogy in archaeological assemblages on the grounds of parsimony .58

Figure 2.3.2: Illustrations comparing microlith forms from India, France and Syria

Figure 2.3.3: Illustrations comparing microlith forms from Hastings, England and the Vindhya Hills, India.

Figure 2.3.4: Illustrations comparing microlith forms from Tanzania, South Africa, India and Sri Lanka .62

\section{Chapter 3}

Figure 3.1.1: Map of key HP sites .....................................................................................66

Figure 3.1.2: The sandstone ridge in which RCC is located ............................................72

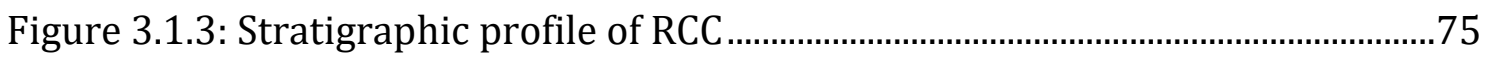

Figure 3.1.4: The gorge containing Umhlatuzana rockshelter ........................................77

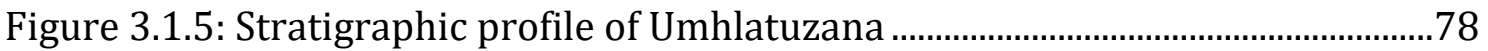

Figure 3.1.6: Diagnostic tool type proportions in lower MSA levels at Umhlatuzana

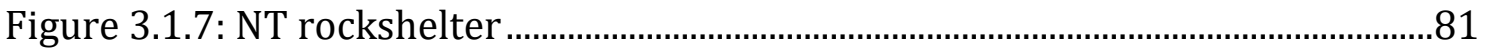

Figure 3.1.8: View from the mouth of NT rockshelter .....................................................82

Figure 3.1.9: Ntloana Tsoana west-facing section ............................................................84

Figure 3.2.1: Map of key Late Palaeolithic sites in India and Sri Lanka........................86

Figure 3.2.2: Excavations at Batadomba-lena in 2005 ...................................................92

Figure 3.2.3: Excavations at Batadomba-lena in 2005...................................................93

Figure 3.2.4: Stratigraphic profile of Batadomba-lena...................................................95

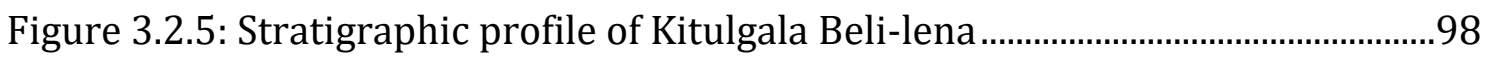

Figure 3.2.6: The alluvial flat in which Patne Area B is situated ..................................102

Figure 3.2.7: Composite stratigraphic profile of Patne ...................................................103

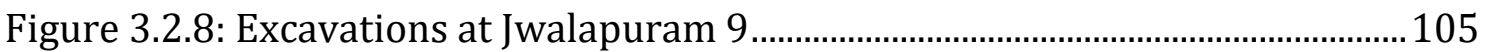


Figure 3.2.9: Stratigraphic profile of Jwalapuram 9 106

\section{Chapter 4}

Figure 4.2.1: Calculation of flake curvature ..................................................................116

Figure 4.2.2: Flake termination types recorded in the attribute analysis ................116

Figure 4.2.3: Features of bipolar percussion...................................................................117

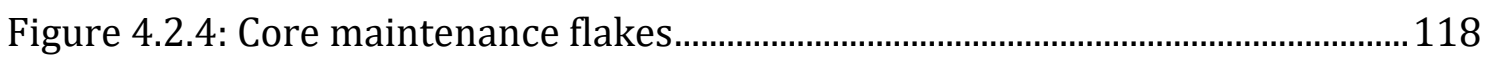

Figure 4.2.5: Retouch outlines recorded in the attribute analysis .............................118

Figure 4.2.6: Retouch types recorded in the attribute analysis..................................119

Figure 4.2.7: Method for measuring Clarkson's Index of Invasiveness ......................120

\section{Chapter 5}

Figure 5.1.1: Raw material proportions of cores at RCC 129

Figure 5.1.2: Examples of recycled lithics from RCC.................................................130

Figure 5.1.3: Proportions of cores manufactured from recycled lithics at RCC .....131

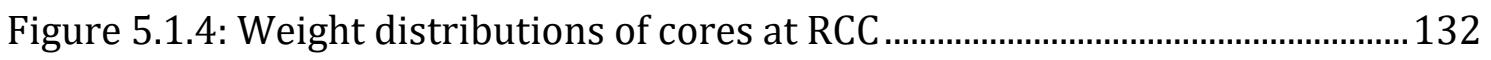

Figure 5.1.5: Proportions of core striking platform types at RCC ................................134

Figure 5.1.6: Average numbers of flake and blade scars on cores at RCC ................134

Figure 5.1.7: Ratios of total removal scar length to core volume at RCC ..................135

Figure 5.1.8: Raw material proportions of debitage at RCC ..........................................137

Figure 5.1.9: Cubic volumes of whole debitage on different raw materials at RCC

Figure 5.1.10: Frequency distributions of whole debitage volumes by raw material

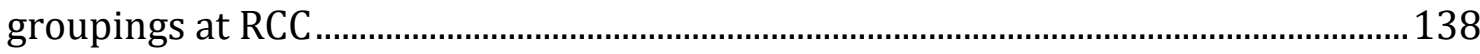

Figure 5.1.11: Examples of non-retouched CCS blades at RCC ....................................139

Figure 5.1.12: Proportions of debitage striking platform types at RCC.....................140

Figure 5.1.13: Proportions of debitage termination types at RCC ................................ 142

Figure 5.1.14: Raw material proportions of tools at RCC.............................................144

Figure 5.1.15: Lengths of whole lithics at RCC .................................................................. 145

Figure 5.1.16: Cubic volumes of lithics on different raw materials at RCC. ..............146

Figure 5.1.17: Examples of HP microliths from RCC .....................................................149

Figure 5.1.18: Raw material proportions of microlith types at RCC ............................149

Figure 5.1.19: Examples of unifacial points from HP layers at RCC ...........................150

Figure 5.1.20: Raw material proportions of non-microlithic tool types at RCC .....151

Figure 5.1.21: Cubic volumes of microlith types at RCC .................................................. 152

Figure 5.1.22: Cubic volumes of non-microlithic tool types at RCC ............................152

Figure 5.1.23: Retouch techniques on microliths at RCC ............................................154 
Figure 5.1.24: Retouch techniques on non-microlithic tools at RCC

Figure 5.1.25: Retouched edge angles of tools according to retouch type at RCC.158

Figure 5.1.26: Frequency distributions of average retouched edge angles on tools

at RCC 159

Figure 5.1.27: Microliths from RCC 160

Figure 5.1.28: Manufacturing trajectories at RCC. 172

Figure 5.2.1: Raw material proportions of cores at Umhlatuzana. 178

Figure 5.2.2: Weight distributions of cores at Umhlatuzana ........................................178

Figure 5.2.3: Proportions of core striking platform types at Umhlatuzana .............180 Figure 5.2.4: Average numbers of flake and blade scars on cores at Umhlatuzana

Figure 5.2.5: Ratios of total removal scar length to core volume at Umhlatuzana 181 Figure 5.2.6: Proportions of debitage categories made on each raw material at Umhlatuzana. 184

Figure 5.2.7: Frequency distributions of lengths of whole hornfels and quartz debitage at Umhlatuzana . 185

Figure 5.2.8: Proportions of debitage striking platform types at Umhlatuzana.....186 Figure 5.2.9: Dorsal scar densities on debitage by raw material at Umhlatuzana 187 Figure 5.2.10: Proportions of debitage termination types at Umhlatuzana .............188

Figure 5.2.11: Examples of 'snapped' hornfels debitage from Umhlatuzana ..........189

Figure 5.2.12: Raw material proportions of tools at Umhlatuzana 192

Figure 5.2.13: Lengths of hornfels and quartz debitage and microliths at Umhlatuzana

Figure 5.2.14: Cubic volumes of lithics on different raw materials at Umhlatuzana 194

Figure 5.2.15: Raw material proportions of microlith types at Umhlatuzana ........196

Figure 5.2.16: Examples of hornfels, siltstone and quartz microliths from Umhlatuzana.

Figure 5.2.17: Raw material proportions of non-microlithic tool types at Umhlatuzana.

Figure 5.2.18: Cubic volumes of microlith types at Umhlatuzana .

Figure 5.2.19: Retouch techniques on microliths at Umhlatuzana 201

Figure 5.2.20: Retouch techniques on non-microlithic tools at Umhlatuzana........202

Figure 5.2.21: Frequency distributions of average retouched edge angles on tools at Umhlatuzana 203

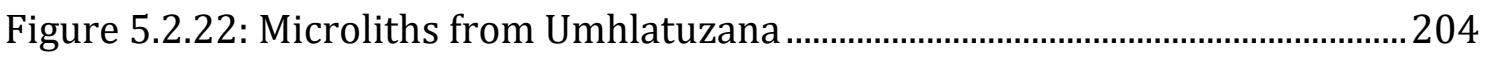

Figure 5.2.23: Manufacturing trajectories at Umhlatuzana .........................................2. 216

Figure 5.3.1: Frequencies of core types made on different raw materials at NT...221

Figure 5.3.2: Weight distributions of cores at NT . 
Figure 5.3.3: Proportions of core striking platforms at NT . .224

Figure 5.3.4: Average numbers of flake and blade scars on cores at NT 224

Figure 5.3.5: Ratios of total removal scar length to core volume at NT 225

Figure 5.3.6: Raw material proportions of debitage at NT 227

Figure 5.3.7: Cubic volumes of whole debitage on different raw materials at NT 227 Figure 5.3.8: Frequency distributions of lengths of whole CCS and quartzite debitage at NT 228

Figure 5.3.9: Proportions of debitage striking platform types at NT 229

Figure 5.3.10: Dorsal scar densities on CCS and quartzite debitage at NT. 231

Figure 5.3.11: Proportions of debitage termination types at NT 232

Figure 5.3.12: Examples of recycled lithics from NT. 233

Figure 5.3.13: Cubic volumes of lithics on different raw materials at NT 235

Figure 5.3.14: Microliths from NT 237

Figure 5.3.15: Microliths from NT 238

Figure 5.3.16: Manufacturing trajectories at NT. 244

\section{Chapter 6}

Figure 6.1.1: Raw material proportions of cores at Batadomba-lena .........................248

Figure 6.1.2: Weight distributions of cores at Batadomba-lena .................................2.249

Figure 6.1.3: Proportions of core striking platform types at Batadomba-lena.......250

Figure 6.1.4: Average numbers of flake and blade scars on cores at Batadomba-lena 251

Figure 6.1.5: Ratios of total removal scar length to core volume at Batadomba-lena 252

Figure 6.1.6: Raw material proportions of debitage at Batadomba-lena 253

Figure 6.1.7: Cubic volumes of whole debitage on different raw materials at Batadomba-lena 255

Figure 6.1.8: Proportions of debitage striking platform types at Batadomba-lena 256

Figure 6.1.9: Proportions of debitage termination types at Batadomba-lena ........258

Figure 6.1.10: Raw material proportions of tools at Batadomba-lena.......................259

Figure 6.1.11: Lengths of whole lithics at Batadomba-lena ..........................................261

Figure 6.1.12: Examples of microliths from Batadomba-lena......................................262

Figure 6.1.13: Dorsal and ventral faces of the Balangoda point from Batadombalena

Figure 6.1.14: Cubic volumes of microlith types at Batadomba-lena..........................265

Figure 6.1.15: Retouch techniques on microliths at Batadomba-lena .......................266

Figure 6.1.16: Retouch techniques on non-microlithic tools at Batadomba-lena .267 
Figure 6.1.17: Frequency distributions of average retouched edge angles on microliths at Batadomba-lena.

Figure 6.1.18: Microliths from Batadomba-lena.

Figure 6.1.19: Frequencies of microlith types in each layer at Batadomba-lena... 278

Figure 6.1.20: Manufacturing trajectories at Batadomba-lena 281

Figure 6.2.1: Double backed pointed microliths from Kitulgala..................................286

Figure 6.2.2: Microliths from Kitulgala 286

Figure 6.3.1: Average numbers of flake and blade scars on different core types at

Patne 290

Figure 6.3.2: Ratios of average removal scar length to core volume on cores at Patne 291

Figure 6.3.3: Proportions of debitage striking platform types at Patne 293

Figure 6.3.4: Proportions of debitage termination types at Patne 295

Figure 6.3.5: Cubic volumes of lithics at Patne..... 297

Figure 6.3.6: Cubic volumes of microlith types at Patne. 299

Figure 6.3.7: Cubic volumes of non-microlithic tool types at Patne 300

Figure 6.3.8: Retouch techniques on microliths at Patne. 301

Figure 6.3.9: Retouch techniques on non-microlithic tools at Patne. 303

Figure 6.3.10: Microliths from Patne 304

Figure 6.3.11: Manufacturing trajectories at Patne. 316

Figure 6.4.1: Raw material proportions of cores at Jwalapuram 321

Figure 6.4.2: Mean cubic volumes of cores on different raw materials at Jwalapuram.

Figure 6.4.3: Proportions of core striking platform types at Jwalapuram 323

Figure 6.4.4: Average numbers of flake and blade scars on cores at Jwalapuram 324 Figure 6.4.5: Frequency distributions of debitage lengths by raw material grouping at Jwalapuram 326

Figure 6.4.6: Proportions of debitage striking platform types at Jwalapuram ........327

Figure 6.4.7: Proportions of debitage termination types at Jwalapuram 329

Figure 6.4.8: Raw material proportions of tools at Jwalapuram 331

Figure 6.4.9: Frequency distributions of tool lengths at Jwalapuram 332

Figure 6.4.10: Cubic volumes of lithics on different raw materials at Jwalapuram

Figure 6.4.11: Raw material proportions of microlith types at Jwalapuram. 335

Figure 6.4.12: Raw material proportions of non-microlithic tool types at Jwalapuram. 336

Figure 6.4.13: Cubic volumes of microlith types at Jwalapuram. 337

Figure 6.4.14: Examples of microliths from Jwalapuram 338 
Figure 6.4.15: Proportions of core types in each context at Jwalapuram

Figure 6.4.16: Proportions of core raw materials in each context at Jwalapuram 343

Figure 6.4.17: Proportions of core striking platform types in each context at Jwalapuram

Figure 6.4.18: Proportions of debitage raw materials in each context at Jwalapuram 348

Figure 6.4.19: Frequencies of microlith types in each layer at Jwalapuram 352

Figure 6.4.20: Manufacturing trajectories at Jwalapuram 354

\section{Chapter 7}

Figure 7.1.1: Scatter graph of bipolar core lengths and widths at HP sites 361

Figure 7.1.2: Cubic volumes of core types at HP sites 362

Figure 7.1.3: Ratios of total removal scar length to core volume on cores at HP sites 363

Figure 7.1.4: Scatter graph of whole debitage lengths and widths at HP sites ........366

Figure 7.1.5: Frequency distributions of whole debitage lengths at HP sites ..........367

Figure 7.1.6: Cubic volumes of whole debitage at HP sites ...........................................368

Figure 7.1.7: Lengths of microlith types at HP sites

Figure 7.2.1: Cubic volumes of cores at Late Palaeolithic sites

Figure 7.2.2: Scatter graph of whole debitage lengths and widths at Late Palaeolithic sites.

Figure 7.2.3: Cubic volumes of whole debitage at Late Palaeolithic sites 384

Figure 7.2.4: Frequency distributions of lengths of whole debitage at Late Palaeolithic sites.. 385

Figure 7.2.5: Lengths of major microlith types at Late Palaeolithic sites 389

Figure 7.3.1: Boxplot of component 1 of the core PCA 399

Figure 7.3.2: Boxplot of component 2 of the core PCA. 400

Figure 7.3.3: Biplot of components 1 and 2 of the core PCA for group centroids of raw material and site groups 401

Figure 7.3.4: Boxplot of component 1 of the debitage PCA .............................................405

Figure 7.3.5: Boxplot of component 2 of the debitage PCA .............................................406

Figure 7.3.6: Biplot of components 1 and 2 of the debitage PCA for group centroids

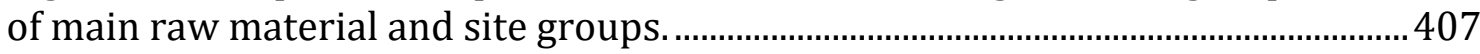

Figure 7.3.7: Boxplot of component 1 of the microlith PCA ..........................................411

Figure 7.3.8: Boxplot of component 2 of the microlith PCA...........................................412

Figure 7.3.9: Biplot of components 1 and 2 of the microlith PCA for group centroids of raw material and site groups 413

Figure 7.3.10: Biplot of functions 1 and 2 of the microlith DFA for group centroids of typological and site groups 


\section{List of tables}

\section{Chapter 2}

Table 2.1.1: Major dated later Pleistocene microlithic industries................................20

Table 2.2.1: Summary of traits associated with modern human behaviour...............38

\section{Chapter 4}

Table 4.2.1: Attributes measured on cores, debitage and tools.

\section{Chapter 5}

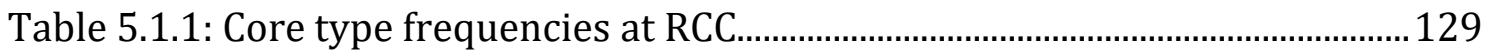

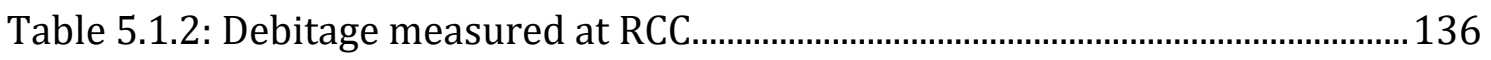

Table 5.1.3: Dorsal scar orientation frequencies on whole debitage at RCC ...........141

Table 5.1.4: Frequencies of core maintenance debitage at RCC ...................................143

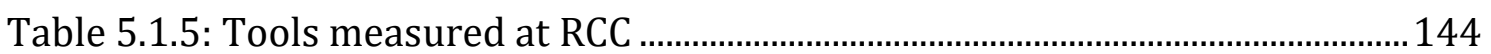

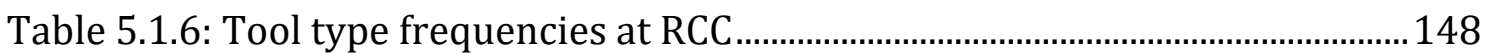

Table 5.1.7: Cores and tools analysed in each layer at RCC............................................. 165

Table 5.1.8: Statistically significant differences between layers in debitage variables at RCC.

Table 5.1.9: Statistically significant differences between layers in microlith variables at RCC.

Table 5.1.10: Statistically significant differences between layers in non-microlithic

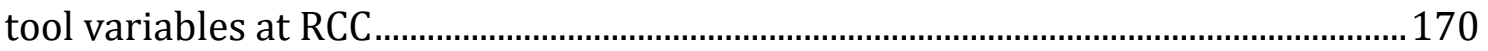

Table 5.2.1: Core type frequencies at Umhlatuzana ........................................................177

Table 5.2.2: Debitage measured at Umhlatuzana.............................................................183

Table 5.2.3: Dorsal scar orientation frequencies on whole debitage at Umhlatuzana

Table 5.2.4: Frequencies of core maintenance debitage at Umhlatuzana ................189

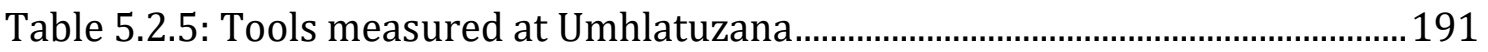

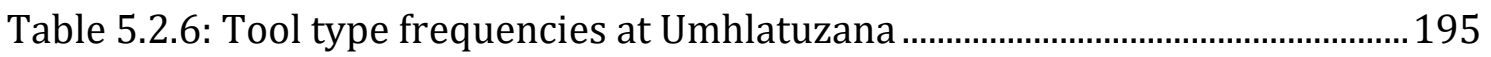

Table 5.2.7: Cores and tools analysed in each layer at Umhlatuzana ..........................208

Table 5.2.8: Statistically significant differences between layers in core variables at

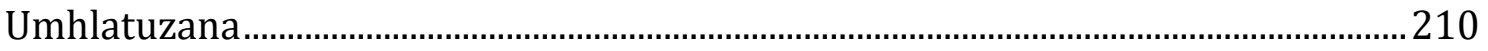

Table 5.2.9: Statistically significant differences between layers in debitage variables at Umhlatuzana

Table 5.2.10: Statistically significant differences between layers in microlith

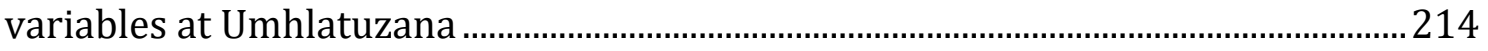

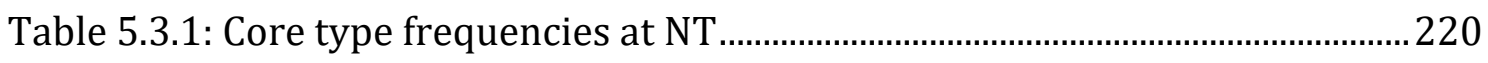


Table 5.3.2: Debitage measured at NT

Table 5.3.3: Dorsal scar orientation frequencies on whole debitage at NT

Table 5.3.4: Frequencies of core maintenance debitage at NT

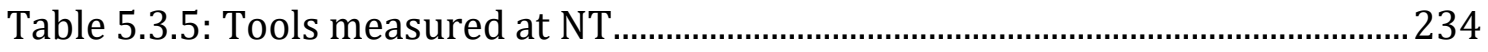

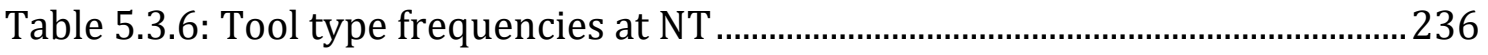

Table 5.3.7: Cores and tools analysed in each layer at NT

Table 5.3.8: Statistically significant differences between layers in core variables at NT

Table 5.3.9: Statistically significant differences between layers in debitage variables at NT

\section{Chapter 6}

Table 6.1.1: Core type frequencies at Batadomba-lena 247

Table 6.1.2: Debitage measured at Batadomba-lena 253

Table 6.1.3: Dorsal scar orientation frequencies on whole debitage at Batadombalena

Table 6.1.4: Frequencies of core maintenance debitage at Batadomba-lena. 258

Table 6.1.5: Tools measured at Batadomba-lena 259

Table 6.1.6: Tool type frequencies at Batadomba-lena. 263

Table 6.1.7: Cores and tools analysed in each layer at Batadomba-lena. 272

Table 6.1.8: Statistically significant differences between layers in debitage variables at Batadomba-lena... 275

Table 6.1.9: Statistically significant differences between layers in microlith variables at Batadomba-lena.. 278

Table 6.2.1: Tools measured at Kitulgala 283

Table 6.2.2: Tool type frequencies at Kitulgala. 285

Table 6.3.1: Core type frequencies at Patne 289

Table 6.3.2: Dorsal scar orientation frequencies on whole debitage at Patne... 295

Table 6.3.3: Tools measured at Patne 296

Table 6.3.4: Tool type frequencies at Patne. 298

Table 6.3.5: Cores and tools analysed in each layer at Patne 305

Table 6.3.6: Statistically significant differences between layers in core variables at Patne.

Table 6.3.7: Statistically significant differences between layers in debitage variables at Patne

Table 6.3.8: Statistically significant differences between layers in microlith variables at Patne

Table 6.3.9: Statistically significant differences between layers in non-microlithic tool variables at Patne 
Table 6.4.1: Core type frequencies at Jwalapuram 320

Table 6.4.2: Debitage measured at Jwalapuram

Table 6.4.3: Dorsal scar orientation frequencies on raw material groupings at Jwalapuram.....

Table 6.4.4: Frequencies of core maintenance debitage at Jwalapuram 330

Table 6.4.5: Tools measured at Jwalapuram 331

Table 6.4.6: Tool type frequencies at Jwalapuram. 335

Table 6.4.7: Cores and tools analysed in each context at Jwalapuram. 340

Table 6.4.8: Statistically significant differences between layers in core variables at Jwalapuram 342

Table 6.4.9: Statistically significant differences between layers in debitage variables at Jwalapuram 348

Table 6.4.10: Statistically significant differences between layers in microlith variables at Jwalapuram

\section{Chapter 7}

Table 7.3.1: Eigenvalues and percentages of variance explained for the core PCA components.

Table 7.3.2: Structure matrix of component scores for the core PCA 398

Table 7.3.3: Variables used in the core DFA, and their loadings on each function 402

Table 7.3.4: Summary of key statistics for the core DFA 402

Table 7.3.5: Results of ANOVA tests of the core DFA functions. 402

Table 7.3.6: Eigenvalues and percentages of variance explained for the debitage PCA.

Table 7.3.7: Structure matrix of component scores for the debitage PCA 404

Table 7.3.8: Variables used in the DFA of debitage, and their loadings on each function. 408

Table 7.3.9: Summary of key statistics for the debitage DFA 408

Table 7.3.10: Results of Kruskal Wallis tests of the debitage DFA functions 408

Table 7.3.11: Eigenvalues and percentages of variance explained for the microlith PCA 410

Table 7.3.12: Structure matrix of component scores for the microlith PCA. 410

Table 7.3.13: Variables used in the DFA of microliths, and their loadings on each function. 414

Table 7.3.14: Summary of key statistics for the microlith DFA 414

Table 7.3.15: Results of Kruskal Wallis tests of the microlith DFA functions 414

\section{Appendix A}

Table A.1.1: Core size measurements at RCC 470 
Table A.1.2: Longest axis length statistics of core types at RCC 470

Table A.1.3: Numbers of striking platforms on core types at RCC.

Table A.1.4: Removal scar numbers and average lengths on core types at RCC.... 471

Table A.1.5: Ratios of removal scar numbers and lengths to core volume at RCC 472

Table A.1.6: Numbers and proportions of broken and whole debitage at RCC ...... 472

Table A.1.7: Size measurements of whole debitage at RCC.

Table A.1.8: Size measurements of whole debitage by raw material groupings at

RCC

Table A.1.9: Proportions of dorsal surface cortex cover on debitage at RCC ..........473

Table A.1.10: Dorsal scar characteristics of debitage types at RCC 474

Table A.1.11: Proportions of recycled lithics in each raw material and debitage

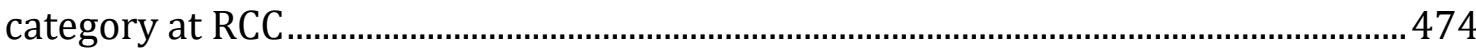

Table A.1.12: Microlith size measurements at RCC .....................................................4 475

Table A.1.13: Non-microlithic tool size measurements at RCC...................................475

Table A.1.14: Length, width and thickness measurements of tool typologies at RCC

Table A.1.15: TCSA measurements of tool typologies at RCC...................................477

Table A.1.16: Microlith haft measurements at RCC.

Table A.1.17: Retouch characteristics of microliths and non-microlithic tools at RCC

Table A.1.18: Retouch lengths, depths and angles of tool typologies at RCC ..........479

Table A.1.19: Clarkson's (2002) IOI measurements of tool typologies at RCC .......480

Table A.2.1: Core size measurements at Umhlatuzana ...................................................480

Table A.2.2: Longest axis length statistics of core types at Umhlatuzana..................481

Table A.2.3: Numbers of striking platforms on core types at Umhlatuzana .............481

Table A.2.4: Removal scar numbers and average lengths on core types at Umhlatuzana

Table A.2.5: Ratios of removal scar numbers and lengths to core volume at Umhlatuzana

Table A.2.6: Numbers and proportions of broken and whole debitage at Umhlatuzana 482

Table A.2.7: Size measurements of whole debitage at Umhlatuzana 483

Table A.2.8: Size statistics of whole debitage in raw material groupings at Umhlatuzana

Table A.2.9: Proportions of dorsal surface cortex cover on debitage at Umhlatuzana 484

Table A.2.10: Dorsal scar characteristics of whole debitage at Umhlatuzana ........ 484

Table A.2.11: Numbers and proportions of snapped debitage at Umhlatuzana .... 485

Table A.2.12: Microlith size measurements at Umhlatuzana 485 
Table A.2.13: Non-microlithic tool size measurements at Umhlatuzana 485

Table A.2.14: Length, width and thickness measurements of tool typologies at Umhlatuzana. 486

Table A.2.15: TCSA measurements of tool typologies at Umhlatuzana 487

Table A.2.16: Size measurements of whole crescent microliths at Umhlatuzana.487

Table A.2.17: Microlith haft measurements at Umhlatuzana 488

Table A.2.18: Retouch characteristics of microliths and non-microlithic tools at Umhlatuzana 488

Table A.2.19: Retouch lengths, depths and angles of tool typologies at Umhlatuzana 489

Table A.2.20: Clarkson's (2002) IOI measurements of tool typologies at Umhlatuzana. 490

Table A.3.1: Core size measurements at NT 490

Table A.3.2: Longest axis length statistics of core types at NT. 491

Table A.3.3: Numbers of striking platforms on core types at NT 491

Table A.3.4: Removal scar numbers and average lengths on core types at NT ......491

Table A.3.5: Ratios of removal scar numbers and lengths to core volume at NT .. 492

Table A.3.6: Numbers and proportions of broken and whole debitage at NT ........492

Table A.3.7: Size measurements of whole debitage at NT 492

Table A.3.8: Proportions of dorsal surface cortex cover on debitage at NT 493

Table A.3.9: Dorsal scar characteristics of whole debitage at NT 493

Table A.3.10: Microlith size measurements at NT 494

Table A.3.11: Non-microlithic tool size measurements at NT 494

Table A.3.12: TCSA measurements of tool typologies at NT. 494

Table A.3.13: Retouch characteristics of microliths and non-microlithic tools at NT 495

Table A.3.14: Retouch lengths, depths and angles of microlithic and non-microlithic tools at NT. 496

Table A.3.15: Clarkson's (2002) IOI measurements of tool types at NT ....................496

Table A.4.1: Core size measurements at Batadomba-lena ................................................496

Table A.4.2: Numbers of striking platforms on core types at Batadomba-lena...... 497

Table A.4.3: Removal scar numbers and average lengths on core types at Batadomba-lena

Table A.4.4: Ratios of removal scar numbers and lengths to core volume at Batadomba-lena 498

Table A.4.5: Numbers and proportions of broken and whole debitage at

Batadomba-lena 498

Table A.4.6: Size measurements of whole debitage at Batadomba-lena 499 
Table A.4.7: Proportions of dorsal surface cortex cover on debitage at Batadombalena.

Table A.4.8: Dorsal scar characteristics of whole debitage at Batadomba-lena.....500

Table A.4.9: Microlith size measurements at Batadomba-lena.. .500

Table A.4.10: Non-microlithic tool size measurements at Batadomba-lena 501

Table A.4.11: Length, width and thickness measurements of microlith typologies at Batadomba-lena 502

Table A.4.12: TCSA measurements of microlith typologies at Batadomba-lena.... 503

Table A.4.13: Microlith haft measurements at Batadomba-lena... 503

Table A.4.14: Retouch characteristics of microliths and non-microlithic tools at Batadomba-lena 504

Table A.4.15: Retouch lengths, depths and angles of tool typologies at Batadombalena. 505

Table A.4.16: Clarkson's (2002) IOI measurements of microlith typologies at Batadomba-lena .506

Table A.5.1: Microlith size measurements at Kitulgala. 506

Table A.5.2: Retouch characteristics of microliths at Kitulgala..... 507

Table A.5.3: Length, width and thickness measurements of tool typologies at Kitulgala

Table A.5.4: TCSA measurements of microlith types at Kitulgala. 508

Table A.5.5: Retouch lengths, depths and angles of tool typologies at Kitulgala ...508 Table A.5.6: Clarkson's (2002) IOI measurements of microlith typologies at Kitulgala. .508

Table A.6.1: Core size measurements at Patne 509

Table A.6.2: Longest axis length statistics of core types at Patne 509

Table A.6.3: Numbers of striking platforms on core types at Patne 509

Table A.6.4: Removal scar numbers and average lengths on core types at Patne.510 Table A.6.5: Ratios of removal scar numbers and lengths to core volume at Patne

Table A.6.6: Numbers and proportions of broken and whole debitage at Patne... 510 Table A.6.7: Size measurements of whole debitage at Patne. 511

Table A.6.8: Proportions of dorsal surface cortex cover on debitage at Patne........511

Table A.6.9: Dorsal scar characteristics of whole debitage at Patne ..........................512

Table A.6.10: Microlith size measurements at Patne....................................................512

Table A.6.11: Non-microlithic tool size measurements at Patne ................................513

Table A.6.12: Length, width and thickness measurements of tool typologies at Patne 514

Table A.6.13: TCSA measurements of tool typologies at Patne 515 
Table A.6.14: Retouch characteristics of microliths and non-microlithic tools at

Patne.

Table A.6.15: Retouch lengths of tool typologies at Patne.

Table A.6.16: Clarkson's (2002) IOI measurements of tool typologies at Patne....517

Table A.7.1: Core size measurements at Jwalapuram....................................................518

Table A.7.2: Longest axis length statistics of core types at Jwalapuram ....................518

Table A.7.3: Removal scar numbers and average lengths on core types at Jwalapuram

Table A.7.4: Ratios of removal scar numbers to core volume at Jwalapuram ........519

Table A.7.5: Mean cortex cover on core types at Jwalapuram ......................................520

Table A.7.6: Size measurements of debitage at Jwalapuram.......................................520

Table A.7.7: Proportions of dorsal surface cortex cover on debitage at Jwalapuram

Table A.7.8: Microlith size measurements at Jwalapuram ............................................ 521

Table A.7.9: Non-microlithic tool size measurements at Jwalapuram ........................522

Table A.7.10: Length, width and thickness measurements of tool typologies at Jwalapuram.

Table A.7.11: Retouched faces of microliths and non-microlithic tools at Jwalapuram

Table A.7.12: Retouch lengths, depths and angles of tool typologies at Jwalapuram

Table A.7.13: Clarkson's (2002) IOI measurements of tool typologies at Jwalapuram.

\section{Appendix B}

Table B.1.1: Results of statistical tests of changes over time in core variables at RCC.

Table B.1.2: Results of statistical tests of changes over time in debitage variables at RCC 530

Table B.1.3: Results of statistical tests of changes over time in microlith variables at RCC .

Table B.1.4: Results of statistical tests of changes over time in non-microlithic tool variables at RCC

Table B.2.1: Results of statistical tests of changes over time in core variables at Umhlatuzana.

Table B.2.2: Results of statistical tests of changes over time in debitage variables at Umhlatuzana.

Table B.2.3: Results of statistical tests of changes over time in microlith variables at Umhlatuzana. 
Table B.3.1: Results of statistical tests of changes over time in core variables at NT

Table B.3.2: Results of statistical tests of changes over time in debitage variables at NT

Table B.4.1: Results of statistical tests of changes over time in debitage variables at Batadomba-lena.

Table B.4.2: Results of statistical tests of changes over time in microlith variables at Batadomba-lena.

Table B.5.1: Results of statistical tests of changes over time in core variables at Patne. 558

Table B.5.2: Results of statistical tests of changes over time in debitage variables at Patne. 560

Table B.5.3: Results of statistical tests of changes over time in microlith variables at Patne. 563

Table B.5.4: Results of statistical tests of changes over time in non-microlithic tool variables at Patne

Table B.6.1: Results of statistical tests of changes over time in core variables at Jwalapuram. 568

Table B.6.2: Results of statistical tests of changes over time in debitage variables at Jwalapuram. 570

Table B.6.3: Results of statistical tests of changes over time in microlith variables at Jwalapuram. 


\section{Chapter 1: Introduction}

Microlith production is a distinctive and significant stone tool technology. However, microlithic industries have tended to be treated as a monolithic entity, without taking into account the variety of different methods of microlith production and use. Variation within and between assemblages can reveal much about past behaviour and technological strategies. This research is the first to demonstrate and explore variability within and between the earliest microlithic industries in southern Africa and South Asia. Analysis of this variation has implications for the long-standing debates concerning modern human behaviour and dispersals.

Microliths are small retouched stone tools, often backed and generally thought to have been hafted as part of composite tools. Despite their diminutive size, they are a substantial topic of discussion in lithic studies. Chapter 2.1 introduces these discussions, including attempts to define microliths and microlithic industries, and related concepts such as geometrics, microblades and backing.

Inter-regional comparative studies of microlithic industries are rare, and tend to contribute towards a homogenising view of microlithic technology. The main examples of this homogenising approach concern the treatment of microliths within the debates concerning modern human behaviour and dispersals. The modern human behaviour debate is one of the keystones of Palaeolithic archaeological enquiry of the last several decades, and concerns the 
characterisation and archaeological correlates of behavioural traits and abilities associated with modern humans. The debate has previously relied upon a trait list of archaeologically visible features derived from the European record (e.g. Mellars 1990, 2006a, Klein 1992, 1995, Bar-Yosef 2002). It is only relatively recently that the earlier development of modern human behaviour in Africa has been fully realised (McBrearty and Brooks 2000), and its exploration in Asia is even more recent (e.g. James and Petraglia 2005). Microlithic technology is a 'trait' that is relevant to both Africa and Asia, but has been misrepresented as homogenous by its inclusion in such lists. This trait list approach has often reduced the investigation of past human behaviour into a simple assessment of the presence or absence of particular traits, which risks ignoring underlying behavioural and technological diversity.

As an alternative to this trait list model, the approach used here draws from the work of researchers who argue that the focus should instead be on behavioural variability (e.g. Shea 2011, Langley et al. 2011). The present work provides a case study for the use of this alternative methodology and analytical focus. It demonstrates the benefits of more thoroughly exploring variability in behaviour, in particular by investigating underlying technological processes in addition to lithic typology. Chapter 2.2 provides an introduction to the modern human behaviour debate, the place of microliths within it, and critiques of the existing framework, before setting out the alternative approach which is used here.

The debate concerning the earliest modern human dispersals out of Africa has similarly amalgamated multiple distinct microlithic industries. The timing and 
routes of Homo sapiens dispersals out of Africa are hotly debated. One particularly contentious area concerns the 'southern arc' of dispersal out of Africa and into Asia and eventually into Australia (e.g. Oppenheimer 2009, Petraglia et al. 2010, Dennell and Petraglia 2012). In particular, the lack of fossil data from the critical time period in the later Pleistocene of South Asia has led some researchers to search for an archaeological signature of early H. sapiens dispersals.

One model for the southern dispersal route concerns microlithic industries. On the basis of artefact illustrations, Mellars (2006b, Mellars et al. 2013) hypothesises that typological similarities between southern African and South Asian Pleistocene microlithic assemblages provide direct evidence of the dispersal of modern humans from the former sub-continent to the latter. This thesis provides the first test of whether microliths can or should be used as a proxy for human dispersals in the Late Pleistocene, and has important implications for the methodology of the debate concerning human dispersals. In contrast to the microlithic dispersal model, the results presented here demonstrate areas of underlying technological diversity that are most parsimoniously explained by independent innovation of microlithic technology, rather than by cultural diffusion. The background to the various models for H. sapiens dispersal is given in Chapter 2.3.

In order to address these issues, the technological systems of two of the earliest microlithic industries in the world are considered - the Howiesons Poort (HP) of southern Africa, and the Late Palaeolithic of South Asia. These industries are cited explicitly as part of the microlithic dispersal model (Mellars 2006b, Mellars et al. 2013). The HP is crucial not only because it represents the earliest industry where 
lithic reduction was organised around the production of microlithic tools, but also because it has been argued to represent the precocious appearance of a suite of 'advanced' or 'modern' human behaviours (e.g. Wurz 1999, Texier et al. 2010, Henshilwood and Dubreuil 2011).

The South Asian Late Palaeolithic also has an important role in our understanding of human behaviour and dispersals. The Indian subcontinent occupies a particularly important position along the 'southern arc' of modern human dispersal. Mitochondrial DNA evidence suggests that c. 45-20 ka the majority of the global human population lived in the region (Atkinson et al. 2008, although cf. the Y chromosome data of Karmin et al. 2015). The industry also provides a valuable counterpoint to the HP. The HP has often been noted for its short-lived appearance, lasting from c. 65-60 ka according to Jacobs et al. (2008) (although this is disputed (Guérin et al. 2013, Tribolo et al. 2013, Feathers 2015, cf. Jacobs and Roberts 2015)). In contrast, microlithic tools are found in South Asia from at least $38 \mathrm{ka}$ and persist in some areas until the adoption of metal technology in the Holocene (Deraniyagala 1992), remaining the dominant tool type through a period of considerable demographic and environmental changes. However, to promote comparability with the hunter-gatherer-produced HP assemblages, this analysis is limited to Pleistocene occupational strata in South Asia. Chapter 3 presents the backgrounds to the HP and the South Asian Late Palaeolithic, and justifies the choice of sites analysed: the HP sites of Rose Cottage Cave and Umhlatuzana in South Africa and Ntloana Tsoana in Lesotho, and the Late Palaeolithic sites of Batadomba-lena and Kitulgala Beli-lena in Sri Lanka, and Patne and Jwalapuram 9 in India. 
In order to investigate variability in lithic technology in detail, quantitative attribute analyses were used. Cores, debitage and retouched tools from the relevant layers of each site were analysed. This is the best way to answer the questions asked by this study as it makes use of all the products of knapping, both intentional and unintentional. Most previous studies of these industries have used only typological analyses, or technological analyses of blanks and retouched tools. However, these generally make up only a small percentage of the assemblages in question. By recording multiple data points for all knapping products it is possible to make much better use of, and gain much more information from, the remaining majority of an assemblage. Attributes were chosen in order to examine raw material preferences, core reduction strategies, blank production and selection, and retouch choices. Chapter 4 describes in detail the methods used, including the attributes measured and the statistical techniques utilised.

The methods described are used to address three specific questions, on different scales of analysis:

1) What degree of variability is evident within individual assemblages, and what can this tell us about the choices made and strategies used by the groups in question?

2) How does microlithic technology change over time, both within individual sites and within regions, and what may have influenced these changes?

3) What differences and similarities are evident between the earliest microlithic industries in southern Africa, India and Sri Lanka, and what factors might explain this? 
The first two questions are answered by the results of analyses of the HP and South Asian Late Palaeolithic assemblages presented in Chapters 5 and 6, respectively. The results highlight multiple aspects of variability, including the existence of distinct manufacturing trajectories for particular tool types and raw materials within sites. They also reveal temporal changes on different scales: gradual and consistent trends, sudden step changes, and layer-specific variation.

Lithic technology is compared between the sites and regions in Chapter 7, including through the use of multivariate statistical techniques. Areas of similarities and differences are highlighted and explored. Variability is found both within regions and between them. Notably, a greater degree of diversity is found to exist in underlying technological processes than in finished tool forms.

Finally, Chapter 8 utilises the contextual information and the results presented in the previous chapters to draw wider conclusions about variability in microlithic technology. Specifically, it answers the three questions posed above and discusses the implications for the debates concerning modern human behaviour and dispersals. It concludes that the most parsimonious explanation for the spatial patterning observed in the lithic assemblages is analogy rather than homology in finished tool forms, implying a model invoking the independent innovation of microliths within local technological traditions. Additionally, it highlights the benefits of using an approach which focuses analytical attention on behavioural variability, in comparison to the currently dominant trait list approach. 
This thesis provides the first technological comparison of early microlithic industries in southern Africa and South Asia. Its original contribution to knowledge is the establishment of the existence of geographic and temporal variability in microlithic technology. The investigation of variability in microlith production is important because it contributes towards our understanding and definitions of microliths. It also has implications for the debates concerning modern human behaviour and dispersals, and specifically for the ways that we attempt to infer these concepts and processes from the archaeological record. Overall, this thesis demonstrates that microlith production is a flexible and diverse technological strategy, which deserves and requires further consideration. 


\section{Chapter 2: Microliths and their theoretical context}

This chapter introduces microlithic technology and the major theoretical debates in which it is invoked. Aspects of microlithic technology (microliths, microlithic industries, microblades, geometrics and backing) are first defined. The history and current state of microlith study are then described, and methods of microlith production and evidence for their utilisation are detailed. The advantages of microlithic tools are discussed, as are explanations for larger-scale processes of 'microlithisation'. These larger-scale explanations are explored further within the two major theoretical debates in which microlithic technology has been cited, concerning modern human behaviour and dispersals.

\subsection{AN INTRODUCTION TO MICROLITHIC TECHNOLOGY}

\section{DEFINITIONS}

Microliths are small retouched stone tools, generally considered to have been hafted as part of composite tools. An accepted standard definition of microliths has failed to materialise despite attempts spanning several decades (e.g. Glover and Lampert 1969, Gould 1969, Clark 1985, Ballin 2000, Elston and Kuhn 2002, Hiscock et al. 2011). Existing definitions often use arbitrary size limits, but can also require the use of microblade blanks, geometric shapes and/or backing retouch (Leplongeon 2014). The collection of definitions in one journal volume alone 
(Elston and Kuhn 2002) reveals the large degree of variation between geographic areas of study.

The most common way to define microlithic tools is through the use of size limits, usually on the length of the tool but sometimes also the width. Two classic and often-used examples are those of Clark and Tixier. Clark (1985) defines microliths as being manufactured on blanks less than $50 \mathrm{~mm}$ long, and preferably less than 30 mm long. Tixier (1963) states that retouched bladelets are those less than $9 \mathrm{~mm}$ wide. However, microlith size definitions vary considerably according to the researcher's area of study. For example, Straus (2002) claims that microliths are less than 2-3 cm long, but often even shorter. This may be true in the European Upper Palaeolithic and Mesolithic which he is discussing, where microlithic tools are often extremely small, but this definition is not necessarily applicable elsewhere. Ambrose (2002), for example, asserts that what he refers to as backed microliths in the early Eburran industry in Kenya have mean lengths of almost 50 $\mathrm{mm}$ and maximum lengths of around $100 \mathrm{~mm}$.

Definitions of microliths are often conflated with other terms, particularly microblades, geometrics and backing. Unretouched microblades (small blades, also referred to as bladelets) suffer from the same problems with size-based definitions as retouched microlithic tools. For example, Tixier (1963) defines microblades as being less than $50 \mathrm{~mm}$ long and $12 \mathrm{~mm}$ wide. Goebel (2002), discussing late Upper Palaeolithic microblades in Siberia, defines them as typically less than $2 \mathrm{~cm}$ long and $1 \mathrm{~cm}$ wide. Ballin (2000) states that microblades are those less than $8 \mathrm{~mm}$ wide. Many other researchers use their own definitions. 
While some researchers use the arbitrary length and/or width measurements proposed by Clark, Tixier and others to define microliths/microblades, others prefer to assess the distribution of tool and debitage lengths within individual assemblages. At the site of Jwalapuram, India, Clarkson et al. (2009) discovered a sharp drop in the distribution of blade lengths at c. $40 \mathrm{~mm}$, and therefore were able to define microblades at the site as being shorter than $40 \mathrm{~mm}$. They also found that virtually all of the microblades defined accordingly were less than $15 \mathrm{~mm}$ wide. Even though these length and width measurements happen to accord with definitions used elsewhere (Clark 1985, Owen 1988, Bar-Yosef and Kuhn 1999, Burdukiewicz 2005), because this definition was based on the actual archaeology at the site rather than an arbitrary size limit it can be used to draw meaningful conclusions about the lithic assemblage at the site.

One particularly relevant study is that conducted by Owen (1988) comparing blade technology between German Upper Palaeolithic and North American Arctic sites. She reports that almost all blades at the Arctic sites were less than $50 \mathrm{~mm}$ long and $20 \mathrm{~mm}$ wide. Larger blades were evident at the German sites, but there was a continuum in blade size with no clear bimodality between smaller and larger blades. She concludes that "No universal criteria were found for distinguishing blades and microblades" (ibid: 192), and recommends that divisions between blades and microblades should be conducted on a site-by-site basis after the entire assemblage has been analysed. Blade and tool lengths should be expected to vary considerably between sites, as they are affected by a wide variety of factors including raw material size, availability and knapping properties, hafting considerations, and stylistic choices (Ambrose 2002). It is therefore preferable to 
assess debitage and tool sizes on a site-by-site basis, for example by looking for bimodality in length and width measurements, in order to construct more meaningful definitions of microblades and microliths at the site in question.

Another problem with the definition of the term 'microblade' is that some researchers use it interchangeably with the term 'microlith', according to differing definitions between regions. For example, Mishra et al. (2013) refer to small blades as microliths. Similarly, Basak et al. (2014: 1167) state that "Microlithic industries are defined by systematic microblade and/or backed artefact production associated with modern humans", i.e. that microblade production is sufficient for an assemblage's designation as part of a microlithic industry.

Aside from confusion caused by the conflation of terms for (retouched) microliths and (unretouched) microblades, an additional problem is that using the terms interchangeably implies that all microliths were manufactured on microblades. Many microliths were in fact manufactured on flake blanks, and many microlith assemblages are predominantly flake-based. For example, Ambrose (2002) notes that many early Later Stone Age (LSA) microlithic assemblages in sub-Saharan Africa are dominated by small flakes, with blades and microblades relatively uncommon. He also explains that blades, backed microliths and microlithisation were separate phenomena in the region. Hiscock (2002) similarly demonstrates that blade production and 'backed artefact' production are separate phenomena in Australia. In order to avoid confusion, the term microlith should be reserved for small retouched tools made on either flakes or blades, while microblades are small non-retouched blades. 
An additional term with which 'microlith' is often conflated is that of 'geometrics'. Microlithic tools in many industries around the world have been found to have been retouched into geometric shapes such as crescents, triangles, trapezoids and rectangles. As a result, it is often assumed that this is true of all microliths, and that this is a defining feature of the tool type. Some researchers even prefer to use geometric microliths as their definitional basis, for example describing the microlithic southern African HP industry as being characterised by 'crescentic' stone tools or segments (e.g. Mellars 2006b, Wadley and Mohapi 2008, Mellars et al. 2013).

Microlith morphologies are not always evident from the published literature, where illustrations of classic and iconic geometric forms tend to dominate. However, when typological counts are available it is sometimes possible to determine the proportions of geometric and non-geometric forms. For example, geometric microliths are often considered a defining feature of the southern African HP. Yet on closer inspection it appears that non-geometric microliths are frequently the dominant morphology. In South Africa, at the site of Klasies River only 143 out of 421 backed tools (34\%) in HP levels are geometric (Villa et al. 2010, Table 4). At Rose Cottage Cave, only 28 out of 292 backed tools (10\%) are segments and trapezes (Harper 1997, Table 1). At Diepkloof the proportions of backed geometric microliths in the early, middle and late HP are $15 \%, 6 \%$ and 45\%, respectively (Porraz et al. 2013). Overall, geometric microliths are best seen as a sub-set of microlithic tools, rather than an equivalent term. 
One final aspect of microlithic technology which requires definition is the use of backing retouch. Backing, sometimes also referred to as blunting, involves the retouch of a tool edge to a near $-90^{\circ}$ angle so as to create a flat edge or back to the tool, rather than a cutting edge. In some cases, removals are made from either the dorsal or the ventral face. Alternatively, one particular type of backing retouch, sometimes referred to as edge or bipolar backing, involves removals being made from both faces of the tool using bipolar percussion conducted on an anvil (Hiscock 2002).

Backing is very common in microlith assemblages. However, again there are terminological differences between archaeologists working in different regions, with some researchers considering backing retouch an inherent part of the definition of microlithic tools. Hiscock (2002, Hiscock et al. 2011) argues that the use of size restrictions alone does not successfully categorise a specific implement type, and that instead of looking for 'microliths' we should be looking for 'backed artefacts', with the focus on the presence of backing rather than small sizes.

However, not all microliths are backed. For example, Belfer-Cohen and GoringMorris (2002) note that semi-abrupt/backing retouch is found primarily on larger points in the Levantine Upper Palaeolithic, while smaller points (of microlithic dimensions) are minimally retouched, often using fine or nibbling retouch. Backing retouch does not occur on microliths in the region until the Epipalaeolithic (from c. 22-20 ka). Additionally, backing is often uncommon in the microlithic industries that proliferated in the early LSA of southern Africa, such as the Robberg (Ambrose 
2002). Again, although backing retouch was frequently applied to microlithic tools, it should not be used as an inherent part of the definition of microliths.

Finally, the issue of what defines a microlithic industry - as distinct from what defines a microlithic tool - must be considered. Small tools (those fitting within the most frequently used size definitions, as discussed above) are found in a number of Lower and Middle Palaeolithic contexts in Europe, Asia and Africa (Barham 2002, Burdukiewicz and Ronen 2003a, Burdukiewicz 2005), and date as far back as c. 1 Ma at the site of Bizat Ruhama in Israel (Zaidner et al. 2003). However, a number of important differences separate these industries from the microlithic industries seen in the later Pleistocene. Firstly, at many of these early sites it is clear that small tools were manufactured from small pebbles of raw material, and so it is not possible to demonstrate whether small tools were produced out of choice or necessity (Burdukiewicz and Ronen 2003b). Secondly, these small tools tend to grade into the larger tools at the sites, and are probably best viewed as the smaller end of the size spectrum of certain tool types; for example, many small tools in Lower Palaeolithic assemblages are small versions of larger denticulated and notched forms (ibid.). And thirdly, these small tools generally only make up tiny proportions of the formal tool assemblages at the sites in question, in contrast to later Pleistocene sites where they usually dominate. A handful of small tools does not a microlithic industry make.

The main reason that the Lower and Middle Palaeolithic industries discussed by Burdukiewicz and Ronen (2003a) do not constitute microlithic industries is that these small tools did not constitute the primary outcome of lithic manufacturing. 
As Kuhn (2002: 84) states, "[t]he simple presence of microlithic artifacts does not define a microlithic assemblage". Many non-microlithic assemblages contain small tools, and many (if not all) microlithic assemblages also contain larger tools.

Kuhn and Elston (2002) constructed a list of five characteristics of late Pleistocene microlithic assemblages which remain a useful starting point in defining microlithic industries: the proliferation of microblade technology, the use of backing retouch, standardisation of tool size and/or shape, the numerical dominance of microlithic tools, and the use of microliths in composite tools (where this is discernible). However, one of the best definitions of microlithic assemblages remains the classic description by J.D. Clark:

"Microlithic industries are those in which the mode for the flaked stone tool equipment is based on the manufacture of bladelets and flakelets, the maximum length of which does not exceed $50 \mathrm{~mm}$ and the great majority are under $30 \mathrm{~mm}$ long. Such industries are characterised by varying proportions of backed tools and small convex scrapers, together with a range of other artefact forms, some of which may be macrolithic in size" (Clark 1985: 95)

Microlithic industries are lithic industries in which production is organised around the manufacture of small flakes and blades suitable for retouch into microlithic tools. It must be demonstrated that the creation of small tools was a deliberate and important manufacturing trajectory within the assemblage. This requires more than a simple statement concerning the numerical dominance of microlithic tools; indeed, microlithic tools may not constitute the majority of the formal tool assemblages of microlithic industries, as a result of various factors contributing towards differential use and transportation of different tool types. Rather, it is necessary to conduct detailed technological assessments of entire assemblages to explore whether microlithic tools were the intended outcome. 
Once this has been discerned, microliths are then able to be defined on a site-bysite basis. These definitions can then be compared between sites and regions, which is a much more informative mode of assessment. If the same arbitrary definition is used everywhere it is not being informed by the actual archaeology. Tool sizes vary between sites for a multitude of reasons, and microliths are no exception. Microblade blanks, geometric shapes and backing retouch are common but not essential. In fact, geographic and temporal variation in the degree to which these three 'optional extras' were used is interesting and worthy of study in itself.

The definitions used in the current study are designed to be general and inclusive, making use of tool size profiles from individual sites rather than imposing arbitrary universal size limits. Microlithic industries are defined here as those where the overall lithic reduction process can be shown to be oriented around the production of blanks subsequently retouched into microlithic tools, as opposed to assemblages that happen to include a handful of tools that are smaller than arbitrary size limits.

Microlithic tools must be viewed and defined in the context of the lithic assemblage as a whole. Given that what exactly a microlith is does vary between sites, it could be argued that this is not in fact a useful term. However, what these different microliths do have in common is their probable use in composite tools, and this is an important innovation in lithic technology that requires specific terminology to discuss it. The definitions of microliths and microlithic industries (as with any other terminology used by lithic researchers) inherently involve subjective 
elements, but they are still useful as long as the researcher is able to explain why they have designated tools or industries as such.

\section{THE HISTORY AND CURRENT STATE OF MICROLITH RESEARCH}

Microliths were among the first Palaeolithic artefacts to attract the attention of amateur archaeologists in the late $19^{\text {th }}$ and early $20^{\text {th }}$ centuries. The first documented microliths, or 'pygmies' (or pygmy flints or implements) as they were often referred to at the time (e.g. Gatty 1895, Smith 1906, Hunt 1911, Hartley 1913, 1914, Wayland 1919), were discovered by A.C. Carleyle in the Vindhya Hills of central India in 1867 (Carleyle's notes quoted in Smith 1906). Much early interest in 'pygmies' came from French anthropologists, for example by M.G. de Mortillet in the 1880s (Brown 1889).

Interest from British anthropologists and naturalists came later, with the first major discovery of 'pygmies' in England being those excavated by W.J.L. Abbot near Hastings (Abbott 1896), which he considered to be very similar to those described by Brown (1889) elsewhere in Europe, Asia and Africa. 'Pygmies' are not mentioned in the first edition of Sir John Evans' classic compendium The Ancient Stone Implements, Weapons and Ornaments of Great Britain published in 1872 (Evans 1872). However, by the time of the second edition published in 1897 there is discussion of "a series of minute tools of flint" (Evans 1897: 324) found in England, India, Egypt, France and Belgium. By 1906 Palestine, Portugal, Spain, north Africa and the Crimean and Sinai peninsulas had been added to the list of 
'pygmy' discovery locations (Smith 1906), as had Australia and Uganda by 1914 (Hartley 1914).

Early descriptions of 'pygmies' were remarkably similar to those often encountered today. Brown (1889: 134-135) states that they rarely exceed 5/8 of an inch $(16 \mathrm{~mm})$ in length, and describes both geometric and non-geometric forms, noting that shapes include "crescent, quadrilateral, scalene and obtusely triangular, while others may be described as thin, acute knifelets, very small spokeshaves, \&c.". Gatty (1895: 36) describes 'pygmies' as “flint implements carefully and perfectly made, no bigger than half an inch, and many much less". He also identified the use of pressure flaking on 'pygmy' tools, and correctly surmised that they would have been hafted. Hartley (1914) began a typological classification scheme for microliths, dividing his 'pygmies' into four main types: crescentic, triangular, pointed and rhomboidal.

Most notably, Smith (Smith 1906: 188) includes in his paper what could be considered to be the first attribute analysis of microliths, conducted by M. Seidler from the Nantes Museum, France. The analysis compares the minimum lengths of different microlith forms (crescents, scalene triangles, rounded and pointed, and rhomboidal and trapezoidal) between two groups of sites (in the Vindhya hills in India and in Scunthorpe in England) (Figure 2.1.1). He concludes that both localities contain the same microlith forms, and that the English artefacts are smaller. 
Figure 2.1.1: M. Seidler's comparison of minimum microlith lengths at sites in the Vindhya hills, India, and Scunthorpe, England (Smith 1906: 188)

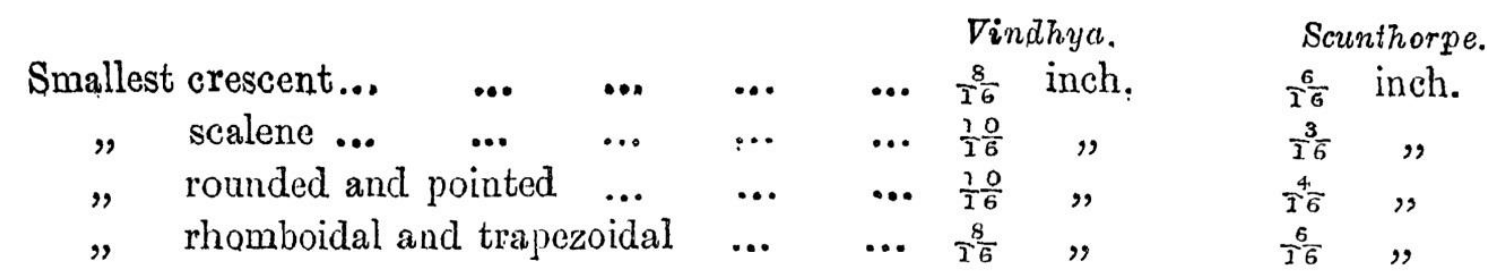

Today, with the benefit of 150 years of survey and excavation, microlithic assemblages have been found across much of the world. Microlithic industries are particularly common in the Holocene, but a trend towards more frequent microlith production has also been noted in the late Pleistocene (Kuhn and Elston 2002). Table 2.1.1 presents the details of some of the major documented and dated late Pleistocene microlithic industries.

\begin{tabular}{|c|c|c|c|c|}
\hline Industry & Region & Dates & Key features & Key references \\
\hline $\begin{array}{l}\text { Howiesons } \\
\text { Poort }\end{array}$ & $\begin{array}{l}\text { Southern } \\
\text { Africa }\end{array}$ & ?65-60 ka & $\begin{array}{l}\text { Relatively large backed } \\
\text { geometric microlith } \\
\text { forms }\end{array}$ & $\begin{array}{l}\text { (Jacobs et al. } \\
\text { 2008, Lombard et } \\
\text { al. 2012, } \\
\text { Henshilwood } \\
\text { 2012, Cochrane et } \\
\text { al. 2013) }\end{array}$ \\
\hline $\begin{array}{l}\text { Transitional } \\
\text { Middle-Later } \\
\text { Stone Age/ } \\
\text { Early Later } \\
\text { Stone Age }\end{array}$ & East Africa & $? 50-35 \mathrm{ka}$ & $\begin{array}{l}\text { Local variants of } \\
\text { industries with backed } \\
\text { microlith components }\end{array}$ & $\begin{array}{l}\text { (Ambrose 1998, } \\
\text { 2002, Diez-Martin } \\
\text { et al. 2009, Brandt } \\
\text { et al. 2012, } \\
\text { Gliganic et al. } \\
\text { 2012, Groucutt et } \\
\text { al. in review) }\end{array}$ \\
\hline $\begin{array}{l}\text { Proto- } \\
\text { Aurignacian }\end{array}$ & $\begin{array}{l}\text { Southern } \\
\text { Europe }\end{array}$ & $45-40 \mathrm{ka}$ & $\begin{array}{l}\text { Non-geometric } \\
\text { microliths } \\
\text { manufactured on } \\
\text { microblades, generally } \\
\text { without backing } \\
\text { retouch }\end{array}$ & $\begin{array}{l}\text { Kuhn 2002, } \\
\text { Straus 2002, } \\
\text { Mellars 2006c) }\end{array}$ \\
\hline $\begin{array}{l}\text { Transitional } \\
\text { Middle-Later } \\
\text { Stone Age/ } \\
\text { Early Later } \\
\text { Stone Age }\end{array}$ & $\begin{array}{l}\text { Southern } \\
\text { Africa }\end{array}$ & $44-18 \mathrm{ka}$ & $\begin{array}{l}\text { Various localised } \\
\text { microlithic industries } \\
\text { including relatively } \\
\text { unstandardized } \\
\text { microliths }\end{array}$ & $\begin{array}{l}\text { (Mitchell 1988a, } \\
\text { Mitchell 1988b, } \\
\text { Wadley 1993, } \\
\text { Lombard et al. } \\
\text { 2012, Villa et al. } \\
\text { 2012) }\end{array}$ \\
\hline
\end{tabular}




\begin{tabular}{|c|c|c|c|c|}
\hline $\begin{array}{l}\text { Upper } \\
\text { Palaeolithic } \\
\text { and Epi- } \\
\text { palaeolithic }\end{array}$ & Levant & $\begin{array}{l}\text { From } 40 \\
\text { ka }\end{array}$ & $\begin{array}{l}\text { Various microblade- } \\
\text { based microlithic } \\
\text { industries, including the } \\
\text { Ahmarian (straight- } \\
\text { edged microblades with } \\
\text { minimal retouch) and } \\
\text { the Kebaran (backed } \\
\text { geometrics) }\end{array}$ & $\begin{array}{l}\text { (Neeley 2002, } \\
\text { Belfer-Cohen and } \\
\text { Goring-Morris } \\
\text { 2002, } \\
\text { Yaroshevich et al. } \\
\text { 2010) }\end{array}$ \\
\hline $\begin{array}{l}\text { Late } \\
\text { Palaeolithic }\end{array}$ & South Asia & $\begin{array}{l}\text { From } 38 \\
\text { ka }\end{array}$ & $\begin{array}{l}\text { Microlithic component } \\
\text { to a mixed microlith and } \\
\text { blade techno-complex, } \\
\text { with much geographic } \\
\text { variation }\end{array}$ & $\begin{array}{l}\text { (Deraniyagala } \\
\text { 1992, James and } \\
\text { Petraglia 2005, } \\
\text { Clarkson et al. } \\
\text { 2009, Petraglia et } \\
\text { al. 2009a, Perera } \\
\text { 2010, Perera et al. } \\
\text { 2011) }\end{array}$ \\
\hline $\begin{array}{l}\text { Late Upper } \\
\text { Palaeolithic }\end{array}$ & East Asia & $\begin{array}{l}\text { From ?25 } \\
\text { ka }\end{array}$ & $\begin{array}{l}\text { Microlithic tools } \\
\text { manufactured on } \\
\text { microblades, including } \\
\text { small tanged points, } \\
\text { occur at some sites } \\
\text { within the Late } \\
\text { Palaeolithic microblade } \\
\text { techno-complex, } \\
\text { alongside the use of } \\
\text { unretouched } \\
\text { microblades }\end{array}$ & $\begin{array}{l}\text { (Bleed 2002, } \\
\text { Seong 2008, 2009, } \\
\text { Bae 2010, Bae and } \\
\text { Bae 2012, Gao } \\
\text { and Dennell } \\
\text { 2014) }\end{array}$ \\
\hline $\begin{array}{l}\text { Late } \\
\text { Palaeolithic }\end{array}$ & North Africa & $\begin{array}{l}\text { From } 22 \\
\text { ka }\end{array}$ & $\begin{array}{l}\text { Backed microliths } \\
\text { manufactured on } \\
\text { microblades dominate } \\
\text { the region }\end{array}$ & (Close 2002) \\
\hline $\begin{array}{l}\text { Epipalaeolithic } \\
\text { and Mesolithic }\end{array}$ & Europe & $\begin{array}{l}\text { From } 20 \\
\text { ka }\end{array}$ & $\begin{array}{l}\text { Widespread } \\
\text { proliferation of } \\
\text { industries containing } \\
\text { microlithic tools, often } \\
\text { very small, with a } \\
\text { transition to backed } \\
\text { retouch during the } \\
\text { Mesolithic }\end{array}$ & $\begin{array}{l}\text { (Straus 2002, } \\
\text { Bailey and Spikins } \\
\text { 2008) }\end{array}$ \\
\hline $\begin{array}{l}\text { Late Upper } \\
\text { Palaeolithic }\end{array}$ & $\begin{array}{l}\text { Northern } \\
\text { Asia and } \\
\text { Beringia }\end{array}$ & $\begin{array}{l}\text { From } 18 \\
\text { ka }\end{array}$ & $\begin{array}{l}\text { Non-geometric } \\
\text { microliths } \\
\text { manufactured on } \\
\text { microblades, alongside } \\
\text { the use of unretouched } \\
\text { microblades }\end{array}$ & $\begin{array}{l}\text { (Elston and } \\
\text { Brantingham } \\
\text { 2002, Goebel } \\
\text { 2002, Yesner and } \\
\text { Pearson 2002) }\end{array}$ \\
\hline Robberg & $\begin{array}{l}\text { Southern } \\
\text { Africa }\end{array}$ & $18-12 \mathrm{ka}$ & $\begin{array}{l}\text { Systematic microblade } \\
\text { production, with } \\
\text { microliths but also } \\
\text { utilised unretouched } \\
\text { microblades }\end{array}$ & $\begin{array}{l}\text { (Mitchell 1988a, } \\
\text { Mitchell 1988b, } \\
\text { Wadley 1993, } \\
\text { Lombard et al. } \\
\text { 2012) }\end{array}$ \\
\hline
\end{tabular}

Table 2.1.1: Major dated later Pleistocene microlithic industries. 'From' designates industries which continue into the Holocene. 


\section{MICROLITH PRODUCTION AND USE}

From the time of their earliest discovery it was recognised that the small size of microliths meant that they must were most likely utilised as part of composite tools (e.g. Brown 1889, Smith 1906, Hartley 1914). Much has been made of the innovation of composite tools, particularly with regards to the development of behavioural and cognitive complexity in our species (e.g. Wadley et al. 2009, Lombard and Parsons 2011, Lombard 2012, Lombard and Haidle 2012). This includes advances such as planning ahead, multi-tasking, abstract thought, conceptualisation of complex action sequences, and mental flexibility.

The first composite tools are very important conceptually, as their production implicates the idea of manufacturing an object that cannot be utilised unless it is combined with other objects, i.e. the viewing of objects as components for the first time. Although hafting using simple adhesives is evident from at least as early as the Middle Palaeolithic (Mazza et al. 2006), complex adhesives and processes of composite tool manufacture are not documented until the microlithic industries of the later Pleistocene (discussed further below).

With the benefit of residue, use-wear and macro-fracture analysis, as well as experimental reconstructions, it has been possible to gain a deeper understanding of the manufacturing methods and functions of composite tools. Analogies with modern hunter-gatherer populations who make use of composite tools have also been made in this regard (e.g. Deraniyagala 1992, d'Errico et al. 2012). However, ethnographic analogies should be treated with extreme caution, especially when dealing with the very distant past. The thorough treatment of these caveats is an 
absolute necessity when drawing on ethnographic comparisons, and lies beyond the scope of this thesis. Therefore only archaeological and experimental data-sets and analyses are discussed here.

Compared to some of the long and complex production sequences documented for other tool types, for example the use-lives of bifacial tools (e.g. Dibble 1995, Odell 2004, Andrefsky 2005, Bar-Yosef and Van Peer 2009), the production of the lithic components to microlithic composite tools often follow relatively simple procedures. Many microlithic industries involve the systematic production of small blanks from small blade cores (Wadley 1993, Neeley 2002, Belfer-Cohen and Goring-Morris 2002, Bleed 2002, Elston and Brantingham 2002, Goebel 2002, Yesner and Pearson 2002, Mackay 2008). These blanks were then often retouched along a lateral margin using backing (e.g. Wurz 1999, Close 2002) or other (e.g. Kuhn 2002, Straus 2002) retouch. As discussed above, small flakes were also used as blanks in a number of assemblages (e.g. Ambrose 2002). Various knapping techniques have been documented in microlithic industries, including the use of bipolar percussion conducted on an anvil (Wadley 1993). In Europe and the Levant an alternative production technique has been described, known as the microburin technique (Henry 1974, Neeley and Barton 1994, Belfer-Cohen and Goring-Morris 2002, Straus 2002). This involves the truncation of a blade blank through controlled snapping, facilitated by the construction of a notch in the side of the blank (Figure 2.1.2).

Once the lithic parts of composite tools were manufactured, they were attached to organic (wood or bone) hafts in a variety of configurations to produce a variety of 
composite tool types. Occasional finds of microlithic composite tools with intact hafts have helped us to understand hafting configurations, such as the discovery in 2009 at a Mesolithic site in Sweden of a broken object which the excavators describe as an arrow shaft (Figure 2.1.3) (Larsson and Sjöström 2011). The use of microliths in bow and arrow systems has been much discussed (e.g. Lombard and Haidle 2012), and recent use-trace analysis is consistent with the use of microliths as arrowheads as far back as 65 ka during the southern African HP (Lombard 2011). Additionally, residue analyses conducted on the edges (particularly backed edges) of microlithic tools reveal that a variety of different materials were utilised in the past to haft microliths, including ochre-based and calcareous mixtures as well as various plant resins and gums (Gibson et al. 2004, Lombard 2006, 2007, 2008, Villa et al. 2012, Charrié-Duhaut et al. 2013, Yaroshevich et al. 2013).

Figure 2.1.2: Examples of different applications of the microburin technique in Levantine Epipalaeolithic industries (Henry 1974, Figure 2)

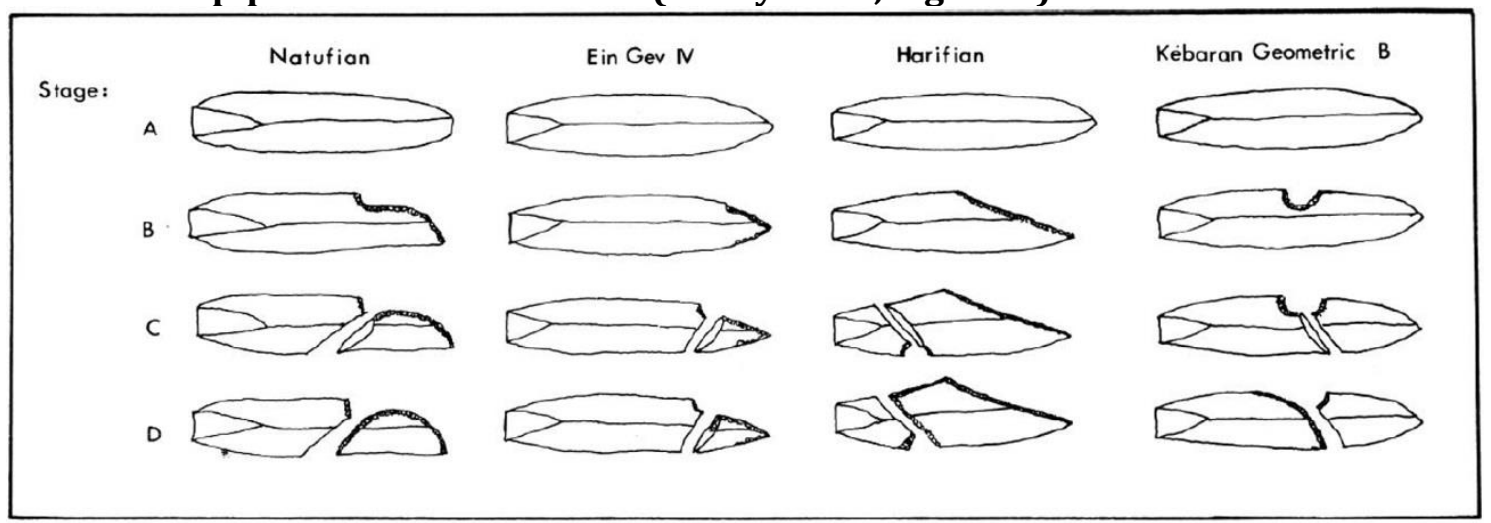

Since their first discovery a multitude of potential uses for microlithic tools have been proposed. Early suggestions include their uses in tattooing with ochre pigment; preparation of arrow shafts, bows, spears and javelins; removal and preparation of animal skins; engraving bone; trepanning; manufacturing of bags 
and carrying devices; skin and shell perforators; surgical instruments; and arrows, harpoons, spears, fishing hooks, multiple scrapers, saws, sickles, carders (for preparing wool for use in textiles), borers, needles and awls; and the wearing of them as ornaments (Brown 1889, Smith 1906, Pole 1907, Read and Smith 1911, Hartley 1914).

Figure 2.1.3: A microlithic composite tool from the Mesolithic assemblage at Rönneholms Mosse, Sweden (Larsson and Sjöström 2011, Figure 2)

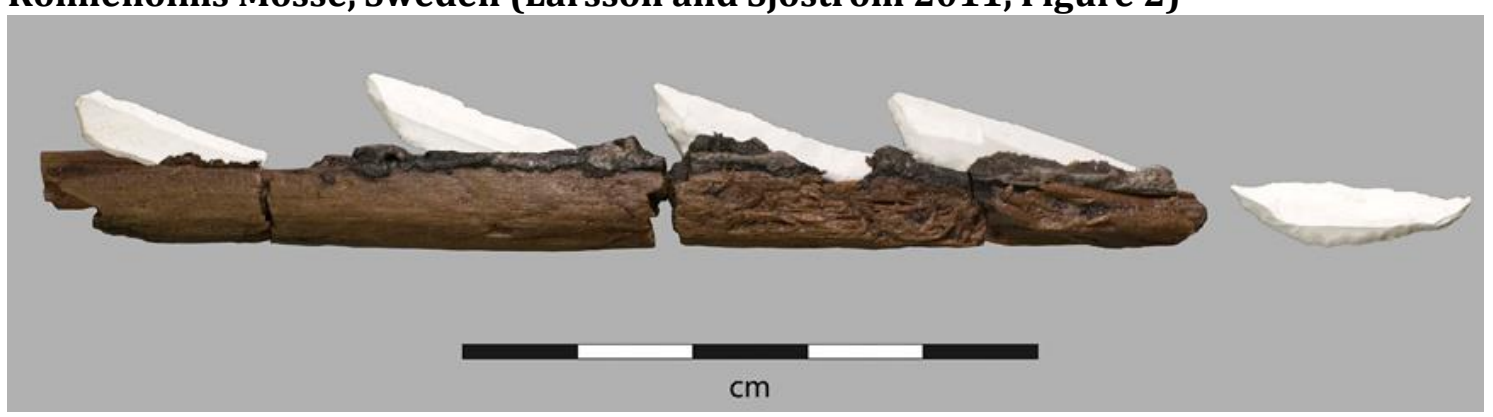

As discussed above, the more recent application of residue, use-wear and macrofracture analyses has advanced our understanding of the various uses to which microlithic tools were put. To a certain extent, it has confirmed earlier suspicions that microliths had a wide variety of uses. For example, use-wear and residue analyses conducted on Holocene Australian backed microliths by Robertson et al. (2009) reveal traces of a wide variety of materials, including wood, non-woody plant materials, bone, hide, blood, skin and feathers.

Whether or not microliths were primarily used as parts of projectile tools is a much debated topic. Use-wear analysis by Igreja and Porraz (2013) on HP geometric backed microliths from Diepkloof, South Africa, implies their use on a variety of materials, including soft and hard animal materials, bone and wood. Only 
two of the 25 microliths analysed exhibit impact fractures possibly indicative of their use as projectiles, leading the authors to conclude that microliths at the site were primarily used as cutting, scraping or other processing tools rather than as projectiles. Similar conclusions have been drawn at Rose Cottage Cave, where usewear and micro-polish analysis conducted by Binneman (1997) suggests that LSA Robberg microblades were mostly used for cutting or sawing soft organic materials, such as vegetal materials or hide.

Lombard's (2008) micro-residue analysis of segment microliths in HP layers at Sibudu, South Africa, suggests that they were mostly used on animal materials, and that there is no evidence for their use in processing plant materials. Animal residues including tissue, fat, collagen, bone, blood and hair are concentrated on the cutting edges of the segments. It is not possible to determine from residue analysis alone whether this was the result of hunting (e.g. with projectile weaponry) or butchery, although Lombard leans towards the former as the cutting edges are generally sharp and not worn through cutting or scraping.

In further support of the use of microliths as projectiles, Pargeter's (2007) experimental reconstructions of composite projectile weapons using HP-like segments demonstrate that HP segments would have made successful projectile tips. Lombard and Pargeter (2008) built on this experiment, and on previous macrofracture analysis of backed HP microliths from Klasies River, South Africa, which revealed impact fractures diagnostic of having been used in projectile weaponry (Wurz and Lombard 2007). Lombard and Pargeter's comparison of macro-fractures on HP segments from the South African sites of Sibudu, 
Umhlatuzana and Klasies River with experimentally-produced equivalents showed that at least some HP segments may have been used as the tips of projectiles.

An experimental approach to microlithic tools was also taken by Yaroshevich et al. (2010) in order to compare experimentally-derived data on the effects of different arrow hafting configurations with Epipalaeolithic assemblages in Israel. They were able to demonstrate the occurrence of fractures diagnostic of projectile impacts on the archaeological samples. Yaroshevich and colleagues (2013) later also conducted analyses of impact fractures and the location of adhesive residues on late Upper Palaeolithic microliths from Ohalo II, Israel, which they view as indicating their use in composite projectiles.

Taken as a whole, the evidence indicates that microliths were used in a wide variety of composite tools for a wide variety of purposes. As Kuhn (2002: 184) states, "the potential for microlithic tools to satisfy multiple needs may be what makes them so attractive and adaptive. The ability to play multiple roles may be what creates the utility that has made them so attractive and widespread".

\section{WHY MICROLITHS?}

Explanations for and meanings of microlithic technology tend to be debated on two different scales. The first, discussed in this section, questions the 'why' of microliths as tools on a smaller scale, i.e. the advantage of manufacturing and using small replaceable lithic parts of composite tools, and uses localised or regional examples and case studies. The second discusses the 'why' of microlithic industries 
on a larger inter-regional scale, addressing the question of why we see the proliferation of microlithic industries in the places and times that we see them in; this is related to the process of 'microlithisation' (Elston and Kuhn 2002 and references therein). These explanations fall into two main areas of archaeological discourse, concerning modern human behaviour and dispersals, and will be discussed below in chapter sections 2.2 and 2.3, respectively. The investigation and comparison of microlithic technologies is able to contribute to examinations of the meanings of microliths and of microlithic industries on both of these scales.

The advantages of the use of microliths and composite tools have most frequently been argued in terms of strategies for risk buffering and management. These arguments derive from what Bamforth and Bleed (1997: 134) term the “optimization perspective”, including archaeological offshoots such as optimal foraging theory. From this standpoint, lithic technology is viewed as a means of managing risk, which involves two distinct components: the probability and the cost of failure.

Bleed (1986) highlights reliability and maintainability as alternatives for the optimal designs of technical systems in order to reduce risk. Reliable systems involve the production of 'over-designed' tools to be used in any situation, and are adaptive in environments where the cost of failure is high. Maintainable systems allow tools to be quickly repaired if broken or adapted if inappropriate, for example through the production of modular tools with standardised replaceable parts, and are adaptive in unpredictable environments where the cost of failure is low. Eerkens (1998) demonstrates that both systems were used at different times 
in microlithic assemblages during the European Mesolithic. Hiscock $(1994,2002)$, however, rejects this dichotomy, arguing that standardisation of microlith shape and size increases both the maintainability and the reliability of composite tools.

Regardless of how technological risk-avoidance strategies are classified, risk often takes centre-stage when explanations for the innovation or adoption of microlithic technology are constructed. For example, Hiscock et al. (2011: 656-657) state that "[r]apidly repaired multi-purpose tools are desirable in riskier conditions, where activities might be unsuccessful and/or where the consequences of failure were severe". Similarly, Elston and Brantingham (2002: 103) describe microlithic technology as a "risk-minimizing strategy" in northern Asia. They tackle the question of what problems microlithic technology solved for hunter-gatherer populations in north Asia at that time by looking at artefact design, and specifically by comparing organic points and 'inset points', i.e. organic points with stone insets. The stone insets are expendable, and the composite inset point is designed to allow the stone inset to be replaced without having to manufacture an entire composite tool from scratch, as the organic component is the more time-intensive part to manufacture (e.g. if wood, it includes nocks, sockets, fletching and/or hafts; or if bone or antler, it includes scraping and/or grinding).

Elston and Brantingham conduct a cost/benefit analysis, noting that the costs of manufacturing inset points are that stone points are less durable (e.g. brittle in cold weather, can shatter on impact), larger manufacturing kits are required for tools that require multiple raw materials, considerable time investment is required to learn how to systematically produce microblades and the hafts for them, and 
that on some metrics the production of microblades is less economical of raw material than the production of bifaces. Overall, however, they conclude that the benefits outweigh the costs, as inset points combine the best design features of organic points (strong) and stone points (lethal).

Torrence (2002) similarly concludes from cost/benefit modelling that the advantages of using multi-purpose microlithic composite tools must have outweighed the time, energy and raw material costs of their manufacture. Most of these costs would have been faced in the production of hafts and the combination of the different elements, rather than in the production of the lithic components (McCall and Thomas 2012). For example, Bleed (2002: 95) states that systematically produced microblades (or microliths blanks more generally) are "cheap, regular and reliable". He also describes the systematic manufacture of microblades at a Japanese Late Palaeolithic site as 'batch processing', which he argues is advantageous because "[i]t reduces set-up costs, it allows the producer to 'get in the groove', and, most important, it can be scheduled at 'down times' that do not conflict with other opportunities" (ibid: 101). Additionally, large numbers of potential blanks can be produced efficiently from single cores; Flenniken's (1987) experimental production of microblades produced an average of 101 per core.

The advantages of microlithic technology as a strategy for dealing with various types of external risk are summarised by Neeley (2002), who explores microlithic technology from an evolutionary perspective. He argues that microliths are advantageous for mobile groups as they are easily transported, they are versatile and can be used in a wide variety of ways, they are interchangeable components in 
composite tools and so make tool maintenance more efficient, they can be processed in large numbers at a single location and then transported as a buffer against lack of suitable raw materials in other locations, and they increase the efficiency with which resources can be exploited in the event of changes in the availability of particular resources.

The adoption of microlithic technology is often seen as a response to various external pressures making risk buffering necessary by increasing the probability or the price of failure. Environmental and/or subsistence pressures are frequently discussed in this context. A classic example is the argument (discussed further in Chapter 3.1) that the microlithic HP in southern Africa occurred in response to deteriorating environmental conditions at the end of MIS 4 which led to changes in mobility and resource use (e.g. Ambrose and Lorenz 1990, Ambrose 2002, McCall 2007, McCall and Thomas 2012, Ziegler et al. 2013, Dusseldorp 2014).

Microlithic technology has also been argued to be adaptive in unpredictable environments or times of increased environmental uncertainty. Hiscock (1994, 2002) uses this line of argument to explain the proliferation of backed microliths in Australia during the mid-Holocene. He contends that the use of composite microlithic tools reduced subsistence risk faced as a result of the colonisation of new landscapes, and also changes in the familiarity of resources due to environmental changes and increased environmental variability.

Subsistence factors have similarly been implicated in the adoption of microlithic technologies in Europe during the Upper Palaeolithic. The development of 
increased dietary breadth and the utilisation of new resources (including the exploitation of small prey species) are argued to have resulted in technological diversification and the adoption of new subsistence technologies, i.e. microliths (Kuhn 2002). A similar argument has been made for the adoption of microlithic technology in Korea during MIS 2 (Seong 2008).

Demographic pressures have also been cited as factors contributing towards increased risk and the adoption of microlithic technologies. For example, BelferCohen and Goring-Morris (2002) note that in the Levant during the Upper Palaeolithic and Epipalaeolithic there is a reduction in the mobility of populations, from small mobile groups of hunter-gatherers with large range sizes to larger sedentary communities. Concomitant increases in population density resulted in the reduction of range sizes, and therefore the more intensive exploitation of raw material sources. They argue that "[t]he introduction of the mass production of composite, hafted, tools should be seen against this background of flint being more efficiently and sparingly exploited" (ibid: 64).

Environmental and demographic pressures have also been implicated in explanations advanced for the adoption of microlithic technology in South Asia (Clarkson et al. 2009, Petraglia et al. 2009). Environmental deterioration towards the end of MIS 3 is postulated to have affected the adoption of new technological and subsistence strategies - including microlithic technology - through the medium of increases in population size and fragmentation (discussed further in Chapter 3.2). 
Social factors have also been included in debates concerning the 'why' of microliths. More specifically, microliths have been discussed as material aspects of the creation of social or exchange networks - what Hiscock et al. (2011: 660) refer to as "the politics of resource sharing". For example, in the microlithic industries of the late Middle Stone Age (MSA) and the MSA/LSA traditional period in East Africa there are relatively high frequencies of long-distance movements of lithic raw materials (defined as being beyond the maximum distance possible for direct procurement within a group's territory), even at sites where more local raw material sources were available (Ambrose 2002). This is interpreted as implying increases in both mobility (for embedded procurement) and exchange (i.e. social networks of information exchange), depending on the circumstances.

In the southern African MSA, Deacon $(1992,1995)$ and Wurz (1999) have likewise proposed that microliths were exchanged as part of delayed reciprocity gift exchange systems, as a social form of risk buffering in unpredictable environments. Wurz analyses HP backed microliths at Klasies River, South Africa, and argues that the production processes involved go beyond functional necessities. She concludes that these artefacts included stylistic elements and were used as material symbols to aid communication with other groups. Similarly, Mackay (2011) compares backed microliths between two HP sites in South Africa, determining that the differences between them are adaptively neutral and so are due to stylistic rather than functional considerations.

Kuhn (2002: 84) disagrees with the symbolic meanings attributed to microliths, stating that "given their size and the manner in which they are likely to have been 
affixed to an armature (i.e., with a partial covering of mastic or glue), microliths themselves were poor media for overtly expressive stylistic messages". However, if, as McCall and Thomas (2012) suggest, microliths themselves were relatively cheap to produce but the rest of the composite tools of which they were a part were expensive, it might be expected that any symbolic meanings, for example in the form of decorations, might be applied to the organic haft rather than the stone insets. Therefore even in the absence of overtly stylistic elements in microlith manufacture we cannot rule out social or cultural meanings attributed to microlithic composite tools.

In their review of the various explanations given for the origins of microlithic technology in Australia, southern Africa and India, Hiscock et al. (2011) conclude that there is no single unifying answer. Although they separate out what they view as the social (use in social/exchange networks) and ecological (use of multifunctional tools as buffering against economic and environmental risk) explanations for microliths, these are in fact often linked and are rarely mutually exclusive (Ambrose 2002).

The costs inherent in the production of composite microlithic tools mean that they are not intrinsically more efficient than the production of other tool types (Kuhn 2002). The benefits of microlithic technology have to outweigh its costs given the particular environmental and social circumstances, in order for it to be adopted. Therefore, in order to explain why this form of technology was adopted in particular places at particular times, microlithic assemblages must be considered within their specific contexts. 


\subsection{MICROLITHS AND MODERN HUMAN BEHAVIOUR}

Microlithic technology has been invoked in two of the biggest areas of recent debate in Palaeolithic archaeology; those concerning the origins and development of modern human behaviour (MHB), and the earliest modern human dispersals out of Africa. The following two sections set out the theoretical background to both of these areas of discourse, and the place of microlithic technology within them.

\section{SUMMARY OF THE MODERN HUMAN BEHAVIOUR DEBATE}

The MHB debate has formed one of the cornerstones of Palaeolithic archaeological enquiry over the last several decades. It concerns the characterisation and archaeological correlates of behavioural traits and abilities associated with modern humans. Although a multitude of explanations for the origins and development of MHB have been proposed, these lie beyond the scope of this thesis. This section therefore discusses only aspects of the methodology of the debate that are pertinent to an understanding of behavioural variability with reference to microlithic technology.

The majority of archaeological attention in the MHB debate has been on disputes between proponents of the 'short range' and 'long range' models (Brumm and Moore 2005). The 'short range' or 'human revolution' model is the traditional view, based on the perceived sudden rapid development of MHB traits in the European Upper Palaeolithic from around $40 \mathrm{ka}$, including systematic production of prismatic blades, tool standardisation, bone and antler tools, grindstones, body 
decorations including beads and personal ornamentation, long-distance exchange of materials, improved hunting tools and art objects, and possibly also storage, structured hearths, spatial organisation of sites and burials with grave goods (e.g. Mellars and Stringer 1989, Mellars 1990, 2006a, 2006b, Klein 1992, 1995, 2000, Bar-Yosef 2002, Mellars et al. 2007). This model has evolved somewhat in recent years, recognizing that some of these behaviours originated in Africa c. 60-50 ka. However, it still revolves around the argument that MHB did not fully appear until dispersing populations brought these behaviours into Eurasia c. 50-40 ka.

In contrast, the 'long range' model highlights the earlier and more gradual appearance of 'modern' behavioural traits in Africa. McBrearty and Brooks (2000, McBrearty 2007) demonstrate that the African evidence exhibits an earlier and more temporally variable development of the various traits usually correlated with MHB, including "blade and microlithic technology, bone tools, increased geographic range, specialized hunting, the use of aquatic resources, long distance trade, systematic processing and use of pigment, and art and decoration" (McBrearty and Brooks 2000: 453). This combination of behaviours is argued to have been gradually assembled in Africa before spreading to the rest of the world, with the earliest evidence for some behaviours traced back to the Acheulean-MSA transition c. 300 ka.

It is clear from the African MSA record that previous theories concerning the origins and development of MHB based solely on the European archaeological record do not hold up in the light of non-European evidence. As excavations in additional geographical regions increasingly add to our knowledge about the 
behaviour of $H$. sapiens over the course of its existence, our models are being replaced and updated to take account of this new material.

However, it is only in the last few years that the archaeological records from Asia and Australasia have been included within the MHB debate. For example, James and Petraglia (2005) examine the later Pleistocene record of South Asia. They conclude that there is no sudden appearance of a 'package' of archaeologically visible 'modern' behavioural traits in South Asia, which would be expected by the 'human revolution' model. Reviews of the archaeological records of Australia (Brumm and Moore 2005, Franklin and Habgood 2007, Habgood and Franklin 2008, Langley et al. 2011) and East Asia (Norton and Jin 2009) come to similar conclusions, i.e. that evidence for MHB traits in these regions is generally patchy and full of discontinuities and anomalies, and that components of the MHB 'package' were assembled gradually and/or in stages.

The same is true of South-East Asia, where complex subsistence behaviours are evident from the earliest modern human occupation, including the use of trapping, the exploitation of a wide range of terrestrial, arboreal and aquatic prey species, the processing of several species of toxic plants for consumption, the successful colonisation of rainforest environments, and the clearing of forest using fire and axes to promote the growth of edible plants (Barker et al. 2007, Summerhayes et al. 2010, Gosden 2010, O'Connor et al. 2011). However, evidence for symbolic behaviours such as art, burials and personal ornamentation is generally rare, patchy and highly regionally variable. A notable exception is the recent discovery of cave art on the island of Sulawesi dating to c. 40 ka BP (Aubert et al. 2014). 
Overall, it is clear that behavioural traits listed in models for MHB appear and develop differently in every region in which they have been assessed.

\section{A CRITIQUE OF THE TRAIT LIST APPROACH TO MODERN HUMAN BEHAVIOUR}

The contrasting 'short range' and 'long range' models for the origins of MHB provide very different interpretations of the archaeological record in relation to understanding the behaviour of H. sapiens. However, what both models have in common is recourse to a trait list of behaviours as a starting point, which researchers from both camps then use to assess the 'modernity' of archaeological assemblages. These traits represent different technological, ecological, social and cultural behaviours, and can be further divided into innovations (newly invented behaviours) and improvements (enhanced or extended versions of behaviours that are already evident).

Table 2.2.1 provides a summary of the behavioural traits cited in classic works on MHB. Improvements are, by definition, extensions to existing behaviours. However, in addition, every innovation listed in Table 2.2 .1 is also evidenced in other hominin species (Gibbons 1998, Burke 2000, Vaquero et al. 2001, Backwell and d'Errico 2001, 2008, d'Errico 2003, d'Errico and Backwell 2003, Joordens et al. 2009, 2015, Courty et al. 2010, Parfitt et al. 2010). Therefore none of these socalled 'modern human behaviours' are in fact unique to modern humans. 


\begin{tabular}{|c|c|c|}
\hline & Innovations & Improvements \\
\hline \multirow[t]{8}{*}{ Technological } & \multirow{2}{*}{$\begin{array}{l}\text { New stone tool types including } \\
\text { end-scrapers, burins and backed } \\
\text { pieces }\end{array}$} & $\begin{array}{l}\text { Greater diversity of stone tool } \\
\text { categories }\end{array}$ \\
\hline & & \multirow{3}{*}{$\begin{array}{l}\text { Greater chronological and } \\
\text { geographical patterning in tool } \\
\text { categories }\end{array}$} \\
\hline & Systematic blade production & \\
\hline & Hafting and composite tools & \\
\hline & Bone and antler tools & $\begin{array}{l}\text { Increased standardisation of } \\
\text { artefact types }\end{array}$ \\
\hline & Systematic use of grindstones & \multirow{3}{*}{ Improved hunting tools } \\
\hline & Complex hearth construction & \\
\hline & Sea crossings and watercraft & \\
\hline \multirow[t]{8}{*}{ Ecological } & \multirow{2}{*}{$\begin{array}{l}\text { Occupation of new and harsh } \\
\text { environments }\end{array}$} & \multirow{3}{*}{$\begin{array}{l}\text { Increased dietary breadth } \\
\text { Longer-distance raw material } \\
\text { procurement }\end{array}$} \\
\hline & & \\
\hline & \multirow{2}{*}{$\begin{array}{l}\text { Specialised hunting of large } \\
\text { mammals }\end{array}$} & \\
\hline & & \multirow{2}{*}{$\begin{array}{l}\text { Intensification of resource } \\
\text { extraction }\end{array}$} \\
\hline & \multirow{2}{*}{$\begin{array}{l}\text { Scheduling and seasonality in } \\
\text { resource exploitation }\end{array}$} & \\
\hline & & \multirow{3}{*}{$\begin{array}{l}\text { Increased curation of exotic raw } \\
\text { materials }\end{array}$} \\
\hline & Site reoccupation & \\
\hline & Exploitation of aquatic resources & \\
\hline \multirow[t]{2}{*}{ Social } & $\begin{array}{l}\text { Individual and group } \\
\text { identification through regional } \\
\text { artefact styles }\end{array}$ & \multirow[t]{2}{*}{$\begin{array}{l}\text { Longer-distance exchange } \\
\text { networks }\end{array}$} \\
\hline & Structured use of domestic space & \\
\hline \multirow[t]{5}{*}{ Cultural } & Personal ornamentation & \\
\hline & Symbolic use of pigment & \\
\hline & Notched and incised objects & \\
\hline & Images and representative art & \\
\hline & Ritual burials with grave goods & \\
\hline
\end{tabular}

Table 2.2.1: Summary of traits associated with MHB (Klein 1995, McBrearty and Brooks 2000, Bar-Yosef 2002, Henshilwood and Marean 2003, Mellars 2007, Norton and Jin 2009, Shea 2011)

Microliths have also been included in such trait lists, either explicitly (e.g.

McBrearty and Brooks 2000) or included within more general technological categories (e.g. Klein 1995). Unlike the innovations listed in Table 2.2.1, fully microlithic industries (as defined in the previous section) are associated only with H. sapiens. They have also been argued to "clearly signal a modern approach to 
technology" (McBrearty and Brooks 2000: 500) and to be demonstrative of the shift to modern cognition and behaviour (Wadley et al. 2009, Lombard and Parsons 2011, Lombard 2012, Lombard and Haidle 2012). For example, it can be argued that they are related to many 'archaeological signatures of MHB' (as listed by McBrearty and Brooks 2000: 492), including the hafting and standardisation of composite tools, the use of projectile weaponry, specialized hunting, complex manufacturing sequences including the procurement of raw materials and the control of fire for the production and use of mastics, exchange networks, and even symbolism in the form of regional artefact styles.

As a result of the inclusion of microliths in lists of MHB traits, the unit of analysis has been the simple assessment of the presence or absence of microlithic technology; what Wadley (2001: 207) terms "a 'shopping list' approach". This has the effect of homogenising microlithic technologies through their discussion largely in terms of the appearance dates of certain microlithic typologies, while ignoring any indications of underlying technological diversity.

Over the course of the later Pleistocene, modern human populations ranged from periglacial to tropical environments, and so it should not be surprising that the material remains of their behavioural adaptations might vary between regions. Yet the same list of archaeological proxies of adaptations to European steppe environments continues to be used to check off aspects of the behavioural record in East African savannahs, Central and East Asian highlands and South and SouthEast Asian rainforests. This is not to deny that attempts to place the archaeological records from these disparate environments into a global framework are a 
potentially fruitful exercise. However, rather than simply looking for evidence of, for example, microliths in each region, it is important to consider what those microliths are telling us about human behaviour, and why that behaviour might have been advantageous in that area at that time.

This highlights one of the main problems with McBrearty and Brooks' (2000) model. Although they push back the dates for certain 'traits' and shift geographical focus from Europe to Africa, their theoretical framework is not entirely distinct from that of the 'human revolution'. They are still stuck within the traditional paradigm of the search for a list of behaviours that have at some point been deemed indicative of modern humans. Furthermore, they continue to view this as an evolutionary and cumulative trend, rather than recognising the patchiness of the archaeological record as an indication of the fact that, although H. sapiens had the ability to use a great variety of adaptations and behaviours in order to cope in a wide range of ecological and social situations, this was context dependent. As Shea (2011: 5) notes, McBrearty and Brooks consider the oldest appearance of a particular behavioural trait anywhere in Africa as evidence for the ability of humans to use that behaviour anywhere else in Africa from that point onwards. Worse, many researchers then expect these behaviours to turn up in the archaeological record across the world from this point (e.g. Franklin and Habgood 2007, Habgood and Franklin 2008).

An additional problem is that many 'hallmarks' of MHB disappear within the archaeological record; for example the ochre-covered marine shell beads in Moroccan Middle Palaeolithic sites - frequently cited as evidence for symbolic 
behaviour - which disappear after MIS 5 and are 'reinvented' c. 40 ka (d'Errico et al. 2009). Likewise, the innovations of the HP industry of southern Africa, including microliths, bone tools and ostrich eggshell beads, have been dated through optically stimulated luminescence dating (OSL) to between 64.8 and $59.5 \mathrm{ka} \mathrm{BP}$ (Jacobs et al. 2008), indicating that these behaviours only existed for $\sim 5,000$ years before disappearing. Although controversy exists as to the accuracy of these dates (Guérin et al. 2013, Tribolo et al. 2013, Feathers 2015, cf. Jacobs and Roberts 2015), counter-arguments tend to push for older dates, and it remains the case that microliths do not reappear in the archaeological record of southern Africa until the LSA (Mitchell 2002). As it is unlikely that these populations 'lost' the cognitive capacity to use symbolism or any other MHB trait, the most parsimonious explanation for these changes is that these behaviours stopped being advantageous (Shea 2011). Models for the emergence of MHB therefore need to take into account the factors that would have led to the origins and maintenance (or disappearance) of these behavioural innovations, including issues such as demography and the mechanisms of cultural transmission.

One of the major critiques of the current models for MHB comes from Henshilwood and Marean's (2003) seminal paper outlining the main problems with the 'behavioural-trait' approach. They begin by highlighting that the creation of such trait lists has come at the cost of a lack of focus on the construction of a coherent body of theory relating to the definition of MHB. Given the lack of such a theoretical body, current test implications concern only the assessment of the presence or absence of certain (often taphonomically-sensitive) behavioural traits. In addition, the implication that these traits are necessarily indicative of MHB lacks 
theoretical justification in many cases, and can be explained more parsimoniously with reference to alternative, and more prosaic, processes, such as demographic and environmental pressures. James and Petraglia (2005: S16) concur, stating that "[g]iven the effect of demographic processes on cultural evolution and innovation, archaeologists have to be careful in selecting the traits considered representative of behavioural modernity".

Henshilwood and Marean (2003) further note that many of these traits are empirically derived from the European record, where human populations would have adapted their behaviour to survive in unique European environmental and climatic conditions. Therefore not only are these traits unsuitable for application in other regions of the world, but they also involve the use of circular reasoning by defining MHB traits as European traits, and then using them as evidence for MHB in Europe. The authors conclude that the trait list approach is inherently flawed, and that the only MHB trait that is methodologically and theoretically sound is that of symbolic behaviour, or more specifically the use of symbolism to organise behaviour. However, Shea (2011) retorts that reducing the debate concerning behavioural modernity into one solely concerning symbolic material culture is problematic for the testing of hypotheses using the archaeological record. In particular, the lack of archaeologically visible or durable symbolic material culture in an archaeological context does not necessarily mean that a particular human group was not making use of behaviours with symbolic meanings, or producing symbolic artefacts on materials that do not survive in the archaeological record. 
Furthermore, in addition to Henshilwood and Marean's (2003) main criticisms of the trait list approach on the grounds of taphonomic bias, ambiguity and theoretical justification, Shea (2011) argues that even if a trait did pass these tests, further issues would arise with regards to the composition of trait lists (for example, with projectile technology omitted), the relative importance of different traits, implied linkages between traits, statistical issues with comparing samples of different sizes, and the limits to how far we can extrapolate about human behaviour from single sites or regions. Shea's overarching arguments are that the current concepts of 'behavioural modernity' and MHB are historically the result of Palaeolithic research in Europe and are inappropriate elsewhere, that they reflect a concern with linear evolutionary and narrative trends that are not representative of actual patterns in behavioural variability, and that they are qualitative and lack analytical precision and therefore cannot form the basis of testable hypotheses about human behaviour.

Langbroek (2011) likewise critiques the MHB debate's adherence to a linear model or 'ladder', which uses primate and ethnographic analogies as the points of polarity and cites 'modern behaviour' as the superior and most sophisticated level of cognition. Instead, he prefers a 'branching tree' model similar to that used in models of hominin phylogeny constructed through cladistic analyses (Figure 2.2.1). This is a refreshing take on models of cognitive evolution, viewing modern human cognition and behaviour as different from, rather than superior to, the cognitive abilities of other hominin species, for example Neanderthals. However, it does not entirely address the question of the evolution of cognitive and 
behavioural abilities within the course of $H$. sapiens evolution, or the specifics of the relationship between cognition and behaviour.

Figure 2.2.1: A linear model (a) and a 'branching tree' model (b) of cognitive evolution (Langbroek 2011, figure 2)

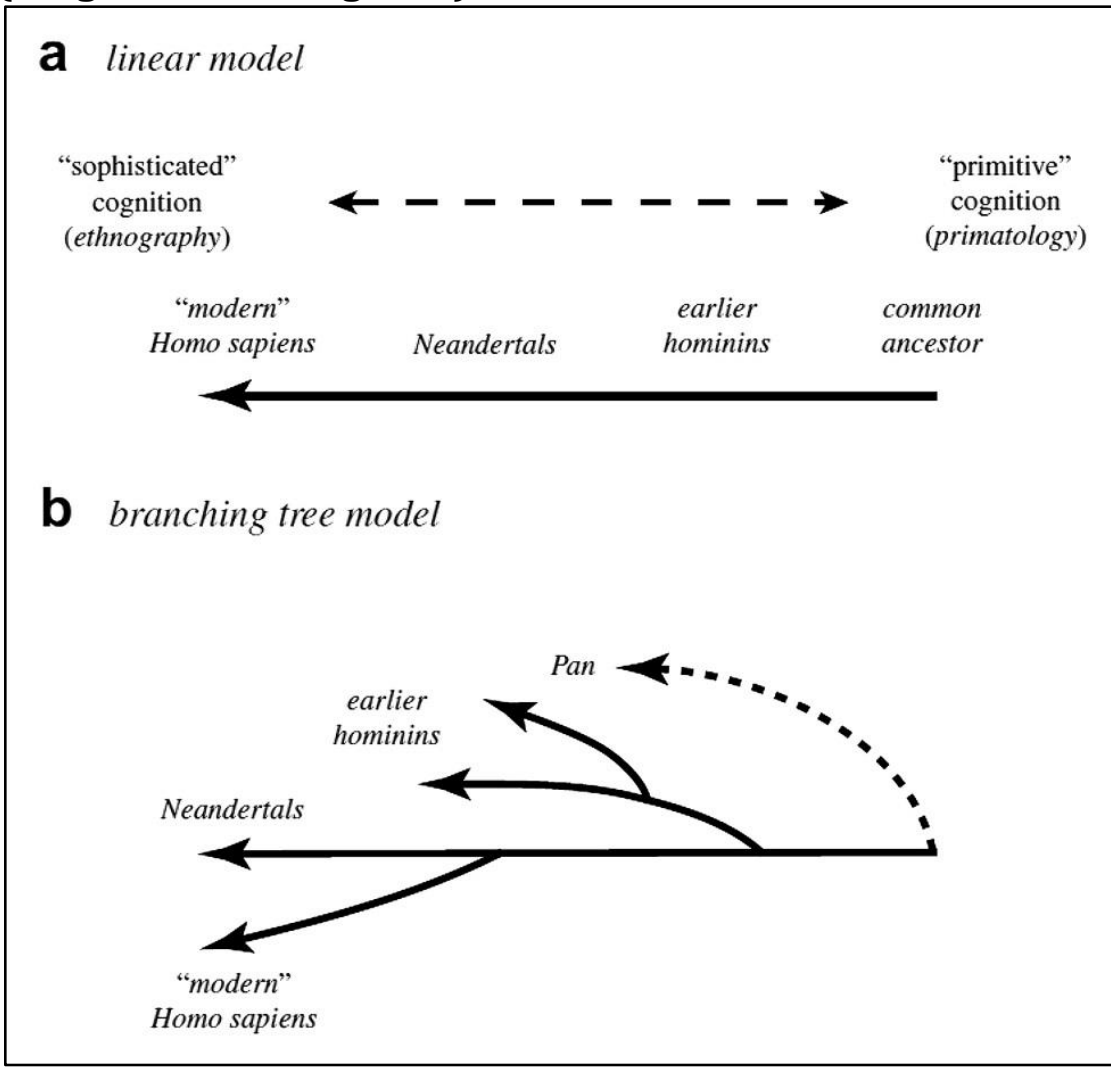

Not all researchers agree that these criticisms of the concept of MHB are justified, however. For example, in his comment on Shea's (2011) paper, Conard asserts that Shea has oversimplified existing models in order to criticise them. Most commentary criticisms, including by Lombard, Potts and Tryon, focus around the operationalization of Shea's arguments, and the methodology he uses in order to demonstrate his point. The example he gives of the uses of different combinations of Grahame Clark's five technological 'Modes' is admittedly not the best illustration of how we can investigate behavioural variability in the archaeological record. 
Future explorations of behavioural variability sensu Shea would hopefully involve more detailed and fine-grained records of behavioural variability. Nevertheless, Shea and others highlight important methodological and theoretical shortfalls in the existing paradigm, and signpost future directions for the way we think about the past behaviour of our species.

\section{ALTERNATIVES TO THE TRAIT LIST APPROACH}

Trait list models for MHB distract us from investigating the variability of human behaviour in the past, by forcing us to direct our studies towards discerning dates at which certain archaeological correlates of behaviours first emerged, and to then extrapolate cognitive and behavioural capacities from this. Viable and useful alternatives to the existing paradigm are difficult to formulate (Langbroek 2011). However, one potential alternative to these conventional models is a more regionally-focused consideration of the various behaviours that different populations of hunter-gatherers chose to adopt at different times, in response to different environmental and social circumstances.

In this vein, Langley et al. (2011) argue for a focus on the 'diversity of complex behaviours', with an emphasis on the overall combination of traits rather than any one individual trait, contra Wadley (2001) who argues that the the discovery of any one of a selection of complex cultural behaviours in an archaeological context is indicative of full cultural modernity. Langley et al.'s approach should also be applauded for its use of each 'instance' of complex cultural behaviour as the analytical unit, i.e. the appearance of a single piece of evidence for a category of 
complex behaviour from a single site with a secure date. Of the 223 Australasian sites they investigated, only 33\% lacked any instances of complex cultural behaviours, indicating that Late Pleistocene sites in Sahul are much richer in evidence for cultural complexity than is usually assumed.

Similarly, Shea's (2011) concept of 'behavioural variability' has parallels with Langley et al.'s (2011) emphasis on the 'diversity of cultural behaviours'. He argues that we should focus our efforts on an investigation of 'behavioural variability' as opposed to 'behavioural modernity', as a way of rephrasing the MHB debate to get beyond a trait list approach and look more analytically at the "strategic sources of variability in particular human behaviors" (Shea 2011: 12), including energetic costs, benefits and risks. This line of reasoning allows us to reject an implicit linear evolutionary framework for the development of certain behaviours, by viewing the capacities for said behaviours as inherent to $H$. sapiens, rather than emerging later during the course of its development (contra Renfrew's 'sapient paradox' (Renfrew 2008)). Additionally, whereas the concept of behavioural modernity can only be assessed on a qualitative scale by looking at the presence and absence of certain traits, behavioural variability is accessible though more detailed and revealing quantitative statistical analyses.

Correspondingly, it has been argued (Potts 1999) that the capacity for behavioural flexibility, plasticity and variability has been selected for over the course of human evolution. The 'ratcheting up' of MHB traits during the latter part of the Late Pleistocene (Mellars 2007) could perhaps be viewed as a result of this selective process. The critical area for analysis would therefore be the utilisation of this 
versatility, i.e. variation in human behaviour, rather than the specific expressions of this ability, i.e. individual behavioural traits.

On the basis of archaeological, palaeoanthropological and genetic evidence, the most parsimonious explanation is that modern human cognitive capacities were in place at or around the time of the beginning of our species, and that no major cognitive changes can have occurred after the first groups that can trace their ancestry back to the major African lineages left Africa (Henshilwood and Marean 2003, Powell et al. 2009, Shea 2011, contra Klein 1992, 1995). This is not to deny that smaller cognitive differences may have existed that were later nullified by further genetic admixture with new populations moving out of Africa. However, the most parsimonious explanation is that these cognitive abilities were in place by the end of MIS 5 at the latest (Petraglia et al. 2010, Dennell and Petraglia 2012, Scally and Durbin 2012, Boivin et al. 2013, Groucutt et al. in review).

Therefore to debate at what time various expressions of these behaviours in archaeologically visible materials happened to appear contemporaneously - as seems to be the case with the traditional Europe-based model - is a moot point. What is more interesting, and more worthy of academic debate, is how these abilities were expressed in different ways in different places at different times, rather than the teleological narrative of how cognitive changes and innovations accumulated in order to result in the pinnacle of the Upper Palaeolithic of Europe. By focussing on the different ways that it is has been possible to be a $H$. sapiens hunter-gatherer in the different climatic and environmental regimes of the later part of the Pleistocene, and by removing our reliance on a linear model of 
behavioural evolution, it is possible to investigate the roots of behavioural variability in much greater detail.

Although we should be looking at Africa for the origins of the cognitive abilities required in order to be able to make use of the behaviours we consider 'human', we must be wary of assuming that these behaviours themselves were transferred from region to region as a 'package' - as James and Petraglia (2005) demonstrated was not the case in South Asia. It is the ability of different populations to use their cognitive capabilities to adapt their behaviour and technology to the environments they find themselves part of that is truly indicative of a modern human way of living.

It is not enough simply to state that a region or period contains evidence for particular behaviours from a predetermined Eurocentric trait list. By accepting that the capacity to use a wide variety of behaviours existed from the first appearance of anatomically modern humans, we can instead begin to examine the choices made and the strategies used by different groups of modern human hunter-gatherers. What is of real interest to archaeological enquiry is why various human populations chose to make use of different technological, ecological, social and cultural adaptations in different places at different times. It is this behavioural variability that is key to understanding our species. 


\subsection{MICROLITHS AND MODERN HUMAN DISPERSALS}

\section{FOSSIL AND GENETIC EVIDENCE FOR HOMO SAPIENS ORIGINS AND DISPERSALS}

The origin and early dispersals of our own species are two of the most thoroughly researched topics in studies of palaeoanthropology and human evolution. The timing and routes of these dispersals are still hotly debated, and these debates are well summarized elsewhere (e.g. Willoughby 2007, Klein 2009, Stringer 2011). This section sets out the current state of knowledge in this area, before exploring in greater depth a particular model for early human dispersals which invokes microlithic technology.

Genetic studies, for example of the coalescence dates of modern human mitochondrial DNA (mtDNA) lineages, indicate a single African origin of our species c. 200 ka (Cann et al. 1987, Stoneking and Cann 1989, Vigilant et al. 1991, Harpending et al. 1993, Ovchinnikov et al. 2000, Ingman et al. 2000, Mishmar et al. 2003, Macaulay et al. 2005, Behar et al. 2008). Y-chromosomal DNA analyses produce roughly similar results (Dorit et al. 1995, Hammer 1995, Karmin et al. 2015). These results accord with the fossil record, as the earliest anatomically modern human (AMH) skeletal remains are also found in Africa. Two sets of cranial remains from Ethiopia are assigned to H. sapiens and dated to $195 \pm 5 \mathrm{ka}$ BP at Omo, Kibish Formation (McDougall et al. 2005), and 160-154 ka BP at Herto, Middle Awash (Clark et al. 2003, White et al. 2003).

The earliest fossil evidence for AMH outside Africa comes from the sites of Qafzeh and Skhul in Israel. The application of numerous dating methods including 
thermoluminescence (TL), U-series and electron spin resonance (ESR) dating indicates that the remains are broadly contemporaneous and date to c. $130-100 \mathrm{ka}$ BP (Valladas et al. 1988, Schwarz et al. 1988, Stringer et al. 1989, McDermott et al. 1993, Mercier et al. 1993, Grün et al. 2005).

To date, no AMH remains have been found in Arabia or South Asia in the crucial time period c. 100-40 ka. The next earliest AMH remains outside Africa are found in south-east Asia. A metatarsal from Callao Cave, Luzon, Philippines is provisionally identified as $H$. sapiens and dated via U-series dating to $66.7 \pm 1 \mathrm{ka}$ BP (Mijares et al. 2010). A cranium assigned to H. sapiens from Tam Pa Ling, Laos, has been given a minimum age of 51-46 ka BP and a maximum age of $63 \mathrm{ka} \mathrm{BP}$ (Demeter et al. 2012). Earlier AMH remains may exist in China, including a mandible from Zhirendong, Guangxi, dated to c. 100 ka BP (Liu et al. 2010), and a molar from Lunadong, Guangxi, bracketed between dates of $126.9 \pm 1.5 \mathrm{ka}$ BP and $70.2 \pm 1.4$ ka BP (Bae et al. 2014). However, generally speaking these fossil remains either cannot be confidently attributed to a specific Homo species, or have dubious stratigraphic and/or chronological associations (Dennell 2014).

H. sapiens was the first hominin species to spread to the Australian mainland. The dates for the oldest human remains found on the island, at Lake Mungo, are contested, but most researchers agree that AMH reached Australia by c. $45 \mathrm{ka}$ (Bowler et al. 2003, O'Connell and Allen 2004, Olley et al. 2006). This date also matches that generally accepted for the earliest colonisation of Europe (Zilhão et al. 2007, Higham et al. 2014, Douka et al. 2014). By 45-40 ka, AMH had spread across Africa, Eurasia and Australasia. 
Similar strategies to those used to determine the age of the origin of our species through analyses of genetic coalescence dates have been used to determine the timing of our dispersal out of Africa. Analyses of mtDNA haplogroup (groups of DNA sequences) distributions in modern populations allow us to calculate the coalescence dates of different lineages. All non-African mtDNA haplogroups are descended from haplogroup L3, which is also found in Africa (Soares et al. 2011).

Estimates for the age of the most recent common ancestor (MRCA) of the L3 haplogroup - and therefore estimates for the earliest 'out of Africa' dispersals vary considerably. Endicott and Ho (2008) give a coalescence age of 50-60 ka, while Macaulay et al. (2005) provide an estimate of c. $84 \mathrm{ka}$. Various other intermediate ages have also been calculated (Forster and Matsumura 2005, Behar et al. 2008, Soares et al. 2009, Endicott et al. 2009, Soares et al. 2011), but there is no universally accepted timeframe.

Additionally, small changes in the way that coalescence dates are calculated can have a large impact on the resultant age range. For example, Scally and Durbin (2012) revised the mitochondrial mutation rate used in their calculations using measurements from next-generation sequencing in modern populations. This produced a mutation rate half that of the commonly used rate, derived from dated fossils, pushing back the date of the MRCA to perhaps 90-130 ka.

It has been argued that DNA coalescence ages can only provide maximum dates for dispersals (Balme et al. 2009). However, as Groucutt et al. (in review) demonstrate, it is theoretically possible for the L3 lineage to have arisen 
subsequent to initial dispersal out of Africa if there was continued gene flow between African and non-African populations. Additionally, earlier dispersals could potentially have been genetically swamped by later dispersals and the proliferation of L3-descended M and $\mathrm{N}$ mtDNA lineages. This possibility is also raised by Reyes-Centeno et al.'s (2014) assessment of the genetic and palaeoanthropological evidence for dispersals, which they argue supports a model involving two stages of dispersal at $\sim 130 \mathrm{ka}$ and $\sim 50 \mathrm{ka}$. However, this conclusion is only reached through the inclusion of modern human cranial phenotype data. Overall, genetic data alone cannot provide us with an accurate picture of the timings of AMH dispersals out of Africa.

The routes of AMH dispersals out of Africa are also hotly debated. One particularly contentious area concerns the 'southern arc' of dispersal out of Africa and into Asia (Oppenheimer 2009, Petraglia et al. 2010, Dennell and Petraglia 2012). This movement is hypothesised to have occurred before the dispersal into Europe, taking AMH out of north-east Africa and along the Indian Ocean rim, eventually ending up in Australia by around 40-50 ka. This dispersal has been assessed through archaeological (e.g. Balme et al. 2009, Armitage et al. 2011), genetic (e.g. Macaulay et al. 2005), geographic (e.g. Field and Lahr 2005, Field et al. 2007) and environmental (e.g. Boivin et al. 2013) means.

Some researchers have highlighted the calculations of mtDNA coalescence dates that place the MRCA c. 50-60 ka, in addition to the evidence for the colonisation of Australia by c. $45 \mathrm{ka}$. Building on these dates, they have argued that AMH dispersed across the Old World in only a few thousand years, and that dispersals must 
therefore have been along the coast as this would provide the most predictable environment (Macaulay et al. 2005, Mellars 2006a, 2006b, Bulbeck 2007, Balme et al. 2009, Mellars et al. 2013). However, if earlier dates for the MRCA are accepted, dispersal does not have to have been so rapid, and therefore does not require the use of 'coastal highways'. In contrast, inland routes making use of river valleys have also been proposed (Korisettar 2007, Boivin et al. 2013).

A recent review of various models for human dispersals (Groucutt et al. in review) divides approaches into two categories - the Early (MIS 5) Onset Dispersal Model, and the Late (post-MIS 5, usually MIS 3) Dispersal Model. They set out their arguments in favour of the former, noting however that this does not preclude the existence of additional later dispersals as advocated by the latter. They conclude that the current evidence base does not allow for the exclusion of either model. However, the inclusion of additional evidence bases may add to the debate.

\section{THE SEARCH FOR AN ARCHAEOLOGICAL SIGNATURE OF EARLY HOMO SAPIENS DISPERSALS}

The fossil evidence for the earliest $H$. sapiens dispersals is patchy at best, and genetic studies cannot definitively tell us when dispersals took place. Some researchers have therefore turned to the archaeological record to look for signatures of human dispersals. In some parts of the world, such as Australia and the Americas, this is straightforward as $\mathrm{AMH}$ are the only hominin species to have reached the region. In Europe the replacement of Neanderthals by AMH over a few thousand years (Higham et al. 2014) is marked by a change in the archaeological record from the Middle to the Upper Palaeolithic. 
However, in Asia and along the 'southern arc' of dispersal there is no obvious package of material culture that heralds the arrival of AMH (as discussed in Chapter 2.2). Proponents of the Late Dispersal Model tend to argue that AMH expansion out of Africa was facilitated by new innovations in stone tool types. Specifically, they look to microliths as the signature of AMH dispersals, which is discussed in the next section. In contrast, researchers who favour the Early Onset Dispersal Model look to the Middle Palaeolithic, focussing on lithic technology rather than typology. This technological approach is explored in this section, as it is currently lacking in - and of potential benefit to - the microlithic model for human dispersal.

One example of the recent use of technological comparisons between Middle Palaeolithic industries in order to discern evidence for human dispersals concerns assemblages in northeast Africa and Arabia. Along with the Levant, Arabia was the first region outside Africa to be colonised by AMH (Beyin 2006, Petraglia et al. 2010). Comparison of lithic assemblages in this region with contemporaneous ones in northeast Africa is therefore of importance to models of human dispersals. Armitage et al. (2011) assess lithic technology in the earliest layers of the site of Jebel Faya, UAE, c. $125 \mathrm{ka} \mathrm{BP}$, and argue that it is more similar to MSA assemblages in northeast Africa than to other Arabian sites. For example, there is a mix of Levallois, volumetric blade and parallel reduction methods. They conclude that this assemblage is indicative of AMH expansion out of Africa and into Arabia in early MIS 5, when sea levels were low and the distance across the Bab al-Mandab strait 
was at its shortest. Petraglia et al. (2011) also report a site in Arabia, dated to c. 75 ka BP, which they consider to be consistent with the evidence from Jebel Faya.

In a similar vein, Levallois core reduction methods found in the Nubian Complex of northeast Africa have also been reported from southern and central Arabia during MIS 5 (Rose et al. 2011, Usik et al. 2013, Crassard and Hilbert 2013). However, it has been called into question whether the Nubian Complex can be considered a marker of a particular population, or whether it represents convergence (Groucutt et al. in review). To complicate the matter further, quantitative attribute analysis of lithic assemblages from the Middle Palaeolithic site of Jubbah in northern Arabia and various northeast African MSA sites during MIS 5 provides a mixed picture (Scerri et al. 2014). Analysis reveals that some elements of early stage core reduction are distinct at Jubbah, while other aspects of later stage core reduction and flake production are similar to the African sites. Scerri and colleagues conclude that more than one Middle Palaeolithic population inhabited Arabia during MIS 5, and that an African origin for the Jubbah assemblages - and therefore potentially a population connection, or dispersal - cannot be ruled out.

As discussed above, Armitage et al. (2011) demonstrate that technological innovation was not necessary to facilitate migration into Arabia. Additionally, several studies of lithic technology in Middle Palaeolithic Indian sites in the Jurreru Valley, India, demonstrate technological continuity in South Asia before and after the 74 ka Toba super-eruption (Petraglia et al. 2007, Haslam et al. 2010, Clarkson et al. 2012). Together with evidence for technological continuity in India between the Middle and Late Palaeolithic (James 2011), there is no sudden change in the 
archaeological record which could be associated with a population replacement or dispersal. Clarkson (2014) reaches the same conclusion in his review of the evidence for archaeological signatures of modern human dispersal. It is therefore entirely possible that there is no aspect of lithic technology or typology that can be used as a marker for human dispersals. Nevertheless, it is still necessary to thoroughly test any proposed archaeological proxies for dispersals.

One major difficulty faced in the assessment of dispersal models is in the distinction between homology and analogy. As O’Brien (2010: 313, author's emphasis) defines them, "[h]omology implies relatedness through some transmission process, whereas analogy implies convergence on a common solution to a problem without transmission". Archaeological tests for either of these explanations for similarities between lithic assemblages are difficult to formulate; witness the depths of the debate concerning whether similarities between variants of the European Neanderthal Mousterian techno-complex represent functional or cultural connections (Bordes 1961a, Mellars 1970, 1996, Binford 1973), and the ongoing debate about whether similarities between European Upper Palaeolithic Solutrean points and North American Clovis points were the result of population dispersal (most recently refuted by O'Brien et al. 2014).

Debates often focus on issues of social learning and cultural transmission, and it can be very difficult to relate this to the archaeological record (O'Brien 2010). Building on Bordes (1969, Bordes and Sonneville-Bordes 1970), Beyin (2006) suggests that typological similarities could be argued to reflect population movements (i.e. dispersal), while technological similarities reflect similar adaptive 
behaviours. However, this ignores the interplay between typology (formal tool forms) and technology (the manufacturing methods used to produce them). A better approach would be one that takes both lithic technology and typology into account.

A basic starting point for the consideration of homology v. analogy is presented in Figure 2.3.1, which recommends expectations of archaeological assemblages based on what form variation between assemblages takes. This approach builds on Clarkson's (2010: 43) assertion that “core technology is a culturally transmitted practice that is more conducive to tracking cultural phylogenies than retouched implements that have undergone strong selection and convergence for functional properties". For example, if the same tools are being produced in the same way at two different sites, cultural diffusion or inheritance (homology) might provide the most parsimonious explanation. However, if the same tool types are being made using different production techniques, equifinality in tool form might be assumed and analogy might be considered a better explanation. I.e. evidence for different groups of people producing the same types of tools due to similarities in tool requirements, but producing them in different ways due to differences in (culturally inherited) traditions of tool production, can most parsimoniously be considered as indicative of independent invention. Other factors must also be taken into account, such as the general contexts of the sites involved, geographical distance, and chronological control. Nevertheless, this is one starting point for the consideration of whether similarities in tool production represent homology or analogy. 
Figure 2.3.1: A basic schematic for distinguishing between homology and analogy in archaeological assemblages on the grounds of parsimony

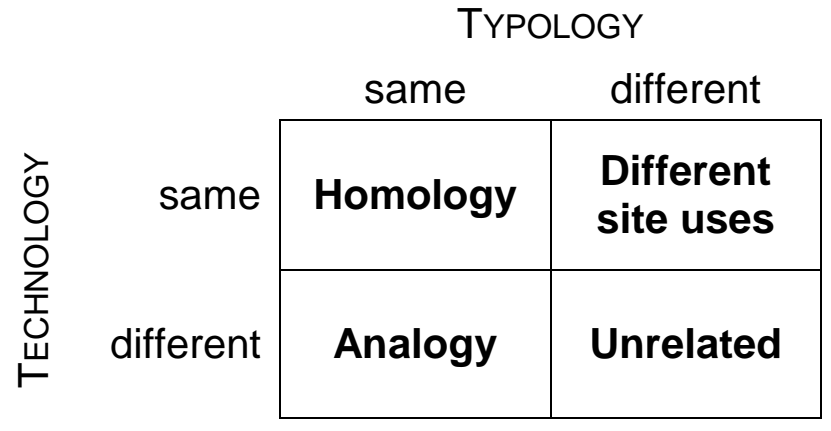

\section{THE MICROLITHIC DISPERSAL MODEL}

Microliths have been cited by proponents of the Late Dispersal Model as a proxy for human dispersals. Most recently, Mellars (2006b, Mellars et al. 2013) hypothesises that microliths can be viewed as a signature of, or a proxy for, the earliest modern human dispersals out of Africa. He is not the first to argue that microliths reflect population movements, however; in fact, the tradition is over 100 years old. Explanations based on cultural diffusion were among the first theoretical models proposed from the archaeological record, and microliths were not immune to such hypothesising.

From the time of their earliest discovery it was argued that similarities between microlith forms in Europe, Africa and Asia implied "an industry so distinctive in character, [which] probably represents the work of a particular race, or wave of population which has migrated westward in the direction in which they have been found" (Brown 1889: 135). This hypothesised movement of microlith-using people was originally viewed as originating in India and spreading west to the Near East and Europe. Comparisons of illustrations of microliths from different regions of the 
world have a similar antiquity; Figure 2.3.2 and Figure 2.3.3 are comparison illustrations from the late $19^{\text {th }}$ century.

Figure 2.3.2: Illustrations comparing microlith forms from India (1-8), France (9-15) and Syria (16) (Brown 1889, Figures 1-16). Scale unavailable.
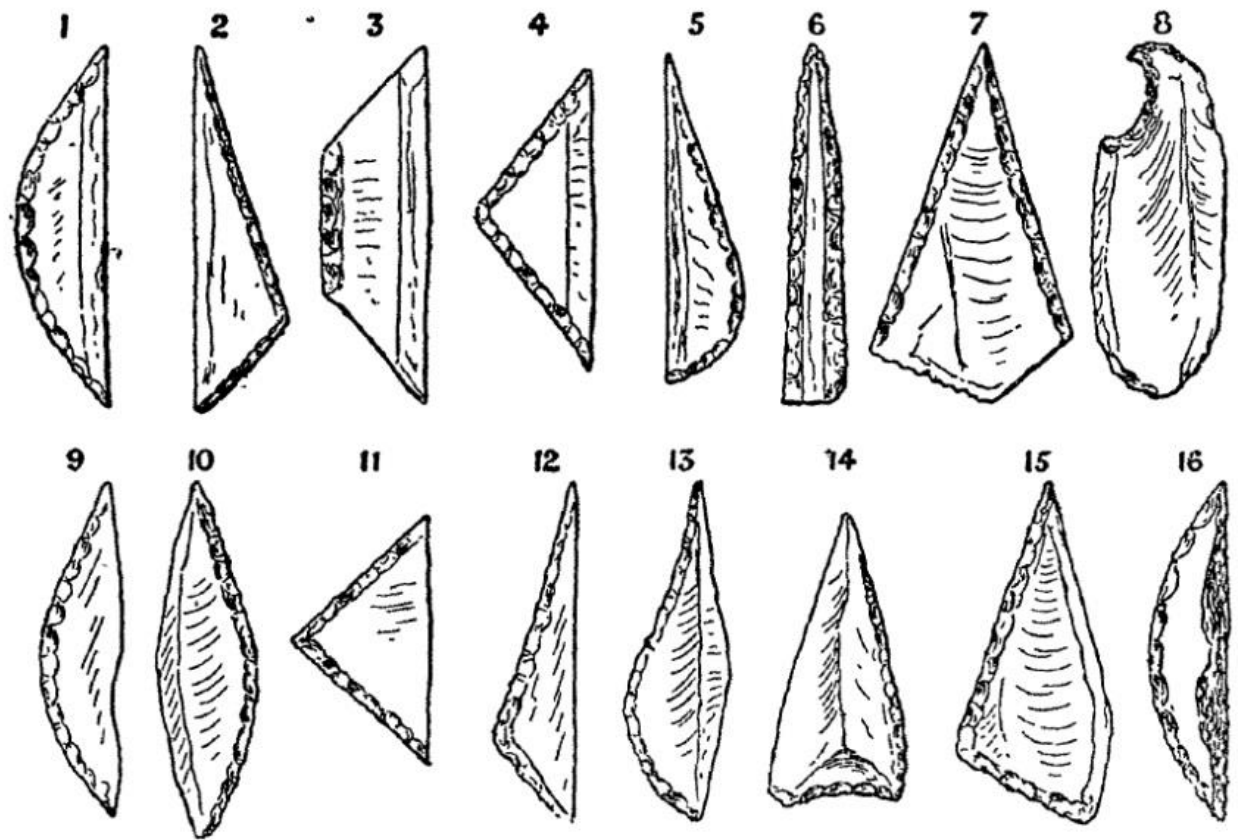

Smali Stone Implements. (Natural Size).

Figure 2.3.3: Illustrations comparing microlith forms from Hastings, England and the Vindhya Hills, India (Evans 1897, Figure 232). Scale unavailable.

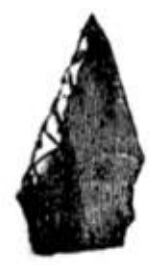

Fig. 232A.

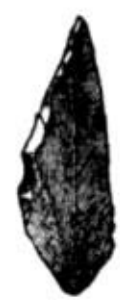

Fig. 232B. Hastings.

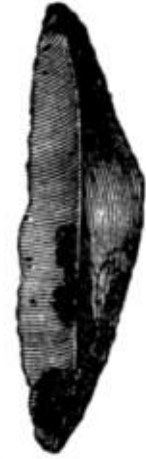

Fig. 232c. $\frac{1}{1}$
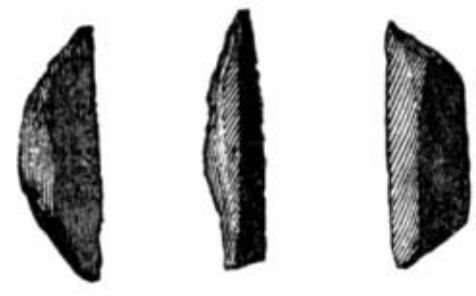

Fig. 232D. Fig. 232E. Fig. 232F. $\frac{1}{1}$ Vindhya Hills.

Not all pioneers of microlith research agreed with the argument advanced by some (e.g. Brown 1889, Gatty 1895) that resemblances between European, African and 
Asian 'pygmy flints' are so extreme that they could only be explained by a migration of people. For example, Evans (1897: 325) argued that "[s]uch an identity of form at places geographically so remote does not imply any actual communication between those who made the tools, but merely shows that some of the requirements of daily life, and the means at command for fulfilling them being the same, tools of the same character have been developed, irrespective of time or space".

Other researchers concluded that the issue deserved further investigation. Read and Smith (1911: 126), for example, noted that "the similarity of [pygmy flint] form is sufficiently striking to deserve careful attention", while cautioning against the automatic assumption that similar tool forms implies production by the same people. Hartley (1914: 55-56) agreed, summarising that "it is unnecessary to suppose that similarity of implements proves identity of race. Stone tools from all parts of the world offer a general similarity of types". Perhaps the best conclusion at the time was reached by Smith:

"The theory of the migration from India to Europe of a peculiar race specially addicted to the manufacture of 'pygmy flints', which settled only in certain widely scattered localities, obviously is at best equally open to objection [as the model of independent innovation] and it is difficult to work out that theory in a plausibly coherent form so as to give a probable explanation of the puzzling facts" (Smith 1906: 191)

A century after Smith concluded that neither the model of cultural diffusion nor that of independent innovation could be satisfactorily accepted, the debate was brought up again. In Mellars' (unrelated) version of the microlithic dispersal hypothesis, he argues that microlith-using people spread from southern Africa to South Asia along a coastal route (Mellars 2006b, Mellars et al. 2013). He draws 
parallels between the morphologies of the small, haftable 'crescentic' stone tools of the southern African HP and those of the Indian and Sri Lankan Microlithic/Late Palaeolithic, arguing that both industries are characterised by "carefully shaped microlithic or larger 'backed-segment' forms of precisely the same range of shapes" (Mellars et al. 2013: 10702). On the basis of published illustrations of backed tools (Figure 2.3.4) and symbolic material culture (beads and engraved pieces), he argues that typological similarities between the two industries are sufficient to support a hypothesis of direct population dispersal between southern Africa and the Indian subcontinent via East Africa.

This dispersal is hypothesised to take place 60-50 ka. Microlithic technology disappears c. 60 ka in southern Africa, and does not appear until c. 38 ka in South Asia. Mellars fills in this chronological gap with a mixture of genetic evidence, and archaeological evidence from East Africa. Unlike earlier propositions of microlithic dispersals, the most recent model is able to make use of supporting genetic data, including studies of mtDNA, Y chromosomes and autosomes. From these data Mellars argues that a model comprising a single dispersal out of Africa is supported, occurring no earlier than c. $60 \mathrm{ka}$. However, genetic estimates of the MRCA are highly controversial, for the reasons outlined above.

Additionally, Mellars states that various East African sites during this time period exhibit HP-like microliths. However, Groucutt et al.'s (2015) review of the East African evidence cited in Mellars' model highlights issues with all of the relevant sites. For example, they argue that there is no HP-like material at the site of Pomongwe Cave, Zimbabwe, and that scrapers are the main tool type. Microliths 
are rare in the Naisiusiu Beds of Olduvai Gorge, Tanzania, which they argue is probably a local Later Stone Age variant. And at Mochena Barago in Ethiopia, Mumba in Tanzania and Enkapune Ya Muto in Kenya they describe the gradual local development of microlithic technology within site sequences. The autochthonous innovation of microlithic technology is also evidenced within South Asia (James 2011, Clarkson et al. 2012), and is discussed further in Chapter 3.2.

Figure 2.3.4: Illustrations comparing microlith forms from Tanzania, South Africa, India and Sri Lanka (Mellars et al. 2013, Figure 3)

AFRICA

Naisiusiu (Olduvai - Tanzania)

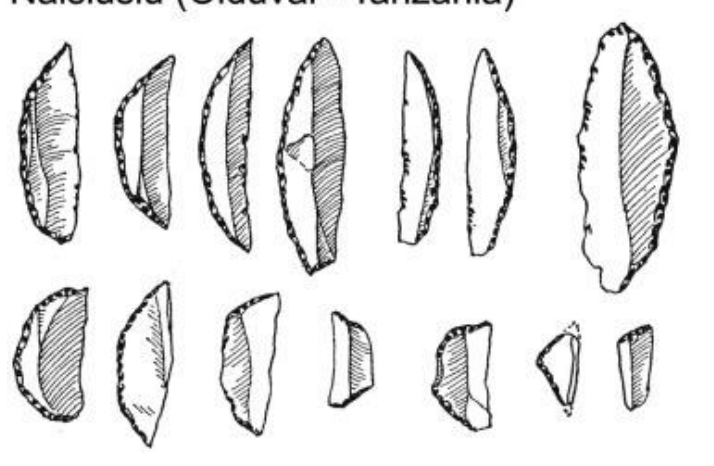

Mumba (Tanzania)
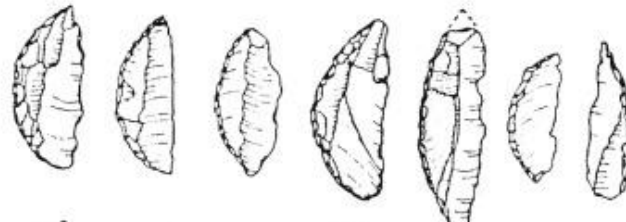

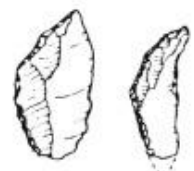
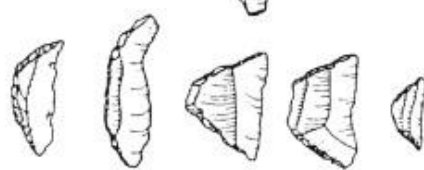

Klasies River (South Africa)
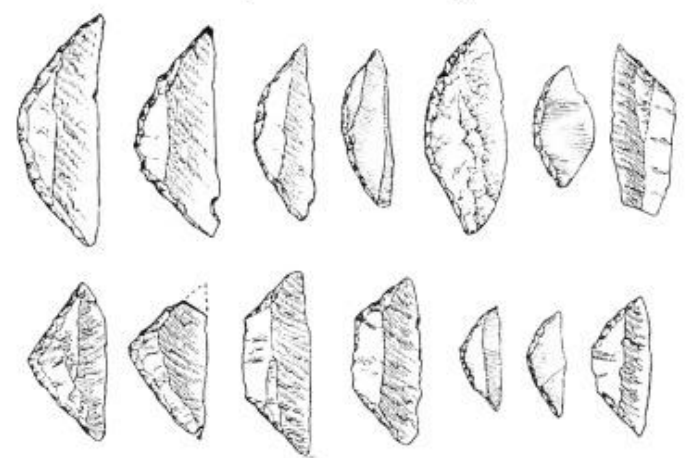

SOUTH ASIA

Jwalapuram (India)
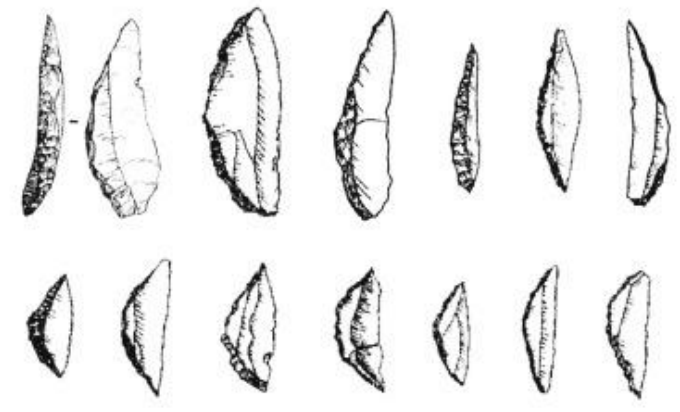

$2 \mathrm{~cm}$

Patne (India)

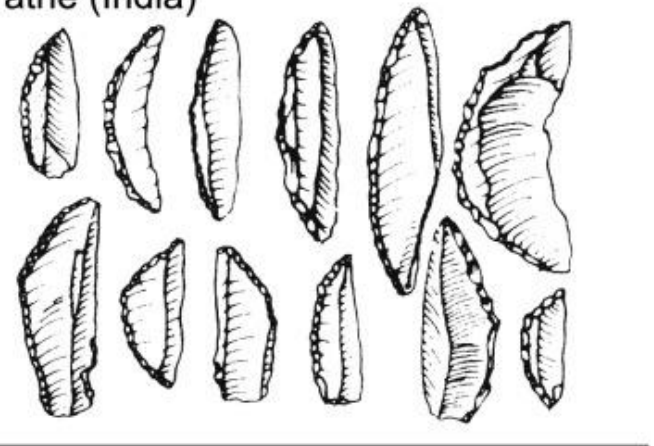

Batadomba-lena (Sri Lanka)

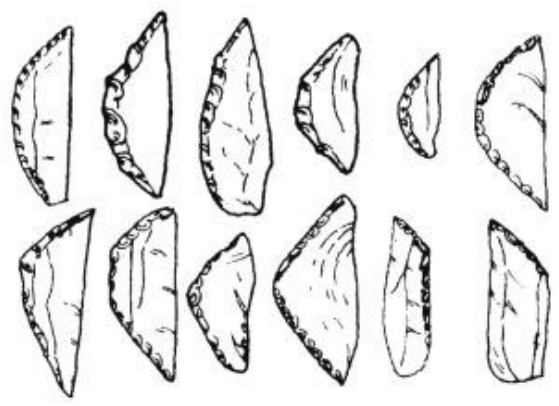


Groucutt and colleagues (2015) conclude that the earliest dispersals out of Africa occurred with MSA/Middle Palaeolithic technology. However, even if the Early Onset Dispersal Model is accepted, this framework still allows for later dispersals, potentially including one associated with microliths at the time hypothesised by the Late Dispersal Model. Therefore the microlithic dispersal model still requires testing. Although studies demonstrating the gradual development of microlithic technologies out of local industries are highly pertinent to the debate concerning microlithic dispersals, they do not directly assess the specific evidence base from which microlithic dispersals are being argued; namely that these microlithic assemblages are directly related because they share the same finished artefact forms, and therefore - by assumption - also the same methods of production.

The use of microlithic technology as an archaeological correlate of the earliest dispersals of modern humans out of Africa and into Asia has not yet been rigorously tested. Equifinality or similarities in stone tool typology could be due to convergence, as the result of many potential factors such as the hunting of similar sized prey or the degree of group mobility. The conflation of South Asian microliths with those of the HP in order to map a model of population dispersals negates the opportunity to explore underlying lithic reduction processes, which would be much more revealing in terms of our understanding of the technological systems of different populations (Clarkson 2010).

This chapter has set out some of the key issues in microlith studies. It has provided two case studies of comparative microlithic work, in the form of the debates concerning modern human behaviour and dispersals, and has explained how these 
over-arching studies have contributed towards a homogenising view of microlithic technology. It is argued here that both areas of discourse would be aided by consideration of microlithic assemblages as unique technological strategies. This can only be achieved through the use of technological attribute analysis, with the aim of highlighting and describing areas of similarity and difference in order to demonstrate the degree of homogeneity or diversity evident within and between microlithic industries. This thesis does exactly that, by comparing microlithic technology in two of the earliest microlithic industries in the world: the HP of southern Africa and the Late Palaeolithic of South Asia. 


\section{Chapter 3: Study areas and site backgrounds}

The previous chapter set out the technological and theoretical backgrounds to microlithic technology. It concluded that in order to investigate behavioural variability and to test the microlithic model of human dispersals detailed technological comparisons of microlithic industries must be conducted. This chapter sets out the general background to the two early microlithic industries analysed, before describing each site and assemblage studied.

\subsection{The Howiesons POORT Of SOUthern Africa}

The HP is one of the best-known and most thoroughly researched parts of the African MSA. Our current state of knowledge defines it as a distinctive stone tool industry found in South Africa, Lesotho and Namibia (Lombard 2005, Henshilwood 2012, Lombard et al. 2012, Cochrane et al. 2013). Figure 3.1.1 maps some of the key HP sites. Some researchers have made much of the seemingly coastal distribution of HP sites (e.g. Mellars et al. 2013). However, the mean distance of HP sites from the modern coast (a minimum value for the distance between HP sites and their contemporary coastline) is $105 \mathrm{~km}$, and ranges up to $350 \mathrm{~km}$ in the case of Rose Cottage Cave (Groucutt et al. in review) or potentially $630 \mathrm{~km}$ in the case of Kathu Pan. Any site patterning near to the coast is most likely simply an artefact of where most research has been conducted. 
Figure 3.1.1: Map of key HP sites, highlighting in bold those analysed in the present study

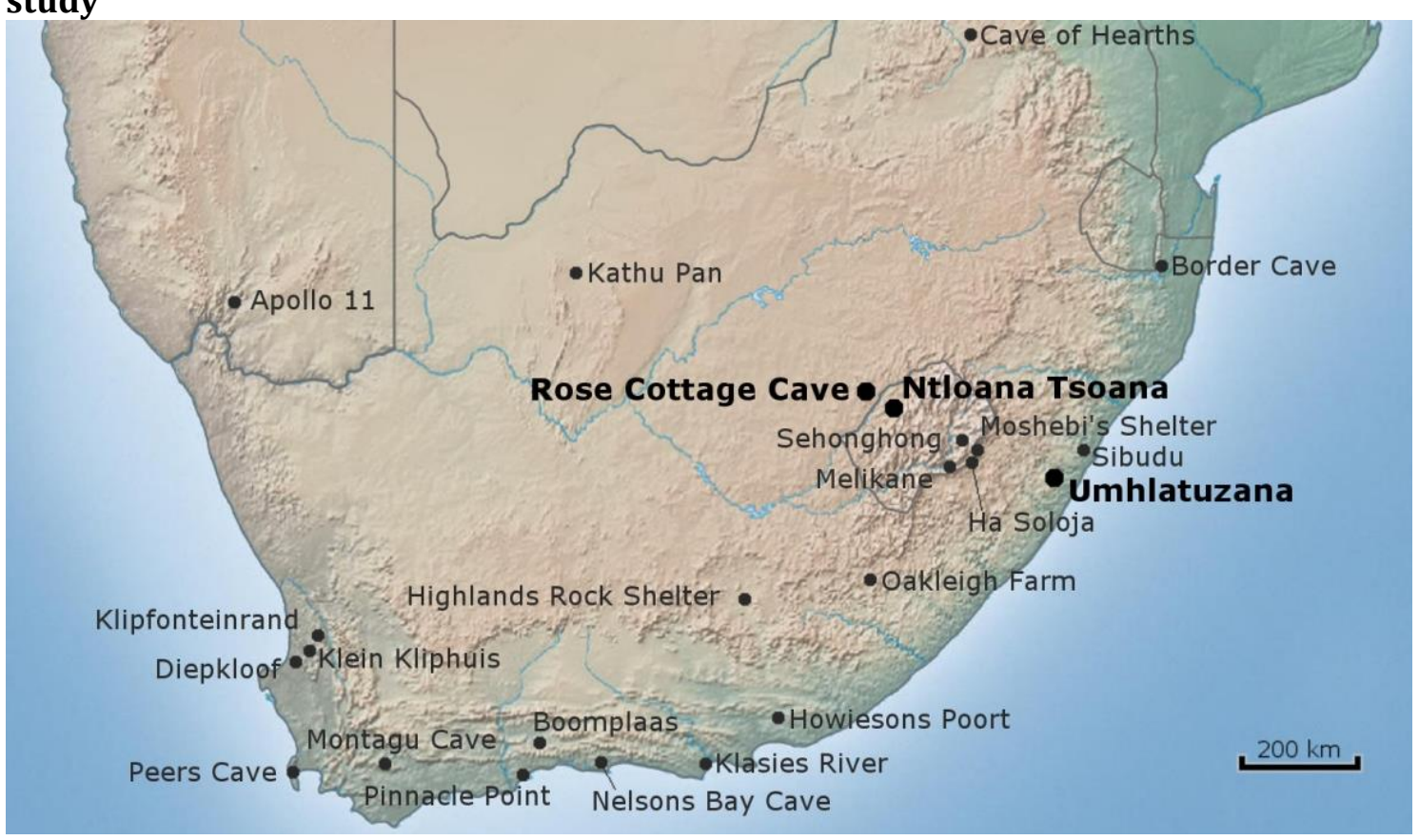

The industry was first defined after the excavation of the Howiesons Poort rockshelter in the 1920s by Stapleton and Hewitt $(1927,1928)$. Similarities were noted with both the Wilton and Still Bay industries, leading the excavators to conclude that the three industries were contemporaneous (Stapleton and Hewitt 1928: 407-409). For several decades researchers continued to argue that the HP was either a stage within the Still Bay industry (Malan 1952) or, more commonly, transitional between the MSA and LSA (Clark 1959, Hole 1959). However, excavations in the 1960s at the site of Klasies River (Singer and Wymer 1982), containing HP layers sandwiched between earlier and later MSA assemblages, demonstrated that the HP was in fact a lithic industry within the MSA.

A wide variety of dating techniques has been applied to HP contexts (most recently summarised by Henshilwood 2012). Today the most commonly accepted dates are those produced through a program of single-grain OSL dating conducted by Jacobs 
et al. (2008). These dates place the HP within a tight chronological boundary between 64.8 and $59.5 \mathrm{ka} \mathrm{BP}$, at the end of MIS 4. A recent attempt by Lombard et al. (2012) to construct a comprehensive technological sequence for the Stone Age of South Africa and Lesotho also makes use of these dates. However, there is some disagreement concerning the dates' reliability and accuracy (Guérin et al. 2013, Tribolo et al. 2013, Feathers 2015, cf. Jacobs and Roberts 2015), and both younger and older dates exist at several sites (Henshilwood 2012, Lombard et al. 2012, Brown et al. 2012, Wurz 2013).

The HP is characterised by small (typically less than $40 \mathrm{~mm}$ ) frequently backed tools, especially on blades (Lombard 2005, Henshilwood 2012), often retouched into geometric shapes such as segments, triangles and trapezes. Some researchers prefer to avoid the term 'microlithic' and instead refer to the HP as being typified by backed 'crescentic' stone tools or segments (Mellars 2006b, Wadley and Mohapi 2008, Mellars et al. 2013), in some cases to distinguish these tool forms from their smaller LSA equivalents (Wadley and Mohapi 2008). However, as HP microliths fit within the size range of those documented elsewhere (Elston and Kuhn 2002, Hiscock et al. 2011) most researchers refer to the HP as a microlithic industry (e.g. Ambrose 2002, Lombard 2005, Cochrane et al. 2013). Furthermore, referring to the HP as being characterised primarily by crescentic stone tools ignores the existence of non-geometric microliths, which at many sites make up the majority of the microlith assemblage (e.g. Kaplan 1990, Mitchell and Steinberg 1992, Villa et al. 2010, Porraz et al. 2013). 
Material culture described as 'symbolic' is rare within the HP. The only concrete examples are the pieces of incised ostrich eggshell at Diepkloof (Texier et al. 2010) and Klipdrift (Henshilwood et al. 2014). Claims have been made for incised or engraved bone at Klasies River (d'Errico and Henshilwood 2007) and Sibudu (Wadley 2006), although the potential decorative and/or symbolic nature of these markings remains to be proved. A fragment of engraved ochre at Klein Kliphuis is more convincing, but originates from a mixed HP and post-HP assemblage (Mackay and Welz 2008).

Multiple explanations have been proposed for the appearance and disappearance of the HP. The most frequently discussed tend to revolve around changes in mobility, resource exploitation and social organisation as a response to changing environmental conditions toward the end of MIS 4 (Ambrose and Lorenz 1990, Ambrose 2002, McCall 2007, McCall and Thomas 2012, Ziegler et al. 2013, Dusseldorp 2014). More specifically, it is also argued that climatic deterioration resulted in the creation of exchange networks as a method of risk buffering, and that aspects of the HP including the increased use of fine-grained materials and the manufacture of both microliths and symbolic material culture played a part in these networks (Deacon 1989, 1995, Wurz 1999, Ambrose 2002).

Following these lines of argument, the ecological pressures resulting in the proposed changes in resource and raw material procurement, and/or the organisation of social networks, declined as a result of climatic amelioration at the onset of MIS 3, resulting in the transition to post-HP industries. However, some palaeoenvironmental studies indicate a disconnect between the timings of 
environmental changes and of the origins and end of the HP (e.g. Clark 2013). Others suggest that the HP actually occurred within relatively warm temperatures at the end of MIS 4, and that the immediate post-HP period in MIS 3 may in fact have been colder and drier (Thackeray 1992, 2009, Bruch et al. 2012).

Not all hypotheses concerning the HP depend solely on environmental and ecological arguments. They also include models of population replacement (Singer and Wymer 1982, McBrearty and Brooks 2000), invocations of cognitive changes (Lombard and Haidle 2012) and spiritual considerations (Lewis-Williams and Pearce 2004). However, it must be noted that the few studies of long-term sequences at HP sites which include pre- and post-HP MSA assemblages tend to highlight overall technological continuity throughout the period on a larger scale (e.g. Thackeray 1989, Harper 1997), rather than technological breaks which might imply the influx of new populations. Overall, the situation is most likely a mix of internal and external factors, the precise relationships of which are impossible to tease out at the level of resolution currently available to us, for example in environmental proxies.

As Cochrane et al. (2013) note, the OSL dates of Jacobs and colleagues (2008) indicate that the HP lasted for a time period perhaps a third of the length of MIS 4, and so we can no longer state that MIS 4 as a whole was the cause of the HP. However, this does not mean that the HP was not necessarily the result of smallerscale environmental or climatic fluctuations; the resolution of our climatic proxies does not at this point allow us to see these potential smaller-scale changes. 
Nevertheless, Cochrane and colleagues argue that the HP is a much more complex cultural entity than is often assumed by explanatory models.

Furthermore, as our understanding of HP chronology and stratigraphy improves, researchers have become increasingly aware of both the temporal and the geographic variability within the industry on a smaller scale (Cochrane et al. 2013). The occurrence of distinctive phases within HP sequences has been suggested at Rose Cottage Cave (Wadley and Harper 1989), Sibudu (Wadley and Mohapi 2008) and Diepkloof (Porraz et al. 2013). There is also evidence for variability in lithic technology between sites. Wadley and Harper (1989) demonstrate several areas of difference between the HP at Rose Cottage Cave and elsewhere in terms of proportions of stone tool types. Lombard (2005) notes further differences in stone tool proportions at other HP sites. Clarkson (2010) analyses core technology from five HP sites using a combination of attribute analysis and 3D analysis of scar patterning, concluding that similar backed microlith forms were being produced from different core reduction methods at each site. Most recently, reduction techniques unique to individual HP assemblages have been reported. For example, Porraz et al. (2013) note the existence of a bifacial chaîne opératoire in the early HP at Diepkloof. Additionally, de la Peña and Wadley (2014) describe the use of different knapping methods for different raw materials in the HP assemblage at Sibudu, including a new method of prismatic blade production.

Another area of technological variability in the HP is in the realm of hafting and adhesives. A recent molecular study of residue found on a quartz flake at the site of 
Diepkloof resulted in the identification of Podocarpus elongatus (Yellowwood) resin (Charrié-Duhaut et al. 2013), in contrast to the ochre-based hafting residues found at Sibudu Cave (Lombard 2006, 2007, 2008), Rose Cottage Cave (Gibson et al. 2004) and Umhlatuzana (Lombard 2007). These glimpses of potential differences between sites indicate that the HP was a variable and dynamic industrial complex, and the investigation of technological variability within this industry is potentially a fruitful avenue for research.

Three HP sites were selected for analysis: Rose Cottage Cave and Umhlatuzana, South Africa, and Ntloana Tsoana, Lesotho (Figure 3.1.1). These sites were chosen as they contain HP deposits that have been excavated in multiple contexts and dated through OSL methods (Jacobs et al. 2008, Lombard et al. 2010, 2012), but have not yet been analysed using the detailed methodology employed here. Technological analysis of the lithics from the original excavations at RCC has not previously been conducted, and the only attribute analysis of the more recent excavations focussed exclusively on blades (Soriano et al. 2007). Umhlatuzana is a particularly rich site (with over one million lithics recovered), but the technology of the HP lithics has not yet been analysed. The lithics from Ntloana Tsoana are from a recent (2009-12) excavation and this study provides the first analysis of the HP assemblage. Ntloana Tsoana was also selected as it is in the same river valley system as RCC and only $35 \mathrm{~km}$ away (in a straight line), providing a useful point of comparison. 


\subsubsection{Rose Cottage Cave, South Africa}

Rose Cottage Cave (RCC) $\left(29^{\circ} 13^{\prime} \mathrm{S}, 27^{\circ} 28^{\prime} \mathrm{E}\right)$ is one of the best known HP sites in southern Africa, containing $6 \mathrm{~m}$ of MSA and LSA deposits. It is located $3 \mathrm{~km}$ east of Ladybrand in the eastern Free State of South Africa, near the Platberg River in the Caledon River corridor (Wadley and Harper 1989, Wadley 1991, 1997). The site has been subject to three periods of excavation; by B.D. Malan in 1943-46, by Peter Beaumont in 1982, and most recently by Lyn Wadley in 1987-98 (Wadley 1991, Soriano et al. 2007). The cave is approximately $20 \mathrm{~m}$ long and $10 \mathrm{~m}$ wide, eroded into the Karoo sandstone. The local environment at the site is currently predominantly grassland (Mitchell 2002) with some scrubland patches (Wadley 1997) (Figure 3.1.2). Unfortunately there is no bone preservation in layers older than 20 ka (Plug and Engela 1992, Jacobs et al. 2008), and organic preservation below this level is rare or absent, with the exception of charcoal. Little is therefore known about the local environment of the site during the MSA.

Figure 3.1.2: The sandstone ridge in which RCC is located

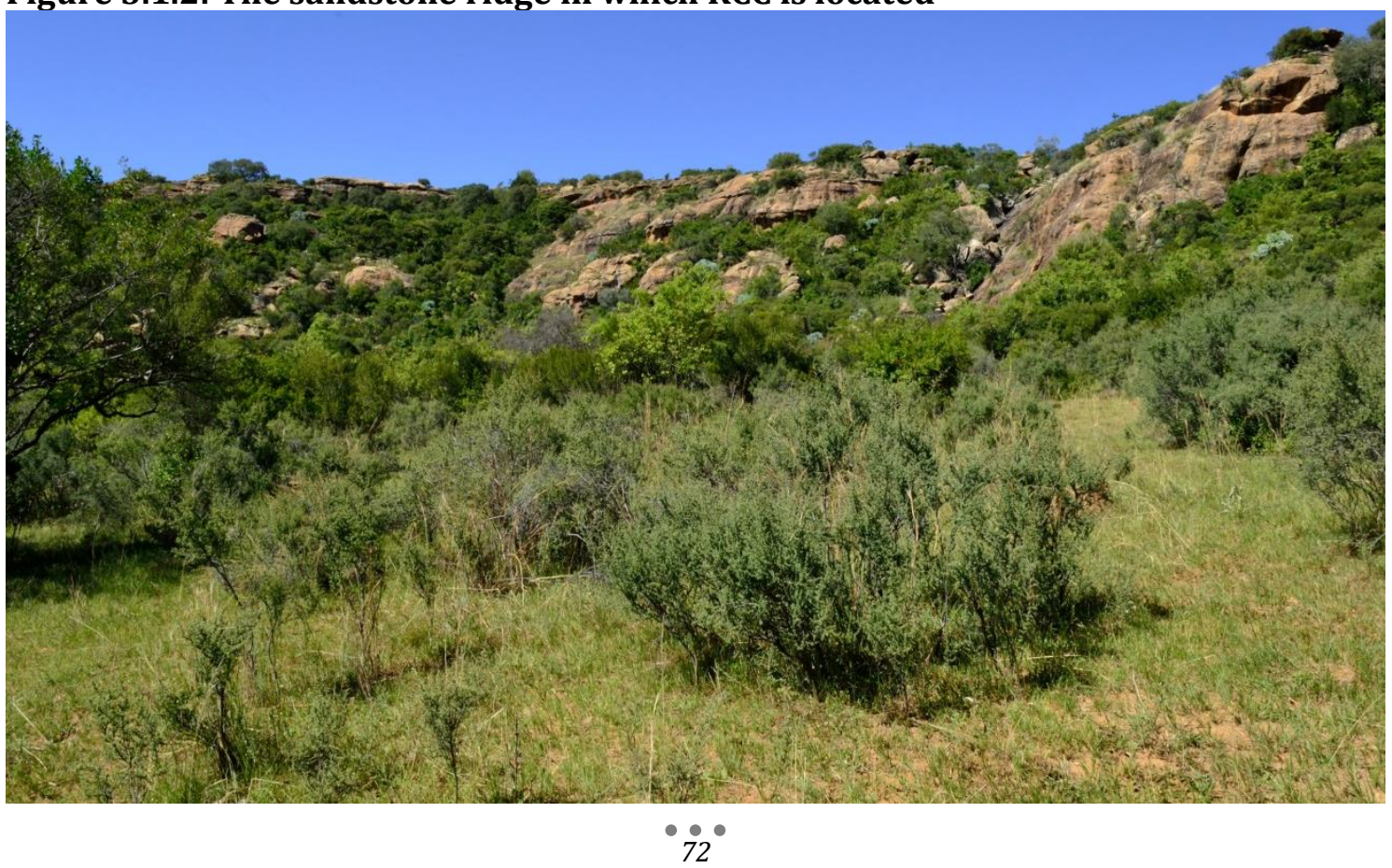


HP layers have been OSL dated; two dates from the lower part of the HP sequence gave ages of $63.3 \pm 2.3 \mathrm{ka} \mathrm{BP}$ and $65.0 \pm 3 \mathrm{ka} \mathrm{BP}$, and a date from the upper part of the sequence gave an age of $63.0 \pm 2.3 \mathrm{ka}$ BP (Jacobs et al. 2008). A date from the oldest post-HP assemblage gave an age of $56.0 \pm 2.3 \mathrm{ka}$ BP. An alternative OSL dating program gave similar end dates for the $\mathrm{HP}$, of $62.5 \pm 2.9 \mathrm{ka}$ BP or $64.1 \pm 3.0$ ka BP, depending on the source of the $\mathrm{U}$ and Th dose measurements (Pienaar et al. 2008). However, it indicated that the earliest HP at the site may date slightly earlier, at $66.9 \pm 2.6 \mathrm{ka}$ BP or $68.7 \pm 2.7 \mathrm{ka}$ BP. The OSL dates roughly correspond with those achieved through TL dating of burnt lithics at the site (Valladas et al. 2005): TL ages between $56.3 \pm 4.5$ and $60.4 \pm 4.6$ ka BP were given for the top of the HP sequence, with a weighted mean age of $58.2 \pm 4.2 \mathrm{ka}$ BP.

Malan only published a short report of his excavations (Malan 1952), with no analysis of the lithics recovered. These were later counted and published by Wadley and Harper (1989), using a typology based on that of Singer and Wymer (1982) and Thackeray and Kelly (1988). They suggested there may be two or three phases within the HP at RCC. Wadley and Harper highlighted differences between the RCC HP and HP assemblages at other sites, including relatively small proportions of segments and trapezes and relatively large proportions of backed and obliquely backed blades, noting that "[m] uch variability is thus evident in Howiesons Poort assemblages, even where sites are geographically close" (Wadley and Harper 1989: 31).

The lithics from Beaumont's excavations in the 1960s were analysed by Kohary in an attempt to investigate aspects of production techniques. However, she mis-read 
the stratigraphy and mixed the assemblages (Wadley and Harper 1989), and the first reliable technological analysis at the site did not occur until almost two decades later (Soriano et al. 2007). The HP lithics from Wadley's excavations in the 1980s and 90s were first published by Harper (1997), whose typological analysis suggested a considerable degree of continuity between the various MSA assemblages at the site, as well as some variability within the HP sequence in terms of tool frequencies.

A technological approach to the lithics excavated by Wadley and Harper was taken by Soriano et al. (2007), who looked at the chaîne opératoire of blades in HP and post-HP assemblages. Their results confirmed Wadley and Harper's (1989, Harper 1997) findings regarding variability within the HP sequence and gradual change between the HP and post-HP. Additionally, Clarkson's (2010) analysis of cores at RCC, as part of a larger comparison study of HP core reduction, found that knapping at the site was much more focussed on blade production compared to other HP sites. Finally, residue analysis has also been conducted at RCC, on backed tools from HP levels, revealing ochre and plant residues on the backed portions of the tools (Gibson et al. 2004). These results add further support to the argument that these microliths were hafted.

A total of 1538 lithics was analysed from the site in the current study. The sample was taken from Malan's excavations rather than the more recent excavations, as the latter did not contain enough lithics to meet the sampling criteria of this study. Analysis focussed on the 'classic' HP as documented by Wadley and Harper (1989), between 144 and 204 inches (Figure 3.1.3), excluding potentially mixed levels 
either side. The squares analysed were $\mathrm{db}, \mathrm{eb}, \mathrm{fb}$, gb and gc (Wadley and Harper 1989), as the majority of spit depths were correlated between these squares.

Figure 3.1.3: Stratigraphic profile of RCC, with the studied section in grey (redrawn from Wadley and Harper 1989).

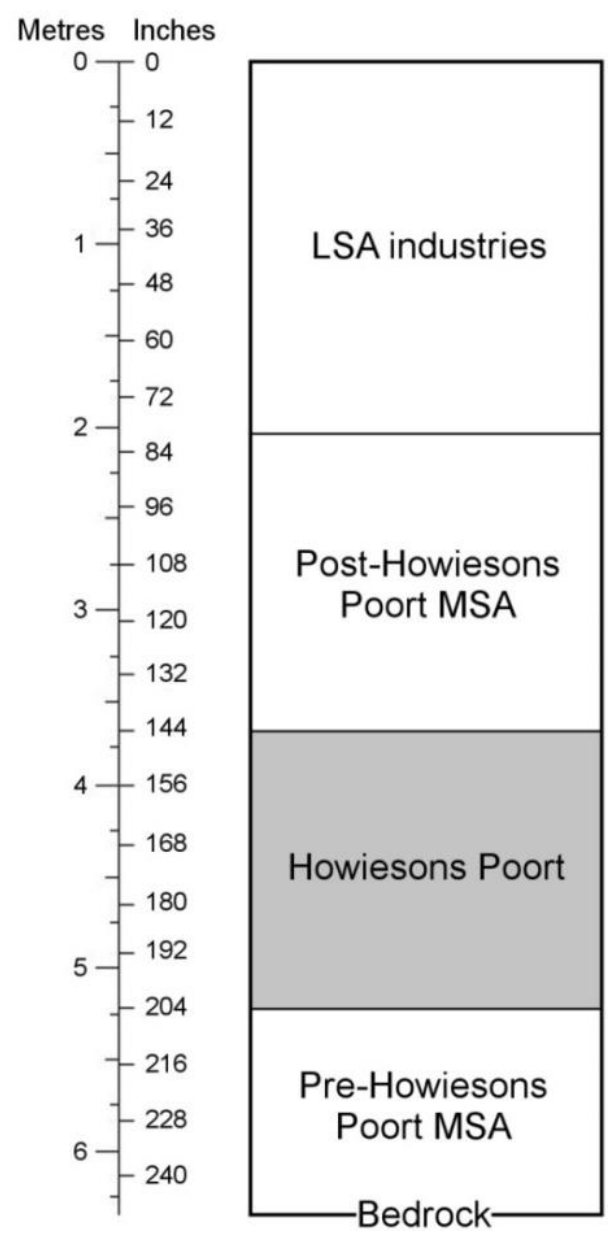

Little is known about the history of Malan's excavation, and it is therefore possible that the integrity of the assemblage is unreliable. However, comparisons with data from Wadley's later excavations at the site (Harper 1997) indicate that debitage proportions are very similar (61\% blades in Harper's report compared to $59 \%$ reported in the current study), and that a greater proportion of broken lithics exists in the current study (18.5\% compared to 5\%), demonstrating that neither flakes nor broken lithics were selectively thrown away. Any selective curation was 
minimal, and has not influenced the results of this study or made it incomparable to the other assemblages analysed here.

\subsubsection{UMHLATUZANA, SOUth AFrica}

Umhlatuzana (29 $48^{\prime} 28^{\prime \prime}$ S, $30^{\circ} 45^{\prime} 22^{\prime \prime}$ ) is a MSA site near Durban in KwaZuluNatal, South Africa, spanning from over 70 ka to the late Holocene and including Still Bay and HP industries. It is a large rockshelter, c. $43 \mathrm{~m}$ wide, located in the ortho-quartzite horizon of the Natal Supergroup, about $100 \mathrm{~m}$ above the Umhlatuzana River (Kaplan 1990). It is in a mixed woodland savannah biome (Mitchell 2002), and today is in an outlying patch of coastal forest (Kaplan 1989, 1990) (Figure 3.1.4). Excavation was conducted in 1985 as part of a rescue project, and reached a depth of $2.6 \mathrm{~m}$ divided into 28 stratigraphic levels (Figure 3.1.5).

Umhlatuzana was long neglected due to confusions over the dating and stratigraphy at the site (Mohapi 2013). However, new OSL dates have helped to clarify these issues in the earlier part of the sequence (Jacobs et al. 2008, Lombard et al. 2010, 2012). Although the dates confirm that some post-depositional mixing occurred between layers, this was confined to discrete, small-scale events. The majority of grains dated were shown to be in their primary context and so the dates are considered to be accurate, although some mixing of sediments and artefacts is anticipated (Lombard et al. 2010). Further mixing is expected due to the excavation of the lag deposits in Layers 5-28 in spits, although Lombard et al. argue that there is still a good degree of internal consistency between the layers. 
Figure 3.1.4: The gorge containing Umhlatuzana rockshelter

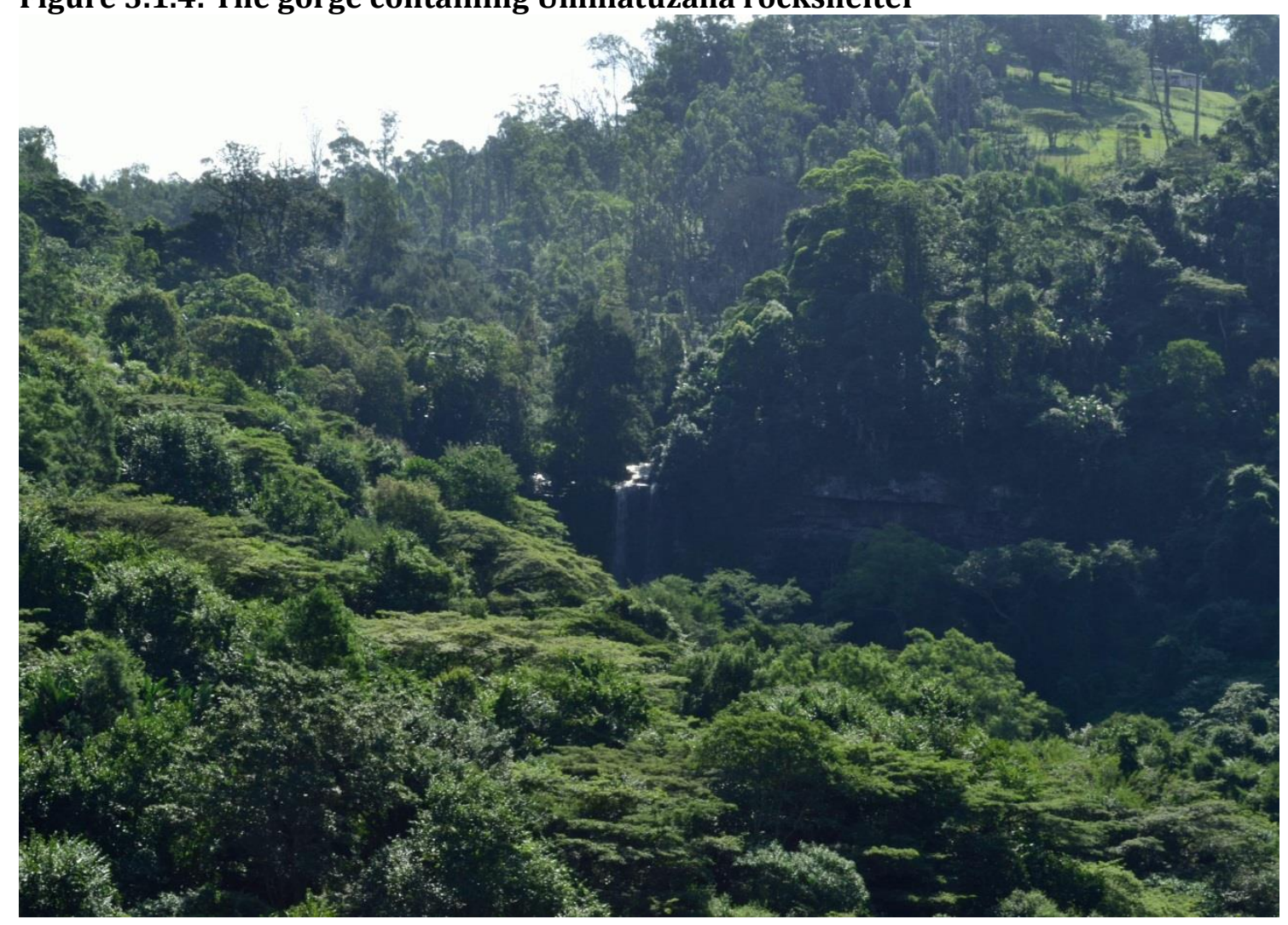

Due to this small-scale mixing there is some disagreement concerning which layers should be attributed to the HP. Layer 24, dated to $60.0 \pm 3.5 \mathrm{ka}$ BP (Figure 3.1.5), is accepted as HP (Kaplan 1990, Lombard et al. 2012, Mohapi 2013, cf. Lombard et al. 2010 who incorrectly report this as Layer 22). However, whereas the excavator states that the HP extends to Layer 26 (Kaplan 1990), Lombard and colleagues (2010) argue that it only extends to Layer 24, on the basis of an OSL date of $70.5 \pm$ 4.7 ka BP that they attribute to Layer 25. They instead assign Layers 25-27 to the Still Bay industry. However, this date actually originates from a unit which is equated to Layer 27 (Kaplan 1990). 
Figure 3.1.5: Stratigraphic profile of Umhlatuzana, with the studied layers in grey (redrawn from Mohapi 2013, with dates from Lombard et al. 2010)

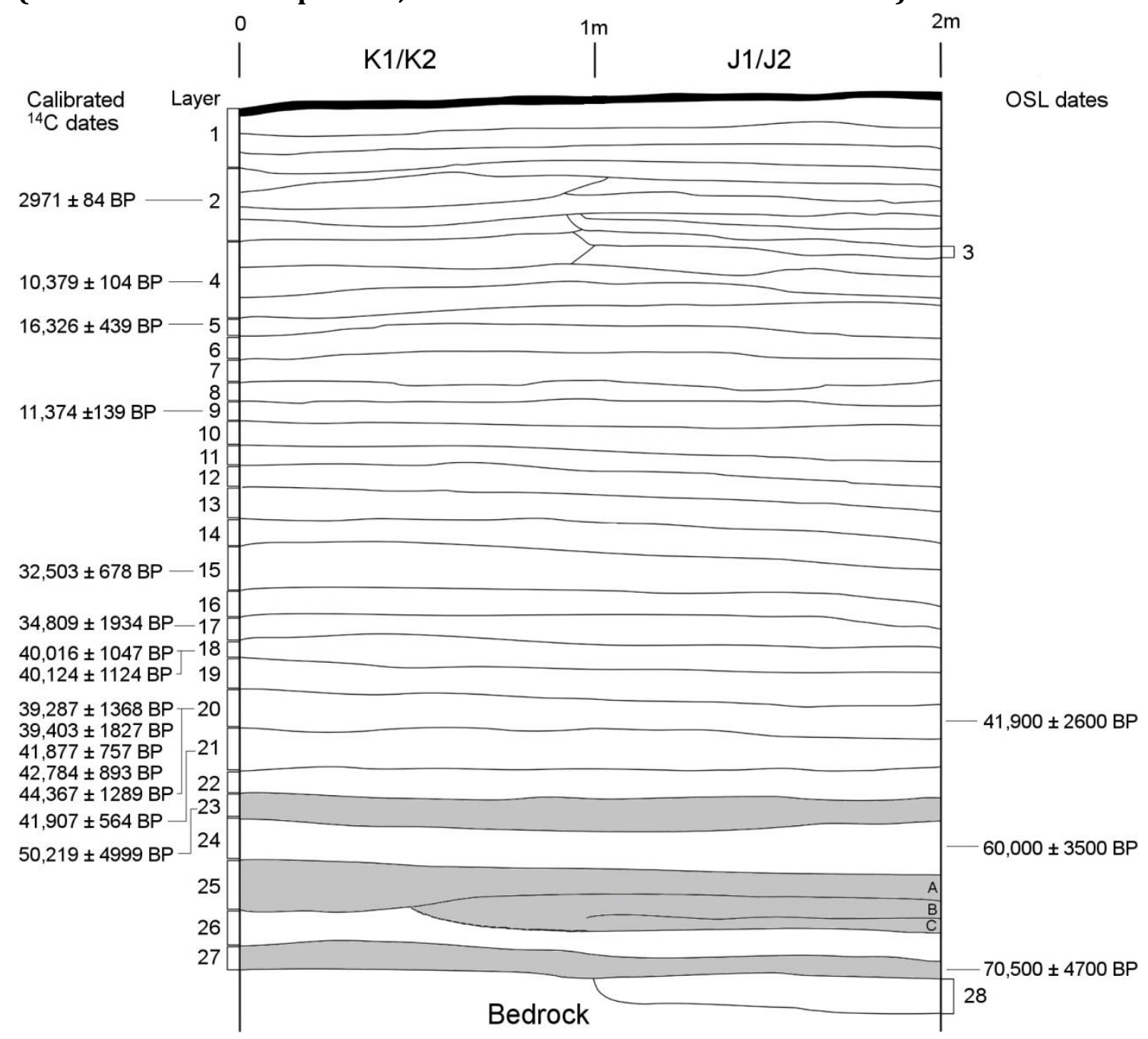

Given recent arguments that the OSL dates of Jacobs et al. (2008) may actually contain a much greater degree of uncertainty (Guérin et al. 2013, Tribolo et al. 2013, Feathers 2015), we cannot rule out the attribution of Layers $25-27$ to the HP on the basis of a single date - nor should we ascribe industries on the sole basis of dates. In fact, when the archaeological evidence is considered, the frequencies of segments, trapezoids and backed pieces remain high below Layer 24 (Figure 3.1.6). Although it is not possible to determine from Kaplan's data which of these backed pieces may fit within the size range of microliths at the site, these technological and morphometric features are typical of HP assemblages. Therefore, allowing for 
some post-depositional mixing of artefacts, it is reasonable to assert that HP occupation at the site extends below Layer 24 .

Figure 3.1.6: Diagnostic tool type proportions in lower MSA levels at Umhlatuzana (data from Kaplan 1990)

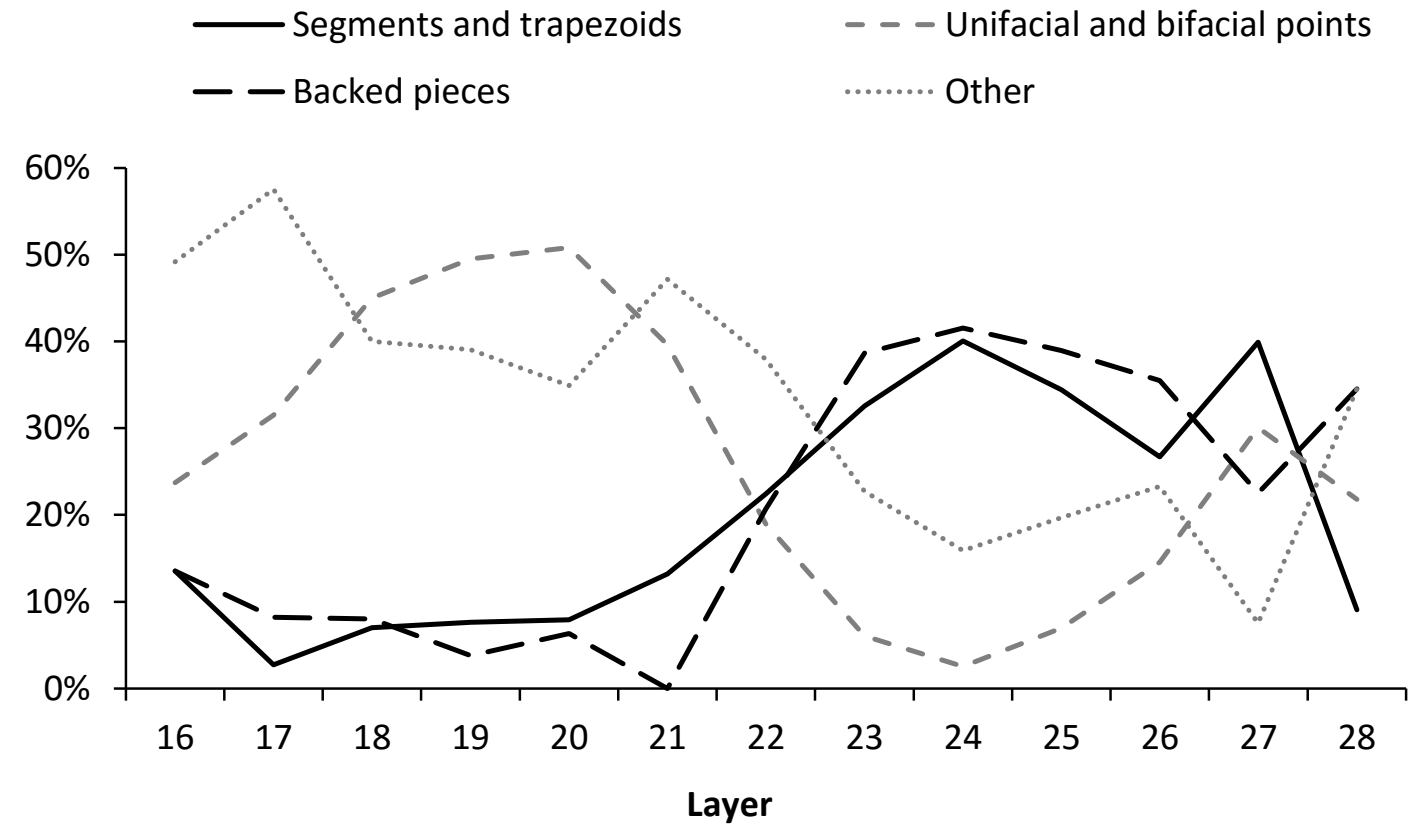

Faunal remains at Umhlatuzana were analysed by Richard Klein and Margaret Avery (reported by Kaplan 1990). Remains in MSA layers are scant and poorly preserved (none were identifiable at all in Layers 28-26), but are taken to indicate the exploitation of a wide range of bovids. Animals represented in HP layers include bushpig and small, medium and large bovids, although in very small numbers. Ground ochre was found in every layer, including those attributed to the HP. Kaplan (1990) interprets this as deliberate and systematic collection of ochre at the site. 
Umhlatuzana is rich in lithic artefacts, with over one million lithics recovered (Kaplan 1990). Until recently lithic analysis at the site consisted solely of the typological classifications conducted by the excavator (Kaplan 1989, 1990), with some length measurements. Subsequent analysis has focussed on the points within the Still Bay layers and on morphological comparisons with points found later in the sequence (Lombard et al. 2010, Mohapi 2013). However, the HP technology at the site remains poorly understood.

The lithics for the present sample were taken from Layers 23, 25 and 27 of squares J2 and K2, comprising a total of 1543 lithics (Figure 3.1.5). This selection of layers allows for an assessment of whether the latter two should be included as part of the HP sequence at the site, on the basis of lithic technological identifications rather than solely typological assertions (c.f. Lombard et al. 2010). The analysis of alternate layers was chosen in order to maximise stratigraphic integrity, given the possibility of mixing at the site. Sediments slope towards the dripline in the rockshelter. Therefore two adjacent squares at the same point on the slope were chosen from the four excavated to bedrock in order to minimise the potential compounding effect of this sloping, given that this section of the stratigraphy was excavated in spits.

\subsubsection{NTLOANA TSOANA, LESOTHO}

Ntloana Tsoana (NT) $\left(29^{\circ} 19^{\prime} \mathrm{S}, 2^{\circ} 49^{\prime} \mathrm{E}\right)$ is a rockshelter containing MSA and LSA deposits, $30 \mathrm{~km}$ east of Maseru on the south bank of the Phuthiatsana River 
(Mitchell and Arthur 2010, 2012). It is a large rockshelter with an area of $280 \mathrm{~m}^{2}$, formed in the exposed Clarens Formation sandstone (Mitchell and Steinberg 1992). The river runs in front of the mouth of the shelter, and was likely never more than a few metres away from the shelter as it is located in a steep gorge with little room for changing of the river course (Figure 3.1.7, Figure 3.1.8). As a result the site is liable to flooding, and it has been suggested that the MSA sequence accumulated as a lag deposit (Mitchell and Steinberg 1992).

\section{Figure 3.1.7: NT rockshelter}

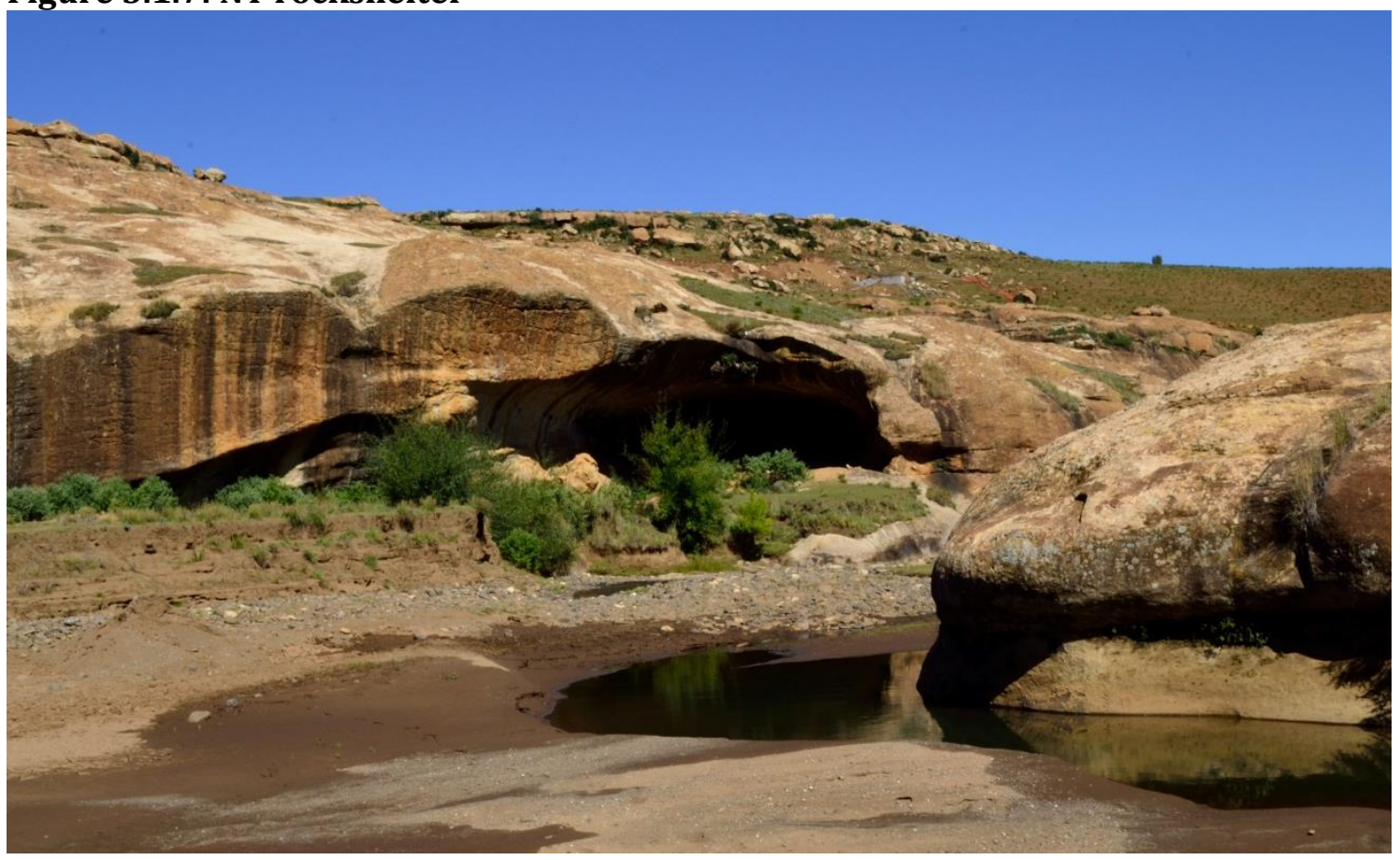

The site was first excavated in 1989 by Mitchell and Steinberg (1992), who identified three major stratigraphically distinct MSA assemblages. Using the lithic typology adopted by Wadley and Harper (1989) at RCC, they noted that the assemblages were principally differentiated by variations in the relative use of raw materials (crypto-crystalline silicas (CCS) and tuff), and by the frequencies of formal tool classes. In particular, the bottom layer (CBS) contained a relatively high 
frequency of backed pieces as well as preferential use of fine-grained CCS. As a result Mitchell and Steinberg attribute this layer to the HP, noting parallels with the HP at the nearby site of RCC. As part of their multiple-site single-grain OSL dating program, Jacobs et al. (2008) produced a date of $60.9 \pm 2.8 \mathrm{ka}$ BP from the upper part of layer CBS, providing further support for Mitchell and Steinberg's attribution of this layer to the HP.

\section{Figure 3.1.8: View from the mouth of NT rockshelter}

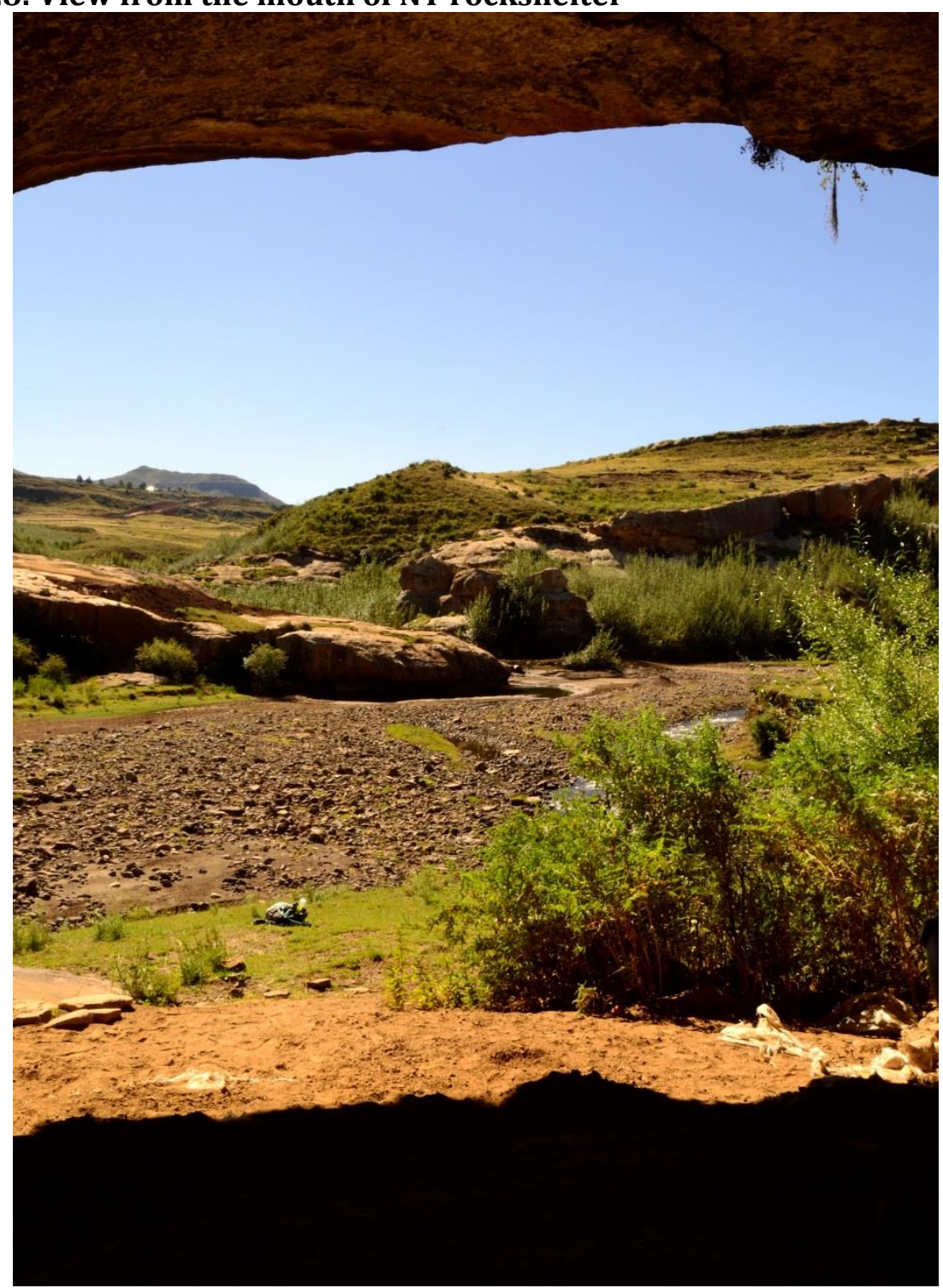


NT was re-excavated in 2009-12 as part of a larger program of survey and excavation lead by Mitchell ahead of the construction of the Metolong Dam (Mitchell and Arthur 2010, 2012). The recent excavations were conducted in particularly fine stratigraphic detail, with 579 separate contexts recorded throughout the sequence. Layers 571 and below are attributed to the MSA (Mohapi 2012). An unpublished report by Mohapi (2012) analysed the lithics from layers 573 and 574 using Mitchell and Steinberg's (1992) typology. She also tabulated the numbers of formal tool types in MSA layers as a whole, noting the existence of segments and backed pieces and the use of fine-grained CCS, which accords with what Mitchell and Steinberg (1992) noted for the HP at the site.

The three earliest layers at the site (577-579) were studied (Figure 3.1.9), with a total of 1027 lithics analysed. As the full MSA sequence from the most recent excavations has not yet been evaluated, it is not currently known which layers can be attributed to the HP. The bottom layers were chosen because the previous work of Mitchell and Steinberg (1992) demonstrated that the HP is the earliest industry present at the site, and Jacobs et al. (2008) consider the underlying sand to be weathered bedrock. Layer 579 is equivalent to layer CBS in the earlier excavations, and corresponds with the 61 ka BP OSL date (Mitchell and Steinberg 1992, Jacobs et al. 2008, C. Arthur pers. comm.). The relationship between layers 577 and 578 and the previous excavation is not known. However, it is possible that they correspond with the middle MSA layer from the previous excavations, associated with an OSL date of $56.0 \pm 1.8 \mathrm{ka}$ BP (Jacobs et al. 2008, C. Arthur pers. comm.). The present study addresses the question of which layers can be attributed to the HP. 


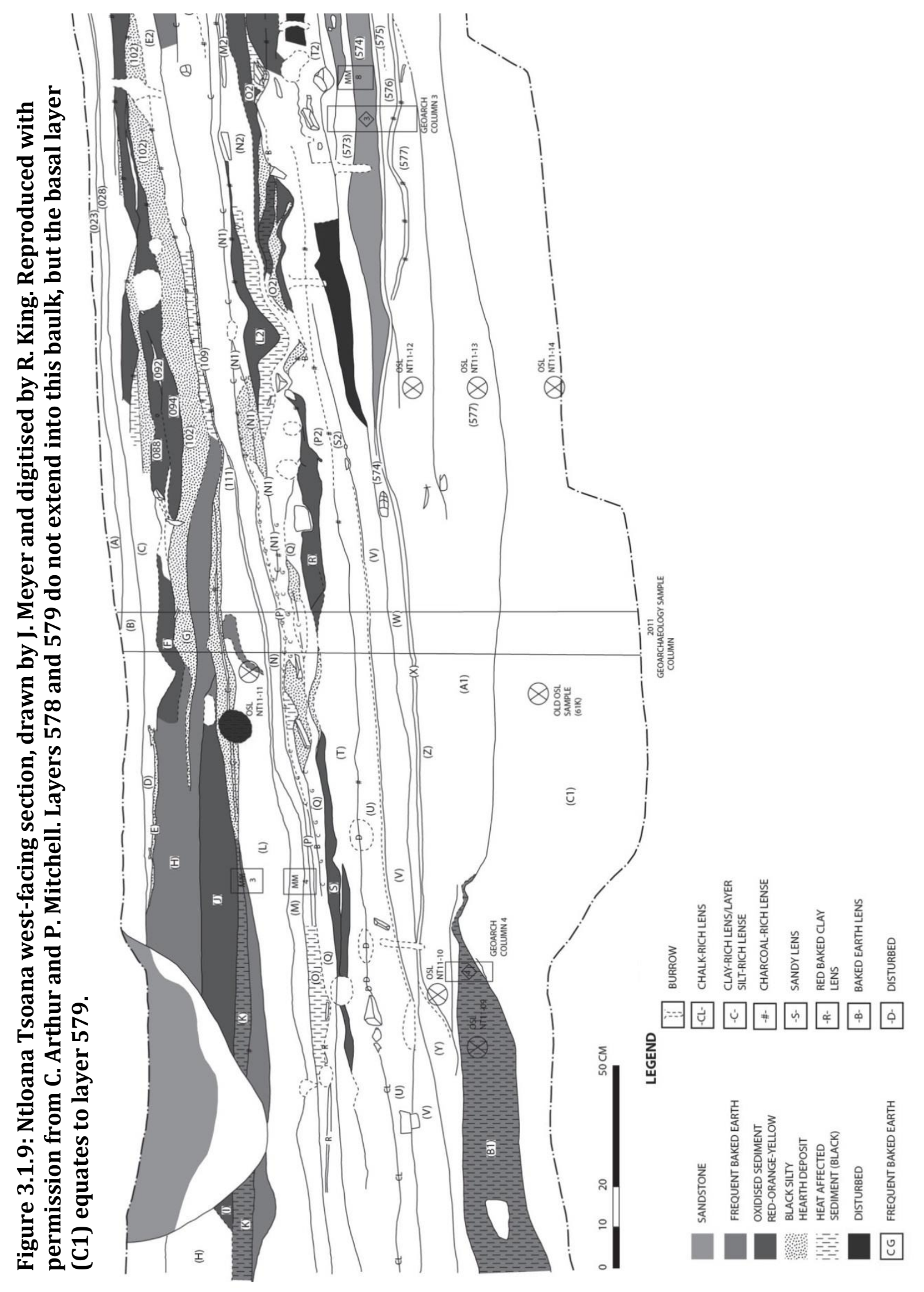




\subsection{The late Palaeolithic of South Asia}

In comparison to the HP of southern Africa, the Late Palaeolithic of South Asia is not as deeply researched or understood. From around 45 ka a variety of blade and microlith-based lithic assemblages begin to appear in the subcontinent, including in India, Pakistan and Sri Lanka (James and Petraglia 2005). As will be discussed further below, although this marks a change from preceding Middle Palaeolithic assemblages there is evidence for overlap and continuity between the two industries. Variously described in the literature as 'Mesolithic', 'Microlithic', 'Upper Palaeolithic', 'Late Stone Age' or, in Sri Lanka, 'Balangoda Culture', James and Petraglia's (2005) convention of referring to this assortment of blade- and microlith-based industries as Late Palaeolithic is followed here. Figure 3.2.1 maps some of the key Late Palaeolithic sites in India and Sri Lanka.

There is a long history of research in Palaeolithic archaeology in South Asia, dating back to the 1860s in India (Foote 1866, Brown 1889) and the 1880s in Sri Lanka (Pole 1907, Sarasin and Sarasin 1908). However, two major difficulties have hampered our understanding of the Palaeolithic of South Asia. Firstly, the scarcity of deep stratified excavations and the reliance on surface samples and surveys has problematised sequence construction. Secondly, and related, is the dearth of chronometric ages from Palaeolithic sites in the subcontinent. Assemblages have often been dated by analogy with industries elsewhere. In the past, this has been further complicated by a reliance on European (e.g. Sarasin and Sarasin 1908) and African (e.g. Allchin 1959, 1963) sequences for comparisons. 
Figure 3.2.1: Map of key Late Palaeolithic sites in India and Sri Lanka, highlighting in bold those analysed in the present study

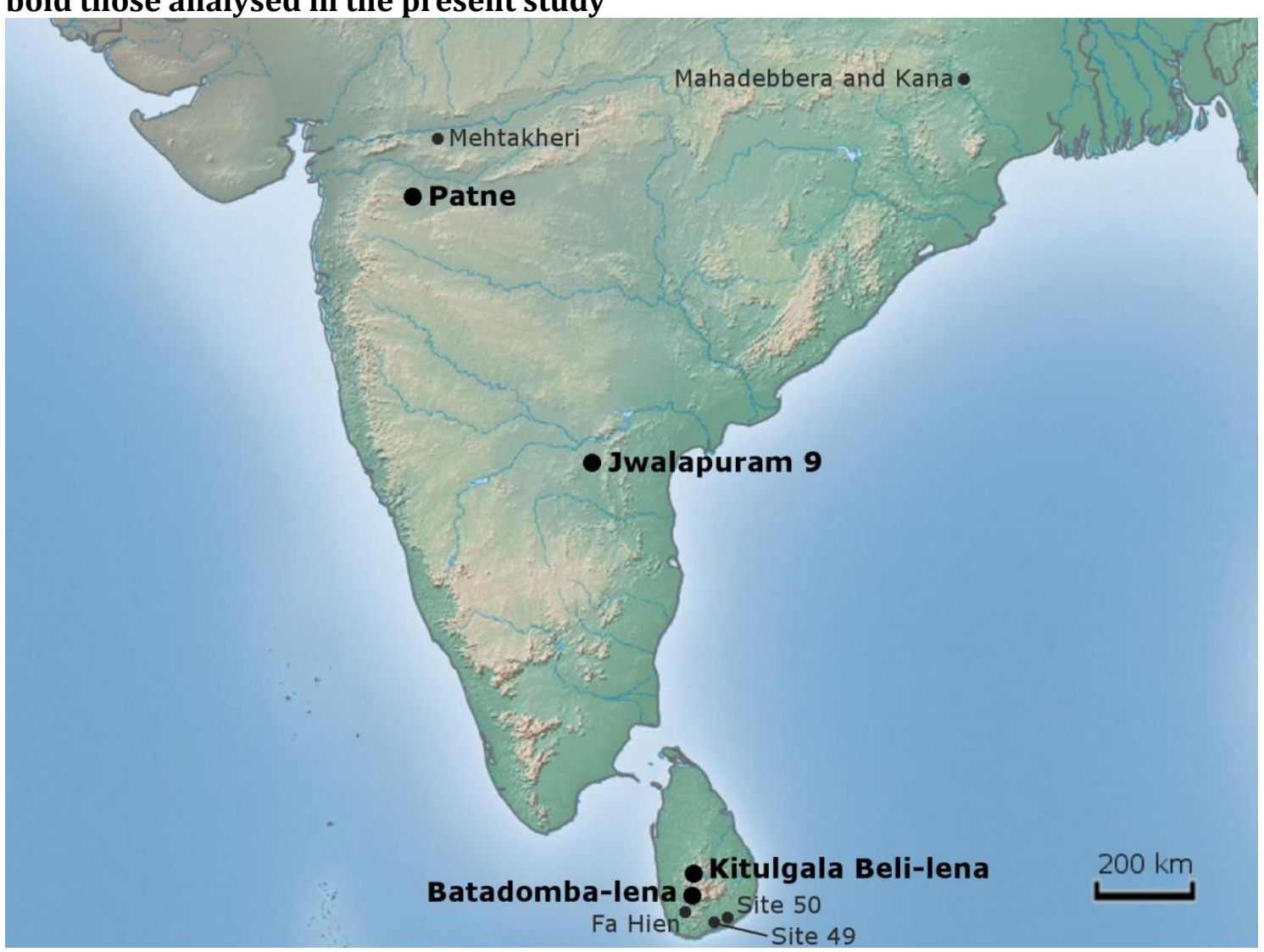

Partly as a result of the dating of sites in relation to sequences elsewhere in the world, microlithic technology in South Asia was originally considered wholly or primarily a Holocene phenomenon (Misra 1985, 2001). However, in the 1980s and early 1990s new chronometric ages indicated that microlithic industries dated back to 28 ka in Sri Lanka at the sites of Batadomba-lena, Bundala Site 49, BundalaPatirajawela Site 50 and Kitulgala Beli-lena (Deraniyagala 1984, 1986, 1992), and at least $25 \mathrm{ka}$ in India at the site of Patne (Sali 1989).

More recently, the discovery of microliths at the site of Fa Hien below a layer dated to c. $38 \mathrm{ka} \mathrm{BP}$, and recalibrated and new dates from Batadomba-lena going back to c. $36 \mathrm{ka} \mathrm{BP}$, confirmed the age of the earliest microlithic industries in Sri Lanka 
(Perera 2010, Perera et al. 2011). In India, the excavation of the site of Jwalapuram 9 pushed the earliest dates for microlithic technology in the country back to a similar age as in Sri Lanka (Clarkson et al. 2009, Petraglia et al. 2009a). Microlithic industries continue into the Holocene in both India and Sri Lanka. In the latter they occur up until the Iron Age in the $2^{\text {nd }}$ millennium BC (Deraniyagala 1992).

The development of microlithic technology in South Asia followed on from the appearance of a variety of blade and microblade assemblages from around $45 \mathrm{ka}$ BP (James and Petraglia 2005). This includes microblades at the Indian sites of Mehtakheri from $44 \pm 2$ ka BP (Mishra et al. 2013), Kana from $42 \pm 4$ ka BP (Basak et al. 2014) and Mahadebbera from $34 \pm 3$ ka BP (Basak et al. 2014). An unknown number of backed artefacts were also retrieved from Mahadebbara, but their size, provenance (whether in the excavated trench or the surface survey) and dating (the sequence spans a further 9000 years to $25 \pm 3 \mathrm{ka}$ ) is not reported. Additionally, a blade-based industry at Site 55, Riwat, Pakistan, which the authors consider to contain elements that are microlithic in size, may date to over $45 \mathrm{ka} \mathrm{BP}$ (Dennell et al. 1992). However, there is no clear or over-riding sequence of technological change within the Late Palaeolithic, and microlithic and blade-based industries coexist throughout this period (James and Petraglia 2005).

Variation within the Late Palaeolithic is poorly understood, but summaries of the existing evidence reveal some areas of difference. In Sri Lanka, the Late Palaeolithic is characterised by very small microliths made on quartz flakes, often less than $20 \mathrm{~mm}$ in length. Both geometric and non-geometric forms are found, including 'classic' segment, trapeze and triangle forms as well as the distinct 
bifacially-retouched tanged Balangoda point, unique to Sri Lanka and southern India (Zeuner and Allchin 1956, Deraniyagala 1992). Bipolar backing of microliths along lateral margins was often used (Perera et al. 2011). In contrast, microliths from Late Palaeolithic sites in India appear to be primarily made on blades and are longer on average, ranging up to c. $40 \mathrm{~mm}$ in length (Clarkson et al. 2009, Petraglia et al. 2009a, James 2011). Although regional comparisons have not previously been conducted on Late Palaeolithic microliths, differences are already evident from the published literature.

Other Late Palaeolithic material culture of note includes possible evidence for symbolic behaviour. Marked ostrich eggshells have been reported from over 40 sites in India between 40-25 ka (Bednarik 2003). However, Bednarik (1992, 1993) asserts that the markings on all but one of these are the result of damage by microorganisms. The only piece not rejected by Bednarik is a geometrically engraved fragment from Patne, dated to $25 \mathrm{ka}$ BP (Sali 1985). One finished and two unfinished ostrich eggshell beads were found in the same layer. Ostrich eggshell beads are also found at Batadomba-lena, Sri Lanka, dating to c. $35 \mathrm{ka} \mathrm{BP}$, alongside beads made from marine mollusc shell (Deraniyagala 1992, Perera et al. 2011). A larger collection of slightly later limestone and bone beads was discovered at Jwalapuram, India, bracketed by dates of 20-12 ka BP (Clarkson et al. 2009). Overall, however, there is no sudden appearance of a package of archaeologically visible 'modern' behavioural traits in South Asia. Novel tool types such as microliths and microblades have their origins much earlier in the subcontinent (for example compared to Europe), while potentially symbolic material culture appears sporadically and relatively recently (James and Petraglia 2005). 
Explicit theorisation concerning the origins of the Late Palaeolithic industries of South Asia is rare. It has previously been assumed that microlithic technology was brought to South Asia as part of the expansion of H. sapiens from Africa to Asia, and that the earliest appearance of microlithic industries therefore represents the first of our species in the continent (Mellars 2006a, 2006b, Mellars et al. 2013, Mishra et al. 2013). However, as discussed in Chapter 2.3, this model depends on a late dispersal out of Africa. New evidence is being uncovered which supports an earlier dispersal date, which would make the earliest microliths found in South Asia too recent to be part of the original dispersal (although this does not rule out any relation to a subsequent dispersal of human populations).

Additionally, although the origins of these microlithic and microblade technologies are currently poorly understood, technological analysis of lithics from Patne, India, one of the few long stratified sequences in South Asia spanning the Middle and Late Palaeolithic, suggests that they emerged locally and gradually out of preceding Middle Palaeolithic industries (James 2011). This is also implied by the results of lithic analyses at sites in the Jurreru Valley (Clarkson et al. 2012). Blade use appears to be a continuation of Middle Palaeolithic technological practices elsewhere in South Asia (Misra and Bellwood 1985). Furthermore, characteristic 'Upper Palaeolithic' tool types (e.g. burins, end-scrapers and microblades) also appear in Middle Palaeolithic contexts in the subcontinent, including at Bhimbetka rockshelter in north-central India (Misra 1985).

Given the evidence for continuity of human populations in South Asia, perhaps a better explanation for the origins of microlithic technology in the region is that 
proposed by Petraglia et al. (2009b, see also Clarkson et al. 2009). They argue that microlithic technology was invented independently in the Indian subcontinent, in response to environmental and demographic pressures. Analysis of mtDNA datasets reveals an increase in population size and/or the fragmentation of population groupings from c. $35 \mathrm{ka}$, most likely related to the deterioration of environmental conditions towards the end of MIS 3. Generally speaking, climatic conditions became more variable and unstable in the latter part of MIS 3, before becoming cooler and especially more arid during MIS 2 and the onset of the LGM. In response, human populations adopted new technological and subsistence strategies, including the use of microlithic technology. Although the model proposed by Petraglia and colleagues best fits the available data, further investigation of technological variability within the Late Palaeolithic would allow these issues to be addressed in greater depth.

Four Late Palaeolithic sites were selected for analysis: Batadomba-lena and Kitulgala Beli-lena, Sri Lanka, and Patne and Jwalapuram 9, India (Figure 3.2.1). These sites were chosen as they are the earliest stratified and dated sites in South Asia containing microlithic industries (with the exception of Fa Hien, Sri Lanka, which was unavailable for study). All but one (Kitulgala) are explicitly cited in Mellars' model of microlithic dispersal (Mellars 2006b, Mellars et al. 2013). The excavations at Batadomba-lena are particularly finely stratified. Metric attribute analysis has been conducted on the assemblage using a small number of variables (Perera 2010), but the more detailed methodology utilised here has not previously been applied. Microlithic technology has not previously been analysed at Kitulgala; only typological counts have been conducted (Wijeyapala 1997). Detailed attribute 
analyses of Patne and Jwalapuram have already been conducted using a

methodology which formed the basis for the one used in the present study (Clarkson et al. 2009, James 2011), and these datasets were made available for comparative purposes by C. Clarkson. The use of the Indian datasets for comparisons allows for similarities and differences to be evaluated between Indian and Sri Lankan Late Palaeolithic industries.

\subsubsection{BATADOMBA-LENA, SRI LANKA}

Batadomba-lena $\left(6^{\circ} 46^{\prime} \mathrm{N}, 80^{\circ} 12^{\prime} \mathrm{E}\right)$ is a small north-east facing rockshelter located on the banks of a stream in Ratnapura District in the wet zone lowland rainforest of south-west Sri Lanka, formed in the gneiss bedrock of the region (Deraniyagala 1992, Perera 2010, Perera et al. 2011) (Figure 3.2.2). It is the richest prehistoric site in Sri Lanka, yielding over 400,000 lithics in addition to human and animal skeletal material and various plant macroremains.

The site was first excavated by P.E.P. Deraniyagala in 1937-40, who interpreted it as belonging to a Mesolithic 'Balangoda' culture between the Pleistocene and Holocene. It was later excavated more extensively by his son S.U. Deraniyagala in 1979-1986 (P.E.P. Deraniyagala 1940, 1963, S.U. Deraniyagala 1992). The systematic excavations of S.U. Deraniyagala - reaching a depth of $2.8 \mathrm{~m}$ - included a comprehensive dating program (radiocarbon, ESR, TL and OSL) which dated the earliest archaeological materials at the site to the Late Pleistocene at around 28.5 ka BP (Agrawal et al. 1985, Deraniyagala 1992, Abeyratne et al. 1997). The most 
recent excavations at the site, conducted by N. Perera in 2005 (Figure 3.2.3),

pushed the earliest dates back further, with a calibrated radiocarbon date from the earliest layer of 36,283-34,609 cal BP (Wk-19963: 30,603 $\pm 400 \mathrm{BP}$, calibrated and reported to the $2 \sigma$ level by Perera et al. 2011). Microlithic occupation at the site continues into the Holocene with no obvious temporal breaks in habitation.

Figure 3.2.2: Excavations at Batadomba-lena in 2005. The gneiss roof of the rockshelter can be seen to the left. Photo courtesy N. Perera.

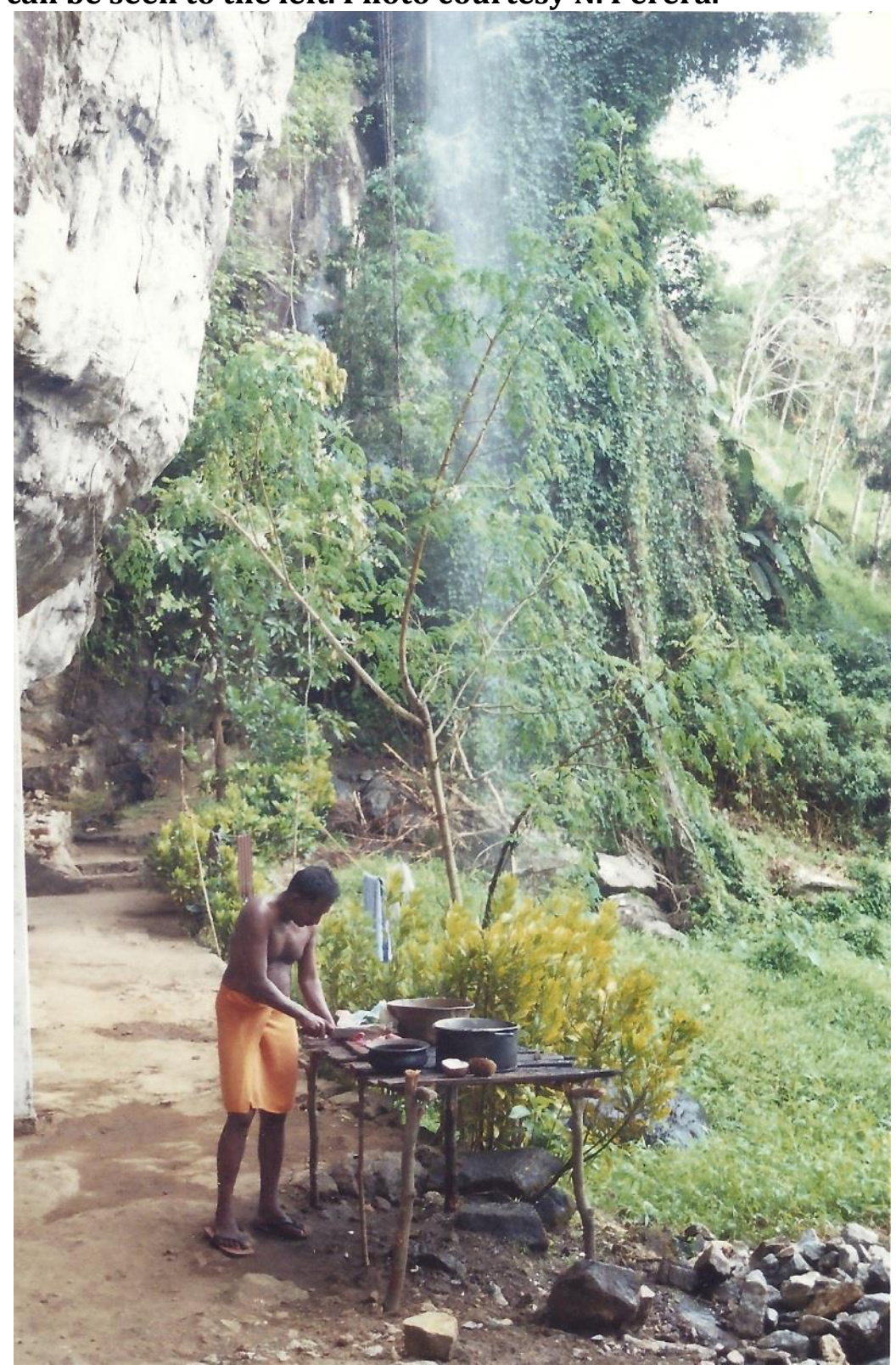


Figure 3.2.3: Excavations at Batadomba-lena in 2005. Photo courtesy N. Perera.

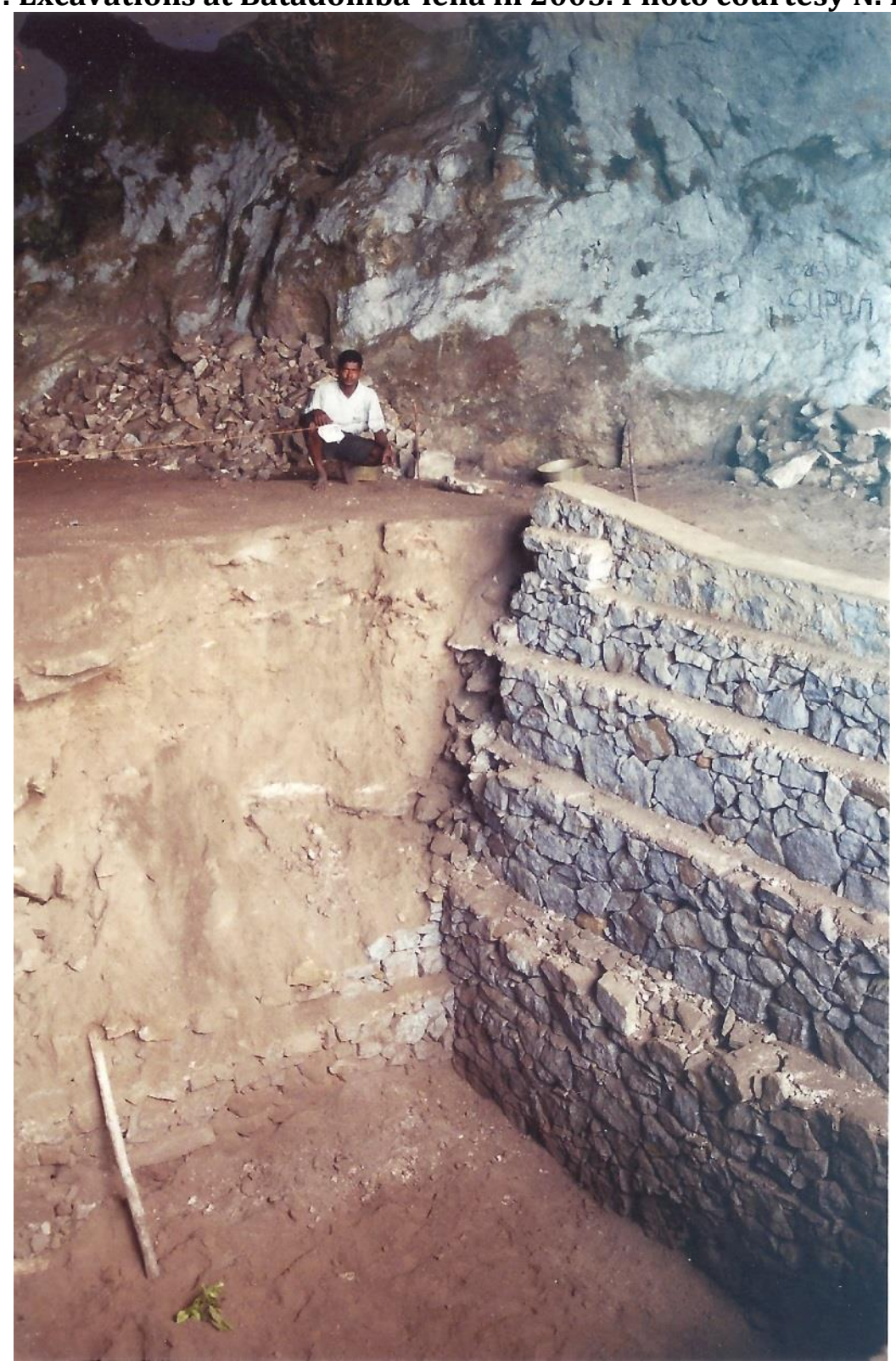

The site is rich in faunal remains, although the highly fragmented and often burnt nature of the remains precludes the taxonomic identification of the majority of excavated bones (Perera et al. 2011). The majority of identifiable remains are of small- and medium-bodied arboreal and mixed terrestrial and arboreal animals. Monkeys dominate the assemblage - typically around $70 \%$ of the vertebrate faunal assemblage in each layer - and squirrels, civets and mongooses are also common. Rarer terrestrial species include mouse deer and boar, although there are no larger 
animals such as elephant, rhinoceros and hippopotamus, even though it is known that they lived in the area at this time (Deraniyagala 1992). A wide variety of other mammalian and non-mammalian vertebrate species were also found in small numbers, including porcupine, rats, bats, jungle fowl, monitor lizards, snakes, terrapins, toads and freshwater fish (Perera et al. 2011). Invertebrate species are also present in large numbers from the earliest layers at the site, in the form of arboreal Acavus spp. gastropods and other terrestrial and freshwater molluscs. Additional use of marine resources is implied by a fragment of marine shell in layer 5 and a ray spine in layer 7a (Deraniyagala 1992) (see Figure 3.2.4 for stratigraphic context). Plant remains are present in all layers, and edible species include wild bananas, breadfruit and Canarium nuts (Perera et al. 2011). Overall, the evidence indicates a broad spectrum diet.

Additionally, the faunal and floral evidence demonstrate that equatorial rainforest persisted in the region even through the Last Glacial Maximum (Perera et al. 2011). For example, monkey species are dominant throughout the period of occupation at the site, and proportions of other arboreal fauna and molluscs remain consistent. The Acavus spp. arboreal molluscs recovered have identical forms to those living in the area today, suggesting that the environment throughout the Late Pleistocene was largely similar to that today, which is dominated by dense evergreen rainforest (Kennedy et al. 1986). Additionally, the Paludomus species of freshwater snail evident throughout the sequence requires conditions that would be achieved in the stream adjacent to the rockshelter under undisturbed rainforest canopy (Perera et al. 2011). Isotope analysis confirms human exploitation of rainforest environments at Batadomba-lena from at least 20 ka (Roberts et al. 2015). 
Figure 3.2.4: Stratigraphic profile of Batadomba-lena with calibrated radiocarbon dates (redrawn from Perera et al. 2011, Figure 2b)

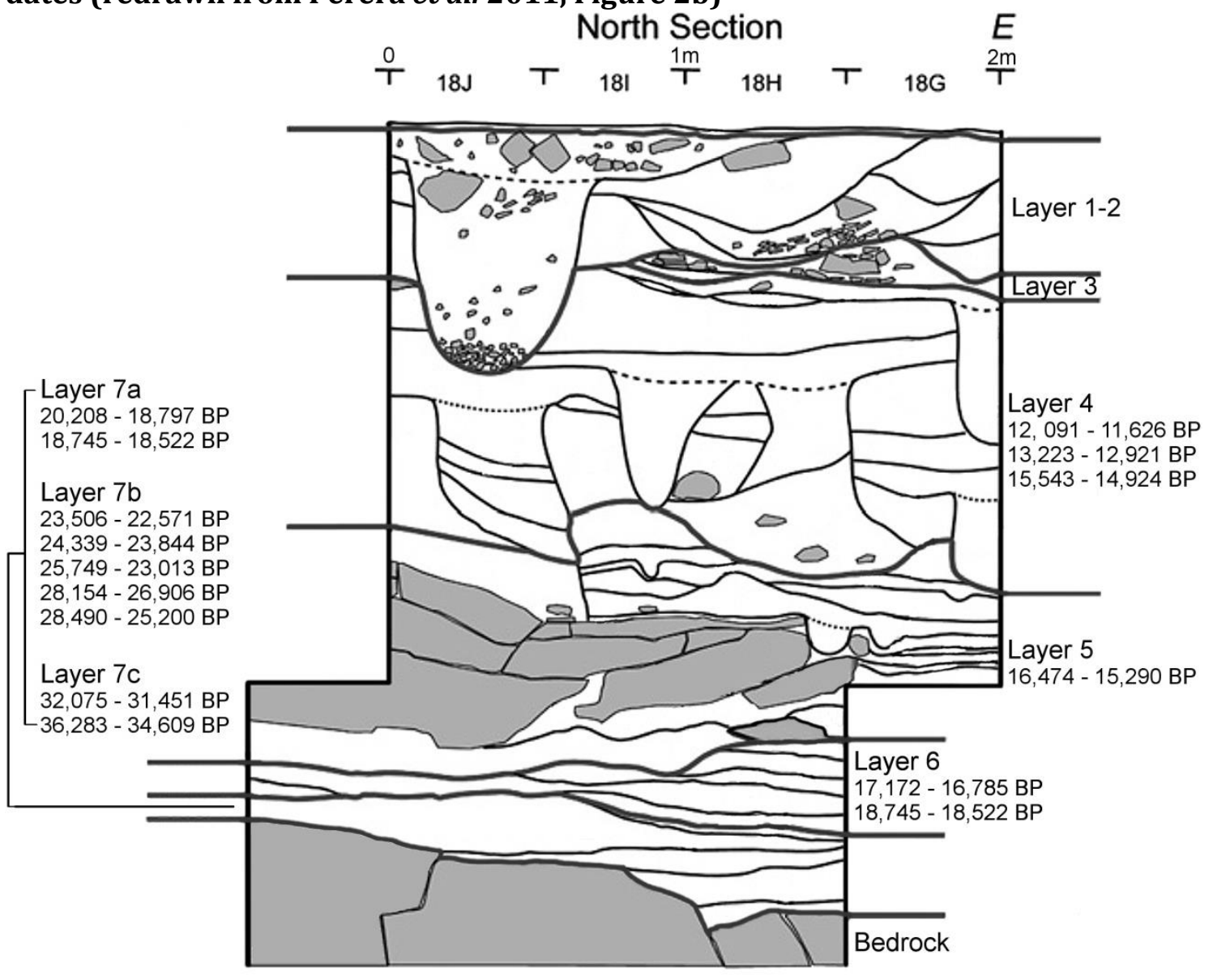

Batadomba-lena also contains important palaeoanthropological evidence,

including some of the earliest $H$. sapiens skeletal remains found in South Asia.

Fragmentary skeletal remains of at least 16 robust $H$. sapiens have been recovered, with both sexes and a range of ages represented (Perera et al. 2011). Human remains were found in layers $4,5,6$ and $7 c$, i.e. from the earliest occupation at the site. Remains were recovered in association with occupational debris including lithics and vegetal and faunal remains (Kennedy et al. 1987). The commingling of individuals is argued to be indicative of fractional and secondary burial practices, and the lack of burial pits is interpreted as implying the laying of bodies in shallow hollows in the cave floor (Kennedy and Deraniyagala 1989, Kennedy 2000). Some 
remains are charred, which Kennedy (2000) argues is the result of coming into contact with hearths or fires in the rockshelter a long time after burial, due to the long sequence of occupation at the site and the lack of evidence for the marking of burial places.

Non-lithic archaeological evidence includes ochre fragments and bone and antler points found throughout the sequence (Perera et al. 2011). Four marine shell beads were also found in layers 5, 6 and 7c (Deraniyagala 1992). Although this constitutes some of the earliest evidence for symbolic behaviour in South Asia, it is not possible to state that these artefacts imply that such behaviour was an important or long-lived element of hunter-gatherer lifeways at this time. An additional point of note, however, is that these marine shells originated from shores at least $80 \mathrm{~km}$ away, potentially implying either the exploitation of a variety of ecozones within the seasonal round, or exchange networks that extended outside of the rainforest environment.

Previous lithic analysis at Batadomba-lena was conducted by S.U. Deraniyagala (1992) and N. Perera (2010). These analyses were primarily typological in nature, with some additional debitage and use-wear analysis conducted by Perera (2010) on smaller samples. The vast majority of lithics are quartz, which was readily available in the vicinity of the rockshelter (N. Perera pers. comm.). A variety of very small geometric and non-geometric microliths are found from the earliest layers onwards, including lunates, triangles, trapezoids and Balangoda points. Microliths were found to be more abundant and morphologically diverse in pre-LGM layers, becoming less abundant during intensified use of the rockshelter after the LGM. 
A total of 1491 lithics was sampled from the 2005 excavations at Batadomba-lena, as these were the best provenanced and curated. In addition, 90 microliths from the 1980s excavations were also included, as the numbers from the 2005 excavation alone were low. The analysis was limited to the Pleistocene section of the profile, comprising layers 4-7c (Figure 3.2.4).

\subsubsection{Kitulgala Beli-Lena, SRI LANKA}

Kitulgala Beli-lena $\left(6^{\circ} 56^{\prime} \mathrm{N}, 80^{\circ} 14^{\prime} \mathrm{E}\right)$ is a large west-facing rockshelter measuring c. $30 \times 10 \mathrm{~m}$, in the Kegalla district of Sabaragamuva province in south-west Sri Lanka (Kennedy et al. 1986, Wijeyapala 1997, Perera 2010). It is located by a stream, on the border of the wet zone lowland and upland rainforest regions. The site was first discovered in 1960 when P.E.P Deraniyagala conducted a small testpit excavation in the rockshelter, although this was never written up (Perera 2010). The site was more extensively excavated by S.U. Deraniyagala in 1978-79 and 1983, and by Wijeyapala in 1986. The excavation reached a depth of $4 \mathrm{~m}$ from surface to bedrock, divided into nine occupational layers (Figure 3.2.5).

As part of S.U. Deraniyagala's program of research, a series of radiocarbon dates on charcoal were produced (Deraniyagala 1992). These dates were recalibrated by Perera and revealed an occupational sequence dating from $>31,070$ cal BP to 38754078 cal BP (Perera 2010, Table 2.2). The lowermost layer did not contain sufficient charcoal for dating, but Perera suggests it dates to perhaps 40-35 ka. 


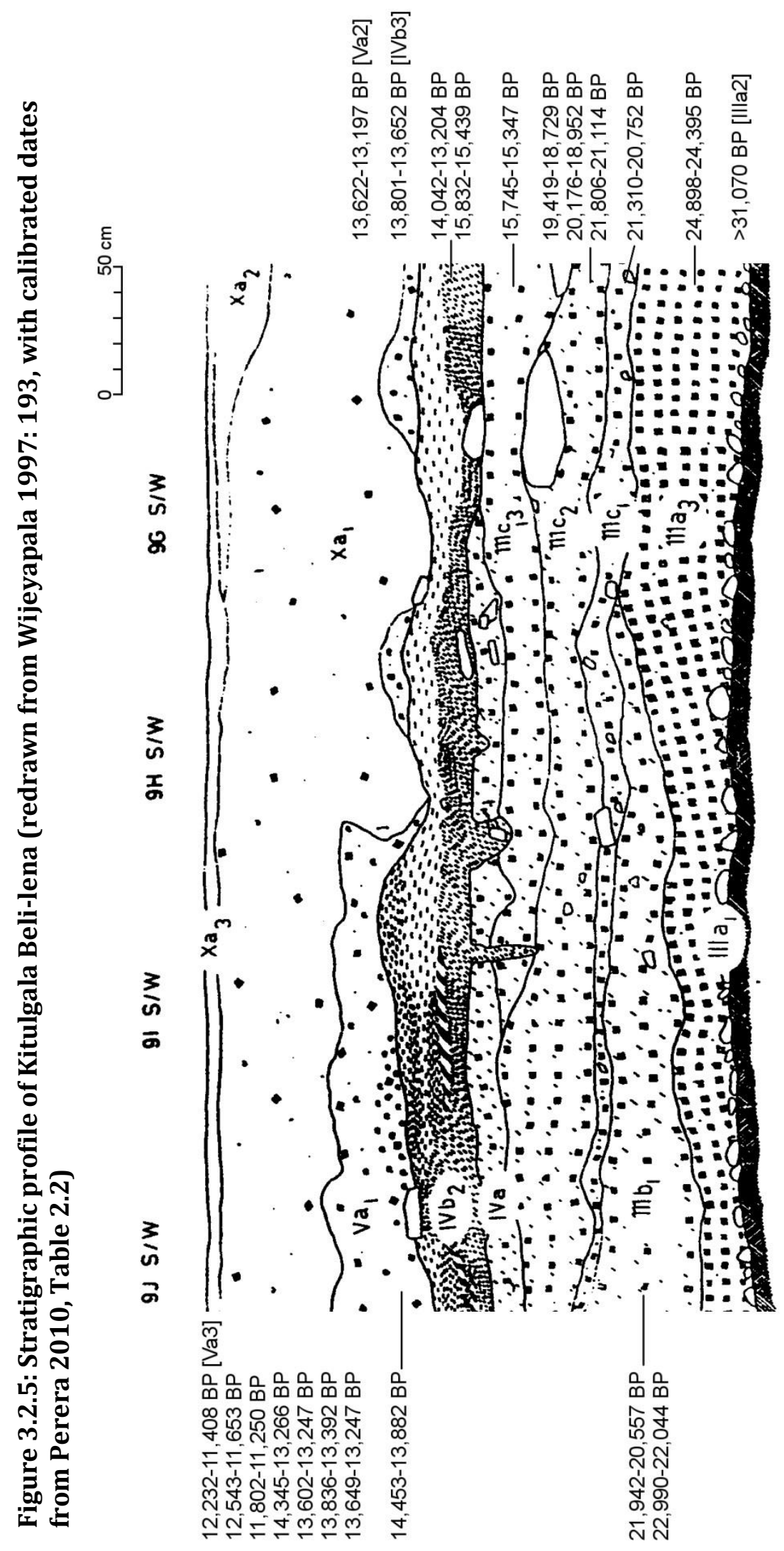


Kitulgala is notable for its human skeletal remains, which were found in all undisturbed layers and include some of the earliest human remains in South Asia. As Perera (2010: 177) notes, considerable disagreement exists between accounts by the excavator (Wijeyapala 1997) and the principal osteologist (Kennedy et al. 1986, Kennedy 1999, 2000) as to the provenance and cataloguing of the human remains from Kitulgala. Perera sides with the latter, concluding that at least 13 individuals are represented at the site. Kennedy (2000: 184) states that some burials were fractional depositions in hollows in the cave floor, and that secondary and disturbed burials are also present.

In terms of non-lithic material culture, ochre and ochre-stained artefacts are found throughout the sequence from the earliest layers upwards (Wijeyapala 1997). Wijeyapala claims that a piece of yellow ochre was found in association with a child burial in the oldest dated layer at the site, although there is no illustration provided to demonstrate this. Bone tools are also found throughout the sequence.

The site is rich in faunal remains. The assemblage is dominated by monkeys and also includes giant and flying squirrel, palm civet, various other mammals, birds and reptiles, and large quantities of arboreal snails, especially Acavus spp. (Kourampas et al. 2009). Kajale (1989) analysed plant remains from the many hearths evident at the site and discovered burnt seeds of an array of rainforest plants, including Canarium zeylanicum nuts, wild banana and breadfruit. Overall, Kennedy et al. (1986) argue that the subsistence strategy at Kitulgala, as at Batadomba-lena, made use of a generalised broad spectrum diet, including a wide variety of plants and animals, especially small game and freshwater shellfish. 
Kourampas et al. (2008) go further, concluding that human populations in Sri Lanka were fully equipped for life in rainforest refugia, and that these environments persisted even through the LGM.

One discovery in the faunal assemblage is of particular note. A shell of the gastropod Potamides cingulatus was found in Layer IIIc1, dating to $21 \mathrm{ka}$ BP. The species is very common on lagoon flats in inter-tidal zones, leading Deraniyagala (1992) to conclude that the only way it could have reached the site was as an inclusion within rock salt which was carried from evaporates located along the coast over $80 \mathrm{~km}$ away (from modern coastlines). He argues that this is evidence for long distance exchange networks at this time. As discussed above, contact with the coast is also evidenced from the earliest occupation at Batadomba-lena.

Previous lithic analysis at Kitulgala consists of the typological counts conducted by Wijeyapala (1997) in his doctoral thesis, using the typology constructed by Deraniyagala (1992) plus a few new tool forms. He analysed 24,772 'stone artefacts' from the excavations in the 1970s and 1980s, and nearly 500,000 'waste lithic flakes' which were counted separately and not considered as lithic artefacts (Wijeyapala 1997: 203). As at Batadomba-lena, the lithic assemblage is almost entirely quartz. Both geometric and non-geometric quartz microliths are reported from the basal layer upwards. Wijeyapala notes that only three types of geometric form were found in this basal layer, while up to 16 forms were found in later levels. Nevertheless, he concludes that "according to new functional necessities, new forms of tools have been introduced [in layer levels] applying the same technological methods which are found in previous habitation levels" (Wijeyapala 
1997: 268), and that overall the sequence at Kitulgala demonstrates technological continuity.

Lithics were sampled from the Pleistocene layers IIIa to V (Figure 3.2.5).

Unfortunately the core and debitage assemblages were unavailable for study, and only the microlithic tools were accessible. A total of 31 microliths were located and analysed. Although this is a small number of artefacts, it accords closely with the 34 geometric microliths recorded from these layers by Wijeyapala (1997: 260). Therefore, bearing in mind potential differences in typological ascriptions caused by differences in analytical approaches, the current study can be considered to include all excavated microliths from Pleistocene levels at the site.

\subsubsection{PATNE, INDIA}

Patne $\left(20^{\circ} 19^{\prime} \mathrm{N}, 74^{\circ} 58^{\prime} \mathrm{E}\right)$ is an open air site located in Jalgaon district of Maharashtra State (Sali 1985, 1989). The site is divided into Areas A and B. Area A contains only a (Holocene) Mesolithic occupation, while Area B also contains Middle and 'Upper' (i.e. Late) Palaeolithic deposits and as such is the area of interest in the present study. The site is situated on a large flat fluvial plain (Figure 3.2.6) in the Deccan Trap region, where "jasper, chalcedony and other allied silicious rock-materials occur in abundance in the traps as veins or small lenticular bodies" (Sali 1989: 7). Area B is located in what is described as an "amphitheatrelike depression carved into the northern scraped face of the Ajanta Hills" (ibid: 7), 
containing the confluence of two small streams. Today the local environment is semi-arid, with some dry deciduous forest.

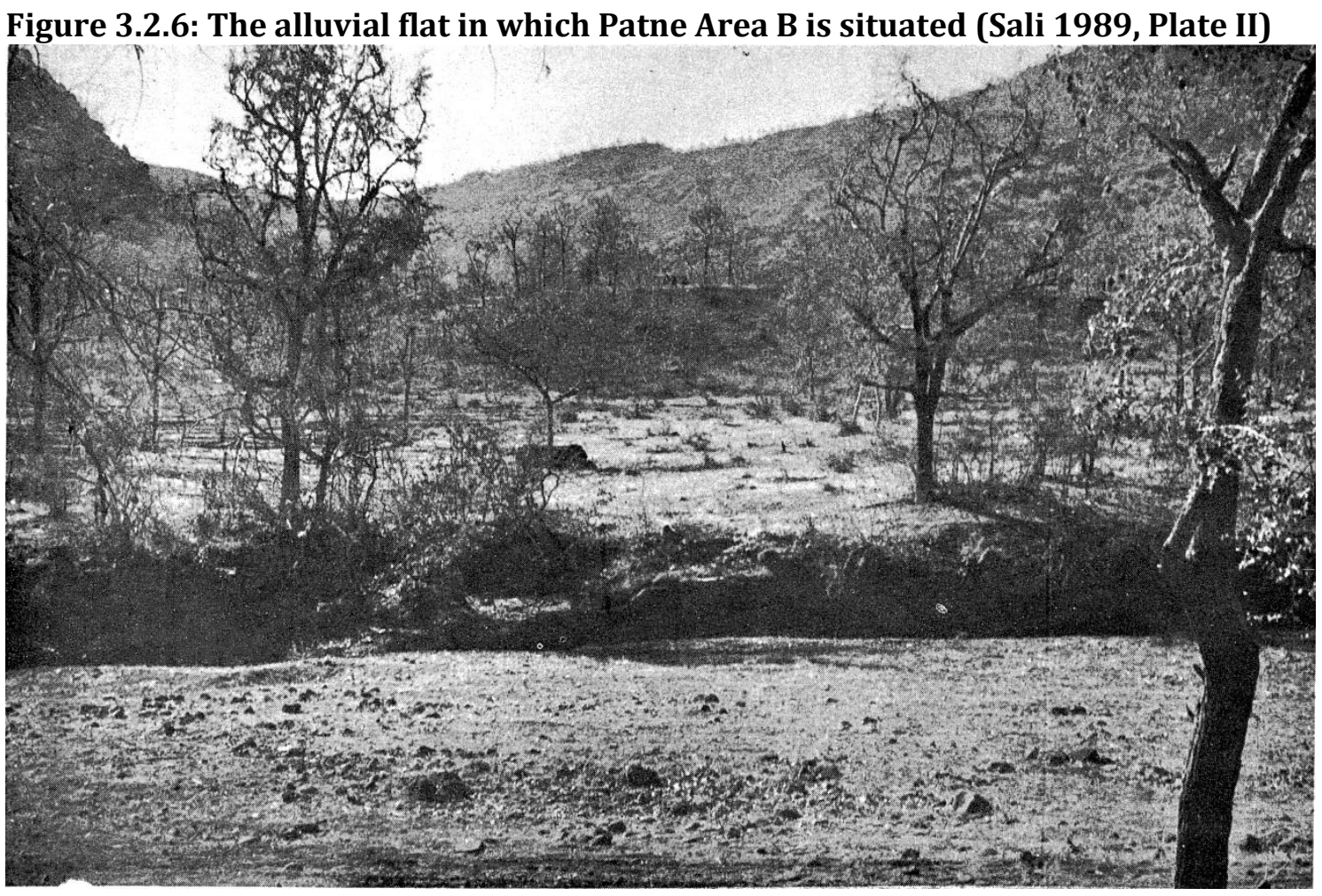

The site was first discovered in 1952 by S.A Sali and M.N. Deshpande. The first small-scale excavation was conducted in 1958, with additional small excavations in subsequent years. More extensive excavations were conducted in 1971-73 under the guidance of H.D. Sankalia. A total of 12 trenches were excavated in Area B in a step trench method. Figure 3.2.7 shows the $10 \mathrm{~m}$ composite stratigraphic profile constructed by matching sediments between trenches. The stratified sequence from the later Middle Palaeolithic to the Holocene at Patne is virtually unique amongst South Asian archaeological sites. 
Figure 3.2.7: Composite stratigraphic profile of Patne with calibrated radiocarbon date (redrawn from the version by James and Petraglia 2005, Figure 4, of Sali 1989, Figure 11)

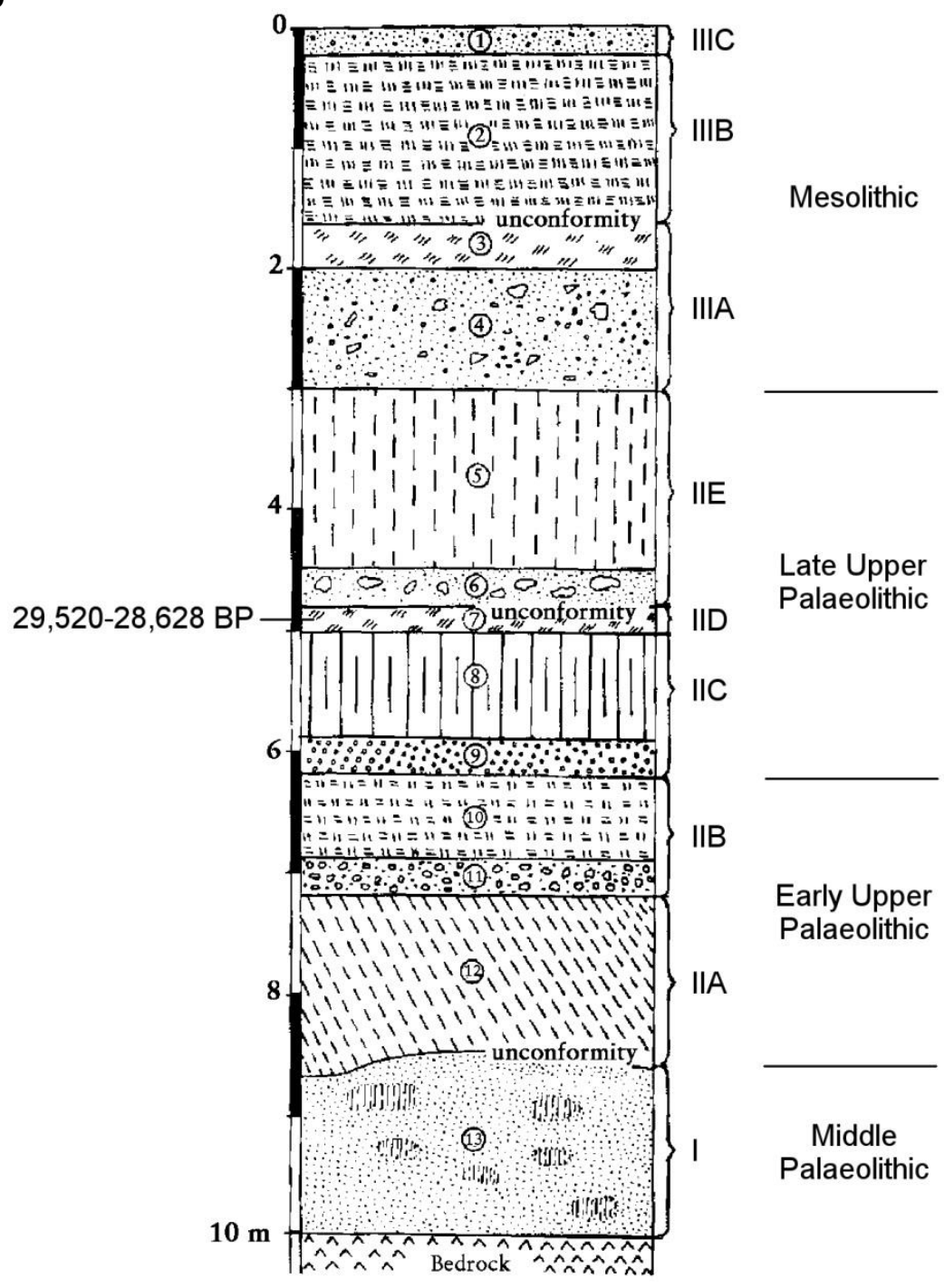

Only one radiometric date is available from the site: a radiocarbon date made on ostrich eggshell from Layer 7 (Sali's Phase IID), which returned a date of 25,000 \pm $200 \mathrm{BP}$. Using the OxCal radiocarbon calibration programme, this date calibrates to 29,520-28,628 cal BP at the $2 \sigma$ level (OxCal v. 4.2.4, IntCal13 calibration curve (Ramsey 2014)). Sali $(1985,1989)$ argues that the Upper Palaeolithic at Patne may go back as far as $35 \mathrm{ka}$, being replaced by the Mesolithic around $10 \mathrm{ka}$. 
Patne contains some of the earliest evidence for symbolic material culture in South Asia. Ostrich eggshell fragments are found throughout Phase II, and include two pieces from Phase IID which are engraved with geometric designs. One finished and two unfinished beads are found in the same layer. The overlying Phase IIE also contains an ostrich eggshell bead and engraved fragment.

Sali conducted typological assessments and descriptions of the lithic assemblage from Patne, totalling 28,336 lithics (Sali 1989, Table 6). He then separated the lithic sequence into different phases on the basis of tool proportions. He also separated the Upper Palaeolithic into an Early and a Late stage, on the basis that geometric and semi-geometric forms first appear in the Late stage. Sali noted that tool sizes are much smaller in the Late Upper Palaeolithic compared to the Early stage, approaching the small tool sizes of the Mesolithic stage.

Building on the work of Sali, James (2011) conducted metric attribute analyses on a sample of 1630 Middle and Upper Palaeolithic lithics, providing the first study of lithic technology at the site. She demonstrated that the Upper/Late Palaeolithic emerges gradually and locally out of the Middle Palaeolithic, inferring for example that there were general trends towards increasingly intensive blade production, and towards increasing use of backing retouch. Access was granted to the database of lithic measurements recorded by James. Lithics from the Upper/Late Palaeolithic Phase II (A-E) were used (Figure 3.2.7), resulting in a total of 1560 lithics. 


\subsubsection{JWALAPURAM 9, INDIA}

Jwalapuram $9\left(15^{\circ} 19.864^{\prime} \mathrm{N}, 78^{\circ} 7.788^{\prime} \mathrm{E}\right)$ is a rockshelter site located on the northern margin of the Jurreru river valley in the Kurnool district of the state of Andhra Pradesh in southern India (Clarkson et al. 2009, Petraglia et al. 2009b). The rockshelter is formed by a large quartzite boulder, which provides shelter over an area of c. $60 \mathrm{~m}^{2}$ (Figure 3.2.8). It was excavated between 2003 and 2009 as part of a larger project of excavation in the river valley, which exposed several Middle and Late Palaeolithic sites (Petraglia et al. 2009b).

Figure 3.2.8: Excavations at Jwalapuram 9 (Clarkson et al. 2009, Figure 1)

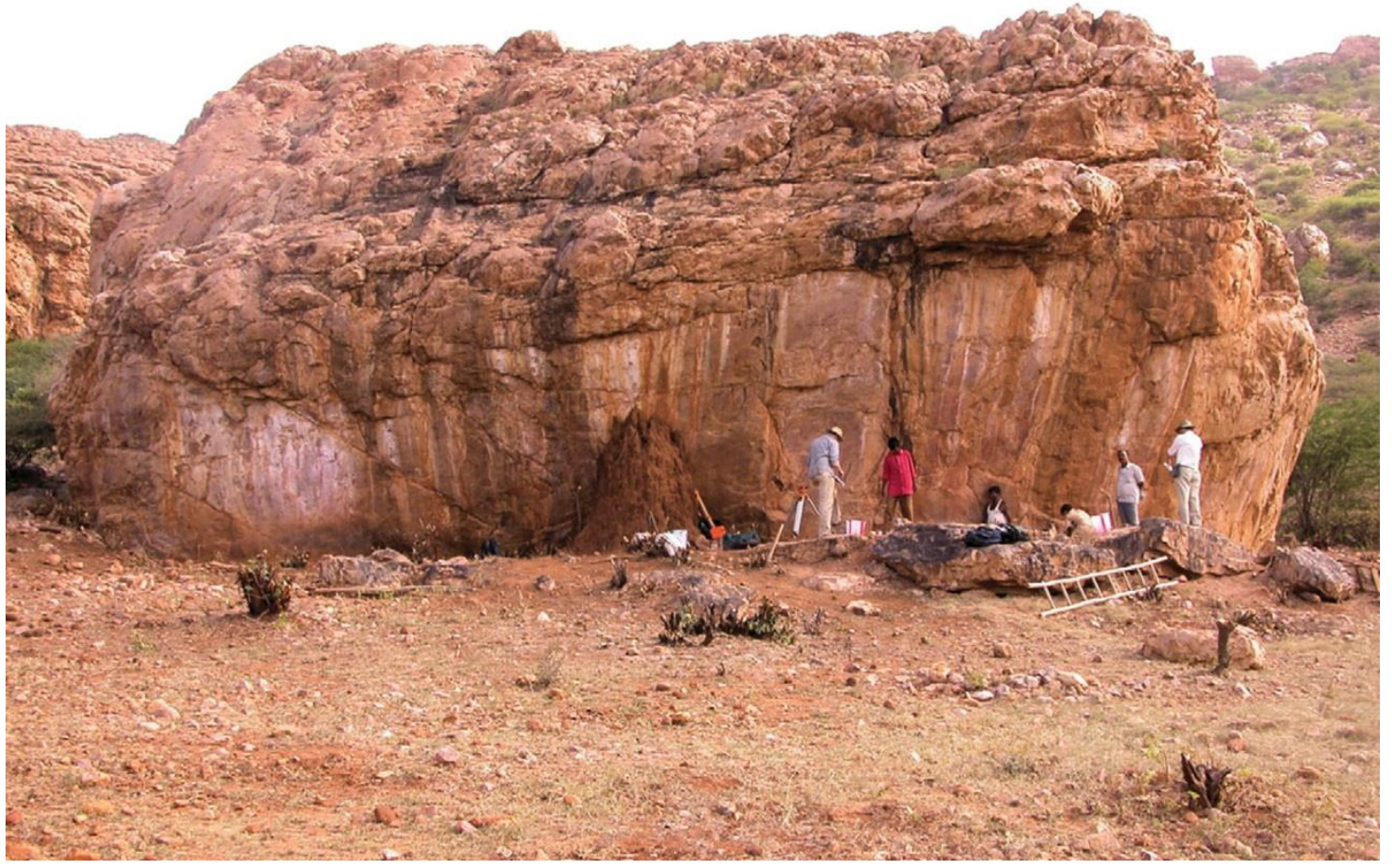

Excavations reached a depth of $3.3 \mathrm{~m}$, containing five occupational units (A-E) and two underlying sterile layers (F-G) (Figure 3.2.9). A series of AMS radiocarbon dates returned calibrated dates of 34-20 ka BP for stratum D and 20-12 ka BP for stratum C (Clarkson et al. 2009). Stratum E could not be dated, but is given an age 
of >34 ka BP. The two upper strata are associated with the late Neolithic and early Iron Age, and therefore date to within the last 3000 years.

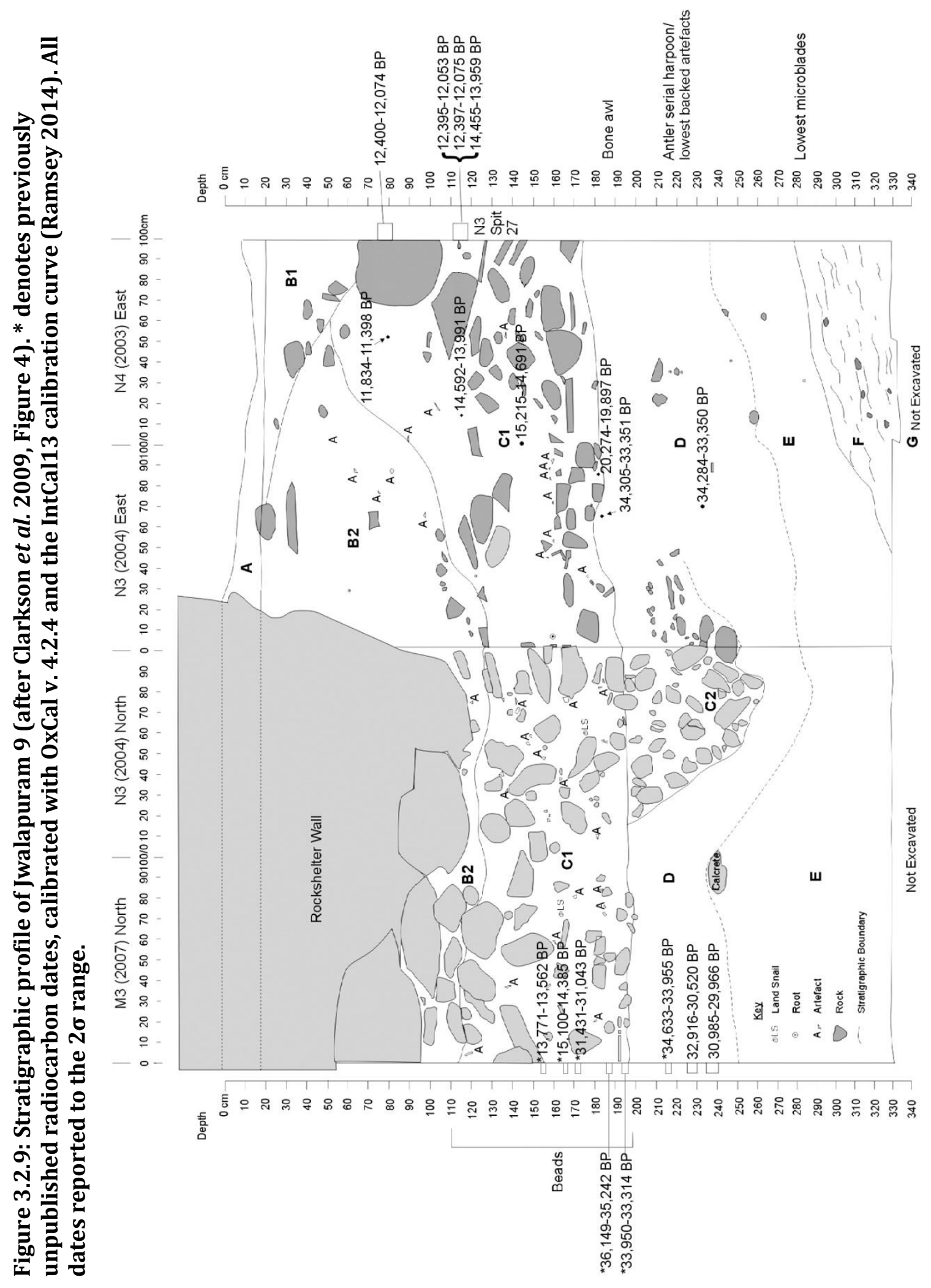


A large faunal assemblage was excavated at the site, comprising 2732 vertebrate remains and 1644 mollusc shells (Clarkson et al. 2009, Petraglia et al. 2009b). Freshwater bivalves were systematically exploited during the entire period of occupation at the site. The mammalian assemblage is dominated by small- and medium-sized ungulates, and indicates a shift in habitat preference from grassland to woodland from strata D to C. At the same time, stable carbon isotope analysis of soil carbonates demonstrates a change from mixed grassland and woodland to more closed woodland environments. Overall, the excavators conclude that there were broad environmental changes in the region during the period of site occupation. These changes correspond with the general drying trend evidenced in South Asia towards the end of MIS 3 and into the LGM (Petraglia et al. 2009a).

Jwalapuram 9 contains the earliest dated $H$. sapiens remains in India. Four burnt cranial fragments and one (unburnt) tooth were found in stratum C, dated to 20-12 ka BP. The same stratum also contains some of the earliest evidence for personal ornamentation in South Asia, in the form of 25 limestone and bone beads. Ochre fragments are found throughout stratum C, and a striated red ochre crayon was found at the interface between strata $C$ and $D$. The excavators hypothesise that these finds may be related to the faded red rock art present on the shelter wall, although there are no dates available for the rock art. Bone tools are evident in the earliest occupation layers at the site, with a harpoon fragment assigned an age of $34 \mathrm{ka} \mathrm{BP}$, and a broken bone point interpreted as an awl associated with dates of 34-20 ka BP. 
A total of 53,162 lithics was excavated at the site, with a peak in density between 20-12 ka. A comprehensive typological and technological analysis of the lithic assemblage was conducted by Clarkson et al. (2009) using a sample of 5580 whole lithics. They noted a dominance of microblades throughout the sequence, and the appearance of backed artefacts from the earliest part of the sequence $(10 \mathrm{~cm}$ above the date of $34 \mathrm{ka}$ ). Backed microliths remain at a high frequency until just before the peak in artefact density c. $20 \mathrm{ka}$. In particular, they drew attention to a number of changes over time in lithic technology at the site: a transition in preferences from asymmetric to symmetric backed microliths; an increase in the number of ridges on microblades; the narrowing of microblades; an increase in the proportion of elongate parallel flake scars on microblade cores; a change in dorsal scar orientations from proximal-to-distal to bidirectional, crested and lateral orientations; a decrease in retouch intensity after $20 \mathrm{ka}$; and a decrease in the use of high quality raw material after $20 \mathrm{ka}$. Overall, their results contradict the view that microlithic industries are homogenous and unchanging.

Access was granted to the database of lithic measurements recorded by Clarkson and colleagues (2009). Lithics from the Pleistocene strata (C-E) were used (Figure 3.2.9), resulting in a total of 3238 lithics (after debitage not meeting the size requirements of the current study was excluded, as defined in Chapter 4 below). 


\section{Chapter 4: Methodology}

The previous chapter introduced the industries and sites studied. This chapter sets out the methods employed in the sampling and collection of data from these sites, the variables used in the attribute analysis, and the statistical techniques used to analyse the data.

\subsection{DATA COLLECTION AND SAMPLING STRATEGIES}

This thesis investigates entire production trajectories by using detailed technological attribute analyses and making use of all the products and byproducts of lithic manufacture. Measurements were made on cores, debitage and both microlithic and non-microlithic tools from the sites described in Chapter 3. The data collection strategy was designed with two purposes in mind. Firstly, the focus was on variables that elucidate technological strategies in raw material preferences, reduction strategies and techniques, blank production and selection, and retouch choices. Secondly, comparability and replicability were key, in order to facilitate comparisons with recoded databases of measurements made at Patne and Jwalapuram 9 by other researchers (Clarkson et al. 2009, James 2011). The methodology used here therefore encompasses that used at the Indian sites.

In order to permit analysis of technological changes over time, multiple layers were studied from the relevant sections at each site. At Umhlatuzana and NT, three 
layers were analysed. The HP section at RCC was excavated in spits, which were able to be separated into three roughly equal sections. These 'layers' are therefore as arbitrary as the spits in which the site was excavated, but they do allow for the comparison of different sections of HP occupation at the site. The Pleistocene section of Batadomba-lena was excavated in six layers. At Kitulgala the small number of microliths recovered was treated as a single sample and not subdivided.

All cores and microliths were measured from each layer studied. This was done both in order to maintain suitable numbers for analysis, and because cores are very informative of manufacturing processes (Clarkson 2010) and microlithic tools are the focus of this study. A sample of debitage and non-microlithic tools was also analysed from each layer. Only lithics over $10 \mathrm{~mm}$ were included in this sample, as smaller lithics are less informative in large numbers (Milne 2009). For example, it can be difficult to discern dorsal scar numbers and characteristics on very small lithics. The number of lithics excavated varied considerably between sites. Statistically speaking, larger samples tend to exhibit greater variation, so an absolute sample number was taken rather than a proportion in order to remove the effect of different sample sizes.

A sample size of 400 was chosen (in cases of less than 400 lithics in a layer, all were measured). For population sizes of more than a few thousand, the sample size required to be representative of the population increases very little as the population size increases (Krejcie and Morgan 1970, Guadagnoli and Velicer 1988). A sample size of 400 per layer and 1,200+ per site (with at least three layers 
per site) therefore meets the standards used by the majority of social science disciplines at the 95\% confidence level (Agresti and Finlay 2009).

Lithics were selected through systematic random sampling (e.g. with the selection of every third lithic). In the case of bags containing large numbers of lithics, these were set out on a table in order to avoid the effects of weight sorting within bags. Where layers were excavated in spits or sub-contexts (as at RCC, NT and Batadomba-lena), these contexts were sampled proportionally. This method, known as stratified sampling, has been shown to produce more representative samples than simple random sampling alone (Agresti and Finlay 2009).

Datasets from Patne and Jwalapuram 9 were cleaned and recoded into the methodological system used here. Only Pleistocene levels were included, and only lithics over $10 \mathrm{~mm}$. Sample sizes were slightly larger but still within the same order of magnitude as at the other sites, so the entire samples were used rather than being further sub-sampled.

\subsection{Attribute ANALYSIS}

There exist a huge number of different approaches to lithic analysis - possibly as many as there are researchers. These range from classifications of tool typology (e.g. Bordes 1961b), to mass analysis of debitage (e.g. Ahler 1989), to hi-tech 3D reconstructions of cores (e.g. Clarkson 2010). There is no 'right' way to conduct 
lithic analysis; the approach taken depends on the questions being asked of the assemblage/s.

As the focus in the present study is the detailed comparison of production techniques, an approach involving quantitative attribute analysis was chosen. The methodology was tested with a pilot study of 234 microliths and debitage pieces from the Sri Lankan Holocene sites of Bandarawela, Maskeliya and Pundalnoya, stored at the Pitt Rivers Museum, Oxford.

Many of the attributes selected for measurement are borrowed from the methods of Clarkson et al. (2009) and James (2011), for the reasons outlined above. The attributes in common are discussed in the relevant site result sections. Key texts on lithic analysis (Shott 1994, Odell 2004, and especially Andrefsky 2005) provided the other attributes used here. The variables measured are listed in Table 4.2.1.

\begin{tabular}{|c|c|}
\hline Attribute & Values/recording method \\
\hline \multicolumn{2}{|l|}{ Cores } \\
\hline Type & $\begin{array}{l}\text { Uni-directional - flake, blade (those where over half of terminal } \\
\text { removals are of blade dimensions) or bipolar } \\
\text { Bi-directional - flake or blade } \\
\text { Multi-directional (with three or more striking platforms) - } \\
\text { amorphous/polyhedral or radial/centripetal }\end{array}$ \\
\hline Raw material & Narrowest raw material category discernible, e.g. clear quartz \\
\hline Colour & Up to two colours recorded, in cases of banded rocks \\
\hline Weight & Measured in grams \\
\hline Longest dimension & Maximum linear dimension \\
\hline Longest axis & $\begin{array}{l}\text { The distance from the centre of the striking platform to the point } \\
\text { where the force exits the nucleus, measured perpendicular to the } \\
\text { striking platform. For cores with more than one platform the } \\
\text { longest removal axis is measured. }\end{array}$ \\
\hline Maximum width & Measured perpendicular to the longest axis \\
\hline Maximum thickness & Measured perpendicular to the longest axis and maximum width \\
\hline Longest face & Length of longest flaked face \\
\hline
\end{tabular}




\begin{tabular}{|c|c|}
\hline $\begin{array}{l}\text { Striking platform } \\
\text { width }\end{array}$ & $\begin{array}{l}\text { Longest measurement across the striking platform. For cores } \\
\text { with more than one platform the largest platform is measured. }\end{array}$ \\
\hline $\begin{array}{l}\text { Striking platform } \\
\text { thickness }\end{array}$ & Measured perpendicular to striking platform width \\
\hline Striking platform type & $\begin{array}{l}\text { Abraded/crushed } \\
\text { Cortical } \\
\text { Flat (1 facet) } \\
\text { Double ( } 2 \text { facets) } \\
\text { Complex ( } 3+\text { facets) }\end{array}$ \\
\hline $\begin{array}{l}\text { Number of striking } \\
\text { platforms }\end{array}$ & $\begin{array}{l}\text { Number of discernible striking platforms. Recorded as } \\
\text { 'circumference' for radial/centripetal cores with flakes removed } \\
\text { around the entire circumference of the core. }\end{array}$ \\
\hline $\begin{array}{l}\text { Perpendicular striking } \\
\text { platforms }\end{array}$ & $\begin{array}{l}\text { For bi-directional cores only, are platforms perpendicular } \\
\text { (rather than opposite)? }\end{array}$ \\
\hline Total number of scars & Number of removal scars \\
\hline Number of flake scars & Number of removal scars with length:width ratios of less than 2 \\
\hline Number of blade scars & Number of removal scars with length:width ratios of more than 2 \\
\hline $\begin{array}{l}\text { Number of aberrant } \\
\text { terminations }\end{array}$ & Number of step terminations \\
\hline $\begin{array}{l}\text { Percentage cortex } \\
\text { cover }\end{array}$ & $\begin{array}{l}\text { Percentage of cortical cover over the entire surface of the core, } \\
\text { excluding striking platform, recorded to the nearest } 10 \% \text { with } \\
\text { additional categories of } 5 \% \text { and } 95 \% \text { for cores with extremely } \\
\text { small or large proportions of cortex cover }\end{array}$ \\
\hline Recycling & $\begin{array}{l}\text { Evidence of being a re-worked older artefact, e.g. exhibiting worn } \\
\text { and/or patinated surfaces with ancient removal scars, alongside } \\
\text { more recent removal scars }\end{array}$ \\
\hline $\begin{array}{l}\text { Length of every } \\
\text { removal scar }\end{array}$ & The oriented length of every removal scar \\
\hline
\end{tabular}

\section{Debitage and tools}

\begin{tabular}{ll} 
Type & \multicolumn{1}{c}{ Debitage: } \\
& Blade (twice as long as it is wide, with parallel arrises) \\
& Flake-blade (exhibits only one of the two blade requirements) \\
& Flake \\
& Tools: \\
& Retouched (non-microlithic) blade \\
& Retouched (non-microlithic) flake \\
& Biface \\
& Microlith on blade \\
& Microlith on flake \\
& Whole \\
& Proximal (striking platform intact) \\
Condition & Medial (lateral margins intact but no striking platform or \\
& termination) \\
& Distal (termination intact) \\
& Longitudinal (one lateral margin intact and all or part of the \\
& striking platform and termination) \\
Rnclear & \\
Raw material & Narrowest raw material category discernible, e.g. clear quartz \\
Colour & Up to two colours recorded, in cases of banded rocks \\
\hline Weight & Measured in grams \\
Longest dimension & Maximum linear dimension \\
Oriented length & The distance from the striking platform to the point where the \\
\hline
\end{tabular}


force exits the nucleus, measured perpendicular to the striking platform

Maximum width
One quarter width

Measured perpendicular to the oriented length

Medial width

Measured perpendicular to the oriented length at the point $1 / 4$ of the length of the artefact from the proximal end

$\begin{array}{ll}\text { Three quarter width } & \begin{array}{l}\text { the proximal and distal ends } \\ \text { Measured perpendicular to the oriented length at the point } 3 / 4 \text { of } \\ \text { the length of the artefact from the proximal end }\end{array} \\ \text { Maximum thickness } & \begin{array}{l}\text { Measured perpendicular to the oriented length and maximum } \\ \text { width }\end{array} \\ \text { Medial thickness } & \begin{array}{l}\text { Measured perpendicular to the oriented length and maximum } \\ \text { width half-way between the proximal and distal ends }\end{array} \\ \text { Striking platform } & \begin{array}{l}\text { Maximum width of the striking platform, measured from lateral } \\ \text { margin to lateral margin }\end{array} \\ \text { width } & \begin{array}{l}\text { Maximum striking platform thickness measured perpendicular } \\ \text { to platform width }\end{array} \\ \text { thickness } & \begin{array}{l}\text { Abraded/crushed } \\ \text { Cortical }\end{array} \\ \text { Striking platform type } & \text { Point } \\ \text { Flat (1 facet) } \\ \text { Double (2 facets) } \\ \text { Complex (3+ facets) }\end{array}$

$\begin{array}{ll}\begin{array}{l}\text { Striking platform } \\ \text { lipping }\end{array} & \begin{array}{l}\text { Presence of platform lipping, recorded as pronounced, slight or } \\ \text { absent }\end{array} \\ \text { Curvature } & \text { The angle of the arc in the isosceles triangle created by the curve }\end{array}$

Curvature The angle of the arc in the isosceles triangle created by the curve of a flake (Figure 4.2.1)

\begin{tabular}{ll}
$\begin{array}{l}\text { Percentage cortex } \\
\text { cover }\end{array}$ & $\begin{array}{l}\text { Percentage of cortical cover on the dorsal surface, recorded to } \\
\text { the nearest } 10 \% \text { with additional categories of } 5 \% \text { and } 95 \% \text { for } \\
\text { artefacts with extremely small or large proportions of cortex } \\
\text { cover }\end{array}$ \\
$\begin{array}{l}\text { Number of dorsal } \\
\text { scars }\end{array}$ & $\begin{array}{l}\text { Number of scars extending over more than } 1 / 4 \text { the length of the } \\
\text { artefact }\end{array}$ \\
$\begin{array}{l}\text { Number of parallel } \\
\text { arrises }\end{array}$ & $\begin{array}{l}\text { Number of arrises/ridges running parallel to the lateral margins } \\
\text { of the artefact and extending all or most of the way along its } \\
\text { length }\end{array}$ \\
\hline Scar orientation & $\begin{array}{l}\text { The number of directions from which dorsal scars originate. } \\
\text { Measured by superimposing diagonal quadrants over an artefact } \\
\text { and summing the number of quadrants with any flakes removed } \\
\text { from the periphery inwards. Of those artefacts with an }\end{array}$ \\
& $\begin{array}{l}\text { orientation of 2, whether the scars are from adjacent or opposite } \\
\text { margins/quadrants is also recorded. }\end{array}$ \\
See Figure 4.2.2 \\
The presence of at least three of the common indicators of \\
bipolar percussion (Cotterell and Kamminga 1987, Odell 2004, \\
percussion
\end{tabular}




\begin{tabular}{|c|c|}
\hline & $\begin{array}{l}\text { and/or patinated surfaces with ancient removal scars, alongside } \\
\text { more recent removal scars }\end{array}$ \\
\hline $\begin{array}{l}\text { Evidence of core } \\
\text { maintenance }\end{array}$ & $\begin{array}{l}\text { Core redirection flake } \\
\text { Core rejuvenation flake } \\
\text { Crested blade } \\
\text { Platform preparation/overhang removal flake (Figure 4.2.4) }\end{array}$ \\
\hline Other classification & $\begin{array}{l}\text { Burin spall } \\
\text { Snapped blade (where intentional snapping is clear) } \\
\text { Obliquely snapped blade } \\
\text { Snapped flake }\end{array}$ \\
\hline \multicolumn{2}{|l|}{ Tools } \\
\hline Typology & $\begin{array}{l}\text { Typological classification based on morphological attributes (e.g. } \\
\text { retouch location and type) }\end{array}$ \\
\hline $\begin{array}{l}\text { Presence of tang or } \\
\text { shoulders }\end{array}$ & $\begin{array}{l}\text { Presence of tang (haft worked on both margins) or shoulder } \\
\text { (haft worked on one margin) }\end{array}$ \\
\hline Haft width & Representative width measurement of hafted section \\
\hline Haft thickness & Representative thickness measurement of hafted section \\
\hline Haft length & Maximum length of hafted section \\
\hline $\begin{array}{l}\text { Presence of haft } \\
\text { notches, abrasion or } \\
\text { polish }\end{array}$ & Presence of notches, abrasion or polish on hafted section \\
\hline $\begin{array}{l}\text { Number of worked } \\
\text { edges }\end{array}$ & $\begin{array}{l}\text { Number of worked edges/incidences of retouch, excluding haft. } \\
\text { For example, a tool worked continuously and in the same } \\
\text { manner along a lateral and distal edge would have a single } \\
\text { incidence of retouch. }\end{array}$ \\
\hline \multicolumn{2}{|l|}{ For each worked edge: } \\
\hline Location & $\begin{array}{l}\text { Entire circumference } \\
\text { Distal } \\
\text { Lateral } \\
\text { Proximal } \\
\text { Any combination of distal, lateral and proximal edges }\end{array}$ \\
\hline Face & $\begin{array}{l}\text { Dorsal } \\
\text { Ventral } \\
\text { Alternating (alternating between dorsal and ventral) } \\
\text { Bifacial (retouched on both the dorsal and ventral faces in the } \\
\text { same location) } \\
\text { Edge (bipolar backing retouch conducted from both the dorsal } \\
\text { and ventral faces to create a perpendicular edge) }\end{array}$ \\
\hline Outline & See Figure 4.2 .5 \\
\hline Type & See Figure 4.2.6 \\
\hline Angle & Angle of the retouched edge, measured to the nearest $5^{\circ}$ \\
\hline Depth & $\begin{array}{l}\text { Maximum depth of retouch, measured from the lateral margin to } \\
\text { the termination of the retouch scars }\end{array}$ \\
\hline Length & $\begin{array}{l}\text { Length of the worked edge, measured following the contours of } \\
\text { the edge }\end{array}$ \\
\hline Index of Invasiveness & $\begin{array}{l}\text { Clarkson's (2002) measurement of the extent of retouch on a tool } \\
\text { (see Figure 4.2.7 and discussion below) }\end{array}$ \\
\hline
\end{tabular}

Table 4.2.1: Attributes measured on cores, debitage and tools 
Figure 4.2.1: Calculation of flake curvature (after Andrefsky 2005, Figure 5.14). $\mathrm{L}$ is the oriented length of the flake, $T$ is the medial thickness, and $A$ (angle height) is measured by pressing calipers together along the dorsal and ventral faces of the flake. The angle of curvature, $c$, is calculated thus: $c=2\left(90-\tan ^{-1}\left(\frac{2(A-T)}{L}\right)\right)$

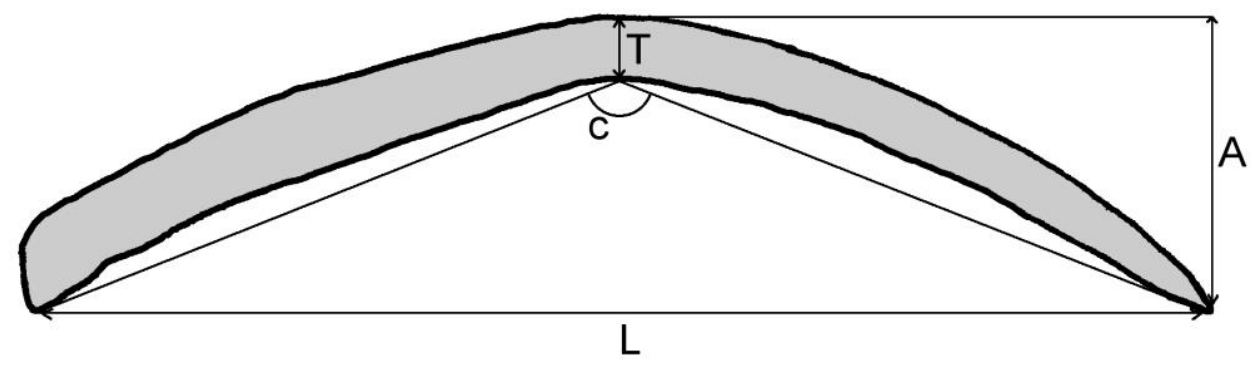

Figure 4.2.2: Flake termination types recorded in the attribute analysis

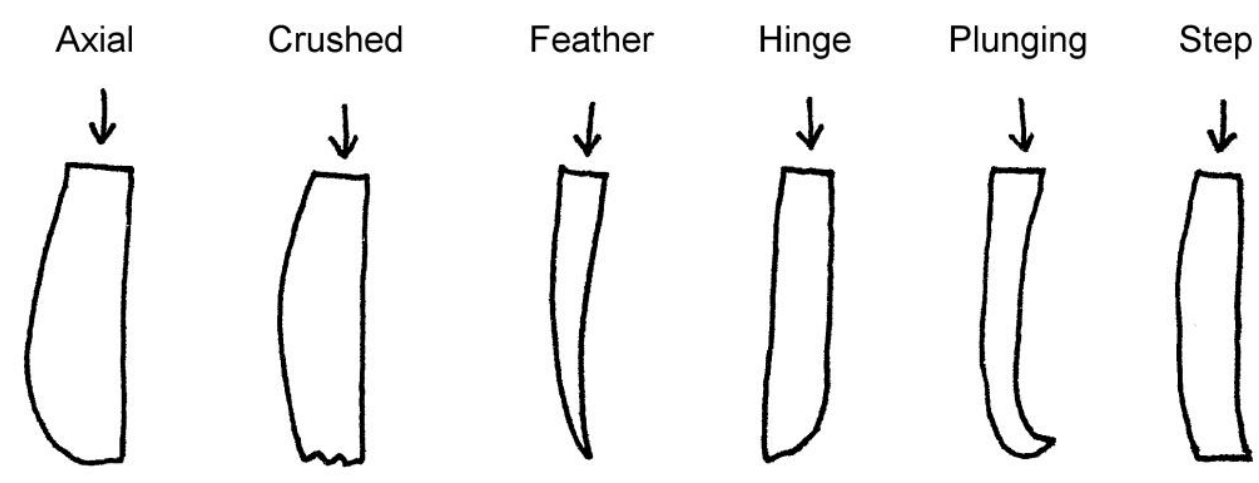

Variables were selected for measurement that help elucidate technological strategies in raw material preferences, core reduction systems, blank production and selection, and retouch choices. For example, by recording fine-scale variations in raw material (such as different qualities of quartz), as well as evidence for lithic recycling and cortex cover, it is possible to discern the form in which raw materials occur. Core types and striking platform numbers can tell us about core reduction techniques, as can the recording of evidence for the use of bipolar percussion. Core reduction intensity can be explored by looking at the numbers of different types of removals and removal lengths, particularly when compared to core size. 
Figure 4.2.3: Features of bipolar percussion, showing wedging initiation, flat fracture planes with perpendicular striking platforms, compression rings, and distal fissuring (after Andrefsky 2005, Figure 6.3)

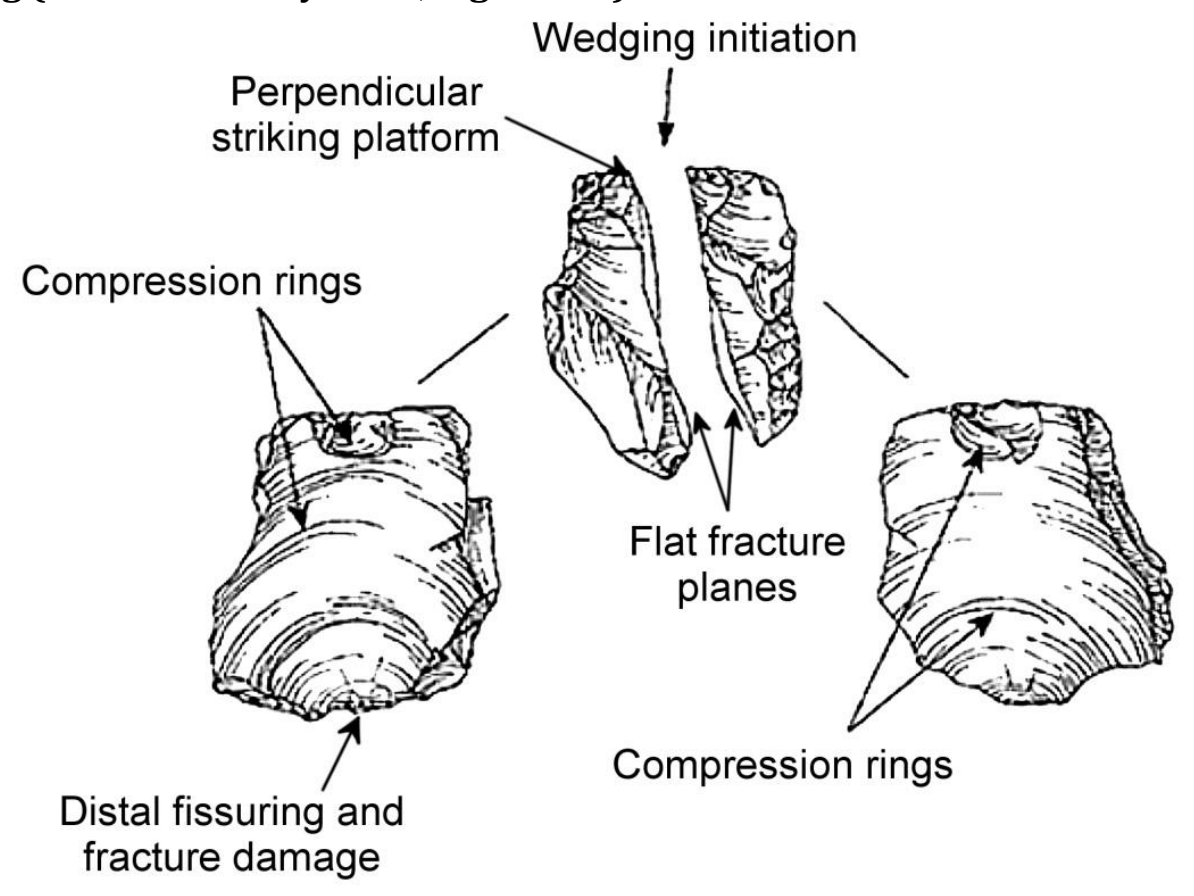

Variables concerning debitage type, size, curvature, cortex cover, striking platforms, dorsal scars and parallel arrises can inform us about stages of core reduction and blank production, as can the recording of evidence for platform preparation, core rejuvenation or platform lipping. For example, some researchers consider platform lipping as indicative of soft-hammer percussion, although others do not consider it to be sufficient evidence in the absence of other indicators (Andrefsky 2005, Driscoll and García-Rojas 2014). When these same variables are considered on retouched tools, inferences can also be made about blank selection. Finally, the recording of specific morphological types as well as the location, face, outline, type, angle, depth and length of each incidence of retouch can provide detailed information about retouch choices and overall tool manufacturing strategies. 
Figure 4.2.4: Core maintenance flakes. A: core redirection flake (James 2011, Figure A.25). B: core rejuvenation flake (example from a blade core) (Inizan et al. 1999, Figure 77). C: crested blade (Inizan et al. 1999, Figure 64). Platform preparation flake/overhang removal (Jones 2007, volume 2: 573)

A

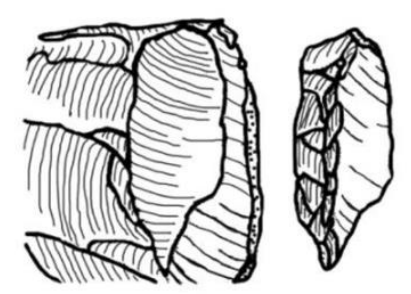

$\mathrm{B}$
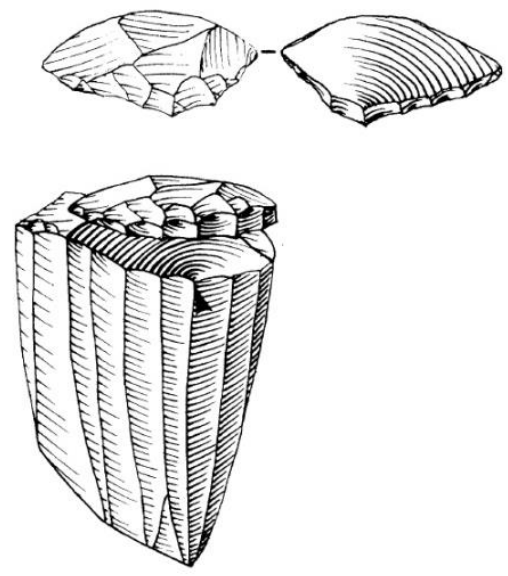

D

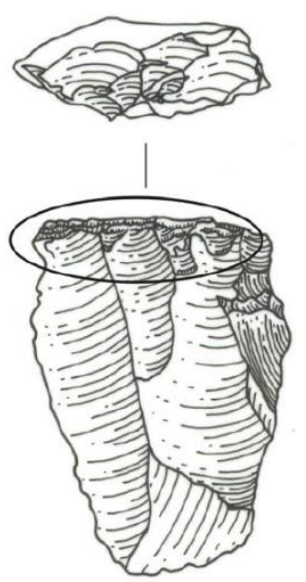

Figure 4.2.5: Retouch outlines recorded in the attribute analysis

Concave Convex Irregular Nosed/beaked Perpendicular Pointed Straight


Figure 4.2.6: Retouch types recorded in the attribute analysis. 'Thinning' retouch refers to a single extensive removal made on one face, often made from the proximal end on the ventral face to remove the bulb of percussion.

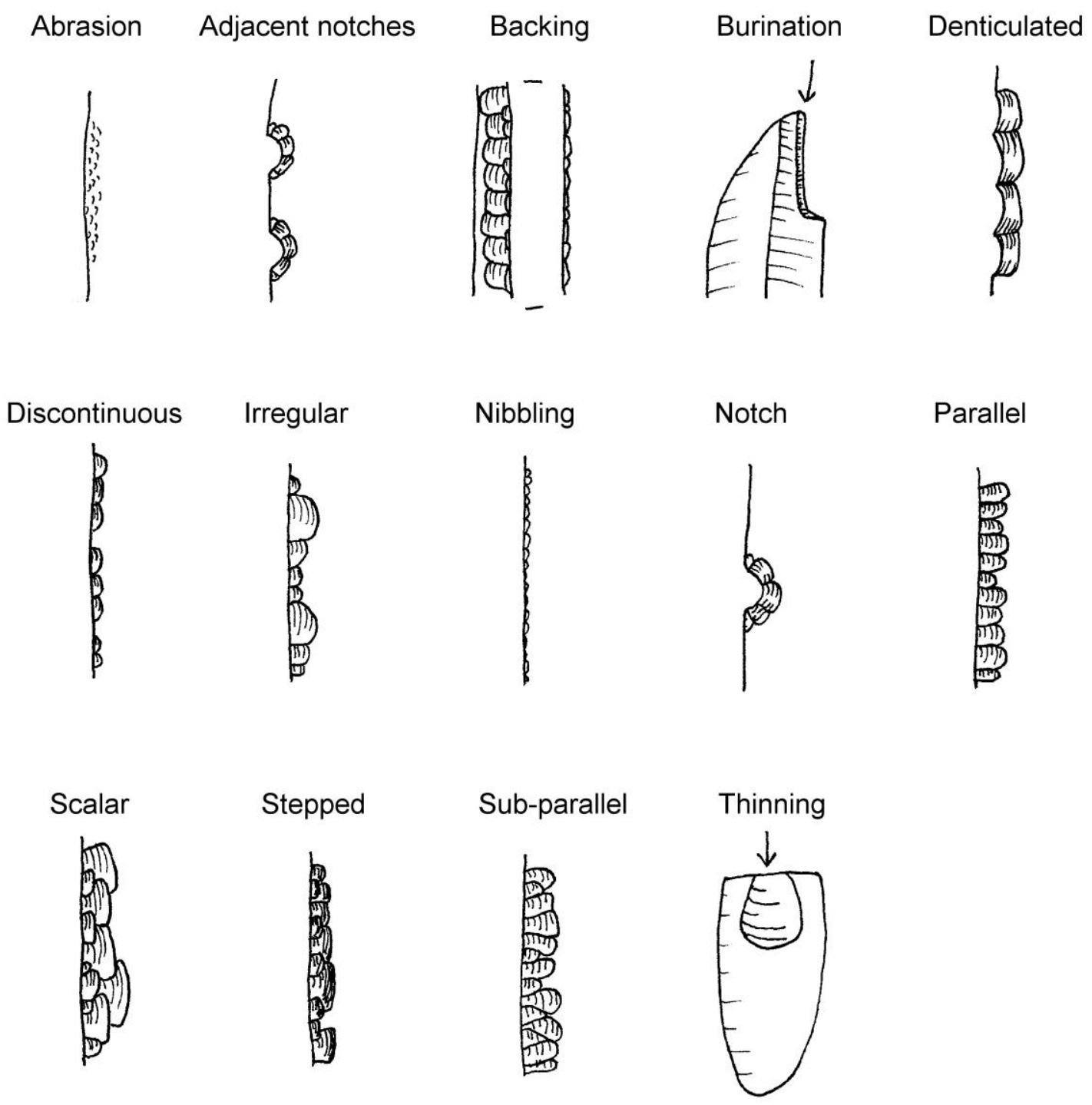

Some differences exist between the methods employed here and those sometimes used by other researchers. The angle of striking platforms is not measured, as although it can be informative it can be problematic to measure and thus can vary considerably between researchers (Andrefsky 2005). It was therefore excluded as replicability and comparability are key design features of this methodology. Also, although proximal and distal flake widths are measured by other researchers (e.g. Clarkson 2007), these are often measured at suitable but arbitrary locations along 
the length of the flake. In order to make the results more directly comparable between pieces, and to ensure that they are replicable, proximal and distal measurements are measured here at $1 / 4$ and 3/4 lengths, respectively. Also, in contrast to many tool classifications but in keeping with some more recent studies (e.g. Jones 2007, Clarkson et al. 2009, Petraglia et al. 2009a), typological classification is based here on morphological attributes, e.g. 'side retouched', rather than potentially misleading functional nomenclature, e.g. 'side scraper'.

Figure 4.2.7: Method for measuring Clarkson's Index of Invasiveness (after Clarkson 2002, Figure 1)

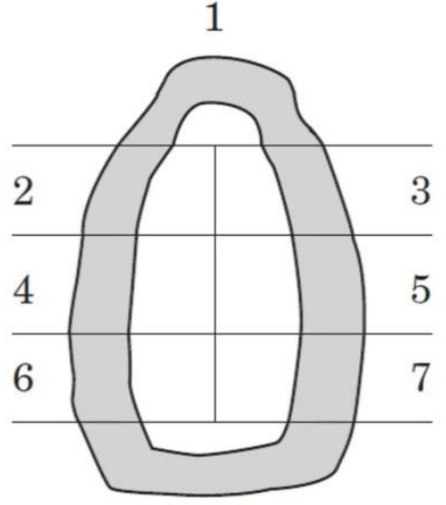

8

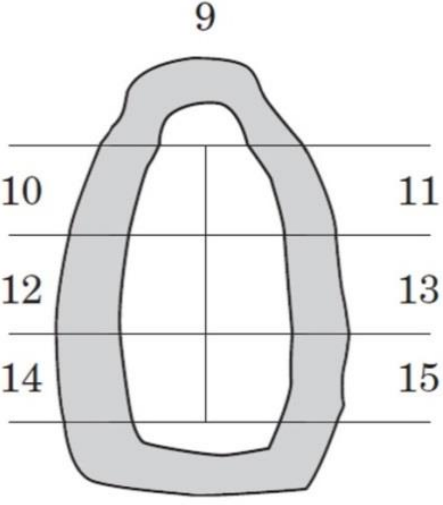

16

Score

Ventral

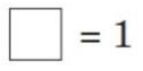

$=0.5$

Several methods exist for quantifying the intensity or extent of retouch on a tool. One is Kuhn's (1990) Geometric Index of Unifacial Reduction (GIUR), which involves the calculation of the ratio between the thickness of the retouched edge of a tool and its maximum thickness. However, this method is best used for the assessment of the re-working of unifacial tools, whereas one of the advantages of microlithic technology is that microliths can be manufactured in large numbers and replaced when broken, rather than requiring re-working (see Chapter 2.1). 
Also, the GIUR does not work well on backed tools (backing being common on microliths), as in these cases the retouched edge is usually also the maximum thickness of the tool.

Clarkson's (2002) Index of Invasiveness (IOI) is therefore preferred in this study. This technique requires the division of the dorsal and ventral faces into eight segments (Figure 4.2.7). A score of 1 is assigned to segments where retouch extends into the inner part of the artefact's surface, a score of 0.5 is given where retouch is confined to the outer part, and segments containing no retouch are given a score of 0 . The total score is then divided by the number of segments (16) to give a result on a scale of 0 to 1 , with 1 indicating a tool that is extensively retouched over all of its dorsal and ventral faces. The IOI has faced some criticism, for example over the fact that the 'inner' and 'outer' parts of tool surfaces are not precisely defined and might vary between researchers (Andrefsky 2005). Nevertheless, it provides the best available method for describing the extent of retouch on microlithic tools with a single comparable number.

The collection of quantitative metric attributes also allows for the calculation of composite variables. For example, ratios are calculated per core of the number of scars, average lengths of scars, and total lengths of scars (i.e. the sum of all scar lengths on a core) to core volume. These allow core reduction intensity to be investigated by looking at the numbers and sizes of scars in relation to the size of the core when it was discarded. 
Two additional composite measurements used are width standard deviation and tip cross-sectional area. A measure of width standard deviation was produced in order to provide a way of quantifying the parallel-ness of the lateral margins of debitage and tools, with lower values indicating greater parallel-ness (and a value of 0 indicating perfectly parallel margins). It is a measure of the standard deviation of widths at different locations on an artefact, calculated with reference to the differences between one-quarter (a), medial (b) and three-quarter (c) width measurements and the mean of width measurements $(\mu)$, such that width standard deviation $=\sqrt{\frac{(a-\mu)^{2}+(b-\mu)^{2}+(c-\mu)^{2}}{3}}$.

Tip cross-sectional area (TCSA) has been shown to be a useful method for separating different types of lithic projectiles, and is calculated as $0.5 \mathrm{x}$ maximum width x maximum thickness (Hughes 1998, Shea 2006). Shea (2006: 829) argues that TCSA may be a less suitable measurement for "certain 'backed pieces' found in a variety of Subsaharan MSA contexts" because they may have been mounted on the sides of wooden shafts as barbs, rather than at the tip. However, TCSA measurements can provide a useful point of comparison between microlithic and non-microlithic tools, and can also be compared against ethnographic and experimental data, such as that reported by Shea (2006). This is especially the case when microlith hafting configurations can be guessed at with a degree of certainty, such as in the case of shouldered or tanged microlithic points. 


\subsection{StATISTICAL ANALYSIS}

Statistical analyses of the attribute data collected were conducted using SPSS v. 21. The use of a quantitative attribute analysis approach allows for the application of various uni- and bi-variate analytical techniques. The results of these analyses are presented in Chapters 5-7, with supplementary data reported in Appendix A. The statistical tests of significance used are justified in Appendix B. An additional statistical measure used in the presentation of results is the coefficient of variation (CV). The CV is a measure of dispersion and standardisation, calculated as the ratio between the standard deviation and the mean of a sample: $\mathrm{CV}=\frac{\sigma}{\mu} \times 100$. Lower $\mathrm{CV}$ values indicate greater degrees of standardisation, although interpretation of values is subjective. Commonly used cut-off points indicative of standardisation are 10 (Wadley and Mohapi 2008, Lombard et al. 2010) and 20 (Fisher 2006). Eerkens and Bettinger (2001) demonstrate mathematically that a CV of 1.7 describes the highest degree of standardisation that is producible and discernible by humans, while a CV of 57.7 would be observed in completely random artefact production. A cut-off point for standardisation of 20 is used in the present study, although this remains an arbitrary guide to whether or not standardised production occurred.

Additionally, multi-variate statistical analyses can be applied, as used successfully elsewhere on similar lithic datasets (e.g. McCall 2006, 2007, Clarkson 2010, Clarkson et al. 2012, Scerri et al. 2014). Specifically, principal component analysis (PCA) and discriminant function analysis (DFA) are made use of here. PCA is a multivariate statistical technique that can be used to reduce large numbers of 
continuous numeric variables to a smaller number of composite factors. It is a useful method for determining which variables constitute each of these latent factors, and what percentage of variation each component explains. It aims to find the smallest number of variables that explain the most variance. PCA is therefore a suitable test to use in order to assess which attributes best describe the differences between sites.

The methods used are from general and archaeology-specific texts on the use of PCA (Baxter 1995, 2003, 2014, Beaumont 2012). PCA makes use of numeric ratio or interval data. There should be more cases than variables used in the analysis. Correlations between variables should be high but not exact (multicollinearity). After these assumptions and prerequisites were verified, the Bartlett Test of Sphericity was used to determine that the correlation matrix is statistically significantly different to an identity matrix (a matrix with zero correlations). Additionally, the Kaiser-Meyer-Olkin Measure of Sampling Adequacy (KMO) was used to determine the proportion of variance that might be indicative of common latent factors. A value of at least 0.5 and preferably over 0.8 is generally taken to indicate that PCA is appropriate.

Because some of the variables used are measured on different scales (for example, size measurements are measured in millimetres while cortex is measured as a percentage of surface cover), principal components were extracted based on the correlation matrix, rather than the covariance matrix. This removes the scaling effect of the variables by standardising data to a mean of 0 and a standard deviation of 1. After PCA was conducted, the factor loadings of variables were 
examined to discern which groups of variables best explain the differences evident between sites. Additionally, component scores for each artefact were calculated in order to assess the degree of similarity or difference between sites. Missing values were substituted with mean values to allow for the inclusion of the Indian sites, where not all variables were recorded.

DFA is a multivariate statistical technique that can be used to predict group memberships based on composite variables (the discriminant functions) constructed from multiple independent variables. It is a useful method for assessing which variables are the best predictors of groupings (in this case sites) and how successful they are at doing so, and which groups are more closely related to each other. It aims to maximally separate groups in the most parsimonious way. DFA is therefore a suitable test to use in order to assess the extent to which the variables measured can predict site identity, and the degree of similarity and difference in lithic technology between sites.

The methods used are from general and archaeology-specific texts on the use of DFA (Klecka 1980, Baxter 2003, Spicer 2004, Burns and Burns 2008, Beer 2011). The variables used in DFA should consist of continuous numeric (ratio) data (although exceptions can be made for interval data), and be normally distributed and independent. There should be fewer predictor variables than there are cases in the smallest group (site) sampled, and the total sample size should have at least ten times as many cases as there are variables (Diekhoff 1992). The variancecovariance matrices for each group should be equal (or should not be statistically significantly unequal), which were assessed through the Box's M test. However, it 
should be noted that Box's M is very sensitive to testing the assumption of multivariate normality. Therefore in large samples it is likely to be significant (i.e. significantly different from normal) due to small deviations from homogeneity. Even if Box's M is significant, if the sample size is large (at least a few hundred) and the log determinants for each group are similar then this violation of the assumption can be safely ignored. Finally, multicollinearity should be avoided between variables, i.e. inter-correlation between predictor variables should be assessed, and redundant variables excluded where appropriate (a correlation of 0.8 is often used as a rule of thumb for exclusion).

First, the composite discriminant functions were derived from the input variables. The stepwise procedure was used, whereby variables are chosen for inclusion based on statistical criteria (specifically Wilks' lambda, which tests whether the variable's contribution is significant), in order to develop the most accurate and parsimonious model. The functions were then used to attempt to classify groups back into themselves, in order to determine the accuracy of the DFA model. The predictive power of the model was compared to the base chance of artefacts belonging to a particular site, known as the prior probability. Prior probabilities were assessed on the basis of group sizes rather than as an equal fraction of the total population, in order to account for the differences in sample sizes between the sites. After DFA was conducted, structure matrices (similar to factor loadings in PCA) were consulted in order to determine which variables are the best predictors of site identity, and how they are grouped. The prediction abilities of the models known as the posterior probabilities - were then compared to the prior probabilities in order to assess the extent to which the variables can predict group 
(site) membership. Finally, function scores were calculated for each artefact in order to examine site clustering.

In the following chapters ( 5 and 6), results will initially be presented on a site by site basis, in order to answer the first question asked by this thesis:

What degree of variability is evident within individual assemblages, and what can this tell us about the choices made and strategies used by the groups in question?

Lithic technology will then be compared between layers at each site, in order to answer the second question:

How does microlithic technology change over time, both within individual sites and within regions, and what may have influenced these changes?

Finally, in Chapter 7 comparisons will be made between the HP sites, and between the Late Palaeolithic sites, before being compared overall between regions, in order to answer the third question:

What differences and similarities are evident between the earliest microlithic industries in southern Africa, India and Sri Lanka, and what factors might explain this? 


\section{Chapter 5: Howiesons Poort microlithic technology}

This chapter presents the results of analyses of lithic assemblages from HP sites. The core, debitage and tool assemblages from each site are considered in turn. Differences between layers are then discussed, and manufacturing trajectories reconstructed. Supplementary data referred to in the text (for example, breaking data down into finer categories) is found in Appendix A, denoted by table references beginning with $A$.

\subsection{Rose Cottage Cave, South Africa}

\section{CORES}

Uni- and bi-directional blade cores are the most common core types (39.7\%) (Table 5.1.1). Other core morphologies include both 'typically MSA' radial cores (Ambrose 1998, Wurz 2013) and 'typically LSA' bipolar cores (Ambrose 2002, Villa et al. 2012, Eren et al. 2013). A variety of raw materials were used, with a dominance of crypto-crystalline silicas (CCS), specifically jasper, chalcedony, agate and chert. Claystone (a fine-grained sedimentary rock similar to mudstone, comprised primarily of clay-sized particles), siltstone, hornfels, silcrete, quartzite and quartz are also evident. Analysis of the proportions of core types made on different raw materials reveals preferences in the use of these materials (Figure 5.1.1). For example, jasper was used preferentially for blade cores. The most likely 
source of the raw materials knapped at RCC is the Caledon River, $8 \mathrm{~km}$ away (Wadley and Harper 1989).

\begin{tabular}{llrr}
\hline Core type & & Frequency & Percentage \\
\hline Uni-directional: & Blade & 46 & 22.5 \\
& Flake & 23 & 11.3 \\
& Bipolar & 20 & 9.8 \\
& & & \\
Bi-directional: & Blade & 35 & 17.2 \\
& Flake & 28 & 13.7 \\
& & & \\
Multi-directional: & Radial & 26 & 12.7 \\
& Amorphous & 26 & 12.7 \\
\hline Total & & 204 & \\
\hline
\end{tabular}

Table 5.1.1: Core type frequencies at RCC

Figure 5.1.1: Raw material proportions of cores at RCC

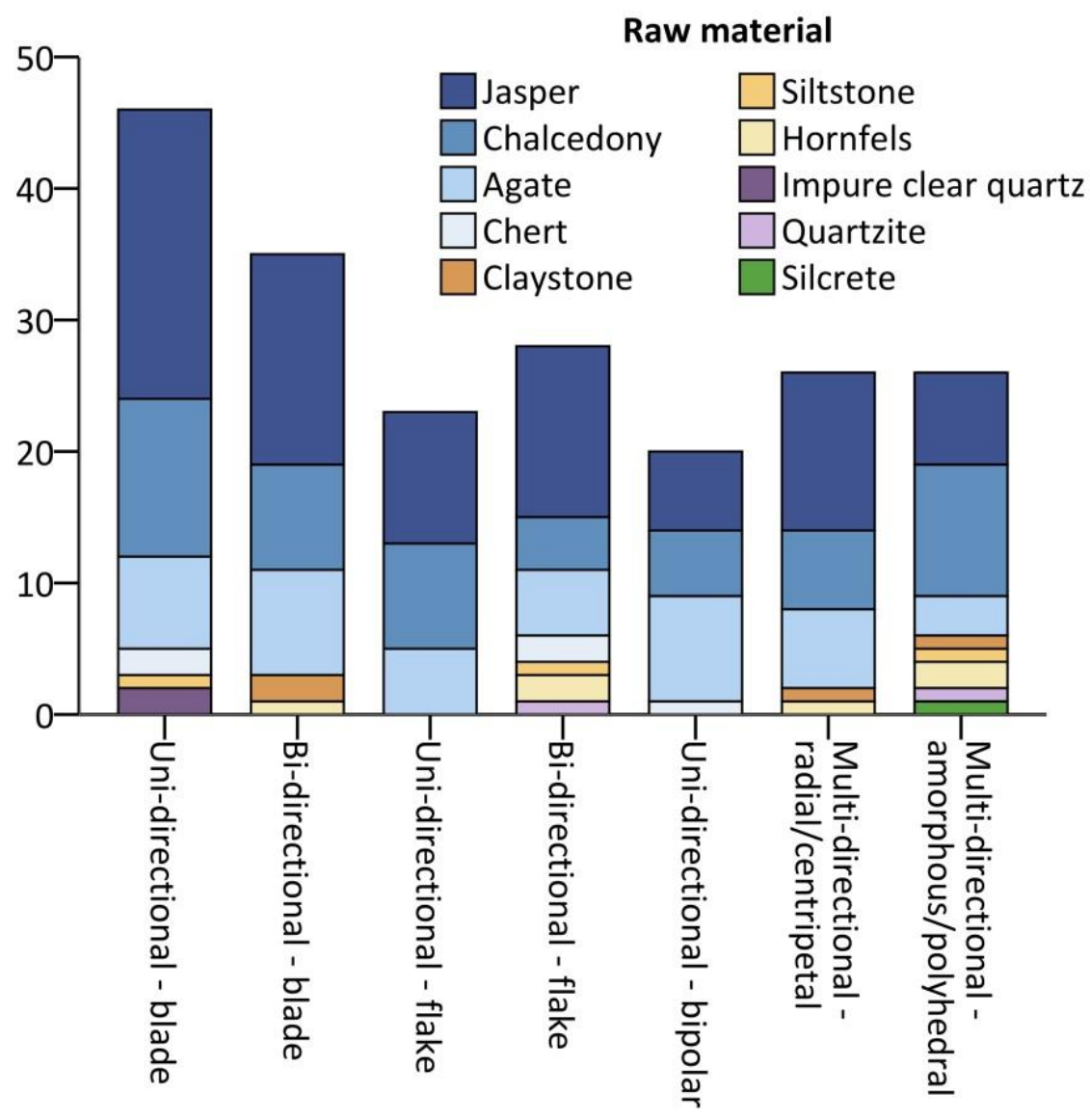


One important source for raw material procurement was through the recycling of older lithics from earlier MSA sites in the area (Figure 5.1.2). Overall, $40.2 \%$ of cores appear to have been recycled in this way; for some core types this figure approaches 60\% (Figure 5.1.3). The "abundance of twice-worked specimens" was also noted by Malan (1952: 192). There are known earlier MSA sites in the area, including one $100 \mathrm{~m}$ away on the hillside above the cave (Wadley, pers. comm.) which are likely sources of the materials. Alternatively, these lithic clasts may have been encountered in the nearby Caledon River, similar to other raw materials (Wadley and Harper 1989, Wadley 1991).

Figure 5.1.2: Examples of recycled lithics from RCC. Top: older artefacts used as tool blanks, which were retouched in order to modify their edges. Bottom: older artefacts used as cores, with flakes removed.

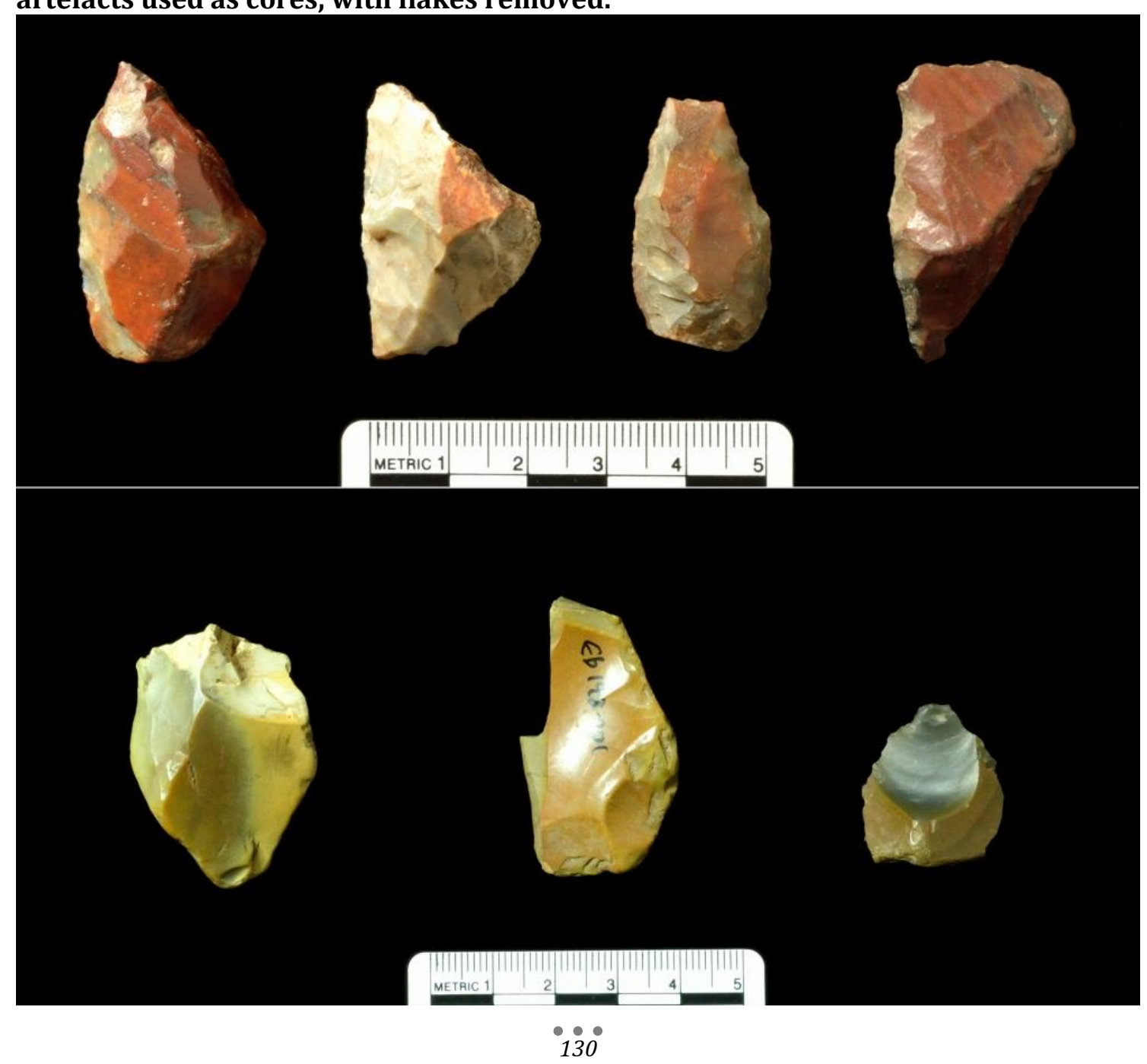


Figure 5.1.3: Proportions of cores manufactured from recycled lithics at RCC

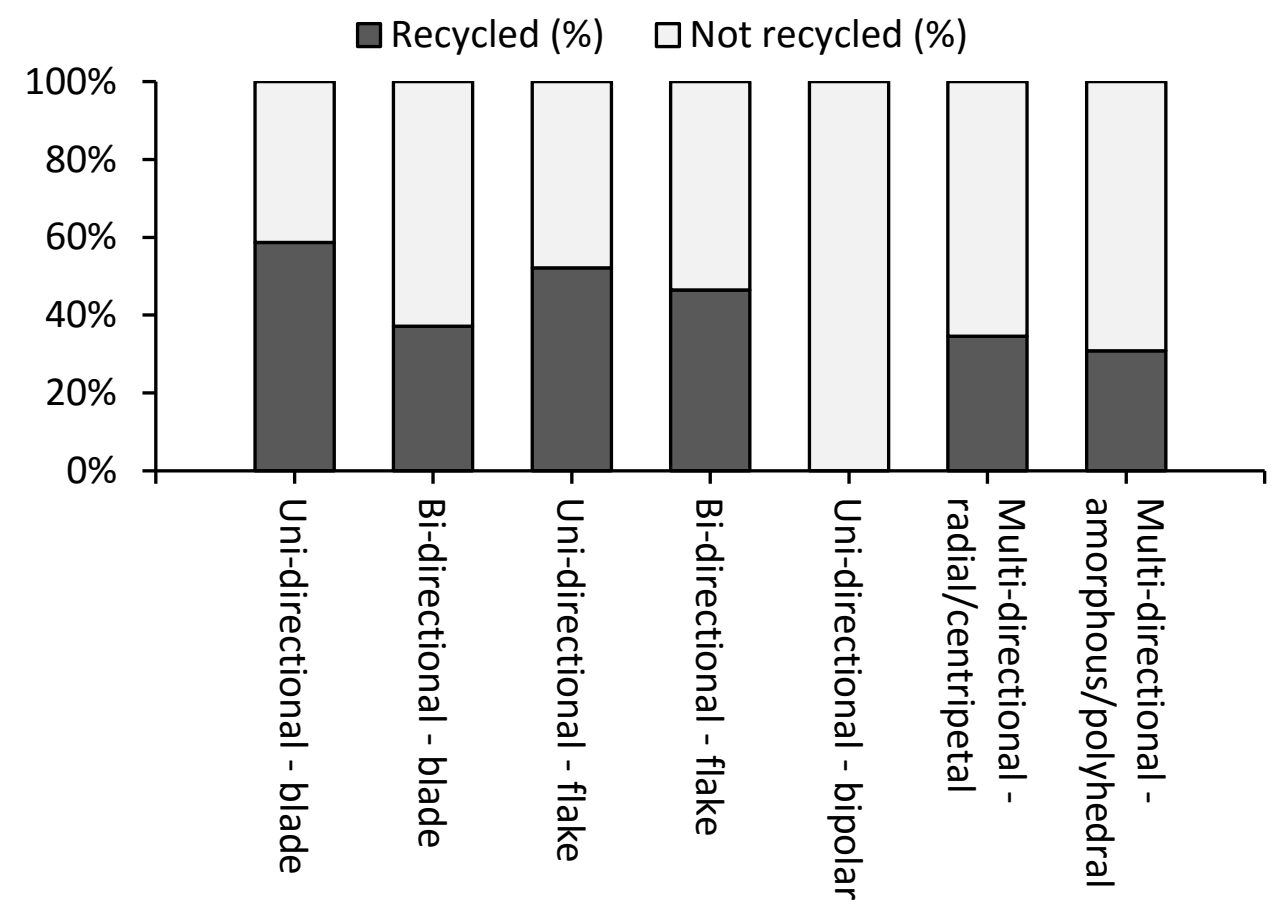

Average core size is $29.3 \times 23.5 \times 12.8 \mathrm{~mm}$, although variation exists between core types (Table A.1.1, Table A.1.2). Uni- and bi-directional blade cores are - as might be expected - longer and thinner on average, with mean measurements of $29.8 \mathrm{x}$ 14.6 × $7.8 \mathrm{~mm}$, compared to $28.1 \times 18.0 \times 8.3 \mathrm{~mm}$ for flake cores. Yet blade cores are also smaller and lighter than flake cores (Figure 5.1.4), with a mean weight of $8.4 \mathrm{~g}$ and mean cubic volume of $8.05 \mathrm{~cm}^{3}$, compared to $11.7 \mathrm{~g}$ and $11.93 \mathrm{~cm}^{3}$. Of these measurements, there are statistically significant differences at the 0.05 level in maximum width $(\mathrm{t}(130)=5.245, \mathrm{p}<.001)$, maximum thickness $(\mathrm{t}(130)=2.377$, $\mathrm{p}=.020)$ and weight $(\mathrm{t}(130)=2.683, \mathrm{p}=.009)$. Flake and blade cores appear to have been worked to different extents, as well as in different ways. Alternatively, blade cores may have been discarded at a later stage in the reduction process. This conclusion is supported by the fact that mean cortex cover is lower on blade cores $(30 \%)$ than on flake cores $(40 \%)$. 
Figure 5.1.4: Weight distributions of cores at RCC

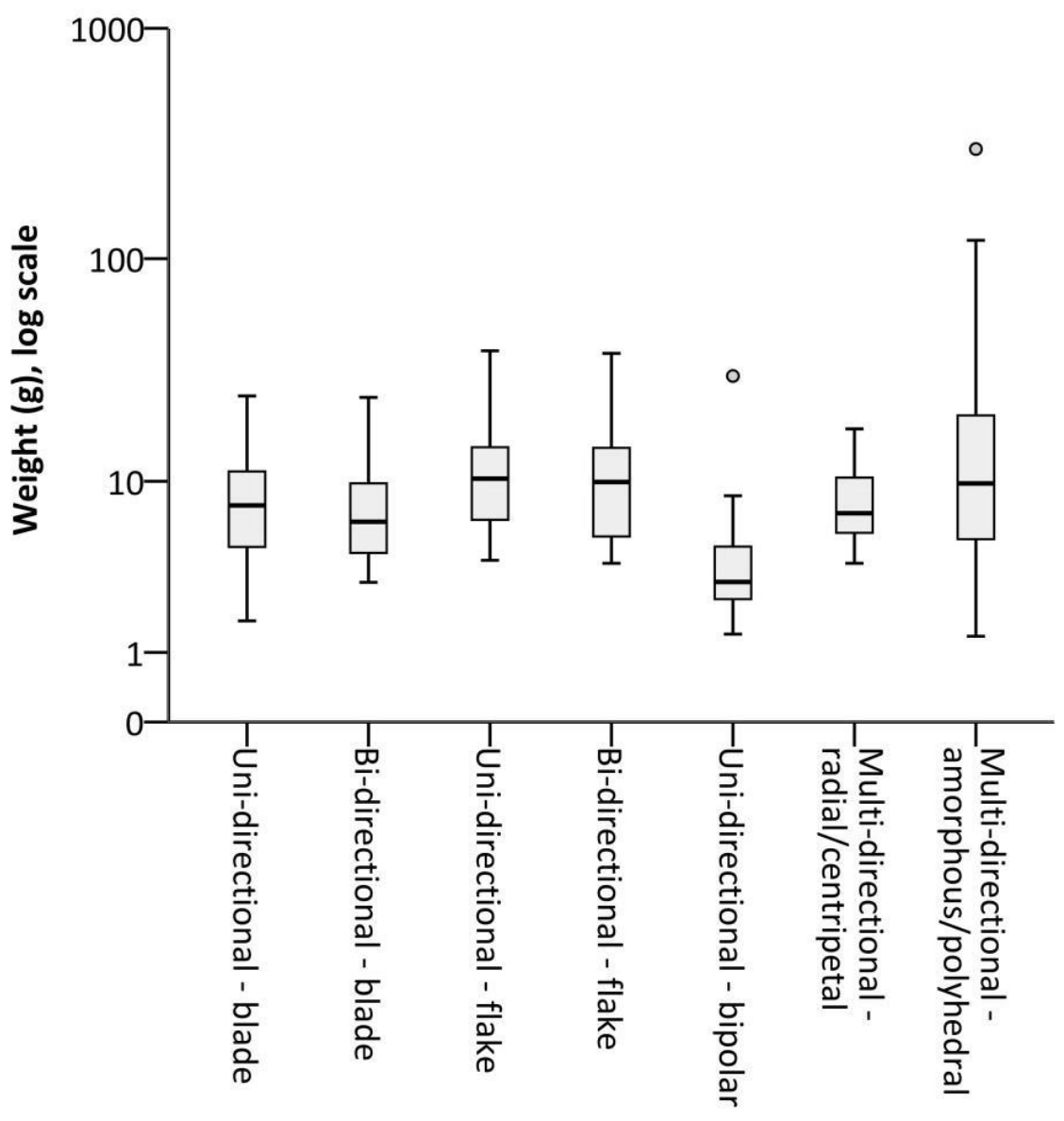

Bipolar cores are considerably smaller than other core types (Figure 5.1.4, Table A.1.2). A bipolar reduction strategy may have been adopted in response to the small size of the nodules being worked. Agate appears to have occurred in small sized nodules in the local environment (indicated by the size of agate cores with high percentages of cortex cover), and was used in higher proportions for bipolar cores (Figure 5.1.1). $40.0 \%$ of bipolar cores were made on agate, compared to $20.6 \%$ in the core assemblage as a whole. Alternatively - or additionally - bipolar percussion may have been used as a final knapping strategy in the reduction sequence of nearly-exhausted cores. 
The majority of multi-directional amorphous cores have three striking platforms (84.6\%) (Table A.1.3). The majority of radial cores were worked from the entire circumference of the core (84.6\%), and only a small proportion had a specific number of discernible platforms around the perimeter, confirming that this was a distinct core reduction technique at the site. An additional difference in the working of two-platform flake and blade cores can be seen in the more frequent rotation of flake cores. While only one blade core was worked from perpendicular platforms, $28.6 \%$ of flake cores were, and all appear to have been worked from both platforms simultaneously.

Striking platform size and type also vary between core types. Overall, flat and cortical striking platforms are most common (29.9\% and 29.4\%, respectively), followed by double-facetted (14.7\%), abraded/crushed (14.2\%) and complex (11.8\%) platforms. Figure 5.1.5 demonstrates that uni-directional cores exhibit a lesser degree of platform preparation, with fewer facetted platforms (double and complex) than bi-directional types.

Figure 5.1.6 reveals that on average multi-directional cores, particularly amorphous cores, exhibit the largest number of removal scars when discarded, and uni-directional cores exhibit the least. In terms of flake/blade scar proportions, the mean number of blade scars on bipolar cores is identical to that of uni-directional blade cores (3.9), while the mean overall number of removals per core is almost identical to that of bi-directional blade cores (7.2 v. 7.1) (Table A.1.4). Therefore, bipolar cores can be considered a type of blade core in this assemblage. 
Figure 5.1.5: Proportions of core striking platform types at RCC

Striking platform type

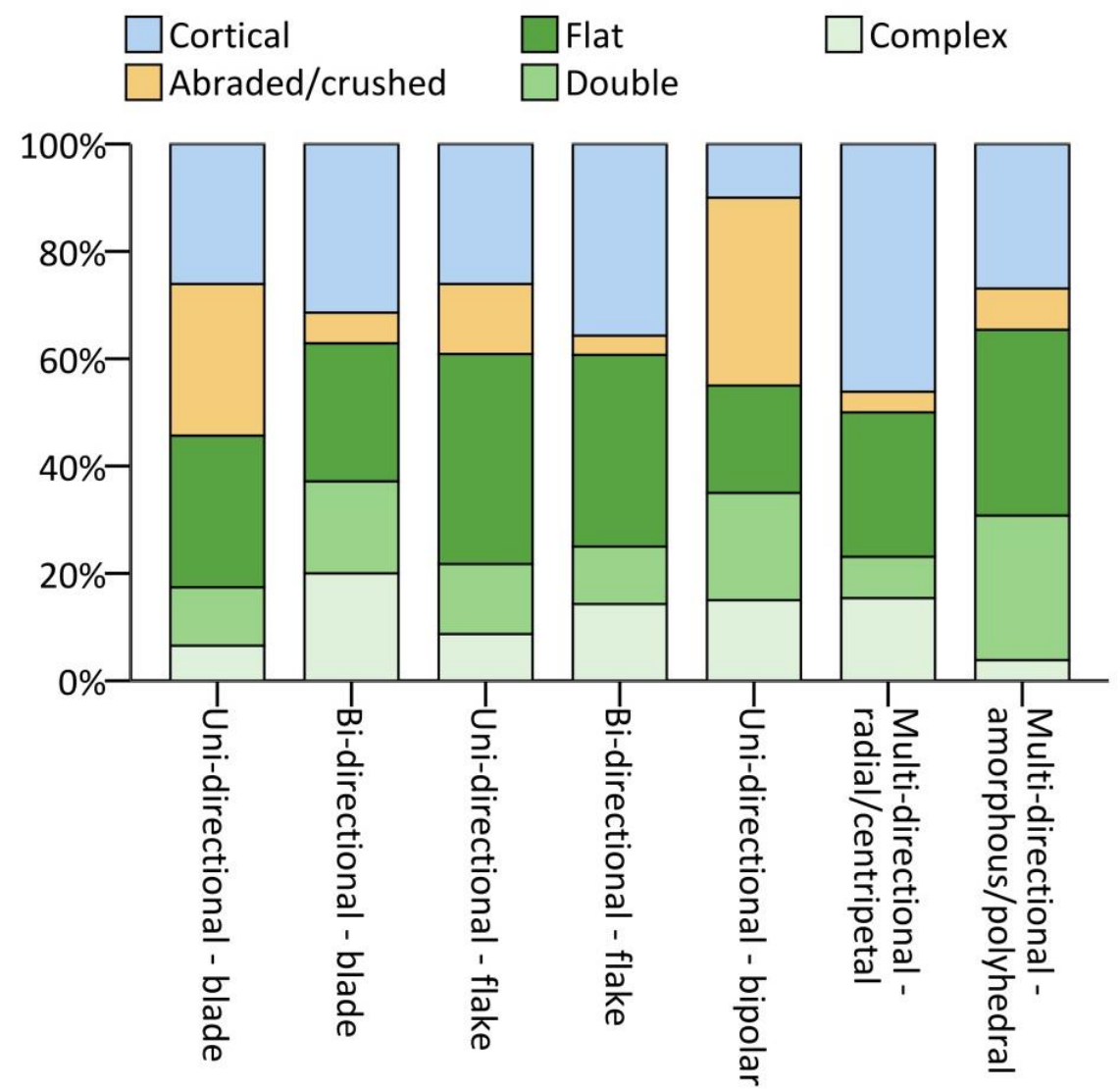

Figure 5.1.6: Average numbers of flake and blade scars on cores at RCC

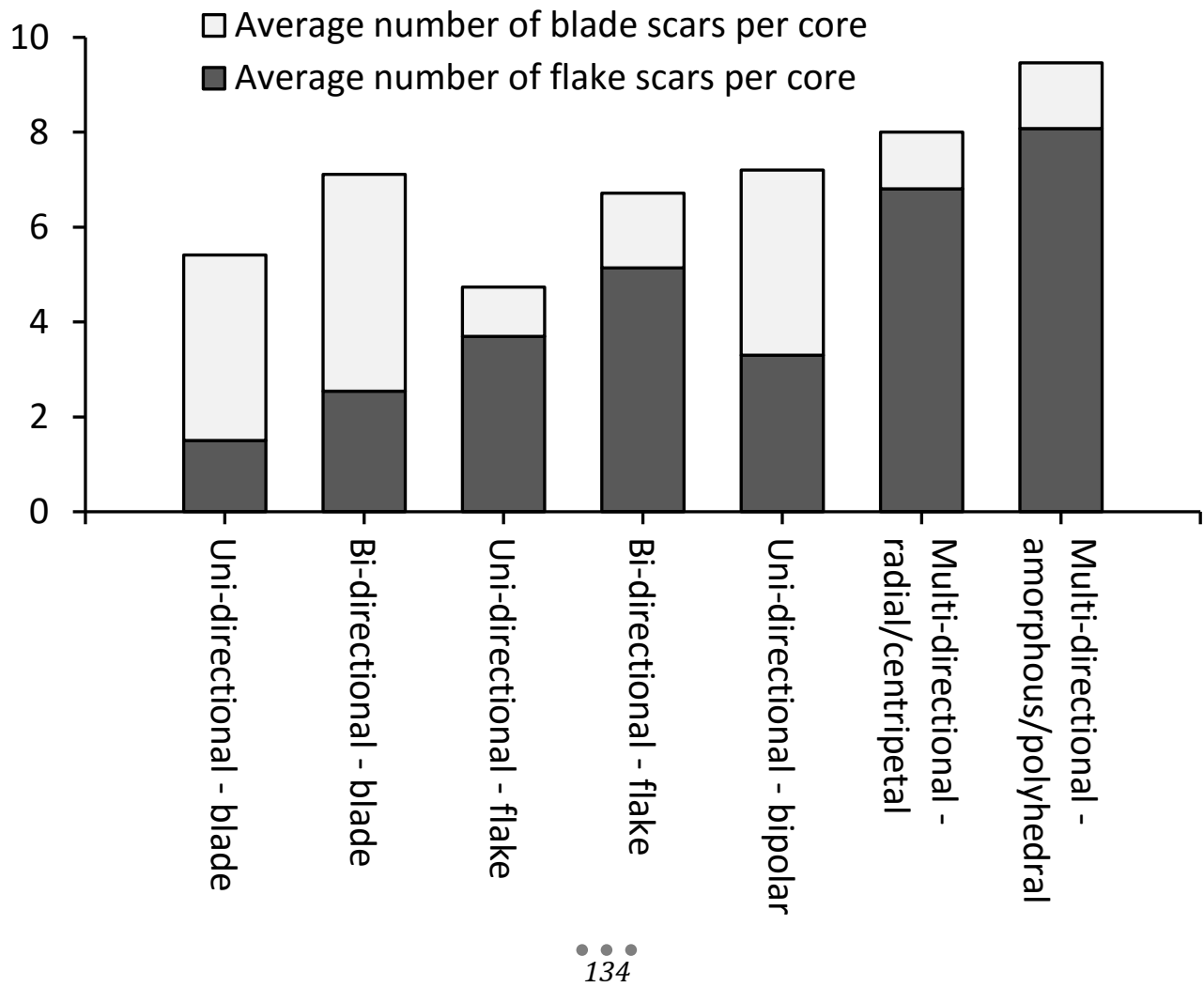


Average terminal removal scar lengths are longer on blade cores $(19.7 \mathrm{~mm})$ than on flake cores (16.3) (Table A.1.4). Figure 5.1.7 compares total scar lengths (the sum of all scar lengths) to core volumes. It demonstrates that removals from blade cores are not only longer than those from flake cores in absolute terms, but are also larger in relation to core sizes. Removals from bipolar cores are particularly extensive. There are both more removals on discarded bipolar cores in relation to their size, and removals of a larger size (Table A.1.5). Combined, this suggests that bipolar percussion was not simply used as a 'last chance' reduction method for exhausted cores, but that it was preferentially used to produce a large number of flakes and, in particular, blades from small cores.

Figure 5.1.7: Ratios of total removal scar length to core volume at RCC

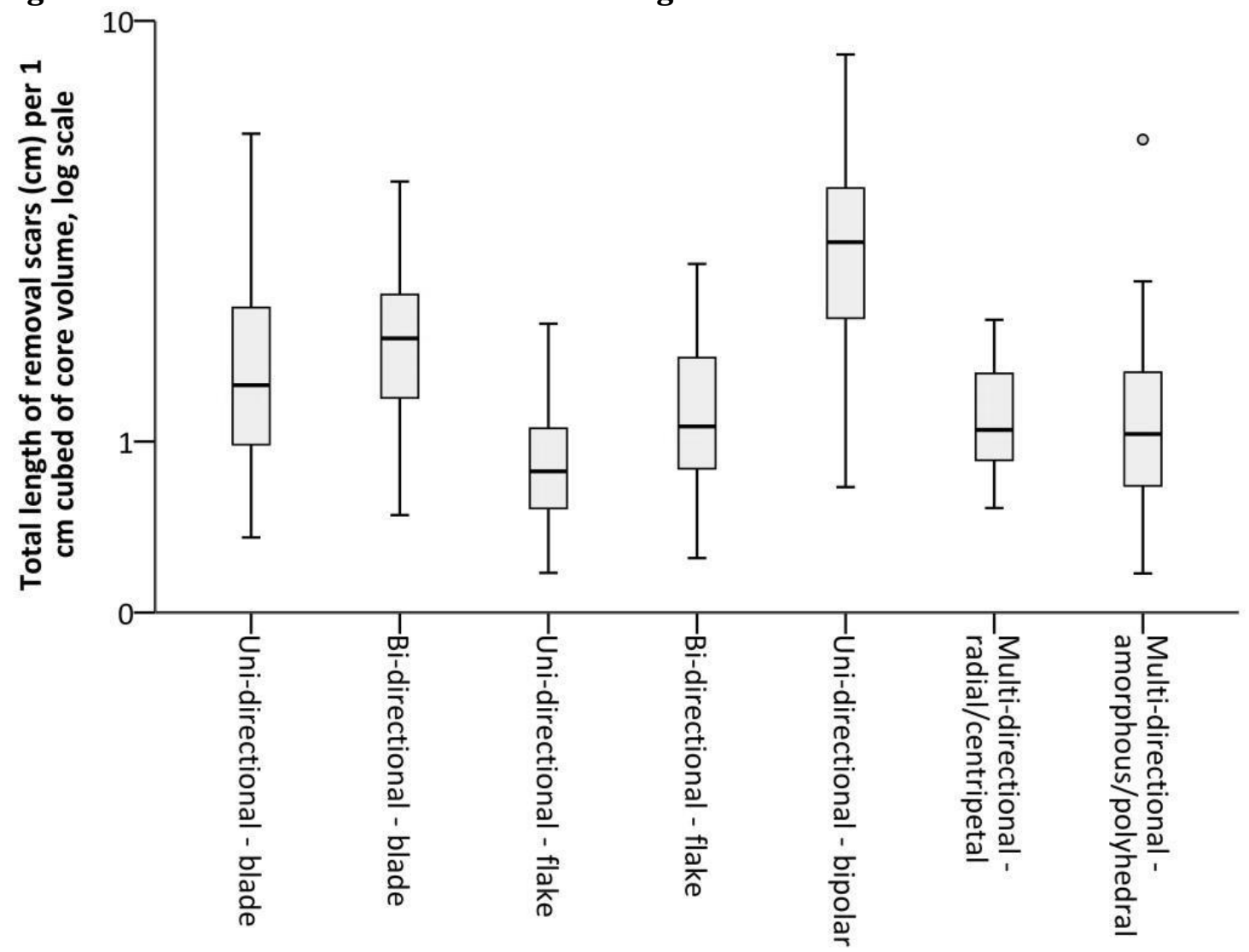




\section{DEBITAGE}

Analysis of the debitage sample agrees with previous reports (Wadley and Harper 1989, Soriano et al. 2007, Clarkson 2010) that the assemblage at RCC is predominantly blade-based (Table 5.1.2). The majority (81.5\%) of lithics are whole (Table A.1.6). As in the core assemblage, the dominant raw material is CCS (75.7\%) (Table 5.1.2, Figure 5.1.8). Additionally, CCS, particularly jasper, was used preferentially for the production of blades.

\begin{tabular}{lrrrrr}
\hline Raw material & Blades & Flake-blades & Flakes & Total & $\mathbf{\%}$ \\
\hline Jasper & 285 & 93 & 42 & 411 & 37.2 \\
Chalcedony & 142 & 64 & 38 & 240 & 21.6 \\
Agate & 82 & 35 & 37 & 153 & 13.7 \\
Silcrete & 41 & 20 & 20 & 80 & 7.2 \\
Claystone & 35 & 12 & 12 & 59 & 5.2 \\
Hornfels & 29 & 16 & 10 & 55 & 4.9 \\
Siltstone & 17 & 19 & 12 & 48 & 4.3 \\
Chert & 20 & 9 & 7 & 35 & 3.2 \\
Quartzite & 15 & 8 & 5 & 28 & 2.5 \\
Milky quartz & & & 2 & 2 & 0.2 \\
Opaque quartz & & 1 & & 1 & 0.1 \\
\hline Total (\%) & $666(59.0)$ & $277(24.6)$ & $185(16.4)$ & 1128 & \\
\hline
\end{tabular}

Table 5.1.2: Debitage measured at RCC

The average size of whole debitage is $31.7 \times 15.3 \times 5.2 \mathrm{~mm}$ (Table A.1.7), although variation exists between debitage and raw material categories. While blades are longer on average (with a mean oriented length of $33.2 \mathrm{~mm}$ ) than flake-blades (30.6 $\mathrm{mm})$ or flakes $(27.6 \mathrm{~mm})$, they are smaller overall (Figure 5.1.9). 
Figure 5.1.8: Raw material proportions of debitage at RCC

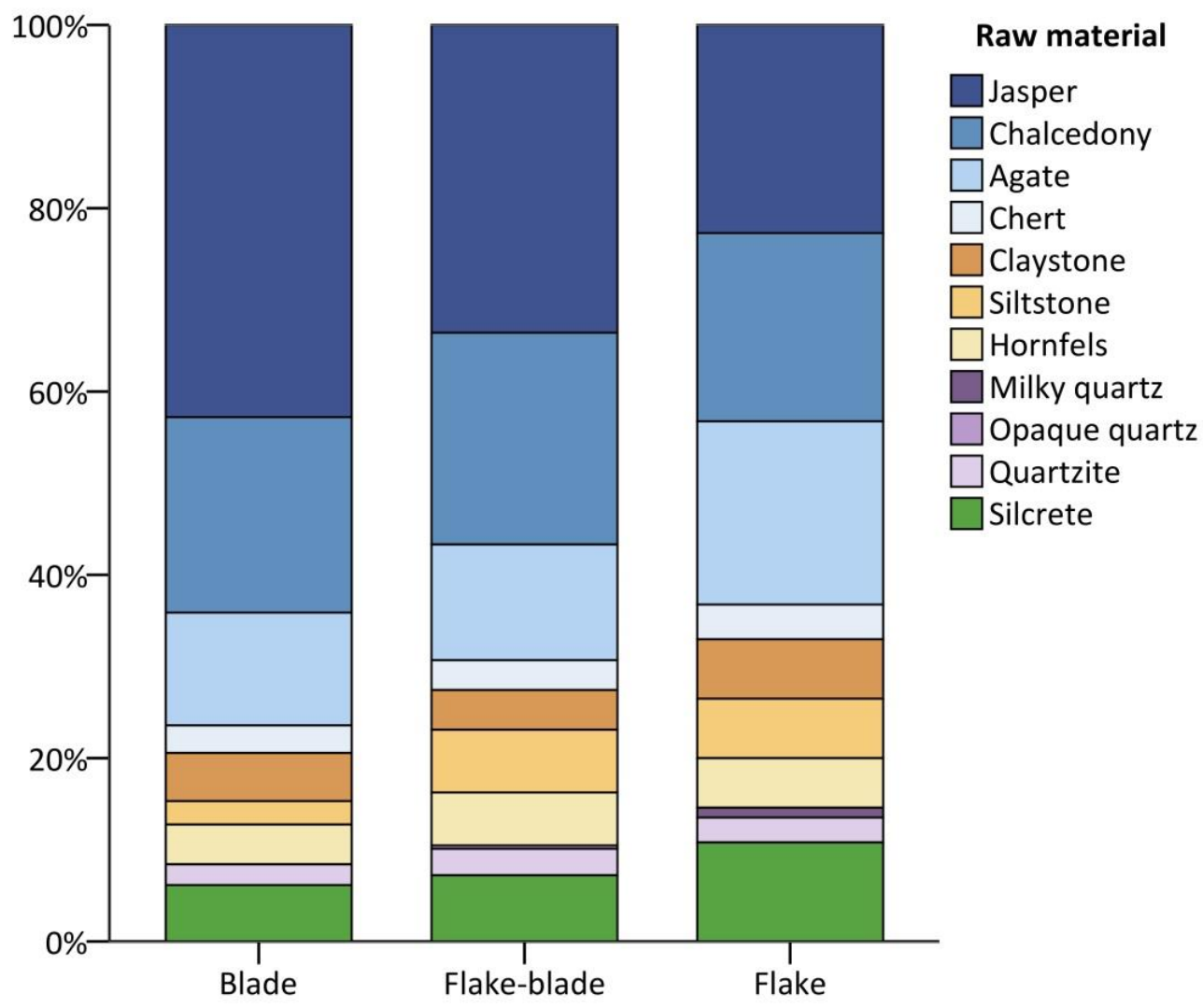

Figure 5.1.9: Cubic volumes of whole debitage on different raw materials at RCC, calculated as oriented length $\mathrm{x}$ maximum width $\mathrm{x}$ maximum thickness

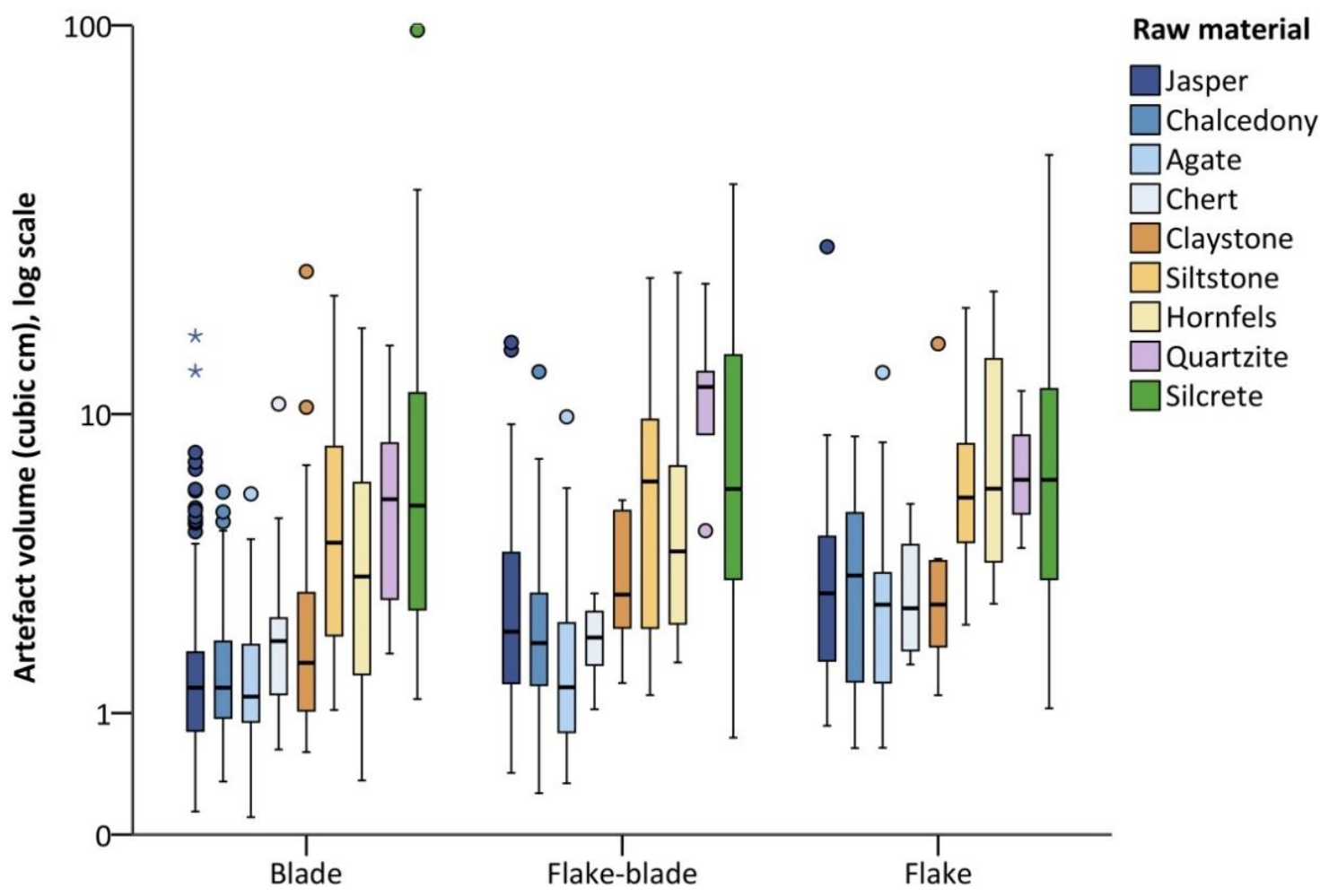


Additionally, Figure 5.1.9 demonstrates a clear grouping of CCS varieties plus claystone to manufacture small flakes and especially blades, while siltstone, quartzite and silcrete were used to manufacture larger flakes and blades. Hornfels appears to have been used for an intermediate size category of blades and flakeblades. Figure 5.1.10 highlights differences in the size distributions of the two main raw material groupings (see also Table A.1.8). CCS and claystone lithics are smaller and more standardised compared to the other raw materials used at the site.

Figure 5.1.10: Frequency distributions of whole debitage volumes by raw material groupings at RCC
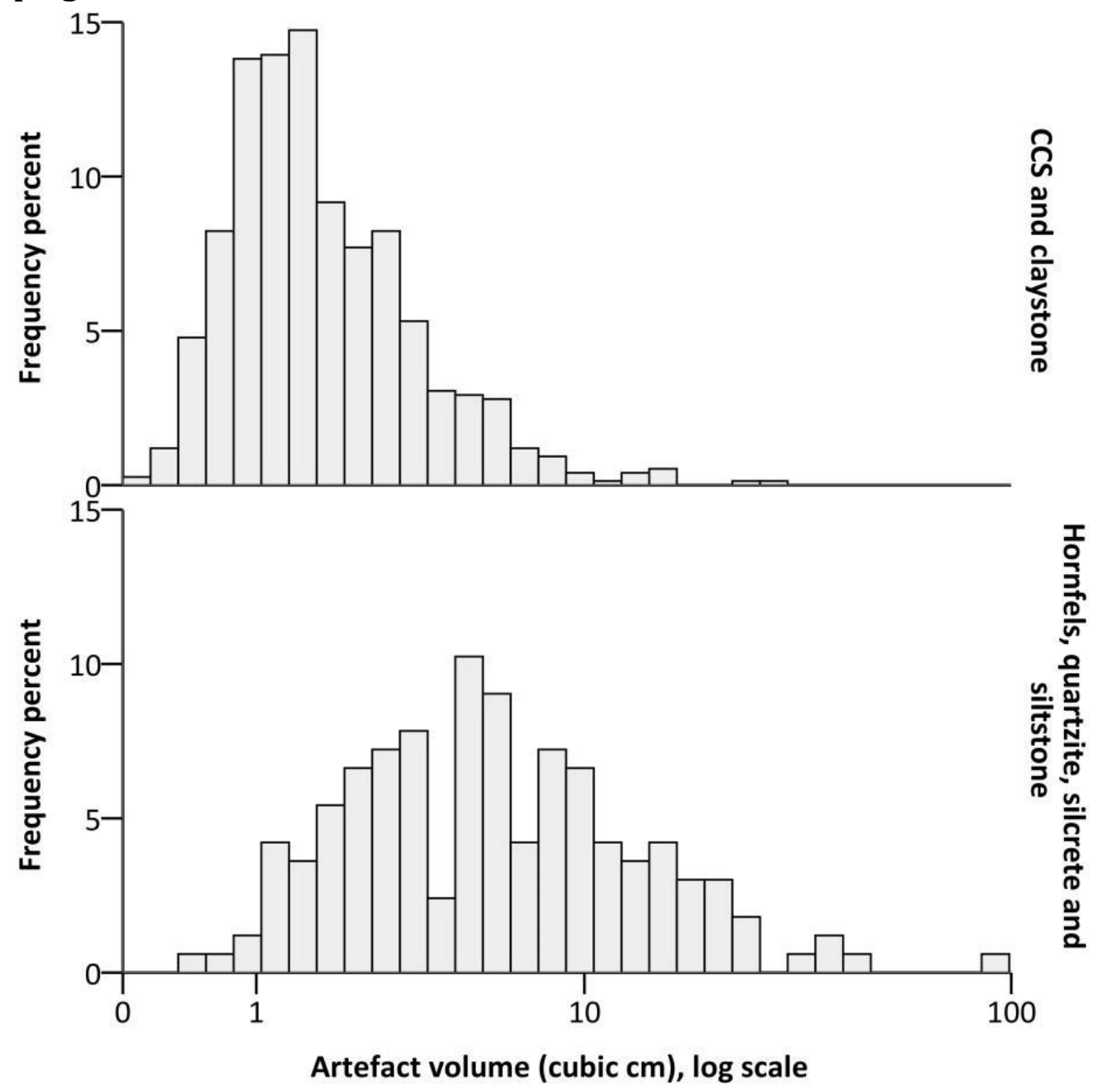
Size groupings are particularly robust in the case of blades, where CCS varieties and claystone were utilised in order to produce small blades of relatively standardised dimensions (Figure 5.1.11). This group of blades have average dimensions of $31.2 \times 11.3 \times 4.3 \mathrm{~mm}$, with a length:width ratio of 2.9. They are also more parallel, with a width standard deviation of 1.07 compared to 1.85 for siltstone, quartzite and silcrete, and 1.65 for hornfels.

Figure 5.1.11: Examples of non-retouched CCS blades at RCC, demonstrating their standardised dimensions and range of colours

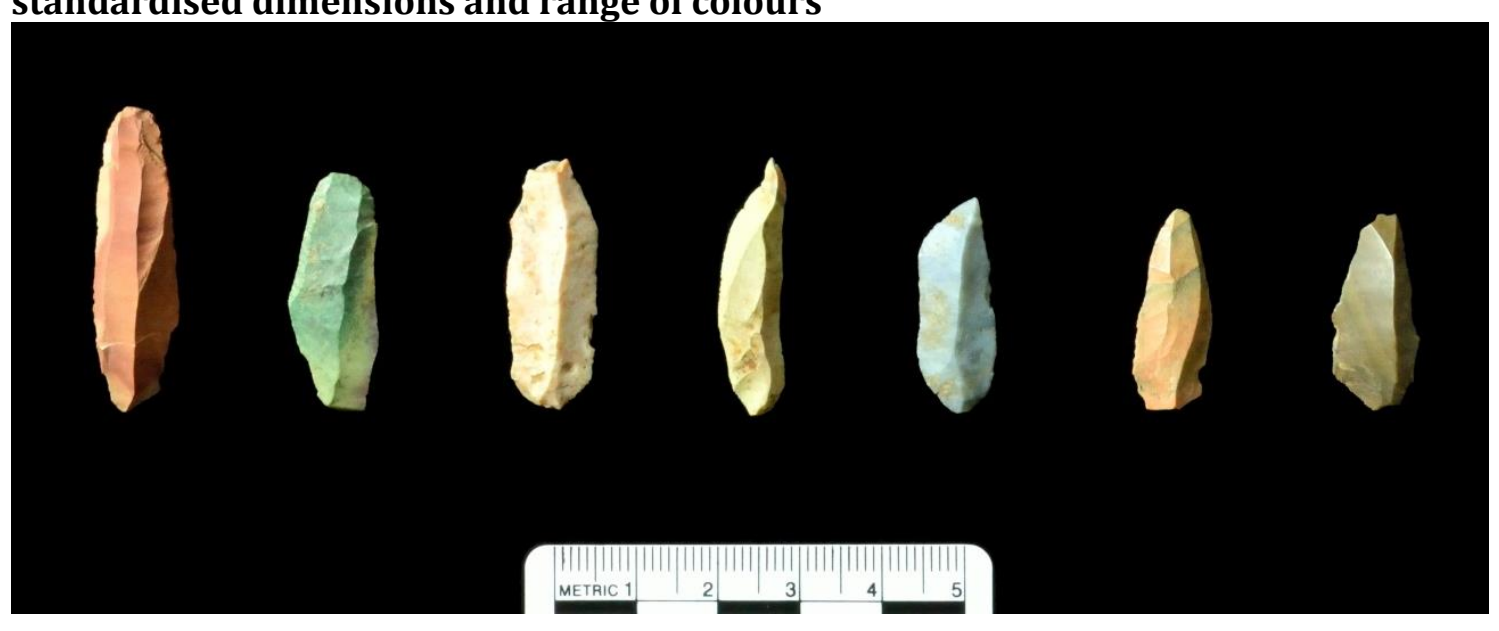

Slight or pronounced lipping is evident on $54.5 \%$ of striking platforms. CCS

varieties and blades exhibit the smallest platforms. For example, CCS blades have average platform dimensions of $5.3 \times 2.0 \mathrm{~mm}$, compared to the overall average of $8.4 \times 3.0 \mathrm{~mm}$. Flat striking platforms are the most common type (Figure 5.1.12). Differences between debitage categories are also evidenced in platform types, with more heavily facetted platforms more common on flakes, and pointed platforms more common on blades. 
Figure 5.1.12: Proportions of debitage striking platform types at RCC Striking platform type

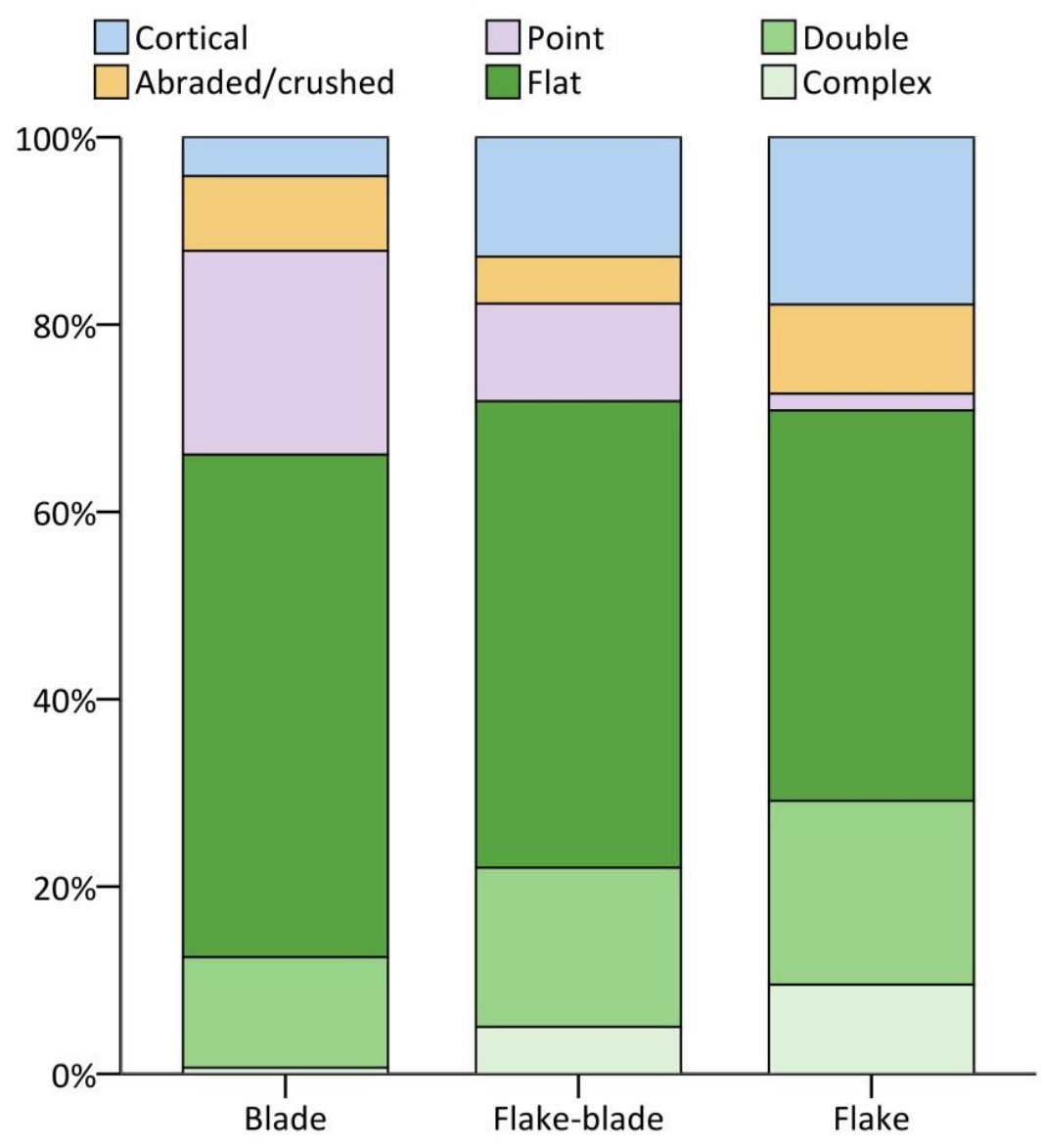

The significantly higher proportion of cortical platforms on flakes suggests that they may have been removed earlier in core reduction sequences, with blades detached once the majority of cortex had already been removed. This is further evidenced by the fact that average cortex cover on the dorsal surfaces of flakes $(23 \%)$ is much higher than that on blades (7\%) (Table A.1.9). Additionally, blades have a slightly more acute mean curvature $\left(166.9^{\circ}\right)$ compared to flake-blades $\left(166.1^{\circ}\right)$ and flakes $\left(165.7^{\circ}\right)$. Overall, given these results and the high proportion of blades at the site, flakes may be evident in the assemblage largely through the process of cortex removal and the preparation of cores for the production of blades. 
Mean dorsal scar counts are similar on different debitage types (3.3 on blades and flake-blades, 3.5 on flakes) (Table A.1.10). Flake-blades have a mean number of parallel arrises (1.3) comparable to that of blades (1.5), but much higher than that of flakes (0.1). Flake-blades were probably removed at around the same stage as blades in the core reduction sequence, once pieces were already being removed in a parallel fashion.

Further support for the conclusion that blades were primarily removed in a later stage of lithic reduction can be seen in the fact that, when dorsal surface area is taken into account, blades exhibit greater mean scar densities ( $1 \mathrm{scar}$ per $1 \mathrm{~cm}^{2}$, compared to 0.7 for flake-blades and 0.6 for flakes) (Table A.1.10). Additionally, while blades and flake-blades have almost identical proportions of dorsal scar orientations, there are much greater numbers of flakes exhibiting scars originating from two adjacent margins or from three directions (Table 5.1.3). This finding again implies that flakes were primarily removed during the process of preparing a core for the removal of blades.

\begin{tabular}{lrr|rr|rr|rrr}
\hline \multicolumn{1}{c}{$\begin{array}{c}\text { Dorsal scar } \\
\text { orientation: }\end{array}$} & \multicolumn{10}{c}{$\mathbf{2}$} & \multicolumn{2}{c}{$\mathbf{2}$} \\
Type & No. & $\%$ & No. & \% & No. & \% & No. & \% & Total \\
\hline Blade & 441 & 80.6 & 48 & 8.8 & 52 & 9.5 & 6 & 1.1 & 547 \\
Flake-blade & 158 & 81.0 & 17 & 8.7 & 17 & 8.7 & 3 & 1.5 & 195 \\
Flake & 73 & 57.5 & 31 & 24.4 & 12 & 9.4 & 11 & 8.7 & 127 \\
\hline Total (\% of total) & 672 & 77.3 & 96 & 11.0 & 81 & 9.3 & 20 & 2.3 & 869 \\
\hline
\end{tabular}

Table 5.1.3: Dorsal scar orientation frequencies on whole debitage at RCC 
Feather terminations are the most common type on debitage (55.1\%) (Figure

5.1.13). Plunging terminations are most common on blades. This may be a result of their removal closer to the end-life of a core, when cores were smaller and blades extended the entire length of the core face, making plunging terminations more likely.

Figure 5.1.13: Proportions of debitage termination types at RCC

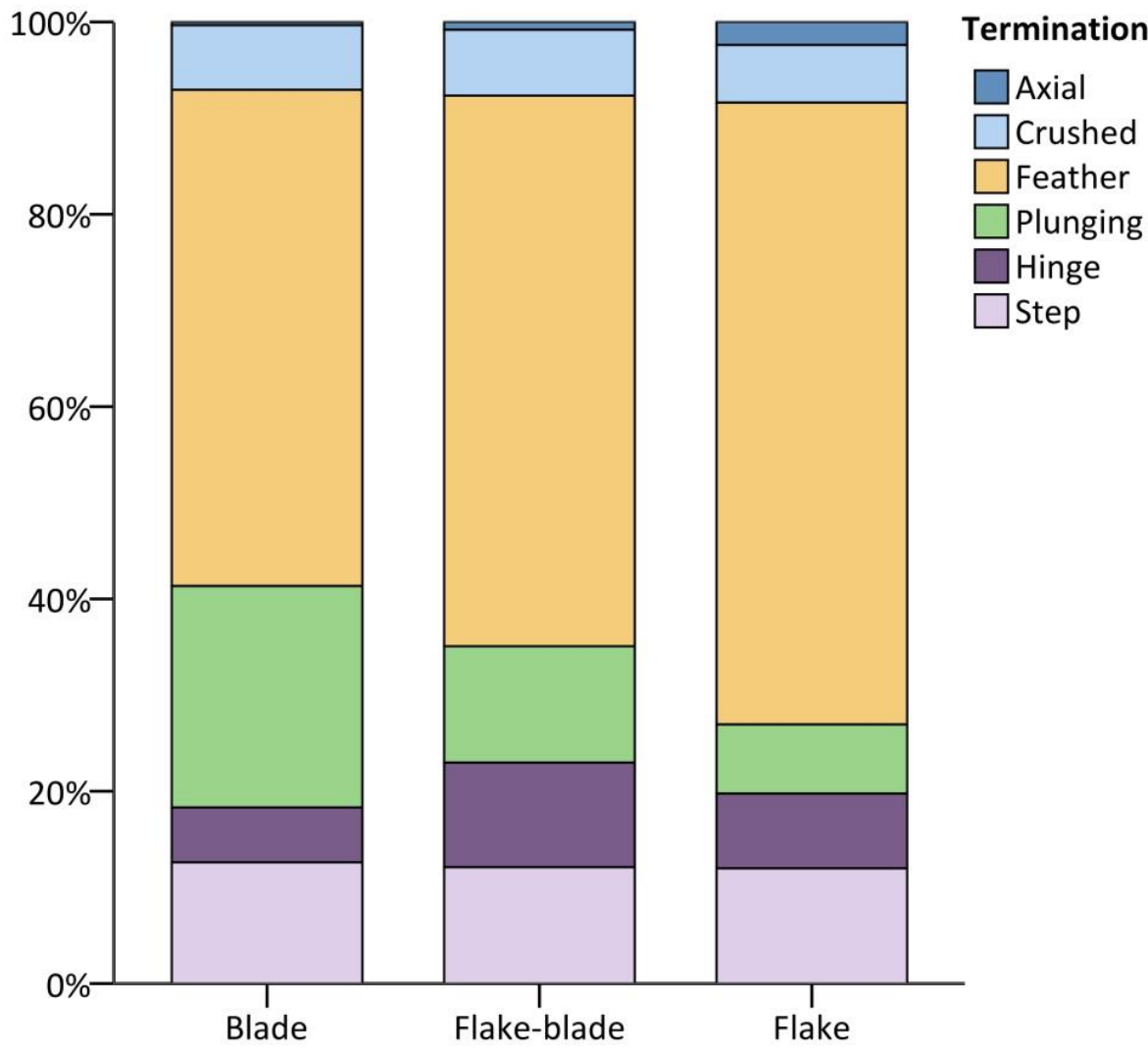

Indications of having been manufactured from recycled older lithics are evident on 11.4\% of debitage (Table A.1.11). The working and maintenance of cores of all raw materials at the site can be seen in the frequencies of core redirection and rejuvenation flakes, platform preparation flakes and crested blades (Table 5.1.4). Crested blades can be considered indicative of a 'laminar' technology alongside other proxies which are evident in the assemblage, including trapezoidal or 
triangular cross-sections, relatively narrow butts, and standardisation of endproducts (Bar-Yosef and Kuhn 1999, Hoggard 2014).

\begin{tabular}{lrrrrr|rr|rr}
\hline & \multicolumn{1}{c}{ Blades } & \multicolumn{1}{c}{ Flake-blades } & \multicolumn{2}{c}{ Flakes } & \multicolumn{3}{c}{ Total } \\
Maintenance flake type & No. & \% & No. & \% & No. & \% & No. & \% \\
\hline Platform preparation flake & 14 & 2.1 & 8 & 2.9 & 9 & 4.9 & 31 & 2.7 \\
Core rejuvenation flake & 20 & 3.0 & 1 & 0.4 & 3 & 1.6 & 24 & 2.1 \\
Core redirection flake & 10 & 1.5 & 2 & 0.7 & 3 & 1.6 & 15 & 1.3 \\
Crested blade & 9 & 1.4 & 1 & 0.4 & & & 10 & 0.9 \\
\hline Total & 53 & 8.0 & 12 & 4.3 & 15 & 8.1 & 80 & 7.1 \\
\hline
\end{tabular}

Table 5.1.4: Frequencies of core maintenance debitage at RCC

TOOLS

Blade blanks were preferred for tool production, with $80.6 \%$ of microliths and $63.9 \%$ of other tools manufactured on blades (Table 5.1.5). CCS varieties are dominant, but were used in much larger proportions for the manufacture of microliths (93.3\%) compared to non-microlithic tools (52.8\%) (Figure 5.1.14). Jasper was especially preferred for microliths made on blade blanks (56.5\%). Although CCS appears in a wide range of colours (Figure 5.1.11), there is no evidence for preferential selection from the debitage assemblage of particular colours of blanks for retouch into tools $\left(\chi^{2}(24)=21.974, p=.376\right)$.

While the reduction of CCS nodules appears to have been primarily for the purpose of producing blanks suitable for retouch into microliths, the higher proportion of non-microlithic tools on other raw materials, especially hornfels and quartzite, explains why these materials were also worked. Two different tool production trajectories were utilised at the site. 


\begin{tabular}{lrrrrrr}
\hline Raw material & $\begin{array}{r}\text { Microliths } \\
\text { on blades }\end{array}$ & $\begin{array}{r}\text { Microliths } \\
\text { on flakes }\end{array}$ & $\begin{array}{r}\text { Retouched } \\
\text { blades }\end{array}$ & $\begin{array}{r}\text { Retouched } \\
\text { flakes }\end{array}$ & Total & \% \\
\hline Jasper & 61 & 7 & 15 & 8 & 91 & 44.2 \\
Chalcedony & 28 & 9 & 6 & 1 & 44 & 21.4 \\
Agate & 12 & 6 & 5 & 2 & 25 & 12.1 \\
Hornfels & 2 & 2 & 6 & 6 & 16 & 7.8 \\
Silcrete & 1 & 1 & 3 & 3 & 8 & 3.9 \\
Siltstone & & & 5 & 2 & 7 & 3.4 \\
Quartzite & 1 & & 3 & 1 & 7 & 3.4 \\
Claystone & 1 & 1 & 2 & & 3 & 2.4 \\
Chert & 2 & & 1 & 26 & 206 & \\
\hline Total & 108 & 26 & 46 & & & \\
\hline
\end{tabular}

Table 5.1.5: Tools measured at RCC

Figure 5.1.14: Raw material proportions of tools at RCC

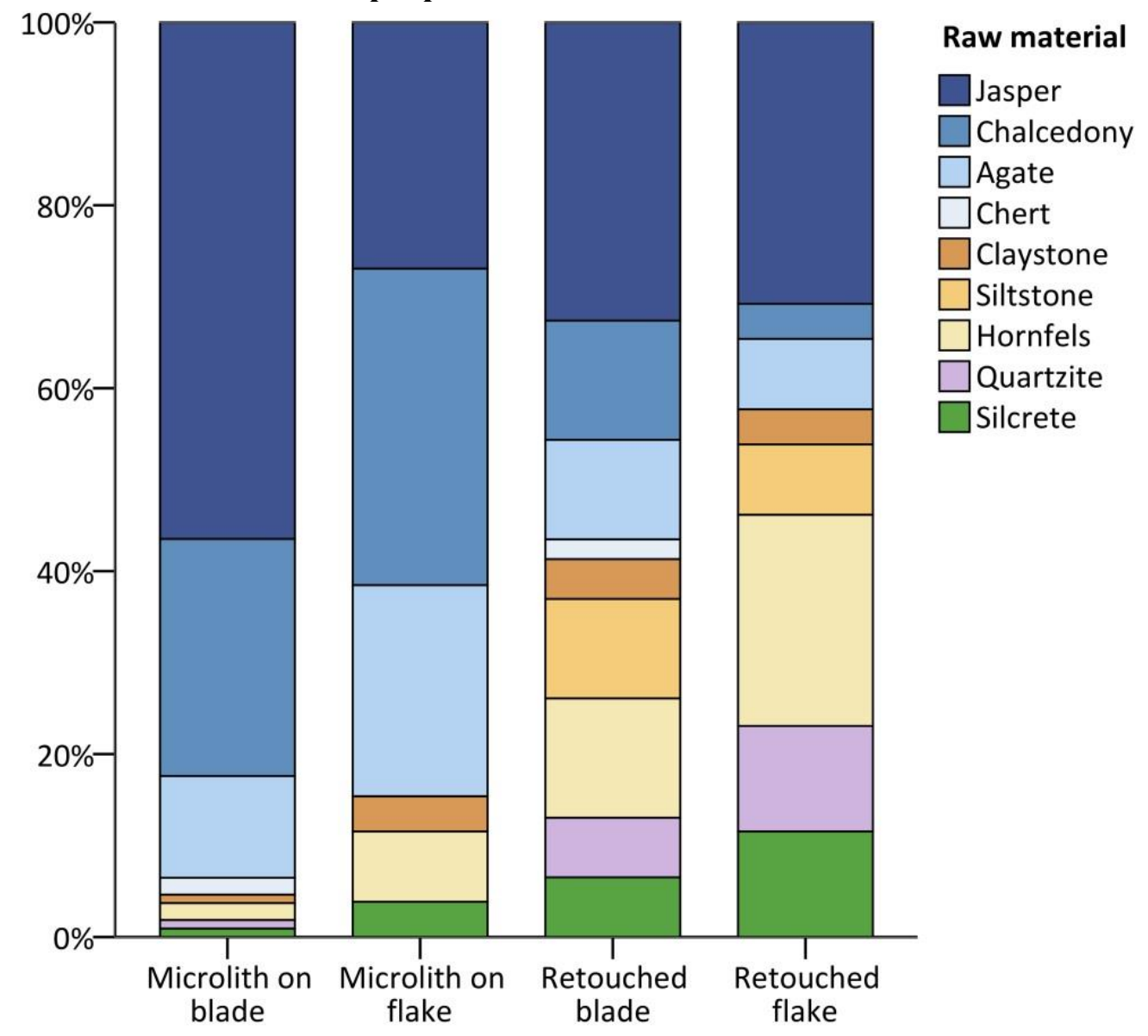


Mean microlith size measurements are 28.4 x 12.5 x $4.1 \mathrm{~mm}$. Size measurements of microlithic and non-microlithic tools (Table A.1.12, Table A.1.13) can be compared with those from the general debitage population to provide insights into blank selection processes. The size profile of microlithic tools broadly matches that of the general debitage population, while non-microlithic tools are consistently larger in every metric (Figure 5.1.15, Figure 5.1.16). Whereas general lithic reduction at the site was geared towards the production of blanks of a suitable size for the manufacture of microliths, only the largest flakes and blades were selected for retouch into other tool forms.

\section{Figure 5.1.15: Lengths of whole lithics at RCC}

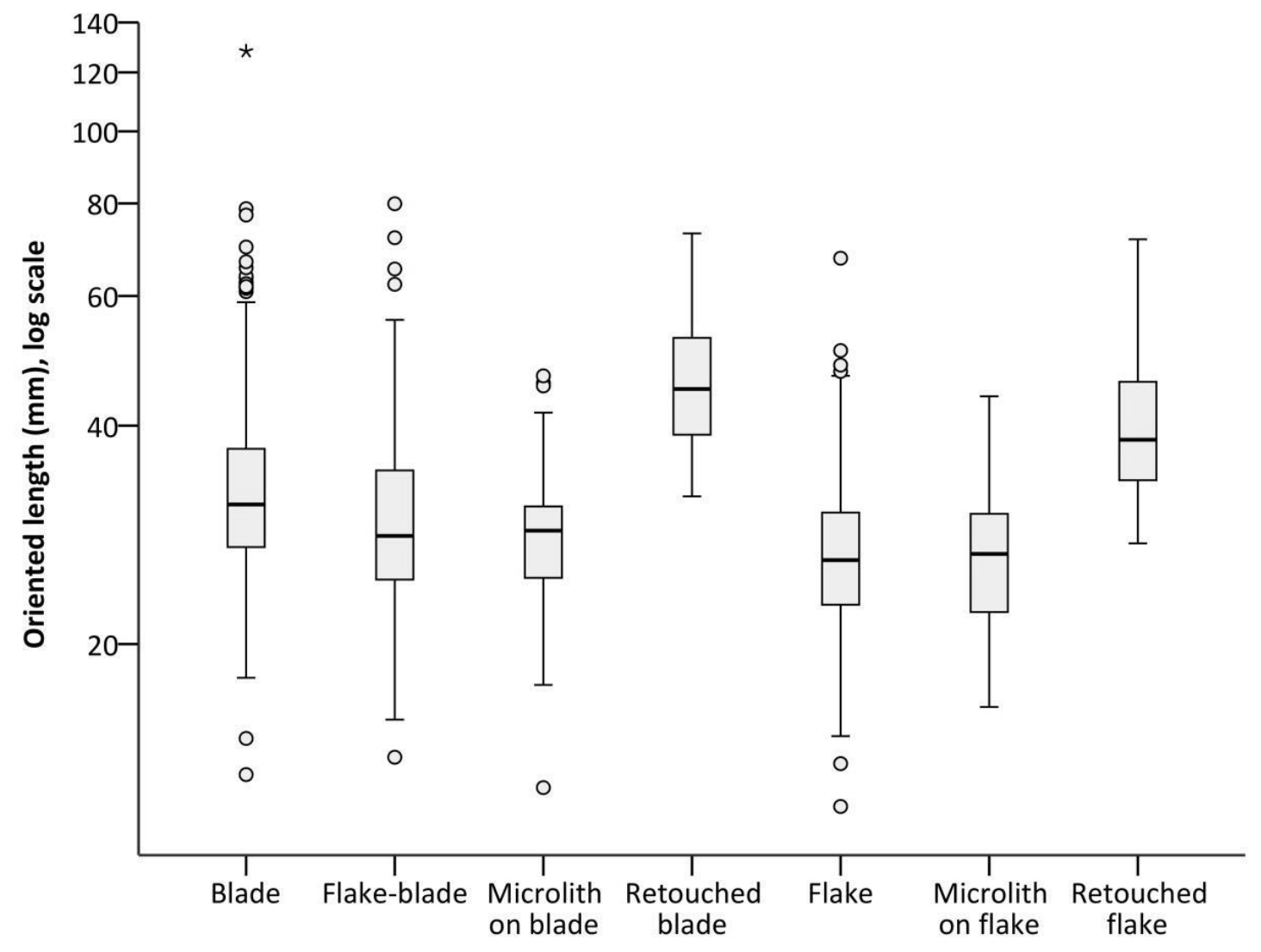


Figure 5.1.16: Cubic volumes of lithics on different raw materials at RCC. All microliths and non-microlithic tools are included, plus all whole debitage.

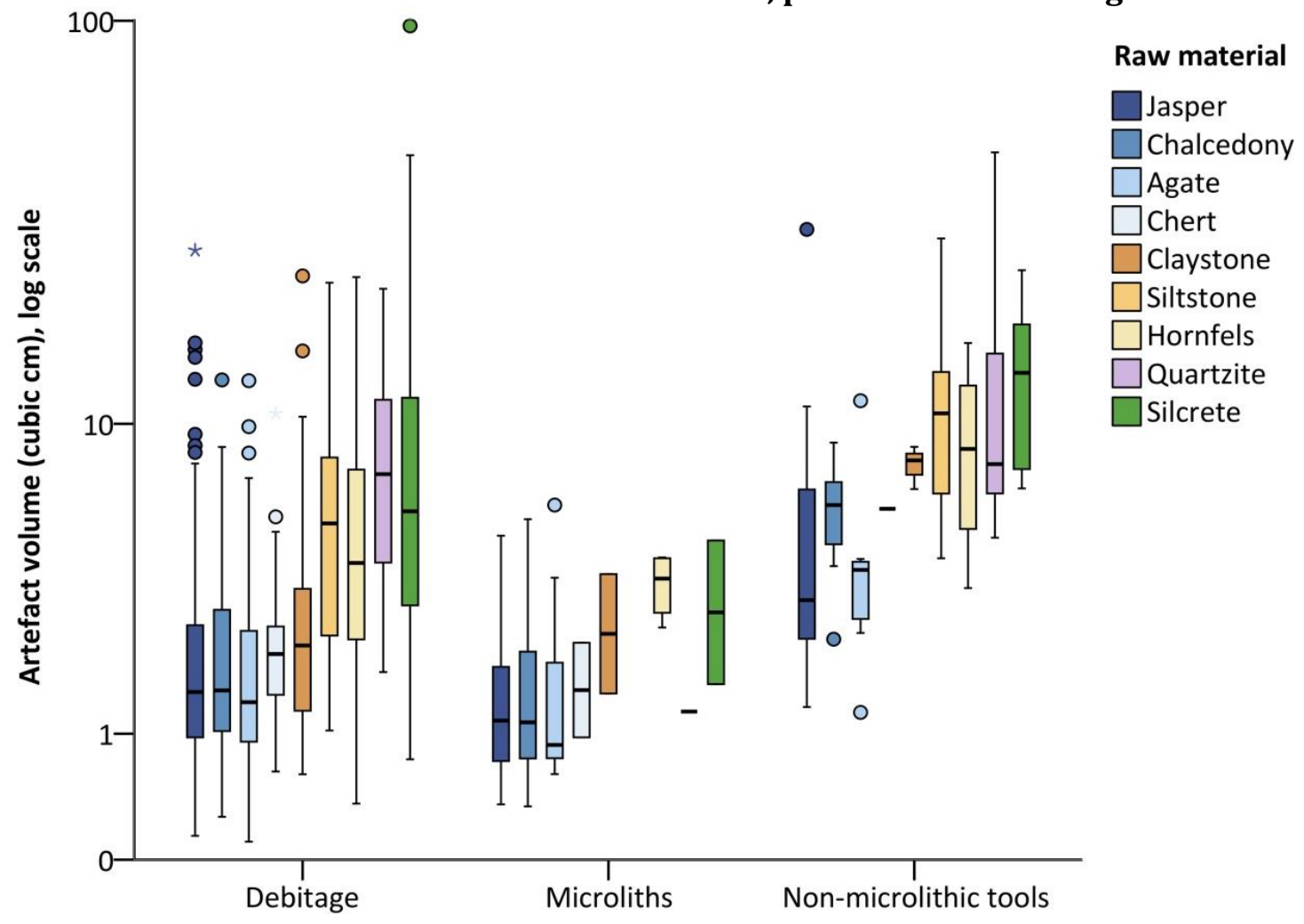

Patterns can also be seen in the selection of blanks from different raw materials.

CCS and claystone microliths have similar size distributions to the corresponding debitage samples (Figure 5.1.16), further indicating that microlith manufacture was the main purpose of CCS and claystone working. However, size distributions of non-microlithic CCS tools barely overlap with the corresponding debitage; their median volume is $4.0 \mathrm{~cm}^{3}$, but only $11 \%$ of CCS debitage is this size or larger. The selection of larger pieces for retouch was most likely opportunistic in comparison to the calculated production of microlith blanks. This pattern is somewhat reversed with the other raw materials, with non-microlithic tool blanks selected from the general size range of siltstone, hornfels, quartzite and silcrete debitage. 
A variety of microlith forms are evident at RCC (Table 5.1.6, Figure 5.1.17). Nongeometric forms are considerably more common than geometric shapes $(81.3 \%$ non-geometric), and the most common types are non-geometric backed pointed and non-pointed forms (33.6\% and 20.9\%, respectively). Of the geometric microliths, crescents are most common (11.9\%). Rectangles and trapezes are also present. There are statistically significant differences in typological frequencies between raw materials $\left(\chi^{2}(56)=92.110, \mathrm{p}=.002\right.$, Cramér's $\left.\mathrm{V}=.313\right)$. This relationship manifests itself primarily in the over-representation of backed nongeometric microlith forms manufactured on jasper (Figure 5.1.18).

\begin{tabular}{lrr}
\hline Coarse typology & Narrow typology & Frequency \\
\hline Microliths: & & 28 \\
Backed non-geometric microlith & &
\end{tabular}

Backed non-geometric microlith point Backed

Double backed

Microlith preform ${ }^{a}$

Non-geometric microlith

Double side retouched

1

Other

9

Side retouched

Non-geometric microlith point

Rectangle microlith

Backed 3

Double backed

Other

Shouldered/tanged microlith

Shouldered 1

Shouldered and backed 2

Shouldered and backed point $\quad 1$

Shouldered point 
Non-microlithic tools:

Burin

Burin

Double side retouched with

burin

End retouched

Misc. $b$

Notched/denticulated

Double notched

1

Side retouched and denticulated $\quad 1$

Side retouched with notch

Point

Backed

Double side retouched

1

Side retouched

Side and end retouched

Circumference retouched 2

Double side and end retouched

Side and double end retouched

Side and end retouched

Side retouched

Double side retouched

Unifacial point

Total

72

Table 5.1.6: Tool type frequencies at RCC. ${ }^{a}$ demonstrating limited initial reshaping (e.g. the removal of the bulb of percussion) but lacking any significant retouch. $b$ exhibiting only abrasion or thinning retouch. 
Figure 5.1.17: Examples of HP microliths from RCC, demonstrating some of the array of shapes and sizes of microlith forms

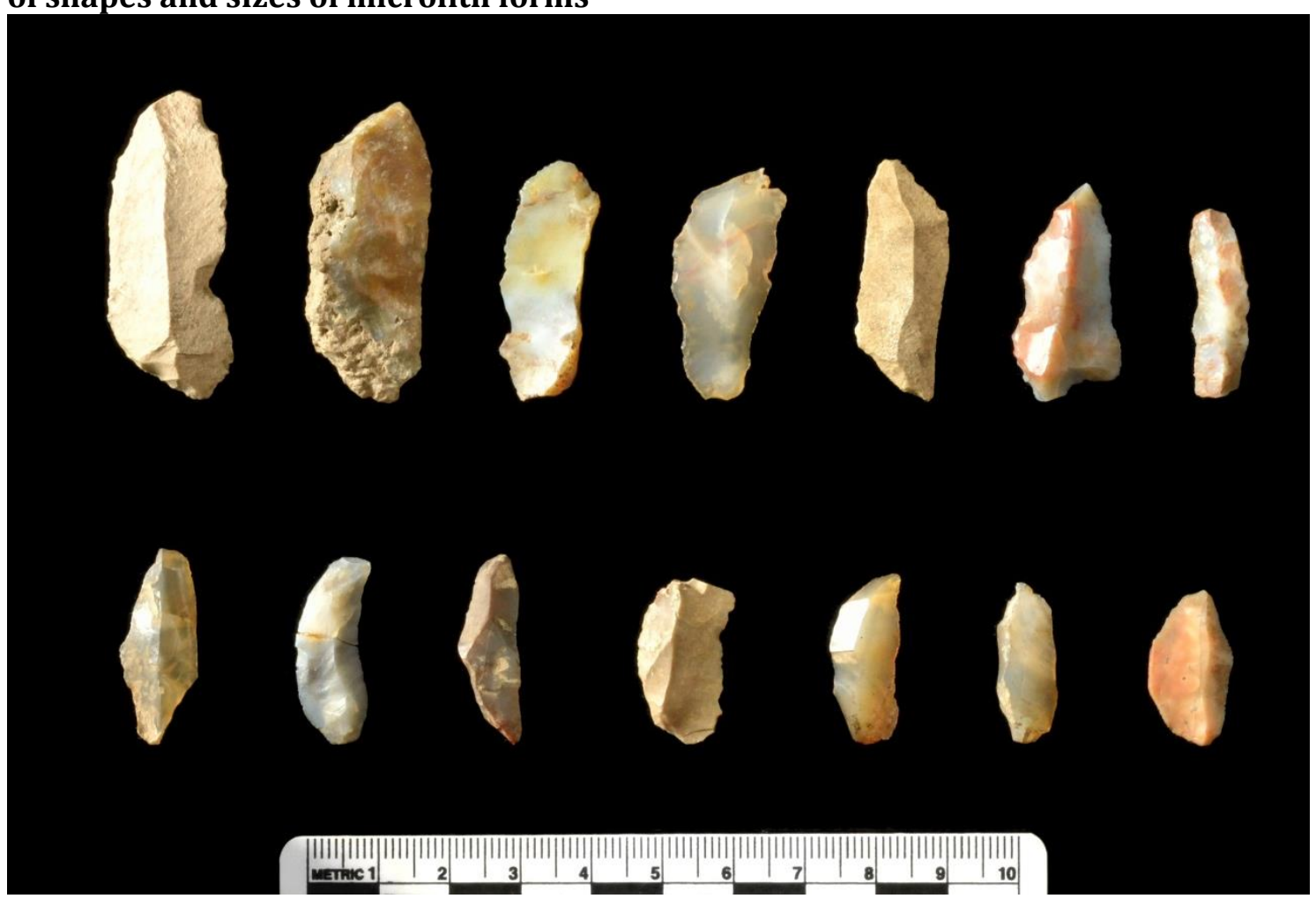

Figure 5.1.18: Raw material proportions of microlith types at RCC

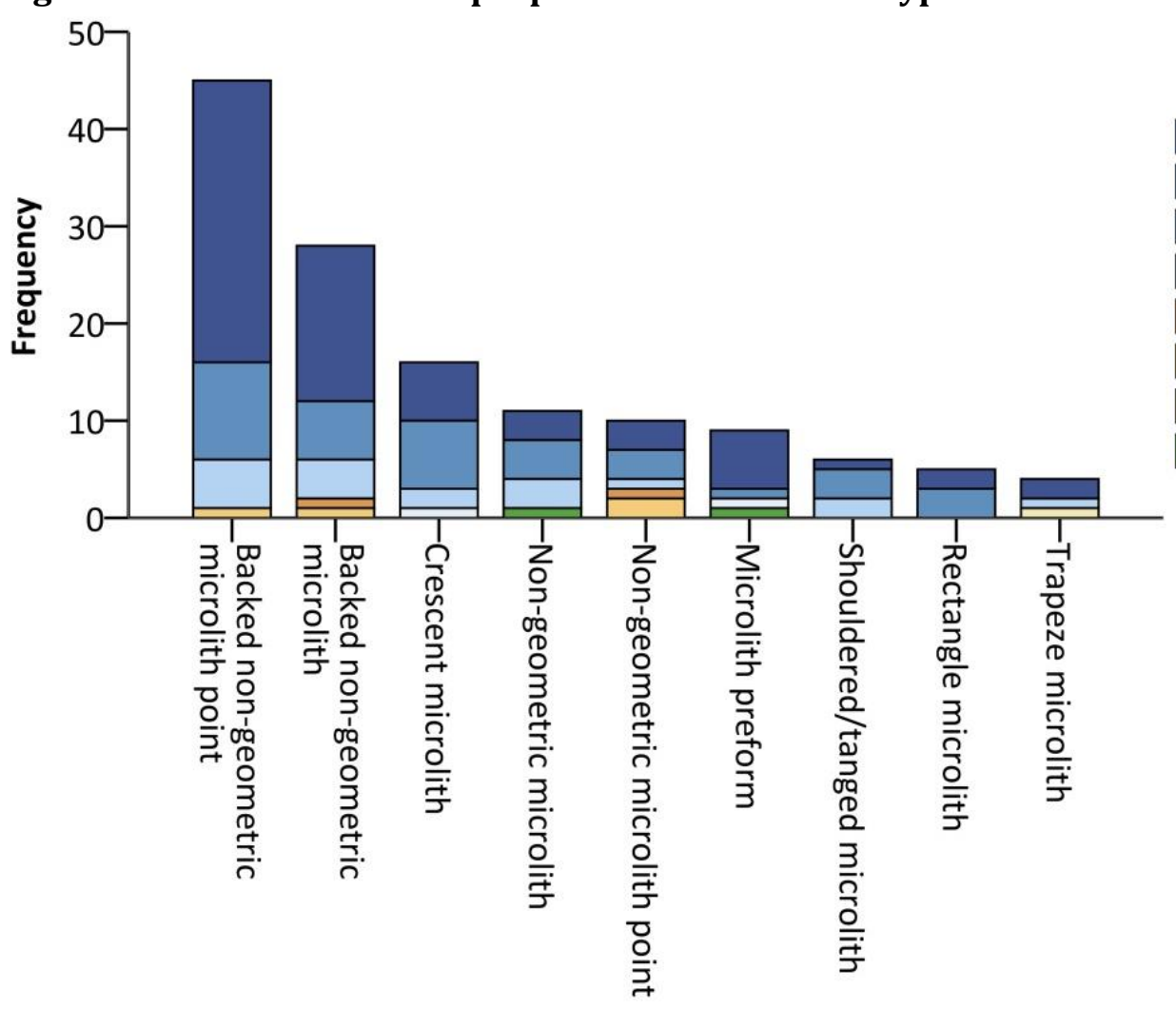

Raw material

$\square$ Jasper

$\square$ Chalcedony

$\square$ Agate

$\square$ Chert

$\square$ Claystone

$\square$ Hornfels

$\square$ Quartzite

$\square$ Silcrete 
The majority of non-microlithic tools (56.9\%) are side or double side retouched, including those that also exhibit points, burins, notches or denticulation (Table 5.1.6). Elements of a more general MSA technology can be seen in the existence of 13 unifacial points (Figure 5.1.19). They are found throughout the HP sequence, and so cannot be attributed solely to mixing between layers. Figure 5.1.20 reports the raw material proportions of different tool typologies.

\section{Figure 5.1.19: Examples of unifacial points from HP layers at RCC}

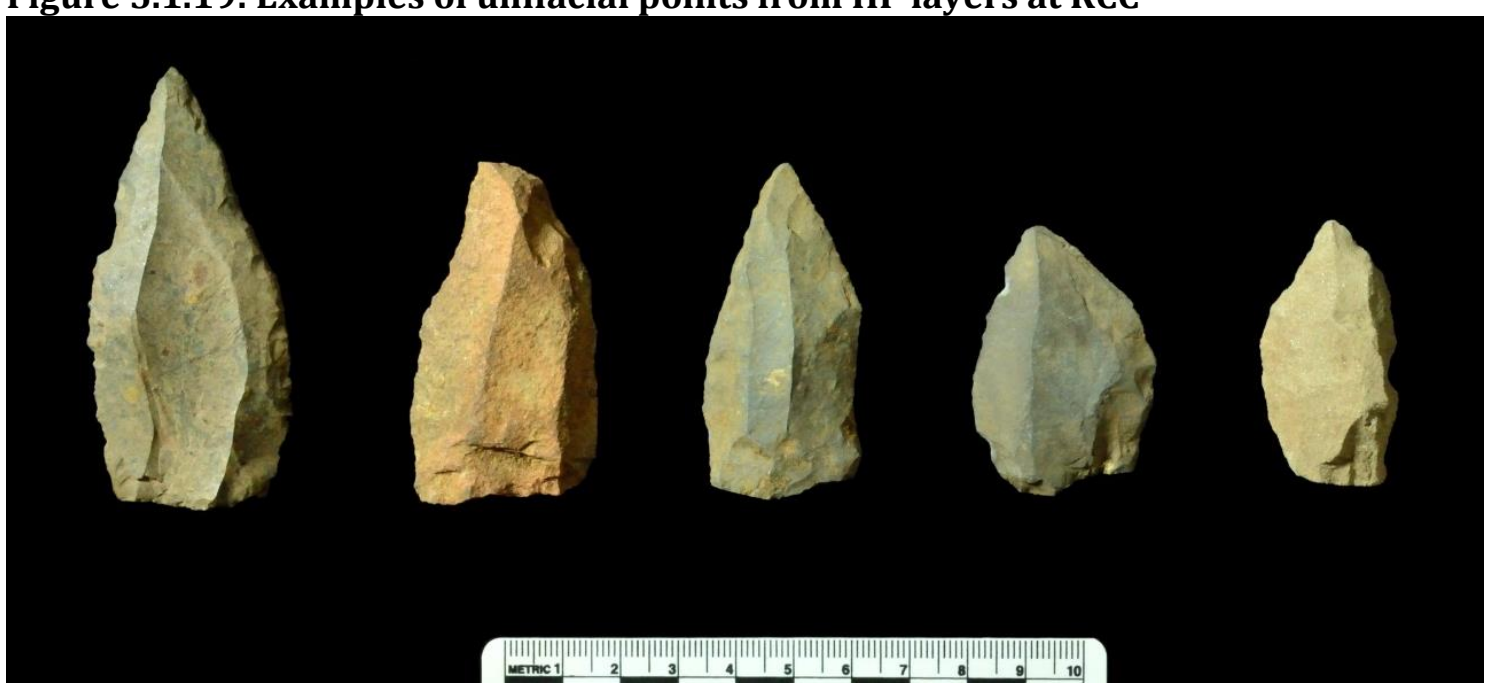

Most microlith forms are of roughly similar sizes, although rectangles are particularly small (Figure 5.1.21, Table A.1.14). Non-backed non-geometric microliths are wider and thicker on average, as they are more likely to be made on flake blanks (38.1\% compared to $15.9 \%$ of other types). No morphology is particularly standardised, although the CV of oriented lengths for most groups is around 20 (Table A.1.14), which can be considered as beginning to indicate a degree of standardisation. Backed non-geometric microliths (pointed or otherwise) appear to be the most standardised. Possibly backed microlithic forms comprised an important subgroup in the minds of HP tool-makers at the site. 
Figure 5.1.20: Raw material proportions of non-microlithic tool types at RCC

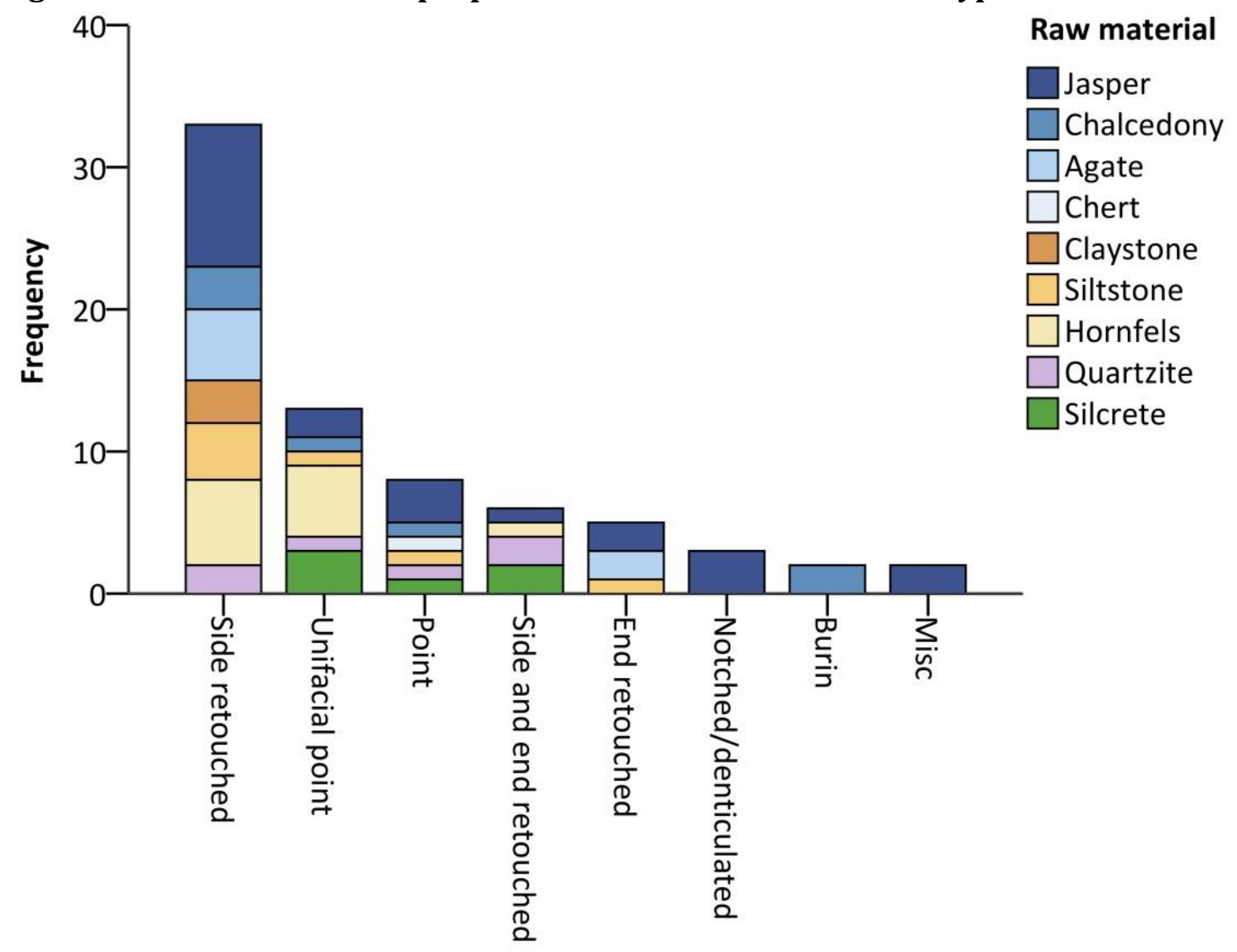

There are no clear groupings in the size measurements of non-microlithic tools although notches are particularly small - and little standardisation in tool sizes (Figure 5.1.22, Table A.1.14). The exception to this is unifacial points, which are the most standardised of all tool types and the only type to have a CV for width of less than 20. Unifacial and other points have mean TCSA measurements of $105.0 \mathrm{~mm}^{2}$ and $64.2 \mathrm{~mm}^{2}$, respectively (Table A.1.15). These values lie between the means of Shea's (2006) dart tip (58 $\mathrm{mm}^{2}$ ) and experimental thrusting spear tip (168 $\mathrm{mm}^{2}$ ) samples, in a range Shea (ibid: 829) considers indicative of use as "tips for handcast spears or thrusting spears, not as projectile points". 
Figure 5.1.21: Cubic volumes of microlith types at RCC

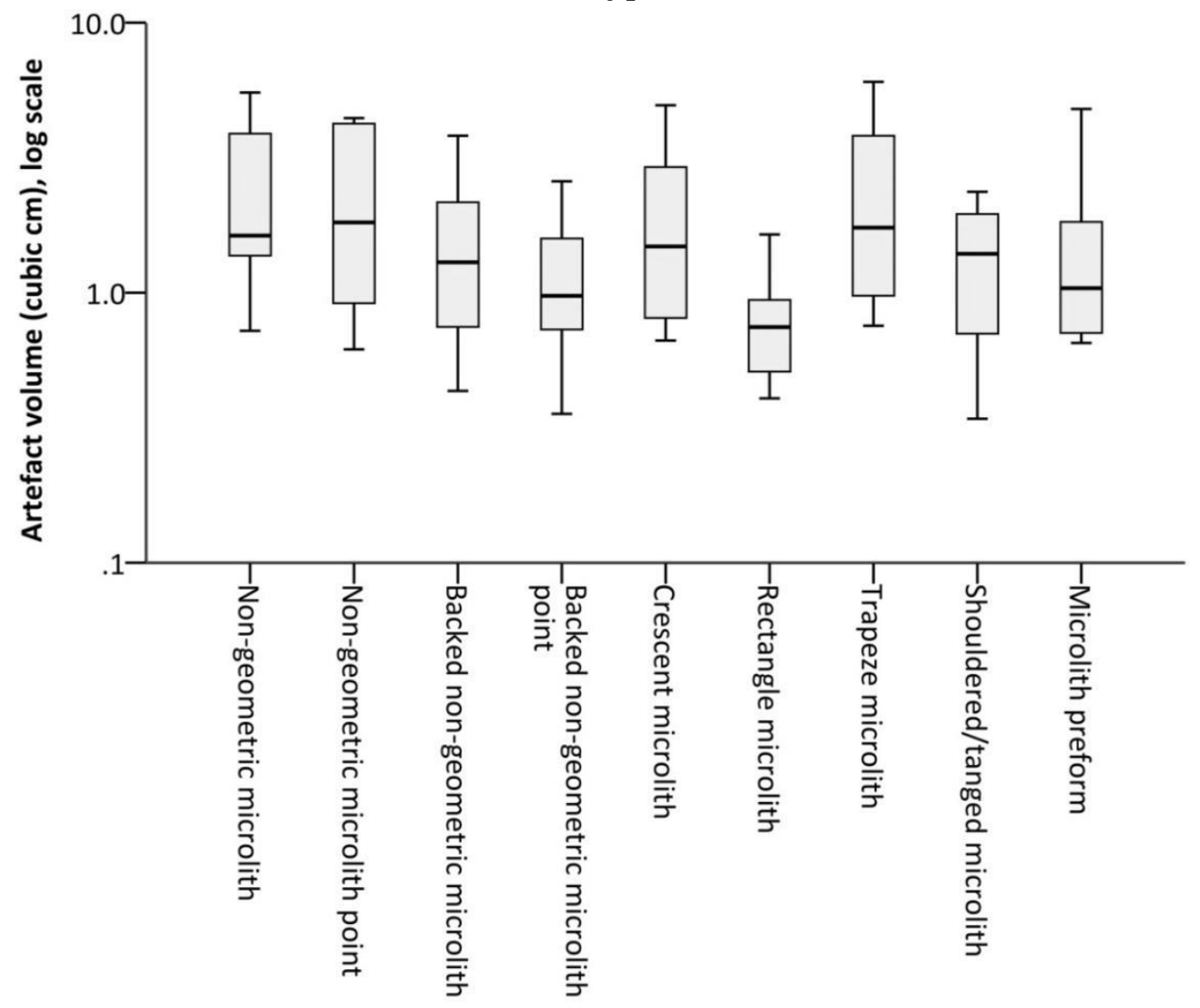

Figure 5.1.22: Cubic volumes of non-microlithic tool types at RCC

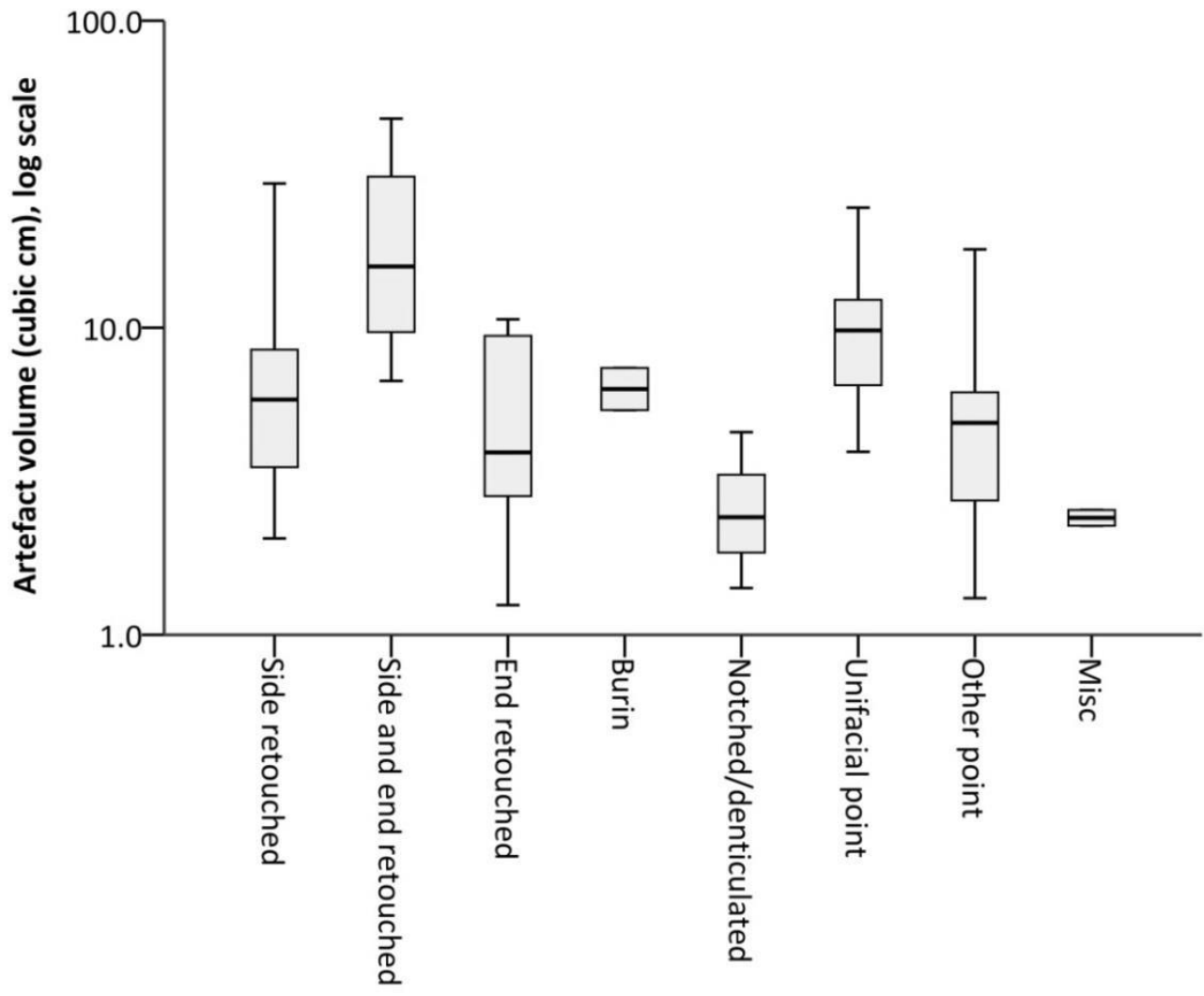


In contrast, mean TCSA measurements for non-geometric microliths $\left(46.8 \mathrm{~mm}^{2}\right)$ lie between Shea's dart tip sample and smaller arrowheads $\left(33 \mathrm{~mm}^{2}\right)$, and those for other microlith varieties are even smaller (mean values range from 18.5-32.9 $\mathrm{mm}^{2}$ ), although these are not standardised (Table A.1.15). Despite Shea's (2006: 829) concerns that TCSA may not be an appropriate measurement on microliths, the fact that microlithic points have consistently smaller TCSA measurements than equivalent non-pointed morphologies suggests that these tools, at least, may have been hafted as points at the ends of composite projectile weaponry. The existence of discrete microlithic and non-microlithic point types in the same assemblage may imply the co-existence of two different hunting strategies during the HP at RCC, although in the absence of use-wear analysis this remains a speculative conclusion.

Obvious shaping of tools to facilitate hafting is uncommon. No non-microlithic tools exhibited tangs or shoulders. Five microliths are shouldered (of which one exhibits abrasion on the hafted portion) and one is tanged, together comprising $4.5 \%$ of the microlithic tool assemblage. Mean haft dimensions are very small (6.6 x $2.5 \times 7.87 \mathrm{~mm}$ ) and not particularly standardised (Table A.1.16). Overall, the construction of haftable appendages does not appear to have constituted a major part of tool production processes.

Backing is the most common retouch type in the microlith assemblage (68.5\%) (Figure 5.1.23). In addition to backed microliths, backing retouch was also used in high proportions on geometric microliths - 23 of 25 geometric microliths $(92.0 \%)$ were backed. Evidence of the initial shaping of blanks for retouch into microlithic tools can be seen in the existence of microlith 'preforms', which exhibit minimal 
retouch. Almost all retouch on microlith preforms consists of minor abrasion, nibbling retouch or thinning removals. The retouch techniques used to produce non-geometric microliths that lack backing are more variable. For example, the most frequent retouch type on these tools (nibbling) constitutes only $21.7 \%$ of retouch incidences.

Figure 5.1.23: Retouch techniques on microliths at RCC

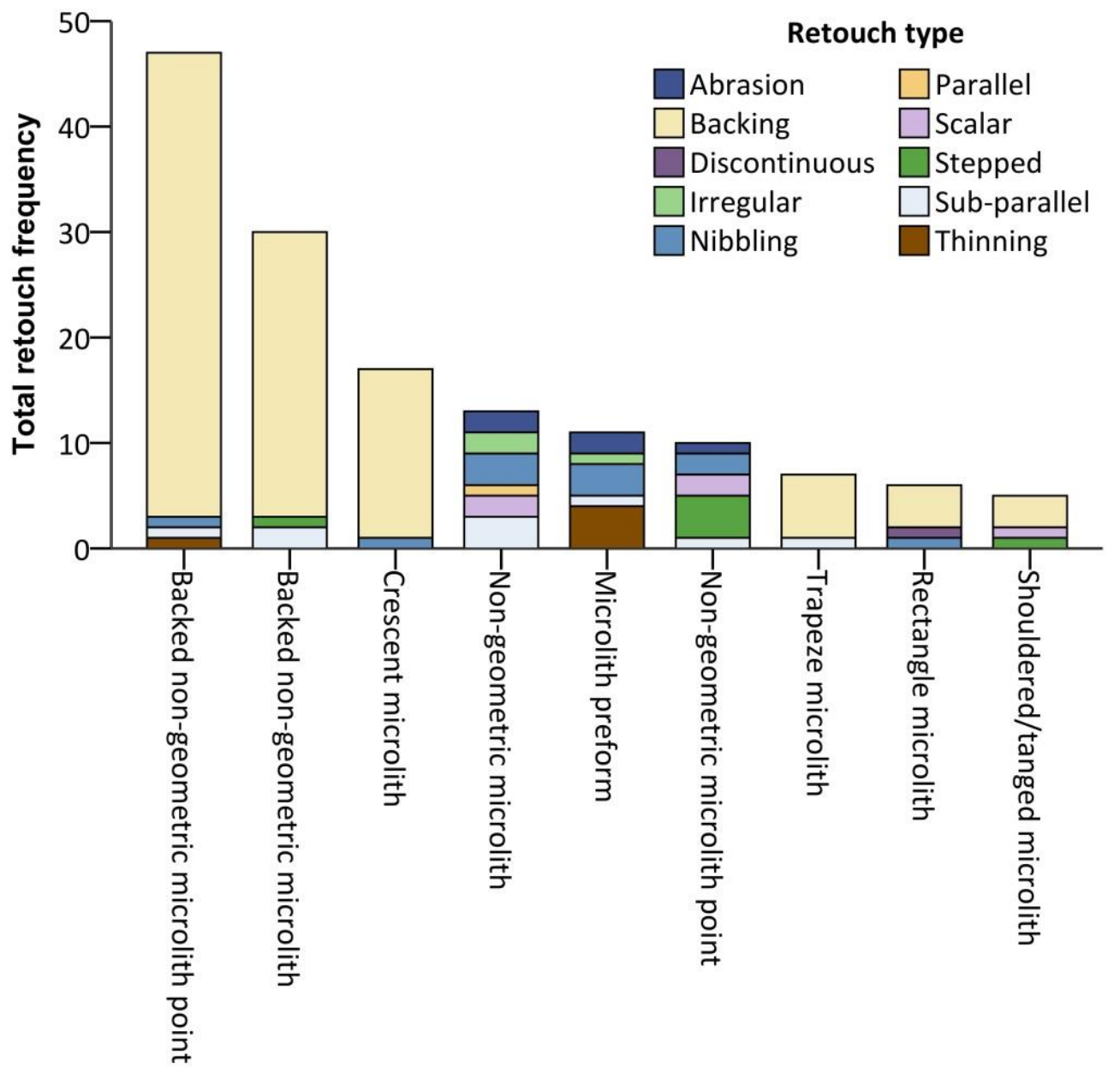

Retouch techniques are also more varied in the non-microlithic tool assemblage. The most common retouch types are scalar and stepped retouch, each comprising $22.7 \%$ of retouch incidences (Figure 5.1.24). Burination, denticulation and notches are found in the non-microlithic tool assemblage but not in the microlith 
assemblage. The opposite is true of parallel retouch. Additionally, backing is only found on a total of five non-microlithic tools, further highlighting the differences between these tool groupings.

Figure 5.1.24: Retouch techniques on non-microlithic tools at RCC

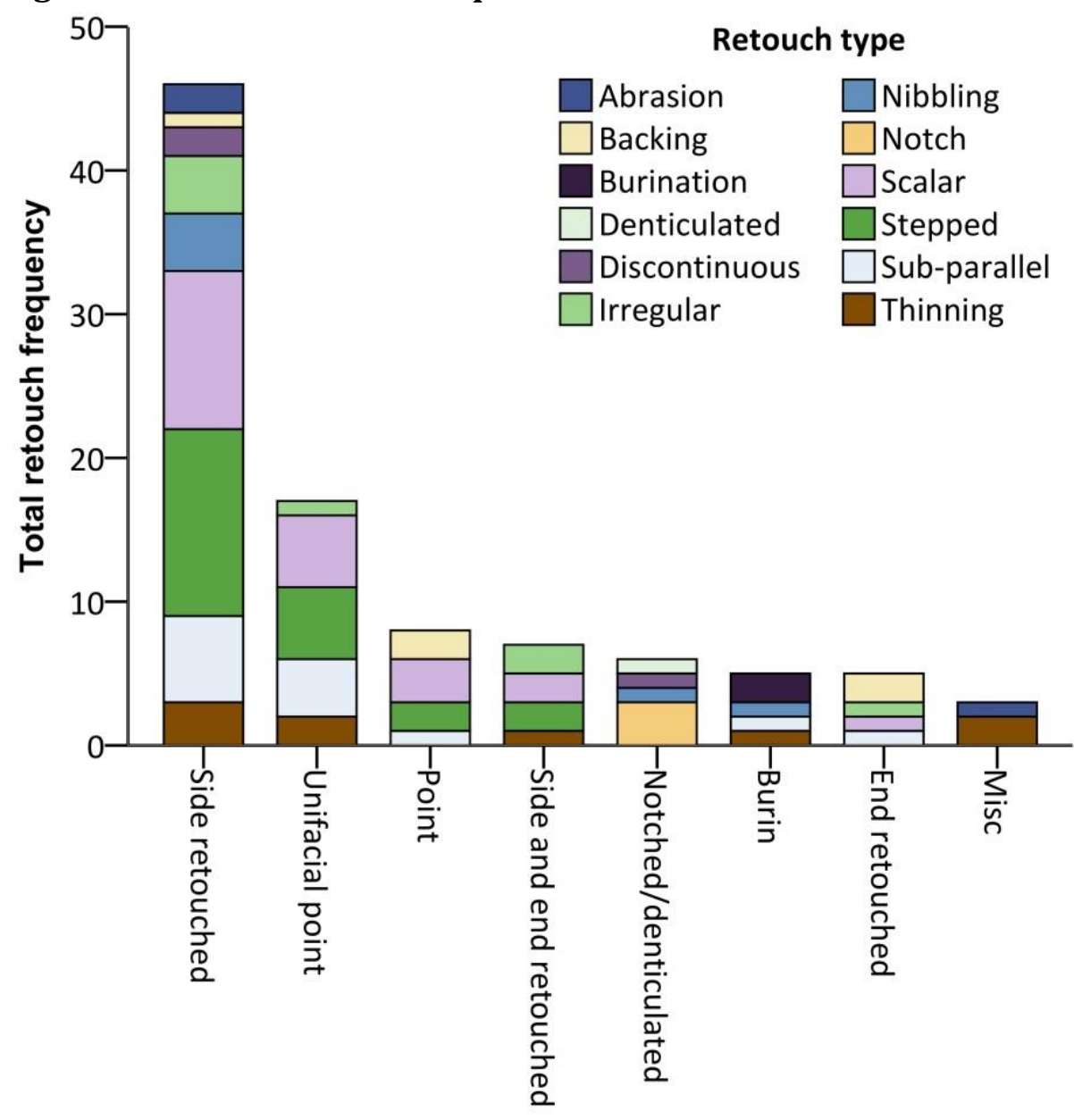

The majority of tools (88.8\% of microliths and $69.4 \%$ of non-microlithic tools) exhibit a single incidence of retouch (Table A.1.17). Retouch in more than one location is more common on non-microlithic tools. The most common retouch location for all tools is along a lateral edge $(71.2 \%$ of microliths and $63.9 \%$ of nonmicrolithic tools). The majority of retouch on non-microlithic tools was made on 
the dorsal face $(78.4 \%)$. The prevalence of edge retouch on microliths is due to the frequent use of bipolar backing retouch; $83.0 \%$ of microlith backing is of this type.

Almost all microliths (92.5\%) exhibit either straight or convex retouch (Table A.1.17). Retouch outlines on non-microlithic tools are more varied. Notably, however, only scalar, stepped and sub-parallel retouch were used to create pointed outlines on non-microlithic tools. Non-microlithic points can be considered an important and distinct tool category at the site.

There is no standardisation apparent in tool retouch length or depth (Table A.1.18). A certain degree of standardisation is evident in the retouch angles of some microlith forms as a result of the lack of variation in the angle of backing retouch. Overall, backing retouch varies between $65^{\circ}$ and $90^{\circ}$, with a mean of $81^{\circ}$. Average retouch lengths and angles are almost identical for pointed and nonpointed non-geometric microliths ( $34.9 \mathrm{~mm}$ and $47^{\circ}$ v. $32.4 \mathrm{~mm}$ and $46^{\circ}$ ), and for the equivalent backed varieties $\left(21.5 \mathrm{~mm}\right.$ and $81^{\circ} \mathrm{v} .21 .4 \mathrm{~mm}$ and $\left.80^{\circ}\right)$. Whether or not a microlith was retouched into a point did not affect the angle or extent of retouch. This is also true with reference to Clarkson's (2002) Index of Invasiveness (IOI), with similarities evident between the mean values for pointed and nonpointed non-geometrics ( 0.147 v. 0.139$)$ and geometrics (0.108 v. 0.117$)$ (Table A.1.19).

Few patterns are evident between non-microlithic tool typologies in retouched edge angles. However, there is a statistically significant difference between sideand end- retouched tools $\left(\chi^{2}(13)=23.105, \mathrm{p}=.040\right)$. Average angles on side- 
retouched tools range from $20-75^{\circ}$, with a mean of $44^{\circ}$, while those on endretouched tools range from $45-90^{\circ}$, with a mean of $68^{\circ}$ (Table A.1.18). As general agreement surrounds the assessment that, on average, smaller edge angles are more frequently associated with cutting activities and larger edge angles with scraping activities (Wilmsen 1968, Cantwell 1979, Andrefsky 2005), this may suggest that side-retouched tools were more likely to be used for cutting activities and end-retouched tools for scraping (although this remains a speculative conclusion).

When individual retouch techniques are considered, edge angles created using the same technique are consistently more acute on non-microlithic tools compared to microliths (Figure 5.1.25). The explanation is unclear, but may be related to a desire not to weaken the edges of microliths and therefore increase the chance of breakage on impact. Figure 5.1.26 further highlights the differences between retouch angles of microlithic and non-microlithic tools.

Clarkson's (2002) IOI varies little between microlith typologies, and the highest value on any individual microlith is 0.344 (Table A.1.19). Non-microlithic tool IOI values vary considerably, however, and include tools with over half of the overall tool surface exhibiting retouch (up to 0.531). Overall, it is clear that microlithic tools were an important and distinct tool type at RCC, for which the majority of the lithic reduction process at the site was designed. Examples of microliths from each layer are illustrated in Figure 5.1.27. 
Figure 5.1.25: Retouched edge angles of tools according to retouch type at RCC

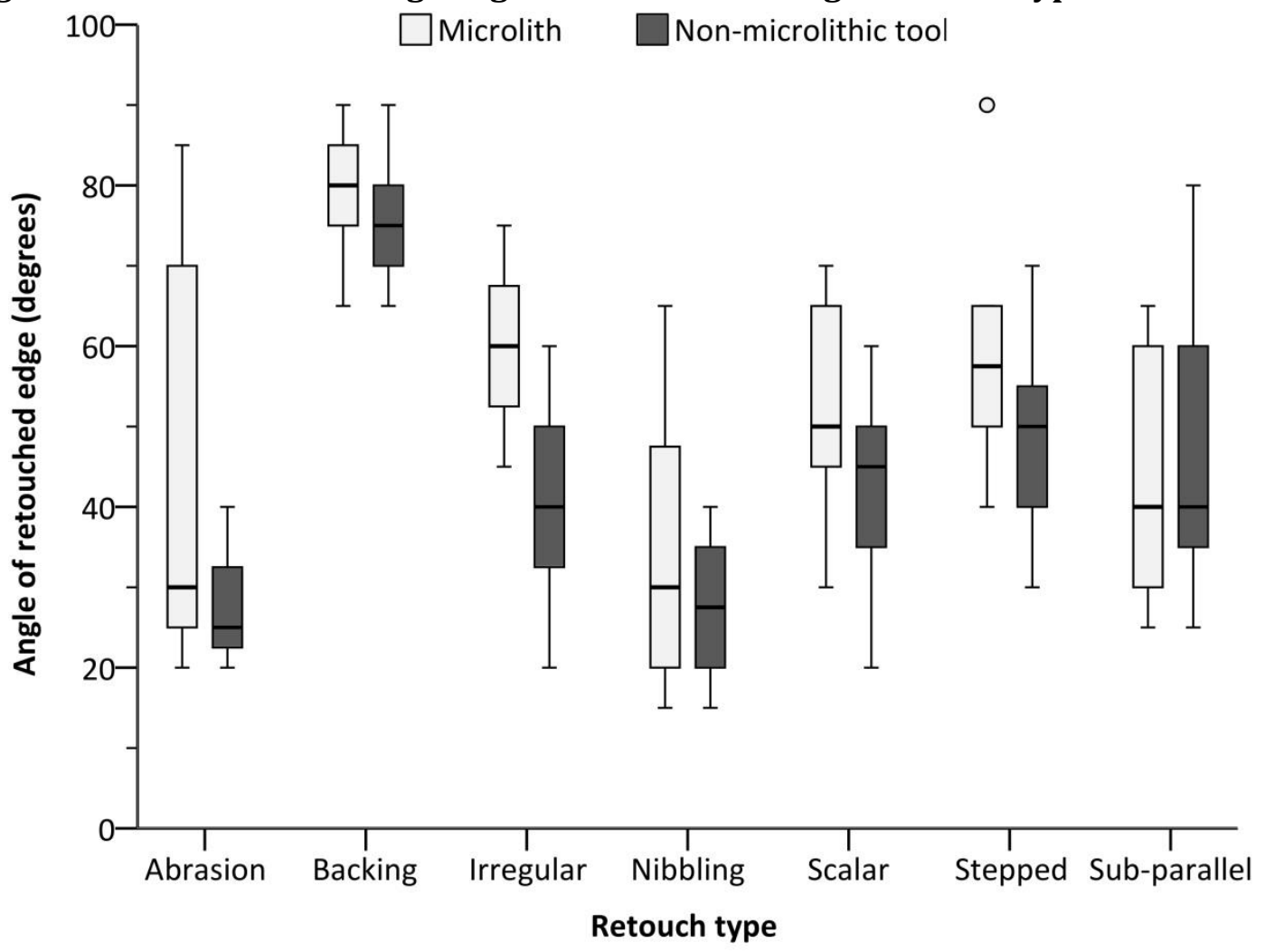


Figure 5.1.26: Frequency distributions of average retouched edge angles on tools at RCC

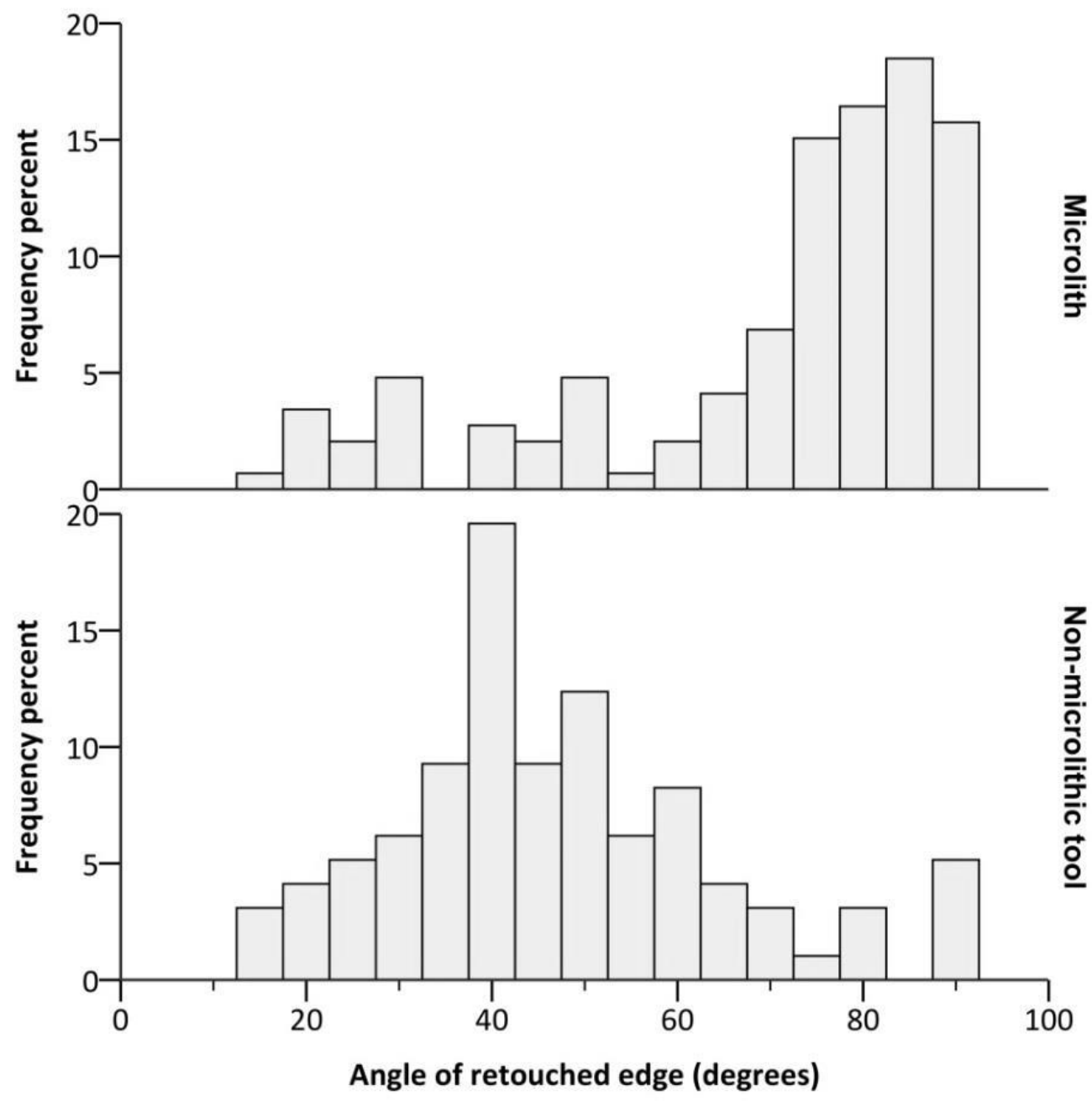


Figure 5.1.27: Microliths from RCC Layer 1 (1-21), 2 (22-45) and 3 (46-69),

demonstrating the range of sizes and forms present. 1-14, 22-31, 46-60: jasper. 1519, 32-41, 61-66: chalcedony. 20, 42, 43, 67-69: agate. 21, 44: hornfels. 45: silcrete. Geometric shapes are relatively uncommon $(17,25,27,45,52)$, while the use of backing retouch to form a point on non-geometric microliths is common $(1,10,13$, $14,16,18,24,29-32,36-39,43,44,46,49,53-56,59,60,62,64,68)$.

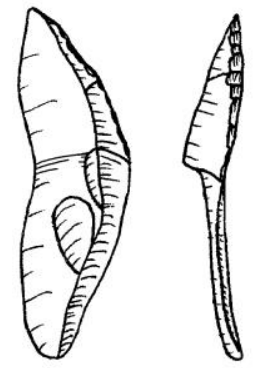

1

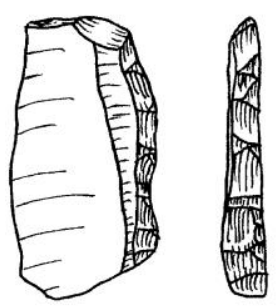

4

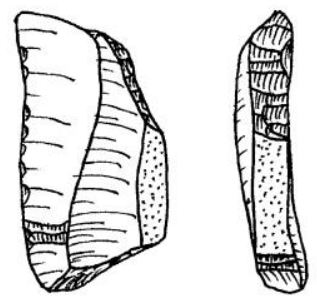

7

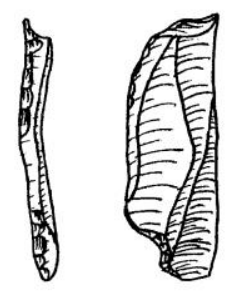

10

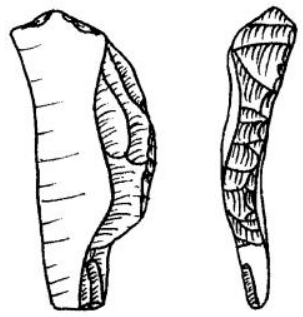

2

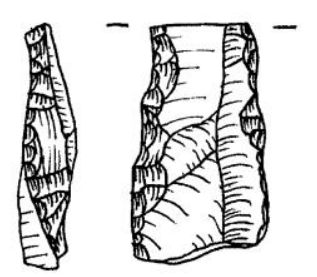

5

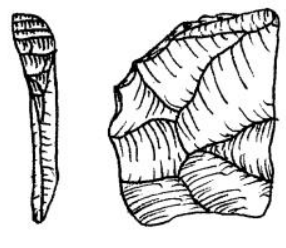

8

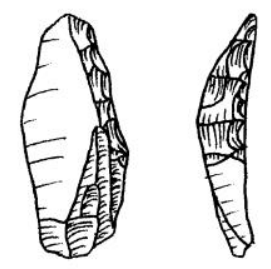

11

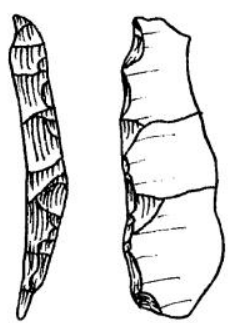

3

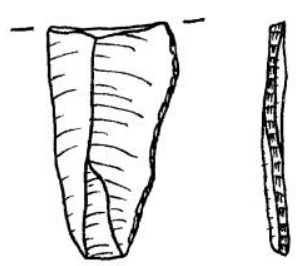

6

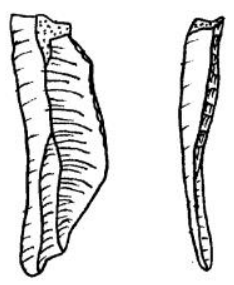

9

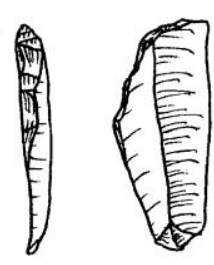

12

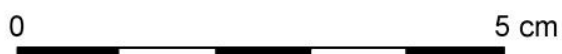




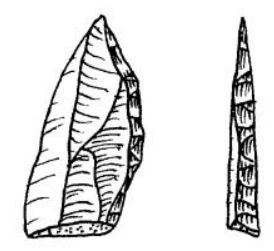

13

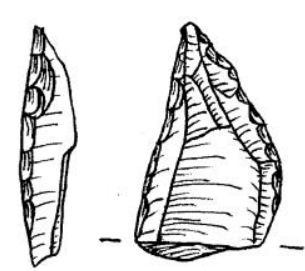

14

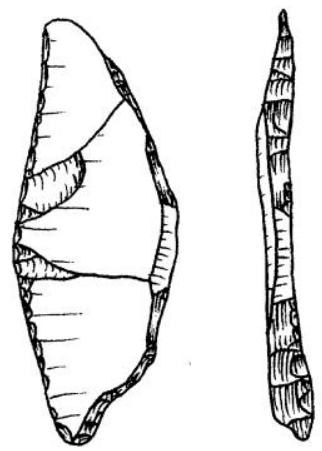

17

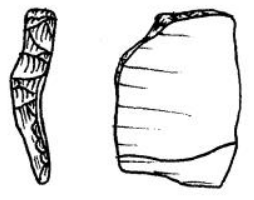

20

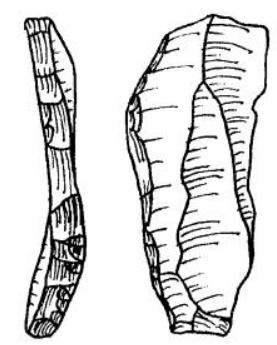

23

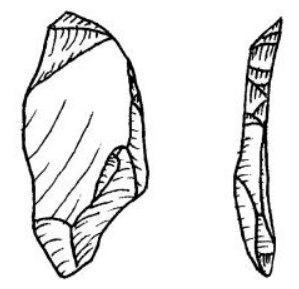

15

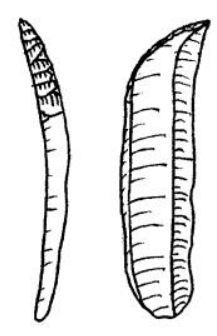

18
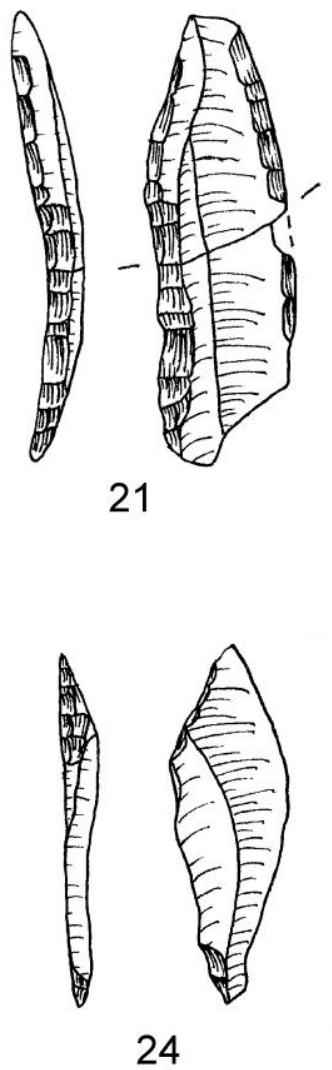

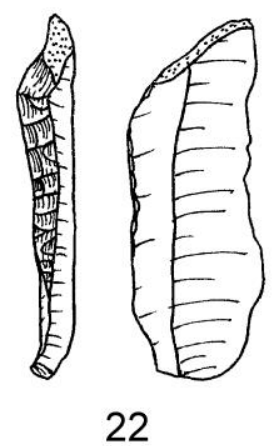




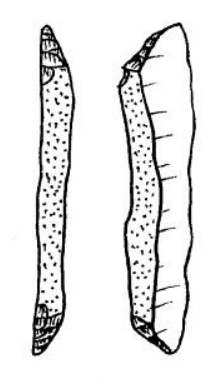

25

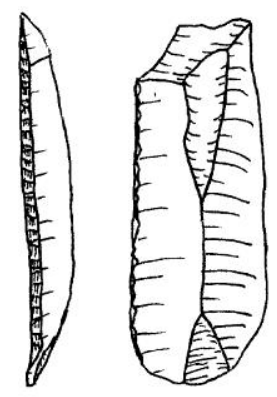

28

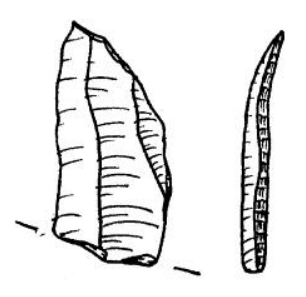

31

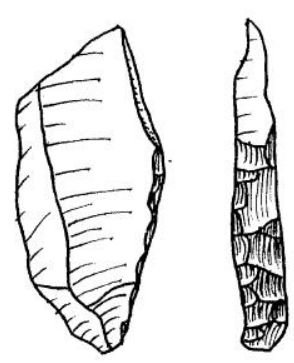

34

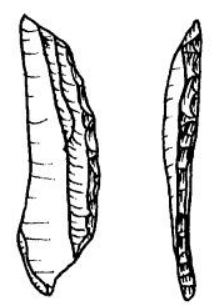

37
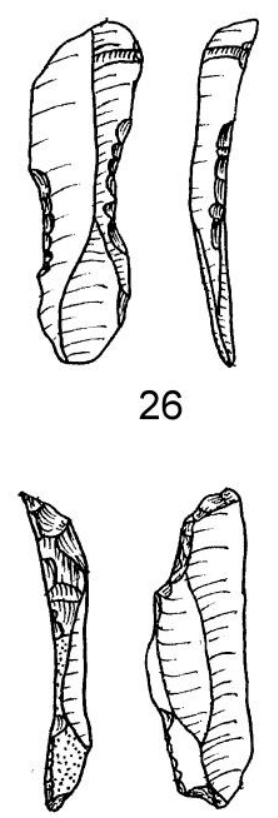

29

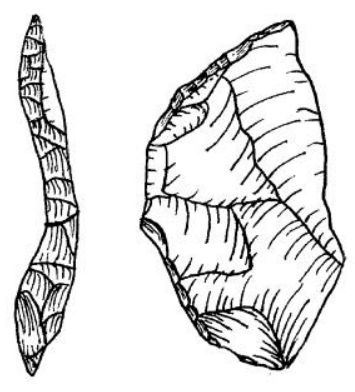

32
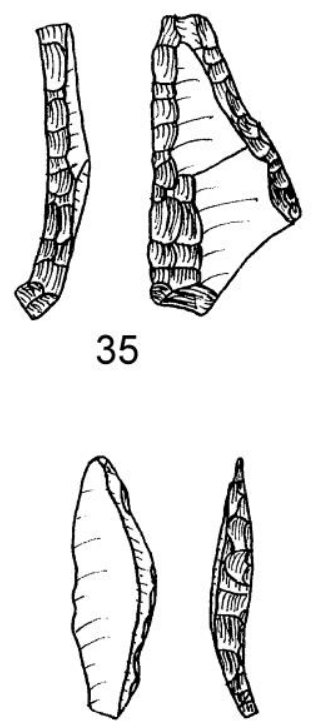

38

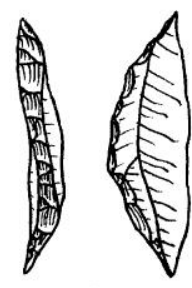

27
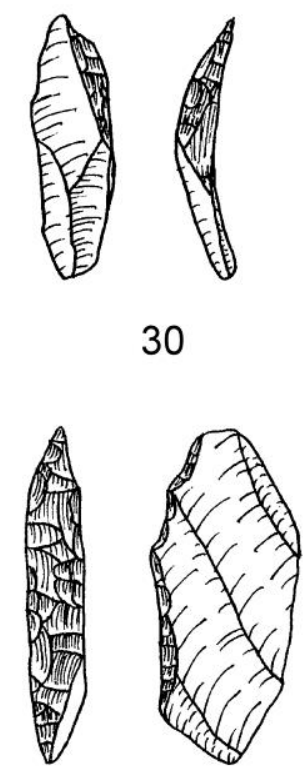

33
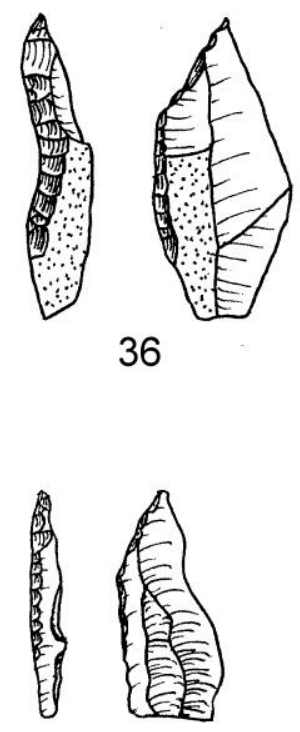

39 

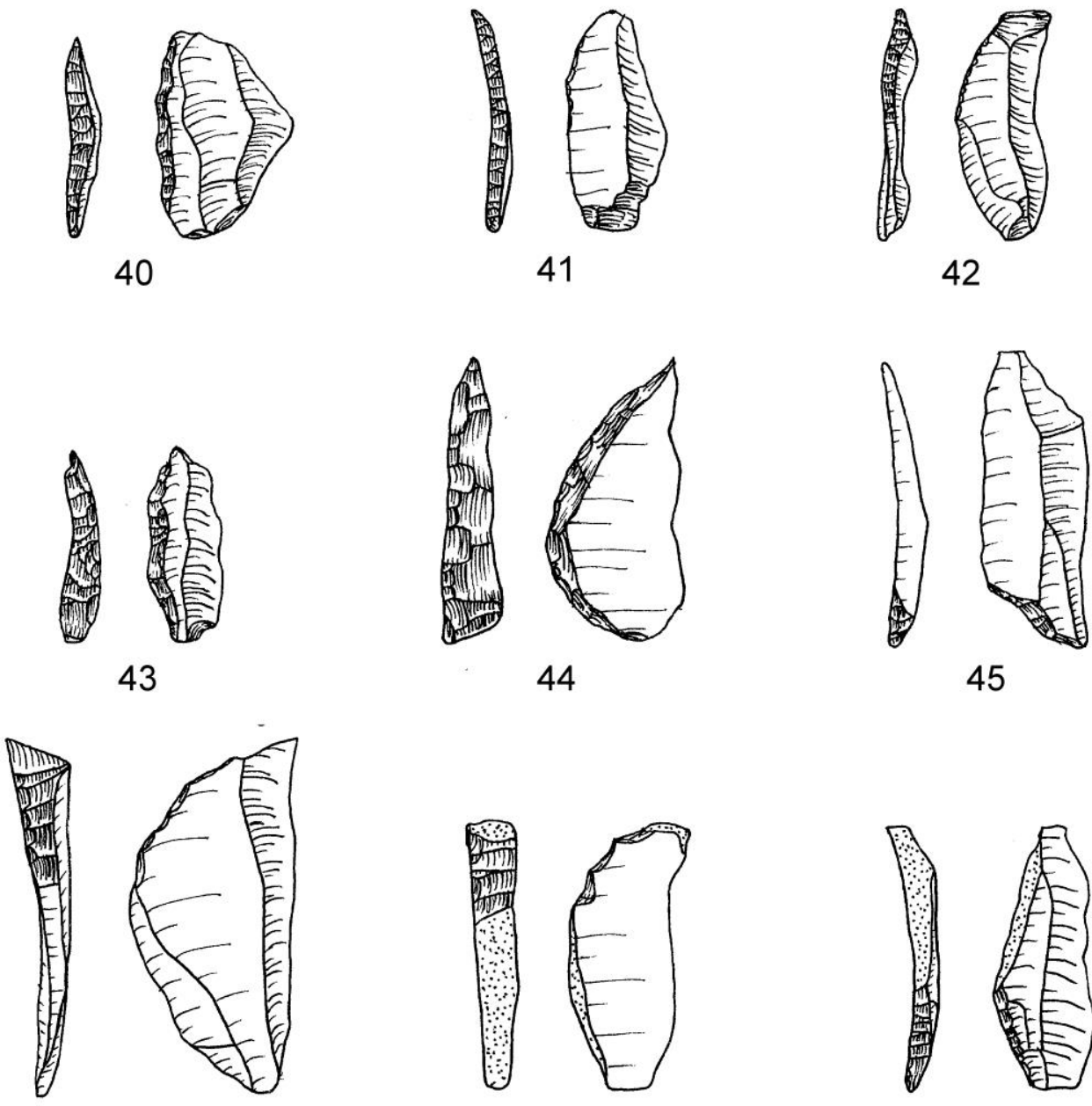

46

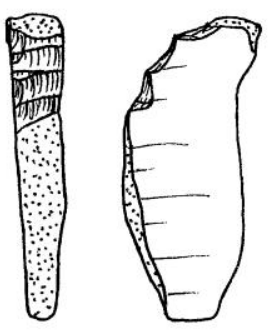

47

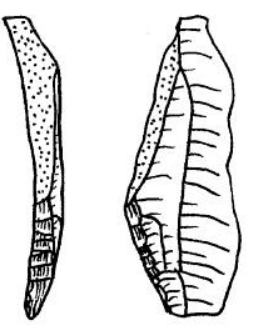

48
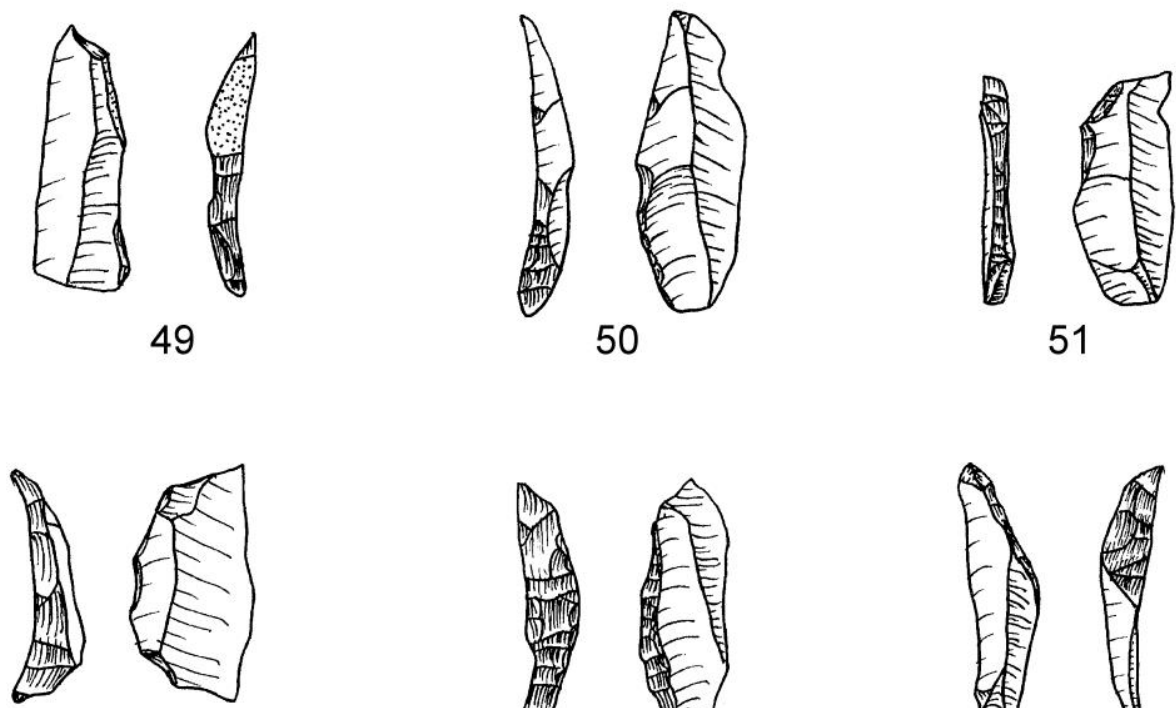

52
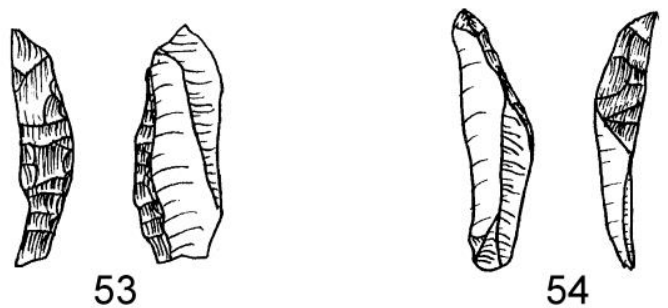


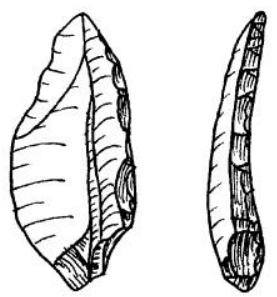

55

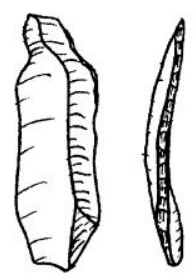

58

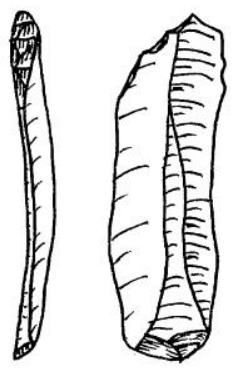

61

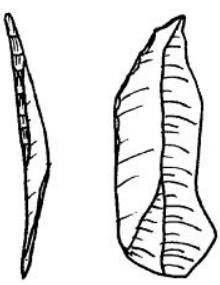

64

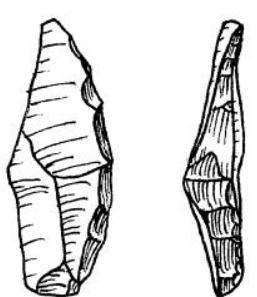

56

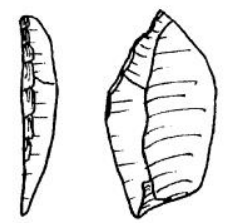

59

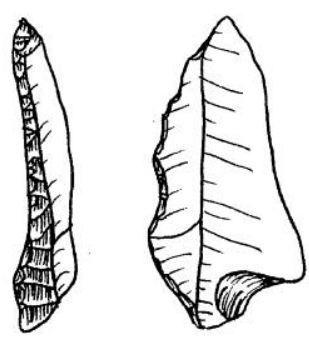

62

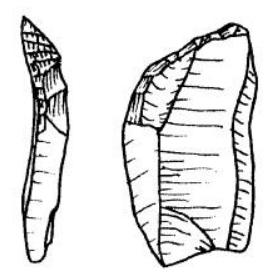

65

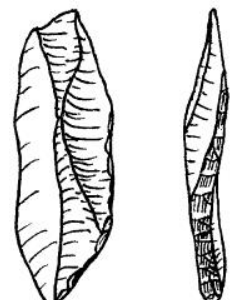

57
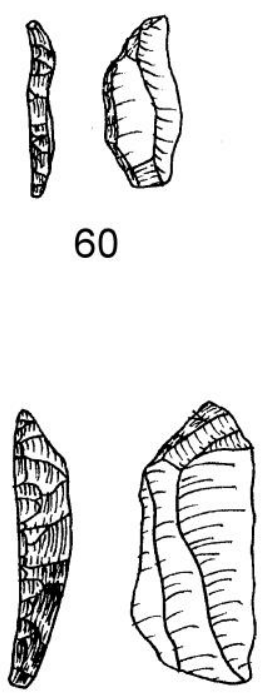

63

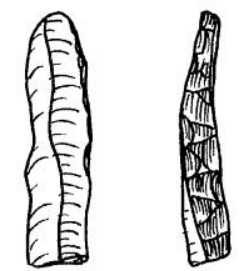
66

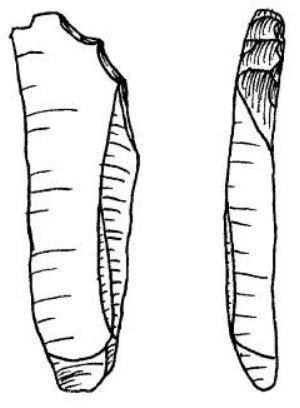

67

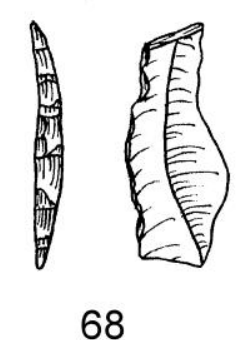

68

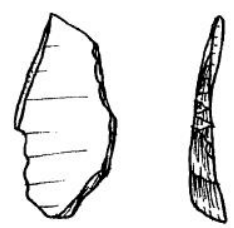

69 
TEMPORAL VARIATION

Results of statistical tests designed to assess whether there are any significant changes over time in the lithic assemblage at RCC are presented in Appendix B.1. The numbers of cores and tools in each layer are reported in Table 5.1.7.

\begin{tabular}{lrrr}
\hline Layer & Cores & Microliths & Non-microlithic tools \\
\hline 1 & 100 & 30 & 29 \\
2 & 60 & 67 & 25 \\
3 & 44 & 37 & 18 \\
\hline
\end{tabular}

Table 5.1.7: Cores and tools analysed in each layer at RCC

\section{Cores}

Results of statistical tests on cores are reported in Table B.1.1. Notably, there are no statistically significant differences between layers in any variable. These results indicate that there were no significant changes in core types, size, reduction intensity or other attributes during HP occupation at the site.

\section{Debitage}

Results of statistical tests on debitage are reported in Table B.1.2 and summarised in Table 5.1.8. Slightly more flakes and flake-blades and fewer blades exist in the top layer of the sequence, although this is not a consistent trend. This minor change in debitage proportions is reflected in the statistically significant differences seen in some of the size variables at the site, as well as parallel arrise numbers and densities. Changes in size are primarily related to width - and to a lesser extent thickness - rather than length, although the parallel-ness of debitage 
does not change significantly. Overall, these changes are indicative of the more flake-like characteristics of the assemblage in the uppermost layer of the HP. Additionally, debitage in Layer 2 is slightly more elongated on average, with smaller striking platforms and higher length:width and length:thickness ratios. Layer 2 is slightly more blade-like, and Layer 1 more flake-like, but overall there are no over-riding trends in debitage dimensions or other characteristics.

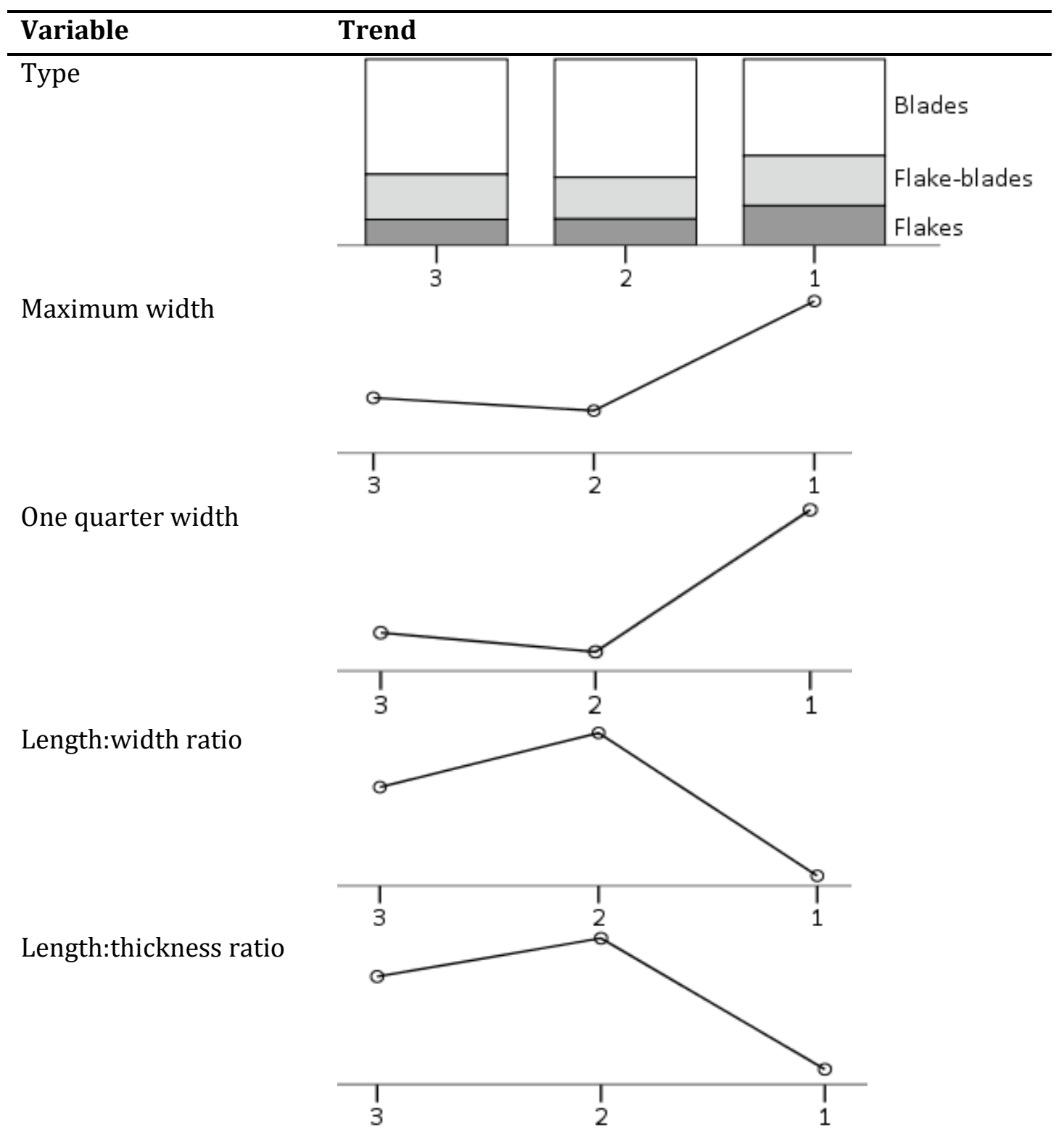


Striking platform width

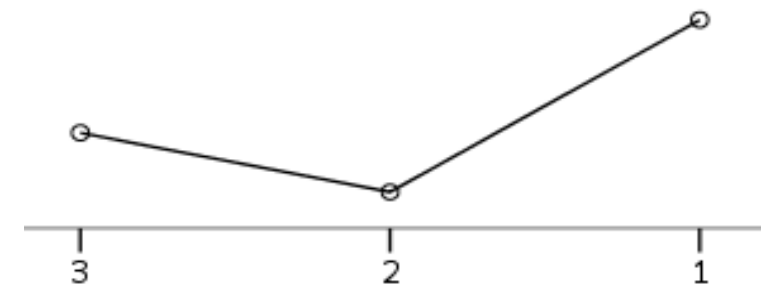

Striking platform

thickness

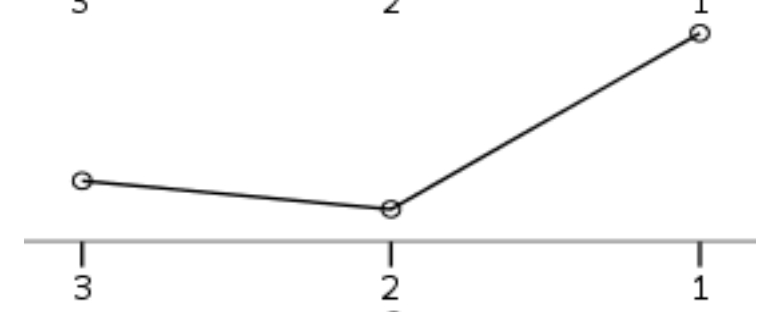

Number of parallel arrises

Arrises per $\mathrm{cm}^{2}$

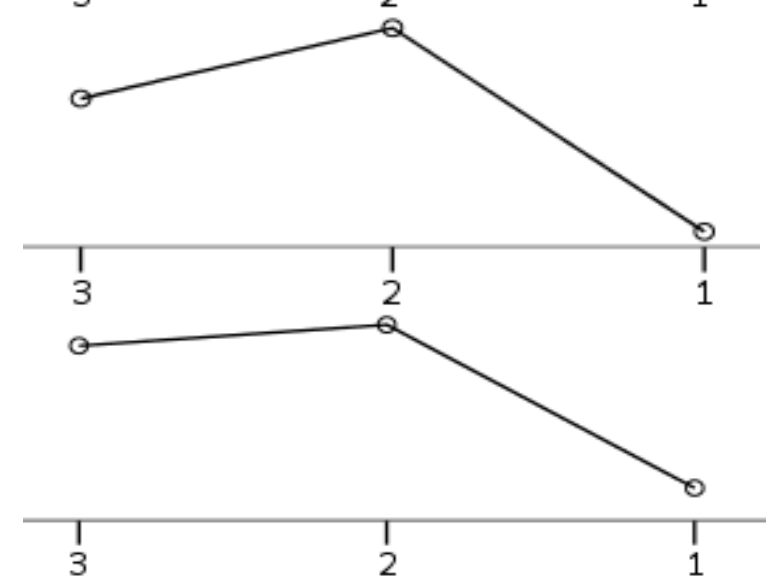

Table 5.1.8: Statistically significant differences between layers in debitage variables at RCC, with simple trendlines of mean values

\section{Microliths}

Results of statistical tests on microliths are reported in Table B.1.3 and summarised in Table 5.1.9. Microliths are wider on average in Layer 1 compared to Layers 2 and 3, reflecting the fact that debitage was also wider on average in this layer. This also impacts on increased TCSA measurements and scar numbers. It is unclear whether wider microliths were the intended outcome, or whether this is a by-product of changes in core reduction which resulted in the production of wider flakes. However, the lack of statistically significant changes in the core assemblage, and the fact that the degree of change in width is stronger in the microlith assemblage than in the debitage sample, implies the former. 


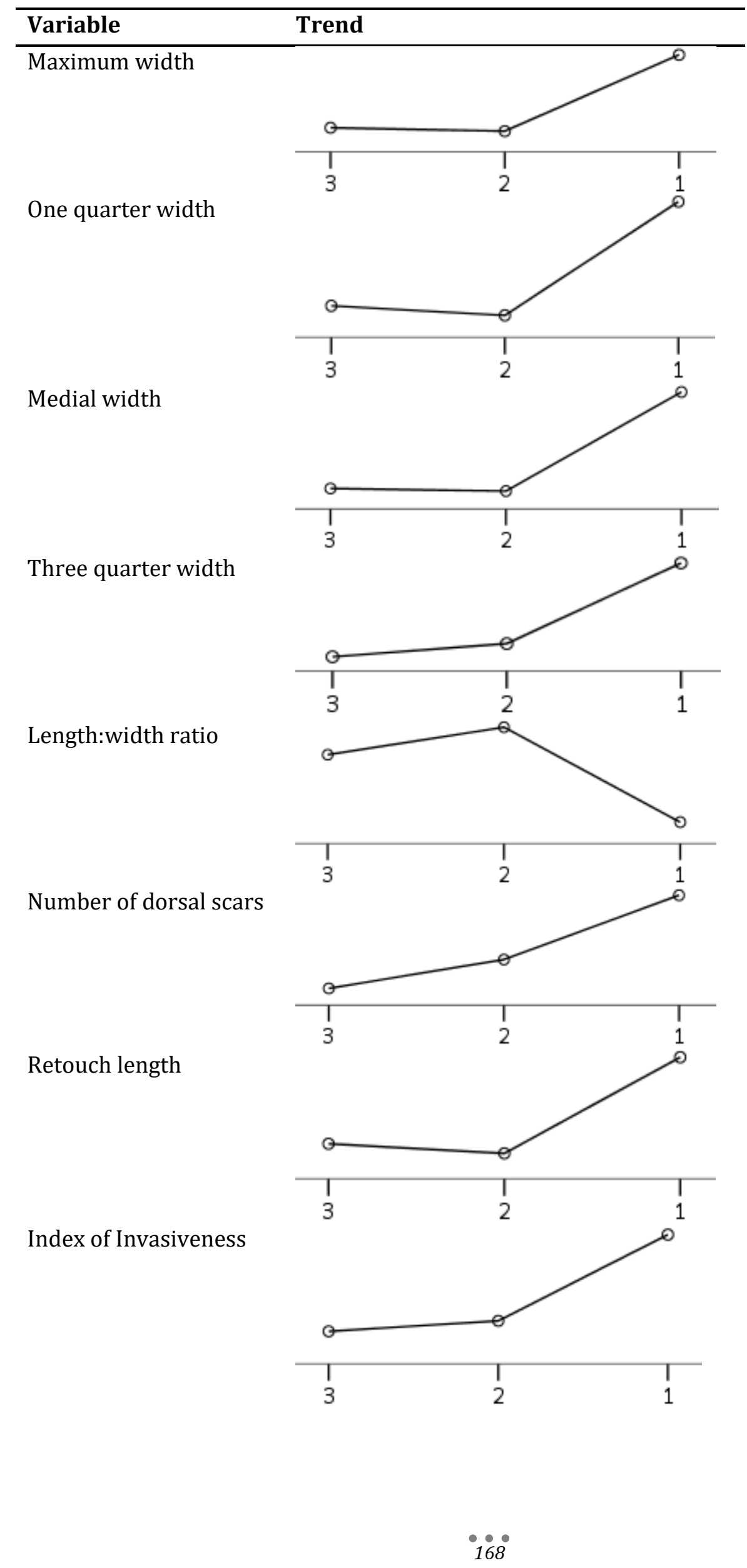


TCSA

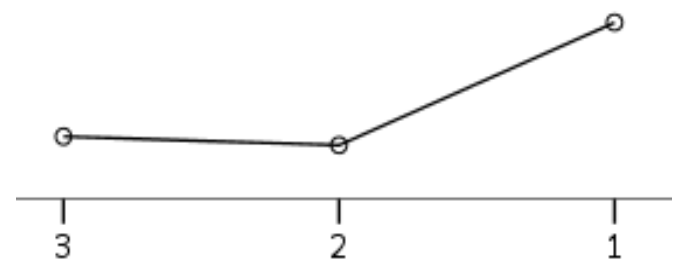

Table 5.1.9: Statistically significant differences between layers in microlith variables at RCC

Additionally, microliths in Layer 1 were more intensively retouched on average. Retouch in this layer tended to be longer but not deeper. Overall, there are some differences in the uppermost HP compared to the rest of the sequence, primarily in the form of wider tools with more invasive retouch, although there are no significant changes in typology.

\section{Non-microlithic tools}

Results of statistical tests on non-microlithic tools are reported in Table B.1.4 and summarised in Table 5.1.10. In similarity with the microlith assemblage, there are relatively few statistically significant differences between layers. There are no changes in tool type proportions or retouch characteristics, with the exception of retouch depth. There is no associated change in IOI values, however, indicating that the impact on the extent of retouch was minimal.

In contrast to the microlith assemblage there are significant differences in blank proportions between layers, with more blade tools in Layer 2. There are also differences evident in length measurements (and the ratios of length to width and thickness), with tools in Layer 2 being more elongated on average. 


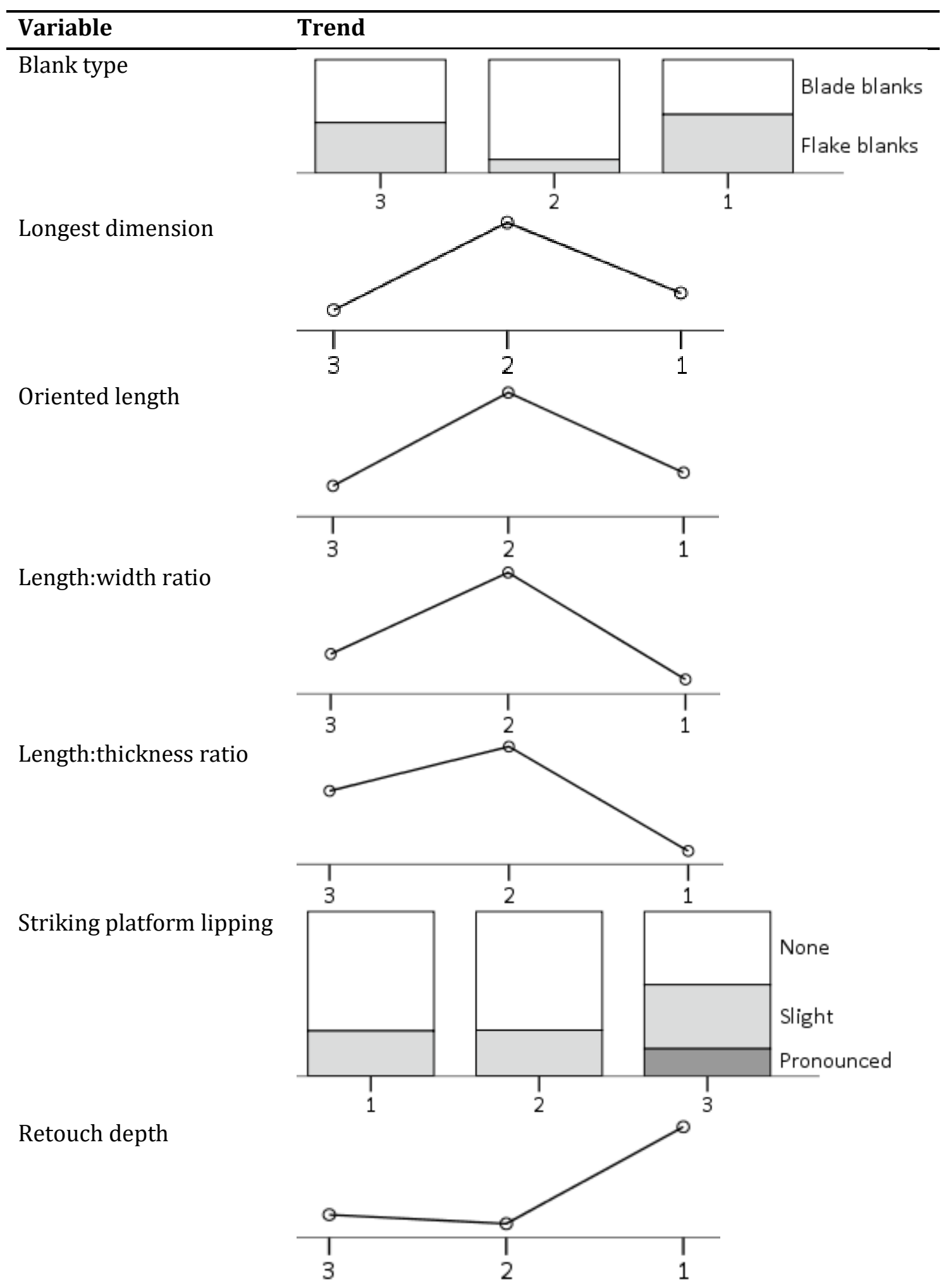

Table 5.1.10: Statistically significant differences between layers in non-microlithic tool variables at RCC 
Non-microlithic tools differ most in Layer 2, whereas in the microlith assemblage they differ most in Layer 1 . The largest differences generally occur between the upper two layers. In their preliminary typological assessment of the same assemblage, Wadley and Harper (1989) suspected that there may be multiple phases within the HP at RCC, although they stated that the excavation of the site in spits precluded their identification. This caveat remains in place. However, the present technological study has found no such evidence for the existence of distinct phases. Furthermore, the lack of any real chronological patterning, regardless of where the spit boundaries happen to be, is meaningful, and demonstrates that there were no substantial technological or typological changes throughout the HP at the site.

\section{MANUFACTURING TRAJECTORIES AND SUMMARY}

Manufacturing trajectories at RCC are summarised in Figure 5.1.28. The primary manufacturing process at the site involved the preparation of cores for the systematic production of small blades, some of which were subsequently selected for retouch into microlithic tools. Raw materials were sourced from the local environment, including the recycling of lithics from nearby earlier MSA sites. CCS varieties are dominant, and jasper was used in particularly high proportions for blade cores, debitage and tools. 
Figure 5.1.28: Manufacturing trajectories at RCC. Solid lines are CCS and claystonedominated trajectories. Dashed lines are a mix of raw materials. Shaded area denotes the primary manufacturing trajectory.

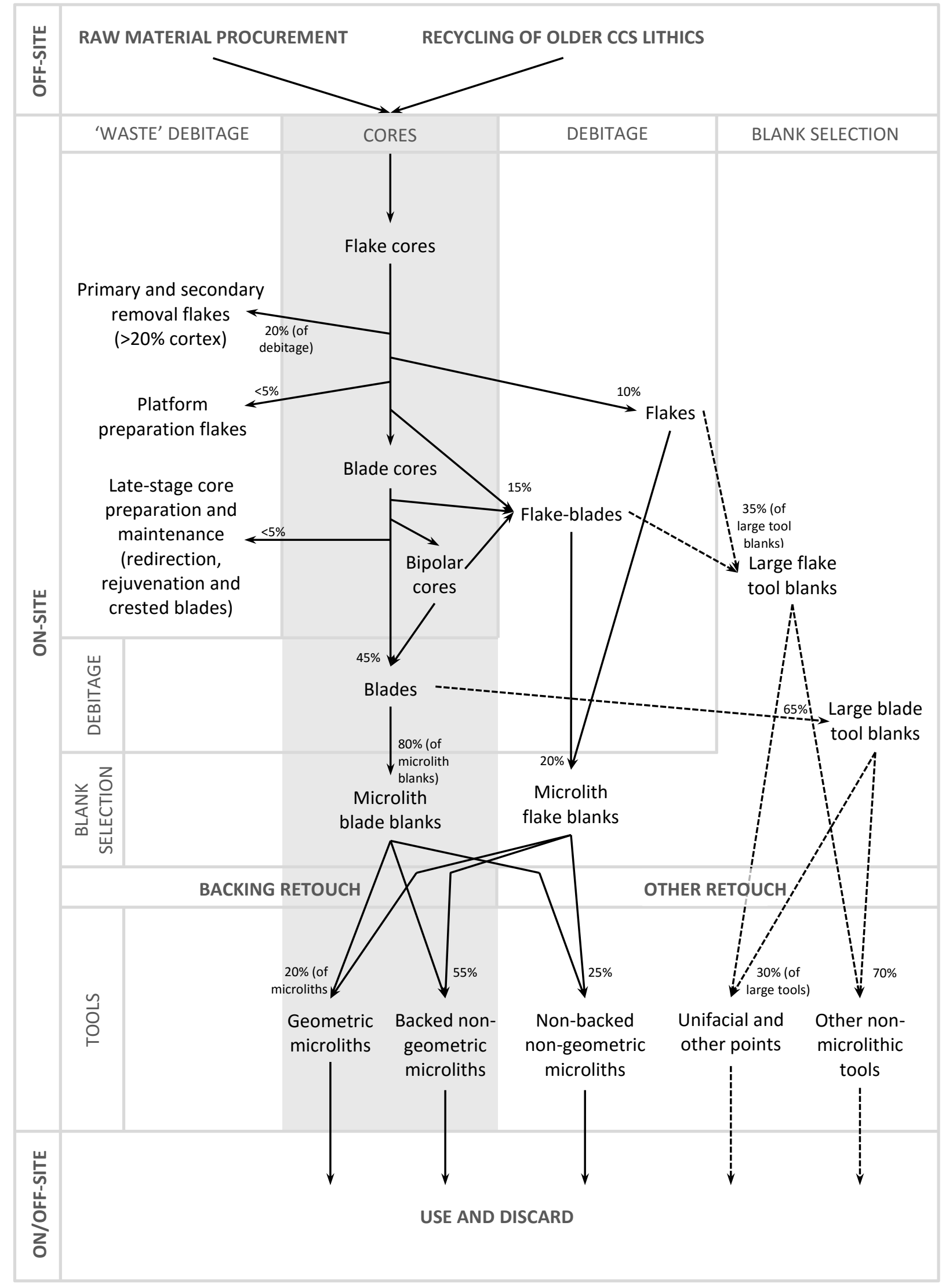


A mix of core types is evident in the assemblage, with no over-riding preferences for working cores from a particular number of striking platforms. Core reduction techniques considered hallmarks of both MSA and LSA technologies are present, with 'typically LSA' bipolar percussion found alongside 'typically MSA' centripetal reduction of radial cores. Bipolar percussion in particular was made use of as an efficient final knapping strategy in the reduction sequences of nearly-exhausted cores, in order to produce large numbers of flakes and especially blades. Bipolar percussion may also have been used partly in response to the small size of some lithic nodules, especially of agate.

Numerous variables indicate that blade cores formed a later stage of core reduction. Blade cores are smaller on average, are more intensively worked (both in absolute terms and relative to core size) and exhibit less cortex cover, while flake cores are more commonly worked from perpendicular striking platforms (i.e. core rotation was common in the preparation of cores for the removal of blades).

In the debitage assemblage an array of variables provide further evidence that blades were the desired and final outcome of the knapping process. Blades exhibit less surface and platform cortex, denser scar concentrations, and more plunging terminations and evidence for late-stage core rejuvenation. Conversely, flakes more frequently exhibit scars from two or three adjacent margins, and evidence for being removed as part of platform preparation. Flakes were primarily removed in the process of preparing cores for the production of blades. A focus on blade production at the site has also been highlighted in previous studies (Soriano et al. 2007, Clarkson 2010). 
The blade production process described applies primarily to CCS and claystone, which were knapped to produce large numbers of relatively standardised blades less than c. $45 \times 20 \mathrm{~mm}$, frequently with flat or pointed striking platforms. These small blades were then selected from for retouch into microlithic tools, with the size distribution of CCS and claystone microliths being very similar to the corresponding debitage samples. The largest CCS and claystone flakes and blades were also selected for retouch into larger (non-microlithic) tools, but this was a subsidiary and possibly more opportunistic process. Only about half of nonmicrolithic tools were made on CCS or claystone blanks, with the rest being made from siltstone, hornfels, quartzite and silcrete. These raw materials were mainly knapped to produce larger flakes and blades. The selection of larger non-CCS tool blanks for retouch into non-microlithic tools was part of a secondary manufacturing process at the site.

The majority of non-microlithic tools are side or double side retouched. Burins, notches and denticulation are evident in the assemblage, as are 'typically MSA' unifacial points which are found throughout the sequence and are the only nonmicrolithic tool form that exhibits any degree of standardisation. Their TCSA measurements fit within those characterised by Shea (2006) as handcast/thrusting spears, in contrast to those of the microlith assemblage at the site which are more similar to projectile arrowheads or dart tips. Non-microlithic points may have constituted an important and distinct tool category at the site, with reference to size and shape but also to retouch technique. 
In the microlith assemblage non-geometric forms are four times more common than geometric shapes. Backed non-geometric microliths are the most common types. Crescents are the most common geometric morphology. Some raw material preferences are evident, with jasper preferred for backed non-geometric microliths. There is little meaningful variation in size between different microlith morphologies, and little evidence for standardisation, with the possible exception of backed non-geometric forms.

Backing (especially bipolar backing) is the most common retouch type used on microliths, and is particularly common on geometric forms. A variety of other retouch techniques were used to manufacture other non-geometric microliths, as well as non-microlithic tools. On the latter, scalar and stepped retouch are most common. Backing is only found on five non-microlithic tools, while several retouch types are unique to either the microlithic or the non-microlithic tool assemblage. Non-microlithic tools also differ somewhat from microliths in retouch angles, which are consistently more acute on non-microlithic tools. This may be related to a desire to decrease the chance of breakage on impact, if used as projectiles.

There are no statistically significant changes over time in the core assemblage, and few in the rest of the assemblage. This is affected by the excavation of the site in spits. However, even when individual spits are considered there are no clear and consistent changes over time. Some changes are evident, for example in the somewhat more flake-like characteristics of the upper layer and the somewhat more blade-like characteristics of the middle layer. However, although there is some degree of variation over time within the sequence, these changes are neither 
consistent over time nor indicative of phases within the sequence. Instead they are the result of inconsistent changes between individual spits which in many cases may simply be ascribed to stochastic processes. Overall, a lithic technological system involving the intensive production of small blades for retouch into microliths remained dominant throughout the HP at RCC.

\subsection{Umhlatuzana, SOUth Africa}

\section{CORES}

A clear preference is evident for working cores from a single platform (Table 5.2.1, Figure 5.2.1). Over half of single platform cores (52.4\%) exhibit signs of the use of bipolar percussion. Bipolar percussion is common in quartz assemblages (Driscoll 2011), and indeed all of the bipolar cores at Umhlatuzana are quartz. In addition to this 'typically LSA' knapping technique, 'typically MSA' radial cores are also present. Blade cores make up only $12.3 \%$ of the core assemblage, in contrast to the argument advanced elsewhere that the HP is predominantly a blade-based industry (e.g. Clark 1985, Soriano et al. 2007, Wadley 2008, Wadley and Mohapi 2008, Cochrane 2008, Wurz 2013).

The majority of cores (95.7\%) are quartz (Figure 5.2.1). Finer qualities of quartz (clear and impure clear quartz, also known as rock crystal) are found primarily as bipolar cores. This may be due to the use of bipolar knapping to maximise the number of flakes that can be removed from a nearly-exhausted core (Knight 1991a, Driscoll 2011), in this case to remove a greater number of flakes of finer quality 
raw material. Alternatively, bipolar knapping may have been used to maximise the proportion of whole flakes that can be produced on quartz cores, as Driscoll (2011) demonstrates experimentally. Lithic raw material sourcing has not been carried out at Umhlatuzana (Mohapi 2013), and so it is not possible to make any further assertions about the sourcing of quartz or other raw materials at the site.

\begin{tabular}{llrr}
\hline Core type & & Frequency & Percentage \\
\hline Uni-directional: & Blade & 14 & 8.6 \\
& Flake & 35 & 21.6 \\
& Bipolar & 54 & 33.3 \\
& & & \\
Bi-directional: & Blade & 6 & 3.7 \\
& Flake & 20 & 12.3 \\
& & & \\
Multi-directional: & Radial & 10 & 6.2 \\
& Amorphous & 23 & 14.2 \\
\hline Total & & 162 & \\
\hline
\end{tabular}

Table 5.2.1: Core type frequencies at Umhlatuzana

Average core size is $24.8 \times 20.9 \times 13.7 \mathrm{~mm}$, although variation exists between types (Table A.2.1, Table A.2.2). Blade cores are smaller than flake cores, with a mean weight of $6.3 \mathrm{~g}$ and cubic volume of $6.1 \mathrm{~cm}^{3}$, compared to $13.8 \mathrm{~g}$ and $12.4 \mathrm{~cm}^{3}$. Radial cores are particularly large (Figure 5.2.2). Bipolar cores are much smaller on average on other core types - with mean dimensions of $17.9 \times 15.7 \times 11.2 \mathrm{~mm}-$ as would be expected if bipolar percussion was utilised as an efficient method for removing flakes from small and/or nearly-exhausted quartz cores. They are also of relatively standardised dimensions (Table A.2.2), suggesting that bipolar cores were all discarded at a similar size, and that bipolar core reduction was a relatively standardised process at Umhlatuzana. 
Figure 5.2.1: Raw material proportions of cores at Umhlatuzana

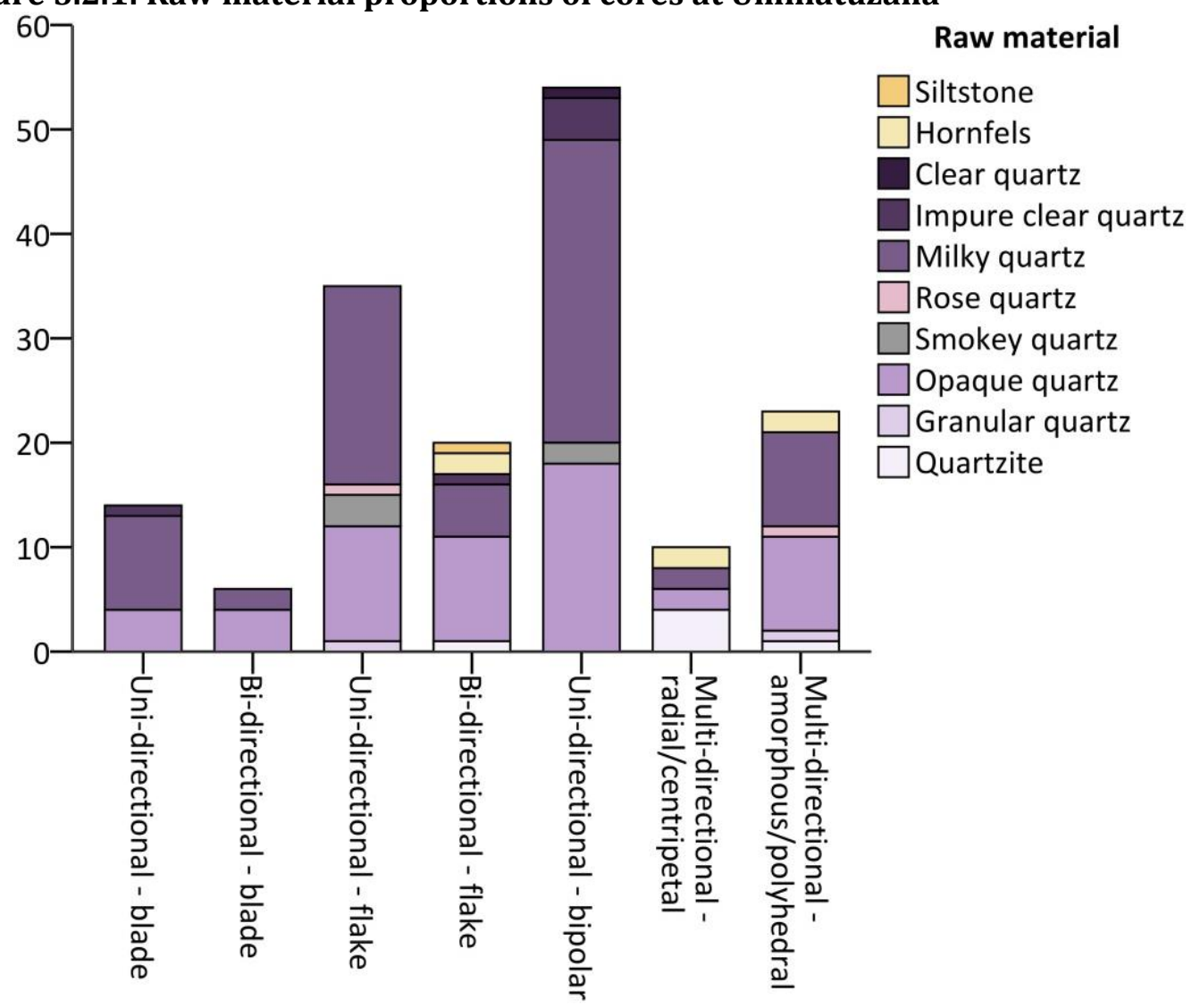

Figure 5.2.2: Weight distributions of cores at Umhlatuzana

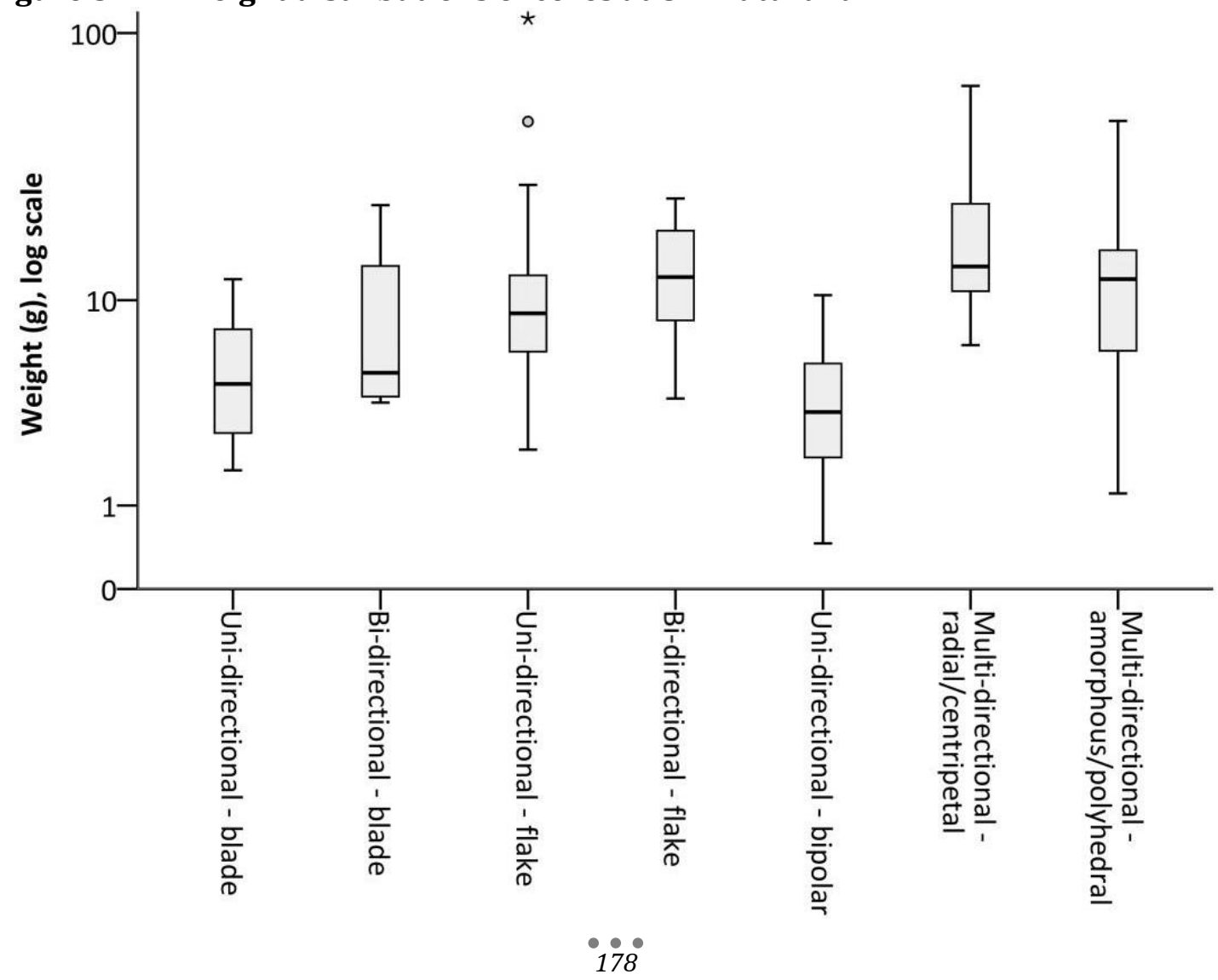


There is little variation within core types in the numbers of striking platforms. All radial cores were worked from the entirety of the circumference, and all but one of the non-radial multi-directional cores were worked from three platforms (Table A.2.3). Radial cores and cores worked from three platforms appear to have been distinct types within the core assemblage at the site. Only two bi-directional cores were worked from perpendicular rather than opposing platforms, indicating that this form of core rotation was uncommon.

Flat striking platforms are dominant (54.9\%), followed by double-facetted (17.9\%) and abraded/crushed platforms (15.4\%) (Figure 5.2.3). Cortical and complex platforms are rare. The low frequency of cortical striking platforms may be partly due to the fact that quartz nodules often lack discernible changes in surface colour or texture. Cortical striking platforms are not found on blade cores, suggesting that these cores may have been more intensively worked, resulting in the removal of more cortex.

Although radial cores are uncommon, they are the most intensively worked core type in terms of absolute scar numbers (Figure 5.2.4, data from Table A.2.4). Very few of these removals are of blade dimensions (3.7\%). Bipolar cores were also used predominantly for the removal of flakes, with only a third (33.3\%) of removals being of blade dimensions. Bipolar cores can be considered most similar to uni-directional flake cores in the assemblage. 
Figure 5.2.3: Proportions of core striking platform types at Umhlatuzana Striking platform type

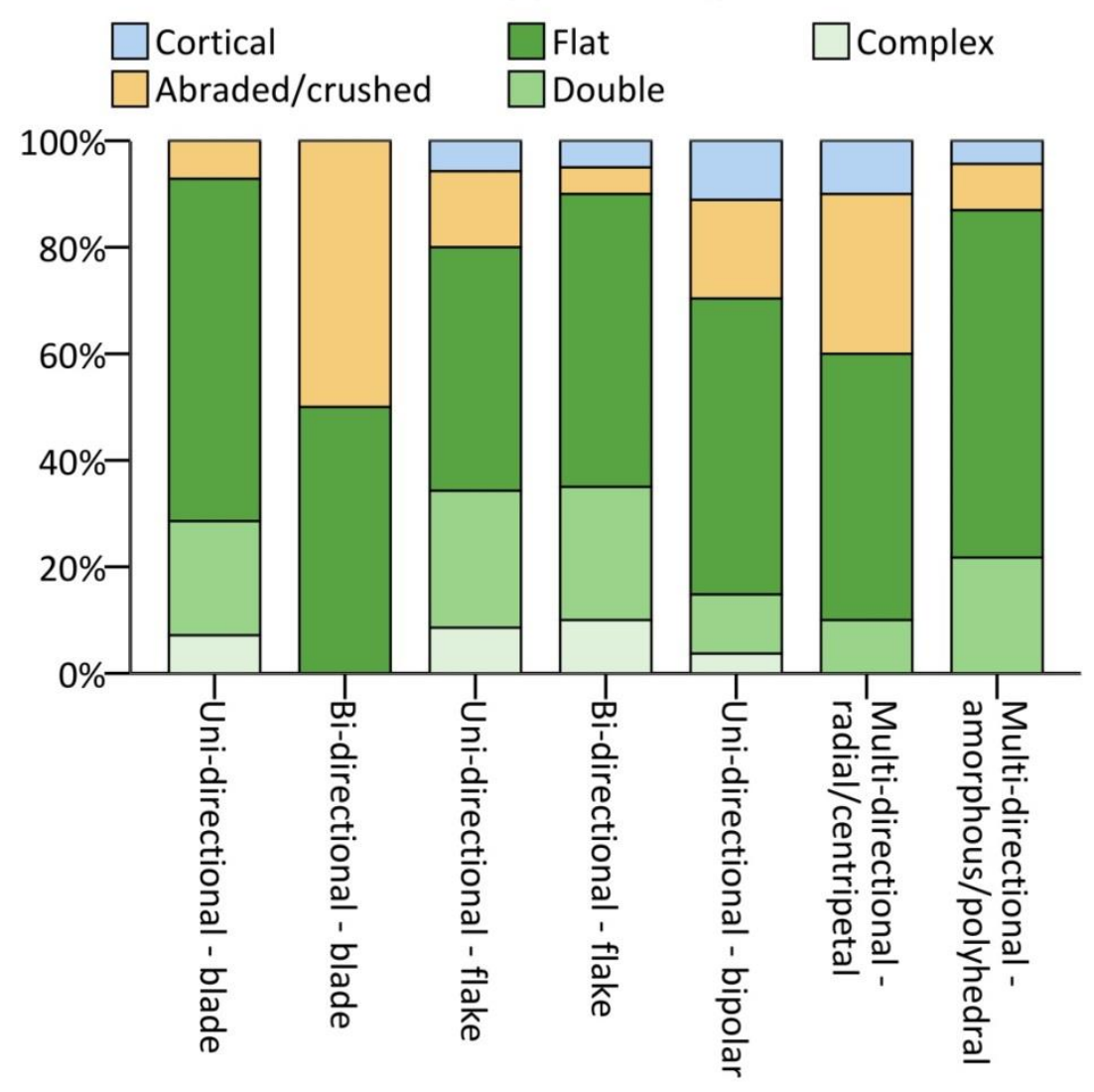

Figure 5.2.4: Average numbers of flake and blade scars on cores at Umhlatuzana

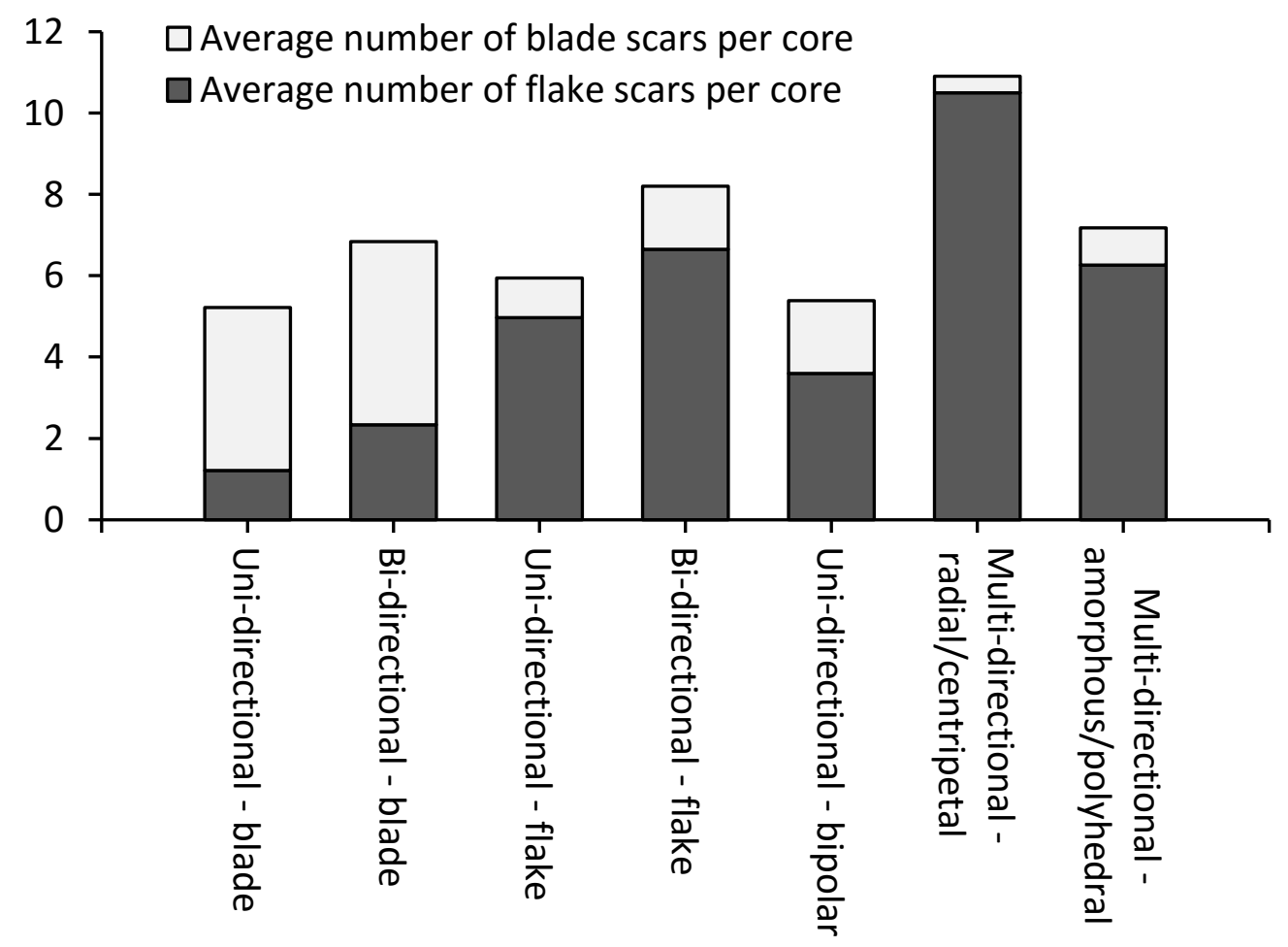


Average terminal removal scar lengths are longer on blade cores $(21.1 \mathrm{~mm})$ than on flake cores $(19.5 \mathrm{~mm})$, and shortest on bipolar $(15.0 \mathrm{~mm})$ and radial cores $(15.4$ $\mathrm{mm}$ ) (Table A.2.4). Removals on blade cores are particularly numerous and large when compared to core volume (Figure 5.2.5, Table A.2.5). Although the absolute number of blade cores is low compared to flake cores, they were worked more intensively to produce a greater number of relatively large removals.

Figure 5.2.5: Ratios of total removal scar length to core volume at Umhlatuzana

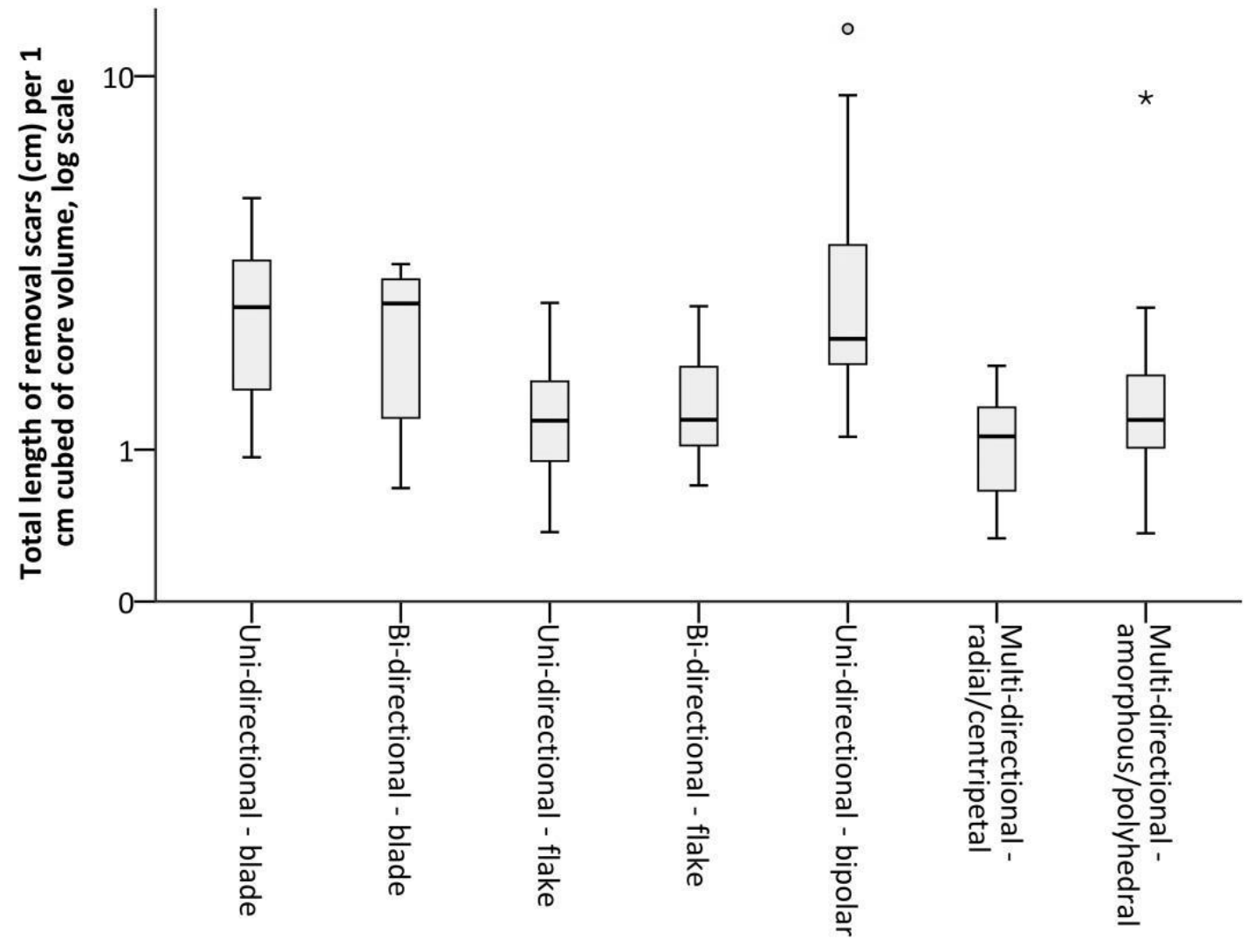

Bipolar cores are the most intensively worked core type. In addition to high mean numbers for all three measures of core reduction intensity, bipolar cores also exhibit particularly high maximum values. These range up to 10.3 scars per $1 \mathrm{~cm}^{3}$ core volume, an average removal length of $25.3 \mathrm{~mm}$ per $1 \mathrm{~cm}^{3}$, and a total removal 
length of $3.2 \mathrm{~cm}$ per $1 \mathrm{~cm}^{3}$ (Table A.2.5). Overall, bipolar cores were intensively and efficiently reduced to produce large numbers of flakes.

\section{DEBITAGE}

In accordance with the core assemblage, debitage exhibits a mix of flakes and blades and cannot be considered to be primarily a blade industry - only $19.6 \%$ are blades (Table 5.2.2). The majority of the debitage assemblage is whole (67.5\%), with striking platforms evident on an additional 14.5\% of debitage (Table A.2.6). The relatively high proportion of broken lithics is to be expected in a predominantly quartz assemblage (Knight 1991b, Driscoll 2011). Quartz constitutes $46.0 \%$ of the debitage assemblage, and was primarily used for the manufacture of flakes (Table 5.2.2, Figure 5.2.6). Hornfels comprises $31.6 \%$ of debitage, and was preferred for blades. However, only six hornfels cores were found in the core assemblage (3.7\%), none of which are blade cores, suggesting that there may have been differential transport of hornfels compared to quartz, potentially with hornfels cores being worked off-site.

The average size of whole debitage is 27.0 × $19.2 \times 6.4 \mathrm{~mm}$ (Table A.2.7). However, differences are apparent between the two dominant raw materials. Quartz debitage is consistently smaller (with a mean volume of $2.7 \mathrm{~cm}^{3}$ ) and more standardised (Figure 5.2.7), while hornfels is larger $\left(5.2 \mathrm{~cm}^{3}\right)$, narrower and thinner on average, with a mean length:width ratio of 1.65 and length:thickness ratio of 5.65, compared to 1.45 and 3.97 for quartz (Table A.2.8). Possible explanations include potential differences in the available sizes of raw material 
clasts, and the difficultly of removing large flakes from cores of low quality quartz (Knight 1991b). However, as will be demonstrated below, a more compelling argument revolves around the use of hornfels and quartz to intentionally produce different types and sizes of blanks for retouch into distinct microlith forms.

\begin{tabular}{lrrrrr}
\hline Raw material & Blades & Flake-blades & Flakes & Total & $\mathbf{\%}$ \\
\hline Hornfels & 99 & 149 & 122 & 370 & 31.2 \\
Milky quartz & 29 & 129 & 158 & 316 & 26.6 \\
Quartzite & 47 & 65 & 53 & 165 & 13.9 \\
Opaque quartz & 14 & 64 & 51 & 129 & 10.9 \\
Impure clear quartz & 8 & 28 & 29 & 65 & 5.5 \\
Siltstone & 7 & 21 & 13 & 41 & 3.5 \\
Silcrete & 4 & 10 & 11 & 25 & 2.1 \\
Jasper & 8 & 3 & 6 & 17 & 1.4 \\
Claystone & 6 & 7 & 3 & 16 & 1.3 \\
Clear quartz & 6 & 4 & 5 & 15 & 1.3 \\
Smokey quartz & 1 & 4 & 7 & 12 & 1.0 \\
Chalcedony & 2 & 4 & 1 & 7 & 0.6 \\
Granular quartz & 2 & 2 & 3 & 7 & 0.6 \\
Rose quartz & & 1 & & 1 & 0.1 \\
\hline Total (\%) & $233(19.6)$ & $491(41.4)$ & $462(39.0)$ & 1186 & \\
\hline
\end{tabular}

Table 5.2.2: Debitage measured at Umhlatuzana

Slight or pronounced lipping is evident on $29.3 \%$ of striking platforms, although on only $2.8 \%$ of platforms is it particularly prominent. Although statistically significant relationships exist between platform lipping and both debitage type $\left(\chi^{2}(4)=12.023, p=.017\right)$ and raw material divided into quartz, hornfels and other $\left(\chi^{2}(4)=15.224, p=.004\right)$, both relationships are negligible (Cramér's $V=.071$ and .080 , respectively). 
Figure 5.2.6: Proportions of debitage categories made on each raw material at Umhlatuzana

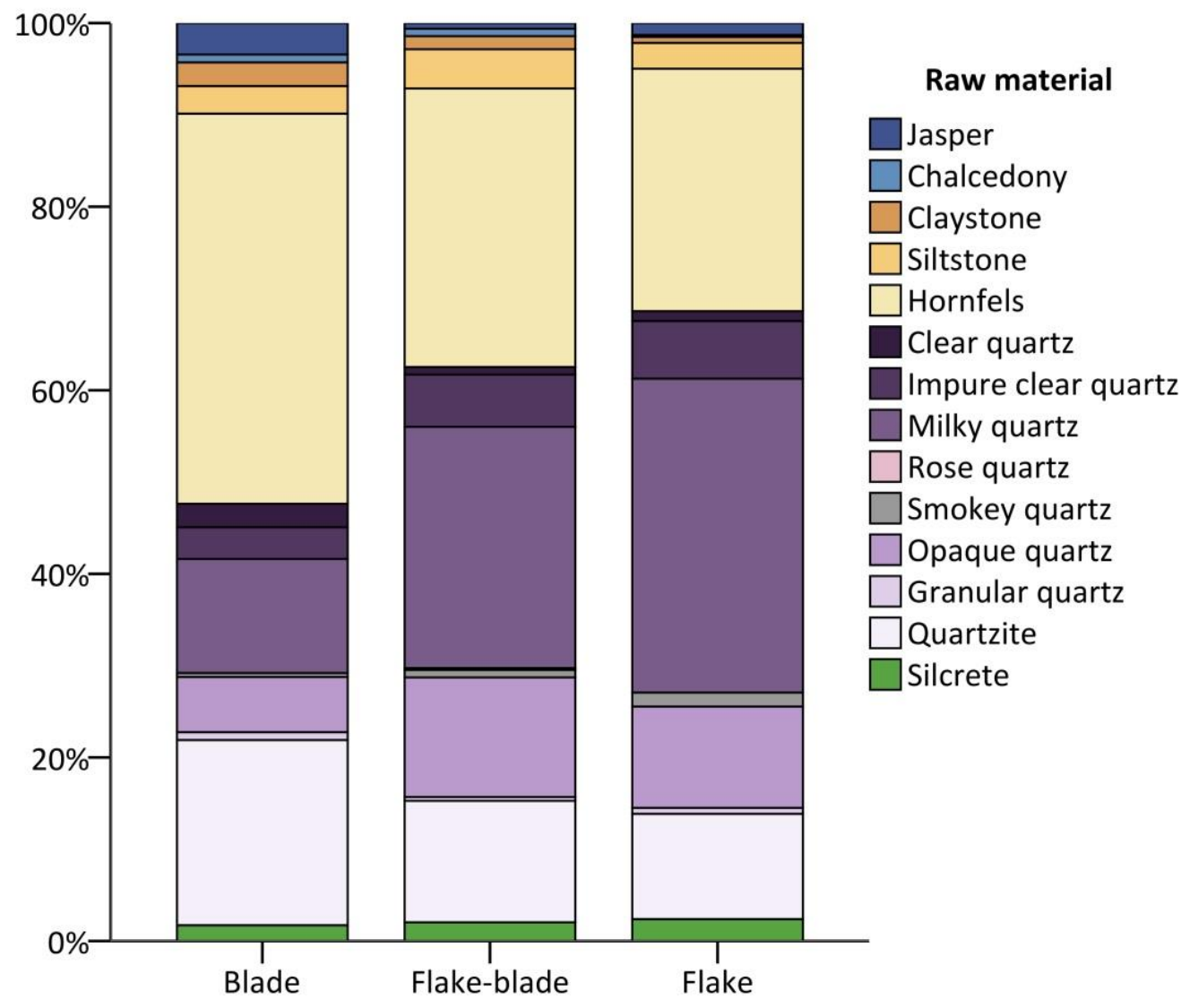

Similar relationships exist with regards to striking platform type. Flat platforms are the most common (70.2\%). However, as Figure 5.2 .8 reveals, there is little variation between debitage types. As with platform lipping, the relationships between platform type and debitage type $\left(\chi^{2}(10)=24.519, \mathrm{p}=.006\right.$, Cramér's V $=$ .108) and raw material $\left(\chi^{2}(10)=24.695, \mathrm{p}=.006\right.$, Cramér's $\left.V=.108\right)$ are statistically significant but weak. Overall, there is no clear indication from the striking platform evidence that hornfels, quartz and other raw materials were worked using different techniques, or that blades and flakes were removed in different ways or at different stages in the reduction process. 
Figure 5.2.7: Frequency distributions of lengths of whole hornfels and quartz debitage at Umhlatuzana

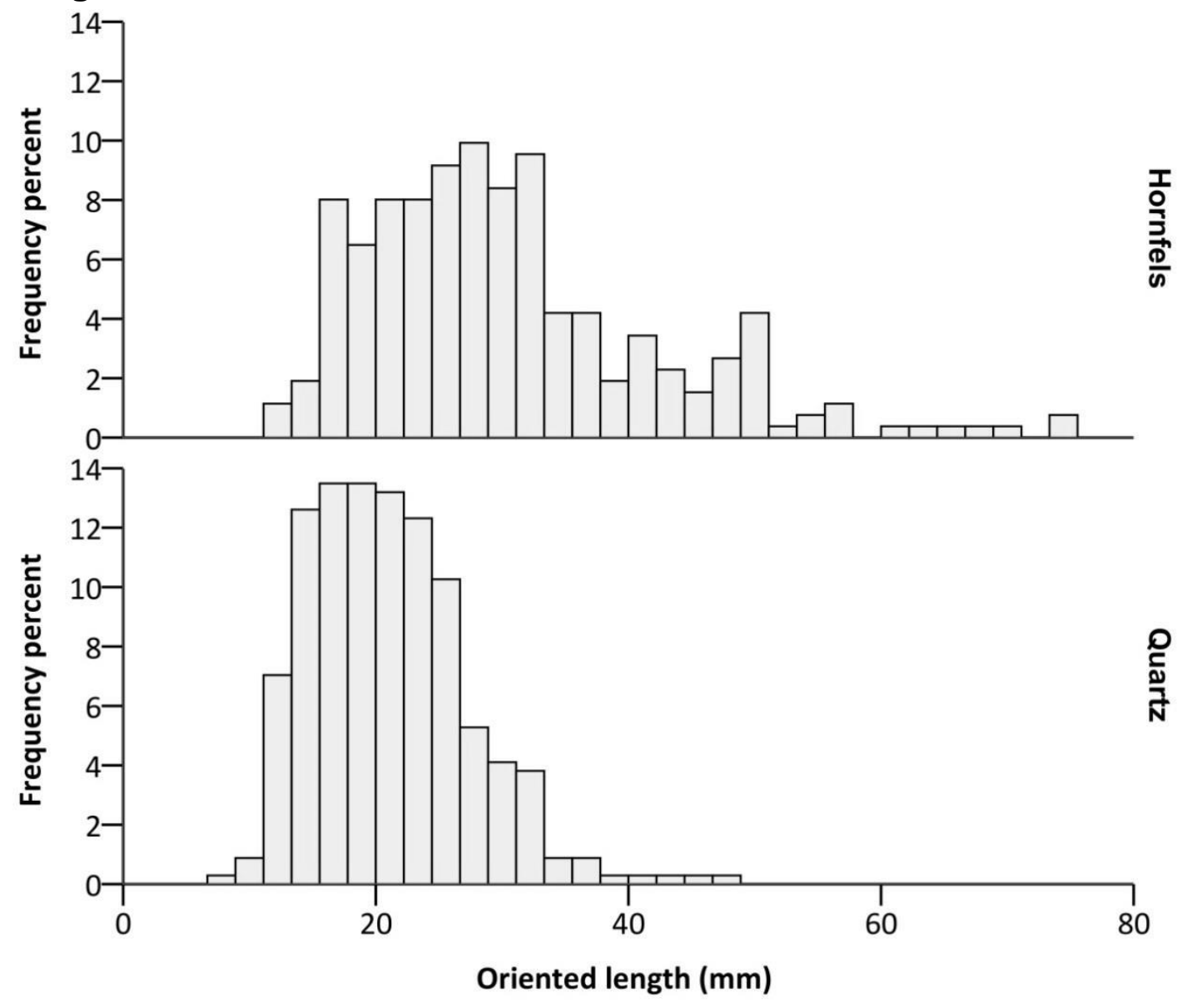

The lack of cortical striking platforms on blades is most likely due to the difficulty of discerning the surface of hornfels, which constitutes the majority of blades. This issue also applies to quartz, explaining the low overall proportions of cortex cover on debitage at the site, with a mean of 3.2\% (Table A.2.9).

Although there are similar mean numbers of dorsal scars on whole blades (2.8) as flakes and flake-blades (2.9), these occur in greater densities on blades (1.1 per $\mathrm{cm}^{2}$, compared to 0.7 for flakes and flake-blades) due to their smaller overall size (Table A.2.10). This could be interpreted as evidence for the removal of blades later in the lithic reduction process, although overall the data is inconclusive. 
Figure 5.2.8: Proportions of debitage striking platform types at Umhlatuzana Striking platform type

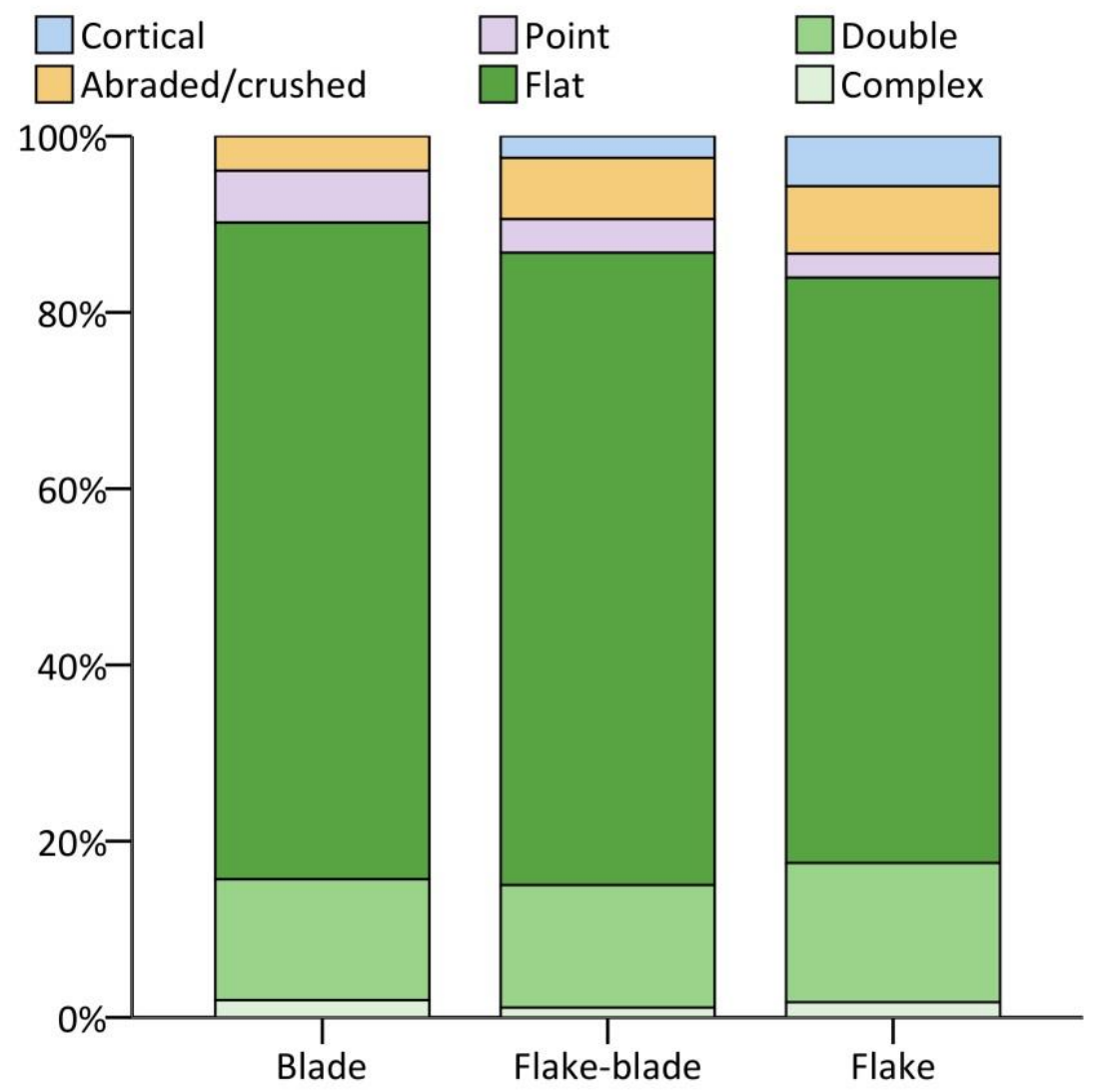

Variation exists in dorsal scar densities when compared between debitage of different raw materials. Quartz debitage exhibits higher densities of dorsal scars (Figure 5.2.9). This is a product of the smaller size of quartz debitage, rather than a result of larger numbers of scars. The differences between raw materials are more pronounced in the case of blades, and least pronounced amongst flakes.

The frequencies of different dorsal scar orientations indicate that scars primarily originate from a single direction (Table 5.2.3). This accords with the conclusions drawn from the core data, where uni-directional cores are dominant. Scars originating from three or four directions are uncommon. Flake-blades form an intermediate category. This is also true with regards to raw material preferences 
and dorsal scar and parallel arrise densities. Overall there is mixed production of flakes and blades at the site, rather than blades being the primary purpose or sole desired end product of lithic reduction.

Figure 5.2.9: Dorsal scar densities on debitage by raw material at Umhlatuzana

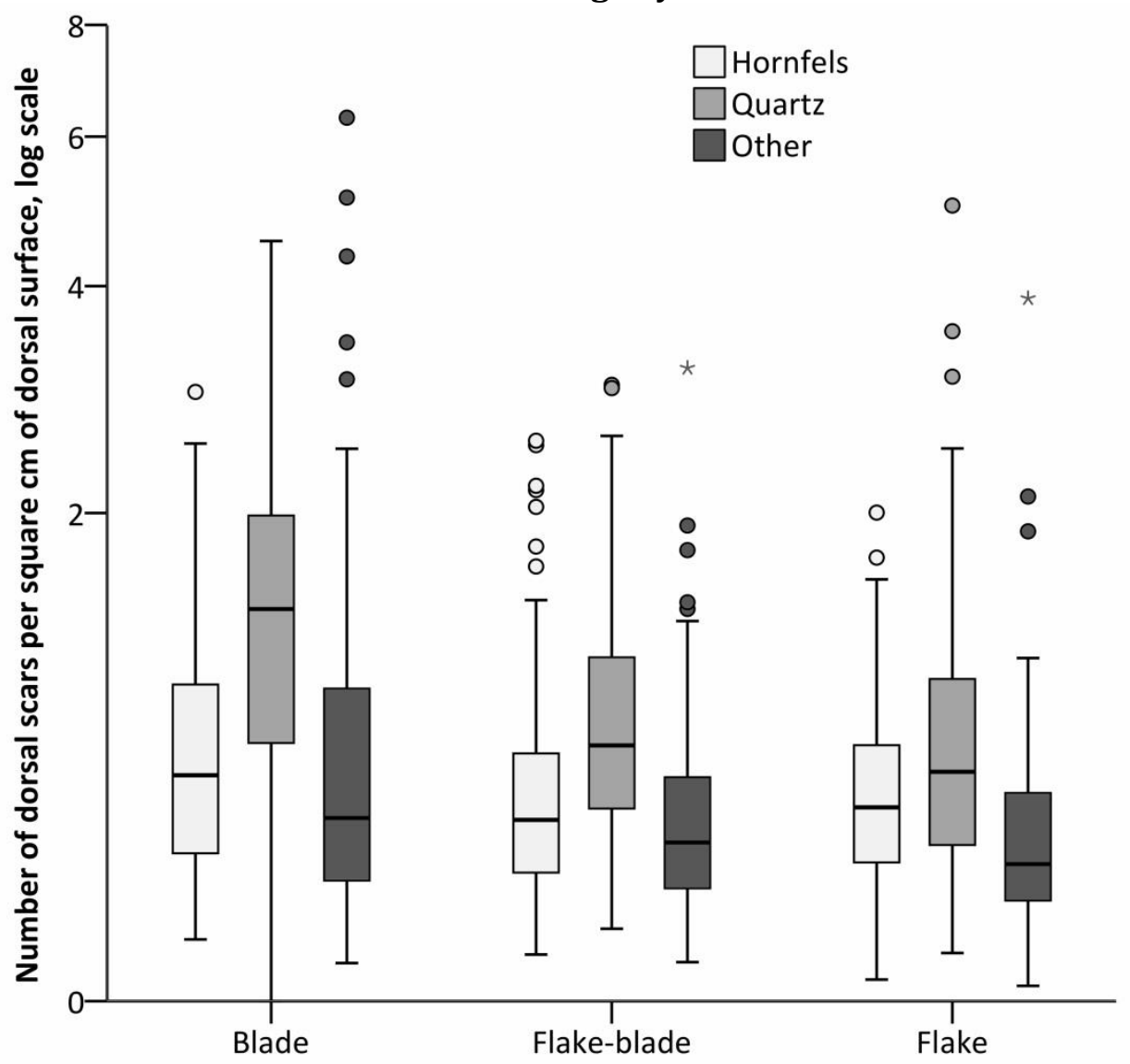

\begin{tabular}{|c|c|c|c|c|c|c|c|c|c|c|c|}
\hline $\begin{array}{l}\text { Dorsal scar } \\
\text { orientation: }\end{array}$ & & 1 & (adj & & (opp & ite) & & & & & \\
\hline Type & No. & $\%$ & No. & $\%$ & No. & $\%$ & No. & $\%$ & No. & $\%$ & Total \\
\hline Blade & 126 & 92.6 & 3 & 2.2 & 7 & 5.1 & & & & & $\overline{136}$ \\
\hline Flake-blade & 292 & 85.1 & 18 & 5.2 & 30 & 8.7 & 3 & 0.9 & & & 343 \\
\hline Flake & 236 & 74.9 & 52 & 16.5 & 22 & 7.0 & 4 & 1.3 & 1 & 0.3 & 315 \\
\hline Total (\% of total) & 654 & 82.4 & 73 & 9.2 & 59 & 7.4 & 7 & 0.9 & 1 & 0.1 & 794 \\
\hline
\end{tabular}

Table 5.2.3: Dorsal scar orientation frequencies on whole debitage at Umhlatuzana 
Feather terminations are the most common termination type (57.9\%). Hinge and step terminations are considerably more common on blades, with flake-blades again an intermediate category (Figure 5.2.10). Roughly a quarter (23.6\%) of quartz flakes and flake-blades have axial or crushed terminations, most likely the result of being removed from cores resting on an anvil during bipolar percussion. Overall, 17.8\% of quartz debitage exhibits convincing evidence of bipolar working, confirming indications of intensive working of quartz at the site through bipolar percussion.

Figure 5.2.10: Proportions of debitage termination types at Umhlatuzana

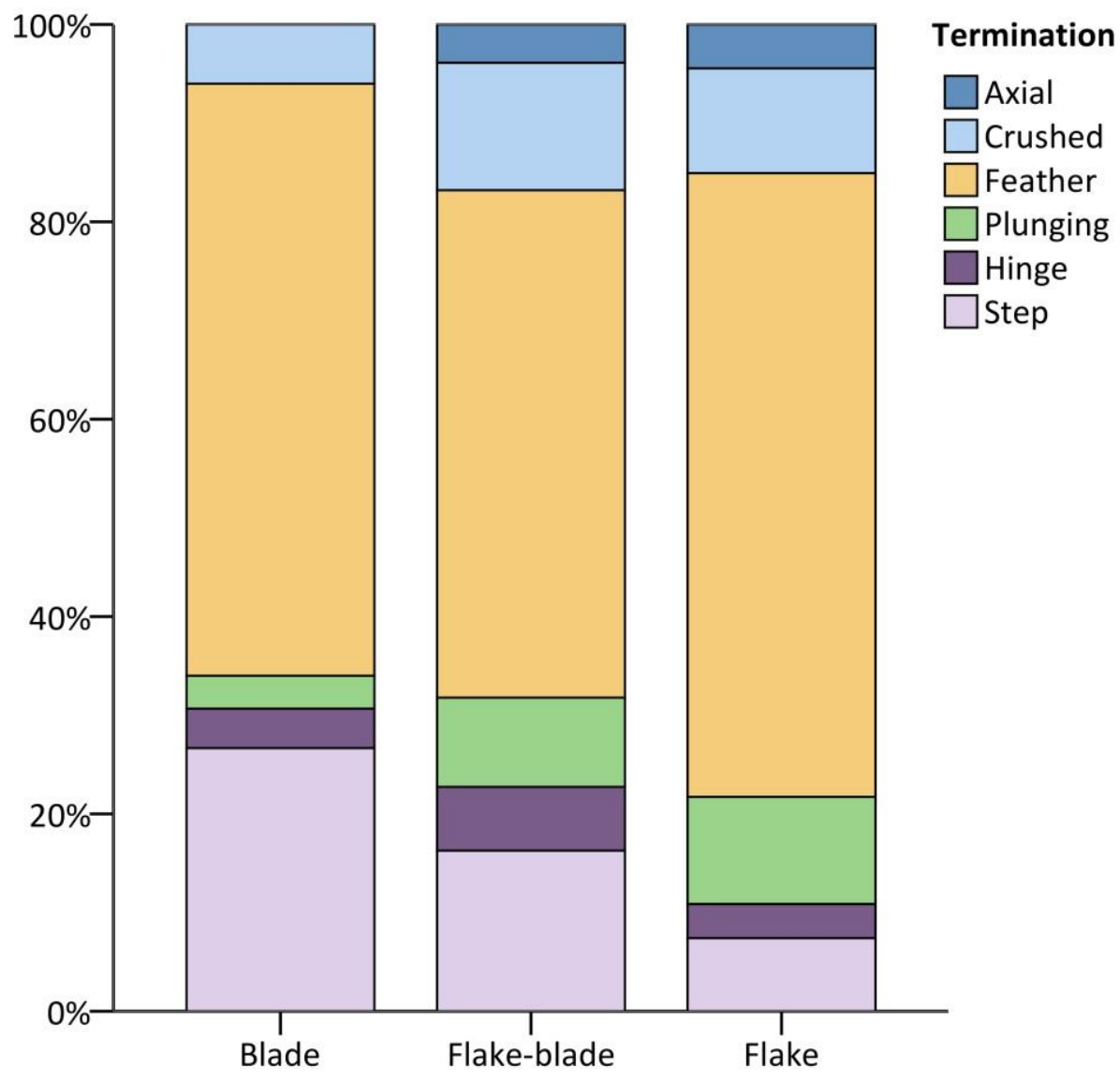

There is minimal evidence for core maintenance in the debitage sample (Table

5.2.4). Crested blades are absent, implying that the 'laminar' method of blade 
production was not utilised at the site. An additional point of note is what appears to be the snapping of blades, and to a lesser extent flakes, of all raw materials (Figure 5.2 .11 ). A total of $13.6 \%$ of the debitage sample exhibits snapped edges that do not appear to be step terminations produced during detachment from a core (Table A.2.11).

\begin{tabular}{l|rr|rrrrr|rr}
\hline & \multicolumn{1}{c}{ Blades } & \multicolumn{3}{c}{ Flake-blades } & \multicolumn{2}{c}{ Flakes } & \multicolumn{3}{c}{ Total } \\
Maintenance flake type & No. & $\mathbf{\%}$ & No. & $\mathbf{\%}$ & No. & \% & No. & \% \\
\hline Platform preparation flake & 3 & 1.3 & 10 & 2.0 & 19 & 4.1 & 32 & 2.7 \\
Core redirection flake & 2 & 0.9 & 2 & 0.4 & 6 & 1.3 & 10 & 0.8 \\
Core rejuvenation flake & & & 1 & 0.2 & 1 & 0.2 & 2 & 0.2 \\
\hline Total & 5 & 2.2 & 13 & 2.6 & 26 & 5.6 & 44 & 3.7 \\
\hline
\end{tabular}

Table 5.2.4: Frequencies of core maintenance debitage at Umhlatuzana

Figure 5.2.11: Examples of 'snapped' hornfels debitage from Umhlatuzana

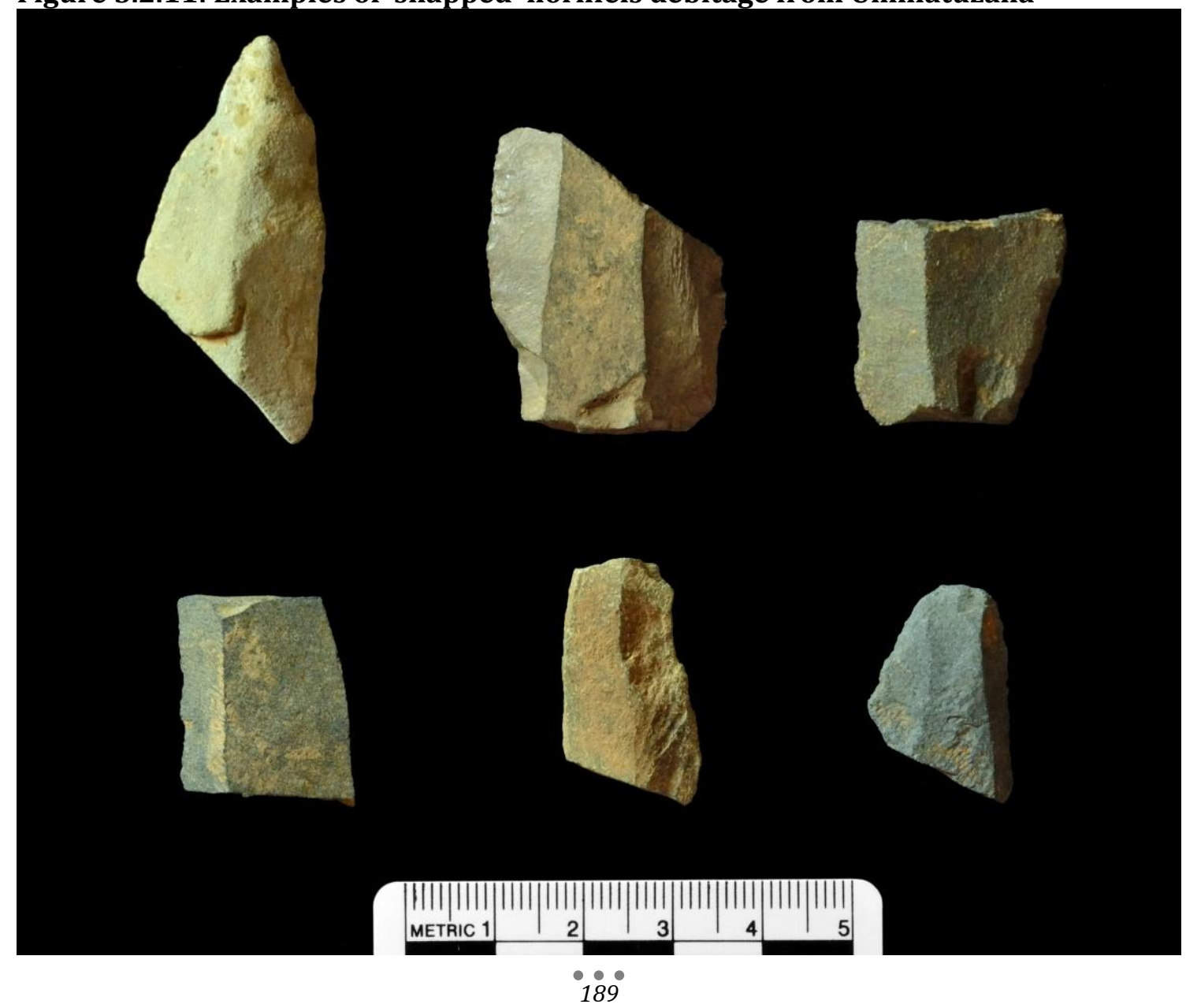


In the absence of detailed microscopy it is impossible to confirm whether these lithics were intentionally snapped, or broken during percussion or peri- or postdepositionally. However, the lithics highlighted here as snapped are those with flat (as opposed to crushed or otherwise clearly damaged) broken edges. Of the snapped pieces, the majority $(77.0 \%)$ are proximal fragments, with most of the remainder being medial fragments (19.9\%). Only five distal snapped fragments are evident (3.1\%). These snapped lithics do not appear to have been removed as part of the microburin technique for the production of microliths, as the snapped edges lack signs of retouch and no typical microburins were found in the debitage sample.

TOOLS

Half of the tool assemblage was manufactured on quartz (49.3\%), and a third on hornfels (34.4\%) (Table 5.2.5). Overall, 51.9\% of microliths and 71.4\% of nonmicrolithic tools were made on blade blanks. Hornfels (and to a lesser extent claystone) was preferred for blade tools, and quartz for flake tools (and quartzite for non-microlithic flake tools) (Figure 5.2.12). Roughly half of tools made on blade blanks (48.1\%) were manufactured on hornfels, compared to $17.8 \%$ of tools produced on flake blanks. For comparison, in the debitage sample, hornfels makes up $42.5 \%$ of the blade assemblage and $26.4 \%$ of the flake assemblage.

In contrast, $76.7 \%$ of tools manufactured on flake blanks were made on quartz, compared to $26.0 \%$ of tools made on blade blanks. In total, $54.8 \%$ of flake debitage is quartz, as is $25.8 \%$ of blade debitage. These raw material differences are 
statistically significant (for blades and blade tools $\chi^{2}(24)=50.597, p=.001$, Cramér's V $=.274$, for flakes and flake tools $\chi^{2}(26)=87.539, \mathrm{p}<.001$, Cramér's V = .205). As such, there is clear evidence for the selection of hornfels blades and quartz flakes as tool blanks. Additionally, higher qualities of quartz were reserved for the manufacture of microliths, with quartzite used almost exclusively for nonmicrolithic tools.

\begin{tabular}{lrrrrrrr}
\hline Raw material & $\begin{array}{r}\text { Microliths } \\
\text { on blades }\end{array}$ & $\begin{array}{r}\text { Microliths } \\
\text { on flakes }\end{array}$ & $\begin{array}{r}\text { Ret. } \\
\text { blades }\end{array}$ & $\begin{array}{r}\text { Ret. } \\
\text { flakes }\end{array}$ & Biface & Total & \% \\
\hline Hornfels & 44 & 15 & 6 & 1 & 1 & 67 & 34.4 \\
Milky quartz & 14 & 31 & & & & 45 & 23.1 \\
Impure clear quartz & 4 & 17 & & & & 21 & 10.8 \\
Clear quartz & 6 & 8 & & & & 14 & 7.2 \\
Opaque quartz & 1 & 13 & & & & 14 & 7.2 \\
Claystone & 10 & 2 & 1 & & & 13 & 6.7 \\
Jasper & 9 & 1 & 1 & & & 11 & 5.6 \\
Quartzite & 3 & & 1 & 2 & & 6 & 3.1 \\
Chalcedony & 2 & & & & & 2 & 1.0 \\
Granular quartz & 1 & & 1 & & & 2 & 1.0 \\
\hline Total & 94 & 87 & 10 & 3 & 1 & 195 & \\
\hline
\end{tabular}

Table 5.2.5: Tools measured at Umhlatuzana

Mean microlith size measurements are 22.2 x 11.8 x 3.9 mm (Table A.2.12).

Differences can be observed between raw materials. Hornfels microliths tend to be made on blade blanks, to be larger on average, and to have a wide range of sizes. These blade blanks were selected from a wide range of the size array of potential hornfels blanks (Figure 5.2.13). Hornfels microliths range up to $53 \mathrm{~mm}$, but even at this size are retouched using the same methods, to the same extent and into the same shapes as smaller examples. Large maximum sizes of specific microlith morphologies are also observed at the site of Klasies River (Villa et al. 2010). 
Indeed, the original excavators of the HP type site noted that "[t]he special type of large crescent often reaching a length of two inches... forms a noticeable feature of the industry" (Stapleton and Hewitt 1928: 407 [original emphasis]).

Figure 5.2.12: Raw material proportions of tools at Umhlatuzana

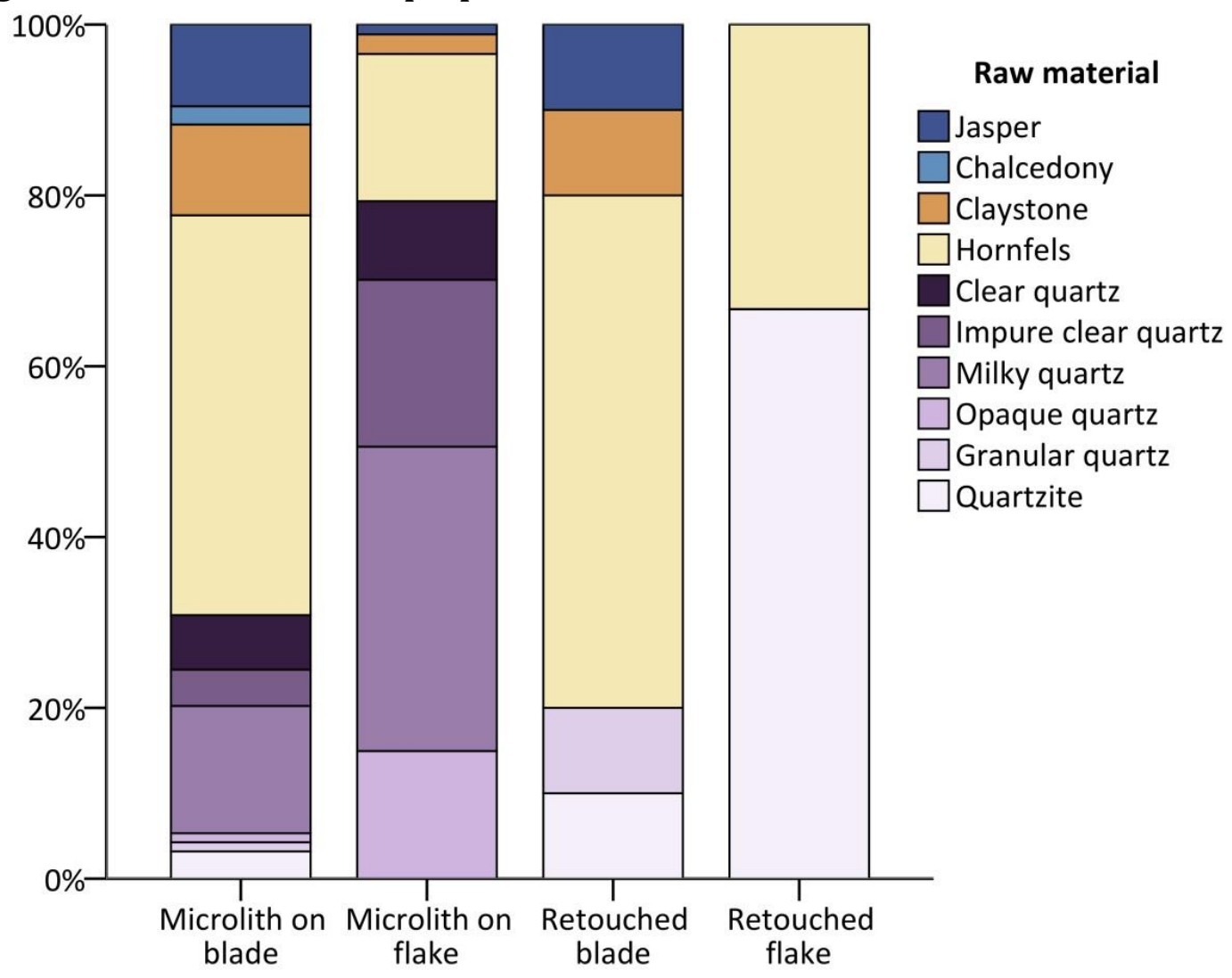

Conversely, quartz microliths tend to be made on flake blanks, to be smaller on average, and, as Figure 5.2.13 reveals, were preferentially made on smaller flake sizes within the range of potential blank sizes. One conclusion is that there was reduced pressure on the size of haftable microliths made on hornfels, possibly because a range of sizes was useful for the task/s for which they were employed, or for the configuration in which they were hafted. Microliths made on quartz may have been used for a different purpose or hafted in a different way, for which a particular size was most useful. 
Figure 5.2.13: Lengths of hornfels and quartz debitage and microliths at Umhlatuzana. All microliths are included, plus whole debitage.

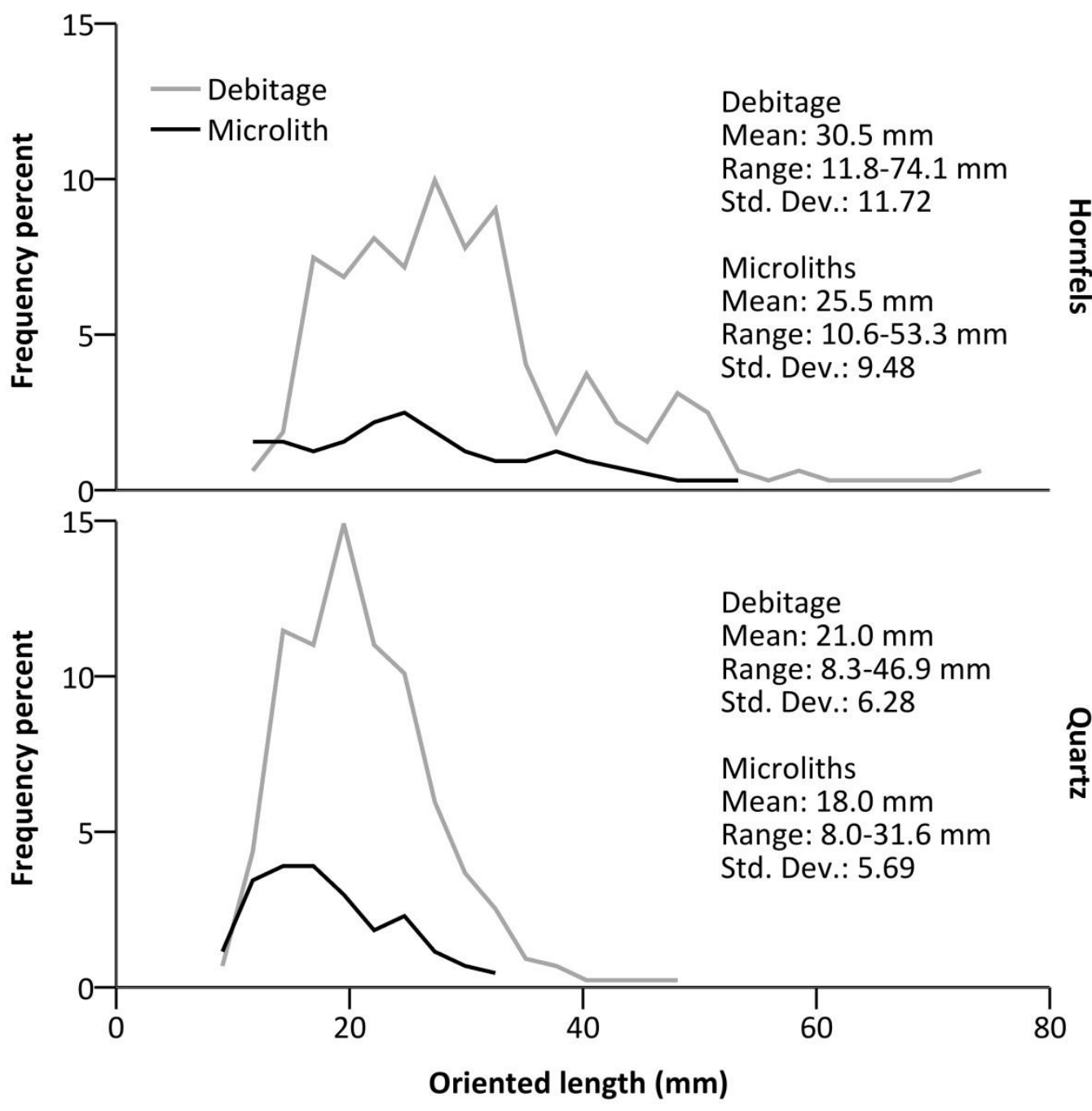

Figure 5.2.14 reveals the size profiles of microlithic and non-microlithic tools compared to debitage of different raw materials. There are no clear groupings of materials. However, non-microlithic tools are considerably and distinctly larger than the microlithic tool assemblage, with little overlap (see also Table A.2.12, Table A.2.13). Blanks for retouch into either tool type were selected from the general debitage population, with microliths selected from smaller sizes and nonmicrolithic tools from larger sizes. Although non-microlithic tools exist only in small numbers at the site, they do appear to have been one of the intended 
outcomes of lithic reduction trajectories, rather than manufactured as the result of an opportunistic process.

Figure 5.2.14: Cubic volumes of lithics on different raw materials at Umhlatuzana. High-quality quartz = clear, impure clear and coloured. Low-quality quartz = milky, opaque and granular.

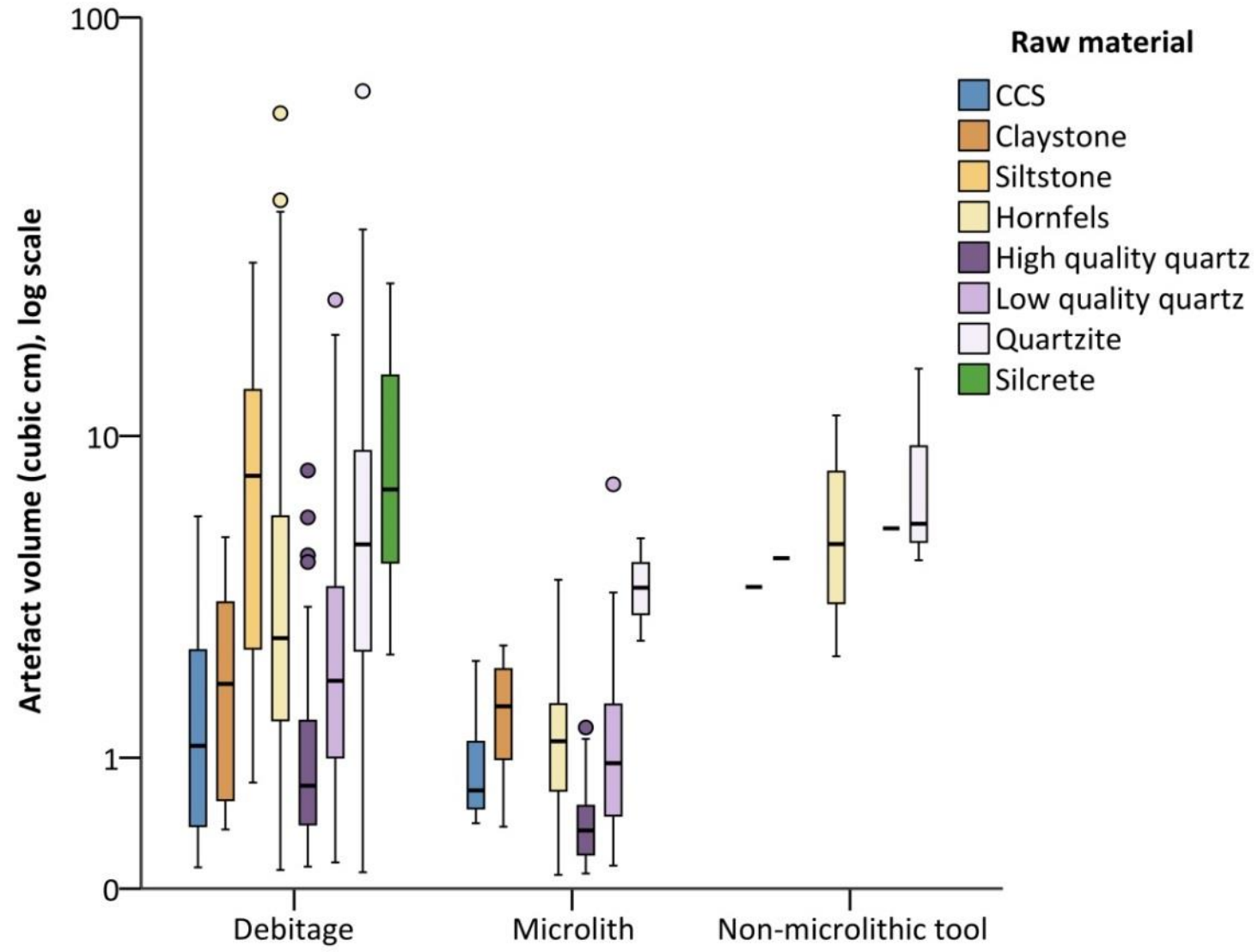

Non-geometric microliths are more common than geometric forms $(61.3 \% \mathrm{v}$.

38.7\%) (Table 5.2.6). The most common forms overall are backed non-geometric points (40.9\%). Crescent microliths are the most common geometric form and the second most common morphology overall, making up $29.8 \%$ of the total microlith assemblage. Trapeze, triangle and rectangle morphologies are also present, as are tanged and shouldered forms. 


\begin{tabular}{llr}
\hline Coarse typology & Narrow typology & Frequency \\
\hline Microliths: & & 20 \\
Backed non-geometric microlith & & \\
Backed non-geometric microlith point & Backed & 71 \\
& Concave point ('awl') & 1 \\
& Double backed & 2
\end{tabular}

$\begin{array}{lr}\text { Crescent microlith } & 54\end{array}$

Non-geometric microlith $\quad$ Other 2

Side retouched 1

$\begin{array}{lr}\text { Non-geometric microlith point } & 8\end{array}$

$\begin{array}{lll}\text { Rectangle microlith } & \text { Backed } & 1\end{array}$

Double backed 1

Shouldered/tanged microlith $\quad$ point 5

Shouldered point 1

$\begin{array}{lr}\text { Trapeze microlith } & 9\end{array}$

Triangle microlith $\quad 5$

$\begin{array}{ll}\text { Total } & 181\end{array}$

Non-microlithic tools:

$\begin{array}{lll}\text { Burin } & \text { Side retouched with burin } & 1\end{array}$

$\begin{array}{lll}\text { Notched/denticulated } & \text { Denticulated } & 1\end{array}$

Side retouched with notches $\quad 1$

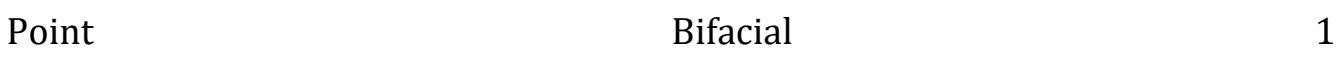

Side retouched 2

$\begin{array}{lll}\text { Side retouched } & \text { Double side retouched } & 6\end{array}$

Side retouched 1

$\begin{array}{lr}\text { Unifacial point } & 1\end{array}$

$\begin{array}{lr}\text { Total } & 14\end{array}$

Table 5.2.6: Tool type frequencies at Umhlatuzana 
In addition to size variations between hornfels and quartz microliths, there are also morphological differences (Figure 5.2.15, Figure 5.2.16). Hornfels was most frequently used for the manufacture of backed non-geometric points $(54.2 \%$ of hornfels microliths v. 31.6\% of quartz), and quartz was more frequently used for geometric microliths, especially crescents (38.9\% of quartz microliths v. $16.9 \%$ of hornfels) as well as trapezes and triangles. Wadley and Mohapi (2008) note a similar pattern in raw material use at the nearby site of Sibudu Cave, i.e. that there are discrete microlithic size categories for separate rock types, including quartz and hornfels, and that these may also have had different functions.

Figure 5.2.15: Raw material proportions of microlith types at Umhlatuzana

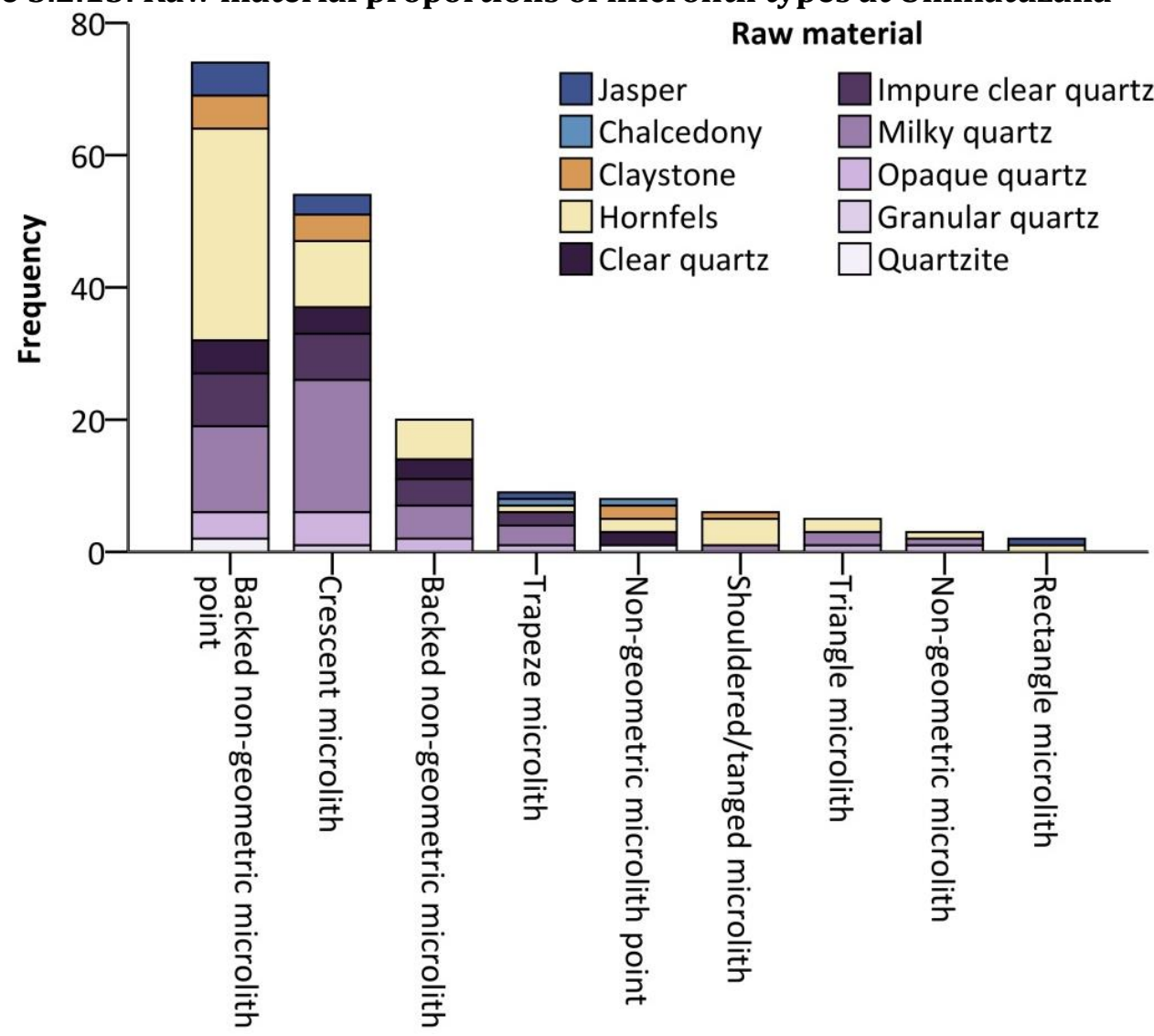


Figure 5.2.16: Examples of hornfels and siltstone (top) and quartz (bottom) microliths from Umhlatuzana. Of particular note are the size differences between crescent forms made on hornfels $(1,3,4,6,8,11,12)$ and quartz $(14-16,18-20,22)$.

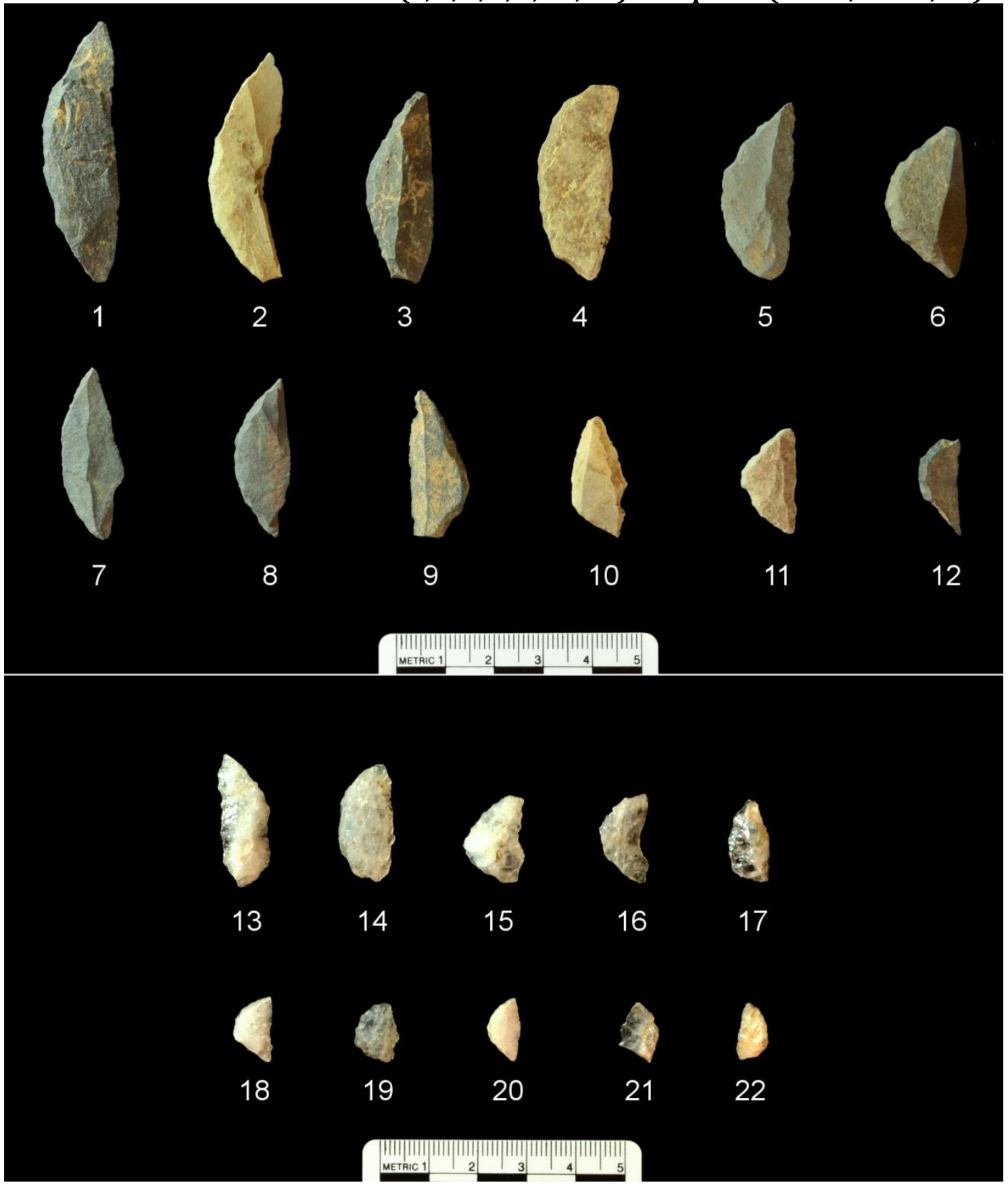

The non-microlithic tool sample is small but contains a variety of forms, including a burin, a denticulated piece and a notched piece (Table 5.2.6). The assemblage also contains a unifacial point and a bifacial point, considered typical of MSA 
assemblages (previously noted by Kaplan 1990, Lombard et al. 2010). Hornfels is dominant in the non-microlithic tool assemblage (Figure 5.2.17).

Figure 5.2.17: Raw material proportions of non-microlithic tool types at Umhlatuzana

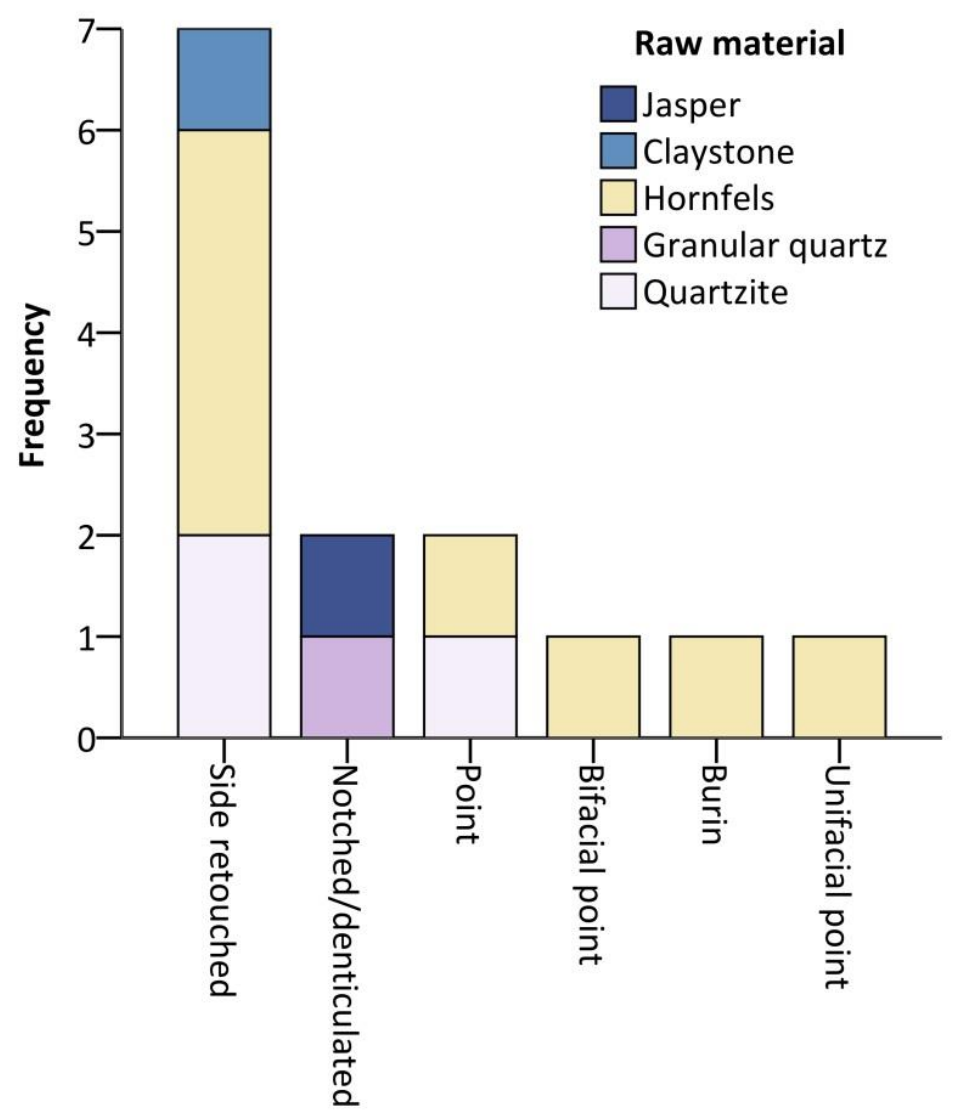

There are no major size differences between different microlith types (Figure 5.2.18, Table A.2.14). Additionally, there is no convincing evidence for standardisation in the size measurements of any microlith typologies. There is, however, substantive size variation within typological categories. An extreme example can be seen in crescent microliths, which range in length between $9.8 \mathrm{~mm}$ and $53.3 \mathrm{~mm}$. 
Figure 5.2.18: Cubic volumes of microlith types at Umhlatuzana

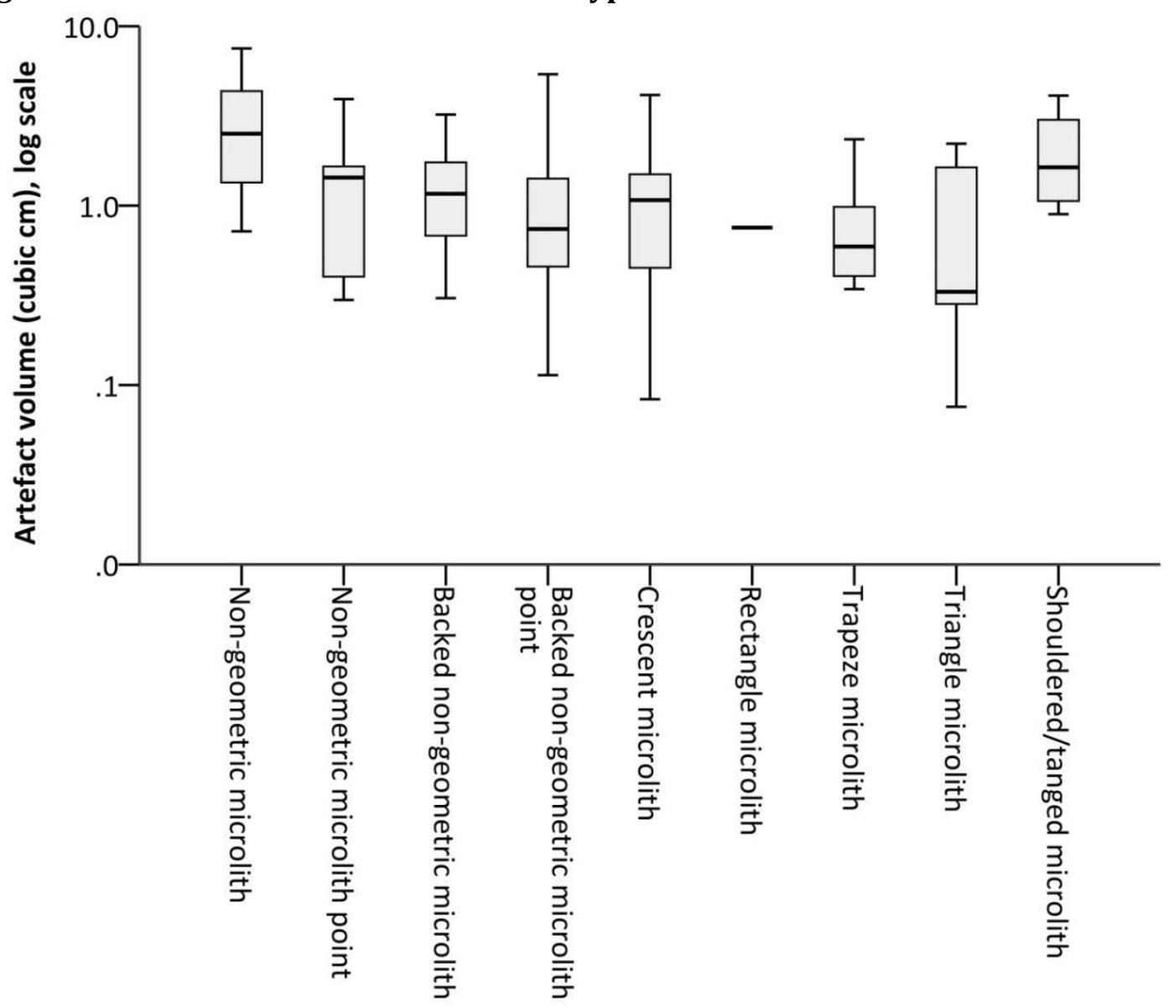

With the exception of non-geometric microliths which are neither backed nor pointed (a small and skewed sample), the TCSA measurements of all microlith types are smaller than the arrowhead sample reported by Shea (2006), with mean values ranging from 19.9-32.6 $\mathrm{mm}^{2}$ (Table A.2.15). However, there is no evidence for the standardisation of any microlith typology with regards to TCSA. There is very little overlap between the TCSA measurements of microliths and nonmicrolithic points. Values for the latter (with a mean of $78.2 \mathrm{~mm}^{2}$ ) would be considered within the range of hand-cast or thrusting spears rather than projectile points. 
Within the category of crescent microliths there exist interesting differences between those manufactured on hornfels and on quartz. As discussed above, hornfels microliths are larger on average. However, while hornfels crescent microliths are considerably longer on average (33.1 mm v. $19.3 \mathrm{~mm}$ ), the difference between mean widths is less pronounced $(12.5 \mathrm{~mm}$ v. $9.1 \mathrm{~mm})$. Additionally, there are no statistically significant differences in their thicknesses or TCSA measurements (Table A.2.16). While hornfels crescent microliths were longer and slightly wider on average than those manufactured on quartz, the crosssection of the tool that would have penetrated a target if they were used as projectiles - assuming they were not mounted transversely, which would seem unlikely given the considerable lengths of some artefacts - was kept to a similar size regardless of raw material.

Obvious shaping of tools to facilitate hafting is rare. No non-microlithic tools exhibit tangs or shoulders. Six microliths are shouldered, comprising 3.3\% of the microlithic tool assemblage. Mean haft dimensions are small $(7.6 \times 3.6 \times 9.0 \mathrm{~mm})$ and do not appear to be standardised (Table A.2.17). Overall, the construction of shoulders to facilitate hafting does not appear to have constituted a major part of stone tool production processes at the site.

Backing is the most common retouch type on microliths (87.2\%) (Figure 5.2.19). Backing is almost ubiquitous on geometric microliths, with 67 out of 70 geometric microliths (95.7\%) exhibiting it. On non-geometric microliths that do not exhibit backing a mix of nibbling, scalar, stepped and sub-parallel retouch was used. A variety of retouch types were also used on non-microlithic tools (Figure 5.2.20), 
with scalar and stepped retouch being most common $(31.8 \%$ and $27.3 \%$, respectively). However, different repertoires of retouch types were used on different tool types, with abrasion, discontinuous and nibbling retouch found only on microliths, and irregular and denticulated retouch as well as burination and the creation of notches found only on non-microlithic tools. Additionally, backing is found on only one non-microlithic tool.

Figure 5.2.19: Retouch techniques on microliths at Umhlatuzana

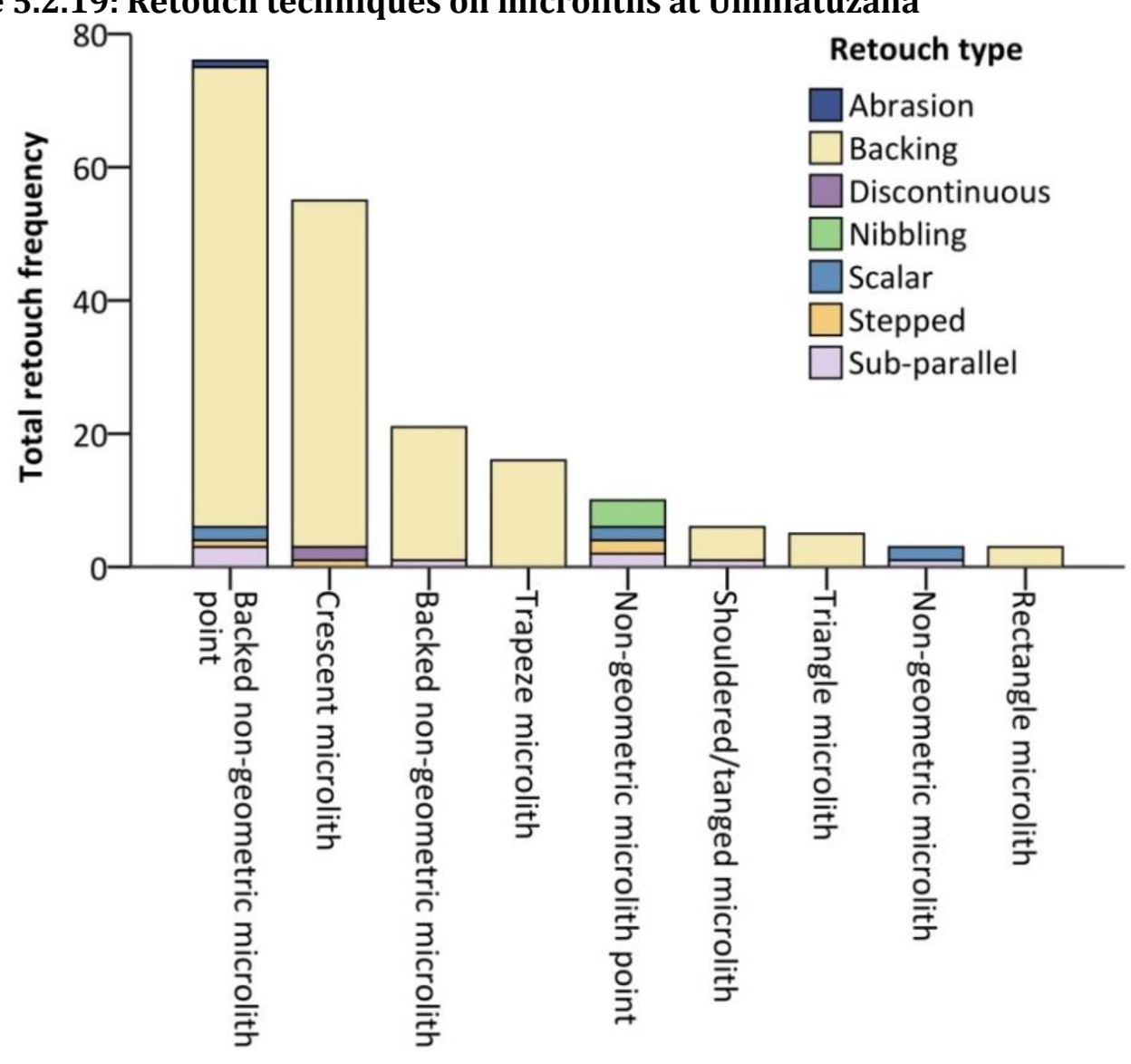

Half of non-microlithic tools (50.0\%) and almost all microliths (92.3\%) are worked along a single edge, primarily along a lateral margin $(82.1 \%$ of microliths and $81.8 \%$ of non-microlithic tools) (Table A.2.18). There is more variation in retouch 
characteristics in the non-microlithic tool assemblage, for example with one retouched blade being worked in three separate locations.

\section{Figure 5.2.20: Retouch techniques on non-microlithic tools at Umhlatuzana}

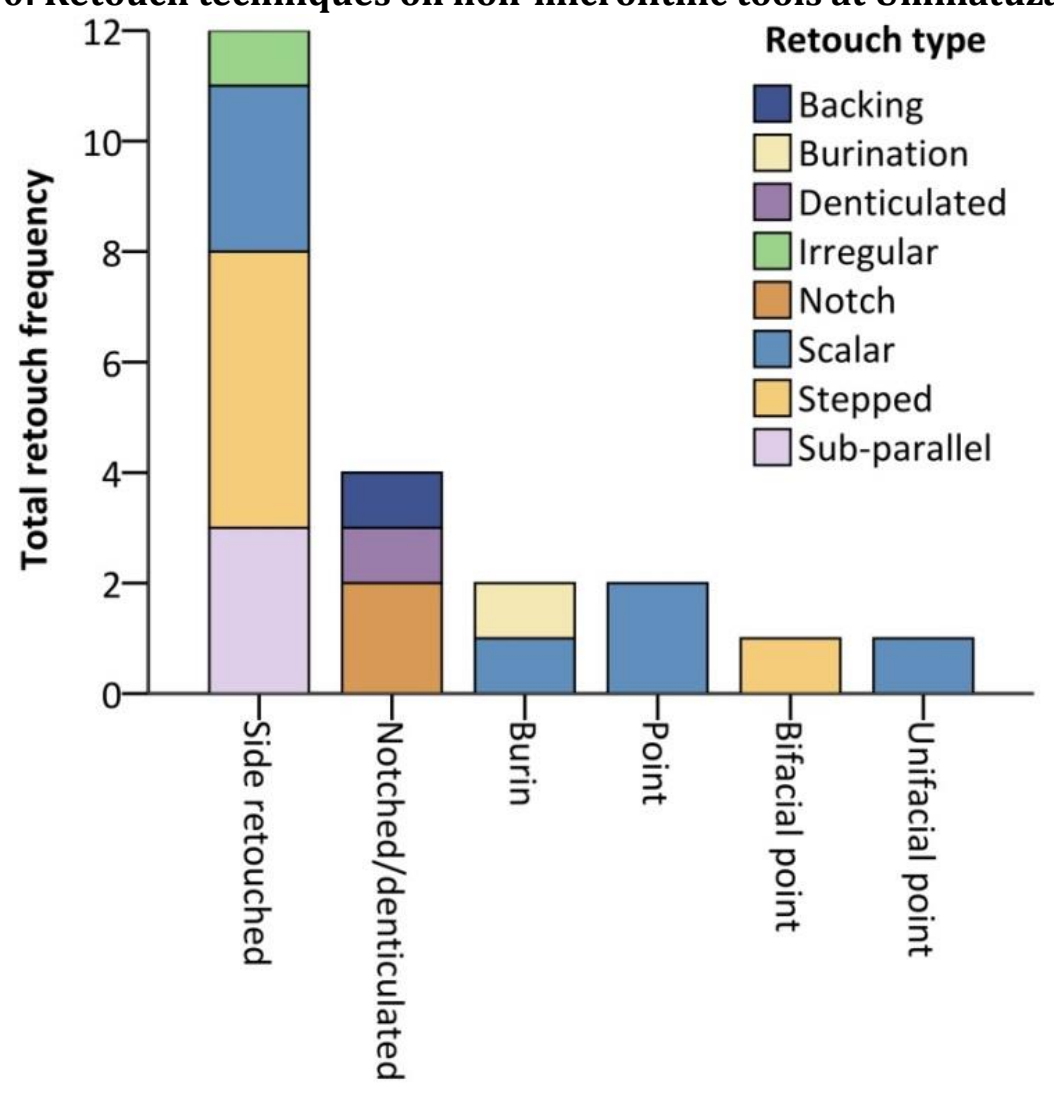

A variety of retouched edge outlines are evident on both microlithic and nonmicrolithic tools. The majority of retouch on non-microlithic tools was made on dorsal faces (72.7\%) (Table A.2.18). In the microlithic tool assemblage edge retouch is more common (59.0\%). This is indicative of the use of the bipolar backing technique, which constitutes $67.6 \%$ of the backing retouch on microliths. It is not associated with any particular raw material, however $\left(\chi^{2}(10)=16.934, p=\right.$ .076). Overall, backing retouch varies between $60^{\circ}$ and $90^{\circ}$, with a mean of $78^{\circ}$ (Table A.2.19). The prevalence of backing retouch in the microlith assemblage also explains the strong negative skew of microlith retouch angles compared to those of 
non-microlithic tools (Figure 5.2.21). Overall, there is little standardisation evident in retouch length, depth or angle (Table A.2.19).

Figure 5.2.21: Frequency distributions of average retouched edge angles on tools at Umhlatuzana

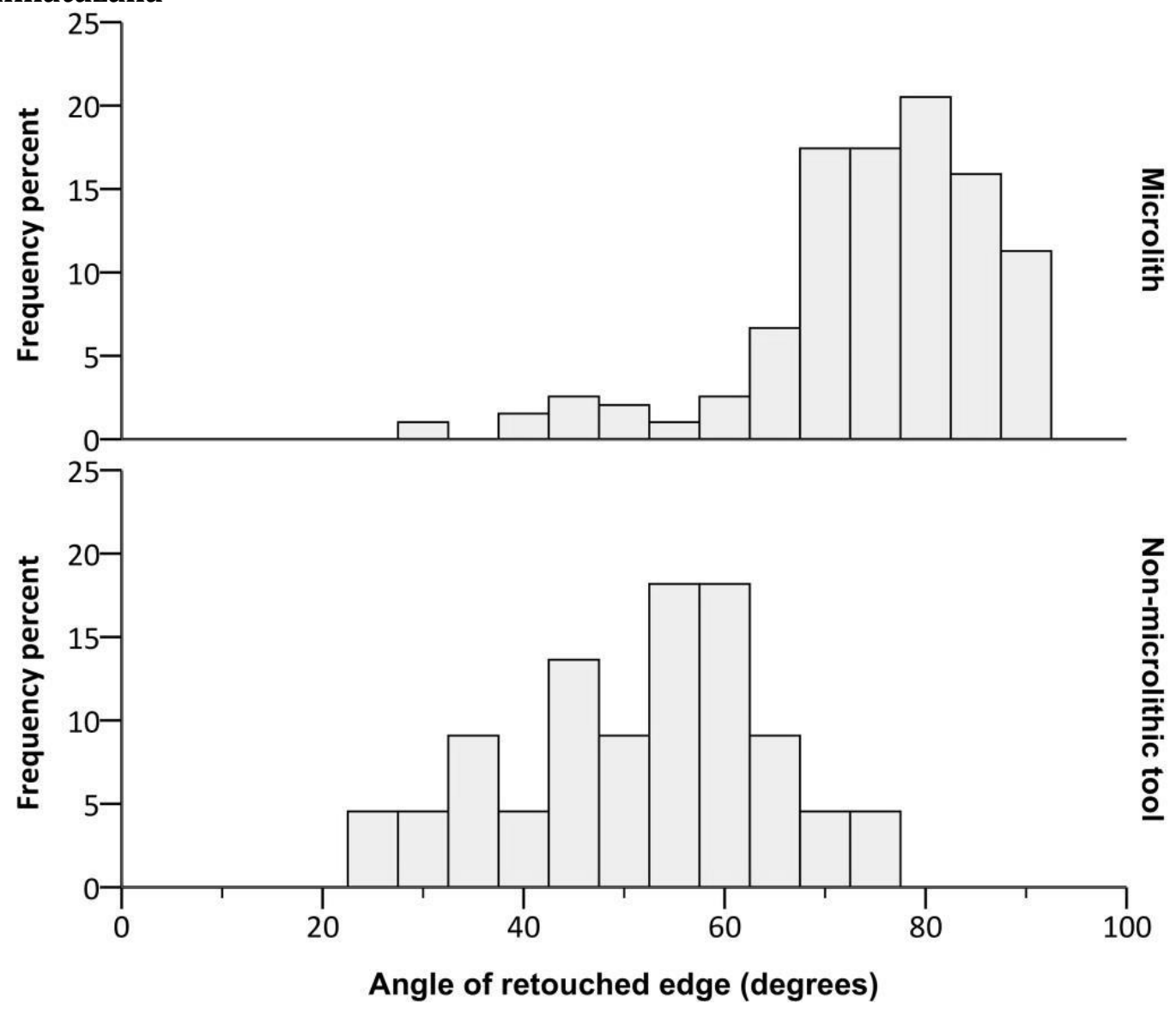

There is also little variation in the mean IOI values for microliths, with a maximum value of 0.313 (Table A.2.20). Non-microlithic tools are more intensively retouched on average. However, no tool exhibits retouch over more than half of its surface area. The most extensively retouched tool - a bifacial point - has an IOI value of 0.438. Examples of microliths from each layer are illustrated in Figure 5.2.22. 
Figure 5.2.22: Microliths from Umhlatuzana Layer 23 (1-24), 25 (25-45) and 27 (4669), demonstrating the range of sizes and forms present. 1, 25-28, 46: jasper. 2, 2932, 47-49: claystone. 3-12, 34-41, 50-57: hornfels. 13-24, 42-45, 58-69: quartz. 33: quartzite. Examples of geometric forms include crescents $(6,13,15,18-21,23-25$, $30,31,34,35,37,38,40,43,45,46,52,55,56,64,69)$ and trapezes $(28,29,42,44$, 61-63). Of particular note are the shouldered points $(5,39,50)$ and the concave point or awl (65).

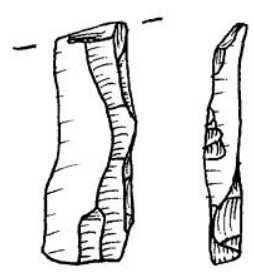

1

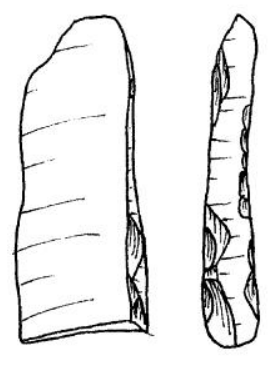

4

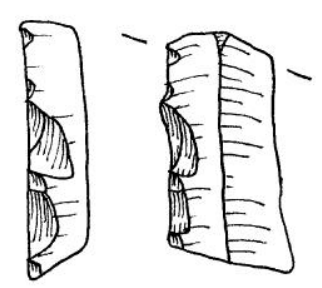

7

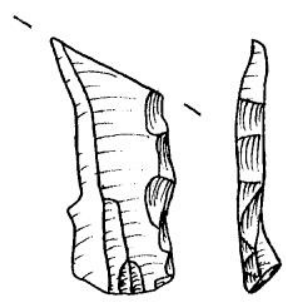

10

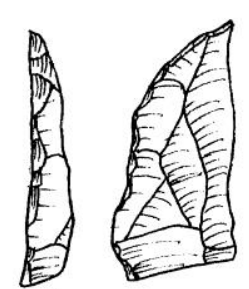

2

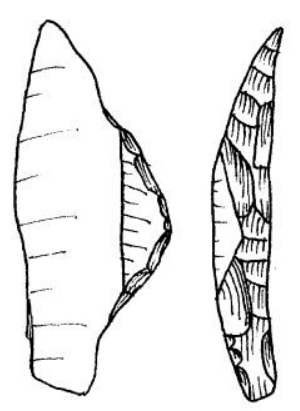

5

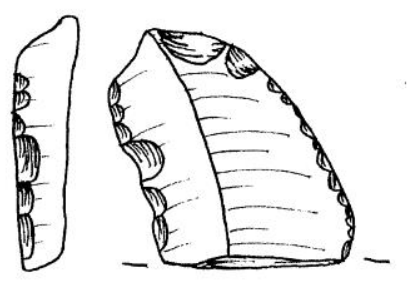

8

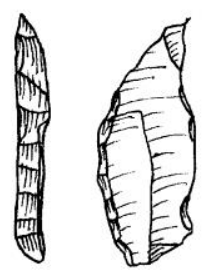

11

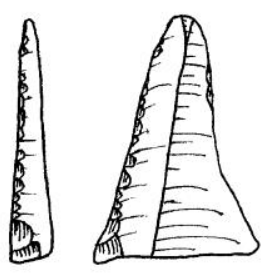

3

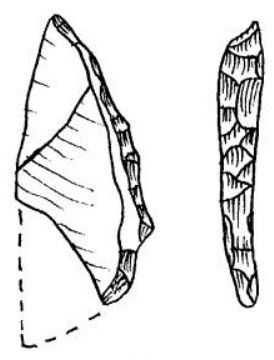

6
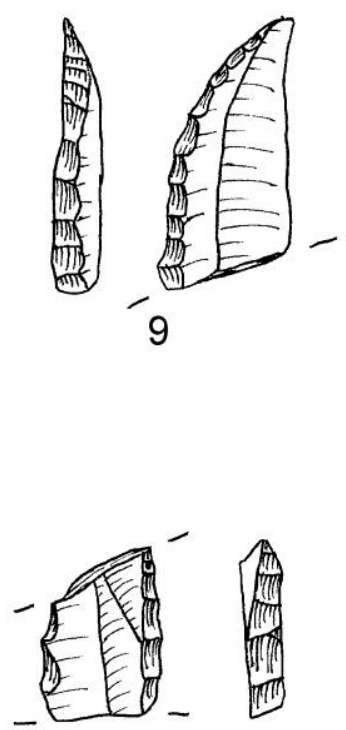

12

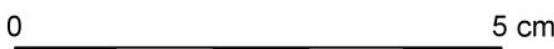




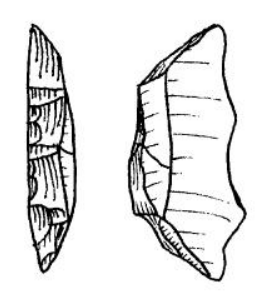

13

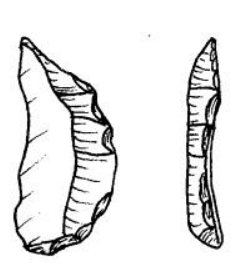

14

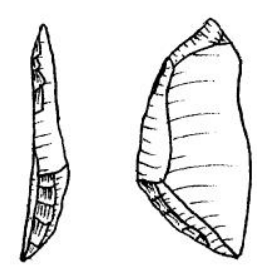

15

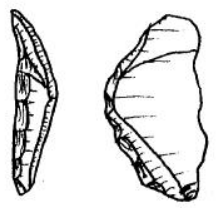

16

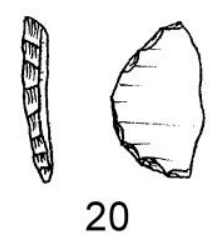

E

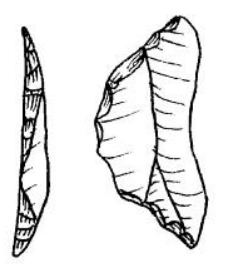

25

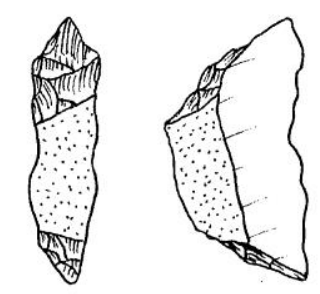

28
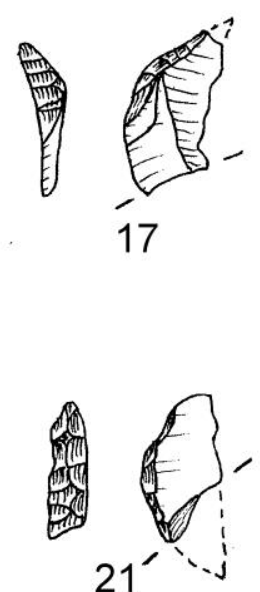

起

19
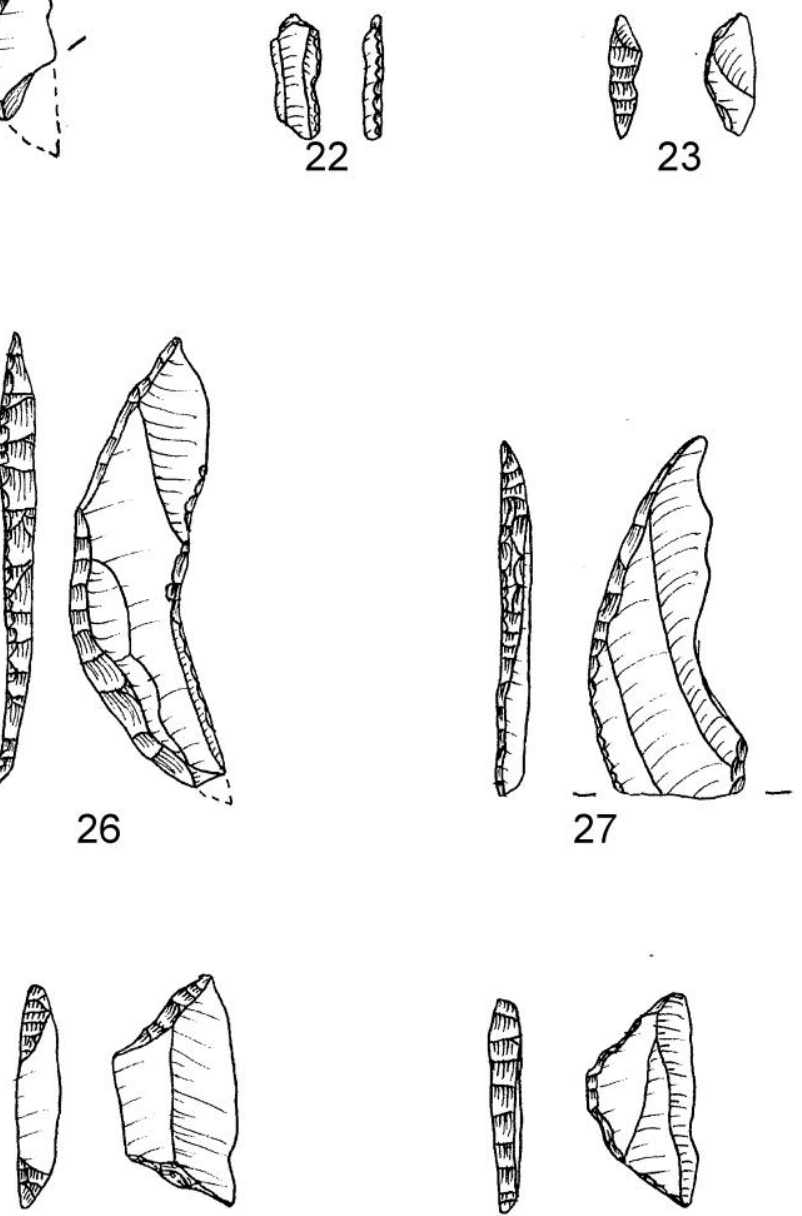

29

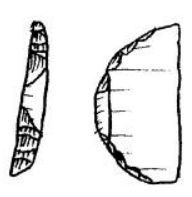

18
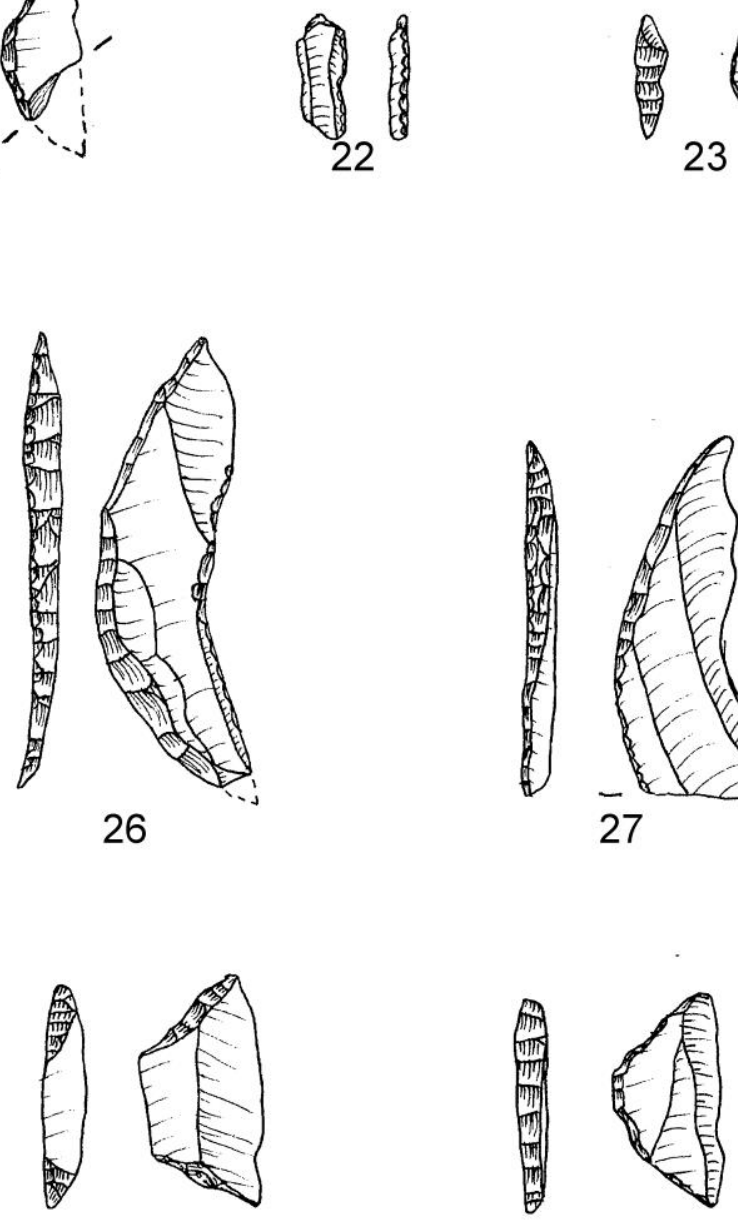

30 


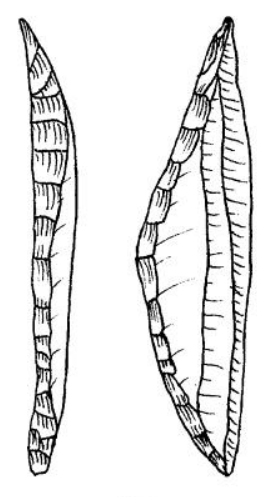

31

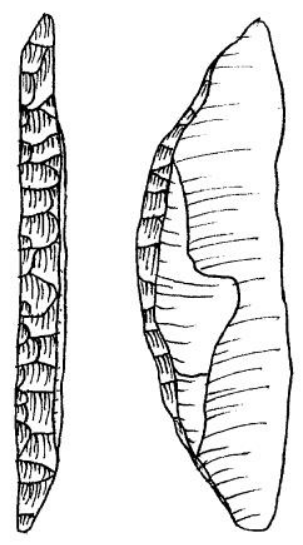

34

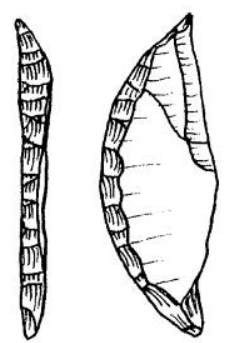

37
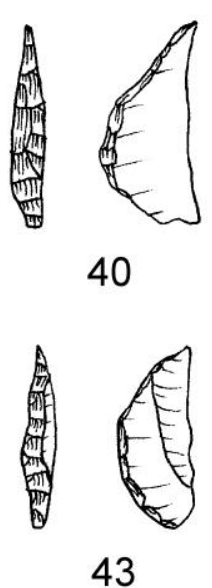
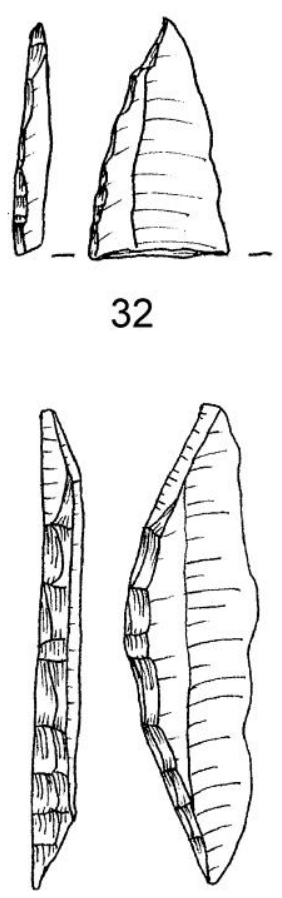

35

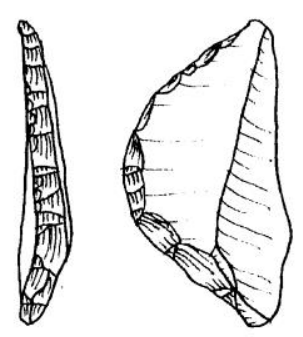

38
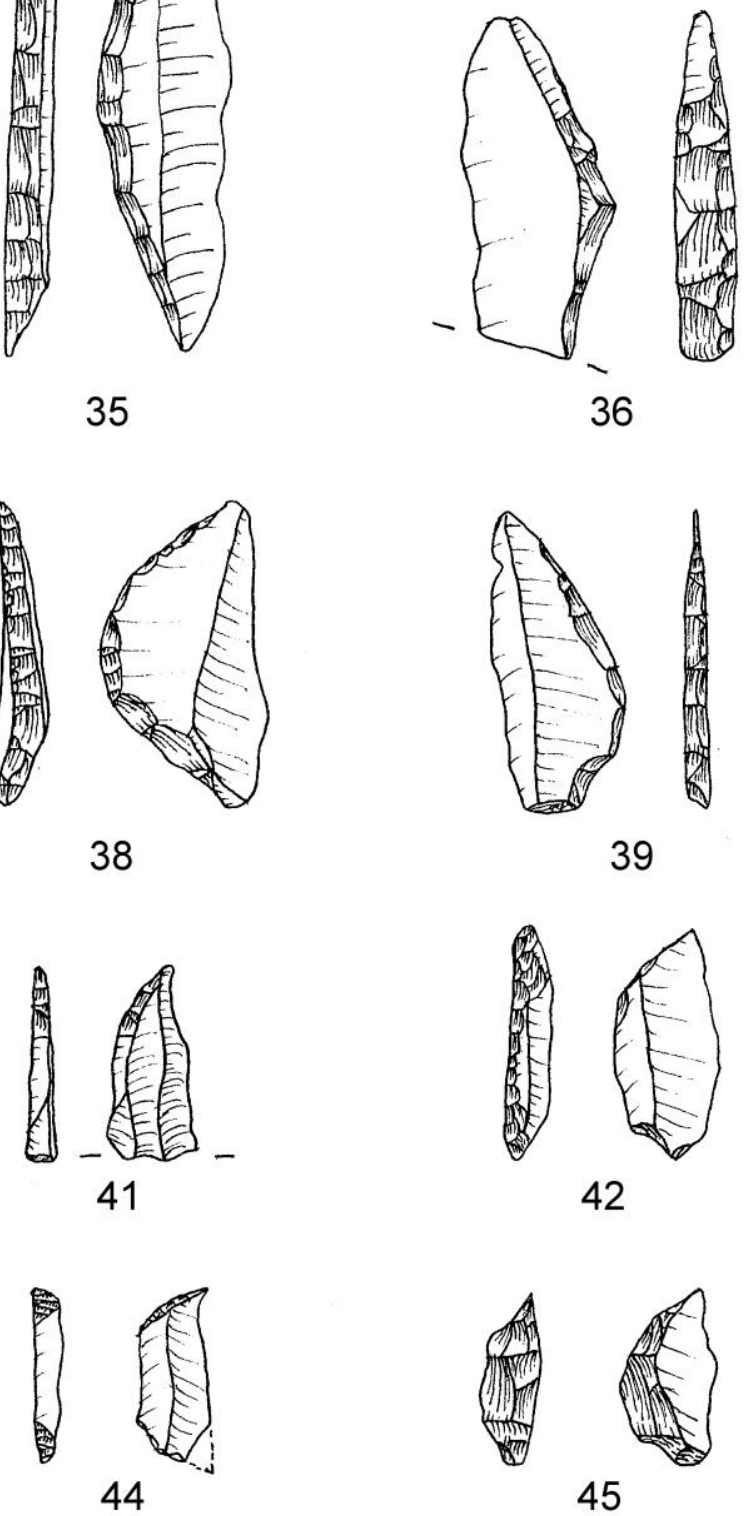


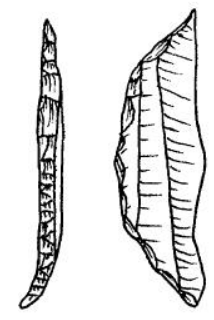

46

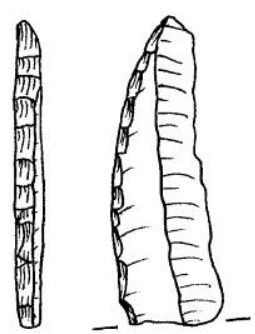

49

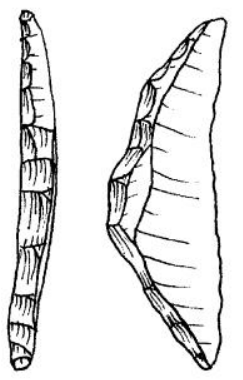

52

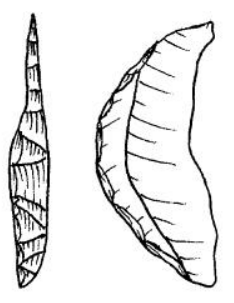

55

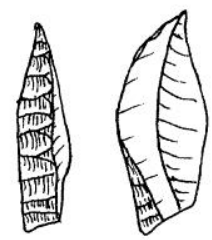

59

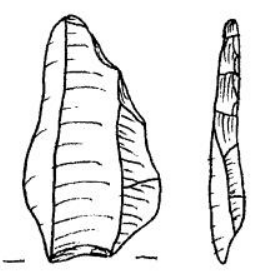

47
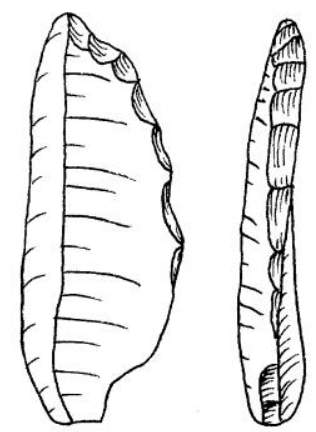

50

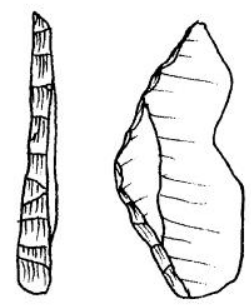

53
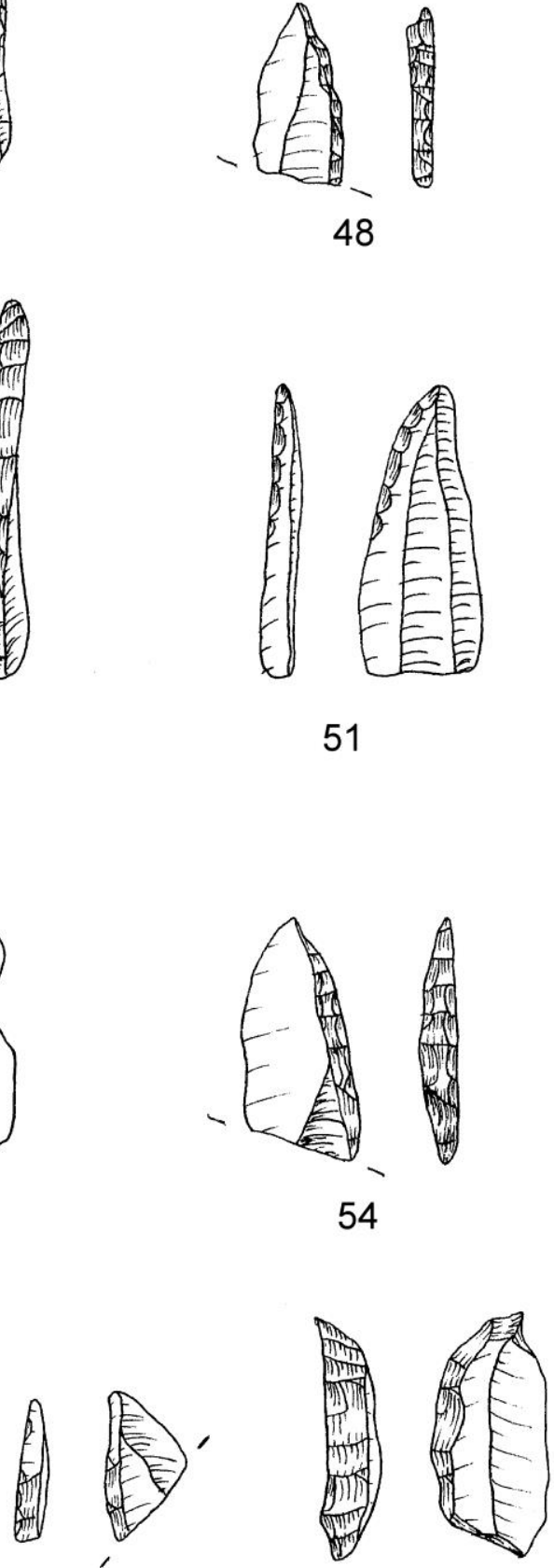

57

58

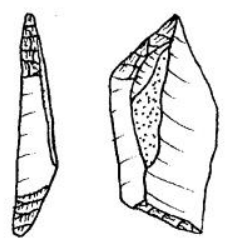

61

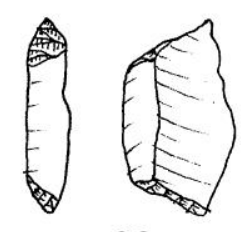

62 


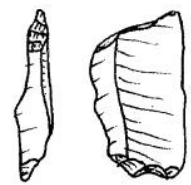

63

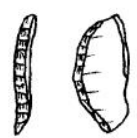

67

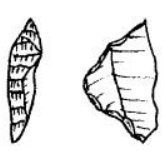

64

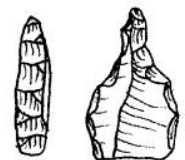

65

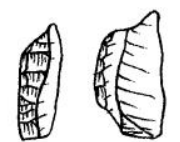

66

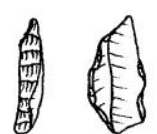

68

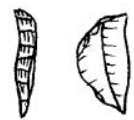

69

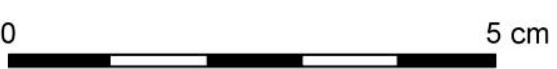

TEMPORAL VARIATION

Results of statistical tests at Umhlatuzana are presented in Appendix B.2. The small number of non-microlithic tools precludes comparisons between layers (Table 5.2.7).

\begin{tabular}{lrrr}
\hline Layer & Cores & Microliths & Non-microlithic tools \\
\hline 23 & 48 & 23 & 2 \\
25 & 103 & 104 & 6 \\
27 & 11 & 54 & 6
\end{tabular}

Table 5.2.7: Cores and tools analysed in each layer at Umhlatuzana

\section{Cores}

Results of statistical tests on cores are reported in Table B.2.1 and summarised in Table 5.2.8. Few variables demonstrate consistent temporal trends. The only potentially revealing trend is the decrease in the proportion of radial cores and increase in amorphous multi-directional cores over time, although the discrepancy in core frequencies between layers must be considered. Cores in Layer 27 exhibit 
more blade removals on average and are more intensively worked, while those in Layer 25 have more flake removals. Cores are smallest in Layer 23.

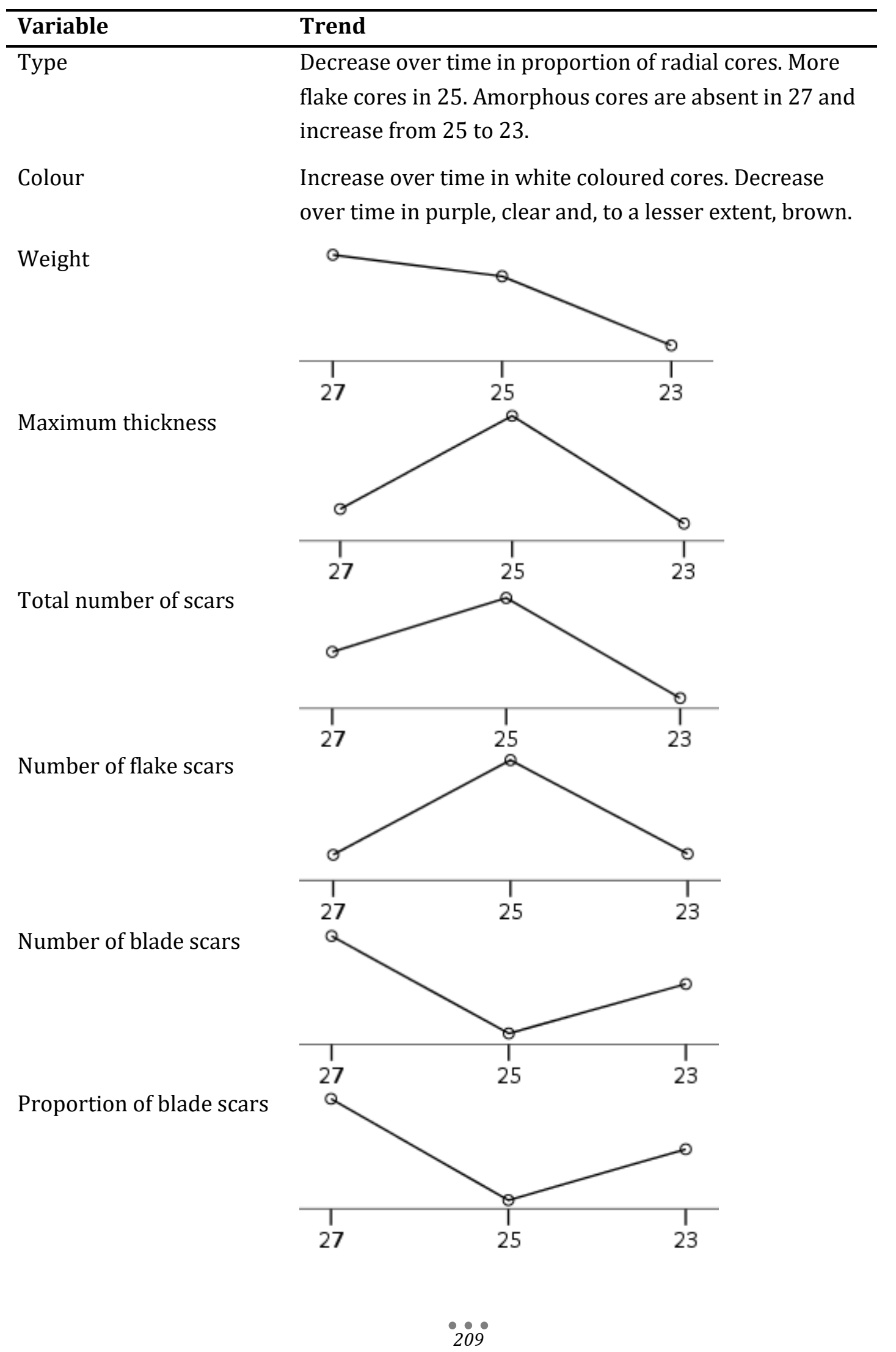


Percentage cortex cover

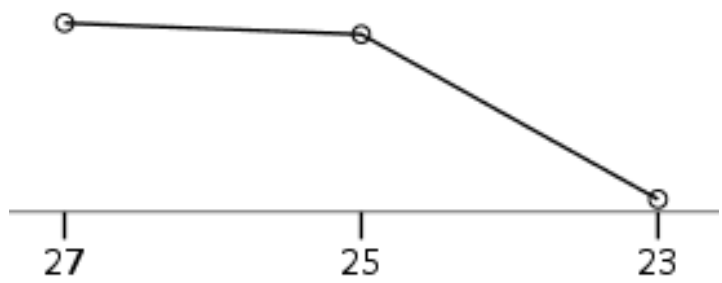

Average scar length per $\mathrm{cm}^{3}$

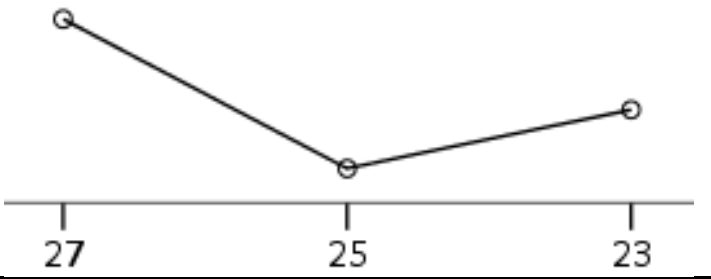

Table 5.2.8: Statistically significant differences between layers in core variables at Umhlatuzana

\section{Debitage}

Results of statistical tests on debitage are reported in Table B.2.2 and summarised in Table 5.2.9. Over the course of the sequence the proportions of hornfels and quartzite decrease while the proportion of quartz increases: Layer 27 contains $43.4 \%$ hornfels, $22.1 \%$ quartzite and $29.7 \%$ quartz, compared to $24.4 \%, 7.8 \%$ and $56.5 \%$, respectively, in Layer 23 . Changes in several other variables, including colour, striking platform lipping, termination type and bipolar percussion, are likely also related to changes in raw materials.

\begin{tabular}{|c|c|c|c|}
\hline Variable & Trend & & \\
\hline \multirow[t]{4}{*}{ Type } & & & \multirow{3}{*}{$\begin{array}{l}\text { Blades } \\
\text { Flake-blades } \\
\text { Flakes }\end{array}$} \\
\hline & & & \\
\hline & & & \\
\hline & $\begin{array}{c}1 \\
27\end{array}$ & $\begin{array}{c}1 \\
25\end{array}$ & \\
\hline Raw material & \multicolumn{3}{|c|}{$\begin{array}{l}\text { Increase over time in use of quartz. Decrease over time in use of } \\
\text { hornfels and, to a lesser extent, quartzite. }\end{array}$} \\
\hline Colour & \multicolumn{3}{|c|}{$\begin{array}{l}\text { Increase over time in proportion of white coloured debitage. } \\
\text { Decrease over time in grey and purple. }\end{array}$} \\
\hline
\end{tabular}




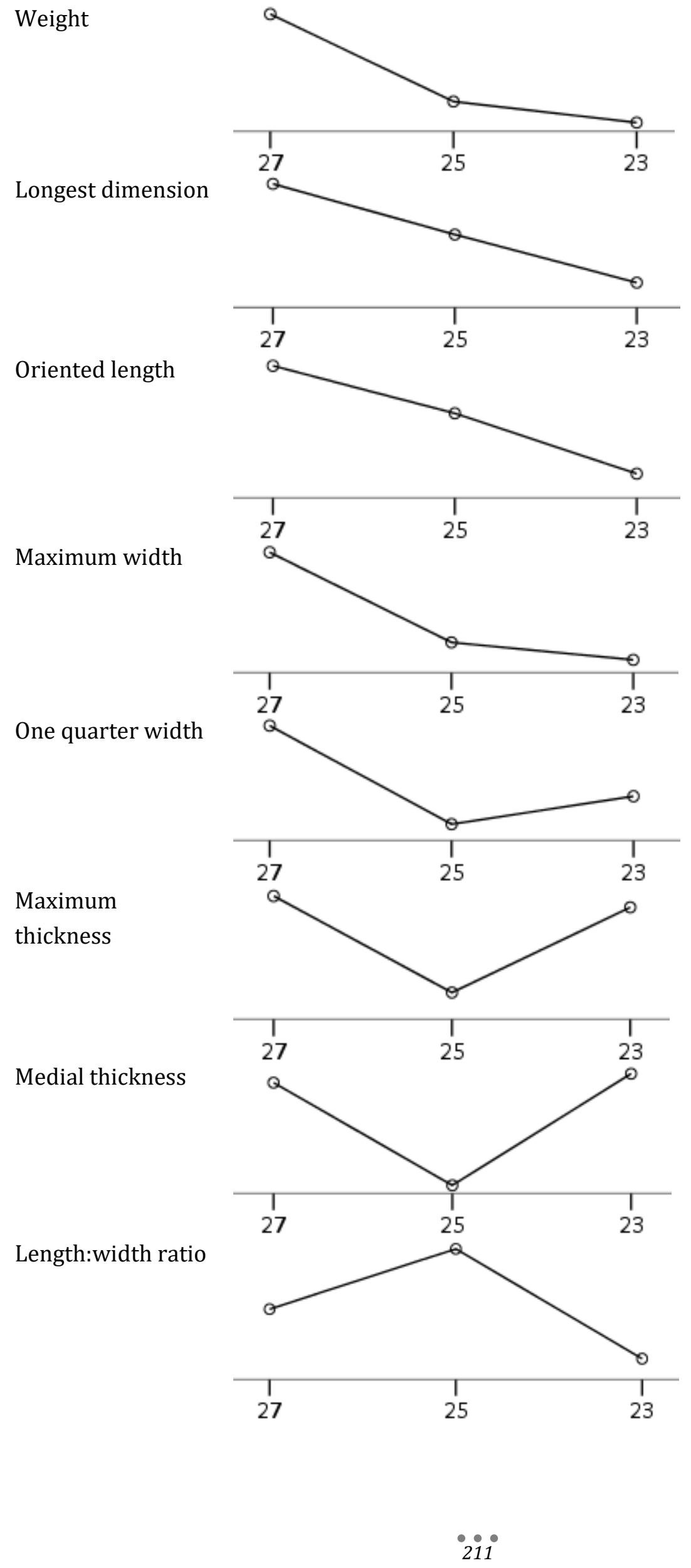


Length:thickness ratio

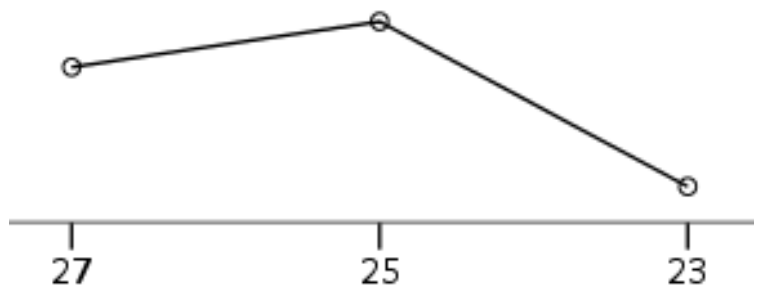

Striking platform width

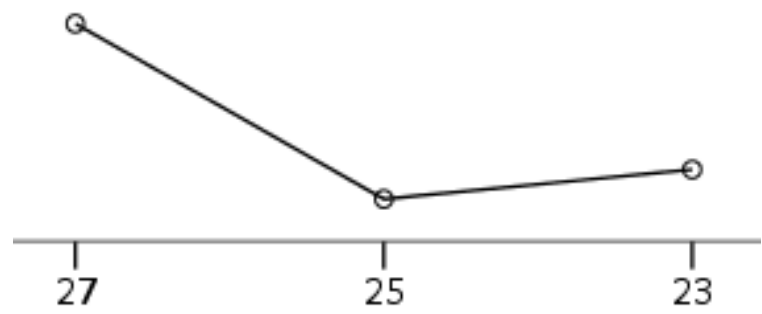

Striking platform thickness

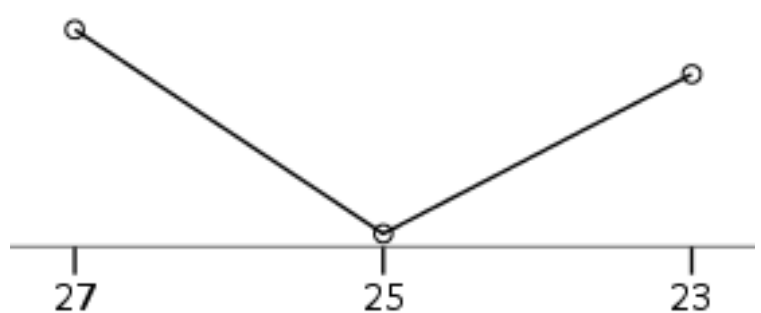

Striking platform lipping

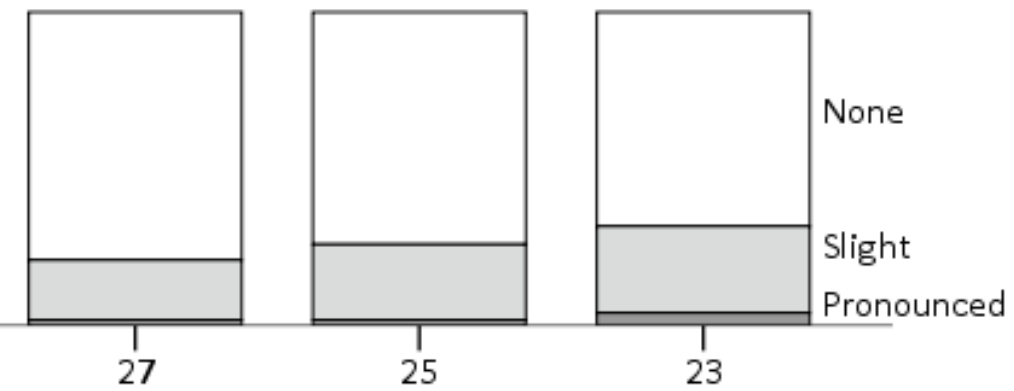

Curvature

Number of parallel arrises

Scars per $\mathrm{cm}^{2}$
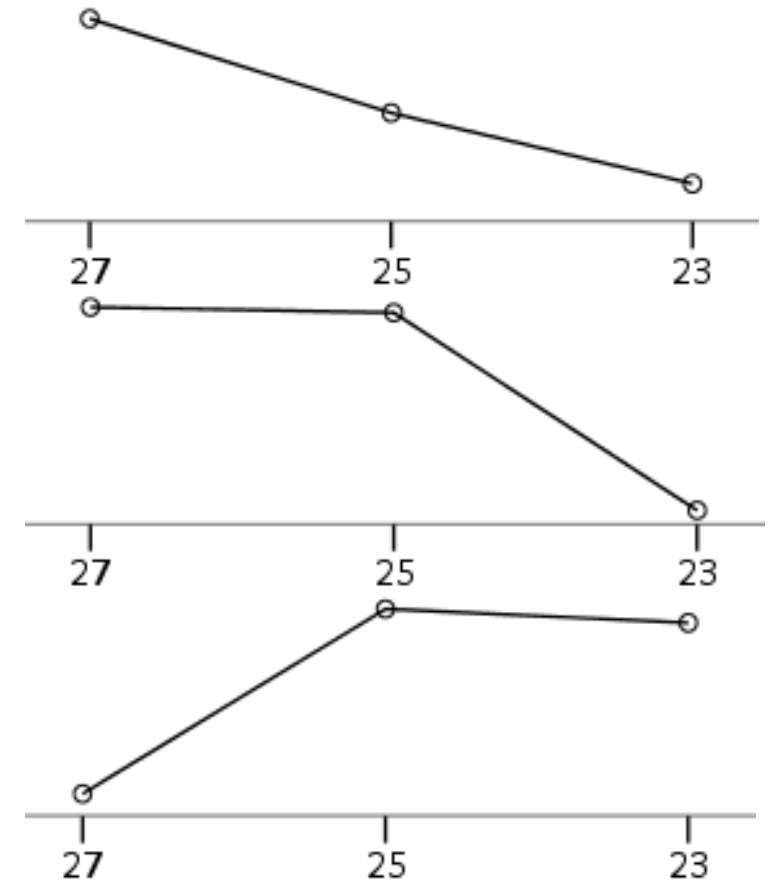
Arrises per $\mathrm{cm}^{2}$

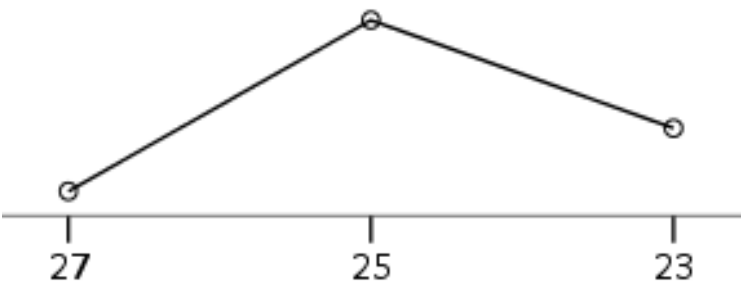

Termination type Slight decrease over time in plunging and step terminations. More axial and crushed terminations in 23.

Evidence of $\quad$ More evidence of bipolar percussion in 23.

bipolar percussion

Other Increase over time in potential snapping. classification

Table 5.2.9: Statistically significant differences between layers in debitage variables at Umhlatuzana

Changes over time are also seen in debitage size. The average length of debitage decreases consistently over time, although debitage is generally much narrower and thinner in Layer 25, which contains a more blade-focussed assemblage (in contrast to the core assemblage). Debitage in Layer 23 is smaller but thicker and more flake-like.

\section{Microliths}

Results of statistical tests on microliths are reported in Table B.2.3 and summarised in Table 5.2.10. Contrary to previous assertions that the HP does not extend to Layer 27 (Lombard et al. 2010), microliths are found in this layer in substantive numbers (Table 5.2.7). Notably, there is only one statistically significant relationship within the variables that also apply to debitage, with more broken tools/tools on broken blanks in later layers. In particular, there are no significant changes in microlith raw material, despite the changes over time 
observed in the debitage sample. Overall there is a considerable degree of consistency in microlith size and general characteristics between layers.

\begin{tabular}{ll}
\hline Variable & Trend \\
\hline Condition & $\begin{array}{l}\text { Decrease over time in the proportion of whole microliths. Increase in the } \\
\text { proportions of proximal breakages and broken blanks of unclear } \\
\text { provenance. }\end{array}$ \\
Retouch face & $\begin{array}{l}\text { Increase over time in the proportion of dorsal retouch. Decrease in the } \\
\text { proportion of edge retouch. }\end{array}$ \\
Retouch type & $\begin{array}{l}\text { Scalar and stepped retouch are only evident in } 27 \text { and 25, abrasion is only } \\
\text { evident in 25, and discontinuous retouch is only evident in 23. However, } \\
\text { these are all at low frequencies, and there is no overall trend. }\end{array}$
\end{tabular}

\section{Table 5.2.10: Statistically significant differences between layers in microlith variables at Umhlatuzana}

In terms of tool-specific variables, there are no statistically significant changes over time in tool morphologies, suggesting that there were also no major changes in microlith tool function and/or hafting arrangements. The only significant differences are those concerning retouch type and face. Patterns in retouch type show no overall trend in technique preferences; the differences observed are in the frequencies of non-backing retouch techniques, which occur in relatively low frequencies.

One notable temporal pattern can be seen in the replacement of edge retouch with retouch made from the dorsal face. This primarily relates to changes in the techniques used to create backing retouch. The proportion of bipolar backing, conducted from both faces, decreases from $93.6 \%$ to $64.2 \%$ to $40.0 \%$. Although backing retouch remains dominant, the method used to produce it changes. 
However, with the exception of this trend the most notable observation in the microlith assemblage is the absence of differences between layers, despite the changes noted in the core and debitage assemblages.

\section{MANUFACTURING TRAJECTORIES AND SUMMARY}

Manufacturing trajectories at Umhlatuzana are summarised in Figure 5.2.23.

Single-platform quartz cores are dominant. Bi- and multi-directional quartz, quartzite, siltstone and hornfels cores were also worked. 'Typically LSA' bipolar percussion was frequently used as an efficient method for removing flakes from nearly-exhausted cores, and as a final stage in the reduction of single- and possibly also two-platform cores. 'Typically MSA' radial working of cores was also used in small frequencies, becoming less prevalent over time. Most terminal removals on cores are of flake dimensions and blade cores are uncommon, although they were more intensively worked than other core types. Although hornfels blades are common, no blade cores are present. Hornfels may have been worked off-site, with the resultant blades brought to the site for further use or retouch.

Cores were worked to produce a mix of debitage products, with the majority of the debitage assemblage being flakes or squat flake-blades. Crested blades, indicative of the 'laminar' method of blade production, are absent. Overall, the assemblage at Umhlatuzana does not accord with arguments that the HP is primarily a bladebased industry. Instead there was mixed production of flakes and blades, with little indication that they were removed using different methods or at different stages in the core reduction process. 
Figure 5.2.23: Manufacturing trajectories at Umhlatuzana. Solid lines are quartzdominated trajectories. Short dash lines are hornfels or other non-quartz raw material dominated trajectories. Long dash lines are a mix of raw materials.

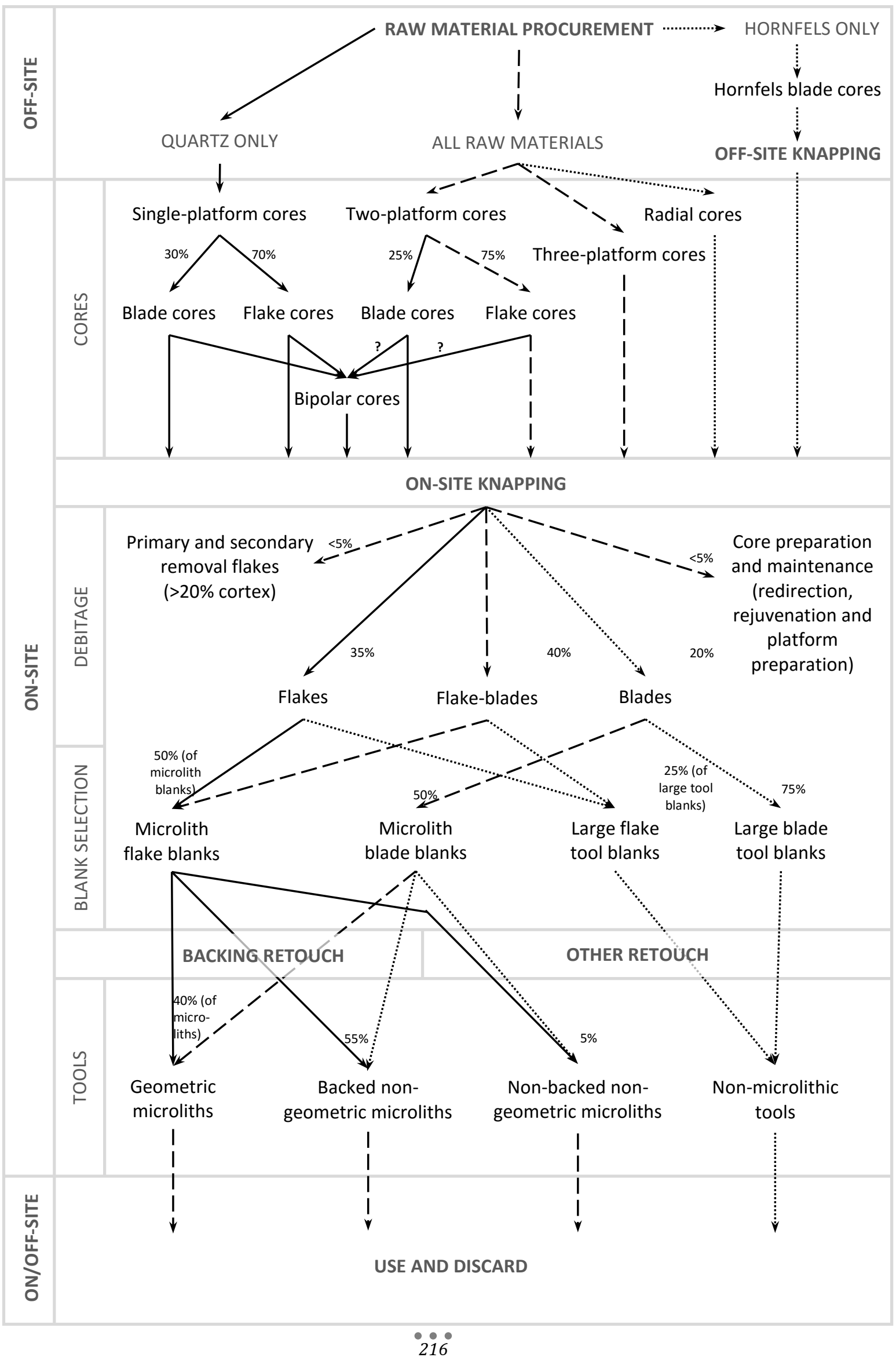


Hornfels was preferentially used to produce blades, and quartz was preferred for flakes. These preferences are amplified further in the selection of blanks for retouch into microlithic tools. Hornfels and quartz blanks are additionally differentiated by size. Hornfels debitage is larger, longer and thinner on average. Hornfels microliths are likewise larger on average, and also exhibit a wider range of sizes as a result of being selected from a wide range of the size array of potential tool blanks. In contrast, quartz microliths are smaller on average, and were manufactured on blanks selected primarily from the smaller end of the range of sizes available, in a more standardised fashion. At the nearby site of Sibudu, Wadley and Mohapi (2008) also note distinct size categories for microliths made on different raw materials, arguing that they may also have had different functions. A similar conclusion is drawn here.

The non-microlithic tool assemblage is small, and was primarily manufactured on hornfels. It includes 'typically MSA' tool forms, including a bifacial and a unifacial point. There is little overlap in the size ranges of microlithic and non-microlithic tools. However, both were selected from the general debitage size range, i.e. both small and large blanks were selected for retouch into different tool types.

In addition to forming a distinct tool category based on size, microlithic tools are also differentiated by the retouch techniques used to produce them. Backing is the most common technique used to shape microlithic tools, while scalar and stepped retouch are most common on non-microlithic tools, only one of which exhibits backing. Furthermore, seven of the 11 retouch techniques documented at the site are unique to either microlithic or non-microlithic tools. Non-microlithic tools are 
also more invasively retouched, on average and in terms of the upper limit of invasiveness.

Non-geometric forms are more common than geometric shapes in the microlith assemblage, especially backed non-geometric points. Crescent microliths are also common. Preferences can be seen for the manufacture of particular morphologies on particular raw materials - hornfels for backed and pointed non-geometric microliths, and quartz for geometric microliths. However, different size blanks do not appear to have been made preferentially into particular microlith forms, with considerable variation evident within individual categories. Differences in microlith size are primarily due to differences in raw material rather than morphology.

There is no evidence for the use of the microburin technique for the production of microliths, although there may have been some intentional snapping of blades, and to a lesser extent flakes, for a similar purpose. One distinct technique that was used in the manufacture of microliths is the frequent use of bipolar backing. However, this technique becomes less common over time, being replaced by backing conducted from the dorsal face alone. This change in technique preference does not appear to have affected any other aspects of microlith production, and should therefore perhaps be equated with a change in local lithic manufacturing traditions rather than a change in tool function.

The most surprising aspect of the microlith assemblage at the site is its consistency over time, with few statistically significant changes evident at this resolution in the 
c. 10,500 year sequence studied. This is particularly unexpected given that there is temporal variation evident in the core and debitage assemblages. In particular, the debitage assemblage indicates that raw material use changed over time - the proportions of hornfels and quartzite debitage decrease over time, while the proportion of quartz increases.

In response to Lombard et al.'s (2010) contention that Layer 27 is mixed and contains an earlier MSA or Still Bay assemblage, the results presented here indicate that this is not the case. Microliths are found throughout the sequence and are very similar between layers, and Layer 27 also does not differ from the other layers in consistent or substantial ways in terms of the underlying technology. As an additional note, there is no evidence at this resolution for the existence of distinct phases within the sequence.

The consistency evident in microlith size and characteristics throughout the sequence implies the production of similar microlithic tools over a long period of time, despite the changes in raw material usage documented in the debitage assemblage. If changes in raw materials were not related to changes in tool production, it could be hypothesised that they were instead related to other external factors. These might include changes in mobility patterns or range sizes, or different landscape use, resulting in differential access to raw materials. Overall, microlith production remained a dominant and stable process throughout the 10,000 year HP sequence at Umhlatuzana. 


\subsection{NtLoAna TSOANA, LeSOTHO}

\section{CORES}

The majority of cores are uni-directional, and flake cores are twice as common as blade cores (Table 5.3.1). This finding does not accord with the common assumption that the HP is primarily a blade industry. Both bipolar percussion and the radial working of cores are present but rare.

\begin{tabular}{llrr}
\hline Core type & & Frequency & Percentage \\
\hline Uni-directional: & Blade & 19 & 25.7 \\
& Flake & 18 & 24.3 \\
& Bipolar & 2 & 2.7 \\
& & & \\
Bi-directional: & Blade & 3 & 4.1 \\
& Flake & 27 & 36.5 \\
& & & \\
Multi-directional: & Radial & 1 & 1.4 \\
& Amorphous & 4 & 5.4 \\
\hline Total & & 74 & \\
\hline
\end{tabular}

Table 5.3.1: Core type frequencies at NT

CCS varieties are dominant (87.8\%), particularly jasper which constitutes $47.3 \%$ of the core assemblage (Figure 5.3.1). Nine (12.2\%) quartzite cores were also found. There are no clear preferences for the working of particular raw materials in particular core forms. Preliminary raw material sourcing surveys indicate that all documented raw materials were available within the local environment of the site (C. Arthur, pers. comm.). Additionally, six cores (8.1\%) exhibit evidence of being recycled from earlier MSA lithics. The recycling of cores does not appear to have been associated with any particular core type or core reduction technique. 
Although the recycling of raw materials appears to have been an uncommon practice, it is notable that it exists here as well as at the nearby site of RCC (see Chapter 5.1).

Figure 5.3.1: Frequencies of core types made on different raw materials at NT

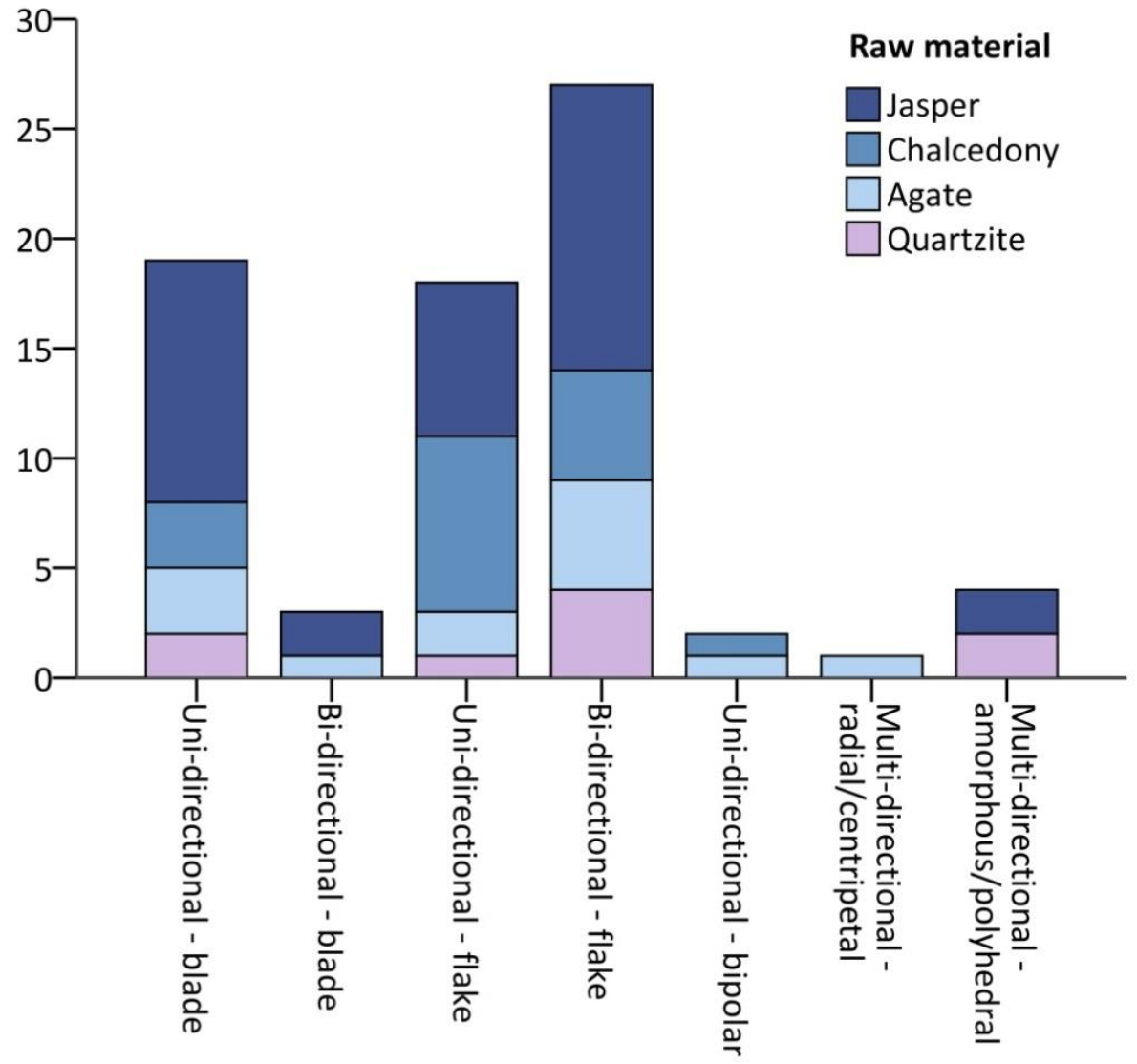

Average core size is $25.6 \times 20.2 \times 12.7 \mathrm{~mm}$. Little variation in size exists between flake and blade cores with one and two platforms, with no indication from the size metrics that blade cores were worked differently or to a different extent than flake cores (Figure 5.3.2, Table A.3.1, Table A.3.2). Bipolar cores are smallest, with mean dimensions of 18.6 × $10.1 \times 4.2 \mathrm{~mm}$, indicating that bipolar percussion was a reduction strategy that was occasionally used to remove flakes from small and/or nearly-exhausted cores. Cores with more than two striking platforms are larger on 
average than other core types, possibly indicating that these formed an earlier stage in the core reduction process. However, there is no evidence for standardisation within any core category. The main differences within the core assemblage are between raw materials. Quartzite cores are larger on average than those on CCS, with a mean weight of $18.4 \mathrm{~g}$ and cubic volume of $19.8 \mathrm{~cm}^{3}$, compared to $7.7 \mathrm{~g}$ and $7.8 \mathrm{~cm}^{3}$.

Figure 5.3.2: Weight distributions of cores at NT

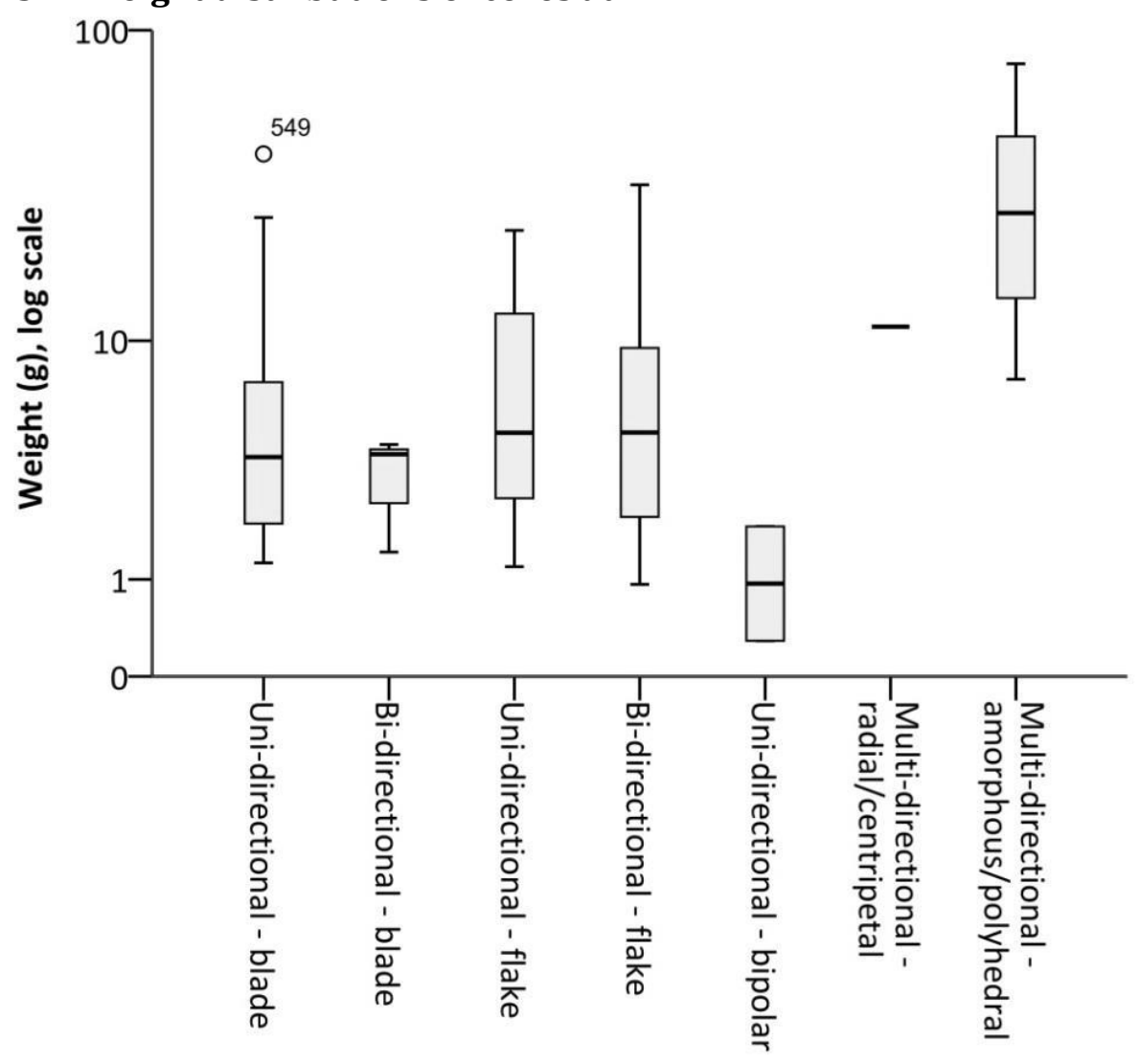

Three of the four amorphous multi-directional cores were worked from three striking platforms (Table A.3.3). All bi-directional blade cores were worked from opposite ends, although a quarter of bi-directional flake cores were worked concurrently from perpendicular striking platforms. Core rotation may have been 
more common for flake cores than for blade cores, although the small frequency of bi-directional blade cores makes comparisons difficult.

The majority of the largest striking platforms on cores are flat (52.7\%), followed by abraded/crushed platforms (16.2\%) (Figure 5.3.3). Facetted (double and complex) striking platforms are relatively uncommon (18.9\% combined). Notably, cortical striking platforms are more common on blade cores than on flake cores (18.2\% compared to $8.9 \%)$. If the aim of core reduction strategies was to prepare cores suitable for the removal of blades (as suggested at RCC in Chapter 5.1), blade cores would be expected to exhibit less cortex as a result of appearing in a later stage of core processing. However, this does not appear to be the case at NT. It should be noted, however, that although cortex is evident on $40.5 \%$ of cores the mean cortex cover is only $10 \%$.

There is no meaningful variation in striking platform size between core types or raw materials. There is also little variation in average removal scar lengths, although terminal removals on blade cores are longer on average than those on flake cores (22.0 mm v. $16.6 \mathrm{~mm})$, and removals on single platform cores are longer on average than those on cores with two or more platforms (20.4 v. 15.9 $\mathrm{mm}$ ) (Table A.3.4). There is similarly little variation in the numbers of terminal removal scars evident on most core types (Figure 5.3.4). The exception is multidirectional amorphous cores, which exhibit much larger numbers of removals than other core types, ranging from 7 to 25 scars. However, when core size is taken into account (Figure 5.3.5, Table A.3.5) they are actually less intensively worked than most other core types. 
Figure 5.3.3: Proportions of core striking platforms at NT. Categories of less than five are combined.

\section{Striking platform type}

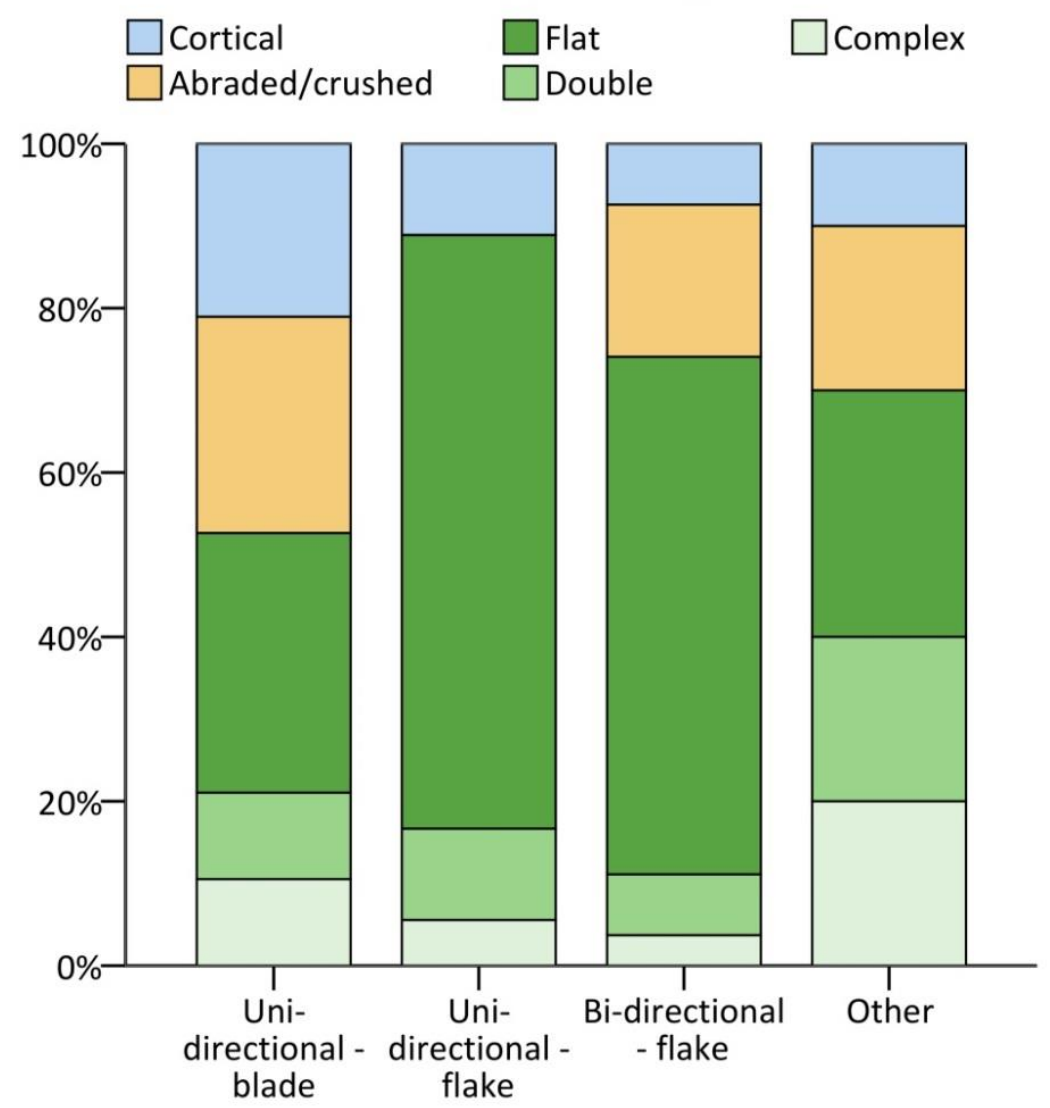

Figure 5.3.4: Average numbers of flake and blade scars on cores at NT

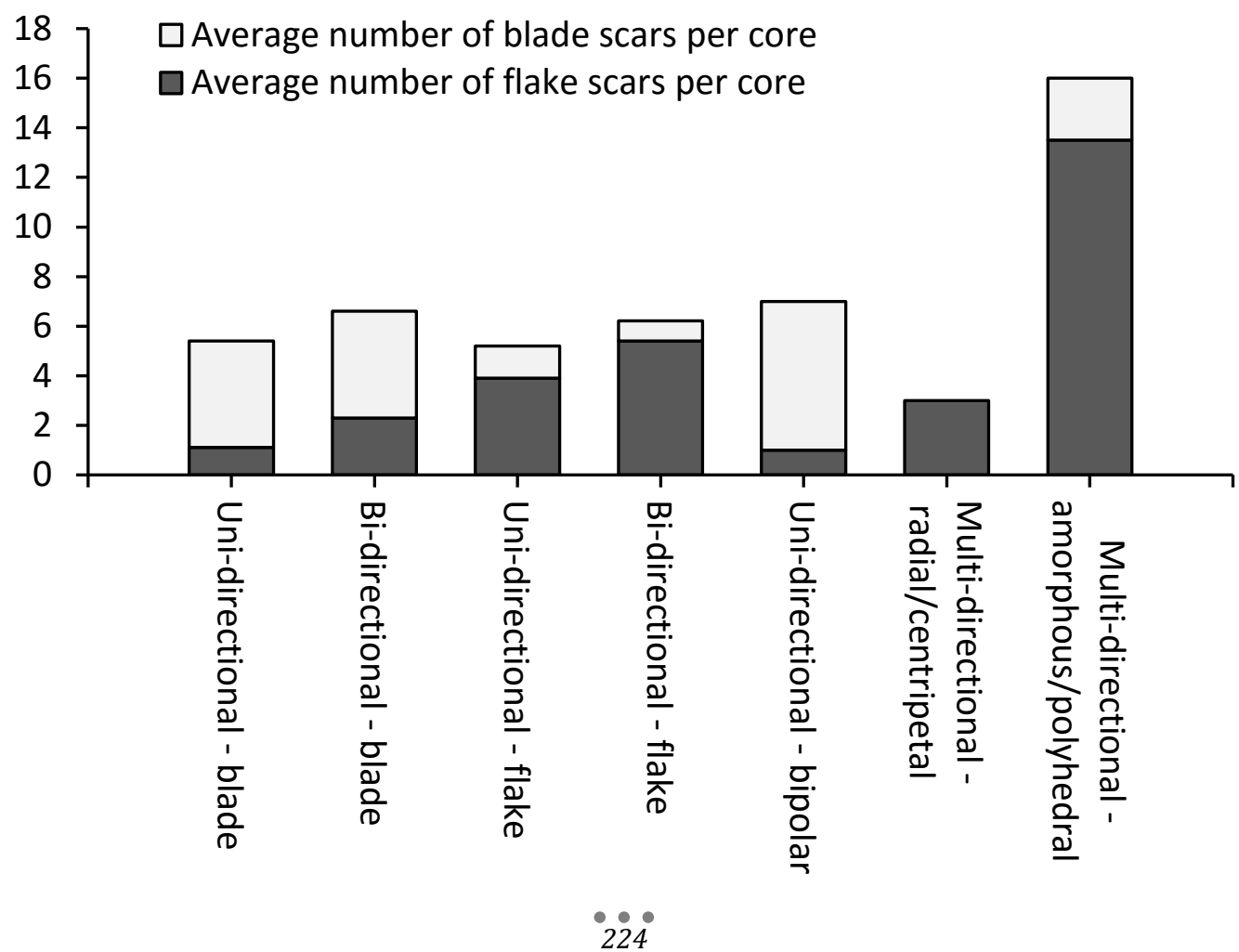


Figure 5.3.5: Ratios of total removal scar length to core volume at NT

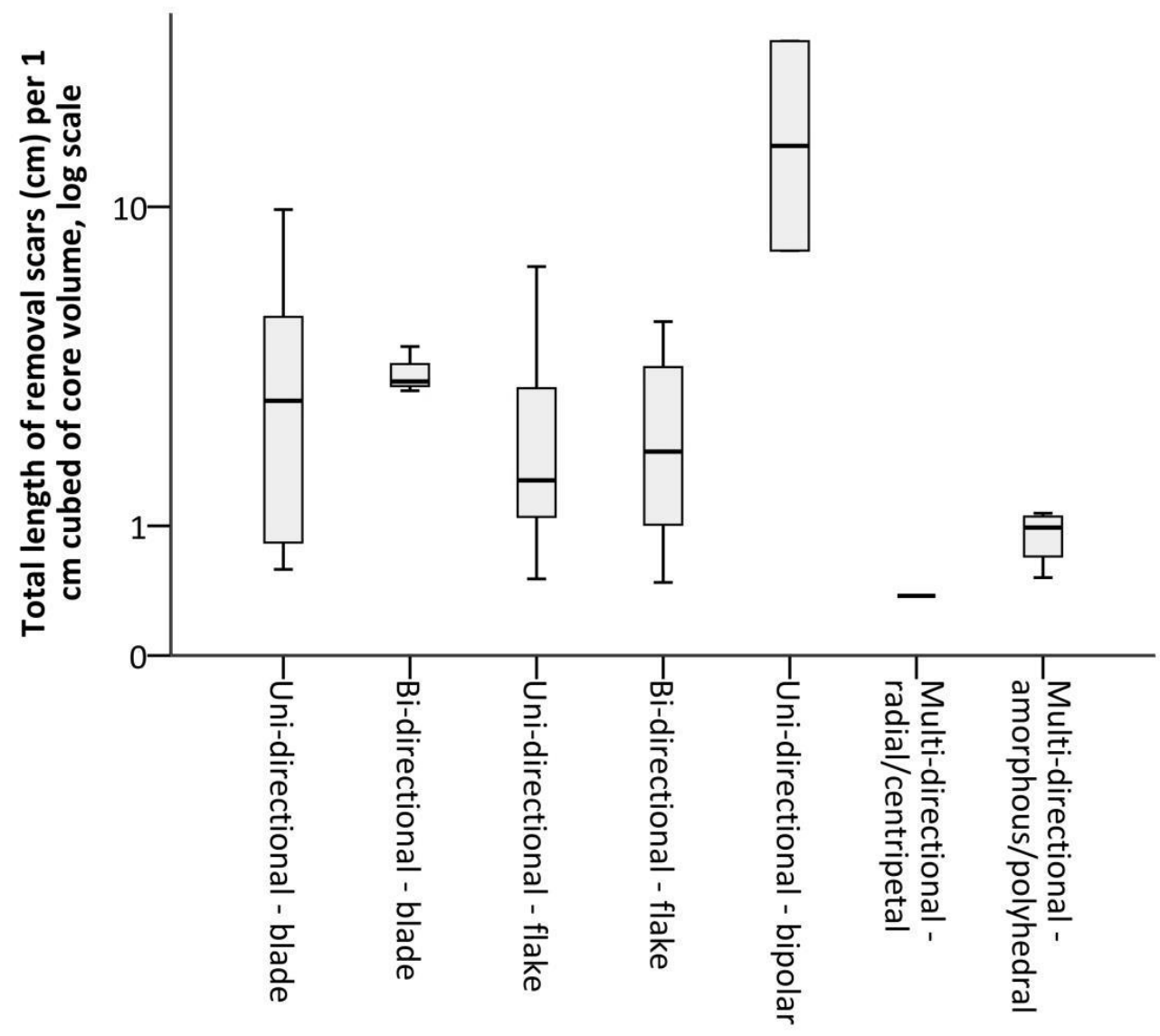

Quartzite cores exhibit more removals on average (9.2) compared to CCS cores (6.1), particularly flake removals (7.3 v. 3.7). However, quartzite cores are also larger on average. When core size is considered, quartzite cores are actually less intensively worked, with a mean total of $1.4 \mathrm{~cm}$ of removals per $1 \mathrm{~cm}^{3}$ of core volume, compared to $3.2 \mathrm{~cm}$ for CCS.

Both bipolar cores exhibit 6 blade scars and 1 flake scar, indicating that in this assemblage bipolar cores can be considered a type of blade core. They are considerably more intensively worked than other core types (Figure 5.3.5, Table A.3.5), as is expected given the prevalent use of bipolar percussion to remove large numbers of flakes from small cores. 


\section{DEBITAGE}

As suggested by the core assemblage, the lithic industry at NT is predominantly flake-based (72.2\%) (Table 5.3.2). Only 50.9\% of debitage is whole (Table A.3.6).

The dominant raw material is CCS, particularly jasper but also including chalcedony and agate. Quartzite constitutes the remaining $36.2 \%$ of the assemblage. CCS was used in slightly higher proportions for blades than for flakes (Figure 5.3.6), but there is no statistically significant relationship between debitage type and raw material $\left(\chi^{2}(6)=12.101, \mathrm{p}=.060\right)$.

\begin{tabular}{lrrrrr}
\hline Raw material & Blades & Flake-blades & Flakes & Total & $\mathbf{\%}$ \\
\hline Jasper & 38 & 51 & 258 & 347 & 37.1 \\
Quartzite & 32 & 56 & 251 & 339 & 36.2 \\
Chalcedony & 27 & 31 & 127 & 185 & 19.8 \\
Agate & 15 & 10 & 40 & 65 & 6.9 \\
\hline Total (\%) & $112(12.0)$ & $148(15.8)$ & $676(72.2)$ & 936 & \\
\hline
\end{tabular}

Table 5.3.2: Debitage measured at NT

Even when broken debitage is excluded, debitage is generally small, with a mean size of 17.8 x 13.0 x $4.7 \mathrm{~mm}$ (Table A.3.7). There are no systematic differences in size between different types. However, differences do exist between raw materials (Figure 5.3.7). Quartzite debitage is larger on average, with a mean weight of $3.0 \mathrm{~g}$ and cubic volume of $3.1 \mathrm{~cm}^{3}$, compared to $1.3 \mathrm{~g}$ and $1.3 \mathrm{~cm}^{3}$ for CCS. Possibly this represents the appearance of earlier stages of quartzite reduction at the site compared to CCS reduction. A more likely explanation, however, is that CCS was available in smaller sized nodules. 
Figure 5.3.6: Raw material proportions of debitage at NT

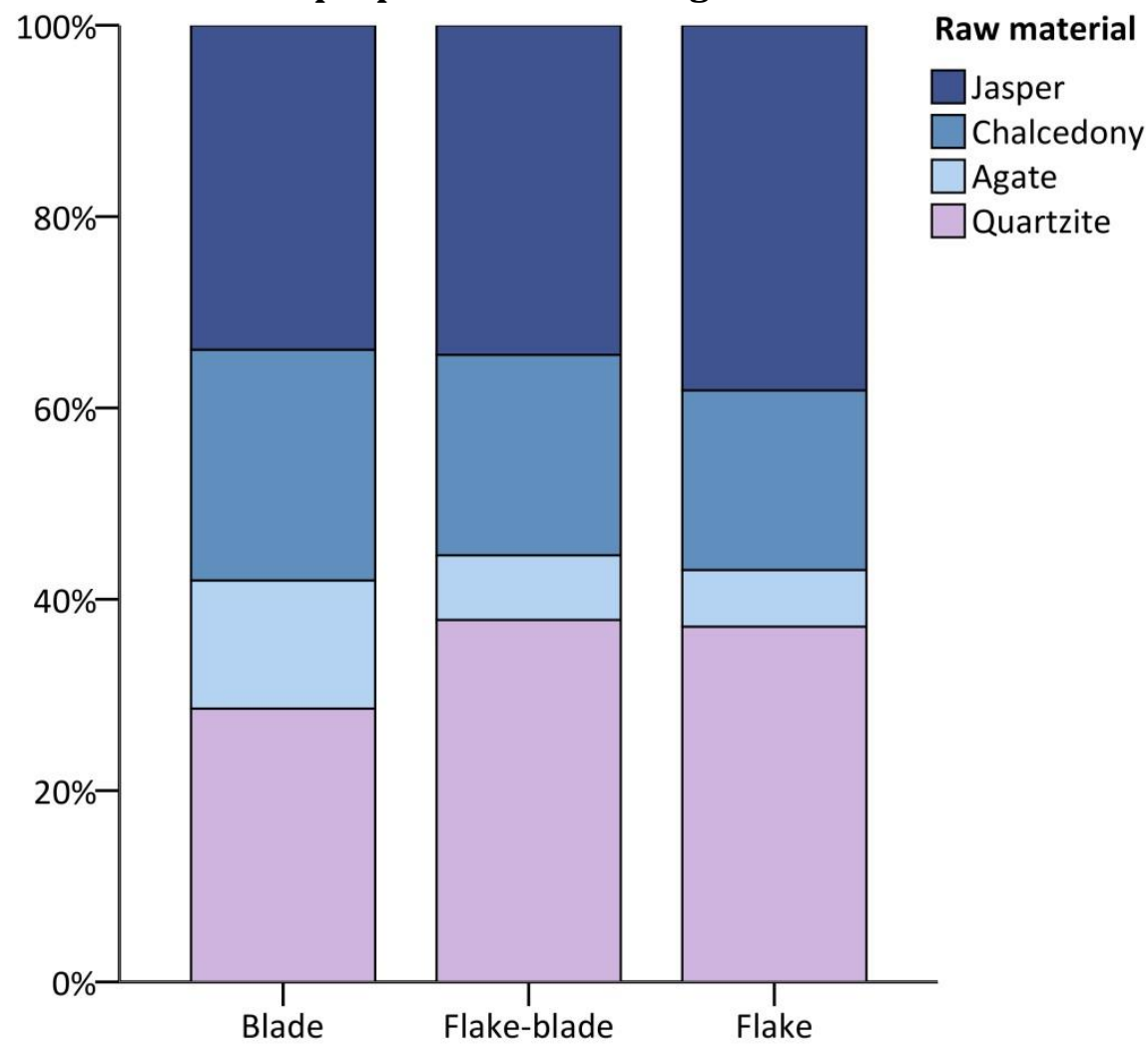

Figure 5.3.7: Cubic volumes of whole debitage on different raw materials at NT

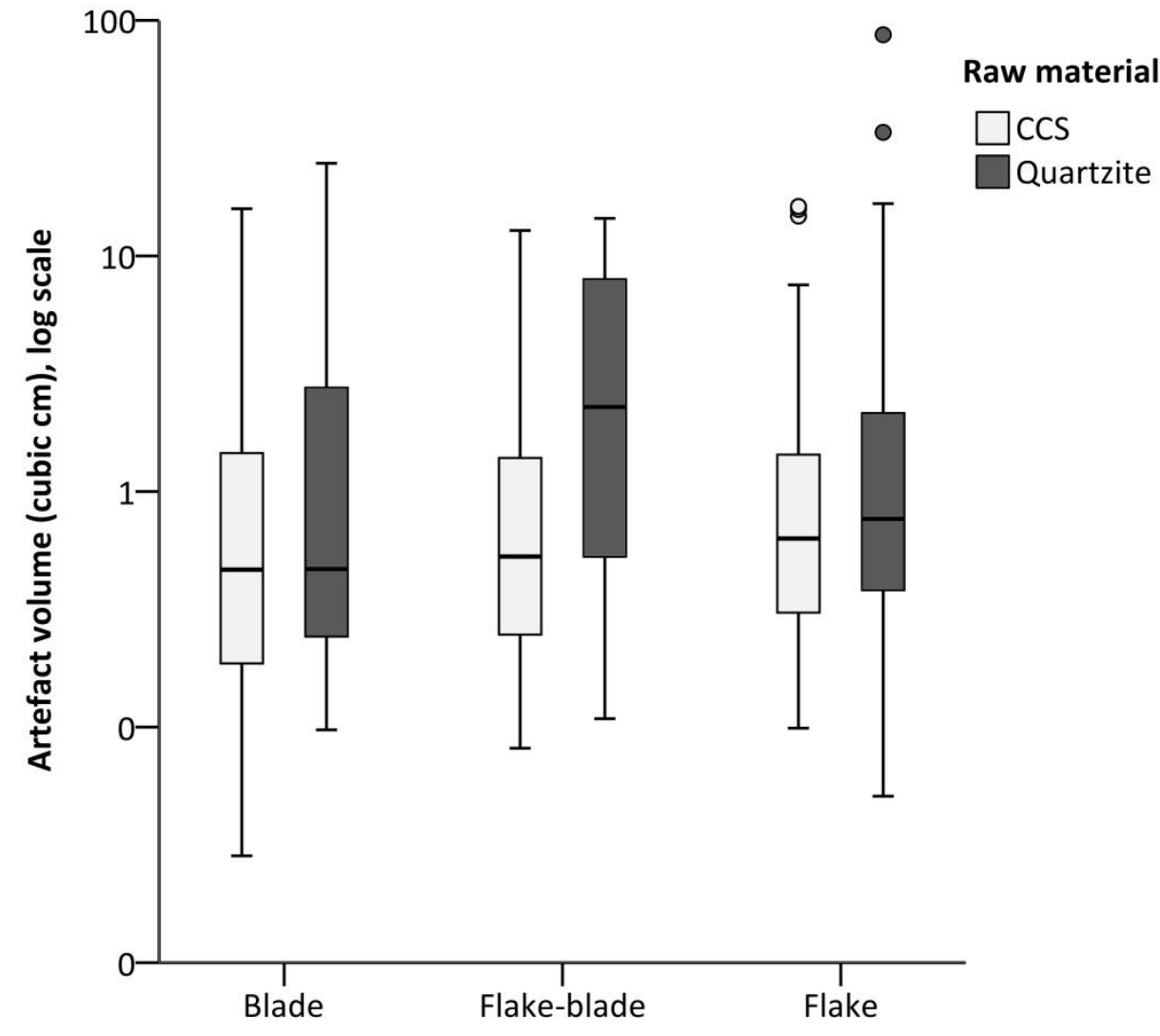


However, when size frequency distributions are compared (Figure 5.3.8) the distribution profiles of CCS and quartzite debitage are actually very similar. The only difference is in the slightly fatter tail of the quartzite distribution, indicating the existence of a small number of much larger lithics. CCS debitage is also slightly more elongated on average, with a mean length:width ratio of 1.6 compared to 1.3 for CCS. However, this is not a substantial difference, and neither raw material grouping could be considered particularly elongated or blade-like. Overall, there is little evidence that CCS and quartzite were worked in significantly different ways.

Figure 5.3.8: Frequency distributions of lengths of whole CCS and quartzite debitage at NT

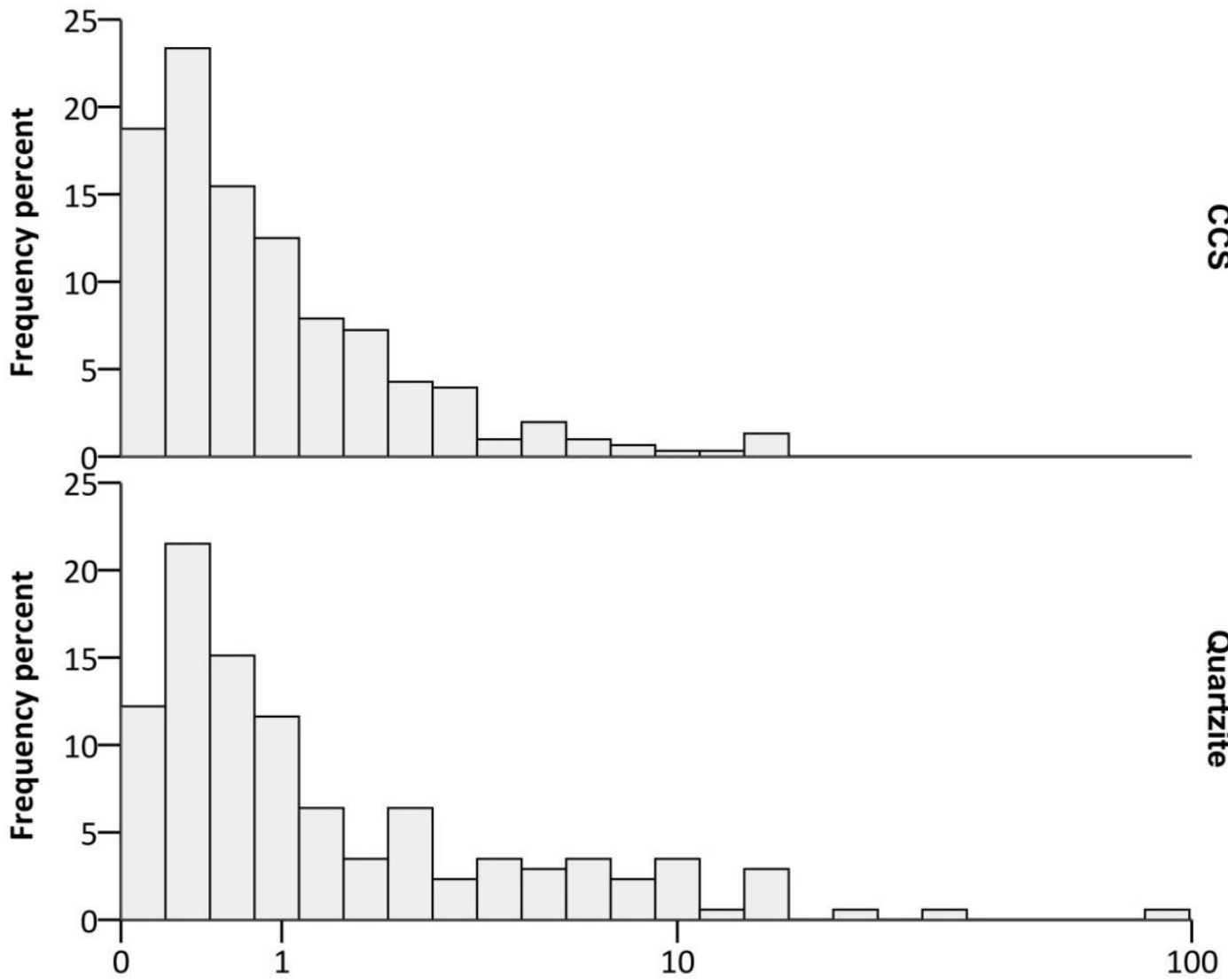

Artefact volume (cubic $\mathrm{cm}$ ), log scale 
Slight or pronounced lipping is evident on $24.3 \%$ of striking platforms, although it is only prominent on $6.5 \%$ of platforms. Lipping is more common on CCS $(29.5 \%)$ than quartzite (16.3\%). Flat striking platforms are the most common type (64.9\%) (Figure 5.3.9). Flat platforms are more common on flakes, while pointed platforms are more common on blades. However, with the exception of pointed platforms flake-blades do not form an intermediate category between flakes and blades in this variable. Cortical platforms are rare overall (3.3\%), and do not vary much between types. There is no indication from the striking platform data that flakes and blades were removed in separate stages during reduction processes.

Figure 5.3.9: Proportions of debitage striking platform types at NT Striking platform type

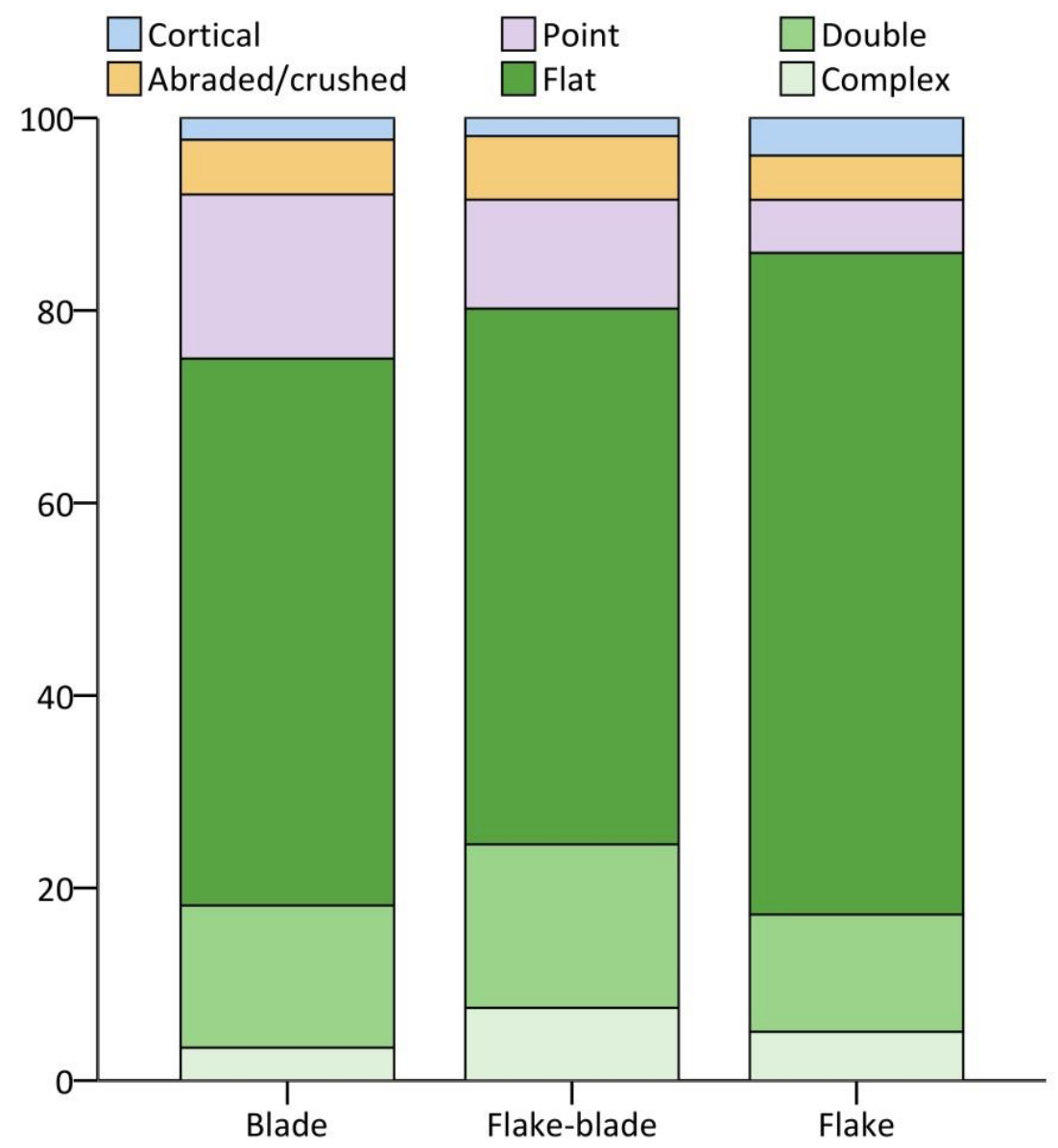


Conversely, slight differences between flakes and blades are evident in dorsal cortex cover. Cortex is more common on flakes, with a mean of $5 \%$ compared to $3 \%$ for flake-blades and $<1 \%$ for blades (Table A.3.8). Additionally, most blades exhibit no cortex and none exhibit over $30 \%$. This could be interpreted as suggesting that blades were removed later in reduction processes than flakes, but blades are much less frequent than flakes and levels of cortex cover are low overall.

Blades, flake-blades and flakes have similar mean numbers of dorsal scars $(2.9,3.0$ and 2.6, respectively). However, when dorsal surface area is taken into account, blades exhibit a greater density of scars than flakes (2.4 per $\mathrm{cm}^{2}$ compared to 1.5$)$, with flake-blades forming an intermediate category (1.9) (Table A.3.9). This may be read as a further possible suggestion that blades may have been removed in a later stage of core reduction. In terms of raw materials, dorsal scar densities are consistently higher on CCS compared to quartzite in all debitage categories (Figure 5.3.10). This is a result of both the larger size of quartzite debitage and smaller absolute numbers of scars.

Over three quarters of whole debitage exhibit removal scars originating from a single direction (Table 5.3.3). Most of the remainder have scars originating from opposite directions (particularly flake-blades), which accords with the evidence for bi-directional working of cores. Scars originating from three directions are rare, and those from four directions are absent. There are no significant differences in dorsal scar orientations between different debitage types or raw materials. 
Figure 5.3.10: Dorsal scar densities on CCS and quartzite debitage at NT

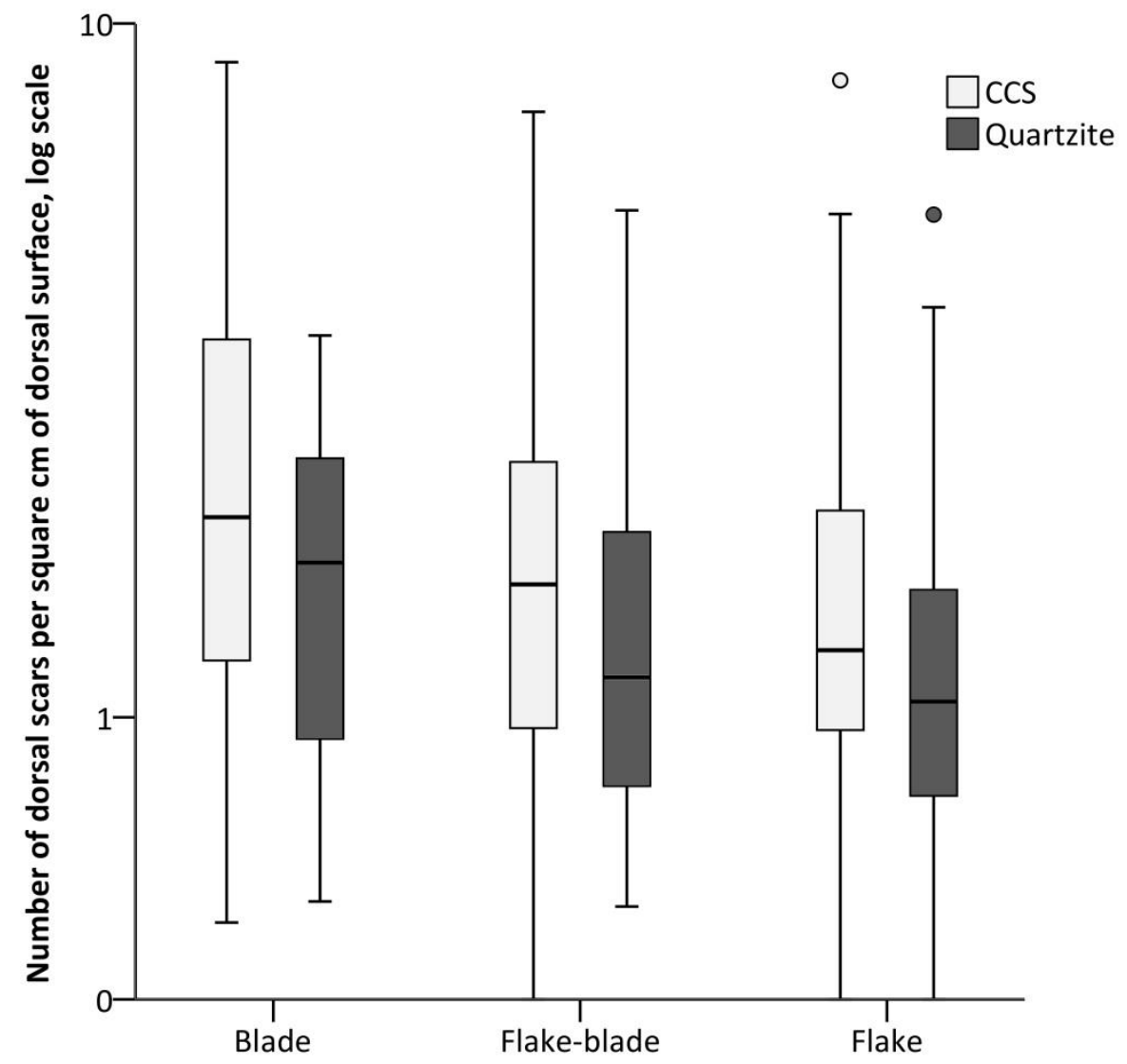

\begin{tabular}{l|rr|rrrrrrr}
\hline Dorsal scar orientation: & \multicolumn{4}{c}{$\mathbf{1}$} & \multicolumn{7}{c}{ 2 (adjacent) } & 2 (opposite) & \multicolumn{3}{l}{ 3 } \\
Type & No. & $\%$ & No. & \% & No. & \% & No. & \% & Total \\
\hline Blade & 58 & 85.3 & 5 & 7.4 & 5 & 7.4 & & & 68 \\
Flake-blade & 66 & 79.5 & 3 & 3.6 & 10 & 12.0 & 4 & 4.8 & 83 \\
Flake & 245 & 76.6 & 35 & 10.9 & 37 & 11.6 & 3 & 0.9 & 320 \\
\hline Total (\% of total) & 369 & 78.3 & 43 & 9.1 & 52 & 11.0 & 7 & 1.5 & 471 \\
\hline
\end{tabular}

Table 5.3.3: Dorsal scar orientation frequencies on whole debitage at NT

Feather terminations are the most common type (57.7\%) and are more common on flakes than on blades (60.4\% v. $48.8 \%$ ), with flake-blades as an intermediate category (Figure 5.3.11). Hinge and step terminations are common (31.9\%), although flake-blades are not intermediate in this category. Axial and crushed terminations, which can be considered indicative of bipolar percussion, are more 
common on blades than on flakes but are rare overall. However, although two cores at the site appear to have been worked using the bipolar technique at the end of their use-lives, no debitage exhibits convincing evidence of having been removed with the use of bipolar percussion.

Figure 5.3.11: Proportions of debitage termination types at NT

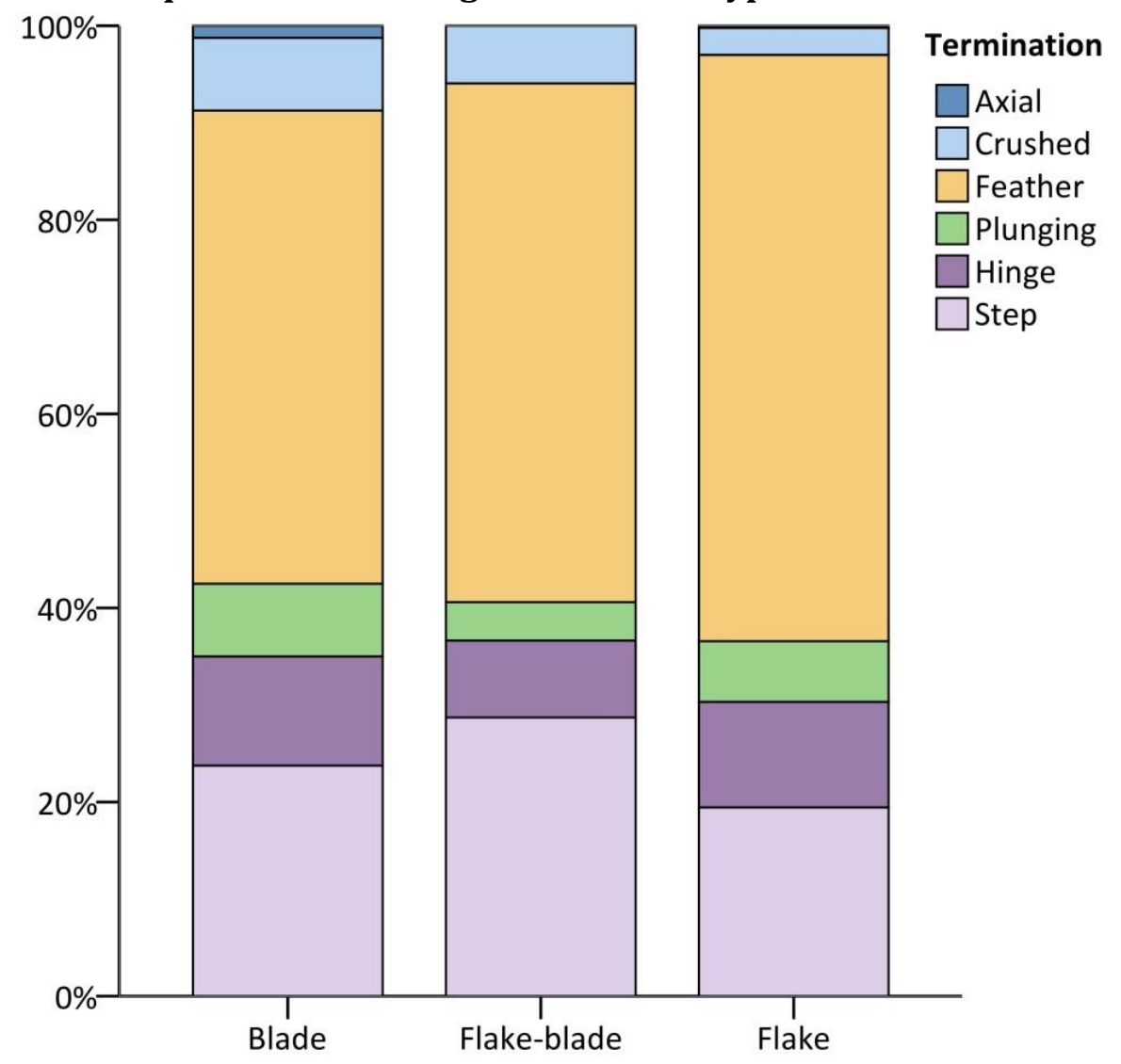

Evidence for the use of recycled raw materials can be seen on 15 chalcedony and jasper flakes and flake-blades (Figure 5.3.12). The low frequency of recycled lithics is in part related to the fact that cortical or recycled surfaces are often not visible on debitage. However, given that only six out of 74 cores exhibit evidence of having been recycled, recycling of older lithics appears to have formed only a minor part of raw material acquisition strategies at NT. 
Figure 5.3.12: Examples of recycled lithics from NT, showing worn scarred surfaces (e.g. the orange surface on 1) alongside fresher scars (at the top and bottom of 1 , and the right of 2).

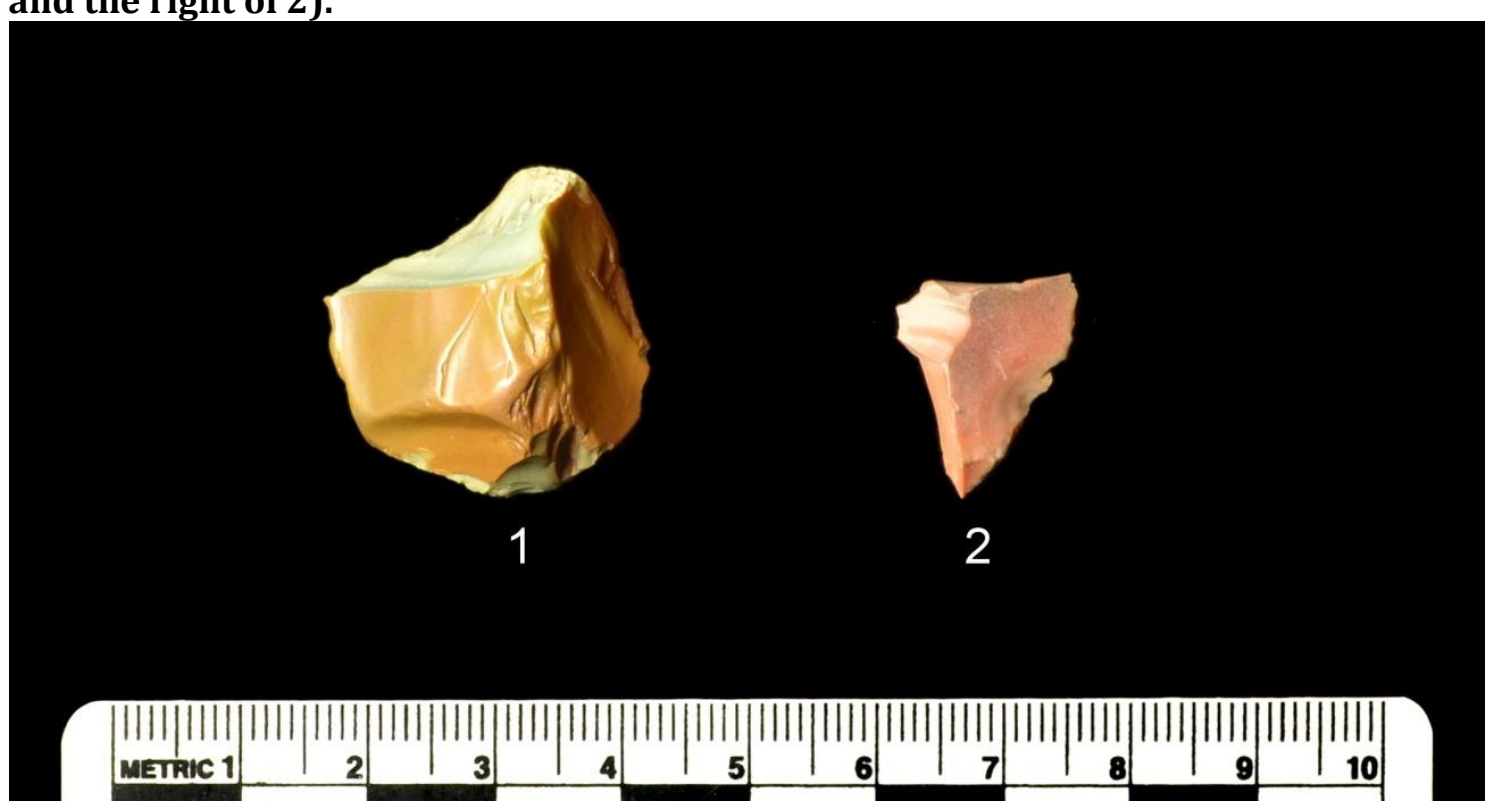

There are few examples of any types of core maintenance flake at the site (Table 5.3.4). This is mostly in the form of platform preparation flakes, but also includes small numbers of core rejuvenation and redirection flakes. Crested blades are absent. Evidence for core maintenance in proportional terms is actually higher among blades compared to flakes, but given the low frequencies overall this is not necessarily meaningful. However, there is no evidence from this data that flakes were primarily used in the preparation of cores for the removal of flakes, as seen at RCC (Chapter 5.1).

\begin{tabular}{l|rr|rrrrr|rr}
\hline & \multicolumn{1}{c}{ Blades } & \multicolumn{3}{c}{ Flake-blades } & \multicolumn{2}{c}{ Flakes } & \multicolumn{3}{c}{ Total } \\
Maintenance flake type & No. & $\mathbf{\%}$ & No. & \% & No. & \% & No. & \% \\
\hline Platform preparation flake & 2 & 1.8 & 2 & 1.4 & 12 & 1.8 & 16 & 1.7 \\
Core rejuvenation flake & 1 & 0.9 & 1 & 0.7 & 2 & 0.3 & 4 & 0.4 \\
Core redirection flake & & & & & 3 & 0.4 & 3 & 0.3 \\
\hline Total & 3 & 2.7 & 3 & 2.1 & 17 & 2.5 & 23 & 2.5 \\
\hline
\end{tabular}

Table 5.3.4: Frequencies of core maintenance debitage at NT 
TOOLS

Tools were manufactured almost equally on flake and blade blanks (55.6\% of microliths and $50.0 \%$ of non-microlithic tools are on blade blanks), even though blades constitute only $12.0 \%$ of the debitage assemblage (Table 5.3 .5 ). CCS is dominant and is found in higher proportions in the tool assemblage compared to the debitage assemblage (82.3\% v. 63.8\%). Differences can be seen in raw material use for different tool types, however. Quartzite was not used for the manufacture of microliths. In contrast, it comprises $37.5 \%$ of the non-microlithic tool assemblage, while agate was not used for these tools. Although minimal differences are evident between quartzite and CCS in the debitage assemblage, they do appear to have been treated differently in terms of their selection as tool blanks.

\begin{tabular}{lrrrrrr}
\hline $\begin{array}{l}\text { Raw } \\
\text { material }\end{array}$ & $\begin{array}{r}\text { Microliths } \\
\text { on blades }\end{array}$ & $\begin{array}{r}\text { Microliths } \\
\text { on flakes }\end{array}$ & $\begin{array}{r}\text { Retouched } \\
\text { blades }\end{array}$ & $\begin{array}{r}\text { Retouched } \\
\text { flakes }\end{array}$ & Total & \% \\
\hline Jasper & 2 & 3 & 1 & 3 & 9 & 52.9 \\
Chalcedony & 3 & & 1 & & 4 & 23.5 \\
Quartzite & & & 2 & 1 & 3 & 17.6 \\
Agate & & 1 & & & 1 & 5.9 \\
\hline Total & 5 & 4 & 4 & 4 & 17 & \\
\hline
\end{tabular}

Table 5.3.5: Tools measured at NT

Microliths at the site are small, with mean dimensions of $20.5 \times 13.9 \times 4.2 \mathrm{~mm}$ (Table A.3.10). None are longer than $30 \mathrm{~mm}$. Non-microlithic tools are more variable in size, ranging up to $66.7 \mathrm{~mm}$ in length (Table A.3.11). There is little overlap between the two tool categories. This can also be seen in Figure 5.3.13, which demonstrates that while microlithic tools fit within the size range of debitage (and therefore potential blanks), non-microlithic tools are considerably 
larger. Although the numbers of tools are very small, it seems reasonable to conclude that the general system of lithic production at the site was geared towards the production of small blanks suitable for retouch into microlithic tools. A number of much larger blanks were also selected for retouch, but these were at the extreme end of the size range available. Additionally, a division is evident in the size ranges of CCS and quartzite non-microlithic tools, with quartzite tools being larger, although as noted above the sample size is small.

Figure 5.3.13: Cubic volumes of lithics on different raw materials at NT

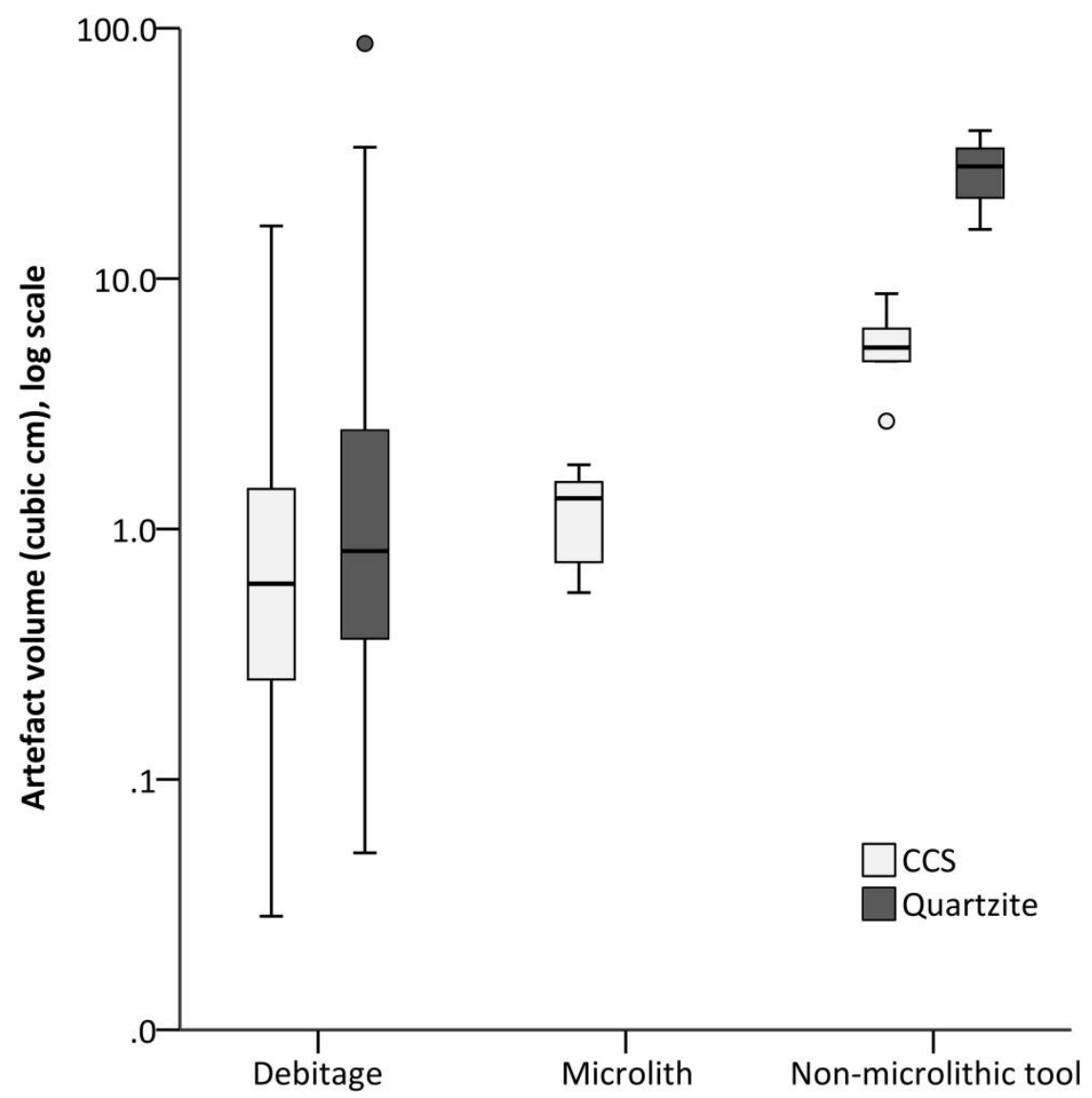

A range of tool forms are present, given the small number of tools recovered

(Table 5.3.6, Figure 5.3.14). Crescents are the most common microlith type and the only geometric form present, and make up a third of the microlith assemblage. The 
remainder are non-geometric forms, of which only one is backed. In the nonmicrolithic tool assemblage one point is present, with the rest being simple side and/or end retouched tools. Notches, denticulates, burins, shoulders and tangs are absent from the tool assemblage.

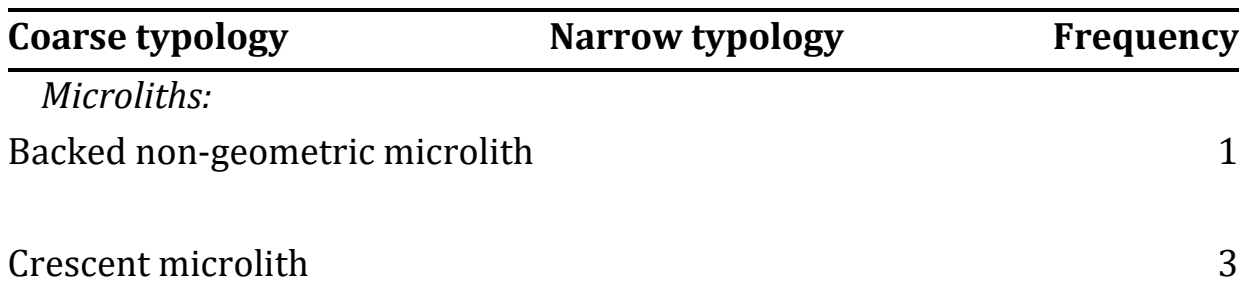

Non-geometric microlith $\quad$ Double side retouched 2

Side retouched 1

Non-geometric microlith point $\quad$ Concave point 1

Double side retouched point $\quad 1$

Total

9

Non-microlithic tools:

End retouched

1

$\begin{array}{lll}\text { Point } & \text { Side retouched concave point } & 1\end{array}$

Side and end retouched $\quad 2$

$\begin{array}{lll}\text { Side retouched } & \text { Double side retouched } & 1\end{array}$

Side retouched 3

Total

8

Table 5.3.6: Tool type frequencies at NT

Small sample sizes preclude comparisons of the sizes of individual tool morphologies. However, when TCSA measurements are considered, mean values for microlith types range from 22.3 to $32.8 \mathrm{~mm}^{2}$ and are therefore similar to Shea's 
(2006) arrowhead sample, although at $58.0 \mathrm{~mm}^{2}$ the largest TCSA of a microlithic tool is the same as his dart tip sample (Table A.3.12). The backed non-geometric microlith has a particularly small TCSA measurement $\left(22.3 \mathrm{~mm}^{2}\right)$. The nonmicrolithic point has a much smaller measurement $\left(109.3 \mathrm{~mm}^{2}\right)$ than the mean for other non-microlithic tools $\left(141.0 \mathrm{~mm}^{2}\right)$.

\section{Figure 5.3.14: Microliths from NT, demonstrating the range of forms evident}

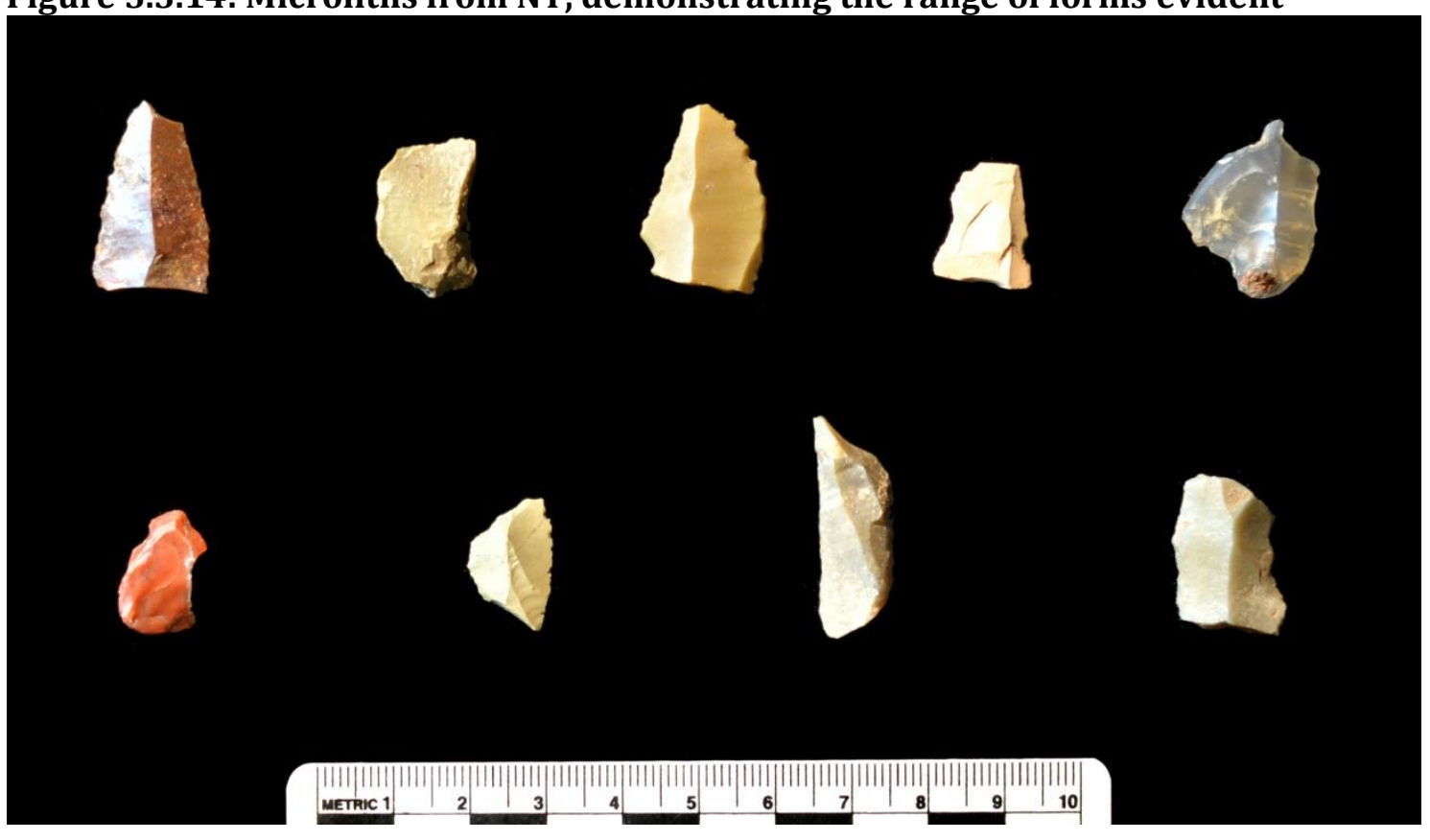

Scalar retouch is the most common retouch type in the tool assemblage, especially among non-microlithic tools (72.7\% of retouch). There is more variation in retouch technique in the microlith assemblage, although again scalar retouch is dominant (33.3\%) (Table A.3.13). Only three microliths (two of the three crescents and one non-geometric form) are backed, none of which made use of bipolar backing. However, much of the scalar retouch was conducted at obtuse angles, and so may have fulfilled a similar function to backing retouch in facilitating hafting. Most tools were retouched along a single lateral edge on the dorsal face. The 
proportions of retouched edges and faces are similar between microlithic and nonmicrolithic tools, while retouch outlines and locations vary more between the categories (Table A.3.13).

Retouch is more extensive on non-microlithic tools, with a mean length of $28.1 \mathrm{~mm}$ and depth of $3.0 \mathrm{~mm}$, compared to $20.3 \mathrm{~mm}$ and $2.7 \mathrm{~mm}$ for microliths (Table A.3.14). Retouch also tends to be conducted to more acute angles on nonmicrolithic tools, with a mean of $47^{\circ}$ compared to $65^{\circ}$ for microliths. There is no evidence for standardisation of retouch extent. Both tool categories have similar mean IOI values ( 0.146 for microliths and 0.152 for non-microlithic tools), and the highest value for any tool is 0.406 (Table A.3.15). The microliths in each layer are illustrated in Figure 5.3.15.

Figure 5.3.15: Microliths from NT Layer 577 (1-6), 578 (7) and $579(8,9)$, demonstrating the lack of standardisation in forms. 1-4, 7: jasper. 5, 8, 9: chalcedony. 6: agate. Particularly unusual is the concave point or 'awl' (6).

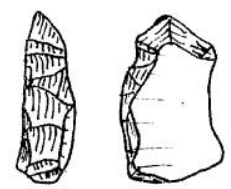

1

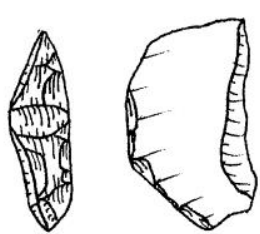

2

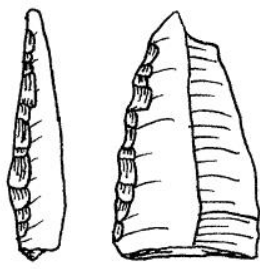

5

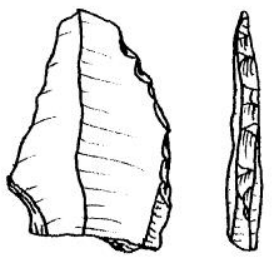

3

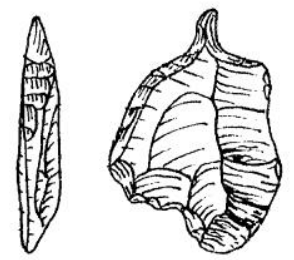

6

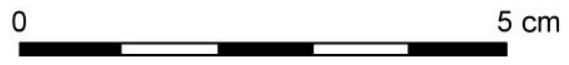




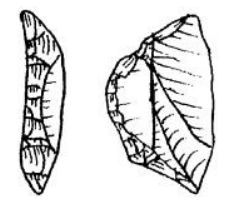

7

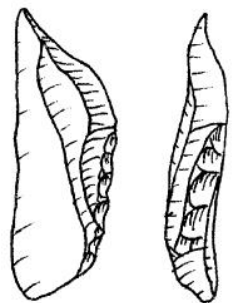

8
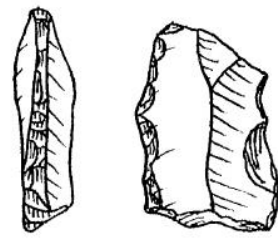

9

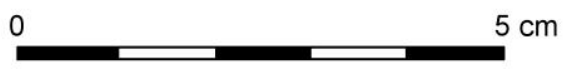

TEMPORAL VARIATION

Results of statistical tests at NT are presented in Appendix B.3. The small number of tools precludes comparisons between layers (Table 5.3.7).

\begin{tabular}{lrrr}
\hline Layer & Cores & Microliths & Non-microlithic tools \\
\hline 577 & 56 & 6 & 3 \\
578 & 11 & 1 & 4 \\
579 & 7 & 2 & 1 \\
\hline
\end{tabular}

Table 5.3.7: Cores and tools analysed in each layer at NT

\section{Cores}

Results of statistical tests on cores are reported in Table B.3.1 and summarised in Table 5.3.8. Only one variable demonstrates statistically significant changes over time in the core assemblage - the number of aberrant terminations. However, this is not an especially useful variable in determining core reduction strategies, and it does not show any clear or meaningful pattern. 


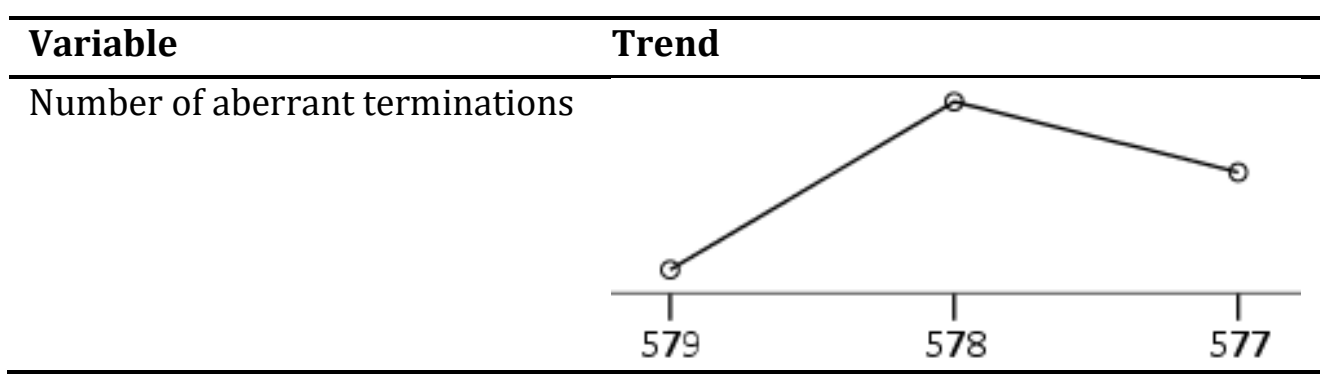

Table 5.3.8: Statistically significant differences between layers in core variables at NT

\section{Debitage}

Results of statistical tests on debitage are reported in Table B.3.2 and summarised in Table 5.3.9. In contrast to the core assemblage there are several areas of temporal variation in the debitage sample, although few are consistent trends. Despite the increase over time in the proportion of flake-blades, the uppermost Layer 577 contains the smallest proportion of true blades. One of the only consistent trends over time is the reduction in mean cortex cover. Some trends can be observed in debitage condition, but overall there is no clear trend and the proportion of whole lithics remains roughly similar. Similarly, although there is a slight increase in the proportion of flat striking platforms over time, most other changes are in the form of minor and inconsistent variations between layers.

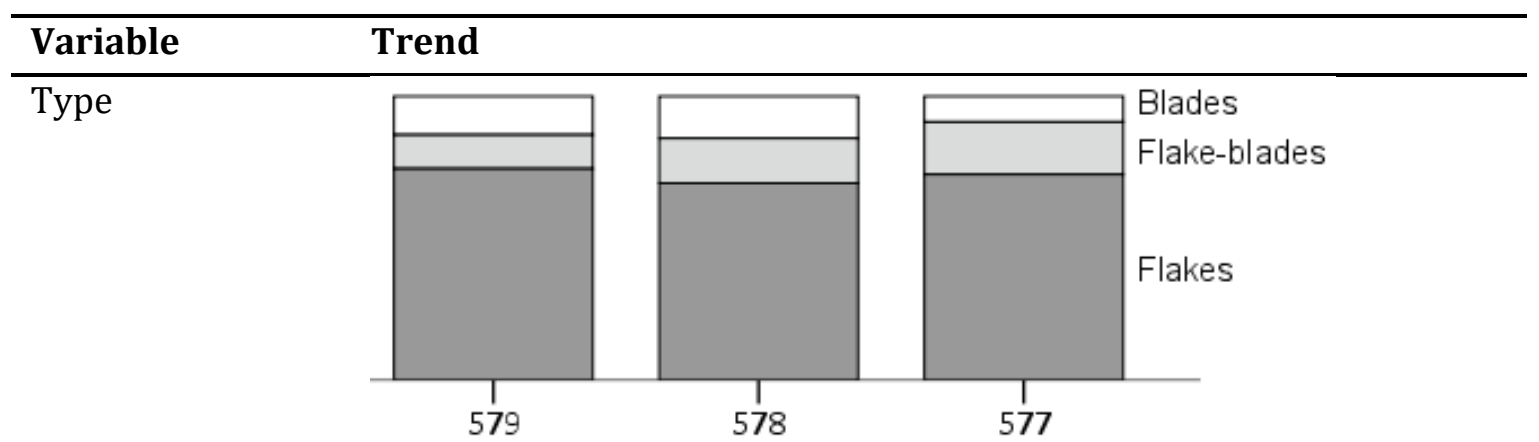

Condition

Slight increase over time in proportion of proximal breakages and decrease in distal breakages. 
Raw material

Colour

Weight

Longest

dimension

Medial width

Oriented length

Three quarter

width

Length:thickness

ratio

Width standard deviation
More quartzite in 579 and fewest in 578.

Increase over time in proportion of brown coloured debitage and decrease in grey.
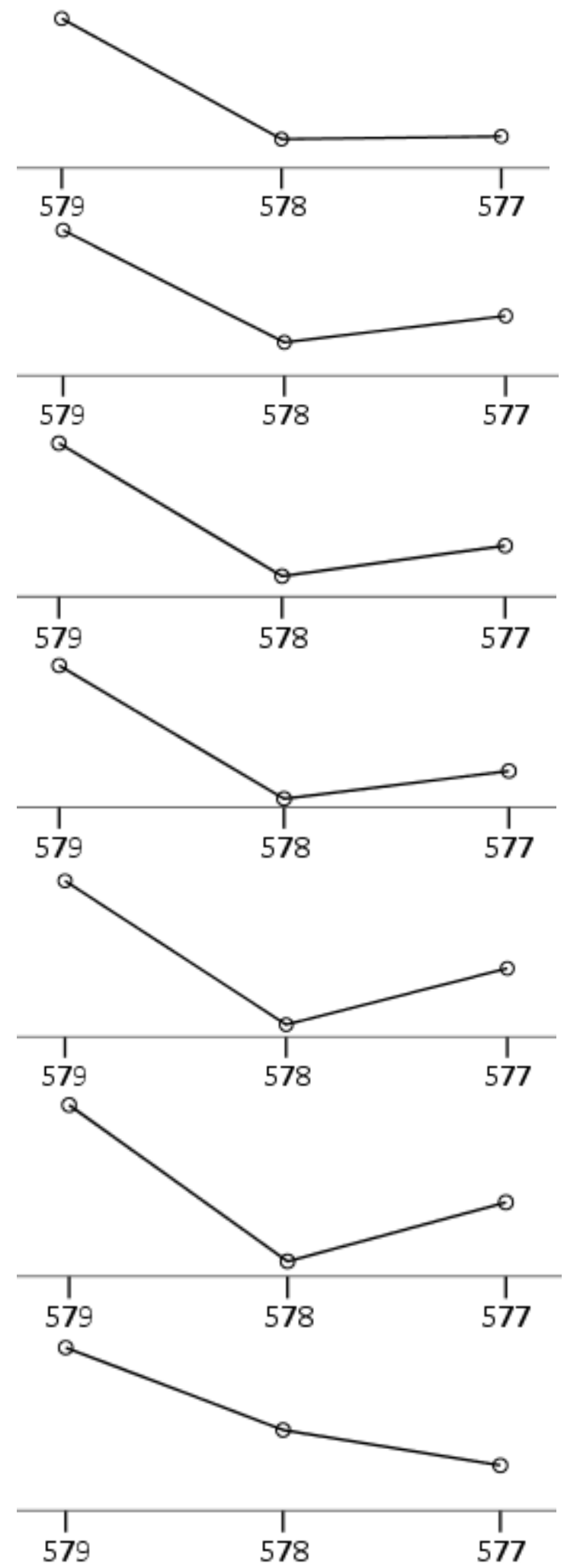

Striking platform Increase in proportion of flat platforms over time. Fewer facetted type

platforms in 578 and more pointed platforms in 577. 


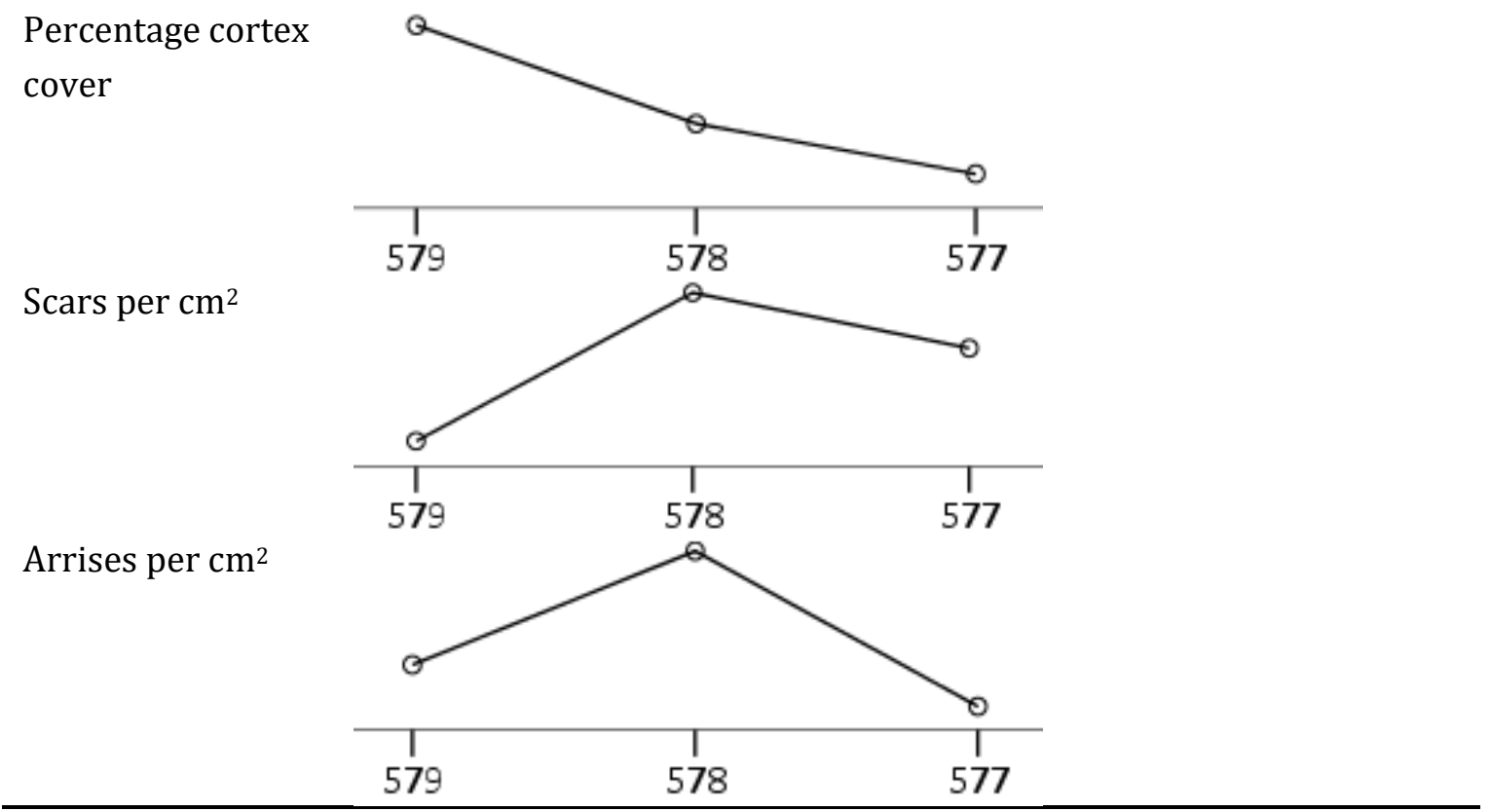

Table 5.3.9: Statistically significant differences between layers in debitage variables at NT

Although the core assemblage does not indicate any significant changes in raw material use over time, there is some variation in the debitage assemblage. More quartzite is found in Layer 579 and less in 578, with 577 being roughly intermediate. Slight variations are evident in the proportions of different CCS varieties, but again there are no consistent trends.

In terms of debitage size, debitage in Layer 579 is larger overall. It tends to be longer and wider, and also thicker in proportional terms. Debitage in Layer 578 is much smaller, and 577 is similar to (although slightly larger than) 578 . The numbers of dorsal scars and parallel arrises on debitage do not change significantly over time, but due to changes in debitage size there are significant changes in scar and arrise density. Size patterns follow patterns in the proportion of quartzite evident in each layer, and so these changes are at least partly linked to 
the fact that quartzite debitage is larger on average. The reverse pattern is true of debitage parallel-ness, and may also be related to changes in raw materials.

\section{MANUFACTURING TRAJECTORIES AND SUMMARY}

Manufacturing trajectories at NT are summarised in Figure 5.3.16. Uni-directional cores and bi-directional flake cores are dominant. Cores with more than two platforms are rare, as is radial working of cores. Bipolar percussion was also occasionally utilised to work small nearly-exhausted cores, primarily for the removal of blades. There is little indication that blade cores were treated in any distinct way compared to flake cores. Although blade cores are slightly more intensively worked, they are generally of a similar size to flake cores. They are also less common, and in fact have a higher prevalence of cortical striking platforms, in contrast to the evidence seen at other sites (e.g. RCC, Chapter 5.1) for blade cores forming a later stage of core reduction.

CCS varieties are dominant at the site, and quartzite was also worked. Recycling of older lithics is evident at low frequencies, indicating that it formed only a minor part of raw material acquisition strategies. Overall there are few indications that raw materials were treated in different ways. However, an exception to this is in the selection of blanks, where quartzite was only used for non-microlithic tools. Additionally, quartzite cores, debitage and tools are all slightly larger than their CCS equivalents. This is probably at least partly due to differences in the sizes of available nodules of raw material, but is still meaningful as it extends to the blanks that were selected for retouch. 
Figure 5.3.16: Manufacturing trajectories at NT. Dashed lines are CCS only. Solid lines are a mix of CCS and quartzite.

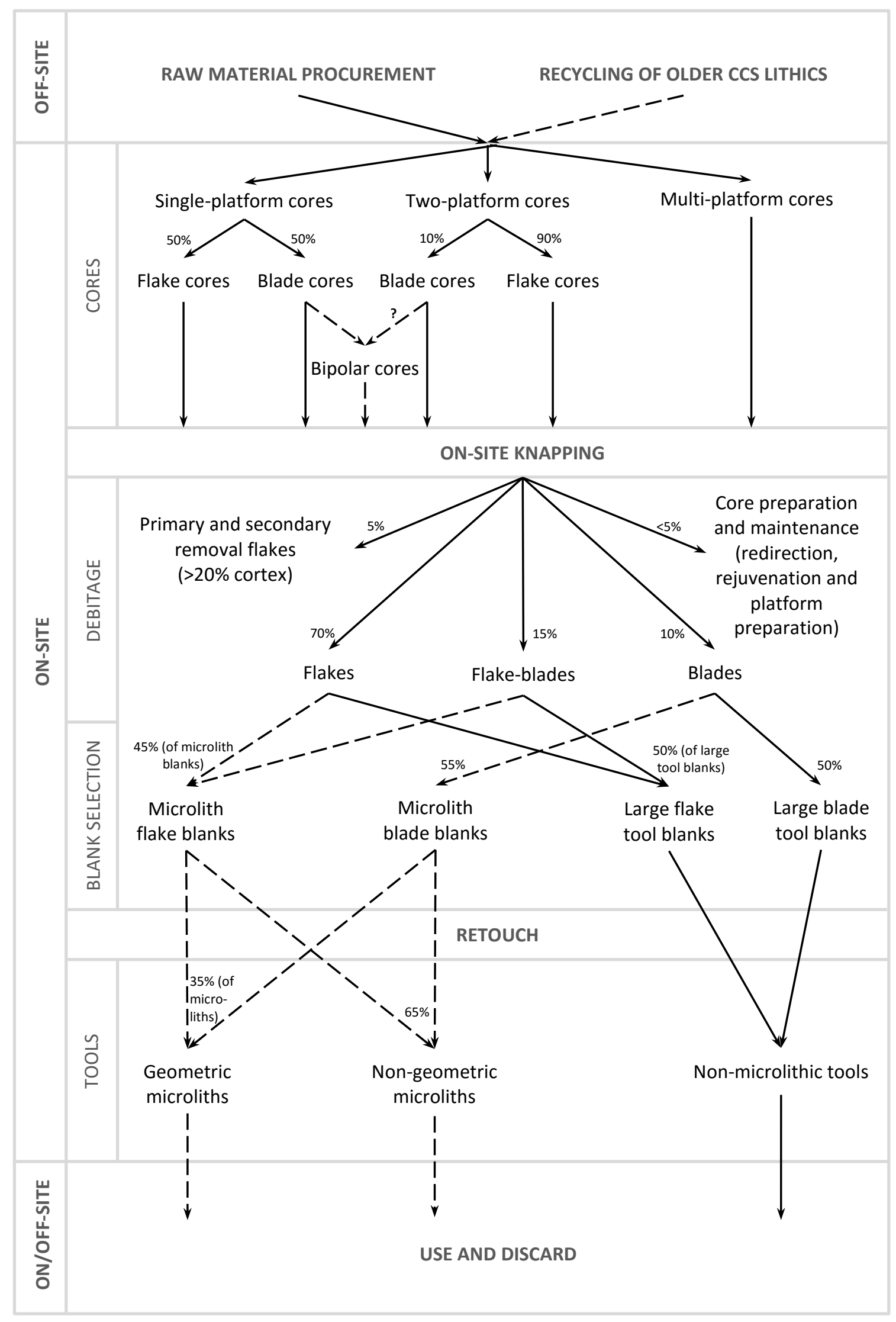


The majority of debitage is of flake dimensions. In addition to the lack of evidence for raw material preferences for flakes and blades, there are also no major differences between flakes and blades in terms of striking platform types and dorsal scar orientations. However, dorsal cortex cover is rarer on blades, and blades exhibit higher densities of dorsal scars on average. Overall, there are some suggestions that blades may have been removed later in reduction processes at NT. However, these indications are not conclusive and are not as strong as seen at RCC.

An additional complicating factor in the distinction between flakes and blades is the fact that flake-blades do not form a clear intermediate category. Flake-blades are intermediate in terms of cortex cover and dorsal scar and parallel arrise densities, but not in absolute numbers of dorsal scars, raw material proportions, dorsal scar orientations, striking platform types or terminations. Possibly this category includes more than one type of debitage product.

The lithic assemblage is small in the layers studied, and so the number of tools is also small. However, both microlithic and non-microlithic tools were recovered. Both tool types are split evenly between flake and blade blanks. Because blades make up only a small proportion of the debitage assemblage, this is indicative of the preferential selection of blades for retouch into tools. Microliths fit within the general size range of available debitage, while non-microlithic tools are much larger. Lithic reduction was aimed at the production of small blanks suitable for retouch into microlithic tools. A few larger blanks at the extreme end of the available size range were also selected for retouch into different tool types. 
A range of tool forms are present within the small tool assemblage. The only geometric microliths present are crescents, which constitute a third of the microlith assemblage. The remainder are non-geometric forms, of which only one is backed. There is one point present in the non-microlithic tool assemblage, with the remainder being side and/or end retouched. Scalar retouch is the most common retouch type on both microlithic and non-microlithic tools. There is more variation in retouch technique in the microlith assemblage, where backing comprises only a quarter of retouch incidences. There is also more variation in retouch outline and location in the microlith assemblage, although as stated above the sample sizes are small.

There are no meaningful changes over time in the core assemblage. In contrast, there are numerous statistically significant differences between layers in the debitage sample. However, most of these differences are weak and do not indicate consistent trends, and can be attributed to small differences in the proportions of quartzite and CCS. There is no evidence for either consistent temporal trends or for the existence of step changes or phases within the layers studied. However, there is evidence for the focussed production of small blanks which were subsequently retouched into microlithic tools, confirming that at least the bottom three layers at NT can be considered part of the HP industry. 


\section{Chapter 6: South Asian Late Palaeolithic microlithic technology}

This chapter presents the results of analyses of lithic assemblages from South Asian Late Palaeolithic sites. Supplementary data referred to in the text is found in Appendix A, denoted by table references beginning with A.

\subsection{Batadomba-LenA, SRI LANKa}

\section{CORES}

The majority of cores are either amorphous multi-directional cores or bipolar cores (Table 6.1.1). Both of these types are common in quartz assemblages, and all but one of the cores are quartz (Figure 6.1.1). Blade cores are rare, and the core assemblage generally implies a heavily flake-dominated core reduction process.

\begin{tabular}{llrr}
\hline Core type & & Frequency & Percentage \\
\hline Uni-directional: & Blade & 2 & 9.5 \\
& Flake & 2 & 9.5 \\
& Bipolar & 7 & 33.3 \\
& & & \\
Bi-directional: & Flake & 2 & 9.5 \\
& & & \\
Multi-directional: & Radial & 2 & 9.5 \\
& Amorphous & 6 & 28.6 \\
\hline Total & & 21 \\
\hline
\end{tabular}

Table 6.1.1: Core type frequencies at Batadomba-lena 
Figure 6.1.1: Raw material proportions of cores at Batadomba-lena

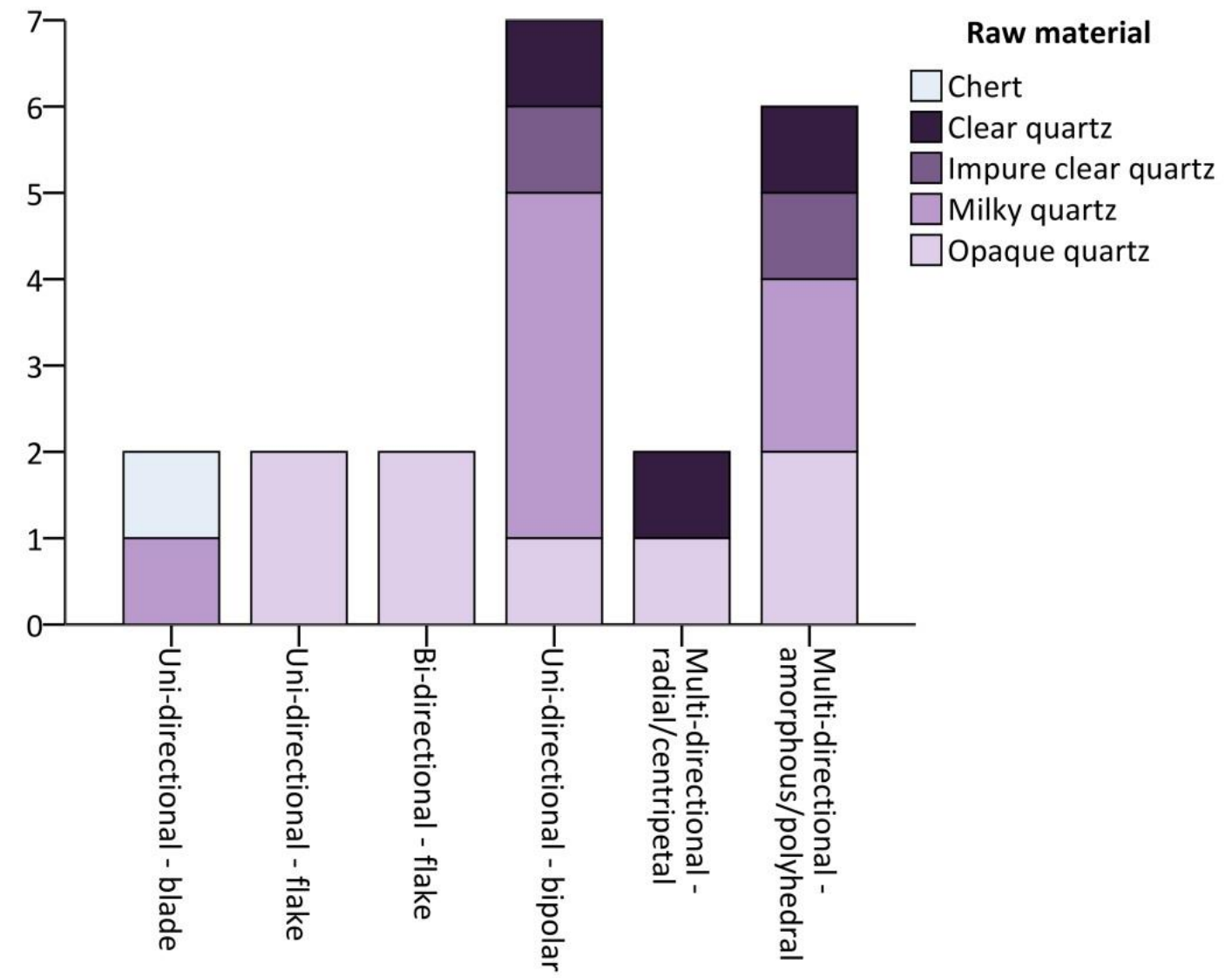

Cores are generally small and squat, with mean dimensions of 24.9 x 26.6 x 17.7 mm (Table A.4.1). There is little variation in core size, with the largest weighing only 27.6 g. Bipolar cores are even smaller (Figure 6.1.2), with mean dimensions of 20.2 x $22.7 \times 15.9 \mathrm{~mm}$. Given their high frequency and small size, bipolar cores may have formed a separate and final stage in the core reduction trajectory, with bipolar percussion being used as a late-stage method for reducing nearlyexhausted cores.

There are no clear patterns in the numbers and directions of striking platforms on different core types, with multi-directional cores being worked from three, four and five platforms (Table A.4.2). Flat striking platforms are most common, with 
complex facetted platforms rare (Figure 6.1.3). Half of the platforms on nonbipolar uni- and bi-directional cores are cortical, while cortex is not seen on the striking platforms of bipolar cores. This provides further evidence that bipolar cores occurred as a later stage of reduction.

Figure 6.1.2: Weight distributions of cores at Batadomba-lena

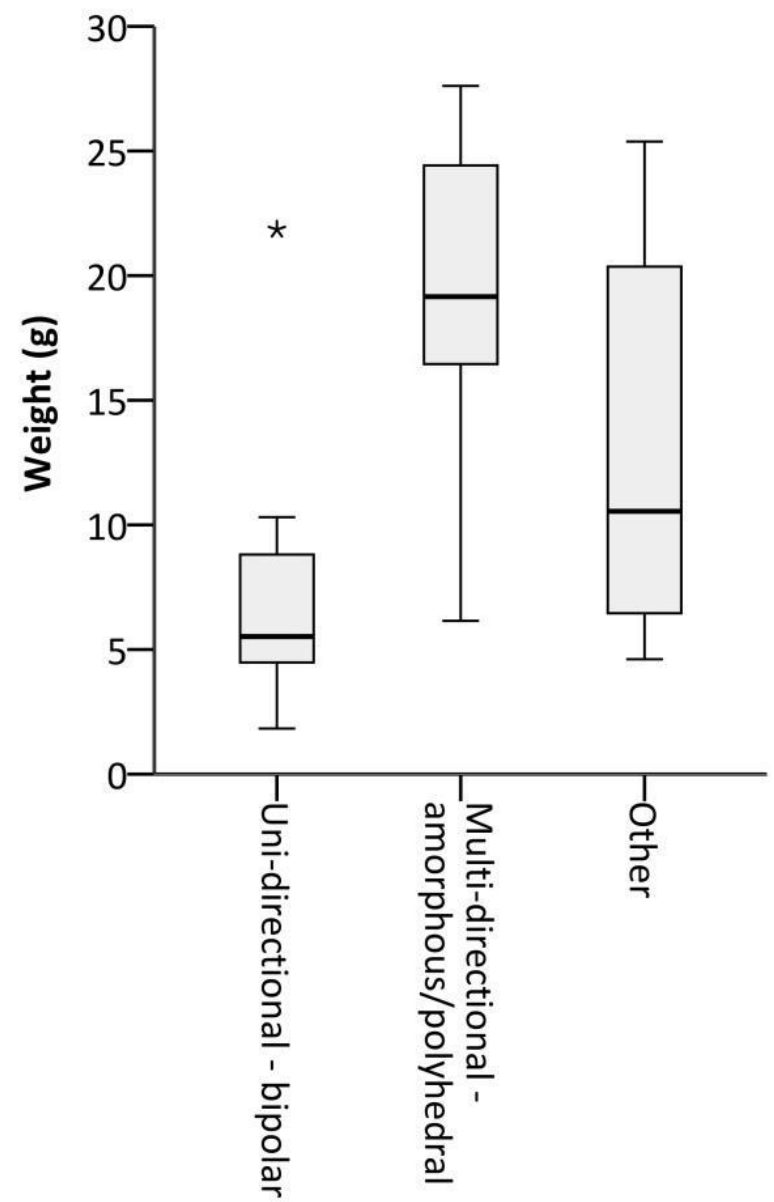

Multi-directional cores exhibit greater numbers of removal scars on average compared to other core types (Figure 6.1.4, Table A.4.3). However, these are almost entirely flake removals. Most terminal removals made on bipolar cores are also of flakes, with $30.4 \%$ of removals being of blade dimensions. Bipolar percussion was primarily used for the removal of flakes from nearly-exhausted 
cores before they were discarded, although more blades were removed through this method of percussion than through the use of freehand knapping.

Figure 6.1.3: Proportions of core striking platform types at Batadomba-lena Striking platform type

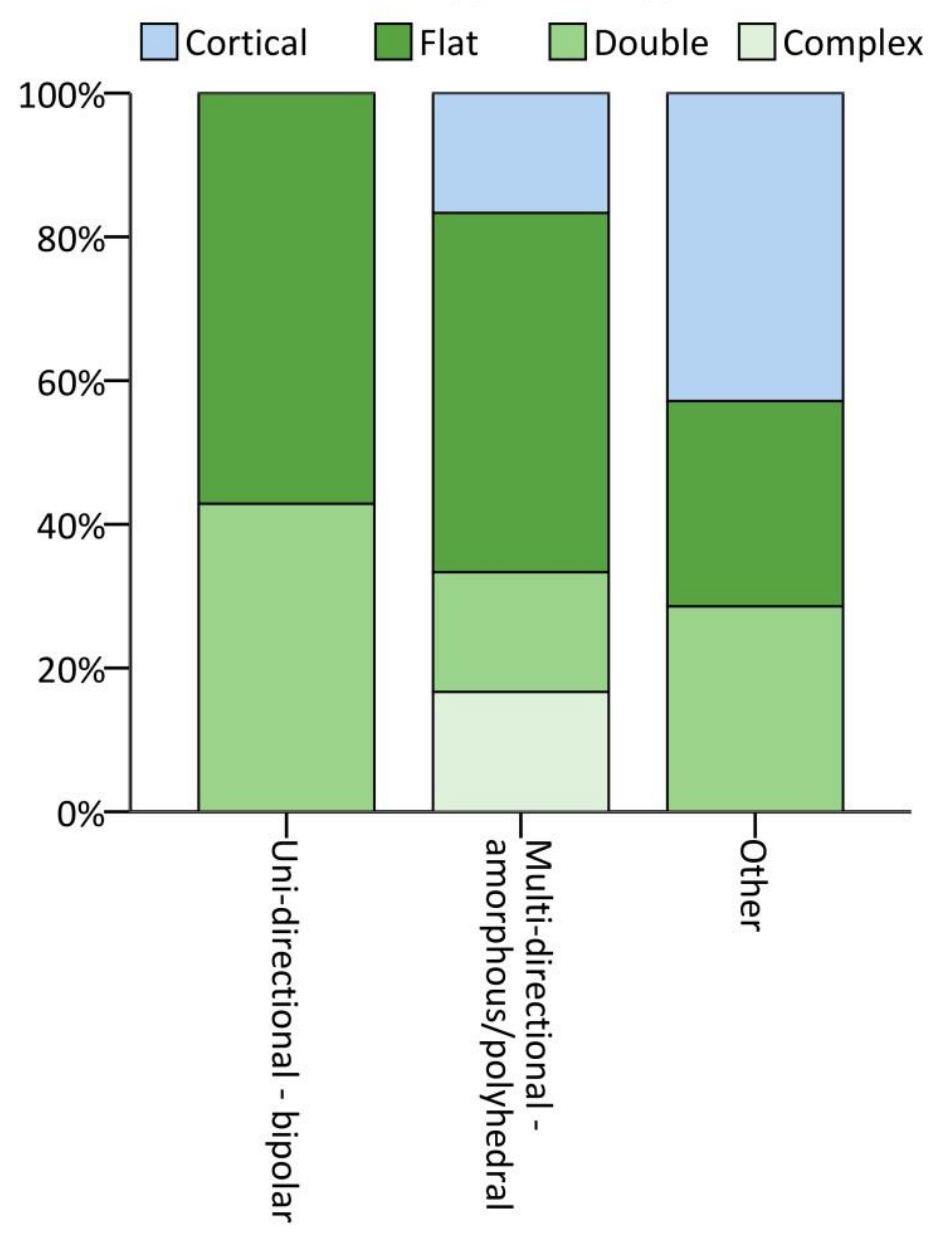

Preferential working of finer qualities of quartz can be seen in the differing degrees of core exhaustion. Discarded cores of high quality quartz (clear and impure clear quartz) have a mode of 8 removal scars with a mean length of $11.9 \mathrm{~mm}$, while those of low quality quartz (milky and opaque quartz) most commonly exhibit 4 removal scars, with a mean length of $18.2 \mathrm{~mm}$. Better qualities of quartz were worked for longer, with more flakes removed until the core was fully exhausted. 
Figure 6.1.4: Average numbers of flake and blade scars on cores at Batadomba-lena

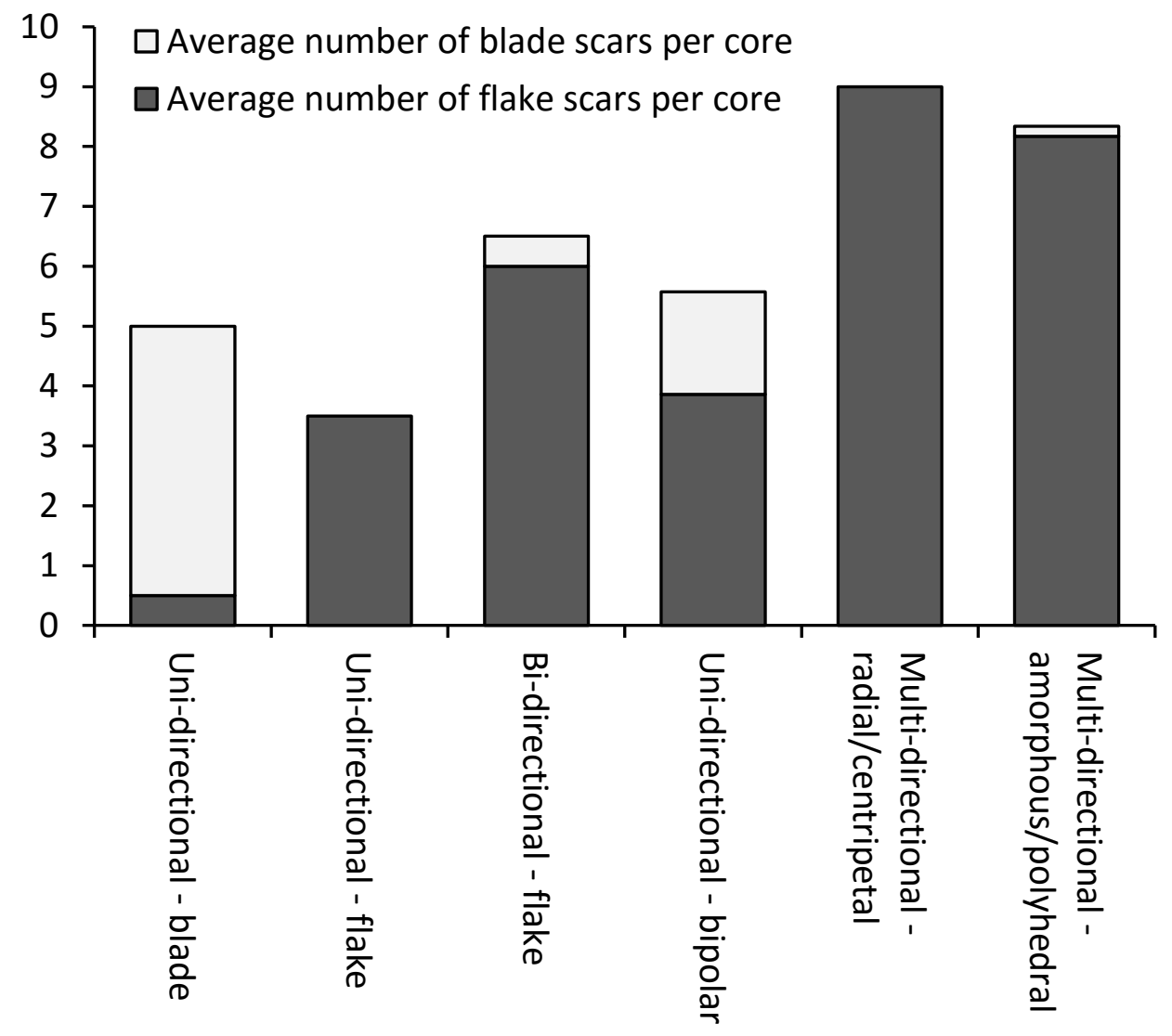

The only two cores with predominantly blade removals also exhibit the largest removal scar lengths on average $(25.7 \mathrm{~mm})$. Blade cores also exhibit the greatest mean $(3.9 \mathrm{~mm})$ and total $(1.9 \mathrm{~cm})$ scar lengths per $1 \mathrm{~cm}^{3}$ core volume (Figure 6.1.5, Table A.4.4). Bipolar cores were also intensively worked in relation to core size, with a mean of 1.1 scars per $\mathrm{cm}^{3}$ core volume compared to $0.5-0.8$ for other core types, confirming that bipolar percussion was utilised as an efficient final stage in core reduction. 
Figure 6.1.5: Ratios of total removal scar length to core volume at Batadomba-lena

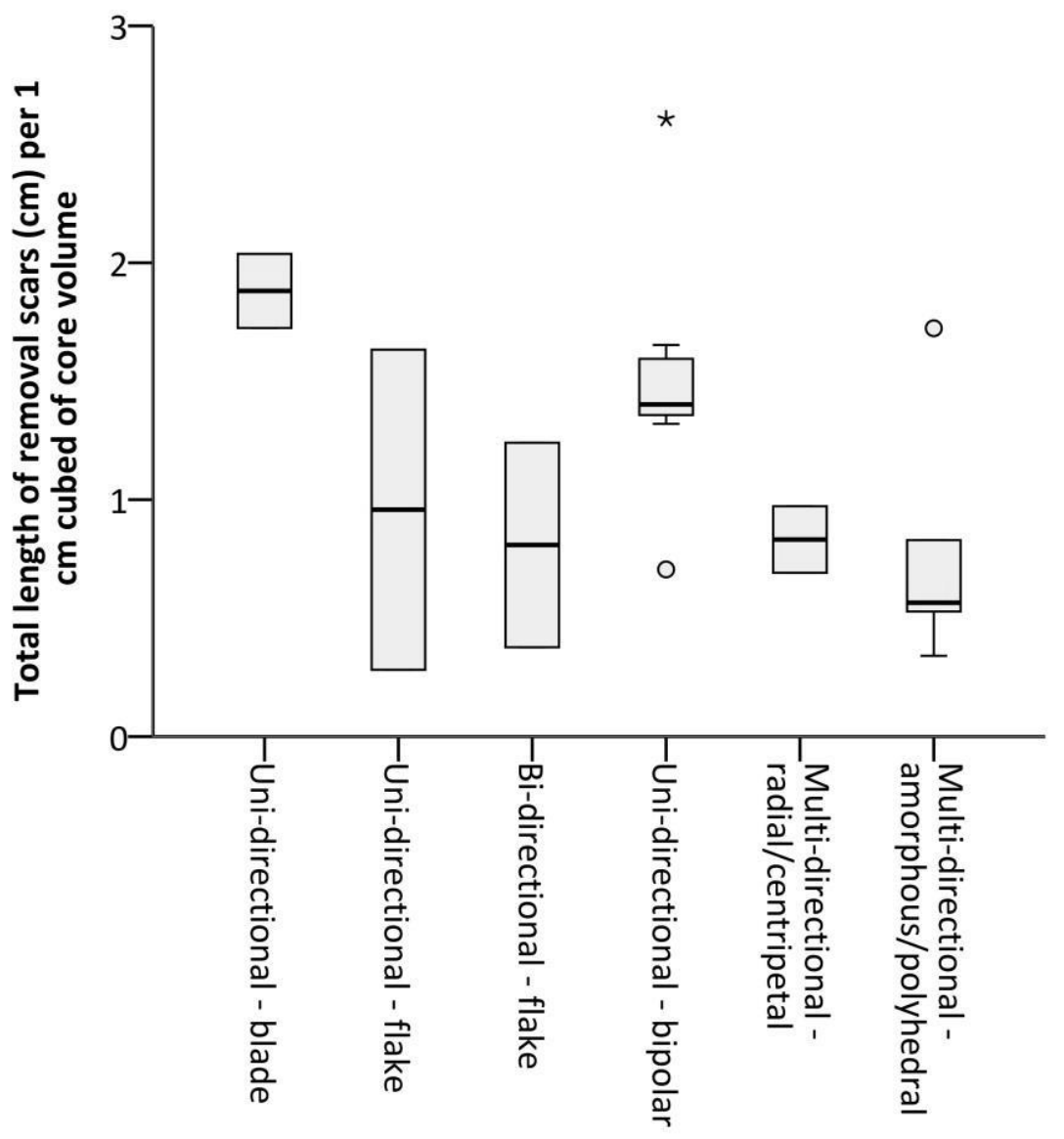

\section{DEBITAGE}

The lithic industry at Batadomba-lena is predominantly flake-based (75.4\% flakes) (Table 6.1.2). Only half of the debitage pieces are whole (49.9\%), with the most common breakage types being proximal (14.0\%) and longitudinal (11.7\%) (Table A.4.5). High proportions of broken lithics are often a feature of quartz assemblages (Knight 1991b, Driscoll 2011). 98.3\% of the debitage sample is quartz, which is locally available in large quantities, with $1.7 \%$ chert, which is not (Table 6.1.2, Figure 6.1.6). The source of the chert is not currently known (N. Perera, pers. comm.). 


\begin{tabular}{lrrrrr}
\hline Raw material & Blades & Flake-blades & Flakes & Total & $\mathbf{\%}$ \\
\hline Clear quartz & 56 & 75 & 352 & 483 & 34.1 \\
Impure clear quartz & 17 & 65 & 295 & 377 & 26.6 \\
Milky quartz & 29 & 64 & 270 & 363 & 25.6 \\
Opaque quartz & 7 & 24 & 111 & 142 & 10.0 \\
Chert & 2 & 5 & 17 & 24 & 1.7 \\
Granular quartz & 1 & 4 & 17 & 22 & 1.6 \\
Smokey quartz & & & 5 & 5 & 0.4 \\
Rose quartz & & & 1 & 1 & 0.1 \\
\hline Total (\%) & $112(7.9)$ & $237(16.7)$ & $1068(75.4)$ & 1417 & \\
\hline
\end{tabular}

Table 6.1.2: Debitage measured at Batadomba-lena

Figure 6.1.6: Raw material proportions of debitage at Batadomba-lena

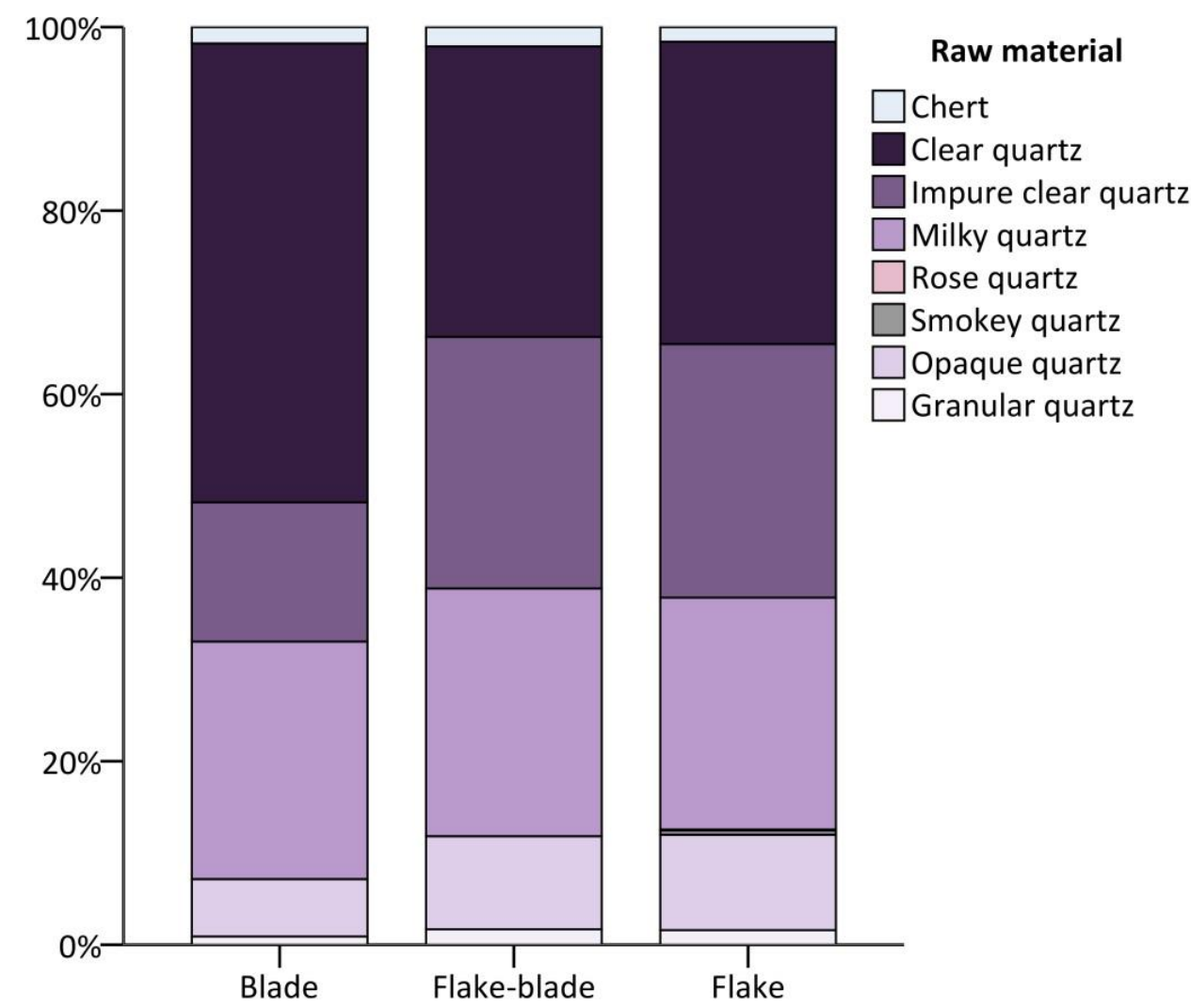

Flakes and flake-blades were made on almost identical raw material proportions

(Figure 6.1.6). High quality quartz was used in higher proportions for the

production of blades, although this relationship is not statistically significant $\left(\chi^{2}(2)\right.$ 
$=1.152, \mathrm{p}=.562)$. Therefore it appears that blades were probably not produced as part of any special process at the site, at least in terms of raw material choices.

The debitage assemblage is generally of very small dimensions, with an average size of whole debitage of $17.6 \times 14.1 \times 4.8 \mathrm{~mm}$ (Table A.4.6). There are no particularly large lithics evident, with the largest dimension of any artefact being 67.1 mm. On average blades are slightly longer and smaller than flakes and flakeblades, and high quality quartz debitage tends to be smaller than that of low quality quartz (Figure 6.1.7). However, there is no significant degree of patterning in size between qualities of quartz or between flakes and blades, further indicating that a single main reduction strategy was utilised at the site. The flake-based nature of the assemblage can be seen in the fact that the mean length:width ratio of whole debitage is only 1:1.4.

Slight or pronounced lipping is evident on $16.4 \%$ of striking platforms, although it is only prominent on $2.3 \%$ of platforms. It is found on both quartz and chert debitage, suggesting that the two raw materials may have been knapped using similar techniques. Flat striking platforms are the most common type (55.7\%) (Figure 6.1.8). The frequent crushing of striking platforms, most likely during the process of bipolar percussion, can also be seen (18.1\%). There is little variation between debitage types in striking platform characteristics, which accords with many of the other variables considered. There is also little variation between raw materials. Overall, there is no indication from the striking platform evidence that quartz and chert were worked in different ways, or that flakes and blades were produced as part of separate reduction trajectories or in separate stages. 
Figure 6.1.7: Cubic volumes of whole debitage on different raw materials at Batadomba-lena

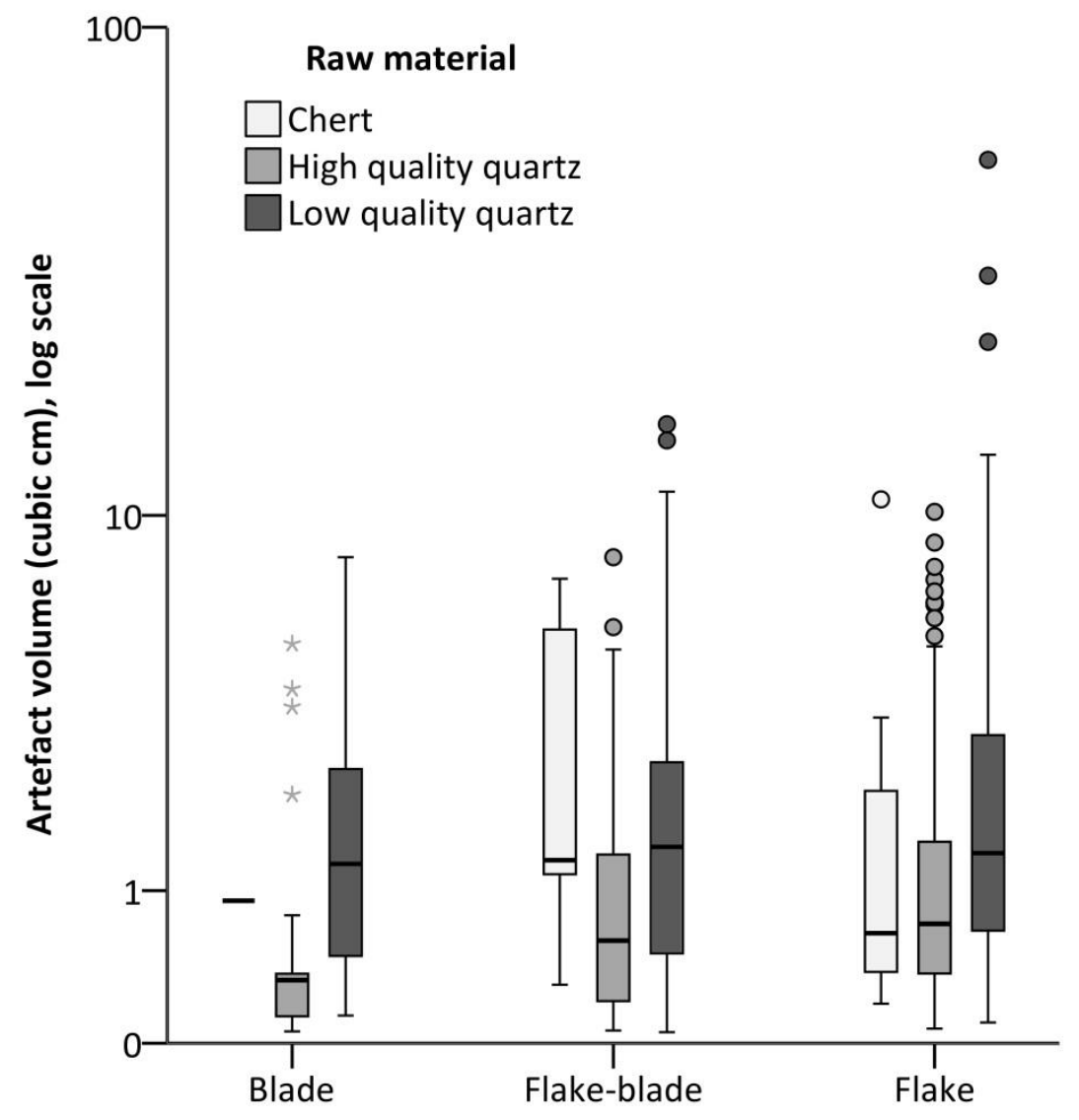

Dorsal surface cortex exists in low frequencies and proportions at the site; only

$3.1 \%$ of the total debitage assemblage exhibits over $50 \%$ dorsal cortex cover (Table A.4.7). While river-worn quartz cobbles do exhibit changes in surface texture and colour which can be described as cortex, vein quartz which erodes out of the gneiss bedrock in the site locality in flat or tabular form does not display such cortex. It may therefore be hypothesised that multiple sources of quartz were utilised in the local environment, including the stream adjacent to the rockshelter as well as locations where tabular quartz was available as part of the erosional facies. Alternatively, primary stage core reduction may have taken place off-site, resulting in under-representation of early-stage removal flakes within the rockshelter. 
Figure 6.1.8: Proportions of debitage striking platform types at Batadomba-lena Striking platform type

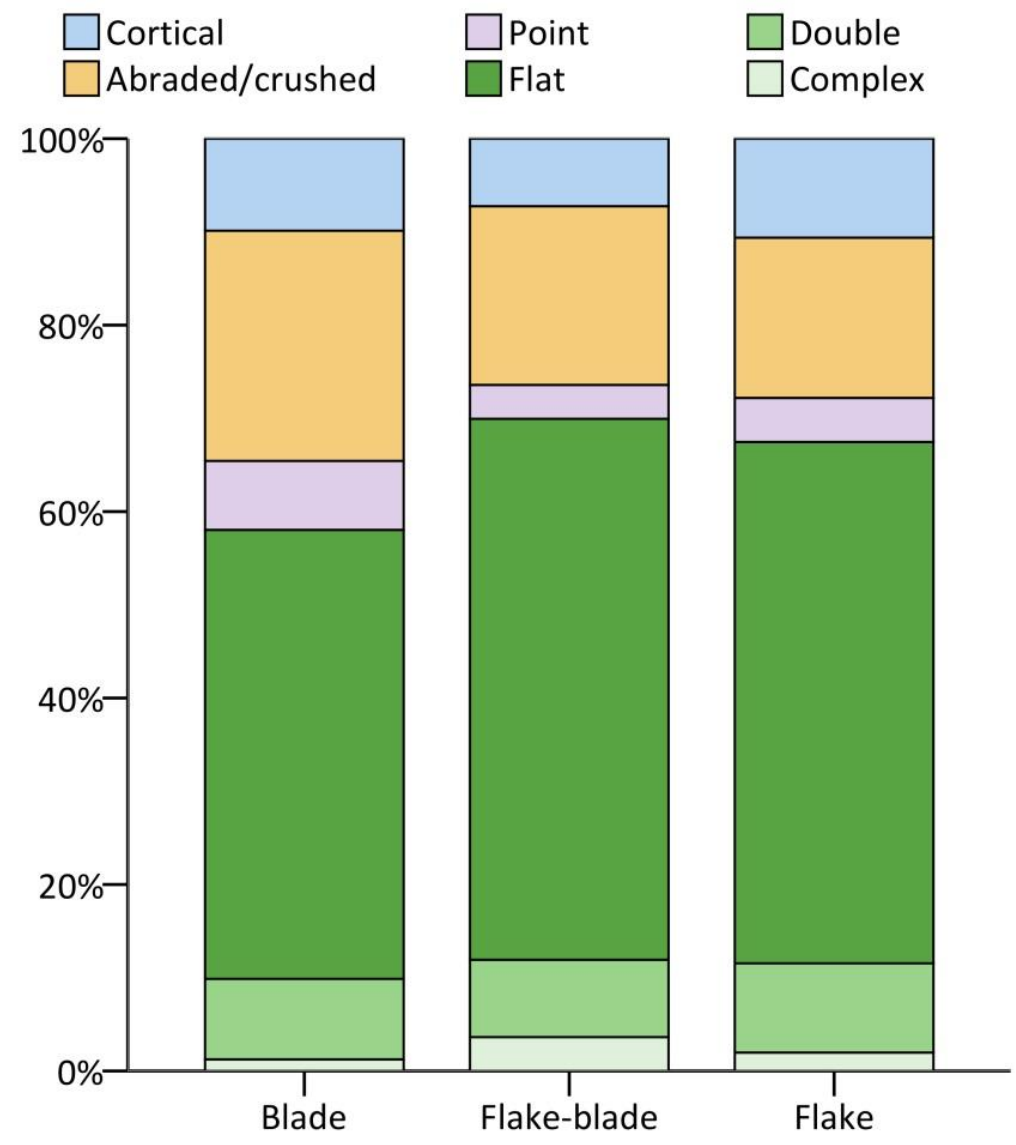

Whole flakes, blades and flake-blades have almost identical numbers of dorsal scars on average (2.6 for blades, 2.7 for flakes and flake-blades) (Table A.4.8). When debitage size is taken into account, blades exhibit slightly higher scar densities (2.3 scars per $\mathrm{cm}^{2}$, compared to 1.7 for flake-blades and 1.4 for flakes). It is therefore possible that blades were removed somewhat later on average in the reduction sequence, when cores had already been heavily reduced. Alternatively, differences in scar densities may be related to the fact that blades were more commonly produced on higher quality quartz, which exhibits higher scar densities on average compared to lower quality quartz (1.79 scars per $\left.1 \mathrm{~cm}^{2} \mathrm{v} .1 .04\right)$. Clear quartz contains fewer internal crystal boundaries, allowing for greater control over and predictability of the size and shape of the final product (Knight 1991b). It 
should therefore be expected that clear quartz would be knapped and worked more intensively.

Over three quarters of debitage exhibits scars made from a single direction (Table 6.1.3). Scars made from more than one direction are more common on flakes than on other debitage types. Of debitage exhibiting scars originating from two directions, these are primarily from opposite directions on blades and from adjacent or perpendicular directions on flakes, suggesting one possible area of difference between the debitage categories.

\begin{tabular}{l|rr|rr|rr|rr|rr|r}
\hline \multicolumn{1}{c}{$\begin{array}{c}\text { Dorsal scar } \\
\text { orientation: }\end{array}$} & \multicolumn{1}{c}{$\mathbf{c}$} & \multicolumn{9}{c}{$\mathbf{2}$} & \multicolumn{2}{c}{} & & \\
Type & No. & $\%$ & No. & \% & No. & \% & No. & \% & No. & \% & Total \\
\hline Blade & 39 & 83.0 & 3 & 6.4 & 5 & 10.5 & & & & & 47 \\
Flake-blade & 128 & 87.1 & 4 & 2.7 & 13 & 8.8 & 2 & 1.4 & & & 147 \\
Flake & 382 & 74.5 & 70 & 13.6 & 37 & 7.2 & 14 & 2.7 & 1 & 0.2 & 504 \\
\hline Total (\% of total) & 549 & 78.7 & 77 & 11.0 & 55 & 7.9 & 16 & 2.3 & 1 & 0.1 & 698 \\
\hline
\end{tabular}

Table 6.1.3: Dorsal scar orientation frequencies on whole debitage at Batadombalena

Feather terminations are the most common termination type (Figure 6.1.9). There are no significant differences between debitage types. The relatively high frequencies of axial and crushed terminations are indicative of the use of bipolar percussion. Overall, $11.0 \%$ of debitage exhibits substantial evidence of bipolar percussion.

There is minimal evidence for core maintenance in the debitage sample. Small numbers of core rejuvenation and redirection flakes and platform preparation 
flakes are evident (Table 6.1.4). A single clear quartz crested blade was also found, which can be considered indicative of 'laminar' blade removal. However, the lack of other proxies for this method of blade removal, plus the general scarcity of blades, suggests that this was not a common manufacturing technique at the site.

Figure 6.1.9: Proportions of debitage termination types at Batadomba-lena

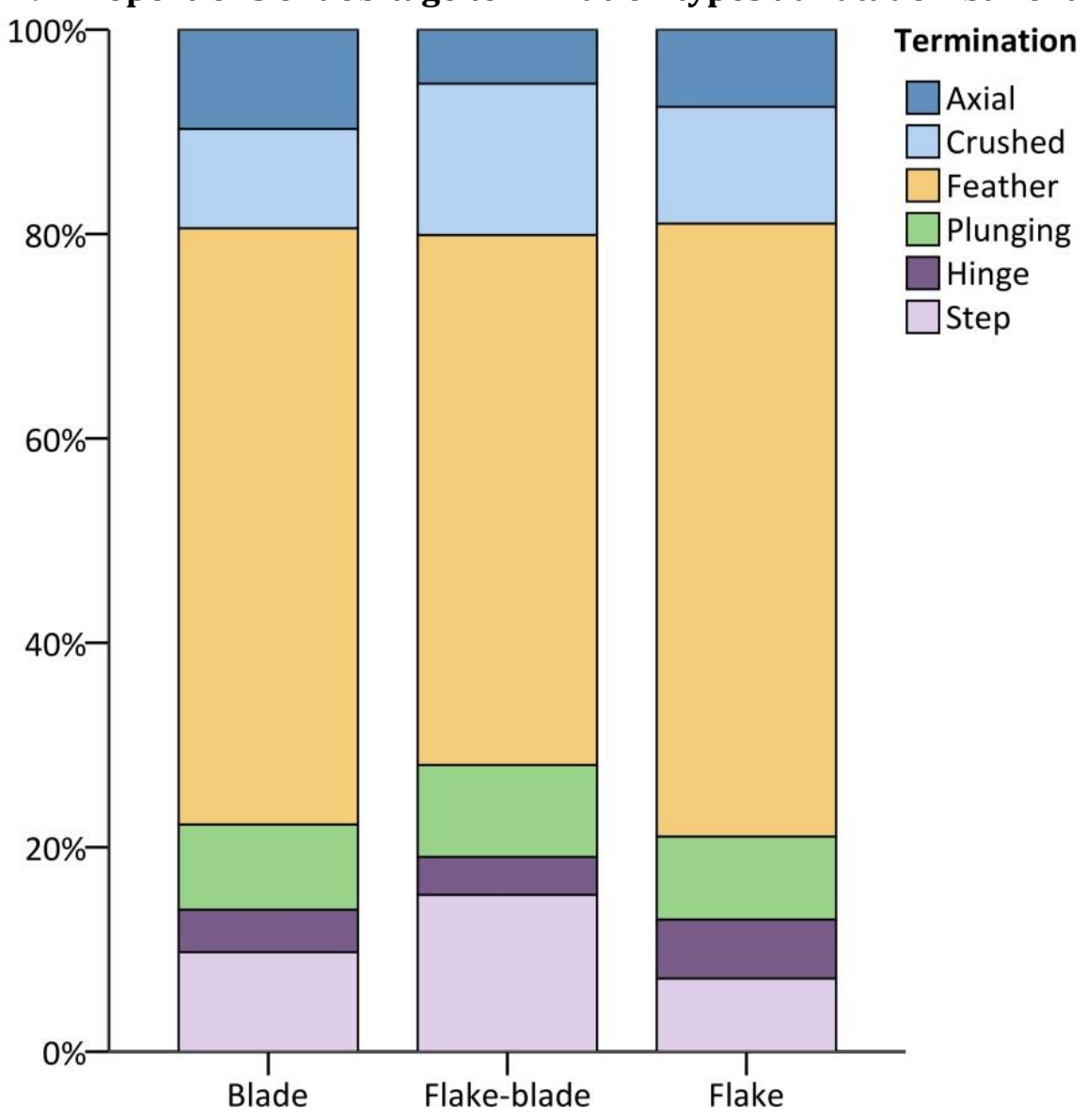

\begin{tabular}{l|cc|rr|rrrrr}
\hline & \multicolumn{1}{c}{ Blades } & \multicolumn{1}{c}{ Flake-blades } & \multicolumn{3}{c}{ Flakes } & \multicolumn{3}{c}{ Total } \\
Maintenance flake type & No. & $\mathbf{\%}$ & No. & $\mathbf{\%}$ & No. & $\mathbf{\%}$ & No. & $\mathbf{\%}$ \\
\hline Platform preparation flake & & & 2 & 0.8 & 35 & 3.3 & 37 & 2.6 \\
Core rejuvenation flake & & & 3 & 1.3 & 22 & 2.1 & 25 & 1.8 \\
Core redirection flake & 2 & 1.8 & 1 & 0.4 & 5 & 0.5 & 8 & 0.6 \\
Crested blade & 1 & 0.9 & & & & & 1 & 0.1 \\
\hline Total & 3 & 2.7 & 6 & 2.5 & 62 & 5.9 & 71 & 5.1 \\
\hline
\end{tabular}

Table 6.1.4: Frequencies of core maintenance debitage at Batadomba-lena 
TOOLS

Tool blanks were intentionally selected from the better qualities of quartz. For example, $87.1 \%$ of microliths and $50.0 \%$ of non-microlithic tools were made on clear quartz blanks (Table 6.1.5, Figure 6.1.10), whereas these form only $34.1 \%$ of the debitage sample. No retouched chert was found in the tool assemblage.

\begin{tabular}{lrrrrrrr}
\hline Raw material & $\begin{array}{r}\text { Microliths } \\
\text { on blades }\end{array}$ & $\begin{array}{r}\text { Microliths } \\
\text { on flakes }\end{array}$ & $\begin{array}{r}\text { Retouched } \\
\text { blades }\end{array}$ & $\begin{array}{r}\text { Retouched } \\
\text { flakes }\end{array}$ & Total & \% \\
\hline Clear quartz & 74 & 61 & 1 & 3 & 139 & 85.3 \\
Impure clear quartz & 6 & 13 & 1 & 1 & 21 & 12.9 \\
Milky quartz & & 1 & 1 & 1 & 3 & 1.8 \\
\hline Total & 80 & 75 & 3 & 5 & 163 & \\
\hline
\end{tabular}

Table 6.1.5: Tools measured at Batadomba-lena

Figure 6.1.10: Raw material proportions of tools at Batadomba-lena

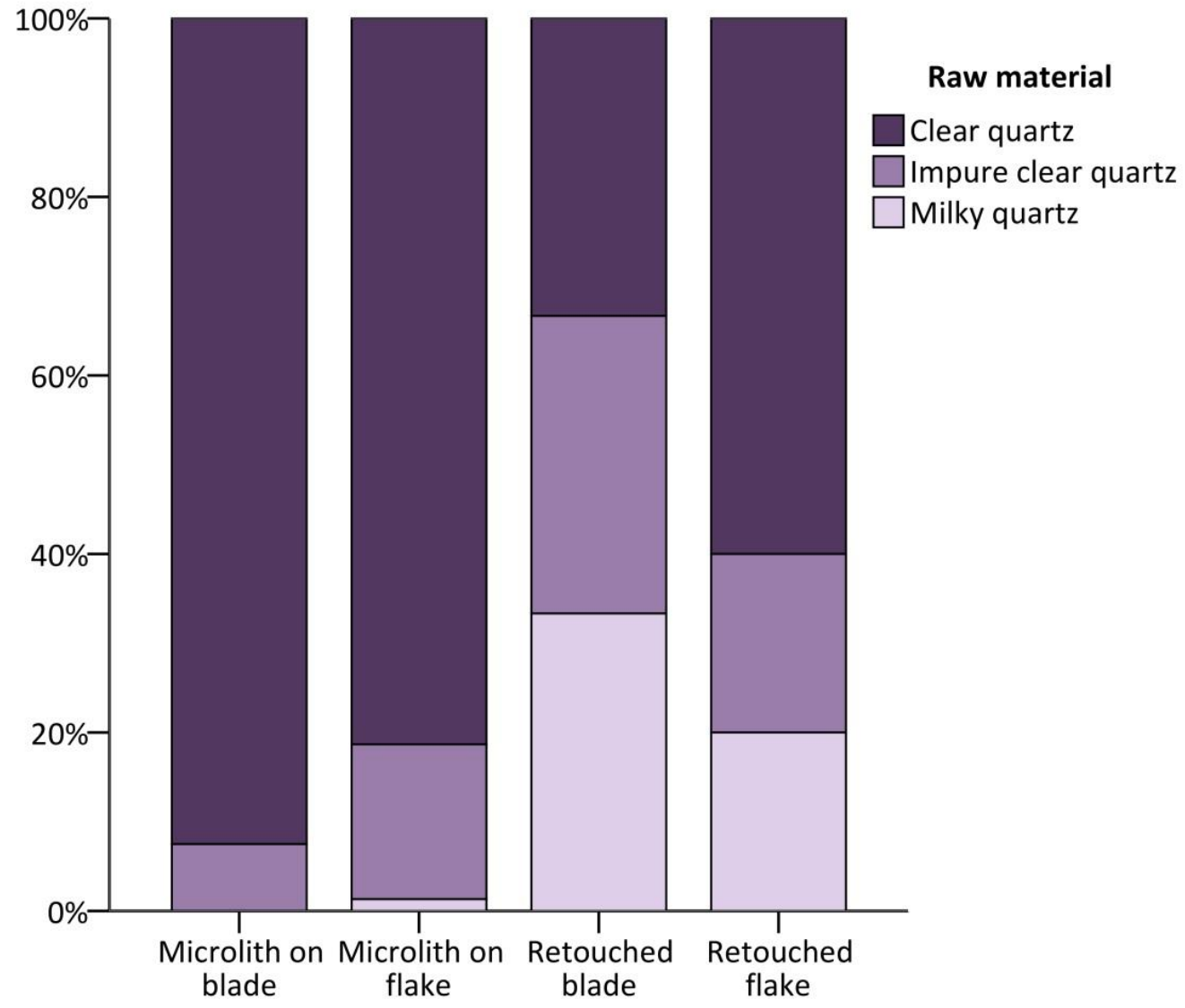


Blades were also selected disproportionately for tool blanks. 51.6\% of microliths and $37.5 \%$ of non-microlithic tools were made on blade blanks (Table 6.1.5), whereas these constitute only $7.9 \%$ of the debitage assemblage. Although blades do not appear to have been produced as part of a distinct manufacturing process at the site, they were more likely to be selected for retouch.

The average size of microliths at the site is very small - 17.0 × $8.9 \times 3.4 \mathrm{~mm}$ (Table A.4.9). The smallest whole microlith measures only $7.4 \times 3.3 \times 1.8 \mathrm{~mm}$, yet was also backed along most of the extent of both lateral edges. However, as previously noted by Perera et al. (2011), the small size of microliths accords with the small size of debitage produced at the site. Rather than intentionally selecting only the smallest potential tool blanks for retouch into microliths, the entire system of lithic reduction at Batadomba-lena was geared towards the production of very small flakes and blades (Figure 6.1.11). This had the effect of widening the base population of lithics from which blanks could be selected, providing a greater range of blank choices.

Non-microlithic tools are uncommon at the site. All of those in the current sample are broken, problematising a clear understanding of their size profiles in relation to both microlithic tools and the debitage sample (data provided in Table A.4.10). Overall, non-microlithic tools were produced in small numbers, and most likely opportunistically as lithic reduction appears to have been conducted with the primary aim of producing microlithic tools. 
Figure 6.1.11: Lengths of whole lithics at Batadomba-lena

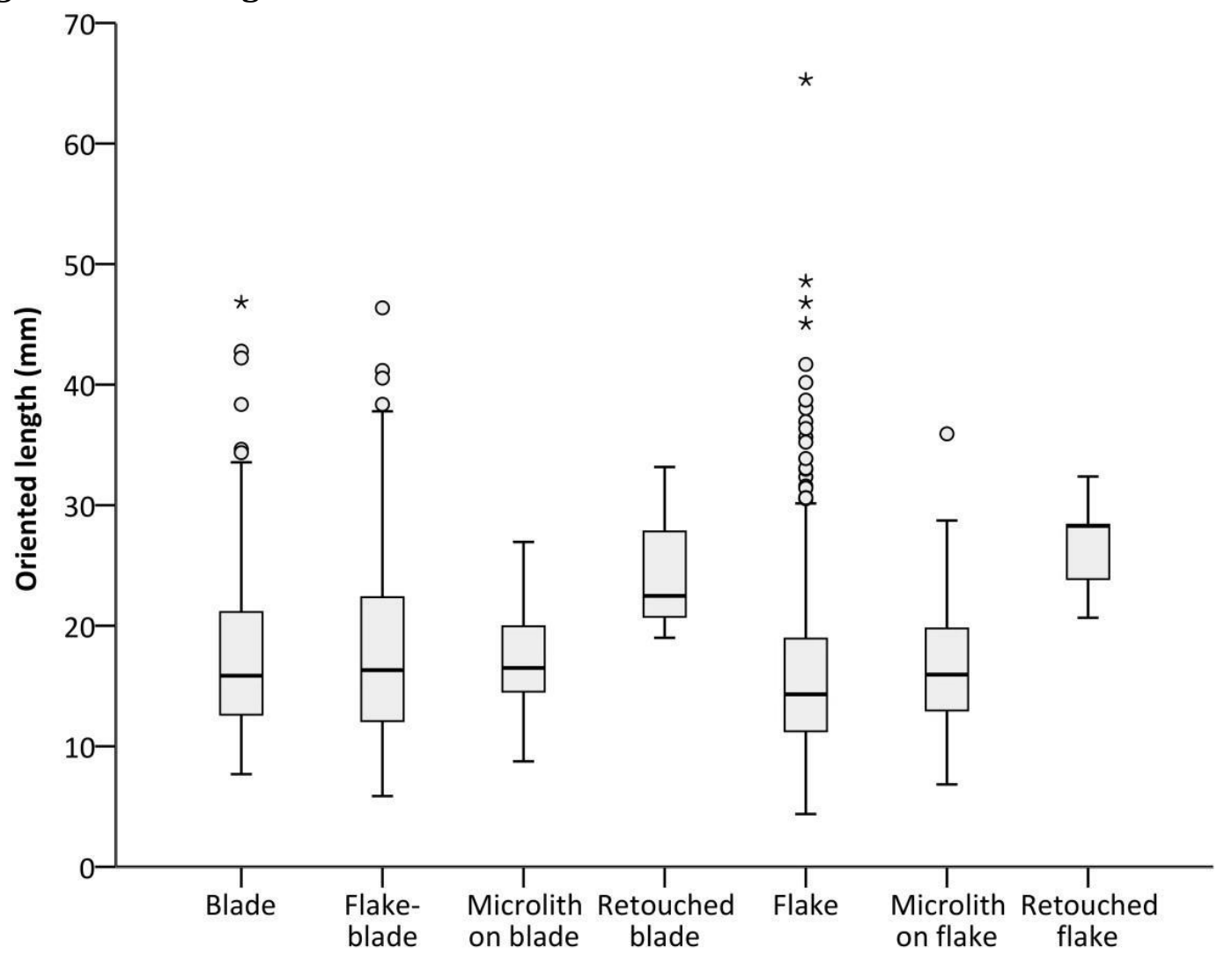

A wide variety of microlith forms are evident (Figure 6.1.12), with backed nongeometric points $(33.5 \%)$ and crescents $(18.1 \%)$ being the most common types (Table 6.1.6). Only $25.2 \%$ of microliths are geometric. Many other morphologies are found in small numbers, including several varieties retouched separately along both lateral margins, as well as various geometric and non-geometric forms exhibiting notches, shoulders or tangs. One example was found in Layer $7 \mathrm{c}$ of the tanged and bifacially worked Balangoda point, previously noted by Perera (2010) (Figure 6.1.13).

There are no preferences evident in the use of particular qualities of quartz for particular microlith morphologies $\left(\chi^{2}(22)=16.302, \mathrm{p}=.801\right)$. However, there is a strong and statistically significant relationship between blank type and microlith 
morphology $\left(\chi^{2}(11)=42.196, \mathrm{p}<.001\right.$, Cramér's $\left.V=.522\right)$. Blade blanks were preferred for backed non-geometric forms (68.6\% blade blanks) and non-backed non-geometric points (90.0\%), while flake blanks were preferred for other nonbacked non-geometric forms (91.7\% flake blanks) and geometric microliths $(69.6 \%)$.

Figure 6.1.12: Examples of microliths from Batadomba-lena, demonstrating their range of shapes and small sizes

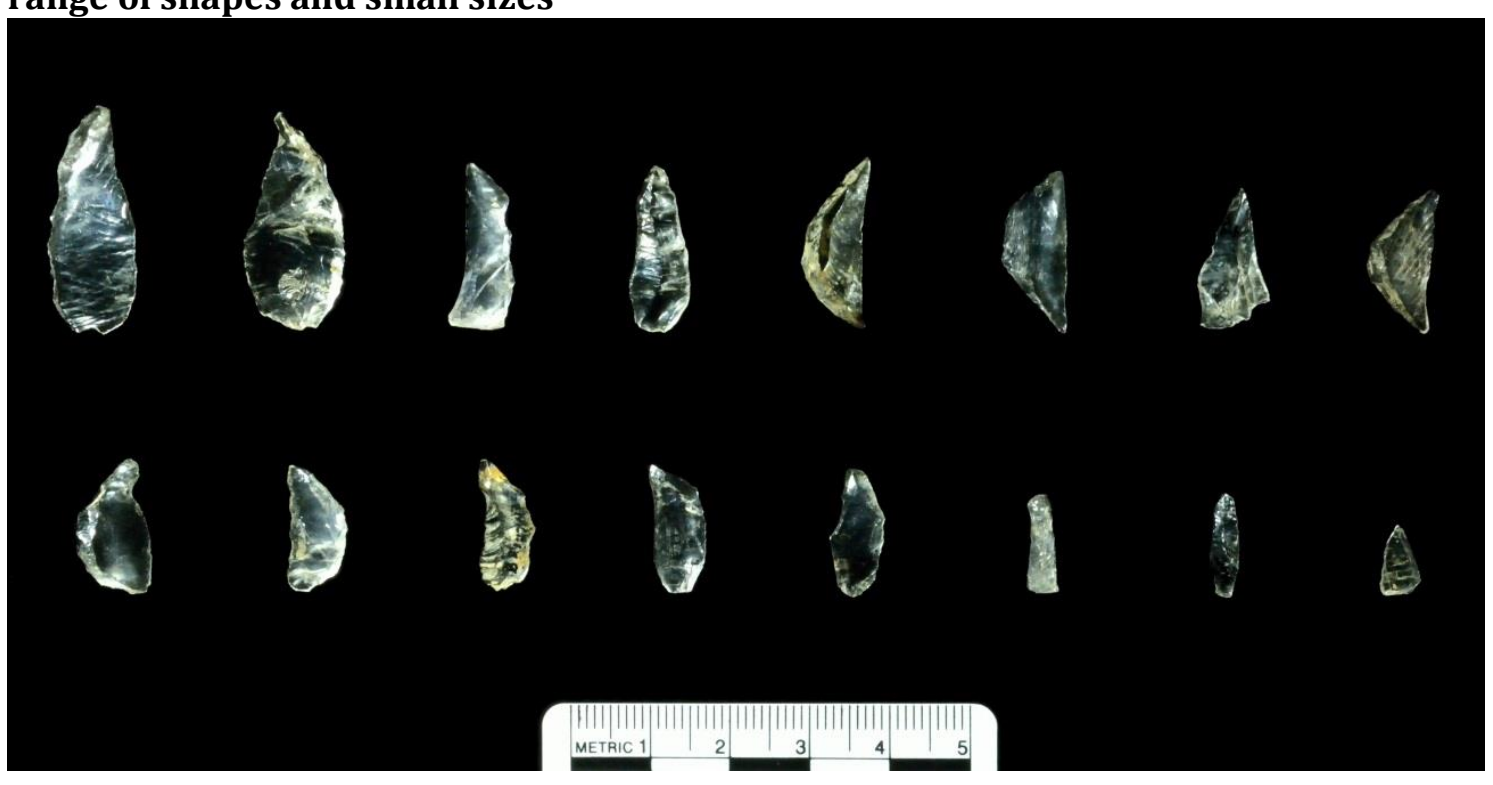

\begin{tabular}{llr}
\hline Coarse typology & Narrow typology & Frequency \\
\hline Microliths: & & \\
Backed non-geometric microlith & Backed & 12 \\
& Backed and side retouched & 3 \\
& Double backed & 1
\end{tabular}

Backed non-geometric microlith point Backed

Double and side retouched

Double backed

Crescent microlith

Crescent 
Non-geometric microlith point

Concave point ('awl') 1

Other

9

Notched microlith

Rectangle microlith

Shouldered/tanged microlith

Balangoda point 1

Shouldered 3

Shouldered and backed point 1

Shouldered with notch 1

Tanged and backed point 1

Tanged point 1

Trapeze microlith 1

$\begin{array}{lr}\text { Triangle microlith } & 7\end{array}$

Total

Non-microlithic tools:

Burin

Notched

Side retouched

Double side retouched 1

Side retouched 4

Total

8

Table 6.1.6: Tool type frequencies at Batadomba-lena

The non-microlithic tool assemblage is small $(n=8)$ and mostly contains side retouched pieces, although it also includes two notched pieces and an artefact exhibiting a burin removal (Table 6.1.6). The small size of the sample precludes further analysis of raw material or blank preferences. 
Figure 6.1.13: Dorsal and ventral faces of the Balangoda point from Batadomba-lena

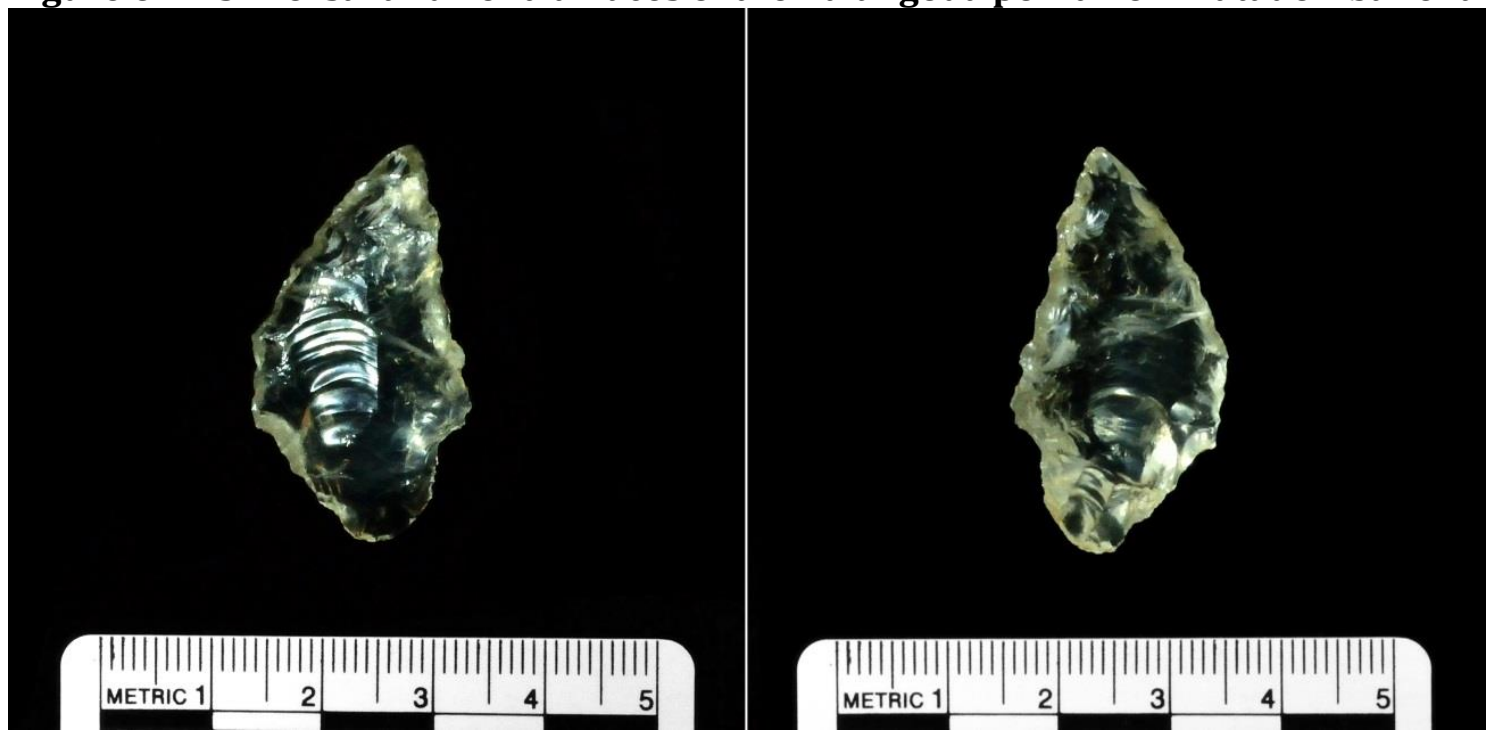

There is little variation in size between microlith forms, with the exception of the relatively large Balangoda point (measuring 35.9 x 19.5 x $7.6 \mathrm{~mm}$ ), further confirming that this is a distinct microlith type (Figure 6.1.14, Table A.4.11). Overall, however, there is little convincing evidence for standardisation in microlith dimensions.

There is also no evidence for standardisation of TCSA measurements. Average TCSA measurements vary considerably between types, although with the exception of the Balangoda point they are all similar to or smaller than Shea's (2006) arrowhead sample, with mean values ranging from 9.4-40.8 $\mathrm{mm}^{2}$ (Table A.4.12). Some are extremely small; the smallest TCSA measurement for a nonbroken microlith is $2.4 \mathrm{~mm}^{2}$. The Balangoda point has a TCSA of $73.7 \mathrm{~mm}^{2}$, which is larger than Shea's dart tip sample. Aside from further indicating the distinctness of this type, this may be indicative of a different function or hafting configuration for similar points. 
Figure 6.1.14: Cubic volumes of microlith types at Batadomba-lena

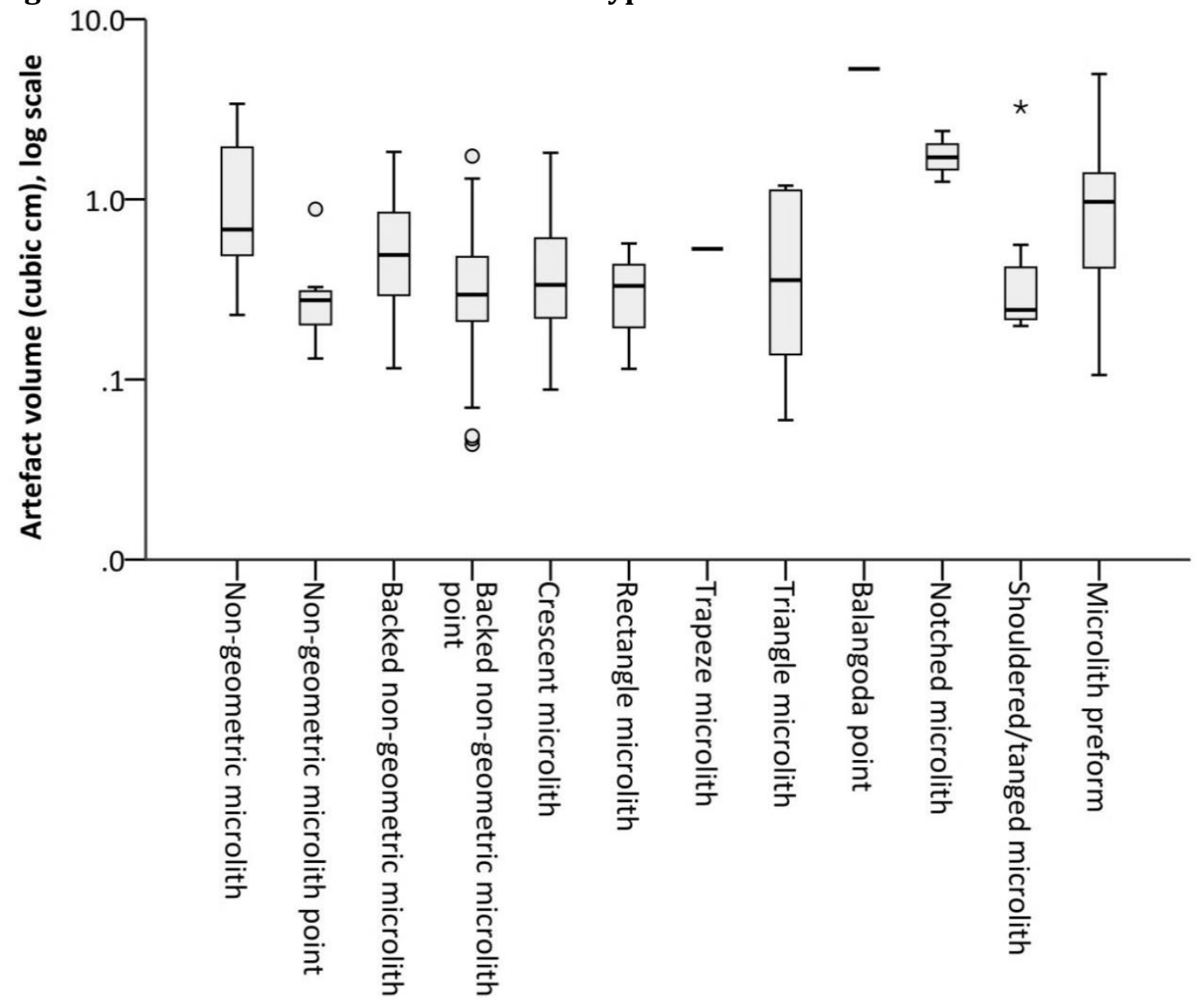

The shaping of shoulders or tangs to facilitate hafting is evident but uncommon (5.2\% of the microlith assemblage). Five microliths are shouldered and three are tanged. Shoulder and tang sizes vary and are not standardised, with mean dimensions of $5.6 \times 2.2 \times 5.7 \mathrm{~mm}$ (Table A.4.13). A variety of shouldered and tanged forms are found, including those exhibiting backing, notches or bifacial working (in the case of the Balangoda point). Microliths with obviously shaped haftable appendages were not manufactured to a standardised template. Instead, a variety of shaping and retouch techniques were utilised.

More than half of retouch applied to microlithic tools is backing retouch (59.8\%) (Figure 6.1.15), 60.2\% of which is bipolar backing. In addition to backed non- 
geometric microliths, 35 out of 39 geometric microliths (89.7\%) exhibit backing. A wide variety of other retouch techniques were also utilised. Thinning removals and sub-parallel, scalar and irregular retouch are particularly common on microlith preforms and non-geometric microliths. Notches are found on a variety of microlith types, including non-geometrics, backed non-geometric points, crescents, and shouldered/tanged forms. Overall, there is considerable variation in the techniques used to retouch different microlith types.

Figure 6.1.15: Retouch techniques on microliths at Batadomba-lena

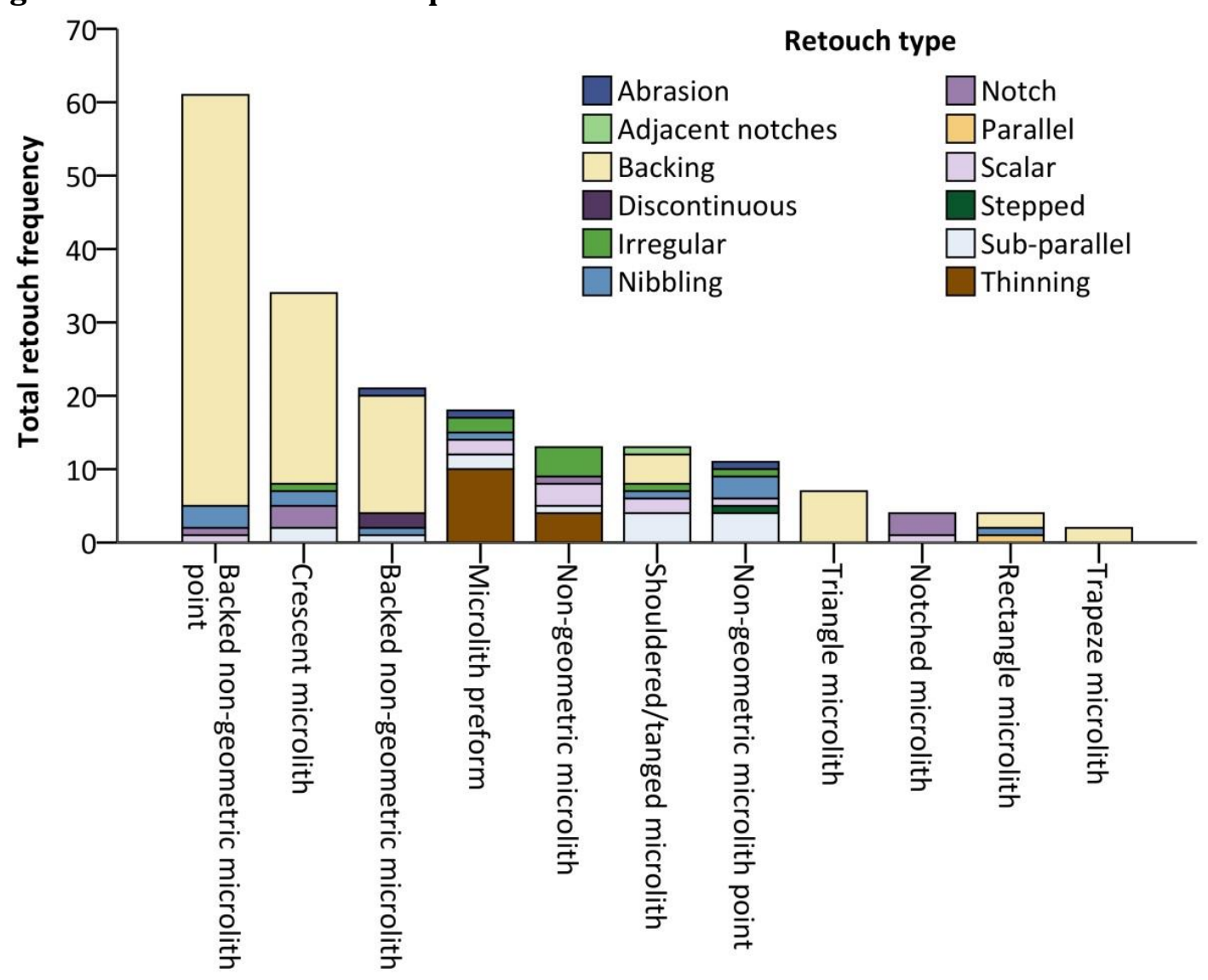

Backing retouch is not found in the non-microlithic tool assemblage, nor are several other retouch techniques that were used to manufacture microliths. A considerable variety of techniques was noted, however, given the small size of the 
non-microlithic tool sample (Figure 6.1.16). Different retouch types were used for blade tools (irregular, parallel and sub-parallel retouch) and flake tools (notches, adjacent notches, burination and scalar and stepped retouch), although this may be an artefact of small sample size $(\mathrm{n}=8)$.

Figure 6.1.16: Retouch techniques on non-microlithic tools at Batadomba-lena

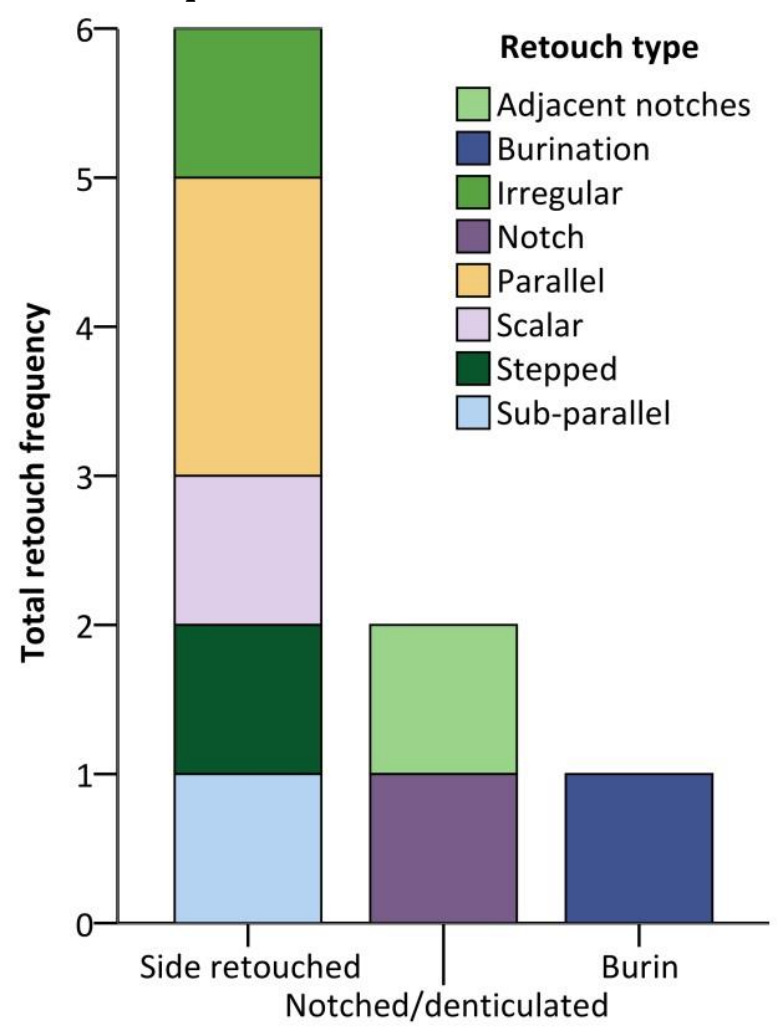

Most tools are worked along a single lateral edge, although many tools $(20.6 \%$ of microliths and $12.5 \%$ of non-microlithic tools) are worked along two distinct margins (Table A.4.14). Dorsal (38.8\%) and edge (37.8\%) retouch are the most common types on microlithic tools. The relatively high proportion of ventral retouch on microliths (19.1\%) is due to the frequent creation of thin, invasive thinning removals on the ventral face to remove the bulb of percussion. Convex (41.0\%) and straight (36.7\%) edges are the most common outlines overall. 
There is no evidence for standardisation in retouch measurements within tool types, with the exception of a certain degree of standardisation in the angles of backing retouch on microlithic tools (Table A.4.15). Overall, backing retouch varies between $65^{\circ}$ and $90^{\circ}$, with a mean of $82^{\circ}$. The high prevalence and angle of backing retouch explains what could almost be described as a bi- or tri-modal distribution of retouch angles on microlithic tools (Figure 6.1.17).

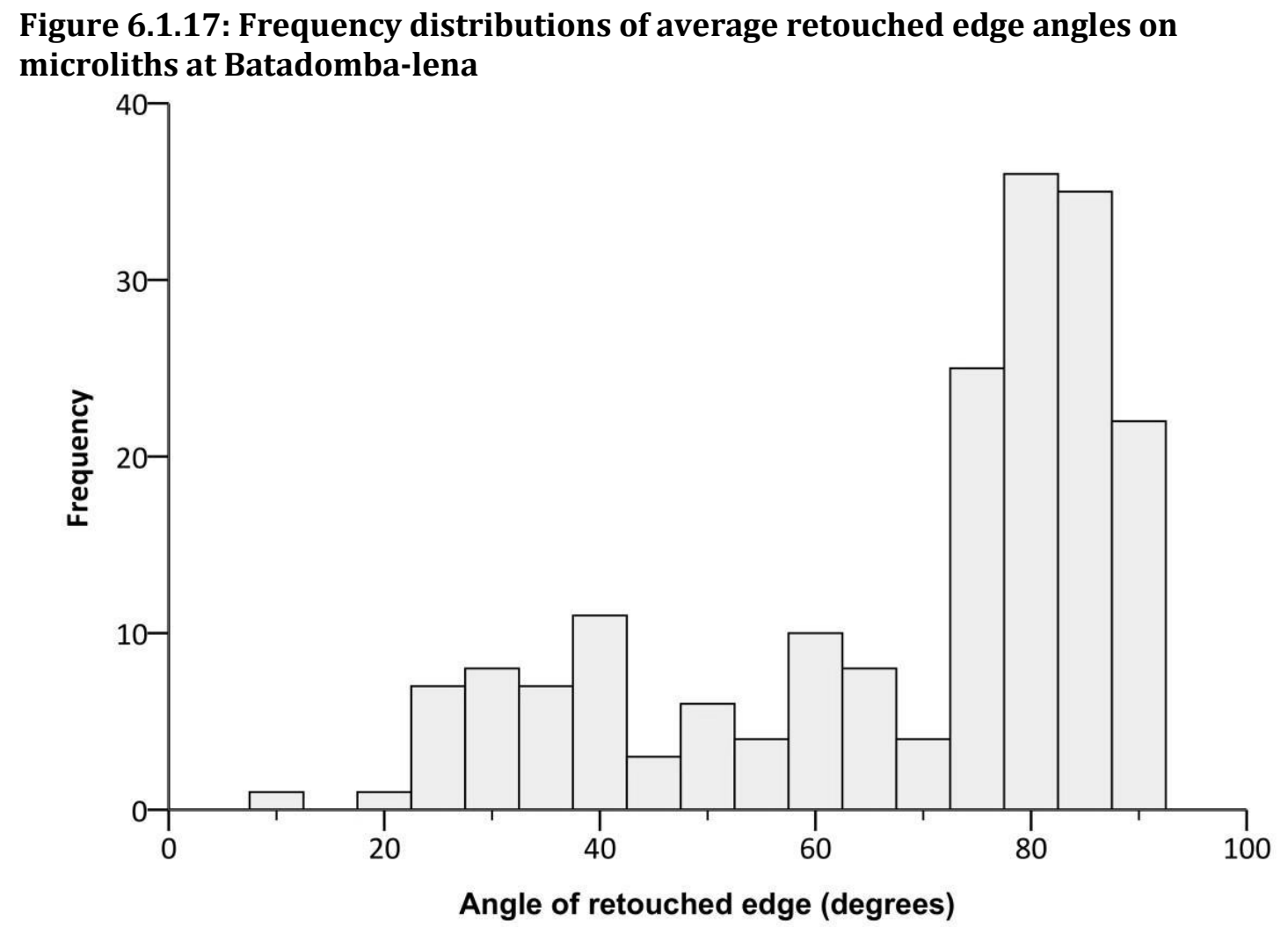

There is little variation in the mean IOI values for microliths, and the highest value on any individual microlith other than the Balangoda point is 0.469 (Table A.4.16). The Balangoda point has a considerably higher IOI value than other microlithic forms (0.719), again highlighting its distinctiveness. Examples of microliths from each layer are illustrated in Figure 6.1.18. 
Figure 6.1.18: Microliths from Batadomba-lena Layer 4 (1,2), 5 (3-8), 6 (9-28), 7a (29-37), 7b (38-51) and 7c (52-74). All are quartz. Examples of geometric forms include crescents $(21,42,61,63,65-67,71)$, triangles $(3,10,16,62)$ and a trapeze (64). A notched crescent can also be seen $(66)$. Tanged $(14,22)$ and shouldered $(12)$ forms are illustrated, as is the Balangoda point (53).

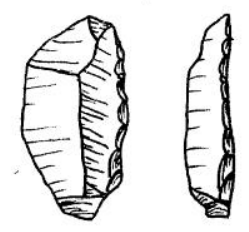

1

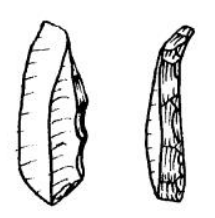

5

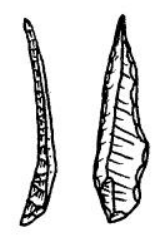

9

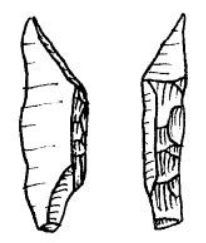

13

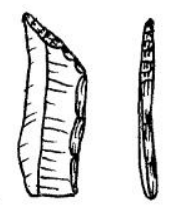

17

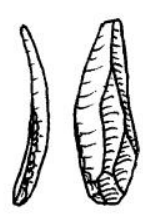

2

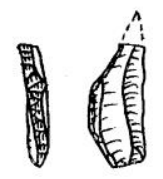

6

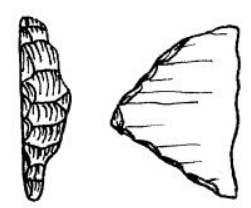

10

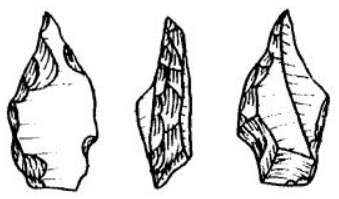

14

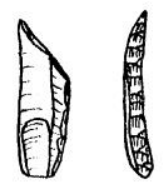

18

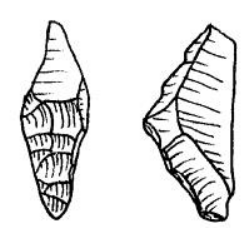

3

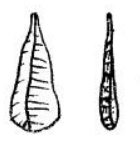

7

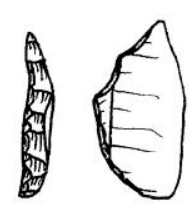

11

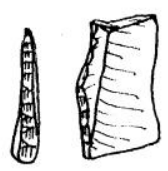

15

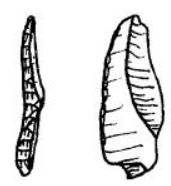

19

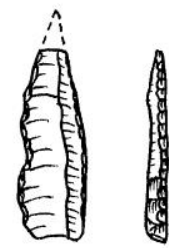

4

of

8

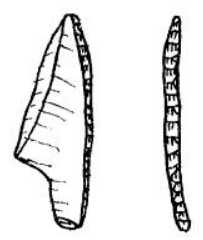

12

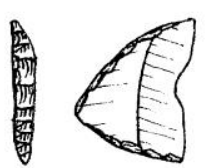

16

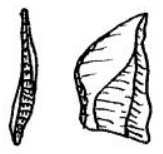

20

$0 \quad 5 \mathrm{~cm}$ 


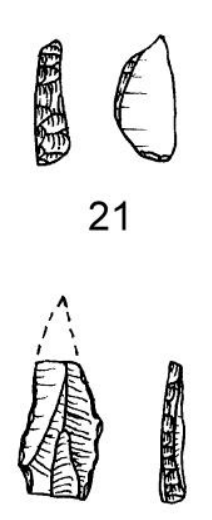

25

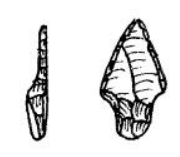

22

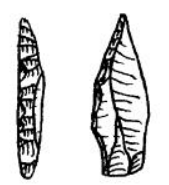

26

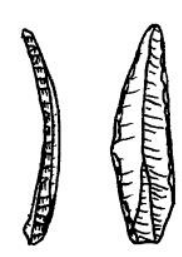

30

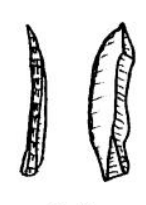

34

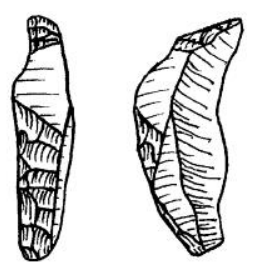

38

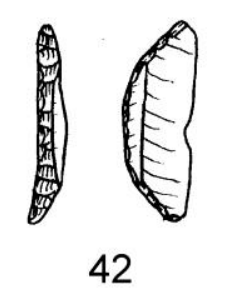

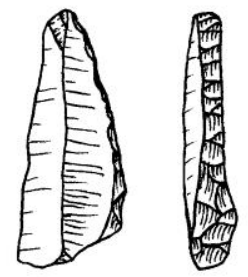

盟

37

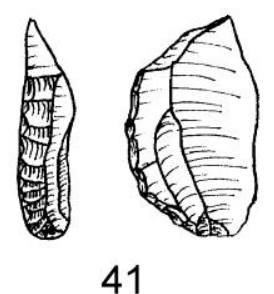

鼻运

23

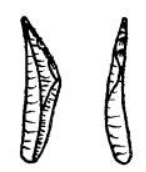

27

31
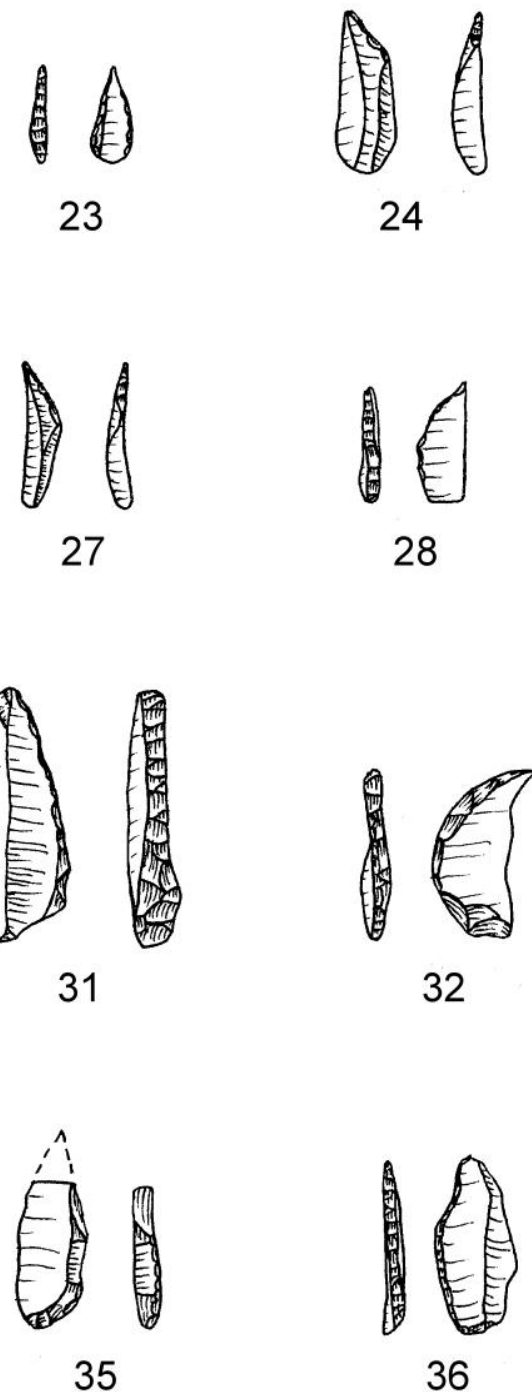

24

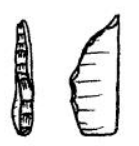

28

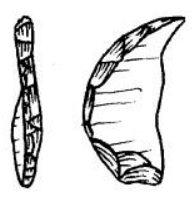

32
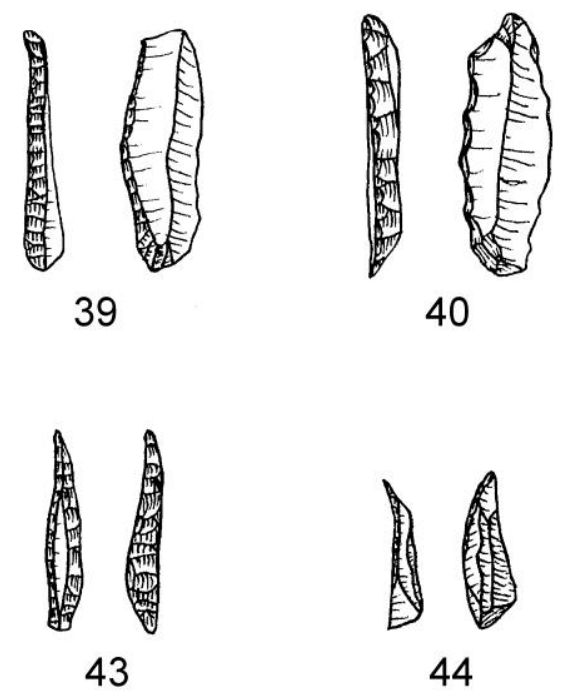

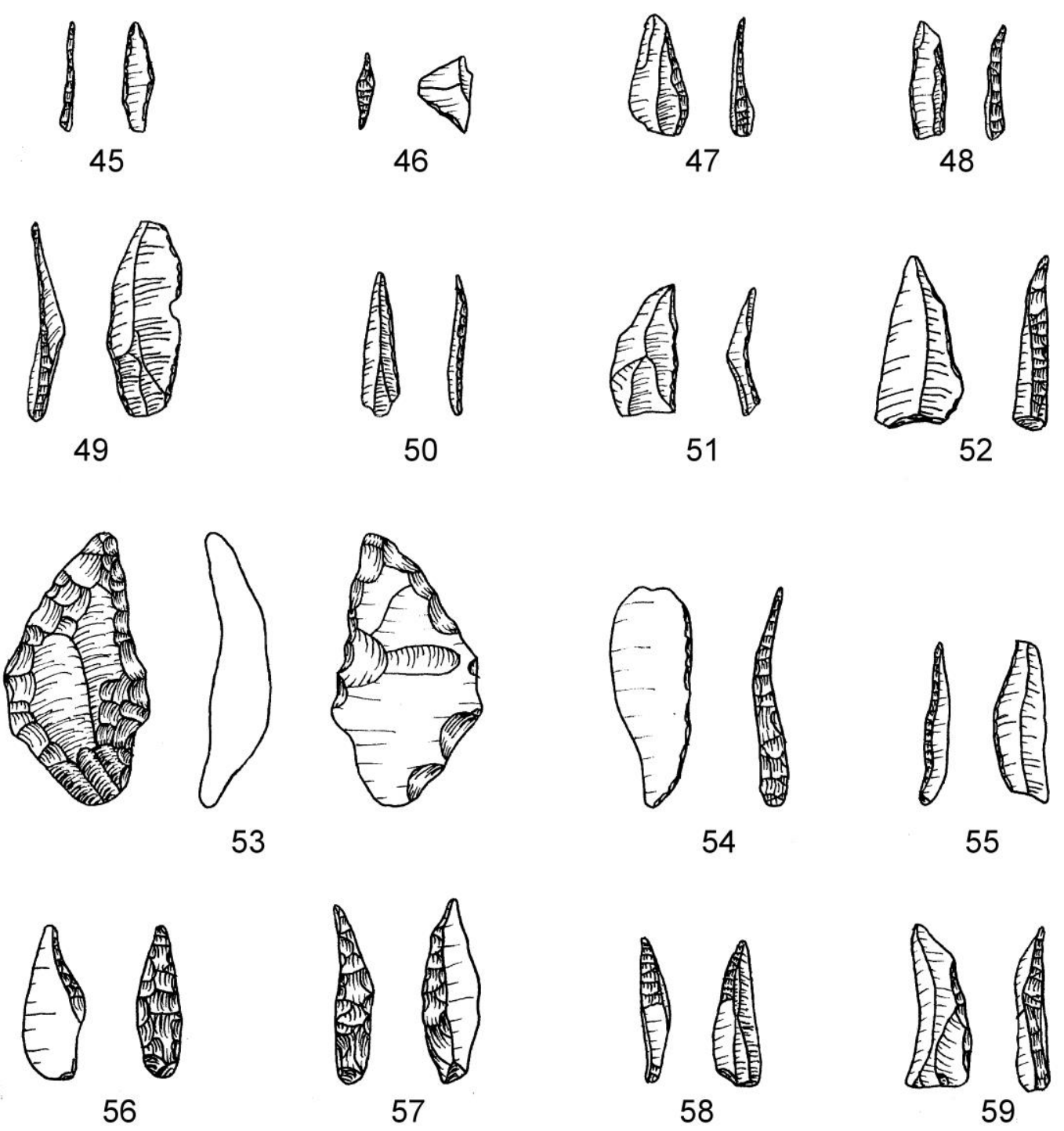

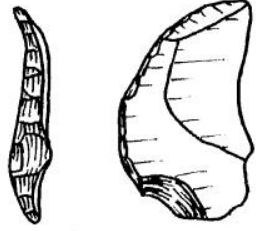

60

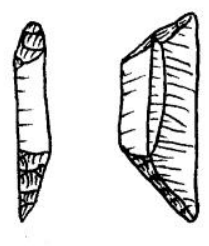

64

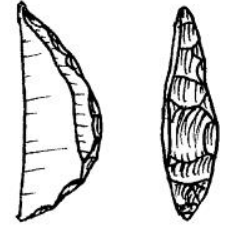

61

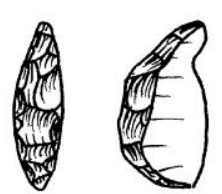

65

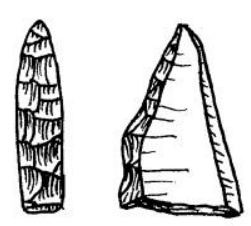

62

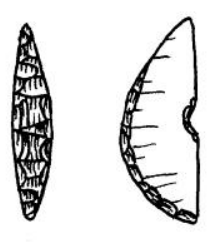

66

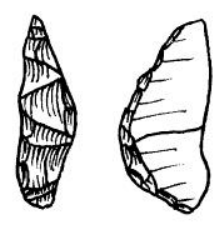

63

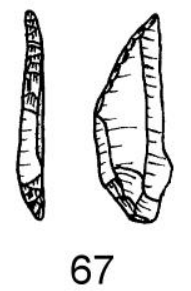




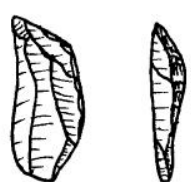

68

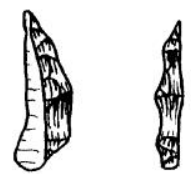

72

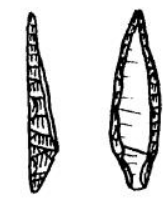

69

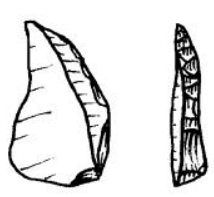

70

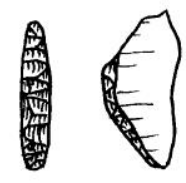

71

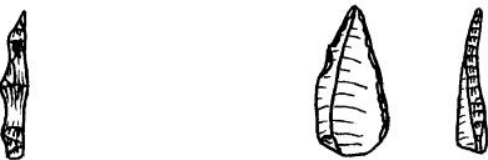

73

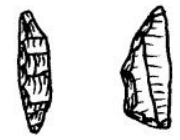

74

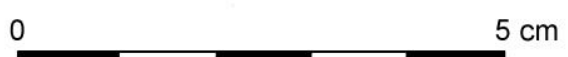

TEMPORAL VARIATION

Results of statistical tests at Batadomba-lena are presented in Appendix B.4. The small numbers of cores and non-microlithic tools preclude comparisons between layers (Table 6.1.7).

\begin{tabular}{lrrr}
\hline Layer & Cores & Microliths & Non-microlithic tools \\
\hline 4 & 3 & 13 & 2 \\
5 & 9 & 24 & 3 \\
6 & 5 & 34 & 2 \\
$7 \mathrm{a}$ & 2 & 19 & \\
$7 \mathrm{~b}$ & 2 & 22 & 1 \\
$7 \mathrm{c}$ & & 43 & \\
\hline
\end{tabular}

Table 6.1.7: Cores and tools analysed in each layer at Batadomba-lena

\section{Debitage}

Results of statistical tests on debitage are reported in Table B.4.1 and summarised in Table 6.1.8. There is no consistent temporal trend in terms of the proportions of 
flakes and blades. However, there is a general trend towards more flake-like characteristics over time, with increases in the proportion of debitage with no parallel arrises, in maximum and medial widths without a concomitant increase in mean length, and in the variation of width measurements. Other consistent changes over time can be seen in the decreases in the proportions of cortex cover and of broken lithics.

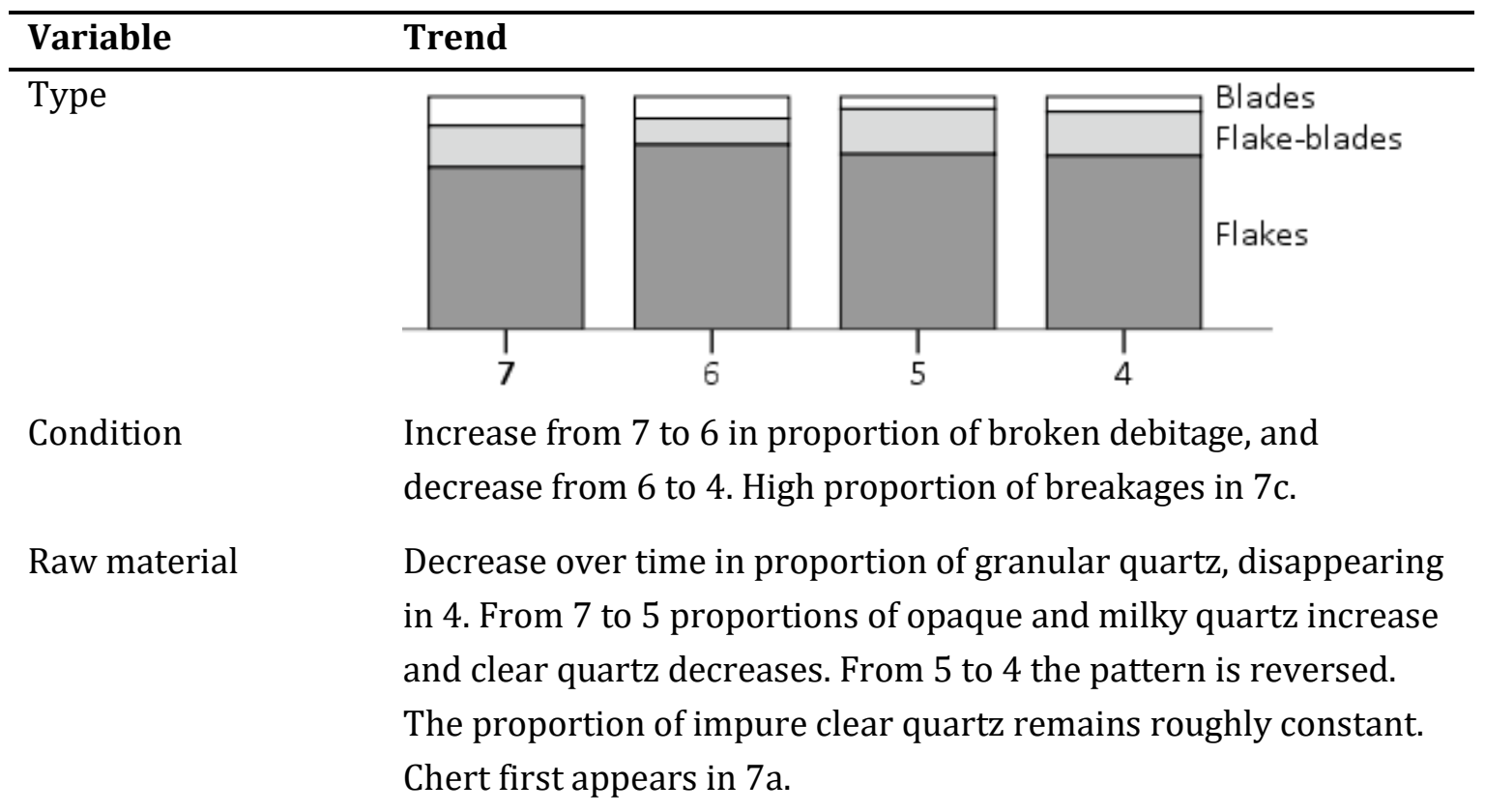

Colour Increase in white quartz and decrease in clear quartz from 7 to 5 . From 5 to 4 the pattern is reversed.

Maximum width

Medial width
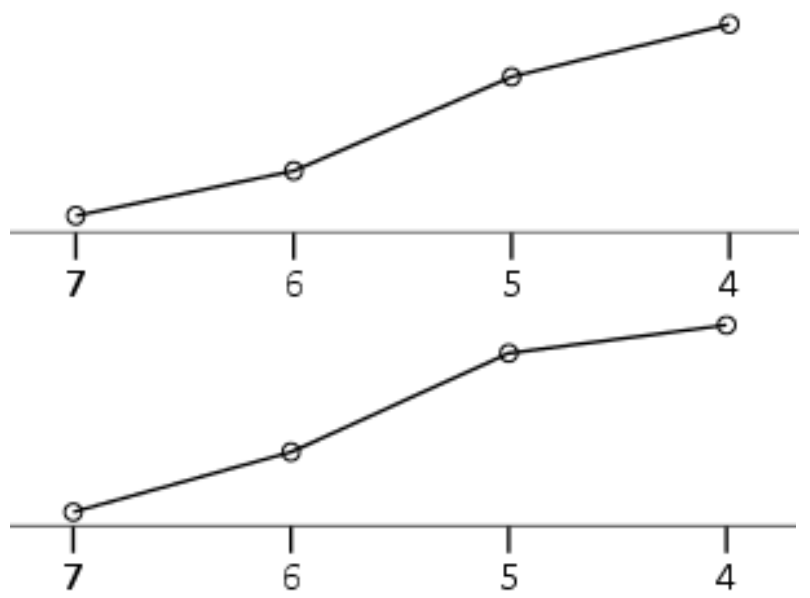
Length:width ratio

Length:thickness

ratio

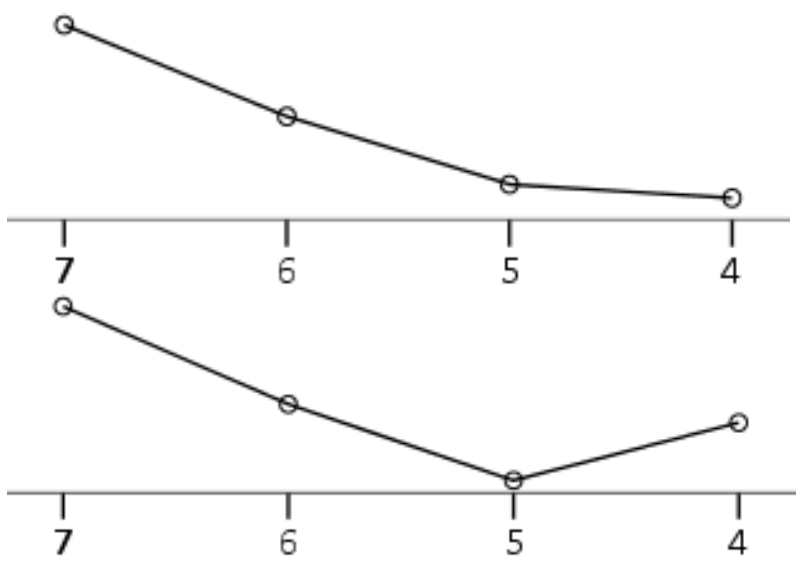

Width standard deviation

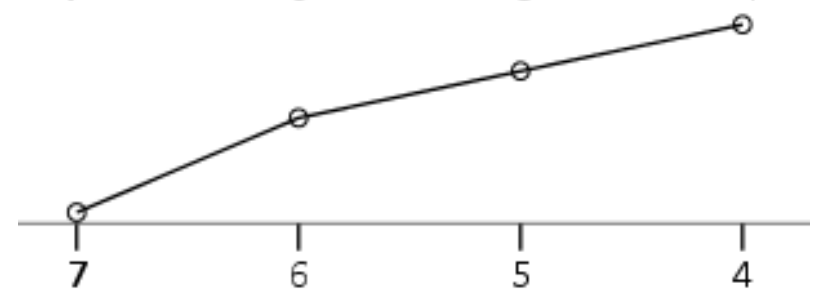

Striking platform width

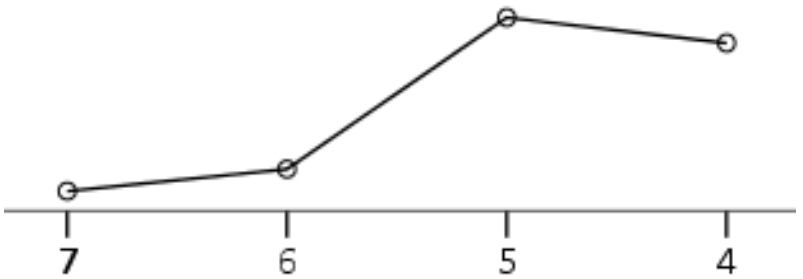

Striking platform thickness

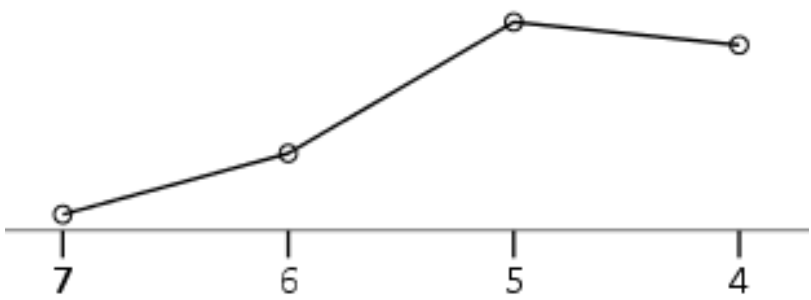

Striking platform Increase from 7 to 5 in proportion of flat striking platforms and type decrease from 5 to 4 .

Striking platform lipping
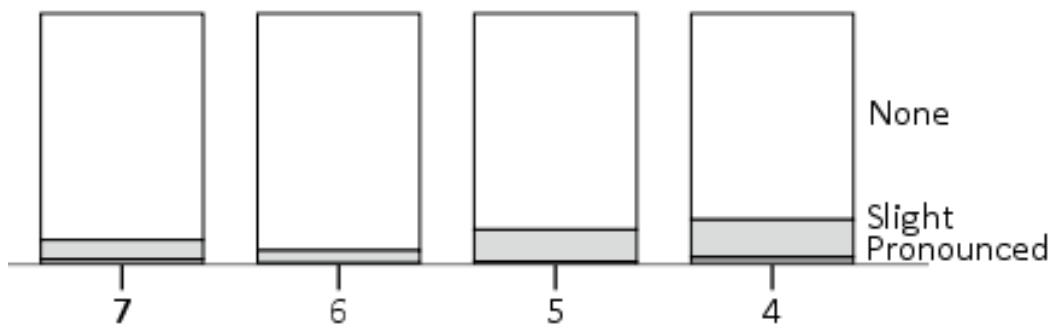

Percentage cortex cover

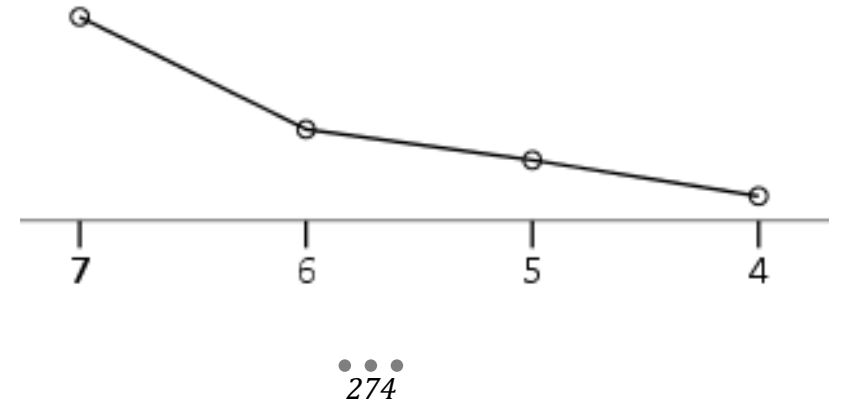


Number of dorsal scars

Number of parallel arrises

Scars per $\mathrm{cm}^{2}$
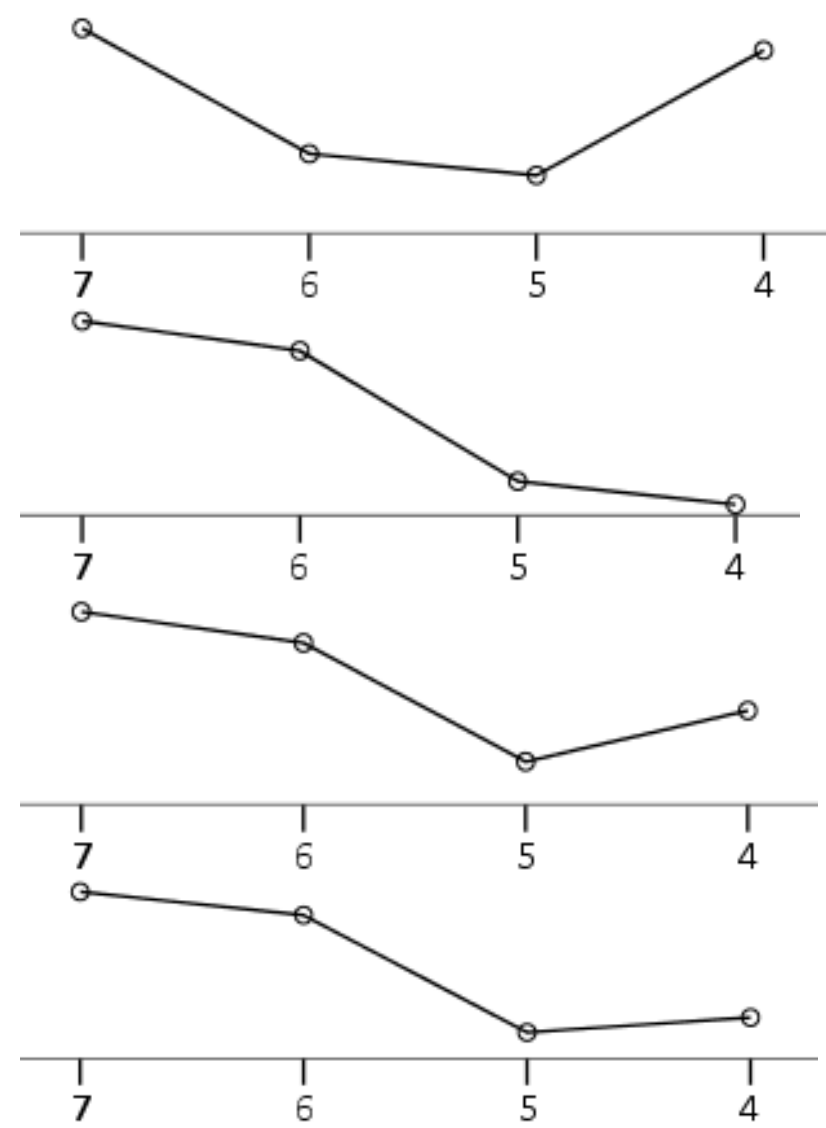

Scar orientation

Slight increase from 7 to 5 in proportion of debitage with scars from a single direction, and decrease from 5 to 4 .

Termination type

Decrease from 7 to 5 in proportion of debitage with feather terminations, and increase from 5 to 4 .

Other classification Slight increase from 7 to 5 in proportion of possibly snapped flakes and blades, and decrease from 5 to 4 .

Table 6.1.8: Statistically significant differences between layers in debitage variables at Batadomba-lena (Layers $7 \mathrm{a}, \mathrm{b}$ and $\mathrm{c}$ combined)

Some variables exhibit more complex trends. For example, several variables exhibit reversals in trends, mostly in the form of an observed trend from Layer 7 to 5 being reversed between Layers 5 and 4 . These variables include raw material, colour, length:thickness ratio, striking platform size and type, dorsal scar numbers, densities and orientation, termination and classification. There are also some patterns that are reversed between Layers 6 and 5, including debitage condition 
and striking platform lipping. Overall, considerable variation is evident over time within the debitage assemblage at Batadomba-lena.

\section{Microliths}

Results of statistical tests on microliths are reported in Table B.4.2 and summarised in Table 6.1.9. There are no statistically significant differences between raw material proportions in different layers. This might be considered surprising as there is considerable change within the debitage sample. However, this should instead be read as an indication that site occupants consistently made use of the best available qualities of quartz for retouch into tools. Absence of change over time is more notable in several other attributes, including length and thickness measurements and striking platform characteristics.

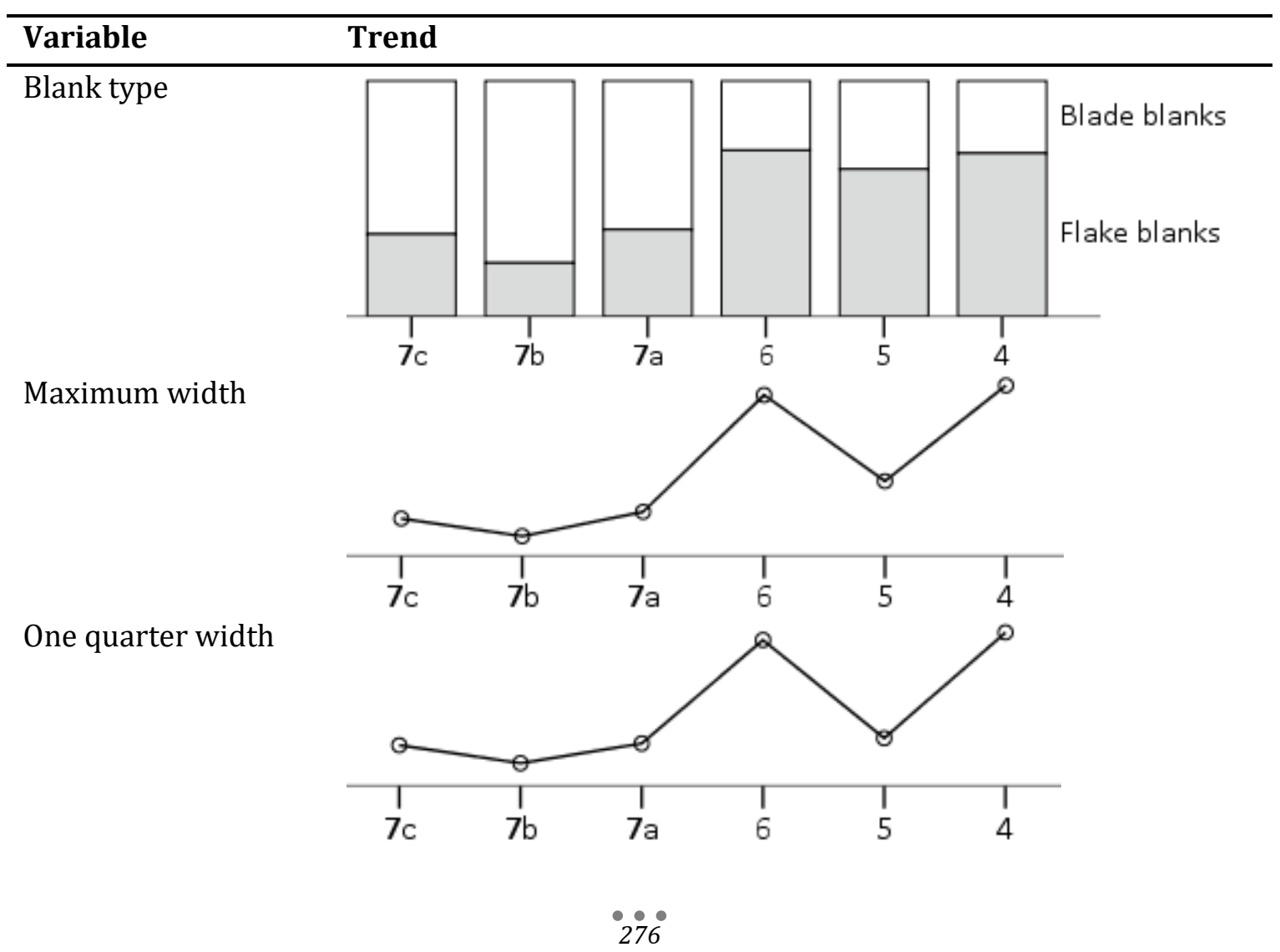


Medial width

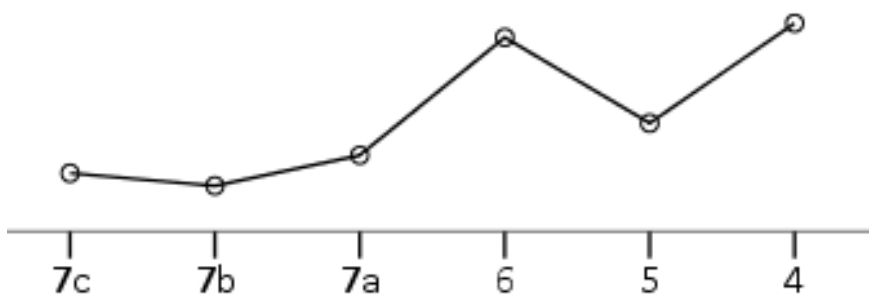

Three quarter width

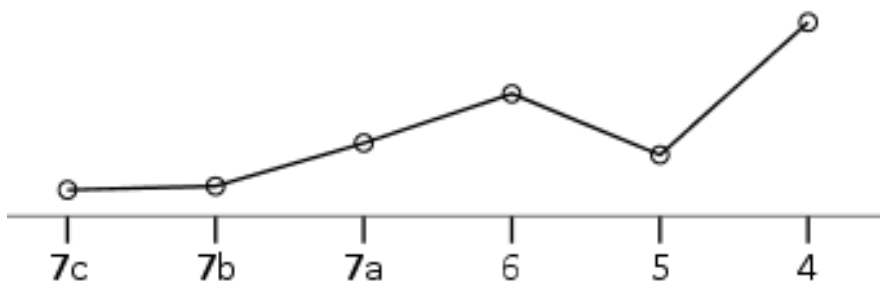

Length:width ratio

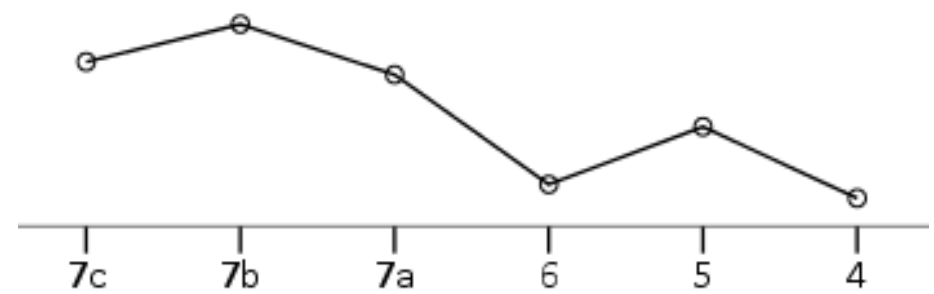

Width standard deviation

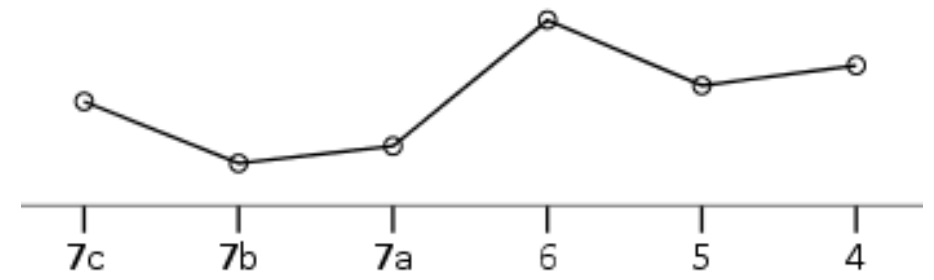

Number of dorsal scars

Scars per $\mathrm{cm}^{2}$
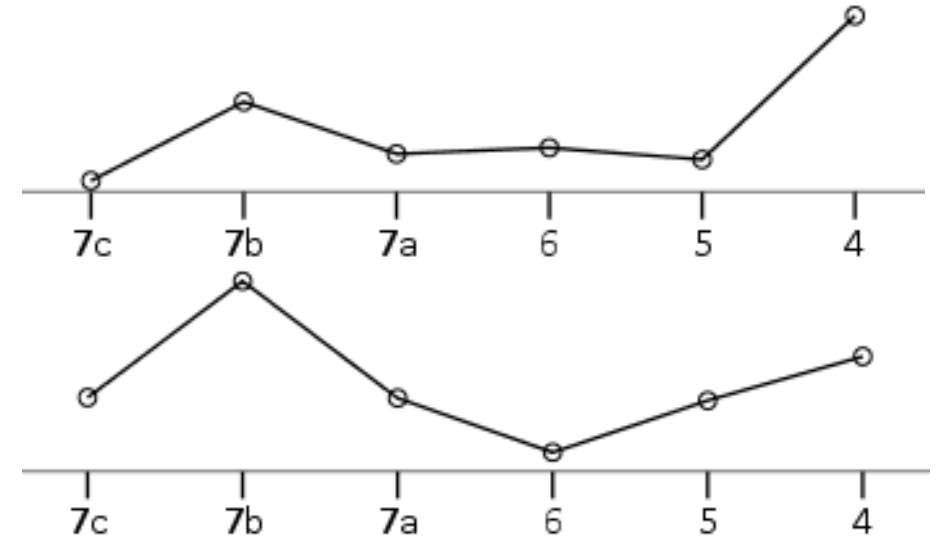

Coarse typology and narrow typology

Retouch location

The proportions of backed non-geometrics decreases over time, non-backed non-geometrics increase over time and geometrics fluctuate. See Figure 6.1.19.

Retouch face

More distal retouch in 6 and less lateral retouch in 4.

Slight and inconsistent increase in ventral retouch over time, and no edge retouch in 4 .

Retouch type

Proportion of backing retouch decreases over time, although with a temporary reversal in the pattern in 5 . 
TCSA

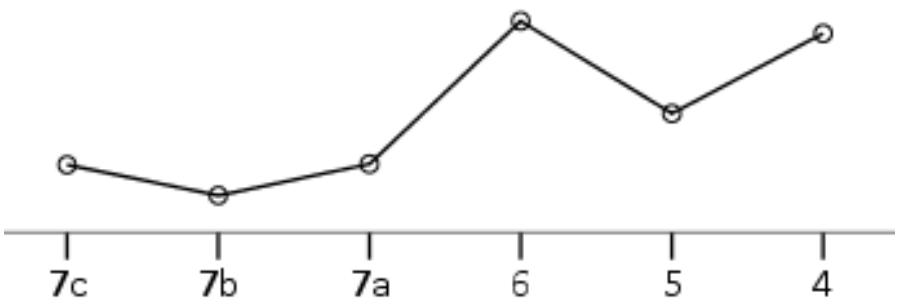

Table 6.1.9: Summary of statistically significant differences between layers in microlith variables at Batadomba-lena

Figure 6.1.19: Frequencies of microlith types in each layer at Batadomba-lena

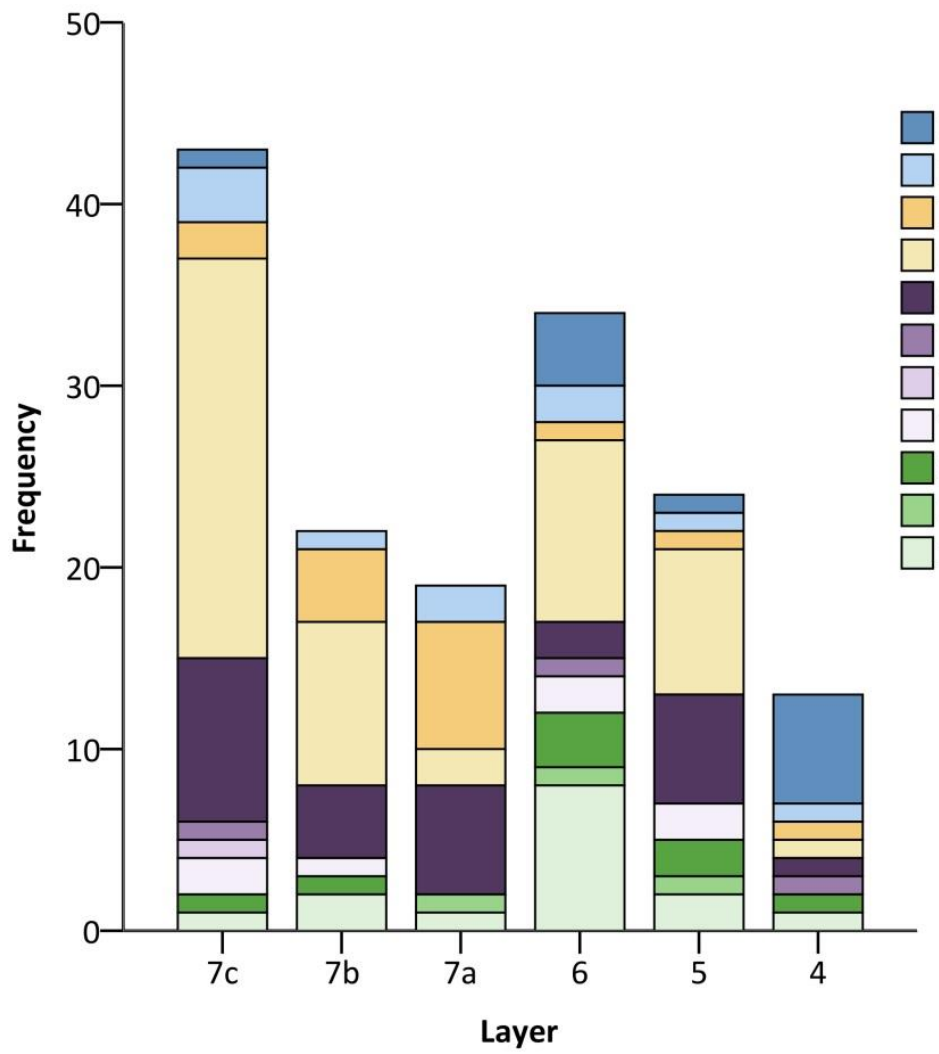

Typology

Non-geometric microlith

Non-geometric microlith point

Backed non-geometric microlith

$\square$ Backed non-geometric microlith point

Crescent microlith

Rectangle microlith

$\square$ Trapeze microlith

$\square$ Triangle microlith

Shouldered/tanged microlith

Notched microlith

Microlith preform

In terms of blank preferences, there are two clear groupings of layers. Microliths are predominantly manufactured on blades in Layers 7c-a (67.9\% blade blanks), and on flakes in Layers 6-4 (67.6\% flake blanks). Changes in blank type are reflected in changes in width measurements and standard deviation, length:width ratios and TCSA measurements. The step change seen between Layers 7a and 6 is not mirrored in the debitage sample, where flakes are dominant throughout and 
change over time is gradual and less pronounced. Preferences for blade tool blanks existed throughout the period of site occupation, but were strongest in the earlier half of the sequence.

The proportions of retouch and microlith types change more gradually. The proportion of backing retouch decreases over time, being dominant in all layers except 6 and 4, although there remains a high proportion of backing in Layer 5 . Likewise, the proportion of backed non-geometric microliths decreases over time, while the proportion of non-backed varieties increases (again with a slight reversal in this trend in Layer 5). The proportion of geometric microliths fluctuates with no clear temporal pattern, indicating that the changes in proportions of backing retouch are primarily related to non-geometric rather than geometric microlith forms.

The angle and extent of retouch does not differ significantly between layers, but the location, face and type does. Variations in retouch location and face, and microlith typology, are not part of overall trends, but instead represent small-scale differences between occupational layers. Overall there is much temporal variation evident within the lithic assemblage at Batadomba-lena, including step changes (like microlith blank preferences), gradual changes over time (as seen in debitage widths) and inconsistent changes between layers (such as in debitage raw material proportions). 


\section{MANUFACTURING TRAJECTORIES AND SUMMARY}

Manufacturing trajectories at Batadomba-lena are summarised in Figure 6.1.20. Cores are frequently worked from multiple platforms and directions. Core reduction strategies made heavy use of the bipolar percussion technique to remove large numbers of flakes from small quartz cores. Bipolar percussion was used as part of a late stage core reduction process to remove flakes from cores that had already been nearly exhausted.

The lithic assemblage at the site is heavily flake-based. Flake-blades are generally more similar to flakes than blades in the assemblage, including in their raw material proportions, striking platform types and dorsal scar densities. Proportionally more blades were removed through bipolar percussion than through the use of freehand knapping. Better qualities of quartz were worked more intensively, and were more frequently used to manufacture blades. However, with the exception of these patterns, blades do not appear to have been removed as part of any distinct or separate process at the site. Overall, lithic reduction at the site was largely undifferentiated.

In contrast to the core and debitage assemblages, the only indication that blades were in any way preferred is in their common use as blanks for retouch into microliths, particularly in Layers 7a-c. Blade blanks were especially preferred for backed non-geometrics and non-backed non-geometric points, while flake blanks were preferred for other non-backed non-geometric forms and for geometric morphologies. 
Figure 6.1.20: Manufacturing trajectories at Batadomba-lena. Solid lines are quartz. Dashed lines are chert.

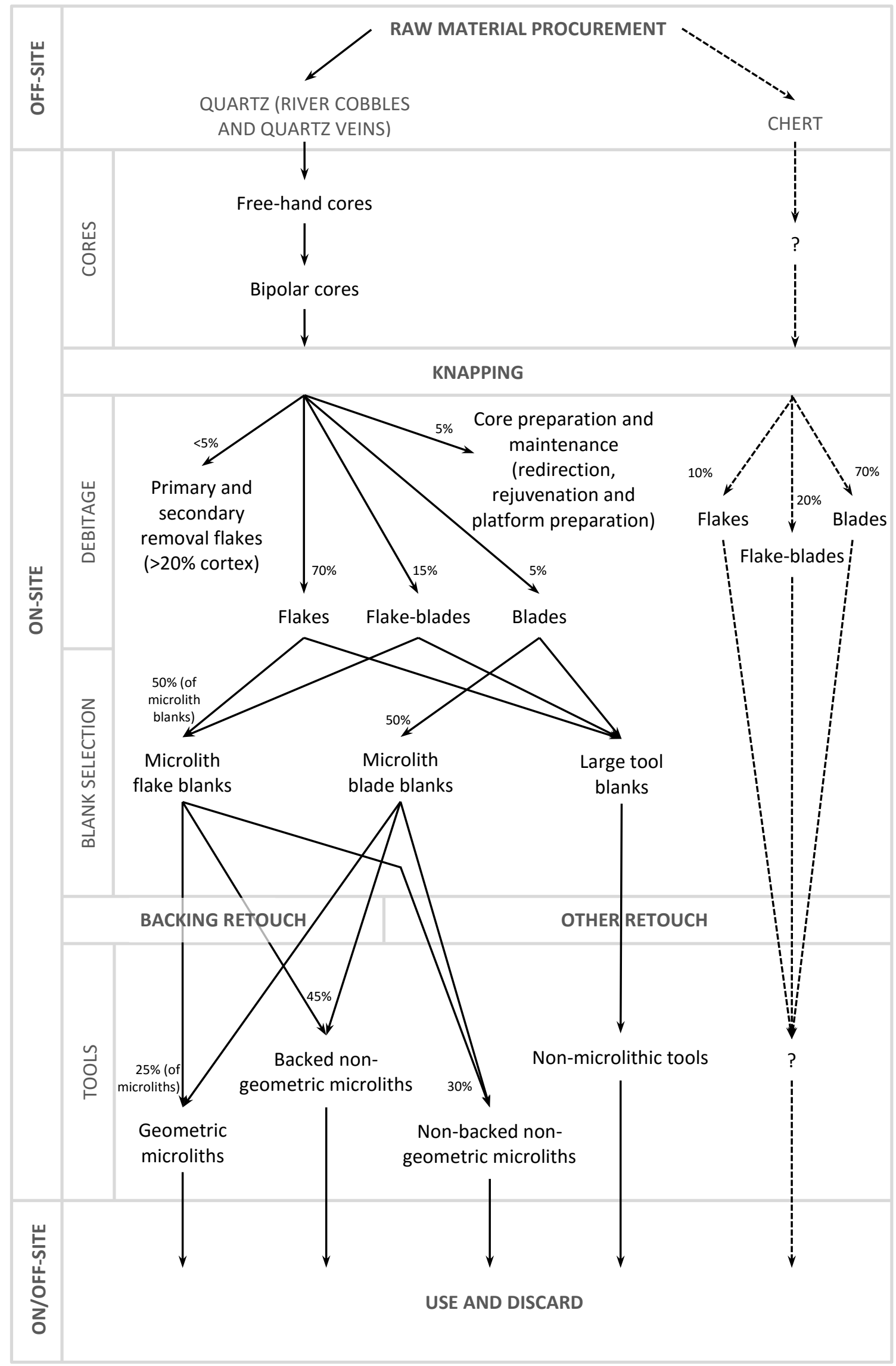


Lithics at the site are generally of very small dimensions, including some exceptionally small microliths. The entire system of lithic reduction at Batadombalena was geared towards the production of very small flakes and blades for retouch into microlithic tools. Non-microlithic tools are rare, and may have been produced more opportunistically rather than as the outcome of a distinct and predetermined reduction trajectory.

In the microlith assemblage, non-geometric forms are three times as common as geometric shapes. Backed non-geometric points and crescents are the most common types. Many other types are found in small numbers, including microliths exhibiting notches, shoulders and tangs. One tanged and bifacially worked Balangoda point was also recovered, which is considerably larger than the rest of the microlith assemblage and is consistent with the conclusions of previous researchers who highlight this form as a distinct type (Deraniyagala 1992, Perera 2010). There is little meaningful variation in size between other microlith types, however, or evidence for standardisation.

Over half of retouch made on microlithic tools is backing, over half of which is bipolar backing, i.e. conducted from both the dorsal and the ventral faces. Most geometric microliths are backed. Backing retouch is absent from the nonmicrolithic tool assemblage, although a variety of techniques are evident. Notches are found on several microlith types, and may be a local adaptation in response to the requirements of hafting such small microliths. Different retouch types appear to have been used for flake tools and for blade tools, although this may be an artefact of the small sample size. 
Considerable variation is evident over time within the debitage and microlith assemblages. This variation is evident in many forms, including sudden step changes, gradual and consistent changes over time, and smaller-scale differences between layers. The assemblage provides a good example of microlith production as a flexible technology comprising a considerable amount of variability on different time-scales.

\subsection{Kitulgala Beli-Lena, SRI Lanka}

\section{MICROLITHS}

Cores and debitage from Kitulgala were unavailable for study. 31 microliths were analysed from Pleistocene levels at Kitulgala: 27 from the basal Layer III, three from Layer IV and one from the terminal Pleistocene Layer V. All microliths are manufactured on quartz, primarily high quality quartz (Table 6.2.1), and all but two are whole. Blade blanks are slightly more common than flake blanks.

\begin{tabular}{lrrrr}
\hline Raw material & Microliths on blades & Microliths on flakes & Total & $\mathbf{\%}$ \\
\hline Clear quartz & 11 & 9 & 20 & 64.5 \\
Impure clear quartz & 7 & 3 & 10 & 32.3 \\
Milky quartz & & 1 & 1 & 3.2 \\
\hline Total & 18 & 13 & 31 & \\
\hline
\end{tabular}

Table 6.2.1: Tools measured at Kitulgala

Microliths at Kitulgala are particularly small, with an average size of 14.5 x $5.7 \times$ $2.7 \mathrm{~mm}$, and none longer than $22 \mathrm{~mm}$ (Table A.5.1). Microliths made on blade blanks are slightly longer and larger on average, with mean dimensions of $15.2 \mathrm{x}$ 
$5.8 \times 2.7 \mathrm{~mm}$ compared to $13.4 \times 5.6 \times 2.6 \mathrm{~mm}$ for those on flake blanks. Striking platforms are visible on four microliths, and are either flat or pointed, with small mean dimensions of $5.0 \times 1.9 \mathrm{~mm}$. Of the 11 microliths with visible terminations, seven are feather terminations and four are plunging.

Despite the small size of microliths, high numbers of dorsal scars are evident. This is particularly true on microliths made on blade blanks, with a mean of 2.4 dorsal scars per tool and a maximum of 5 (compared to a mean of 1.2 and a maximum of 2 for those made on flake blanks). When surface area is taken into account, this equates to a mean density of 3.4 scars per $\mathrm{cm}^{2}$ and a maximum of 6.4 . Microliths were manufactured on small, fine flakes and blades, the production of which demonstrates a considerable degree of technological finesse.

Just under half of microliths are of geometric forms (45.2\%), particularly crescents (38.7\%) (Table 6.2.2), all of which exhibit backing retouch. Most of the remainder are backed non-geometric forms (48.4\%), with only two microliths (6.5\%) being neither backed nor geometric. All geometric and backed non-geometric microlith forms exhibit only backing retouch, while non-backed types exhibit only subparallel retouch. Of the backing retouch, $58.8 \%$ is bipolar backing. Most retouch is conducted along a single convex (67.6\%) or straight (24.3\%) edge (Table A.5.2).

The majority (80.6\%) of microlithic tools are retouched along one edge, with six retouched along two separate edges (Table A.5.2). Of these, five are small pointed, double pointed or crescent forms backed along both the convex and the straight edges (Figure 6.2.1, no. 1-5), and one was retouched around the entire 
circumference to create a similar shape (Figure 6.2.1, no. 6). These forms may have been hafted as points rather than transversely, as they lack an acute cutting edge. They are smaller than other microlith types, with mean dimensions of $13.7 \times 4.2 \mathrm{x}$ $2.2 \mathrm{~mm}$, although they are not particularly standardised (Table A.5.3). They also have very small TCSA measurements, with a mean of $4.7 \mathrm{~mm}^{2}$ which is smaller than the smallest value listed in Shea's (2006) arrowhead sample (Table A.5.4).

\begin{tabular}{llr}
\hline Coarse typology & Narrow typology & Frequency \\
\hline Backed non-geometric microlith & Backed & 2 \\
& Double backed & 1
\end{tabular}

$\begin{array}{lll}\text { Backed non-geometric microlith point } & \text { Backed } & 10\end{array}$

Double backed 2

$\begin{array}{lr}\text { Crescent microlith } & 12\end{array}$

$\begin{array}{lll}\text { Non-geometric microlith } & \text { Double side retouched } & 1\end{array}$

$\begin{array}{ll}\text { Non-geometric microlith point } & 1\end{array}$

$\begin{array}{ll}\text { Trapeze microlith } & 1\end{array}$

$\begin{array}{ll}\text { Triangle microlith } & 1\end{array}$

$\begin{array}{ll}\text { Total } & 31\end{array}$

Table 6.2.2: Tool type frequencies at Kitulgala

Backed non-geometric microliths are the most standardised type in terms of retouch extent, with the least variation in retouch length and depth (Table A.5.5). However, no typological group is in any way extensively retouched, with low mean and maximum values recorded for each type. For example, the maximum IOI value 
for any microlith is 0.250 (Table A.5.6). The microliths in each layer are illustrated in Figure 6.2.2.

Figure 6.2.1: Double backed pointed microliths from Kitulgala (1-5) and one microlith backed along the entire circumference (6)

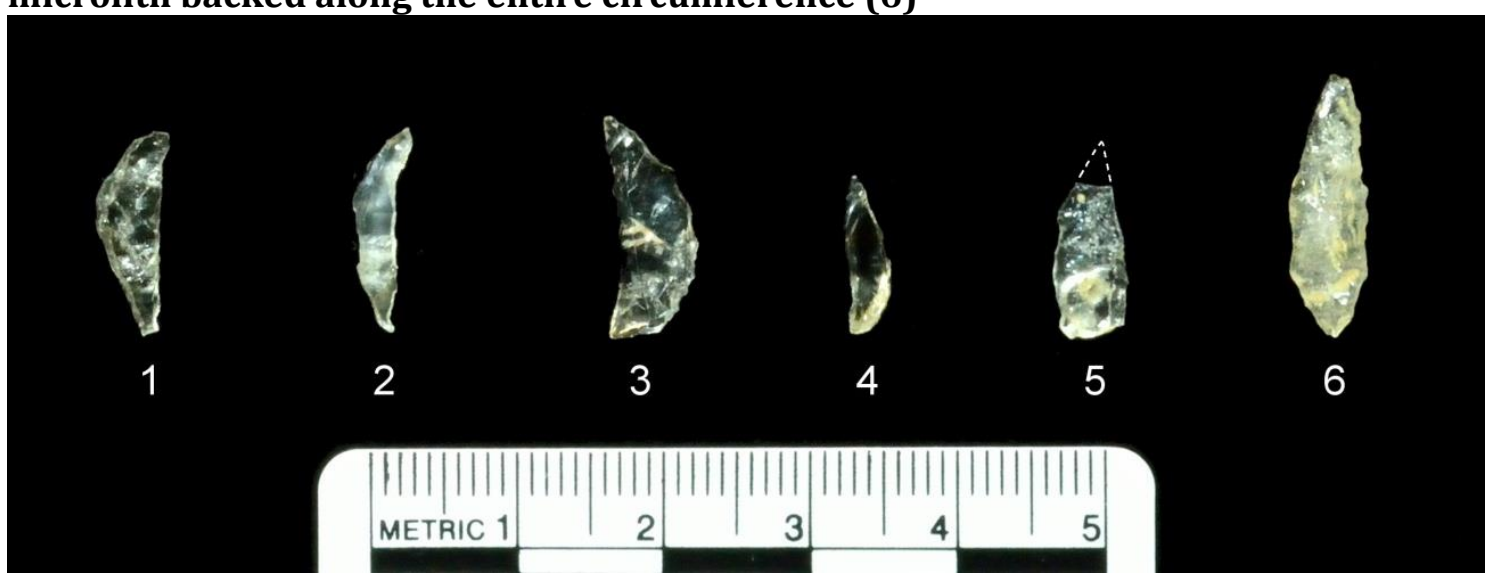

Figure 6.2.2: Microliths from Kitulgala Layer V (1), IV (2-4), IIIc (5-22), IIIb (23) and IIIa (24-31). All are quartz. Of particular note are the pointed double backed forms $(8,15,22,26,29,30)$.

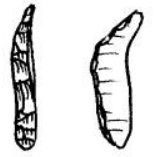

1

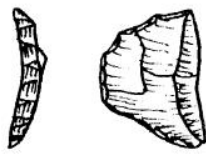

5

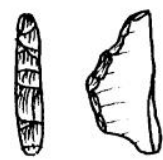

9

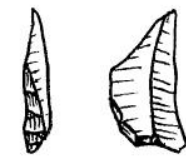

2

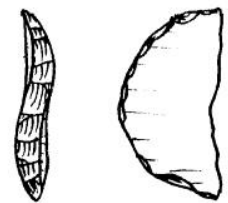

6

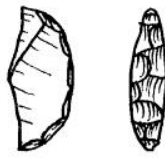

10
E

3

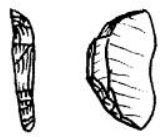

7

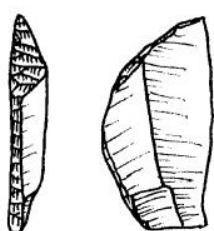

11

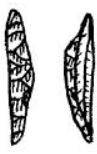

4

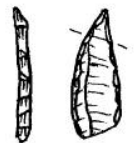

8

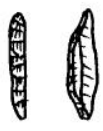

12 


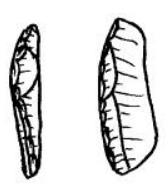

13

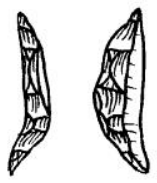

17

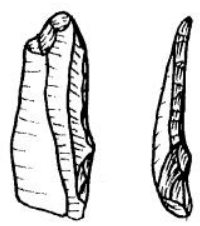

21

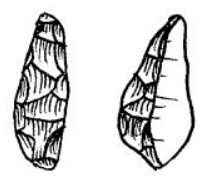

25

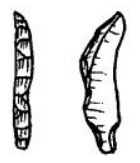

29

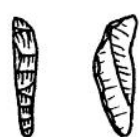

14

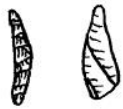

18

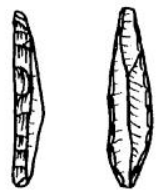

22

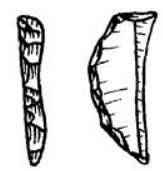

26

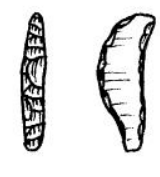

30
5

15

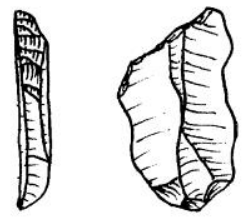

19

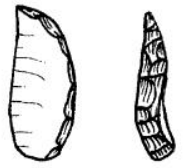

23

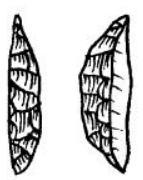

27

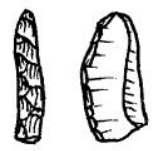

20

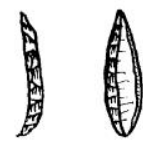

24

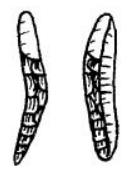

28

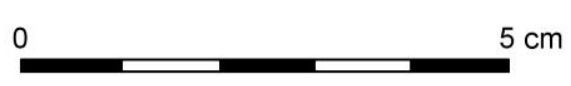

\section{SUMMARY}

In the absence of data from cores and debitage at the site it is impossible to reconstruct full manufacturing trajectories. However, high dorsal scar counts and densities on the microliths studied are indicative of a considerable degree of 
knapping finesse, used to create very small, fine flakes and blades from a raw material that can be very difficult to work in this manner due to its internal inconsistencies (Knight 1991b, Driscoll 2011). Microliths at the site are small and have typical dimensions of only $15 \times 5 \mathrm{~mm}$. Nearly all microliths are backed, and are roughly split between geometric and non-geometric forms. Of particular note are six thin pointed crescent-like microliths which were backed along both lateral margins. These artefacts are particularly small, with exceptionally small TCSA measurements, and were most likely hafted as points as they lack an acute cutting angle. It is possible that these formed a distinct tool category at Kitulgala.

\subsection{PATNE, INDIA}

The results presented below are based on analysis of data collected by James (2011). Differences between James' methodology and the one used in the current study are described in Appendix C.1. The main difference is that James did not record raw material, although Sali (1989) notes that the majority of the assemblage was produced on locally-available jasper and chalcedony, with chert, quartz and quartzite also present.

\section{CORES}

Uni- and bi-directional flake cores are the most common core types (Table 6.3.1). Cores worked from two platforms are more common than those worked from one 
or from three or more. One core demonstrates evidence for the use of bipolar percussion. Radial working of cores is absent.

\begin{tabular}{llrr}
\hline Core type & & Frequency & Percentage \\
\hline Uni-directional: & Blade & 5 & 8.8 \\
& Flake & 16 & 28.1 \\
& Bipolar & 1 & 1.8 \\
& & & \\
Bi-directional: & Blade & 8 & 14.0 \\
& Flake & 19 & 33.3 \\
Multi-directional: & Amorphous & \multicolumn{2}{c}{} \\
\hline Total & & 57 & 14.0 \\
\hline
\end{tabular}

Table 6.3.1: Core type frequencies at Patne

Cores were generally discarded while still relatively large; average core size is 40.0 x $33.0 \times 23.5 \mathrm{~mm}$, and the largest core measured $79.5 \times 54.4 \times 71.3 \mathrm{~mm}$ when discarded (Table A.6.1). Differences are evident between core types. Blade cores are smaller on average than equivalent flake cores, with an average size of $34.2 \mathrm{x}$ $28.4 \times 18.7 \mathrm{~mm}$ compared to $41.3 \times 34.5$ x $24.6 \mathrm{~mm}$ (Table A.6.2). The bipolar core is particularly long, with dimensions of $51.9 \times 27.3 \times 24.8 \mathrm{~mm}$. Possibly it was held on a surface (anvil) for knapping due to the difficulty of working such a long, thin core. No core type shows any convincing evidence for standardisation.

Bi-directional flake cores were often worked from perpendicular striking platforms (in $26.3 \%$ of cases), indicating a considerable degree of perpendicular core rotation (Table A.6.3). However, bi-directional blade cores were only worked from opposite ends, potentially suggesting that flake and blade cores formed 
separate stages of core reduction. Cores may have been shaped by removing flakes from the end and from the side, before they were used for the removal of blades.

More removals were made on average on bi-directional cores (10.9) compared to single platform cores (9.1) (Figure 6.3.1, Table A.6.4). Blade removals are relatively common even on cores that were not primarily used to remove blades. In total, $35.1 \%$ of all terminal removals are of blades. The range of core types evident at Patne is therefore possibly more of a continuum, rather than clearly divided into flake cores and blade cores as inferred at other sites in this study.

Figure 6.3.1: Average numbers of flake and blade scars on different core types at Patne

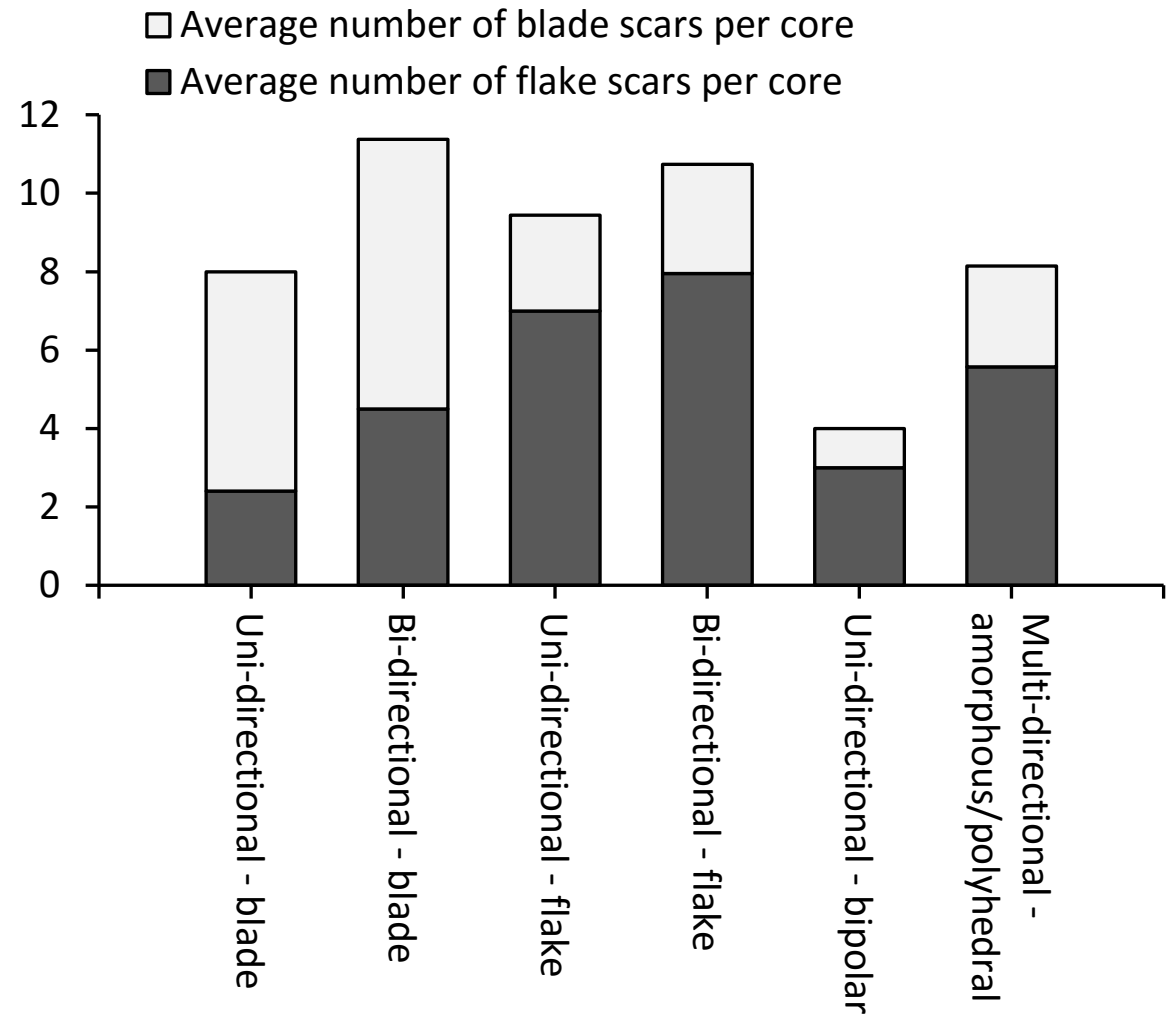

Only four removals are recorded on the bipolar core (Table A.6.4). This is unusual, as bipolar percussion is generally used to remove large numbers of flakes and/or 
blades from relatively small cores, whereas here it is used on a relatively large core to make relatively few removals. The bipolar core is not intensively worked in relation to core size (Figure 6.3.2, Table A.6.5).

Figure 6.3.2: Ratios of average removal scar length to core volume on cores at Patne

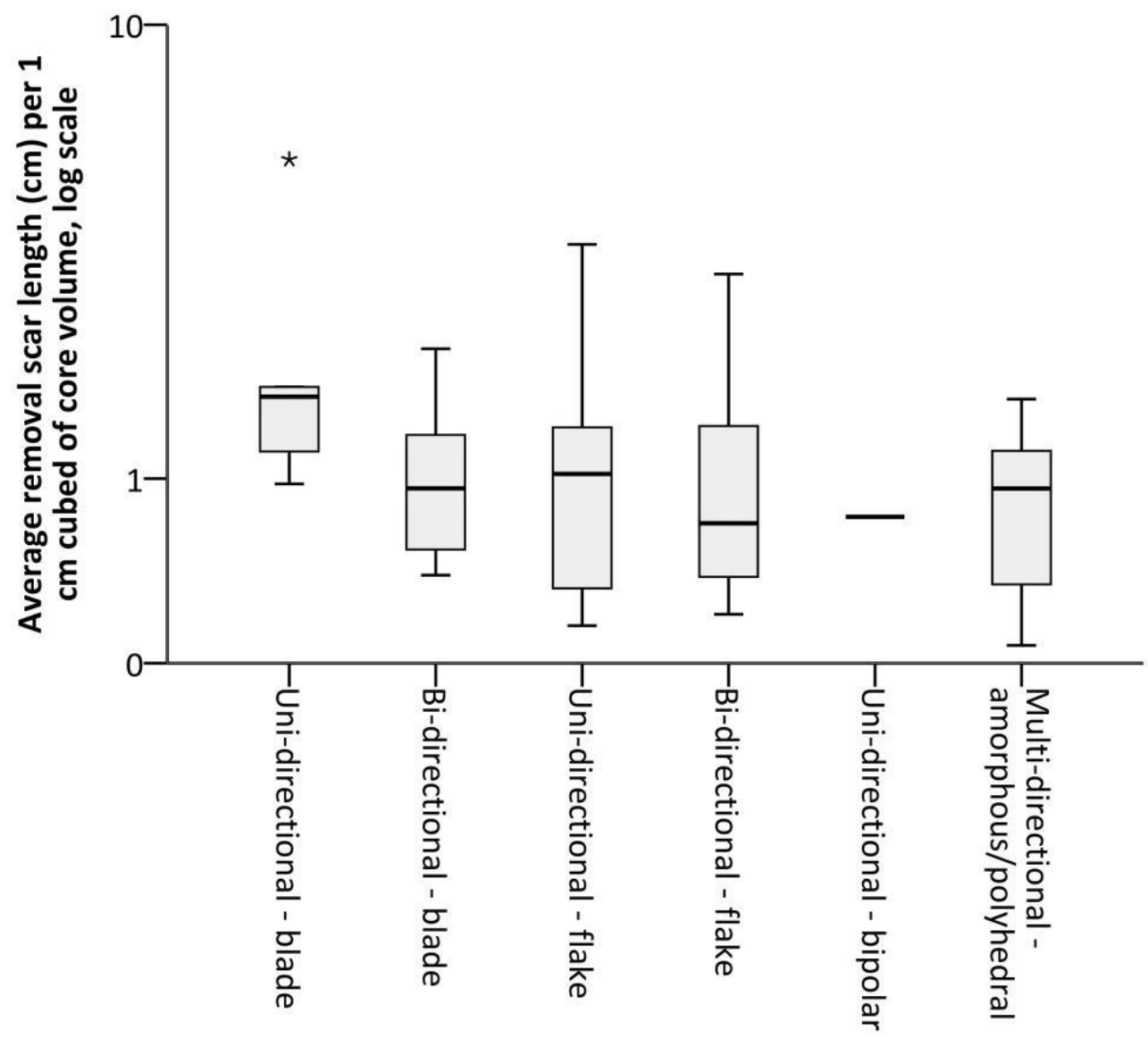

No core type was particularly heavily worked, suggesting that efficient use of raw material was not a pressing concern. Indeed, Sali (1989) notes that jasper, chalcedony and other similar raw materials were locally available in abundance. This would also explain the near absence of bipolar reduction, and the fact that bipolar percussion appears to have been adopted in this case in order to deal with the challenge of working a particularly long and thin core, rather than as an efficient method for removing large numbers of flakes and/or blades. 
Further evidence of the low levels of core reduction intensity at the site can be seen in the fact that cortex is evident on the majority (68.5\%) of cores, with a mean cortex cover of $30 \%$. Mean cortex cover is lower on blade cores compared to flake cores (20\% v. 35\%), highlighting a small difference between the core types. Overall, these core type designations represent different ends of the reduction continuum, with blade removals tending to be made slightly later in reduction sequences.

\section{DEBITAGE}

The debitage assemblage is dominated by flakes (60.9\%), with some flake-blades (20.5\%) and blades (18.6\%). The majority of debitage for which condition was recorded is broken, with only $3.7 \%$ recorded as whole (although $43.2 \%$ did not have their condition recorded) (Table A.6.6), precluding meaningful size comparisons between debitage categories. Average debitage size is $29.7 \mathrm{x} 17.0 \mathrm{x}$ $6.5 \mathrm{~mm}$ (Table A.6.7). Size measurements generally indicate an emphasis on relatively long and thin end products. For example, the mean length:width ratio of all whole debitage is 2.1 , and whole blades have a mean of 3.0 and a maximum of 6.6. Although blades are not the dominant debitage type at the site, there is nevertheless considerable elongation of some debitage overall.

Slight or pronounced lipping is evident on $10.4 \%$ of striking platforms. It is slightly less common on blades (5.9\%), although the differences between debitage types are not statistically significant $\left(\chi^{2}(2)=4.025, \mathrm{p}=.134\right)$. Flat striking platforms are the most common type (Figure 6.3.3). More heavily facetted striking platforms are 
more common on flakes than on blades, with flake-blades as an intermediate category. Crushed/abraded platforms were not recorded by James (2011).

Figure 6.3.3: Proportions of debitage striking platform types at Patne Striking platform type

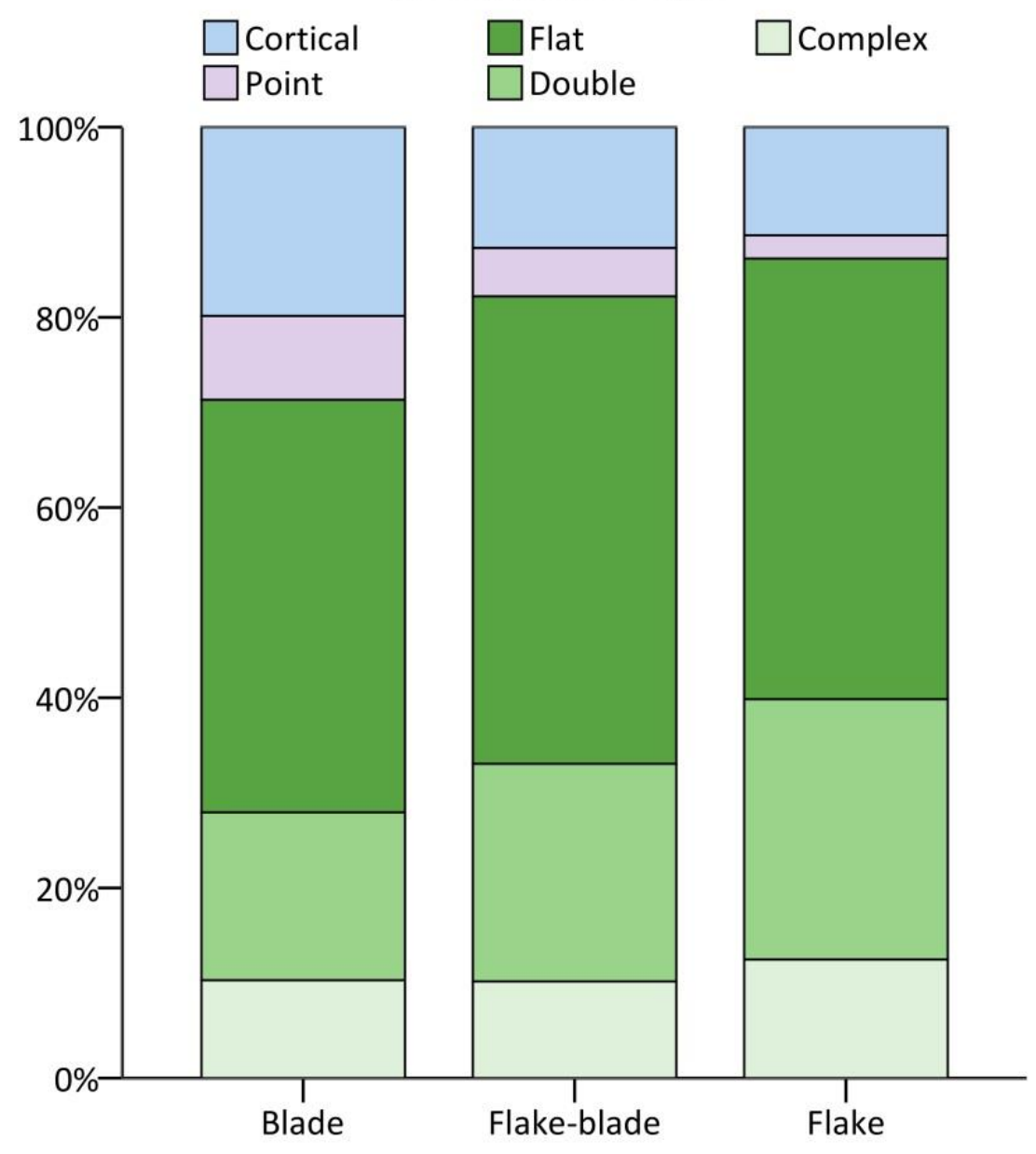

Pointed striking platforms are more common on blades, and the striking platforms of blades are smaller on average $(6.2 \times 2.6 \mathrm{~mm})$ than those of flakes $(12.7 \times 4.7$ $\mathrm{mm}$ ) or flake-blades ( $8.8 \times 3.7 \mathrm{~mm})$. These size differences might suggest that blades were removed later in the core reduction sequence. However, cortical striking platforms are also more common on blades, suggesting they may have been removed earlier. These differences in striking platform types between 
debitage categories are statistically significant, although the relationship is not strong $\left(\chi^{2}(8)=19.983, p=.010\right.$, Cramér's $\left.V=.127\right)$.

As a further complicating factor, in contrast to the higher proportion of cortical striking platforms amongst blades, blades exhibit lower proportions of dorsal cortex cover, with a mean of $4 \%$ compared to $12 \%$ for flake-blades and $11 \%$ for flakes (Table A.6.8). Additionally, blades exhibit denser concentrations of dorsal scars (with a mean of 1.3 per $1 \mathrm{~cm}^{2}$ compared to 1.0 for flakes) (Table A.6.9). Overall, it is unclear whether blades were produced at a separate stage to flakes in the reduction process, and it appears most likely that a variety of lithic products were intentionally produced.

In terms of scar orientation, scars made from a single direction are most common (Table 6.3.2). Within termination proportions, feather terminations are most common (70.9\%) (Figure 6.3.4). Axial or crushed terminations are not recorded, but this is likely to have a negligible impact on termination proportions as these types are generally indicative of bipolar percussion, which is very rare at the site. There are few differences between debitage types in termination proportions, further indicating that flakes and blades formed part of the same process of core reduction. 


\begin{tabular}{|c|c|c|c|c|c|c|c|c|c|c|c|}
\hline \multirow{2}{*}{$\begin{array}{l}\text { Dorsal scar } \\
\text { orientation: } \\
\text { Type }\end{array}$} & \multicolumn{2}{|c|}{1} & \multicolumn{2}{|c|}{2} & \multicolumn{2}{|c|}{2} & \multicolumn{2}{|c|}{3 or 4} & \multicolumn{2}{|c|}{ Unknown } & \multirow[b]{2}{*}{ Total } \\
\hline & No. & $\%$ & No. & $\%$ & No. & $\%$ & No. & $\%$ & No. & $\%$ & \\
\hline Blade & 21 & 80.8 & 2 & 7.7 & 3 & 11.5 & & & & & 26 \\
\hline Flake-blade & 3 & 50.0 & & & & & 3 & 50.0 & & & 6 \\
\hline Flake & 10 & 52.6 & 5 & 26.3 & 1 & 5.3 & 2 & 10.5 & 1 & 5.3 & 19 \\
\hline $\begin{array}{l}\text { Total (\% of } \\
\text { total) }\end{array}$ & 34 & 66.7 & 7 & 13.7 & 4 & 7.8 & 5 & 9.8 & 1 & 2.0 & 51 \\
\hline
\end{tabular}

Table 6.3.2: Dorsal scar orientation frequencies on whole debitage at Patne

Figure 6.3.4: Proportions of debitage termination types at Patne

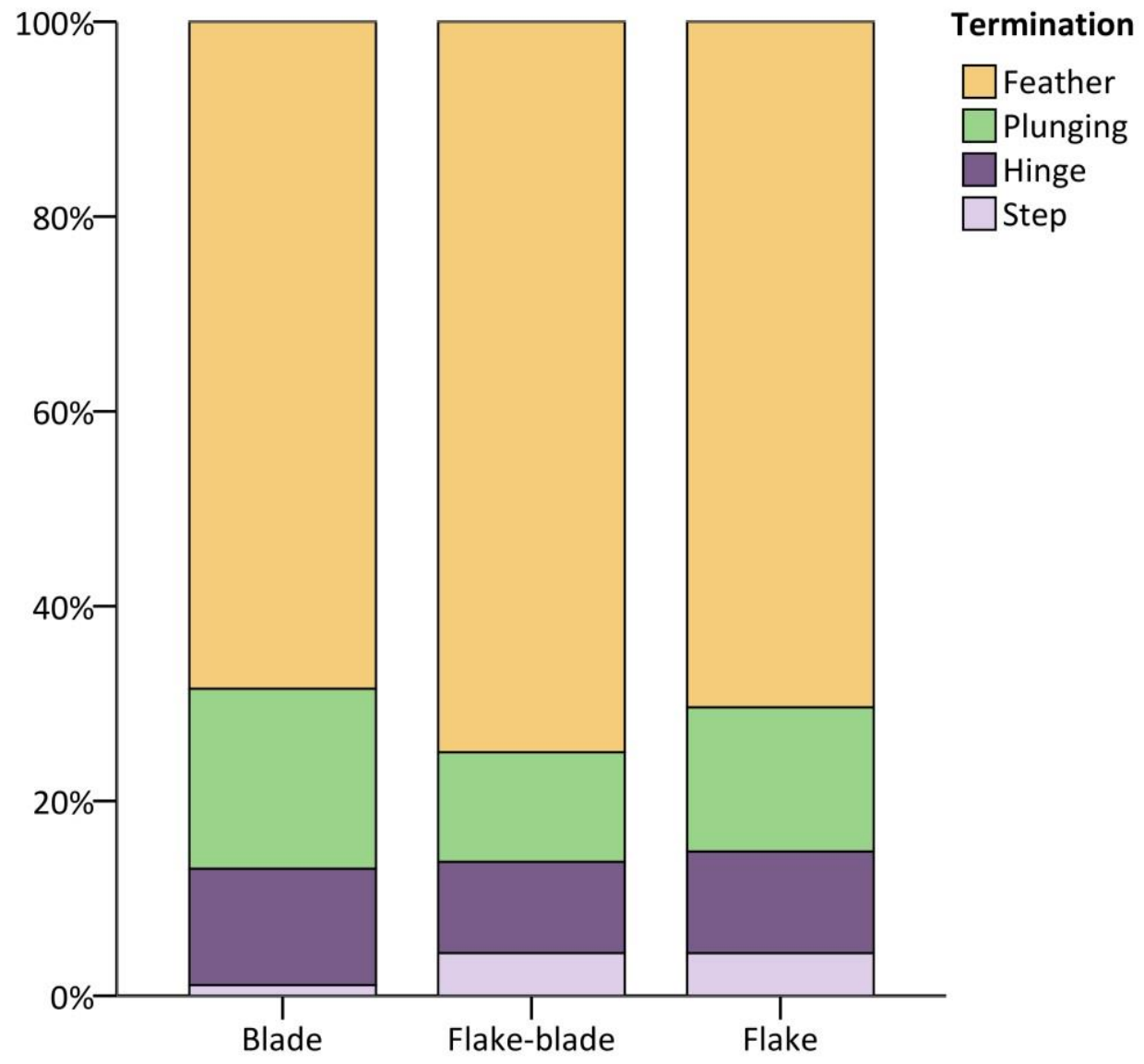

TOOLS

In contrast to the flake-dominated debitage assemblage, retouched tools were primarily manufactured on blade blanks $(82.5 \%$ of microliths and $58.2 \%$ of non- 
microlithic tools) (Table 6.3.3). Although blades do not appear to have been produced as part of a distinct manufacturing trajectory at the site, they were selected preferentially for retouch.

\begin{tabular}{lrr}
\hline & Frequency & $\mathbf{\%}$ \\
\hline \multicolumn{1}{c}{ Microliths: } & & \\
On blades & 52 & 82.5 \\
On flakes & 11 & 17.5 \\
& & \\
$\quad$ Non-microlithic tools: & & \\
On blades & 32 & 58.2 \\
On flakes & 23 & 41.8 \\
\hline Total & 118 & \\
\hline
\end{tabular}

Table 6.3.3: Tools measured at Patne

Average microlith size is $22.9 \times 9.1 \times 3.8 \mathrm{~mm}$ (Table A.6.10). Although there are slightly more microliths in the sample than non-microlithic tools, debitage size profiles more closely match those of non-microlithic tools than microliths (Figure 6.3.5, Table A.6.10, Table A.6.11). Blanks for retouch into non-microlithic tools were selected from the general debitage population, while microlith blanks were selected from only the smallest available debitage.

Non-geometric microliths constitute 79.4\% of the microlithic assemblage (Table 6.3.4). Single backed non-geometric microliths are dominant (63.5\%). Geometric forms include crescents, triangles and trapezes. Tanged or shouldered forms are absent. In the non-microlithic tool assemblage, most tools are side or end retouched. Burinations are also common. Points are present, including unifaciallyretouched points, as are a small number of notched and denticulated tool forms. 


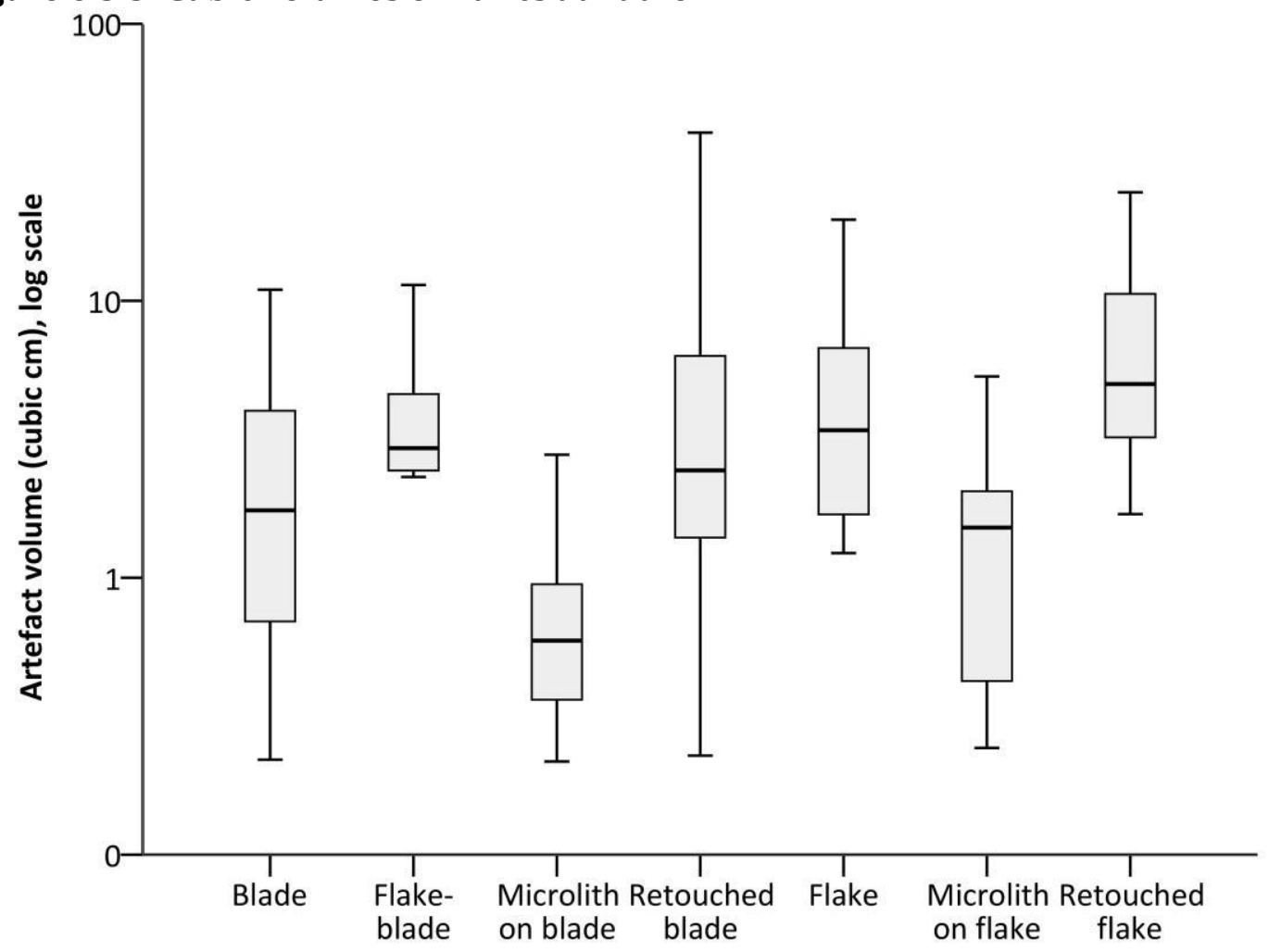

There are no statistically significant differences in the proportions of nonmicrolithic tool types manufactured on flake and on blade blanks $\left(\chi^{2}(6)=5.435, p\right.$ $=.489)$. However, strong and significant differences are evident in the microlith assemblage $\left(\chi^{2}(5)=32.035, \mathrm{p}<.001\right.$, Cramér's $\left.V=.713\right)$. Blade blanks were predominantly used for backed non-geometric microliths (78.8\%), while flake blanks were predominantly used for non-backed non-geometric forms (54.5\%).

Most microlith forms are of similar dimensions on average, although non-backed non-geometric microliths and burins form a distinct group on the basis of size, being slightly shorter and considerably wider and thicker than other types (Figure 6.3.6, Table A.6.12). Microliths exhibit more standardisation in size than nonmicrolithic tools, although no type can be considered particularly standardised (Figure 6.3.6, Figure 6.3.7, Table A.6.12). 


\begin{tabular}{llr}
\hline Coarse typology & Narrow typology & Frequency \\
\hline Microliths: & & 1 \\
Backed non-geometric microlith & Backed and side retouched & 40 \\
& Backed & 1
\end{tabular}

$\begin{array}{lr}\text { Microlith with burin } & 2\end{array}$

$\begin{array}{lr}\text { Crescent microlith } & 9\end{array}$

Non-geometric microlith $\quad 5$

Trapeze microlith 2

Triangle microlith 2

$\begin{array}{lr}\text { Total } & 63\end{array}$

Non-microlithic tools:

$\begin{array}{ll}\text { Burin } & \text { Burin } \\ & 12\end{array}$

Double burin $\quad 1$

Triple burin 1

$\begin{array}{lll}\text { End retouched } & \text { Double end retouched } & 1\end{array}$

End retouched $\quad 11$

Notched/denticulated Denticulated 2

Side and end retouched with notch $\quad 1$

$\begin{array}{lll}\text { Point } & \text { Backed point } & 1\end{array}$

Concave point ('awl') 3

Side and end retouched $\quad$ Double side and end retouched 4

Side and end retouched 2

Side retouched $\quad$ Double side retouched 2

Side retouched 12

$\begin{array}{ll}\text { Unifacial point } & 2\end{array}$

$\begin{array}{lr}\text { Total } & 55\end{array}$

Table 6.3.4: Tool type frequencies at Patne 
Figure 6.3.6: Cubic volumes of microlith types at Patne

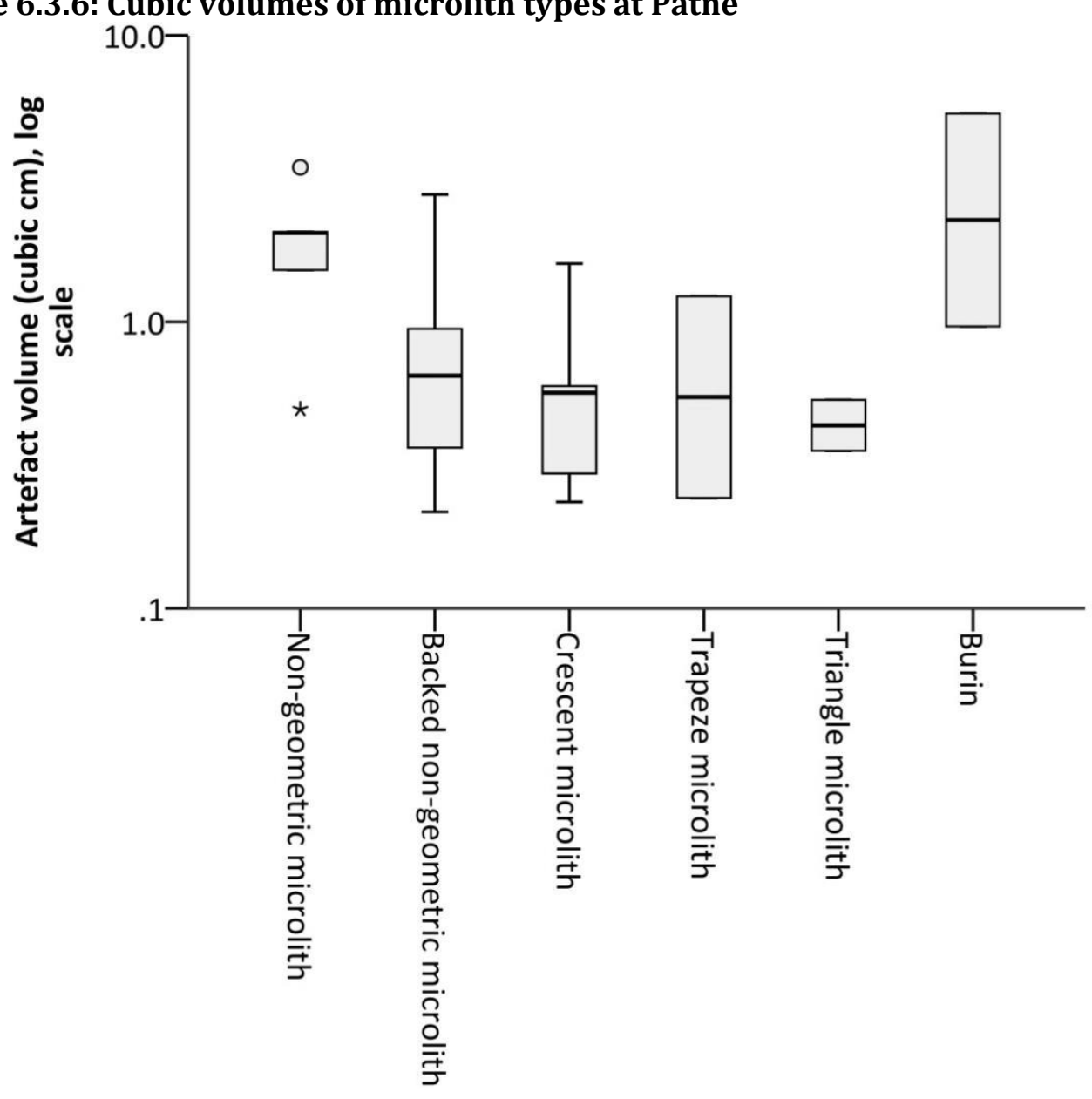

In the microlith assemblage there is little variation in mean lengths, but slightly more variation in width and thickness measurements (Table A.6.12). Mean dimensions of non-microlithic tool types vary less, although there is variation within individual types. For example, the lengths of points vary from $23.9 \mathrm{~mm}$ to $75.8 \mathrm{~mm}$. When non-microlithic point categories are combined to create a group of sufficient size to calculate $\mathrm{CV}$ values, no standardisation is evident, with $\mathrm{CV}$ values of 41.87, 38.69 and 55.49 for length, width and thickness, respectively.

Geometric and backed non-geometric microliths (excluding burins) mostly exhibit very small TCSA measurements, with group means ranging from 10.0-15.9 $\mathrm{mm}^{2}$ 
(Table A.6.13). Non-backed non-geometric microliths have much larger TCSA measurements, with a mean of $49.0 \mathrm{~mm}^{2}$, and only the smallest overlaps with those of the geometric and backed non-geometric microlith sample. The mean TCSA of non-backed non-geometric microliths lies between those of Shea's (2006) arrowhead and dart tip samples, and so it cannot be ruled out that these tools were also used as projectiles. They may, however, have formed a different category of projectile, although it is perhaps more likely that they fulfilled a different function, as discussed in Chapter 2.1.

Figure 6.3.7: Cubic volumes of non-microlithic tool types at Patne

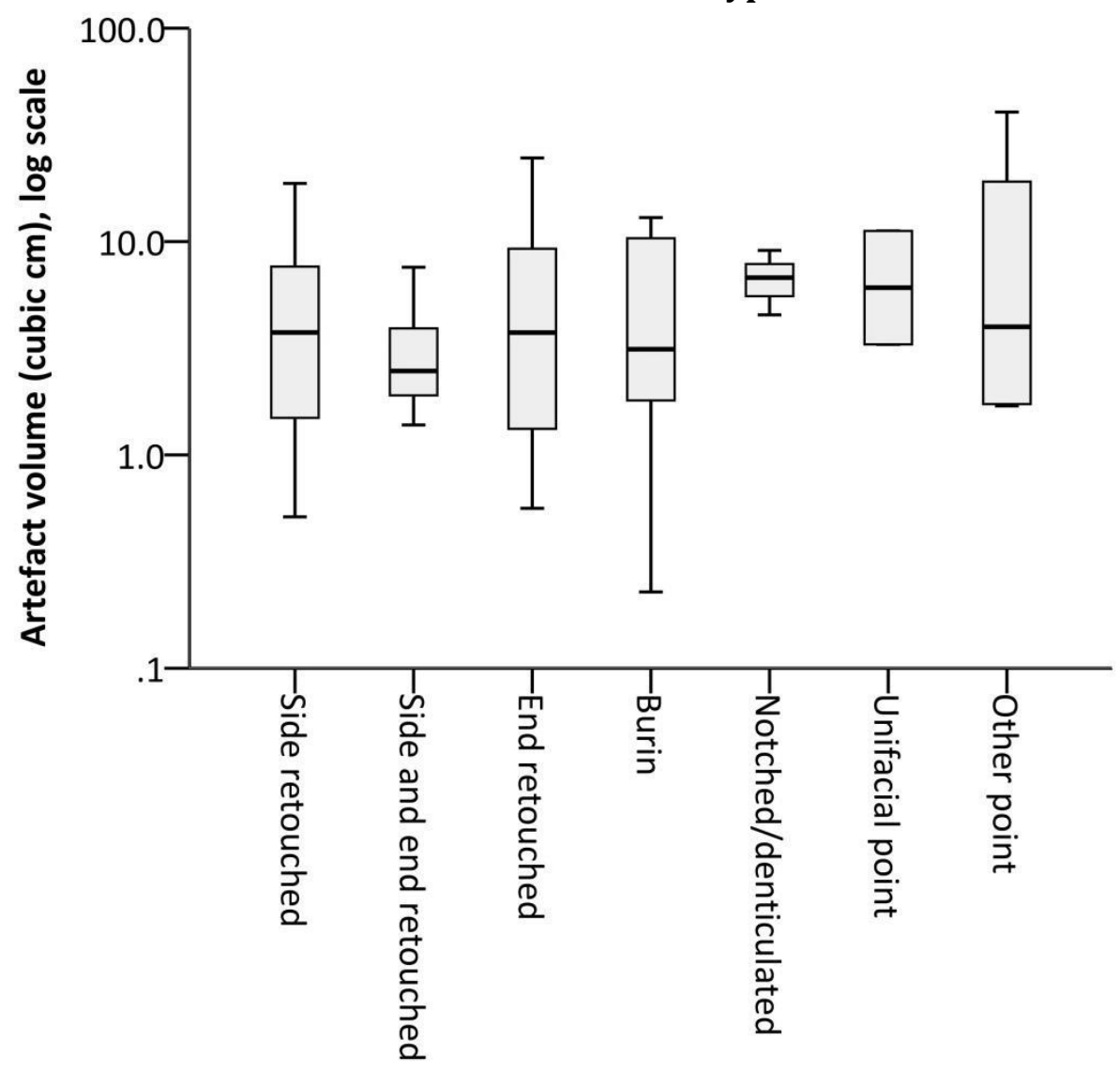

Within the non-microlithic tool sample the mean TCSA measurements for points where use as projectiles might be expected - lies in the range that Shea (2006) 
considers indicative of hand-cast or thrusting spears $\left(85.3 \mathrm{~mm}^{2}\right.$ for unifacial points and $102.7 \mathrm{~mm}^{2}$ for other points) (Table A.6.13). However, even the point assemblage does not exhibit any degree of standardisation.

Most retouch conducted on microlithic tools is backing (83.3\%), of which $16.7 \%$ is bipolar backing. All retouch conducted on geometric microliths is backing (Figure 6.3.8). Parallel, sub-parallel and scalar retouch techniques were used on nongeometric microliths. One incidence each of parallel and sub-parallel retouch is found on the second retouched edges of backed non-geometric microliths.

Figure 6.3.8: Retouch techniques on microliths at Patne

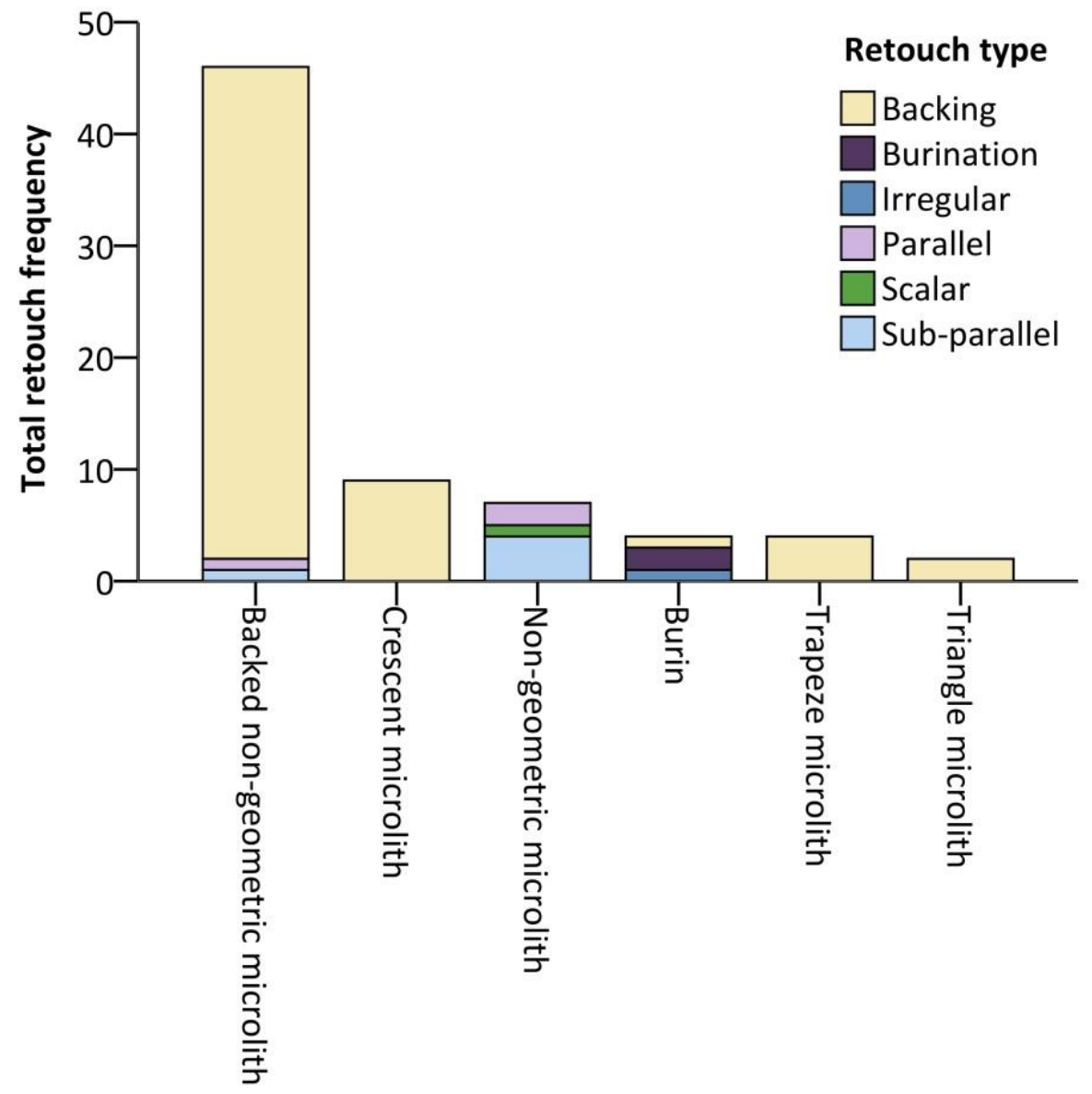


Some differences can be seen in retouch technique between microliths manufactured on flake and on blade blanks. While backing retouch constitutes almost all (94.7\%) retouch on microliths manufactured on blade blanks, it makes up less than half (40.0\%) of retouch on those manufactured on flake blanks. In contrast, sub-parallel and parallel retouch are much more common on the latter, and together are as common as backing retouch (40.0\%). Scalar and irregular retouch are present on flake-based microliths but not on blade-based microliths.

Retouch techniques are more varied in the non-microlithic tool assemblage (Figure 6.3.9). Sub-parallel retouch is dominant (39.4\%), and burinations are also common (23.9\%). Backing retouch is also found (9.9\%). In addition to the distinct size profiles of microlithic and non-microlithic tools, these tool categories were also worked and shaped using different repertoires of retouch techniques. For example, whereas both microlithic burins exhibit one burination and one edge retouched using a different technique, all non-microlithic burins exhibit only burinations, including one double and one triple burin.

The majority of tools are worked along a single lateral edge, although some are retouched in two separate locations $(14.3 \%$ of microliths and $14.5 \%$ of nonmicrolithic tools) and four non-microlithic tools are retouched in three locations (Table A.6.14). While lateral retouch is dominant on microlithic tools (83.3\%), end retouch is more common on non-microlithic tools (50.7\%). Dorsal retouch is most common overall (83.3\% of microliths and $93.1 \%$ of non-microlithic tools), followed by ventral retouch on non-microlithic tools $(12.7 \%)$ and edge retouch on microliths (13.9\%). Edge retouch in the microlith assemblage appears in the form 
of bipolar backing, and is found on non-geometric microliths only. Bipolar backing is also evident in the non-microlithic tool sample, where it was used to retouch a point along the distal and both lateral edges. The shapes of retouched edges of microlithic tools are almost entirely straight $(52.8 \%)$ or convex $(41.7 \%)$. There is more variation in the non-microlithic tool sample, including nosed/beaked, perpendicular and irregular outlines which are absent from the microlith sample.

Figure 6.3.9: Retouch techniques on non-microlithic tools at Patne

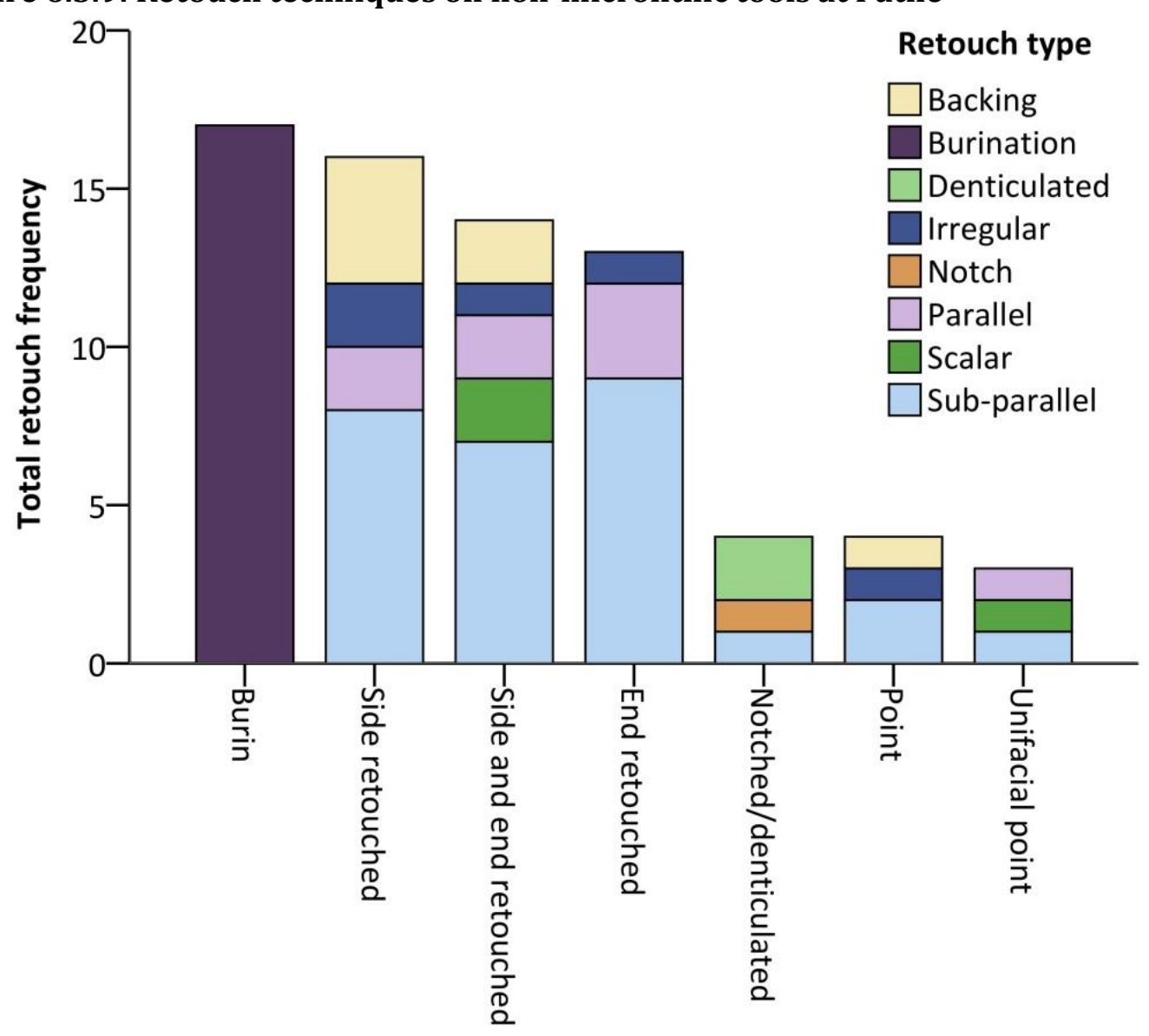

There is no evidence for standardisation of retouch lengths within specific tool types, with the possible exception of crescent microliths which have a CV of 17.98 (Table A.6.15). IOI values are generally low, with no tools retouched over more than half of their surface area (Table A.6.16). Unusually, microliths are retouched 
slightly more extensively on average than non-microlithic tools, with a mean IOI of 0.110 compared to 0.091. Examples of microliths are illustrated in Figure 6.3.10.

Figure 6.3.10: Microliths from Patne Layer IIE (1-19), IID (20-37), IIC (38-41) and IIB (42) (compiled from Sali 1989, Figures 21-23 and 25). According to Sali's descriptions, these forms include various forms of backed microblades (1-7, 20-25, 38-42), tanged blades $(8,26,27)$ and points $(9,10,28-31)$, as well as crescents $(11-$ $14,32-37)$, triangles $(15-17)$ and trapezes $(18,19)$.

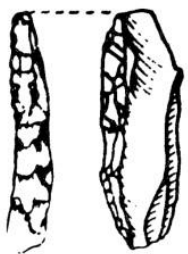

1

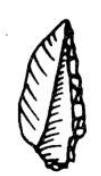

8

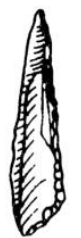

15

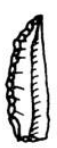

22

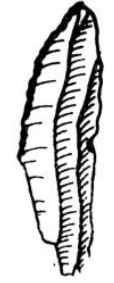

2

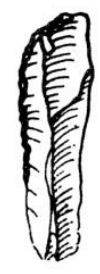

3

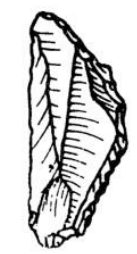

4
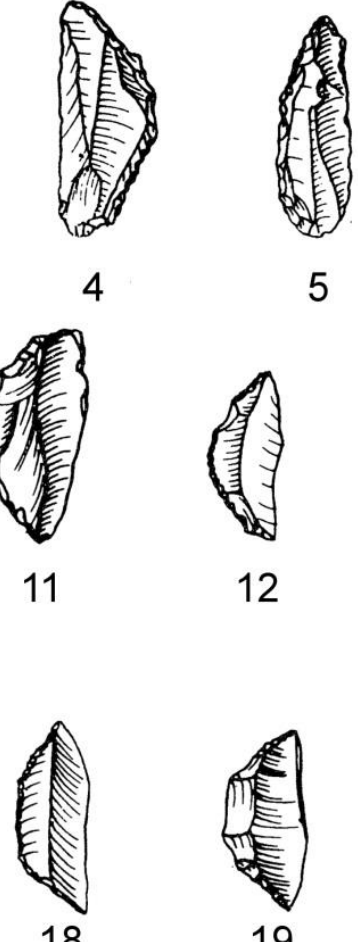

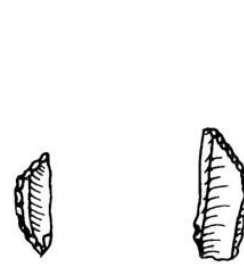

17

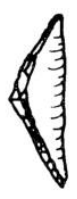

16

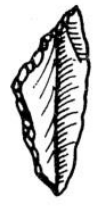

23

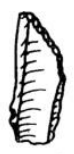

24

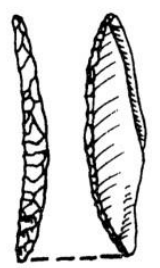

25

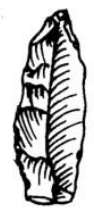

31

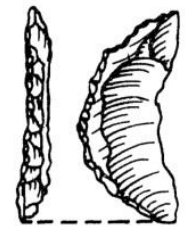

32
5

12
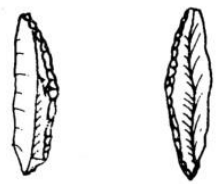

6

7

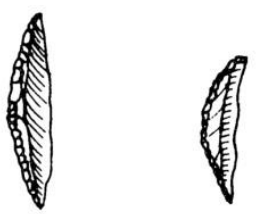

13

14
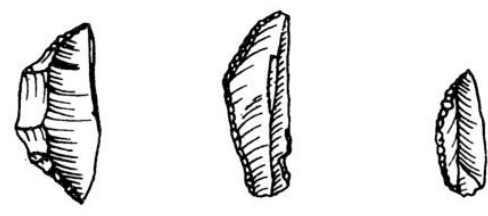

20

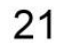

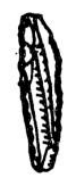

26

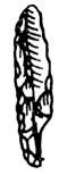

27

28
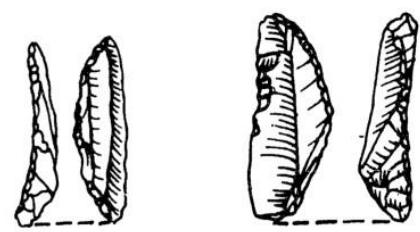

33

34

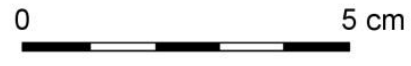




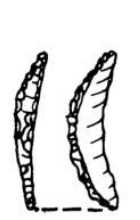

35

40

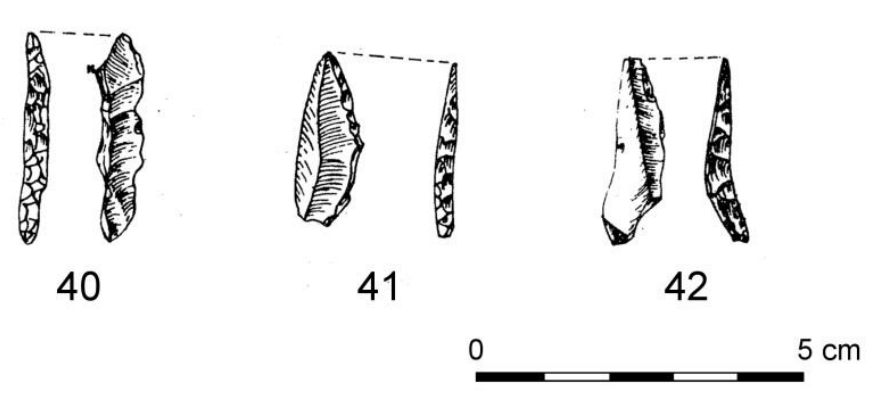

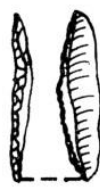

36

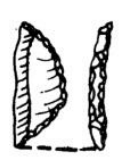

37

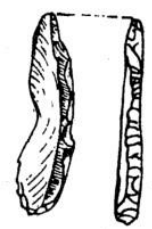

38

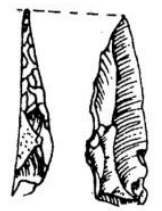

39

TEMPORAL VARIATION

Results of statistical tests at Patne are presented in Appendix B.5. The numbers of cores and tools in each layer are reported in Table 6.3.5.

\begin{tabular}{lrrr}
\hline Layer & Cores & Microliths & Non-microlithic tools \\
\hline IIE & 8 & 14 & 5 \\
IID & 19 & 35 & 25 \\
IIC & 12 & 10 & 6 \\
IIB & 18 & 4 & 19 \\
\hline
\end{tabular}

Table 6.3.5: Cores and tools analysed in each layer at Patne

\section{Cores}

Results of statistical tests on cores are reported in Table B.5.1 and summarised in Table 6.3.6. There is remarkable consistency in core size over time (also noted by James 2011), with the length of the longest face being the only size variable that differs significantly between layers. 


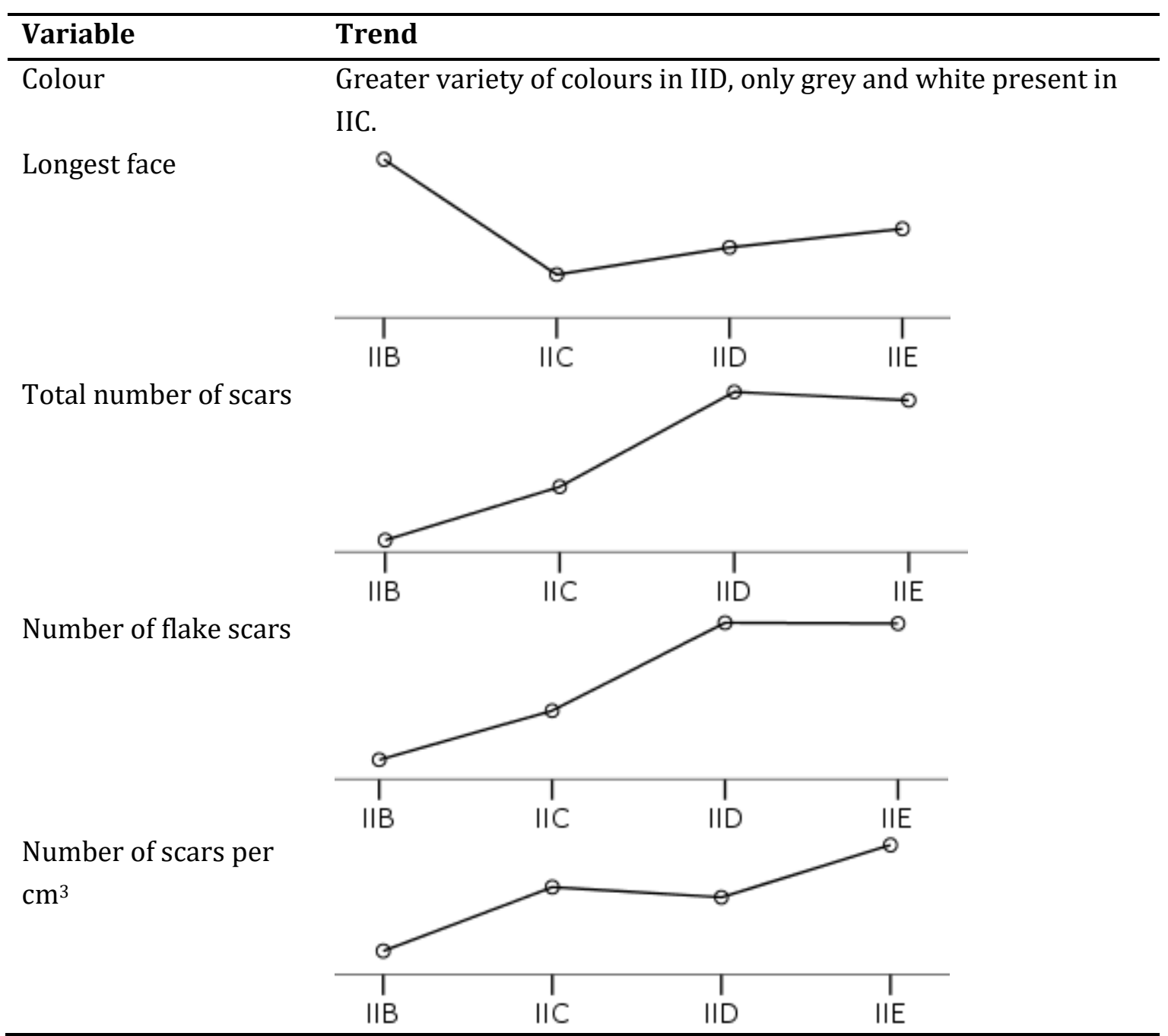

Table 6.3.6: Statistically significant differences between layers in core variables at Patne

Changes in raw material are hinted at through the proxy of changes in colour. The main changes observed in the core sample are in reduction intensity and flake and blade production. There is a considerable increase over time in the number and density of terminal removal scars on each core. However, the average length of removals does not vary significantly over time. More removals were being made on each core, but not of substantially different sizes. Therefore this pattern could be explained by changes in the pressures made on the efficient use of raw materials, rather than, for example, a desire to remove smaller flakes and blades for the 
production of microlithic tools. The increase in removal numbers and densities is mostly due to an increase in the number of flake scars per core, which is in contrast to the argument put forward by James (2011) that the lithic assemblage exhibits an intensification of blade production over time.

\section{Debitage}

Results of statistical tests on debitage are reported in Table B.5.2 and summarised in Table 6.3.7. Notably, the only variable that does not differ significantly between layers is debitage type. A variety of complex patterns exist in the changes over time observed, with few variables exhibiting consistent temporal trends. These include trends towards more broken lithics over time, and more cortex cover. The remainder of the variables exhibit more complex differences between layers. For example, there are slight differences in striking platform types and lipping between layers, but no overall trend.

\begin{tabular}{ll}
\hline Variable & Trend \\
\hline Condition & Increase over time in the proportion of broken debitage, \\
especially of unclear breakages. \\
Higher proportions of blue and green debitage in IIB. \\
Oriented length
\end{tabular}


Medial width

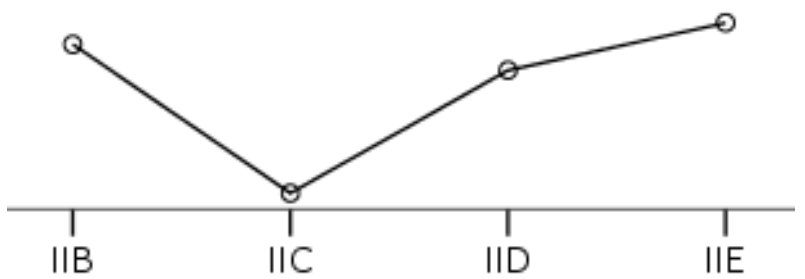

Maximum thickness

Medial thickness
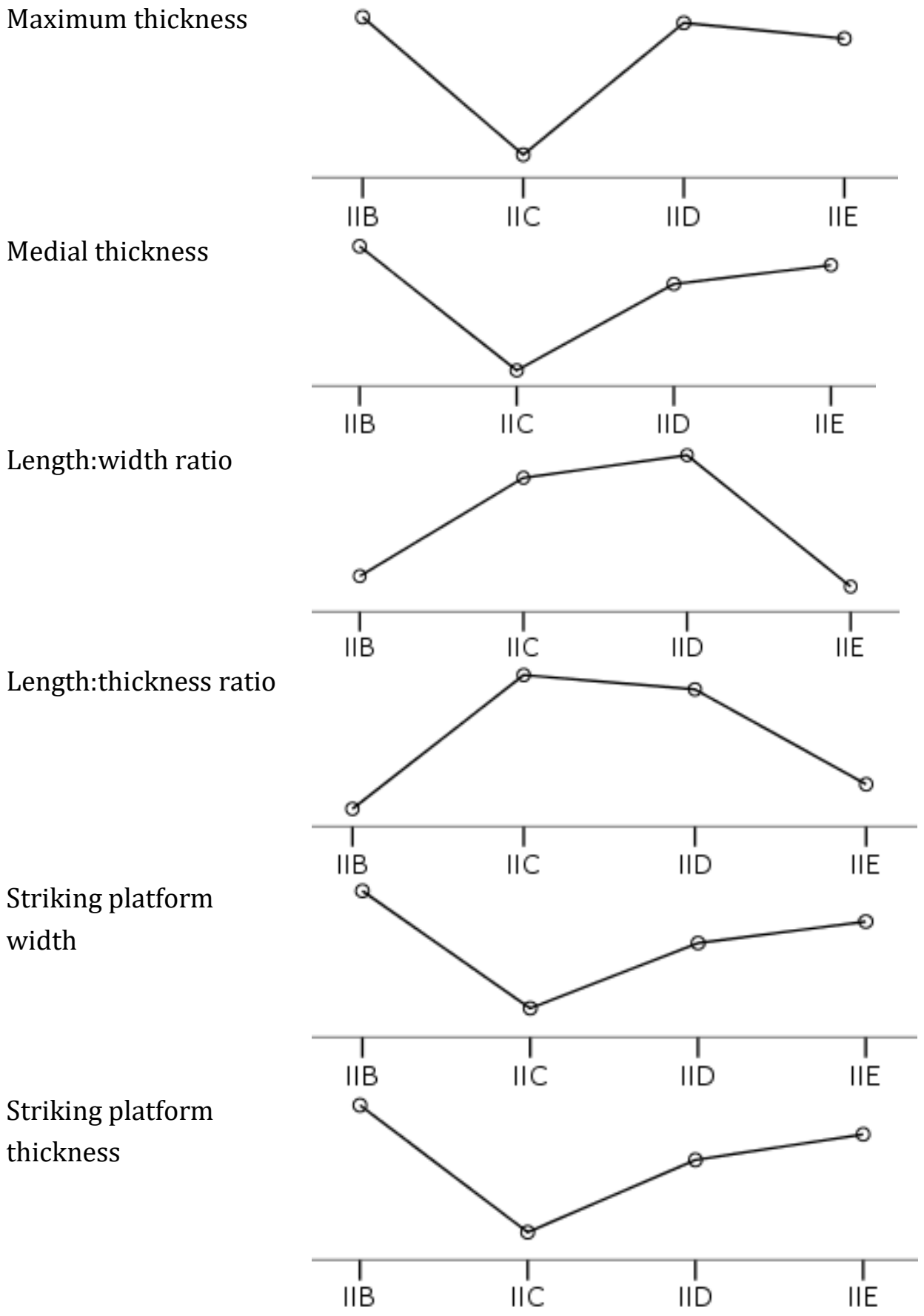

Striking platform type More cortical striking platforms in IIB. 
Striking platform lipping

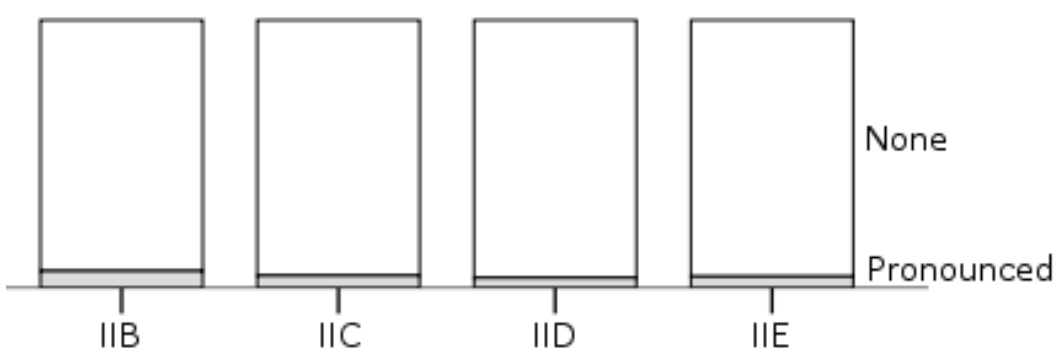

Percentage cortex cover

Number of dorsal scars

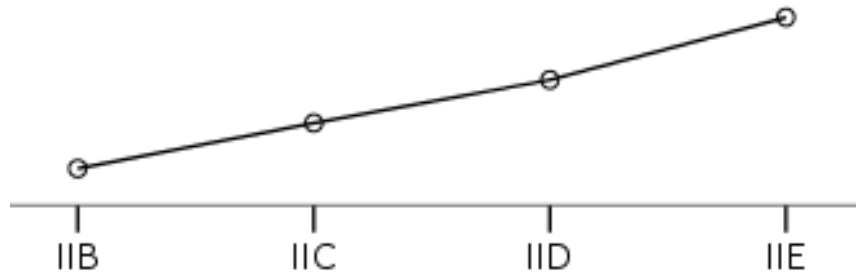

Number of parallel arrises

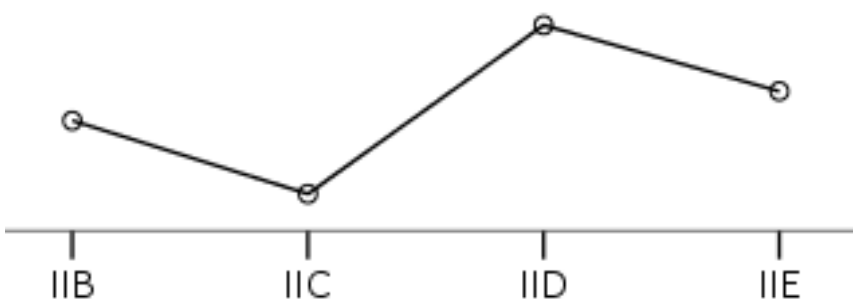

Scars per $\mathrm{cm}^{2}$

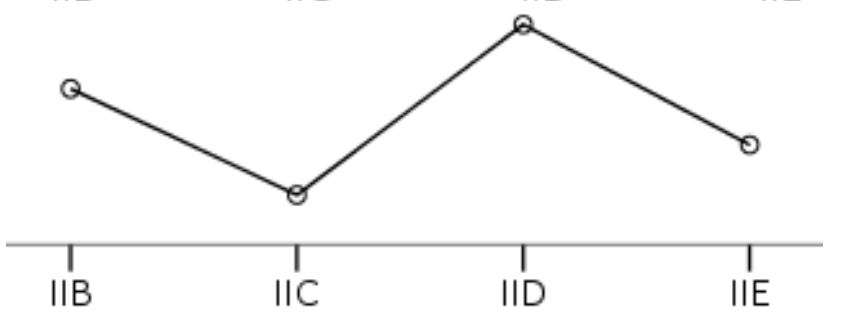

Arrises per $\mathrm{cm}^{2}$
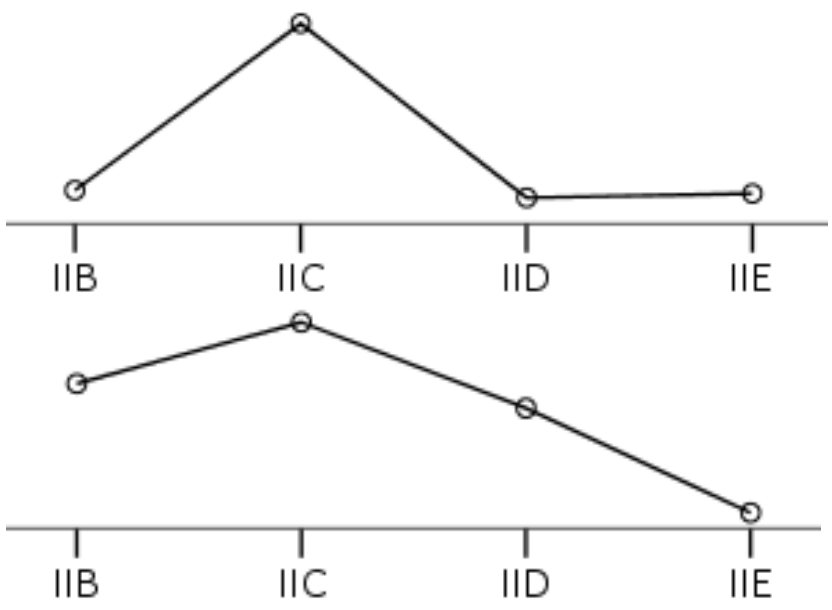

Scar orientation

Increase over time in proportion of debitage with scars from a single direction. Decrease over time in proportions of debitage with scars from two opposite and four directions.

Termination type

Decrease over time in proportions of hinge and step terminations.

Table 6.3.7: Statistically significant differences between layers in debitage variables at Patne 
In terms of size and scar numbers and densities, debitage in Layer IIB is longer, wider and thicker, and therefore larger, overall, and has low length:width and length:thickness ratios indicating squatter and thicker debitage in relation to length - the assemblage in Layer IIB is larger and more flake-like. Debitage in Layer IIC is significantly smaller on average than the other layers, but has high length:width and length:thickness ratios - the assemblage in Layer IIC is smaller and more blade-like. In Layer IID there is a return to an overall size similar to that in Layer IIB, although it retains high length:width and length:thickness ratios similar to Layer IIC - the assemblage in Layer IID is larger and more blade-like. The Layer IIE debitage assemblage is similar to that in Layer IIB, although it exhibits an even lower density of parallel arrises - the assemblage in Layer IIE is larger and more flake-like.

These observations are generally in accordance with those made by James (2011). However, James concludes that the debitage assemblage indicates an overall pattern of increasing intensity of lithic reduction over time, especially of blade production. In contrast, it is argued here that although the core assemblage does attest to a greater number of removals being made per core over time, the debitage assemblage does not demonstrate a reduction over time in the size of debitage, an increase over time in the density of dorsal scars, or any other change that could be attributed to increased intensity of lithic production. Instead, the debitage sample reveals the existence of four different assemblages, distinguished by multiple variables but especially by size and by emphases on flake- or blade-like characteristics, with few overall trends evident. 


\section{Microliths}

Results of statistical tests on microliths are reported in Table B.5.3 and summarised in Table 6.3.8. With the exception of Layer IIB, microliths are consistently more common than non-microlithic tools, and increase in proportion with the frequencies of non-microlithic tools (Table 6.3.5). Although flake debitage remains dominant in all layers, there is a near-consistent increase in the density of parallel arrises over time, indicating an increase in the use of more blade-like blanks for microliths.

\begin{tabular}{ll}
\hline Variable & Trend \\
\hline Colour & Blue microliths appear only in IIB, purple only in IIC and yellow only \\
in IID and IIE. & Arrises per cm ${ }^{2}$
\end{tabular}

Table 6.3.8: Statistically significant differences between layers in microlith variables at Patne 
Many variables exhibit no significant differences between layers, including size measurements and typology. There are, however, significant changes in some retouch variables (face and type), as well as in retouch intensity (IOI). Changes in these variables appear to reflect retouch choices which are specific to individual layer assemblages. For example, bipolar backing only occurs in Layer IID. Although this may be partly related to the larger sample size in this layer, it may also be a true indication of the use of a distinct retouch technique during one particular period in the site's history. Differences in retouch type may likewise be related to technological choices specific to particular occupational timeframes.

\section{Non-microlithic tools}

Results of statistical tests on non-microlithic tools are reported in Table B.5.4 and summarised in Table 6.3.9. The non-microlithic tool assemblage is the only lithic type that displays statistically significant differences between layers in the proportions of flakes $\mathrm{v}$. blades, although these changes are not part of a consistent trend. Indeed, no variables indicate consistent trends over time. For example, there are layer-specific combinations of termination types, and a particular emphasis on convex retouched edges in Layer IIB in comparison to the dominance of straight retouched edges in the other layers.

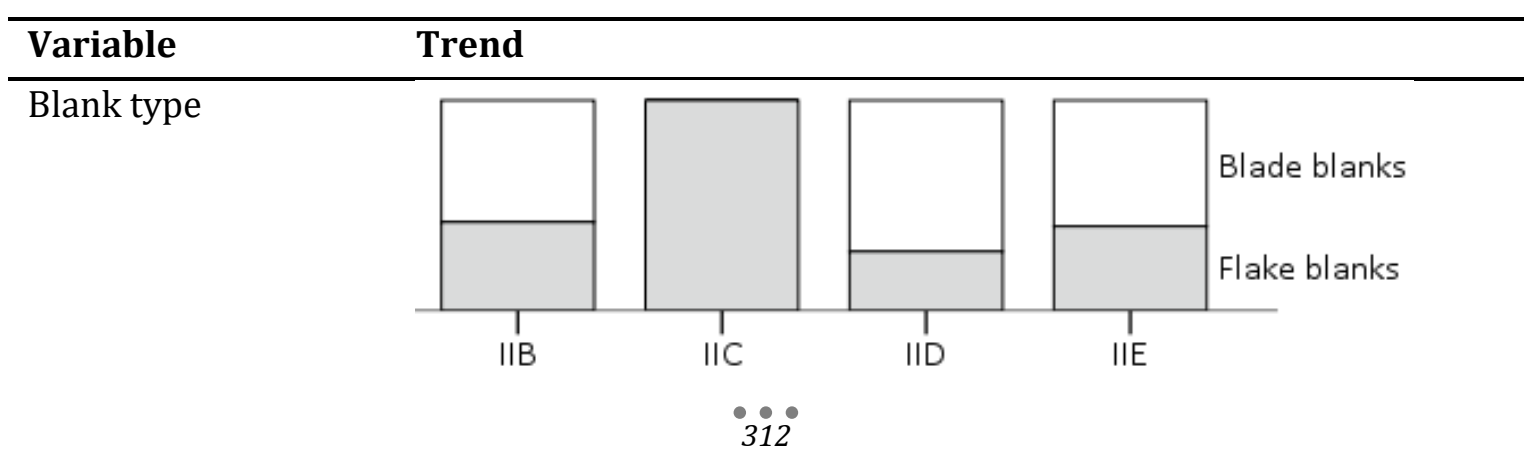


Colour

No overall trends, but slightly different colour profiles in each layer, with the least diversity in IIC.

Maximum width

Medial width

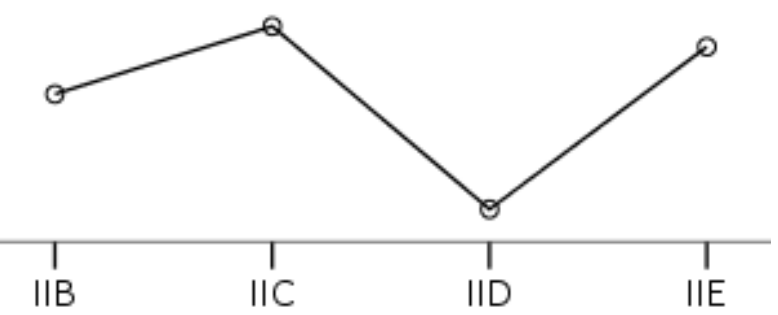

Maximum thickness

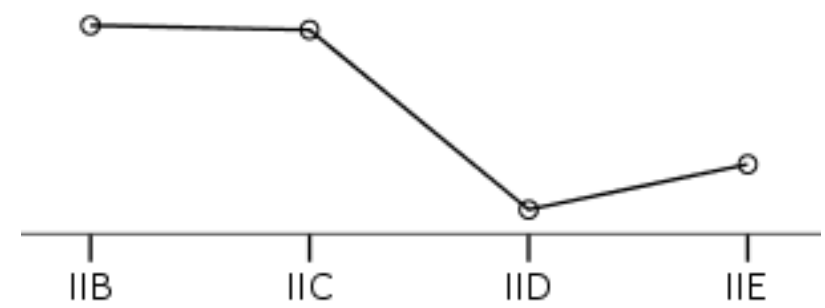

Medial thickness

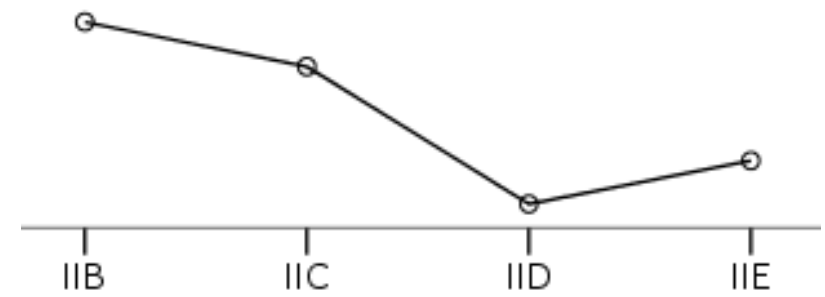

Striking platform type

Scars per $\mathrm{cm}^{2}$

Arrises per $\mathrm{cm}^{2}$

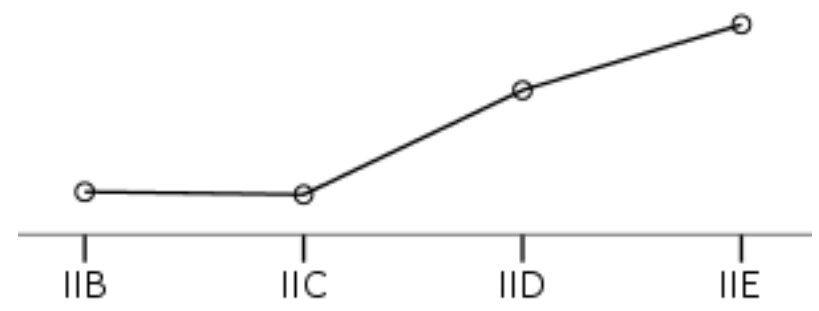

Termination type
IIC contains only flat platforms. Cortical platforms appear only in IIB.
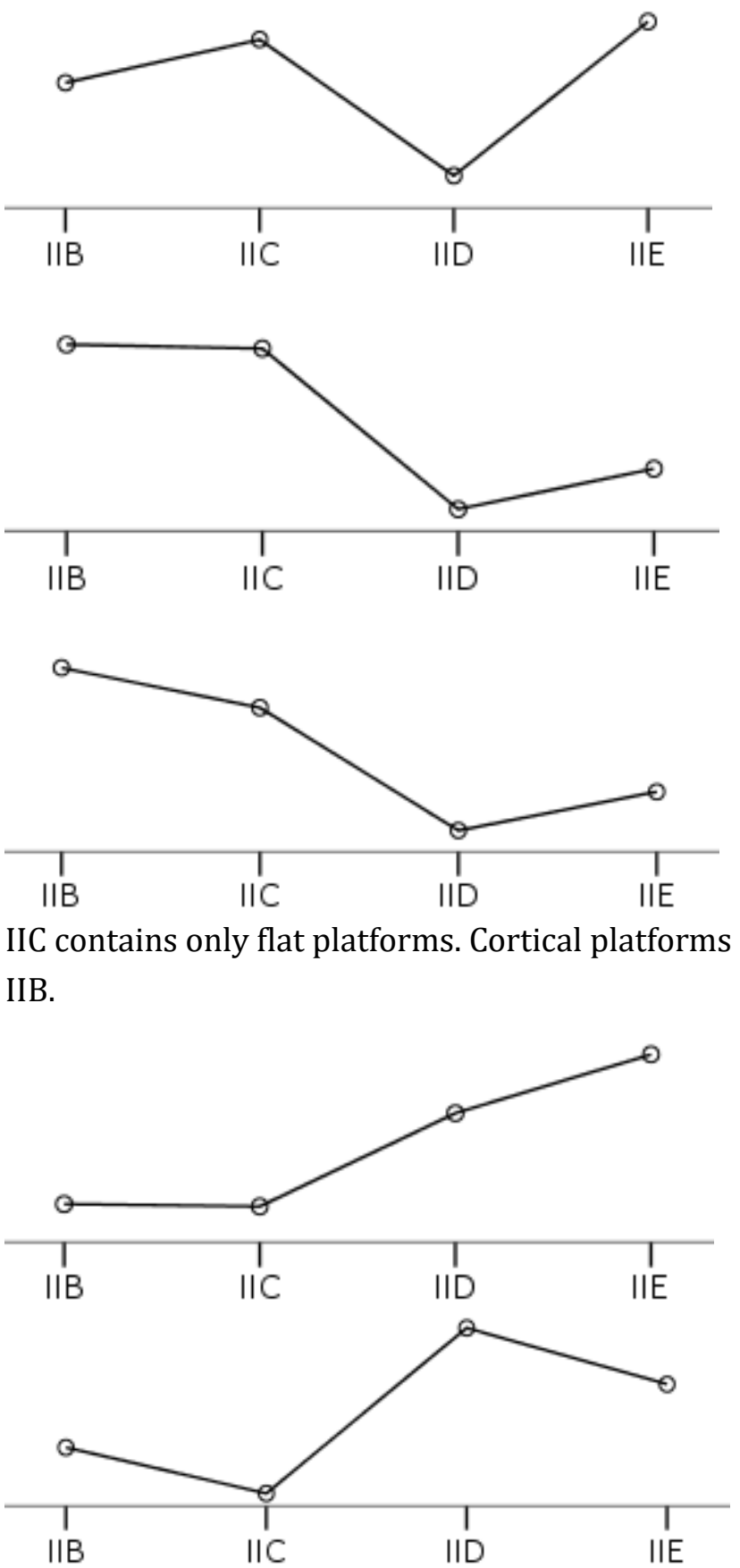

IIE contains only feather terminations. Step terminations appear only in IIB and IIC, plunging in IIB and IID and hinge in IIC and IID. 
Retouch outline

Index of Invasiveness

TCSA
Straight retouched edges are dominant in all layers except IIB, where convex edges are dominant.

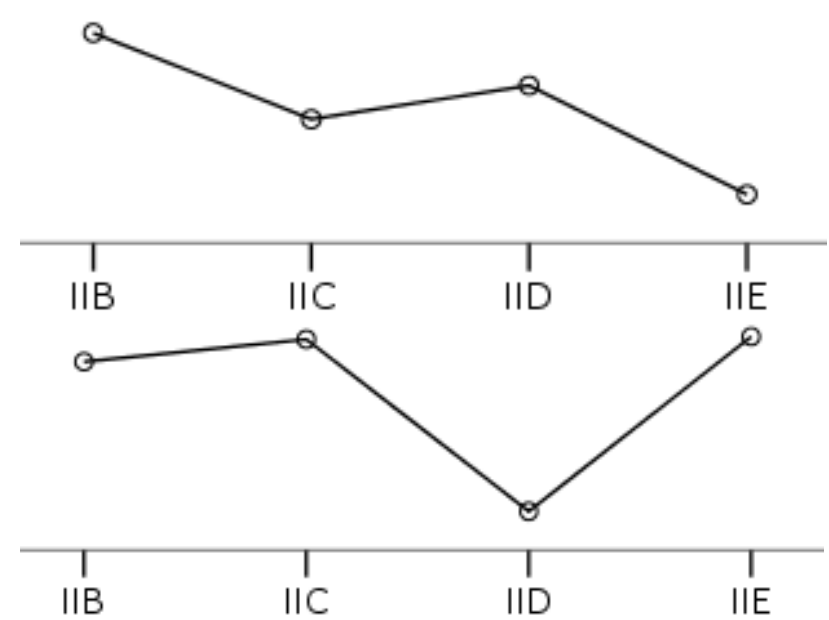

Table 6.3.9: Statistically significant differences between layers in non-microlithic tool variables at Patne

The mean values of many metric variables oscillate between layers, with no overriding trajectory. Mean width and thickness measurements are similar in IIB and IIC, then decrease substantially from IIC to IID, before increasing somewhat from IID to IIE. This is essentially the opposite of the pattern seen in the debitage assemblage, where lithics in IIC are considerably smaller on all size metrics, and those in IID are considerably larger. Possibly during site occupation in IIC only the largest and thickest lithics were selected for retouch into non-microlithic tool forms, while relatively small blanks were selected in IID. The non-microlithic tool assemblage in IIC also looks different to those in other layers in other ways, with only flake blanks used and only flat striking platforms evident, although this may be an artefact of small sample size. Overall, a complex picture of temporal variation is apparent at Patne. 


\section{MANUFACTURING TRAJECTORIES AND SUMMARY}

Manufacturing trajectories at Patne are summarised in Figure 6.3.11. Single and double platform cores are dominant, with multi-directional cores rare and radial cores absent. There are some indications that an earlier stage of core reduction may have involved the working of cores from perpendicular platforms and the removal of flakes in order to prepare cores for the removal of blades. However, flake cores are considerably more common than blade cores, and size differences between types are small. Additionally, blade removals are relatively common even on flake cores, suggesting more of a continuum of core types at the site rather than a separation of flake and blade cores in the reduction sequence.

The relatively large size of discarded cores suggests that efficient use of raw material was not a pressing concern. Low levels of core reduction intensity can also be seen in the low numbers of terminal removal scars in relation to core size, and in the high proportions of cortex cover. Related to this is the near absence of bipolar percussion at the site, which is a technique often utilised to more efficiently reduce stone raw materials. Only one core with evidence for bipolar percussion was recorded, which was particularly large and not very intensively worked.

However, there is some evidence to suggest that cores became more intensively worked in later levels. There is an increase over time in the number of terminal removal scars on cores both in absolute terms and relative to core size, although the average length of these removals does not change significantly. More removals were being made on each core, but not of substantially different sizes, suggesting 
that this trend was related to changes in pressures made on the efficiency of raw material use, rather than changes in reduction strategies.

Figure 6.3.11: Manufacturing trajectories at Patne

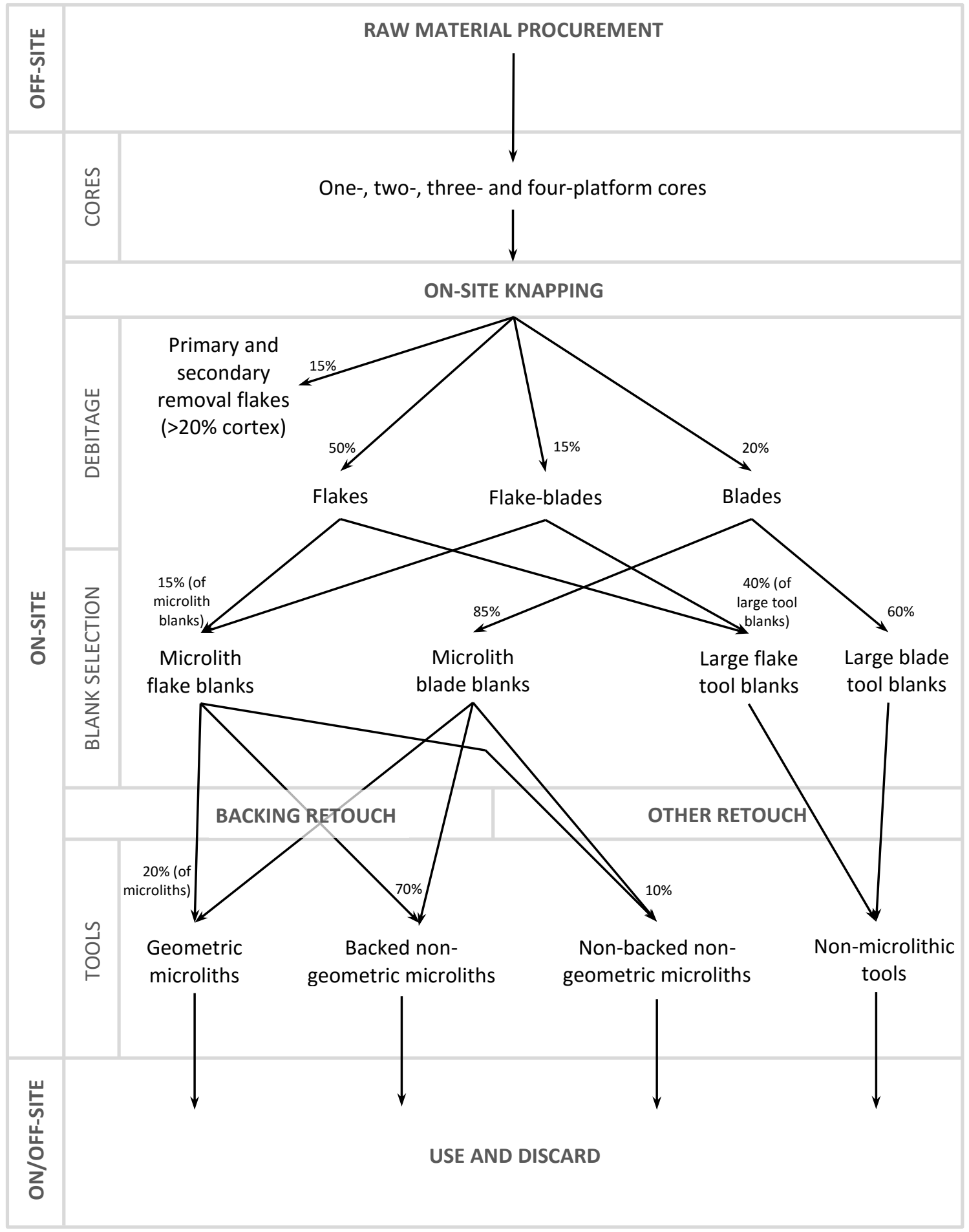


The increases in scar numbers and densities are largely due to an increase in the number of flakes removed per core. The majority of the products of lithic reduction are flakes. It is unclear whether blades formed a separate stage to flakes in the reduction sequence. Blades exhibit less dorsal cortex, smaller striking platforms and denser scar concentrations, but more cortical striking platforms. Other variables show no significant differences between flakes and blades, including platform lipping and termination types.

Considerable variation between layers is evident in the debitage sample, the majority of which is in the form of layer-specific variation rather than consistent trajectories of change. In contrast to the conclusion of James (2011), the debitage assemblage does not demonstrate a clear trend towards an increased intensity of lithic reduction or of blade production. Instead, it reveals the existence of four somewhat different lithic assemblages, each used to produce similar microlithic and non-microlithic tools.

Unusually, the size profiles of non-microlithic tools more closely match those of the debitage assemblage than do microlithic tool size profiles. While blanks for retouch into non-microlithic tools were selected from the general debitage population, microlith blanks were selected from only the smallest available debitage. Additionally, while blades do not appear to have been afforded any special treatment or attention in the core reduction process, they were heavily preferred for selection as blanks in both the microlith and non-microlithic tool assemblages. 
In the microlith assemblage, non-geometric forms are four times as common as geometric shapes. The variety of forms is relatively non-diverse, with nongeometric microliths backed along a single edge making up almost two thirds of the assemblage. Geometric forms include crescents, triangles and trapezes. Tanged and shouldered forms are absent. Much of the size variation between microlith types is in the form of differences in width and thickness measurements, although no microlith type is particularly standardised.

In the microlith assemblage blade blanks were primarily used for the production of backed forms, while flake blanks were preferred for non-backed forms. In contrast, there are no preferences evident in the use of flake or blade blanks for the production of particular non-microlithic tool forms. Non-microlithic tools retouched along a single end or lateral margin are common, as are burins. Points are present, including unifacially-retouched points, as are a small number of notched and denticulated tool forms. Most of the variation in size within the nonmicrolithic tool assemblage is within specific tool types, rather than between them. No tool category demonstrates standardisation, including the points.

Most retouch conducted on microliths is backing - including all retouch on geometric microliths - less than a fifth of which is bipolar backing. Backing is more common on microliths manufactured on blade blanks than those on flake blanks, where parallel and sub-parallel retouch was also frequently used. Scalar and irregular retouch are present on flake-based microliths but not on blade-based equivalents. Although there are few differences between flakes and blades in the debitage assemblage, their differences as blank types are more pronounced. 
Backing retouch is also evident in low frequencies in the non-microlithic tool assemblage, although bipolar backing is absent. Retouch techniques and outlines are more varied on non-microlithic tools. Sub-parallel retouch is dominant, and burinations are also common. However, these larger burins were worked differently to the microlithic burins in the lithic assemblage.

Another difference between the microlithic and non-microlithic tool assemblages is that non-microlithic tools differ more between layers than microliths. The most notable difference in microliths between layers is the fact that bipolar backing only occurs in Layer IID, which may be an indication of the use of a distinct retouch technique during one particular period of site occupation. The non-microlithic tool assemblage is the only lithic type that displays statistically significant differences between layers in the proportions of flakes v. blades, but again these changes do not form a consistent trend. This is also the case for all other variables that exhibit statistically significant differences. Overall, the lithic assemblage at Patne is best viewed as a complex assortment of different strategies for the production of both microlithic and non-microlithic tools.

\subsection{JWALAPURAM 9, INDIA}

The results presented below are based on analysis of data collected by Clarkson et al. (2009). Differences between Clarkson's methodology and the one used in the current study are described in Appendix C.2. The main differences are that all debitage measured is whole, and that flake and blade designations were not 
recorded for all debitage. Therefore the classic definition of blades as having a length:width ratio above 2 was applied.

\section{CORES}

Multi-directional cores are more common than those with one or two platforms, although only a small number were worked in a radial manner (Table 6.4.1). Single platform cores are more common than two-platform cores, and the majority of cores can be classified as flake cores. Bipolar cores are also common, especially on quartz and quartzite (Figure 6.4.1). Most of the remainder of the quartz cores are amorphous multi-directional cores.

\begin{tabular}{llrr}
\hline Core type & & Frequency & Percentage \\
\hline Uni-directional: & Blade & 13 & 5.2 \\
& Flake & 44 & 17.5 \\
& Bipolar & 60 & 23.8 \\
& & & \\
Bi-directional: & Blade & 5 & 2.0 \\
& Flake & 24 & 9.5 \\
& & & \\
Multi-directional: & Radial & 5 & 2.0 \\
& Amorphous & 78 & 31.0 \\
& & & \\
Platforms unknown: & Blade & 8 & 3.2 \\
& Flake & 15 & 6.0 \\
\hline Total & & 252 & \\
\hline
\end{tabular}

Table 6.4.1: Core type frequencies at Jwalapuram

Limestone, dolerite and CCS varieties (chalcedony and chert) are also seen in the core assemblage. No single raw material makes up the majority of the assemblage, 
but chert (30.6\%) and limestone (29.0\%) are most common. Although the sources of the raw materials used at the site are not currently known, the most likely candidates are very close to the site (Clarkson et al. 2009).

Figure 6.4.1: Raw material proportions of cores at Jwalapuram

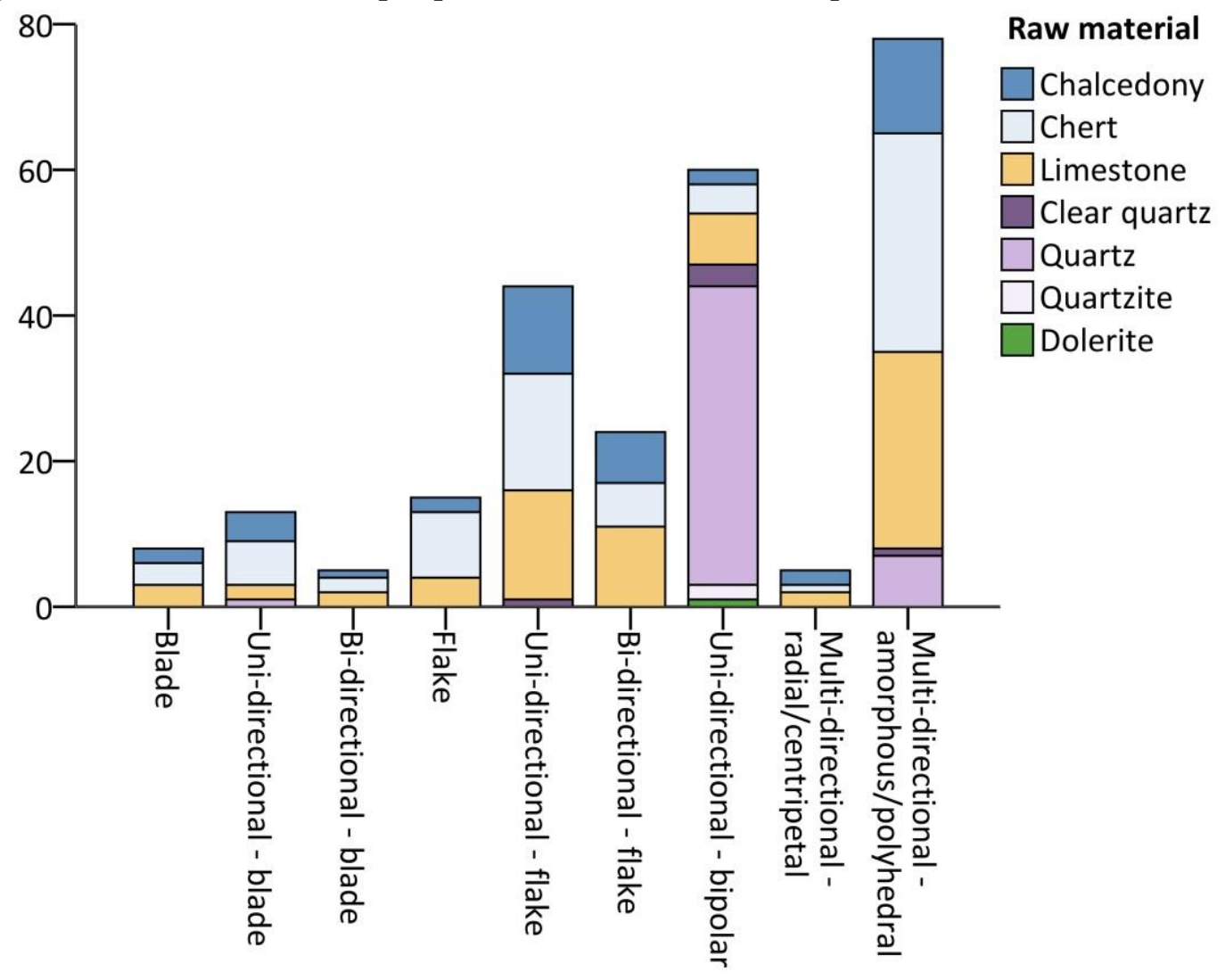

Cores are small on average, with mean dimensions of 20.8 x 17.7 x $13.4 \mathrm{~mm}$ (Table A.7.1). There is also considerable variation in core sizes, for example with cores weighing between $0.1 \mathrm{~g}$ and $475 \mathrm{~g}$. Much of this variation is due to differences between core types rather than within them; for example, bipolar cores are much smaller on average $(16.6$ x $12.4 \times 7.7 \mathrm{~mm})$ (Table A.7.2). No core type appears particularly standardised in terms of the size at which they were discarded. Some variation can also be attributed to differences in raw materials. As Figure 6.4.2 demonstrates, even when core type is held constant quartz cores are smaller on 
average, while limestone and chalcedony cores are larger. This may be related to different sizes of available raw material nodules.

Figure 6.4.2: Mean cubic volumes of cores on different raw materials at Jwalapuram. The single dolerite core is excluded.

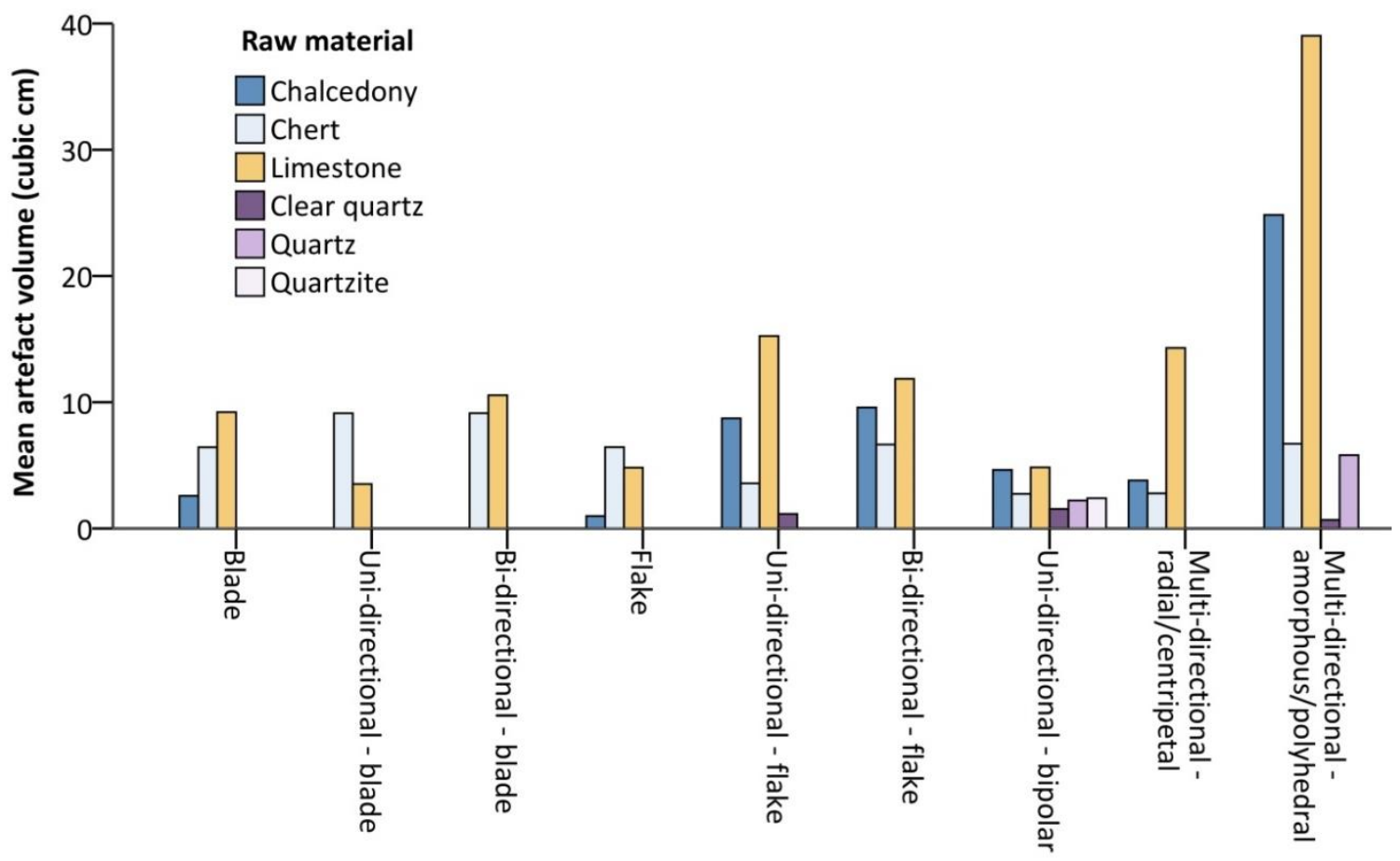

The most common striking platform types are double/complex (32.7\%) and flat platforms (29.0\%) (Figure 6.4.3). Cortical platforms are evident on a fifth of cores (20.4\%), indicating that primary stage core reduction was conducted at the site. The main difference between types is that abraded/crushed striking platforms are almost exclusively found on bipolar cores, where they make up 71.7\% of platforms. This is a classic feature of bipolar percussion.

Blade removals are uncommon on most cores that were not primarily used for the removal of blades (Figure 6.4.4, Table A.7.3). For example, no removals of blade dimensions were made on radial cores, at least at the end of their use-lives. Blade 
removals are also uncommon on other multi-platform cores, as well as on bipolar cores. Bipolar cores in this assemblage can be viewed as a type of flake core.

Figure 6.4.3: Proportions of core striking platform types at Jwalapuram

Striking platform type

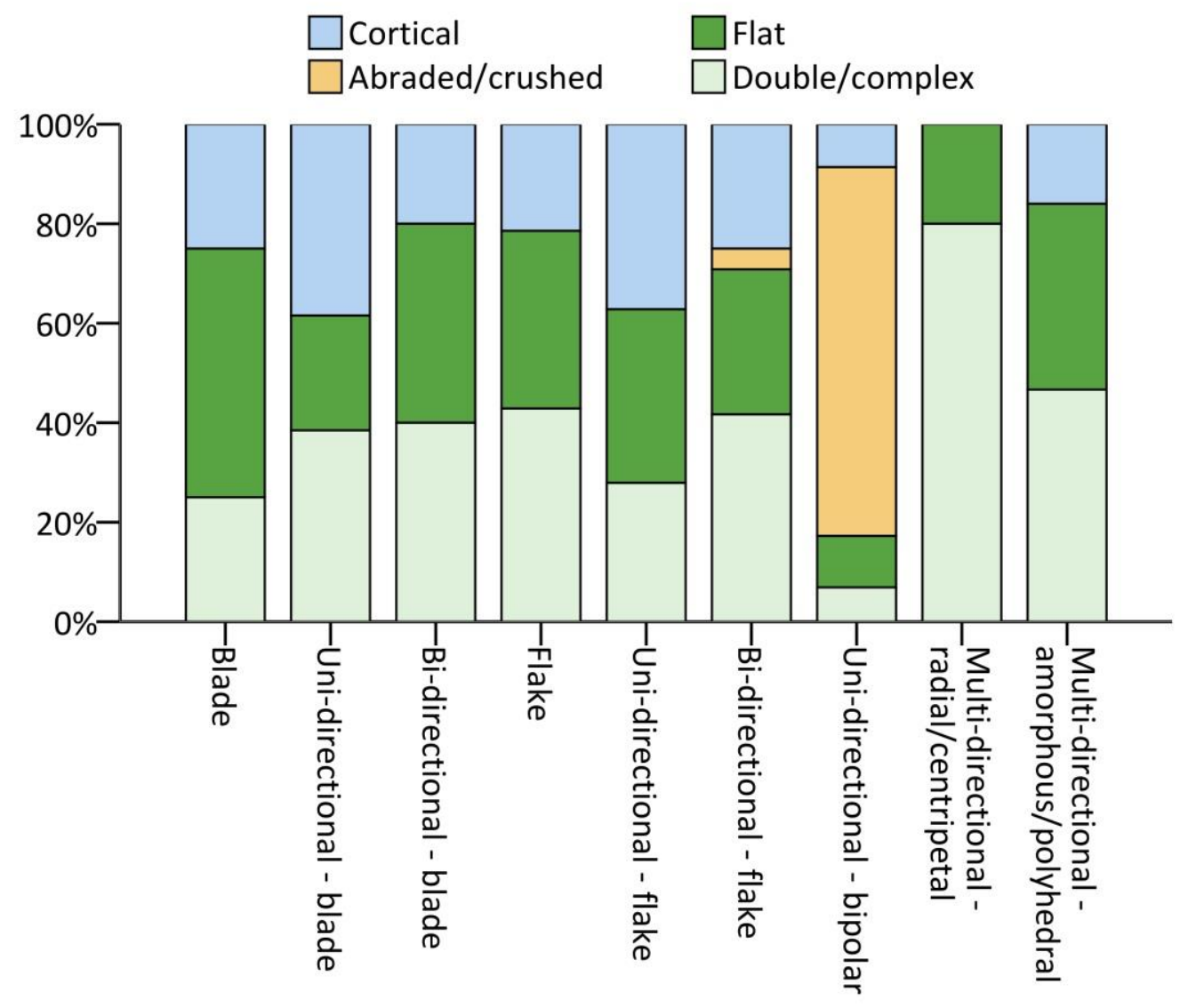

In the assemblage as a whole, cores are relatively intensively worked. This is especially true of bipolar cores, where particularly large numbers of removals were made relative to core size ( 8.4 scars per $1 \mathrm{~cm}^{3}$ ) (Table A.7.4). Flake cores are more intensively worked on average than blade cores both in absolute numbers and relative to core size. Radial cores are the most intensively worked core type in terms of absolute numbers of removals, but not when core size is taken into account. They also had particularly small flakes removed from them. Mean removal 
scar lengths are shortest on radial cores $(9.9 \mathrm{~mm})$ and longest on bi-directional cores (17.4 mm) (Table A.7.3).

Figure 6.4.4: Average numbers of flake and blade scars on cores at Jwalapuram

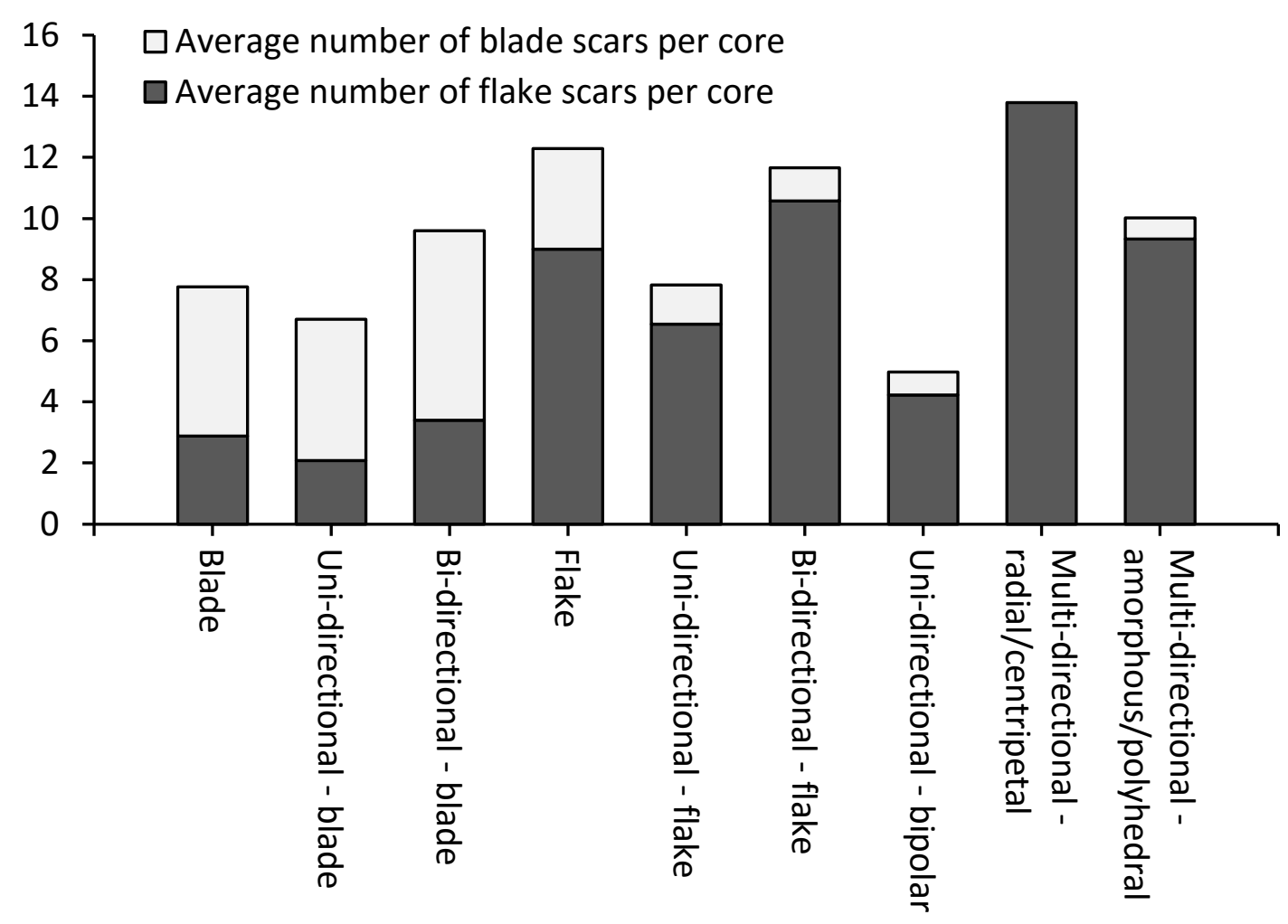

Evidence for the primary working of cores at the site can be seen in the fact that $57.7 \%$ of cores display cortex, with a mean cortex cover of $25 \%$. The highest levels of cortex cover are evident on single platform cores (excluding bipolar cores), with a mean of 35\% (Table A.7.5) - furthermore, none of this category is devoid of cortex. Multi-directional cores exhibit the smallest mean proportion of cortex cover (17\%), while two-platform cores are intermediate (25\%). Possibly cores worked from one platform formed an earlier stage in the core reduction process to those worked from two or more platforms, with additional striking platforms being created and maintained later in the knapping process, although this does not 
accord with the evidence from core size measurements. Bipolar cores have relatively low levels of cortex cover, further indicating that bipolar percussion was a technique used at the site to reduce cores at the end of their use-lives.

DEBITAGE

Flakes comprise two thirds of the debitage assemblage (Table 6.4.2). Half of the assemblage is limestone, with 36.5\% made on CCS varieties (chert, chalcedony and jasper) and most of the remainder being quartz or quartzite. Raw material proportions of blades and flakes are very similar, with the main difference being the absence of jasper blades.

\begin{tabular}{lrrrr}
\hline Raw material & Blades & Flakes & Total & $\mathbf{\%}$ \\
\hline Limestone & 436 & 970 & 1406 & 52.0 \\
Chert & 214 & 361 & 575 & 21.2 \\
Chalcedony & 143 & 264 & 407 & 15.0 \\
Quartz & 72 & 125 & 197 & 7.3 \\
Quartzite & 20 & 48 & 68 & 2.5 \\
Clear quartz & 5 & 16 & 21 & 0.8 \\
Dolerite & 4 & 19 & 23 & 0.8 \\
Jasper & & 9 & 9 & 0.3 \\
\hline Total (\%) & $894(33.0)$ & $1812(67.0)$ & 2706 & \\
\hline
\end{tabular}

Table 6.4.2: Debitage measured at Jwalapuram

The mean dimensions of debitage are 18.5 x $11.9 \times 4.0 \mathrm{~mm}$ (Table A.7.6). As noted by Clarkson et al. (2009), the frequency distribution of blade sizes drops off at 40 $\mathrm{mm}$, and this also applies to debitage as a whole. However, some differences in size distributions can be seen between raw material groupings (Figure 6.4.5). Quartz 
and quartzite debitage is more tightly distributed with a drop-off of $17 \mathrm{~mm} ; 75.5 \%$ of the quartz debitage analysed (i.e. $>10 \mathrm{~mm}$ long) is less than $17 \mathrm{~mm}$ long. The size distributions of other raw materials are more spread out. Amongst CCS debitage only $58.8 \%$ of debitage is shorter than $17 \mathrm{~mm}$, and amongst limestone and dolerite the proportion is $46.7 \%$. The distribution of CCS is more peaked, whereas limestone and dolerite have very flat distributions, with higher maximum lengths than other raw materials.

Figure 6.4.5: Frequency distributions of debitage lengths by raw material grouping at Jwalapuram
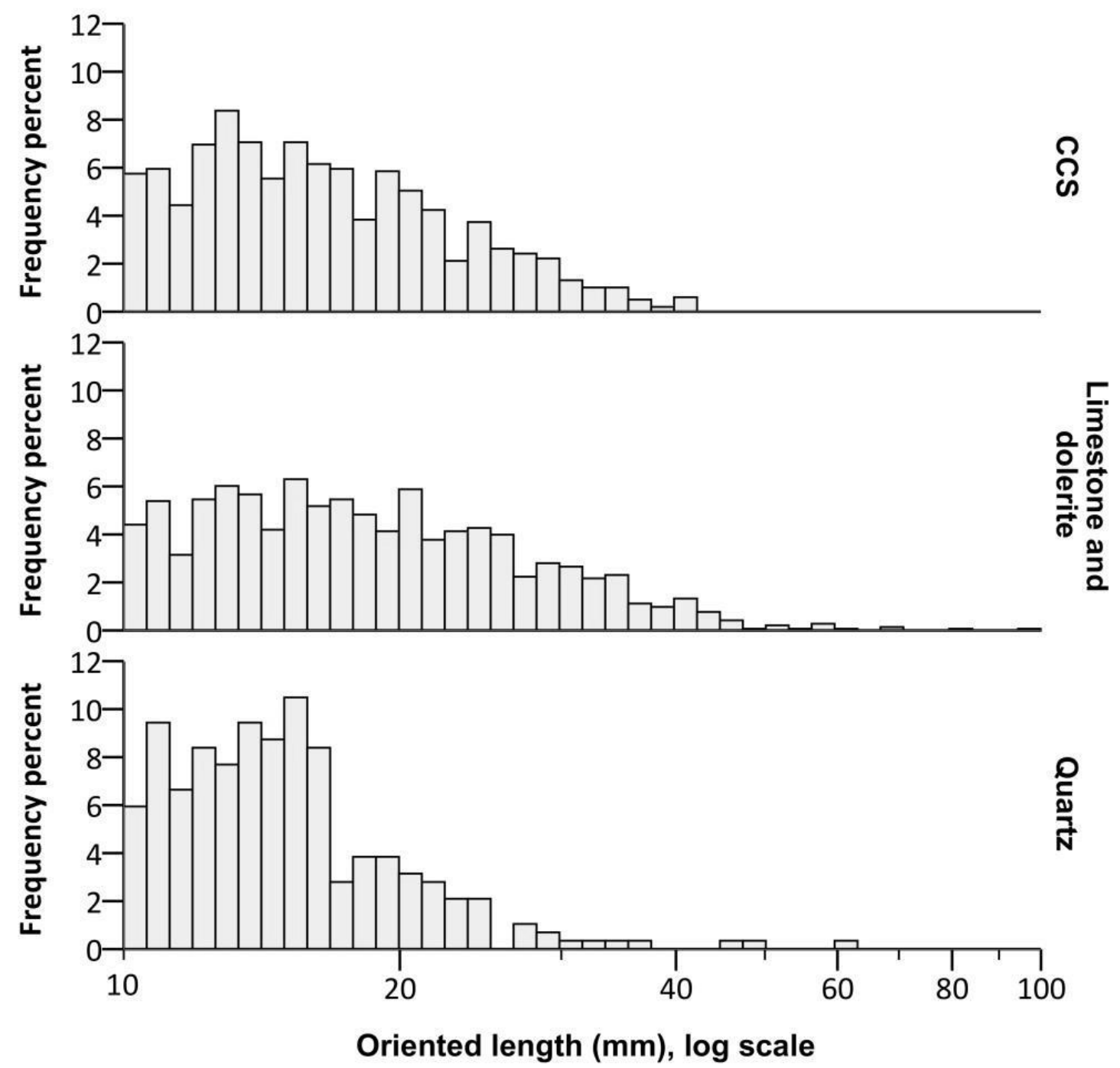
As might be expected, blades are longer on average than flakes $(21.8 \mathrm{~mm} \mathrm{v} 16.9$ $\mathrm{mm})$. However, as they are also thinner on average $(7.9 \mathrm{~mm} \mathrm{v} 13.9 \mathrm{~mm})$ they have a smaller mean overall volume $\left(1.0 \mathrm{~cm}^{3} \mathrm{v} 1.6 \mathrm{~cm}^{3}\right)$. Perhaps the most notable difference in size is in mean striking platform size, which is much smaller on blades (14.2 $\mathrm{mm}^{2} \mathrm{v} 39.3 \mathrm{~mm}^{2}$ ). Platform types also differ between flakes and blades (Figure 6.4.6). Pointed and crushed platforms are more common on blades, while more heavily facetted platforms are more common on flakes. Flat platforms are the most common type overall (52.0\%).

Figure 6.4.6: Proportions of debitage striking platform types at Jwalapuram

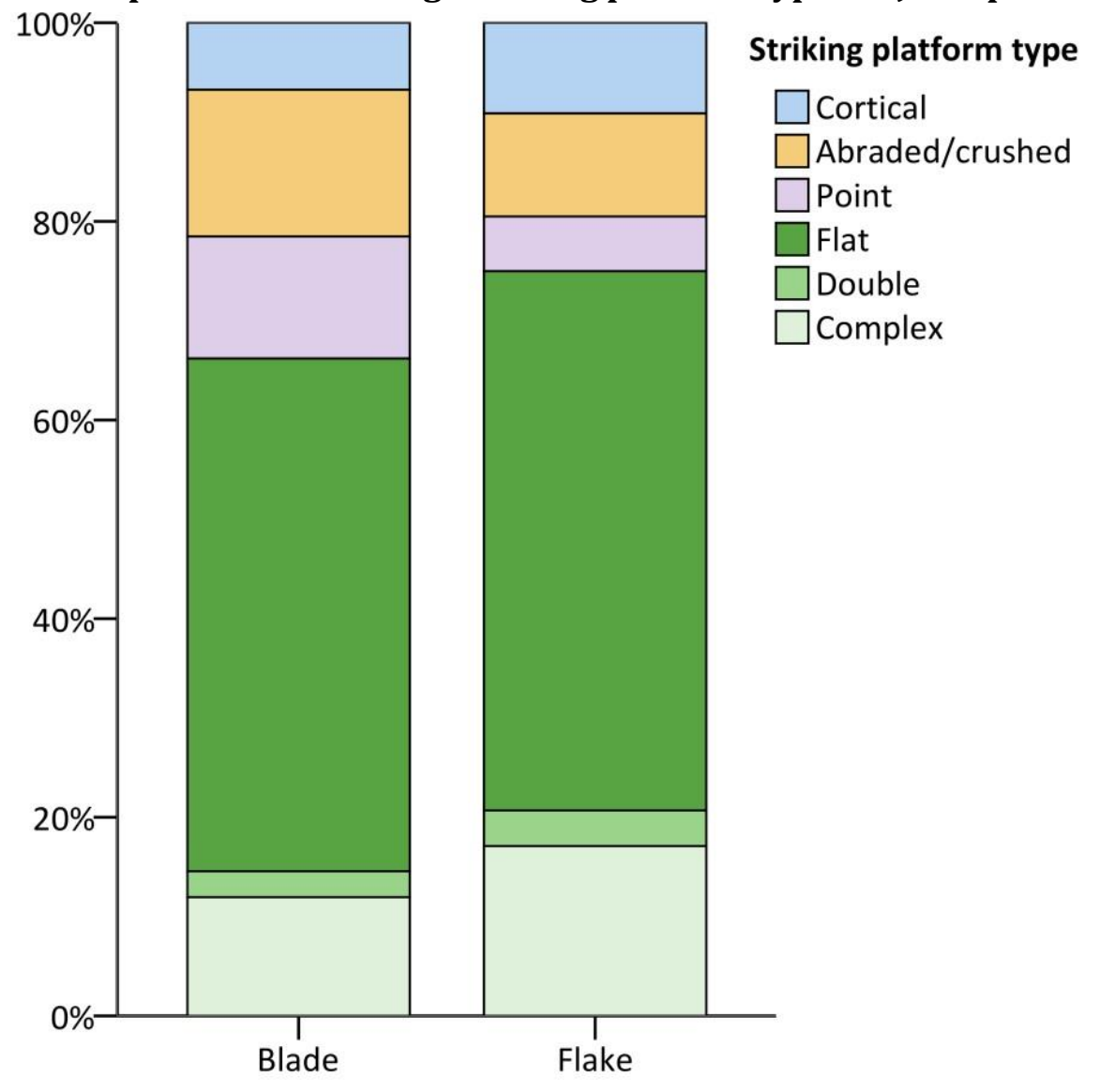

The higher proportion of crushed striking platforms on blades is related to the higher frequency of evidence of bipolar percussion on blades (3.8\%, compared to 
$2.9 \%$ of flakes). For the same reason, crushed platforms are considerably more common on quartz debitage (41.2\%). Almost a third (31.7\%) of quartz debitage exhibits evidence of bipolar percussion. Removal through the use of bipolar percussion is also noted on chalcedony, chert, limestone and quartzite, although it is rare $(0.7 \%)$.

In terms of surface characteristics, cortex exists in relatively high frequencies, with a mean cortex cover of $11 \%$ on blades and $14 \%$ on flakes (Table A.7.7). In combination with the similarly high proportions of cortex in the core assemblage, this indicates the occurrence of primary reduction activities at the site. The proportions of dorsal scar orientations for flakes and blades are almost identical, so Table 6.4.3 instead compares the proportions between raw material groupings. Scars predominantly originate from a single direction. Where scars originate from two directions, these are mostly from opposite margins. Orientation proportions on CCS and on limestone and dolerite debitage are roughly similar. However, scars originating from opposite directions are much more common on quartz and quartzite debitage. This may be related to the frequent use of bipolar percussion, resulting in the removal of scars from the end of the core in contact with the anvil. Alternatively, bipolar percussion may sometimes been used alongside a core rotation strategy that involved the working of the core from two ends.

The majority of debitage terminations are feather terminations (78.0\%) (Figure 6.4.7). Aberrant terminations (hinge and step fractures) are more common on flakes than on blades, but otherwise there is little difference between the two debitage categories. Although axial terminations were not recorded, crushed 
terminations are also indicative of bipolar percussion. Unsurprisingly, therefore, they are considerably more common on quartz debitage $(31.8 \%$, compared to $3.2 \%$ in the assemblage overall).

\begin{tabular}{|c|c|c|c|c|c|c|c|c|c|c|c|}
\hline $\begin{array}{l}\text { Dorsal scar } \\
\text { orientation: }\end{array}$ & 1 & & (adja & $n t)$ & (opp & site) & 30 & & Unkn & wn & \\
\hline Raw material & No. & $\%$ & No. & $\%$ & No. & $\%$ & No. & $\%$ & No. & $\%$ & Total \\
\hline $\mathrm{CCS}$ & 741 & 74.8 & 43 & 4.3 & 95 & 9.6 & 70 & 7.1 & 42 & 4.2 & 991 \\
\hline $\begin{array}{l}\text { Limestone and } \\
\text { dolerite }\end{array}$ & 1140 & 79.8 & 26 & 1.8 & 115 & 8.0 & 85 & 6.0 & 63 & 4.4 & 1429 \\
\hline $\begin{array}{l}\text { Quartz and } \\
\text { quartzite }\end{array}$ & 180 & 62.9 & 1 & 0.3 & 74 & 25.9 & 11 & 3.8 & 20 & 7.0 & 286 \\
\hline $\begin{array}{l}\text { Total (\% of } \\
\text { total) }\end{array}$ & 2061 & 76.2 & 70 & 2.6 & 284 & 10.5 & 166 & 6.2 & 125 & 4.6 & 2706 \\
\hline
\end{tabular}

Table 6.4.3: Dorsal scar orientation frequencies on raw material groupings at Jwalapuram

Figure 6.4.7: Proportions of debitage termination types at Jwalapuram

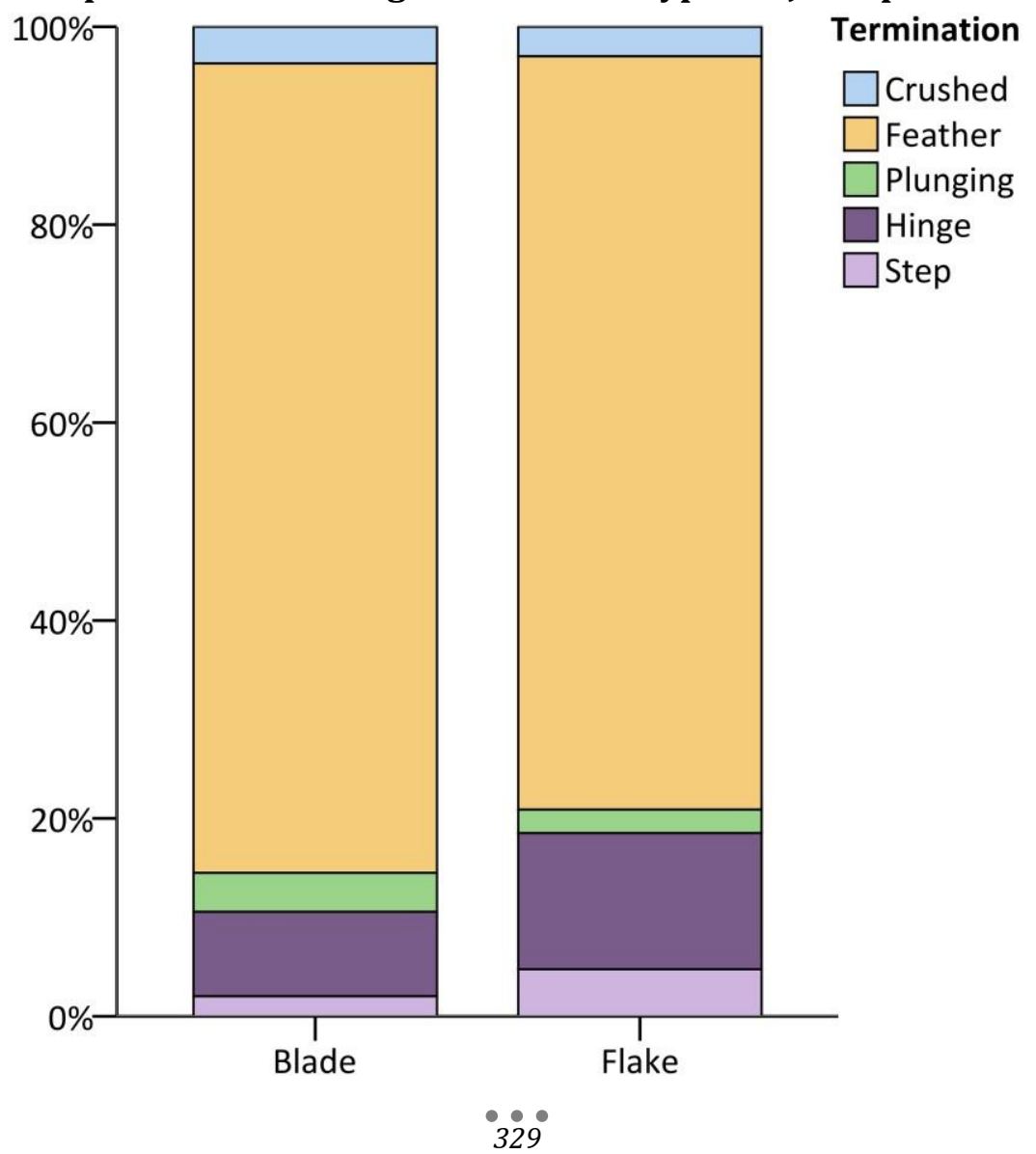


As discussed above, there is evidence for primary stage core reduction activities at the site. Later-stage core reduction and maintenance is also evident from the numbers of core redirection and rejuvenation flakes recorded (Table 6.4.4). Laminar blades were not recorded and platform preparation flakes were recorded less conservatively. Nevertheless, it is clear that the lithic assemblage at the site represents multiple stages of lithic reduction processes.

\begin{tabular}{l|rr|rr|rr}
\hline & \multicolumn{2}{c}{ Blades } & \multicolumn{2}{c}{ Flakes } & \multicolumn{2}{c}{ Total } \\
Maintenance flake type & No. & $\mathbf{\%}$ & No. & \% & No. & \% \\
\hline Core rejuvenation flake & 12 & 1.3 & 1 & 0.1 & 13 & 0.5 \\
Core redirection flake & 44 & 4.9 & 44 & 2.4 & 88 & 3.3 \\
\hline Total & 56 & 6.2 & 45 & 2.5 & 101 & 3.7 \\
\hline
\end{tabular}

Table 6.4.4: Frequencies of core maintenance debitage at Jwalapuram

TOOLS

As in the debitage assemblage, limestone, chalcedony and chert are the most common raw materials in the tool assemblage, although no single raw material comprises over half of the assemblage (Table 6.4.5). Limestone and quartzite were preferentially used for the manufacture of non-microlithic tools (Figure 6.4.8). CCS was preferred for microliths, especially chert blades and chalcedony flakes. Blade blanks were preferred overall for microliths (73.4\%), and flake blanks for nonmicrolithic tools (92.3\%). As flake and blade designations here refer only to elongation (as opposed to elongation plus parallel arrises), this implies that elongation was considered an important and desired aspect of microlith production, especially as blades make up only a third of the debitage assemblage. 


\begin{tabular}{lrrrrrr}
\hline Raw & $\begin{array}{r}\text { Microliths } \\
\text { on blades }\end{array}$ & $\begin{array}{r}\text { Microliths } \\
\text { on flakes }\end{array}$ & $\begin{array}{r}\text { Retouched } \\
\text { blades }\end{array}$ & $\begin{array}{r}\text { Retouched } \\
\text { flakes }\end{array}$ & Total & \% \\
\hline Limestone & 86 & 28 & & 10 & 124 & 44.3 \\
Chert & 72 & 14 & & & 86 & 30.7 \\
Chalcedony & 32 & 22 & 1 & & 55 & 19.6 \\
Quartzite & 3 & 2 & & & 7 & 2.5 \\
Quartz & 1 & 3 & & & 4 & 1.4 \\
Clear quartz & 2 & 1 & & & 3 & 1.1 \\
Dolerite & & 1 & 1 & 12 & 280 & 0.4 \\
\hline Total & 196 & 71 & & &
\end{tabular}

Table 6.4.5: Tools measured at Jwalapuram

Figure 6.4.8: Raw material proportions of tools at Jwalapuram

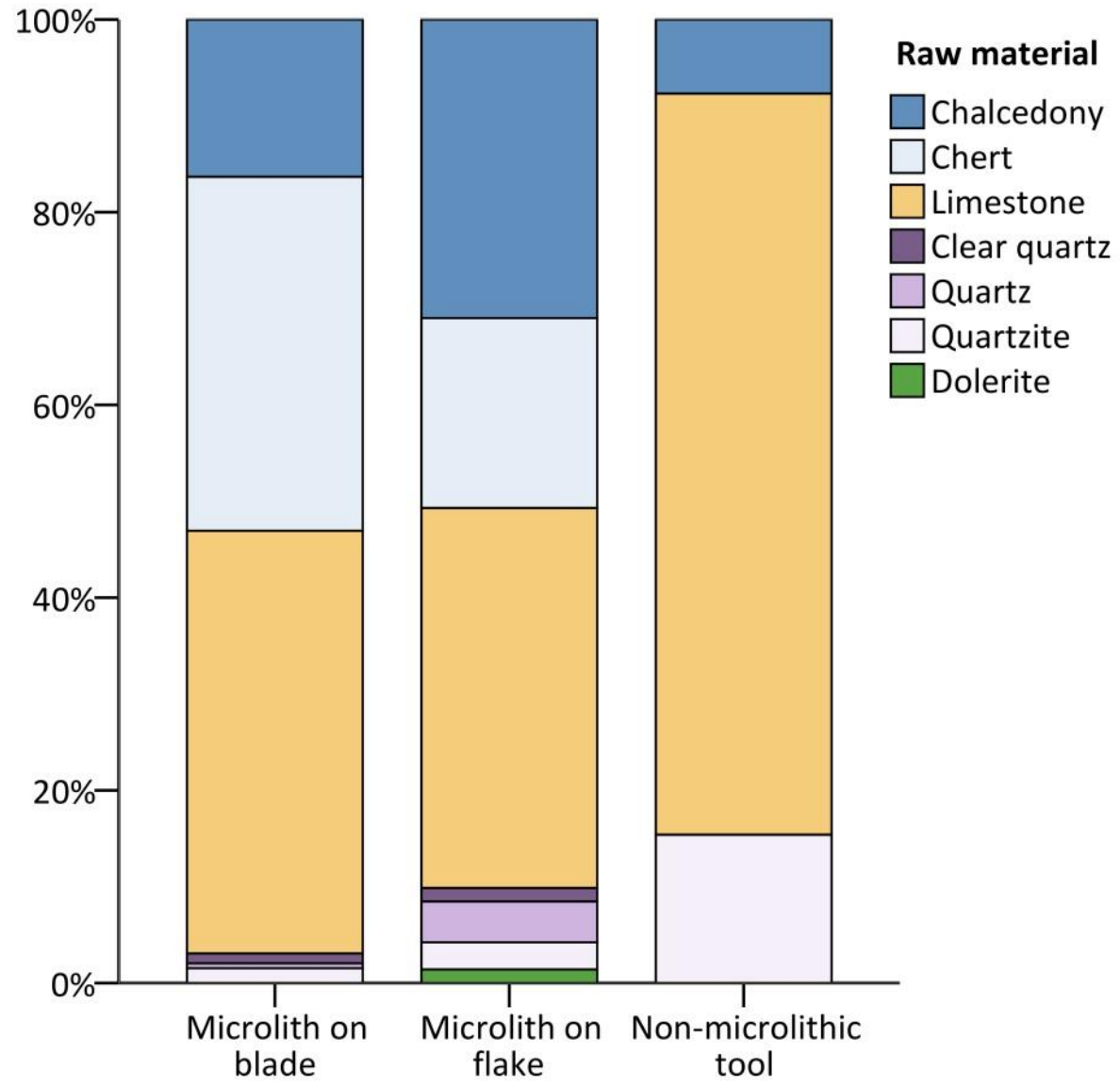

Average microlith size measurements are 20.4 x $9.2 \times 4.3 \mathrm{~mm}$ (Table A.7.8).

Preferences for elongated microliths are further evidenced by tool size

measurements. The mean length:width ratio of microliths at the site is 2.9 , 
compared to 1.4 for non-microlithic tools and 1.8 for debitage (Table A.7.9). There is also a considerable size disparity between microliths and non-microlithic tools. For example, there is a near-bimodal distribution of oriented length measurements, with a separation at c. $40 \mathrm{~mm}$ (Figure 6.4.9). This distribution confirms the conclusion of Clarkson et al. (2009) that this is a suitable cut-off point for microlithic tools at the site, although quartzite microliths tend to be larger than those made on other raw materials (Figure 6.4.10).

Figure 6.4.9: Frequency distributions of tool lengths at Jwalapuram

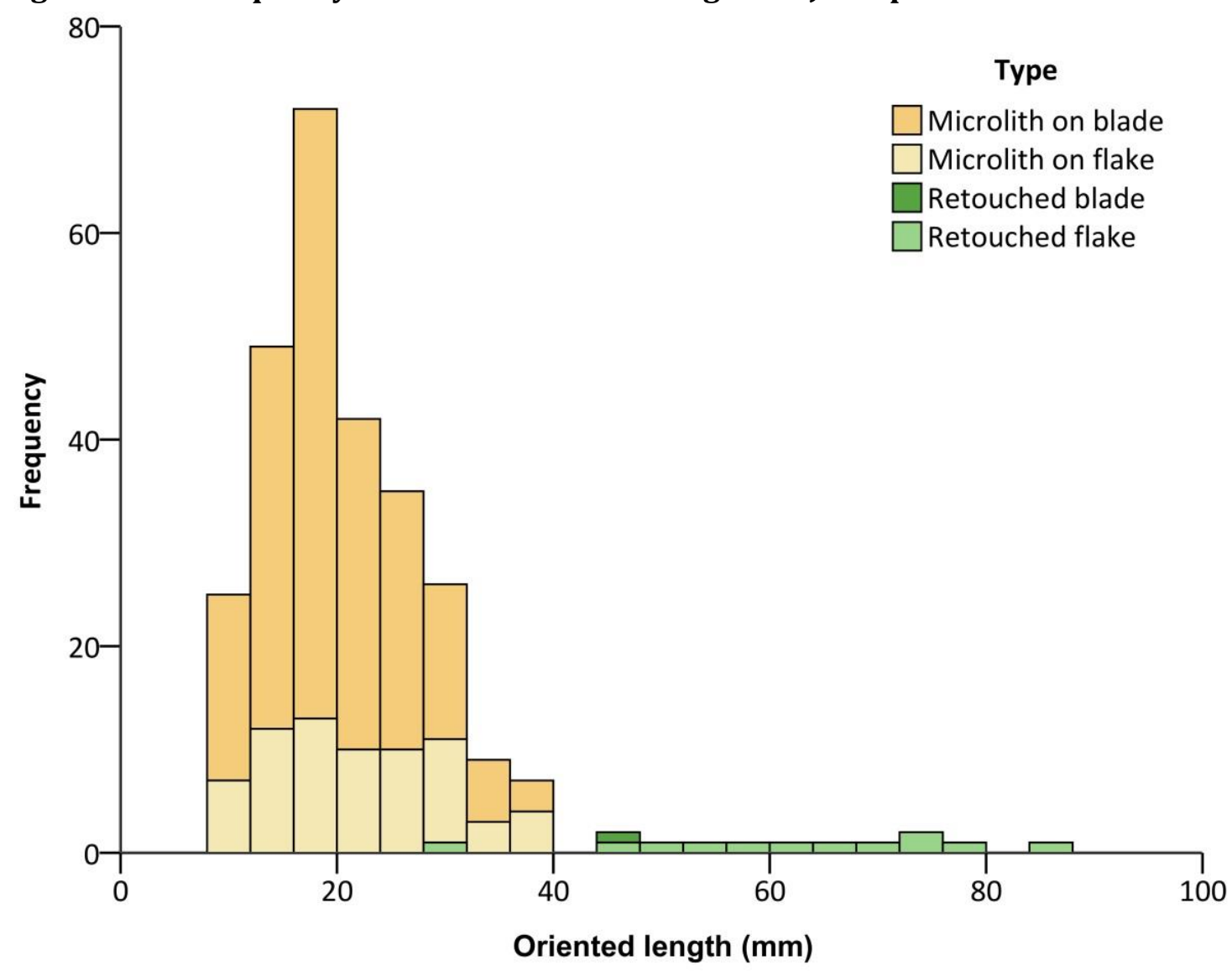

Figure 6.4.10 reveals that the size distributions of microlithic tools closely match those of the debitage assemblage, with blanks selected from the general debitage population for retouch into microlithic tools. Conversely, blanks selected for 
retouch into larger tools were selected from only the largest debitage available. Lithic technology at Jwalapuram was organised with the aim of producing microlithic tools, and the very largest debitage was also selected for retouch on occasion.

Figure 6.4.10: Cubic volumes of lithics on different raw materials at Jwalapuram

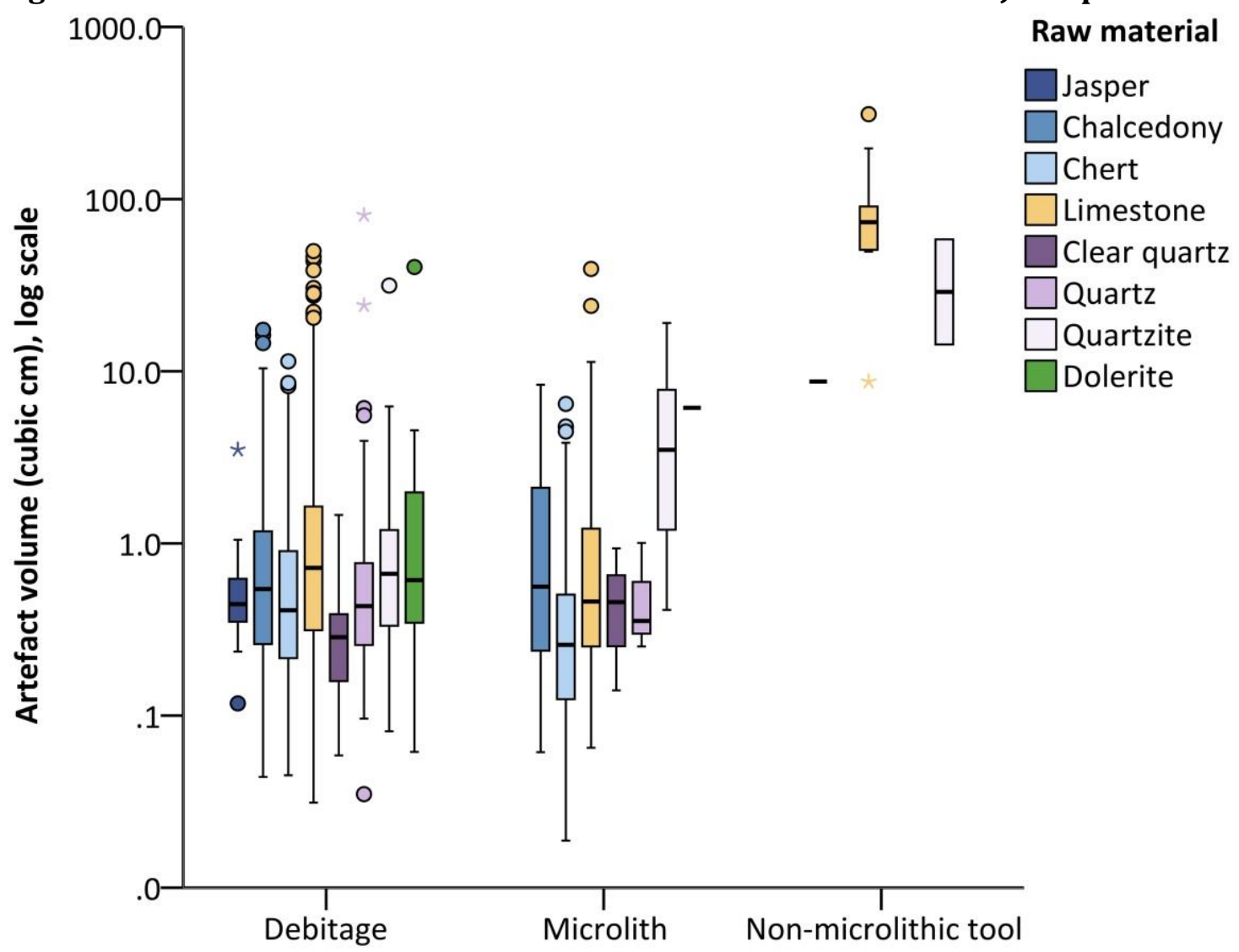

A variety of microlith forms are present (Table 6.4.6), with backed non-geometric microliths dominant (55.8\%). Burinated and notched pieces are also common, including two microlithic tools exhibiting both burinations and notches.

Shouldered and tanged tools are absent. Geometric microliths make up only $17.6 \%$ of the microlith assemblage. Unfortunately, other than one artefact recorded as being trapezoidal it is unclear what shape these geometric tools took. All geometric 
microliths are backed. Although the number of retouched edges on each tool was not directly recorded, typological descriptions indicate that at least 37 microliths (13.9\%) were retouched in two or more separate locations. There are no statistically significant differences in raw material proportions of different microlith typologies $(x 2(30)=32.111, \mathrm{p}=.362)($ Figure 6.4.11) .

\begin{tabular}{lll}
\hline Coarse typology & Narrow typology & Frequency \\
\hline Microliths: & & \\
Backed geometric microlith &
\end{tabular}

Backed non-geometric microlith Backed

Double backed

Microlith with burin

Burin

Side retouched with burin

Non-geometric microlith

Circumference retouched $\quad 2$

Double end retouched 1

Double side and end retouched 4

Double side retouched 2

End retouched 11

Side and end retouched $\quad 5$

Side retouched 10

$\begin{array}{lll}\text { Notched microlith } & \text { End retouched with burin and notch } & 1\end{array}$

End retouched with notch $\quad 5$

Notched $\quad 7$

Side and end retouched with burin and notch $\quad 1$

Side and end retouched with notch 2

Side retouched with notches $\quad 1$

$\begin{array}{lll}\text { Trapeze microlith } & \text { Trapeze microlith with notch } & 1\end{array}$

Total

Non-microlithic tools:

Burin

End retouched 
Notched/denticulated

Double side and end retouched with notch

End retouched with notch

Notched

Side retouched with notch

Side and end retouched

Double side and end retouched

Side retouched

Total

Table 6.4.6: Tool type frequencies at Jwalapuram

Figure 6.4.11: Raw material proportions of microlith types at Jwalapuram

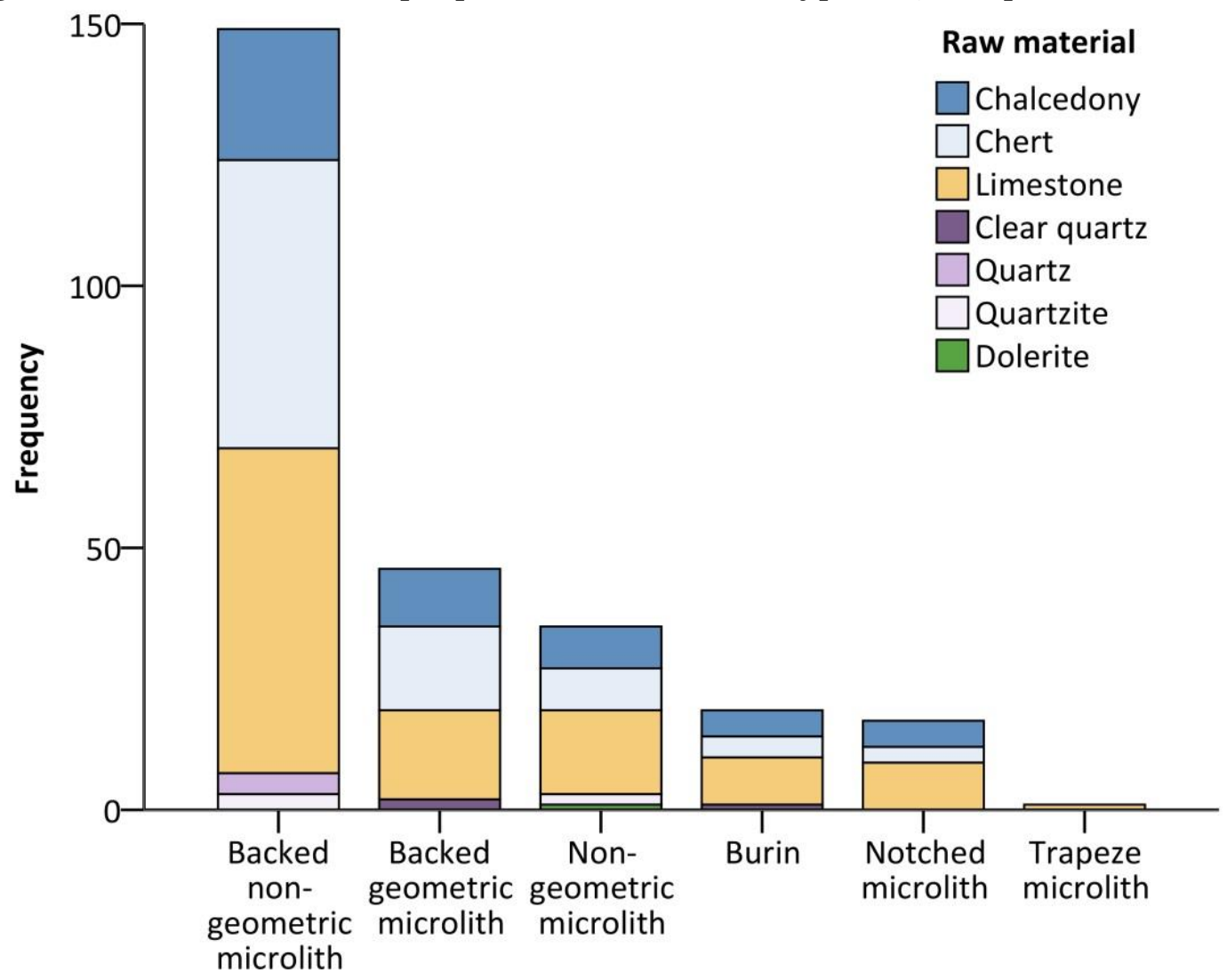

Notched artefacts are common in the non-microlithic tool assemblage (38.5\%)

(Table 6.4.6). A single burin is present, which is the only non-microlithic tool not 
manufactured on either limestone or quartzite (Figure 6.4.12). The remainder of the small non-microlithic tool assemblage are side and/or end retouched tools, with no points recorded.

Figure 6.4.12: Raw material proportions of non-microlithic tool types at Jwalapuram

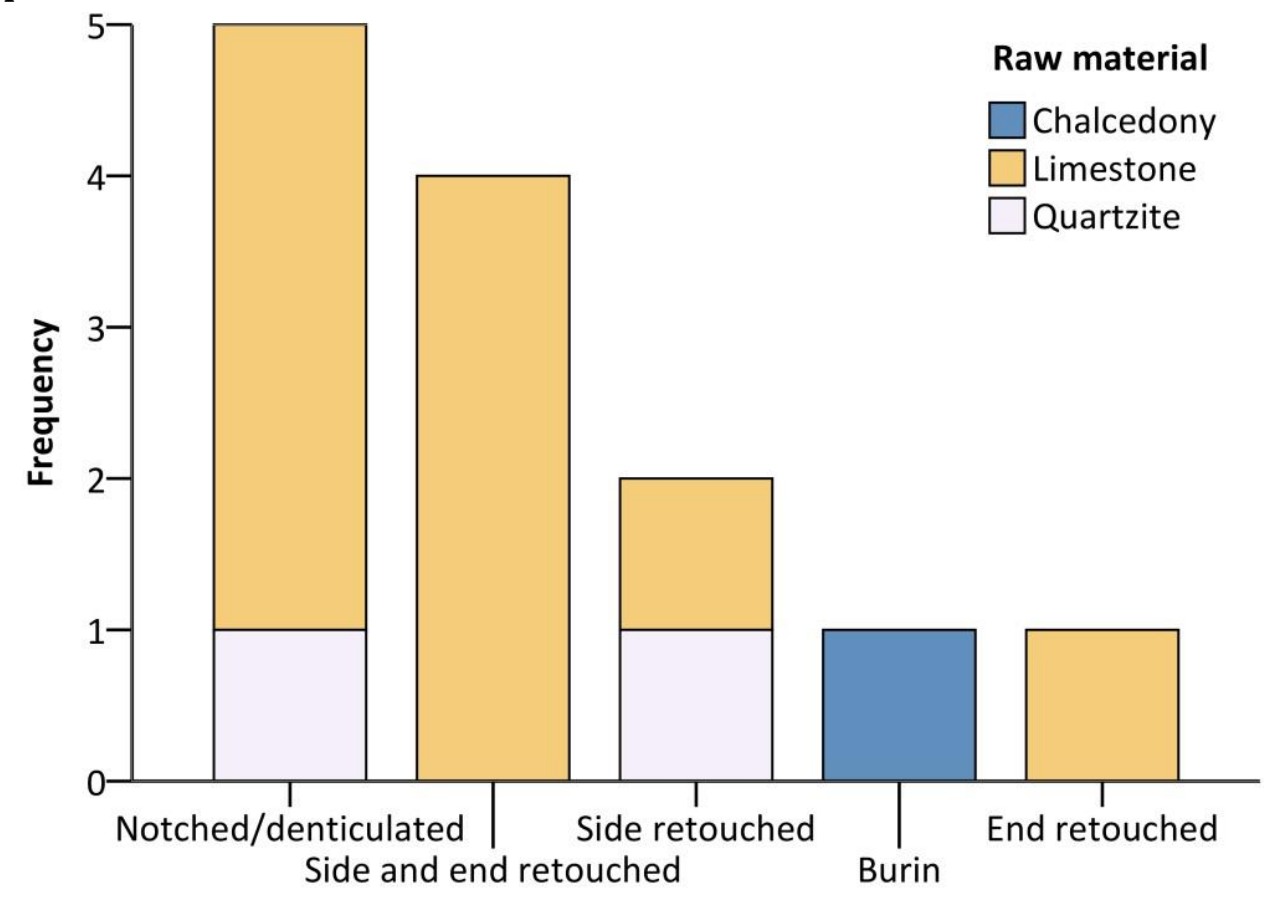

Although there is no evidence for size standardisation within any tool category, differences between types are evident. Backed forms are much smaller on average than non-backed forms (Figure 6.4.13, Table A.7.10). For example, the largest medial width and thickness measurements on backed microliths are both smaller than the mean measurements on non-backed microliths. The most meaningful categorisation of microliths in this case may therefore be according to whether or not backing retouch is present, rather than whether or not the tool was retouched into a geometric shape. Almost three quarters (73.0\%) of microliths are backed, 
and the high incidence of edge retouch (44.2\%) is indicative of the frequent use of bipolar backing (Table A.7.11).

Figure 6.4.13: Cubic volumes of microlith types at Jwalapuram

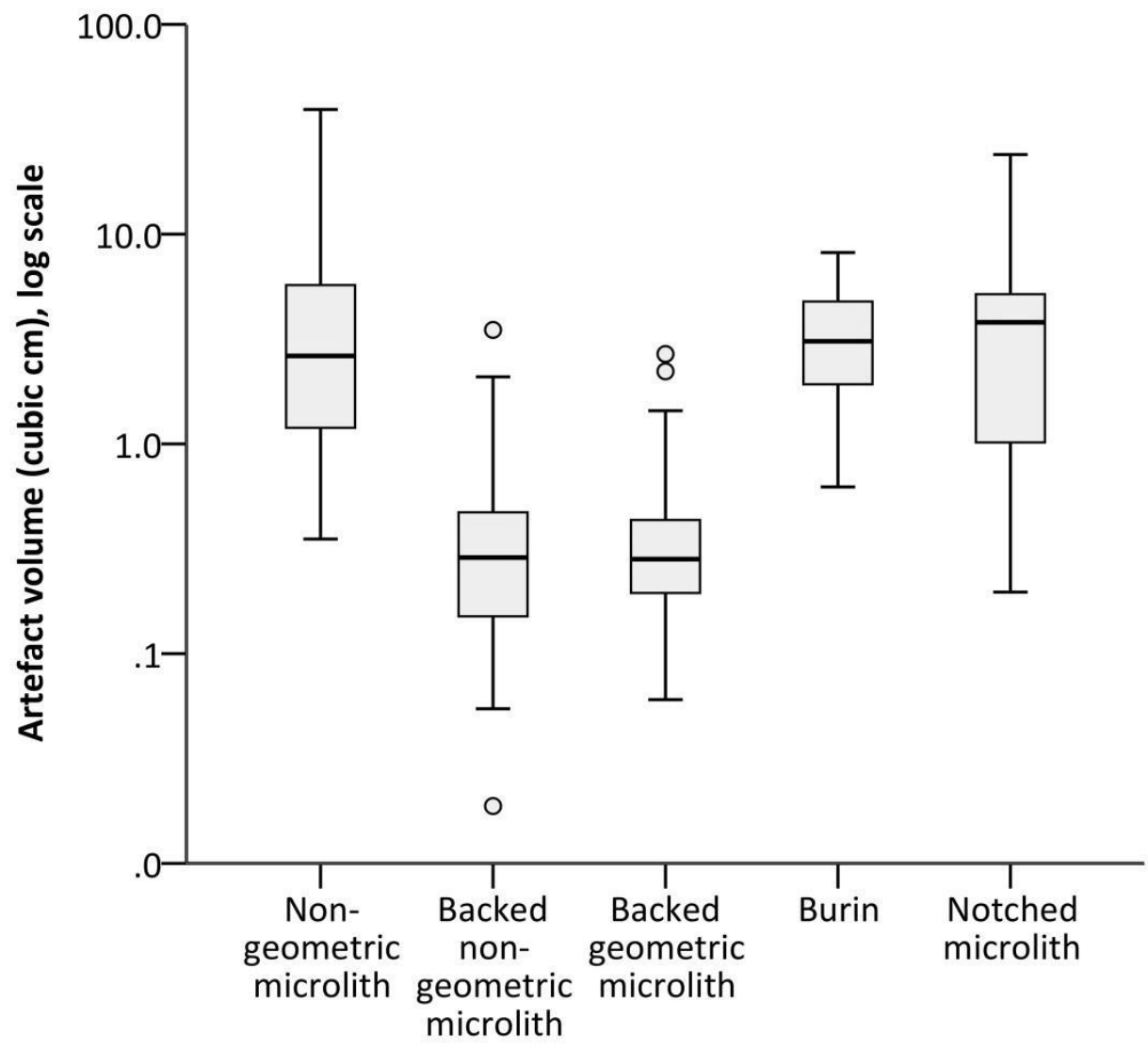

Additionally, $31.5 \%$ of retouch on microliths other than notches and burinations is described as stepped (other retouch types were not recorded). The equivalent proportion on non-microlithic tools is $30.8 \%$. Most retouch on microliths was conducted either on the dorsal face (37.3\%) or along the edge (44.2\%) in the form of bipolar backing (Table A.7.11). Conversely, dorsal (28.6\%) and bifacial (23.8\%) retouch are most common on non-microlithic tools. These differences provide further support for the distinctions made between microlithic and non-microlithic tools. 
There is considerable standardisation of retouch angles on all tool categories considered, with mean values ranging between $70-75^{\circ}$ on both microlithic and non-microlithic tools (Table A.7.12). This can only partly be explained by the prevalence of backing retouch. Differences in the lengths and depths of retouch on microlithic tools are in keeping with differences in overall size, i.e. the smaller size of backed microliths. Retouch on non-microlithic tools is more extensive, particularly in terms of retouch length (with a mean length of $82.4 \mathrm{~mm}$ compared to $21.1 \mathrm{~mm}$ for microliths). There is considerable variation in IOI values, including microliths with values of up to 0.750 , i.e. with retouch extending over $75 \%$ of the total surface (Table A.7.13). Non-microlithic tools are retouched slightly more extensively on average, with a mean value of 0.216 compared to 0.182 . Examples of microliths are illustrated in Figure 6.4.14.

Figure 6.4.14: Examples of microliths from Jwalapuram (after Clarkson et al. 2012, Figure 5), including backed geometrics (1-15), backed non-geometrics (16-22) and a double backed artefact (23).

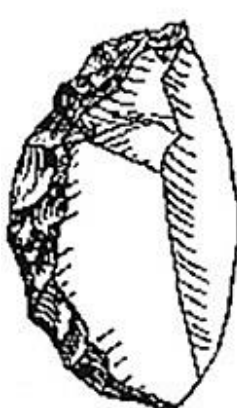

1

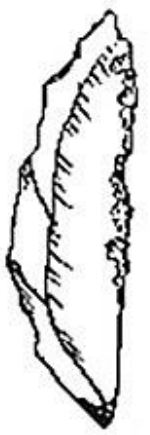

2

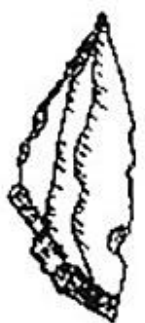

3

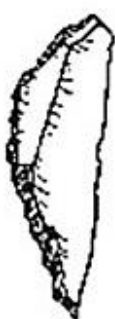

4

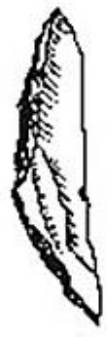

5

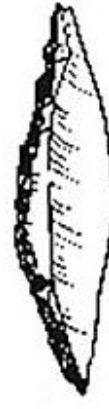

6

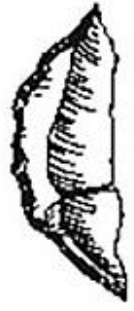

7

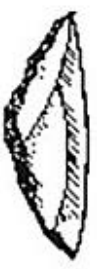

8

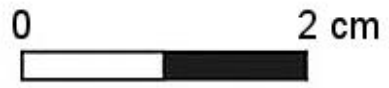



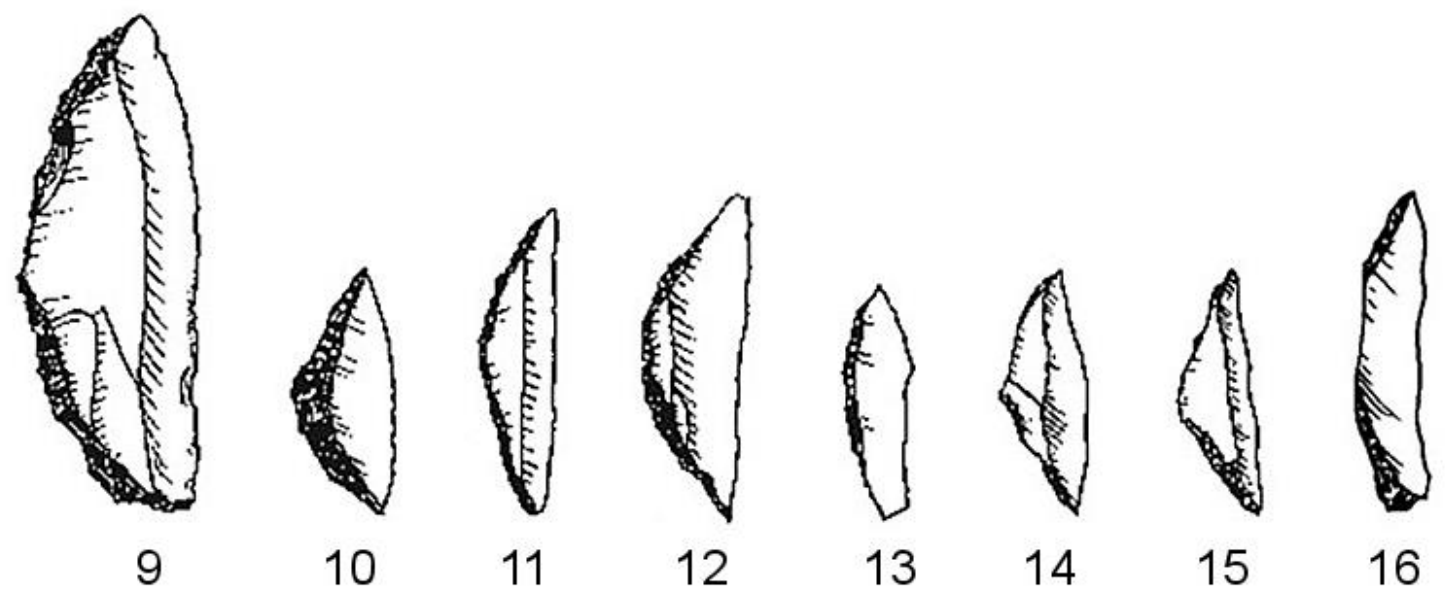

10

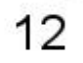

$13 \quad 14$

15

16

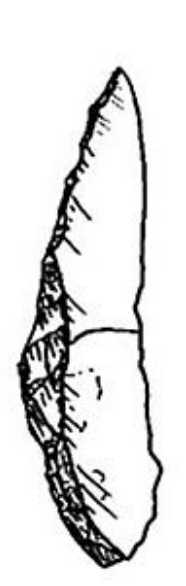

17

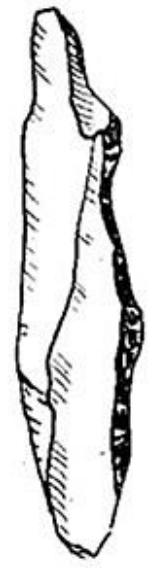

18

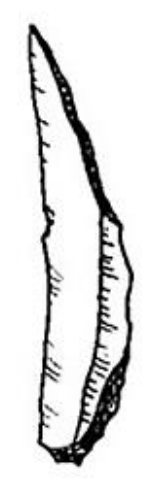

19

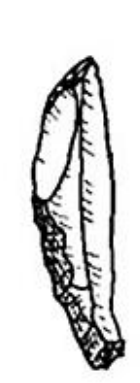

20

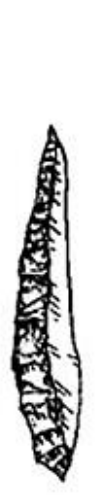

21

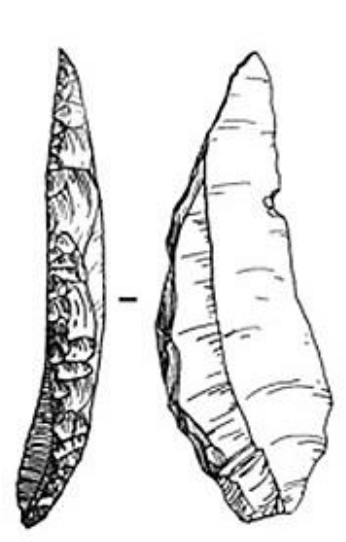

22

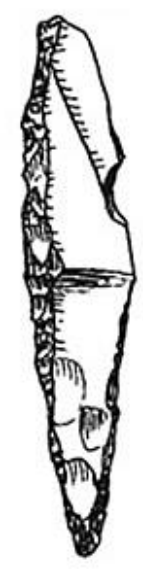

23

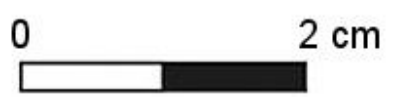

TEMPORAL VARIATION

Results of statistical tests at Jwalapuram are presented in Appendix B.6. The small number of non-microlithic tools precludes comparisons between layers (Table 6.4.7). 


\begin{tabular}{lllrrr}
\hline Context & Depth & Stratum & Cores & Microliths & Non-microlithic tools \\
\hline 1 & $1.0-1.2 \mathrm{~m}$ & $\mathrm{C}$ & 34 & 29 & 1 \\
2 & $1.2-1.4$ & $\mathrm{C}$ & 102 & 68 & 5 \\
3 & $1.4-1.6 \mathrm{~m}$ & $\mathrm{C}$ & 41 & 27 & 3 \\
4 & $1.6-1.8 \mathrm{~m}$ & $\mathrm{C}$ & 26 & 45 & 1 \\
5 & $1.8-2.0 \mathrm{~m}$ & $\mathrm{C} / \mathrm{D}$ & 34 & 75 & 2 \\
6 & $>2.0 \mathrm{~m}$ & C/D/E & 15 & 23 & 1 \\
\hline
\end{tabular}

Table 6.4.7: Cores and tools analysed in each context at Jwalapuram

\section{Cores}

Results of statistical tests on cores are reported in Table B.6.1 and summarised in Table 6.4.8. Statistically significant differences between contexts are evident in all variables except for cortex cover, which is on the border of the 0.05 level. Many of the observed differences can be attributed to the use of different core reduction techniques to work different raw materials. For example, context 6 contains the highest proportion of quartz (73.3\% compared to an average of $18.1 \%$ in the other contexts). This explains why the context also contains the highest proportions of bipolar cores (53.3\% v. 21.9\%) and abraded/crushed striking platforms (46.7\% v. $15.6 \%)$, and also the smallest and most intensively worked cores - these variables are all associated with the use of bipolar percussion to work quartz. Subsequently, the proportion of quartz decreases fairly steadily over time, as do the proportions of bipolar cores and abraded/crushed striking platforms.

\begin{tabular}{ll}
\hline Variable & Trend \\
\hline Type & Decrease over time in proportion of bipolar cores, and increase \\
& $\begin{array}{l}\text { over time in proportion of flake cores. Complex picture of } \\
\text { changes over time in other core types, with considerable } \\
\text { variations in the proportion of blade cores. See Figure 6.4.15. }\end{array}$
\end{tabular}

Raw material Decrease over time in proportion of quartz, with a complex 
picture of variations in other raw material proportions. See Figure 6.4.16.

Weight

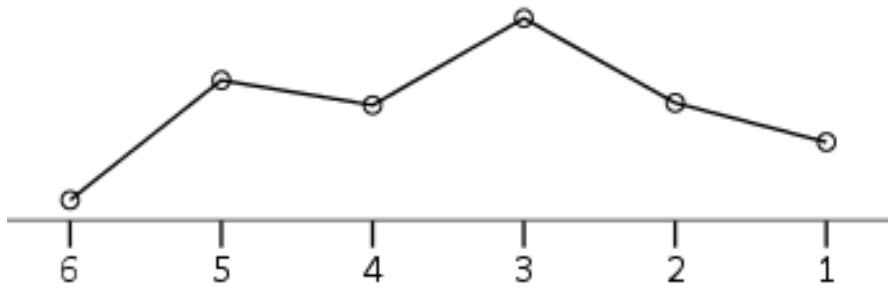

Longest axis

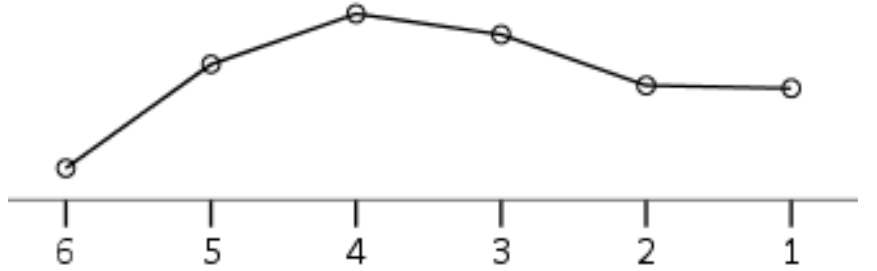

Maximum width

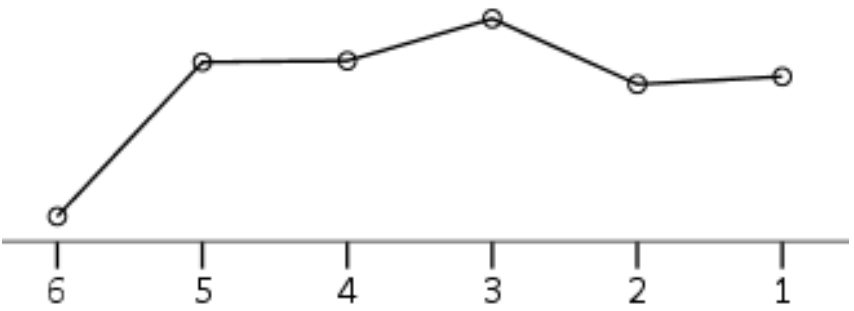

Maximum thickness

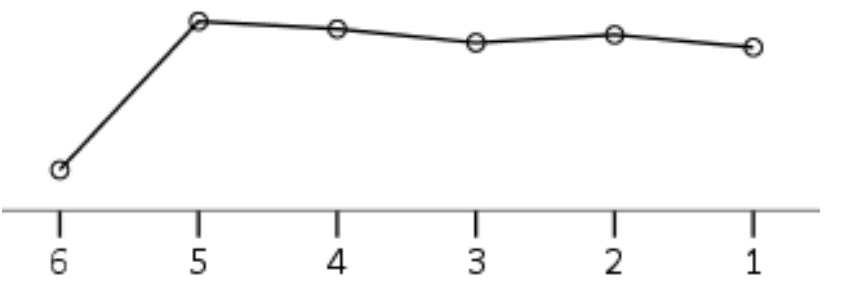

Longest face

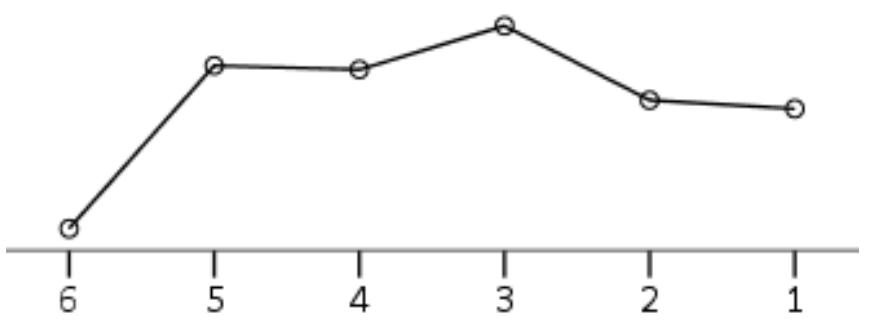

Striking platform width

Striking platform

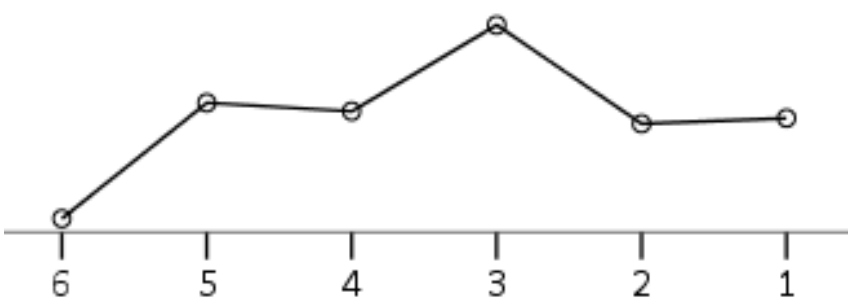

thickness

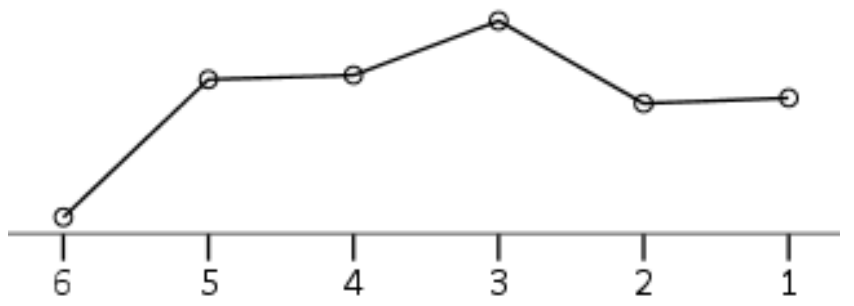

Striking platform type

Complex pictures of changes over time, with no overall trends.

See Figure 6.4.17. 
Total number of scars

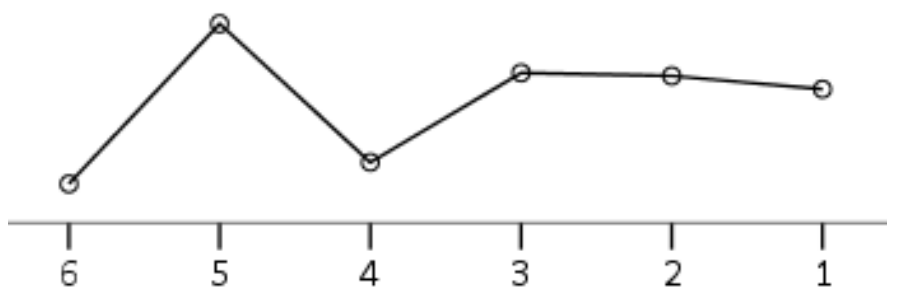

Number of flake scars

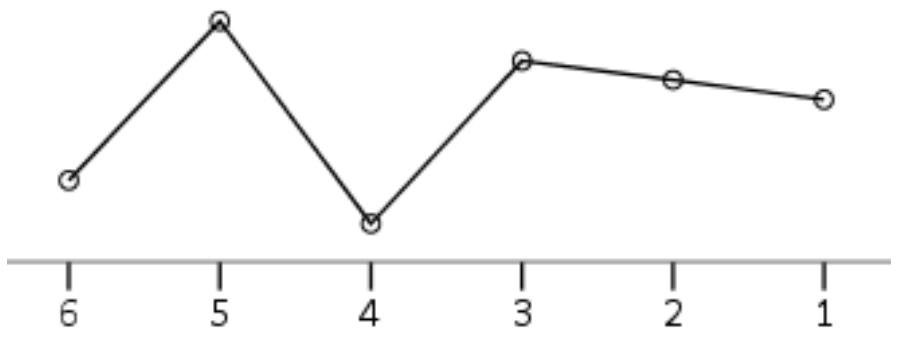

Number of blade scars

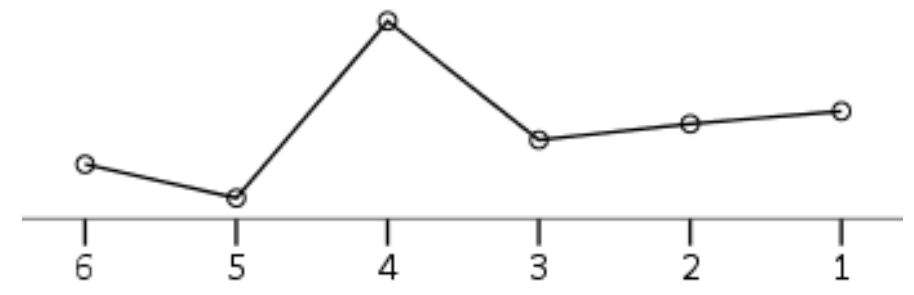

Proportion of blade scars

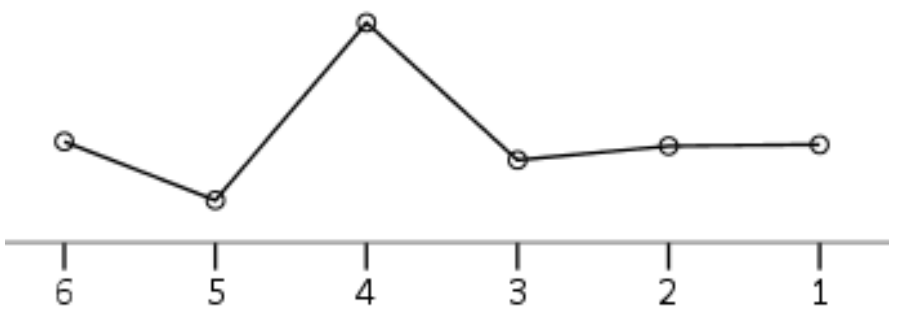

Number of aberrant terminations

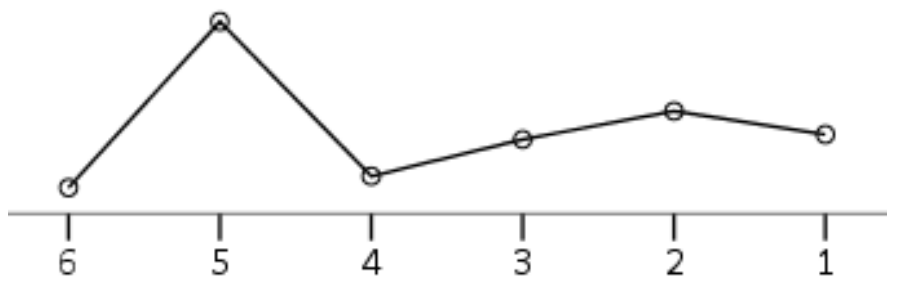

Average removal length

Number of scars per

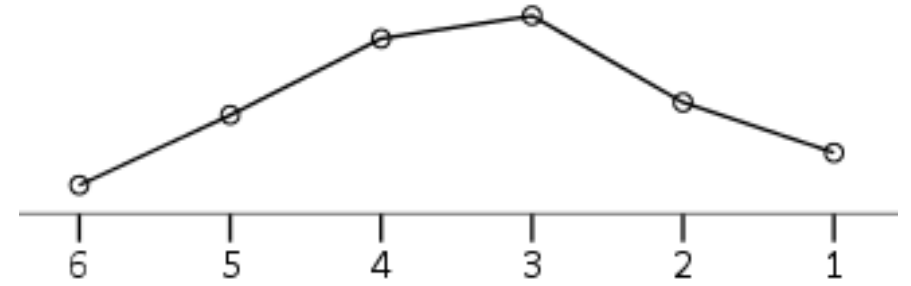
$\mathrm{cm}^{3}$

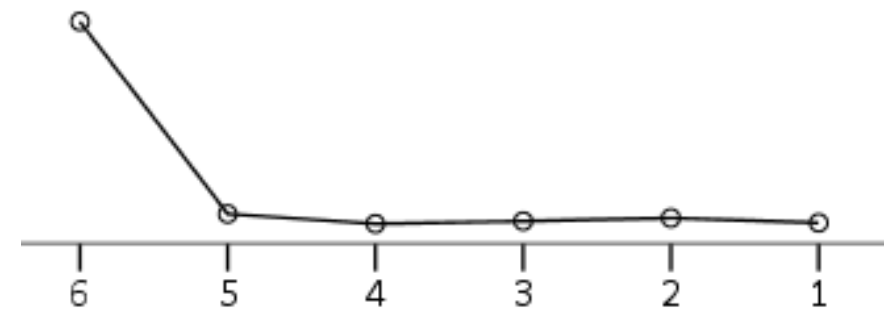

Table 6.4.8: Statistically significant differences between layers in core variables at Jwalapuram 
Figure 6.4.15: Proportions of core types in each context at Jwalapuram

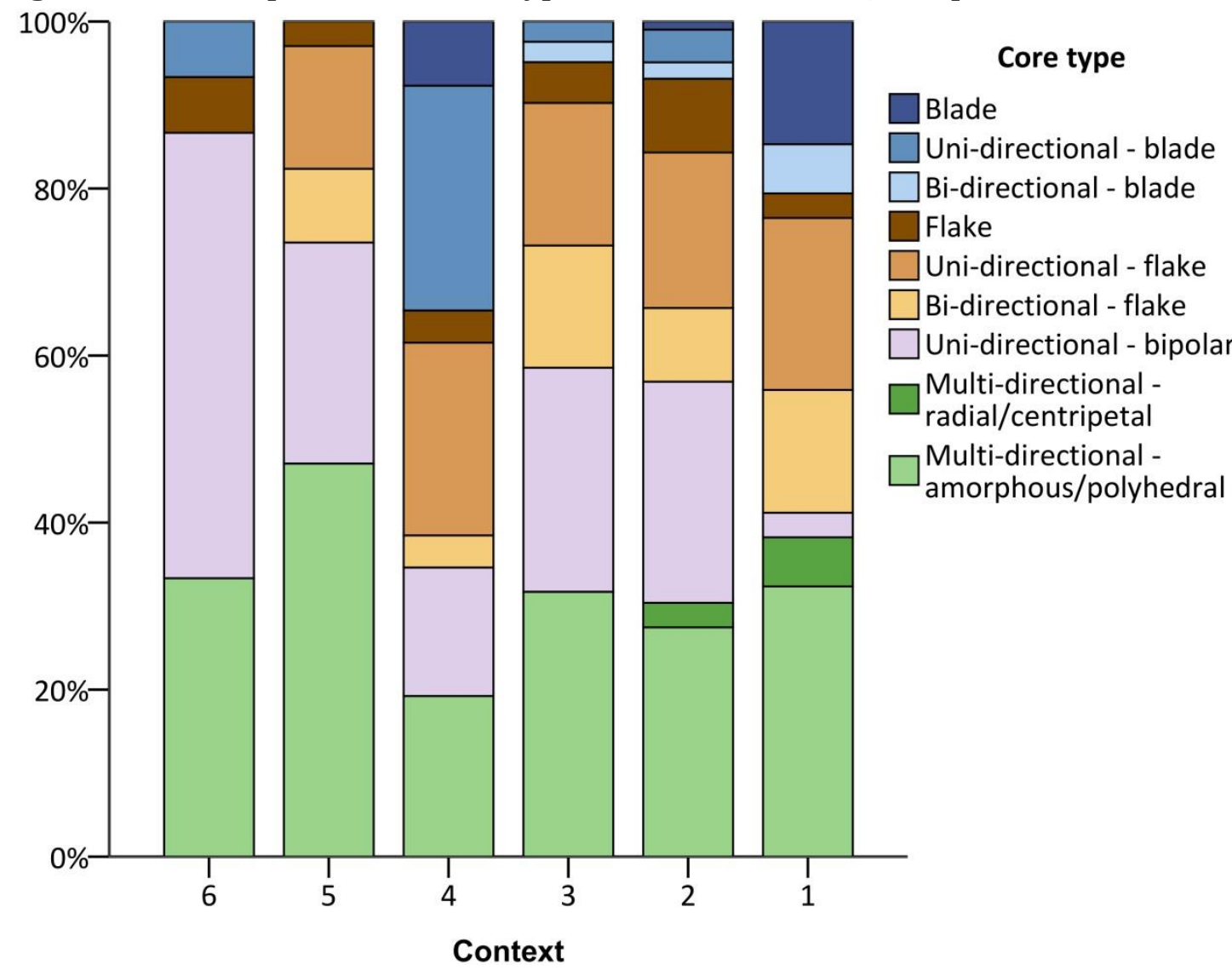

Figure 6.4.16: Proportions of core raw materials in each context at Jwalapuram

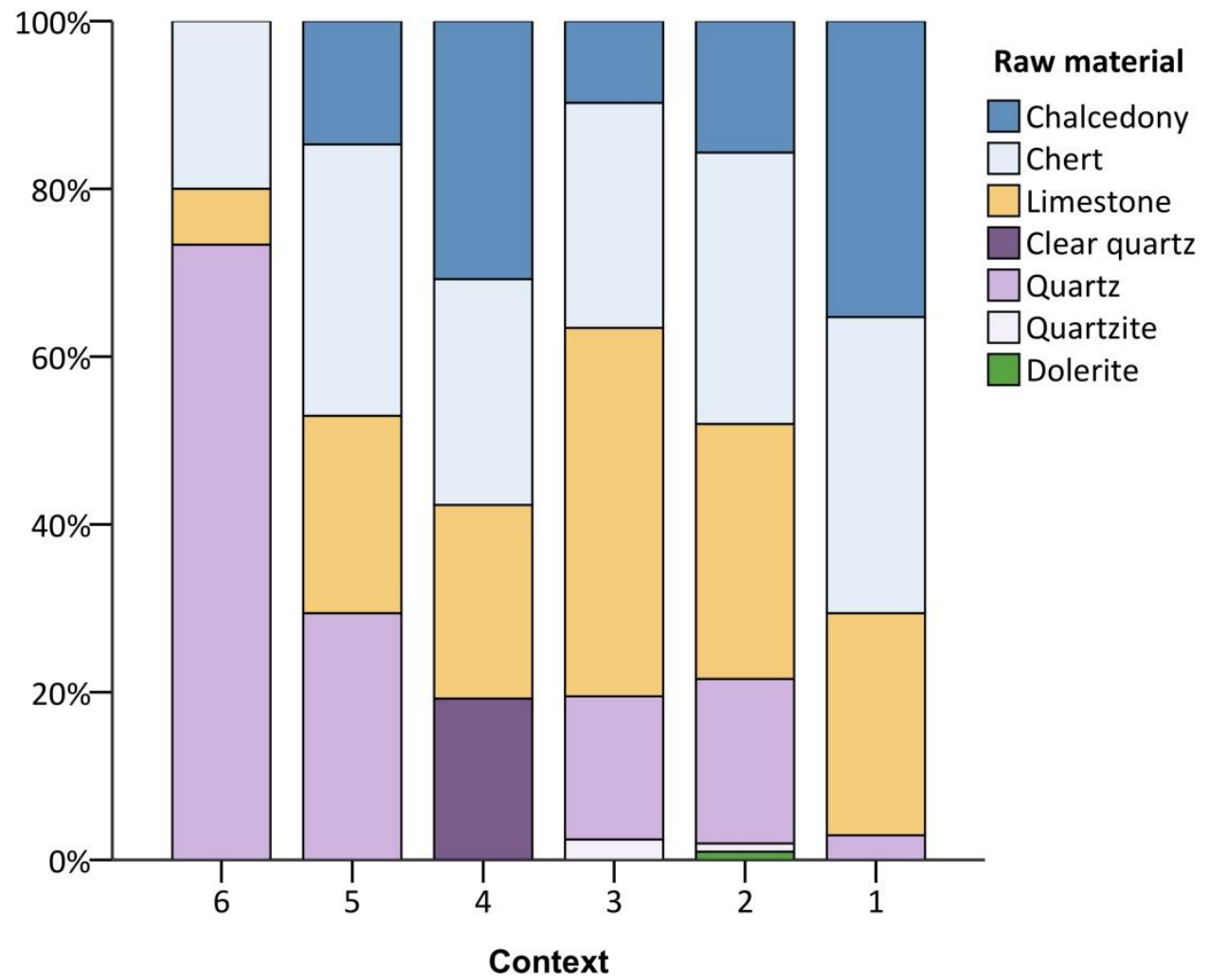




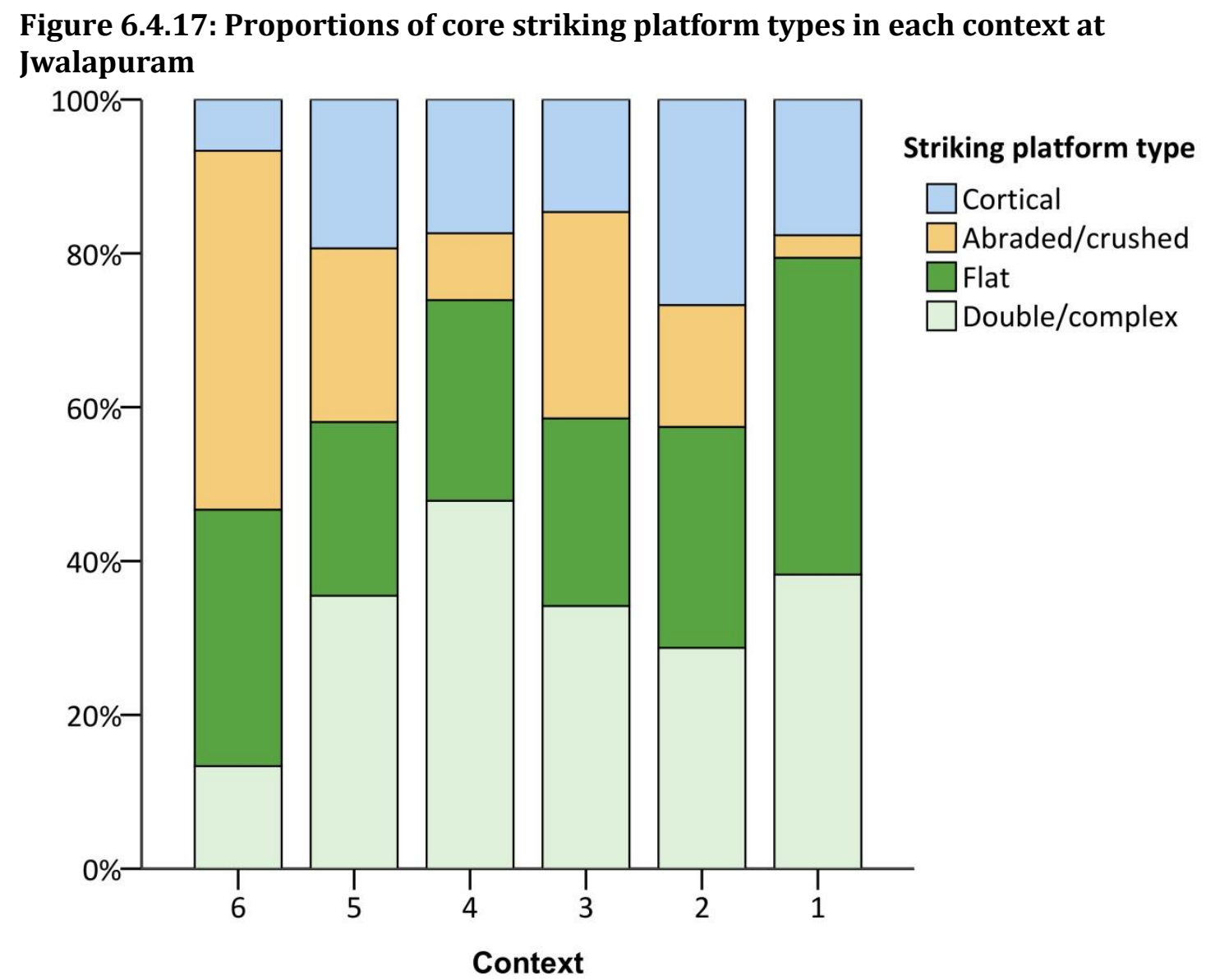

Although the proportion of chert remains relatively stable throughout the sequence, the proportion of chalcedony varies between $0 \%$ and $35.3 \%$. Chalcedony was used much more commonly in contexts 4 and 1 . The proportions of blade cores and facetted striking platforms also peak in these contexts. Whether more blades were able to be removed from cores because the use of high quality CCS permitted it, or CCS was sought out in order to produce higher frequencies of blades, is unclear.

Patterns in size metrics can also be seen. Cores tend to be smallest in context 6 before becoming considerably larger in context 5 and reaching a peak in size in contexts 4 and 3 , then reducing in size in contexts 2 and 1 . For example, mean 
weight, maximum width, longest face, striking platform width and thickness, and removal scar length measurements are largest in context 3 , while longest axis measurements are largest in context 4 . These size variations may be partly related to differing proportions of raw materials, specifically limestone, although the trends are still evident even when limestone is excluded. Overall, context 6 contains a very different core assemblage, with many intensively worked small quartz bipolar cores. Cores become much larger on average in context 5 and 4, although they are more flake-dominated in the former and blade-dominated in the latter. Context 3 contains the largest cores, and a mix of flake and blade cores. Cores then become smaller and more blade-oriented between contexts 3 and 1 .

A few final points of note concern the short-lived appearances of particular raw materials and reduction strategies. Clear quartz only appears in context 4 , dolerite in context 2, and quartzite in contexts 3 and 2 . Additionally, although amorphous multi-directional cores are present in all contexts, radial cores only appear in contexts 1 and 2 . It is interesting to note that although radial core reduction is typically considered a Middle Palaeolithic/Stone Age strategy, it only appears in the uppermost section of the Jwalapuram sequence, dated to 14,592-13,991 BP (Clarkson et al. 2009).

\section{Debitage}

Results of statistical tests on debitage are reported in Table B.6.2 and summarised in Table 6.4.9. Statistically significant differences between contexts are evident in all variables except for evidence for core maintenance. The results provide a 
slightly different picture to that given by the core assemblage. There is a roughly consistent decrease over time in the proportion of debitage of blade dimensions, in contrast to the core assemblage which shows peaks in blade core production in contexts 4 and 1 . Striking platform types also vary less dramatically than in the core assemblage, although abraded/crushed striking platforms are again more common in quartz-dominated context 6. Differences are also evident in raw material proportions. In the debitage sample there is a consistent increase over time in the proportion of CCS (mostly in the lower layers), again in contrast to the peaks seen in the core assemblage in contexts 4 and 1 . There is also less variation over time in the debitage assemblage in the proportion of limestone.

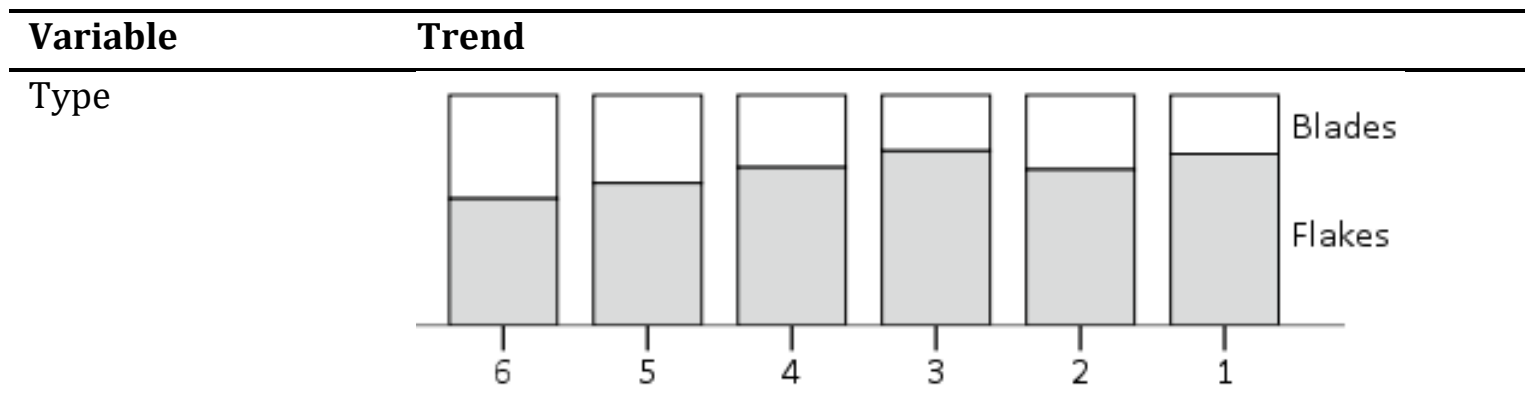

Raw material Consistent increase over time in the proportion of CCS. Other small inconsistent variations can be seen in Figure 6.4.18.

Weight

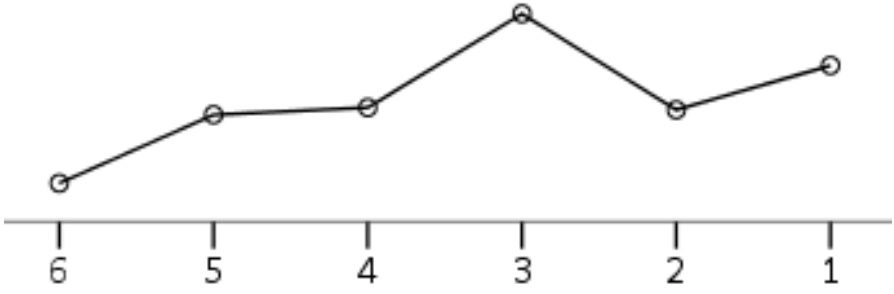

Longest dimension

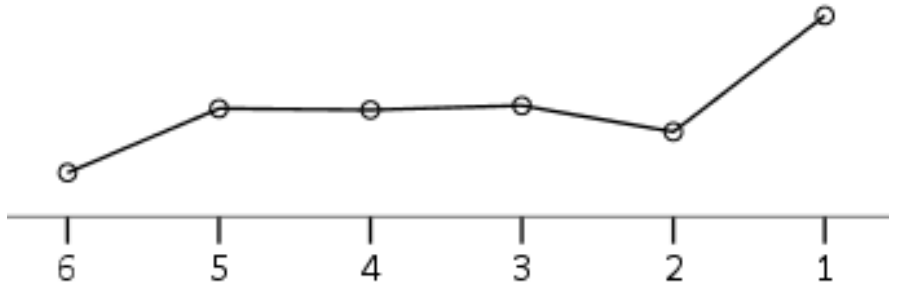


Oriented length

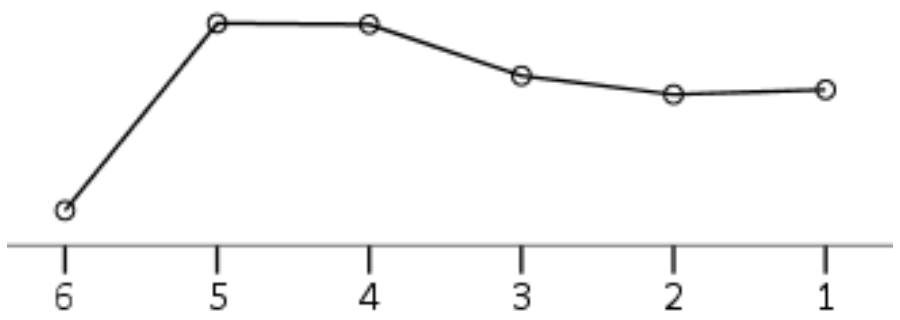

Medial width

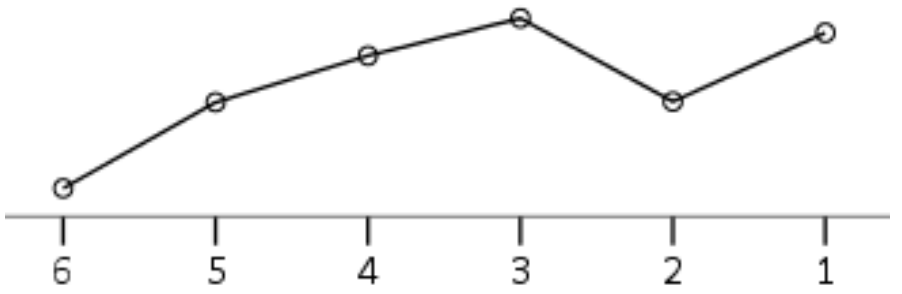

Medial thickness

Length:width ratio

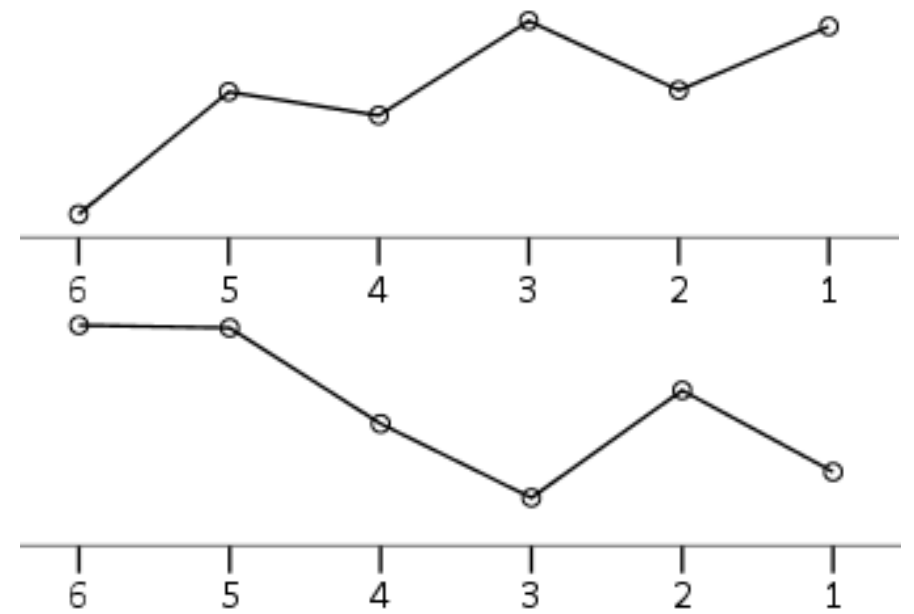

Length:thickness

ratio

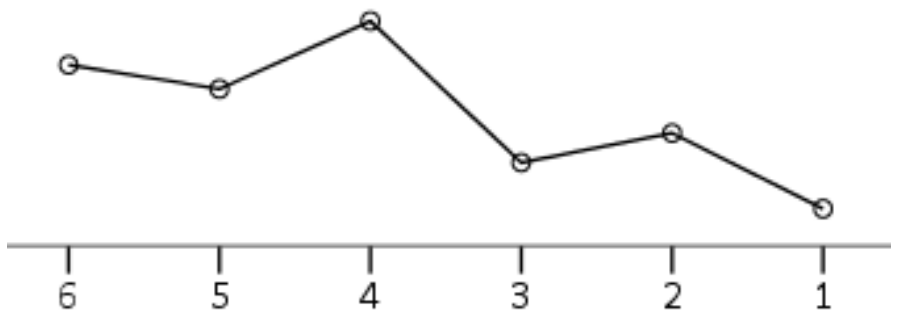

Striking platform width

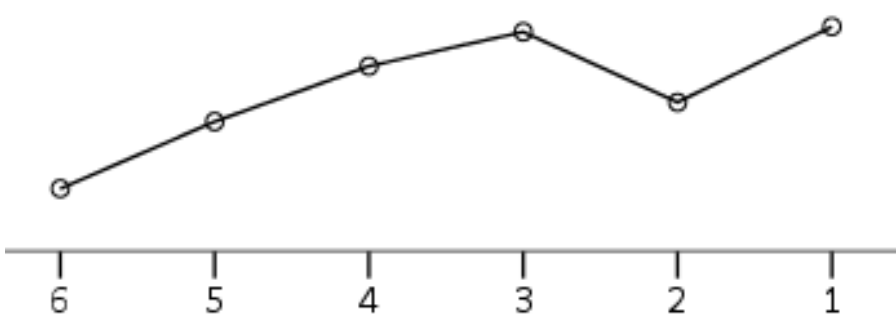

Striking platform thickness

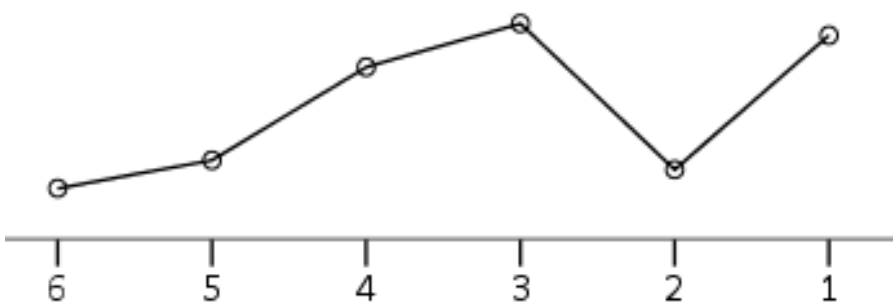

Striking platform Consistent increase over time in the proportion of flat striking type platforms, with a slight reversal in 2 , but no overall trends. 
Percentage cortex cover

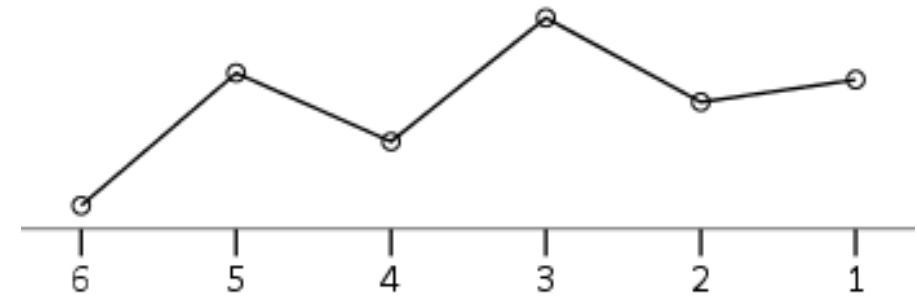

Scar orientation

More debitage with scars from two opposite directions in 6, 3 and 1 , from three directions in 5 , and from a single direction in 4 and 2 .

Termination type

Slight and inconsistent increase over time in proportion of feather terminations. More crushed terminations in 6 and 5, and more plunging terminations in 3 .

Evidence of bipolar percussion

More evidence of bipolar percussion in 6 and 5, least in 4, and intermediate in 3-1.

Table 6.4.9: Statistically significant differences between layers in debitage variables at Jwalapuram

Figure 6.4.18: Proportions of debitage raw materials in each context at Jwalapuram

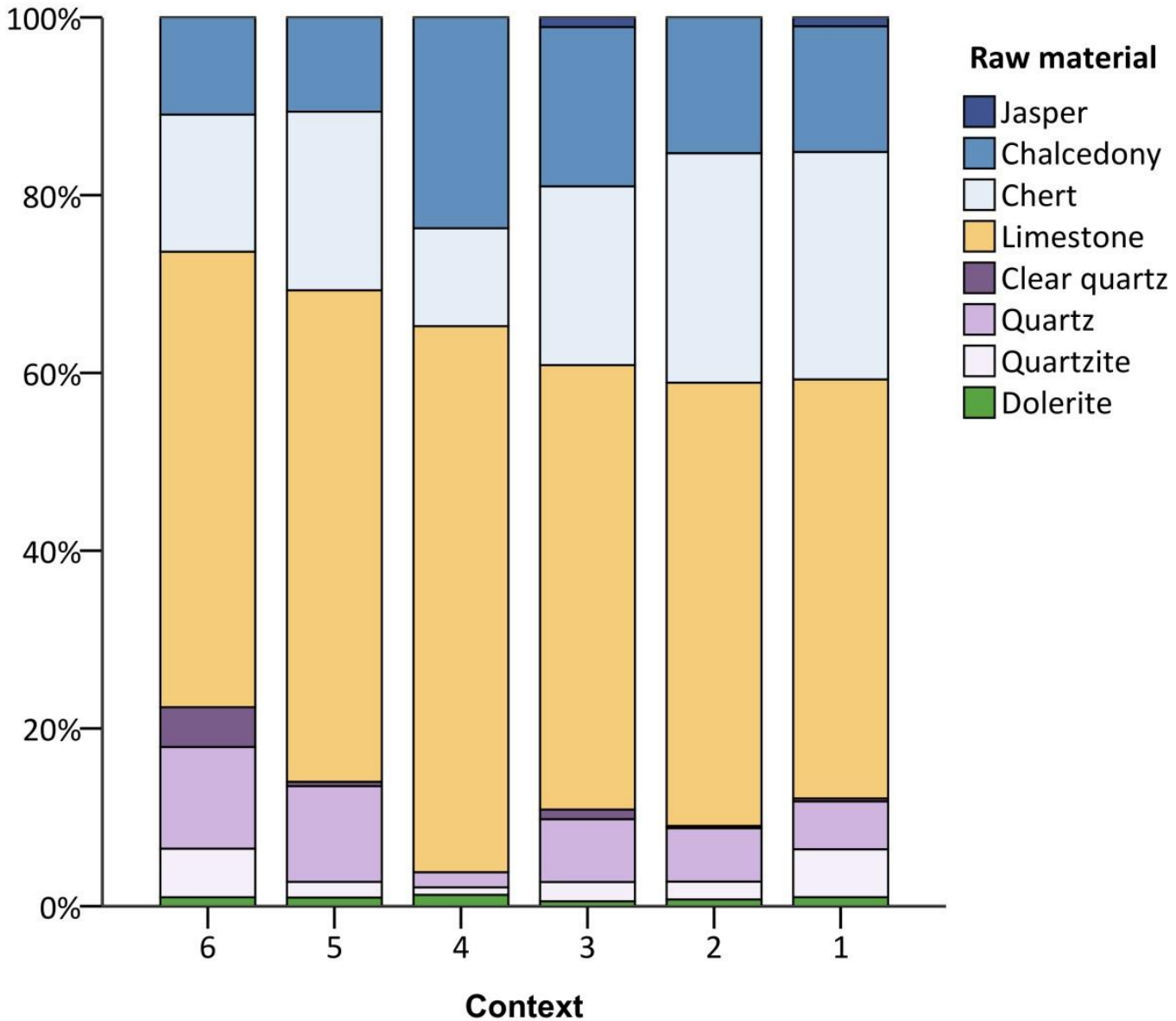


Although most quartz is again found in context 6 , the difference between this and other contexts is not enormous. In the core assemblage quartz is dominant in context 6 , but in the debitage assemblage it never makes up more than a small proportion of each context. The proportion of debitage with evidence for bipolar percussion (including the proportion of crushed terminations) varies in accordance with the proportion of quartz in each layer.

Size metrics reveal a complex picture of variation. As in the core assemblage, debitage in context 6 is smaller on all metrics, and is also the most blade-like in terms of length:width ratios. Debitage increases in size between contexts 6 and 3, and also becomes less elongated. This trend is reversed in context 2 , when debitage becomes smaller and more blade-like. The debitage in context 1 is larger on average than context 2 , and is also particularly thick, squat and flake-like.

\section{Microliths}

Results of statistical tests on microliths are reported in Table B.6.3 and summarised in Table 6.4.10. In contrast to the core and debitage assemblages, there are no statistically significant differences in raw material proportions. Despite changes in the nodules of raw materials being knapped, microliths were consistently manufactured on similar proportions of raw materials. The variations evident in the majority of other variables therefore cannot be attributed primarily to changes in raw material use. Variations in striking platform type, scar orientation, termination type and retouch length and depth appear in the form of small-scale differences between contexts. Another example of this context-specific 
variation can be seen in the particularly high frequencies of burins/burinations in context 3 , and of notches/notched microlith types in context 1 .

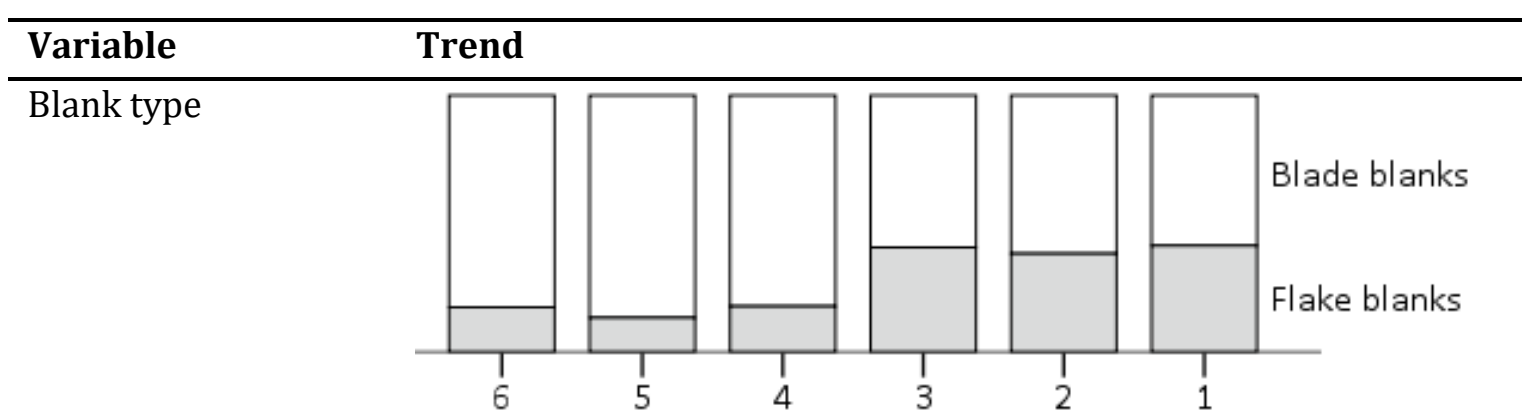

Longest dimension

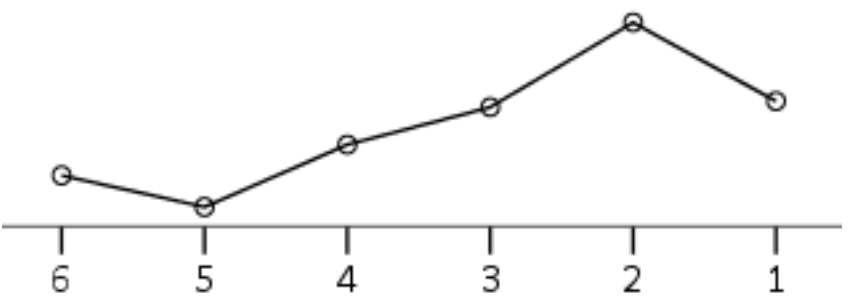

Oriented length

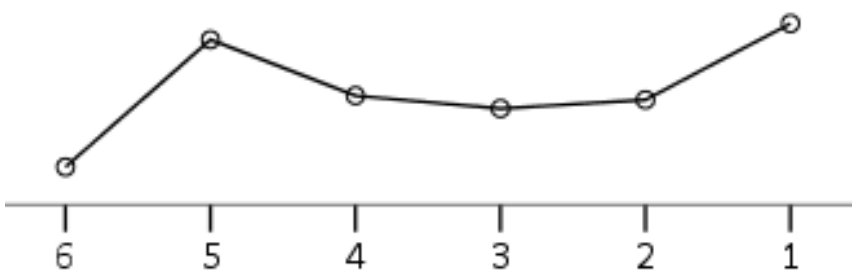

Medial width

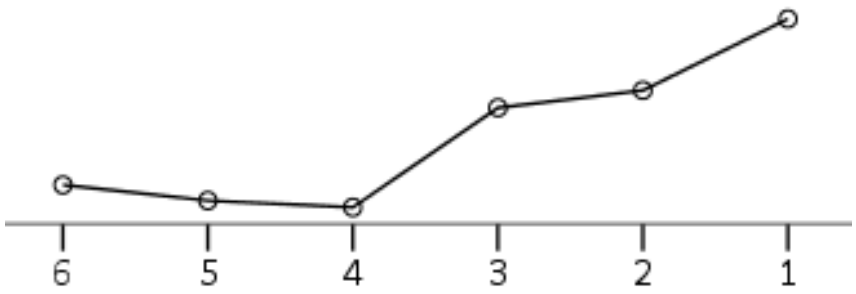

Length:width ratio

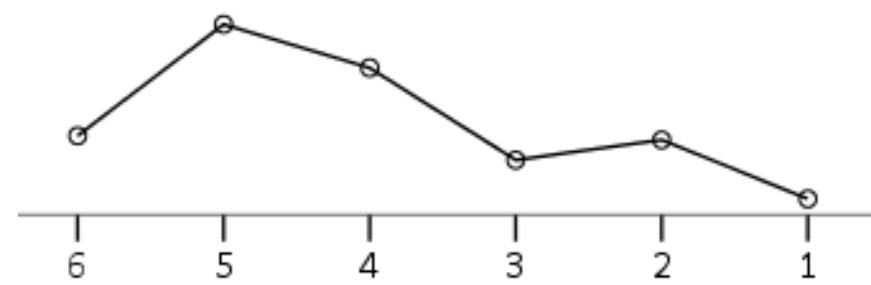

Striking platform

More pointed platforms in 5 and more abraded/crushed and type complex platforms in 4.

Percentage cortex

cover

Scar orientation

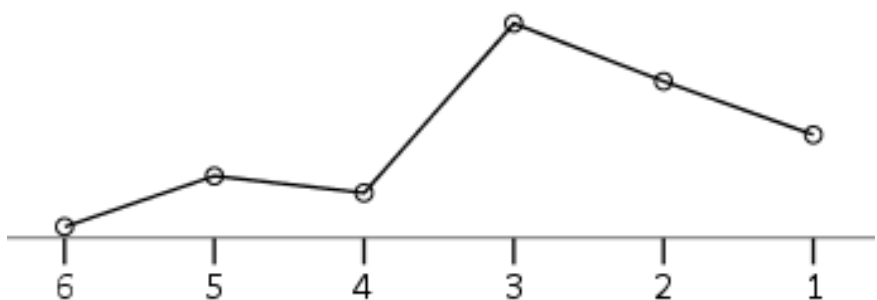

More microliths with scars originating from three directions in 6, and only those from one direction in 3. 
Termination type

Coarse typology and narrow typology

Retouch face

Retouch type

Retouch depth

Retouch length

Index of Invasiveness

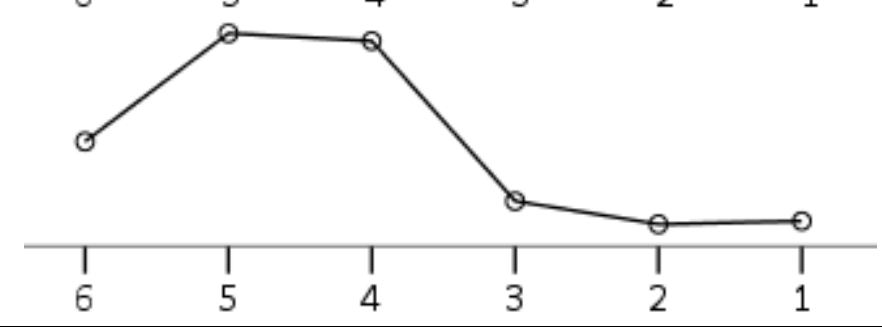

Table 6.4.10: Statistically significant differences between layers in microlith variables at Jwalapuram

However, a different structure of variation can be seen in several variables, and comes in the form of a step change between contexts 4 and 3 at $160 \mathrm{~cm}$, forming two clear groupings of contexts. Blade blanks were used in much higher proportions in contexts 6-4 (with a mean of 84.6\%) compared to contexts 3-1 (60.5\%). Additionally, backed geometric microliths are much more common in contexts 6-4 (28.0\% compared to $5.6 \%$ in contexts $3-1)$, as are backed non- 
geometric microliths, to a lesser extent (59.4\% v. 51.6\%). Conversely, non-backed microliths are more common in the top part of the sequence. A sudden decrease at this point in the numbers of microblades and backed pieces was also noted by Clarkson et al. (2009, Figure 9). This point in the sequence occurs between radiocarbon dates calibrated to 20,274-19,897 $\mathrm{BP}$ and 14,691-15,215 BP, corresponding roughly to the end of the LGM.

Figure 6.4.19: Frequencies of microlith types in each layer at Jwalapuram

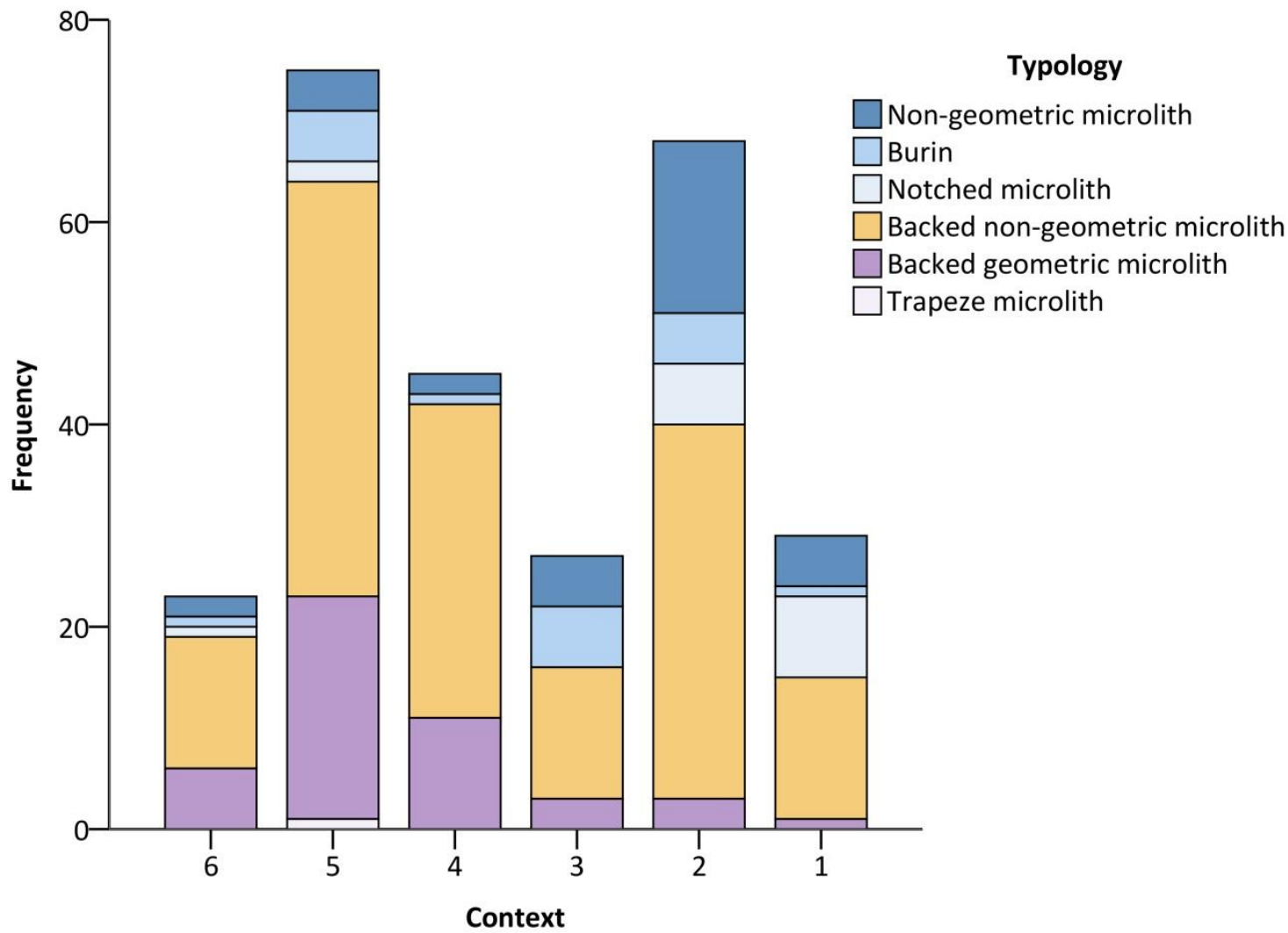

There is more variation in microlith typology in the top of the sequence compared to the bottom; six types are found in contexts 1-3 that do not occur in contexts 4-6, but the reverse is only true of one type. There is also more variation in later layers in terms of the choice of face to retouch, with bifacial retouch first appearing in context 5 and ventral retouch in 4 , both of which increase in proportion over time. 
In terms of size, microliths generally become larger and squatter over time, with width rather than length increasing the most. This is slightly different to the patterns seen in the core and debitage assemblages, where the largest average sizes were found in the middle of the sequence. One explanation involves the types of tool that were being produced. As demonstrated above, non-backed microliths are larger on average than backed forms, resulting in the production of larger microliths in the later part of the sequence.

Overall, contexts 6-4 are dominated by smaller backed geometric and nongeometric microliths made on blade blanks, and contexts 3-1 are dominated by larger non-backed and especially non-geometric microliths made on more flakelike blanks. The resulting picture is of two distinct phases of microlith production, with classic backed geometric and non-geometric microliths being replaced in the upper post-LGM part of the sequence by a wider variety of non-geometric types, with less of an emphasis on backing retouch. However, no such step changes are visible in the core and debitage assemblages, indicating that the division of the sequence into two separate phases would be inappropriate.

\section{MANUFACTURING TRAJECTORIES}

Manufacturing trajectories at Jwalapuram are summarised in Figure 6.4.20.

Limestone and CCS are the most commonly worked raw materials, with quartz, quartzite and dolerite also present. Cores with three or more platforms occur most frequently, although these were rarely worked centripetally. Some one- and, to a lesser extent, two-platform cores are also present. 
Figure 6.4.20: Manufacturing trajectories at Jwalapuram

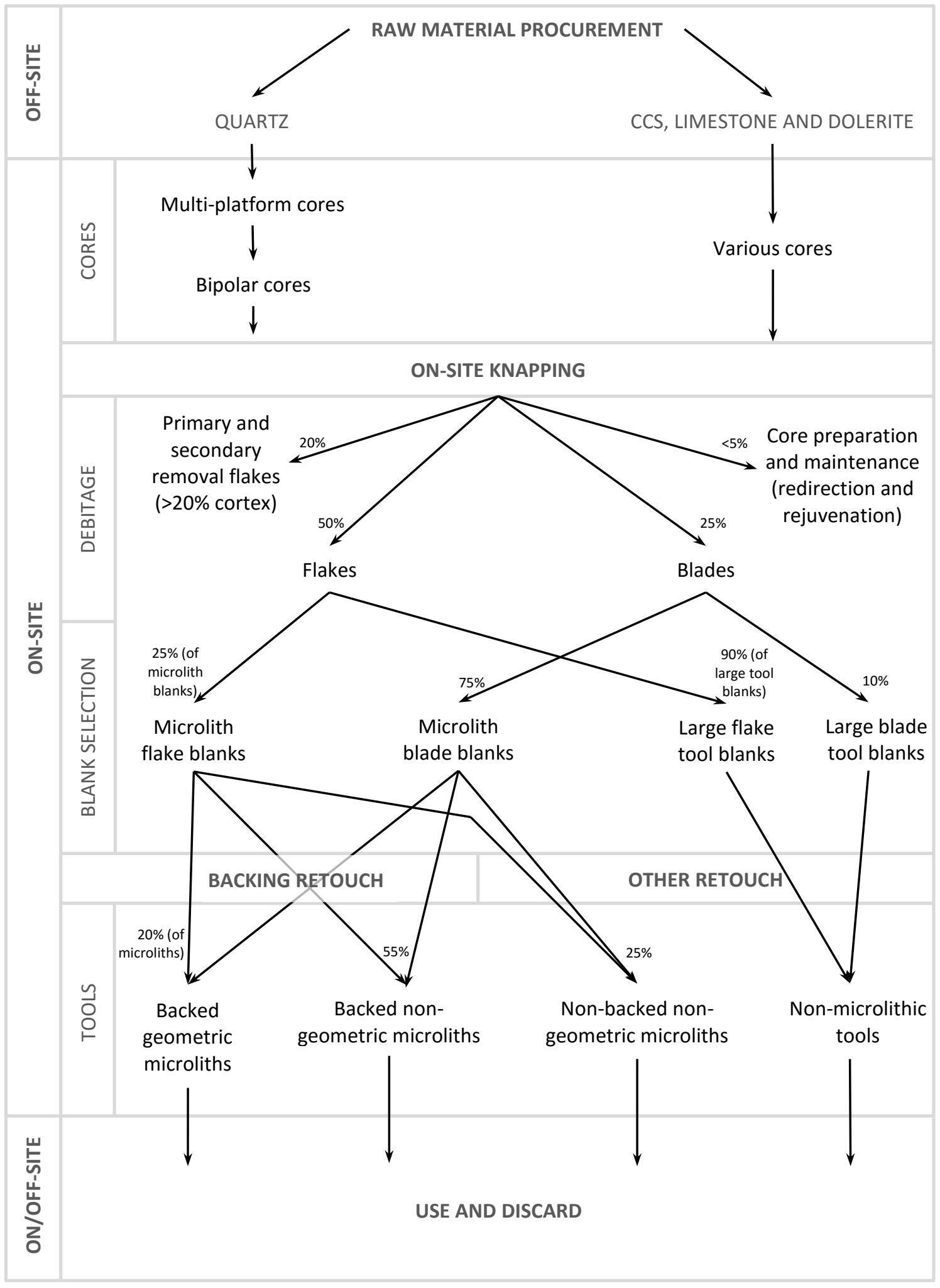


Bipolar cores are common. The vast majority of quartz cores were reduced using bipolar percussion at the end of their use-lives, whereas only a minority of other raw materials were, suggesting the existence of separate manufacturing trajectories for quartz and non-quartz raw materials. Bipolar cores were intensively worked at the site, primarily for the removal of flakes, and are particularly common in the earliest context.

The products of knapping are primarily flakes, which are twice as common as blades. Flakes and blades have very similar cortex cover proportions, suggesting that they were probably not removed in separate stages during the reduction process. There are also very similar raw material, termination and scar orientation proportions for flakes and blades (although differences are evident in striking platform sizes and types). Overall, these similarities suggest that the ' $2: 1$ ' ratio rule for separating flake and blade categories used here is not altogether meaningful when applied retrospectively, as few significant differences are noted between these groupings.

Although flakes are dominant in the core, debitage and non-microlithic tool assemblages, blades are dominant in the microlith assemblage. Yet while there are notable peaks in the proportions of blade cores in contexts 4 and 1, the debitage assemblage exhibits a gradual decrease over time in the production of blades, and the microlith assemblage shows a step change between a heavy focus on blade blanks in contexts 6-4 and a mix of flake and blade blanks in contexts $3-1$. Blades were clearly being preferentially selected for retouch into microlithic tools 
throughout the sequence, but the practices concerning blade production in a more general sense are more complex.

CCS blades were especially preferred for microliths, while limestone and dolerite flakes were preferred for the manufacture of non-microlithic tools. Elongation was considered an important aspect of the selection of blanks for retouch into microlithic tool forms, even though blades formed only a minority of the debitage assemblage.

In terms of size, microliths were chosen from the general debitage population, while non-microlithic tools are very large in comparison. As noted by Clarkson et al. (2009) there is a cut-off point in the frequency distribution of both debitage and microlith lengths at $40 \mathrm{~mm}$. However, this applies mostly to CCS, limestone and dolerite. Quartz debitage is more tightly distributed, with over three quarters being less than $17 \mathrm{~mm}$. Nevertheless, there is a near-bimodal distribution of tool lengths, separated at c. $40 \mathrm{~mm}$, which provides a useful separation of tool types. Differences also exist between microlithic and non-microlithic tools in their retouched faces, with edge and dorsal retouch most common on microliths, and dorsal and bifacial retouch most common on non-microlithic tools.

Microliths are twenty times more common than non-microlithic tools. Notches and burins occur frequently, especially in the microlith assemblage. Around three quarters of microliths are backed, and backed non-geometric microliths are dominant. Geometric microliths are also all backed, but are relatively uncommon. Backed geometric and non-geometric microliths are much smaller on average than 
non-backed types. It may therefore be concluded that the most meaningful categorisation of microliths at Jwalapuram is not whether a tool was retouched into a geometric or a non-geometric shape, but whether or not it was retouched using backing. Overall, however, despite considerable temporal variations in the core, debitage and microlith assemblages, it is clear that the overall aim of the lithic industry at Jwalapuram was the intensive production of microlithic tools. 


\section{Chapter 7: The southern African and South Asian early microlithic industries compared}

The previous two chapters presented the results of analyses of the lithic industries at southern African HP and South Asian Late Palaeolithic sites. This chapter first compares these sites within regions, in order to assess the degree of variability within the two industries. Microlithic technology is then compared between regions, making use of multivariate statistical techniques, to highlight areas of similarity and difference between them.

\subsection{COMPARISON BETWEEN SITES AND SUMMARY OF VARIABILITY IN THE SOUTHERn AfRICAN HOWIESONS POORT}

\section{RAW MATERIAL PREFERENCES}

The nearby sites of RCC and NT are both dominated by CCS, with evidence for the use of recycled older CCS lithics at both. There are differences in the other raw materials used at the sites, with quartzite making up the remainder of the assemblage at NT and a wider variety of materials (quartzite, claystone, siltstone, hornfels, quartz and silcrete) present at RCC. However, there is sufficient evidence to suggest that occupants of both sites may have been making use of the same or similar sources of raw materials. 
At RCC, CCS and claystone were worked in order to produce large numbers of relatively standardised small blades less than c. $45 \times 20 \mathrm{~mm}$, which were used as blanks for the production of microliths. The other raw materials found at the site were used to produce larger flakes and blades, which were selected for retouch into non-microlithic tools. Different manufacturing trajectories were utilised for different raw material groupings. However, at NT there are few indications that different raw materials were treated or worked differently, although there are some preferences evident in blank selection, as quartzite does not appear to have been used for microlithic tools.

CCS is rare at the more distant site of Umhlatuzana. The main raw materials used were hornfels and quartz, with quartzite also present. As at RCC (but in contrast to NT), strong preferences can be seen in the uses of different raw materials.

Differences between hornfels and quartz exist in terms of reduction processes, blank selection and retouch. For example, quartz was primarily worked to produce flakes, and hornfels for blades. Both were used to manufacture microliths, but most non-microlithic tools were made on hornfels.

Raw material proportions remained at similar levels throughout the sequence at RCC. Small variations exist between layers at NT in the proportion of quartzite in each layer. Only at Umhlatuzana is there a consistent change over time in raw material use. Here there is a decrease over time in the proportions of hornfels and quartzite debitage, and a concomitant increase in quartz, which becomes dominant in the upper layer. 
However, these changes are not reflected in the tool assemblage. In contrast to arguments that the explosion of microlith production seen in the HP was related to an increased use of fine-grained raw materials (Ambrose and Lorenz 1990, Ambrose 2002), the evidence from Umhlatuzana would seem to suggest that the same form of microlithic technology continued to be produced at the site despite changes in raw materials. Therefore these changes in raw materials were most likely due to other external factors unrelated to technological choices, such as changes in range sizes, mobility patterns or landscape use.

\section{CORE REDUCTION STRATEGIES}

Different core types are dominant at each site studied. Blade cores are common at RCC, whereas flake cores make up the majority of the assemblage at nearby NT. Bipolar cores are the most numerous type at Umhlatuzana, with single platform flake cores also common. However, the use of radial/centripetal reduction - often considered a typically MSA phenomenon (Ambrose 1998, Wurz 2013) - is found at all three sites. At Umhlatuzana it becomes less common over time, but at RCC and NT it persists throughout the sequence (although it is rare at the latter).

Bipolar technology is typically considered an LSA phenomenon in sub-Saharan Africa (Ambrose 2002, Villa et al. 2012, Eren et al. 2013), although silcrete and chert bipolar cores have been documented in HP levels at Klein Kliphuis, South Africa (Hiscock 2015). Bipolar percussion is evident at all three sites studied here. However, it was made use of in different ways. At RCC and NT, bipolar percussion was sometimes used as a final knapping strategy in the reduction of nearly- 
exhausted CCS cores in order to produce small blades. It may also have been used in response to the small size of raw material nodules, particularly of agate at RCC. In contrast, however, bipolar percussion was used frequently on quartz at Umhlatuzana as an efficient way to maximise the number of whole flakes that can be removed from a small core (Knight 1991a, Driscoll 2011).

Figure 7.1.1 highlights the differences between bipolar cores at the sites. Those at RCC are more elongated, with a wide range of core lengths but a relatively restricted range of widths, in keeping with the general elongation of cores and core products at the site. Conversely, those at Umhlatuzana are more rounded, with a greater range of widths compared to lengths.

Figure 7.1.1: Scatter graph of bipolar core lengths and widths at HP sites

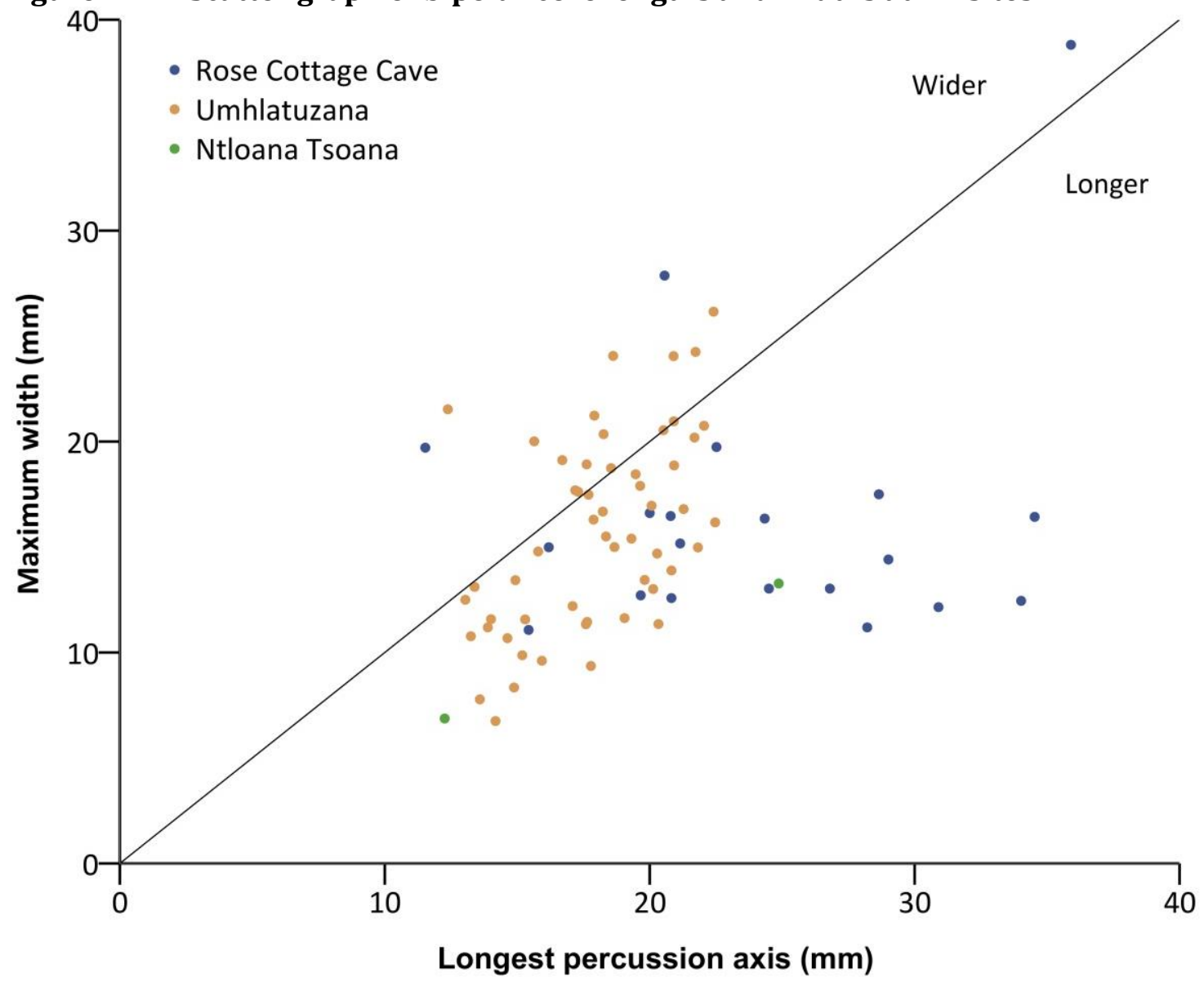


Bipolar cores are not the only core type that differs in size between the sites.

Figure 7.1.2 reveals that cores of most types are smallest at NT. As the raw materials used at NT are very similar to those at RCC, these size differences cannot be equated solely with differences in material. Instead they may reflect the existence of different stages of core reduction at the sites (with either less early stage reduction or more late stage present at NT), or differences in reduction intensity. Although the former explanation cannot be ruled out, Figure 7.1.3 demonstrates that the degree of reduction intensity is indeed higher on average at NT, and lowest at RCC. Overall, the largest differences between the sites in terms of core size and reduction intensity can be seen between the geographically proximate sites of RCC and NT.

Figure 7.1.2: Cubic volumes of core types at HP sites

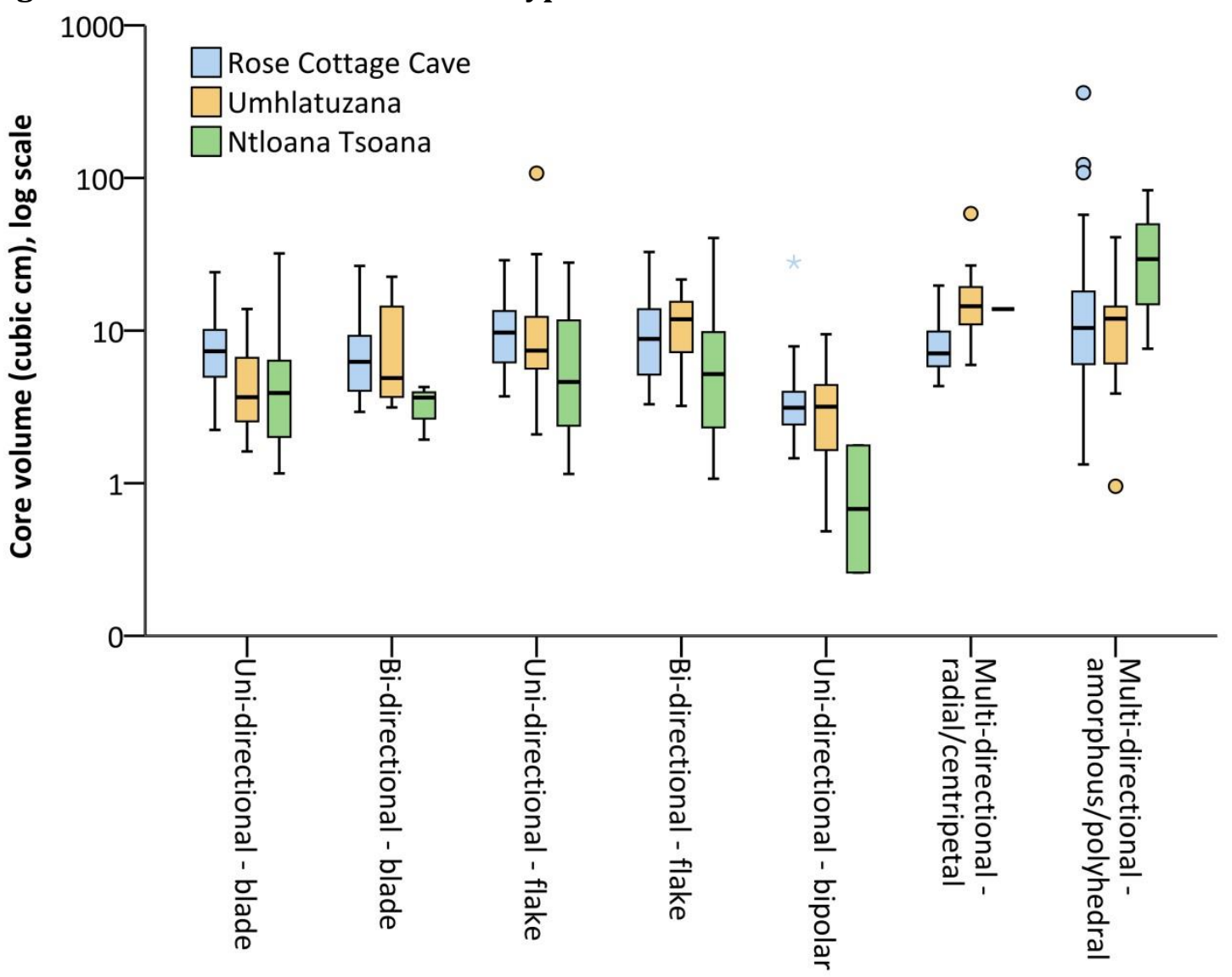


Figure 7.1.3: Ratios of total removal scar length to core volume on cores at HP sites

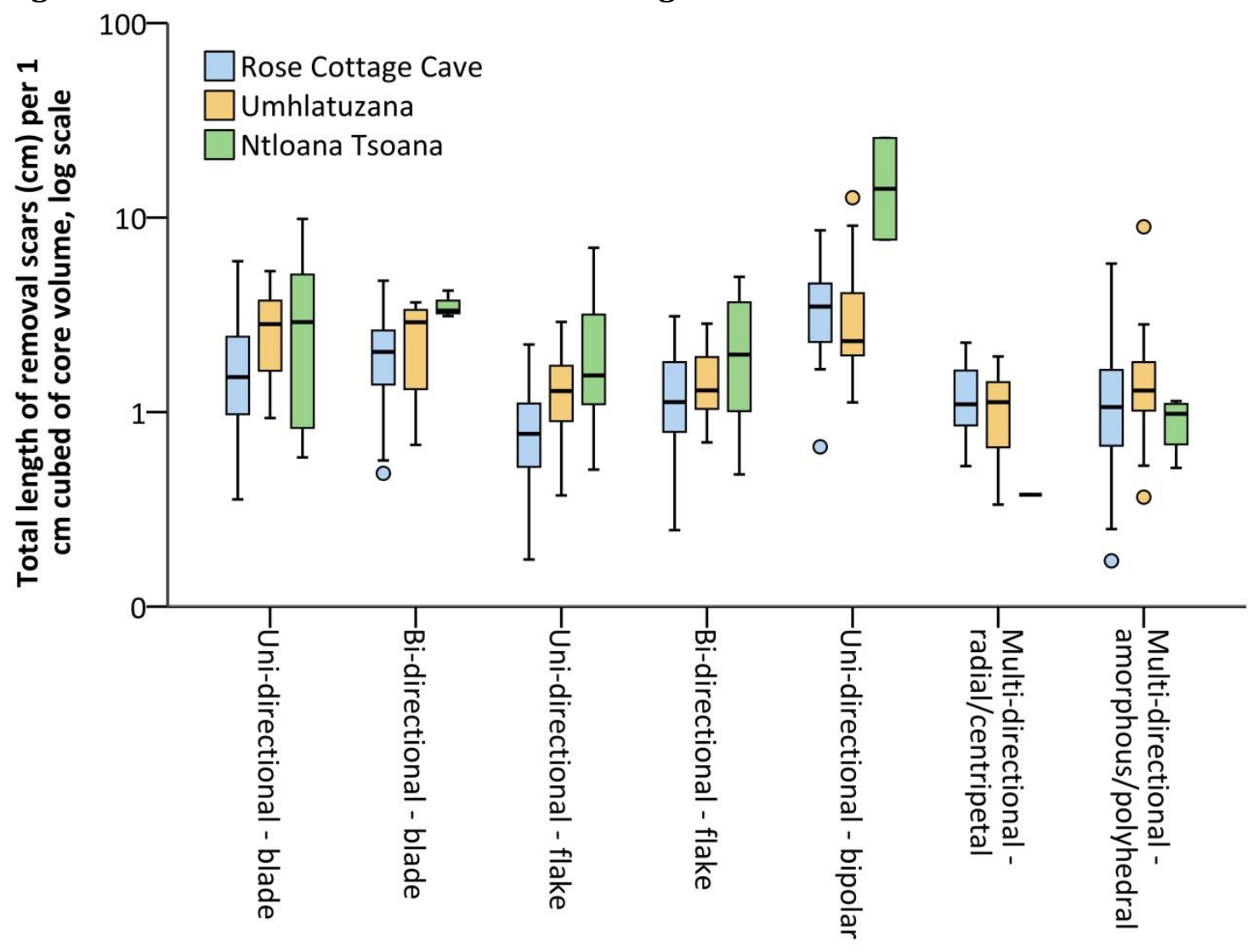

Major differences also exist between RCC and NT in terms of the sequences of lithic reduction, with more similarities evident between NT and Umhlatuzana despite the almost total lack of overlap in raw material types. At NT and Umhlatuzana, there are some possible indications that blades may have been removed slightly later within reduction sequences, but overall there is little indication that flakes and blades were removed using different methods or at different stages. At Umhlatuzana the main differences in flake and blade production are related to raw material, as discussed above. At NT the data is inconclusive, and is complicated by the fact that flake-blades do not appear to constitute a clear intermediate category. Conversely, blades at RCC were clearly removed as part of a later stage of reduction, and were the desired outcome of lithic production at the site. 
Core reduction strategies did not alter in any significant way during the sequences of HP occupation at RCC and NT, and remain remarkably consistent. However, at Umhlatuzana there are numerous small-scale variations between layers. Cores are larger on average in the middle part of the sequence, with correspondingly more removals. Yet with the exception of a decrease over time in the proportion of radial cores, temporal changes are predominantly short-lived and non-directional, and simply represent small context-specific variations.

Overall, comparisons of core reduction strategies confirm Clarkson's (2010) findings concerning regional and site-specific core reduction methods in the HP. For example, different core types are dominant at each site. RCC is the only site that exhibits manufacturing trajectories that were designed around the production of blades. Although bipolar percussion was utilised at all three sites, it was used in different ways. And although NT is geographically close to RCC, and the same raw materials were utilised at both sites, both exhibit different processes of core reduction - for example, cores are smaller and more intensively worked at NT. These differences in core reduction strategies - particularly when raw material can be controlled for, as in the case of RCC and NT - are suggestive of regional traditions of core reduction (Clarkson 2010), and certainly imply that more variation is evident within the HP than is often assumed.

\section{BLANK PRODUCTION AND SELECTION}

As with core types, different debitage products are dominant at each site. Blades predominate in the RCC assemblage, while flakes are most common at NT, and 
Umhlatuzana exhibits more of a mix of flakes, flake-blades and blades. Crested blades, indicative of laminar technologies (Bar-Yosef and Kuhn 1999, Hoggard 2014), are only present at RCC. Overall, the assemblage at RCC is the only one which corresponds with the popular assumption that the HP is primarily a bladebased industry (e.g. Clark 1985, Soriano et al. 2007, Wadley 2008, Wadley and Mohapi 2008, Cochrane 2008, Wurz 2013), while the assemblages from Umhlatuzana and NT indicate that more variability in blank production is evident within the industry.

As a result, size differences are evident between debitage at the sites (Figure 7.1.4). Whole debitage at NT is consistently smaller on all size metrics, while that at RCC and Umhlatuzana is relatively similar. RCC has a particularly tightly peaked debitage size distribution, whereas more variation is evident at Umhlatuzana due to the differences in size between quartz and hornfels debitage (Figure 7.1.5). Notably, these size differences remain constant even when debitage type (flake, blade, or flake-blade) is controlled for, as Figure 7.1.6 demonstrates in the case of debitage volume. Additionally, debitage is more elongated (length:width ratio) and thinner (length:thickness ratio) at RCC.

In terms of specific blank production techniques utilised at the sites, there is no evidence at any site of the use of the microburin technique to manufacture blanks for microlith production. However, at Umhlatuzana there is some possible intentional snapping of blanks (without the construction of notches as in the microburin technique), especially hornfels blades, for the preliminary shaping of microlith blanks. 
Figure 7.1.4: Scatter graph of whole debitage lengths and widths at HP sites

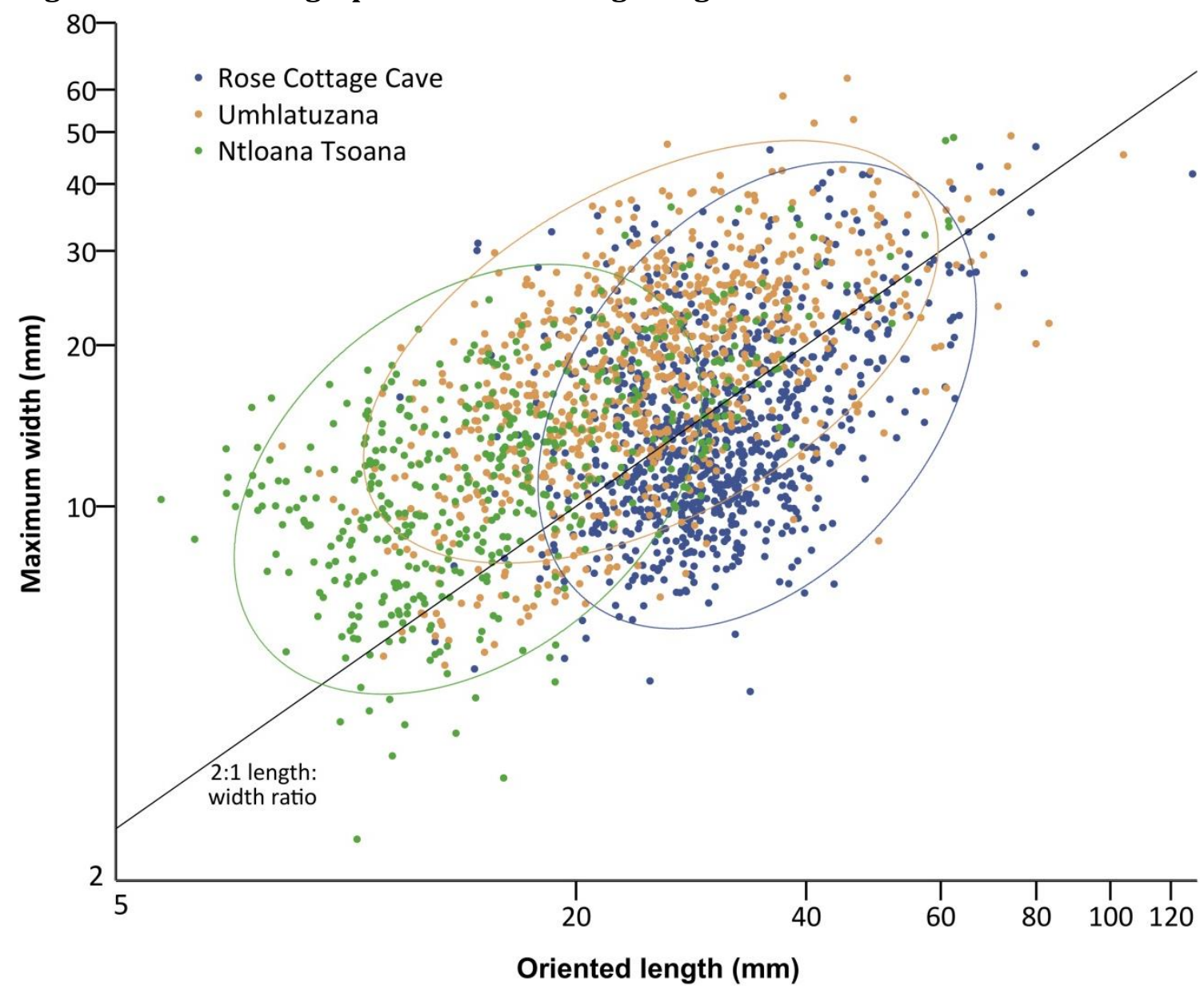

One similarity between the sites is in the overall preference for blade blanks for tools. At NT, flake and blade blanks were used equally for both microlithic and nonmicrolithic tools. However, because blades make up only a minority of the debitage assemblage, this indicates the preferential selection of blades. At RCC blade debitage is dominant, but blade blanks are even more common in the tool assemblage, especially for microliths. At Umhlatuzana blades constitute less than a quarter of the debitage assemblage but half of microlithic tool blanks and almost three quarters of non-microlithic tool blanks. However, at Umhlatuzana blade preferences are only evident on hornfels. While hornfels blades were preferentially selected for retouch, flakes were preferentially selected from the available quartz debitage, particularly for microliths. 
Figure 7.1.5: Frequency distributions of whole debitage lengths at HP sites

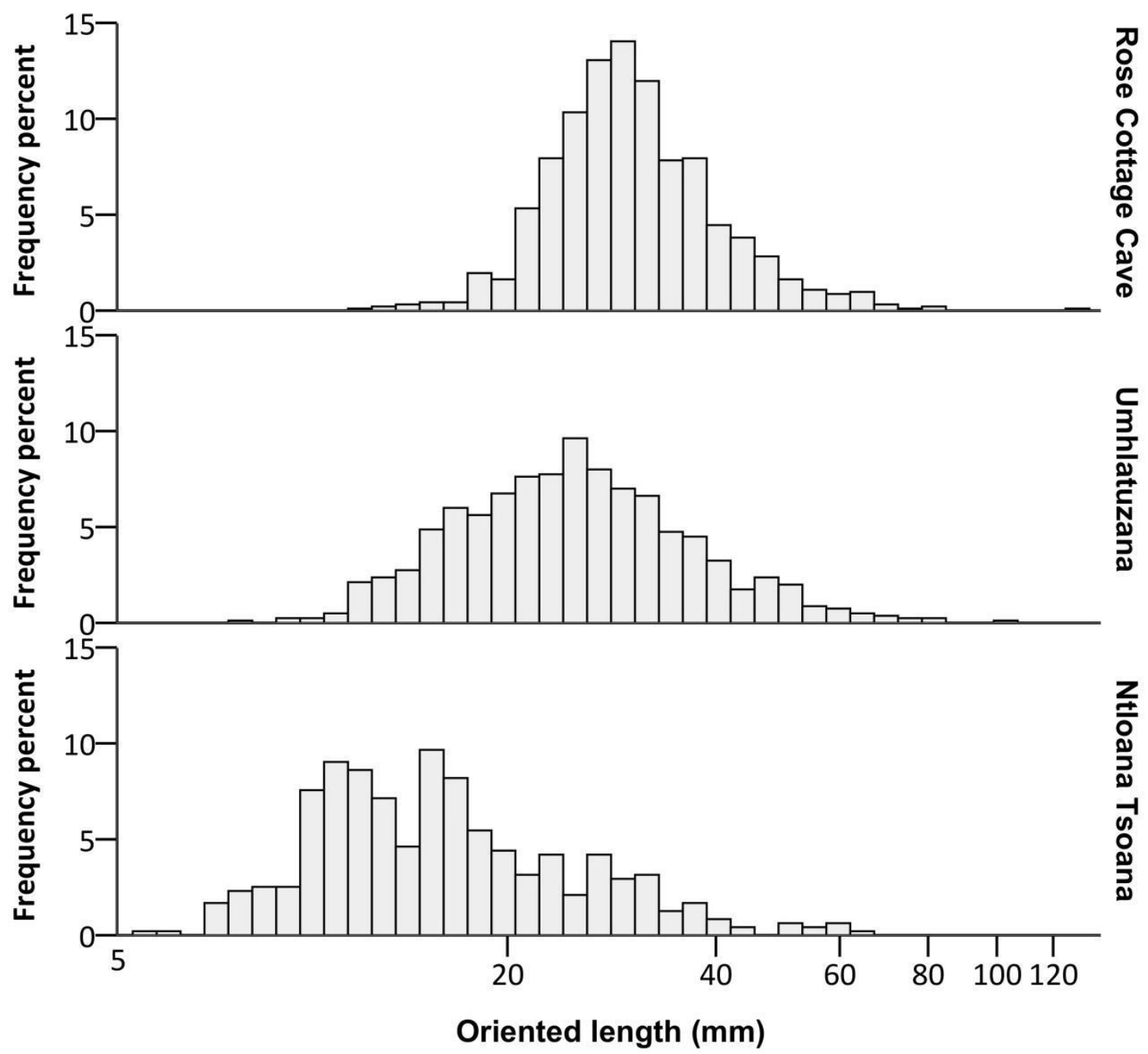

Differences are also evident between the sites in the selection of blanks for retouch into tools of different sizes. At RCC and NT, microliths fit within the general debitage size ranges, while non-microlithic tools are significantly larger. Small blanks were produced in large numbers and selected for retouch into microlithic tools, while larger flakes and blades were more opportunistically selected for retouch into different tool types. Lithic reduction was aimed primarily at the production of small blanks suitable for retouch into microlithic tools. However, at Umhlatuzana both microlithic and non-microlithic tools were selected from the general array of available debitage sizes, although there is little overlap between 
microlithic and non-microlithic tool sizes (i.e. both small and large blanks were selected for retouch into different tool types, but few of an intermediate size). This distinction implies that non-microlithic tool production differed between the sites.

Figure 7.1.6: Cubic volumes of whole debitage at HP sites

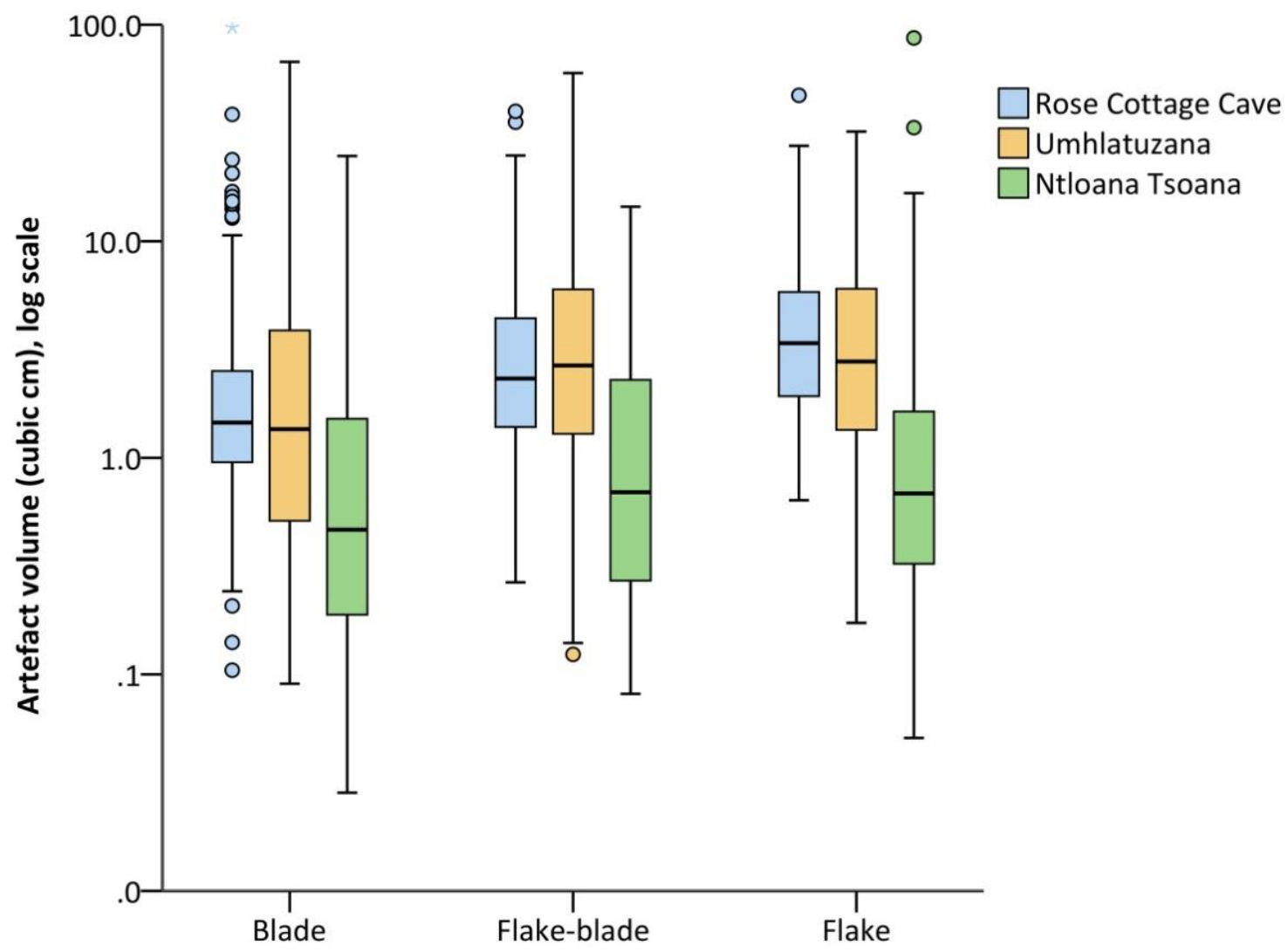

The main determining factor in microlith blank selection at Umhlatuzana appears to have been raw material. Hornfels microlith blanks were selected from a wide range of potential blank sizes, whereas quartz microlith blanks were chosen from the smaller end of the sizes available and are more standardised. Overall, hornfels was used for larger blade blanks, and quartz for smaller flake blanks. Although raw material preferences are evident in blank selection at both RCC and NT, only at Umhlatuzana do these preferences extend to blank size. 
There are no major changes evident in blank production or selection methods during the sequences at any of the sites, although small-scale temporal variability in debitage characteristics can be seen. For example, the middle layer at RCC is more blade-like, while the upper layer is more flake-like. Debitage at Umhlatuzana and NT is more variable over time compared to RCC. However, most of this variability is weak and inconsistent, and much can be related to changes in raw material proportions (of quartzite and CCS at NT, and of quartz at Umhlatuzana). Overall, despite some small-scale changes, manufacturing trajectories did not vary much over time at the HP sites analysed, although they do differ between sites; most variation is therefore geographical rather than chronological.

\section{RETOUCH CHOICES}

Variation exists between sites both in tool typology and technology. For example, although microliths are more common than non-microlithic tools at all sites, nonmicrolithic tools are more common at RCC. Conversely, one similarity can be seen in the existence of non-microlithic points at each site. At RCC, typically MSA unifacial points occur throughout the sequence, and at Umhlatuzana a unifacial and a bifacial point are present. These invasively retouched point types are absent at NT, although a side retouched point is present. Unifacial points are found across South and East Africa during the MSA (Clark 1988, Brooks et al. 2006, Wurz 2014). For example, unifacial points characterise the post-HP MSA at Sibudu (Wadley 2005), and are also found in pre-HP deposits at the site (Wadley and Jacobs 2006). Classic MSA macro-point technology existed alongside HP microlithic technology at all three sites studied here. The manufacture of two discrete point types at the 
sites may suggest the co-existence of separate and distinct hunting strategies, assuming that microlithic points were utilised as projectile weaponry.

Notably, geometric microliths are not the dominant microlith type at any of the three sites. They vary between $18.7 \%$ of the microlith assemblage at RCC to $38.7 \%$ at Umhlatuzana. Crescents are the most common geometric form at all sites. Backed non-geometrics are dominant at RCC and Umhlatuzana, particularly laterally backed microlithic points. Non-backed non-geometrics are dominant at $\mathrm{NT}$, although the recovered tool assemblage is small. The dominance of nongeometric microliths at RCC was previously noted by Wadley and Harper (1989) as an unusual aspect of the HP at the site. However, the present analysis indicates that this may in fact be common to many - if not most - other HP sites.

Differences exist in the microlithic forms present at RCC and Umhlatuzana (excluding the small microlith assemblage at NT). Although the microlith assemblage studied at RCC is slightly smaller (134 v. 181), six microlith forms were found at RCC but not at Umhlatuzana (double side retouched, preforms, rectangles, shouldered, shouldered and backed, and tanged and pointed), compared to two in the opposite case (concave points and triangles). Notably, microlith preforms (those where only initial shaping has been conducted, such as the removal of the bulb of percussion) are only present at RCC, possibly implying differences in the stages of microlith production between the sites.

A slightly greater degree of variability is evident in microlith typology at RCC compared to Umhlatuzana, although at neither is there any convincing evidence for 
standardisation within types. The only possible exception to this is in backed nongeometrics at RCC. At Umhlatuzana in particular there is considerable variation within categories. Different sized blanks were not preferentially made into particular forms, and differences in size and shape are primarily due to differences in raw material (similar to the pattern noted at the nearby site of Sibudu by Wadley and Mohapi (2008)).

Microlith size differences can also be seen between the sites. Microliths at RCC are consistently larger than at Umhlatuzana and NT, even when typology is controlled for, with the exception of shouldered/tanged forms (Figure 7.1.7) . Following on from the discussion of microlith definitions in Chapter 2.1, this analysis demonstrates that the application of a universal and arbitrary cut-off point for the lengths of microlithic tools would be neither accurate nor meaningful.

On the other hand, TCSA measurements exhibit similarities between the sites. Microlithic tools at each site cluster between what have been defined elsewhere as arrowheads and dart tips (Shea 2006), and tend to fall closer to the smaller arrowhead sample. In contrast, non-microlithic points have similar TCSA measurements to Shea's hand-cast/thrusting spear sample. Shea warns against calculating TCSA measurements on microlithic tools where the hafting orientation is unclear, and in the absence of use-trace analysis it is not possible to confirm the use of any individual microlith as a projectile. Nevertheless, TCSA measurements can provide a useful point of comparison between potential microlithic and nonmicrolithic projectile points in the HP. 
Figure 7.1.7: Lengths of microlith types at HP sites

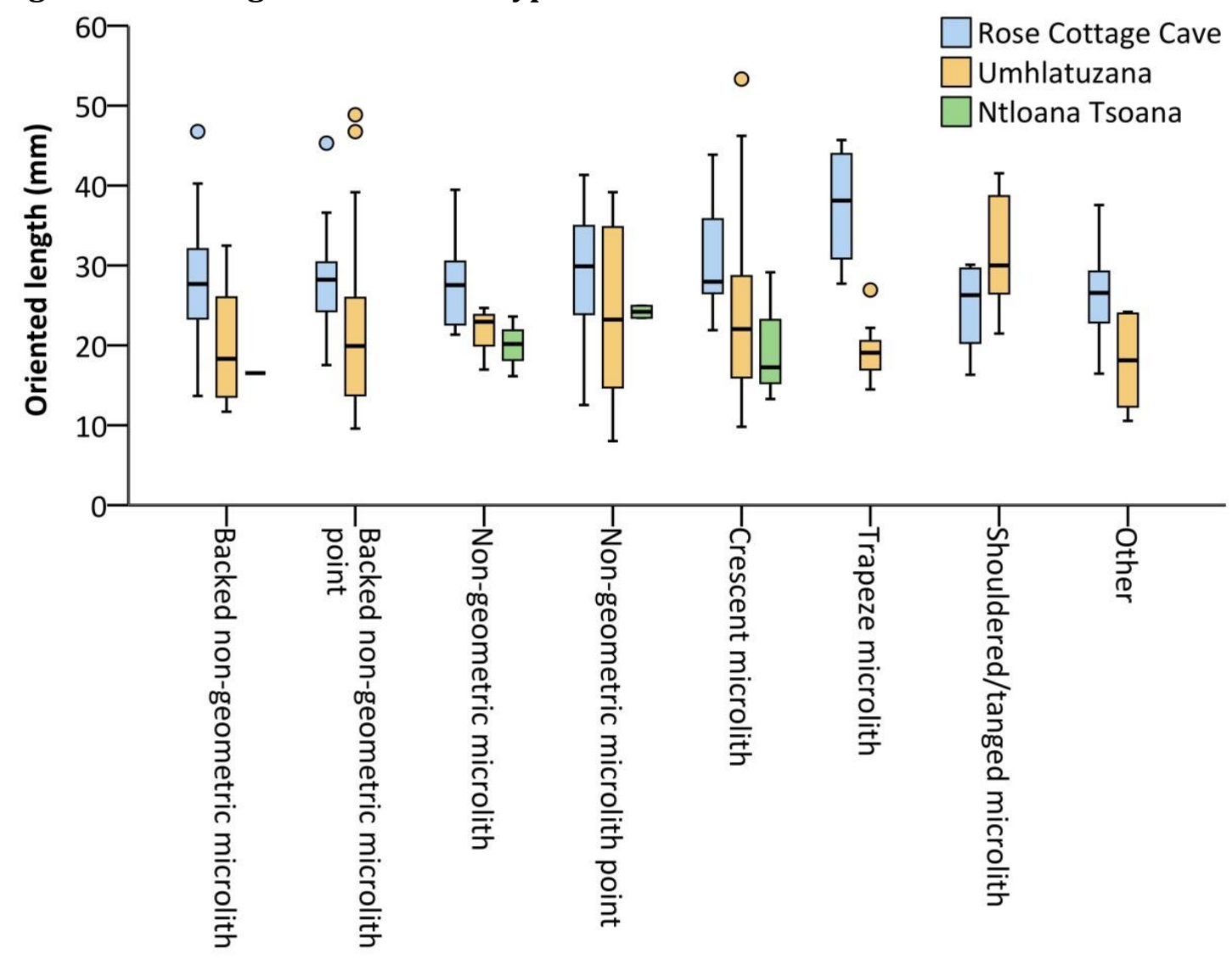

Of the classic attributes used to define microliths (length, width, geometric shapes, blade blanks and backing), the use of backing retouch is perhaps the most useful at the HP sites studied. Backing is by far the most common retouch type at Umhlatuzana (87.2\% of retouch incidences) and RCC (68.5\%). At NT it only constitutes a quarter of retouch incidences, but the small number of microlithic tools recovered means that this may not be representative. Backing is present on almost all geometric microliths at all sites, in addition to the majority of nongeometric microliths at RCC and Umhlatuzana, and thus supersedes geometric shape as a primary categorisation of microlithic tools. Rare occurrences of backing retouch also occur in the non-microlithic tool assemblages (five cases at RCC and one at Umhlatuzana). However, given the dramatically different frequencies with which backing retouch was used in the manufacture of microlithic and of non- 
microlithic tools, backing remains a useful distinguishing feature of microlith production.

Two slightly different methods of backing retouch were utilised during the HP backing conducted from a single face (dorsal or ventral), and bipolar backing conducted from both faces. Bipolar backing is absent in the small sample at NT, but is dominant at RCC and Umhlatuzana. The use of bipolar backing remained at a consistently high level throughout the HP at RCC. At Umhlatuzana, however, bipolar backing becomes less common over time (decreasing from $93.6 \%$ to 40.0\%), being replaced by backing conducted from a single face. Although the use of backing retouch relative to other retouch types does not change considerably over time, the method to produce it does. There are no concomitant changes in other aspects of microlith production over time, and so this is best viewed as a sitespecific temporal change in retouch technique.

At RCC and Umhlatuzana the retouch techniques used on non-microlithic tools are considerably more varied than those in the microlith assemblages, with scalar and stepped being the most common types but no one technique constituting more than a third of retouch incidences. At NT the opposite is true, with almost three quarters of retouch on non-microlithic tools being of scalar type, but no single retouch type constituting over a third of retouch incidences on microlithic tools.

Several retouch techniques are unique to either the microlithic or the nonmicrolithic tool assemblages at each site. Some of these differ between sites, but at the sites where they occur (RCC and Umhlatuzana) burination, denticulation and 
notches are only found in the non-microlithic tool assemblages and were not used to create microliths. Overall, almost a third of retouch types at RCC (4/13), half at NT (3/6) and almost two thirds at Umhlatuzana (7/11) were utilised only for either microlithic or non-microlithic tools. Distinctions between microlithic and macrolithic tools in the HP sites studied can be made on the basis of retouch type as well as size.

One of the most notable features of tool retouching processes at the sites is in the considerable degree of consistency and stability evident in the tool assemblages. At RCC the uppermost layer contains slightly wider microliths on average compared to the preceding layers, mirroring the debitage assemblage, and also contains slightly more intensively retouched microliths. Also, non-microlithic tools in the middle section are somewhat longer and more blade-like compared to other layers. However, there are no over-riding chronological trends and the data does not indicate any substantial changes in tool typology or technology.

At Umhlatuzana, other than the trend from bipolar to single-face backing retouch, the only temporal variation is in the form of small layer-specific changes in tool completeness and proportions of non-backing retouch types. This is despite considerable temporal changes in the core and debitage assemblages. Most notably, there are no real changes in the raw materials used in microlith production, despite significant changes in the raw material proportions of available blanks. Site occupants were producing the same microlith forms in the same ways despite changes in raw materials. Overall, the retouch processes 
involved in the production and shaping of microlithic tools remained relatively uniform throughout the HP sequences at the sites studied.

\section{SUMMARY}

Both similarities and differences between the HP sites studied have been documented. Similarities include the presence of radial/centripetal core working, bipolar percussion, and non-microlithic points at all sites. Blades were preferred as tool blanks at all sites. Geometric microlith forms are not dominant at any site, although crescents are the most common geometric shape at each. At both sites with large microlith assemblages (RCC and Umhlatuzana), backing retouch (particularly bipolar backing) and backed non-geometric microliths are dominant. Distinctions appear to have been made at all sites in the types of retouch technique used to manufacture microliths and non-microlithic tools. Yet there is no convincing evidence for standardisation within specific microlith typologies at any site, although microlith TCSA measurements are all similar to smaller projectiles such as arrowheads, and are distinct from larger points. An overall stability in lithic reduction sequences over time is evident at all sites, particularly in the tool assemblages, and there is no evidence for any distinct phases within the HP.

On the other hand, each site exhibits different dominant debitage and core types: blades and blade cores at RCC, flakes and flake cores at NT, and bipolar cores and a mix of debitage products at Umhlatuzana. Bipolar percussion was used differently at each site, with bipolar cores more elongated at RCC and more variable in size at Umhlatuzana. Additionally, several microlith forms are only present at one site. 
Each local variant of the HP is distinct. Only RCC can be described as containing a blade-based industry. Blades at RCC were removed later in reduction sequences, as the intended final outcome of lithic reduction, whereas evidence for the production of blades in this way at Umhlatuzana and NT is inconclusive. Additionally, nonmicrolithic tools are considerably more common at RCC, and microliths are larger and more typologically variable.

The sites of RCC and NT are geographically close, and there is evidence to suggest that similar sources of raw materials were used. Yet there are numerous differences between the two sites, and several areas where RCC exhibits more similarities with Umhlatuzana despite differences in raw material. For example, cores are smaller and more intensively worked at NT. At RCC and Umhlatuzana there are distinct strategies for working different raw materials, but there is little evidence for this at NT. Also, NT is the only site which exhibits greater variation in the retouch techniques used to produce microliths (rather than non-microlithic tools, as at the other sites).

Different raw materials were worked at Umhlatuzana compared to RCC and NT, which may account for many - but not all - of the distinct features of the HP at the site. Only at Umhlatuzana do raw material preferences extend to blank size and type. Snapping of tool blanks as part of the initial shaping process is also unique to the site. Umhlatuzana is the only site demonstrating a consistent change over time in raw material preferences, although this is not the case in the tool assemblage. Finally, while microliths fit within the general size range of debitage at RCC and NT, at Umhlatuzana this is true of both microlithic and non-microlithic tools. 
In contrast to commonly accepted descriptions of the HP, the sites analysed here indicate that the HP is not primarily a blade-dominated industry. Additionally, geometric microliths are not the dominant tool type. Microlith shapes and sizes vary considerably between sites, and the presence of geometric shapes or the use of a universal cut-off length are not useful definitions of microliths or of microlithic industries. Although it remains an insufficient definition when used alone, the most notable feature that microlithic tools have in common between the assemblages studied is the use of backing retouch.

Overall, there is considerable evidence for variability within the HP, as seen in the numerous differences in raw material preferences, core reduction strategies, blank production and selection, and retouch choices between the sites. There is more geographic variability evident than chronological variability at the sites studied. Some variability can be tied to differences in raw material, but some is unrelated to the materials used; for example, there are several areas of difference in manufacturing trajectories between RCC and NT despite the likely use of the same or similar raw material sources. These local differences can therefore be tied to localised technological or cultural factors instead, such as tool functions or hafting requirements. On the basis of the results presented here, there is no convincing evidence for the existence of phases within the HP, or of the 'matching up' of changes over time between the sites (for example, there is no overall trend towards the increased use of fine-grained raw materials, or in the production of blades). Instead, the HP is characterised by considerable variability between sites. 


\subsection{COMPARISON BETWEEN SITES AND SUMMARY OF VARIABILITY IN THE SOUth Asian late Palaeolithic}

\section{RAW MATERIAL PREFERENCES}

Lithic manufacture was conducted almost solely on quartz at Batadomba-lena and Kitulgala, which was locally available in large quantities. Higher qualities of quartz were worked more intensively, and were preferentially selected as tool blanks. Quartz was also worked at Jwalapuram, although CCS and limestone are dominant. No core type is particularly heavily worked at Patne, suggesting that efficient use of raw materials was not a pressing concern; Sali (1989) notes that CCS and similar raw materials were locally available in abundance.

The raw materials utilised at Jwalapuram were also available in the local environment (Clarkson et al. 2009). Although no single raw material is dominant overall, some raw material preferences are evident in the assemblage. Limestone and quartzite were preferred for non-microlithic tools, and CCS for microliths. Separate reduction trajectories are hinted at for different raw materials, with bipolar percussion common on small quartz cores. Many technological changes over time can be tied to changes in the uses of raw materials. However, despite significant changes in the raw material proportions of cores and debitage, there is not one in the microlith assemblage. Microliths were produced on the same types of raw materials despite differences in availability, and other changes in microlith features (including size and typology) cannot be attributed solely to changes in raw material use. The same is true of different qualities of quartz at Batadombalena. 


\section{CORE REDUCTION STRATEGIES}

Flake cores are more common than blade cores at all sites. Amorphous multidirectional and bipolar cores make up the majority of the assemblages at both Batadomba-lena and Jwalapuram, largely due to the common working of quartz. One- and two-platform flake cores are dominant at Patne. Radial working of cores is present at Batadomba-lena and Jwalapuram, although it is uncommon. Cores at Batadomba-lena and Jwalapuram are small on average, and are consistently smaller than those at Patne (Figure 7.2.1).

\section{Figure 7.2.1: Cubic volumes of cores at Late Palaeolithic sites}

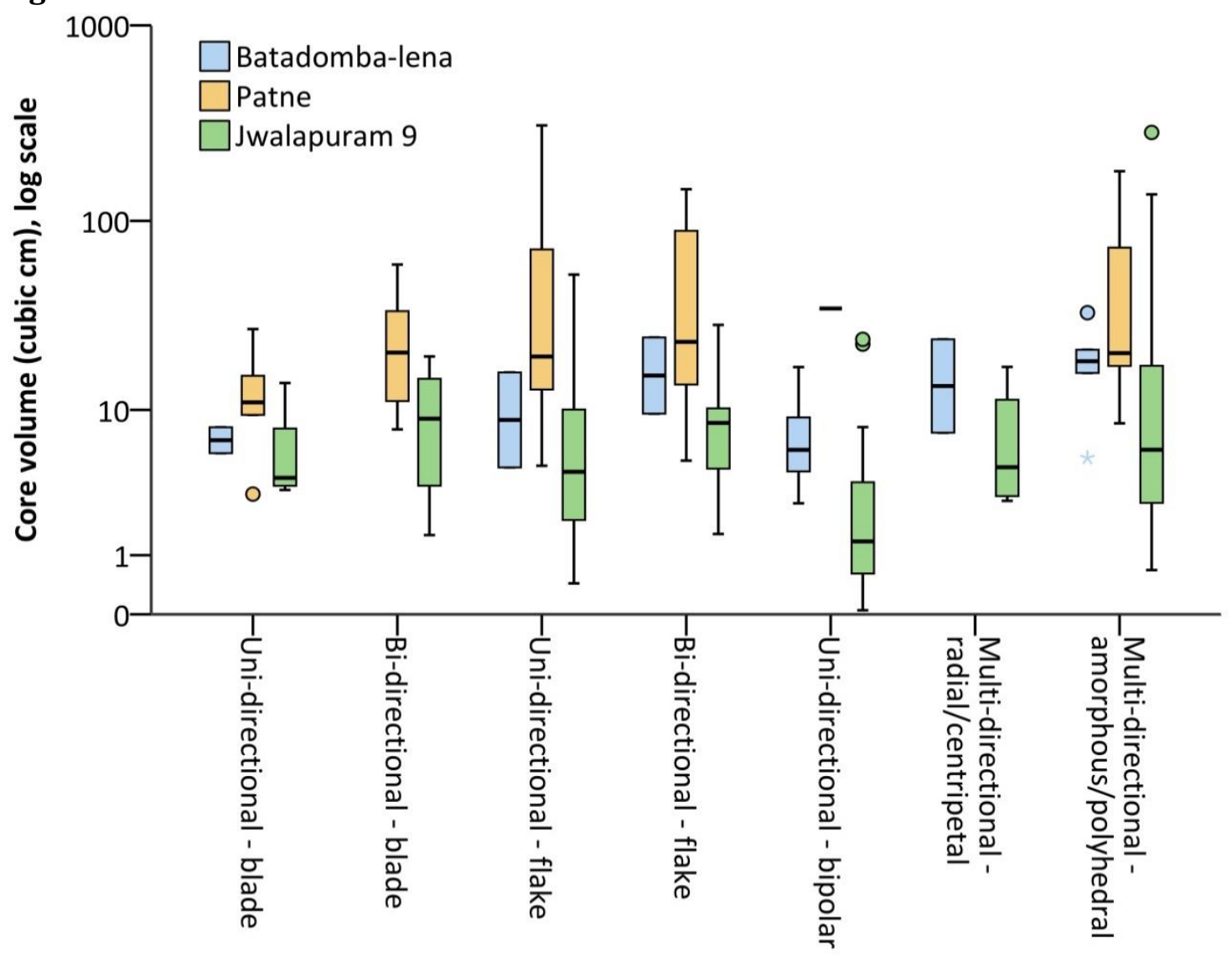

Bipolar percussion is evident at all sites on quartz (where material is recorded). Bipolar percussion was primarily used to remove flakes at all sites. However, at 
Batadomba-lena more blades were removed from bipolar cores than from other non-blade cores, whereas at Patne they have the lowest proportion of blade removals of any core type, and at Jwalapuram they also exhibit particularly low proportions of blade removals.

Bipolar percussion was used frequently and intensively at Batadomba-lena and Jwalapuram as a late-stage core reduction method for the removal of flakes (and some blades) from nearly-exhausted quartz cores. However, the one bipolar core at Patne is long, thin and not intensively worked. Possibly it was worked on an anvil due to the difficulty of making removals from such a long, thin core. In contrast to Batadomba-lena and Jwalapuram, where bipolar percussion was used to remove relatively large numbers of removals from relatively small cores, at Patne the opposite is true. The use of bipolar percussion at Batadomba-lena and Jwalapuram is indicative of the existence of a distinct manufacturing process for quartz. However, there is no convincing evidence at Patne for the existence of multiple reduction trajectories.

Largely undifferentiated production of flakes and blades occurred at Batadombalena. However, some differences between flake and blade cores are evident at the Indian sites. For example, at Jwalapuram cores which were not specifically used for the primary removal of blades exhibit very few blade removals, with almost half of cores exhibiting no blade removals. Also, flake cores are more intensively worked than blade cores in both absolute and relative terms. 
At Patne, blade cores are smaller and more intensively worked, and also exhibit less cortex. Perpendicular striking platforms are common on flake cores but not blade cores, possibly suggesting that these cores formed separate stages of core reduction, with cores being shaped by removing flakes from the end and from the side before they were used for the removal of blades. However, in contrast to the more concrete distinction at Jwalapuram, blade removals are common even on flake cores at Patne, suggesting that flake and blade core type designations are more likely different ends of a reduction continuum at the site.

Relatively little change over time is evident in the core assemblage at Patne. Although core size remains relatively stable, there is however an increase over time in the number (but not the average size) of removals. This increase is mostly due to an increase in the number of flake removals, and is best interpreted as increased pressure to make more efficient use of raw materials by working cores more intensively.

In contrast, virtually all core variables differ significantly between layers at Jwalapuram. Many changes can be attributed to variations in raw material proportions, and the fact that different core reduction techniques were used to work these different raw materials (e.g. bipolar percussion of quartz, and CCS frequently used for blade cores). There is a particularly strong focus on small intensively-worked quartz bipolar cores in the earliest part of the sequence.

At all sites flake cores are more common than blade cores, and bipolar percussion was utilised, primarily for the removal of flakes. Many additional similarities can 
be seen between Batadomba-lena and Jwalapuram. While some similarities are due to correlations in raw materials (i.e. quartz), others cannot be explained by raw material alone. Differences between Batadomba-lena and the Indian sites can also be seen, in the undifferentiated working of flake and blade cores at the former compared to the several areas of difference evidenced between core types at the latter. Overall, both differences and similarities are evident in core reduction strategies between the sites.

\section{BLANK PRODUCTION AND SELECTION}

Flakes are the most common debitage product at all sites. Blades occur in the largest proportion at Jwalapuram (bearing in mind the post hoc distinction imposed), yet even here they constitute only a third of the assemblage. Although flakes are dominant at Patne, blades are especially long and thin at the site.

No clear picture emerges at any site of whether blades were produced as part of any special process, or at a particular stage in reduction sequences. Although some variables differ at each site between flakes and blades (and flake-blades), overall there is no indication that flakes were removed primarily to prepare cores for the removal of blades or that blades were removed as part of a distinct reduction process, as seen for example at RCC (discussed above). Instead, largely undifferentiated production of lithic products of different degrees of elongation occurred. 
Some size differences in debitage production are evident between the sites, although they are not particularly pronounced (Figure 7.2.2). Debitage at Batadomba-lena and Jwalapuram is of a similar size (with slightly more variation at the latter). Debitage at Patne is larger on average, even when debitage type is held constant (Figure 7.2.3). As Clarkson et al. (2009) note, the frequency distribution of blades at Jwalapuram drops off at c. $40 \mathrm{~mm}$, and this also extends to debitage as a whole. A similar drop-off in size can be seen at Batadomba-lena, although this is slightly smaller at c. $30-35 \mathrm{~mm}$ (Figure 7.2.4). However, at Patne there is no such drop-off, with a less peaked distribution of debitage extending into much larger sizes.

Figure 7.2.2: Scatter graph of whole debitage lengths and widths at Late Palaeolithic sites

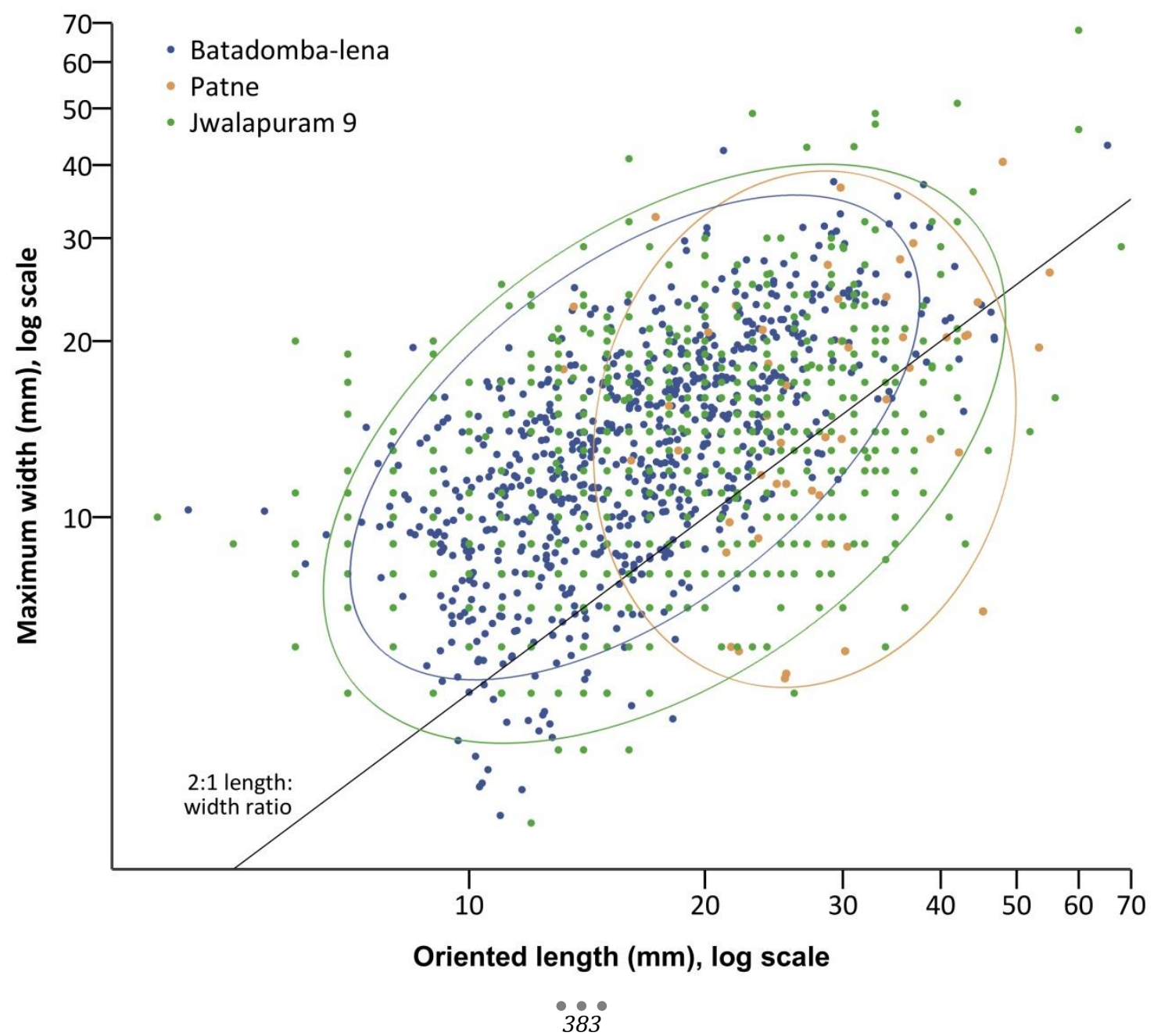


Figure 7.2.3: Cubic volumes of whole debitage at Late Palaeolithic sites

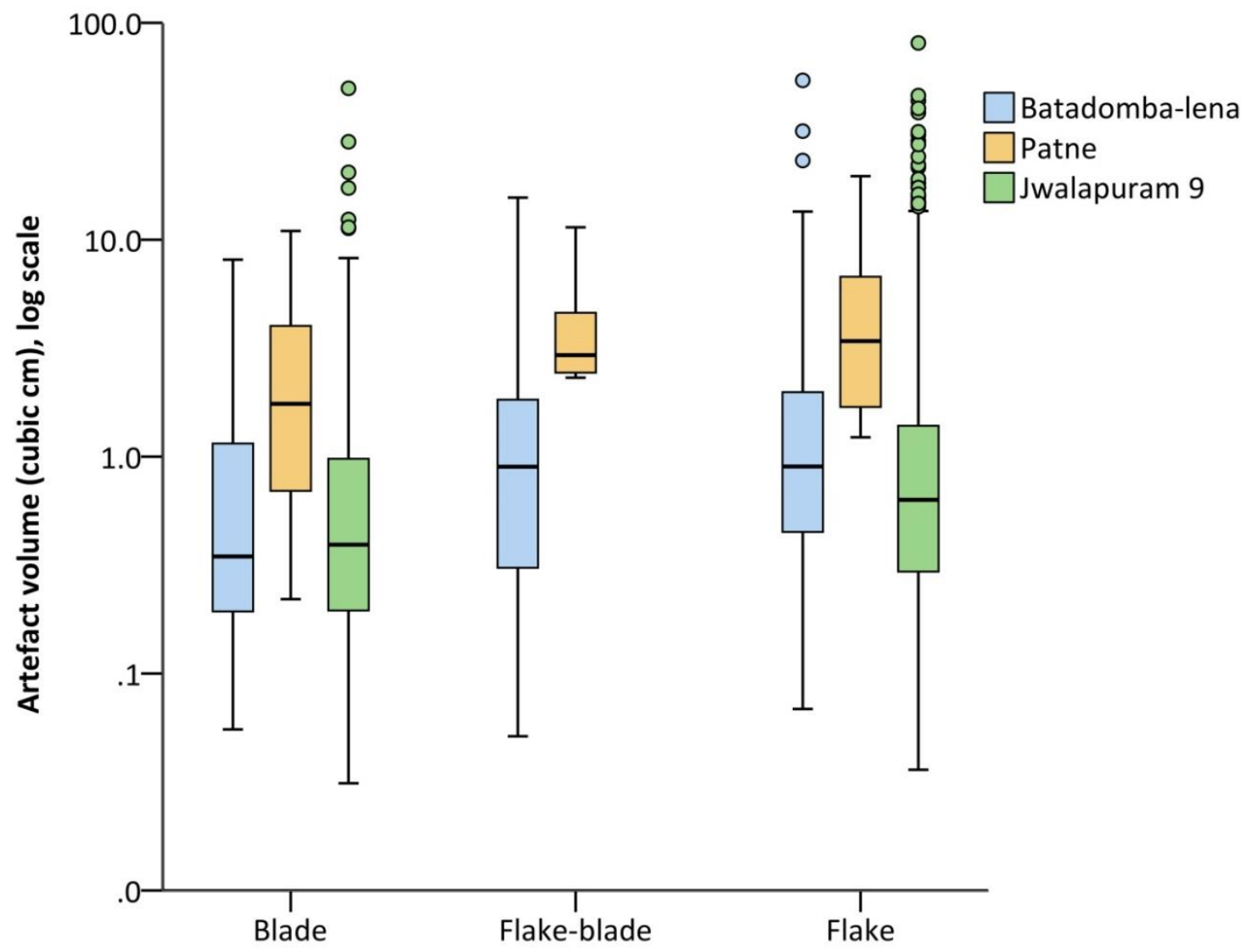

Another similarity between sites can be seen in the fact that although all sites have flake-dominated core and debitage assemblage, blade blanks were preferentially selected for microliths. The only exception to this is in the upper three layers of Batadomba-lena. At Batadomba-lena and Patne, blade blanks were also selected out of proportion for non-microlithic tools. Elongation was an important factor in the choice of blanks for tool production.

One important area of difference is in the relationship between debitage and tool size distributions. At Batadomba-lena and Jwalapuram microlith blanks were chosen from within the general size range of available debitage, while blanks for non-microlithic tools were selected from only the largest debitage. Lithic 
production was primarily geared towards the production of blanks of a suitable size for retouch into microliths. However, at Patne non-microlithic tool sizes more closely match the distribution of debitage size, while microlith blanks sere selected from only the smallest available debitage. Although the Late Palaeolithic at Patne can still be described as containing a microlithic industry, this discrepancy in size distributions highlights the fact that macrolithic tools can nevertheless form important components of microlithic assemblages.

Figure 7.2.4: Frequency distributions of lengths of whole debitage at Late Palaeolithic sites. Due to a small sample size, debitage from Patne where condition is unrecorded has been included.
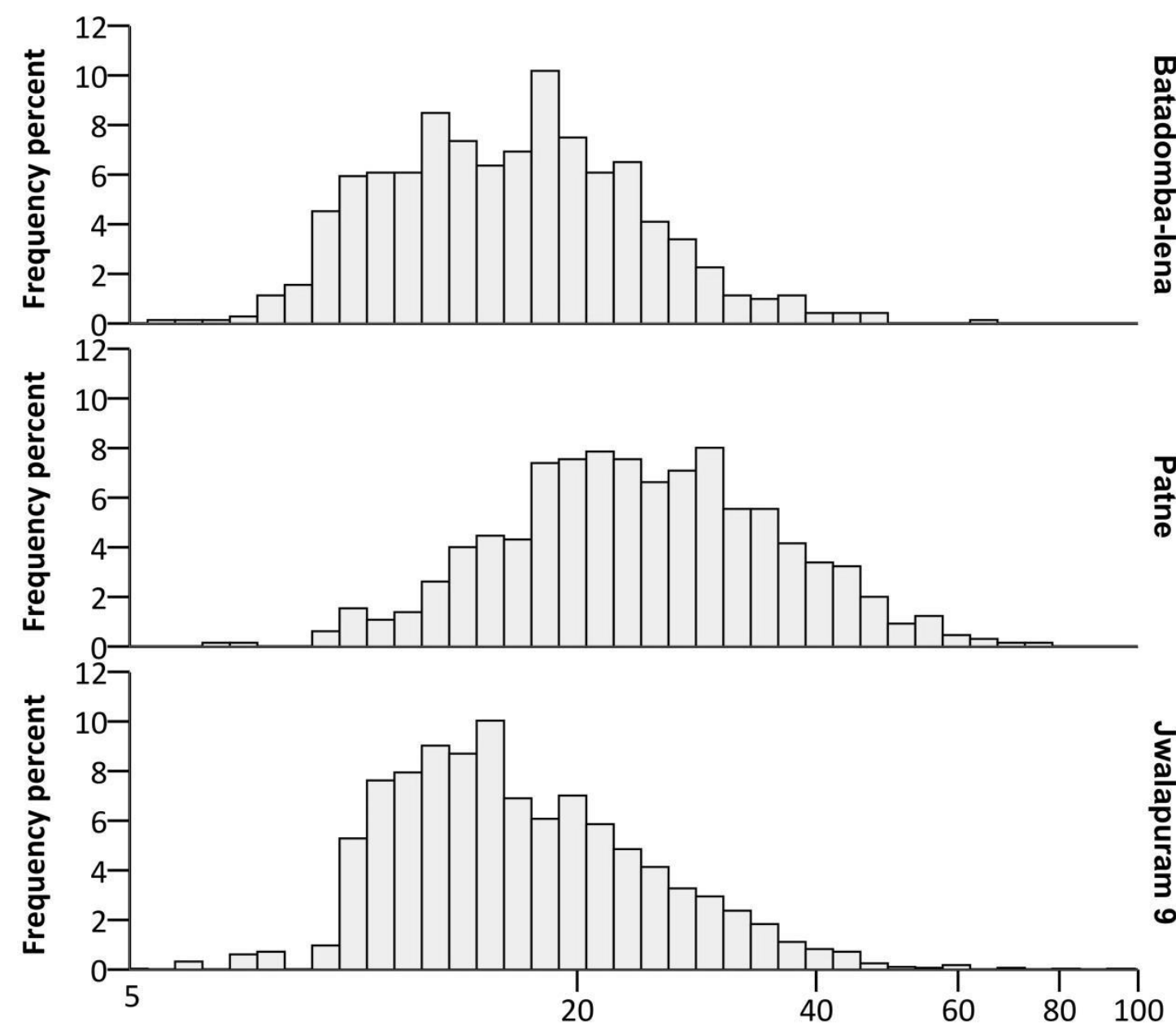

Oriented length $(\mathrm{mm})$, log scale 
Considerable changes occur throughout the debitage sequences at all sites, although much of it is in the form of small-scale variations between individual layers rather than over-riding patterns. One exception is in the general trend towards fewer blades and more flake-like debitage characteristics observed at both Batadomba-lena and Jwalapuram. At Patne, there is no conclusive evidence from either the core or the debitage assemblages to definitively support James' (2011) argument for an increased intensity of blade production over time.

However, in contrast to the other sites there is an increase over time in the use of blade blanks for microliths, despite no more blades being produced.

One similarity between the Indian sites is in the fact that there are few consistent changes in debitage variables over time, resulting in complex pictures of variation in size and debitage type. As argued above, given the variability within the sites, these sequences are perhaps best viewed as a series of distinct assemblages rather than representing any over-riding transition. Overall, differences are evident between the sites, as well as many similarities, particularly between Batadombalena and Jwalapuram. More chronological variation is evident than geographical variation, due to the long timespans of the sites analysed.

\section{RETOUCH CHOICES}

Microliths outnumber non-microlithic tools at each site, although they are much rarer at Jwalapuram (4.6\%) and Batadomba-lena (the equivalent of $15.3 \%$ when

the sample size is accounted for) compared to Patne where they constitute almost half of the tool assemblage (46.6\%). Another similarity between the sites is that 
non-microlithic burins and notched pieces are found at each. Burinations are particularly prevalent at Patne, which is also the only site to contain concave points ('awls'). Possibly perforating activities were common at the site. Patne is also the only site to contain non-microlithic points.

Geometric forms are not the dominant microlith type at any site, varying from $17.6 \%$ of the microlith assemblage at Jwalapuram to $45.2 \%$ at Kitulgala. Backed non-geometric points and crescents are the most common types at the Sri Lankan sites. At the Indian sites, backed non-geometrics make up the considerable majority of the assemblages, followed by crescents at Patne and the category of backed geometrics (which likely includes many crescents) at Jwalapuram.

Several differences in microlith forms exist between the sites. Microlithic burins are only found at the Indian sites, especially at Jwalapuram. Shouldered and tanged forms, bifacially-retouched microliths and microlith preforms are unique to Batadomba-lena; the site contains the greatest degree of variation in microlith forms, despite containing a microlith assemblage almost half the size of that at Jwalapuram. Numerous notched forms are found at both Batadomba-lena and Jwalapuram, but are absent from Kitulgala and Patne. At Patne, unlike the other sites, notched and denticulated artefacts are only found in the non-microlithic tool assemblage. Finally, only two microliths are neither backed nor geometric at Kitulgala, and six small double-backed pointed forms are found, with especially small TCSA measurements. These were most likely hafted as points as they lack an acute cutting edge, and may form a distinct and previously undocumented microlith type at the site. 
At Batadomba-lena and Patne, preferences are evident in the selection of blanks for retouch into particular microlith forms. At both sites blade blanks were preferred for backed non-geometrics, and flake blanks for non-backed nongeometrics. Additionally, at Batadomba-lena blade blanks were also preferred for non-backed non-geometric points, and flake blanks for geometric forms. At Jwalapuram no such preferences for particular microlith forms are evident, although blade blanks were preferred for microliths generally, and flake blanks for non-microlithic tools. Raw material also played a part in blank selection at Jwalapuram, with limestone and quartzite preferentially used for non-microlithic tools, and CCS for microliths. At Batadomba-lena the highest quality quartz available was used for tool blanks, especially for microliths. Although the debitage assemblage is not available for comparison, the same appears to be true at Kitulgala.

Differences in microlith sizes are also evident between the sites. Microliths at the Sri Lankan sites are consistently smaller than their Indian equivalents (Figure 7.2.5). Those at Kitulgala are especially small, while those at Patne are generally the largest and those at Jwalapuram tend to be more variable. However, at no site is there any convincing evidence for standardisation in the size of particular microlith forms, with more variation evident within types than between them.

At Kitulgala, blank-based variation is evident in microlith lengths, with bladebased microliths being longer on average than flake-based equivalents but only slightly wider and thicker. In contrast, at Batadomba-lena, Patne and Jwalapuram, more size variation between microliths made on different blanks is in width and 
thickness measurements than in length. In addition to demonstrating that a universal cut-off point for microlith lengths would be unsuitable at the sites analysed, the comparison of microlith sizes between sites also demonstrates that the reliance on length measurements alone is often insufficient.

Figure 7.2.5: Lengths of major microlith types at Late Palaeolithic sites

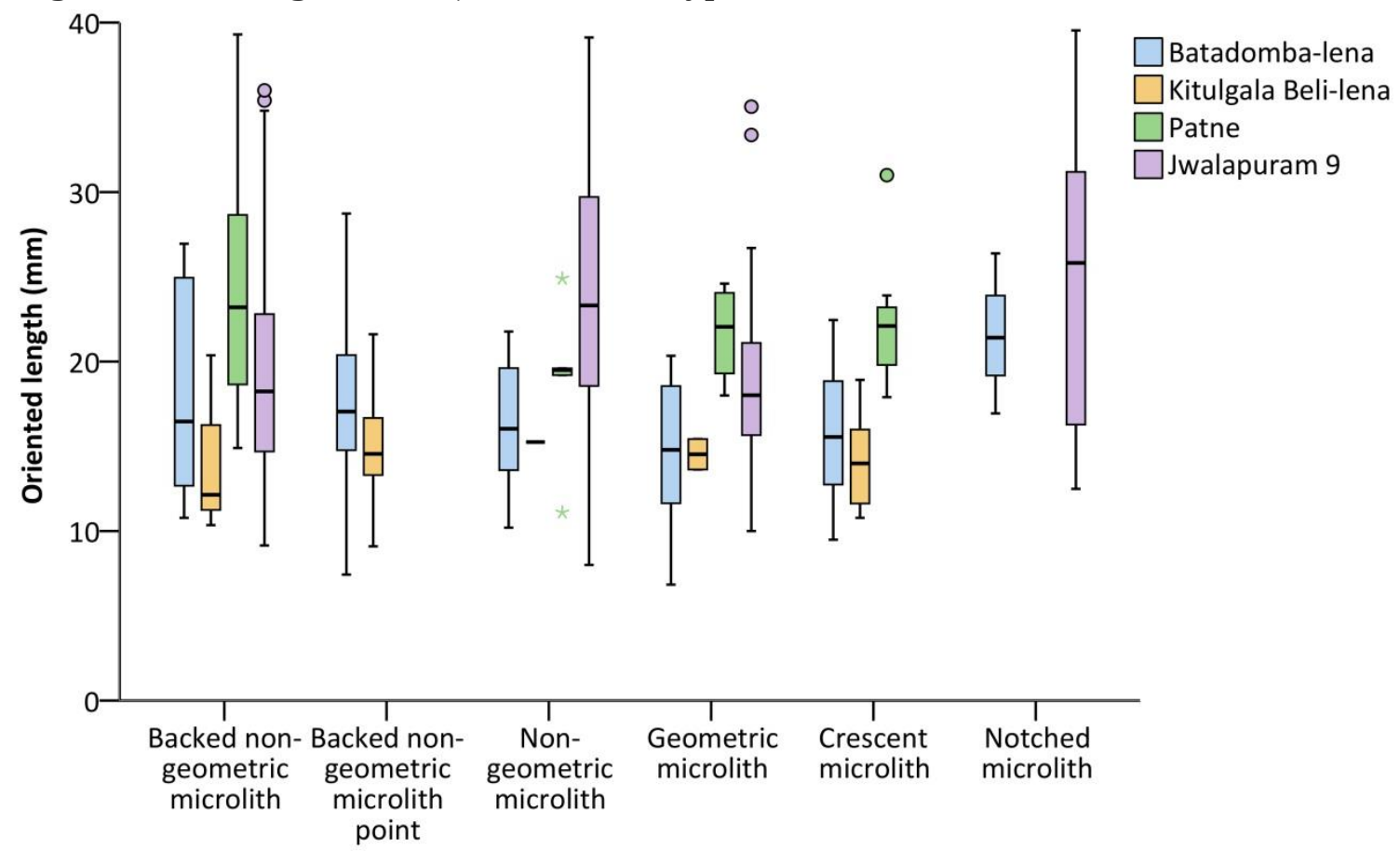

TCSA measurements are very small at all of the sites at which they could be calculated. Mean measurements for most types at each site are similar to or smaller than Shea's (2006) arrowhead sample. Those at Kitulgala are especially small. At Batadomba-lena the one exception is the Balangoda point, which has a TCSA measurement larger than Shea's dart tip sample. Possibly this point type had a distinct function or hafting configuration. At Patne, there is a notable separation in the distribution of TCSA measurements between (smaller) backed and (larger) non-backed microliths. This may similarly indicate distinct functions for the two 
groupings, or possibly distinct categories of projectiles. Non-microlithic points at Patne form a third grouping on the basis of TCSA measurements, in the range that Shea considers indicative of hand-cast or thrusting spears, although these points do not appear to be standardised.

Backing retouch is the most common retouch type on microliths at all sites. At Kitulgala, Jwalapuram and Patne all geometric microliths are backed (where this is recorded). At Batadomba-lena, only four of 39 microliths do not exhibit backing retouch. At the Sri Lankan sites, bipolar backing is the dominant backing technique used, and was used to a similar degree ( $\sim 60 \%$ of backing retouch). Additionally, at the Indian sites there is a distinction between the sizes of backed and non-backed microliths, with backed forms much smaller. Here, the presence of backing retouch may be a particularly meaningful categorisation of microliths, compared to whether or not a geometric shape was produced.

Several unique aspects of the use of backing retouch are evident at Patne. Bipolar backing only occurs in one layer of occupation, and only on non-geometric microliths. At Patne, bipolar backing was a distinct retouch technique used during one particular period in the site's history. It is also the only site where backing retouch was used in the non-microlithic tool assemblage, including one incidence of bipolar backing. Additionally, almost all retouch used to manufacture microliths on blade blanks is backing, whereas this constitutes a minority of retouch on flakebased microliths (where parallel and sub-parallel retouch are preferred instead). No major differences in retouch technique between microliths made on different blank types are evident at the other sites. 
Batadomba-lena exhibits the greatest diversity of retouch techniques. In the microlith assemblages of all sites, abrasion and discontinuous, nibbling and thinning retouch are only found at Batadomba-lena. Burinations are the only retouch type absent from the site, and are found only at the Indian sites. Even taking into account the much smaller assemblage size, retouch techniques at Kitulgala are the least varied; all geometric and backed non-geometric microliths exhibit only backing retouch, and all non-backed microliths exhibit only subparallel retouch. In the non-microlithic tool assemblages, adjacent notches are unique to Batadomba-lena, while backing and denticulation are unique to Patne although the assemblage at the latter is an order of magnitude larger than the former. At the Indian sites, retouch characteristics are more varied in the nonmicrolithic tool assemblages. Although slight differences in the way that retouch types were recorded at the Indian sites mean that this is not conclusive, more variability is evident overall in the retouch choices made at Batadomba-lena compared to the other sites.

At all sites where temporal variation could be considered, tool assemblages exhibit less variation over time than core and debitage assemblages. Many temporal changes are small-scale variations between individual layers rather than consistent trends. Larger-scale patterns and step changes are also evident, however. The most notable of these is that at both Batadomba-lena and Jwalapuram step changes occur in preferences for microlith blanks, with blade blanks preferred in the bottom halves of the sequences and more flake blanks present in the upper halves, concurrent with sudden decreases in the proportions 
of backed microliths. These changes occur between dates of 20-15 ka BP at Jwalapuram and 19.5-18.5 ka BP at Batadomba-lena, i.e. at the end of the LGM. Although there are no associated step changes in the core and debitage assemblages (the shift towards more flake-like debitage is gradual at both sites), these results may suggest the existence of two separate phases of microlith production, with classic backed geometric and non-geometric microliths manufactured on blade blanks being replaced by a wider variety of non-geometric forms with less of an emphasis on backing retouch. In contrast, however, the sequence at Patne demonstrates a gradual and consistent increase in the use of blade blanks over time. In the future, analysis of additional South Asian sites during this time period may be able to clarify these patterns.

\section{SUMMARY}

Similarities and differences between the Late Palaeolithic sites have been documented. All sites contain flake-dominated core and debitage assemblages, but preferential selection of blade blanks for microliths and, at Batadomba-lena and Patne, non-microlithic tools. There is no definitive evidence at any site that blades were produced at a particular stage in reduction sequences; instead, relatively undifferentiated production of debitage products of varying degrees of elongation occurred. Microliths outnumber non-microlithic tools at all sites, and nongeometric microliths outnumber geometric forms. Backing retouch is the most common retouch type on microliths. Almost all microlithic forms exhibit very small TCSA measurements. Non-microlithic burins and notched pieces are present at all sites, although there is no convincing evidence for standardisation of any tool 
forms. Generally speaking, most temporal change is observed in the core and debitage assemblages, with less variation evident in the tool assemblages. In particular, considerable change is evident throughout the debitage sequences at all sites, although the majority of variation is in the form of small-scale variations rather than consistent trends.

Differences are apparent between the Indian and Sri Lankan sites. Although there is largely undifferentiated production of flakes and blades at Batadomba-lena, there are some areas of difference between flake and blade cores at the Indian sites. Furthermore, bipolar cores have especially low proportions of flake removals at the Indian sites, whereas at Batadomba-lena, although they were still primarily used for the removal of blades, they were used to remove more blades than equivalent freehand cores.

Additional geographical differences are evident in the tool assemblages. At the Sri Lankan sites backed non-geometric points and crescents are the dominant microlith forms, but no single type makes up the majority of the assemblages. At the Indian sites, in contrast, backed non-geometrics make up the considerable majority of the microlith assemblages. Microlithic burins are found at the Indian sites but not in Sri Lanka. Bipolar backing is the dominant backing technique at the Sri Lankan sites but not in India. The Sri Lankan microliths are consistently smaller than their Indian equivalents. Finally, at Jwalapuram, there is a particularly strong distinction between the sizes of (smaller) backed and (larger) non-backed microliths. Similar distinctions are also apparent at Patne in the form of TCSA measurements, although they are not clearly evident at the Sri Lankan sites. 
Several differences can also be seen between the Indian sites, however. For example, there is little change over time in the core assemblage at Patne, with the main change being a general trend towards an increased density of removal scars on cores. In contrast, at Jwalapuram considerable temporal changes are evident in the core assemblage, most of which are small-scale variations between layers and not general trends.

Other differences between the sites also transcend geographical proximity, and may instead be related to differences in raw materials or other factors. Amorphous multi-directional and bipolar cores are dominant at Batadomba-lena and Jwalapuram - commonly found on quartz - while one- and two-platform flake cores are dominant at Patne. Bipolar cores are evident at all sites and were primarily used to remove flakes, but bipolar percussion was utilised differently at Patne compared to Batadomba-lena and Jwalapuram.

Many other similarities in the core and debitage assemblages can also be seen between Batadomba-lena and Jwalapuram, in contrast to Patne. For example, radial working of cores is present at the former two sites but not the latter, and cores were discarded at much larger sizes at Patne. Additionally, while distinct manufacturing processes for quartz are apparent at the former sites, there is no clear evidence for the existence of distinct reduction trajectories at Patne. Debitage sizes at Batadomba-lena and Jwalapuram are smaller on average and drop off at similar lengths, whereas debitage at Patne ranges into much larger sizes. While microlith blanks were chosen from the general size range of available debitage at Batadomba-lena and Jwalapuram, at Patne non-microlithic tool sizes more closely 
match the distribution of debitage size. Lastly, the sequences at Batadomba-lena and Jwalapuram demonstrate trends towards fewer blades and more flake-like debitage, whereas at Patne there is an increase in the use of blade blanks for microliths over time. This distinction is even stronger in the tool assemblage, where the former two sites exhibit step changes from more to fewer blade blanks half-way through their sequences, compared to the gradual inverse trend at Patne.

In the tool assemblage, an additional similarity between Batadomba-lena and Jwalapuram is that both sites contain numerous notched microliths, whereas notched microlith forms are absent from Patne and Kitulgala. However, preferences for retouching particular blanks into particular microlith forms are aspects of tool production that are shared between Batadomba-lena and Patne, and are not apparent at Jwalapuram or Kitulgala.

Other differences are unique to individual sites. For example, Batadomba-lena is the only site to contain shouldered, tanged or bifacially-retouched microliths, and microlith preforms. The site also displays the greatest degree of variation in microlith forms and retouch techniques. Kitulgala exhibits the least variability in retouch techniques, although it contains six very small double-backed pointed forms which are not documented elsewhere. Patne is the only site to contain nonmicrolithic points, and is also the only site to exhibit backing retouch in the nonmicrolithic tool assemblage and to contain notched and denticulated tools only in the non-microlithic tool assemblage. Unusually, bipolar backing only occurs in one layer of occupation at the site, and only on non-geometric microliths. It is also the 
only site to exhibit major differences in retouch techniques between microliths made on different blank types.

As in the HP sites discussed in the previous section, it is again demonstrated here that microblades are not a prerequisite for the production of microliths, that the majority of microliths are not geometric forms, and that a universal cut-off length for microliths is not applicable. Backing retouch has again been shown to be a useful, albeit insufficient, part of the designation of microlithic tools.

Overall, there is considerable evidence for variability within the South Asian Late Palaeolithic, as well as many areas of similarity between the sites studied. Much of the variation explored is chronological rather than geographical. In particular, chronological variation has been described on several different timescales, including step changes, consistent temporal trends, and inconsistent small-scale variations, revealing microlith production as a particularly flexible technology. Some of the variability discussed appears to be geographical, some may be related to raw material (particularly the similarities between Batadomba-lena and Jwalapuram), and some may be due to other factors. The following section explores similarities and differences between all of the sites studied, and explicitly considers the impacts of both geography and raw material. 


\subsection{COMPARISON BETWEEN EARLY MICROLITHIC TECHNOLOGY IN SOUTHERN AFRICA AND SOUTH ASIA}

To avoid redundancy with the previous sections, individual variables are not considered in this section. Instead, it sets out the results of multivariate analyses of the core, debitage and microlith datasets (relatively small frequencies of nonmicrolithic tools preclude their analysis using multivariate statistical techniques), using the methods and tests described in Chapter 4.3.

\section{CORES}

A PCA of cores from each site was conducted, in order to reduce the large number of variables measured on cores to a smaller number of composite variables, and to use these to compare cores between sites. The KMO test returns a value of .755, and Bartlett's Test of Sphericity is statistically significant $(\mathrm{p}<.001)$, indicating that PCA is appropriate. Two components were extracted with eigenvalues greater than 1 (i.e. accounting for more variance than a single variable), and components 1 and 2 together explain $67.9 \%$ of the variation (Table 7.3.1). An oblique rotation method (promax) was used as there is overlap in variance among factors. Component 1 is most strongly influenced by large length, width and thickness measurements, and distinguishes between large cores (high values) and small cores (low values) (Table 7.3.2). Component 2 is most strongly influenced by high numbers and proportions of blade removals alongside low numbers of flake removals, and distinguishes between blade cores (high values) and flake cores (low values). 


\begin{tabular}{lrrr}
\hline Component & Eigenvalue & \% of variance & Cumulative \% \\
\hline 1 & 4.29 & 42.9 & 42.9 \\
2 & 2.49 & 24.9 & 67.9 \\
3 & 0.99 & 9.9 & 77.7 \\
4 & 0.73 & 7.3 & 85.0 \\
5 & 0.49 & 4.9 & 89.9 \\
6 & 0.40 & 4.0 & 93.9 \\
7 & 0.27 & 2.7 & 96.6 \\
8 & 0.18 & 1.8 & 98.4 \\
9 & 0.12 & 1.2 & 99.6 \\
10 & 0.04 & 0.4 & 100.0 \\
\hline
\end{tabular}

Table 7.3.1: Eigenvalues and percentages of variance explained for the core PCA components

\begin{tabular}{lrr}
\hline Variable & Component 1 & Component 2 \\
\hline Maximum width & $\mathbf{. 8 9 5}$ & -.026 \\
Longest face & $\mathbf{. 8 6 5}$ & .310 \\
Longest axis & $\mathbf{. 8 3 4}$ & .365 \\
Maximum thickness & $\mathbf{. 8 0 4}$ & -.111 \\
Striking platform width & $\mathbf{. 6 5 4}$ & -.131 \\
Average removal scar length & $\mathbf{. 6 2 7}$ & .529 \\
Striking platform thickness & $\mathbf{. 6 0 4}$ & -.238 \\
Proportion of blade removals & -.233 & $\mathbf{. 8 9 6}$ \\
Number of blade scars & -.122 & $\mathbf{. 8 0 7}$ \\
Number of flake scars & .382 & -.667 \\
\hline
\end{tabular}

Table 7.3.2: Structure matrix of component scores for the core PCA. Loadings above .6 are in bold.

Figure 7.3.1 and Figure 7.3.2 plot the values of components 1 and 2, respectively, for cores at each site. They reveal that there is more variation between the South Asian sites than between those in southern Africa, particularly in terms of size (component 1), and that the HP cores are more blade-like on average (component 2). Overlaps are evident in scores between the regions, with no discrete regional groups. However, this is a result of the fact that the material constraints of 
knapping (for example, there is a size limit at which cores become too small to be worked) place limits on the amount of variability that is able to exist between different lithic traditions. Regardless, the results of a Kruskal Wallis test on the component scores from each site (as the data is non-parametric) demonstrate that the differences between sites are statistically significant (for component $1 \chi^{2}(5)=$ 135.115, $\mathrm{p}<.001$; for component $\left.2 \chi^{2}(5)=111.651, \mathrm{p}<.001\right)$.

Figure 7.3.1: Boxplot of component 1 of the core PCA. Higher values indicate larger core sizes.

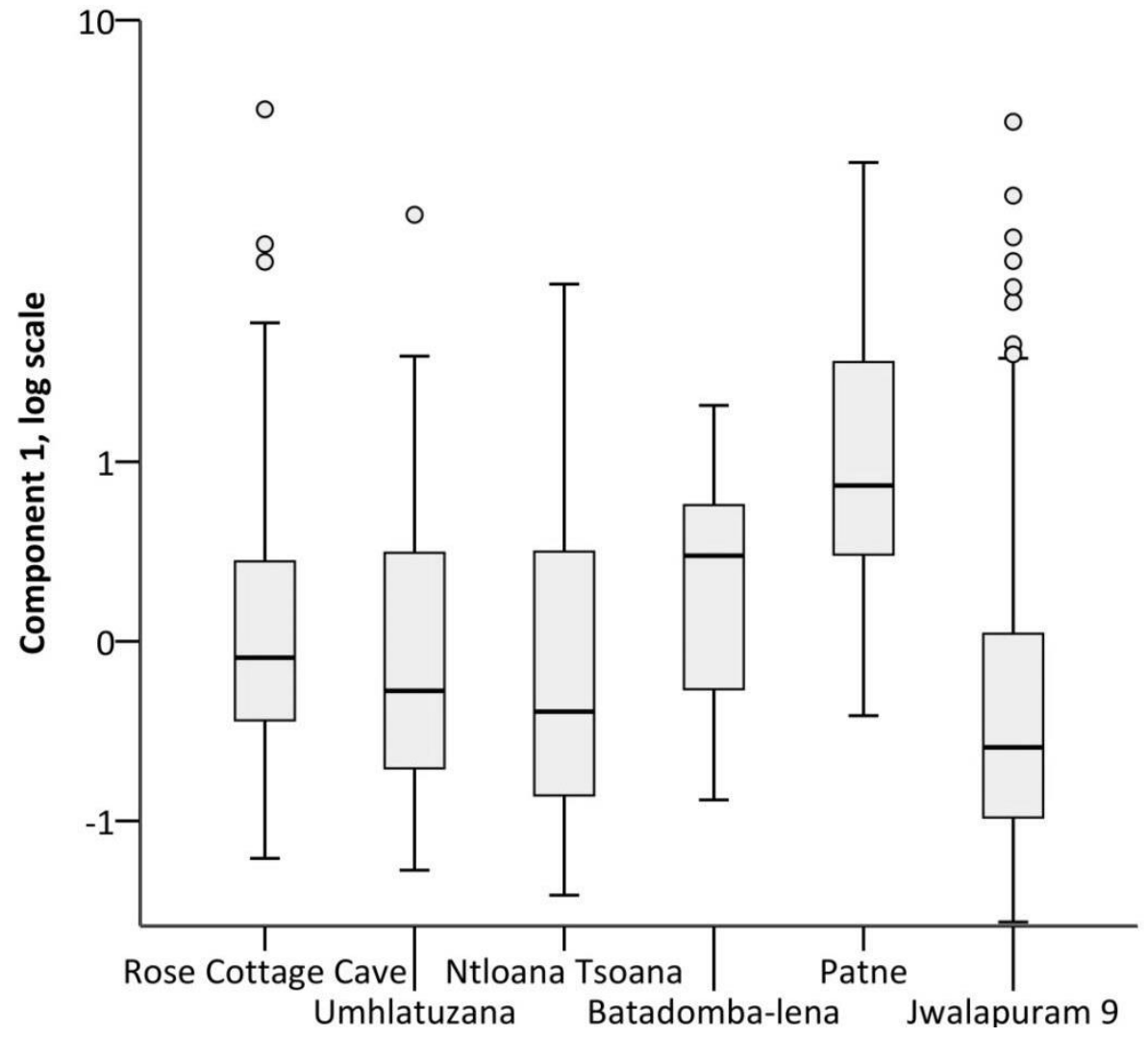

Figure 7.3.3 plots the group centroids of the major site and raw material groups for components 1 and 2. When both components are considered together, sites group within regions. The HP group centroids tend to cluster in the larger and more blade-like quadrants, while the South Asian sites generally have smaller and more flake-like cores (the exception is high quality quartz cores at Jwalapuram, although 
this is a small group $(n=5)$ ). Additionally, group centroids tend to cluster more closely within sites than within raw material groups, indicating that most variability is not due to differences in raw material.

Figure 7.3.2: Boxplot of component 2 of the core PCA. Higher values indicate cores with more blade removals.

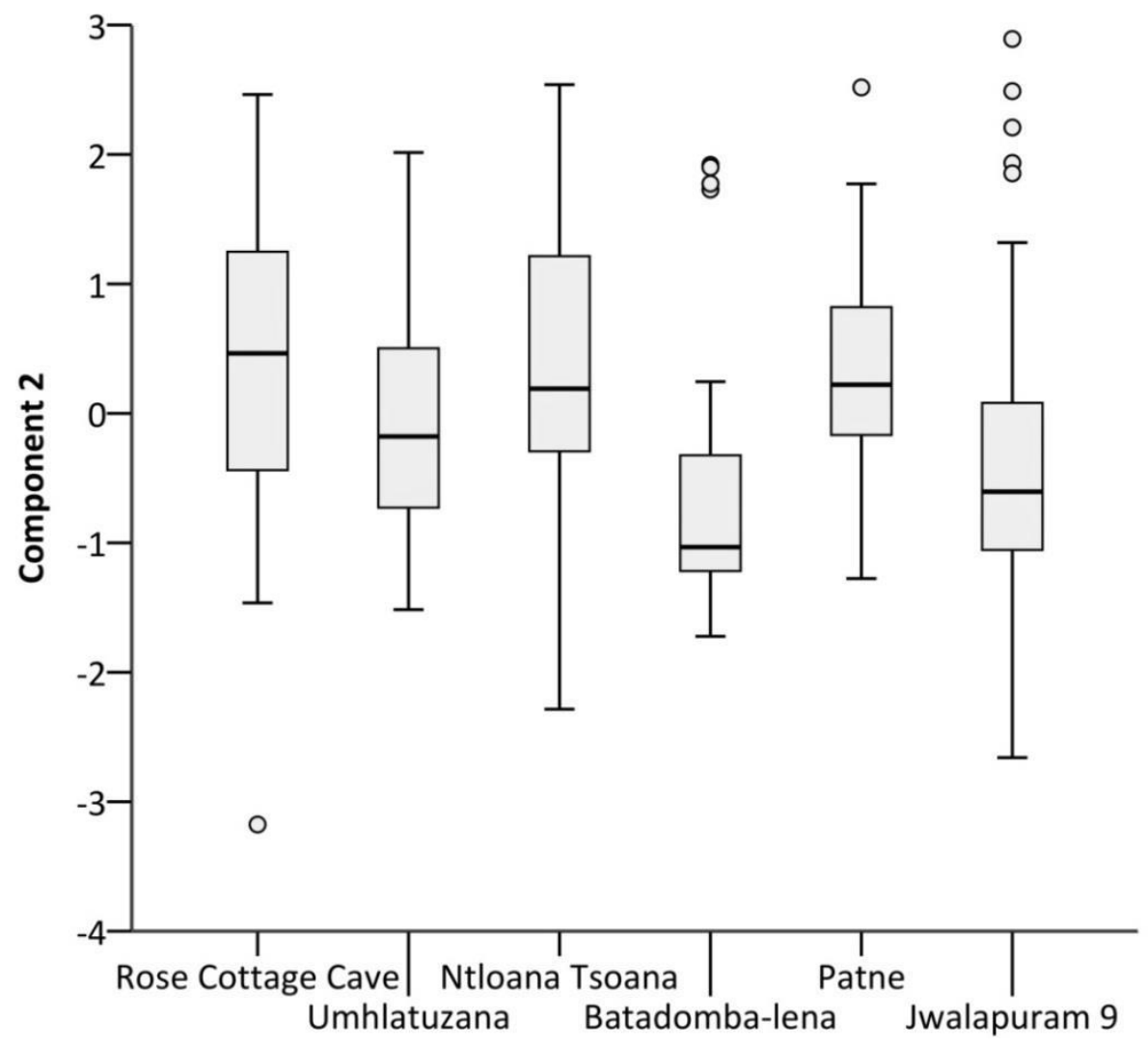

This conclusion is further supported by the results of the DFA of cores. Stepwise DFA produced a model which comprised 10 variables (Table 7.3.3). Five functions were extracted, of which the first four are statistically significant $(\mathrm{p}<.001$ in all cases). This indicates that, for the first four functions, cores vary more between groups than within them. Functions 1 and 2 explain $75.4 \%$ of the variability between sites (calculated by squaring their canonical correlations) (Table 7.3.4). Cores are correctly classified by the model into their sites in $69.3 \%$ of cases. 
Compared to the prior probability of correct site attribution of $23.7 \%$, this is a $192.4 \%$ increase in prediction power. When the function scores are calculated for each core, ANOVA tests reveal that statistically significant differences exist between sites in all four significant functions (Table 7.3.5).

Figure 7.3.3: Biplot of components 1 and 2 of the core PCA for group centroids of raw material and site groups. $\mathrm{BDL}=$ Batadomba-lena, $\mathrm{JWP}=$ Jwalapuram, $\mathrm{NT}=$ Ntloana Tsoana, RCC $=$ Rose Cottage Cave, ULZ = Umhlatuzana. Circles are CCS varieties, diamonds are quartz, and triangles are other raw materials.

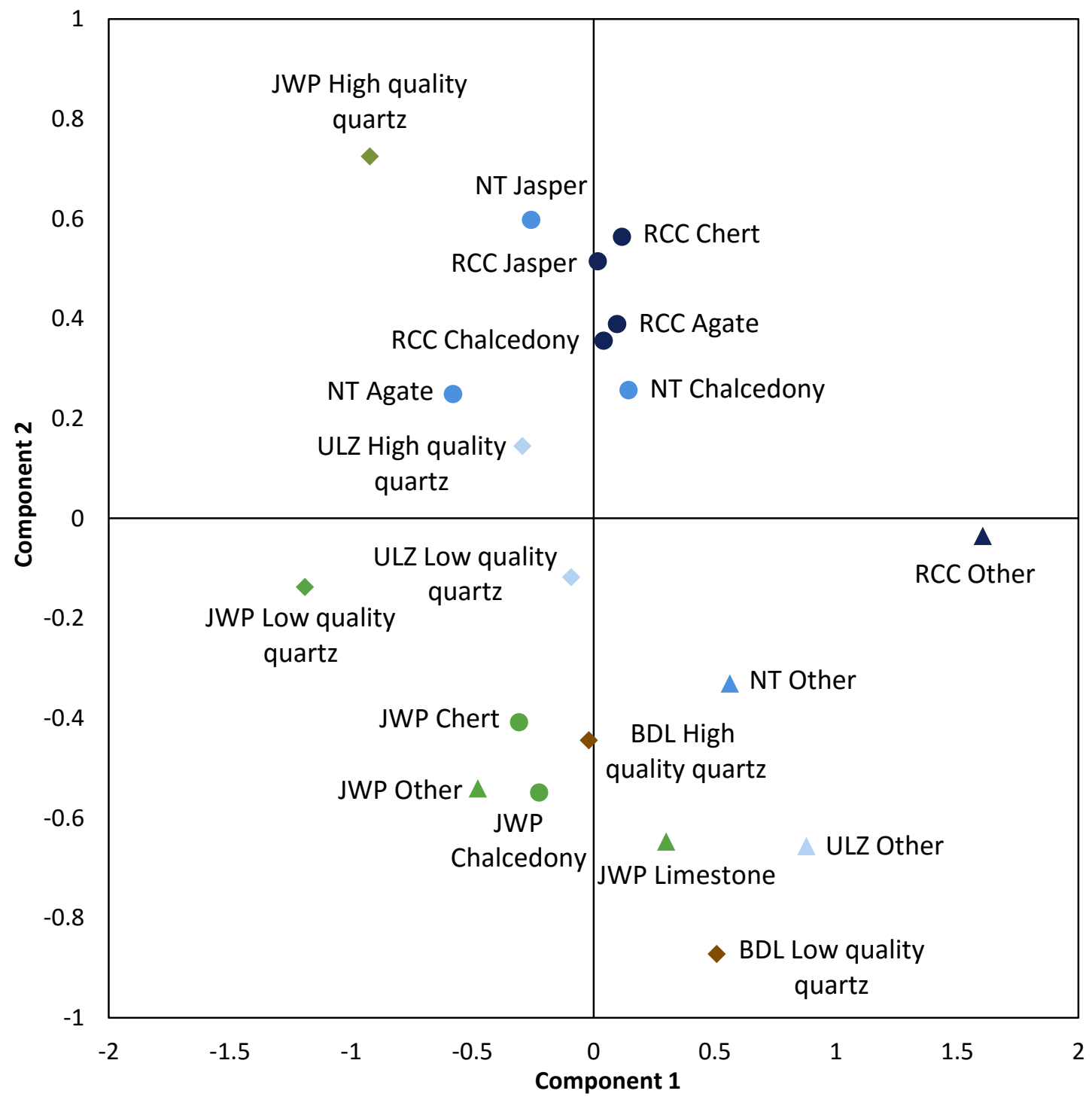




\begin{tabular}{lcrrrr}
\hline & \multicolumn{5}{c}{ Function } \\
Variable & $\mathbf{1}$ & $\mathbf{2}$ & $\mathbf{3}$ & $\mathbf{4}$ & $\mathbf{5}$ \\
\hline Longest axis a & .562 & -.061 & .459 & .159 & -.410 \\
Cortex & .411 & .470 & -.106 & -.383 & .005 \\
Number of aberrant terminations & .008 & .436 & .322 & -.230 & -.083 \\
Maximum thickness a & .153 & -.085 & .826 & -.438 & .041 \\
Number of scars & .021 & .339 & .415 & .135 & .320 \\
Average removal scar length a & .123 & -.175 & .375 & .290 & -.296 \\
Number of blade scars & .345 & .092 & .090 & .557 & .783 \\
Maximum width a & .356 & -.072 & .479 & -.482 & .350 \\
Proportion of blade removals & .310 & -.090 & -.226 & .413 & .291 \\
Bipolar b & -.288 & -.218 & -.086 & .301 & .776 \\
\hline
\end{tabular}

Table 7.3.3: Variables used in the core DFA, and their loadings on each function. ${ }^{a}$ log-transformed to maintain normality. $b$ coded as an interval variable, where $1=$ present and $0=$ absent.

\begin{tabular}{lrrrr}
\hline Function & Eigenvalue & \% of variance & Cumulative \% & Canonical correlation \\
\hline 1 & .779 & 49.2 & 49.2 & .662 \\
2 & .462 & 29.1 & 78.3 & .562 \\
3 & .270 & 17.1 & 95.4 & .461 \\
4 & .051 & 3.2 & 98.6 & .220 \\
5 & .023 & 1.4 & 100.0 & .149 \\
\hline
\end{tabular}

Table 7.3.4: Summary of key statistics for the core DFA

\begin{tabular}{ll}
\hline Function & Test result \\
\hline 1 & $\mathrm{~F}(5,280)=5.820, \mathbf{p}<. \mathbf{0 0 1}$ \\
2 & $\mathrm{~F}(5,252)=8.129, \mathbf{p}<. \mathbf{0 0 1}$ \\
3 & $\mathrm{~F}(4,280)=9.070, \mathbf{p}<. \mathbf{0 0 1}$ \\
4 & $\mathrm{~F}(5,268)=3.854, \mathbf{p}=\mathbf{. 0 0 2}$ \\
\hline
\end{tabular}

Table 7.3.5: Results of ANOVA tests of the core DFA functions. Data was logtransformed to maintain normality.

Notably, when raw material is used as the grouping variable, cores only correctly classify into their groups in $39.9 \%$ of cases, compared to an equivalent for grouping into sites (i.e. excluding Patne, which lacks raw material classifications) 
of $72.2 \%$. The variables utilised classify cores more effectively into their sites than into their raw materials. Clarkson (2010: 53) reached the same conclusion in his assessment of HP cores, using this result to assert that "raw material differences... appear to be subservient to other causes of variation in creating differences between regions".

The results of the PCA and DFA indicate that there are significant differences between the sites. PCA reveals that the most important groups of variables for distinguishing between sites are size characteristics (length, width and thickness), and the numbers and proportions of blade scars. The HP cores are more similar to each other, and are larger and more blade-like on average. The South Asian cores are more variable, and are frequently smaller and more flake-like. Additionally, both analytical techniques indicate that although raw material does constrain knapping options and has an effect on core technology, the majority of the variability documented is not due to raw material differences and is instead related to other local cultural or technological factors.

\section{DEBITAGE}

A PCA of debitage from each site was conducted. The KMO test returns a value of .740 , and Bartlett's Test of Sphericity is statistically significant $(\mathrm{p}<.001)$, indicating that PCA is appropriate. Two components were extracted with eigenvalues greater than 1, and components 1 and 2 together explain $80.3 \%$ of the variation (Table 7.3.6). Again, promax rotation was used. Component 1 is most strongly influenced by width and thickness measurements, including of striking 
platforms, and distinguishes between large and more flake-like debitage (high

values) and smaller and less chunky debitage (low values) (Table 7.3.7).

Component 2 is most strongly influenced by length and elongation, and

distinguishes between long and blade-like debitage (high values) and short and

flake-like debitage (low values). The components therefore reflect similar aspects

of lithic technology to those described above for cores.

\begin{tabular}{lrrr}
\hline Component & Eigenvalue & \% of variance & Cumulative \% \\
\hline 1 & 4.17 & 59.6 & 59.6 \\
2 & 1.45 & 20.7 & 80.3 \\
3 & 0.61 & 8.8 & 89.1 \\
4 & 0.43 & 6.2 & 95.2 \\
5 & 0.18 & 2.6 & 97.9 \\
6 & 0.12 & 1.8 & 99.6 \\
7 & 0.03 & 0.4 & 100.0
\end{tabular}

Table 7.3.6: Eigenvalues and percentages of variance explained for the debitage PCA

\begin{tabular}{lrr}
\hline Variable & Component 1 & Component 2 \\
\hline Maximum width & $\mathbf{. 9 3 2}$ & -.069 \\
Medial width & $\mathbf{. 9 3 2}$ & -.109 \\
Striking platform width & $\mathbf{. 8 5 3}$ & -.143 \\
Striking platform thickness & $\mathbf{. 8 0 1}$ & .017 \\
Medial thickness & .777 & .251 \\
Length:width ratio & -.390 & .877 \\
Oriented length & .556 &. $\mathbf{7 5 9}$ \\
\hline
\end{tabular}

Table 7.3.7: Structure matrix of component scores for the debitage PCA. Loadings above .6 are in bold.

Figure 7.3.4 and Figure 7.3.5 plot the values of components 1 and 2, respectively, for debitage at each site. There are no discrete regional groupings in the distribution of either component, although the HP sites have longer and more 
elongated debitage on average. Nevertheless, the differences between sites are statistically significant (Kruskal Wallis test for component $1 \chi^{2}(5)=413.383, p<$ .001 ; for component $\left.2 \chi^{2}(5)=1016.078, \mathrm{p}<.001\right)$.

Figure 7.3.4: Boxplot of component 1 of the debitage PCA. Higher values indicate larger and more flake-like debitage.

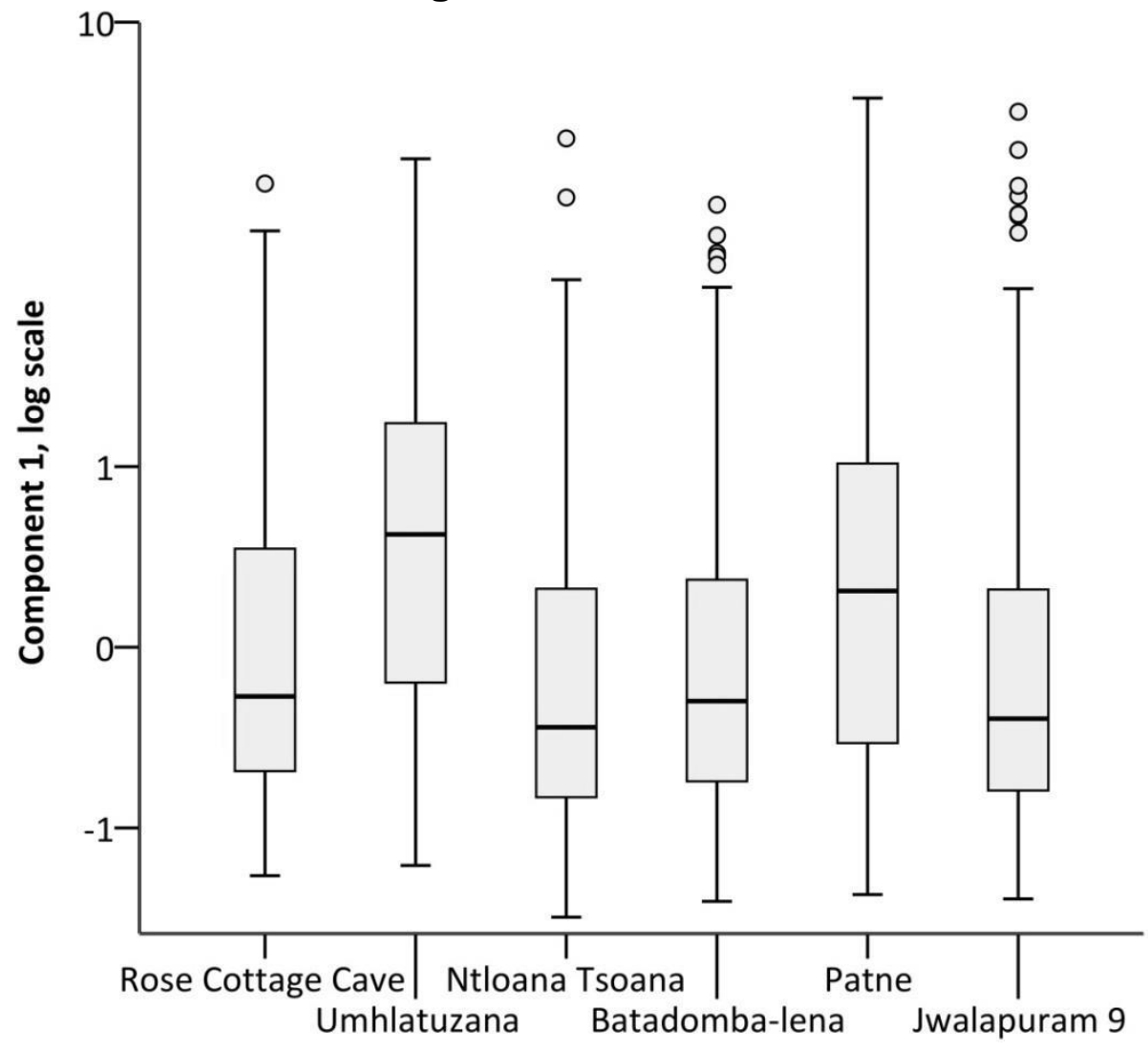

Figure 7.3.6 plots the group centroids of the major site and raw material groups for components 1 and 2. Regional groupings are less clear than in the core PCA. While the group centroids for Umhlatuzana and RCC tend to cluster together in the larger (especially Umhlatuzana) and more blade-like (especially RCC) quadrants, there are overlaps in scores between the other sites. The separation of the group centroids for CCS varieties at RCC and NT is notable, given the fact that these raw materials may have been obtained from the same sources. The same raw materials 
were worked at two nearby and contemporaneous sites to produce different debitage forms, with the main differences being in length and elongation.

Figure 7.3.5: Boxplot of component 2 of the debitage PCA. Higher values indicate longer and more blade-like debitage.

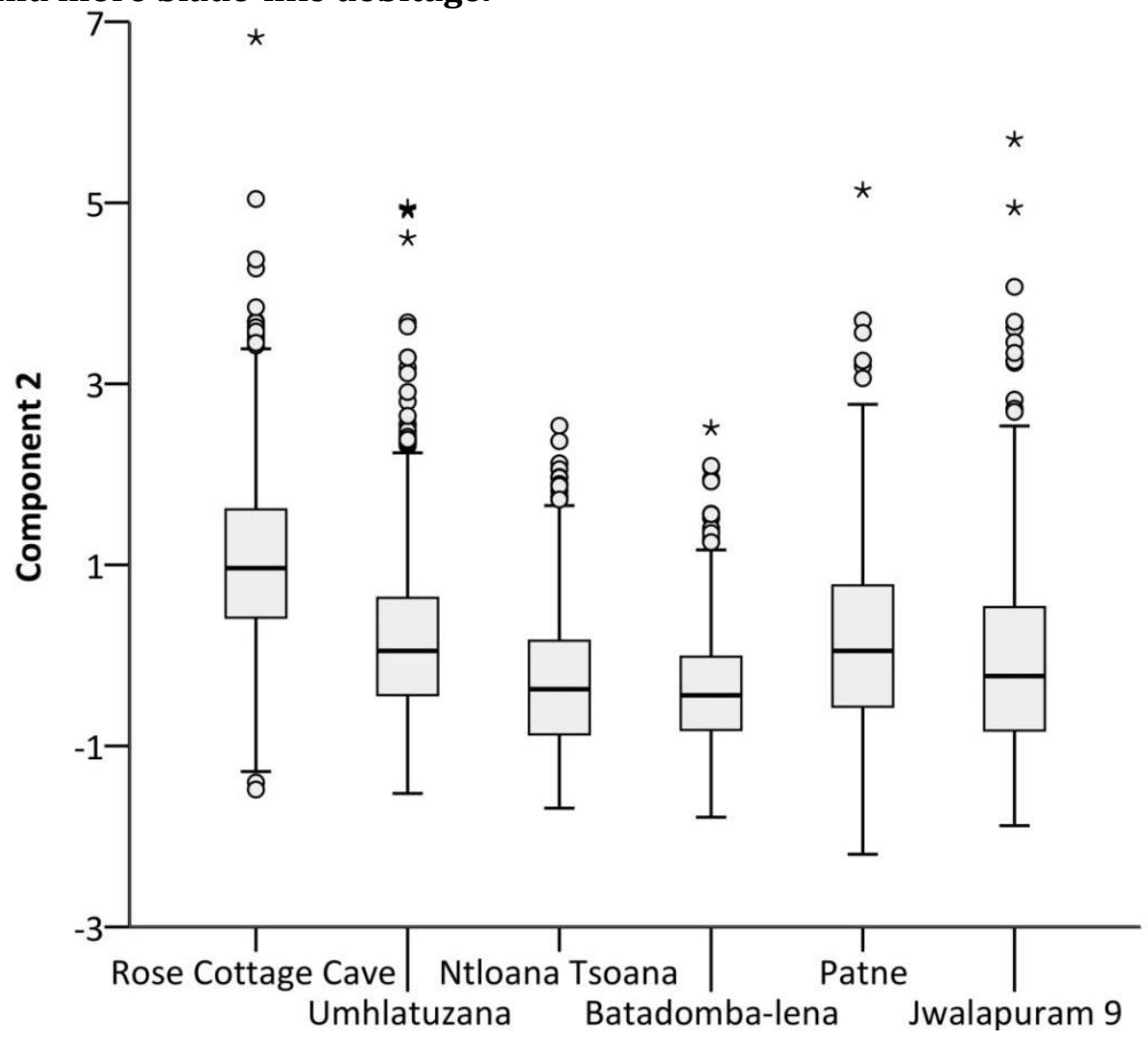

Stepwise DFA was also conducted, producing a model which comprised 12 variables (Table 7.3.8). Five functions were extracted, all of which are statistically significant ( $p<.001$ in all cases). Functions 1 and 2 explain $56.6 \%$ of the variability between sites (Table 7.3.9). Debitage is correctly classified by the model into their sites in $48.9 \%$ of cases. Compared to the prior probability of correct site attribution of $19.5 \%$, this is a $150.8 \%$ increase in prediction power. When the function scores are calculated for each core, Kruskal Wallis tests (as the data is non-parametric) reveal that statistically significant differences exist between sites in all functions (Table 7.3.10). 
Figure 7.3.6: Biplot of components 1 and 2 of the debitage PCA for group centroids of main raw material and site groups. Circles are CCS varieties, diamonds are quartz, and triangles are other raw materials.

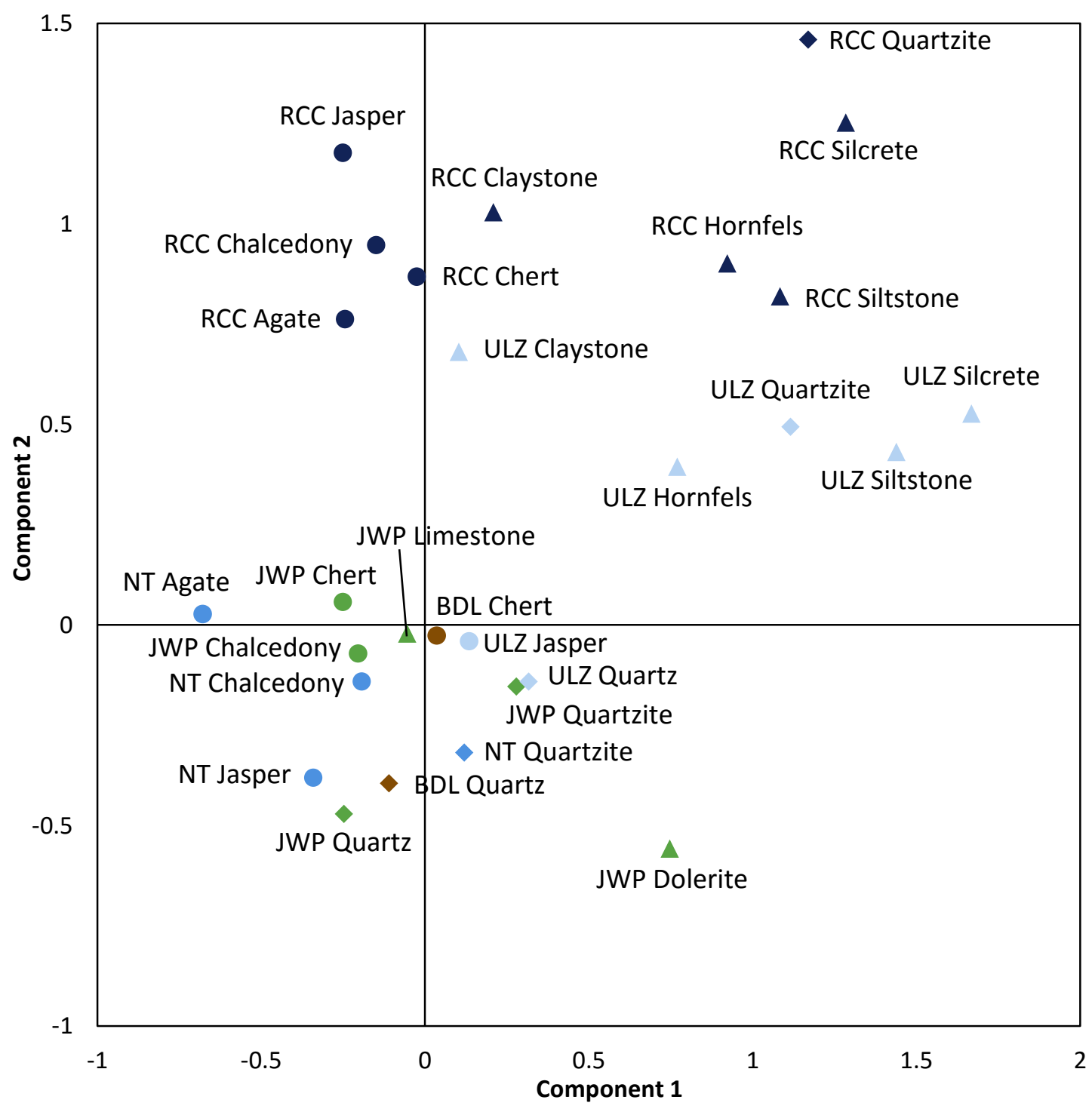

As in the core dataset, debitage is more effectively classified by site than by raw material. When raw material is used as the grouping variable, cores only correctly classify into their groups in $31.2 \%$ of cases, compared to an equivalent for site groupings of $56.3 \%$. The variables utilised classify cores more effectively into their sites than into their raw materials. 


\begin{tabular}{lrrrrr}
\hline & \multicolumn{5}{c}{ Function } \\
Variable & $\mathbf{1}$ & $\mathbf{2}$ & $\mathbf{3}$ & $\mathbf{4}$ & $\mathbf{5}$ \\
\hline Oriented length ${ }^{*}$ & .842 & -.079 & .407 & .090 & -.052 \\
Length:width ratio & .554 & .295 & -.365 & -.488 & -.054 \\
Length:thickness ratio $^{*}$ & .494 & .386 & -.210 & -.300 & .106 \\
Striking platform width $^{*}$ & .008 & -.158 & .772 & .218 & -.371 \\
Medial width $^{*}$ & .175 & -.151 & .707 & .592 & .110 \\
Maximum width $^{*}$ & .189 & -.148 & .668 & .595 & .025 \\
Striking platform thickness $^{*}$ & -.045 & -.199 & .648 & .310 & -.085 \\
Dorsal scars per cm $^{2}{ }^{*}$ & -.406 & .247 & -.634 & -.091 & -.194 \\
Medial thickness * & .138 & -.191 & .570 & .259 & -.203 \\
Cortex & .119 & .141 & -.158 & .037 & -.065 \\
Number of dorsal scars & .257 & .075 & -.104 & .622 & -.456 \\
Scar orientation & .060 & -.130 & -.151 & .382 & -.178 \\
Oriented length * & .842 & -.079 & .407 & .090 & -.052 \\
\hline
\end{tabular}

Table 7.3.8: Variables used in the DFA of debitage, and their loadings on each function. * log-transformed to maintain normality.

\begin{tabular}{lrrrr}
\hline Function & Eigenvalue & \% of variance & Cumulative \% & Canonical correlation \\
\hline 1 & .564 & 54.6 & 54.6 & .601 \\
2 & .257 & 24.8 & 79.4 & .452 \\
3 & .129 & 12.5 & 91.9 & .338 \\
4 & .065 & 6.3 & 98.2 & .247 \\
5 & .019 & 1.8 & 100.0 & .137 \\
\hline
\end{tabular}

Table 7.3.9: Summary of key statistics for the debitage DFA

\begin{tabular}{ll}
\hline Function & Test result \\
\hline 1 & $\chi^{2}(5)=1474.113, \mathbf{p}<.001$ \\
2 & $\chi^{2}(5)=773.194, \mathbf{p}<.001$ \\
3 & $\chi^{2}(5)=465.163, \mathbf{p}<.001$ \\
4 & $\chi^{2}(5)=192.409, \mathbf{p}<.001$ \\
5 & $\chi^{2}(5)=76.089, \mathbf{p}<.001$ \\
\hline
\end{tabular}

Table 7.3.10: Results of Kruskal Wallis tests of the debitage DFA functions 
The results of the PCA and DFA indicate that there are significant differences between the sites. PCA reveals that the most important groups of variables for distinguishing between sites are width and thickness measurements (i.e. overall size and chunkiness), and length and elongation. Overlaps are evident in PCA component scores between regions, and regional groupings are not entirely clear, although the HP sites have larger and more blade-like debitage on average (especially RCC and Umhlatuzana). Site and region groupings are less discrete in the debitage PCA compared to cores, which is also reflected in the results of the DFA where the model has less predictive power than that for cores. Nevertheless, debitage groups more easily into site than into raw material categories, as in the core assemblage, again indicating that raw material is a relatively minor source of variability between sites.

\section{MICROLITHS}

A PCA of microliths from each site was conducted. The KMO test returns a value of .671 , and Bartlett's Test of Sphericity is statistically significant $(\mathrm{p}<.001)$, indicating that PCA is appropriate. Oriented length was not used in the analysis surprisingly, this reduced the KMO test result to an unacceptably low value of .585. This result indicates that length is less useful than other variables as an indicator for discriminating between microliths from different sites.

Two components were extracted with eigenvalues greater than 1 , and components 1 and 2 together explain $69.5 \%$ of the variation (Table 7.3.11). Promax rotation was used. Component 1 is most strongly influenced by width and thickness 
measurements, length:width ratios and retouch depth, and distinguishes between large and squat/flake-like microliths (high values) and smaller more elongated/blade-like microliths (low values) (Table 7.3.12). Component 2 is most strongly influenced by retouch length and IOI measurements, and distinguishes between microliths with extensive (high values) and minimal (low values) retouch.

\begin{tabular}{lrrr}
\hline Component & Eigenvalue & \% of variance & Cumulative \% \\
\hline 1 & 4.74 & 52.7 & 52.7 \\
2 & 1.51 & 16.8 & 69.5 \\
3 & .83 & 9.3 & 78.8 \\
4 & .69 & 7.7 & 86.5 \\
5 & .57 & 6.3 & 92.8 \\
6 & .32 & 3.6 & 96.3 \\
7 & .21 & 2.4 & 98.7 \\
8 & .11 & 1.2 & 99.9 \\
9 & .01 & .1 & 100.0 \\
\hline
\end{tabular}

Table 7.3.11: Eigenvalues and percentages of variance explained for the microlith PCA

\begin{tabular}{lrr}
\hline Variable & Component 1 & Component 2 \\
\hline Medial width & $\mathbf{. 9 4 1}$ & -.011 \\
Maximum width & $\mathbf{. 8 7 7}$ & -.007 \\
Striking platform width & $\mathbf{. 8 5 6}$ & .185 \\
Medial thickness & $\mathbf{. 8 4 2}$ & .212 \\
Striking platform thickness & $\mathbf{. 8 3 4}$ & .179 \\
Length:width ratio & $\mathbf{. 6 4 9}$ & .406 \\
Average retouch depth & .638 & .102 \\
Index of Invasiveness & -.036 & $\mathbf{. 8 3 1}$ \\
Average retouch length & .334 & $\mathbf{. 7 6 5}$ \\
\hline
\end{tabular}

Table 7.3.12: Structure matrix of component scores for the microlith PCA. Loadings above .6 are in bold. 
Figure 7.3.7 and Figure 7.3.8 plot the values of components 1 and 2, respectively, for microliths at each site. A small degree of regional grouping is apparent in component 1, with HP microliths shown to be larger (particularly wider and thicker) on average. No such regional groupings are evident in component 2, with considerable overlap between regions. Nevertheless, the differences between sites are statistically significant (Kruskal Wallis test for component $1 \chi^{2}(6)=114.039, p$ $<.001$; for component $\left.2 \chi^{2}(6)=45.749, \mathrm{p}<.001\right)$.

Figure 7.3.7: Boxplot of component 1 of the microlith PCA. Higher values indicate larger and more flake-like microliths.

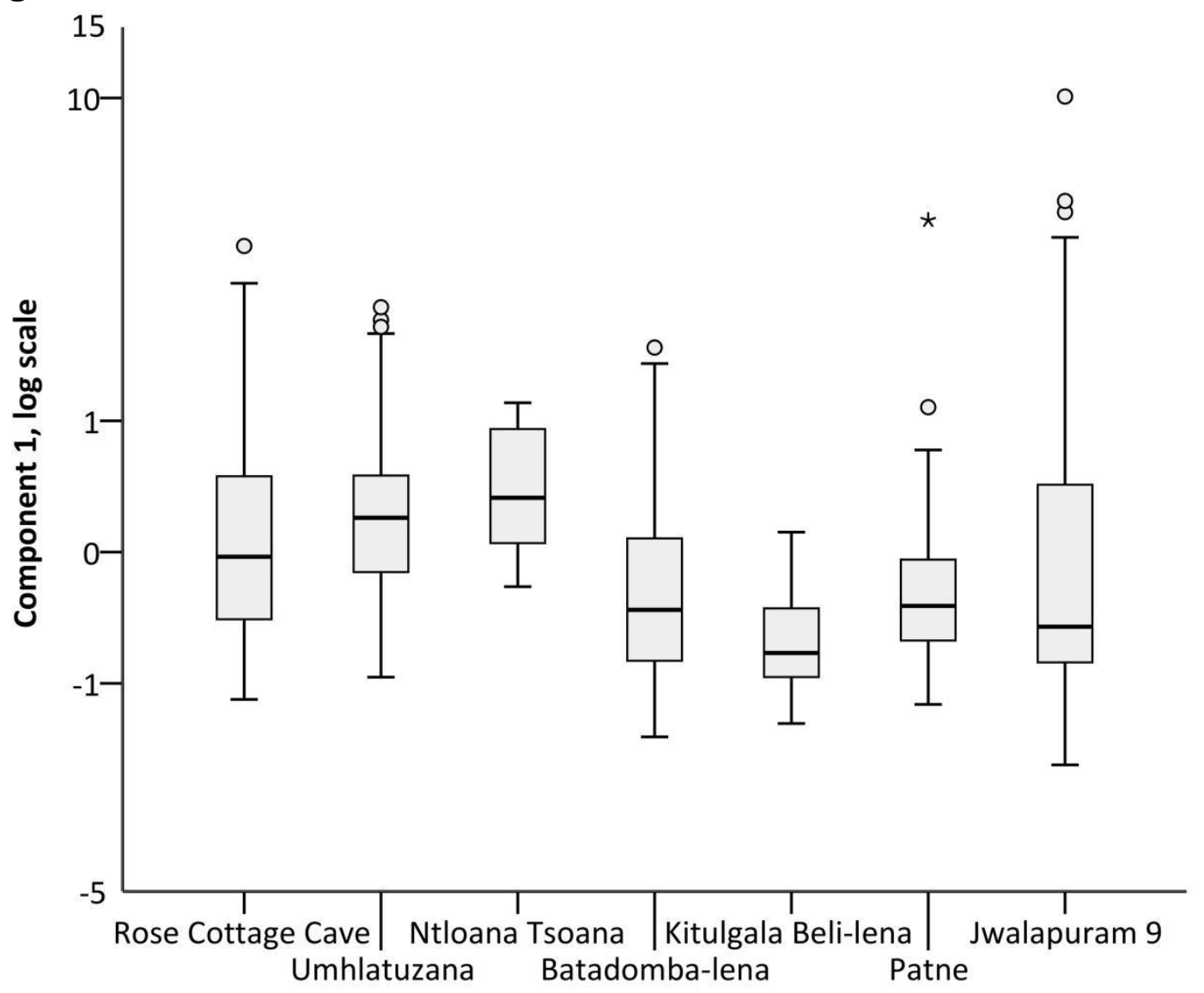

Figure 7.3.9 plots the group centroids of the major site and raw material groups for components 1 and 2. Regional and site groupings are less clear than in both the core and debitage PCAs, and as noted above the main difference is along 
component 1 , which predominantly relates to blank size measurements (especially width and thickness) rather than retouch characteristics. In contrast, a similarity with the core and debitage PCAs can be seen in the fact that clustering of group centroids by raw material is also lacking, indicating that differences between sites are not primarily due to differences in raw materials.

Figure 7.3.8: Boxplot of component 2 of the microlith PCA. Higher values indicate more extensive retouch.

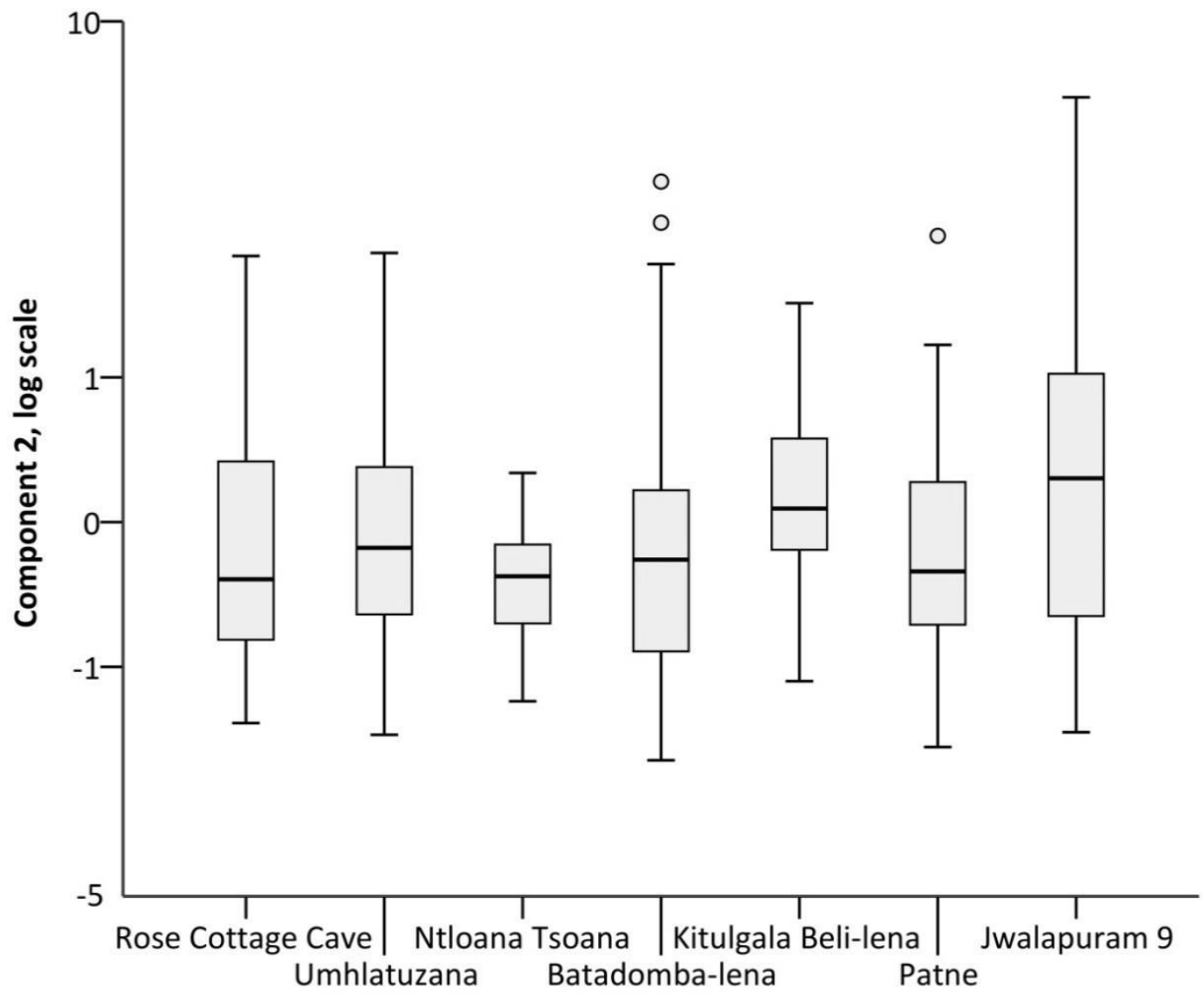

DFA further supports this conclusion. Stepwise DFA produced a model which comprised 11 variables (Table 7.3.13). Six functions were extracted, of which the first five are statistically significant $(\mathrm{p}<.001$ for functions $1-4, \mathrm{p}=.024$ for function 5). Functions 1 and 2 explain 75.2\% of the variability between sites (Table 7.3.14). Microliths are correctly classified by the model into their sites in $57.1 \%$ of cases. 
Compared to the prior probability of correct site attribution of $21.3 \%$, this is a $168.1 \%$ increase in prediction power. When the function scores are calculated for each core, Kruskal Wallis tests (as the data is non-parametric) reveal that statistically significant differences exist between sites in all five significant functions (Table 7.3.15).

Figure 7.3.9: Biplot of components 1 and 2 of the microlith PCA for group centroids of raw material and site groups. Circles are CCS varieties, diamonds are quartz, and triangles are other raw materials.

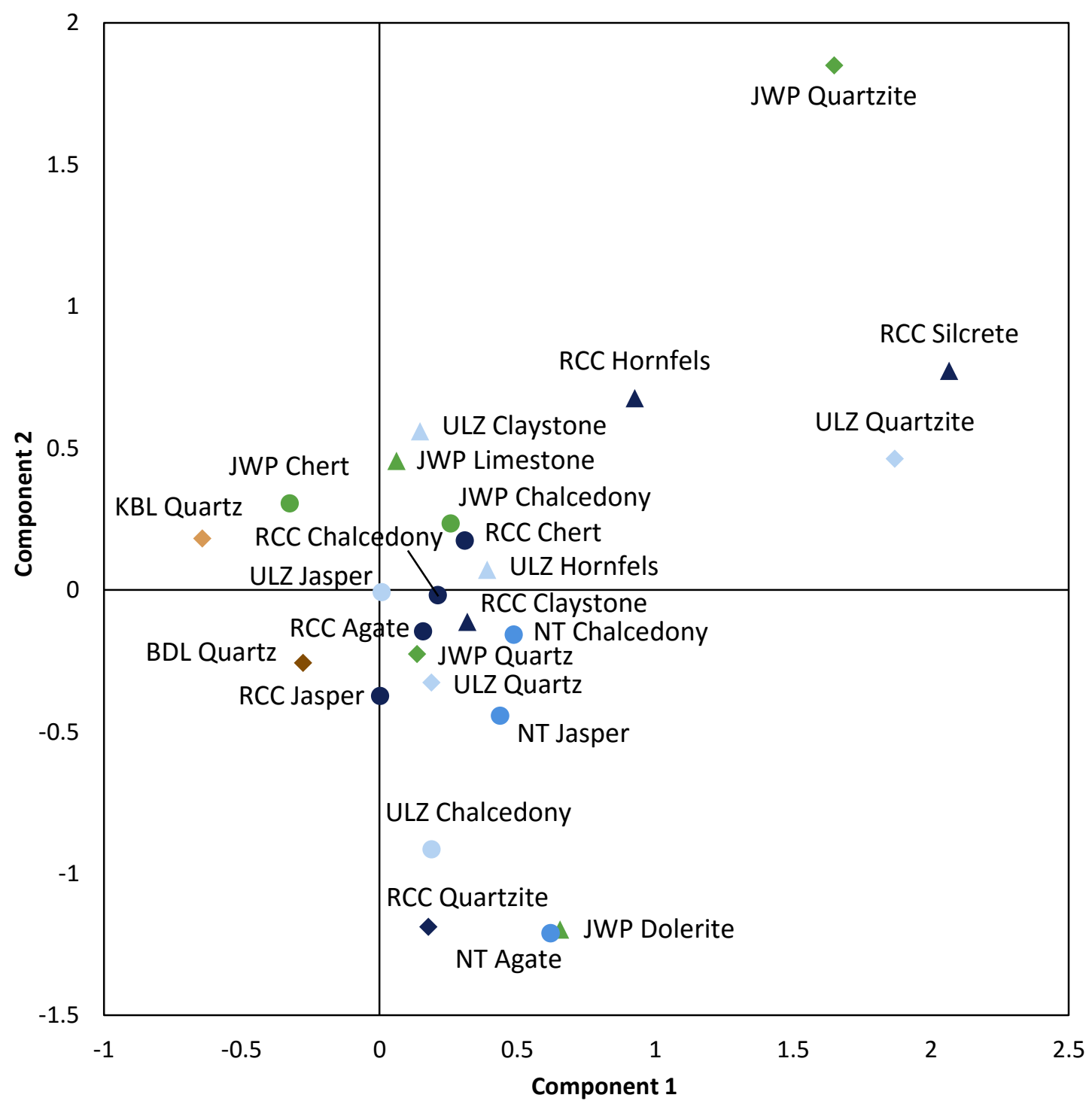




\begin{tabular}{lrrrrrr}
\hline & \multicolumn{7}{c}{ Function } \\
Variable & $\mathbf{1}$ & $\mathbf{2}$ & $\mathbf{3}$ & $\mathbf{4}$ & $\mathbf{5}$ & $\mathbf{6}$ \\
\hline Dorsal scars per cm ${ }^{2}$ & .574 & -.217 & -.022 & .069 & .366 & .069 \\
Oriented length & -.325 & .832 & .044 & .007 & .161 & -.270 \\
Length:thickness ratio & -.048 & .425 & -.058 & -.038 & .386 & .091 \\
Average retouch length & -.206 & .191 & .356 & -.196 & .206 & -.164 \\
Number of dorsal scars & .320 & .410 & -.160 & .449 & -.402 & -.042 \\
Length:width ratio & .366 & .336 & -.192 & -.382 & .508 & -.270 \\
Medial width * & -.425 & .349 & .304 & .387 & -.488 & .117 \\
Medial thickness * & -.054 & .263 & .314 & -.013 & -.395 & -.128 \\
Index of Invasiveness & .086 & -.327 & .210 & .277 & .391 & -.236 \\
Scar orientation & .066 & .148 & -.298 & .049 & -.130 & .606 \\
Cortex & .066 & .164 & .033 & .301 & .215 & .415 \\
\hline
\end{tabular}

Table 7.3.13: Variables used in the DFA of microliths, and their loadings on each function. * log-transformed to maintain normality.

\begin{tabular}{lrrrr}
\hline Function & Eigenvalue & \% of variance & Cumulative \% & Canonical correlation \\
\hline 1 & .944 & 61.4 & 61.4 & .697 \\
2 & .363 & 23.6 & 85.0 & .516 \\
3 & .142 & 9.2 & 94.2 & .353 \\
4 & .054 & 3.5 & 97.7 & .226 \\
5 & .033 & 2.1 & 99.8 & .178 \\
6 & .003 & 0.2 & 100.0 & .053 \\
\hline
\end{tabular}

Table 7.3.14: Summary of key statistics for the DFA of microliths

\begin{tabular}{ll}
\hline Function & Test result \\
\hline 1 & $\chi^{2}(6)=400.720, \mathbf{p}<.001$ \\
2 & $\chi^{2}(6)=215.637, \mathbf{p}<.001$ \\
3 & $\chi^{2}(6)=101.484, \mathbf{p}<.001$ \\
4 & $\chi^{2}(6)=34.829, \mathbf{p}<.001$ \\
5 & $\chi^{2}(6)=35.692, \mathbf{p}<. \mathbf{0 0 1}$ \\
\hline
\end{tabular}

Table 7.3.15: Results of Kruskal Wallis tests of the microlith DFA functions

As with the core and debitage datasets, variables are better predictors of site than material. When raw material is used as the grouping variable, cores only correctly 
classify into their groups in $42.6 \%$ of cases, compared to an equivalent for grouping into sites of $60.7 \%$. The site at which a microlith was manufactured is more easily determined than its raw material.

An additional conclusion that can be drawn from the results of the DFA concerns microlith typology. When group centroids are plotted for sites and major microlith groups (geometric, non-geometric and backed non-geometric) using the first two functions, considerable site- and region-based grouping occurs (Figure 7.3.10). Microliths with higher values for function 1 are short and narrow, with high scar numbers and densities. Those with higher values for function 2 are long, thick and elongated. The southern African and South Asian microliths are primarily distinguished by function 1 . The Sri Lankan centroids cluster in the region of the graph associated with small, elongated microliths with dense scar concentrations. Microliths at the Indian sites are especially elongated, but are larger on average. HP microliths are relatively long and wide, with lower scar concentrations.

Southern African and Sri Lankan microlith centroids cluster closely within their regions (except for NT, which is complicated by small frequencies), while those at the Indian sites are more variable. However, there is no clustering by microlith type. There is no evidence for any geometric or other microlith 'template' that was exported or transferred between sites. Rather, microliths of different types were manufactured within local site-specific manufacturing traditions. 
Figure 7.3.10: Biplot of functions 1 and 2 of the microlith DFA for group centroids of typological and site groups. Circles are geometric forms (including both backed and non-backed varieties due to the rare occurrences of the latter), triangles are backed non-geometrics, and diamonds are non-backed non-geometrics.

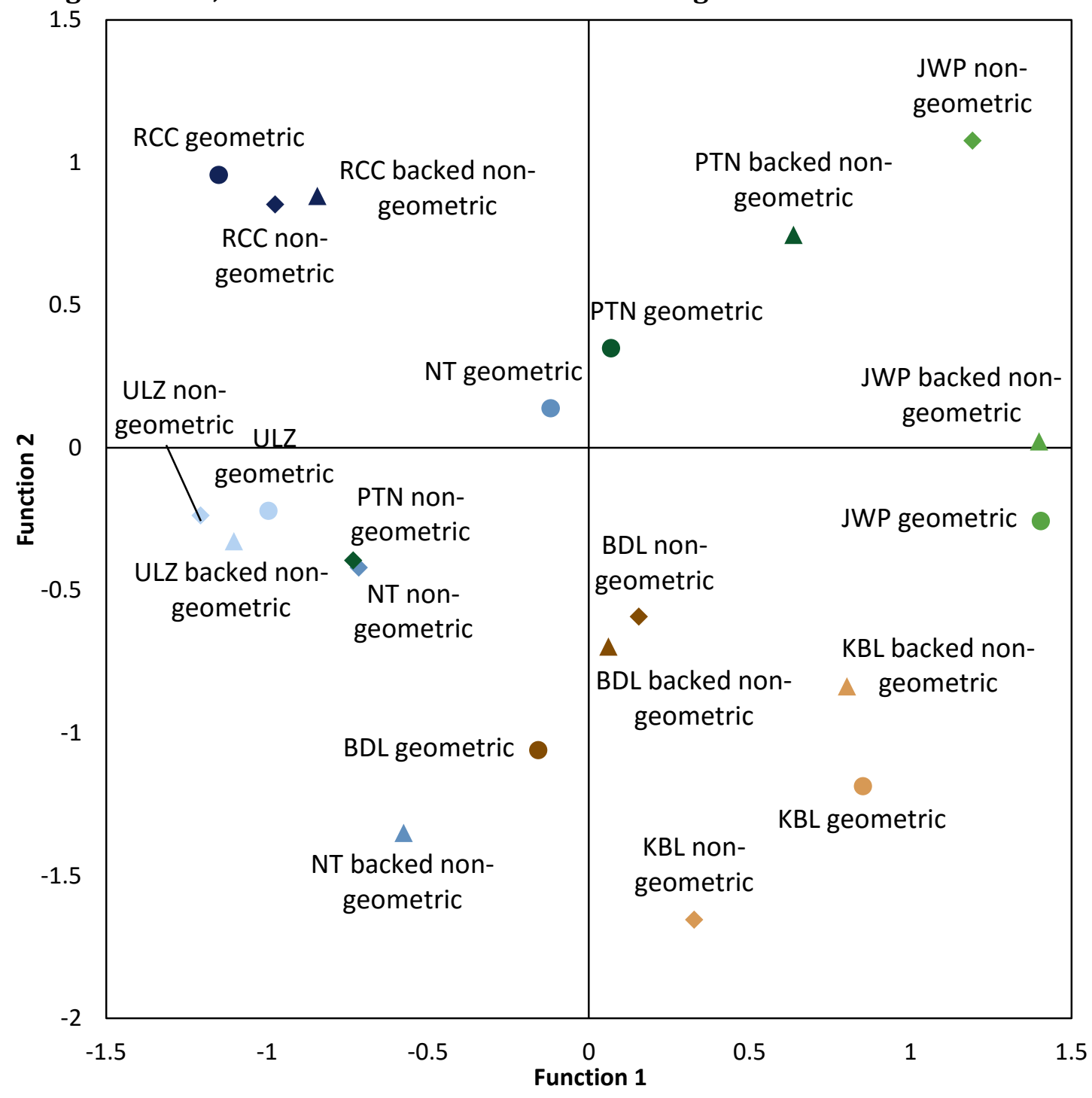

Typological differences are also evident between sites and regions. For example, notched microliths are only found among the South Asian sites (Batadomba-lena and Jwalapuram), and microlithic burins only at the Indian sites. A greater number of unique forms occur in the South Asian dataset, although the sample size is larger. Shouldered backed microliths are only found at RCC, double side retouched points at NT and notched trapezes at Jwalapuram. Batadomba-lena contains several forms not found elsewhere: notched crescents, a backed and side 
retouched point, a tanged bifacial (Balangoda) point, and a notched shouldered microlith. The interpretation of these typological differences is complicated by unequal assemblage sizes. However, one possible conclusion is that the notch and tang innovations at Batadomba-lena may be related to the functional requirements of hafting such small microliths.

The results of the PCA and DFA indicate that there are significant differences between the sites. PCA reveals that the most important groups of variables for distinguishing between sites are size characteristics (width and thickness) and elongation, and retouch extent (length and invasiveness). Interestingly, in the PCA length proved to be a relatively poor indicator for distinguishing between microliths from different sites. Overlap exists between regions in the PCA components, although HP microliths are wider and thicker on average. Regional and site groupings are less clear than in the core and debitage PCAs, which is also reflected in the results of the DFA where the model has less predictive power than that for cores (although slightly greater than that for debitage). As with cores and debitage, microliths are more effectively categorised into sites than into raw materials. Finally, DFA demonstrates that when both sites and major microlith categories are considered, microliths cluster strongly within sites but not within microlith types. There is no universal template for particular microlith forms, and instead microliths vary according to site- and region-based technological traditions. 


\section{SUMMARY}

Through the use of two multivariate statistical techniques - DFA and PCA - a number of underlying technological factors/components have been produced, which have been shown to distinguish between sites in a statistically significant manner. One consistent result from each of the analyses is that all factors distinguish between sites more clearly than between raw materials. Although raw material differences contribute towards the variation observed between lithic assemblages, they are outweighed by other cultural or geographic sources of technological variation. There is no reason to believe a priori that this might be the case. Indeed, this is in contrast to a previous inter-regional comparative study of differences in blade and microblade production (Owen 1988), which concluded that raw material differences were more important than culture in determining blade size. These results are therefore meaningful, and highlight that the similarities and differences observed are valid in terms of technological variability.

Cores are mainly distinguished on the basis of size and in the numbers and proportions of blade removals. Regional groupings are not entirely discrete, due to the inherent limitations imposed on technological variation by the material constraints of knapping. Generally, however, the HP cores are larger and more blade-like, while the South Asian cores are smaller and more flake-like. There is more variation between the South Asian sites than between the southern Africa sites, especially in terms of size.

Debitage is primarily distinguished between sites on the basis of width and thickness, and length and elongation, although regional groupings are less clear 
than for cores. Debitage at the HP sites is larger (especially longer) and more elongated on average. However, this is mostly the case at RCC and Umhlatuzana; differences are apparent between RCC and NT despite their geographical proximity, indicating the existence of variability on small as well as large geographic scales.

Surprisingly, in the microlith assemblage length is shown not to be a particularly useful indicator for discriminating between microliths from different sites, implying that the use of cut-off lengths for microliths is not a useful method for defining them. Instead, microliths are primarily distinguished between sites on the basis of width and thickness, as well as retouch extent.

In terms of microlith size, Sri Lankan microliths are smallest, with those from Kitulgala being especially diminutive. The Indian microliths are larger, particularly in terms of length, and they are also particularly elongated. The southern African microliths are larger on average than any of the South Asian sites, especially in width and thickness. However, regional and site groupings are less clear than in the core and debitage analyses. The differences in microlith size characteristics between sites contrast somewhat with differences in debitage characteristics - all South Asian sites have predominantly flake debitage, but they have particularly elongated microliths.

In the DFA analyses, the predictive power of the model for cores is highest (a 190\% improvement), followed by microliths (170\%) and debitage (150\%). That cores are a better predictor of site identity highlights that the byproducts of lithic 
reduction can be as or more revealing in terms of site or cultural groupings than retouched tool assemblages, a point previously argued by Clarkson (2010). Underlying technological variability is obscured when only finished microlithic tools are considered.

These results also indicate that cores are more clearly differentiated between sites. Site and regional clustering in the PCAs is greatest in the core assemblage, followed by the debitage assemblage, with least clustering evident in the microlith assemblage. It can be inferred from this that the differences between sites are greater in terms of the underlying technological aspects of lithic manufacturing than in the retouched tool assemblages. This pattern was predicted in Chapter 2 as being more likely to occur in cases of analogy rather than homology, and will be discussed in further detail in the following chapter.

Finally, microliths have been shown to cluster much more closely by site and region than by typology. A geometric microlith (predominantly backed crescents) at one site has more in common with a non-backed non-geometric microlith which exhibits a completely different shape and retouch technique - at the same site, than it does with a microlith of the same form at a different site. There is no geometric or other microlith 'template' that was shared between sites, and microliths of different types were manufactured within local site-specific manufacturing traditions. 


\section{Chapter 8: Conclusions and wider implications}

\section{INTRODUCTION}

This thesis set out to investigate similarities and differences between early microlithic assemblages, and to provide the first technological comparison of southern African HP and South Asian Late Palaeolithic industries. Previous research has tended to homogenise these industries, by focussing only on tool typology and by primarily considering microlithic technology as a proxy for modern human behaviour or dispersals, rather than an informative aspect of human behaviour in itself. Instead, this study makes use of detailed technological attribute analyses of cores and debitage, as well as tools, in order to fully investigate technological processes and manufacturing trajectories. The original contribution to knowledge of this thesis is the demonstration that considerable spatial and temporal variability exists in microlithic technology, and the detailing of the forms that this variability takes. This is important because such variability has implications for the debates concerning modern human behaviour and dispersals, as well as for the way that we define microlithic industries.

The preceding chapters (Chapters 5-7) presented the results of analyses of lithic technology at seven southern African and South Asian sites. This chapter sets out how these results answer the research questions asked at the beginning of this thesis, and what implications this has for the wider debates concerning microlithic industries and their place in discussions of modern human behaviour and 
dispersals. It also evaluates the success and limitations of the current project, and sets out directions for future work to further address these questions.

QUESTION 1: WHAT DEGREE OF VARIABILITY IS EVIDENT WITHIN INDIVIDUAL ASSEMBLAGES, AND WHAT CAN THIS TELL US ABOUT THE CHOICES MADE AND STRATEGIES USED BY THE GROUPS IN QUESTION?

The results presented in Chapters 5 and 6 consistently highlight areas of variability within individual sites, for example in the form of distinct manufacturing trajectories for different tool types or raw materials. One particularly relevant area is in the existence at all sites where full assemblages were analysed (i.e. excluding Kitulgala) of macrolithic tools alongside microliths. Size differences are inherent in these definitions, and bimodal size distributions are often evident. For example, at Jwalapuram there is a clear drop-off of microlith lengths at c. $40 \mathrm{~mm}$, with other tools generally much larger. Width and thickness measurements in particular proved useful in distinguishing between tool types.

Differences in widths and thicknesses between microlithic and macrolithic tools are reflected in TCSA measurements, with microliths at all sites generally falling in the range of arrowheads and dart tips as defined by Shea (2006), and larger points falling in the range considered indicative of hand-cast/thrusting spears. Although TCSA measurements alone cannot be used to determine the function of any individual microlithic tool, the fact that pointed, tanged and shouldered forms often have particularly small measurements suggests that these artefacts, at least, would have been suitably formed for use as projectiles. It could be extrapolated that size differences between microlithic and macrolithic tools imply the existence 
of distinct exploitation strategies involving different categories of projectile weaponry, for example for different sized prey. Alternatively, given numerous examples of microliths being used for functions other than as projectile weaponry (discussed in Chapter 2.1), they may have fulfilled other distinct technological needs.

Interestingly, retouch techniques were also revealed to be a useful method for distinguishing between tool types. The techniques used on microlithic and macrolithic tools at each site are often very different, with backing retouch in particular used in high frequencies for microliths but often absent from nonmicrolithic tool assemblages. These multiple distinguishing factors demonstrate the validity of the separation into microlithic and macrolithic tools.

Following on from this, separate manufacturing trajectories for different tool types are often evident. At RCC, NT, Batadomba-lena and Jwalapuram, comparisons of the size distributions of debitage, microliths and non-microlithic tools demonstrate that general lithic reduction was geared towards the production of small flakes and blades suitable for retouch into microlithic tools. Blanks for retouch into larger tools were selected as part of a secondary and perhaps more opportunistic process. This is most evident at RCC, where relatively standardised small blades less than c. $45 \times 20 \mathrm{~mm}$ were produced in large numbers and used as microlith blanks. However, at Umhlatuzana and Patne both microlithic and macrolithic tools were selected from among the general size range of debitage. Although both tool varieties were produced at all sites, differences exist as to the importance placed on the manufacture of larger tools. 
In particular, larger points are found in each of the HP tool assemblages. These include unifacial points at RCC and Umhlatuzana, and a bifacial point at the latter. Radial reduction of cores is also present at all HP sites. Microlithic technology and bipolar percussion - typically considered a LSA phenomenon - occur alongside these aspects of typical MSA technology throughout the site sequences studied. This combination of technological traits accords with arguments that the HP is situated within regional lithic traditions (discussed in Chapter 3.1), thus providing an example of the autochthonous innovation of microlithic technology.

Variability within individual assemblages is also evident in the existence of distinct manufacturing trajectories for the working of different raw materials. This is clearest at Umhlatuzana, where quartz was primarily worked for the production of small flakes, and hornfels for larger blades, with some primary reduction of hornfels - but not quartz - possibly occurring off-site. These differences are amplified in the microlith assemblage. Additionally, bipolar percussion was used to reduce quartz at the site but not other raw materials. The same pattern concerning bipolar percussion is evident at Jwalapuram. Also, at RCC and NT CCS varieties were preferentially used to produce microliths, especially on blade blanks, while non-microlithic tools were manufactured on a mix of raw materials, including lower-quality and coarser materials.

The in-depth exploration of lithic technology at each site reveals multiple areas of variability within sites. These include the elucidation of exactly what strategies were used to manufacture microlithic tools, how they were manufactured in different ways to larger tools, to what extent they were considered the main aim of 
lithic manufacture, and how different raw materials were utilised in order to achieve these goals. This is significant because it can tell us exactly what it was about microliths that was deemed important or advantageous, and what technological and behavioural needs they fulfilled.

One example of this is that cross-sections of microlithic tools (the area that would have penetrated a target, if used as a projectile) were made to be distinctly smaller than those of other points, often regardless of the length of the tool. Additionally, backing was clearly an important aspect of microlith production. If this type of retouch can be assumed to be related to hafting requirements (i.e. creating a flat edge to insert into a haft or handle), it can be concluded that the vast majority of retouch on microliths was conducted in order to facilitate hafting, rather than to manufacture points or cutting edges. These insights would not be revealed through solely typological studies. Consideration of underlying technological processes and manufacturing trajectories reveals considerable variability within sites.

\section{QUESTION 2: HOW DOES MICROLITHIC TECHNOLOGY CHANGE OVER TIME, BOTH WITHIN INDIVIDUAL SITES AND WITHIN REGIONS, AND WHAT MAY HAVE INFLUENCED THESE CHANGES?}

A surprising degree of consistency over time has been demonstrated in the HP sites studied, through the use of statistical comparisons between layers reported in Chapter 5 (and Appendix B). Most of the visible changes in the assemblages are small-scale variations between layers, rather than over-arching trends or transitions. No evidence for the existence of distinct phases has been produced at any of the sites, contrary to previous suggestions (e.g. Wadley and Harper 1989) - 
although the possibility remains that the consideration of finer-grained sequences might reveal additional patterns. The smallest degree of variation occurs in the core and microlith assemblages. For example, there are no statistically significant changes over time in the core assemblage at RCC, and virtually none at NT.

Additionally, no HP site exhibits significant changes in tool typology. This is particularly notable at Umhlatuzana, which demonstrates a considerable degree of consistency in the microlith assemblage over the c. 10,500 year sequence. This occurs despite significant changes in the raw material proportions of debitage, and therefore available blanks. Changes in raw material use appear unrelated to changes in tool production, and so may be hypothesised to be related to other external factors such as changes in mobility patterns or range sizes, or different landscape use, resulting in differential access to raw materials.

A greater degree of temporal variability is evident within the South Asian sites (Chapter 6 and Appendix B). However, this is a result of their longer temporal spans. If the HP had continued over another 15-20,000 years, with a similar timespan to some of the Late Palaeolithic sites studied, we should expect to see similar degrees of temporal change in the two industries. Yet although the overall degree of change over time cannot be compared between the industries on the same scale, the form that this variation takes can be considered.

Through analysis of the temporal changes among the South Asian Late Palaeolithic sites it is possible to detect temporal variability on three different scales. Firstly, some general trends are evident within sites. One example can be seen at Patne, 
where an increase in core reduction intensity can be observed over time, particularly in terms of the numbers of flakes removed from each core. Batadomba-lena is the only site that exhibits a consistent trend in the production of particular microlith forms, with backed non-geometric forms becoming less prevalent over time, being replaced by non-backed non-geometric forms.

Secondly, as at the HP sites many small-scale variations between layers are evident in the South Asian assemblages. Particularly complex differences between layers are evident at Patne, with each layer forming a distinct debitage assemblage distinguished primarily by size and emphasis on flakes or blades. Some differences occur that are specific to a single period of occupation - for example, bipolar backing only occurs in one layer. Similarly, at Jwalapuram there are particularly large numbers of microlithic burins in one layer, and notched microliths in another.

Thirdly, some temporal variability occurs in the form of more abrupt changes. At both Batadomba-lena and Jwalapuram blade blanks were preferred for the manufacture of microliths in the lower halves of the sequences, with a shift to more flake blanks being used in the upper halves. Both sites also exhibit a decrease in the use of backing retouch at the same time, although at Batadomba-lena this is more gradual.

This pattern is in contrast to Patne, where blade blanks became increasingly preferred for microliths over time. It is also of relevance with reference to previous work which has highlighted the technological and typological diversity evident 
within the Late Palaeolithic of South Asia (James and Petraglia 2005, and discussion in Chapter 3.2). Not only is there no clear regionalisation on the basis of geography, but there is also no clear chronological patterning; for example, there is no gradual transition from flake-based assemblages to blade-based ones. The results presented here accord with the conclusion that the South Asian Late Palaeolithic is a diverse and variable industry.

The timing of changes in the South Asian sites is potentially revealing about their causes. The fact that the most dramatic changes at Jwalapuram and Batadombalena occur at the end of the LGM (20-15 ka BP at the former and 19.5-18.5 ka BP at the latter) adds support to the argument that environmental changes were one of the prime factors contributing to technological variation in the microlithic industries of South Asia, perhaps coupled with its impact on, or acting through the proxy of, demographic changes (Petraglia et al. 2009a, and discussion in Chapter 3.2).

Although temporal changes are evident within both the HP and the South Asian assemblages, in general there are few consistent uni-directional trends over time either within individual sites or within the regions as a whole. Most chronological changes occur in the form of small-scale variations between layers rather than consistent patterns. Instead, each assemblage in each layer is best viewed as a complex assortment of different strategies for tool production, reflecting technological adaptations to the unique and specific social and ecological circumstances of site occupants at that time. 
QUESTION 3: WHAT DIFFERENCES AND SIMILARITIES ARE EVIDENT BETWEEN THE EARLIEST MICROLITHIC INDUSTRIES IN SOUTHERN AFRICA, INDIA AND SRI LANKA, AND WHAT FACTORS MIGHT EXPLAIN THIS?

Both similarities and differences within and between the HP and South Asian sites studied are revealed in Chapter 7. Much variation is evident within industries/regions, as well as between them. The degree of intra-regional variability makes generalising about differences between the HP and South Asian sites difficult. However, in broad terms the main differences are that cores and debitage at the HP sites tend to be larger and more elongated or blade-like, while those at the South Asian sites are smaller and more flake-like on average. The same holds true of microlithic tools, although the Sri Lankan microliths are smaller on average than those from the Indian sites, while the Indian microliths also tend to be more elongated. The HP microliths are larger on average, particularly in terms of width and thickness.

In addition to differences between regions, there is also considerable variability within regions. For example, the results presented in Chapter 5 demonstrate that the HP is not entirely a blade-based industry, as is often argued (see Chapter 3.1). Only RCC meets this description, with flakes primarily removed in order to prepare cores for the removal of blades. No such process is evident at the other HP sites studied, with flakes dominant at NT and a mix of debitage products at Umhlatuzana. The differences between RCC and NT are particularly notable given their geographic proximity and the fact that they may have made use of the same sources of raw materials. Geographic variability in lithic production is evident on small as well as large scales. 
Differences are also evident between the South Asian sites, for example in the ways in which bipolar percussion was utilised, and tool type proportions. However, there is no clear geographic patterning in variation, with many differences evident within the Indian and the Sri Lankan assemblages. One example of differences transcending geographic proximity can be seen in the many technological similarities between Batadomba-lena and Jwalapuram, which is at least partly related to the use of quartz at both sites. Differences are evident even within the Sri Lankan quartz microlith assemblage, with microliths from Kitulgala being considerably smaller than those from Batadomba-lena. Again, these results highlight the technological diversity evident within the South Asian Late Palaeolithic, and the fact that this term encompasses a variety of lithic traditions.

Additionally, several novel microlith forms occur at Batadomba-lena, including tanged and shouldered forms, and notches are particularly common. These innovations may be related to the functional requirements of hafting such small microliths, and specifically appear designed to facilitate hafting as projectiles or points. Against a background of long-term habitation of a dense rainforest environment, including the frequent exploitation of monkeys and other arboreal prey (discussed in Chapter 3.2), it might further be hypothesised that microliths at the site were produced as part of a specific hunting strategy for such prey. A similar conclusion might be drawn from the existence of several very small doublebacked pointed microlith forms at Kitulgala, which are not seen elsewhere.

Raw material has been demonstrated to be only a minor factor contributing towards differences in lithic technology between sites and regions. Instead, other 
factors influencing technological variability are more influential. As discussed in Chapter 2.1, numerous explanations have been proposed for the forms that microliths took. It is not possible from the data available to state exactly which factors resulted in particular aspects of microlith variability. However, environmental and ecological factors have been highlighted above as potentially important factors impacting upon technological and behavioural variability. Some of the most dramatic technological changes described in the South Asian Late Palaeolithic coincide with major periods of climatic change. Additionally, the different ecological settings of the sites considered (described in Chapter 3) would have posed different technological challenges and requirements, for example in the different prey species that might have been encountered (assuming that at least some microlithic tools were used in hunting weaponry).

Consideration of the similarities and differences between microlithic tool assemblages can contribute towards our understanding of microliths as a tool type more generally. Following on from the discussion in Chapter 2.1 concerning microlith definitions, it has been demonstrated here that microliths are not in fact necessarily retouched into geometric forms. In fact, geometric morphologies are not dominant at any site, and make up only around a quarter of microlith assemblages on average. The dominance of non-geometric microliths was previously noted at RCC by Wadley and Harper (1989). However, the results presented here demonstrate that this is not unique to RCC but may extend to the rest of the HP and to microlithic assemblages more generally. 
It has also been highlighted that there is no universal size definition or criteria for microliths. If we were to attempt to apply length cut-off points for microliths, these would be at $40 \mathrm{~mm}$ at RCC, Umhlatuzana, Patne and Jwalapuram, $30 \mathrm{~mm}$ at NT and Batadomba-lena, and $20 \mathrm{~mm}$ at Kitulgala. Therefore these limits are not even applicable on a regional level, and should instead be interpreted on a site-by-site basis. Interestingly, width appears to be more important than length in defining microliths at many sites. Microliths were often made to be of similar widths at a site regardless of length. Perhaps this is not surprising, given that it is the width of a tool that influences the area that would penetrate a target if it was used as a projectile. Additionally, in the case of laterally-hafted microliths, this might indicate a degree of standardisation in the width of tools which were inserted into hafts.

The use of blade blanks has been demonstrated to be a common factor in microlith production, with blades often being preferentially selected from the range of available blanks. However, flake blanks were also used in varying proportions for microlithic tools, and at Umhlatuzana quartz flakes were preferentially selected as tool blanks. Although the use of blade blanks is common, it cannot be considered a prerequisite feature of microlithic technology.

Similarly, the use of backing retouch has been shown to be a common but nonessential feature of microlithic technology at all sites. Backing and geometric forms are two features that often occur together, with the vast majority of geometric microliths being backed. However, a wide variety of non-geometric forms also 
exhibit backing retouch at each site. Backing therefore supersedes geometric shapes as a primary categorisation of microlithic tools.

Overall, while small sizes, geometric forms, the use of blade blanks and the presence of backing retouch are all important features of microlithic technology, the importance of each one varies between sites. Although consideration of these features still form a necessary step in the definition of microliths on a site-by-site basis (for example by looking for bimodal distributions of tool lengths, widths or TCSAs), it is not possible - and unwise - to attempt to impose a universal definition of microliths between sites and regions. The fact that the defining features of microliths vary between sites may make life difficult for lithic analysts, but in fact it is exactly the sort of feature of lithic technology that should be being investigated. It is important because it tells us exactly which aspects of microlithic tools were considered key at specific places and times; for example, whether the benefits of processes such as the bipolar backing of the lateral edge of a small tool outweighed the time and energy costs involved.

\section{IMPLICATIONS FOR THE MODERN HUMAN BEHAVIOUR DEBATE}

The modern human behaviour debate was introduced in Chapter 2.2 as a theoretical framework which involves an inter-regional comparative approach to microliths. The debate's current focus on the presence or absence of particular archaeologically-visible behavioural traits was shown to have contributed towards a homogenising view of microlithic technology. It was argued that a better alternative approach would instead aim to investigate behavioural variability 
through a more regionally-focussed consideration of the variety of technological strategies that different populations chose to adopt in different places and at different times. It is this approach that has been adopted throughout this thesis.

The results of analyses presented in Chapters 5-7 provide a comprehensive account of the variability evident within and between microlithic industries in southern Africa and South Asia. The answers to the questions presented above reveal aspects of variability within sites, over time, and between sites and regions. More specifically, variability has been demonstrated in raw material preferences, core reduction strategies, blank production and selection, and retouch choices.

Exploration and interpretation of this variability has important implications for our approach to understanding human behaviour in the archaeological record. It demonstrates that populations were adapting their technological behaviours to specific local circumstances and requirements. For example, various microlithic innovations in Sri Lanka were possibly made in response to the requirements of hunting arboreal prey in dense rainforest environments. Distinct technological signatures are evident at each site, even when raw material and typology are taken into account, revealing that microlithic technology is far from homogeneous. By demonstrating that microlith production varies between sites and regions in response to local conditions, it has been established that microlithic technology is a diverse and flexible technological strategy. Furthermore, the temporal dimension of behavioural variability has also been highlighted, revealing that microlithic industries are not homogenous over time and that periods of both stasis and change occur. 
This thesis contributes to the methodology of the modern human behaviour debate by demonstrating the benefits of focussing on behavioural variability through the detailed investigation of microlithic technology, rather than the traditional trait list approach. For example, the use of technological attribute analyses allows for the use of a variety of statistical techniques. These methods provide additional ways of exploring and quantifying variability within, and especially between, sites.

Additionally, there are benefits to shifting the unit of analysis from the presence or absence of microliths at specific sites to the microliths themselves and their associated by-products. There are two ways of looking at variability in behaviour. One is to count up all the different things that different populations do, and compare the numbers. However, this approach is strongly influenced by taphonomy and preservation, especially for behaviours which are discerned through the existence of organic materials (including many artistic or symbolic endeavours) (Henshilwood and Marean 2003). The alternative method is to control for taphonomic effects by investigating variability in a single behaviour. The choice of microlithic technology means that assemblages of any age or geographic location can be assessed equitably. As a result, the investigation of variability in microlithic technology presented here provides a particularly effective case study of how behavioural variability can be considered.

Finally, the use of this approach means that areas of underlying technological variability can be discerned that would not otherwise be observable. Examples include different uses of bipolar percussion, different blank production and selection processes, and different methods for working the same raw materials. 
This approach provides a better and more nuanced way of understanding actual technological behaviour in the past, and how it varies. It is this high degree of behavioural variability, flexibility and innovativeness that truly characterises modern human behaviour.

\section{IMPLICATIONS FOR MODERN HUMAN DISPERSALS}

Chapter 2.3 introduced the debate concerning the earliest modern human dispersals out of Africa, and the role that archaeological evidence has played in these debates. Specifically, it discussed the microlithic dispersal model, as posited by Mellars (2006b, Mellars et al. 2013). The comparative analyses conducted here (Chapter 7) contribute to our understanding of human dispersals by providing the first test of the microlithic dispersal model on the basis of lithic technology.

Specifically, the results presented here contribute towards the methodology of the debate concerning human dispersals. Cores are shown to be more clearly differentiated between sites than are microliths. An implication of this finding is that, in order to fully test the microlithic dispersal model, we should perhaps be focussing our attention on cores in order to look at the underlying diversity of lithic technology. Underlying technological variability is obscured when only retouched tools are considered, which is one of the problems with previous approaches that this study was designed to address.

To some degree the results of comparisons between sites and regions confirm Mellars' point concerning the ostensive similarities between microlithic tool forms. 
The uses of backing and blade blanks are common in both southern Africa and South Asia. Many microlith morphologies are found in both regions, although in contrast to Mellars' arguments 'crescentic' and geometric shapes are actually much less common than non-geometric forms. Also, size differences between sites are more pronounced than is often assumed.

Yet what is most notable about the results of the analyses is that the differences between sites are greater in terms of the underlying technological aspects of lithic manufacturing than in the retouched tool assemblages. Cores (and to a lesser extent debitage) cluster more clearly by site and by region than microlithic tools, which overlap more between regions. Somewhat similar microlith forms were produced by different groups, presumably due to similarities in, for example, functional requirements and hafting restrictions. However, they were produced using different methods and as part of different manufacturing trajectories due to differences in local culturally-inherited traditions of tool production.

This pattern of equifinality in final tool form coupled with underlying technological variation was predicted in Chapter 2.3 as being more likely to occur in cases of analogy than homology. The implication from the comparisons of microlithic assemblages presented here is that independent innovation of microlithic technology rather than cultural diffusion best explains the observed patterns, especially when further supported by evidence for gradual autochthonous development of microlithic technology within South Asia (James 2011). This is in disagreement with Mellars' microlithic dispersal model. 
The results of multivariate statistical analyses further indicate that microliths predominantly cluster by site and region rather than by typology. There are no universal templates for particular microlith forms that were exported or transferred between sites, and microliths of different types were manufactured within local technological traditions. Again, this conclusion accords more closely with arguments for independent innovation of microliths, rather than models which consider microliths a proxy for human dispersals.

\section{LIMITATIONS OF THE STUDY AND RECOMMENDATIONS FOR FUTURE RESEARCH}

Overall, the attribute methodology utilised was effective both at reconstructing manufacturing trajectories and in discriminating between lithic products from each site. The inclusion of debitage and especially cores proved to be extremely useful, and in many ways much more revealing than the analysis of tools alone. However, some variables were more useful than others. For example, although up to two colours were recorded for each lithic, at no site was there any statistical indication for any degree of preferential selection of particular colours of raw material for retouch into tools. Additionally, flake curvature and striking platform lipping proved not to be very useful in the reconstruction of manufacturing trajectories.

There are inevitable problems and limitations when other researchers' datasets are utilised. The methodology was designed to overlap with those used in the studies of the Indian sites wherever possible, and great care was taken in the recoding of data. However, some variables were not recorded at these sites. Most 
problematically, raw material was not recorded systematically at Patne. Even when the same variables are recorded, caveats are still unavoidable when comparing some measurements between sites. For example, core reduction intensity could not be compared directly between sites on some metrics because removal lengths were recorded differently at the Indian sites. Differences in typology were also apparent on occasion, for example with specific geometric shapes not recorded at Jwalapuram. Inter-observer variation can also affect the recording of variables more generally. Nevertheless, the data collected proved sufficient to address the questions posed by this study.

All research projects must necessarily be circumscribed in order to focus analytical attention on the specific questions under consideration. For example, because this research project was designed to investigate fine-grained technological variability, it was decided to use a relatively small number of sites and to analyse them using a detailed attribute methodology, as opposed to a larger number of sites with a less detailed methodology. There are no research projects that would not benefit from the addition of new data. Specifically, however, data from early microlithic sites in East Africa could be added to the data collected here in order to more fully test the microlithic dispersal model. These data could be included in multivariate analyses in order to test whether they group with any of the assemblages already included, and to consider what this might mean in terms of human dispersals.

This study has successfully demonstrated that variability exists within and between the earliest microlithic technologies in southern Africa and South Asia, and has highlighted what form this variability takes. However, the specifics of the 
external factors that resulted in this variability lie beyond the scope of this thesis. Future research could expand upon this by addressing exactly what the factors were that affected the innovation of microlithic technology in different areas, for example by consolidating more detailed dating and environmental datasets to track how technological changes might be related to ecological changes. However, this would also require the creation of more detailed chronological sequences at several sites, especially at the southern African sites studied but also at Patne.

\section{CONCLUSION}

Microlith production is a variable and nuanced lithic technology, but has often been homogenised by previous comparative studies. By applying detailed attribute analyses to microlith assemblages, this thesis set out to better investigate past technological and behavioural variability. The original contribution of this work is its demonstration and exploration of variability within and between sites in the first technological analysis conducted on both southern African and South Asian early microlithic industries.

This thesis demonstrates that microlith production is not a homogenous technology. These results have implications for our definitions of microliths and microlithic technology, highlighting that universal definitions are inappropriate and that differences in microlith sizes, blanks, morphologies and retouch between sites are in many ways what is most deserving of analytical attention. The results of the analyses presented here also have important implications for the debates concerning modern human behaviour and dispersals. Although work remains to be 
done to definitively confirm or disprove the microlithic dispersal model, the comparative analyses undertaken here produce results which are more parsimoniously explained by independent innovation rather than cultural diffusion. The results also demonstrate the benefits of using a theoretical approach which focuses on variability in underlying technological strategies rather than simply the presence or absence of particular tool types. This method of investigating behavioural variability has been shown to be highly revealing in terms of the technological strategies adopted by past populations. It is this behavioural and technological variability that is key to understanding our species. 


\section{Bibliography}

Abbott, W.J.L. (1896) The Hastings kitchen middens. The Journal of the Anthropological Institute of Great Britain and Ireland XXV: 122-129

Abeyratne, M., Spooner, N.A., Grun, R. and Head, J. (1997) Multidating studies of Batadomba cave, Sri Lanka. Quaternary Science Reviews 16: 243-255

Agrawal, D.P., Krishnamurthy, R.V. and Kusumgar, S. (1985) Physical Research Laboratory radiocarbon date list V. Radiocarbon 27: 95-110

Agresti, A. and Finlay, B. (2009) Statistical Methods for the Social Sciences. 4th Edition. London: Pearson

Ahler, S.A. (1989) Mass analysis of flaking debris: studying the forest rather than the tree. In: Henry, D. and Odell, G., eds. Alternative Approaches to Lithic Analysis. Archaeological Papers of the American Anthropological Association no. 1.pp. 85-118

Allchin, B. (1959) The Indian Middle Stone Age. Bulletin of the Institute of Archaeology 2: 1-36

Allchin, B. (1963) The Indian Stone Age sequence. The Journal of the Royal Anthropological Institute of Great Britain and Ireland 93 (2): 210-234

Ambrose, S.H. (1998) Chronology of the Later Stone Age and food production in East Africa. Journal of Archaeological Science 25: 377-392

Ambrose, S.H. (2002) Small things remembered: origins of early microlithic industries in Sub-Saharan Africa. Archeological Papers of the American Anthropological Association 12 (1): 9-29

Ambrose, S.H. and Lorenz, K.G. (1990) Social and ecological models for the Middle Stone Age in southern Africa. In: Mellars, P., ed. The Emergence of Modern Humans: An Archaeological Perspective. Ithaca: Cornell University Press. pp. 333

Andrefsky, W. (2005) Lithics: Macroscopic Approaches to Analysis. 2nd Edition. Cambridge: Cambridge University Press

Armitage, S.J., Jasim, S.A., Marks, A.E., Parker, A.G., Usik, V.I. and Uerpmann, H.P. (2011) The southern route 'out of Africa': evidence for an early expansion of modern humans into Arabia. Science 331 (6016): 453-456

Atkinson, Q.D., Gray, R.D. and Drummond, A.J. (2008) mtDNA variation predicts population size in humans and reveals a major southern Asian chapter in human prehistory. Molecular Biology and Evolution 25 (2): 468-474 
Aubert, M., Brumm, A., Ramli, M., Sutikna, T., Saptomo, E.W., Hakim, B., Morwood, M.J., Van den Bergh, G.D., Kinsley, L. and Dosseto, A. (2014) Pleistocene cave art from Sulawesi, Indonesia. Nature 514: 223-227

Backwell, L.R. and d'Errico, F. (2001) Evidence of termite foraging by Swartkrans early hominids. Proceedings of the National Academy of Sciences 98 (4): 13581363

Backwell, L.,R. and d'Errico, F. (2008) Early hominid bone tools from Drimolen, South Africa. Journal of Archaeological Science 35 (11): 2880-2894

Bae, C.J. and Bae, K. (2012) The nature of the Early to Late Palaeolithic transition in Korea: current perspectives. Quaternary International 281: 26-35

Bae, C.J., Wang, W., Zhao, J., Huang, S., Tian, F. and Shen, G. (2014) Modern human teeth from Late Pleistocene Luna Cave (Guangxi, China). Quaternary International 354: 169-183

Bae, K. (2010) Origin and patterns of the Upper Paleolithic industries in the Korean Peninsula and movement of modern humans in East Asia. Quaternary International 211 (1-2): 103-112

Bailey, G. and Spikins, P. eds. (2008) Mesolithic Europe. Cambridge: Cambridge University Press

Ballin, T.B. (2000) Classification and description of lithic artefacts: a discussion of the basic lithic terminology. Lithics 21: 9-15

Balme, J., Davidson, I., McDonald, J., Stern, N. and Veth, P. (2009) Symbolic behaviour and the peopling of the southern arc route to Australia. Quaternary International 202 (1): 59-68

Bamforth, D.B. and Bleed, P. (1997) Technology, flaked stone technology, and risk. Archeological Papers of the American Anthropological Association 7: 109-139

Barham, L. (2002) Backed tools in Middle Pleistocene central Africa and their evolutionary significance. Journal of Human Evolution 43: 585-603

Barker, G., Barton, H., Bird, M., Daly, P., Datan, I., Dykes, A., Farr, L., Gilbertson, D., Harrisson, B., Hunt, C., Higham, T., Kealhofer, L., Krigbaum, J., Lewis, H., McLaren, S., Paz, V., Pike, A., Piper, P., Pyatt, B., Rabett, R., Reynolds, T., Rose, J., Rushworth, G., Stephens, M., Stringer, C., Thompson, J. and Turney, C. (2007) The 'human revolution' in lowland tropical Southeast Asia: the antiquity and behavior of anatomically modern humans at Niah Cave (Sarawak, Borneo). Journal of Human Evolution 52 (3): 243-261

Bar-Yosef, 0. (2002) The Upper Paleolithic revolution. Annual Review of Anthropology 31: 363-393 
Bar-Yosef, O. and Kuhn, S.L. (1999) The big deal about blades: laminar technologies and human evolution. American Anthropologist 101 (2): 322-338

Bar-Yosef, O. and Van Peer, P. (2009) The chaîne opératoire approach in Middle Paleolithic archaeology. Current Anthropology 50 (1): 103-131

Basak, B., Srivastava, P., Dasgupta, S., Kumar, A. and Rajaguru, S.N. (2014) Earliest dates and implications of Microlithic industries of Late Pleistocene from Mahadebbera and Kana, Purulia district, West Bengal. Current Science 107 (7): 1167-1171

Baxter, M.J. (1995) Standardization and transformation in Principal Component Analysis, with applications to archaeometry. Journal of the Royal Statistical Society 44 (4): 513-527

Baxter, M.J. (2003) Statistics in Archaeology. London: Arnold

Baxter, M.J. (2014) Multivariate analysis of archaeometric data - lecture notes [Online] URL: http://www.academia.edu/7721938/Multivariate_Analysis_of_Archaeometric _Data_--_Lecture_Notes (Accessed 20th March, 2015)

Beaumont, R. (2012) Principal Component Analysis and Factor Analysis using SPSS 19 and $R$ (psych package) [Online] URL:

http://www.floppybunny.org/robin/web/virtualclassroom/stats/statistics2/ pca1.pdf (Accessed 21st March, 2015)

Bednarik, R.G. (1992) Natural line markings on Palaeolithic objects. Anthropologie 30 (3): 233-240

Bednarik, R.G. (1993) About Palaeolithic ostrich eggshell in India. Bulletin of the Indo-Pacific Prehistory Association 13: 34-43

Bednarik, R.G. (2003) The earliest evidence of palaeoart. Rock Art Research 20 (2): 89-136

Beer, N. (2011) Discriminant function analysis [Online] URL: http://www.nils-beerstatistics.com/documents/3\%20Discriminant\%20Function\%20Analysis.pdf (Accessed 20th September, 2014)

Behar, D.M., Villems, R., Soodyall, H., Blue-Smith, J., Pereira, L., Metspalu, E., Scozzari, R., Makkan, H., Tzur, S., Comas, D., Bertranpetit, J., Quintana-Murcia, L., Tyler-Smith, C., Wells, R.S., Rosset, S. and The Genographic Consortium (2008) The dawn of human matrilineal diversity. The American Journal of Human Genetics 82 (5): 1130-1140

Belfer-Cohen, A. and Goring-Morris, N. (2002) Why microliths? Microlithization in the Levant. Archeological Papers of the American Anthropological Association 12 (1): $57-68$ 
Beyin, A. (2006) The Bab al Mandab vs the Nile-Levant: an appraisal of the two dispersal routes for early modern humans out of Africa. African Archaeology Review 23: 5-30

Binford, L. (1973) Interassemblage variability - the Mousterian and the 'functional' argument. In: Renfrew, C., ed. The Explanation of Culture Changes: Models in Prehistory. London: Duckworth. pp. 227-254

Binneman, J. (1997) Usewear traces on Robberg bladelets from Rose Cottage Cave. South African Journal of Science 93: 479-481

Bleed, P. (1986) The optimal design of hunting weapons: maintainability or reliability. American Antiquity 51 (4): 737-747

Bleed, P. (2002) Cheap, regular, and reliable: implications of design variation in Late Pleistocene Japanese microblade technology. Archeological Papers of the American Anthropological Association 12 (1): 95-102

Boivin, N., Fuller, D.Q., Dennell, R., Allaby, R. and Petraglia, M.D. (2013) Human dispersal across diverse environments of Asia during the Upper Pleistocene. Quaternary International 300: 32-47

Bordes, F. (1961a) Mousterian cultures in France. Science 134 (3482): 803-810

Bordes, F. (1961b) Typologie du Paléolithique Ancien et Moyen. Bordeaux: Publications de l'Institut de Préhistoire de l'Université de Bordeaux

Bordes, F. (1969) Reflections on typology and techniques in the Palaeolithic. Arctic Anthropology 6 (1): 1-29

Bordes, F. and Sonneville-Bordes, D. (1970) The significance of variability in Palaeolithic assemblages. World Archaeology 2 (1): 61-73

Bowler, J.M., Johnston, H., Olley, J.M., Prescott, J.R., Roberts, R.G., Shawcross, W. and Spooner, N.A. (2003) New ages for human occupation and climatic change at Lake Mungo, Australia. Nature 421 (6925): 837-840

Brandt, S.A., Fisher, E.C., Hildebrand, E.A., Vogelsang, R., Ambrose, S.H., Lesur, J. and Wang, H. (2012) Early MIS 3 occupation of Mochena Barago rockshelter, southwest Ethiopian highlands: implications for Late Pleistocene archaeology, palaeoenvironments and modern human dispersals. Quaternary International 274: $38-54$

Brooks, A.S., Nevell, L., Yellen, J.E. and Hartman, G. (2006) Projectile technologies of the African MSA: implications for modern human origins. In: Hovers, E. and Kuhn, S.L., eds. Transitions Before the Transition: Evolution and Stability in the Middle Paleolithic and Middle Stone Age. New York: Springer. pp. 233-255 
Brown, K.S., Marean, C.W., Jacobs, Z., Schoville, B.J., Oestmo, S., Fisher, E.C., Bernatchez, J., Karkanas, P. and Matthews, T. (2012) An early and enduring advanced technology originating 71,000 years ago in South Africa. Nature 491: 590-593

Brown, J.A. (1889) On some small highly specialized forms of stone implements, found in Asia, North Africa and Europe. The Journal of the Anthropological Institute of Great Britain and Ireland 18: 1324-139

Bruch, A.A., Sievers, C. and Wadley, L. (2012) Quantification of climate and vegetation from southern African Middle Stone Age sites - an application using Late Pleistocene plant material from Sibudu, South Africa. Quaternary Science Reviews 45: 7-17

Brumm, A. and Moore, M.W. (2005) Symbolic revolutions and the Australian archaeological record. Cambridge Archaeological Journal 15 (2): 157-175

Bulbeck, D. (2007) Where river meets sea: a parsimonious model for Homo sapiens colonization of the Indian Ocean Rim and Sahul. Current Anthropology 48 (2): 315-321

Burdukiewicz, J.M. (2005) Microlithic technology in the Stone Age. Journal of the Israel Prehistoric Society 35: 337-351

Burdukiewicz, J.M. and Ronen, A. eds. (2003a) Lower Palaeolithic Small Tools in Europe and the Levant. Oxford: British Archaeological Reports International Series

Burdukiewicz, J.M. and Ronen, A. (2003b) Research problems of the Lower and Middle Palaeolithic small tool assemblages. In: Burdukiewicz, J.M. and Ronen, A., eds. Lower Palaeolithic Small Tools in Europe and the Levant. Oxford: British Archaeological Reports International Series. pp. 235-239

Burke, A. (2000) The view from Starosele: faunal exploitation at a Middle Palaeolithic site in western Crimea. International Journal of Osteoarchaeology 10 (5): 325-335

Burns, R. and Burns, R. (2008) Business Research Methods and Statistics Using SPSS. Thousand Oaks, CA: Sage Publications

Cann, R.L., Stoneking, M. and Wilson, A.C. (1987) Mitochondrial DNA and human evolution. Nature 325: 31-36

Cantwell, A. (1979) The functional analysis of scrapers: problems, new techniques, and cautions. Lithic Technology 8 (1): 5-11

Charrié-Duhaut, A., Porraz, G., Cartwright, C.R., Igreja, M., Connan, J., Poggenpoel, C. and Texier, P.J. (2013) First molecular identification of a hafting adhesive in 
the Late Howiesons Poort at Diepkloof Rock Shelter (Western Cape, South Africa). Journal of Archaeological Science 40: 3506-3518

Clark, J.D. (1959) The Prehistory of Southern Africa. Harmondsworth: Penguin Press

Clark, J.D. (1985) The microlithic industries of Africa: their antiquity and possible economic implications. In: Misra, V.N. and Bellwood, P.S., eds. Recent Advances in Indo-Pacific Prehistory: Proceedings of the International Symposium held at Poona, December 19-21, 1978. New Delhi: Oxford \& IBH Publishing Co. pp. 95103

Clark, J.D. (1988) The Middle Stone Age of East Africa and the beginnings of regional identity. Journal of World Prehistory 2 (3): 235-305

Clark, J.D., Beyene, Y., WoldeGabriel, G., Hart, W.K., Renne, P.R., Gilbert, H., Defleur, A., Suwa, G., Katoh, S., Ludwig, K.R., Boisserie, J.R., Asfaw, B. and White, T.D. (2003) Stratigraphic, chronological and behavioural contexts of Pleistocene Homo sapiens from Middle Awash, Ethiopia. Nature 423 (6941): 747-752

Clark, J.L. (2013) Exploring the relationship between climate change and the decline of the Howieson's Poort at Sibudu Cave (South Africa). In: Clark, J.L. and Speth, J.D., eds. Zooarchaeology and Modern Human Origins: Human Hunting Behavior During the Later Pleistocene. Dordrecht: Springer. pp. 9-18

Clarkson, C. (2002) An Index of Invasiveness for the measurement of unifacial and bifacial retouch: a theoretical, experimental and archaeological verification. Journal of Archaeological Science 29 (1): 65-75

Clarkson, C. (2007) Lithics in the Land of the Lightening Brothers: The Archaeology of Wardaman Country, Northern Territory. Canberra: ANU E Press

Clarkson, C. (2010) Regional diversity within the core technology of the Howiesons Poort techno-complex. In: Lycett, S.J. and Chauhan, P.R., eds. New Perspectives on Old Stones: Analytical Approaches to Paleolithic Technologies. New York: Springer. pp. 43-59

Clarkson, C. (2014) East of Eden: founder effects and the archaeological signature of modern human dispersal. In: Dennell, R. and Porr, M., eds. Southern Asia, Australia, and the Search for Human Origins. Cambridge: Cambridge University Press. pp. 76-89

Clarkson, C., Jones, S. and Harris, C. (2012) Continuity and change in the lithic industries of the Jurreru Valley, India, before and after the Toba eruption. Quaternary International 258: 165-179

Clarkson, C., Petraglia, M.D., Korisettar, R., Haslam, M., Boivin, N., Crowther, A., Ditchfield, P., Fuller, D., Miracle, P., Harris, C., Connell, K., James, H. and Koshy, J. (2009) The oldest and longest enduring microlithic sequence in India: 
35,000 years of modern human occupation and change at the Jwalapuram Locality 9 rockshelter. Antiquity 83 (320): 326-348

Close, A.E. (2002) Backed bladelets are a foreign country. Archeological Papers of the American Anthropological Association 12 (1): 31-44

Cochrane, G.W.G. (2008) The transition from Howieson's Poort to post-Howieson's Poort industries in Southern Africa. In: Lombard, M., Sievers, C. and Ward, V., eds. Current Themes in Middle Stone Age Research. Cape Town: South African Archaeological Society Goodwin Series 10. pp. 157-167

Cochrane, G.W.G., Doelman, T. and Wadley, L. (2013) Another dating revolution for prehistoric archaeology? Journal of Archaeological Method and Theory 20: 4260

Cotterell, B. and Kamminga, J. (1987) The formation of flakes. American Antiquity 52: 675-708

Courty, M.A., Carbonell, E., Vallverdú Poch, J. and Banerjee, R. (2010) Microstratigraphic and multi-analytical evidence for advanced Neanderthal pyrotechnology at Abric Romani (Capellades, Spain). Quaternary International 247: 294-312

Crassard, R. and Hilbert, Y.H. (2013) A Nubian Complex site from central Arabia: implications for Levallois taxonomy and human dispersals during the Upper Pleistocene. PLOS ONE 8 (7): e69221

de la Peña, P. and Wadley, L. (2014) New knapping methods in the Howiesons Poort at Sibudu. Quaternary International 350: 26-42

Deacon, H.J. (1989) Late Pleistocene palaeoecology and archaeology in the southern Cape, South Africa. In: Mellars, P. and Stringer, C.B., eds. The Human Revolution: Behavioural and Biological Perspectives on the Origin of Modern Humans. Edinburgh: Edinburgh University Press. pp. 547-564

Deacon, H.J. (1992) Southern Africa and modern human origins. Philosophical Transactions of the Royal Society of London. Series B: Biological Sciences 337 (1280): 177-183

Deacon, H.J. (1995) Two Late Pleistocene-Holocene archaeological depositories from the Southern Cape, South Africa. The South African Archaeological Bulletin 50 (162): 121-131

Demeter, F., Shackelford, L.L., Bacon, A., Duringer, P., Westaway, K., Sayavongkhamdy, T., Braga, J., Sichanthongtip, P., Khamdalavong, P., Ponche, J., Wang, H., Lundstrom, C., Patole-Edoumba, E. and Karpoff, A. (2012) Anatomically modern human in Southeast Asia (Laos) by $46 \mathrm{ka}$. Proceedings of the National Academy of Sciences 109 (36): 14375-14380 
Dennell, R. (2014) The fossil record for Homo sapiens between Arabia and Australia. In: Dennell, R. and Porr, M., eds. Southern Asia, Australia and the Search for Human Origins. Cambridge: Cambridge University Press. pp. 33-50

Dennell, R. and Petraglia, M.D. (2012) The dispersal of Homo sapiens across southern Asia: how early, how often, how complex? Quaternary Science Reviews 47: 15-22

Dennell, R., Rendell, H.M., Halim, M. and Moth, E. (1992) A 45,000-year-old openair Paleolithic site at Riwat, northern Pakistan. Journal of Field Archaeology 19 (1): $17-33$

Deraniyagala, P.E.P. (1940) The Stone Age and cave men of Ceylon. Part I. Journal of the Royal Asiatic Society, Ceylon Branch 34 (92): 351-373

Deraniyagala, P.E.P. (1963) Prehistoric archaeology in Ceylon. Asian Perspectives 7 (1-2): 189-192

Deraniyagala, S.U. (1984) Mesolithic stone tool technology at 28,000 BP in Sri Lanka. Ancient Ceylon 5: 105-108

Deraniyagala, S.U. (1986) Pleistocene coastal sediments in the Dry Zone of Sri Lanka: chronology, palaeo-environment and technology. Ancient Ceylon (6): $49-62$

Deraniyagala, S.U. (1992) The Prehistory of Sri Lanka: An Ecological Perspective. Colombo: Department of Archaeological Survey, Government of Sri Lanka

d'Errico, F. (2003) The invisible frontier. A multiple species model for the origin of behavioral modernity. Evolutionary Anthropology 12 (4): 188-202

d'Errico, F. and Backwell, L.R. (2003) Possible evidence of bone tool shaping by Swartkrans early hominids. Journal of Archaeological Science 30 (12): 15591576

d'Errico, F. and Henshilwood, C.S. (2007) Additional evidence for bone technology in the southern African Middle Stone Age. Journal of Human Evolution 52: 142163

d'Errico, F., Vanhaeren, M., Barton, N., Bouzouggar, A., Mienis, H., Richter, D., Hublin, J.J., McPherron, S.P. and Lozouet, P. (2009) Additional evidence on the use of personal ornaments in the Middle Paleolithic of North Africa. Proceedings of the National Academy of Sciences 106 (38): 16051-16056

d'Errico, F., Backwell, L., Villa, P., Degano, I., Lucejko, J.J., Bamford, M.K., Higham, T.F.G., Colombini, M.P. and Beaumont, P.B. (2012) Early evidence of San material culture represented by organic artifacts from Border Cave, South Africa. Proceedings of the National Academy of Sciences 109 (33): 13214-13219 
Dibble, H.L. (1995) Middle Paleolithic scraper reduction: background, clarification, and review of the evidence to date. Journal of Archaeological Method and Theory 2 (4): 299-368

Diekhoff, G. (1992) Statistics for the Social and Behavioural Sciences: Univariate, Bivariate, Multivariate. New York: McGraw-Hill

Diez-Martin, F., Domínguez-Rodrigo, M., Sánchez, P., Mabulla, A.Z.P., Tarriño, A., Barba, R., Prendergast, M.E. and De Luque, L. (2009) The Middle to Later Stone Age technological transition in East Africa: new data from Mumba rockshelter bed $\mathrm{V}$ (Tanzania) and their implications for the origin of modern human behavior. Journal of African Archaeology 7 (2): 147-173

Dorit, R.L., Akashi, H. and Gilbert, W. (1995) Absence of polymorphism at the ZFY locus on the human Y chromosome. Science 268 (5214): 1183-1185

Douka, K., Higham, T.F., Wood, R., Boscato, P., Gambassini, P., Karkanas, P., Peresani, M. and Ronchitelli, A.M. (2014) On the chronology of the Uluzzian. Journal of Human Evolution 68: 1-13

Driscoll, K. (2011) Vein quartz in lithic traditions: an analysis based on experimental archaeology. Journal of Archaeological Science 38: 734-745

Driscoll, K. and García-Rojas, M. (2014) Their lips are sealed: identifying hard stone, soft stone, and antler hammer direct percussion in Palaeolithic prismatic blade production. Journal of Archaeological Science 47: 134-141

Dusseldorp, G.L. (2014) Explaining the Howiesons Poort to post-Howiesons Poort transition: a review of demographic and foraging adaptation models. Azania: Archaeological Research in Africa 49 (3): 317-353

Eerkens, J.W. (1998) Reliable and maintainable technologies: artifact standardization and the Early to Later Mesolithic transition in northern England. Lithic Technology 23 (1): 42-53

Eerkens, J.W. and Bettinger, R.L. (2001) Techniques for assessing standardization in artifact assemblages: can we scale material variability? American Antiquity 66 (3): 493-504

Elston, R.G. and Kuhn, S. eds. (2002) Thinking Small: Global Perspectives on Microlithization. Arlington, VA: American Anthropological Association

Elston, R.G. and Brantingham, P.J. (2002) Microlithic technology in northern Asia: a risk-minimizing strategy of the Late Paleolithic and Early Holocene. Archeological Papers of the American Anthropological Association 12 (1): 103116

Endicott, P. and Ho, Y.W. (2008) A Bayesian evaluation of human mitochondrial substitution rates. The American Journal of Human Genetics 82 (4): 895-902 
Endicott, P., Ho, S.Y.W., Metspalu, M. and Stringer, C. (2009) Evaluating the mitochondrial timescale of human evolution. Trends in Ecology and Evolution 24 (9): 515-521

Eren, M.I., Diez-Martin, F. and Dominguez-Rodrigo, M. (2013) An empirical test of the relative frequency of bipolar reduction in Beds VI, V, and III at Mumba Rockshelter, Tanzania: implications for the East African Middle to Late Stone Age transition. Journal of Archaeological Science 40 (1): 248-256

Evans, J. (1872) The Ancient Stone Implements, Weapons and Ornaments of Great Britain. London: Longmans, Green, Reader and Dyer

Evans, J. (1897) The Ancient Stone Implements, Weapons and Ornaments of Great Britain. 2nd Edition. London: Longmans, Green and Co.

Feathers, J. (2015) Luminescence dating at Diepkloof Rock Shelter - new dates from single-grain quartz. Journal of Archaeological Science : doi:10.1016/j.jas.2015.02.012

Field, J.S. and Lahr, M.M. (2005) Assessment of the southern dispersal: GIS-based analyses of potential routes at Oxygen Isotopic Stage 4. Journal of World Prehistory 19 (1): 1-45

Field, J.S., Petraglia, M.D. and Lahr, M.M. (2007) The southern dispersal hypothesis and the South Asian archaeological record: examination of dispersal routes through GIS analysis. Journal of Anthropological Archaeology 26: 88-108

Fisher, L.E. (2006) Blades and microliths: changing contexts of tool production from Magdalenian to Early Mesolithic in southern Germany. Journal of Anthropological Archaeology 25: 226-238

Flenniken, J.J. (1987) The Paleolithic Dyuktai pressure blade technique of Siberia. Arctic Anthropology 24 (2): 117-132

Foote, R.B. (1866) On the occurrence of stone implements in lateritic formations in various parts of the Madras and North Arcot Districts. The Madras Journal of Literature and Science 3rd Series (Part 2): 1-35

Forster, P. and Matsumura, S. (2005) Did early humans go north or south? Science 308 (5724): 965-966

Franklin, N.R. and Habgood, P.J. (2007) Modern human behaviour and Pleistocene Sahul in review. Australian Archaeology 65: 1-16

Gao, X. and Dennell, R. eds. (2014) Recent Advances in Studies of the Late Pleistocene and Palaeolithic of Northeast Asia. Quaternary International 347

Gatty, R.A. (1895) Pigmy flints. Science-Gossip II: 36-37 
Gibbons, A. (1998) Ancient island tools suggest Homo erectus was a seafarer. Science 279 (5357): 1635-1637

Gibson, N.E., Wadley, L. and Williamson, B.S. (2004) Microscopic residues as evidence of hafting on backed tools from the 60,000 to 68,000 year-old Howiesons Poort layers of Rose Cottage Cave, South Africa. Southern African Humanities 16: 1-11

Gliganic, L.A., Jacobs, Z., Roberts, R.G., Domínguez-Rodrigo, M. and Mabulla, A.Z.P. (2012) New ages for Middle and Later Stone Age deposits at Mumba rockshelter, Tanzania: optically stimulated luminescence dating of quartz and feldspar grains. Journal of Human Evolution 62: 533-547

Glover, I.C. and Lampert, R.J. (1969) Puntutjarpa rockshelter excavations by R.A. Gould: a critical review. Archaeology and Physical Anthropology in Oceania 4 (3): $222-228$

Goebel, T. (2002) The 'microblade adaptation' and recolonization of Siberia during the late Upper Pleistocene. Archeological Papers of the American Anthropological Association 12 (1): 117-131

Gosden, C. (2010) When humans arrived in the New Guinea highlands. Science 330 (6000): 41-42

Gould, R.A. (1969) Puntutjarpa rockshelter: a reply to Messrs. Glover and Lamper. Archaeology and Physical Anthropology in Oceania 4 (3): 229-237

Groucutt, H.S., Scerri, E.M.L., Lewis, L., Clark-Balzan, L., Blinkhorn, J., Jennings, R.P., Parton, A. and Petraglia, M.D. (2015) Stone tool assemblages and models for the dispersal of Homo sapiens out of Africa. Quaternary International : doi:10.1016/j.quaint.2015.01.039

Groucutt, H.S., Petraglia, M.D., Bailey, G., Scerri, E.M.L., Parton, A., Clark-Balzan, L., Jennings, R., Lewis, L., Blinkhorn, J., Drake, N.A., Breeze, P., Boivin, N., Inglis, R.H., Devès, M.H., Meredith-Williams, M., Thomas, M.G. and Scally, A. (in review) Rethinking the dispersal of Homo sapiens out of Africa. Evolutionary Anthropology

Grün, R., Stringer, C., McDermott, F., Nathan, R., Porat, N., Robertson, S., Talor, L., Mortimer, G., Eggins, S. and McCulloch, M. (2005) U-series and ESR analyses of bones and teeth relating to the human burials from Skhul. Journal of Human Evolution 49: 316-334

Guadagnoli, E. and Velicer, W.F. (1988) Relation of sample size to the stability of component patterns. Psychological Bulletin 103 (2): 265-275

Guérin, G., Murray, A.S., Jain, M., Thomsen, K.J. and Mercier, N. (2013) How confident are we in the chronology of the transition between Howieson's Poort and Still Bay? Journal of Human Evolution 64: 314-317 
Habgood, P.J. and Franklin, N.R. (2008) The revolution that didn't arrive: a review of Pleistocene Sahul. Journal of Human Evolution 55 (2): 187-222

Hammer, M.F. (1995) A recent common ancestry for human Y chromosomes. Nature 378: 376-378

Harpending, H.C., Sherry, S.T., Rogers, A.R. and Stoneking, M. (1993) The genetic structure of ancient human populations. Current Anthropology 34 (4): 483-496

Harper, P. (1997) The Middle Stone Age sequences at Rose Cottage Cave: a search for continuity and discontinuity. South African Journal of Science 93 (10): 470475

Hartley, C. (1913) The stone implements of Ceylon. Spolia Zeylanica 9: 117-123

Hartley, C. (1914) On the occurrence of pigmy implements in Ceylon. Spolia Zeylanica 10 (36): 54-67

Haslam, M., Clarkson, C., Petraglia, M., Korisettar, R., Jones, S., Shipton, C., Ditchfield, P. and Ambrose, S.H. (2010) The 74 ka Toba super-eruption and southern Indian hominins: archaeology, lithic technology and environments at Jwalapuram Locality 3. Journal of Archaeological Science 37: 3370-3384

Henry, D.O. (1974) The utilization of the microburin technique in the Levant. Paléorient 2 (2): 389-398

Henshilwood, C.S. (2012) Late Pleistocene techno-traditions in Southern Africa: a review of the Still Bay and Howiesons Poort, c. 75-59 ka. Journal of World Prehistory 25: 205-237

Henshilwood, C.S. and Marean, C.W. (2003) The origin of modern human behavior. Current Anthropology 44 (5): 627-651

Henshilwood, C.S. and Dubreuil, B. (2011) The Still Bay and Howiesons Poort, 7759 ka: symbolic material culture and the evolution of the mind during the African Middle Stone Age. Current Anthropology 52 (3): 361-400

Henshilwood, C., van Niekerk, K.L., Wurz, S., Delagnes, A., Armitage, S.J., Rifkin, R.F., Douze, K., Keene, P., Haaland, M.M., Reynard, R., Discamps, E. and Mienies, S.S. (2014) Klipdrift Shelter, southern Cape, South Africa: preliminary report on the Howiesons Poort layers. Journal of Archaeological Science 45: 284-303

Higham, T., Douka, K., Wood, R., Ramsey, C.B., Brock, F., Basell, L., Camps, M., Arrizabalaga, A., Baena, J., Barroso-Ruiz, C., Bergman, C., Boitard, C., Boscato, P., Caparros, M., Conard, N.J., Draily, C., Froment, A., Galvan, B., Gambassini, P., Garcia-Moreno, A., Grimaldi, S., Haesaerts, P., Holt, B., Iriarte-Chiapusso, M.J., Jelinek, A., Jorda Pardo, J.F., Maillo-Fernandez, J.M., Marom, A., Maroto, J., Menendez, M., Metz, L., Morin, E., Moroni, A., Negrino, F., Panagopoulou, E., Peresani, M., Pirson, S., de la Rasilla, M., Riel-Salvatore, J., Ronchitelli, A., 
Santamaria, D., Semal, P., Slimak, L., Soler, J., Soler, N., Villaluenga, A., Pinhasi, R. and Jacobi, R. (2014) The timing and spatiotemporal patterning of Neanderthal disappearance. Nature 512 (7514): 306-309

Hiscock, P. (1994) Technological responses to risk in Holocene Australia. Journal of World Prehistory 8 (3): 267-292

Hiscock, P. (2002) Pattern and context in the Holocene proliferation of backed artifacts in Australia. Archeological Papers of the American Anthropological Association 12 (1): 163-177

Hiscock, P. (2015) Making it small in the Palaeolithic: bipolar stone-working, miniature artefacts and models of core recycling. World Archaeology 47 (1): 158-169

Hiscock, P., Clarkson, C. and Mackay, A. (2011) Big debates over little tools: ongoing disputes over microliths on three continents. World Archaeology 43 (4): 660-671

Hoggard, C.S. (2014) Revisiting the 'Big Deal about Blades': a full contextualisation of prismatic (volumetric laminar) technology before MIS 5. The Post Hole 35: 28-35

Hole, F. (1959) A critical analysis of the Magosian. The South African Archaeological Bulletin 14 (56): 126-134

Hughes, S.S. (1998) Getting to the point: evolutionary change in prehistoric weaponry. Journal of Archaeological Method and Theory 5 (4): 345-408

Hunt, A. (1911) Prehistoric Lincolnshire. In: Sympson, E.M., ed. Memorials of Old Lincolnshire. London: George Allen \& Sons. pp. 1-23

Igreja, M. and Porraz, G. (2013) Functional insights into the innovative Early Howiesons Poort technology at Diepkloof Rock Shelter (Western Cape, South Africa). Journal of Archaeological Science 40: 3475-3491

Ingman, M., Kaessmann, H., Pääbo, S. and Gyllensten, U. (2000) Mitochondrial genome variation and the origin of modern humans. Nature 408: 708-713

Inizan, M.L., Reduron-Ballinger, M., Roche, H. and Tixier, J. (1999) Technology and Terminology of Knapped Stone. Translated by J. Féblot-Augustins. Nanterre: Cercle de Recherches et d'Etudes Prehistoriques

Jacobs, Z. and Roberts, R.G. (2015) An improved single grain OSL chronology for the sedimentary deposits from Diepkloof Rockshelter, Western Cape, South Africa. Journal of Archaeological Science : doi:10.1016/j.jas.2015.01.023

Jacobs, Z., Roberts, R.G., Galbraith, R.F., Deacon, H.J., Grün, R., Mackay, A., Mitchell, P., Vogelsang, R. and Wadley, L. (2008) Ages for the Middle Stone Age of 
southern Africa: implications for human behavior and dispersal. Science 322 (5902): 733-735

James, H.V.A. (2011) Becoming human: the emergence of modern human behaviour within South Asia. PhD thesis. University of Cambridge

James, H.V.A. and Petraglia, M.D. (2005) Modern human origins and the evolution of behavior in the later Pleistocene record of South Asia. Current Anthropology 46: S3-S27

Jones, S. (2007) A human catastrophe? The impact of the 74,000 year-old supervolcanic eruption of Toba on hominin populations in India. PhD thesis. University of Cambridge

Joordens, J.C.A., Wesselingh, F., de Vos, J., Vonhof, H. and Kroon, D. (2009) Relevance of aquatic environments for hominins: a case study from Trinil (Java, Indonesia). Journal of Human Evolution 57 (6): 656-671

Joordens, J.C.A., d'Errico, F., Wesselingh, F.P., Munro, S., De Vos, J., Wallinga, J., Ankjaergaard, C., Reimann, T., Wijbrans, J.R., Kuiper, K.F., Mücher, H.J., Coqueugniot, H., Prié, V., Joosten, I., Van Os, B., Schulp, A.S., Panuel, M., Van der Haas, V., Lustenhouwer, W., Reijmer, J.J.G. and Roebroeks, W. (2015) Homo erectus at Trinil on Java used shells for tool production and engraving. Nature 518: $228-231$

Kajale, M.D. (1989) Mesolithic exploitation of wild plants in Sri Lanka: archaebotanical study at the cave site of Beli-Lena. In: Harris, D.R. and Hillman, G.C., eds. Foraging and Farming: The Evolution of Plant Exploitation. London: Unwin Hyman Ltd. pp. 269-281

Kaplan, J. (1989) 45,000 years of hunter-gatherer history in Natal as seen from Umhlatuzana Rock Shelter. South African Archaeological Society Goodwin Series 6: 7-16

Kaplan, J. (1990) The Umhlatuzana Rock Shelter sequence: 100,000 years of Stone Age history. Natal Museum Journal of Humanities 2: 1-94

Karmin, M., Saag, L., Vicente, M., Wilson Sayres, M.A., Jarve, M., Talas, U.G., Rootsi, S., Ilumae, A.M., Magi, R., Mitt, M., Pagani, L., Puurand, T., Faltyskova, Z., Clemente, F., Cardona, A., Metspalu, E., Sahakyan, H., Yunusbayev, B., Hudjashov, G., DeGiorgio, M., Loogvali, E.L., Eichstaedt, C., Eelmets, M., Chaubey, G., Tambets, K., Litvinov, S., Mormina, M., Xue, Y., Ayub, Q., Zoraqi, G., Korneliussen, T.S., Akhatova, F., Lachance, J., Tishkoff, S., Momynaliev, K., Ricaut, F.X., Kusuma, P., Razafindrazaka, H., Pierron, D., Cox, M.P., Sultana, G.N., Willerslev, R., Muller, C., Westaway, M., Lambert, D., Skaro, V., Kovacevic, L., Turdikulova, S., Dalimova, D., Khusainova, R., Trofimova, N., Akhmetova, V., Khidiyatova, I., Lichman, D.V., Isakova, J., Pocheshkhova, E., Sabitov, Z., Barashkov, N.A., Nymadawa, P., Mihailov, E., Seng, J.W., Evseeva, I., Migliano, A.B., Abdullah, S., Andriadze, G., Primorac, D., Atramentova, L., Utevska, O., Yepiskoposyan, L., Marjanovic, D., 
Kushniarevich, A., Behar, D.M., Gilissen, C., Vissers, L., Veltman, J.A., Balanovska, E., Derenko, M., Malyarchuk, B., Metspalu, A., Fedorova, S., Eriksson, A., Manica, A., Mendez, F.L., Karafet, T.M., Veeramah, K.R., Bradman, N., Hammer, M.F., Osipova, L.P., Balanovsky, O., Khusnutdinova, E.K., Johnsen, K., Remm, M., Thomas, M.G., Tyler-Smith, C., Underhill, P.A., Willerslev, E., Nielsen, R., Metspalu, M., Villems, R. and Kivisild, T. (2015) A recent bottleneck of Y chromosome diversity coincides with a global change in culture. Genome Research 25 (4): 459-466

Kennedy, K.A.R. (1999) Palaeoanthropology of South Asia. Evolutionary Anthropology 8: 165-185

Kennedy, K.A.R. (2000) God-Apes and Fossil Men. Ann Arbor: University of Michigan Press

Kennedy, K.A.R. and Deraniyagala, S.U. (1989) Fossil remains of 28,000-year-old hominids from Sri Lanka. Current Anthropology 30 (3): 394-399

Kennedy, K.A.R., Disotell, T., Roertgen, W.J., Chiment, J. and Sherry, J. (1986) Biological anthropology of Upper Pleistocene hominids from Sri Lanka: Batadomba Lena and Beli Lena caves. Ancient Ceylon 6: 165-265

Kennedy, K.A.R., Deraniyagala, S.U., Roertgen, W.J., Chiment, J. and Disotell, T. (1987) Upper Pleistocene fossil hominids from Sri Lanka. American Journal of Physical Anthropology 72 (4): 441-461

Klecka, W.R. (1980) Discriminant Analysis. Sage University Paper Series on Quantitative Applications in the Social Sciences. Thousand Oaks, CA: Sage Publications

Klein, R.G. (1992) The archeology of modern human origins. Evolutionary Anthropology 1 (1): 5-14

Klein, R.G. (1995) Anatomy, behavior, and modern human origins. Journal of World Prehistory 9 (2): 167-198

Klein, R.G. (2000) Archeology and the evolution of human behavior. Evolutionary Anthropology 9: 17-36

Klein, R.G. (2009) The Human Career: Human Biological and Cultural Origins. 3rd Edition. Chicago: University of Chicago Press

Knight, J. (1991a) Technological analysis of the anvil (bipolar) technique. Lithics 12: $57-87$

Knight, J. (1991b) Vein Quartz. Lithics 12: 37-56

Korisettar, R. (2007) Toward developing a basin model for Palaeolithic settlement of the Indian subcontinent: geodynamics, monsoon dynamics, habitat diversity 
and dispersal routes. In: Petraglia, M.D. and Allchin, B., eds. The Evolution and History of Human Populations in South Asia: Inter-disciplinary Studies in Archaeology, Biological Anthropology, Linguistics and Genetics. Dordrecht: Springer. pp. 69-96

Kourampas, N., Simpson, I.A., Perera, N. and Deraniyagala, S.U. (2008) Late Pleistocene hunter-gatherers in the South Asian rainforest: geoarchaeology of inhabited rockshelters in southwestern Sri Lanka. Antiquity 82 (316)

Kourampas, N., Simpson, I.A., Perera, N., Deraniyagala, S.U. and Wijeyapala, W.H. (2009) Rockshelter sedimentation in a dynamic tropical landscape: Late Pleistocene-Early Holocene archaeological deposits in Kitulgala Beli-lena, southwestern Sri Lanka. Geoarchaeology 24 (6): 677-714

Krejcie, R.V. and Morgan, D.W. (1970) Determining sample size for research activities. Educational and Psychological Measurement 30: 607-610

Kuhn, S.L. (1990) A geometric index of reduction for unifacial stone tools. Journal of Archaeological Science 17 (5): 583-593

Kuhn, S.L. (2002) Pioneers of microlithization: the "Proto-Aurignacian" of southern Europe. Archeological Papers of the American Anthropological Association 12 (1): 83-93

Kuhn, S.L. and Elston, R.G. (2002) Introduction: thinking small globally. Archeological Papers of the American Anthropological Association 12 (1): 1-7

Langbroek, M. (2011) Trees and ladders: a critique of the theory of human cognitive and behavioural evolution in Palaeolithic archaeology. Quaternary International 270: 4-14

Langley, M.C., Clarkson, C. and Ulm, S. (2011) From small holes to grand narratives: the impact of taphonomy and sample size on the modernity debate in Australia and New Guinea. Journal of Human Evolution 61 (2): 197-208

Larsson, L. and Sjöström, A. (2011) Early Mesolithic flint-tipped arrows from Sweden. Antiquity 85: 330

Leplongeon, A. (2014) Microliths in the Middle and Later Stone Age of eastern Africa: new data from Porc-Epic and Goda Buticha cave sites, Ethiopia. Quaternary International 343: 100-116

Lewis-Williams, J.D. and Pearce, D.G. (2004) San Spirituality: Roots, Expressions and Social Consequences. Cape Town: Double Storey Books

Liu, W., Jin, C., Zhang, Y., Cai, Y., Xing, S., Wu, X., Cheng, H., Edwards, R.L., Pan, W., Qin, D., An, Z., Trinkaus, E. and Wu, X. (2010) Human remains from Zhirendong, South China, and modern human emergence in East Asia. Proceedings of the National Academy of Sciences 107 (45): 19201-19206 
Lombard, M. (2005) The Howiesons Poort of South Africa: what we know, what we think we know, what we need to know. Southern African Humanities 17: 33-55

Lombard, M. (2006) Direct evidence for the use of ochre in the hafting technology of Middle Stone Age tools from Sibudu Cave. Southern African Humanities 18 (1): 57-67

Lombard, M. (2007) The gripping nature of ochre: the association of ochre with Howiesons Poort adhesives and Later Stone Age mastics from South Africa. Journal of Human Evolution 53: 406-419

Lombard, M. (2008) Finding resolution for the Howiesons Poort through the microscope: micro-residue analysis of segments from Sibudu Cave, South Africa. Journal of Archaeological Science 35: 26-41

Lombard, M. (2011) Quartz-tipped arrows older than 60 ka: further use-trace evidence from Sibudu, KwaZulu-Natal, South Africa. Journal of Archaeological Science 38: 1918-1930

Lombard, M. (2012) Thinking through the Middle Stone Age of sub-Saharan Africa. Quaternary International 270: 140-155

Lombard, M. and Parsons, I. (2011) What happened to the human mind after the Howiesons Poort? Antiquity 85: 1433-1443

Lombard, M. and Haidle, M.N. (2012) Thinking a bow-and-arrow set: cognitive implications of Middle Stone Age bow and stone-tipped arrow technology. Cambridge Archaeological Journal 22 (2): 237-264

Lombard, M., Wadley, L., Jacobs, Z., Mohapi, M. and Roberts, R.G. (2010) Still Bay and serrated points from Umhlatuzana Rock Shelter, Kwazulu-Natal, South Africa. Journal of Archaeological Science 37: 1773-1784

Lombard, M., Wadley, L., Deacon, J., Wurz, S., Parsons, I., Mohapi, M., Swart, J. and Mitchell, P. (2012) South African and Lesotho Stone Age sequence updated (I). South African Archaeological Bulletin 67: 120-144

Macaulay, V., Hill, C., Achilli, A., Rengo, C., Clarke, D., Meehan, W., Blackburn, J., Semino, O., Scozzari, R., Cruciani, F., Taha, A., Shaari, N.K., Raja, J.M., Ismail, P., Zainuddin, Z., Goodwin, W., Bulbeck, D., Bandelt, H., Oppenheimer, S., Torroni, A. and Richards, M. (2005) Single, rapid coastal settlement of Asia revealed by analysis of complete mitochondrial genomes. Science 308 (5724): 1034-1036

Mackay, A. (2008) On the production of blades and its relationship to backed artefacts in the Howiesons Poort at Diepkloof, South Africa. Lithic Technology 33 (1): 87-99 
Mackay, A. (2011) Potentially stylistic differences between backed artefacts from two nearby sites occupied $\sim 60,000$ years before present in South Africa. Journal of Anthropological Archaeology 30: 235-245

Mackay, A. and Welz, A. (2008) Engraved ochre from a Middle Stone Age context at Klein Kliphuis in the Western Cape of South Africa. Journal of Archaeological Science 35: 1521-1532

Malan, B.D. (1952) The final phase of the Middle Stone Age in South Africa. In: Leakey, L.S.B. and Cole, S., eds. Proceedings of the Pan-African Congress on Prehistory, 1947. Oxford: Blackwell. pp. 188-194

Mazza, P.P.A., Martini, F., Sala, B., Magi, M., Colombini, M.P., Giachi, G., Landucci, F., Lemorini, C., Modugno, F. and Ribechini, E. (2006) A new Palaeolithic discovery: tar-hafted stone tools in a European Mid-Pleistocene bone-bearing bed. Journal of Archaeological Science 33: 1310-1318

McBrearty, S. (2007) Down with the revolution. In: Mellars, P., Boyle, K., Bar-Yosef, O. and Stringer, C., eds. Rethinking the Human Revolution. Cambridge: McDonald Institute for Archaeological Research. pp. 133-151

McBrearty, S. and Brooks, A.S. (2000) The revolution that wasn't: a new interpretation of the origin of modern human behavior. Journal of Human Evolution 39 (5): 453-563

McCall, G.S. (2006) Multivariate perspectives on change and continuity in the Middle Stone Age lithics from Klasies River Mouth, South Africa. Journal of Human Evolution 51: 429-439

McCall, G.S. (2007) Behavioral ecological models of lithic technological change during the later Middle Stone Age of South Africa. Journal of Archaeological Science 34: 1738-1751

McCall, G.S. and Thomas, J.T. (2012) Still Bay and Howiesons Poort foraging strategies: recent research and models of culture change. African Archaeological Review 29 (1): 1-44

McDermott, F., Grün, R., Stringer, C.B. and Hawkesworth, C.J. (1993) Massspectrometric U-series dates for Israeli Neanderthal/early modern hominid sites. Nature 363: 252-255

McDougall, I., Brown, F.H. and Fleagle, J.G. (2005) Stratigraphic placement and age of modern humans from Kibish, Ethiopia. Nature 433: 733-736

Mellars, P. (1970) Some comments on the notion of 'functional variability' in stone tool assemblages. World Archaeology 2 (1): 74-89

Mellars, P. ed. (1990) The Emergence of Modern Humans: An Archaeological Perspective. New York: Cornell University Press 
Mellars, P. (1996) The Neanderthal Legacy. New Jersey: Princeton University Press

Mellars, P. (2006a) Why did modern human populations disperse from Africa ca. 60,000 years ago? A new model. Proceedings of the National Academy of Sciences 103 (25): 9381-9386

Mellars, P. (2006b) Going East: new genetic and archaeological perspectives on the modern human colonization of Eurasia. Science 313 (5788): 796-800

Mellars, P. (2006c) Archeology and the dispersal of modern humans in Europe: deconstructing the 'Aurignacian'. Evolutionary Anthropology 15: 167-182

Mellars, P. (2007) Rethinking the human revolution: Eurasian and African perspectives. In: Mellars, P., Boyle, K., Bar-Yosef, O. and Stringer, C., eds. Rethinking the Human Revolution. Cambridge: McDonald Institute for Archaeological Research. pp. 1-14

Mellars, P. and Stringer, C. eds. (1989) The Human Revolution: Behavioural and Biological Perspectives on the Origins of Modern Humans. Edinburgh: Edinburgh University Press

Mellars, P., Boyle, K., Bar-Yosef, O. and Stringer, C. eds. (2007) Rethinking the Human Revolution: New Behavioural and Biological Perspectives on the Origin and Dispersal of Modern Humans. Cambridge: McDonald Institute of Archaeological Research

Mellars, P., Gorí, K.C., Carr, M., Soares, P.A. and Richards, M.B. (2013) Genetic and archaeological perspectives on the initial modern human colonization of southern Asia. Proceedings of the National Academy of Sciences 110 (26): 10699-10704

Mercier, N., Valladas, H., Bar-Yosef, O., Vandermeersch, B., Stringer, C. and Joron, J.L. (1993) Thermoluminescence date for the Mousterian burial site of EsSkhul, Mt. Carmel. Journal of Archaeological Science 20: 169-174

Mijares, A.S., Détroit, F., Grün, R., Bellwood, P., Aubert, M., Champion, G., Cuevas, N., De Leon, A. and Dizon, E. (2010) New evidence for a 67,000-year-old human presence at Callao Cave, Luzon, Philippines. Journal of Human Evolution 59 (1): 123-132

Milne, S.B. (2009) Debitage sample size and its implications for understanding lithic assemblage variability. Canadian Journal of Archaeology 33: 40-64

Mishmar, D., Ruiz-Pesini, E., Golik, P., Macaulay, V., Clark, A.G., Hosseini, S., Brandon, M., Easley, K., Chen, E., Brown, M.D., Sukernik, R.I., Olckers, A. and Wallace, D.C. (2003) Natural selection shaped regional mtDNA variation in humans. Proceedings of the National Academy of Sciences 100 (1): 171-176 
Mishra, S., Chauhan, N. and Singhvi, A.K. (2013) Continuity of microblade technology in the Indian subcontinent since $45 \mathrm{ka}$ : implications for the dispersal of modern humans. PLOS ONE 8 (7): e69280

Misra, V.N. (1985) Microlithic industries in India. In: Misra, V. and Bellwood, P., eds. Recent Advances in Indo-Pacific Prehistory: Proceedings of the International Symposium held at Poona, December 19-21, 1978. Leiden: Brill. pp. 111-122

Misra, V.N. (2001) Prehistoric human colonization of India. Journal of Biosciences 26 (4): 491-531

Misra, V.N. and Bellwood, P. eds. (1985) Recent Advances in Indo-Pacific Prehistory: Proceedings of the International Symposium held at Poona, December 19-21, 1978. New Delhi: Oxford \& IBH Publishing Co.

Mitchell, P. (1988a) The Late Pleistocene early microlithic assemblages of southern Africa. World Archaeology 20 (1): 27-39

Mitchell, P. (1988b) The Early Microlithic Assemblages of Southern Africa. Oxford: British Archaeological Reports International Series

Mitchell, P. (2002) The Archaeology of Southern Africa. Cambridge: Cambridge University Press

Mitchell, P. and Steinberg, J.M. (1992) Ntloana Tsoana: a Middle Stone Age sequence from western Lesotho. The South African Archaeological Bulletin 47 (155): 26-33

Mitchell, P. and Arthur, C. (2010) Archaeological fieldwork in the Metolong Dam catchment, Lesotho, 2008-10. Nyame Akuma 74: 51-62

Mitchell, P. and Arthur, C. (2012) The archaeology of the Metolong Dam, Lesotho. The Digging Stick 29 (1): 1-5

Mohapi, M. (2012) Ntloana Tsoana Middle Stone Age lithics: preliminary results of a technological analysis. Unpublished report. University of Johannesburg

Mohapi, M. (2013) The Middle Stone Age point assemblage from Umhlatuzana Rock Shelter: a morphometric study. Southern African Humanities 25: 25-51

Neeley, M.P. (2002) Going microlithic: a Levantine perspective on the adoption of microlithic technologies. Archeological Papers of the American Anthropological Association 12 (1): 45-55

Neeley, M.P. and Barton, C.M. (1994) A new approach to interpreting late Pleistocene microlith industries in southwest Asia. Antiquity 68: 275-288

Norton, C.J. and Jin, J.J.H. (2009) The evolution of modern human behavior in East Asia: current perspectives. Evolutionary Anthropology 18 (6): 247-260 
O'Brien, M.J. (2010) The future of Paleolithic studies: a view from the New World. In: Lycett, S. and Chauhan, P., eds. New Perspectives on Old Stones: Analytical Approaches to Paleolithic Technologies. New York: Springer. pp. 311-334

O'Brien, M.J., Boulanger, M.T., Collard, M., Buchanan, B., Tarle, L., Straus, L.G. and Eren, M.I. (2014) On thin ice: problems with Stanford and Bradley's proposed Solutrean colonisation of North America. Antiquity 88 (340): 606-624

O'Connell, J.F. and Allen, J. (2004) Dating the colonization of Sahul (Pleistocene Australia-New Guinea): a review of recent research. Journal of Archaeological Science 31 (6): 835-853

O'Connor, S., Ono, R. and Clarkson, C. (2011) Pelagic fishing at 42,000 years before the present and the maritime skills of modern humans. Science 334: 11171121

Odell, G.H. (2004) Lithic Analysis. New York: Kluwer Academic/Plenum Publishers

Olley, J.M., Roberts, R.G., Yoshida, H. and Bowler, J.,M. (2006) Single-grain optical dating of grave-infill associated with human burials at Lake Mungo, Australia. Quaternary Science Reviews 25: 2469-2474

Oppenheimer, S. (2009) The great arc of dispersal of modern humans: Africa to Australia. Quaternary International 202 (1-2): 2-13

Ovchinnikov, I.V., Götherström, A., Romanova, G.P., Kharitonov, V.M., Lidén, K. and Goodwin, W. (2000) Molecular analysis of Neanderthal DNA from the northern Caucasus. Nature 404: 490-493

Owen, L. (1988) Blade and microblade technology: selected assemblages from the North American Arctic and Upper Paleolithic of southwest Germany. Oxford: British Archaeological Reports International Series

Parfitt, S.A., Ashton, N.M., Lewis, S.G., Abel, R.L., Coope, G.R., Field, M.H., Gale, R., Hoare, P.G., Larkin, N.R. and Lewis, M.D. (2010) Early Pleistocene human occupation at the edge of the boreal zone in northwest Europe. Nature 466 (7303): 229-233

Perera, N. (2010) Prehistoric Sri Lanka: Late Pleistocene Rockshelters and an OpenAir Site. Oxford: British Archaeological Reports International Series

Perera, N., Kourampas, N., Simpson, I.A., Deraniyagala, S.U., Bulbeck, D., Kamminga, J., Perera, J., Fuller, D.Q., Szabo, K. and Oliveira, N.V. (2011) People of the ancient rainforest: Late Pleistocene foragers at the Batadomba-lena rockshelter, Sri Lanka. Journal of Human Evolution 61 (3): 254-269

Petraglia, M.D., Haslam, M., Fuller, D.Q., Boivin, N. and Clarkson, C. (2010) Out of Africa: new hypotheses and evidence for the dispersal of Homo sapiens along the Indian Ocean rim. Annals of Human Biology 37 (3): 288-311 
Petraglia, M.D., Alsharekh, A.M., Crassard, R., Drake, N.A., Groucutt, H., Parker, A.G. and Roberts, R.G. (2011) Middle Palaeolithic occupation on a Marine Isotope Stage 5 lakeshore in the Nefud Desert, Saudi Arabia. Quaternary Science Reviews 30: 1555-1559

Petraglia, M.D., Korisettar, R., Boivin, N., Clarkson, C., Ditchfield, P., Jones, S., Koshy, J., Lahr, M.M., Oppenheimer, C., Pyle, D., Roberts, R., Schwenninger, J., Arnold, L. and White, K. (2007) Middle Palaeolithic assemblages from the Indian subcontinent before and after the Toba super-eruption. Science 317: 114-116

Petraglia, M.D., Clarkson, C., Boivin, N., Haslam, M., Korisettar, R., Chaubey, G., Ditchfield, P., Fuller, D., James, H., Jones, S., Kivisild, T., Koshy, J., Lahr, M.M., Metspalu, M., Roberts, R. and Arnold, L. (2009a) Population increase and environmental deterioration correspond with microlithic innovations in South Asia ca. 35,000 years ago. Proceedings of the National Academy of Sciences 106 (30): 12261-12266

Petraglia, M.D., Korisettar, R., Bai, M.K., Boivin, N., Janardhana, B., Clarkson, C., Cunningham, K., Ditchfield, P., Fuller, D., Hampson, J., Haslam, M., Jones, S., Koshy, J., Miracle, P., Oppenheimer, C., Roberts, R. and White, K. (2009b) Human occupation, adaptation and behavioral change in the Pleistocene and Holocene of South India: recent investigations in the Kurnool District, Andhra Pradesh. Eurasian Prehistory 6 (1-2): 119-166

Pienaar, M., Woodborne, S. and Wadley, L. (2008) Optically stimulated luminescence dating at Rose Cottage Cave. South African Journal of Science 104: $65-70$

Plug, I. and Engela, R. (1992) The macrofaunal remains from recent excavations at Rose Cottage Cave, Orange Free State. The South African Archaeological Bulletin 47: 16-25

Pole, J. (1907) A few remarks on prehistoric stones in Ceylon. Journal of the Ceylon Branch of the Royal Asiatic Society 19 (58): 272-281

Porraz, G., Texier, P.J., Archer, W., Piboule, M., Rigaud, J.P. and Tribolo, C. (2013) Technological successions in the Middle Stone Age sequence of Diepkloof Rock Shelter, Western Cape, South Africa. Journal of Archaeological Science 40: 3376-3400

Potts, R. (1999) Variability selection in hominid evolution. Evolutionary Anthropology 7 (3): 81-96

Powell, A., Shennan, S. and Thomas, M.G. (2009) Late Pleistocene demography and the appearance of modern human behavior. Science 324 (5932): 1298-1301

Ramsey, C.B. (2014) OxCal 4.2.4 [Online] URL: https://c14.arch.ox.ac.uk/oxcal/OxCal.html (Accessed January 16th, 2015) 
Read, C.H. and Smith, R.A. (1911) A Guide to the Antiquities of the Stone Age in the Department of British and Mediaeval Antiquities. 2nd Edition. London: The British Museum

Renfrew, C. (2008) Neuroscience, evolution and the sapient paradox: the factuality of value and of the sacred. Philosophical Transactions of the Royal Society B: Biological Sciences 363 (1499): 2041-2047

Reyes-Centeno, H., Ghirotto, S., Detroit, F., Grimaud-Herve, D., Barbujani, G. and Harvati, K. (2014) Genomic and cranial phenotype data support multiple modern human dispersals from Africa and a southern route into Asia. Proceedings of the National Academy of Sciences 111 (20): 7248-7253

Roberts, P., Perera, N., Wedage, O., Deraniyagala, S., Perera, J., Eregama, S., Gledhill, A., Petraglia, M.D. and Lee-Thorp, J.A. (2015) Direct evidence for human reliance on rainforest resources in late Pleistocene Sri Lanka. Science 347 (6227): 1246-1249

Robertson, G., Attenbrow, V. and Hiscock, P. (2009) Multiple uses for Australian backed artefacts. Antiquity 83: 296-308

Rose, J.I., Usik, V.I., Marks, A.E., Hilbert, Y.H., Galletti, C.S., Parton, A., Geiling, J.M., Černý, V., Morley, M.W. and Roberts, R.G. (2011) The Nubian Complex of Dhofar, Oman: an African Middle Stone Age industry in southern Arabia. PLOS ONE 6 (11): e28239

Sali, S.A. (1985) The Upper Palaeolithic culture at Patne, District Jalgaon, Maharashtra. In: Misra, V.N. and Bellwood, P., eds. Recent Advances in IndoPacific Prehistory: Proceedings of the International Symposium held at Poona, December 19-21, 1978. New Delhi: Oxford \& IBH Publishing Co. pp. 137-146

Sali, S.A. (1989) The Upper Palaeolithic and Mesolithic Cultures of Maharashtra. Pune: Deccan College Post Graduate and Research Institute

Sarasin, P. and Sarasin, F. (1908) Results of natural history research in Ceylon. Translated by D. Bulbeck. Wiesbaden: C. W. Kreidel's Verlag

Scally, A. and Durbin, R. (2012) Revising the human mutation rate: implications for understanding human evolution. Nature Reviews Genetics 13 (824): 745-753

Scerri, E.M.L., Groucutt, H.S., Jennings, R.P. and Petraglia, M.D. (2014) Unexpected technological heterogeneity in northern Arabia indicates complex Late Pleistocene demography at the gateway to Asia. Journal of Human Evolution 75: $125-142$

Schwarz, H.P., Grün, R., Vandermeersch, B., Bar-Yosef, O., Valladas, H. and Tchernov, E. (1988) ESR dates for the hominid burial site of Qafzeh in Israel. Journal of Human Evolution 17: 733-737 
Seong, C. (2008) Tanged points, microblades and Late Palaeolithic hunting in Korea. Antiquity 82 (318): 871-883

Seong, C. (2009) Emergence of a blade industry and evolution of Late Palaeolithic technology in the Republic of Korea. Journal of Anthropological Research 65 (3): 417-451

Shea, J.J. (2006) The origins of lithic projectile point technology: evidence from Africa, the Levant, and Europe. Journal of Archaeological Science 33: 823-846

Shea, J.J. (2011) Homo sapiens is as Homo sapiens was. Current Anthropology 52 (1): 1-35

Shott, M.J. (1994) Size and form in the analysis of flake debris: Review and recent approaches. Journal of Archaeological Method and Theory 1 (1): 69-110

Singer, R. and Wymer, J. (1982) The Middle Stone Age at Klasies River Mouth in South Africa. Chicago: University of Chicago Press

Smith, V.A. (1906) Pygmy flints. Indian Antiquary: A Journal of Oriental Research XXXV: 185-195

Soares, P., Ermini, L., Thomson, N., Mormina, M., Rito, T., Röhl, A., Salas, A., Oppenheimer, S., Macaulay, V. and Richards, M.B. (2009) Correcting for purifying selection: an improved human mitochondrial molecular clock. The American Journal of Human Genetics 84 (6): 740-459

Soares, P., Alshamali, F., Pereira, J.B., Fernandes, V., Silva, N.M., Afonso, C., Costa, M.D., Musilová, E., Macaulay, V., Richards, M.B., Černý, V. and Pereira, L. (2011) The expansion of mtDNA haplogroup L3 within and out of Africa. Molecular Biology and Evolution 29 (3): 915-927

Soriano, S., Villa, P. and Wadley, L. (2007) Blade technology and tool forms in the Middle Stone Age of South Africa: the Howiesons Poort and post-Howiesons Poort at Rose Cottage Cave. Journal of Archaeological Science 34: 681-703

Spicer, J. (2004) Making Sense of Multivariate Data Analysis. Thousand Oaks, CA: Sage Publications

Stapleton, P. and Hewitt, J. (1927) Stone implements from a rockshelter at Howieson's Poort near Grahamstown. South African Journal of Science 24: 574587

Stapleton, P. and Hewitt, J. (1928) Stone implements from Howieson's Poort, near Grahamstown. South African Journal of Science 25: 399-409

Stoneking, M. and Cann, R.L. (1989) African origin of human mitochondrial DNA. In: Mellars, P. and Stringer, C., eds. The Human Revolution: Behavioural and 
Biological Perspectives on the Origins of Modern Humans. Edinburgh: University of Edinburgh Press. pp. 17-30

Straus, L.G. (2002) Selecting small: microlithic musings for the Upper Paleolithic and Mesolithic of western Europe. Archeological Papers of the American Anthropological Association 12 (1): 69-81

Stringer, C. (2011) The Origin of Our Species. London: Allen Lane

Stringer, C., Grün, R., Schwarz, H.P. and Goldberg, P. (1989) ESR dates for the hominid burial site of Es Skhul in Israel. Nature 338: 756-758

Summerhayes, G.R., Leavesley, M., Fairbairn, A., Mandui, H., Field, J., Ford, A. and Fullagar, R. (2010) Human adaptation and plant use in highland New Guinea 49,000 to 44,000 years ago. Science 330 (6000): 78-81

Texier, P., Porraz, G., Parkington, J., Rigaud, J., Poggenpoel, C., Miller, C., Tribolo, C., Cartwright, C.R., Coudenneau, A., Klein, R., Steele, T. and Verna, C. (2010) A Howiesons Poort tradition of engraving ostrich eggshell containers dated to 60,000 years ago at Diepkloof Rock Shelter, South Africa. Proceedings of the National Academy of Sciences 107 (14): 6180-6185

Thackeray, A.I. (1989) Changing fashions in the Middle Stone Age: the stone artefact sequence from Klasies River main site, South Africa. The African Archaeological Review 7: 33-57

Thackeray, A.I. and Kelly, A.J. (1988) A technological and typological analysis of Middle Stone Age assemblages antecedent to the Howiesons Poort at Klasies River Mouth. South African Archaeological Bulletin 43: 15-26

Thackeray, J.F. (1992) Chronology of Late Pleistocene deposits associated with Homo sapiens at Klasies River Mouth, South Africa. Palaeoecology of Africa and the Surrounding Islands 23: 177-191

Thackeray, J.F. (2009) Chronology, climate and technological innovation associated with the Howieson's Poort and Still Bay industries in South Africa. South African Journal of Science 105: 90

Tixier, J. (1963) Typologie de l'Epipaléolithique du Maghreb. Mémoires du Centre de Recherches Anthropologiques, Préhistoriques et Ethnographiques 2. Paris: Arts et Métiers Graphiques

Torrence, R. (2002) Thinking big about small tools. Archeological Papers of the American Anthropological Association 12 (1): 179-189

Tribolo, C., Mercier, N., Douville, E., Joron, J.L., Reyss, J.L., Dufer, D., Cantin, N., Lefrais, Y., Miller, C.E., Porraz, G., Parkington, J., Rigaud, J.P. and Texier, P.J. (2013) OSL and TL dating of the Middle Stone Age sequence at Diepkloof Rock 
Shelter (South Africa): a clarification. Journal of Archaeological Science 40: 3401-3411

Usik, V.I., Rose, J.I., Hilbert, Y.H., Van Peer, P. and Marks, A.E. (2013) Nubian Complex reduction strategies in Dhofar, southern Oman. Quaternary International 300: 244-266

Valladas, H., Reyss, J.L., Joron, J.L., Valladas, G., Bar-Yosef, O. and Vandermeersch, B. (1988) Thermoluminescence dating of Mousterian 'Proto-Cro-Magnon' remains from Israel and the origin of modern man. Nature 331: 614-616

Valladas, H., Wadley, L., Mercier, N., Froget, L., Tribolo, C., Reyss, J.L. and Joron, J.L. (2005) Thermoluminescence dating on burnt lithics from Middle Stone Age layers at Rose Cottage Cave. South African Journal of Science 101: 169-174

Vaquero, M., Vallverdú, J., Rosell, J., Pastó, I. and Allué, E. (2001) Neandertal behavior at the middle Palaeolithic site of Abric Romani, Capellades, Spain. Journal of Field Archaeology 28 (1-2): 1-2

Vigilant, L., Stoneking, M., Harpending, H., Hawkes, K. and Wilson, A.C. (1991) African populations and the evolution of human mitochondrial DNA. Science 253 (5027): 1503-1507

Villa, P., Soriano, S., Teyssandier, N. and Wurz, S. (2010) The Howiesons Poort and MSA III at Klasies River main site, Cave 1A. Journal of Archaeological Science 37: 630-655

Villa, P., Soriano, S., Tsanova, T., Degano, I., Higham, T.F.G., d'Errico, F., Backwell, L., Lucejko, J.J., Colombini, M.P. and Beaumont, P.B. (2012) Border Cave and the beginning of the Later Stone Age in South Africa. Proceedings of the National Academy of Sciences 109 (33): 13208-13213

Wadley, L. (1991) Rose Cottage Cave: background and a preliminary report on the recent excavations. The South African Archaeological Bulletin 46 (154): 125130

Wadley, L. (1993) The Pleistocene Later Stone Age south of the Limpopo river. Journal of World Prehistory 7 (3): 243-296

Wadley, L. (1997) Rose Cottage Cave: archaeological work 1987 to 1997. South African Journal of Science 93 (10): 439-444

Wadley, L. (2001) What is cultural modernity? A general view and a South African perspective from Rose Cottage Cave. Cambridge Archaeological Journal 11 (2): 201-221

Wadley, L. (2005) A typological study of the final Middle Stone Age stone tools from Sibudu Cave, Kwazulu-Natal. The South African Archaeological Bulletin 60 (182): $51-63$ 
Wadley, L. (2006) Partners in grime: results of multi-disciplinary archaeology at Sibudu Cave. Southern African Humanities 18 (1): 315-341

Wadley, L. (2008) The Howieson's Poort industry of Sibudu Cave. South African Archaeological Society Goodwin Series 10: 122-132

Wadley, L. and Harper, P. (1989) Rose Cottage Cave revisited: Malan's Middle Stone Age collection. The South African Archaeological Bulletin 44 (149): 23-32

Wadley, L. and Jacobs, Z. (2006) Sibudu Cave: background to the excavations, stratigraphy and dating. Southern African Humanities 18 (1): 1-26

Wadley, L. and Mohapi, M. (2008) A segment is not a monolith: evidence from the Howiesons Poort of Sibudu, South Africa. Journal of Archaeological Science 35 (9): 2594-2605

Wadley, L., Hodgskiss, T. and Grant, M. (2009) Implications for complex cognition from the hafting of tools with compound adhesives in the Middle Stone Age, South Africa. Proceedings of the National Academy of Sciences 106 (24): 95909594

Wayland, E.J. (1919) Outlines of the Stone Age of Ceylon. Spolia Zeylanica 11 (41): 85-125

White, T.D., Asfaw, B., DeGusta, D., Gilbert, H., Richards, G.D., Suwa, G. and Howell, F.C. (2003) Pleistocene Homo sapiens from Middle Awash, Ethiopia. Nature 423: $742-747$

Wijeyapala, W.H. (1997) New light on the prehistory of Sri Lanka in the context of recent investigations of cave sites. PhD thesis. University of Peradeniya

Willoughby, P.R. (2007) The Evolution of Modern Humans in Africa: A Comprehensive Guide. New York: Altamira

Wilmsen, E.N. (1968) Functional analysis of flaked stone artifacts. American Antiquity 33 (2): 156-161

Wurz, S. (1999) The Howiesons Poort backed artefacts from Klasies River: an argument for symbolic behaviour. The South African Archaeological Bulletin 54: $38-50$

Wurz, S. (2013) Technological trends in the Middle Stone Age of South Africa between MIS 7 and MIS 3. Current Anthropology 54 (S8): S305-S319

Wurz, S. (2014) Southern and East African Middle Stone Age: geography and culture. In: Smith, C., ed. Encyclopedia of Global Archaeology. New York: Springer. pp. 6890-6912 
Yaroshevich, A., Nadel, D. and Tsatskin, A. (2013) Composite projectiles and hafting technologies at Ohalo II (23 ka, Israel): analyses of impact fractures, morphometric characteristics and adhesive remains on microlithic tools. Journal of Archaeological Science 40: 4009-4023

Yaroshevich, A., Kaufman, D., Nuzhnyy, D., Bar-Yosef, O. and Weinstein-Evron, M. (2010) Design and performance of microlith implemented projectiles during the Middle and the Late Epipalaeolithic of the Levant: experimental and archaeological evidence. Journal of Archaeological Science 37: 368-388

Yesner, D.R. and Pearson, G. (2002) Microblades and migrations: ethnic and economic models in the peopling of the Americas. Archeological Papers of the American Anthropological Association 12 (1): 133-161

Zaidner, Y., Ronen, A. and Burdukiewicz, J.M. (2003) The Lower Palaeolithic microlithic industry of Bizat Ruhama, Israel. L'Anthropologie 107 (2): 203-222

Zeuner, F.F. and Allchin, B. (1956) The microlithic sites of Tinnevelly District, Madras State. Ancient India 12: 4-20

Ziegler, M., Simon, M.H., Hall, I.R., Barker, S., Stringer, C. and Zahn, R. (2013) Development of Middle Stone Age innovation linked to rapid climate change. Nature Communications 4 (1905): doi:10.1038/ncomms2897

Zilhão, J., Trinkaus, E., Constantin, S., Milota, S., Gherase, M., Sarcina, L., Danciu, A., Rougier, H., Quilès, J. and Rodrigo, R. (2007) The Peștera cu Oase people, Europe's earliest modern humans. In: Mellars, P., Boyle, K., Bar-Yosef, O. and Stringer, C., eds. Rethinking the Human Revolution: New Behavioural and Biological Perspectives on the Origin and Dispersal of Modern Humans. Cambridge: McDonald Institute of Archaeology Monographs. pp. 249-262 


\title{
Appendix A: Supplementary data tables
}

\author{
A.1 Rose Cottage Cave, South Africa
}

\section{CORES}

\begin{tabular}{lrrrr}
\hline Statistic & Min & \multicolumn{1}{c}{ Max } & Mean & Std. Dev. \\
\hline Longest dimension (mm) & 14.6 & 101.4 & 31.8 & 9.04 \\
Longest axis (mm) & 11.5 & 75.8 & 29.3 & 8.50 \\
Longest face (mm) & 12.3 & 73.7 & 28.4 & 8.51 \\
Maximum width (mm) & 11.1 & 101.0 & 23.5 & 8.91 \\
Maximum thickness (mm) & 5.3 & 47.3 & 12.8 & 5.15 \\
Weight (g) & 1.4 & 300.0 & 11.6 & 23.23 \\
Cubic volume (cm ${ }^{3}$ ) & 1.3 & 361.6 & 11.7 & 27.64 \\
Largest striking platform width (mm) & 3.6 & 77.5 & 15.9 & 7.84 \\
Largest striking platform thickness (mm) & 0.8 & 68.2 & 8.2 & 6.22 \\
Largest striking platform area $\left(\mathrm{cm}^{2}\right)$ & 0.1 & 52.8 & 1.7 & 3.82 \\
\hline
\end{tabular}

Table A.1.1: Core size measurements at RCC

\begin{tabular}{llrrr}
\hline Core type & & Mean & Std. Dev. & CV \\
\hline Uni-directional: & Blade & 29.5 & 6.26 & 21.23 \\
& Flake & 26.7 & 6.34 & 23.70 \\
& Bipolar & 24.3 & 6.65 & 27.37 \\
& & & & \\
Bi-directional: & Blade & 30.1 & 6.76 & 22.43 \\
& Flake & 29.2 & 7.38 & 25.32 \\
& & & & \\
Multi-directional: & Radial & 29.5 & 4.15 & 14.08 \\
& Amorphous & 34.0 & 16.05 & 47.15 \\
\hline
\end{tabular}

Table A.1.2: Longest axis length statistics of core types at RCC 


\begin{tabular}{|c|c|c|c|c|}
\hline Core type & & No. striking platforms & Frequency & \% of type \\
\hline \multirow[t]{4}{*}{ Bi-directional: } & Blade & 2 - opposite & 34 & 97.1 \\
\hline & & 2 - perpendicular & 1 & 2.9 \\
\hline & Flake & 2 - opposite & 20 & 71.4 \\
\hline & & 2 - perpendicular & 8 & 28.6 \\
\hline \multirow[t]{6}{*}{ Multi-directional: } & Radial & 3 & 1 & 3.8 \\
\hline & & 4 & 3 & 11.5 \\
\hline & & Circumference & 22 & 84.6 \\
\hline & Amorphous & 3 & 22 & 84.6 \\
\hline & & 4 & 3 & 11.5 \\
\hline & & 6 & 1 & 3.8 \\
\hline
\end{tabular}

Table A.1.3: Numbers of striking platforms on core types at RCC

\begin{tabular}{llrr|rrrr}
\hline \multirow{2}{*}{$\begin{array}{l}\text { Core type } \\
\end{array}$} & Per core: & \multicolumn{2}{c}{ Flake scars } & \multicolumn{3}{c}{ Blade scars } & \multicolumn{2}{c}{ Scar length (mm) } \\
& & $\begin{array}{r}\text { Mean } \\
\text { no. }\end{array}$ & $\begin{array}{r}\text { Std. } \\
\text { Dev. }\end{array}$ & Mean & Std. & Mean & Std. Dev. \\
& & 1.5 & 1.39 & 3.9 & 1.40 & 20.5 & 4.33 \\
\hline Uni-directional: & Blade & 3.7 & 1.22 & 1.0 & 0.93 & 15.7 & 3.77 \\
& Flake & 3.3 & 2.27 & 3.9 & 2.22 & 16.6 & 4.59 \\
& Bipolar & & & & & & \\
Bi-directional: & Blade & 2.5 & 1.46 & 4.6 & 1.75 & 18.7 & 4.47 \\
& Flake & 5.1 & 2.29 & 1.6 & 1.07 & 16.9 & 3.80 \\
& & & & & & & \\
Multi-directional: & Radial & 6.8 & 2.80 & 1.2 & 1.63 & 11.9 & 2.77 \\
& Amorphous & 8.1 & 4.34 & 1.4 & 1.81 & 15.2 & 5.69 \\
\hline
\end{tabular}

Table A.1.4: Removal scar numbers and average lengths on core types at RCC 


\begin{tabular}{|c|c|c|c|c|}
\hline Core type & Mean per core: & $\begin{array}{r}\text { No. scars } \\
\text { per } \mathrm{cm}^{3}\end{array}$ & $\begin{array}{r}\text { Average scar length } \\
\text { per } \mathrm{cm}^{3}(\mathrm{~mm})\end{array}$ & 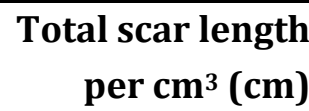 \\
\hline \multirow[t]{3}{*}{ Uni-directional: } & Blade & 0.9 & 3.4 & $\overline{1.8}$ \\
\hline & Flake & 0.6 & 1.8 & 0.9 \\
\hline & Bipolar & 2.4 & 5.4 & 3.8 \\
\hline \multirow[t]{2}{*}{ Bi-directional: } & Blade & 1.2 & 3.0 & 2.1 \\
\hline & Flake & 0.8 & 2.1 & 1.3 \\
\hline \multirow[t]{2}{*}{ Multi-directional: } & Radial & 1.1 & 1.6 & 1.2 \\
\hline & Amorphous & 1.1 & 1.6 & 1.3 \\
\hline
\end{tabular}

Table A.1.5: Ratios of removal scar numbers and lengths to core volume at RCC

DEBITAGE

\begin{tabular}{lrr}
\hline Condition & Frequency & $\mathbf{\%}$ \\
\hline Whole & 919 & 81.5 \\
Proximal & 93 & 8.2 \\
Distal & 55 & 4.9 \\
Longitudinal & 39 & 3.5 \\
Medial & 22 & 2.0 \\
\hline Total & 1128 & \\
\hline
\end{tabular}

Table A.1.6: Numbers and proportions of broken and whole debitage at RCC

\begin{tabular}{lrrrr}
\hline Statistic & Minimum & Maximum & Mean & Std. Dev. \\
\hline Weight (g) & 0.1 & 76.9 & 2.8 & 4.25 \\
Longest dimension (mm) & 13.3 & 128.1 & 32.9 & 10.02 \\
Oriented length (mm) & 11.8 & 128.1 & 31.7 & 9.96 \\
Maximum width (mm) & 4.5 & 47.0 & 15.3 & 6.87 \\
Maximum thickness (mm) & 1.4 & 18.2 & 5.2 & 2.33 \\
Dorsal surface area (cm $\left.{ }^{2}\right)$ & 0.7 & 53.5 & 5.1 & 4.06 \\
Volume (cm ${ }^{3}$ ) & 0.1 & 97.2 & 3.4 & 5.38 \\
Striking platform width (mm) & 1.4 & 36.5 & 8.4 & 5.72 \\
Striking platform thickness (mm) & 0.4 & 14.7 & 3.0 & 2.19 \\
Striking platform area (mm $\left.{ }^{2}\right)$ & 1.0 & 417.6 & 35.4 & 52.02 \\
\hline
\end{tabular}

Table A.1.7: Size measurements of whole debitage at RCC 


\begin{tabular}{llrrr}
\hline Raw material & Measurement & Mean & Std. Dev. & Kurtosis \\
\hline CCS and claystone & Oriented length $(\mathrm{mm})$ & 29.8 & 7.29 & 2.89 \\
& Weight $(\mathrm{g})$ & 2.0 & 1.96 & 19.14 \\
& Dorsal surface area $\left(\mathrm{cm}^{2}\right)$ & 4.2 & 2.21 & 9.12 \\
& Volume $\left(\mathrm{cm}^{3}\right)$ & 2.3 & 2.36 & 32.65 \\
& & & \\
Hornfels, quartz, & Oriented length $(\mathrm{mm})$ & 40.1 & 14.91 & 6.58 \\
quartzite, silcrete and & Weight $(\mathrm{g})$ & 6.5 & 8.14 & 35.72 \\
siltstone & Dorsal surface area $\left(\mathrm{cm}^{2}\right)$ & 9.6 & 6.68 & 12.37 \\
& Volume $\left(\mathrm{cm}^{3}\right)$ & 8.4 & 10.23 & 35.67 \\
\hline
\end{tabular}

Table A.1.8: Size measurements of whole debitage by raw material groupings at RCC

\begin{tabular}{lrrr}
\hline Dorsal cortex (\%) & \% of blades & \% of flake-blades & \% of flakes \\
\hline 0 & 74.3 & 67.9 & 66.5 \\
5 & 4.7 & 3.2 & 1.6 \\
10 & 5.6 & 4.3 & 3.2 \\
20 & 2.6 & 2.9 & 1.1 \\
30 & 4.2 & 1.4 & 2.2 \\
40 & 2.3 & 1.4 & 2.2 \\
50 & 4.1 & 4.0 & 3.2 \\
60 & 1.1 & 0.7 & 0.5 \\
70 & 0.8 & 0.4 & 2.2 \\
80 & 0.3 & 0.4 & 1.1 \\
90 & 0.3 & 2.2 & 1.6 \\
100 & & 11.2 & 14.6 \\
\hline Average cortex cover (\%): & 7 & 18 & 23 \\
\hline
\end{tabular}

Table A.1.9: Proportions of dorsal surface cortex cover on debitage at RCC 


\begin{tabular}{|c|c|c|c|c|c|}
\hline Debitage type & Statistic & Minimum & Maximum & Mean & Std. Dev. \\
\hline \multirow[t]{4}{*}{ Blades } & No. dorsal scars & 1 & 10 & 3.3 & 1.35 \\
\hline & No. parallel arrises & 1 & 4 & 1.5 & 0.59 \\
\hline & Scars per $\mathrm{cm}^{2}$ & 0.1 & 4.1 & 1.0 & 0.56 \\
\hline & Arrises per $\mathrm{cm}^{2}$ & $<0.1$ & 1.7 & 0.5 & 0.28 \\
\hline \multirow[t]{4}{*}{ Flake-blades } & No. dorsal scars & 1 & 8 & 3.3 & 1.29 \\
\hline & No. parallel arrises & 0 & 4 & 1.3 & 0.59 \\
\hline & Scars per $\mathrm{cm}^{2}$ & 0.1 & 2.0 & 0.7 & 0.43 \\
\hline & Arrises per $\mathrm{cm}^{2}$ & 0 & 1.1 & 0.3 & 0.20 \\
\hline \multirow[t]{4}{*}{ Flakes } & No. dorsal scars & 1 & 8 & 3.5 & 1.59 \\
\hline & No. parallel arrises & 0 & 2 & 0.1 & 0.36 \\
\hline & Scars per $\mathrm{cm}^{2}$ & 0.1 & 2.7 & 0.6 & 0.41 \\
\hline & Arrises per $\mathrm{cm}^{2}$ & 0 & 0.9 & $<0.1$ & 0.11 \\
\hline
\end{tabular}

Table A.1.10: Dorsal scar characteristics of debitage types at RCC

\begin{tabular}{lrrrr}
\hline Raw material & Blades (\%) & Flake-blade (\%) & Flake (\%) & Total (\%) \\
\hline Jasper & 11.9 & 36.6 & 21.4 & 18.3 \\
Chalcedony & 9.2 & 15.6 & 26.3 & 13.5 \\
Agate & 7.3 & 8.6 & 21.6 & 11.0 \\
Chert & & 11.1 & 14.3 & 5.6 \\
\hline Overall \% & 8.0 & 17.3 & 15.1 & 11.4
\end{tabular}

Table A.1.11: Proportions of recycled lithics in each raw material and debitage category at RCC 
TOOLS

\begin{tabular}{lrrrr}
\hline Statistic & Minimum & Maximum & Mean & Std. Dev. \\
\hline Weight (g) & 0.3 & 5.8 & 1.5 & 1.09 \\
Longest dimension (mm) & 16.7 & 46.8 & 29.7 & 6.15 \\
Oriented length (mm) & 12.5 & 46.8 & 28.4 & 6.34 \\
Maximum width (mm) & 6.5 & 34.9 & 12.5 & 4.46 \\
Maximum thickness (mm) & 2.1 & 8.4 & 4.1 & 1.41 \\
Dorsal surface area (cm $\left.{ }^{2}\right)$ & 1.1 & 8.4 & 3.6 & 1.55 \\
Volume (cm ${ }^{3}$ ) & 0.3 & 6.0 & 1.6 & 1.18 \\
Striking platform width (mm) & 1.8 & 18.3 & 5.7 & 3.63 \\
Striking platform thickness (mm) & 0.8 & 7.2 & 2.0 & 1.25 \\
Striking platform area $\left(\mathrm{mm}^{2}\right)$ & 1.7 & 121.4 & 15.2 & 21.95 \\
\hline
\end{tabular}

Table A.1.12: Microlith size measurements at RCC

\begin{tabular}{lrrrr}
\hline Statistic & Minimum & Maximum & Mean & Std. Dev. \\
\hline Weight (g) & 1.4 & 55.1 & 7.7 & 8.14 \\
Longest dimension (mm) & 26.6 & 76.0 & 44.5 & 10.38 \\
Oriented length (mm) & 23.9 & 72.9 & 43.0 & 10.89 \\
Maximum width (mm) & 7.5 & 42.5 & 21.9 & 7.09 \\
Maximum thickness (mm) & 2.8 & 18.1 & 7.6 & 2.86 \\
Dorsal surface area (cm $\left.{ }^{2}\right)$ & 2.8 & 26.5 & 9.7 & 4.92 \\
Volume (cm ${ }^{3}$ ) & 1.2 & 48.0 & 8.3 & 7.64 \\
Striking platform width (mm) & 3.2 & 27.7 & 12.8 & 6.78 \\
Striking platform thickness (mm) & 1.1 & 11.2 & 4.7 & 2.64 \\
Striking platform area $\left(\mathrm{mm}^{2}\right)$ & 3.8 & 309.6 & 74.8 & 71.61 \\
\hline
\end{tabular}

Table A.1.13: Non-microlithic tool size measurements at RCC 


\begin{tabular}{|c|c|c|c|c|c|c|c|c|c|c|c|c|c|c|c|}
\hline \multirow[b]{2}{*}{ Tool type } & \multicolumn{5}{|c|}{ Oriented length (mm) } & \multicolumn{5}{|c|}{ Maximum width (mm) } & \multicolumn{5}{|c|}{ Maximum thickness (mm) } \\
\hline & Min & Max & Mean & SD & $\mathbf{C V}$ & Min & $\operatorname{Max}$ & Mean & SD & $\mathbf{C V}$ & Min & $\operatorname{Max}$ & Mean & SD & $\mathbf{C V}$ \\
\hline \multicolumn{16}{|l|}{ Microliths: } \\
\hline Non-geometric point & 12.5 & 41.3 & 28.8 & 8.39 & 29.13 & 10.2 & 24.4 & 15.3 & 4.33 & 28.32 & 2.3 & 8.1 & 4.9 & 1.85 & 37.42 \\
\hline Backed non-geometric & 13.7 & 46.8 & 28.7 & 6.94 & 24.20 & 6.5 & 17.7 & 12.0 & 3.09 & 25.64 & 2.2 & 6.2 & 4.1 & 1.18 & 28.66 \\
\hline Backed non-geometric point & 17.5 & 45.3 & 28.0 & 5.23 & 18.70 & 6.6 & 17.2 & 10.8 & 2.61 & 24.06 & 2.2 & 5.7 & 3.7 & 0.97 & 26.15 \\
\hline Crescent & 21.9 & 43.9 & 30.5 & 6.43 & 21.11 & 7.4 & 20.1 & 12.5 & 3.76 & 30.20 & 2.8 & 7.0 & 4.5 & 1.37 & 30.74 \\
\hline Rectangle & 16.5 & 28.6 & 22.5 & - & - & 6.5 & 15.1 & 10.3 & - & - & 2.3 & 4.5 & 3.5 & - & - \\
\hline Trapeze & 27.7 & 45.7 & 37.4 & - & - & 7.3 & 17.1 & 12.9 & - & - & 3.0 & 8.4 & 4.7 & - & - \\
\hline Shouldered/tanged & 16.3 & 30.1 & 24.8 & 5.48 & 22.09 & 7.2 & 19.3 & 12.9 & 4.35 & 33.86 & 2.3 & 6.2 & 4.1 & 1.61 & 39.26 \\
\hline Preform & 20.3 & 37.6 & 28.4 & 4.81 & 16.92 & 9.8 & 26.3 & 13.5 & 5.35 & 39.74 & 2.1 & 7.3 & 3.9 & 1.62 & 41.75 \\
\hline \multicolumn{16}{|l|}{ Non-microlithic tools: } \\
\hline Side retouched & 24.4 & 72.9 & 40.7 & 10.13 & 24.89 & 7.5 & 34.3 & 20.9 & 5.84 & 27.98 & 3.7 & 14.9 & 7.7 & 2.62 & 34.07 \\
\hline Side and end retouched & 32.5 & 71.6 & 52.1 & 12.94 & 24.84 & 22.5 & 42.5 & 32.1 & 6.92 & 21.52 & 7.1 & 18.1 & 11.2 & 3.92 & 35.12 \\
\hline End retouched & 23.9 & 44.8 & 33.4 & - & - & 14.9 & 33.4 & 23.5 & - & - & 3.5 & 10.2 & 6.3 & - & - \\
\hline Burin & 47.8 & 60.3 & 54.1 & - & - & 17.4 & 21.4 & 19.4 & - & - & 5.7 & 6.5 & 6.1 & - & - \\
\hline Notched/denticulated & 30.1 & 42.1 & 35.6 & - & - & 10.7 & 19.8 & 14.3 & - & - & 3.8 & 7.7 & 5.4 & - & - \\
\hline Unifacial point & 35.1 & 58.5 & 47.2 & 8.07 & 17.12 & 17.9 & 32.3 & 24.9 & 4.83 & 19.40 & 4.6 & 13.0 & 8.3 & 2.12 & 25.60 \\
\hline Other point & 24.8 & 69.6 & 46.2 & 12.74 & 27.56 & 11.3 & 30.8 & 18.8 & 7.69 & 40.87 & 2.8 & 12.2 & 6.2 & 2.77 & 44.65 \\
\hline Misc. & 33.4 & 38.3 & 35.9 & - & - & 9.1 & 16.4 & 12.7 & - & - & 4.7 & 6.5 & 5.6 & - & - \\
\hline
\end{tabular}

Table A.1.14: Length, width and thickness measurements of tool typologies at RCC. SD and CV values for counts of 5 or fewer are excluded 


\begin{tabular}{lrrrrr}
\hline Tool type & Minimum & Maximum & Mean & Std. Dev. & CV \\
\hline Microliths: & & & & & \\
Non-geometric & 12.1 & 129.1 & 53.0 & 39.93 & 75.32 \\
Non-geometric point & 14.9 & 72.8 & 40.1 & 22.54 & 56.27 \\
Backed non-geometric & 9.0 & 47.4 & 25.3 & 10.81 & 42.75 \\
Backed non-geometric point & 8.6 & 41.1 & 20.5 & 8.82 & 42.97 \\
Crescent & 11.8 & 59.3 & 29.3 & 15.74 & 53.79 \\
Rectangle & 11.2 & 34.1 & 18.5 & - & - \\
Trapeze & 11.1 & 71.5 & 32.9 & - & - \\
Shouldered/tanged & 8.4 & 59.9 & 28.7 & 19.04 & 66.41 \\
Preform & 11.2 & 95.8 & 29.1 & 26.34 & 90.64 \\
& & & & & \\
$\quad$ Non-microlithic tools: & & & & & \\
Side retouched & 30.4 & 239.8 & 83.9 & 48.39 & 57.70 \\
Side and end retouched & 103.2 & 335.1 & 186.5 & 96.51 & 51.75 \\
End retouched & 26.2 & 170.6 & 82.1 & - & - \\
Burin & 56.4 & 61.3 & 58.9 & - & - \\
Notched/denticulated & 20.5 & 75.8 & 41.7 & - & - \\
Unifacial point & 51.5 & 210.3 & 105.0 & 41.40 & 39.42 \\
Other point & 16.2 & 170.9 & 64.2 & 50.48 & 78.64 \\
Misc. & 29.6 & 170.6 & 82.1 & - & - \\
\hline Table A.1.15: TCSA measur.
\end{tabular}

Table A.1.15: TCSA measurements of tool typologies at RCC

\begin{tabular}{lrrrrr}
\hline Haft measurement & Minimum & Maximum & Mean & Std. Dev. & CV \\
\hline Width (mm) & 4.2 & 10.1 & 6.6 & 2.04 & 30.79 \\
Thickness (mm) & 2.2 & 3.0 & 2.5 & 0.35 & 13.99 \\
Length (mm) & 1.9 & 11.5 & 7.8 & 3.68 & 47.37 \\
\hline
\end{tabular}

Table A.1.16: Microlith haft measurements at RCC 


\begin{tabular}{|c|c|c|}
\hline & Microliths (\%) & Non-microlithic tools (\%) \\
\hline \multicolumn{3}{|l|}{ No. worked edges: } \\
\hline 1 & 90.2 & 69.4 \\
\hline 2 & 9.1 & 27.8 \\
\hline 3 & 0.8 & 1.4 \\
\hline 4 & & 1.4 \\
\hline \multicolumn{3}{|l|}{ Retouch location: } \\
\hline Circumference & & 3.1 \\
\hline Distal & 2.1 & 3.1 \\
\hline Distal and lateral & 11.6 & 4.1 \\
\hline Distal, lateral and lateral & 1.4 & 1.0 \\
\hline Lateral & 71.2 & 63.9 \\
\hline Lateral and lateral & 2.1 & 9.3 \\
\hline Proximal & 6.8 & 12.4 \\
\hline Proximal and lateral & 4.1 & 1.0 \\
\hline Proximal, distal and lateral & 0.7 & 1.0 \\
\hline Proximal, lateral and lateral & & 1.0 \\
\hline \multicolumn{3}{|l|}{ Retouch face: } \\
\hline Alternating & & 1.0 \\
\hline Bifacial & & 1.0 \\
\hline Dorsal & 31.5 & 78.4 \\
\hline Edge & 58.9 & 4.1 \\
\hline Ventral & 9.6 & 15.5 \\
\hline \multicolumn{3}{|l|}{ Retouch outline: } \\
\hline Concave & & 4.1 \\
\hline Convex & 46.6 & 23.7 \\
\hline Irregular & 3.4 & 3.1 \\
\hline Perpendicular & 2.1 & \\
\hline Pointed & 2.1 & 10.3 \\
\hline Straight & 45.9 & 58.8 \\
\hline
\end{tabular}

Table A.1.17: Retouch characteristics of microliths and non-microlithic tools at RCC. Number of worked edges is as a percentage of the number of tools. Other measures are a percentage of the total number of incidences of retouch. 


\begin{tabular}{|c|c|c|c|c|c|c|c|c|c|c|c|c|c|c|c|}
\hline \multirow[b]{2}{*}{ Tool type } & \multicolumn{5}{|c|}{ Retouch length (mm) } & \multicolumn{5}{|c|}{ Retouch depth (mm) } & \multicolumn{5}{|c|}{ Retouch angle $\left({ }^{\circ}\right)$} \\
\hline & Min & Max & Mean & SD & CV & Min & Max & Mean & SD & CV & Min & Max & Mean & SD & CV \\
\hline \multicolumn{16}{|l|}{ Microliths: } \\
\hline Non-geometric & 12.5 & 68.7 & 32.4 & 19.03 & 58.77 & 0.7 & 8.0 & 2.9 & 2.16 & 73.72 & 20 & 85 & 46 & 22.92 & 49.44 \\
\hline Non-geometric point & 16.1 & 68.9 & 34.9 & 17.40 & 49.80 & 0.5 & 5.9 & 2.6 & 1.71 & 65.10 & 20 & 65 & 47 & 18.11 & 38.95 \\
\hline Backed non-geometric & 11.7 & 39.9 & 21.4 & 7.20 & 33.73 & 1.0 & 6.3 & 3.3 & 1.53 & 46.02 & 58 & 90 & 80 & 9.19 & 11.48 \\
\hline Backed non-geometric point & 9.4 & 45.4 & 21.5 & 7.61 & 35.39 & 1.1 & 5.5 & 3.1 & 1.31 & 41.68 & 50 & 90 & 81 & 8.19 & 10.13 \\
\hline Crescent & 13.7 & 52.3 & 31.4 & 12.11 & 38.56 & 1.2 & 7.4 & 3.6 & 1.90 & 52.52 & 48 & 90 & 81 & 10.71 & 13.26 \\
\hline Rectangle & 14.9 & 25.3 & 18.9 & - & - & 1.3 & 3.5 & 2.8 & - & - & 40 & 85 & 65 & - & - \\
\hline Trapeze & 7.7 & 25.6 & 15.4 & - & - & 2.9 & 3.9 & 3.6 & - & - & 48 & 75 & 67 & - & - \\
\hline Shouldered/tanged & 27.7 & 33.8 & 29.9 & - & - & 2.0 & 6.1 & 4.9 & - & - & 48 & 90 & 74 & - & - \\
\hline Preform & 2.5 & 35.6 & 13.2 & 10.78 & 81.70 & 0.8 & 14.4 & 5.9 & 5.72 & 96.49 & 20 & 90 & 50 & 22.60 & 45.21 \\
\hline \multicolumn{16}{|l|}{ Non-microlithic tools: } \\
\hline Side retouched & 14.5 & 74.1 & 31.7 & 11.72 & 36.93 & 0.8 & 13.3 & 4.4 & 2.72 & 62.01 & 20 & 75 & 44 & 14.62 & 33.39 \\
\hline Side and end retouched & 55.8 & 143.0 & 100.6 & 37.12 & 36.91 & 2.5 & 20.8 & 9.5 & 8.22 & 86.56 & 40 & 60 & 47 & 6.83 & 14.64 \\
\hline End retouched & 11.9 & 38.4 & 22.8 & - & - & 2.5 & 9.8 & 5.2 & - & - & 45 & 90 & 68 & - & - \\
\hline Burin & 2.4 & 17.2 & 9.8 & - & - & 10.3 & 15.1 & 12.7 & - & - & 59 & 80 & 69 & - & - \\
\hline Notched/denticulated & 12.1 & 32.3 & 19.4 & - & - & 1.5 & 1.9 & 1.7 & - & - & 40 & 53 & 44 & - & - \\
\hline Unifacial point & 25.4 & 119.7 & 61.1 & 25.35 & 41.52 & 2.0 & 17.4 & 7.0 & 4.88 & 69.26 & 23 & 55 & 43 & 9.92 & 22.99 \\
\hline Other point & 18.3 & 67.8 & 37.1 & 15.78 & 42.50 & 2.9 & 6.7 & 4.8 & 1.53 & 32.25 & 20 & 80 & 51 & 20.43 & 40.36 \\
\hline Misc. & 5.6 & 15.2 & 10.4 & - & - & 0.7 & 9.3 & 5.0 & - & - & 40 & 78 & 59 & - & - \\
\hline
\end{tabular}

Table A.1.18: Retouch lengths, depths and angles of tool typologies at RCC 


\begin{tabular}{lrrrr}
\hline Tool type & Minimum & Maximum & Mean & Std. Dev. \\
\hline \multicolumn{1}{c}{ Microliths: } & & & & \\
Non-geometric & 0.063 & 0.281 & 0.139 & 0.078 \\
Non-geometric point & 0.063 & 0.281 & 0.147 & 0.066 \\
Backed non-geometric & 0.063 & 0.250 & 0.117 & 0.044 \\
Backed non-geometric point & 0.031 & 0.188 & 0.108 & 0.041 \\
Crescent & 0.063 & 0.250 & 0.133 & 0.054 \\
Rectangle & 0.125 & 0.156 & 0.138 & - \\
Trapeze & 0.031 & 0.125 & 0.070 & - \\
Shouldered/tanged & 0.063 & 0.344 & 0.161 & 0.010 \\
Preform & 0.063 & 0.156 & 0.097 & 0.037 \\
& & & & \\
$\quad$ Non-microlithic tools: & & & & \\
Side retouched & 0.063 & 0.438 & 0.172 & 0.077 \\
Side and end retouched & 0.156 & 0.531 & 0.286 & 0.136 \\
End retouched & 0.031 & 0.063 & 0.044 & - \\
Burin & 0.063 & 0.313 & 0.188 & - \\
Notched/denticulated & 0.156 & 0.219 & 0.177 & - \\
Unifacial point & 0.094 & 0.500 & 0.252 & 0.125 \\
Other point & 0.031 & 0.219 & 0.133 & 0.057 \\
Misc. & 0.063 & 0.125 & 0.094 & - \\
\hline Table A.19: Clarkson & $(2002)$ & I0I measurem
\end{tabular}

Table A.1.19: Clarkson's (2002) IOI measurements of tool typologies at RCC

\section{A.2 Umhlatuzana, SOUth Africa}

\section{CORES}

\begin{tabular}{lrrrr}
\hline Statistic & Min & Max & Mean & Std. Dev. \\
\hline Longest dimension (mm) & 13.8 & 64.0 & 27.2 & 8.58 \\
Longest axis (mm) & 12.4 & 53.2 & 24.8 & 7.71 \\
Longest face (mm) & 12.7 & 57.0 & 25.2 & 7.73 \\
Maximum width (mm) & 6.8 & 62.7 & 20.9 & 8.03 \\
Maximum thickness (mm) & 5.1 & 32.2 & 13.7 & 4.72 \\
Weight (g) & 0.5 & 113.1 & 9.9 & 12.20 \\
Cubic volume (cm $\left.{ }^{3}\right)$ & 0.5 & 107.5 & 9.1 & 11.15 \\
Largest striking platform width (mm) & 2.5 & 61.7 & 15.5 & 7.78 \\
Largest striking platform thickness (mm) & 1.1 & 33.4 & 9.8 & 5.45 \\
Largest striking platform area $\left(\mathrm{cm}^{2}\right)$ & 0.1 & 20.6 & 1.9 & 2.36 \\
\hline
\end{tabular}

Table A.2.1: Core size measurements at Umhlatuzana 


\begin{tabular}{llrrr}
\hline Core type & & Mean & Std. Dev. & CV \\
\hline Uni-directional: & Blade & 25.2 & 5.12 & 20.32 \\
& Flake & 26.6 & 6.82 & 25.64 \\
& Bipolar & 17.9 & 2.79 & 15.59 \\
& & & & \\
Bi-directional: & Blade & 31.1 & 10.73 & 34.50 \\
& Flake & 27.7 & 4.86 & 28.38 \\
& & & & \\
Multi-directional: & Radial & 36.6 & 8.27 & 22.60 \\
& Amorphous & 28.4 & 6.16 & 21.69 \\
\hline
\end{tabular}

Table A.2.2: Longest axis length statistics of core types at Umhlatuzana

\begin{tabular}{lllrr}
\hline Core type & & No. striking platforms & Frequency & \% of type \\
\hline Bi-directional: & Blade & 2 - opposite & 6 & 100.0 \\
& & & & \\
& Flake & 2- opposite & 18 & 90.0 \\
& & $2-$ perpendicular & 2 & 10.0 \\
& & & 10 & 100.0 \\
Multi-directional: & Radial & Circumference & & \\
& & & 22 & 95.7 \\
& \multirow{2}{*}{ Amorphous } & 3 & 1 & 4.3 \\
\hline
\end{tabular}

Table A.2.3: Numbers of striking platforms on core types at Umhlatuzana

\begin{tabular}{|c|c|c|c|c|c|c|c|}
\hline \multirow[b]{2}{*}{ Core type } & \multirow[t]{2}{*}{ Per core: } & \multicolumn{2}{|c|}{ Flake scars } & \multicolumn{2}{|c|}{ Blade scars } & \multicolumn{2}{|c|}{ Scar length (mm) } \\
\hline & & $\begin{array}{c}\text { Mean } \\
\text { no. }\end{array}$ & $\begin{array}{l}\text { Std. } \\
\text { Dev. }\end{array}$ & $\begin{array}{c}\text { Mean } \\
\text { no. }\end{array}$ & $\begin{array}{l}\text { Std. } \\
\text { Dev }\end{array}$ & Mean & Std. Dev. \\
\hline \multirow[t]{3}{*}{ Uni-directional: } & Blade & 1.2 & 0.70 & 4.0 & 0.78 & 20.8 & 4.43 \\
\hline & Flake & 5.0 & 1.69 & 1.0 & 0.86 & 20.2 & 5.15 \\
\hline & Bipolar & 3.6 & 1.98 & 1.8 & 1.58 & 15.0 & 2.40 \\
\hline \multirow[t]{2}{*}{ Bi-directional: } & Blade & 2.3 & 1.21 & 4.5 & 1.52 & 21.9 & 6.00 \\
\hline & Flake & 6.7 & 1.69 & 1.6 & 1.36 & 18.2 & 2.99 \\
\hline \multirow[t]{2}{*}{ Multi-directional: } & Radial & 10.5 & 4.17 & 0.4 & 0.97 & 15.4 & 3.74 \\
\hline & Amorphous & 6.3 & 2.51 & 0.9 & 1.08 & 18.6 & 2.74 \\
\hline
\end{tabular}

Table A.2.4: Removal scar numbers and average lengths on core types at Umhlatuzana 


\begin{tabular}{llrrr}
\hline Mean per core: & $\begin{array}{r}\text { No. scars } \\
\text { per } \mathbf{~ c m}^{3}\end{array}$ & $\begin{array}{r}\text { Average scar length } \\
\text { per } \mathbf{~ c m}^{\mathbf{3}} \mathbf{( \mathbf { m m } )}\end{array}$ & $\begin{array}{r}\text { Total scar length } \\
\left.\mathbf{p e r}_{\mathbf{~ c m}} \mathbf{3} \mathbf{( c m}\right)\end{array}$ \\
\hline Uni-directional: & Blade & 1.5 & 5.7 & 2.9 \\
& Flake & 0.8 & 2.6 & 1.4 \\
& Bipolar & 2.3 & 6.5 & 3.2 \\
& & & & 2.5 \\
Bi-directional: & Blade & 1.3 & 3.6 & 1.5 \\
& Flake & 0.9 & 1.9 & \\
& & & & 1.1 \\
Multi-directional: & Radial & 0.8 & 1.1 & 1.7 \\
& Amorphous & 0.9 & 2.9 & \\
\hline
\end{tabular}

Table A.2.5: Ratios of removal scar numbers and lengths to core volume at Umhlatuzana

DEBITAGE

\begin{tabular}{lrr}
\hline Condition & Frequency & $\mathbf{\%}$ \\
\hline Whole & 800 & 67.5 \\
Proximal & 172 & 14.5 \\
Longitudinal & 92 & 7.8 \\
Medial & 58 & 4.9 \\
Distal & 55 & 4.6 \\
Unclear & 9 & 0.8 \\
\hline Total & 1186 &
\end{tabular}

Table A.2.6: Numbers and proportions of broken and whole debitage at Umhlatuzana 


\begin{tabular}{lrrrr}
\hline Statistic & Minimum & Maximum & Mean & Std. Dev. \\
\hline Weight (g) & 0.1 & 64.5 & 4.5 & 6.23 \\
Longest dimension (mm) & 11.6 & 105.3 & 29.6 & 11.71 \\
Oriented length (mm) & 8.3 & 104.1 & 27.0 & 11.49 \\
Maximum width (mm) & 2.7 & 63.0 & 19.2 & 8.36 \\
Maximum thickness (mm) & 1.0 & 17.2 & 6.4 & 2.98 \\
Dorsal surface area (cm $\left.{ }^{2}\right)$ & 0.6 & 47.2 & 5.7 & 4.70 \\
Volume (cm ${ }^{3}$ ) & 0.1 & 67.4 & 4.6 & 5.99 \\
Striking platform width (mm) & 2.1 & 47.4 & 12.3 & 6.33 \\
Striking platform thickness (mm) & 0.8 & 17.1 & 4.6 & 2.66 \\
Striking platform area (mm²) & 1.9 & 674.3 & 69.4 & 74.61 \\
\hline
\end{tabular}

Table A.2.7: Size measurements of whole debitage at Umhlatuzana

\begin{tabular}{llrrr}
\hline Raw material & Measurement & Mean & Std. Dev. & Kurtosis \\
\hline Hornfels & Oriented length $(\mathrm{mm})$ & 30.5 & 11.72 & 1.50 \\
& Weight $(\mathrm{g})$ & 5.3 & 7.47 & 19.44 \\
& Dorsal surface area $\left(\mathrm{cm}^{2}\right)$ & 6.8 & 5.06 & 7.79 \\
& Volume $\left(\mathrm{cm}^{3}\right)$ & 5.2 & 6.93 & 19.30 \\
& & & \\
Quartz & Oriented length (mm) & 21.0 & 6.28 & 1.16 \\
& Weight (g) & 2.6 & 2.94 & 30.83 \\
& Dorsal surface area $\left(\mathrm{cm}^{2}\right)$ & 3.6 & 2.20 & 3.12 \\
& Volume $\left(\mathrm{cm}^{3}\right)$ & 2.7 & 2.93 & 9.68 \\
\hline
\end{tabular}

Table A.2.8: Size statistics of whole debitage in raw material groupings at Umhlatuzana 


\begin{tabular}{lrrr}
\hline Dorsal cortex (\%) & \% of blades & \% of flake-blades & \% of flakes \\
\hline 0 & 97.0 & 94.3 & 90.5 \\
5 & & 0.4 & 0.6 \\
10 & 0.9 & 1.6 & 1.5 \\
20 & 0.4 & 0.4 & 0.4 \\
30 & & 0.4 & 0.6 \\
40 & & 0.2 & 0.6 \\
50 & 1.3 & 1.8 & 0.2 \\
60 & & 0.2 & 0.6 \\
70 & & 0.4 & 0.2 \\
80 & & 0 & 0.4 \\
90 & 0.4 & 0.2 & 2.4 \\
100 & & & 1.7 \\
\hline Average cortex cover (\%): & 1 & 2 & 6 \\
\hline
\end{tabular}

Table A.2.9: Proportions of dorsal surface cortex cover on debitage at Umhlatuzana

\begin{tabular}{llrrrr}
\hline Debitage type & Statistic & Minimum & Maximum & Mean & Std. Dev. \\
\hline Blades & No. dorsal scars & 1 & 7 & 2.8 & 1.05 \\
& No. parallel arrises & 1 & 3 & 1.3 & 0.48 \\
& Scars per cm ${ }^{2}$ & $<0.1$ & 5.1 & 1.1 & 0.94 \\
& Arrises per cm ${ }^{2}$ & $<0.1$ & 3.4 & 0.5 & 0.48 \\
& & & & & \\
Flake-blades & No. dorsal scars & 1 & 9 & 2.9 & 1.12 \\
& No. parallel arrises & 0 & 2 & 1.1 & 0.38 \\
& Scars per cm ${ }^{2}$ & 0.1 & 3.2 & 0.7 & 0.52 \\
& Arrises per cm ${ }^{2}$ & 0 & 2.0 & 0.3 & 0.25 \\
& & & & & \\
Flakes & No. dorsal scars & 1 & 7 & 2.9 & 1.32 \\
& No. parallel arrises & 0 & 2 & 0.1 & 0.28 \\
& Scars per cm ${ }^{2}$ & 0.04 & 4.99 & 0.7 & 0.54 \\
& Arrises per cm ${ }^{2}$ & 0 & 2.1 & $<0.1$ & 0.17 \\
\hline
\end{tabular}

Table A.2.10: Dorsal scar characteristics of whole debitage at Umhlatuzana 


\begin{tabular}{l|rr|rr|rr|rr}
\hline & \multicolumn{1}{c}{ Blades } & \multicolumn{1}{c}{ Flake-blades } & \multicolumn{2}{c}{ Flakes } & \multicolumn{2}{c}{ Total } \\
Breakage type & No. & \% & No. & \% & No. & \% & No. & \% \\
\hline Snapped & 67 & 28.8 & 59 & 12.1 & 16 & 3.5 & 142 & 12.0 \\
Obliquely snapped & 10 & 4.3 & 9 & 1.8 & & & 19 & 1.6 \\
\hline Total & 77 & 33.1 & 68 & 13.9 & 16 & 3.5 & 161 & 13.6 \\
\hline
\end{tabular}

Table A.2.11: Numbers and proportions of snapped debitage at Umhlatuzana

TOOLS

\begin{tabular}{lrrrr}
\hline Statistic & Minimum & Maximum & Mean & Std. Dev. \\
\hline Weight $(\mathrm{g})$ & 0.1 & 6.0 & 1.2 & 0.99 \\
Longest dimension (mm) & 9.8 & 53.3 & 23.2 & 8.66 \\
Oriented length (mm) & 8.0 & 53.3 & 22.2 & 8.86 \\
Maximum width (mm) & 4.0 & 26.0 & 11.8 & 3.80 \\
Maximum thickness (mm) & 1.5 & 12.2 & 3.9 & 1.42 \\
Dorsal surface area (cm $\left.{ }^{2}\right)$ & 0.5 & 8.0 & 2.7 & 1.63 \\
Volume (cm $\left.{ }^{3}\right)$ & 0.1 & 7.5 & 1.2 & 1.05 \\
Striking platform width (mm) & 1.6 & 20.4 & 7.5 & 4.02 \\
Striking platform thickness (mm) & 0.8 & 7.6 & 2.9 & 1.44 \\
Striking platform area $\left(\mathrm{mm}^{2}\right)$ & 2.1 & 154.5 & 25.7 & 27.25 \\
\hline
\end{tabular}

Table A.2.12: Microlith size measurements at Umhlatuzana

\begin{tabular}{lrrrr}
\hline Statistic & Minimum & Maximum & Mean & Std. Dev. \\
\hline Weight (g) & 2.8 & 14.7 & 6.7 & 3.14 \\
Longest dimension (mm) & 29.2 & 59.8 & 39.2 & 9.05 \\
Oriented length (mm) & 25.0 & 59.8 & 36.6 & 10.24 \\
Maximum width (mm) & 12.1 & 33.0 & 21.8 & 5.00 \\
Maximum thickness (mm) & 3.9 & 12.7 & 7.8 & 2.64 \\
Dorsal surface area (cm $\left.{ }^{2}\right)$ & 4.7 & 11.6 & 7.8 & 2.25 \\
Volume (cm $^{3}$ ) & 2.4 & 14.7 & 6.2 & 3.36 \\
Striking platform width (mm) & 3.4 & 22.4 & 13.1 & 6.93 \\
Striking platform thickness (mm) & 1.6 & 10.4 & 5.6 & 3.40 \\
Striking platform area (mm $\left.{ }^{2}\right)$ & 11.2 & 231.6 & 95.5 & 96.46 \\
\hline
\end{tabular}

Table A.2.13: Non-microlithic tool size measurements at Umhlatuzana 


\begin{tabular}{|c|c|c|c|c|c|c|c|c|c|c|c|c|c|c|c|}
\hline \multirow[b]{2}{*}{ Tool type } & \multicolumn{5}{|c|}{ Oriented length (mm) } & \multicolumn{5}{|c|}{ Maximum width (mm) } & \multicolumn{5}{|c|}{ Maximum thickness (mm) } \\
\hline & Min & Max & Mean & SD & CV & Min & Max & Mean & SD & CV & Min & Max & Mean & SD & CV \\
\hline \multicolumn{16}{|l|}{ Microliths: } \\
\hline Non-geometric & 17.0 & 24.7 & 21.5 & - & - & 11.5 & 25.1 & 18.3 & - & - & 3.7 & 12.2 & 7.3 & - & 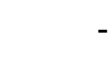 \\
\hline Non-geometric point & 8.0 & 39.2 & 24.1 & 11.37 & 47.20 & 8.3 & 20.5 & 14.1 & 4.05 & 28.76 & 2.7 & 5.8 & 3.6 & 10.2 & 28.36 \\
\hline Backed non-geometric & 11.7 & 32.5 & 19.9 & 6.67 & 33.55 & 7.3 & 26.0 & 14.3 & 4.91 & 34.28 & 2.4 & 6.6 & 4.3 & 1.11 & 25.56 \\
\hline Backed non-geometric point & 9.6 & 48.9 & 21.2 & 8.87 & 41.85 & 4.0 & 20.9 & 11.6 & 3.46 & 29.86 & 2.0 & 7.8 & 3.7 & 1.35 & 36.70 \\
\hline Crescent & 9.8 & 53.3 & 23.7 & 9.50 & 40.03 & 4.7 & 16.6 & 10.5 & 2.97 & 28.26 & 1.5 & 6.8 & 3.8 & 1.19 & 31.10 \\
\hline Rectangle & 24.2 & 24.3 & 24.2 & - & . & 8.9 & 12.5 & 10.7 & - & - & 3.5 & 3.5 & 3.5 & - & - \\
\hline Trapeze & 14.5 & 26.9 & 19.5 & 3.62 & 18.60 & 7.6 & 13.6 & 10.3 & 2.19 & 21.30 & 2.4 & 6.4 & 3.6 & 1.26 & 34.70 \\
\hline Triangle & 10.6 & 24.0 & 16.6 & - & - & 4.7 & 14.9 & 10.0 & - & - & 1.5 & 6.5 & 3.8 & - & - \\
\hline Shouldered/tanged & 21.5 & 41.5 & 33.2 & 7.75 & 23.37 & 12.2 & 16.5 & 14.1 & 1.70 & 12.08 & 2.9 & 6.0 & 4.5 & 1.29 & 28.75 \\
\hline \multicolumn{16}{|l|}{ Non-microlithic tools: } \\
\hline Side retouched & 25.0 & 37.5 & 31.2 & 4.92 & 15.76 & 19.2 & 33.0 & 23.8 & 4.64 & 19.53 & 5.5 & 12.7 & 8.4 & 2.70 & 32.17 \\
\hline Burin & 59.8 & 59.8 & 59.8 & - & - & 19.2 & 19.2 & 19.2 & - & - & 7.6 & 7.6 & 7.6 & - & - \\
\hline Notched/denticulated & 30.0 & 50.8 & 40.4 & - & - & 20.1 & 22.9 & 21.5 & - & - & 3.9 & 8.4 & 6.1 & - & - \\
\hline Points & 29.0 & 49.3 & 38.1 & - & - & 12.1 & 27.5 & 19.1 & - & - & 5.1 & 12.0 & 7.7 & - & - \\
\hline
\end{tabular}

Table A.2.14: Length, width and thickness measurements of tool typologies at Umhlatuzana 


\begin{tabular}{lrrrrr}
\hline Tool type & Minimum & Maximum & Mean & Std. Dev. & CV \\
\hline \multicolumn{1}{c}{ Microliths: } & & & & & \\
Non-geometric & 21.2 & 152.3 & 76.1 & - & - \\
Non-geometric point & 11.3 & 59.2 & 26.5 & 14.95 & 56.50 \\
Backed non-geometric & 10.1 & 68.4 & 32.1 & 14.88 & 46.36 \\
Backed non-geometric point & 4.2 & 80.0 & 22.8 & 14.35 & 63.03 \\
Crescent & 4.3 & 39.1 & 21.2 & 9.92 & 46.79 \\
Rectangle & 15.6 & 22.0 & 19.9 & - & - \\
Trapeze & 9.1 & 43.5 & 21.1 & 10.59 & 50.19 \\
Triangle & 3.6 & 46.1 & 22.2 & - & - \\
Shouldered/tanged & 17.6 & 49.4 & 32.6 & 13.04 & 40.00 \\
& & & & & \\
$\quad$ Non-microlithic tools: & & & & & \\
Side retouched & 52.9 & 210.1 & 93.8 & 43.96 & 46.87 \\
Burin & 72.8 & 72.8 & 72.8 & - & - \\
Notched/denticulated & 38.8 & 95.7 & 53.1 & - & - \\
Points & 41.8 & 164.5 & 78.2 & - & - \\
\hline Table A.2.15: TCSA measurem
\end{tabular}

Table A.2.15: TCSA measurements of tool typologies at Umhlatuzana

\begin{tabular}{|c|c|c|c|c|c|c|}
\hline $\begin{array}{l}\text { Measurement } \\
(\mathrm{mm})\end{array}$ & $\begin{array}{l}\text { Raw } \\
\text { material }\end{array}$ & Min. & Max. & Mean & $\begin{array}{l}\text { Std. } \\
\text { Dev. }\end{array}$ & $\begin{array}{r}\text { Significance of } \\
\text { difference }\end{array}$ \\
\hline \multirow[t]{2}{*}{ Oriented length } & Hornfels & 22.6 & 53.3 & 33.1 & 8.55 & $\mathrm{t}(42)=5.783, \mathbf{p}<. \mathbf{0 0 1}$ \\
\hline & Quartz & 9.8 & 31.6 & 19.3 & 6.02 & (independent t-test) \\
\hline \multirow[t]{2}{*}{ Maximum width } & Hornfels & 8.8 & 16.6 & 12.5 & 2.16 & $\mathrm{t}(42)=2.842, \mathbf{p}=. \mathbf{0 0 7}$ \\
\hline & Quartz & 4.7 & 15.9 & 9.7 & 2.93 & (independent t-test) \\
\hline \multirow{3}{*}{$\begin{array}{l}\text { Maximum } \\
\text { thickness }\end{array}$} & & & & & & $U(42)=152, p=.629$ \\
\hline & Hornfels & 2.6 & 5.1 & 3.8 & 0.91 & (Mann-Whitney U test*) \\
\hline & Quartz & 1.5 & 6.8 & 3.9 & 1.32 & \\
\hline \multirow[t]{2}{*}{ TCSA } & Hornfels & 11.6 & 39.1 & 24.0 & 8.99 & $\mathrm{U}(42)=132, \mathrm{p}=.299$ \\
\hline & Quartz & 4.3 & 38.0 & 20.3 & 10.76 & (Mann-Whitney U test*) \\
\hline
\end{tabular}

Table A.2.16: Size measurements of whole crescent microliths at Umhlatuzana.

Hornfels $n=10$, quartz $n=34 .^{*}$ non-parametric distributions 


\begin{tabular}{lrrrrr}
\hline Haft measurement & Minimum & Maximum & Mean & Std. Dev. & CV \\
\hline Width (mm) & 6.0 & 9.8 & 7.6 & 1.38 & 18.15 \\
Thickness (mm) & 2.2 & 4.9 & 3.6 & 1.25 & 34.63 \\
Length (mm) & 2.6 & 20.8 & 9.0 & 7.05 & 78.43 \\
\hline
\end{tabular}

Table A.2.17: Microlith haft measurements at Umhlatuzana

\begin{tabular}{|c|c|c|}
\hline & Microliths (\%) & Non-microlithic tools (\%) \\
\hline \multicolumn{3}{|c|}{ No. worked edges: } \\
\hline 1 & 92.3 & 50.0 \\
\hline 2 & 7.7 & 42.9 \\
\hline 3 & & 7.1 \\
\hline
\end{tabular}

Retouch location:

5.6

Distal and lateral $\quad 1.0$

Distal, lateral and lateral $\quad 0.5$

Lateral $\quad 82.1$

81.8

Lateral and lateral

4.6

16.3

Proximal

5.1

4.5

Proximal and lateral

1.0

Retouch face:

Bifacial

Dorsal

Edge

59.0

Ventral

Retouch outline:

$\begin{array}{lrr}\text { Concave } & 1.0 & 13.6 \\ \text { Convex } & 52.8 & 13.6 \\ \text { Irregular } & 1.0 & 9.1 \\ \text { Nosed/beaked } & 1.0 & \\ \text { Perpendicular } & 1.5 & 4.5 \\ \text { Pointed } & 4.6 & 9.1 \\ \text { Straight } & 37.9 & 50.0\end{array}$

Table A.2.18: Retouch characteristics of microliths and non-microlithic tools at Umhlatuzana 


\begin{tabular}{|c|c|c|c|c|c|c|c|c|c|c|c|c|c|c|c|}
\hline \multirow[b]{2}{*}{ Tool type } & \multicolumn{5}{|c|}{ Retouch length (mm) } & \multicolumn{5}{|c|}{ Retouch depth (mm) } & \multicolumn{5}{|c|}{ Retouch angle $\left({ }^{\circ}\right)$} \\
\hline & Min & Max & Mean & SD & CV & Min & Max & Mean & SD & CV & Min & Max & Mean & SD & CV \\
\hline \multicolumn{16}{|l|}{ Microliths: } \\
\hline Non-geometric & 14.5 & 38.3 & 29.3 & - & - & 2.9 & 7.6 & 5.9 & - & - & 45 & 65 & 55 & - & - \\
\hline Non-geometric point & 8.7 & 41.9 & 24.8 & 12.15 & 48.97 & 1.1 & 7.6 & 2.8 & 2.11 & 74.30 & 30 & 73 & 46 & 13.88 & 30.22 \\
\hline Backed non-geometric & 10.2 & 32.4 & 21.0 & 7.64 & 36.34 & 2.2 & 6.2 & 3.8 & 1.01 & 26.79 & 70 & 90 & 80 & 6.38 & 8.00 \\
\hline Backed non-geometric point & 7.2 & 56.9 & 23.6 & 11.40 & 48.41 & 1.6 & 6.6 & 3.3 & 1.04 & 31.63 & 40 & 90 & 76 & 9.69 & 12.72 \\
\hline Crescent & 13.5 & 60.8 & 29.3 & 10.24 & 34.92 & 1.5 & 6.9 & 3.6 & 1.18 & 32.24 & 40 & 90 & 77 & 9.84 & 12.73 \\
\hline Rectangle & 22.1 & 24.3 & 23.2 & - & - & 3.5 & 3.5 & 3.5 & - & - & 70 & 90 & 80 & - & - \\
\hline Trapeze & 6.4 & 26.4 & 11.4 & 6.22 & 54.77 & 1.8 & 5.6 & 3.1 & 1.14 & 36.61 & 65 & 83 & 75 & 5.51 & 7.38 \\
\hline Triangle & 9.3 & 38.6 & 21.9 & - & - & 1.7 & 6.5 & 3.9 & - & - & 70 & 90 & 80 & - & - \\
\hline Shouldered/tanged & 10.4 & 30.1 & 24.9 & 7.48 & 30.07 & 2.8 & 4.8 & 3.9 & 0.87 & 22.59 & 50 & 90 & 75 & 13.78 & 18.38 \\
\hline \multicolumn{16}{|l|}{ Non-microlithic tools: } \\
\hline Side retouched & 22.0 & 72.7 & 33.6 & 18.04 & 53.60 & 3.1 & 8.7 & 5.8 & 2.03 & 34.94 & 33 & 63 & 49 & 12.32 & 25.36 \\
\hline Burin & 15.3 & 15.3 & 15.3 & - & - & 14.4 & 14.4 & 14.4 & - & - & 55 & 55 & 55 & - & - \\
\hline Notched/denticulated & 21.5 & 22.6 & 22.1 & - & - & 2.8 & 4.8 & 3.8 & - & - & 45 & 75 & 60 & - & - \\
\hline Points & 21.7 & 64.0 & 44.6 & - & - & 3.8 & 8.4 & 5.5 & - & - & 45.0 & 70.0 & 52.5 & - & - \\
\hline
\end{tabular}

Table A.2.19: Retouch lengths, depths and angles of tool typologies at Umhlatuzana 


\begin{tabular}{lrrrr}
\hline Tool type & Minimum & Maximum & Mean & Std. Dev. \\
\hline \multicolumn{1}{c}{ Microliths: } & & & & \\
Non-geometric & 0.031 & 0.250 & 0.146 & - \\
Non-geometric point & 0.094 & 0.250 & 0.156 & 0.055 \\
Backed non-geometric & 0.063 & 0.219 & 0.123 & 0.037 \\
Backed non-geometric point & 0.031 & 0.313 & 0.140 & 0.043 \\
Crescent & 0.063 & 0.219 & 0.153 & 0.018 \\
Rectangle & 0.156 & 0.250 & 0.203 & - \\
Trapeze & 0.031 & 0.156 & 0.094 & 0.041 \\
Triangle & 0.094 & 0.156 & 0.144 & - \\
Shouldered/tanged & 0.063 & 0.156 & 0.120 & 0.037
\end{tabular}

Non-microlithic tools:

$\begin{array}{llllr}\text { Side retouched } & 0.156 & 0.406 & 0.232 & 0.084 \\ \text { Burin } & 0.219 & 0.219 & 0.219 & - \\ \text { Notched/denticulated } & 0.156 & 0.281 & 0.219 & - \\ \text { Points } & 0.063 & 0.438 & 0.227 & -\end{array}$

Table A.2.20: Clarkson's (2002) IOI measurements of tool typologies at Umhlatuzana

\section{A.3 NtLoana TsOANA, Lesotho}

\section{CORES}

\begin{tabular}{lrrrr}
\hline Statistic & Min & Max & Mean & Std. Dev. \\
\hline Longest dimension (mm) & 12.3 & 58.1 & 28.3 & 9.45 \\
Longest axis (mm) & 12.3 & 55.1 & 25.6 & 8.84 \\
Longest face (mm) & 12.1 & 54.5 & 25.5 & 8.66 \\
Maximum width (mm) & 6.9 & 48.4 & 20.2 & 8.98 \\
Maximum thickness (mm) & 3.1 & 34.0 & 12.7 & 6.14 \\
Weight (g) & 0.3 & 78.5 & 9.0 & 11.96 \\
Cubic volume (cm3) & 0.3 & 83.1 & 9.3 & 12.58 \\
Largest striking platform width (mm) & 3.7 & 38.3 & 14.1 & 8.34 \\
Largest striking platform thickness (mm) & 0.9 & 34.5 & 8.6 & 6.73 \\
Largest striking platform area $\left(\mathrm{cm}^{2}\right)$ & 0.1 & 13.1 & 1.7 & 2.43 \\
\hline
\end{tabular}

Table A.3.1: Core size measurements at NT 


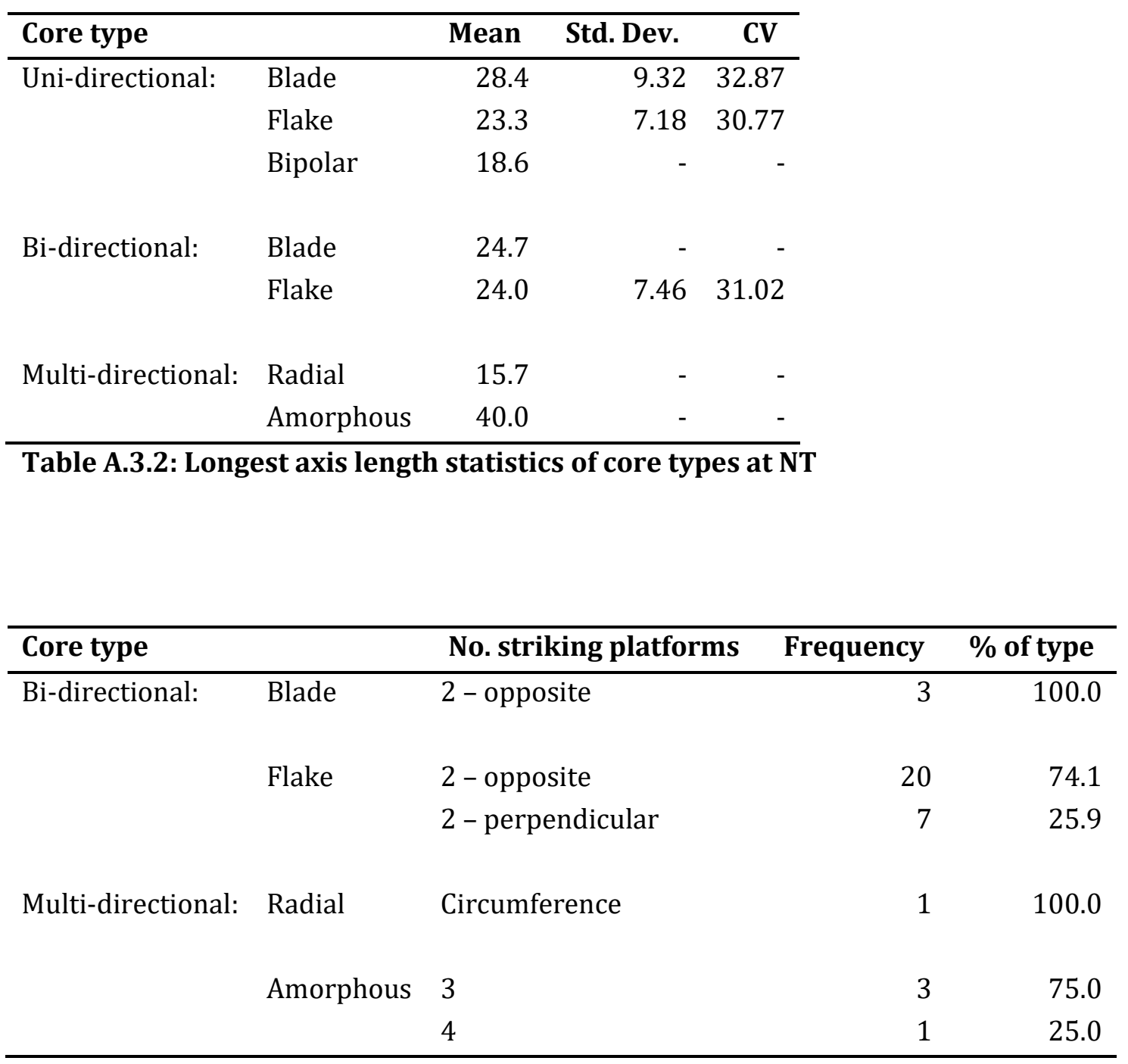

Table A.3.3: Numbers of striking platforms on core types at NT

\begin{tabular}{|c|c|c|c|c|c|c|c|}
\hline \multirow[b]{2}{*}{ Core type } & \multirow[t]{2}{*}{ Per core: } & \multicolumn{2}{|c|}{ Flake scars } & \multicolumn{2}{|c|}{ Blade scars } & \multicolumn{2}{|c|}{ Scar length (mm) } \\
\hline & & $\begin{array}{r}\text { Mean } \\
\text { no. }\end{array}$ & $\begin{array}{l}\text { Std. } \\
\text { Dev. }\end{array}$ & $\begin{array}{r}\text { Mean } \\
\text { no. }\end{array}$ & $\begin{array}{l}\text { Std. } \\
\text { Dev }\end{array}$ & Mean & Std. Dev. \\
\hline \multirow[t]{3}{*}{ Uni-directional: } & Blade & 1.1 & 1.18 & 4.3 & 1.77 & 22.8 & 7.57 \\
\hline & Flake & 3.9 & 0.96 & 1.3 & 0.84 & 18.0 & 5.79 \\
\hline & Bipolar & 1.0 & - & 6.0 & - & 14.5 & - \\
\hline \multirow[t]{2}{*}{ Bi-directional: } & Blade & 2.3 & - & 4.3 & - & 17.3 & - \\
\hline & Flake & 5.4 & 1.99 & 1.3 & 0.81 & 15.7 & 4.75 \\
\hline \multirow[t]{2}{*}{ Multi-directional: } & Radial & 3.0 & - & 0.0 & - & 17.3 & - \\
\hline & Amorphous & 13.5 & - & 2.5 & - & 16.6 & - \\
\hline
\end{tabular}

Table A.3.4: Removal scar numbers and average lengths on core types at NT 


\begin{tabular}{|c|c|c|c|c|}
\hline Core type & Mean per core: & $\begin{array}{r}\text { No. scars } \\
\text { per } \mathrm{cm}^{3}\end{array}$ & $\begin{array}{r}\text { Average scar length } \\
\text { per } \mathrm{cm}^{3}(\mathrm{~mm})\end{array}$ & 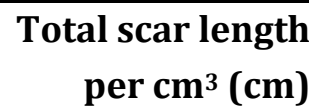 \\
\hline \multirow[t]{3}{*}{ Uni-directional: } & Blade & 1.9 & 6.4 & $\overline{3.6}$ \\
\hline & Flake & 1.5 & 4.3 & 2.2 \\
\hline & Bipolar & 15.4 & 23.8 & 16.7 \\
\hline \multirow[t]{2}{*}{ Bi-directional: } & Blade & 2.1 & 5.5 & 3.6 \\
\hline & Flake & 1.7 & 3.7 & 2.3 \\
\hline \multirow[t]{2}{*}{ Multi-directional: } & Radial & 0.2 & 1.3 & 0.4 \\
\hline & Amorphous & 0.6 & 0.8 & 0.9 \\
\hline
\end{tabular}

Table A.3.5: Ratios of removal scar numbers and lengths to core volume at NT

DEBITAGE

\begin{tabular}{lrr}
\hline Condition & Frequency & $\mathbf{\%}$ \\
\hline Whole & 476 & 50.9 \\
Proximal & 118 & 12.6 \\
Unclear & 104 & 11.1 \\
Distal & 97 & 10.4 \\
Medial & 95 & 10.1 \\
Longitudinal & 46 & 4.9 \\
\hline Total (\%) & 936 & \\
\hline
\end{tabular}

Table A.3.6: Numbers and proportions of broken and whole debitage at NT

\begin{tabular}{lrrrr}
\hline Statistic & Minimum & Maximum & Mean & Std. Dev. \\
\hline Weight (g) & $<0.1$ & 84.5 & 1.9 & 4.99 \\
Longest dimension (mm) & 10.0 & 74.5 & 19.9 & 9.45 \\
Oriented length (mm) & 5.7 & 62.4 & 17.8 & 9.28 \\
Maximum width (mm) & 2.4 & 48.9 & 13.0 & 6.30 \\
Maximum thickness (mm) & 0.5 & 28.5 & 4.7 & 2.85 \\
Dorsal surface area (cm $\left.{ }^{2}\right)$ & 0.2 & 30.5 & 2.7 & 3.25 \\
Volume (cm ${ }^{3}$ ) & $<0.1$ & 86.9 & 2.0 & 5.10 \\
Striking platform width (mm) & 1.7 & 39.7 & 8.3 & 4.74 \\
Striking platform thickness (mm) & 0.5 & 19.7 & 3.2 & 2.17 \\
Striking platform area $\left(\mathrm{mm}^{2}\right)$ & 1.0 & 577.3 & 34.0 & 48.69 \\
\hline
\end{tabular}

Table A.3.7: Size measurements of whole debitage at NT 


\begin{tabular}{lrrr}
\hline Dorsal cortex (\%) & \% of blades & \% of flake-blades & \% of flakes \\
\hline 0 & 97.3 & 89.9 & 89.5 \\
5 & 0.9 & 3.4 & 1.9 \\
10 & 0.9 & 1.4 & 1.2 \\
20 & & 0.7 & 1.0 \\
30 & 0.9 & 1.4 & 0.3 \\
40 & & 1.4 & 0.1 \\
50 & & & 0.9 \\
60 & & & 1.8 \\
70 & & 0.7 & 0.6 \\
80 & & 0.7 & 0.7 \\
90 & & & 0.3 \\
95 & & & 0.1 \\
100 & $<1$ & 3 & 1.5 \\
\hline Average cortex cover (\%): & & & 5 \\
\hline
\end{tabular}

Table A.3.8: Proportions of dorsal surface cortex cover on debitage at NT

\begin{tabular}{|c|c|c|c|c|c|}
\hline Debitage type & Statistic & Minimum & Maximum & Mean & Std. Dev. \\
\hline \multirow[t]{4}{*}{ Blades } & No. dorsal scars & 2 & 7 & 2.9 & 1.05 \\
\hline & No. parallel arrises & 1 & 3 & 1.3 & 0.52 \\
\hline & Scars per $\mathrm{cm}^{2}$ & 0.2 & 8.1 & 2.4 & 1.68 \\
\hline & Arrises per $\mathrm{cm}^{2}$ & 0.1 & 4.3 & 1.2 & 0.97 \\
\hline \multirow[t]{4}{*}{ Flake-blades } & No. dorsal scars & 1 & 9 & 3.0 & 1.33 \\
\hline & No. parallel arrises & 0 & 2 & 1.1 & 0.44 \\
\hline & Scars per $\mathrm{cm}^{2}$ & 0.2 & 7.9 & 1.9 & 1.49 \\
\hline & Arrises per $\mathrm{cm}^{2}$ & 0.0 & 3.4 & 0.8 & 0.65 \\
\hline \multirow[t]{4}{*}{ Flakes } & No. dorsal scars & 1 & 8 & 2.6 & 1.31 \\
\hline & No. parallel arrises & 0 & 2 & 0.3 & 0.49 \\
\hline & Scars per $\mathrm{cm}^{2}$ & 0.0 & 8.6 & 1.5 & 1.11 \\
\hline & Arrises per $\mathrm{cm}^{2}$ & 0.0 & 1.8 & 0.2 & 0.36 \\
\hline
\end{tabular}

Table A.3.9: Dorsal scar characteristics of whole debitage at NT 


\begin{tabular}{lrrrr}
\hline Statistic & Minimum & Maximum & Mean & Std. Dev. \\
\hline Weight (g) & 0.6 & 2.0 & 1.3 & 0.51 \\
Longest dimension (mm) & 16.8 & 29.1 & 22.3 & 4.39 \\
Oriented length (mm) & 13.3 & 29.1 & 20.5 & 5.13 \\
Maximum width (mm) & 9.5 & 20.1 & 13.9 & 3.54 \\
Maximum thickness (mm) & 2.7 & 5.8 & 4.2 & 0.98 \\
Dorsal surface area (cm $\left.{ }^{2}\right)$ & 1.6 & 4.0 & 2.8 & 0.91 \\
Volume (cm ${ }^{3}$ ) & 0.6 & 1.8 & 1.2 & 0.49 \\
Striking platform width (mm) & 5.9 & 14.5 & 11.2 & 3.47 \\
Striking platform thickness (mm) & $<0.1$ & 4.2 & 1.7 & 1.75 \\
Striking platform area $\left(\mathrm{mm}^{2}\right)$ & 7.9 & 61.3 & 36.1 & 19.62 \\
\hline
\end{tabular}

Table A.3.10: Microlith size measurements at NT

\begin{tabular}{lrrrr}
\hline Statistic & Minimum & Maximum & Mean & Std. Dev. \\
\hline Weight (g) & 2.7 & 34.4 & 13.0 & 11.63 \\
Longest dimension (mm) & 28.5 & 69.1 & 46.7 & 15.21 \\
Oriented length (mm) & 23.8 & 66.7 & 43.1 & 16.16 \\
Maximum width (mm) & 16.0 & 35.1 & 26.8 & 7.08 \\
Maximum thickness (mm) & 5.9 & 17.8 & 9.7 & 4.08 \\
Dorsal surface area (cm $\left.{ }^{2}\right)$ & 4.4 & 22.0 & 12.0 & 6.73 \\
Volume (cm ${ }^{3}$ ) & 2.7 & 39.0 & 13.8 & 13.12 \\
Striking platform width (mm) & 7.2 & 27.8 & 17.6 & 6.97 \\
Striking platform thickness (mm) & 3.6 & 12.2 & 6.4 & 2.75 \\
Striking platform area $\left(\mathrm{mm}^{2}\right)$ & 26.2 & 338.6 & 126.0 & 98.48 \\
\hline
\end{tabular}

Table A.3.11: Non-microlithic tool size measurements at NT

\begin{tabular}{lrrr}
\hline Tool type & Minimum & Maximum & Mean \\
\hline \multicolumn{1}{c}{ Microliths: } & & & \\
Non-geometric & 17.3 & 37.4 & 30.3 \\
Backed non-geometric & 22.3 & 22.3 & 22.3 \\
Crescent & 16.3 & 58.0 & 32.8
\end{tabular}

Non-microlithic tools:

$\begin{array}{lrrr}\text { Point } & 109.3 & 109.3 & 109.3 \\ \text { Side and/or end retouched } & 48.5 & 292.3 & 141.0\end{array}$

Table A.3.12: TCSA measurements of tool typologies at NT 


\begin{tabular}{|c|c|c|}
\hline & Microliths (\%) & Non-microlithic tools (\%) \\
\hline \multicolumn{3}{|l|}{ No. worked edges: } \\
\hline 1 & 66.7 & 62.5 \\
\hline 2 & 33.3 & 37.5 \\
\hline \multicolumn{3}{|l|}{ Retouch type: } \\
\hline Backing & 25.0 & \\
\hline Discontinuous & 8.3 & 9.1 \\
\hline Irregular & 8.3 & \\
\hline Nibbling & 25.0 & 9.1 \\
\hline Scalar & 33.3 & 72.7 \\
\hline Sub-parallel & & 9.1 \\
\hline \multicolumn{3}{|l|}{ Retouch location: } \\
\hline Distal & 8.3 & 18.2 \\
\hline Distal and lateral & & 18.2 \\
\hline Lateral & 75.0 & 63.6 \\
\hline Proximal & 8.3 & \\
\hline Proximal, distal and lateral & 8.3 & \\
\hline \multicolumn{3}{|l|}{ Retouch face: } \\
\hline Dorsal & 83.3 & 81.8 \\
\hline Ventral & 16.7 & 18.2 \\
\hline \multicolumn{3}{|l|}{ Retouch outline: } \\
\hline Concave & & 18.2 \\
\hline Convex & 41.7 & 36.4 \\
\hline Irregular & 16.7 & \\
\hline Nosed/beaked & 8.3 & \\
\hline Straight & 33.3 & 45.5 \\
\hline
\end{tabular}

Table A.3.13: Retouch characteristics of microliths and non-microlithic tools at NT 


\begin{tabular}{lrrrrr}
\hline Statistic & Minimum & Maximum & Mean & Std. Dev. & CV \\
\hline Microliths: & & & & & \\
Retouch length (mm) & 11.3 & 28.3 & 20.3 & 5.97 & 29.44 \\
Retouch depth (mm) & 0.8 & 5.2 & 2.7 & 1.56 & 58.54 \\
Retouch angle ( ${ }^{\circ}$ ) & 37.5 & 80.0 & 64.7 & 13.60 & 21.02 \\
& & & & & \\
$\quad$ Non-microlithic tools: & & & & & \\
Retouch length (mm) & 15.4 & 45.9 & 28.1 & 11.83 & 42.10 \\
Retouch depth (mm) & 1.3 & 6.8 & 3.0 & 1.79 & 59.09 \\
Retouch angle $\left({ }^{\circ}\right)$ & 40.0 & 60.0 & 47.2 & 8.28 & 17.56 \\
\hline
\end{tabular}

Table A.3.14: Retouch lengths, depths and angles of microlithic and non-microlithic tools at NT

\begin{tabular}{lrrrr}
\hline Tool type & Minimum & Maximum & Mean & Std. Dev. \\
\hline Microliths & 0.063 & 0.250 & 0.146 & 0.073 \\
Non-microlithic tools & 0.094 & 0.406 & 0.152 & 0.104 \\
\hline
\end{tabular}

Table A.3.15: Clarkson's (2002) IOI measurements of tool types at NT

\section{A.4 BATAdomba-LenA, SRI LANKA}

\section{CORES}

\begin{tabular}{lrrrr}
\hline Statistic & Min & Max & Mean & Std. Dev. \\
\hline Longest dimension (mm) & 20.0 & 49.1 & 31.4 & 7.17 \\
Longest axis (mm) & 9.6 & 35.1 & 24.9 & 7.90 \\
Longest face (mm) & 10.9 & 46.4 & 25.6 & 8.36 \\
Maximum width (mm) & 17.8 & 49.1 & 26.6 & 7.87 \\
Maximum thickness (mm) & 9.4 & 28.2 & 17.7 & 5.51 \\
Weight (g) & 1.8 & 27.6 & 13.0 & 8.40 \\
Cubic volume (cm $\left.{ }^{3}\right)$ & 2.7 & 33.4 & 12.7 & 8.47 \\
Largest striking platform width (mm) & 13.8 & 33.8 & 21.9 & 5.99 \\
Largest striking platform thickness (mm) & 2.7 & 21.9 & 14.6 & 4.33 \\
Largest striking platform area $\left(\mathrm{cm}^{2}\right)$ & 0.4 & 7.4 & 3.3 & 1.68 \\
\hline
\end{tabular}

Table A.4.1: Core size measurements at Batadomba-lena 


\begin{tabular}{lllrr}
\hline Core type & & No. striking platforms & Frequency & \% of type \\
\hline Bi-directional: & Flake & 2 - opposite & 2 & 100.0 \\
Multi-directional: & \multirow{2}{*}{ Radial } & 3 & 1 & 50.0 \\
& & Circumference & 1 & 50.0 \\
& \multirow{3}{*}{ Amorphous } & Unclear & 2 & 33.3 \\
& 3 & 2 & 33.3 \\
& 4 & 1 & 16.7 \\
& & 5 & 1 & 16.7 \\
\hline
\end{tabular}

Table A.4.2: Numbers of striking platforms on core types at Batadomba-lena

\begin{tabular}{|c|c|c|c|c|c|c|c|}
\hline \multirow[b]{2}{*}{ Core type } & \multirow[t]{2}{*}{ Per core: } & \multicolumn{2}{|c|}{ Flake scars } & \multicolumn{2}{|c|}{ Blade scars } & \multicolumn{2}{|c|}{ Scar length (mm) } \\
\hline & & $\begin{array}{r}\text { Mean } \\
\text { no. }\end{array}$ & $\begin{array}{l}\text { Std. } \\
\text { Dev. }\end{array}$ & $\begin{array}{r}\text { Mean } \\
\text { no. }\end{array}$ & $\begin{array}{l}\text { Std. } \\
\text { Dev }\end{array}$ & Mean & Std. Dev. \\
\hline \multirow[t]{3}{*}{ Uni-directional: } & Blade & 0.5 & 0.71 & 4.5 & 0.71 & 25.7 & 2.01 \\
\hline & Flake & 3.5 & 0.71 & 0 & 0 & 16.9 & 2.61 \\
\hline & Bipolar & 3.9 & 3.02 & 1.7 & 2.43 & 17.9 & 7.06 \\
\hline Bi-directional: & Flake & 6.0 & 1.41 & 0.5 & 0.71 & 16.5 & 4.51 \\
\hline \multirow[t]{2}{*}{ Multi-directional: } & Radial & 9.0 & 1.41 & 0 & 0 & 14.0 & 9.67 \\
\hline & Amorphous & 8.2 & 4.54 & 0.2 & 0.41 & 14.6 & 1.90 \\
\hline
\end{tabular}

Table A.4.3: Removal scar numbers and average lengths on core types at Batadomba-lena 


\begin{tabular}{|c|c|c|c|c|}
\hline Core type & Mean per core: & $\begin{array}{r}\text { No. scars } \\
\text { per } \mathrm{cm}^{3}\end{array}$ & $\begin{array}{r}\text { Average scar length } \\
\text { per } \mathrm{cm}^{3}(\mathrm{~mm})\end{array}$ & 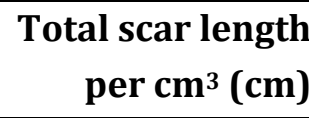 \\
\hline \multirow[t]{3}{*}{ Uni-directional: } & Blade & 0.7 & 3.9 & 1.9 \\
\hline & Flake & 0.5 & 2.5 & 1.0 \\
\hline & Bipolar & 1.1 & 2.7 & 1.5 \\
\hline \multirow[t]{3}{*}{ Bi-directional: } & Blade & 0.5 & 1.3 & 0.8 \\
\hline & Flake & & & \\
\hline & & 0.8 & 0.9 & 0.8 \\
\hline \multirow[t]{2}{*}{ Multi-directional: } & Radial & 0.6 & 1.0 & 0.8 \\
\hline & Amorphous & 0.7 & 3.9 & 1.9 \\
\hline
\end{tabular}

Table A.4.4: Ratios of removal scar numbers and lengths to core volume at Batadomba-lena

DEBITAGE

\begin{tabular}{lrr}
\hline Condition & Frequency & $\mathbf{\%}$ \\
\hline Whole & 707 & 49.9 \\
Proximal & 198 & 14.0 \\
Longitudinal & 166 & 11.7 \\
Distal & 132 & 9.3 \\
Unclear & 128 & 9.0 \\
Medial & 86 & 6.1 \\
\hline Total & 1417 &
\end{tabular}

Table A.4.5: Numbers and proportions of broken and whole debitage at Batadombalena 


\begin{tabular}{lrrrr}
\hline Statistic & Minimum & Maximum & Mean & Std. Dev. \\
\hline Weight (g) & $<0.1$ & 62.7 & 1.7 & 3.38 \\
Longest dimension (mm) & 10.0 & 67.1 & 19.9 & 7.63 \\
Oriented length (mm) & 4.4 & 65.3 & 17.6 & 7.11 \\
Maximum width (mm) & 3.1 & 43.2 & 14.1 & 6.11 \\
Maximum thickness (mm) & 1.0 & 25.1 & 4.8 & 2.67 \\
Dorsal surface area (cm $\left.{ }^{2}\right)$ & 0.3 & 28.2 & 2.8 & 2.34 \\
Volume (cm ${ }^{3}$ ) & 0.1 & 54.3 & 1.8 & 3.24 \\
Striking platform width (mm) & 1.1 & 37.1 & 7.9 & 4.48 \\
Striking platform thickness (mm) & 0.4 & 16.1 & 3.3 & 1.99 \\
Striking platform area (mm $\left.{ }^{2}\right)$ & 0.7 & 412.9 & 32.8 & 41.76 \\
\hline
\end{tabular}

Table A.4.6: Size measurements of whole debitage at Batadomba-lena

\begin{tabular}{lrrr}
\hline Dorsal cortex (\%) & \% of blades & \% of flake-blades & \% of flakes \\
\hline 0 & 92.0 & 92.4 & 88.1 \\
5 & 0.9 & 1.3 & 1.4 \\
10 & 1.8 & 1.3 & 2.3 \\
20 & & 0.8 & 1.3 \\
30 & 0.9 & 1.3 & 0.7 \\
40 & 3.6 & 1.3 & 0.6 \\
50 & 0.9 & 1.3 & 1.6 \\
60 & & 0.4 & 0.6 \\
70 & & & 0.4 \\
80 & & & 0.6 \\
90 & & & 1.0 \\
95 & & & 0.2 \\
100 & 2 & 2 & 1.3 \\
\hline Average cortex cover (\%): & & & 5 \\
\hline
\end{tabular}

Table A.4.7: Proportions of dorsal surface cortex cover on debitage at Batadombalena 


\begin{tabular}{llrrrr}
\hline Debitage type & Statistic & Minimum & Maximum & Mean & Std. Dev. \\
\hline Blades & No. dorsal scars & 2 & 6 & 2.6 & 0.95 \\
& No. parallel arrises & 1 & 3 & 1.2 & 0.45 \\
& Scars per cm ${ }^{2}$ & 0.3 & 5.9 & 2.3 & 1.59 \\
& Arrises per cm ${ }^{2}$ & 0.1 & 3.7 & 1.1 & 0.83 \\
& & & & & \\
Flake-blades & No. dorsal scars & 1 & 6 & 2.7 & 0.88 \\
& No. parallel arrises & 0 & 2 & 1.1 & 0.48 \\
& Scars per cm ${ }^{2}$ & 0.2 & 7.4 & 1.7 & 1.32 \\
& Arrises per cm & & & & \\
& & 0 & 3.3 & 0.7 & 0.59 \\
Flakes & No. dorsal scars & 1 & & & \\
& No. parallel arrises & 0 & 3 & 2.7 & 1.22 \\
& Scars per cm ${ }^{2}$ & 0.1 & 6.9 & 1.4 & 1.01 \\
& Arrises per cm ${ }^{2}$ & 0 & 2.7 & 0.2 & 0.43 \\
\hline
\end{tabular}

Table A.4.8: Dorsal scar characteristics of whole debitage at Batadomba-lena

TOOLS

\begin{tabular}{lrrrr}
\hline Statistic & Minimum & Maximum & Mean & Std. Dev. \\
\hline Weight (g) & $<0.1$ & 6.5 & 0.6 & 0.84 \\
Longest dimension (mm) & 7.4 & 35.9 & 17.8 & 4.75 \\
Oriented length (mm) & 6.8 & 35.9 & 17.0 & 4.73 \\
Maximum width (mm) & 3.3 & 25.4 & 8.9 & 4.24 \\
Maximum thickness (mm) & 1.1 & 9.2 & 3.4 & 1.51 \\
Dorsal surface area (cm $\left.{ }^{2}\right)$ & 0.2 & 7.0 & 1.6 & 1.07 \\
Volume (cm ${ }^{3}$ ) & $<0.1$ & 5.3 & 0.7 & 0.82 \\
Striking platform width (mm) & 1.0 & 11.6 & 4.3 & 2.29 \\
Striking platform thickness (mm) & 0.4 & 5.7 & 1.9 & 1.01 \\
Striking platform area $\left(\mathrm{mm}^{2}\right)$ & 0.8 & 62.9 & 10.1 & 10.85 \\
\hline
\end{tabular}

Table A.4.9: Microlith size measurements at Batadomba-lena 


\begin{tabular}{lrrrr}
\hline Statistic & Minimum & Maximum & Mean & Std. Dev. \\
\hline Weight (g) & 1.5 & 6.2 & 3.4 & 1.95 \\
Longest dimension (mm) & 20.8 & 35.8 & 29.0 & 5.08 \\
Oriented length (mm) & 19.0 & 33.2 & 26.0 & 5.31 \\
Maximum width (mm) & 9.9 & 29.8 & 19.8 & 7.75 \\
Maximum thickness (mm) & 5.2 & 10.6 & 6.9 & 1.98 \\
Dorsal surface area (cm ${ }^{2}$ ) & 2.5 & 7.7 & 5.1 & 2.10 \\
Volume (cm $^{3}$ ) & 1.3 & 6.6 & 3.7 & 2.10 \\
Striking platform width (mm) & 2.1 & 8.9 & 6.0 & 2.69 \\
Striking platform thickness (mm) & 1.8 & 4.8 & 3.4 & 1.27 \\
Striking platform area (mm $\left.{ }^{2}\right)$ & 4.6 & 37.1 & 21.4 & 13.54 \\
\hline
\end{tabular}

Table A.4.10: Non-microlithic tool size measurements at Batadomba-lena 


\begin{tabular}{|c|c|c|c|c|c|c|c|c|c|c|c|c|c|c|c|}
\hline \multirow[b]{2}{*}{ Microlith type } & \multicolumn{5}{|c|}{ Oriented length (mm) } & \multicolumn{5}{|c|}{ Maximum width (mm) } & \multicolumn{5}{|c|}{ Maximum thickness (mm) } \\
\hline & Min & Max & Mean & SD & CV & Min & Max & Mean & SD & CV & Min & Max & Mean & SD & CV \\
\hline Non-geometric & 10.2 & 21.8 & 16.3 & 3.65 & 22.48 & 7.7 & 25.4 & 14.4 & 5.53 & 38.55 & 2.3 & 8.8 & 4.6 & 1.83 & 40.15 \\
\hline Non-geometric point & 14.3 & 19.8 & 16.6 & 1.81 & 10.90 & 4.2 & 8.8 & 6.5 & 1.34 & 20.74 & 1.8 & 5.6 & 2.7 & 1.08 & 39.16 \\
\hline Backed non-geometric & 10.8 & 27.0 & 18.2 & 6.05 & 33.25 & 5.6 & 16.3 & 9.5 & 3.06 & 32.33 & 1.7 & 6.1 & 3.3 & 1.16 & 35.49 \\
\hline Backed non-geometric point & 7.4 & 28.7 & 17.5 & 4.07 & 23.34 & 3.3 & 14.1 & 6.9 & 2.49 & 36.27 & 1.1 & 6.8 & 3.0 & 1.24 & 41.80 \\
\hline Crescent & 9.5 & 22.5 & 15.8 & 3.71 & 23.41 & 4.0 & 13.6 & 7.5 & 2.04 & 27.27 & 1.7 & 5.9 & 3.4 & 1.08 & 31.42 \\
\hline Rectangle & 12.3 & 17.6 & 14.8 & - & - & 3.6 & 9.3 & 7.0 & - & - & 2.6 & 3.5 & 3.0 & - & - \\
\hline Trapeze & 20.3 & 20.3 & 20.3 & - & - & 8.0 & 8.0 & 8.0 & - & - & 3.3 & 3.3 & 3.3 & - & - \\
\hline Triangle & 6.8 & 19.9 & 14.1 & 5.25 & 37.35 & 5.2 & 13.2 & 9.7 & 2.98 & 30.87 & 1.3 & 6.1 & 3.3 & 1.88 & 56.90 \\
\hline 'Balangoda point' & 35.9 & 35.9 & 35.9 & - & - & 19.5 & 19.5 & 19.5 & - & - & 7.6 & 7.6 & 7.6 & - & - \\
\hline Notched & 16.9 & 26.4 & 21.6 & - & - & 16.2 & 19.5 & 17.5 & - & - & 4.1 & 5.6 & 4.7 & - & - \\
\hline Shouldered/tanged & 11.0 & 26.8 & 18.7 & 7.00 & 37.51 & 4.9 & 16.4 & 8.4 & 3.81 & 45.41 & 1.9 & 7.4 & 3.2 & 1.95 & 61.55 \\
\hline Preform & 9.6 & 27.2 & 15.8 & 5.00 & 31.76 & 4.9 & 20.9 & 13.3 & 4.97 & 37.33 & 2.1 & 9.2 & 4.7 & 1.90 & 40.64 \\
\hline
\end{tabular}

Table A.4.11: Length, width and thickness measurements of microlith typologies at Batadomba-lena 


\begin{tabular}{lrrrrr}
\hline Microlith type & Minimum & Maximum & Mean & Std. Dev. & CV \\
\hline Non-geometric & 11.2 & 88.6 & 36.0 & 27.45 & 76.32 \\
Non-geometric point & 4.2 & 24.7 & 9.4 & 5.79 & 61.75 \\
Backed non-geometric & 4.9 & 34.6 & 16.4 & 9.46 & 57.60 \\
Backed non-geometric point & 2.2 & 39.3 & 11.2 & 8.55 & 76.43 \\
Crescent & 4.6 & 40.4 & 13.6 & 7.83 & 57.78 \\
Rectangle & 4.7 & 16.2 & 10.8 & - & - \\
Trapeze & 13.0 & 13.0 & 13.0 & - & - \\
Triangle & 3.4 & 33.2 & 17.4 & 12.36 & 70.94 \\
'Balangoda point' & 73.7 & 73.7 & 73.7 & - & - \\
Notched & 37.0 & 45.4 & 40.8 & - & - \\
Shouldered/tanged & 4.8 & 61.0 & 16.1 & 19.89 & 123.61 \\
Preform & 5.3 & 91.2 & 34.4 & 23.80 & 69.21 \\
\hline
\end{tabular}

Table A.4.12: TCSA measurements of microlith typologies at Batadomba-lena

\begin{tabular}{lrrrrr}
\hline Haft measurement & Minimum & Maximum & Mean & Std. Dev. & CV \\
\hline Width (mm) & 3.2 & 10.6 & 5.6 & 3.12 & 55.61 \\
Thickness (mm) & 1.2 & 3.4 & 2.2 & 0.87 & 39.04 \\
Length (mm) & 2.1 & 9.9 & 5.7 & 3.38 & 58.96 \\
\hline
\end{tabular}

Table A.4.13: Microlith haft measurements at Batadomba-lena 


\begin{tabular}{|c|c|c|}
\hline & Microliths (\%) & Non-microlithic tools (\%) \\
\hline \multicolumn{3}{|l|}{ No. worked edges: } \\
\hline $0^{*}$ & 0.6 & \\
\hline 1 & 78.1 & 87.5 \\
\hline 2 & 20.6 & 12.5 \\
\hline 3 & 0.6 & \\
\hline \multicolumn{3}{|l|}{ Retouch location: } \\
\hline Distal & 8.0 & \\
\hline Distal and lateral & 2.1 & \\
\hline Lateral & 75.0 & 88.9 \\
\hline Lateral and lateral & 5.3 & \\
\hline Proximal & 6.4 & 11.1 \\
\hline Proximal and lateral & 2.1 & \\
\hline Proximal, distal and lateral & 0.5 & \\
\hline Proximal, lateral and lateral & 0.5 & \\
\hline \multicolumn{3}{|l|}{ Retouch face: } \\
\hline Alternating & 1.1 & 11.1 \\
\hline Bifacial & 3.2 & \\
\hline Dorsal & 38.8 & 55.6 \\
\hline Edge & 37.8 & \\
\hline Ventral & 19.1 & 33.3 \\
\hline \multicolumn{3}{|l|}{ Retouch outline: } \\
\hline Concave & 9.6 & 33.3 \\
\hline Convex & 41.0 & 22.2 \\
\hline Irregular & 4.3 & \\
\hline Perpendicular & 3.2 & \\
\hline Pointed & 5.3 & \\
\hline Straight & 36.7 & 44.4 \\
\hline
\end{tabular}

Table A.4.14: Retouch characteristics of microliths and non-microlithic tools at Batadomba-lena. * Tanged/shouldered with no additional retouch. 


\begin{tabular}{|c|c|c|c|c|c|c|c|c|c|c|c|c|c|c|c|}
\hline \multirow[b]{2}{*}{ Tool type } & \multicolumn{5}{|c|}{ Retouch length (mm) } & \multicolumn{5}{|c|}{ Retouch depth (mm) } & \multicolumn{5}{|c|}{ Retouch angle $\left({ }^{\circ}\right)$} \\
\hline & Min & Max & Mean & SD & CV & Min & Max & Mean & SD & CV & Min & Max & Mean & SD & CV \\
\hline \multicolumn{16}{|l|}{ Microliths: } \\
\hline Non-geometric & 1.9 & 40.4 & 13.3 & 10.99 & 82.32 & 1.3 & 7.3 & 4.0 & 1.95 & 48.99 & 25 & 90 & 49 & 18.60 & 38.16 \\
\hline Non-geometric point & 7.5 & 44.9 & 16.2 & 10.60 & 65.53 & 0.4 & 6.0 & 2.3 & 1.85 & 79.15 & 25 & 70 & 46 & 16.30 & 35.43 \\
\hline Backed non-geometric & 4.9 & 36.5 & 18.8 & 9.27 & 49.36 & 0.8 & 6.3 & 2.0 & 1.48 & 74.37 & 53 & 90 & 75 & 10.39 & 13.88 \\
\hline Backed non-geometric point & 4.7 & 42.5 & 19.4 & 9.11 & 46.99 & 0.6 & 6.2 & 2.0 & 1.37 & 69.18 & 50 & 90 & 80 & 8.80 & 11.01 \\
\hline Crescent & 4.5 & 28.7 & 19.0 & 6.97 & 36.65 & 1.0 & 5.5 & 2.8 & 1.05 & 37.49 & 53 & 90 & 78 & 13.11 & 16.91 \\
\hline Rectangle & 11.0 & 14.4 & 12.9 & - & - & 1.3 & 2.9 & 2.2 & - & - & 70 & 80 & 75 & - & - \\
\hline Trapeze & 10.4 & 10.4 & 10.4 & - & - & 3.2 & 3.2 & 3.2 & - & - & 80 & 80 & 80 & - & - \\
\hline Triangle & 6.7 & 34.1 & 18.1 & 11.20 & 61.81 & 0.9 & 6.2 & 3.1 & 2.17 & 70.11 & 75 & 90 & 85 & 6.45 & 7.59 \\
\hline 'Balangoda point' & 41.2 & 41.2 & 41.2 & - & - & 9.4 & 9.4 & 9.4 & - & - & 38 & 38 & 38 & - & - \\
\hline Notched & 7.2 & 9.4 & 8.5 & - & - & 2.3 & 3.7 & 2.9 & - & - & 60 & 80 & 72 & - & - \\
\hline Shouldered/tanged & 7.9 & 21.0 & 14.7 & 6.11 & 41.50 & 0.8 & 7.4 & 2.3 & 2.49 & 107.56 & 30 & 85 & 55 & 24.65 & 44.81 \\
\hline Preform & 3.0 & 57.8 & 12.9 & 13.46 & 104.06 & 1.4 & 12.5 & 4.9 & 3.24 & 65.55 & 30 & 90 & 49 & 15.75 & 32.47 \\
\hline \multicolumn{16}{|l|}{ Non-microlithic tools: } \\
\hline Burin & 15.0 & 15.0 & 15.0 & - & - & 5.3 & 5.3 & 5.3 & - & - & 65 & 65 & 65 & - & - \\
\hline Notched & 14.8 & 25.8 & 20.3 & - & - & 0.8 & 5.7 & 3.2 & - & - & 25 & 35 & 30 & - & - \\
\hline Side retouched & 9.7 & 26.3 & 16.9 & - & - & 2.3 & 9.1 & 4.7 & - & - & 30 & 65 & 52 & - & - \\
\hline
\end{tabular}

Table A.4.15: Retouch lengths, depths and angles of tool typologies at Batadomba-lena 


\begin{tabular}{lrrrr}
\hline Tool type & Minimum & Maximum & Mean & Std. Dev. \\
\hline Non-geometric & 0.031 & 0.219 & 0.120 & 0.064 \\
Non-geometric point & 0.063 & 0.281 & 0.147 & 0.077 \\
Backed non-geometric & 0.063 & 0.250 & 0.158 & 0.049 \\
Backed non-geometric point & 0.063 & 0.281 & 0.154 & 0.056 \\
Crescent & 0.031 & 0.188 & 0.142 & 0.039 \\
Rectangle & 0.156 & 0.250 & 0.188 & - \\
Trapeze & 0.125 & 0.125 & 0.125 & - \\
Triangle & 0.063 & 0.156 & 0.125 & 0.036 \\
'Balangoda point' & 0.719 & 0.719 & 0.719 & - \\
Notched & 0.031 & 0.094 & 0.052 & 0.036 \\
Shouldered/tanged & 0.063 & 0.406 & 0.210 & 0.115 \\
Preform & 0.031 & 0.469 & 0.167 & 0.131 \\
\hline
\end{tabular}

Table A.4.16: Clarkson's (2002) IOI measurements of microlith typologies at Batadomba-lena. Non-microlithic tools are excluded as none are whole.

\section{A.5 Kitulgala Beli-lena, SRI Lanka}

\section{MICROLITHS}

\begin{tabular}{lrrrr}
\hline Statistic & Minimum & Maximum & Mean & Std. Dev. \\
\hline Weight (g) & $<0.1$ & 0.9 & 0.3 & 0.23 \\
Longest dimension (mm) & 9.1 & 22.3 & 14.6 & 3.14 \\
Oriented length (mm) & 9.1 & 21.6 & 14.5 & 3.01 \\
Maximum width (mm) & 2.5 & 11.2 & 5.7 & 2.43 \\
Maximum thickness (mm) & 1.2 & 5.1 & 2.7 & 0.73 \\
Dorsal surface area (cm $\left.{ }^{2}\right)$ & 0.3 & 2.4 & 0.9 & 0.55 \\
Volume (cm ${ }^{3}$ ) & 0.1 & 0.8 & 0.3 & 0.21 \\
Striking platform width (mm) & 2.2 & 6.3 & 5.0 & 1.93 \\
Striking platform thickness (mm) & 1.0 & 2.6 & 1.9 & 0.67 \\
Striking platform area $\left(\mathrm{mm}^{2}\right)$ & 2.1 & 13.9 & 10.1 & 5.41 \\
\hline
\end{tabular}

Table A.5.1: Microlith size measurements at Kitulgala 


\begin{tabular}{lrlr}
\hline & \% & \% \\
\hline \multicolumn{1}{c}{ No. worked edges: } & \multicolumn{3}{c}{ Retouch outline: } \\
1 & 80.6 & Concave & 2.7 \\
2 & 19.4 & Convex & 67.6 \\
& & Perpendicular & 2.7 \\
$\quad$ Retouch type: & & Pointed & 2.7 \\
Backing & 91.9 & Straight & 24.3 \\
Sub-parallel & 8.1 & & \\
& & Retouch location: \\
$\quad$ Retouch face: & & Circumference & 2.7 \\
Dorsal & 40.5 & Distal & 2.7 \\
Edge & 54.1 & Distal and lateral & 2.7 \\
Ventral & 5.4 & Lateral & 89.2 \\
& & Proximal and lateral & 2.7 \\
\hline
\end{tabular}

Table A.5.2: Retouch characteristics of microliths at Kitulgala

\begin{tabular}{lrrrrr}
\hline & \multicolumn{6}{c}{ Oriented length (mm) } \\
Microlith type & Min & Max & Mean & SD & CV \\
\hline Non-geometric & 11.2 & 15.3 & 13.2 & - & - \\
Backed non-geometric & 9.1 & 21.6 & 15.2 & 3.73 & 24.58 \\
Geometric & 11.2 & 18.9 & 14.4 & 2.41 & 16.74 \\
Double backed & 10.4 & 17.9 & 13.7 & 2.83 & 21.65
\end{tabular}

Maximum width (mm)

\begin{tabular}{lrrrrr} 
Microlith type & Min & Max & Mean & SD & CV \\
\hline Non-geometric & 4.5 & 5.6 & 5.1 & - & - \\
Backed non-geometric & 2.5 & 11.2 & 6.2 & 3.03 & 48.90 \\
Geometric & 3.3 & 10.1 & 6.1 & 2.23 & 36.67 \\
Double backed & 2.7 & 5.3 & 4.2 & 1.20 & 28.31
\end{tabular}

Maximum thickness (mm)

\begin{tabular}{lrrrrr} 
Microlith type & Min & Max & Mean & SD & CV \\
\hline Non-geometric & 2.3 & 2.5 & 2.4 & - & - \\
Backed non-geometric & 1.8 & 4.0 & 2.7 & 0.63 & 23.30 \\
Geometric & 1.7 & 5.1 & 2.9 & 0.83 & 28.57 \\
Double backed & 1.2 & 2.8 & 2.2 & 0.66 & 30.75 \\
\hline
\end{tabular}

Table A.5.3: Length, width and thickness measurements of tool typologies at Kitulgala 


\begin{tabular}{lrrrrr}
\hline Microlith type & Minimum & Maximum & Mean & Std. Dev. & CV \\
\hline Non-geometric & 5.5 & 6.6 & 6.0 & - & - \\
Backed non-geometric & 2.2 & 19.6 & 9.1 & 6.03 & 66.57 \\
Geometric & 3.1 & 17.9 & 9.1 & 4.49 & 49.34 \\
Double backed & 2.0 & 7.5 & 4.7 & 2.32 & 49.47 \\
\hline
\end{tabular}

Table A.5.4: TCSA measurements of microlith types at Kitulgala

\begin{tabular}{lrrrrr}
\hline & \multicolumn{6}{c}{ Retouch length (mm) } \\
Microlith type & Min & Max & Mean & SD & CV \\
\hline Non-geometric & 13.3 & 14.8 & 14.1 & - & - \\
Backed non-geometric & 10.9 & 22.8 & 16.6 & 3.25 & 19.51 \\
Geometric & 13.9 & 31.8 & 21.1 & 5.05 & 23.99 \\
Double backed & 9.7 & 41.8 & 19.0 & 11.62 & 61.16 \\
& & & & & \\
Microlith type & & Retouch depth (mm) & \\
Non-geometric & 1.2 & 2.6 & 1.9 & - & - \\
Backed non-geometric & 1.8 & 3.1 & 2.5 & 0.47 & 18.54 \\
Geometric & 1.8 & 5.1 & 2.8 & 0.88 & 31.41 \\
Double backed & 1.0 & 2.3 & 1.7 & 0.53 & 31.35
\end{tabular}

Retouch angle $\left({ }^{\circ}\right)$

\begin{tabular}{lrrrrr} 
Microlith type & Min & Max & Mean & SD & CV \\
\hline Non-geometric & 28 & 60 & 44 & - & - \\
Backed non-geometric & 75 & 90 & 82 & 5.82 & 7.09 \\
Geometric & 80 & 90 & 86 & 3.93 & 4.55 \\
Double backed & 80 & 88 & 85 & 2.74 & 3.22 \\
\hline
\end{tabular}

Table A.5.5: Retouch lengths, depths and angles of tool typologies at Kitulgala

\begin{tabular}{lrrrr}
\hline Microlith type & Minimum & Maximum & Mean & Std. Dev. \\
\hline Non-geometric & 0.156 & 0.219 & 0.188 & - \\
Backed non-geometric & 0.063 & 0.156 & 0.133 & 0.038 \\
Geometric & 0.156 & 0.156 & 0.156 & - \\
Double backed & 0.250 & 0.250 & 0.250 & - \\
\hline
\end{tabular}

Table A.5.6: Clarkson's (2002) IOI measurements of microlith typologies at Kitulgala 


\section{A.6 PATNE, INDIA}

\section{CORES}

\begin{tabular}{lrrrr}
\hline Statistic & Minimum & Maximum & Mean & Std. Dev. \\
\hline Longest axis (mm) & 21.3 & 91.5 & 40.0 & 13.78 \\
Longest face (mm) & 23.2 & 91.5 & 40.9 & 13.49 \\
Maximum width (mm) & 11.0 & 65.6 & 33.0 & 13.30 \\
Maximum thickness (mm) & 7.3 & 71.3 & 23.5 & 10.99 \\
Cubic volume $\left(\mathrm{cm}^{3}\right)$ & 3.1 & 308.4 & 42.4 & 52.89 \\
\hline
\end{tabular}

Table A.6.1: Core size measurements at Patne

\begin{tabular}{llrrr}
\hline Core type & & Mean & Std. Dev. & CV \\
\hline Uni-directional: & Blade & 29.8 & - & - \\
& Flake & 39.7 & 13.44 & 33.85 \\
& Bipolar & 51.9 & - & - \\
& & & & \\
Bi-directional: & Blade & 36.9 & 10.75 & 29.12 \\
& Flake & 42.7 & 12.14 & 28.44
\end{tabular}

Multi-directional: $\quad$ Amorphous $\quad 42.2 \quad 21.83 \quad 51.69$

Table A.6.2: Longest axis length statistics of core types at Patne

\begin{tabular}{lllrr}
\hline Core type & & No. striking platforms & Frequency & \% of type \\
\hline Bi-directional: & Blade & 2 - opposite & 8 & 100.0 \\
& \multirow{3}{*}{ Flake } & 2- opposite & 14 & 73.7 \\
& & $2-$ perpendicular & 5 & 26.3 \\
& & & & \\
Multi-directional: & Amorphous & Unclear & 2 & 25.0 \\
& & 3 & 4 & 50.0 \\
& & 4 & 2 & 25.0 \\
\hline
\end{tabular}

Table A.6.3: Numbers of striking platforms on core types at Patne 


\begin{tabular}{llrr|rr|rrr}
\hline \multirow{2}{*}{ Core type } & Per core: & \multicolumn{2}{c}{ Flake scars } & \multicolumn{2}{c}{ Blade scars } & \multicolumn{2}{c}{ Scar length (mm) } \\
& & $\begin{array}{r}\text { Mean } \\
\text { no. }\end{array}$ & $\begin{aligned} \text { Std. } \\
\text { Dev. }\end{aligned}$ & $\begin{array}{r}\text { Mean } \\
\text { no. }\end{array}$ & $\begin{array}{r}\text { Std. } \\
\text { Dev }\end{array}$ & Mean & Std. Dev. \\
\hline Uni-directional: & Blade & 2.4 & - & 5.6 & - & 19.7 & - \\
& Flake & 7.0 & 3.63 & 2.4 & 1.59 & 21.1 & 7.86 \\
& Bipolar & 3.0 & - & 1.0 & - & 25.8 & - \\
Bi-directional: & Blade & 4.5 & 1.69 & 6.9 & 2.80 & 18.8 & 6.21 \\
& Flake & 7.9 & 3.49 & 2.8 & 2.12 & 22.2 & 7.63 \\
& & & & & & & \\
Multi-directional: & Amorphous & 5.6 & 3.82 & 2.6 & 4.69 & 19.7 & 5.20 \\
\hline
\end{tabular}

Table A.6.4: Removal scar numbers and average lengths on core types at Patne

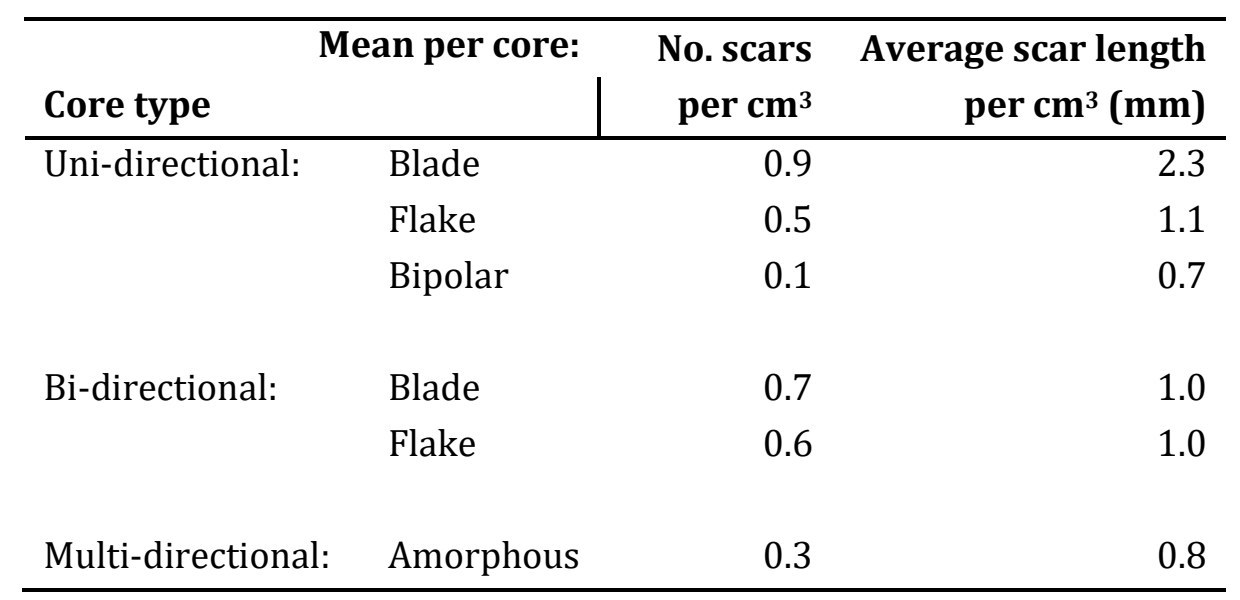

Table A.6.5: Ratios of removal scar numbers and lengths to core volume at Patne

DEBITAGE

\begin{tabular}{lrr}
\hline Condition & Frequency & $\mathbf{\%}$ \\
\hline Not recorded & 598 & 43.2 \\
Unclear & 417 & 30.1 \\
Distal & 175 & 12.6 \\
Proximal & 84 & 6.1 \\
Whole & 51 & 3.7 \\
Longitudinal & 30 & 2.2 \\
Medial & 30 & 2.2 \\
\hline Total & 1385 &
\end{tabular}

Table A.6.6: Numbers and proportions of broken and whole debitage at Patne 


\begin{tabular}{lrrrr}
\hline Statistic & Minimum & Maximum & Mean & Std. Dev. \\
\hline Oriented length (mm) & 13.2 & 55.1 & 29.7 & 9.79 \\
Maximum width (mm) & 5.3 & 40.5 & 17.0 & 8.06 \\
Maximum thickness (mm) & 1.7 & 15.7 & 6.5 & 2.76 \\
Dorsal surface area (cm $\left.{ }^{2}\right)$ & 1.3 & 19.4 & 5.3 & 3.69 \\
Volume (cm ${ }^{3}$ ) & 0.2 & 19.6 & 3.9 & 3.88 \\
Striking platform width (mm) & 1.0 & 43.3 & 10.5 & 6.87 \\
Striking platform thickness (mm) & 0.3 & 27.8 & 4.0 & 3.10 \\
Striking platform area (mm $\left.{ }^{2}\right)$ & 0.6 & 1125.9 & 57.8 & 91.60 \\
\hline
\end{tabular}

Table A.6.7: Size measurements of whole debitage at Patne

\begin{tabular}{lrrr}
\hline Dorsal cortex (\%) & \% of blades & \% of flake-blades & \% of flakes \\
\hline 0 & 88.3 & 75.4 & 77.0 \\
5 & 2.3 & 0.7 & 0.4 \\
10 & 2.7 & 3.9 & 5.0 \\
20 & 2.3 & 2.5 & 2.5 \\
30 & 0.4 & 3.2 & 3.3 \\
40 & 0.4 & 1.4 & 0.9 \\
50 & 1.9 & 6.0 & 3.2 \\
60 & & 0.4 & 0.2 \\
70 & & 0.7 & 0.4 \\
80 & & 2.8 & 1.3 \\
90 & & 0.7 & 1.8 \\
100 & 1.6 & 2.5 & 3.6 \\
Not recorded & & & 0.5 \\
\hline Average cortex cover: & 4 & 12 & 11 \\
\hline
\end{tabular}

Table A.6.8: Proportions of dorsal surface cortex cover on debitage at Patne 


\begin{tabular}{llrrrr}
\hline Debitage type & Statistic & Minimum & Maximum & Mean & Std. Dev. \\
\hline Blades & No. dorsal scars & 2 & 9 & 4.0 & 1.82 \\
& No. parallel arrises & 1 & 7 & 2.3 & 1.38 \\
& Scars per cm ${ }^{2}$ & 0.2 & 3.6 & 1.3 & 0.82 \\
& Arrises per cm ${ }^{2}$ & 0.1 & 2.9 & 0.8 & 0.65 \\
& & & & \\
Flake-blades & No. dorsal scars & 1 & 7 & 3.4 & 1.80 \\
& No. parallel arrises & 0 & 6 & 0.9 & 1.39 \\
& Scars per cm ${ }^{2}$ & 0.1 & 2.0 & 0.7 & 0.55 \\
& Arrises per cm ${ }^{2}$ & 0 & 1.2 & 0.2 & 0.29 \\
& & & & & \\
Flakes & No. dorsal scars & 1 & 7 & 4.5 & 2.43 \\
& No. parallel arrises & 0 & 5 & 1.5 & 1.76 \\
& Scars per cm ${ }^{2}$ & 0.3 & 1.8 & 1.0 & 0.65 \\
& Arrises per cm ${ }^{2}$ & 0 & 1.5 & 0.4 & 0.56 \\
\hline
\end{tabular}

Table A.6.9: Dorsal scar characteristics of whole debitage at Patne

TOOLS

\begin{tabular}{lrrrr}
\hline Statistic & Minimum & Maximum & Mean & Std. Dev. \\
\hline Oriented length (mm) & 11.1 & 39.3 & 22.9 & 5.60 \\
Maximum width (mm) & 3.9 & 31.1 & 9.1 & 4.82 \\
Maximum thickness (mm) & 1.8 & 10.2 & 3.8 & 1.49 \\
Dorsal surface area $\left(\mathrm{cm}^{2}\right)$ & 0.6 & 6.0 & 2.1 & 1.03 \\
Volume $\left(\mathrm{cm}^{3}\right)$ & 0.2 & 5.3 & 0.9 & 0.85 \\
Striking platform width (mm) & 1.4 & 29.4 & 5.5 & 6.04 \\
Striking platform thickness (mm) & 0.5 & 9.7 & 2.2 & 2.04 \\
Striking platform area $\left(\mathrm{mm}^{2}\right)$ & 1.1 & 285.2 & 23.2 & 63.98 \\
\hline
\end{tabular}

Table A.6.10: Microlith size measurements at Patne 


\begin{tabular}{lrrrr}
\hline Statistic & Minimum & Maximum & Mean & Std. Dev. \\
\hline Oriented length (mm) & 20.1 & 75.8 & 34.8 & 10.96 \\
Maximum width (mm) & 6.7 & 64.2 & 19.4 & 10.13 \\
Maximum thickness (mm) & 1.4 & 17.8 & 7.0 & 3.09 \\
Dorsal surface area $\left(\mathrm{cm}^{2}\right)$ & 1.6 & 22.7 & 7.0 & 4.50 \\
Volume (cm $\left.{ }^{3}\right)$ & 0.2 & 40.5 & 6.1 & 6.96 \\
Striking platform width (mm) & 2.9 & 37.2 & 13.3 & 9.40 \\
Striking platform thickness (mm) & 1.1 & 12.9 & 4.9 & 3.21 \\
Striking platform area $\left(\mathrm{mm}^{2}\right)$ & 4.1 & 408.9 & 92.4 & 118.60 \\
\hline
\end{tabular}

Table A.6.11: Non-microlithic tool size measurements at Patne 


\begin{tabular}{|c|c|c|c|c|c|c|c|c|c|c|c|c|c|c|c|}
\hline \multirow[b]{2}{*}{ Tool type } & \multicolumn{5}{|c|}{ Oriented length (mm) } & \multicolumn{5}{|c|}{ Maximum width (mm) } & \multicolumn{5}{|c|}{ Maximum thickness (mm) } \\
\hline & Min & Max & Mean & SD & CV & Min & Max & Mean & SD & CV & Min & $\operatorname{Max}$ & Mean & SD & CV \\
\hline \multicolumn{16}{|l|}{ Microliths: } \\
\hline Non-geometric & 11.1 & 24.9 & 18.9 & - & - & 11.9 & 31.1 & 19.1 & - & - & 2.3 & 7.0 & 5.3 & - & . \\
\hline Backed non-geometric & 14.9 & 39.3 & 23.8 & 6.04 & 25.37 & 3.9 & 12.0 & 7.9 & 1.86 & 23.59 & 1.9 & 6.2 & 3.7 & 1.10 & 29.97 \\
\hline Crescent & 17.9 & 31.0 & 22.3 & 3.92 & 17.55 & 4.8 & 11.2 & 7.6 & 2.06 & 27.30 & 2.2 & 4.8 & 3.3 & 0.93 & 28.06 \\
\hline Trapeze & 18.0 & 24.6 & 21.3 & - & - & 7.5 & 10.4 & 9.0 & - & - & 1.8 & 4.8 & 3.3 & - & - \\
\hline Triangle & 20.6 & 23.5 & 22.1 & - & - & 7.1 & 8.2 & 7.7 & - & - & 2.1 & 3.2 & 2.7 & - & - \\
\hline Burin & 17.2 & 20.2 & 18.7 & - & - & 9.0 & 30.4 & 19.7 & - & - & 5.3 & 10.2 & 7.8 & - & - \\
\hline \multicolumn{16}{|l|}{ Non-microlithic tools: } \\
\hline Side retouched & 22.0 & 53.4 & 34.8 & 8.35 & 24.04 & 6.7 & 34.5 & 18.8 & 9.14 & 48.74 & 2.7 & 13.5 & 6.7 & 3.49 & 52.27 \\
\hline Side and end retouched & 22.0 & 29.4 & 26.2 & 3.44 & 13.10 & 9.4 & 38.8 & 18.9 & 10.34 & 54.83 & 5.0 & 8.8 & 6.4 & 1.44 & 22.43 \\
\hline End retouched & 21.3 & 56.8 & 33.6 & 11.62 & 34.56 & 7.2 & 64.2 & 21.7 & 15.09 & 69.44 & 3.4 & 12.0 & 7.1 & 3.11 & 44.06 \\
\hline Burin & 20.1 & 53.5 & 34.1 & 8.32 & 24.40 & 8.1 & 37.5 & 18.1 & 8.91 & 49.22 & 1.4 & 11.4 & 6.6 & 2.68 & 40.60 \\
\hline Notched/denticulated & 28.8 & 49.8 & 40.6 & - & - & 18.2 & 22.5 & 20.9 & - & - & 7.0 & 8.6 & 8.0 & - & - \\
\hline Unifacial point & 32.5 & 46.8 & 39.7 & - & - & 15.6 & 22.2 & 18.9 & - & - & 6.5 & 10.8 & 8.7 & - & - \\
\hline Other point & 23.9 & 75.8 & 47.2 & - & - & 10.0 & 30.0 & 19.1 & - & - & 5.1 & 17.8 & 8.9 & - & - \\
\hline
\end{tabular}

Table A.6.12: Length, width and thickness measurements of tool typologies at Patne 


\begin{tabular}{lrrrrr}
\hline Tool type & Minimum & Maximum & Mean & Std. Dev. & CV \\
\hline \multicolumn{1}{c}{ Microliths: } & & & & & \\
Non-geometric & 22.3 & 90.2 & 49.0 & - & - \\
Backed non-geometric & 6.2 & 35.7 & 14.9 & 7.31 & 48.97 \\
Crescent & 6.5 & 25.8 & 13.1 & 6.99 & 53.26 \\
Trapeze & 6.8 & 25.0 & 15.9 & - & - \\
Triangle & 8.6 & 11.4 & 10.0 & - & - \\
Burin & 23.9 & 155.0 & 89.4 & - & - \\
& & & & & \\
$\quad$ Non-microlithic tools: & & & & & \\
Side retouched & 10.3 & 232.9 & 74.9 & 67.57 & 90.22 \\
Side and end retouched & 23.5 & 170.7 & 66.1 & 53.31 & 80.69 \\
End retouched & 12.6 & 385.2 & 93.9 & 105.19 & 112.06 \\
Burin & 5.7 & 213.8 & 68.8 & 59.10 & 85.86 \\
Notched/denticulated & 78.3 & 91.3 & 82.8 & - & - \\
Unifacial point & 50.7 & 119.9 & 85.3 & - & - \\
Other point & 25.5 & 267.0 & 102.7 & - & - \\
\hline
\end{tabular}

Table A.6.13: TCSA measurements of tool typologies at Patne 


\begin{tabular}{|c|c|c|}
\hline & Microliths (\%) & Non-microlithic tools (\%) \\
\hline \multicolumn{3}{|c|}{ No. worked edges: } \\
\hline 1 & 85.7 & 78.2 \\
\hline 2 & 14.3 & 14.5 \\
\hline 3 & & 7.3 \\
\hline
\end{tabular}

Retouch location:

Distal $\quad 8.3$

21.1

Distal and lateral

$\begin{array}{ll}4.2 & 1.4\end{array}$

Distal, lateral and lateral

$1.4 \quad 2.8$

Lateral

83.3

45.1

Proximal

2.8

29.6

Retouch face:

Alternating

1.4

Bifacial

1.4

Dorsal

83.3

93.1

Edge

13.9

1.4

Ventral

2.8

12.7

Retouch outline:

Concave

Convex

41.7

26.8

Irregular

Nosed/beaked

11.3

Perpendicular

1.4

Pointed

2.8

Straight

52.8

49.3

Table A.6.14: Retouch characteristics of microliths and non-microlithic tools at Patne 


\begin{tabular}{lrrrrr}
\hline Tool type & Minimum & Maximum & Mean & Std. Dev. & CV \\
\hline Microliths: & & & & & \\
Non-geometric & 8.7 & 17.6 & 14.4 & - & - \\
Backed non-geometric & 0.1 & 38.7 & 19.5 & 7.37 & 37.75 \\
Crescent & 17.5 & 30.5 & 22.0 & 3.95 & 17.98 \\
Trapeze & 10.5 & 17.2 & 13.8 & - & - \\
Triangle & 20.3 & 23.3 & 21.8 & - & - \\
Burin & 10.5 & 22.1 & 16.3 & - & - \\
& & & & & \\
$\quad$ Non-microlithic tools: & & & & & \\
Side retouched & 6.5 & 40.3 & 23.8 & 11.39 & 47.84 \\
Side and end retouched & 11.4 & 21.8 & 16.4 & 4.41 & 26.85 \\
End retouched & 5.5 & 28.8 & 14.4 & 8.07 & 55.88 \\
Burin & 5.1 & 31.7 & 14.5 & 8.20 & 56.53 \\
Notched/denticulated & 14.7 & 25.8 & 19.9 & - & - \\
Unifacial point & 7.1 & 15.5 & 11.3 & - & - \\
Other point & 10.9 & 44.0 & 28.2 & - & - \\
\hline Table A.6.15: Retouch lengths & &
\end{tabular}

Table A.6.15: Retouch lengths of tool typologies at Patne

\begin{tabular}{lrrrr}
\hline Tool type & Minimum & Maximum & Mean & Std. Dev. \\
\hline \multicolumn{1}{c}{ Microliths: } & & & & \\
Non-geometric & 0.031 & 0.125 & 0.069 & - \\
Backed non-geometric & 0.031 & 0.438 & 0.104 & 0.067 \\
Crescent & 0.094 & 0.219 & 0.115 & 0.044 \\
Trapeze & 0.125 & 0.188 & 0.156 & - \\
Triangle & 0.094 & 0.156 & 0.125 & - \\
Burin & 0.156 & 0.344 & 0.250 & -
\end{tabular}

Non-microlithic tools:

\begin{tabular}{llllr} 
Side retouched & 0.031 & 0.188 & 0.087 & 0.056 \\
Side and end retouched & 0.094 & 0.219 & 0.151 & 0.042 \\
End retouched & 0.031 & 0.188 & 0.076 & 0.063 \\
Burin & 0.031 & 0.125 & 0.063 & 0.030 \\
Notched/denticulated & 0.063 & 0.094 & 0.083 & - \\
Unifacial point & 0.063 & 0.219 & 0.141 & - \\
Other point & 0.063 & 0.313 & 0.141 & - \\
\hline
\end{tabular}

Table A.6.16: Clarkson's (2002) IOI measurements of tool typologies at Patne 


\section{A.7 JWALAPURAM 9, INDIA}

CORES

\begin{tabular}{lrrrr}
\hline Statistic & Min & Max & Mean & Std. Dev. \\
\hline Longest axis (mm) & 5.3 & 80.0 & 20.8 & 10.27 \\
Longest face (mm) & 5.2 & 75.0 & 22.3 & 10.31 \\
Maximum width (mm) & 3.9 & 91.0 & 17.7 & 9.91 \\
Maximum thickness (mm) & 1.7 & 48.0 & 13.4 & 7.53 \\
Weight (g) & 0.1 & 475.0 & 12.4 & 36.40 \\
Cubic volume (cm ${ }^{3}$ ) & $<0.1$ & 283.9 & 10.5 & 27.70 \\
Largest striking platform width (mm) & 2.1 & 167.0 & 15.8 & 13.21 \\
Largest striking platform thickness (mm) & 0.3 & 56.5 & 12.1 & 9.02 \\
Largest striking platform area $\left(\mathrm{cm}^{2}\right)$ & $<0.1$ & 25.2 & 2.5 & 3.43 \\
\hline
\end{tabular}

Table A.7.1: Core size measurements at Jwalapuram

\begin{tabular}{llrrr}
\hline Core type & & Mean & Std. Dev. & CV \\
\hline Uni-directional: & Blade & 21.4 & 5.67 & 26.51 \\
& Flake & 20.1 & 9.75 & 48.43 \\
& Bipolar & 16.6 & 7.03 & 42.27
\end{tabular}

Bi-directional: $\quad$ Blade $\quad 28$

$\begin{array}{llll}\text { Flake } & 26.5 & 10.01 & 37.74\end{array}$

$\begin{array}{lllrr}\text { Multi-directional: } & \text { Radial } & 19.3 & - & - \\ & \text { Amorphous } & 22.2 & 12.59 & 56.66\end{array}$

Platforms unknown: $\quad$ Blade $\quad 20.5 \quad 6.87 \quad 33.56$

$\begin{array}{llll}\text { Flake } & 20.0 & 6.59 & 32.97\end{array}$

Table A.7.2: Longest axis length statistics of core types at Jwalapuram 


\begin{tabular}{|c|c|c|c|c|c|c|c|}
\hline \multirow[b]{2}{*}{ Core type } & \multirow[t]{2}{*}{ Per core: } & \multicolumn{2}{|c|}{ Flake scars } & \multicolumn{2}{|c|}{ Blade scars } & \multicolumn{2}{|c|}{ Scar length (mm) } \\
\hline & & $\begin{array}{r}\text { Mean } \\
\text { no. }\end{array}$ & $\begin{array}{l}\text { Std. } \\
\text { Dev. }\end{array}$ & $\begin{array}{r}\text { Mean } \\
\text { no. }\end{array}$ & $\begin{array}{l}\text { Std. } \\
\text { Dev. }\end{array}$ & Mean & Std. Dev. \\
\hline \multirow[t]{3}{*}{ Uni-directional: } & Blade & 2.1 & 1.80 & 4.6 & 3.23 & 16.2 & 3.40 \\
\hline & Flake & 6.5 & 3.63 & 1.3 & 1.64 & 14.3 & 8.52 \\
\hline & Bipolar & 4.2 & 2.32 & 0.8 & 1.29 & 14.7 & 5.18 \\
\hline \multirow[t]{2}{*}{ Bi-directional: } & Blade & 3.4 & - & 6.2 & - & 17.7 & - \\
\hline & Flake & 10.6 & 7.75 & 1.1 & 1.21 & 17.4 & 8.56 \\
\hline \multirow[t]{2}{*}{ Multi-directional: } & Radial & 13.8 & - & 0.0 & - & 9.9 & 0 \\
\hline & Amorphous & 9.3 & 4.29 & 0.7 & 1.17 & 14.3 & 7.51 \\
\hline \multirow{3}{*}{$\begin{array}{l}\text { Platforms } \\
\text { unknown: }\end{array}$} & Blade & & & & & & \\
\hline & & 2.9 & 1.81 & 4.9 & 1.25 & 14.1 & 6.09 \\
\hline & Flake & 9.0 & 3.35 & 3.3 & 1.14 & 13.1 & 5.50 \\
\hline
\end{tabular}

Table A.7.3: Removal scar numbers and average lengths on core types at Jwalapuram

\begin{tabular}{llr}
\hline Core type & & No. scars per $\mathbf{~ c m}^{\mathbf{3}}$ (mean per core) \\
\hline Uni-directional: & Blade & 2.1 \\
& Flake & 2.9 \\
& Bipolar & 8.4 \\
& & \\
Bi-directional: & Blade & 1.9 \\
& Flake & 1.6 \\
Multi-directional: & Radial & \\
& Amorphous & 2.9 \\
& & 2.6 \\
Platforms unknown: & Blade & 1.9 \\
& Flake & 6.8 \\
\hline
\end{tabular}

Table A.7.4: Ratios of removal scar numbers to core volume at Jwalapuram 


\begin{tabular}{llr}
\hline Core type & & Mean cortex cover (\%) \\
\hline Uni-directional: & Blade & 46 \\
& Flake & 32 \\
& Bipolar & 14 \\
& & \\
Bi-directional: & Blade & 26 \\
& Flake & 25 \\
& & \\
Multi-directional: & Radial & 8 \\
& Amorphous & 18 \\
& & \\
Platforms unknown: & Blade & 29 \\
& Flake & 19 \\
\hline
\end{tabular}

Table A.7.5: Mean cortex cover on core types at Jwalapuram

DEBITAGE

\begin{tabular}{|c|c|c|c|c|}
\hline Statistic & Minimum & Maximum & Mean & Std. Dev. \\
\hline Weight (g) & 0.1 & 172.2 & 1.9 & 5.12 \\
\hline Longest dimension (mm) & 10.0 & 122.0 & 23.1 & 10.88 \\
\hline Oriented length (mm) & 10.0 & 96.6 & 18.5 & 7.84 \\
\hline Medial width (mm) & 1.9 & 64.4 & 11.9 & 6.24 \\
\hline Medial thickness (mm) & 0.8 & 81.0 & 4.0 & 2.91 \\
\hline Dorsal surface area $\left(\mathrm{cm}^{2}\right)^{*}$ & 0.3 & 33.6 & 2.4 & 2.33 \\
\hline Volume $\left(\mathrm{cm}^{3}\right) *$ & $<0.1$ & 80.8 & 1.4 & 3.37 \\
\hline Striking platform width (mm) & 0.5 & 72.6 & 7.7 & 5.57 \\
\hline Striking platform thickness (mm) & $<0.1$ & 30.7 & 2.7 & 2.37 \\
\hline Striking platform area $\left(\mathrm{mm}^{2}\right)$ & 0.1 & 2228.4 & 31.3 & 72.96 \\
\hline
\end{tabular}

Table A.7.6: Size measurements of debitage at Jwalapuram. * calculated using medial width and thickness measurements. 


\begin{tabular}{lrr}
\hline Dorsal cortex (\%) & \% of blades & \% of flakes \\
\hline 0 & 73.9 & 69.4 \\
5 & 0.6 & 0.4 \\
10 & 4.8 & 5.0 \\
20 & 2.9 & 4.2 \\
30 & 3.0 & 3.5 \\
40 & 2.0 & 3.1 \\
50 & 2.1 & 1.6 \\
60 & 1.1 & 1.8 \\
70 & 0.9 & 1.3 \\
80 & 1.0 & 1.3 \\
90 & 0.8 & 1.5 \\
95 & & 0.1 \\
100 & 4.5 & 4.8 \\
Not recorded & 2.3 & 1.9 \\
\hline Average cortex cover (\%): & 11 & 14 \\
\hline
\end{tabular}

Table A.7.7: Proportions of dorsal surface cortex cover on debitage at Jwalapuram

TOOLS

\begin{tabular}{lrrrr}
\hline Statistic & Minimum & Maximum & Mean & Std. Dev. \\
\hline Weight (g) & 0.1 & 69.5 & 2.1 & 5.49 \\
Longest dimension (mm) & 10.0 & 36.0 & 20.8 & 6.18 \\
Oriented length (mm) & 8.0 & 39.5 & 20.4 & 6.79 \\
Medial width (mm) & 1.4 & 47.6 & 9.2 & 6.93 \\
Medial thickness (mm) & 1.0 & 21.8 & 4.3 & 3.16 \\
Dorsal surface area (cm $\left.{ }^{2}\right)$ & 0.2 & 18.0 & 2.1 & 2.30 \\
Volume (cm ${ }^{3}$ ) & $<0.1$ & 39.3 & 1.5 & 3.47 \\
Striking platform width (mm) & 1.0 & 50.8 & 6.3 & 6.55 \\
Striking platform thickness (mm) & 0.2 & 24.5 & 2.8 & 3.08 \\
Striking platform area $\left(\mathrm{mm}^{2}\right)$ & 0.5 & 1245.6 & 36.5 & 120.57 \\
\hline
\end{tabular}

Table A.7.8: Microlith size measurements at Jwalapuram 


\begin{tabular}{lcccc}
\hline Statistic & Minimum & Maximum & Mean & Std. Dev. \\
\hline Weight (g) & 8.8 & 480.0 & 112.9 & 123.81 \\
Longest dimension (mm) & 41.0 & 110.0 & 70.6 & 23.63 \\
Oriented length (mm) & 30.0 & 86.9 & 60.5 & 15.67 \\
Medial width (mm) & 22.0 & 92.0 & 45.5 & 17.93 \\
Medial thickness (mm) & 9.0 & 47.0 & 23.7 & 11.44 \\
Dorsal surface area (cm $\left.{ }^{2}\right)$ & 8.7 & 66.2 & 28.9 & 15.71 \\
Volume (cm ${ }^{3}$ ) & 8.7 & 311.3 & 83.1 & 83.96 \\
Striking platform width (mm) & 7.8 & 75.0 & 26.9 & 18.13 \\
Striking platform thickness (mm) & 3.3 & 31.0 & 11.3 & 7.93 \\
Striking platform area (mm $\left.{ }^{2}\right)$ & 30.8 & 2325.0 & 413.9 & 631.68 \\
\hline
\end{tabular}

Table A.7.9: Non-microlithic tool size measurements at Jwalapuram 


\begin{tabular}{|c|c|c|c|c|c|c|c|c|c|c|c|c|c|c|c|}
\hline \multirow[b]{2}{*}{ Tool type } & \multicolumn{5}{|c|}{ Oriented length (mm) } & \multicolumn{5}{|c|}{ Medial width (mm) } & \multicolumn{5}{|c|}{ Medial thickness (mm) } \\
\hline & Min & $\operatorname{Max}$ & Mean & SD & $\mathbf{C V}$ & Min & $\operatorname{Max}$ & Mean & SD & $\mathbf{C V}$ & Min & Max & Mean & SD & $\mathbf{C V}$ \\
\hline \multicolumn{16}{|l|}{ Microliths: } \\
\hline Non-geometric & 8.0 & 39.1 & 25.1 & 7.63 & 30.44 & 6.0 & 47.6 & 18.4 & 8.27 & 44.89 & 2.8 & 21.8 & 7.7 & 4.06 & 52.59 \\
\hline Backed non-geometric & 9.2 & 36.0 & 19.0 & 6.00 & 31.52 & 1.4 & 13.8 & 6.0 & 2.15 & 35.65 & 1.1 & 7.4 & 2.9 & 1.19 & 40.75 \\
\hline Backed geometric & 10.0 & 35.0 & 18.7 & 5.08 & 27.17 & 3.7 & 15.3 & 6.1 & 2.51 & 40.87 & 1.0 & 6.3 & 3.0 & 1.13 & 38.02 \\
\hline Burin & 9.0 & 37.6 & 21.5 & 7.17 & 33.34 & 8.6 & 27.0 & 16.5 & 5.23 & 31.62 & 4.0 & 20.3 & 9.0 & 3.64 & 40.60 \\
\hline Notched & 12.5 & 39.5 & 25.3 & 8.56 & 33.87 & 4.9 & 40.7 & 17.8 & 10.33 & 57.96 & 2.8 & 15.0 & 7.3 & 3.83 & 52.16 \\
\hline \multicolumn{16}{|l|}{ Non-microlithic tools: } \\
\hline Side and/or end retouched & 30.0 & 72.0 & 56.0 & 13.63 & 24.32 & 29.0 & 92.0 & 50.9 & 20.22 & 39.69 & 10.0 & 47.0 & 24.7 & 11.44 & 46.33 \\
\hline Burin & 44.0 & 44.0 & 44.0 & - & - & 22.0 & 22.0 & 22.0 & - & - & 9.0 & 9.0 & 9.0 & - & - \\
\hline Notched/denticulated & 45.0 & 86.9 & 70.0 & - & - & 24.8 & 55.5 & 42.5 & - & - & 12.8 & 43.1 & 25.2 & - & - \\
\hline
\end{tabular}

Table A.7.10: Length, width and thickness measurements of tool typologies at Jwalapuram

\begin{tabular}{lrr}
\hline Retouched face & Microliths (\%) & Non-microlithic tools (\%) \\
\hline Bifacial & 2.9 & 23.8 \\
Dorsal & 37.3 & 28.6 \\
Edge & 44.2 & 4.8 \\
Ventral & 4.9 & 4.8 \\
Not recorded & 10.7 & 38.1 \\
\hline
\end{tabular}

Table A.7.11: Retouched faces of microliths and non-microlithic tools at Jwalapuram 


\begin{tabular}{|c|c|c|c|c|c|c|c|c|c|c|c|c|c|c|c|}
\hline \multirow[b]{2}{*}{ Tool type } & \multicolumn{5}{|c|}{ Retouch length (mm) } & \multicolumn{5}{|c|}{ Retouch depth (mm) } & \multicolumn{5}{|c|}{ Retouch angle $\left(^{\circ}\right)$} \\
\hline & Min & $\operatorname{Max}$ & Mean & SD & $\mathbf{C V}$ & Min & $\operatorname{Max}$ & Mean & SD & $\mathbf{C V}$ & Min & $\operatorname{Max}$ & Mean & SD & CV \\
\hline \multicolumn{16}{|l|}{ Microliths: } \\
\hline Non-geometric & 3.7 & 97.0 & 25.3 & 20.14 & 79.56 & 1.2 & 19.7 & 4.5 & 3.45 & 77.35 & 45.0 & 85.0 & 72.9 & 8.89 & 12.20 \\
\hline Backed non-geometric & 4.8 & 67.7 & 20.4 & 9.41 & 46.08 & 0.4 & 4.8 & 2.2 & 0.94 & 43.09 & 60.0 & 90.0 & 75.0 & 6.89 & 9.19 \\
\hline Backed geometric & 8.4 & 44.6 & 21.0 & 7.18 & 34.17 & 1.0 & 5.2 & 2.4 & 1.03 & 42.26 & 59.7 & 85.0 & 74.7 & 7.39 & 9.88 \\
\hline Burin & 2.6 & 86.0 & 15.2 & 19.27 & 126.41 & 7.2 & 22.9 & 15.2 & 5.23 & 34.29 & 70.0 & 70.0 & 70.0 & - & - \\
\hline Notched & 5.0 & 61.2 & 24.8 & 15.02 & 60.46 & 1.9 & 14.4 & 8.0 & 3.52 & 44.02 & 55.0 & 85.0 & 70.6 & 9.68 & 13.71 \\
\hline \multicolumn{16}{|l|}{ Non-microlithic tools: } \\
\hline Side and/or end retouched & 41.0 & 127.9 & 77.6 & 33.98 & 43.78 & 2.7 & 19.5 & 11.4 & 6.08 & 53.39 & 60.0 & 80.0 & 74.2 & 7.31 & 9.85 \\
\hline Burin & 9.0 & 9.0 & 9.0 & - & - & 24.0 & 24.0 & 24.0 & - & - & - & - & - & - & - \\
\hline Notched/denticulated & 39.1 & 164.0 & 103.9 & - & - & 4.8 & 18.2 & 11.1 & - & - & 60.0 & 85.3 & 72.1 & - & - \\
\hline
\end{tabular}

Table A.7.12: Retouch lengths, depths and angles of tool typologies at Jwalapuram 


\begin{tabular}{lrrrr}
\hline Microlith type & Minimum & Maximum & Mean & Std. Dev. \\
\hline \multicolumn{1}{c}{ Microliths: } & & & & \\
Non-geometric & 0.031 & 0.438 & 0.124 & 0.100 \\
Backed non-geometric & 0.063 & 0.750 & 0.181 & 0.104 \\
Backed geometric & 0.125 & 0.531 & 0.272 & 0.135 \\
Burin & 0.063 & 0.250 & 0.104 & 0.052 \\
Notched & 0.031 & 0.344 & 0.155 & 0.093
\end{tabular}

Non-microlithic tools:

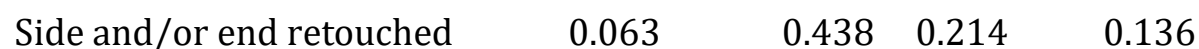

$\begin{array}{llll}\text { Burin } & 0.125 & 0.125 & 0.125\end{array}$

$\begin{array}{llll}\text { Notched/denticulated } & 0.063 & 0.344 & 0.238\end{array}$

Table A.7.13: Clarkson's (2002) IOI measurements of tool typologies at Jwalapuram 


\section{Appendix B: Results of statistical tests of temporal changes}

\section{B.1 Rose Cottage Cave, South Africa}

CORES

\begin{tabular}{|c|c|c|c|c|}
\hline Variable & Test used & Test result & Significance (at 0.05 level) & Trend \\
\hline Type & Chi-square & $\chi^{2}(12)=11.890, p=.455$ & Not significant & \\
\hline Raw material & Chi-square & $\chi^{2}(18)=12.011, p=.847$ & Not significant & \\
\hline Colour & Chi-square & $\chi^{2}(20)=13.970, p=.832$ & Not significant & \\
\hline Weight & Kruskal-Wallis & $\chi^{2}(2)=0.513, p=.774$ & Not significant & \\
\hline Longest dimension & Kruskal-Wallis & $\chi^{2}(2)=1.977, p=.230$ & Not significant & \\
\hline Longest axis & Kruskal-Wallis & $\chi^{2}(2)=0.230, p=.988$ & Not significant & \\
\hline Maximum width & $\begin{array}{l}\text { Log transformation }+ \\
\text { ANOVA }^{\text {a }}\end{array}$ & $\mathrm{F}(2,201)=0.054, \mathrm{p}=.948$ & Not significant & \\
\hline Maximum thickness & $\begin{array}{l}\text { Log transformation }+ \\
\text { ANOVA a }\end{array}$ & $F(2,201)=1.785, p=.170$ & Not significant & \\
\hline Longest face & $\begin{array}{l}\text { Log transformation }+ \\
\text { ANOVA }^{\text {a }}\end{array}$ & $\mathrm{F}(2,200)=1.979, \mathrm{p}=.141$ & Not significant & \\
\hline Striking platform width & Kruskal-Wallis ${ }^{b}$ & $\chi^{2}(2)=0.602, p=.740$ & Not significant & \\
\hline Striking platform thickness & $\begin{array}{l}\text { Log transformation }+ \\
\text { ANOVA }^{\text {a }}\end{array}$ & $\mathrm{F}(2,201)=0.263, \mathrm{p}=.769$ & Not significant & \\
\hline Striking platform type & Chi-square & $\chi^{2}(8)=11.164, p=.193$ & Not significant & \\
\hline Number of striking platforms & Chi-square & $\chi^{2}(10)=7.481, p=.679$ & Not significant & \\
\hline Perpendicular striking platforms & Chi-square & $\chi^{2}(2)=1.241, p=.538$ & Not significant & \\
\hline
\end{tabular}




\begin{tabular}{|c|c|c|c|}
\hline Total number of scars & Kruskal-Wallis & $\chi^{2}(2)=0.790 p=.674$ & Not significant \\
\hline Number of flake scars & Kruskal-Wallis & $\chi^{2}(2)=0.078, p=.962$ & Not significant \\
\hline Number of blade scars & Kruskal-Wallis & $\chi^{2}(2)=0.348, p=.840$ & Not significant \\
\hline Proportion of blade scars & Kruskal-Wallis & $\chi^{2}(2)=0.004, p=.998$ & Not significant \\
\hline Number of aberrant terminations & Kruskal-Wallis & $\chi^{2}(2)=4.720, p=.094$ & Not significant \\
\hline Percentage cortex cover & Kruskal-Wallis & $\chi^{2}(2)=1.662, p=.436$ & Not significant \\
\hline Recycling & Chi-square & $\chi^{2}(2)=0.904, p=.636$ & Not significant \\
\hline Average removal length & $\begin{array}{l}\text { Log transformation }+ \\
\text { ANOVA }^{a}\end{array}$ & $F(2,201)=0.625, p=.536$ & Not significant \\
\hline $\begin{array}{l}\text { Standard deviation of removal scar } \\
\text { lengths }\end{array}$ & ANOVA & $F(2,201)=0.673, p=.511$ & Not significant \\
\hline Number of scars per $\mathrm{cm}^{3}$ core volume & $\begin{array}{l}\text { Log transformation }+ \\
\text { ANOVA }^{\text {a }}\end{array}$ & $F(2,201)=0.086, p=.917$ & Not significant \\
\hline $\begin{array}{l}\text { Average scar length per } \mathrm{cm}^{3} \text { core } \\
\text { volume }\end{array}$ & Kruskal-Wallis & $\chi^{2}(2)=0.982, p=.612$ & Not significant \\
\hline Total scar length per $\mathrm{cm}^{3}$ core volume & $\begin{array}{l}\text { Log transformation }+ \\
\text { ANOVA }^{a}\end{array}$ & $F(2,201)=0.082, p=.922$ & Not significant \\
\hline \multicolumn{4}{|c|}{$\begin{array}{l}\text { Table B.1.1: Results of statistical tests of changes over time in core variables at RCC. } \\
\text { a significance confirmed with non-parametric Kruskal-Wallis test conducted on non-transformed data. } \\
\text { b although log transformation of the variable data results in normal distributions, both overall and within each layer (as determined by } \\
\text { the Shapiro-Wilk test), Levene's tests indicate that the variances are unequal. As a result of this violation of the assumption of } \\
\text { homoscedasticity, combined with the fact that there are considerable differences in sample sizes between the layers, the non-parametric } \\
\text { Kruskal-Wallis test is preferred as it is robust to these violations of the ANOVA test assumptions. The results of the Kruskal-Wallis test are } \\
\text { then confirmed with the Welch F-statistic for the log-transformed data, which can be used in cases of unequal variances. }\end{array}$} \\
\hline
\end{tabular}




\section{DEBITAGE}

\begin{tabular}{|c|c|c|c|c|}
\hline Variable & Test used & Test result & $\begin{array}{l}\text { Significance (at } 0.05 \\
\text { level) }\end{array}$ & Trend \\
\hline Type & Chi-square & $\begin{array}{l}\chi^{2}(4)=14.732, \mathbf{p}=. \mathbf{0 0 5} \\
\text { Cramér's V =.081 }\end{array}$ & $\begin{array}{l}\text { Significant but very weak } \\
\text { differences between layers }\end{array}$ & $\begin{array}{l}\text { More flakes and fewer blades in } \\
\text { Layer } 1 \text {, but no difference between } \\
\text { Layers } 2 \text { and } 3 \text {. }\end{array}$ \\
\hline Condition & Chi-square & $\chi^{2}(8)=9.258, p=.321$ & Not significant & \\
\hline Raw material & Chi-square & $\chi^{2}(20)=23.057, p=.286$ & Not significant & \\
\hline Colour & Chi-square & $\chi^{2}(24)=33.307, p=.098$ & Not significant & \\
\hline Weight & Kruskal-Wallis & $\chi^{2}(2)=5.355, p=.069$ & Not significant & \\
\hline Longest dimension & Kruskal-Wallis & $\chi^{2}(2)=1.270, p=.530$ & Not significant & \\
\hline Oriented length & Kruskal-Wallis & $\chi^{2}(2)=2.625, p=.269$ & Not significant & \\
\hline Maximum width & Kruskal-Wallis & $\chi^{2}(2)=8.891, \mathbf{p}=. \mathbf{0 1 2}$ & $\begin{array}{l}\text { Significant differences } \\
\text { between layers }\end{array}$ & $\begin{array}{l}\text { Increase from Layer } 2 \text { to } 1 \text {. Layers } \\
3 \text { and } 2 \text { are similar, and there is no } \\
\text { overall trend. }\end{array}$ \\
\hline One quarter width & Kruskal-Wallis & $\chi^{2}(2)=9.569, \mathbf{p}=.008$ & $\begin{array}{l}\text { Significant differences } \\
\text { between layers }\end{array}$ & $\begin{array}{l}\text { Increase from Layer } 2 \text { to } 1 \text {. Layers } \\
3 \text { and } 2 \text { are similar, and there is no } \\
\text { overall trend. }\end{array}$ \\
\hline Medial width & Kruskal-Wallis & $\chi^{2}(2)=5.578, p=.061$ & Not significant & \\
\hline Three quarter width & Kruskal-Wallis & $\chi^{2}(2)=2.514, p=.285$ & Not significant & \\
\hline Maximum thickness & $\begin{array}{l}\text { Log transformation }+ \\
\text { ANOVA }^{\text {a }}\end{array}$ & $F(2,1125)=2.855, p=.058$ & Not significant & \\
\hline Medial thickness & $\begin{array}{l}\text { Log transformation }+ \\
\text { ANOVA }^{a}\end{array}$ & $F(2,955)=1.308, p=.271$ & Not significant & \\
\hline Length:width ratio & Kruskal-Wallis & $\chi^{2}(2)=13.656, \mathbf{p}=. \mathbf{0 0 1}$ & $\begin{array}{l}\text { Significant differences } \\
\text { between layers }\end{array}$ & $\begin{array}{l}\text { Decrease from Layer } 2 \text { to } 1 \text {. Layers } \\
3 \text { and } 2 \text { are similar, and there is no } \\
\text { overall trend. }\end{array}$ \\
\hline Length:thickness ratio & Log transformation + & $F(2,1125)=6.208, p=.002$ & Significant differences & Increase from Layer 3 to 2 , and \\
\hline
\end{tabular}




\begin{tabular}{|c|c|c|c|c|}
\hline & ANOVA $^{a}$ & & between layers & $\begin{array}{l}\text { decrease from Layer } 2 \text { to } 1 \text {. No } \\
\text { overall trend. }\end{array}$ \\
\hline Striking platform width & Kruskal-Wallis & $\chi^{2}(2)=13.386, \mathbf{p}=. \mathbf{0 0 1}$ & $\begin{array}{l}\text { Significant differences } \\
\text { between layers }\end{array}$ & $\begin{array}{l}\text { Decrease from Layer } 3 \text { to } 2 \text {, and } \\
\text { increase from Layer } 2 \text { to } 1 \text {. No } \\
\text { overall trend. }\end{array}$ \\
\hline $\begin{array}{l}\text { Striking platform } \\
\text { thickness }\end{array}$ & Kruskal-Wallis & $\chi^{2}(2)=11.976, \mathbf{p}=.003$ & $\begin{array}{l}\text { Significant differences } \\
\text { between layers }\end{array}$ & $\begin{array}{l}\text { Decrease from Layer } 3 \text { to } 2 \text {, and } \\
\text { increase from Layer } 2 \text { to } 1 \text {. No } \\
\text { overall trend. }\end{array}$ \\
\hline Striking platform lipping & Chi-square & $\chi^{2}(4)=3.907, p=.419$ & Not significant & \\
\hline Curvature & Kruskal-Wallis & $\chi^{2}(2)=4.411, p=.110$ & Not significant & \\
\hline Percentage cortex cover & Kruskal-Wallis & $\chi^{2}(2)=2.214, p=.330$ & Not significant & \\
\hline Number of dorsal scars & Kruskal-Wallis & $\chi^{2}(2)=0.502, p=.778$ & Not significant & \\
\hline $\begin{array}{l}\text { Number of parallel } \\
\text { arrises }\end{array}$ & Kruskal-Wallis & $\chi^{2}(2)=10.204, \mathbf{p}=.006$ & $\begin{array}{l}\text { Significant differences } \\
\text { between layers }\end{array}$ & $\begin{array}{l}\text { Decrease from Layer } 2 \text { to } 1 \text {. Layers } \\
3 \text { and } 2 \text { are similar, and there is no } \\
\text { overall trend. }\end{array}$ \\
\hline Scar orientation & Chi-square & $\chi^{2}(8)=4.058, p=.852$ & Not significant & \\
\hline Termination type & Chi-square & $\chi^{2}(12)=13.688, p=.321$ & Not significant & \\
\hline $\begin{array}{l}\text { Evidence of bipolar } \\
\text { percussion }\end{array}$ & Chi-square & $\chi^{2}(2)=3.035, p=.219$ & Not significant & \\
\hline Recycling & Chi-square & $\chi^{2}(2)=0.277, p=.871$ & Not significant & \\
\hline $\begin{array}{l}\text { Evidence of core } \\
\text { maintenance }\end{array}$ & Chi-square & $\chi^{2}(8)=3.781, p=.876$ & Not significant & \\
\hline
\end{tabular}




\section{Table B.1.2: Results of statistical tests of changes over time in debitage variables at RCC}

a significance confirmed with non-parametric Kruskal-Wallis test conducted on non-transformed data

\section{MICROLITHS}

\begin{tabular}{|c|c|c|c|c|}
\hline Variable & Test used & Test result & Significance (at 0.05 level) & Trend \\
\hline Blank type & Chi-square & $\chi^{2}(2)=5.225, p=.072$ & Not significant & \\
\hline Condition & Chi-square & $\chi^{2}(8)=12.093, p=.147$ & Not significant & \\
\hline Raw material & Chi-square & $\chi^{2}(14)=21.074, p=.100$ & Not significant & \\
\hline Colour & Chi-square & $\chi^{2}(20)=25.441, p=.185$ & Not significant & \\
\hline Weight & $\begin{array}{l}\text { Log transformation } \\
+ \text { ANOVA }^{\text {a }}\end{array}$ & $F(2,131)=2.901, p=.058$ & Not significant & \\
\hline Longest dimension & ANOVA & $F(2,131)=0.349, p=.706$ & Not significant & \\
\hline Oriented length & ANOVA & $F(2,131)=1.460, p=.236$ & Not significant & \\
\hline Maximum width & Kruskal-Wallis ${ }^{\text {b }}$ & $\chi^{2}(2)=6.669, \mathbf{p}=.036$ & $\begin{array}{l}\text { Significant differences } \\
\text { between layers }\end{array}$ & $\begin{array}{l}\text { Increase from Layer } 2 \text { to } 1 \text {. Layers } 3 \\
\text { and } 2 \text { are similar, and there is no } \\
\text { overall trend. }\end{array}$ \\
\hline One quarter width & Kruskal-Wallis b & $\chi^{2}(2)=6.573, \mathbf{p}=.037$ & $\begin{array}{l}\text { Significant differences } \\
\text { between layers }\end{array}$ & $\begin{array}{l}\text { Increase from Layer } 2 \text { to } 1 \text {. Layers } 3 \\
\text { and } 2 \text { are similar, and there is no } \\
\text { overall trend. }\end{array}$ \\
\hline Medial width & $\begin{array}{l}\text { Log transformation } \\
+ \text { ANOVA }^{a}\end{array}$ & $F(2,131)=6.109, \mathbf{p}=.003$ & $\begin{array}{l}\text { Significant differences } \\
\text { between layers }\end{array}$ & $\begin{array}{l}\text { Increase from Layer } 2 \text { to } 1 \text {. Layers } 3 \\
\text { and } 2 \text { are similar, and there is no } \\
\text { overall trend. }\end{array}$ \\
\hline Three quarter width & $\begin{array}{l}\text { Log transformation } \\
+ \text { ANOVA }\end{array}$ & $F(2,131)=3.764, p=.026$ & $\begin{array}{l}\text { ANOVA and KW tests give } \\
\text { different results at the } 0.05\end{array}$ & $\begin{array}{l}\text { Increase from Layer } 2 \text { to } 1 \text {. Layers } 3 \\
\text { and } 2 \text { are similar, and there is no }\end{array}$ \\
\hline
\end{tabular}




\begin{tabular}{|c|c|c|c|c|}
\hline & Kruskal-Wallis & $\chi^{2}(2)=4.903, p=.086$ & $\begin{array}{l}\text { level, but the overall result } \\
\text { will be considered to be } \\
\text { significant }\end{array}$ & overall trend. \\
\hline Maximum thickness & $\begin{array}{l}\text { Log transformation } \\
+ \text { ANOVA }^{\text {a }}\end{array}$ & $\mathrm{F}(2,131)=1.293, \mathrm{p}=.278$ & Not significant & \\
\hline Medial thickness & $\begin{array}{l}\text { Log transformation } \\
+ \text { ANOVA }\end{array}$ & $\mathrm{F}(2,131)=0.871, \mathrm{p}=.421$ & Not significant & \\
\hline Length:width ratio & Kruskal-Wallis b & $\chi^{2}(2)=8.399, \mathbf{p}=\mathbf{. 0 1 5}$ & $\begin{array}{l}\text { Significant differences } \\
\text { between layers }\end{array}$ & $\begin{array}{l}\text { Decrease from Layer } 2 \text { to } 1 \text {. Layers } 3 \\
\text { and } 2 \text { are similar, and there is no } \\
\text { overall trend. }\end{array}$ \\
\hline Length:thickness ratio & Kruskal-Wallis ${ }^{b}$ & $\chi^{2}(2)=5.408, p=.067$ & Not significant & \\
\hline $\begin{array}{l}\text { Width standard } \\
\text { deviation }\end{array}$ & ANOVA & $F(2,112)=2.119, p=.125$ & Not significant & \\
\hline $\begin{array}{l}\text { Striking platform } \\
\text { width }\end{array}$ & Kruskal-Wallis b & $\chi^{2}(2)=4.323, p=.115$ & Not significant & \\
\hline $\begin{array}{l}\text { Striking platform } \\
\text { thickness }\end{array}$ & Kruskal-Wallis & $\chi^{2}(2)=5.496, p=.064$ & Not significant & \\
\hline Curvature & ANOVA & $F(2,131)=1.766, p=.175$ & Not significant & \\
\hline $\begin{array}{l}\text { Percentage cortex } \\
\text { cover }\end{array}$ & Kruskal-Wallis & $\chi^{2}(2)=0.252, p=.882$ & Not significant & \\
\hline $\begin{array}{l}\text { Number of dorsal } \\
\text { scars }\end{array}$ & Kruskal-Wallis & $\chi^{2}(2)=6.714, \mathbf{p}=.035$ & $\begin{array}{l}\text { Significant differences } \\
\text { between layers }\end{array}$ & $\begin{array}{l}\text { Increase over time in mean number of } \\
\text { dorsal scars, with most of the } \\
\text { difference between Layers } 2 \text { and } 1 .\end{array}$ \\
\hline $\begin{array}{l}\text { Number of parallel } \\
\text { arrises }\end{array}$ & Kruskal-Wallis & $\chi^{2}(2)=1.281, p=.527$ & Not significant & \\
\hline $\begin{array}{l}\text { Number of scars per } \\
\mathrm{cm}^{2} \text { surface area }\end{array}$ & Kruskal-Wallis ${ }^{b}$ & $\chi^{2}(2)=0.233, p=.890$ & Not significant & \\
\hline
\end{tabular}




\begin{tabular}{|c|c|c|c|c|}
\hline $\begin{array}{l}\text { Number of parallel } \\
\text { arrises per } \mathrm{cm}^{2} \text { surface } \\
\text { area }\end{array}$ & $\begin{array}{l}\text { Log transformation } \\
+ \text { ANOVA }^{a}\end{array}$ & $F(2,113)=0.442, p=.644$ & Not significant & \\
\hline Scar orientation & Chi-square & $\chi^{2}(6)=12.536, p=.051$ & Not significant & \\
\hline Termination type & Chi-square & $\chi^{2}(10)=11.829, p=.297$ & Not significant & \\
\hline Recycling & $\mathrm{N} / \mathrm{A}^{\mathrm{c}}$ & & & \\
\hline Other classification & $\mathrm{N} / \mathrm{A}^{\mathrm{c}}$ & & & \\
\hline Coarse typology & Chi-square & $\chi^{2}(16)=12.692, p=.695$ & Not significant & \\
\hline Narrow typology & Chi-square & $\chi^{2}(34)=28.820, p=.719$ & Not significant & \\
\hline $\begin{array}{l}\text { Presence of tangs or } \\
\text { shoulders }\end{array}$ & $\mathrm{N} / \mathrm{A}^{\mathrm{c}}$ & & & \\
\hline Haft width & $\mathrm{N} / \mathrm{A}^{\mathrm{c}}$ & & & \\
\hline Haft thickness & $\mathrm{N} / \mathrm{A}^{\mathrm{c}}$ & & & \\
\hline Haft length & $\mathrm{N} / \mathrm{A}^{\mathrm{c}}$ & & & \\
\hline $\begin{array}{l}\text { Number of worked } \\
\text { edges }\end{array}$ & Kruskal-Wallis & $\chi^{2}(2)=1.142, p=.565$ & Not significant & \\
\hline Retouch location & Chi-square & $\chi^{2}(16)=22.290, p=.134$ & Not significant & \\
\hline Retouch face & Chi-square & $\chi^{2}(6)=3.996, p=.677$ & Not significant & \\
\hline Retouch outline & Chi-square & $\chi^{2}(10)=16.149, p=.095$ & Not significant & \\
\hline Retouch type & Chi-square & $\chi^{2}(20)=22.184, p=.331$ & Not significant & \\
\hline Retouch angle & Kruskal-Wallis & $\chi^{2}(2)=4.267, p=.118$ & Not significant & \\
\hline Retouch depth & $\begin{array}{l}\text { Log transformation } \\
+ \text { ANOVA }^{\text {a }}\end{array}$ & $F(2,143)=1.286, p=.279$ & Not significant & \\
\hline Retouch length & $\begin{array}{l}\text { Log transformation } \\
+ \text { ANOVA }^{\text {a }}\end{array}$ & $F(2,143)=7.193, \mathbf{p}=. \mathbf{0 0 1}$ & $\begin{array}{l}\text { Significant differences } \\
\text { between layers }\end{array}$ & $\begin{array}{l}\text { Decrease from Layer } 3 \text { to } 2 \text {, and } \\
\text { increase from Layer } 2 \text { to } 1 \text {. No overall } \\
\text { trend. }\end{array}$ \\
\hline Index of Invasiveness & Kruskal-Wallis & $\chi^{2}(2)=8.416, \mathbf{p}=.015$ & $\begin{array}{l}\text { Significant differences } \\
\text { between layers }\end{array}$ & $\begin{array}{l}\text { Increase over time, with most of the } \\
\text { difference between Layers } 2 \text { and } 1 .\end{array}$ \\
\hline
\end{tabular}


ANOVA and KW tests give different results at the 0.05 level, but the overall result

$$
\text { Kruskal-Wallis } \quad \chi^{2}(2)=5.342, p=.069
$$

will be considered to be

significant
Increase from Layer 2 to 1 . Layers 3 and 2 are similar, and there is no overall trend.

\begin{abstract}
Table B.1.3: Results of statistical tests of changes over time in microlith variables at RCC.
a significance confirmed with non-parametric Kruskal-Wallis test conducted on non-transformed data.

b although log transformation of the variable data results in normal distributions, both overall and within each layer (as determined by the Shapiro-Wilk test), Levene's tests based on means indicate that the variances are unequal. As a result of this violation of the assumption of homoscedasticity, combined with the fact that there are considerable differences in sample sizes between the layers, the non-parametric Kruskal-Wallis test is preferred as it is robust to these violations of the ANOVA test assumptions. The results of the Kruskal-Wallis test are then confirmed with the Welch F-statistic for the log-transformed data, which can be used in cases of unequal variances.

c variable is evident in the sample but not in every layer and/or in numbers too small to be statistically viable, or variable that exhibits only one category in two layers and so cannot be compared meaningfully between layers.
\end{abstract}

NON-MICROLITHIC TOOLS

\begin{tabular}{|c|c|c|c|c|}
\hline Variable & Test used & Test result & Significance (at 0.05 level) & Trend \\
\hline Blank type & Chi-square & $\begin{array}{l}\chi^{2}(2)=9.905, \mathbf{p}=. \mathbf{0 0 7} \\
\text { Cramér's } V=.371\end{array}$ & $\begin{array}{l}\text { Significant and strong } \\
\text { differences between layers }\end{array}$ & $\begin{array}{l}\text { Layers } 3 \text { and } 1 \text { contain roughly equal } \\
\text { proportions of flake and blade blanks. } \\
\text { Layer } 2 \text { contains predominantly blade } \\
\text { blanks. No overall trend. }\end{array}$ \\
\hline Condition & Chi-square & $\chi^{2}(8)=9.167, p=.328$ & Not significant & \\
\hline Raw material & Chi-square & $\chi^{2}(16)=17.636, p=.346$ & Not significant & \\
\hline Colour & Chi-square & $\chi^{2}(16)=18.971, p=.270$ & Not significant & \\
\hline
\end{tabular}




\begin{tabular}{|c|c|c|c|c|}
\hline & + ANOVAa $^{a}$ & & & \\
\hline Longest dimension & $\begin{array}{l}\text { Log transformation } \\
+ \text { ANOVAa }\end{array}$ & $F(2,69)=3.723, \mathbf{p}=. \mathbf{0 2 9}$ & $\begin{array}{l}\text { Significant differences } \\
\text { between layers }\end{array}$ & $\begin{array}{l}\text { Layers } 3 \text { and } 1 \text { are similar, but tools in } \\
\text { Layer } 2 \text { are much longer on average. }\end{array}$ \\
\hline Oriented length & $\begin{array}{l}\text { Log transformation } \\
+ \text { ANOVA a }\end{array}$ & $F(2,69)=3.189, \mathbf{p}=. \mathbf{0 4 7}$ & $\begin{array}{l}\text { Significant differences } \\
\text { between layers }\end{array}$ & $\begin{array}{l}\text { Layers } 3 \text { and } 1 \text { are similar, but tools in } \\
\text { Layer } 2 \text { are much longer on average. }\end{array}$ \\
\hline Maximum width & ANOVA & $F(2,69)=0.390, p=.678$ & Not significant & \\
\hline One quarter width & ANOVA & $F(2,69)=1.656, p=.198$ & Not significant & \\
\hline Medial width & ANOVA & $F(2,69)=0.310, p=.735$ & Not significant & \\
\hline Maximum thickness & $\begin{array}{l}\text { Log transformation } \\
+ \text { ANOVA }^{a}\end{array}$ & $F(2,69)=2.504, p=.089$ & Not significant & \\
\hline Medial thickness & $\begin{array}{l}\text { Log transformation } \\
+ \text { ANOVA }\end{array}$ & $F(2,69)=1.385, p=.257$ & Not significant & \\
\hline Length:width ratio & $\begin{array}{l}\text { Log transformation } \\
+ \text { ANOVA } \\
\text { Kruskal-Wallis }\end{array}$ & $\chi^{2}(2)=6.965, \mathbf{p}=. \mathbf{0 3 1}$ & $\begin{array}{l}\text { ANOVA and KW tests give } \\
\text { different results at the } 0.05 \\
\text { level, but the overall result will } \\
\text { be considered to be significant }\end{array}$ & $\begin{array}{l}\text { Layers } 3 \text { and } 1 \text { are similar, but the } \\
\text { mean ratio in Layer } 2 \text { is much higher. }\end{array}$ \\
\hline $\begin{array}{l}\text { Width standard } \\
\text { deviation }\end{array}$ & Kruskal-Wallis & $\chi^{2}(2)=3.647, p=.161$ & Not significant & \\
\hline $\begin{array}{l}\text { Striking platform } \\
\text { width }\end{array}$ & Kruskal-Wallis & $\chi^{2}(2)=4.734, p=.094$ & Not significant & \\
\hline $\begin{array}{l}\text { Striking platform } \\
\text { thickness }\end{array}$ & Kruskal-Wallis & $\chi^{2}(2)=2.885, p=.236$ & Not significant & \\
\hline Striking platform type & Chi-square & $\chi^{2}(12)=9.157, p=.689$ & Not significant & \\
\hline Striking platform & Chi-square & $\chi^{2}(4)=11.142, \mathbf{p}=. \mathbf{0 2 5}$ & Significant and moderately & More platform lipping in Layer 3. \\
\hline
\end{tabular}




\begin{tabular}{|c|c|c|c|}
\hline & & & layers \\
\hline Curvature & Kruskal-Wallis & $\chi^{2}(2)=1.780, p=.411$ & Not significant \\
\hline $\begin{array}{l}\text { Percentage cortex } \\
\text { cover }\end{array}$ & Kruskal-Wallis & $\chi^{2}(2)=0.178, p=.915$ & Not significant \\
\hline $\begin{array}{l}\text { Number of dorsal } \\
\text { scars }\end{array}$ & Kruskal-Wallis & $\chi^{2}(2)=1.051, p=.591$ & Not significant \\
\hline $\begin{array}{l}\text { Number of parallel } \\
\text { arrises }\end{array}$ & Kruskal-Wallis & $\chi^{2}(2)=3.350, p=.187$ & Not significant \\
\hline $\begin{array}{l}\text { Number of scars per } \\
\mathrm{cm}^{2} \text { surface area }\end{array}$ & $\begin{array}{l}\text { Log transformation } \\
+ \text { ANOVA }\end{array}$ & $F(2,67)=0.867, p=.425$ & Not significant \\
\hline $\begin{array}{l}\text { Number of parallel } \\
\text { arrises per } \mathrm{cm}^{2} \text { surface } \\
\text { area }\end{array}$ & $\begin{array}{l}\text { Log transformation } \\
+ \text { ANOVA a }\end{array}$ & $\mathrm{F}(2,54)=1.542, \mathrm{p}=.223$ & Not significant \\
\hline Scar orientation & Chi-square & $\chi^{2}(8)=14.359, p=.073$ & Not significant \\
\hline Termination type & Chi-square & $\chi^{2}(12)=12.708, p=.391$ & Not significant \\
\hline $\begin{array}{l}\text { Evidence of bipolar } \\
\text { percussion }\end{array}$ & $\mathrm{N} / \mathrm{A}^{\mathrm{b}}$ & & \\
\hline Recycling & $\mathrm{N} / \mathrm{A}^{\mathrm{b}}$ & & \\
\hline $\begin{array}{l}\text { Evidence of core } \\
\text { maintenance }\end{array}$ & $\mathrm{N} / \mathrm{A}^{\mathrm{b}}$ & & \\
\hline Other classification & $\mathrm{N} / \mathrm{A}^{\mathrm{b}}$ & & \\
\hline Coarse typology & Chi-square & $\chi^{2}(14)=13.883, p=.458$ & Not significant \\
\hline Narrow typology & Chi-square & $\chi^{2}(32)=32.618, p=.436$ & Not significant \\
\hline $\begin{array}{l}\text { Number of worked } \\
\text { edges }\end{array}$ & Kruskal-Wallis & $\chi^{2}(2)=0.089, p=.956$ & Not significant \\
\hline Retouch location & Chi-square & $\chi^{2}(18)=17.670, p=.478$ & Not significant \\
\hline Retouch face & Chi-square & $\chi^{2}(8)=11.809, p=.160$ & Not significant \\
\hline Retouch outline & Chi-square & $\chi^{2}(8)=8.430, p=.393$ & Not significant \\
\hline
\end{tabular}




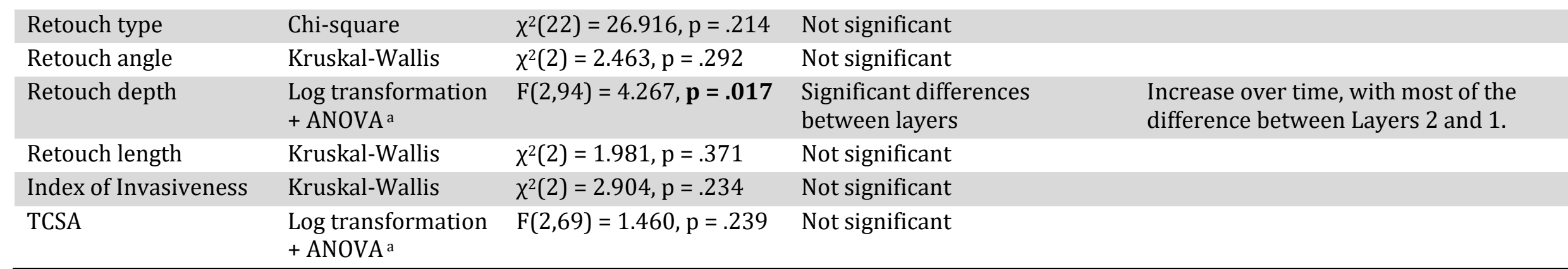

Table B.1.4: Results of statistical tests of changes over time in non-microlithic tool variables at RCC.

a significance confirmed with non-parametric Kruskal-Wallis test conducted on non-transformed data.

b variable is evident in the sample but not in every layer and/or in numbers too small to be statistically viable, or variable that exhibits only one category in two layers and so cannot be compared meaningfully between layers.

\section{B.2 Umhlatuzana, South Africa}

\section{CORES}

\begin{tabular}{|c|c|c|c|c|}
\hline Variable & Test used & Test result & Significance (at 0.05 level) & Trend \\
\hline Type & Chi-square & $\begin{array}{l}\chi^{2}(12)=29.064, p= \\
.004, \text { Cramér's } V=.300\end{array}$ & $\begin{array}{l}\text { Significant and strong } \\
\text { differences between layers }\end{array}$ & $\begin{array}{l}\text { Decrease over time in proportion of radial } \\
\text { cores. More flake cores (uni- and bi- } \\
\text { directional) in Layer } 25 \text {. Amorphous cores } \\
\text { are absent in Layer } 27 \text { and increase from } \\
\text { Layer } 25 \text { to } 23 \text {. }\end{array}$ \\
\hline Raw material & Chi-square & $\chi^{2}(18)=28.019, p=.062$ & Not significant & \\
\hline Colour & Chi-square & $\chi^{2}(10)=20.308, p=$ & $\begin{array}{l}\text { Significant and moderately } \\
\text { strong differences between }\end{array}$ & $\begin{array}{l}\text { Increase over time in white coloured cores } \\
\text { Decrease over time in purple, clear and, to }\end{array}$ \\
\hline
\end{tabular}




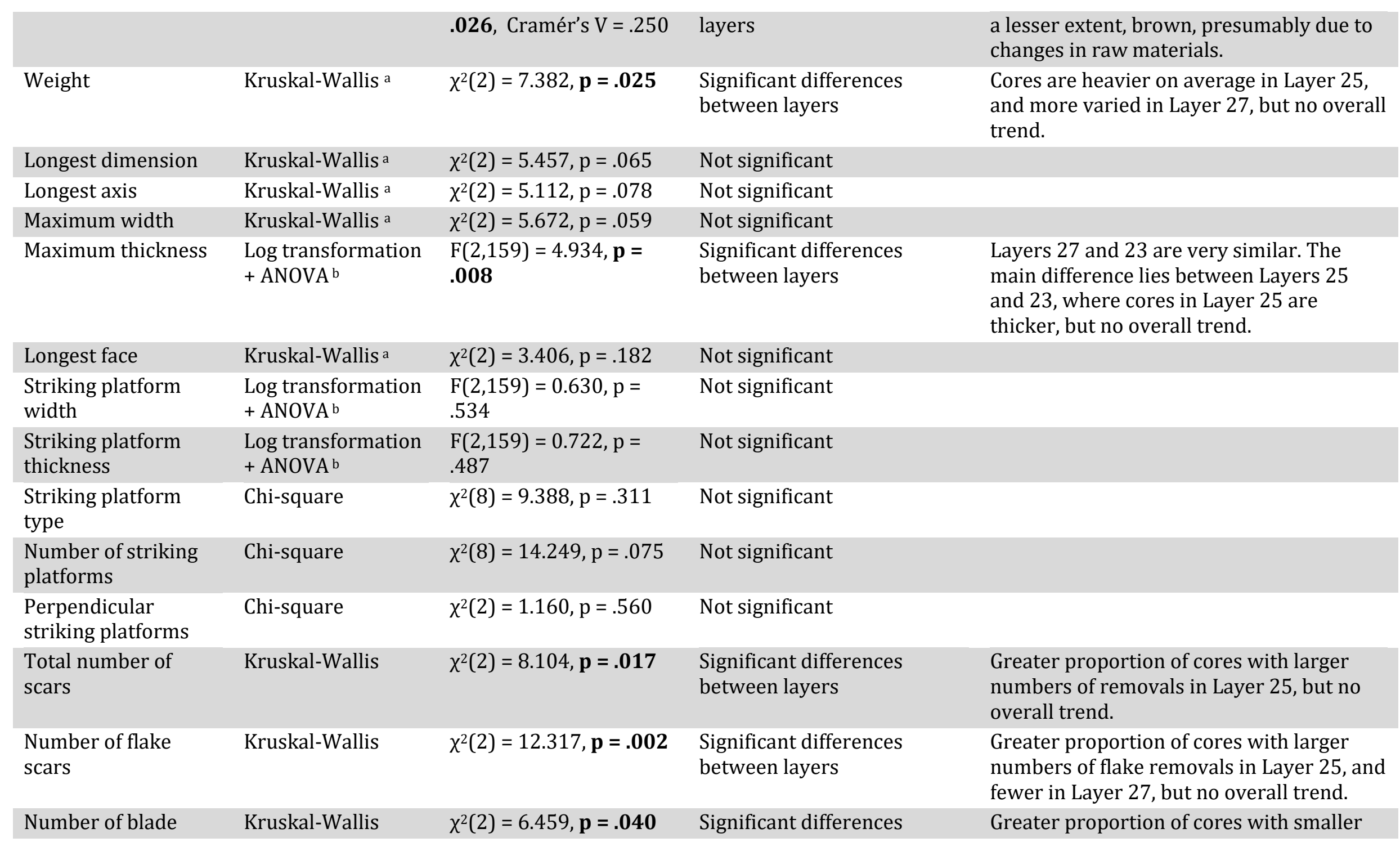




\begin{tabular}{|c|c|c|c|c|}
\hline scars & & & between layers & $\begin{array}{l}\text { numbers of blade removals in Layer } 25 \text {, } \\
\text { but no overall trend. }\end{array}$ \\
\hline $\begin{array}{l}\text { Proportion of blade } \\
\text { scars }\end{array}$ & Kruskal-Wallis & $\chi^{2}(2)=7.222, \mathbf{p}=. \mathbf{0 2 7}$ & $\begin{array}{l}\text { Significant differences } \\
\text { between layers }\end{array}$ & $\begin{array}{l}\text { Blade removal proportions are lower in } \\
\text { Layer } 25 \text { and slightly higher in Layer } 27 \text {, } \\
\text { but no overall trend. }\end{array}$ \\
\hline $\begin{array}{l}\text { Number of aberrant } \\
\text { terminations }\end{array}$ & Kruskal-Wallis & $\chi^{2}(2)=0.477, p=.788$ & Not significant & \\
\hline $\begin{array}{l}\text { Percentage cortex } \\
\text { cover }\end{array}$ & Kruskal-Wallis & $\chi^{2}(2)=10.128, \mathbf{p}=.006$ & $\begin{array}{l}\text { Significant differences } \\
\text { between layers }\end{array}$ & $\begin{array}{l}\text { Increase in average cortex cover over time, } \\
\text { with most of the difference between Layers } \\
25 \text { and } 23 \text {. }\end{array}$ \\
\hline $\begin{array}{l}\text { Average removal } \\
\text { length }\end{array}$ & $\begin{array}{l}\text { Log transformation } \\
+ \text { ANOVA }\end{array}$ & $\begin{array}{l}F(2,159)=0.582, p= \\
.560\end{array}$ & Not significant & \\
\hline $\begin{array}{l}\text { Standard deviation of } \\
\text { removal scar lengths }\end{array}$ & Kruskal-Wallis a & $\chi^{2}(2)=0.226, p=.893$ & Not significant & \\
\hline $\begin{array}{l}\text { Number of scars per } \\
\mathrm{cm}^{3} \text { core volume }\end{array}$ & Kruskal-Wallis a & $\chi^{2}(2)=4.498, p=.106$ & Not significant & \\
\hline $\begin{array}{l}\text { Average scar length } \\
\text { per } \mathrm{cm}^{3} \text { core volume }\end{array}$ & Kruskal-Wallis a & $\chi^{2}(2)=7.994, \mathbf{p}=. \mathbf{0 1 8}$ & $\begin{array}{l}\text { Significant differences } \\
\text { between layers }\end{array}$ & $\begin{array}{l}\text { Lower average ratio in Layer } 25 \text {, but no } \\
\text { overall trend. }\end{array}$ \\
\hline $\begin{array}{l}\text { Total scar length per } \\
\mathrm{cm}^{3} \text { core volume }\end{array}$ & Kruskal-Wallis a & $\chi^{2}(2)=5.032, p=.081$ & Not significant & \\
\hline
\end{tabular}

Table B.2.1: Results of statistical tests of changes over time in core variables at Umhlatuzana.

a although log transformation of the variable data results in normal distributions, both overall and within each layer (as determined by the Shapiro-Wilk test), Levene's tests indicate that the variances are unequal. As a result of this violation of the assumption of

homoscedasticity, combined with the fact that there are considerable differences in sample sizes between the layers $\left(n_{25} \approx 10 * n_{27}\right)$, the non-parametric Kruskal-Wallis test is preferred as it is robust to these violations of the ANOVA test assumptions. The results of the

Kruskal-Wallis test are then confirmed with the Welch F-statistic for the log-transformed data, which can be used in cases of unequal variances.

b significance confirmed with non-parametric Kruskal-Wallis test conducted on non-transformed data. 


\section{DEBITAGE}

\begin{tabular}{|c|c|c|c|c|}
\hline Variable & Test used & Test result & Significance (at 0.05 level) & Trend \\
\hline Type & Chi-square & $\begin{array}{l}\chi^{2}(4)=17.950, \mathbf{p}=. \mathbf{0 0 1} \\
\text { Cramér's V =.087 }\end{array}$ & $\begin{array}{l}\text { Significant but very weak } \\
\text { differences between layers }\end{array}$ & $\begin{array}{l}\text { More flakes in Layer } 27 \text { and more } \\
\text { blades in Layer } 25 \text {, but no overall } \\
\text { trend. }\end{array}$ \\
\hline Condition & Chi-square & $\chi^{2}(10)=7.382, p=.689$ & Not significant & \\
\hline Raw material & Chi-square & $\begin{array}{l}\chi^{2}(26)=147.355, \mathbf{p}<. \mathbf{0 0 1} \\
\text { Cramér's V }=.249\end{array}$ & $\begin{array}{l}\text { Significant and moderately } \\
\text { strong differences between } \\
\text { layers }\end{array}$ & $\begin{array}{l}\text { Increase over time in use of quartz. } \\
\text { Decrease over time in use of } \\
\text { hornfels and, to a lesser extent, } \\
\text { quartzite. }\end{array}$ \\
\hline Colour & Chi-square & $\begin{array}{l}\chi^{2}(16)=116.156, \mathbf{p}<. \mathbf{0 0 1} \\
\text { Cramér's V = .221 }\end{array}$ & $\begin{array}{l}\text { Significant and moderately } \\
\text { strong differences between } \\
\text { layers }\end{array}$ & $\begin{array}{l}\text { Increase over time in proportion of } \\
\text { white coloured debitage. Decrease } \\
\text { over time in grey and purple, as a } \\
\text { result of changes in raw materials. }\end{array}$ \\
\hline Weight & $\begin{array}{l}\text { Log transformation } \\
+ \text { ANOVA }^{\mathrm{a}}\end{array}$ & $F(2,783.401)=6.513, p=.002 b$ & $\begin{array}{l}\text { Significant differences } \\
\text { between layers }\end{array}$ & $\begin{array}{l}\text { Decrease from Layer } 27 \text { to } 25 . \\
\text { Layers } 25 \text { and } 23 \text { are similar. }\end{array}$ \\
\hline Longest dimension & $\begin{array}{l}\text { Log transformation } \\
+ \text { ANOVA }^{\mathrm{a}}\end{array}$ & $F(2,774.436)=10.671, \mathbf{p}<.001 b$ & $\begin{array}{l}\text { Significant differences } \\
\text { between layers }\end{array}$ & Consistent decrease over time. \\
\hline Oriented length & $\begin{array}{l}\text { Log transformation } \\
+ \text { ANOVA }^{\text {a }}\end{array}$ & $F(2,772.750)=12.398, \mathbf{p}<.001 b$ & $\begin{array}{l}\text { Significant differences } \\
\text { between layers }\end{array}$ & Consistent decrease over time. \\
\hline Maximum width & $\begin{array}{l}\text { Log transformation } \\
+ \text { ANOVA }^{\text {a }}\end{array}$ & $F(2,1183)=7.498, \mathbf{p}=. \mathbf{0 0 1}$ & $\begin{array}{l}\text { Significant differences } \\
\text { between layers }\end{array}$ & $\begin{array}{l}\text { Decrease from Layer } 27 \text { to } 25 . \\
\text { Layers } 25 \text { and } 23 \text { are similar. }\end{array}$ \\
\hline One quarter width & $\begin{array}{l}\text { Log transformation } \\
+ \text { ANOVA } \\
\text { Kruskal-Wallis }\end{array}$ & $\chi^{2}(2)=6.918, \mathbf{p}=.031$ & $\begin{array}{l}\text { ANOVA and KW tests give } \\
\text { different results at the } 0.05 \\
\text { level, but the overall result } \\
\text { will be considered to be } \\
\text { significant }\end{array}$ & $\begin{array}{l}\text { Decrease from Layer } 27 \text { to } 25 \text {, and } \\
\text { increase from Layer } 25 \text { to } 23 \text {. No } \\
\text { overall trend. }\end{array}$ \\
\hline Medial width & $\begin{array}{l}\text { Log transformation } \\
+ \text { ANOVA }\end{array}$ & $F(2,796)=2.738, p=.065$ & Not significant & \\
\hline
\end{tabular}




\begin{tabular}{|c|c|c|c|c|}
\hline $\begin{array}{l}\text { Three quarter } \\
\text { width }\end{array}$ & $\begin{array}{l}\text { Log transformation } \\
+ \text { ANOVA }^{\text {a }}\end{array}$ & $F(2,528.755)=2.682, p=.069 b$ & Not significant & \\
\hline $\begin{array}{l}\text { Maximum } \\
\text { thickness }\end{array}$ & $\begin{array}{l}\text { Log transformation } \\
+ \text { ANOVA } \\
\text { Kruskal-Wallis }\end{array}$ & $\chi^{2}(2)=4.459, p=.108$ & $\begin{array}{l}\text { ANOVA and KW tests give } \\
\text { different results at the } 0.05 \\
\text { level, but the overall result } \\
\text { will be considered to be } \\
\text { significant }\end{array}$ & $\begin{array}{l}\text { Decrease from Layer } 27 \text { to } 25 \text {, and } \\
\text { increase from Layer } 25 \text { to } 23 \text {. No } \\
\text { overall trend. }\end{array}$ \\
\hline Medial thickness & $\begin{array}{l}\text { Log transformation } \\
+ \text { ANOVA }^{\text {a }}\end{array}$ & $F(2,889)=5.164, p=.006$ & $\begin{array}{l}\text { Significant differences } \\
\text { between layers }\end{array}$ & $\begin{array}{l}\text { Decrease from Layer } 27 \text { to } 25 \text {, and } \\
\text { increase from Layer } 25 \text { to } 23 \text {. No } \\
\text { overall trend. }\end{array}$ \\
\hline Length:width ratio & $\begin{array}{l}\text { Log transformation } \\
+ \text { ANOVA }^{\text {a }}\end{array}$ & $F(2,1174)=3.195, \mathbf{p}=. \mathbf{0 4 1}$ & $\begin{array}{l}\text { Significant differences } \\
\text { between layers }\end{array}$ & $\begin{array}{l}\text { Increase from Layer } 27 \text { to } 25 \text {, and } \\
\text { decrease from Layer } 25 \text { to } 23 \text {. No } \\
\text { overall trend. }\end{array}$ \\
\hline $\begin{array}{l}\text { Length:thickness } \\
\text { ratio }\end{array}$ & $\begin{array}{l}\text { Log transformation } \\
+ \text { ANOVA }^{a}\end{array}$ & $F(2,796)=12.858, \mathbf{p}<.001$ & $\begin{array}{l}\text { Significant differences } \\
\text { between layers }\end{array}$ & $\begin{array}{l}\text { Increase from Layer } 27 \text { to } 25 \text {, and } \\
\text { decrease from Layer } 25 \text { to } 23 \text {. No } \\
\text { overall trend. }\end{array}$ \\
\hline $\begin{array}{l}\text { Width standard } \\
\text { deviation }\end{array}$ & Kruskal-Wallis & $\chi^{2}(2)=2.565, p=.277$ & Not significant & \\
\hline $\begin{array}{l}\text { Striking platform } \\
\text { width }\end{array}$ & $\begin{array}{l}\text { Log transformation } \\
+ \text { ANOVA }^{\text {a }}\end{array}$ & $\mathrm{F}(2,699.721)=8.884, \mathbf{p}<. \mathbf{0 0 1} b$ & $\begin{array}{l}\text { Significant differences } \\
\text { between layers }\end{array}$ & $\begin{array}{l}\text { Decrease in mean striking platform } \\
\text { width from Layer } 27 \text { to } 25 \text {. Layers } \\
25 \text { and } 23 \text { are similar. }\end{array}$ \\
\hline $\begin{array}{l}\text { Striking platform } \\
\text { thickness }\end{array}$ & $\begin{array}{l}\text { Log transformation } \\
+ \text { ANOVA }^{\text {a }}\end{array}$ & $F(2,1052)=3.049, \mathbf{p}=. \mathbf{0 4 8}$ & $\begin{array}{l}\text { Significant differences } \\
\text { between layers }\end{array}$ & $\begin{array}{l}\text { Decrease in mean striking platform } \\
\text { thickness from Layer } 27 \text { to } 25 \text {. } \\
\text { Layers } 25 \text { and } 23 \text { are similar. }\end{array}$ \\
\hline $\begin{array}{l}\text { Striking platform } \\
\text { type }\end{array}$ & Chi-square & $\chi^{2}(12)=13.848, p=.310$ & Not significant & \\
\hline $\begin{array}{l}\text { Striking platform } \\
\text { lipping }\end{array}$ & Chi-square & $\begin{array}{l}\chi^{2}(4)=14.690, \mathbf{p}=. \mathbf{0 0 5} \\
\text { Cramér's V =.079 }\end{array}$ & $\begin{array}{l}\text { Significant but very weak } \\
\text { differences between layers }\end{array}$ & $\begin{array}{l}\text { Increase over time in proportion of } \\
\text { debitage with slight or pronounced } \\
\text { lipping. }\end{array}$ \\
\hline Curvature & Kruskal-Wallis & $\chi^{2}(2)=8.975, \mathbf{p}=. \mathbf{0 1 1}$ & Significant differences & Slight decrease over time, i.e. \\
\hline
\end{tabular}




\begin{tabular}{|c|c|c|c|c|}
\hline & & & between layers & increasingly more curved. \\
\hline $\begin{array}{l}\text { Percentage cortex } \\
\text { cover }\end{array}$ & Kruskal-Wallis & $\chi^{2}(2)=5.453, p=.065$ & Not significant & \\
\hline $\begin{array}{l}\text { Number of dorsal } \\
\text { scars }\end{array}$ & Kruskal-Wallis & $\chi^{2}(2)=2.750, p=.253$ & Not significant & \\
\hline $\begin{array}{l}\text { Number of parallel } \\
\text { arrises }\end{array}$ & Kruskal-Wallis & $\chi^{2}(2)=7.119, \mathbf{p}=.027$ & $\begin{array}{l}\text { Significant differences } \\
\text { between layers }\end{array}$ & $\begin{array}{l}\text { Decrease over time, with most of } \\
\text { the difference between Layers } 25 \\
\text { and } 23 .\end{array}$ \\
\hline $\begin{array}{l}\text { Number of scars } \\
\text { per } \mathrm{cm}^{2} \text { surface } \\
\text { area }\end{array}$ & $\begin{array}{l}\text { Log transformation } \\
+ \text { ANOVA }^{a}\end{array}$ & $\mathrm{~F}(2,771.799)=17.387, \mathbf{p}<. \mathbf{0 0 1} b$ & $\begin{array}{l}\text { Significant differences } \\
\text { between layers }\end{array}$ & $\begin{array}{l}\text { Increase over time, with most of } \\
\text { the difference between Layers } 27 \\
\text { and } 25 .\end{array}$ \\
\hline $\begin{array}{l}\text { Number of parallel } \\
\text { arrises per } \mathrm{cm}^{2} \\
\text { surface area }\end{array}$ & $\begin{array}{l}\text { Log transformation } \\
+ \text { ANOVA }^{a}\end{array}$ & $F(2,740)=8.992, \mathbf{p}<. \mathbf{0 0 1}$ & $\begin{array}{l}\text { Significant differences } \\
\text { between layers }\end{array}$ & $\begin{array}{l}\text { Increase over time, with most of } \\
\text { the difference between Layers } 27 \\
\text { and } 25 .\end{array}$ \\
\hline Scar orientation & Chi-square & $\chi^{2}(10)=5.366, p=.865$ & Not significant & \\
\hline Termination type & Chi-square & $\begin{array}{l}\chi^{2}(12)=37.146, \mathbf{p}<. \mathbf{0 0 1} \\
\text { Cramér's } V=.125\end{array}$ & $\begin{array}{l}\text { Significant but weak } \\
\text { differences between layers }\end{array}$ & $\begin{array}{l}\text { Slight decrease over time in } \\
\text { proportion of plunging and step } \\
\text { terminations. More axial and } \\
\text { crushed terminations in Layer } 23 \text {. }\end{array}$ \\
\hline $\begin{array}{l}\text { Evidence of bipolar } \\
\text { percussion }\end{array}$ & Chi-square & $\begin{array}{l}\chi^{2}(4)=23.060, \mathbf{p}<.001 \\
\text { Cramér's } V=.099\end{array}$ & $\begin{array}{l}\text { Significant but very weak } \\
\text { differences between layers }\end{array}$ & $\begin{array}{l}\text { More evidence of bipolar } \\
\text { percussion in Layer } 23 \text {, but no } \\
\text { overall trend. }\end{array}$ \\
\hline $\begin{array}{l}\text { Evidence of core } \\
\text { maintenance }\end{array}$ & Chi-square & $\chi^{2}(6)=6.635, p=.356$ & Not significant & \\
\hline Other classification & Chi-square & $\begin{array}{l}\chi^{2}(6)=30.027, \mathbf{p}<.001 \\
\text { Cramér's } V=.113\end{array}$ & $\begin{array}{l}\text { Significant but weak } \\
\text { differences between layers }\end{array}$ & $\begin{array}{l}\text { Increase over time in proportions } \\
\text { of possibly snapped flakes and } \\
\text { blades. }\end{array}$ \\
\hline
\end{tabular}

\footnotetext{
Table B.2.2: Results of statistical tests of changes over time in debitage variables at Umhlatuzana.

a significance confirmed with non-parametric Kruskal-Wallis test conducted on non-transformed data.

b Welch's F statistic reported due to unequal variances.
} 


\section{MICROLITHS}

\begin{tabular}{|c|c|c|c|c|}
\hline Variable & Test used & Test result & Significance (at 0.05 level) & Trend \\
\hline Blank type & Chi-square & $\chi^{2}(2)=1.731, p=.421$ & Not significant & \\
\hline Condition & Chi-square & $\begin{array}{l}\chi^{2}(10)=26.864, \mathbf{p}=. \mathbf{0 0 3} \\
\text { Cramér's } V=.272\end{array}$ & $\begin{array}{l}\text { Significant and moderately } \\
\text { strong differences between } \\
\text { layers }\end{array}$ & $\begin{array}{l}\text { Decrease over time in the } \\
\text { proportion of whole microliths. } \\
\text { Increase over time in the } \\
\text { proportions of proximal breakages } \\
\text { and broken blanks of unclear } \\
\text { provenance. }\end{array}$ \\
\hline Raw material & Chi-square & $\chi^{2}(18)=19.390, p=.368$ & Not significant & \\
\hline Colour & Chi-square & $\chi^{2}(12)=7.815, p=.799$ & Not significant & \\
\hline Weight & $\begin{array}{l}\text { Log transformation } \\
+ \text { ANOVA }^{a}\end{array}$ & $F(2,178)=2.159, p=.119$ & Not significant & \\
\hline Longest dimension & $\begin{array}{l}\text { Log transformation } \\
+ \text { ANOVA }^{a}\end{array}$ & $F(2,178)=2.361, p=.097$ & Not significant & \\
\hline Oriented length & $\begin{array}{l}\text { Log transformation } \\
+ \text { ANOVA }^{a}\end{array}$ & $F(2,178)=1.440, p=.240$ & Not significant & \\
\hline Maximum width & $\begin{array}{l}\text { Log transformation } \\
+ \text { ANOVA }^{\text {a }}\end{array}$ & $F(2,178)=1.604, p=.204$ & Not significant & \\
\hline One quarter width & $\begin{array}{l}\text { Log transformation } \\
+ \text { ANOVA }^{a}\end{array}$ & $F(2,178)=0.469, p=.627$ & Not significant & \\
\hline Medial width & $\begin{array}{l}\text { Log transformation } \\
+ \text { ANOVA }^{a}\end{array}$ & $F(2,178)=1.686, p=.188$ & Not significant & \\
\hline Three quarter width & $\begin{array}{l}\text { Log transformation } \\
+ \text { ANOVA }^{a}\end{array}$ & $F(2,178)=2.181, p=.116$ & Not significant & \\
\hline Maximum thickness & $\begin{array}{l}\text { Log transformation } \\
+ \text { ANOVA }^{\text {a }}\end{array}$ & $F(2,178)=0.742, p=.477$ & Not significant & \\
\hline Medial thickness & Log transformation & $F(2,178)=0.726, p=.485$ & Not significant & \\
\hline
\end{tabular}




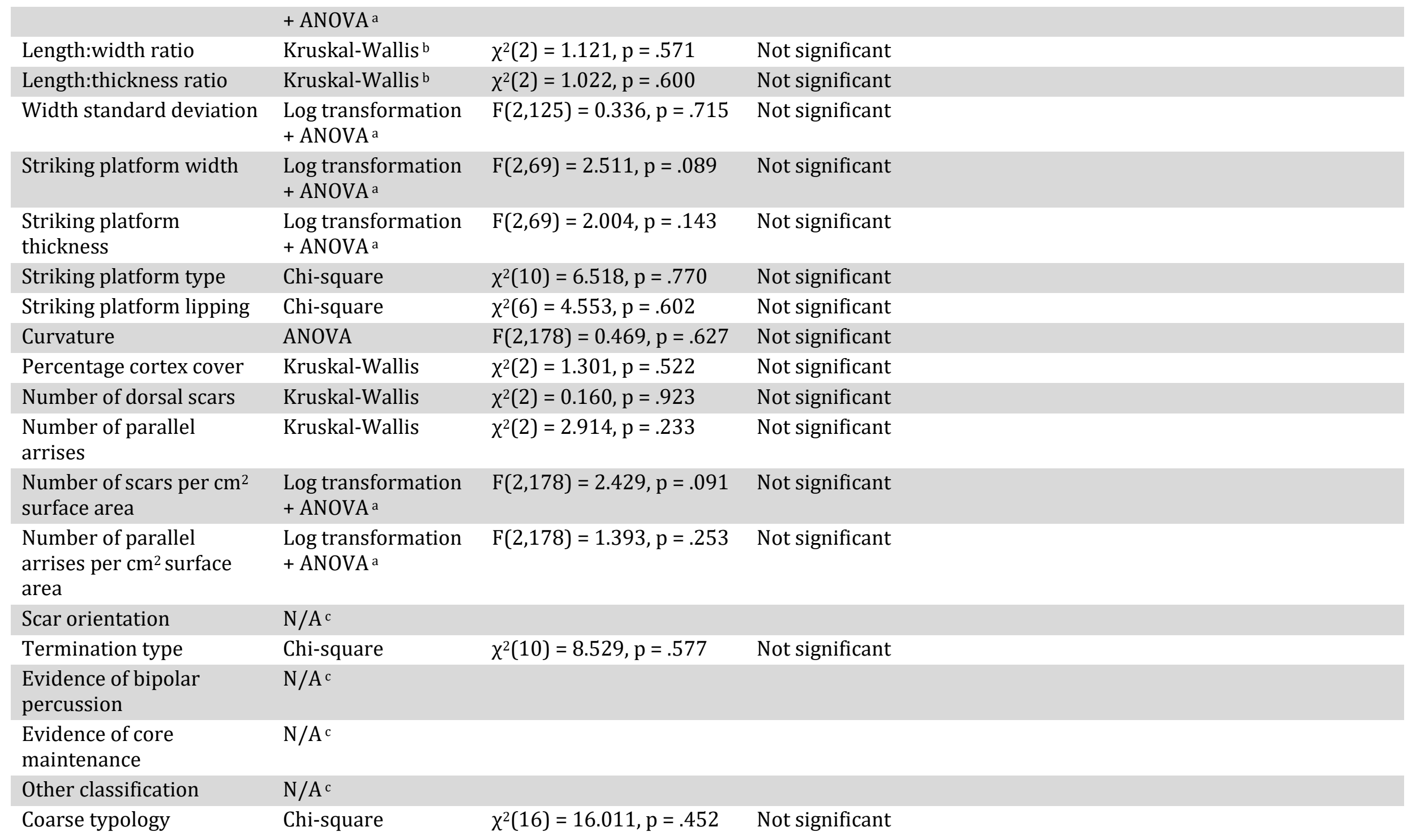




\begin{tabular}{|c|c|c|c|c|}
\hline Narrow typology & Chi-square & $\chi^{2}(26)=32.839, p=.167$ & Not significant & \\
\hline $\begin{array}{l}\text { Presence of tangs or } \\
\text { shoulders }\end{array}$ & $\mathrm{N} / \mathrm{A}^{\mathrm{c}}$ & & & \\
\hline Haft width & $\mathrm{N} / \mathrm{A}^{\mathrm{c}}$ & & & \\
\hline Haft thickness & $\mathrm{N} / \mathrm{A}^{\mathrm{c}}$ & & & \\
\hline Haft length & $\mathrm{N} / \mathrm{A}^{\mathrm{c}}$ & & & \\
\hline $\begin{array}{l}\text { Haft notches, abrasion or } \\
\text { polish }\end{array}$ & $\mathrm{N} / \mathrm{A}^{\mathrm{c}}$ & & & \\
\hline Number of worked edges & Kruskal-Wallis & $\chi^{2}(2)=0.498, p=.780$ & Not significant & \\
\hline Retouch location & Chi-square & $\chi^{2}(12)=9.462, p=.663$ & Not significant & \\
\hline Retouch face & Chi-square & $\begin{array}{l}\chi^{2}(4)=14.624, \mathbf{p}=. \mathbf{0 0 6} \\
\text { Cramér's V =.194 }\end{array}$ & $\begin{array}{l}\text { Significant but weak } \\
\text { differences between layers }\end{array}$ & $\begin{array}{l}\text { Increase over time in the } \\
\text { proportion of dorsal retouch. } \\
\text { Decrease over time in the } \\
\text { proportion of edge retouch. } \\
\text { Proportion of ventral retouch } \\
\text { remains low and constant. }\end{array}$ \\
\hline Retouch outline & Chi-square & $\chi^{2}(12)=7.242, p=.841$ & Not significant & \\
\hline Retouch type & Chi-square & $\begin{array}{l}\chi^{2}(12)=23.817, \mathbf{p}=. \mathbf{0 2 2}, \\
\text { Cramér's } V=.247\end{array}$ & $\begin{array}{l}\text { Significant and moderately } \\
\text { strong differences between } \\
\text { layers }\end{array}$ & $\begin{array}{l}\text { Scalar and stepped retouch are } \\
\text { only evident in Layers } 27 \text { and } 25, \\
\text { abrasion is only evident in Layer } \\
25 \text {, and discontinuous retouch is } \\
\text { only evident in Layer } 23 \text {. However, } \\
\text { these are all at low frequencies, and } \\
\text { there is no overall trend. }\end{array}$ \\
\hline Retouch angle & Kruskal-Wallis & $\chi^{2}(2)=1.410, p=.494$ & Not significant & \\
\hline Retouch depth & $\begin{array}{l}\text { Log transformation } \\
+ \text { ANOVA }^{\text {a }}\end{array}$ & $F(2,192)=2.393, p=.094$ & Not significant & \\
\hline Retouch length & Kruskal-Wallis & $\chi^{2}(2)=2.468, p=.291$ & Not significant & \\
\hline Index of Invasiveness & Kruskal-Wallis & $\chi^{2}(2)=0.067, p=.967$ & Not significant & \\
\hline
\end{tabular}


Table B.2.3: Results of statistical tests of changes over time in microlith variables at Umhlatuzana.

a significance confirmed with non-parametric Kruskal-Wallis test conducted on non-transformed data.

b although log transformation of the variable data results in normal distributions, both overall and within each layer (as determined by the Shapiro-Wilk test), Levene's tests indicate that the variances are unequal. As a result of this violation of the assumption of

homoscedasticity, combined with the fact that there are considerable differences in sample sizes between the layers, the non-parametric Kruskal-Wallis test is preferred as it is robust to these violations of the ANOVA test assumptions. The results of the Kruskal-Wallis test are then confirmed with the Welch F-statistic for the log-transformed data, which can be used in cases of unequal variances.

c variable is evident in the sample but not in every layer and/or in numbers too small to be statistically viable, or variable that exhibits only one category in two layers and so cannot be compared meaningfully between layers.

\section{B.3 NtLoAna TSOANA, Lesotho}

\section{CORES}

\begin{tabular}{|c|c|c|c|}
\hline Variable & Test used & Test result & $\begin{array}{ll}\text { Significance (at 0.05 level) } & \text { Trend }\end{array}$ \\
\hline Type & Chi-square & $\chi^{2}(12)=9.637, p=.648$ & Not significant \\
\hline Raw material & Chi-square & $\chi^{2}(6)=11.173, p=.083$ & Not significant \\
\hline Colour & Chi-square & $\chi^{2}(16)=21.132, p=.174$ & Not significant \\
\hline Longest dimension & $\begin{array}{l}\text { Log transformation } \\
+ \text { ANOVA }^{a}\end{array}$ & $F(2,71)=0.549, p=.580$ & Not significant \\
\hline
\end{tabular}


+ ANOVA $^{a}$

\begin{tabular}{|c|c|c|c|c|}
\hline Maximum width & $\begin{array}{l}\text { Log transformation } \\
+ \text { ANOVA }^{\text {a }}\end{array}$ & $F(2,71)=1.019, p=.366$ & Not significant & \\
\hline Maximum thickness & $\begin{array}{l}\text { Log transformation } \\
+ \text { ANOVA }{ }^{a}\end{array}$ & $F(2,71)=1.019, p=.366$ & Not significant & \\
\hline Longest face & $\begin{array}{l}\text { Log transformation } \\
+ \text { ANOVA }^{\text {a }}\end{array}$ & $\mathrm{F}(2,71)=0.229, \mathrm{p}=.796$ & Not significant & \\
\hline Striking platform width & $\begin{array}{l}\text { Log transformation } \\
+ \text { ANOVA a }\end{array}$ & $F(2,71)=2.208, p=.117$ & Not significant & \\
\hline $\begin{array}{l}\text { Striking platform } \\
\text { thickness }\end{array}$ & $\begin{array}{l}\text { Log transformation } \\
+ \text { ANOVAa }\end{array}$ & $F(2,71)=0.444, p=.643$ & Not significant & \\
\hline Striking platform type & Chi-square & $\chi^{2}(8)=3.237, p=.919$ & Not significant & \\
\hline $\begin{array}{l}\text { Number of striking } \\
\text { platforms }\end{array}$ & Chi-square & $\chi^{2}(8)=4.968, p=.761$ & Not significant & \\
\hline $\begin{array}{l}\text { Perpendicular striking } \\
\text { platforms }\end{array}$ & Chi-square & $\chi^{2}(2)=2.485, p=.289$ & Not significant & \\
\hline Total number of scars & Kruskal-Wallis & $\chi^{2}(2)=0.191, p=.909$ & Not significant & \\
\hline Number of flake scars & Kruskal-Wallis & $\chi^{2}(2)=3.832, p=.147$ & Not significant & \\
\hline Number of blade scars & Kruskal-Wallis & $\chi^{2}(2)=3.931, p=.140$ & Not significant & \\
\hline Proportion of blade scars & Kruskal-Wallis & $\chi^{2}(2)=5.146, p=.076$ & Not significant & \\
\hline $\begin{array}{l}\text { Number of aberrant } \\
\text { terminations }\end{array}$ & Kruskal-Wallis & $\chi^{2}(2)=7.757, \mathbf{p}=. \mathbf{0 2 1}$ & $\begin{array}{l}\text { Significant differences } \\
\text { between layers }\end{array}$ & $\begin{array}{l}\text { Considerable increase from Layer } 579 \\
\text { to } 578 \text {, and decrease from Layer } 578 \\
\text { to } 577 \text {, but no overall trend. }\end{array}$ \\
\hline Percentage cortex cover & Kruskal-Wallis & $\chi^{2}(2)=4.301, p=.116$ & Not significant & \\
\hline Recycling & Chi-square & $\chi^{2}(2)=0.683, p=.711$ & Not significant & \\
\hline Average removal length & $\begin{array}{l}\text { Log transformation } \\
+ \text { ANOVAa }\end{array}$ & $F(2,71)=0.407, p=.667$ & Not significant & \\
\hline $\begin{array}{l}\text { Standard deviation of } \\
\text { removal scar lengths }\end{array}$ & ANOVA & $F(2,71)=1.107, p=.336$ & Not significant & \\
\hline
\end{tabular}


Number of scars per $\mathrm{cm}^{3}$ core volume

Average scar length per

$\mathrm{cm}^{3}$ core volume

Total scar length per $\mathrm{cm}^{3}$

core volume
Log transformation $\quad \mathrm{F}(2,71)=0.546, \mathrm{p}=.582 \quad$ Not significant + ANOVA $^{a}$

Log transformation $\quad F(2,71)=1.479, p=.235 \quad$ Not significant

+ ANOVA a

Log transformation $\quad F(2,71)=1.274, p=.286 \quad$ Not significant + ANOVA $^{\text {a }}$

\section{Table B.3.1: Results of statistical tests of changes over time in core variables at NT.}

a significance confirmed with non-parametric Kruskal-Wallis test conducted on non-transformed data.

\section{DEBITAGE}

\begin{tabular}{|c|c|c|c|c|}
\hline Variable & Test used & Test result & Significance (at 0.05 level) & Trend \\
\hline Type & Chi-square & $\begin{array}{l}\chi^{2}(4)=9.708, \mathbf{p}=. \mathbf{0 4 6} \\
\text { Cramér's V =.072 }\end{array}$ & $\begin{array}{l}\text { Significant but very weak } \\
\text { differences between layers }\end{array}$ & $\begin{array}{l}\text { Increase over time in proportion of } \\
\text { flake-blades. Fewer blades in Layer } \\
577 .\end{array}$ \\
\hline Condition & Chi-square & $\begin{array}{l}\chi^{2}(10)=18.446, \mathbf{p}=. \mathbf{0 4 8}, \\
\text { Cramér's V =.099 }\end{array}$ & $\begin{array}{l}\text { Significant but very weak } \\
\text { differences between layers }\end{array}$ & $\begin{array}{l}\text { Slight increase over time in proportion } \\
\text { of proximal breakages and decrease in } \\
\text { distal breakages. }\end{array}$ \\
\hline Raw material & Chi-square & $\begin{array}{l}\chi^{2}(6)=13.428, \mathbf{p}=. \mathbf{0 3 7} \\
\text { Cramér's V =.085 }\end{array}$ & $\begin{array}{l}\text { Significant but very weak } \\
\text { differences between layers }\end{array}$ & $\begin{array}{l}\text { More quartzite in Layer } 579 \text { and } \\
\text { fewest in Layer } 578 \text {, but no overall } \\
\text { trend. }\end{array}$ \\
\hline Colour & Chi-square & $\begin{array}{l}\chi^{2}(22)=68.980, \mathbf{p}<. \mathbf{0 0 1} \\
\text { Cramér's V =.192 }\end{array}$ & $\begin{array}{l}\text { Significant but weak } \\
\text { differences between layers }\end{array}$ & $\begin{array}{l}\text { Increase over time in proportion of } \\
\text { brown coloured debitage and decrease } \\
\text { in grey, as a result of changes in raw } \\
\text { materials. }\end{array}$ \\
\hline Weight & Kruskal-Wallis & $\chi^{2}(2)=6.272, \mathbf{p}=.043$ & $\begin{array}{l}\text { Significant differences } \\
\text { between layers }\end{array}$ & $\begin{array}{l}\text { Decrease from Layer } 579 \text { to } 578 \text {. } \\
\text { Layers } 578 \text { and } 577 \text { are similar, and }\end{array}$ \\
\hline
\end{tabular}




\begin{tabular}{|c|c|c|c|c|}
\hline & & & & there is no overall trend. \\
\hline Longest dimension & Kruskal-Wallis & $\chi^{2}(2)=11.603, \mathbf{p}=.003$ & $\begin{array}{l}\text { Significant differences } \\
\text { between layers }\end{array}$ & $\begin{array}{l}\text { Decrease from Layer } 579 \text { to } 578 \text {, and } \\
\text { slight increase from Layer } 578 \text { to } 577 \text {. } \\
\text { No overall trend. }\end{array}$ \\
\hline Oriented length & Kruskal-Wallis & $\chi^{2}(2)=17.531, p<.001$ & $\begin{array}{l}\text { Significant differences } \\
\text { between layers }\end{array}$ & $\begin{array}{l}\text { Decrease from Layer } 579 \text { to } 578 \text {, and } \\
\text { slight increase from Layer } 578 \text { to } 577 \text {. } \\
\text { No overall trend. }\end{array}$ \\
\hline Maximum width & $\begin{array}{l}\text { Log transformation } \\
+ \text { ANOVA }^{a}\end{array}$ & $\begin{array}{l}F(2,556.295)=1.894, p= \\
.151 b\end{array}$ & Not significant & \\
\hline One quarter width & $\begin{array}{l}\text { Log transformation } \\
+ \text { ANOVA }^{a}\end{array}$ & $F(2,473)=1.764, p=.172$ & Not significant & \\
\hline Medial width & $\begin{array}{l}\text { Log transformation } \\
+ \text { ANOVA }^{a}\end{array}$ & $F(2,473)=3.743, \mathbf{p}=\mathbf{. 0 2 4}$ & $\begin{array}{l}\text { Significant differences } \\
\text { between layers }\end{array}$ & $\begin{array}{l}\text { Decrease from Layer } 579 \text { to } 578 \text {, and } \\
\text { slight increase from Layer } 578 \text { to } 577 \text {. } \\
\text { No overall trend. }\end{array}$ \\
\hline Three quarter width & $\begin{array}{l}\text { Log transformation } \\
+ \text { ANOVA }^{a}\end{array}$ & $F(2,473)=6.696, p=.001$ & $\begin{array}{l}\text { Significant differences } \\
\text { between layers }\end{array}$ & $\begin{array}{l}\text { Decrease from Layer } 579 \text { to } 578 \text {, and } \\
\text { slight increase from Layer } 578 \text { to } 577 \text {. } \\
\text { No overall trend. }\end{array}$ \\
\hline Maximum thickness & $\begin{array}{l}\text { Log transformation } \\
+ \text { ANOVA }^{a}\end{array}$ & $F(2,933)=0.721, p=.487$ & Not significant & \\
\hline Medial thickness & Kruskal-Wallis & $\chi^{2}(2)=2.076, p=.354$ & Not significant & \\
\hline Length:width ratio & $\begin{array}{l}\text { Log transformation } \\
+ \text { ANOVA }^{a}\end{array}$ & $F(2,829)=2.616, p=.074$ & Not significant & \\
\hline $\begin{array}{l}\text { Length:thickness } \\
\text { ratio }\end{array}$ & $\begin{array}{l}\text { Log transformation } \\
+ \text { ANOVA }^{a}\end{array}$ & $F(2,829)=6.592, \mathbf{p}=.001$ & $\begin{array}{l}\text { Significant differences } \\
\text { between layers }\end{array}$ & $\begin{array}{l}\text { Decrease from Layer } 579 \text { to } 578 \text {, and } \\
\text { slight increase from Layer } 578 \text { to } 577 \text {. } \\
\text { No overall trend. }\end{array}$ \\
\hline $\begin{array}{l}\text { Width standard } \\
\text { deviation }\end{array}$ & Kruskal-Wallis & $\chi^{2}(2)=6.368, \mathbf{p}=.041$ & $\begin{array}{l}\text { Significant differences } \\
\text { between layers }\end{array}$ & $\begin{array}{l}\text { Increase from Layer } 579 \text { to } 578 \text {, and } \\
\text { slight decrease from Layer } 578 \text { to } 577 \text {. } \\
\text { No overall trend. }\end{array}$ \\
\hline $\begin{array}{l}\text { Striking platform } \\
\text { width }\end{array}$ & Kruskal-Wallis & $\chi^{2}(2)=3.291, p=.193$ & Not significant & \\
\hline
\end{tabular}




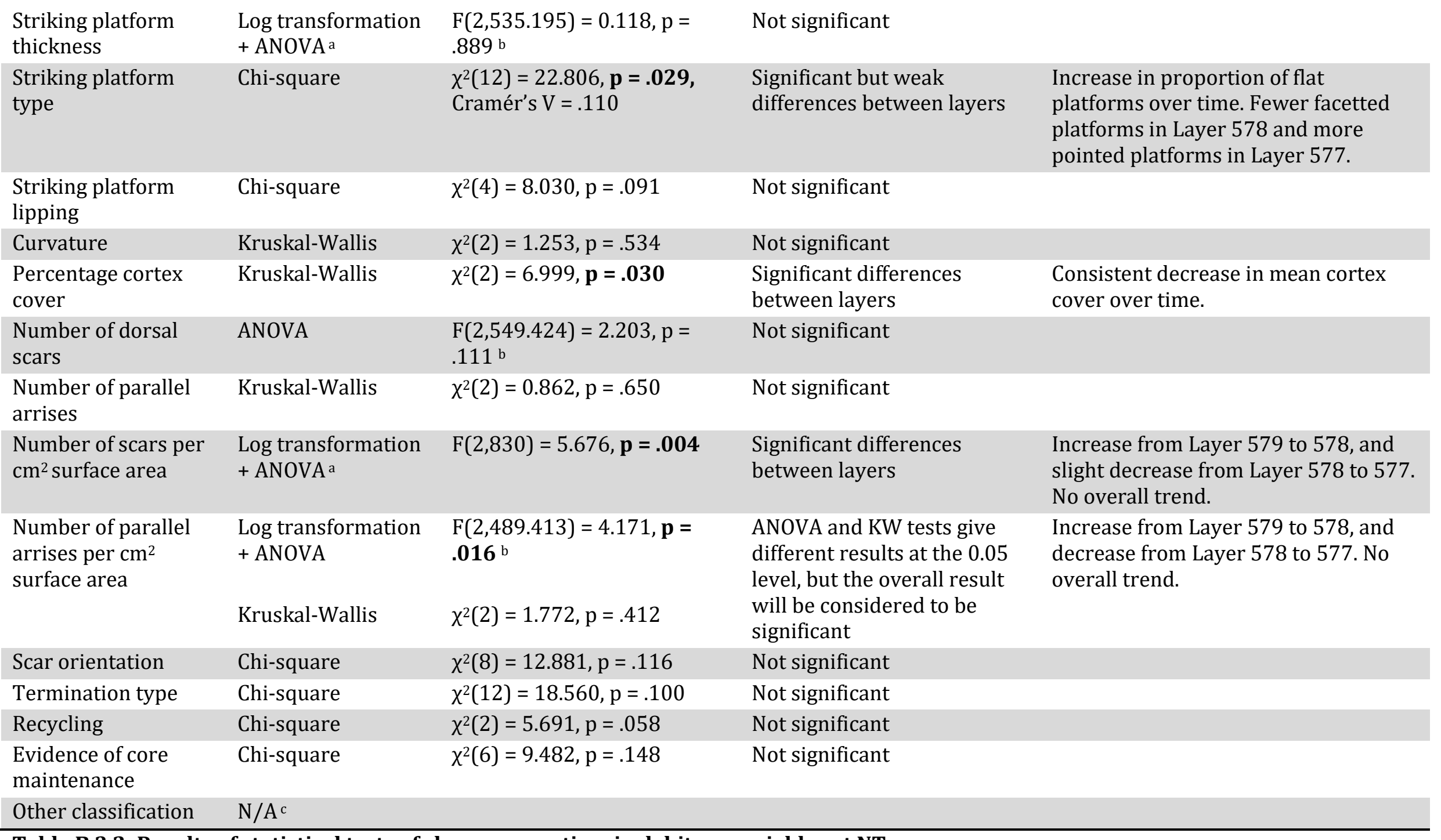

Table B.3.2: Results of statistical tests of changes over time in debitage variables at NT. 
a significance confirmed with non-parametric Kruskal-Wallis test conducted on non-transformed data.

b Welch's F statistic reported due to unequal variances.

${ }^{c}$ variable is evident in the sample, but not in every layer and in numbers too small to be statistically viable.

\section{B.4 Batadomba-LENA, SRI LANKa}

\section{DEBITAGE}

\begin{tabular}{|c|c|c|c|c|}
\hline Variable & Test used & Test result & Significance (at 0.05 level) & Trend \\
\hline Type & Chi-square & $\begin{array}{l}\chi^{2}(6)=23.123, \mathbf{p}=. \mathbf{0 0 1}, \\
\text { Cramér's V =.090 }\end{array}$ & $\begin{array}{l}\text { Significant but very weak } \\
\text { differences between layers }\end{array}$ & $\begin{array}{l}\text { More blades in Layer } 7 \text { (especially 7a) } \\
\text { and more flakes in Layer 6, but no overall } \\
\text { trend. }\end{array}$ \\
\hline Condition & Chi-square & $\begin{array}{l}\chi^{2}(15)=64.698, \mathbf{p}<.001 \\
\text { Cramér's } V=.123\end{array}$ & $\begin{array}{l}\text { Significant but weak } \\
\text { differences between layers }\end{array}$ & $\begin{array}{l}\text { Increase from Layer } 7 \text { to } 6 \text { in proportion } \\
\text { of broken debitage, and decrease from } \\
\text { Layer } 6 \text { to } 4 \text {. High proportion of } \\
\text { breakages in Layer } 7 \mathrm{c} \text {. }\end{array}$ \\
\hline Raw material & Chi-square & $\begin{array}{l}\chi^{2}(21)=182.451, \mathbf{p}<. \mathbf{0 0 1} \\
\text { Cramér's V = .207 }\end{array}$ & $\begin{array}{l}\text { Significant and moderately } \\
\text { strong differences between } \\
\text { layers }\end{array}$ & $\begin{array}{l}\text { Decrease over time in proportion of } \\
\text { granular quartz, disappearing in Layer } 4 . \\
\text { From Layer } 7 \text { to } 5 \text { proportions of opaque } \\
\text { and milky quartz increase and clear } \\
\text { quartz decreases. From Layer } 5 \text { to } 4 \text { the } \\
\text { pattern is reversed. The proportion of } \\
\text { impure clear quartz remains roughly } \\
\text { constant. Chert first appears in Layer } 7 \mathrm{a} \text {. }\end{array}$ \\
\hline Colour & Chi-square & $\begin{array}{l}\chi^{2}(9)=108.552, \mathbf{p}<.001, \\
\text { Cramér's V =.160 }\end{array}$ & $\begin{array}{l}\text { Significant but weak } \\
\text { differences between layers }\end{array}$ & $\begin{array}{l}\text { Increase in white quartz and decrease in } \\
\text { clear quartz from Layer } 7 \text { to } 5 . \text { From }\end{array}$ \\
\hline
\end{tabular}


Layer 5 to 4 the pattern is reversed.

\begin{tabular}{|c|c|c|c|c|}
\hline Weight & ANOVA & $\begin{array}{l}\mathrm{F}(3,706.471)=0.967, \mathrm{p}= \\
.408^{\mathrm{a}}\end{array}$ & Not significant & \\
\hline Longest dimension & ANOVA & $\mathrm{F}(3,1413)=1.549, \mathrm{p}=.200$ & Not significant & \\
\hline Oriented length & ANOVA & $F(3,1284)=0.252, p=.680$ & Not significant & \\
\hline Maximum width & ANOVA & $F(3,1412)=7.228, \mathbf{p}<.001$ & $\begin{array}{l}\text { Significant differences } \\
\text { between layers }\end{array}$ & Consistent increase over time. \\
\hline One quarter width & ANOVA & $F(3,703)=2.284, p=.078$ & Not significant & \\
\hline Medial width & ANOVA & $F(3,703)=3.030, \mathbf{p}=.029$ & $\begin{array}{l}\text { Significant differences } \\
\text { between layers }\end{array}$ & Consistent increase over time. \\
\hline $\begin{array}{l}\text { Three quarter } \\
\text { width }\end{array}$ & ANOVA & $F(3,703)=1.658, p=.175$ & Not significant & \\
\hline $\begin{array}{l}\text { Maximum } \\
\text { thickness }\end{array}$ & ANOVA & $\mathrm{F}(3,1413)=1.447, \mathrm{p}=.228$ & Not significant & \\
\hline Medial thickness & ANOVA & $F(3,867)=1.574, p=.194$ & Not significant & \\
\hline Length:width ratio & ANOVA & $\begin{array}{l}\mathrm{F}(3,612,864)=10.094, \mathbf{p}< \\
.001 \text { a }\end{array}$ & $\begin{array}{l}\text { Significant differences } \\
\text { between layers }\end{array}$ & Consistent decrease over time. \\
\hline $\begin{array}{l}\text { Length:thickness } \\
\text { ratio }\end{array}$ & ANOVA & $F(3,1292)=2.649, p=.048$ & $\begin{array}{l}\text { Significant differences } \\
\text { between layers }\end{array}$ & $\begin{array}{l}\text { Decrease from Layer } 7 \text { to } 5 \text { and increase } \\
\text { from Layer } 5 \text { to } 4 \text {. }\end{array}$ \\
\hline $\begin{array}{l}\text { Width standard } \\
\text { deviation }\end{array}$ & Kruskal-Wallis & $\chi^{2}(3)=21.705, \mathbf{p}<. \mathbf{0 0 1}$ & $\begin{array}{l}\text { Significant differences } \\
\text { between layers }\end{array}$ & Consistent increase over time. \\
\hline $\begin{array}{l}\text { Striking platform } \\
\text { width }\end{array}$ & ANOVA & $\begin{array}{l}F(3,500.135)=9.019, \mathbf{p}< \\
.001 \text { a }\end{array}$ & $\begin{array}{l}\text { Significant differences } \\
\text { between layers }\end{array}$ & $\begin{array}{l}\text { Increase from Layer } 7 \text { to } 5 \text { with most of } \\
\text { the difference between Layers } 6 \text { and } 5 \text {, } \\
\text { and decrease from Layer } 5 \text { to } 4 \text {. }\end{array}$ \\
\hline $\begin{array}{l}\text { Striking platform } \\
\text { thickness }\end{array}$ & ANOVA & $\mathrm{F}(3,1032)=2.814, \mathbf{p}=\mathbf{. 0 3 8}$ & $\begin{array}{l}\text { Significant differences } \\
\text { between layers }\end{array}$ & $\begin{array}{l}\text { Increase from Layer } 7 \text { to } 5 \text { with most of } \\
\text { the difference between Layers } 6 \text { and } 5 \text {, } \\
\text { and decrease from Layer } 5 \text { to } 4 \text {. }\end{array}$ \\
\hline $\begin{array}{l}\text { Striking platform } \\
\text { type }\end{array}$ & Chi-square & $\begin{array}{l}\chi^{2}(18)=70.795, \mathbf{p}<.001, \\
\text { Cramér's } V=.129\end{array}$ & $\begin{array}{l}\text { Significant but weak } \\
\text { differences between layers }\end{array}$ & $\begin{array}{l}\text { Increase from Layer } 7 \text { to } 5 \text { in proportion } \\
\text { of flat striking platforms and decrease }\end{array}$ \\
\hline
\end{tabular}




\begin{tabular}{|c|c|c|c|c|}
\hline & & & & from Layer 5 to 4 . \\
\hline $\begin{array}{l}\text { Striking platform } \\
\text { lipping }\end{array}$ & Chi-square & $\begin{array}{l}\chi^{2}(6)=34.255, \mathbf{p}<. \mathbf{0 0 1} \\
\text { Cramér's V =.110 }\end{array}$ & $\begin{array}{l}\text { Significant but weak } \\
\text { differences between layers }\end{array}$ & $\begin{array}{l}\text { Decrease from Layer } 7 \text { to } 6 \text { in proportion } \\
\text { of debitage with slight or pronounced } \\
\text { lipping, and increase from Layer } 6 \text { to } 4 \text {. }\end{array}$ \\
\hline Curvature & ANOVA & $F(3,867)=0.706, p=.549$ & Not significant & \\
\hline $\begin{array}{l}\text { Percentage cortex } \\
\text { cover }\end{array}$ & Kruskal-Wallis & $\chi^{2}(3)=14.316, \mathbf{p}=.003$ & $\begin{array}{l}\text { Significant differences } \\
\text { between layers }\end{array}$ & $\begin{array}{l}\text { Decrease over time, with the biggest } \\
\text { change from Layer } 7 \text { to } 6 .\end{array}$ \\
\hline $\begin{array}{l}\text { Number of dorsal } \\
\text { scars }\end{array}$ & Kruskal-Wallis & $\chi^{2}(3)=15.194, \mathbf{p}=.002$ & $\begin{array}{l}\text { Significant differences } \\
\text { between layers }\end{array}$ & $\begin{array}{l}\text { Decrease from Layer } 7 \text { to } 6 \text { and increase } \\
\text { from Layer } 5 \text { to } 4 \text {. }\end{array}$ \\
\hline $\begin{array}{l}\text { Number of parallel } \\
\text { arrises }\end{array}$ & Kruskal-Wallis & $\chi^{2}(3)=23.812, \mathbf{p}<. \mathbf{0 0 1}$ & $\begin{array}{l}\text { Significant differences } \\
\text { between layers }\end{array}$ & $\begin{array}{l}\text { Decrease over time, with the biggest } \\
\text { change from Layer } 6 \text { to } 5 .\end{array}$ \\
\hline $\begin{array}{l}\text { Number of scars } \\
\text { per } \mathrm{cm}^{2} \text { surface } \\
\text { area }\end{array}$ & ANOVA & $\begin{array}{l}F(3,602.940)=8.434, \mathbf{p}< \\
.001 \text { a }\end{array}$ & $\begin{array}{l}\text { Significant differences } \\
\text { between layers }\end{array}$ & $\begin{array}{l}\text { Decrease from Layer } 7 \text { to } 5 \text { and increase } \\
\text { from Layer } 5 \text { to } 4 \text {. }\end{array}$ \\
\hline $\begin{array}{l}\text { Number of parallel } \\
\text { arrises per } \mathrm{cm}^{2} \\
\text { surface area }\end{array}$ & $\begin{array}{l}\text { Log transformation } \\
+ \text { ANOVA } \\
\text { Kruskal-Wallis }\end{array}$ & $\chi^{2}(3)=29.454, \mathbf{p}<. \mathbf{0 0 1}$ & $\begin{array}{l}\text { ANOVA and KW tests give } \\
\text { different results at the } 0.05 \\
\text { level, but the overall result } \\
\text { will be considered to be } \\
\text { significant }\end{array}$ & $\begin{array}{l}\text { Decrease from Layer } 7 \text { to } 5 \text { and increase } \\
\text { from Layer } 5 \text { to } 4 \text {. }\end{array}$ \\
\hline Scar orientation & Chi-square & $\begin{array}{l}\chi^{2}(15)=55.073, \mathbf{p}<. \mathbf{0 0 1} \\
\text { Cramér's } V=.114\end{array}$ & $\begin{array}{l}\text { Significant but weak } \\
\text { differences between layers }\end{array}$ & $\begin{array}{l}\text { Slight increase from Layer } 7 \text { to } 5 \text { in } \\
\text { proportion of debitage with scars from a } \\
\text { single direction, and decrease from Layer } \\
5 \text { to } 4 \text {. }\end{array}$ \\
\hline Termination type & Chi-square & $\begin{array}{l}\chi^{2}(18)=41.293, \mathbf{p}=. \mathbf{0 0 1} \\
\text { Cramér's V = .099 }\end{array}$ & $\begin{array}{l}\text { Significant but very weak } \\
\text { differences between layers }\end{array}$ & $\begin{array}{l}\text { Decrease from Layer } 7 \text { to } 5 \text { in proportion } \\
\text { of debitage with feather terminations, } \\
\text { and increase from Layer } 5 \text { to } 4 \text {. }\end{array}$ \\
\hline $\begin{array}{l}\text { Evidence of bipolar } \\
\text { percussion }\end{array}$ & Chi-square & $\chi^{2}(6)=6.927, p=.328$ & Not significant & \\
\hline $\begin{array}{l}\text { Evidence of core } \\
\text { maintenance }\end{array}$ & Chi-square & $\chi^{2}(12)=18.956, p=.090$ & Not significant & \\
\hline
\end{tabular}




\begin{tabular}{|c|c|c|c|}
\hline Other classification & Chi-square & $\begin{array}{l}\chi^{2}(15)=30.559, \mathbf{p}=. \mathbf{0 1 0} \\
\text { Cramér's } V=.085\end{array}$ & $\begin{array}{l}\text { Significant but very weak } \\
\text { differences between layers }\end{array}$ \\
\hline
\end{tabular}

Slight increase from Layer 7 to 5 in proportion of possibly snapped flakes and blades, and decrease from Layer 5 to 4.

Table B.4.1: Results of statistical tests of changes over time in debitage variables at Batadomba-lena.

a Welch's F statistic reported due to unequal variances.

\section{MICROLITHS}

\begin{tabular}{|c|c|c|c|c|}
\hline Variable & Test used & Test result & Significance (at 0.05 level) & Trend \\
\hline Blank type & Chi-square & $\begin{array}{l}\chi^{2}(5)=20.840, \mathbf{p}=. \mathbf{0 0 1} \\
\text { Cramér's V =.367 }\end{array}$ & $\begin{array}{l}\text { Significant and strong } \\
\text { differences between layers }\end{array}$ & $\begin{array}{l}\text { Blade blanks dominant in Layers } 7 c, b \\
\text { and a. Flake blanks dominant in Layers } \\
6,5 \text { and } 4 .\end{array}$ \\
\hline Condition & Chi-square & $\chi^{2}(25)=27.119, p=.350$ & Not significant & \\
\hline Raw material & Chi-square & $\chi^{2}(10)=11.916, p=.291$ & Not significant & \\
\hline Colour & Chi-square & $\chi^{2}(5)=7.204, p=.206$ & Not significant & \\
\hline Weight & $\begin{array}{l}\text { Log transformation } \\
+ \text { ANOVA }^{\text {a }}\end{array}$ & $F(5,149)=1.398, p=.228$ & Not significant & \\
\hline Longest dimension & $\begin{array}{l}\text { Log transformation } \\
+ \text { ANOVA }^{\text {a }}\end{array}$ & $F(5,149)=0.596, p=.703$ & Not significant & \\
\hline Oriented length & $\begin{array}{l}\text { Log transformation } \\
+ \text { ANOVA }^{\text {a }}\end{array}$ & $F(5,149)=0.678, p=.641$ & Not significant & \\
\hline Maximum width & $\begin{array}{l}\text { Log transformation } \\
+ \text { ANOVA }^{\text {a }}\end{array}$ & $F(5,149)=4.596, \mathbf{p}=\mathbf{. 0 0 1}$ & $\begin{array}{l}\text { Significant differences } \\
\text { between layers }\end{array}$ & \multirow{2}{*}{$\begin{array}{l}\text { Decrease from Layer } 7 c \text { to } 7 b \text {, followed } \\
\text { by an increase over time, although with } \\
\text { a temporary reversal in the pattern in } \\
\text { Layer } 5 \text {. The largest difference is }\end{array}$} \\
\hline One quarter width & $\begin{array}{l}\text { Log transformation } \\
+ \text { ANOVA }^{\text {a }}\end{array}$ & $F(5,149)=3.439, \mathbf{p}=.006$ & $\begin{array}{l}\text { Significant differences } \\
\text { between layers }\end{array}$ & \\
\hline
\end{tabular}




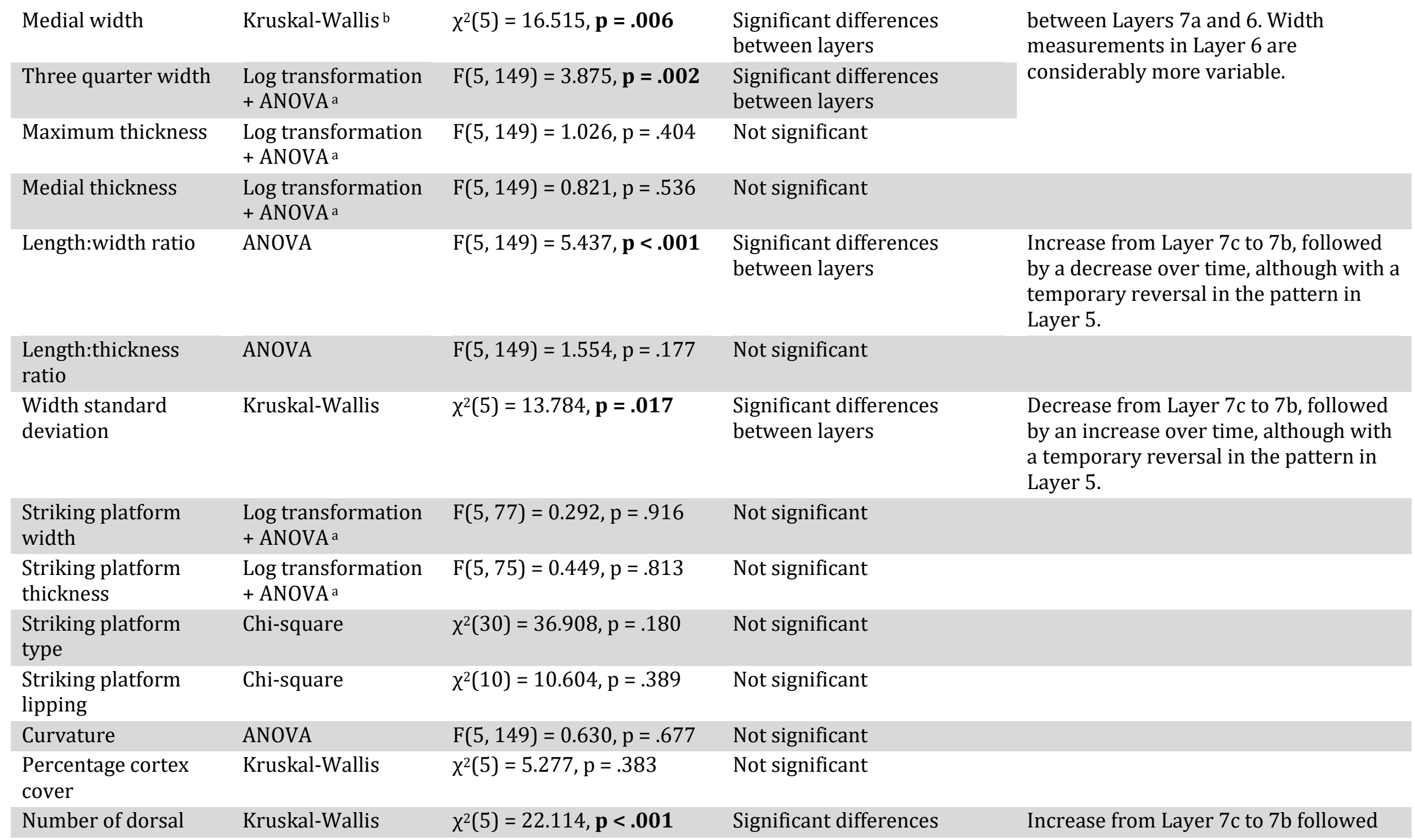




\begin{tabular}{|c|c|c|c|c|}
\hline scars & & & between layers & $\begin{array}{l}\text { by a decrease to } 7 a \text {, stasis between } \\
\text { Layers } 7 a \text { and } 5 \text {, and a sudden increase } \\
\text { in Layer } 4 .\end{array}$ \\
\hline $\begin{array}{l}\text { Number of parallel } \\
\text { arrises }\end{array}$ & Kruskal-Wallis & $\chi^{2}(5)=6.958, p=.224$ & Not significant & \\
\hline $\begin{array}{l}\text { Number of scars per } \\
\mathrm{cm}^{2} \text { surface area }\end{array}$ & $\begin{array}{l}\text { Log transformation } \\
+ \text { ANOVA }^{\mathrm{a}}\end{array}$ & $F(5,148)=2.661, \mathbf{p}=.025$ & $\begin{array}{l}\text { Significant differences } \\
\text { between layers }\end{array}$ & $\begin{array}{l}\text { Increase from Layer } 7 c \text { to } 7 b \text {, decrease } \\
\text { from Layer } 7 b \text { to } 6 \text {, and increase from } \\
\text { Layer } 6 \text { to } 4 \text {. }\end{array}$ \\
\hline $\begin{array}{l}\text { Number of parallel } \\
\text { arrises per } \mathrm{cm}^{2} \\
\text { surface area }\end{array}$ & $\begin{array}{l}\text { Log transformation } \\
+ \text { ANOVA }^{\text {a }}\end{array}$ & $F(5,90)=0.807, p=.547$ & Not significant & \\
\hline Scar orientation & $\mathrm{N} / \mathrm{A}^{\mathrm{c}}$ & & & \\
\hline Termination type & Chi-square & $\chi^{2}(30)=41.190, p=.084$ & Not significant & \\
\hline $\begin{array}{l}\text { Evidence of bipolar } \\
\text { percussion }\end{array}$ & $\mathrm{N} / \mathrm{A}^{\mathrm{c}}$ & & & \\
\hline $\begin{array}{l}\text { Evidence of core } \\
\text { maintenance }\end{array}$ & $\mathrm{N} / \mathrm{A}^{\mathrm{c}}$ & & & \\
\hline Coarse typology & Chi-square & $\begin{array}{l}\chi^{2}(50)=91.209, \mathbf{p}<. \mathbf{0 0 1} \\
\text { Cramér's } V=.343\end{array}$ & $\begin{array}{l}\text { Significant and strong } \\
\text { differences between layers }\end{array}$ & \multirow{3}{*}{$\begin{array}{l}\text { Complex picture of changes in microlith } \\
\text { typology over time. Overall, the } \\
\text { proportions of backed non-geometrics } \\
\text { decreases over time, non-backed non- } \\
\text { geometrics increase over time and } \\
\text { geometrics fluctuate. See Figure } 6.1 .19 \text {. }\end{array}$} \\
\hline Narrow typology & Chi-square & $\begin{array}{l}\chi^{2}(105)=129.914, \mathbf{p}= \\
\mathbf{. 0 5 0}, \text { Cramér's } V=.409\end{array}$ & $\begin{array}{l}\text { Significant and very strong } \\
\text { differences between layers }\end{array}$ & \\
\hline $\begin{array}{l}\text { Presence of tangs or } \\
\text { shoulders }\end{array}$ & $\mathrm{N} / \mathrm{A}^{\mathrm{c}}$ & & & \\
\hline Haft width & $\mathrm{N} / \mathrm{A}^{\mathrm{c}}$ & & & \\
\hline Haft thickness & $\mathrm{N} / \mathrm{A}^{\mathrm{c}}$ & & & \\
\hline Haft length & $\mathrm{N} / \mathrm{A}^{\mathrm{c}}$ & & & \\
\hline $\begin{array}{l}\text { Number of worked } \\
\text { edges }\end{array}$ & Kruskal-Wallis & $\chi^{2}(5)=0.730, p=.981$ & Not significant & \\
\hline
\end{tabular}


Retouch location

Chi-square

$\chi^{2}(40)=69.135, \mathbf{p}=. \mathbf{0 0 3}$,

Cramér's V = .271

\begin{tabular}{|c|c|c|}
\hline Retouch face & Chi-square & $\begin{array}{l}\chi^{2}(25)=40.888, \mathbf{p}=. \mathbf{0 2 4} \\
\text { Cramér's } V=.208\end{array}$ \\
\hline Retouch outline & Chi-square & $\chi^{2}(30)=39.964, p=.106$ \\
\hline Retouch type & Chi-square & $\begin{array}{l}\chi^{2}(60)=82.190, \mathbf{p}=. \mathbf{0 3 0}, \\
\text { Cramér's V =.295 }\end{array}$ \\
\hline Retouch angle & Kruskal-Wallis & $\chi^{2}(5)=8.228, p=.144$ \\
\hline Retouch depth & $\begin{array}{l}\text { Log transformation } \\
+ \text { ANOVA }^{\text {a }}\end{array}$ & $F(5,182)=1.279, p=.275$ \\
\hline Retouch length & $\begin{array}{l}\text { Log transformation } \\
+ \text { ANOVA }^{\text {a }}\end{array}$ & $F(5,182)=1.517, p=.187$ \\
\hline Index of Invasiveness & Kruskal-Wallis & $\chi^{2}(5)=3.418, p=.636$ \\
\hline TCSA & $\begin{array}{l}\text { Log transformation } \\
+ \text { ANOVA a }\end{array}$ & $F(5,149)=2.876, \mathbf{p}=\mathbf{. 0 1 6}$ \\
\hline
\end{tabular}

Significant and moderately strong differences between layers

Significant and moderately strong differences between layers

Not significant

Significant and moderately strong differences between layers

Not significant

Not significant

Not significant

Not significant

Significant differences between layers
More distal retouch in Layer 6 and less lateral retouch in Layer 4 , but no overall trend.

Slight and inconsistent increase in ventral retouch over time, and no edge retouch in Layer 4, but no clear overall trend.

Proportion of backing retouch

decreases over time, although with a temporary reversal in the pattern in Layer 5.

Table B.4.2: Results of statistical tests of changes over time in microlith variables at Batadomba-lena.

a significance confirmed with non-parametric Kruskal-Wallis test conducted on non-transformed data.

b although log transformation of the variable data results in normal distributions, both overall and within each layer (as determined by the Shapiro-Wilk test), Levene's tests indicate that the variances are unequal. As a result of this violation of the assumption of homoscedasticity, combined with the fact that there are considerable differences in sample sizes between the layers, the non-parametric Kruskal-Wallis test is preferred as it is robust to these violations of the ANOVA test assumptions. The results of the Kruskal-Wallis test are then confirmed with the Welch F-statistic for the log-transformed data, which can be used in cases of unequal variances. c variable is evident in the sample, but not in every layer and in numbers too small to be statistically viable. 


\section{B.5 PATNE, INDIA}

CORES

\begin{tabular}{|c|c|c|c|c|}
\hline Variable & Test used & Test result & Significance (at 0.05 level) & Trend \\
\hline Type & Chi-square & $\chi^{2}(15)=15.121, p=.443$ & Not significant & \\
\hline Colour & Chi-square & $\begin{array}{l}\chi^{2}(21)=34.242, \mathbf{p}=. \mathbf{0 3 4} \\
\text { Cramér's } V=.447\end{array}$ & $\begin{array}{l}\text { Significant and very strong } \\
\text { differences between layers }\end{array}$ & $\begin{array}{l}\text { Greater variety of colours in D, only } \\
\text { grey and white present in C. } \\
\text { Presumably indicative of changes in raw } \\
\text { materials. }\end{array}$ \\
\hline Longest axis & Kruskal-Wallis a & $\chi^{2}(3)=7.781, p=.051$ & Not significant & \\
\hline Maximum width & ANOVA & $F(3,53)=0.085, p=.968$ & Not significant & \\
\hline Maximum thickness & Kruskal-Wallis a & $\chi^{2}(3)=3.668, p=.300$ & Not significant & \\
\hline Longest face & Kruskal-Wallis a & $\chi^{2}(3)=8.110, \mathbf{p}=.044$ & $\begin{array}{l}\text { Significant differences } \\
\text { between layers }\end{array}$ & $\begin{array}{l}\text { Decrease in mean from } B \text { to } C \text {, and } \\
\text { increase from } C \text { to } E \text {. The largest } \\
\text { difference is between } B \text { and } C \text {. }\end{array}$ \\
\hline $\begin{array}{l}\text { Number of striking } \\
\text { platforms }\end{array}$ & Chi-square & $\chi^{2}(12)=18.977, p=.089$ & Not significant & \\
\hline $\begin{array}{l}\text { Perpendicular striking } \\
\text { platforms }\end{array}$ & Chi-square & $\chi^{2}(3)=0.557, p=.906$ & Not significant & \\
\hline Total number of scars & ANOVA & $F(3,53)=6.162, \mathbf{p}=.001$ & $\begin{array}{l}\text { Significant differences } \\
\text { between layers }\end{array}$ & $\begin{array}{l}\text { Large increase from B to D. D and } E \text { are } \\
\text { similar. The largest difference is } \\
\text { between } C \text { and } D \text {. }\end{array}$ \\
\hline Number of flake scars & ANOVA & $F(3,52)=4.409, \mathbf{p}=.008$ & $\begin{array}{l}\text { Significant differences } \\
\text { between layers }\end{array}$ & $\begin{array}{l}\text { Large increase from } B \text { to } D . D \text { and } E \text { are } \\
\text { similar. The largest difference is } \\
\text { between } C \text { and } D \text {. }\end{array}$ \\
\hline Number of blade scars & $\begin{array}{l}\text { Log transformation } \\
+ \text { ANOVA }\end{array}$ & $F(3,42)=0.614, p=.610$ & Not significant & \\
\hline
\end{tabular}




\begin{tabular}{|c|c|c|c|c|}
\hline $\begin{array}{l}\text { Proportion of blade } \\
\text { scars }\end{array}$ & ANOVA & $F(3,52)=0.071, p=.975$ & Not significant & \\
\hline $\begin{array}{l}\text { Number of aberrant } \\
\text { terminations }\end{array}$ & Kruskal-Wallis & $\chi^{2}(3)=4.642, p=.200$ & Not significant & \\
\hline $\begin{array}{l}\text { Percentage cortex } \\
\text { cover }\end{array}$ & ANOVA & $\mathrm{F}(3,50)=2.007, \mathrm{p}=.125$ & Not significant & \\
\hline $\begin{array}{l}\text { Average removal } \\
\text { length }\end{array}$ & ANOVA & $F(3,53)=2.092, p=.112$ & Not significant & \\
\hline $\begin{array}{l}\text { Number of scars per } \\
\mathrm{cm}^{3} \text { core volume }\end{array}$ & $\begin{array}{l}\text { Log transformation } \\
+ \text { ANOVA }^{\mathrm{b}}\end{array}$ & $F(3,53)=3.337, \mathbf{p}=\mathbf{. 0 2 6}$ & $\begin{array}{l}\text { Significant differences } \\
\text { between layers }\end{array}$ & $\begin{array}{l}\text { Increase from } B \text { to } C \text {, slight decrease } \\
\text { from } C \text { to } D \text {, and increase from } D \text { to } E \text {. }\end{array}$ \\
\hline $\begin{array}{l}\text { Average scar length } \\
\text { per } \mathrm{cm}^{3} \text { core volume }\end{array}$ & ANOVA & $F(3,53)=0.750, p=.527$ & Not significant & \\
\hline
\end{tabular}

Table B.5.1: Results of statistical tests of changes over time in core variables at Patne.

a although log transformation of the variable data results in normal distributions, both overall and within each layer (as determined by the Shapiro-Wilk test), Levene's tests indicate that the variances are unequal. As a result of this violation of the assumption of

homoscedasticity, combined with the fact that there are considerable differences in sample sizes between the layers, the non-parametric Kruskal-Wallis test is preferred as it is robust to these violations of the ANOVA test assumptions. The results of the Kruskal-Wallis test are then confirmed with the Welch F-statistic for the log-transformed data, which can be used in cases of unequal variances.

b significance confirmed with non-parametric Kruskal-Wallis test conducted on non-transformed data.

\section{DEBITAGE}

\begin{tabular}{lllll}
\hline Variable & Test used & Test result & Significance (at 0.05 level) & Trend \\
\hline Type & Chi-square & $\chi^{2}(6)=11.859, p=.065$ & Not significant & \\
Condition & Chi-square & $\chi^{2}(15)=141.446, \mathbf{p}<.001$, & $\begin{array}{l}\text { Significant and moderately } \\
\text { strong differences between }\end{array}$ & $\begin{array}{l}\text { Increase over time in the proportion } \\
\text { of broken debitage, especially of } \\
\text { unclear breakages. }\end{array}$
\end{tabular}




\begin{tabular}{|c|c|c|c|c|}
\hline Colour & Chi-square & $\begin{array}{l}\chi^{2}(36)=224.111, \mathbf{p}<.001 \\
\text { Cramér's V = .232 }\end{array}$ & $\begin{array}{l}\text { Significant and moderately } \\
\text { strong differences between } \\
\text { layers }\end{array}$ & $\begin{array}{l}\text { Much higher proportions of blue and } \\
\text { green debitage in B. }\end{array}$ \\
\hline Oriented length & Kruskal-Wallis & $\chi^{2}(3)=106.751, \mathbf{p}<.001$ & $\begin{array}{l}\text { Significant differences } \\
\text { between layers }\end{array}$ & $\begin{array}{l}\text { Decrease from } B \text { to } C \text {, increase from } C \\
\text { to } D \text {, and decrease from } D \text { to } E \text {. The } \\
\text { largest difference is between } C \text { and } D \text {. }\end{array}$ \\
\hline Maximum width & $\begin{array}{l}\text { Log transformation } \\
+ \text { ANOVA a }\end{array}$ & $\begin{array}{l}F(3,762.502)=53.990, \mathbf{p}< \\
.001 b\end{array}$ & $\begin{array}{l}\text { Significant differences } \\
\text { between layers }\end{array}$ & $\begin{array}{l}\text { Decrease from } B \text { to } C \text { and increase } \\
\text { from } C \text { to } E \text {, with most of the increase } \\
\text { between } C \text { and } D \text {. }\end{array}$ \\
\hline Maximum thickness & Kruskal-Wallis & $\chi^{2}(3)=133.788, p<.001$ & $\begin{array}{l}\text { Significant differences } \\
\text { between layers }\end{array}$ & $\begin{array}{l}\text { Decrease from } B \text { to } C \text {, increase from } C \\
\text { to } D \text {, and slight decrease from } D \text { to } E \text {. }\end{array}$ \\
\hline Medial thickness & Kruskal-Wallis & $\chi^{2}(3)=56.164, p<.001$ & $\begin{array}{l}\text { Significant differences } \\
\text { between layers }\end{array}$ & $\begin{array}{l}\text { Decrease from } B \text { to } C \text { and increase } \\
\text { from } C \text { to } E \text {, with most of the increase } \\
\text { between } C \text { and } D \text {. }\end{array}$ \\
\hline $\begin{array}{l}\text { Striking platform } \\
\text { width }\end{array}$ & $\begin{array}{l}\text { Log transformation } \\
+ \text { ANOVA }\end{array}$ & $\begin{array}{l}F(3,338.036)=15.722, \mathbf{p}< \\
. \mathbf{0 0 1}\end{array}$ & $\begin{array}{l}\text { Significant differences } \\
\text { between layers }\end{array}$ & $\begin{array}{l}\text { Decrease from } B \text { to } C \text { and increase } \\
\text { from } C \text { to } E \text {, with most of the increase } \\
\text { between } C \text { and } D \text {. }\end{array}$ \\
\hline $\begin{array}{l}\text { Striking platform } \\
\text { thickness }\end{array}$ & $\begin{array}{l}\text { Log transformation } \\
+ \text { ANOVAa }\end{array}$ & $\mathrm{F}(3,627)=14.057, \mathbf{p}<. \mathbf{0 0 1}$ & $\begin{array}{l}\text { Significant differences } \\
\text { between layers }\end{array}$ & $\begin{array}{l}\text { Decrease from } B \text { to } C \text { and increase } \\
\text { from } C \text { to } E \text {, with most of the increase } \\
\text { between } C \text { and } D \text {. }\end{array}$ \\
\hline Striking platform type & Chi-square & $\chi^{2}(15)=27.961, \mathbf{p}=. \mathbf{0 2 2}$ & Significant but very weak & More cortical striking platforms in B, \\
\hline
\end{tabular}




\begin{tabular}{|c|c|c|c|c|}
\hline & & Cramér's V = .082 & differences between layers & but no overall trend. \\
\hline $\begin{array}{l}\text { Striking platform } \\
\text { lipping }\end{array}$ & Chi-square & $\begin{array}{l}\chi^{2}(6)=22.474, \mathbf{p}=. \mathbf{0 0 1} \\
\text { Cramér's V =.090 }\end{array}$ & $\begin{array}{l}\text { Significant but very weak } \\
\text { differences between layers }\end{array}$ & $\begin{array}{l}\text { Fewer lipped platforms in } \mathrm{D} \text { and, to a } \\
\text { lesser extent, E, but no overall trend. }\end{array}$ \\
\hline $\begin{array}{l}\text { Percentage cortex } \\
\text { cover }\end{array}$ & Kruskal-Wallis & $\chi^{2}(3)=104.615, \mathbf{p}<.001$ & $\begin{array}{l}\text { Significant differences } \\
\text { between layers }\end{array}$ & Consistent increase over time. \\
\hline $\begin{array}{l}\text { Number of dorsal } \\
\text { scars }\end{array}$ & Kruskal-Wallis & $\chi^{2}(3)=21.091, \mathbf{p}<.001$ & $\begin{array}{l}\text { Significant differences } \\
\text { between layers }\end{array}$ & $\begin{array}{l}\text { Decrease from } B \text { to } C \text {, increase from } C \\
\text { to } D \text {, and decrease from } D \text { to } E \text {. The } \\
\text { largest difference is between } C \text { and D. }\end{array}$ \\
\hline $\begin{array}{l}\text { Number of parallel } \\
\text { arrises }\end{array}$ & Kruskal-Wallis & $\chi^{2}(3)=17.109, \mathbf{p}=.001$ & $\begin{array}{l}\text { Significant differences } \\
\text { between layers }\end{array}$ & $\begin{array}{l}\text { Decrease from B to C, increase from C } \\
\text { to } D \text {, and decrease from } D \text { to } E \text {. The } \\
\text { largest difference is between C and D. }\end{array}$ \\
\hline $\begin{array}{l}\text { Number of scars per } \\
\mathrm{cm}^{2} \text { surface area }\end{array}$ & Kruskal-Wallis & $\chi^{2}(3)=107.996, \mathbf{p}<.001$ & $\begin{array}{l}\text { Significant differences } \\
\text { between layers }\end{array}$ & $\begin{array}{l}\text { Increase from } B \text { to } C \text { and decrease } \\
\text { from } C \text { to } D . D \text { and } E \text { are similar. }\end{array}$ \\
\hline $\begin{array}{l}\text { Number of parallel } \\
\text { arrises per } \mathrm{cm}^{2} \text { surface } \\
\text { area }\end{array}$ & Kruskal-Wallis & $\chi^{2}(3)=12.411, \mathbf{p}=.006$ & $\begin{array}{l}\text { Significant differences } \\
\text { between layers }\end{array}$ & $\begin{array}{l}\text { Increase from } B \text { to } C \text { and decrease } \\
\text { from } C \text { to } E \text {. }\end{array}$ \\
\hline Scar orientation & Chi-square & $\begin{array}{l}\chi^{2}(15)=97.766, \mathbf{p}<. \mathbf{0 0 1} \\
\text { Cramér's } V=.153\end{array}$ & $\begin{array}{l}\text { Significant but weak } \\
\text { differences between layers }\end{array}$ & $\begin{array}{l}\text { Increase over time in proportion of } \\
\text { debitage with scars from a single } \\
\text { direction. Decrease over time in } \\
\text { proportion of debitage with scars from } \\
\text { two opposite and four directions. }\end{array}$ \\
\hline Termination type & Chi-square & $\begin{array}{l}\chi^{2}(12)=31.380, \mathbf{p}=.002 \\
\text { Cramér's } V=.087\end{array}$ & $\begin{array}{l}\text { Significant but very weak } \\
\text { differences between layers }\end{array}$ & $\begin{array}{l}\text { Decrease over time in proportion of } \\
\text { hinge and step terminations. }\end{array}$ \\
\hline
\end{tabular}

\footnotetext{
Table B.5.2: Results of statistical tests of changes over time in debitage variables at Patne.

a significance confirmed with non-parametric Kruskal-Wallis test conducted on non-transformed data.

b Welch's F statistic reported due to unequal variances.
} 


\section{MICROLITHS}

\begin{tabular}{|c|c|c|c|c|}
\hline Variable & Test used & Test result & Significance (at 0.05 level) & Trend \\
\hline Blank type & Chi-square & $\chi^{2}(3)=5.309, p=.151$ & Not significant & \\
\hline Condition & $\mathrm{N} / \mathrm{A}^{\mathrm{a}}$ & & & \\
\hline Colour & Chi-square & $\begin{array}{l}\chi^{2}(24)=63.226, \mathbf{p}<. \mathbf{0 0 1} \\
\text { Cramér's } V=.578\end{array}$ & $\begin{array}{l}\text { Significant and very strong } \\
\text { differences between layers }\end{array}$ & $\begin{array}{l}\text { Blue microliths appear only in B, purple } \\
\text { appear only in } C \text { and yellow appear only } \\
\text { in } D \text { and } E \text {, but no overall trend. }\end{array}$ \\
\hline Oriented length & Kruskal-Wallis & $\chi^{2}(3)=6.832, p=.077$ & Not significant & \\
\hline Maximum width & Kruskal-Wallis & $\chi^{2}(3)=6.848, p=.077$ & Not significant & \\
\hline Medial width & Kruskal-Wallis & $\chi^{2}(3)=3.965, p=.265$ & Not significant & \\
\hline Maximum thickness & $\begin{array}{l}\text { Log transformation } \\
+ \text { ANOVA }{ }^{b}\end{array}$ & $F(3,59)=1.506, p=.222$ & Not significant & \\
\hline Medial thickness & Kruskal-Wallis & $\chi^{2}(3)=1.240, p=.744$ & Not significant & \\
\hline Length:width ratio & Kruskal-Wallis c & $\chi^{2}(3)=1.615, p=.656$ & Not significant & \\
\hline Length:thickness ratio & ANOVA & $F(3,59)=0.770, p=.516$ & Not significant & \\
\hline $\begin{array}{l}\text { Striking platform } \\
\text { width }\end{array}$ & $\mathrm{N} / \mathrm{A}^{\mathrm{a}}$ & & & \\
\hline $\begin{array}{l}\text { Striking platform } \\
\text { thickness }\end{array}$ & $\mathrm{N} / \mathrm{A}^{\mathrm{a}}$ & & & \\
\hline Striking platform type & $\mathrm{N} / \mathrm{A}^{\mathrm{a}}$ & & & \\
\hline $\begin{array}{l}\text { Striking platform } \\
\text { lipping }\end{array}$ & $\mathrm{N} / \mathrm{A}^{\mathrm{a}}$ & & & \\
\hline $\begin{array}{l}\text { Percentage cortex } \\
\text { cover }\end{array}$ & Kruskal-Wallis & $\chi^{2}(3)=2.012, p=.570$ & Not significant & \\
\hline $\begin{array}{l}\text { Number of dorsal } \\
\text { scars }\end{array}$ & Kruskal-Wallis & $\chi^{2}(3)=4.868, p=.182$ & Not significant & \\
\hline $\begin{array}{l}\text { Number of parallel } \\
\text { arrises }\end{array}$ & Kruskal-Wallis & $\chi^{2}(3)=5.110, p=.164$ & Not significant & \\
\hline
\end{tabular}


Number of scars per $\mathrm{cm}^{2}$ surface area

\section{Number of parallel Log transformation}

arrises per $\mathrm{cm}^{2}$ surface + ANOVA ${ }^{\mathrm{b}}$

area

Scar orientation $\chi^{2}(3)=7.044, p=.071$

Not significant

$F(3,58)=2.284, \mathbf{p}=.006$

$\chi^{2}(12)=28.042, \mathbf{p}=\mathbf{. 0 0 5}, \quad$ Significant and strong

Cramér's V = .385
Chi-square
Significant differences

between layers

differences between layers
Increase from $B$ to $D$ and slight decrease from $\mathrm{D}$ to $\mathrm{E}$.

Increase over time in the proportion of microliths with scars from a single direction, although there is a slight reversal of this trend between $C$ and $D$. Decrease over time in the proportion of microliths with scars from three directions - these are absent in D and $\mathrm{E}$.

\begin{tabular}{|c|c|c|c|c|}
\hline Termination type & Chi-square & $\chi^{2}(9)=7.721, p=.562$ & Not significant & \\
\hline Coarse typology & Chi-square & $\chi^{2}(15)=16.927, p=.323$ & Not significant & \\
\hline Narrow typology & Chi-square & $\chi^{2}(21)=21.150, p=.450$ & Not significant & \\
\hline $\begin{array}{l}\text { Presence of tangs or } \\
\text { shoulders }\end{array}$ & $\mathrm{N} / \mathrm{A}^{\mathrm{a}}$ & & & \\
\hline $\begin{array}{l}\text { Number of worked } \\
\text { edges }\end{array}$ & Kruskal-Wallis & $\chi^{2}(3)=1.320, p=.724$ & Not significant & \\
\hline Retouch location & Chi-square & $\chi^{2}(12)=9.850, p=.629$ & Not significant & \\
\hline Retouch face & Chi-square & $\begin{array}{l}\chi^{2}(6)=36.898, \mathbf{p}<. \mathbf{0 0 1} \\
\text { Cramér's V =.506 }\end{array}$ & $\begin{array}{l}\text { Significant and very strong } \\
\text { differences between layers }\end{array}$ & $\begin{array}{l}\text { Ventral retouch only appears in B and } \\
\text { edge retouch only appears in D. }\end{array}$ \\
\hline Retouch outline & Chi-square & $\chi^{2}(9)=4.051, p=.908$ & Not significant & \\
\hline Retouch type & Chi-square & $\begin{array}{l}\chi^{2}(15)=33.290, \mathbf{p}=. \mathbf{0 0 4} \\
\text { Cramér's V =.393 }\end{array}$ & $\begin{array}{l}\text { Significant and strong } \\
\text { differences between layers }\end{array}$ & $\begin{array}{l}\text { Backing retouch is less common in B } \\
\text { and more common in } \mathrm{D} \text {, but no overall } \\
\text { trends. }\end{array}$ \\
\hline Retouch length & ANOVA & $F(3,68)=32.532, p=.667$ & Not significant & \\
\hline Index of Invasiveness & Kruskal-Wallis & $\chi^{2}(3)=9.628, p=.022$ & $\begin{array}{l}\text { Significant differences } \\
\text { between layers }\end{array}$ & $\begin{array}{l}\text { Decrease from } B \text { to } C \text {, increase from } C \text { to } \\
D \text { and decrease from } D \text { to } E \text {, but no } \\
\text { overall trend. }\end{array}$ \\
\hline
\end{tabular}


Table B.5.3: Results of statistical tests of changes over time in microlith variables at Patne.

a variable is evident in the sample, but not in every layer and in numbers too small to be statistically viable.

b significance confirmed with non-parametric Kruskal-Wallis test conducted on non-transformed data.

c although the data has a normal distribution (as determined by the Shapiro-Wilk test), Levene's tests indicate that the variances are unequal. As a result of this violation of the assumption of homoscedasticity, combined with the fact that there are considerable differences in sample sizes between the layers, the non-parametric Kruskal-Wallis test is preferred as it is robust to these violations of the ANOVA test assumptions. The results of the Kruskal-Wallis test are then confirmed with the Welch F-statistic for the log-transformed data, which can be used in cases of unequal variances.

d as with c, above, but the data is log-normal.

\section{NON-MICROLITHIC TOOLS}

\begin{tabular}{|c|c|c|c|c|}
\hline Variable & Test used & Test result & Significance (at 0.05 level) & Trend \\
\hline Blank type & Chi-square & $\begin{array}{l}\chi^{2}(3)=10.317, \mathbf{p}=. \mathbf{0 1 6}, \\
\text { Cramér's V =.433 }\end{array}$ & $\begin{array}{l}\text { Significant and very strong } \\
\text { differences between layers }\end{array}$ & $\begin{array}{l}\text { C contains only microliths on flake } \\
\text { blanks. B and E are similar, and D } \\
\text { contains fewer flake blanks. }\end{array}$ \\
\hline Condition & $\mathrm{N} / \mathrm{A}^{\mathrm{a}}$ & & & \\
\hline Colour & Chi-square & $\begin{array}{l}\chi^{2}(30)=55.298, \mathbf{p}=. \mathbf{0 0 3} \\
\text { Cramér's } V=.579\end{array}$ & $\begin{array}{l}\text { Significant and very strong } \\
\text { differences between layers }\end{array}$ & $\begin{array}{l}\text { No overall trends, but slightly different } \\
\text { colour profiles in each layer, with the } \\
\text { least diversity in C. }\end{array}$ \\
\hline Oriented length & $\begin{array}{l}\text { Log transformation } \\
+ \text { ANOVA }\end{array}$ & $F(3,51)=2.290, p=.089$ & Not significant & \\
\hline Maximum width & Kruskal-Wallis & $\chi^{2}(3)=12.403, \mathbf{p}=.006$ & $\begin{array}{l}\text { Significant differences } \\
\text { between layers }\end{array}$ & $\begin{array}{l}\text { Increase from } B \text { to } C \text {, decrease from } C \\
\text { to } D \text {, and increase from } D \text { to } E \text {. The } \\
\text { largest difference is between } C \text { and } D \text {. }\end{array}$ \\
\hline Medial width & Kruskal-Wallis c & $\chi^{2}(3)=12.114, \mathbf{p}=.007$ & Significant differences & Increase from $B$ to $C$, decrease from $C$ \\
\hline
\end{tabular}


between layers

\begin{tabular}{|c|c|c|c|c|}
\hline & & & & \\
\hline Maximum thickness & ANOVA & $F(3,51)=3.930, \mathbf{p}=. \mathbf{0 1 3}$ & $\begin{array}{l}\text { Significant differences } \\
\text { between layers }\end{array}$ & $\begin{array}{l}\mathrm{B} \text { and } \mathrm{C} \text { are similar. Decrease from } \mathrm{C} \text { to } \\
\mathrm{D} \text { and slight increase from } \mathrm{D} \text { to } \mathrm{E} \text {. The } \\
\text { largest difference is between } \mathrm{C} \text { and } \mathrm{D} \text {. }\end{array}$ \\
\hline Medial thickness & Kruskal-Wallis & $\chi^{2}(3)=13.422, \mathbf{p}=. \mathbf{0 0 4}$ & $\begin{array}{l}\text { Significant differences } \\
\text { between layers }\end{array}$ & $\begin{array}{l}\text { Decrease from } B \text { to } D \text { and slight } \\
\text { increase from } D \text { to } E \text {. The largest } \\
\text { difference is between } C \text { and D. }\end{array}$ \\
\hline Length:width ratio & Kruskal-Wallis c & $\chi^{2}(3)=7.552, p=.056$ & Not significant & \\
\hline Length:thickness ratio & Kruskal-Wallis ${ }^{c}$ & $\chi^{2}(3)=6.561, p=.087$ & Not significant & \\
\hline Striking platform width & $\begin{array}{l}\text { Log transformation } \\
+ \text { ANOVA }{ }^{b}\end{array}$ & $F(3,24)=1.675, p=.199$ & Not significant & \\
\hline $\begin{array}{l}\text { Striking platform } \\
\text { thickness }\end{array}$ & $\begin{array}{l}\text { Log transformation } \\
+ \text { ANOVA } \mathrm{b}\end{array}$ & $F(3,51)=1.501, p=.240$ & Not significant & \\
\hline Striking platform type & Chi-square & $\begin{array}{l}\chi^{2}(12)=22.888, \mathbf{p}=. \mathbf{0 2 9} \\
\text { Cramér's V =.372 }\end{array}$ & $\begin{array}{l}\text { Significant and strong } \\
\text { differences between layers }\end{array}$ & $\begin{array}{l}\text { C contains only flat platforms. Cortical } \\
\text { platforms appear only in B. }\end{array}$ \\
\hline $\begin{array}{l}\text { Striking platform } \\
\text { lipping }\end{array}$ & Chi-square & $\chi^{2}(6)=7.236, p=.300$ & Not significant & \\
\hline Percentage cortex cover & Kruskal-Wallis & $\chi^{2}(3)=2.958, p=.398$ & Not significant & \\
\hline Number of dorsal scars & Kruskal-Wallis & $\chi^{2}(3)=7.560, p=.056$ & Not significant & \\
\hline $\begin{array}{l}\text { Number of parallel } \\
\text { arrises }\end{array}$ & Kruskal-Wallis & $\chi^{2}(3)=2.829, p=.419$ & Not significant & \\
\hline $\begin{array}{l}\text { Number of scars per } \\
\mathrm{cm}^{2} \text { surface area }\end{array}$ & $\begin{array}{l}\text { Log transformation } \\
+ \text { ANOVA }{ }^{b}\end{array}$ & $F(3,51)=4.126, \mathbf{p}=\mathbf{. 0 1 1}$ & $\begin{array}{l}\text { Significant differences } \\
\text { between layers }\end{array}$ & $\begin{array}{l}B \text { and } C \text { are similar. Increase from } C \text { to } \\
\text { E, with most of the difference between } \\
\text { C and D. }\end{array}$ \\
\hline $\begin{array}{l}\text { Number of parallel } \\
\text { arrises per } \mathrm{cm}^{2} \text { surface } \\
\text { area }\end{array}$ & $\begin{array}{l}\text { Log transformation } \\
+ \text { ANOVA } \\
\text { Kruskal-Wallis }\end{array}$ & $\mathrm{F}(3,38)=1.804, \mathrm{p}=.163$ & $\begin{array}{l}\text { ANOVA and KW tests give } \\
\text { different results at the } 0.05 \\
\text { level, but the overall result } \\
\text { will be considered to be } \\
\text { significant }\end{array}$ & $\begin{array}{l}\text { Decrease from } B \text { to } C \text {, increase from } C \\
\text { to } D \text {, and decrease from } D \text { to } E \text {. The } \\
\text { largest difference is between } C \text { and } D \text {. }\end{array}$ \\
\hline
\end{tabular}

to $\mathrm{D}$, and increase from $\mathrm{D}$ to $\mathrm{E}$. The o

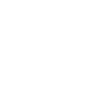




\begin{tabular}{|c|c|c|c|c|}
\hline Scar orientation & Chi-square & $\chi^{2}(15)=16.222, p=.342$ & Not significant & \\
\hline Coarse typology & Chi-square & $\chi^{2}(18)=20.372, p=.312$ & Not significant & \\
\hline Narrow typology & Chi-square & $\chi^{2}(39)=41.669, p=.355$ & Not significant & \\
\hline $\begin{array}{l}\text { Number of worked } \\
\text { edges }\end{array}$ & Kruskal-Wallis & $\chi^{2}(3)=3.729, p=.292$ & Not significant & \\
\hline Retouch face & Chi-square & $\chi^{2}(12)=8.047, p=.781$ & Not significant & \\
\hline Retouch outline & Chi-square & $\begin{array}{l}\chi^{2}(15)=28.163, \mathbf{p}=. \mathbf{0 2 1}, \\
\text { Cramér's V =.364 }\end{array}$ & $\begin{array}{l}\text { Significant and strong } \\
\text { differences between layers }\end{array}$ & $\begin{array}{l}\text { Straight retouched edges are dominant } \\
\text { in all layers except B, where convex } \\
\text { edges are dominant. }\end{array}$ \\
\hline Retouch type & Chi-square & $\chi^{2}(21)=28.690, p=.122$ & Not significant & \\
\hline Retouch length & $\begin{array}{l}\text { Log transformation } \\
+ \text { ANOVA }{ }^{\mathrm{b}}\end{array}$ & $F(3,66)=1.675, p=.181$ & Not significant & \\
\hline \multicolumn{5}{|c|}{$\begin{array}{l}\text { Table B.5.4: Results of statistical tests of changes over time in non-microlithic tool variables at Patne. } \\
\text { a variable is evident in the sample, but not in every layer and in numbers too small to be statistically viable. } \\
\text { b significance confirmed with non-parametric Kruskal-Wallis test conducted on non-transformed data. } \\
\text { c although log transformation of the variable data results in normal distributions, both overall and within each layer (as determined by the } \\
\text { Shapiro-Wilk test), Levene's tests indicate that the variances are unequal. As a result of this violation of the assumption of } \\
\text { homoscedasticity, combined with the fact that there are considerable differences in sample sizes between the layers, the non-parametric } \\
\text { Kruskal-Wallis test is preferred as it is robust to these violations of the ANOVA test assumptions. The results of the Kruskal-Wallis test are } \\
\text { then confirmed with the Welch F-statistic for the log-transformed data, which can be used in cases of unequal variances. }\end{array}$} \\
\hline
\end{tabular}




\section{B.6 JWALAPURAM 9, INDIA}

\section{CORES}

\begin{tabular}{|c|c|c|c|c|}
\hline Variable & Test used & Test result & Significance (at 0.05 level) & Trend \\
\hline Type & Chi-square & $\begin{array}{l}\chi^{2}(40)=86.384, \mathbf{p}<.001 \\
\text { Cramér's } V=.262\end{array}$ & $\begin{array}{l}\text { Significant and moderately } \\
\text { strong differences between } \\
\text { layers }\end{array}$ & $\begin{array}{l}\text { Decrease over time in proportion of bipolar } \\
\text { cores, and increase over time in proportion of } \\
\text { flake cores. Complex picture of changes over } \\
\text { time in other core types, with considerable } \\
\text { fluctuations in proportion of blade cores. See } \\
\text { Figure 6.4.15. }\end{array}$ \\
\hline Raw material & Chi-square & $\begin{array}{l}\chi^{2}(30)=101.953, \mathbf{p} \\
<.001, \text { Cramér's V =.284 }\end{array}$ & $\begin{array}{l}\text { Significant and moderately } \\
\text { strong differences between } \\
\text { layers }\end{array}$ & $\begin{array}{l}\text { Decrease over time in proportion of quartz, } \\
\text { with a complex picture of variations in other } \\
\text { raw material proportions. See Figure 6.4.16. }\end{array}$ \\
\hline Weight & Kruskal-Wallis a & $\chi^{2}(5)=35.487, p<.001$ & $\begin{array}{l}\text { Significant differences } \\
\text { between layers }\end{array}$ & $\begin{array}{l}\text { Increase from context } 6 \text { to } 3 \text {, and decrease } \\
\text { from } 3 \text { to } 1 \text {, although with a temporary slight } \\
\text { reversal in the pattern in } 4 \text {. Most of the } \\
\text { difference is between } 6 \text { and } 5 \text {. }\end{array}$ \\
\hline Longest axis & $\begin{array}{l}\text { Log transformation } \\
+ \text { ANOVA }{ }^{b}\end{array}$ & $F(5,232)=7.389, \mathbf{p}<.001$ & $\begin{array}{l}\text { Significant differences } \\
\text { between layers }\end{array}$ & $\begin{array}{l}\text { Increase from context } 6 \text { to } 4 \text {, and decrease } \\
\text { from } 4 \text { to } 1 \text {, with most of the difference } \\
\text { between } 6 \text { and } 5 \text {. }\end{array}$ \\
\hline $\begin{array}{l}\text { Maximum } \\
\text { thickness }\end{array}$ & $\begin{array}{l}\text { Log transformation } \\
+ \text { ANOVA } \\
\text { Kruskal-Wallis }\end{array}$ & $F(5,139)=2.464, \mathbf{p}=\mathbf{. 0 3 6}$ & $\begin{array}{l}\text { ANOVA and KW tests give } \\
\text { different results at the } 0.05 \\
\text { level, but the overall result } \\
\text { will be considered to be }\end{array}$ & $\begin{array}{l}\text { Considerable increase from context } 6 \text { to } 5 \text {, and } \\
\text { a slight decrease from } 5 \text { to } 1 \text {. }\end{array}$ \\
\hline
\end{tabular}


significant

\begin{tabular}{|c|c|c|c|c|}
\hline Longest face & Kruskal-Wallis & $\chi^{2}(5)=27.999, p<.001$ & $\begin{array}{l}\text { Significant differences } \\
\text { between layers }\end{array}$ & $\begin{array}{l}\text { Increase from context } 6 \text { to } 3 \text {, and decrease } \\
\text { from } 3 \text { to } 1 \text {, although with a temporary slight } \\
\text { reversal in the pattern in } 4 \text {. Most of the } \\
\text { difference is between } 6 \text { and } 5 \text {. }\end{array}$ \\
\hline $\begin{array}{l}\text { Striking platform } \\
\text { width }\end{array}$ & Kruskal-Wallis & $\chi^{2}(5)=23.077, p<.001$ & $\begin{array}{l}\text { Significant differences } \\
\text { between layers }\end{array}$ & $\begin{array}{l}\text { Increase from context } 6 \text { to } 3 \text {, and decrease } \\
\text { from } 3 \text { to } 1 \text {, although with temporary slight } \\
\text { reversals in the pattern in } 2 \text { and } 4 \text {. Most of the } \\
\text { difference is between } 6 \text { and } 5 \text {. }\end{array}$ \\
\hline $\begin{array}{l}\text { Striking platform } \\
\text { thickness }\end{array}$ & Kruskal-Wallis & $\chi^{2}(5)=20.545, p=.001$ & $\begin{array}{l}\text { Significant differences } \\
\text { between layers }\end{array}$ & $\begin{array}{l}\text { Increase from context } 6 \text { to } 3 \text {, and decrease } \\
\text { from } 3 \text { to } 1 \text {, although with a temporary slight } \\
\text { reversal in the pattern in } 2 \text {. Most of the } \\
\text { difference is between } 6 \text { and } 5 \text {. }\end{array}$ \\
\hline $\begin{array}{l}\text { Striking platform } \\
\text { type }\end{array}$ & Chi-square & $\begin{array}{l}\chi^{2}(20)=41.915, \mathbf{p}=. \mathbf{0 0 3} \\
\text { Cramér's V = .204 }\end{array}$ & $\begin{array}{l}\text { Significant and moderately } \\
\text { strong differences between } \\
\text { layers }\end{array}$ & $\begin{array}{l}\text { Complex pictures of changes over time, with } \\
\text { no overall trends. See Figure 6.4.17. }\end{array}$ \\
\hline $\begin{array}{l}\text { Total number of } \\
\text { scars }\end{array}$ & Kruskal-Wallis & $\chi^{2}(5)=12.636, p=.027$ & $\begin{array}{l}\text { Significant differences } \\
\text { between layers }\end{array}$ & $\begin{array}{l}\text { Considerable increase from context } 6 \text { to } 5 \text {, } \\
\text { considerable decrease from } 5 \text { to } 4 \text {, increase } \\
\text { from } 4 \text { to } 3 \text {, and a slight decrease from } 3 \text { to } 1 \text {. }\end{array}$ \\
\hline $\begin{array}{l}\text { Number of flake } \\
\text { scars }\end{array}$ & Kruskal-Wallis & $\chi^{2}(5)=22.153, p<.001$ & $\begin{array}{l}\text { Significant differences } \\
\text { between layers }\end{array}$ & $\begin{array}{l}\text { Considerable increase from context } 6 \text { to } 5 \text {, } \\
\text { considerable decrease from } 5 \text { to } 4 \text {, } \\
\text { considerable increase from } 4 \text { to } 3 \text {, and a slight } \\
\text { decrease from } 3 \text { to } 1 \text {. }\end{array}$ \\
\hline $\begin{array}{l}\text { Number of blade } \\
\text { scars }\end{array}$ & Kruskal-Wallis & $\chi^{2}(5)=17.436, p=.004$ & $\begin{array}{l}\text { Significant differences } \\
\text { between layers }\end{array}$ & $\begin{array}{l}\text { Decrease from context } 6 \text { to } 5 \text {, considerable } \\
\text { increase from } 5 \text { to } 4 \text {, considerable decrease } \\
\text { from } 4 \text { to } 3 \text {, and a slight increase from } 3 \text { to } 1 \text {. }\end{array}$ \\
\hline $\begin{array}{l}\text { Proportion of } \\
\text { blade scars }\end{array}$ & Kruskal-Wallis & $\chi^{2}(5)=22.605, p<.001$ & $\begin{array}{l}\text { Significant differences } \\
\text { between layers }\end{array}$ & $\begin{array}{l}\text { Decrease from context } 6 \text { to } 5 \text {, considerable } \\
\text { increase from } 5 \text { to } 4 \text {, considerable decrease } \\
\text { from } 4 \text { to } 3 \text {, and a slight increase from } 3 \text { to } 1 \text {. }\end{array}$ \\
\hline $\begin{array}{l}\text { Number of } \\
\text { aberrant }\end{array}$ & Kruskal-Wallis & $\chi^{2}(5)=21.662, p=.001$ & $\begin{array}{l}\text { Significant differences } \\
\text { between layers }\end{array}$ & $\begin{array}{l}\text { Considerable increase from context } 6 \text { to } 5 \text {, } \\
\text { considerably decrease from } 5 \text { to } 4 \text {, slight }\end{array}$ \\
\hline
\end{tabular}




\begin{tabular}{|c|c|c|c|c|}
\hline terminations & & & & $\begin{array}{l}\text { increase from } 4 \text { to } 2 \text { and a slight decrease } \\
\text { from } 2 \text { to } 1 \text {. }\end{array}$ \\
\hline $\begin{array}{l}\text { Percentage } \\
\text { cortex cover }\end{array}$ & Kruskal-Wallis & $\chi^{2}(5)=10.988, p=.052$ & Not significant & \\
\hline $\begin{array}{l}\text { Average removal } \\
\text { length }\end{array}$ & $\begin{array}{l}\text { Log transformation } \\
+ \text { ANOVA }\end{array}$ & $\mathrm{F}(5,241)=6.396, \mathbf{p}<. \mathbf{0 0 1}$ & $\begin{array}{l}\text { Significant differences } \\
\text { between layers }\end{array}$ & $\begin{array}{l}\text { Increase from context } 6 \text { to } 3 \text {, and decrease } \\
\text { from } 3 \text { to } 1 \text {. }\end{array}$ \\
\hline $\begin{array}{l}\text { Number of scars } \\
\text { per } \mathrm{cm}^{3} \text { core } \\
\text { volume }\end{array}$ & $\begin{array}{l}\text { Log transformation } \\
+ \text { ANOVA }{ }^{b}\end{array}$ & $\mathrm{~F}(5,138)=4.973, \mathbf{p}<.001$ & $\begin{array}{l}\text { Significant differences } \\
\text { between layers }\end{array}$ & $\begin{array}{l}\text { Considerably higher in context } 6 \text {, but } \\
\text { relatively similar between } 5-1 \text {. }\end{array}$ \\
\hline
\end{tabular}

\section{DEBITAGE}

\begin{tabular}{|c|c|c|c|c|}
\hline Variable & Test used & Test result & Significance (at 0.05 level) & Trend \\
\hline Type & Chi-square & $\begin{array}{l}\chi^{2}(5)=56.434, \mathbf{p}<. \mathbf{0 0 1} \\
\text { Cramér's V }=.144\end{array}$ & $\begin{array}{l}\text { Significant but weak } \\
\text { differences between layers }\end{array}$ & $\begin{array}{l}\text { Consistent decrease over time in proportion of } \\
\text { blades, with a slight reversal in context } 2 \text {. }\end{array}$ \\
\hline Raw material & Chi-square & $\begin{array}{l}\chi^{2}(35)=173.146, \mathbf{p}< \\
. \mathbf{0 0 1}, \text { Cramér's V =.113 }\end{array}$ & $\begin{array}{l}\text { Significant but weak } \\
\text { differences between layers }\end{array}$ & $\begin{array}{l}\text { Consistent increase over time in the proportion } \\
\text { of CCS. Other small inconsistent variations in raw } \\
\text { material proportions can be seen in Figure } \\
6.4 .18 \text {. }\end{array}$ \\
\hline Weight & Kruskal-Wallis & $\chi^{2}(5)=69.632, p<.001$ & Significant differences & Increase from context 6 to 3 , decrease from 3 to \\
\hline
\end{tabular}




\begin{tabular}{|c|c|c|c|c|}
\hline & & & between layers & 2 , and increase from 2 to 1. \\
\hline $\begin{array}{l}\text { Longest } \\
\text { dimension }\end{array}$ & Kruskal-Wallis & $\chi^{2}(5)=16.155, \mathbf{p}=.006$ & $\begin{array}{l}\text { Significant differences } \\
\text { between layers }\end{array}$ & $\begin{array}{l}\text { Increase from context } 6 \text { to } 3 \text {, slight decrease from } \\
3 \text { to } 2 \text {, and a considerable increase from } 2 \text { to } 1 \text {. }\end{array}$ \\
\hline Oriented length & Kruskal-Wallis & $\chi^{2}(5)=35.683, p<.001$ & $\begin{array}{l}\text { Significant differences } \\
\text { between layers }\end{array}$ & $\begin{array}{l}\text { Considerable increase from context } 6 \text { to } 4 \text {, and } \\
\text { decrease from } 4 \text { to } 1 \text {. Most of the difference is } \\
\text { between } 6 \text { and } 5 \text {. }\end{array}$ \\
\hline Medial width & Kruskal-Wallis & $\chi^{2}(5)=94.979, \mathbf{p}<. \mathbf{0 0 1}$ & $\begin{array}{l}\text { Significant differences } \\
\text { between layers }\end{array}$ & $\begin{array}{l}\text { Considerable increase from context } 6 \text { to } 3 \text {, } \\
\text { decrease from } 3 \text { to } 2 \text {, and increase from } 2 \text { to } 1 \text {. }\end{array}$ \\
\hline Medial thickness & Kruskal-Wallis & $\chi^{2}(5)=34.008, p<.001$ & $\begin{array}{l}\text { Significant differences } \\
\text { between layers }\end{array}$ & $\begin{array}{l}\text { Increase from context } 6 \text { to } 5 \text {, slight decrease from } \\
5 \text { to } 4 \text {, increase from } 4 \text { to } 3 \text {, decrease from } 3 \text { to } 2 \\
\text { and increase from } 2 \text { to } 1 \text {. Generally increasing } \\
\text { over time. }\end{array}$ \\
\hline $\begin{array}{l}\text { Length:width } \\
\text { ratio a }^{\text {a }}\end{array}$ & $\begin{array}{l}\text { Log } \\
\text { transformation } \\
+ \text { ANOVA }^{b}\end{array}$ & $\begin{array}{l}\mathrm{F}(5,2700)=15.165, \mathbf{p}< \\
. \mathbf{0 0 1}\end{array}$ & $\begin{array}{l}\text { Significant differences } \\
\text { between layers }\end{array}$ & $\begin{array}{l}\text { Considerable decrease from context } 6 \text { to } 3 \text {, } \\
\text { increase from } 3 \text { to } 2 \text { and decrease from } 2 \text { to } 1 \text {. }\end{array}$ \\
\hline $\begin{array}{l}\text { Length:thickness } \\
\text { ratio a }^{\text {a }}\end{array}$ & Kruskal-Wallis & $\chi^{2}(5)=49.208, p<.001$ & $\begin{array}{l}\text { Significant differences } \\
\text { between layers }\end{array}$ & $\begin{array}{l}\text { Decrease from context } 6 \text { to } 5 \text {, increase from } 5 \text { to } \\
4 \text {, decrease from } 4 \text { to } 3 \text {, increase from } 3 \text { to } 2 \text { and } \\
\text { increase from } 2 \text { to } 1 \text {. Generally decreasing over } \\
\text { time. }\end{array}$ \\
\hline $\begin{array}{l}\text { Striking platform } \\
\text { width }\end{array}$ & Kruskal-Wallis & $\chi^{2}(5)=75.235, \mathbf{p}<. \mathbf{0 0 1}$ & $\begin{array}{l}\text { Significant differences } \\
\text { between layers }\end{array}$ & $\begin{array}{l}\text { Considerable increase from context } 6 \text { to } 3 \text {, } \\
\text { decrease from } 3 \text { to } 2 \text { and increase from } 2 \text { to } 1 \text {. }\end{array}$ \\
\hline $\begin{array}{l}\text { Striking platform } \\
\text { thickness }\end{array}$ & Kruskal-Wallis & $\chi^{2}(5)=55.407, p<.001$ & $\begin{array}{l}\text { Significant differences } \\
\text { between layers }\end{array}$ & $\begin{array}{l}\text { Considerable increase from context } 6 \text { to } 3 \text {, } \\
\text { considerable decrease from } 3 \text { to } 2 \text { and } \\
\text { considerable increase from } 2 \text { to } 1 \text {. }\end{array}$ \\
\hline $\begin{array}{l}\text { Striking platform } \\
\text { type }\end{array}$ & Chi-square & $\begin{array}{l}\chi^{2}(30)=204.462, \mathbf{p}< \\
. \mathbf{0 0 1}, \text { Cramér's V =.123 }\end{array}$ & $\begin{array}{l}\text { Significant but weak } \\
\text { differences between layers }\end{array}$ & $\begin{array}{l}\text { Consistent increase over time in proportion of } \\
\text { flat striking platforms, with a slight reversal in } \\
\text { context } 2 \text {. Some other small variations between } \\
\text { contexts, but no other overall trends. }\end{array}$ \\
\hline $\begin{array}{l}\text { Percentage cortex } \\
\text { cover }\end{array}$ & Kruskal-Wallis & $\chi^{2}(5)=45.388, p<.001$ & $\begin{array}{l}\text { Significant differences } \\
\text { between layers }\end{array}$ & $\begin{array}{l}\text { Much lower mean cortex cover in context } 6 \text {. } \\
\text { Oscillating variations between } 5 \text { and } 1 \text { but no }\end{array}$ \\
\hline
\end{tabular}




\begin{tabular}{|c|c|c|c|c|}
\hline & & & & overall trend. \\
\hline Termination type & Chi-square & $\begin{array}{l}\chi^{2}(25)=103.761, \mathbf{p}< \\
. \mathbf{0 0 1}, \text { Cramér's V =.088 }\end{array}$ & $\begin{array}{l}\text { Significant but very weak } \\
\text { differences between layers }\end{array}$ & $\begin{array}{l}\text { Slight inconsistent increase over time in } \\
\text { proportion of feather terminations. More } \\
\text { crushed terminations in contexts } 6 \text { and } 5 \text {, and } \\
\text { more plunging terminations in context } 3 \text {. }\end{array}$ \\
\hline $\begin{array}{l}\text { Evidence of core } \\
\text { maintenance }\end{array}$ & Chi-square & $\chi^{2}(10)=8.224, p=.607$ & Not significant & \\
\hline $\begin{array}{l}\text { Other } \\
\text { classification }\end{array}$ & $\mathrm{N} / \mathrm{A}^{\mathrm{c}}$ & & & \\
\hline
\end{tabular}

Table B.6.2: Results of statistical tests of changes over time in debitage variables at Jwalapuram.

a calculated using medial width or thickness measurements.

b significance confirmed with non-parametric Kruskal-Wallis test conducted on non-transformed data.

c variable is evident in the sample, but not in every layer and in numbers too small to be statistically viable.

\section{MICROLITHS}

\begin{tabular}{lllll}
\hline Variable & Test used & Test result & Significance (at 0.05 level) & Trend \\
\hline Blank type & Chi-square & $\chi^{2}(5)=20.282, \mathbf{p}=.001$, & $\begin{array}{l}\text { Significant and moderately } \\
\text { strong differences between } \\
\text { layers }\end{array}$ & $\begin{array}{l}\text { More blade blanks in contexts 6, 5 and 4, } \\
\text { and fewer in 3, } 2 \text { and } 1 .\end{array}$
\end{tabular}




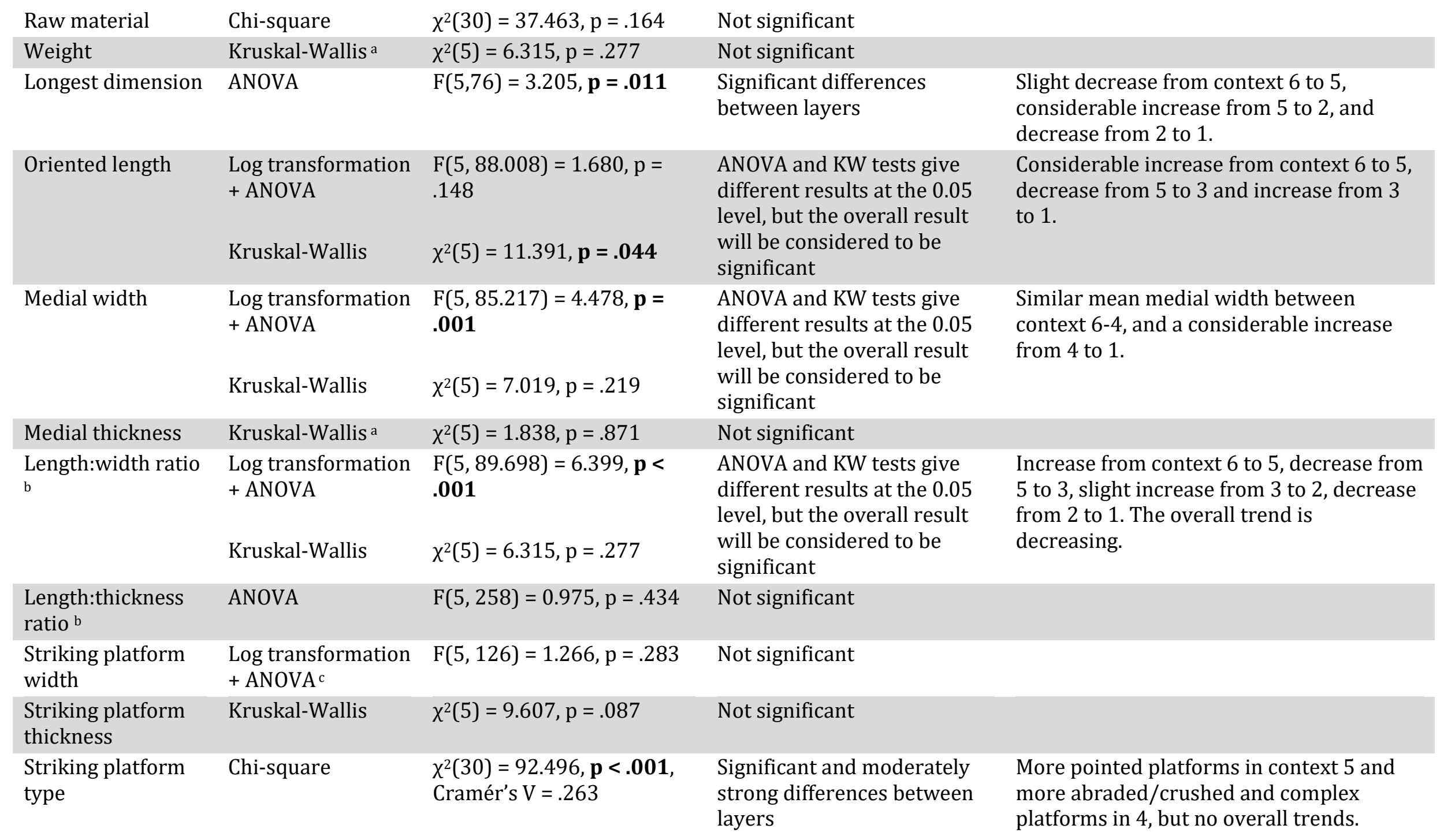




\begin{tabular}{|c|c|c|c|c|}
\hline $\begin{array}{l}\text { Percentage cortex } \\
\text { cover }\end{array}$ & Kruskal-Wallis & $\chi^{2}(5)=11.786, \mathbf{p}=.038$ & $\begin{array}{l}\text { Significant differences } \\
\text { between layers }\end{array}$ & $\begin{array}{l}\text { Slight increase from context } 6 \text { to } 5 \text {, slight } \\
\text { decrease from } 5 \text { to } 4 \text {, considerable increase } \\
\text { from } 4 \text { to } 3 \text {, and decrease from } 3 \text { to } 1 \text {. }\end{array}$ \\
\hline Scar orientation & Chi-square & $\begin{array}{l}\chi^{2}(25)=50.921, \mathbf{p}=\mathbf{. 0 0 2} \\
\text { Cramér's V =.195 }\end{array}$ & $\begin{array}{l}\text { Significant but weak } \\
\text { differences between layers }\end{array}$ & $\begin{array}{l}\text { More microliths with scars originating } \\
\text { from three directions in context } 6 \text {, and only } \\
\text { those from one direction in } 3 \text {, but no } \\
\text { overall trends. }\end{array}$ \\
\hline Termination type & Chi-square & $\begin{array}{l}\chi^{2}(15)=40.866, \mathbf{p}<.001 \\
\text { Cramér's } V=.226\end{array}$ & $\begin{array}{l}\text { Significant and moderately } \\
\text { strong differences between } \\
\text { layers }\end{array}$ & $\begin{array}{l}\text { Only feather terminations are found in } \\
\text { contexts } 6,4 \text { and } 3 \text {, with small numbers of } \\
\text { hinge and step terminations found in } 5,2 \\
\text { and } 1 \text {, but no overall trends. }\end{array}$ \\
\hline $\begin{array}{l}\text { Evidence of core } \\
\text { maintenance }\end{array}$ & $\mathrm{N} / \mathrm{A}^{\mathrm{d}}$ & & & \\
\hline Coarse typology & Chi-square & $\begin{array}{l}\chi^{2}(25)=76.800, \mathbf{p}<. \mathbf{0 0 1}, \\
\text { Cramér's } V=.240\end{array}$ & $\begin{array}{l}\text { Significant and moderately } \\
\text { strong differences between } \\
\text { layers }\end{array}$ & \multirow{2}{*}{$\begin{array}{l}\text { Complex picture of changes in microlith } \\
\text { typology over time. Overall, backed } \\
\text { geometric microliths are much more } \\
\text { common in contexts } 6-4 \text {, as are to a lesser } \\
\text { extent backed non-geometric microliths. } \\
\text { See Figure 6.4.19. }\end{array}$} \\
\hline Narrow typology & Chi-square & $\begin{array}{l}\chi^{2}(90)=148.885, \mathbf{p}<.001 \\
\text { Cramér's V = .334 }\end{array}$ & $\begin{array}{l}\text { Significant and strong } \\
\text { differences between layers }\end{array}$ & \\
\hline Retouch type & Chi-square & $\begin{array}{l}\chi^{2}(20)=71.191, \mathbf{p}<. \mathbf{0 0 1} \\
\text { Cramér's V }=.240\end{array}$ & $\begin{array}{l}\text { Significant and moderately } \\
\text { strong differences between } \\
\text { layers }\end{array}$ & $\begin{array}{l}\text { More burinations in context } 3 \text { and more } \\
\text { notches in } 1 \text {. Adjacent notches only appear } \\
\text { in } 2 \text {, and stepped retouch is absent from } 4 \\
\text { and } 3 \text {. }\end{array}$ \\
\hline Retouch angle & Kruskal-Wallis & $\chi^{2}(5)=9.226, p=.099$ & Not significant & \\
\hline Retouch depth & Kruskal-Wallis & $\chi^{2}(5)=19.148, \mathbf{p}=. \mathbf{0 0 2}$ & $\begin{array}{l}\text { Significant differences } \\
\text { between layers }\end{array}$ & $\begin{array}{l}\text { Similar mean retouch depth between } \\
\text { contexts } 6-4 \text {, considerable increase from } 4 \\
\text { to } 3 \text {, considerable decrease from } 3 \text { to } 2 \text { and }\end{array}$ \\
\hline
\end{tabular}


slight increase from 2 to 1 .

\begin{tabular}{lllll} 
Retouch length & Kruskal-Wallis & $\chi^{2}(5)=27.720, \mathbf{p}<.001$ & $\begin{array}{l}\text { Significant differences } \\
\text { between layers }\end{array}$ & $\begin{array}{l}\text { Increase from } 6 \text { to 5, decrease from } 5 \text { to } 2 \\
\text { and increase from } 2 \text { to } 1 .\end{array}$ \\
$\begin{array}{llll}\text { Index of } \\
\text { Invasiveness }\end{array}$ & $\begin{array}{l}\text { Log transformation } \\
+ \text { ANOVAc }\end{array}$ & $\begin{array}{l}\mathrm{F}(5,261)=14.116, \mathbf{p}< \\
\mathbf{. 0 0 1}\end{array}$ & $\begin{array}{l}\text { Significant differences } \\
\text { between layers }\end{array}$ & $\begin{array}{l}\text { Significant increase from context } 6 \text { to 5, } \\
\text { significant decrease from 5 to 2, and slight } \\
\text { increase from 2 to 1. }\end{array}$ \\
\hline
\end{tabular}

Table B.6.3: Results of statistical tests of changes over time in microlith variables at Jwalapuram.

a although the data has a normal or log normal distribution (as determined by the Shapiro-Wilk test), Levene's tests indicate that the variances are unequal. As a result of this violation of the assumption of homoscedasticity, combined with the fact that there are considerable differences in sample sizes between the layers, the non-parametric Kruskal-Wallis test is preferred as it is robust to these violations of the ANOVA test assumptions. The results of the Kruskal-Wallis test are then confirmed with the Welch F-statistic for the logtransformed data, which can be used in cases of unequal variances.

b calculated using medial width or thickness measurements.

c significance confirmed with non-parametric Kruskal-Wallis test conducted on non-transformed data.

${ }^{d}$ variable is evident in the sample, but not in every layer and in numbers too small to be statistically viable. 


\section{Appendix C: Notes on comparisons with the Indian datasets}

\section{C.1 Patne, INDiA}

\section{CORES}

The following variables were not included in James' (2011) dataset or did not have analogous variables: raw material, weight, longest dimension, striking platform width, striking platform thickness and striking platform type. The lengths of the initial, final, biggest and smallest removal scars were recorded, and so average length is calculated but the standard deviation of removal lengths and the ratio of total scar length to core volume cannot be considered. It should also be noted that James adopted a random sampling strategy for the core assemblage, rather than measuring all cores as at the other sites analysed here. Additionally, James used a unique raw material classificatory scheme, which does not translate into recognised geological types.

\section{DEBITAGE}

The following variables were not included in James' (2011) dataset or did not have analogous variables: raw material, weight, longest dimension, one and three quarter widths, curvature, evidence of bipolar percussion, and evidence of core maintenance. Other variables based on these measurements are therefore also excluded, for example the calculation of width standard deviation. 
TOOLS

In contrast to the other sites analysed, both the microlithic and the non-microlithic tool assemblages analysed at Patne are samples (rather than whole populations). In common with the debitage sample, the following variables were not recorded by James (2011): raw material, weight, longest dimension, one and three quarter widths, curvature, evidence of bipolar percussion, and evidence of core maintenance. Additionally, retouch angle, retouch depth, and variables related to tangs and shoulders were not recorded. Whether or not microlith forms were retouched into points is also not recorded.

\section{C.2 JWALAPURAM 9, INDIA}

\section{CORES}

The following variables were not included in Clarkson's et al.'s (2009) dataset: longest dimension, number of striking platforms and colour. Although the absence of most of these variables has only a minimum impact on the usefulness of the dataset, the absence of the number of striking platforms means that some cores are classified simply as blade or flake cores.

Some variables were not recorded for all cores: longest axis, maximum thickness, and striking platform width, thickness and type. A few differences exist in the recording of some other variables. Double and complex striking platforms were recorded as a single category. The number of aberrant terminations includes all non-feather terminations. And the number of scars recorded refers only to scars 
which are over $15 \mathrm{~mm}$ long (Petraglia et al. 2009). Finally, only the lengths of the final four removal scars are recorded. Although the average removal length can still be considered, this measurement should be treated as a minimum as final removal scars should be expected to be smaller than the average length of removal scars on the core. Additionally, the standard deviation of removal lengths and the ratios of average and total scar length to core volume are not considered.

\section{DEBITAGE}

All whole lithics from Jwalapuram were measured by Clarkson et al. (2009). Only those longer than $10 \mathrm{~mm}$ from late Pleistocene Strata C, D and E are considered here. The following variables were not included in Clarkson's dataset: colour, maximum width, one and three quarter widths, maximum thickness, presence of striking platform lipping, curvature, and number of parallel arrises. Anomalously large dorsal scar numbers precluded the inclusion of this variable. No single size attribute was recorded on every artefact.

The main problem faced in the recoding of Clarkson's dataset is that flakes and blades were either not recorded or not recorded consistently. For the sake of consistency and to facilitate comparisons with the other sites analysed, the classic definition of blades as having a length:width ratio above 2 was applied where this was able to be calculated (using oriented length and medial width). All other debitage was classified as flakes. Flake-blades could not be calculated as a category due to the fact that numbers of parallel arrises were not recorded. 
TOOLS

Unlike at the other sites analysed, both the microlithic and the non-microlithic tool assemblages measured at Jwalapuram are entire populations of whole tools. In common with the debitage sample, the following variables were not recorded by Clarkson et al. (2009): colour, maximum width, one and three quarter widths, maximum thickness, presence of striking platform lipping, curvature, and number of parallel arrises. TCSA measurements could not be calculated due to the absence of maximum thickness measurements. For the same reasons outlined above, dorsal scar counts and densities are also not considered. The same caveats apply with regards to the fact that no single size attribute was recorded on every artefact.

Additionally, the following tool-specific variables were not recorded or were not recorded in a comparable manner: number of worked edges, retouch location, retouch outline, and variables related to tangs and shoulders. As with debitage, the definition of blade blanks as having length:width ratios above 2 has been applied here. The only retouch type recorded was stepped retouch, although backing was also noted as part of typological designations (i.e. the presence of any backing on a tool was noted, but not for individual retouched sections). Retouch measurements were recorded at three locations along retouched margins, and so averages were taken. With regards to typological designations, points are not noted and geometric forms (e.g. crescent, trapeze) are not specified. 